


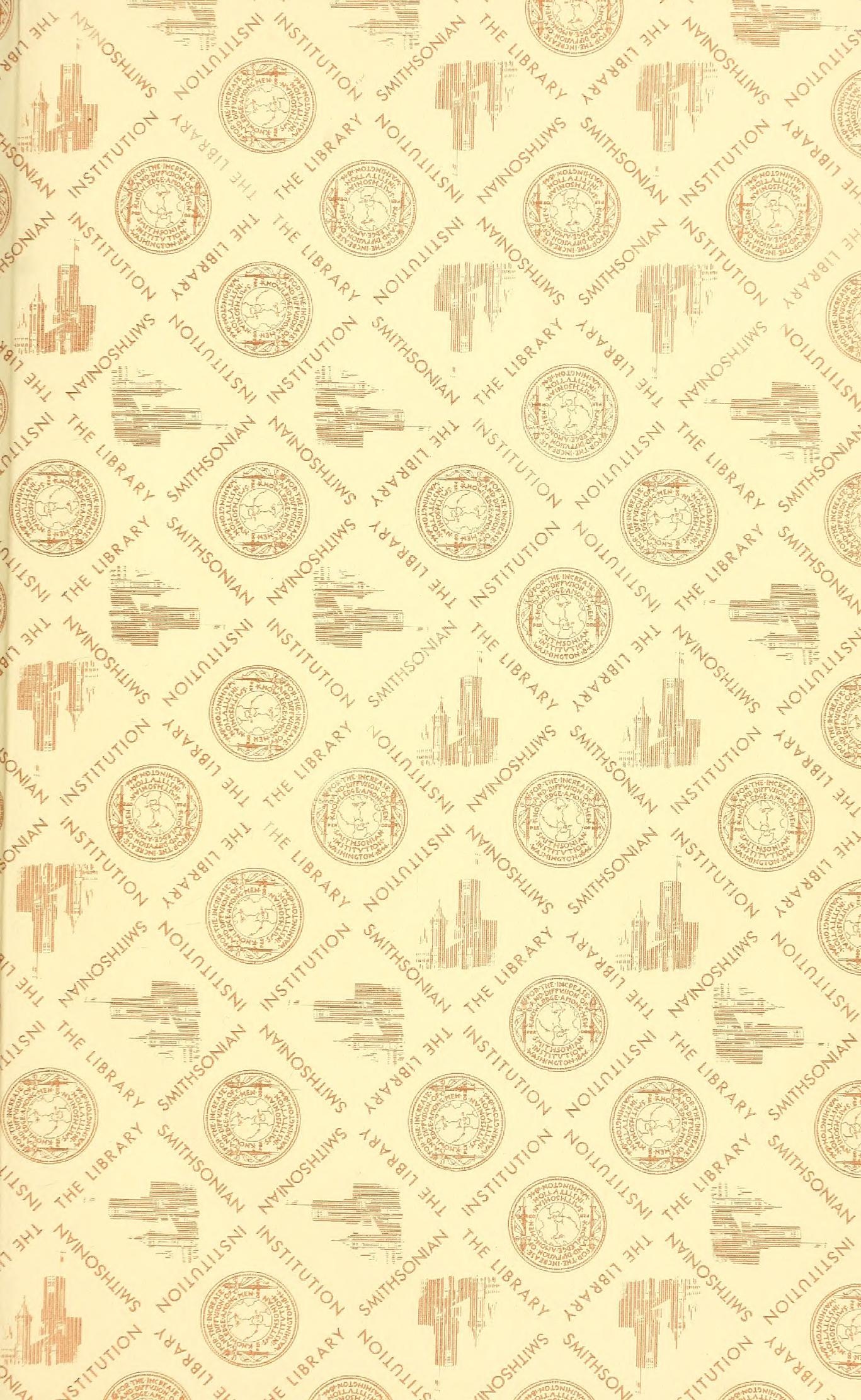





Bailey, F. M., Comprehensive catalogue of Queensland plants.

cf. E. D. Merrill, J. Bot. 58 (1920) 200; C. T.

White, Proc. R. Soc. Queensl. 61 (1950) 112; H. S. Marshall, Kew Bull. (1953) 279.

According to WhITE the first few copies were received from the printer before Christmas 1912, but the general issue was not made until early 1913, according to Merrill March 1913. 

Bailey, F.M.

Died is Buiboul gou 26, 19/5 
PLATE I.

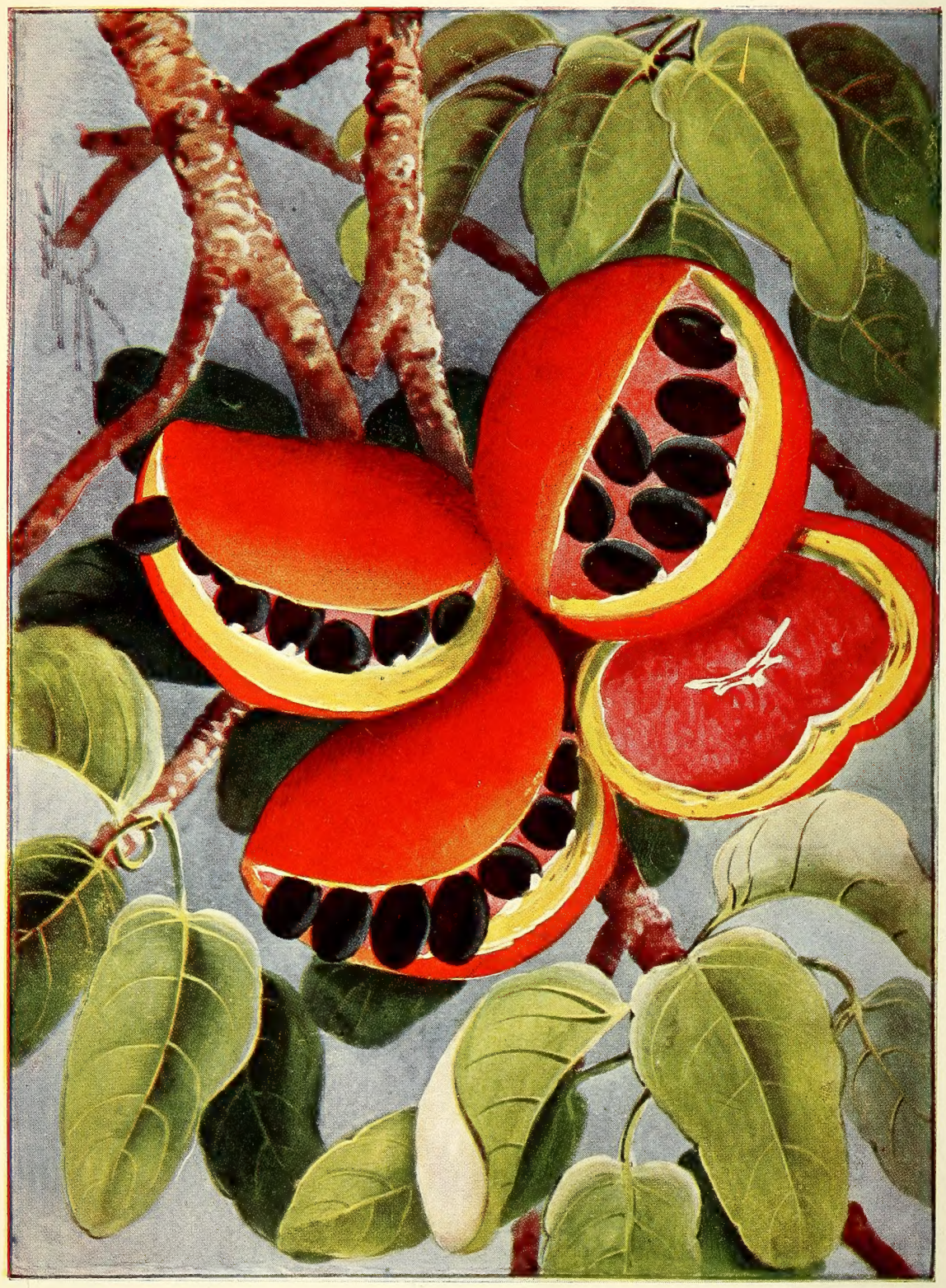

Sterculia Quadrifida, R. Br. 


\section{COMPREHENSIVE CATALOGUE}

\section{$\mathrm{OF}$ \\ QUEENSLAND PLANTS}

Both Indigenous and Naturalised.

To which are added, where known, the aboriginal and other vernacular names; with numerous illustrations, and copious notes on the properties, features \&c., of the plants.

BY

\section{F. MANSON BAILEY, C.M.G., F.L.S.}

Colonial Botanist, Queensland.

As long as plants are cultivated, and it is necessary to speak of them and write of them, even so long will some form of classificaticn and nomenclature be necessary.-M. T. Masters, F.R.S.

Published under the Authority of the Queensland Government.

A. J. CUMMING, GOVERNMENT PRINTER, WILLIAM STREET, BRISBANE. 


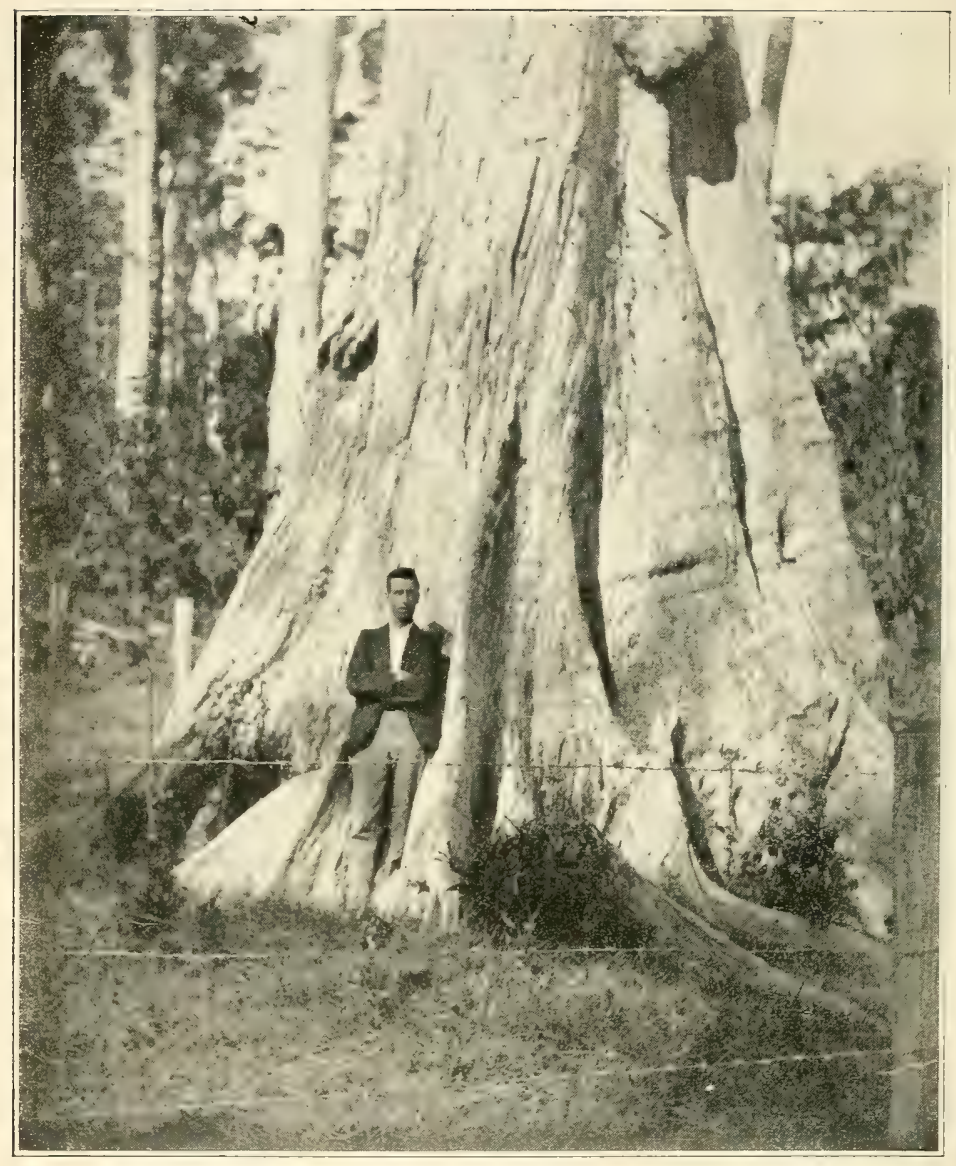

Photo. by W. D. Francis.

Eugenia Francisir, Bail.

Base of trunk. The figure in the above photograph was $5 \mathrm{ft}$. $10 \mathrm{in}$. in height. 


\section{PREFATORY AND OTHER NOTES.}

THE present publication is a second edition of the "Catalogue of the Indigenous and Naturalised Plants of Queensland," which was published in June, I8go.

The system followed is, for the Phanerogams and higher vascular Cryptogams, Bentham and Hooker's Genera Plantarum and the Flora Australiensis; for the other Cryptogamic Orders, various authors of high reputation.

As one may easily suppose, a vast number of the State's plants have been brought to our knowledge since then. These will be found recorded, and in most instances with descriptions, in my various publications of later years in the "Queensland Agricultural Journal." This has been of great advantage to all persons wishing to keep up a knowledge of the State's Flora,-for, within a few weeks of a plant's discovery, a description of it has been given in the pages of that journal. However, this Catalogue, in which may be consulted all the known plants to a given date, will always be of very great value, not only to Queenslanders but to botanists generally.

With the idea of extending its usefulness, great changes have been made, both in the arrangement and contents of the work. The figures have been given at the special desire of Dr. the Hon. Wm. Kidston, who considered they would be helpful to persons possessing but a slight knowledge of botany.

In all plants marked as containing "Prussic Acid," a Hydrocyanic (Prussic) Acid yielding glucoside had been found by Mr. J. C. Brünnich, F.C.S., Queensland Agricultural Chemist, or other chemists. Where a plant is marked "Poisonous" it must be understood that it has been suspected of being poisonous to stock; but in many cases this has not been satisfactorily proved. 


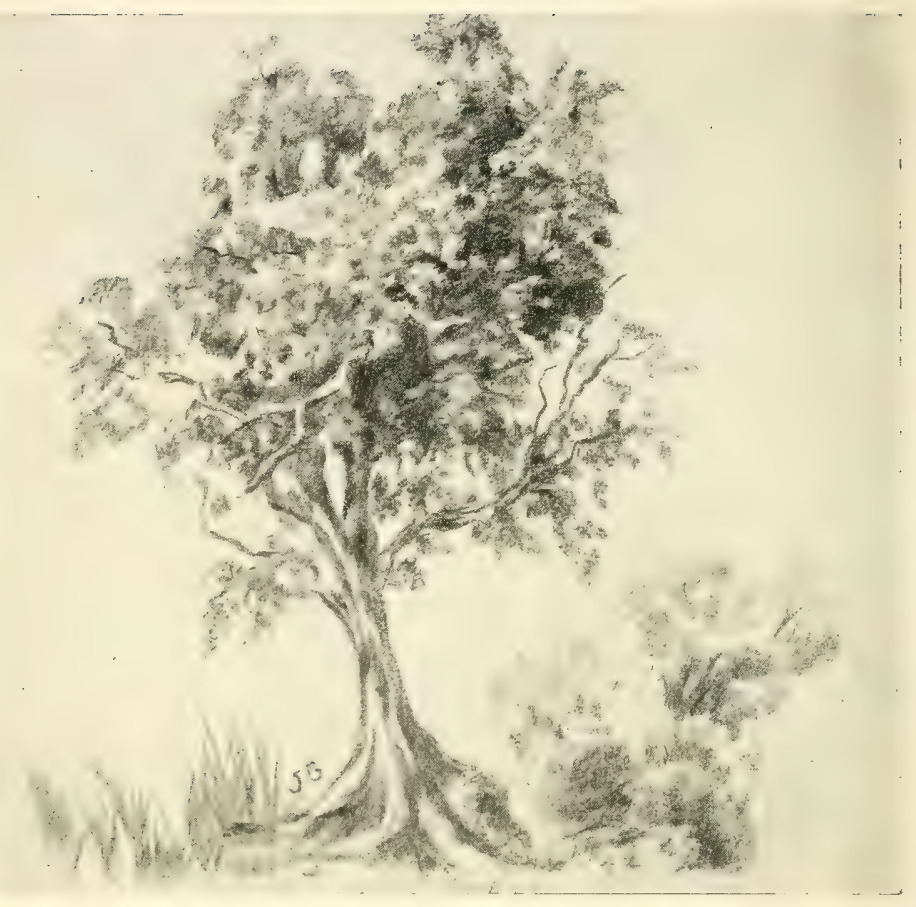

Bauhinia Hawkesiana, Bail.

From a sketch by Mrs. R. W. Garraway. 
The known wholesome species of Fungi are marked "Edible"; this does not imply that they are all used for food in Queensland, but that they have been or are so used in other countries. In all cases where blight-fungi are mentioned the names of the hosts or plants they usually infest are also given ; and as Bordeaux mixture is the usual remedy in these cases its composition is here given:-“ $4 \mathrm{lb}$. sulphate of copper, $4 \mathrm{lb}$. of fresh lime, 40 gallons of water. Dissolve the copper in, say, 5 gallons of water in a wooden vessel; the best way is to tie it in a loosely woven bag, and suspend the bag from a stick laid across the tub; or, if in a hurry, use hot water. Slake the lime in another vessel, and add it to the copper solution; stir well till thoroughly mixed, then add sufficient water to bring the whole up to 40 gallons. Now strain out any dirt. The addition of $3 \mathrm{lb}$. of treacle makes the mixture more adhesive, and should be used if damp weather is feared."Kirk.

Where plants are designated varieties I do not consider it correct to suppose that all or any great proportion of such deviations are the effect of cross-fertilisation, especially in the indigenous plants; it would be better that they should be looked upon rather as sports having nothing whatever to do with sexuality. Cross-fertilisation may, and in all probability does, play a principal part in causing the varieties of plants like the melon, pumpkin, cabbage, turnip, \&c., as well as various garden flowers, while many of these may have originated from sports. That sports may originate from the seed is, I think, shown in the matter of the dwarf China Scarlet Peach. Among seedlings of this plant it is not uncommon to find some of a very dwarf, spreading, bushy habit, which have the flowers and fruit of the parent, and are perfectly healthy as a rule, and are, in my opinion, sports, not hybrids; and these we find are commonly perpetuated by means of budding and grafting for the sake of their so-called double blooms as well as the colour of the flowers, and it may be that all the tall-growing double-flowering kinds have originated from a dwarf one in China many years back. The first I can remember of these sports were among some plants raised in 1844 from stones borne by a tree brought out to South Australia by my father in 


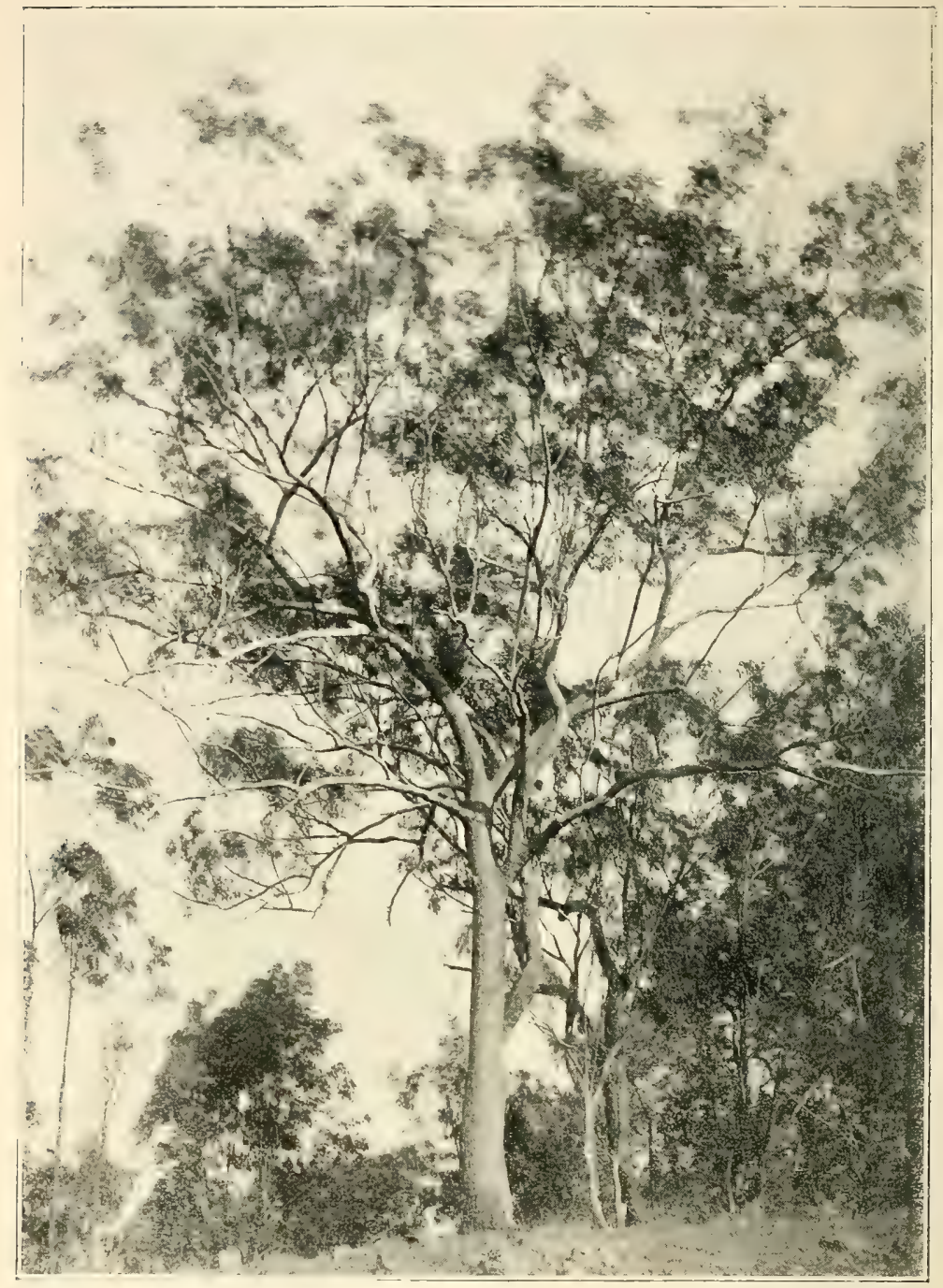

Photo. by Dr. T. L. Bancroft.

Eucalyptus stannariensis, Bail.

"Dead Finish" 
IS39. These dwarf peach-trees are very common, and vary much in colour of flower as well as flavour of fruit. In the above idea I do not ask others to agree with me: this is only thrown out as an idea of my own.

Two other remarkable sports from the seed may be mentioned - the one Robinia pseudoacacia var. tortuosa, and our own Barklya syringifolia var. tortuosa.

While on this subject mention might be made of one genus (Eucalyptus) of our forest-trees in which I believe the numerous kinds have all originated from sports, even those which we now call species having originated in this manner at a much earlier period. For instance, one need only refer to one species, the socalled "Blue Gum," from the supposed blue colour of the bark, or "Red Gum" when referring to the timber. This tree varies so much in its flowers that the species cannot be safely determined by flowers alone; the bark, wood, and foliage are all similar, and all these forms, where the flowers seem so different, run so closely the one into the other that we can only consider them as belonging to one species, with very variously shaped flower-buds. The immortal Bentham in the "Flora Australiensis" gives three varieties, but, doubtless, these owe their differences to soil or climate more than anything else. Thus I always think it unsafe, in this species especially, to name any supposed forms or varieties.

The feature of the vernacular names following the systematic is one which will be likely to give satisfaction to a large number of the public, but the utility of these names is not of the advantage which one might suppose, for in many cases they are only local, and often a plant will be found to be known in the same district by more than one name. Take for example our "Blue Gum," so called from the colouring of the bark, while persons using the timber designate it " Red Gum," both of these names being used in other Australian States for entirely different trees. This is the case with a large number of our plants, especially if they enjoy a wide range. In the case of aboriginal names the district where each name is used has been added. In many country districts these aboriginal names will be found of great assistance to persons 


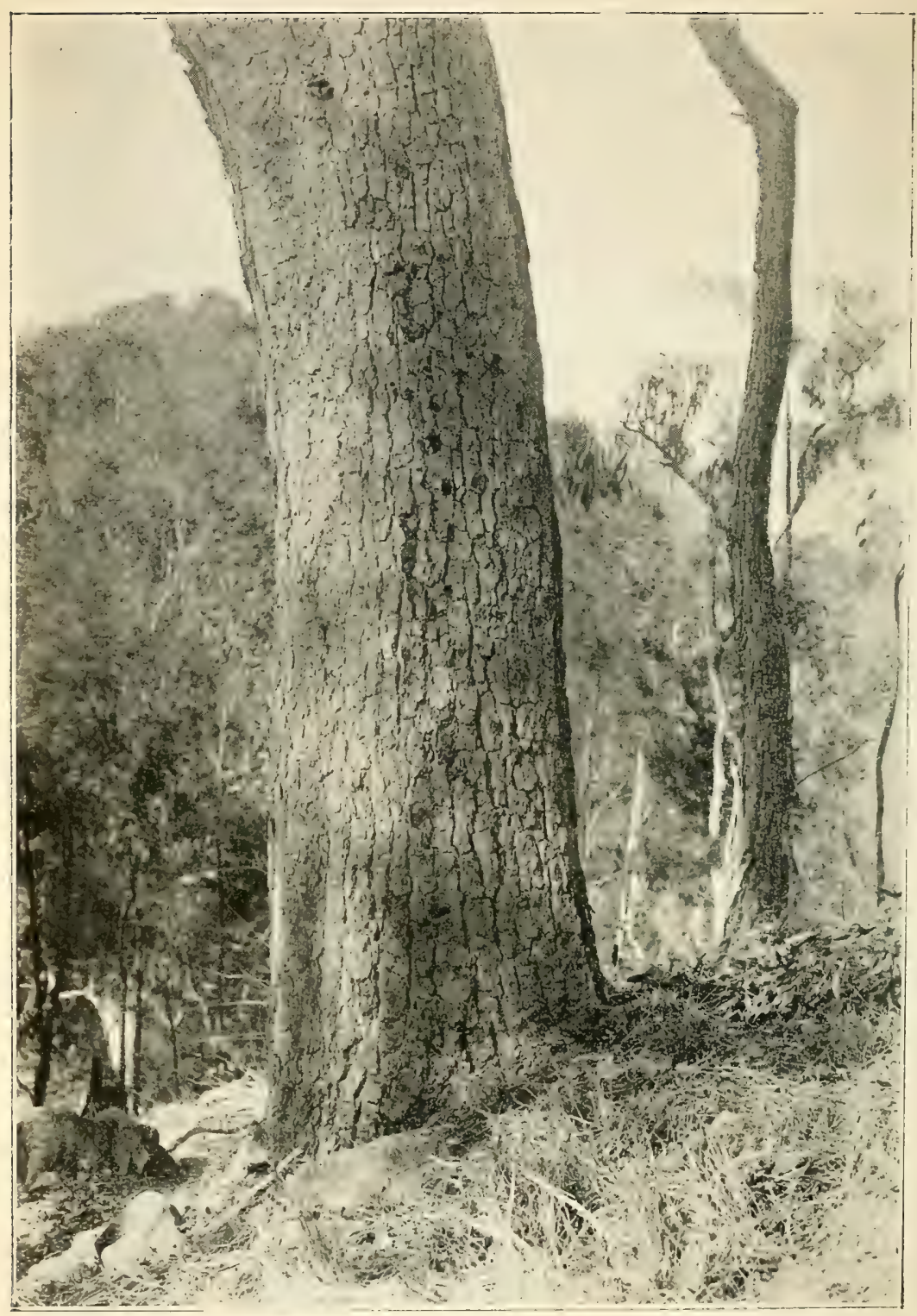

Photo. by Dr. T. L. Bancroft.

Eucalyptus stannariensis, Bail.

"Dead Finish." 
desirous of obtaining information regarding the native plants from the aboriginal inhabitants. The initial letters "R.B.H." indicate that the aboriginal name was received from Mr. R. B. Howard, the present Protector of Aborigines, and that such names have not appeared previously in my publications. It appears that these people have names for such plants only as they make use of. The uses made of the plants by the natives will mostly be found recorded in the "Queensland Flora."

Again, an account of a country's vegetation speaks more directly and truthfully to the intelligent man who is thinking of leaving Europe to settle in one or other of the British possessions than all the lecturers that have ever addressed a public meeting. From the plants of a country one gains a good idea of climate; the plants speak the truth, they have nothing to gain or lose, and from them the intending settler can easily judge if the country which he desires to settle in would grow the crops which he intends to cultivate or use in his trade. Let me point out just one instance, say the article rubber: By casting the eye over this Catalogue it will be seen that a very large number of our indigenous plants contain the crude matter from which this article is made, and, as a proper and careful testing of the milky sap has not been carried out, we are still in the dark as to the value of our native trees for this material.

It is probable that for all time, as at present, there will be differences of opinion as to the relative value of the words " genus," "species," "variety," and "form," and, as there is no positive rule to guide one in the matter, each botanist in classifying his plants follows what he thinks best. Thus in the present work it may be found that some of the plants are riven as forms which another botanist would have placed as varieties, and others as varieties which by some would have been placed as species, and so on. This is of little importance so long as the plants are not hidden, for the main use of classification and nomenclature is to make it possible to converse and write abou^ plants in a manner easily understood. To illustrate the matter let us take the wellknown plant Hardenbergia monophylla, the Bushman's Sarsapa- 


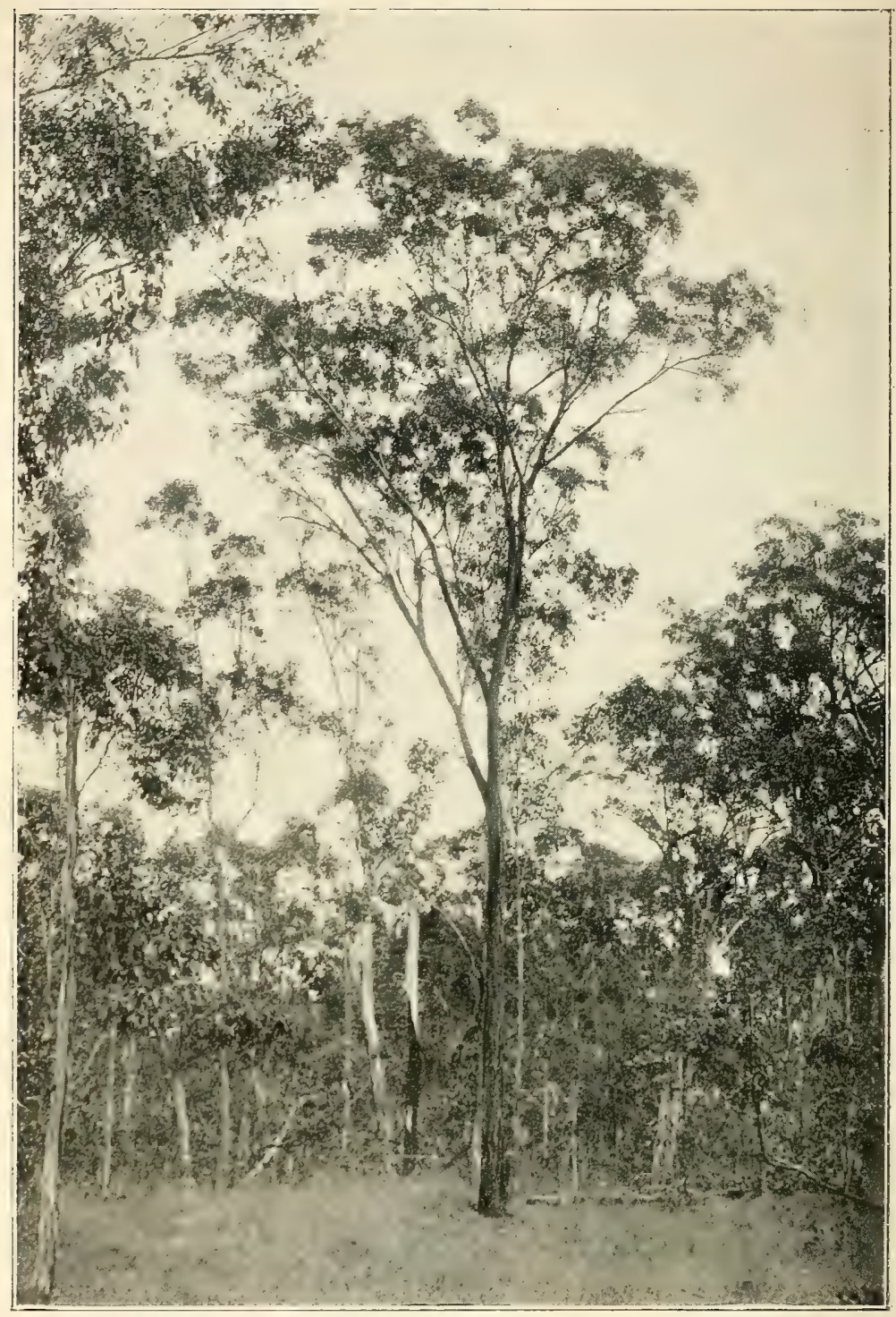

Photo. by Dr. T. L. Bancroft.

Eucalyptus sideropilloia, Benth.; forma decorticans, Bail.

"Naked-top Ironbark." 
rilla: The normal form in habit differs so much from $H$. ovata, known as "Native Lilac" in South Australia, that the two plants could not be grown in a garden the one as a substitute for the other; yet Bentham in "Flora Australiensis" places H. ovata only as a synonym. Both are met with in Queensland, and the latter is. given as a variety of the other. Another instance might be mentioned where two grasses, on account of their beauty, must sooner or later be placed among those recommended for ornamental planting or cultivating for use in a dry state for decorative purposes. These are only mentioned in the "Flora Australiensis" as synonyms of Pappophorum nigricans. R. Br. in Prod. gave them as $P$. purpurascens and $P$.gracile; and as they scarcely differ from the first-named species in anything more than stature and colour, Bentham, from a botanical point of view, was perfectly correct; but when grown for special purposes a special name is required for each; thus they will be found mentioned here as forms. In the case of some new plants where perhaps my specimens might scarcely warrant their being given as species, they have been placed as such because.I fancied that when further specimens were obtained they would so prove.

As my writings on botanical matters principally deal with plants of Queensland, I may be allowed to refer in these notes to the subject of seeds and the very short time many retain their power of germination, and the probable cause of this. All persons who have resided in Queensland for a number of years will have observed that as a rule January and February are our wet months, that then the whole atmosphere is often charged with moisture, and that in most houses it becomes difficult to keep even clothes hanging up in a room from becoming mildewed. Now, this moisture has the effect upon seeds of causing an incipient germination, particularly if kept in paper or bags and placed away in box or drawer, and with some seeds when placed in a bottle and corked; and as germination can only take place once all such seeds are destroyed. Thus it will be understood that climate has much to do with the length of time seeds will retain their powers of germination. An instance to illustrate what is here pointed out may be given. The late Baron Mueller, in one of his writings; 


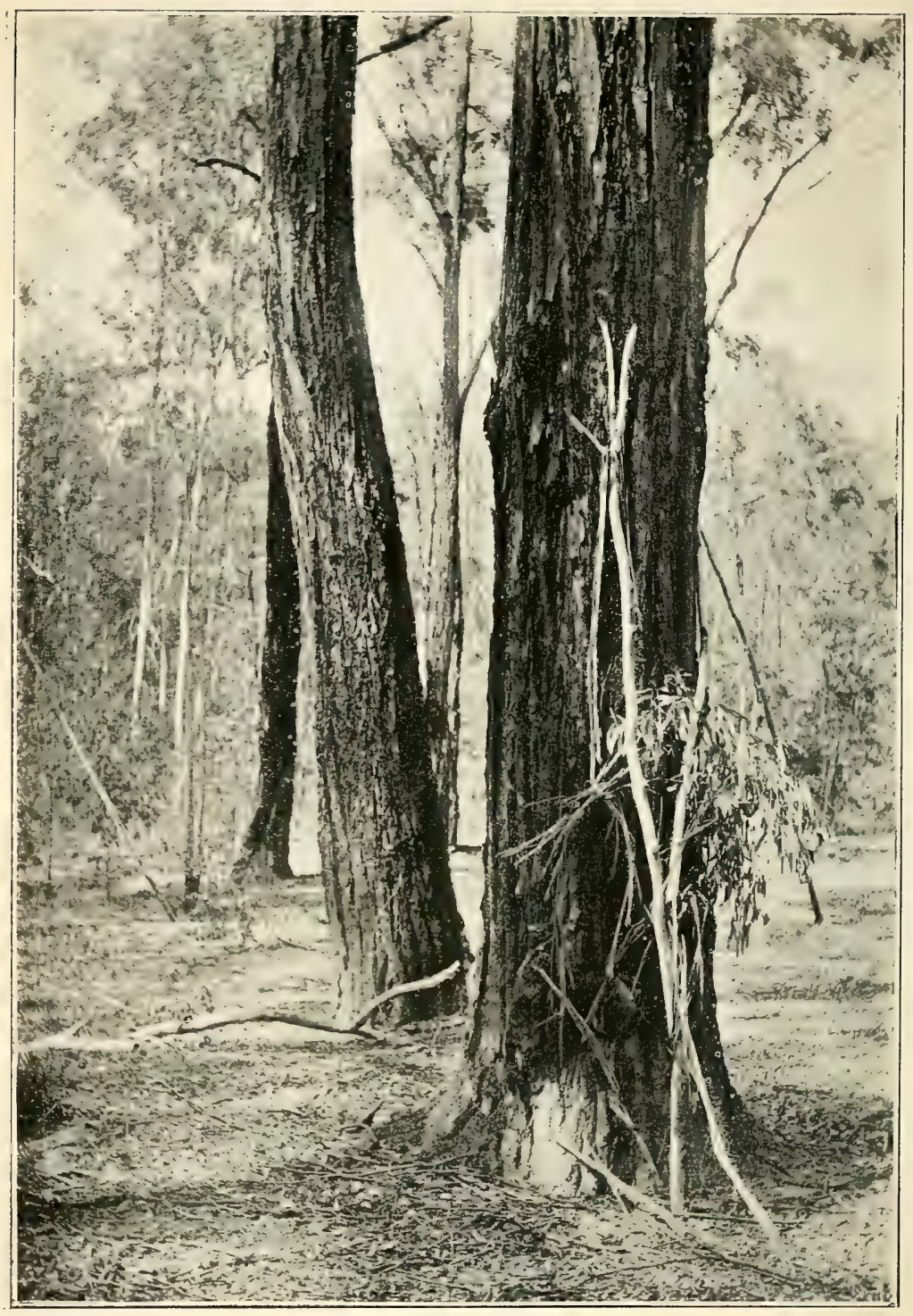

Photo. by Dr. T. L. Bancroft.

Eucalyptus siderophlota, Benth.; forma decorticans, Bail.

"Naked-top Ironbark." Base of trunk, smaller branches, shed bark, and foliage. 
mentions that the seed of a certain Eucalypt had with him retained its power of germination for fourteen years, whereas with me seed of the same species could not be made to germinate the second year, yet these seeds were kept in paper in a drawer from the one year to the other in my office. I may say every seed grew the first and none the second year, which is a pretty general rule with Eucalypts in Queensland.

Besides those marked in the Catalogue as possessing certain valuable economic properties, there are probably a number of others equally rich; in fact the Queensland flora contains plants which yield almost all the substances obtained from the Vegetabie Kingdom. In food for stock it is uncommonly well supplied, and, what is of the greatest importance, the indigenous plants are suitable to the climate in a remarkable degree. For instance, what grasses other than indigenous ones could be found to sleep througin years without rain, and then, at the advent of a week or so of good rain, spring into life and cover the country with fresh green herbage, not only, let it be understood, from the seed which might be preserved in the earth, but also from the old roots which to all appearance had been destitute of life? It may be safely said that very few, if any, parts of the world could furnish grasses equally good with such a tenacity of life.

There are a great many more than the species noted which fossess medicinal virtues; and when these are better known some may be found worthy of cultivation for their properties; and it will be found that when a plant is known to possess any particular virtue, be it medicinal or other, a note referring to the fact is given. The same may be said of the plants yielding tanning barks, oils, dyes, gums, resins, \&c. The kinds of woods number about I,000, and among them are those suitable for all purposes for which the material is used. A very large number of the indigenous. plants furnish material suitable for paper-making, rope, twine, and hessian cloth.

When any particular plant is said to furnish a useful fruit, it must not be imagined that the fruit equals the Apple, Pear, or Peach of the present day, but all so marked are superior to the fruits known to our far-back forefathers. 


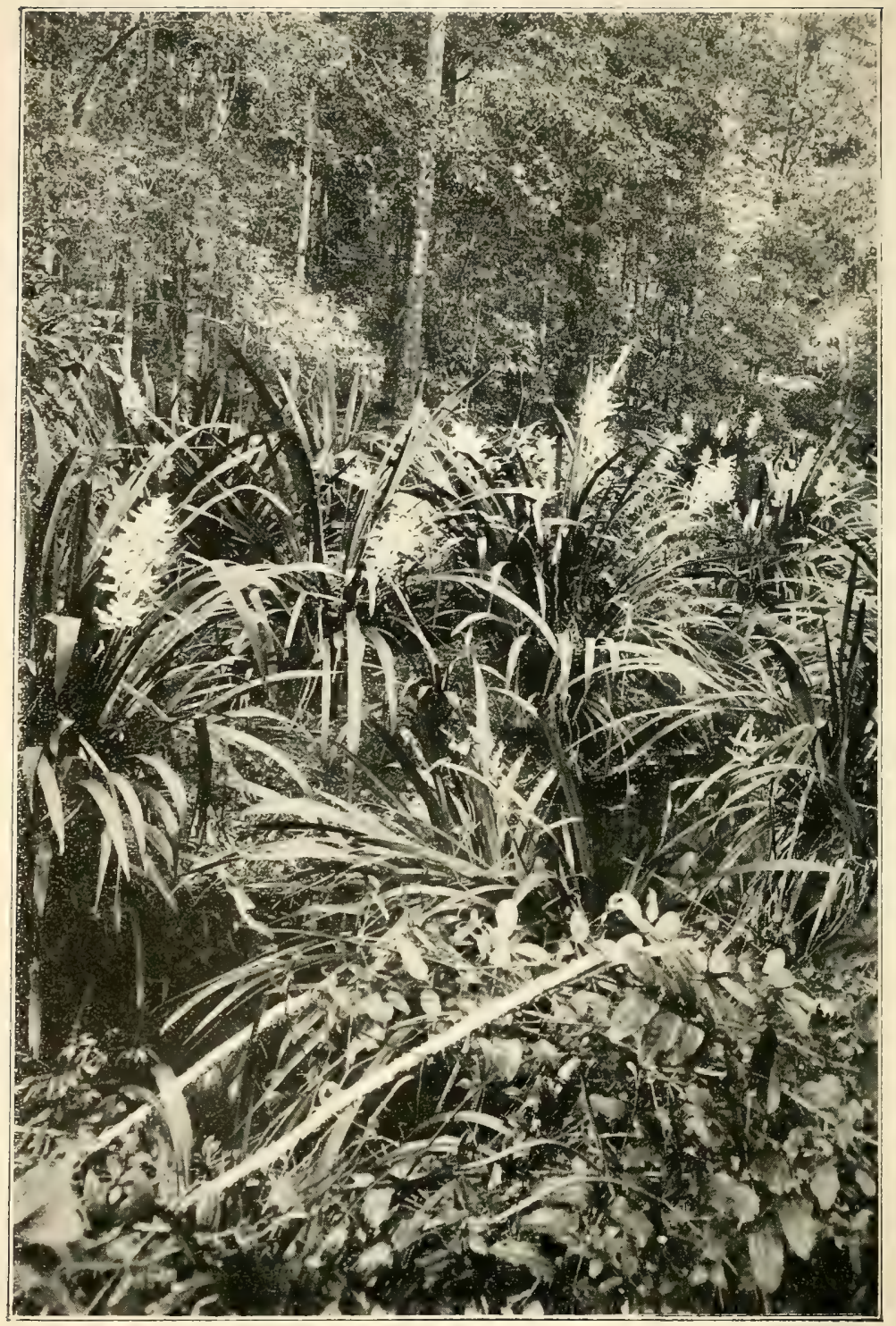

Photo. by A. H. Burbank.

Helmholtzia glaberrima, $T$. Caruel. 
The whole of the present book is taken up in enumerating the forms of vegetable life which meet the eye of the traveller in passing over the vast lands and waters of Queensland, and if one has an eye for the beauties of Flora's Kingdom we may safely imagine one repeating the following lines of Cowper:-

Lovely indeed the mimic works of Art, But Nature's works far lovelier: I admire,

None more admires, the painter's magic skill,

But imitative strokes can do no more

Than please the eye, sweet Nature ev'ry sense,

The air salubrious of her lofty hills,

The cheery fragrance of her dewy vales, And music of her woods-no works of man May rival these; these all bespeak a power Peculiar, and exclusively her own.

22nd November, I909.

F.M.B. 


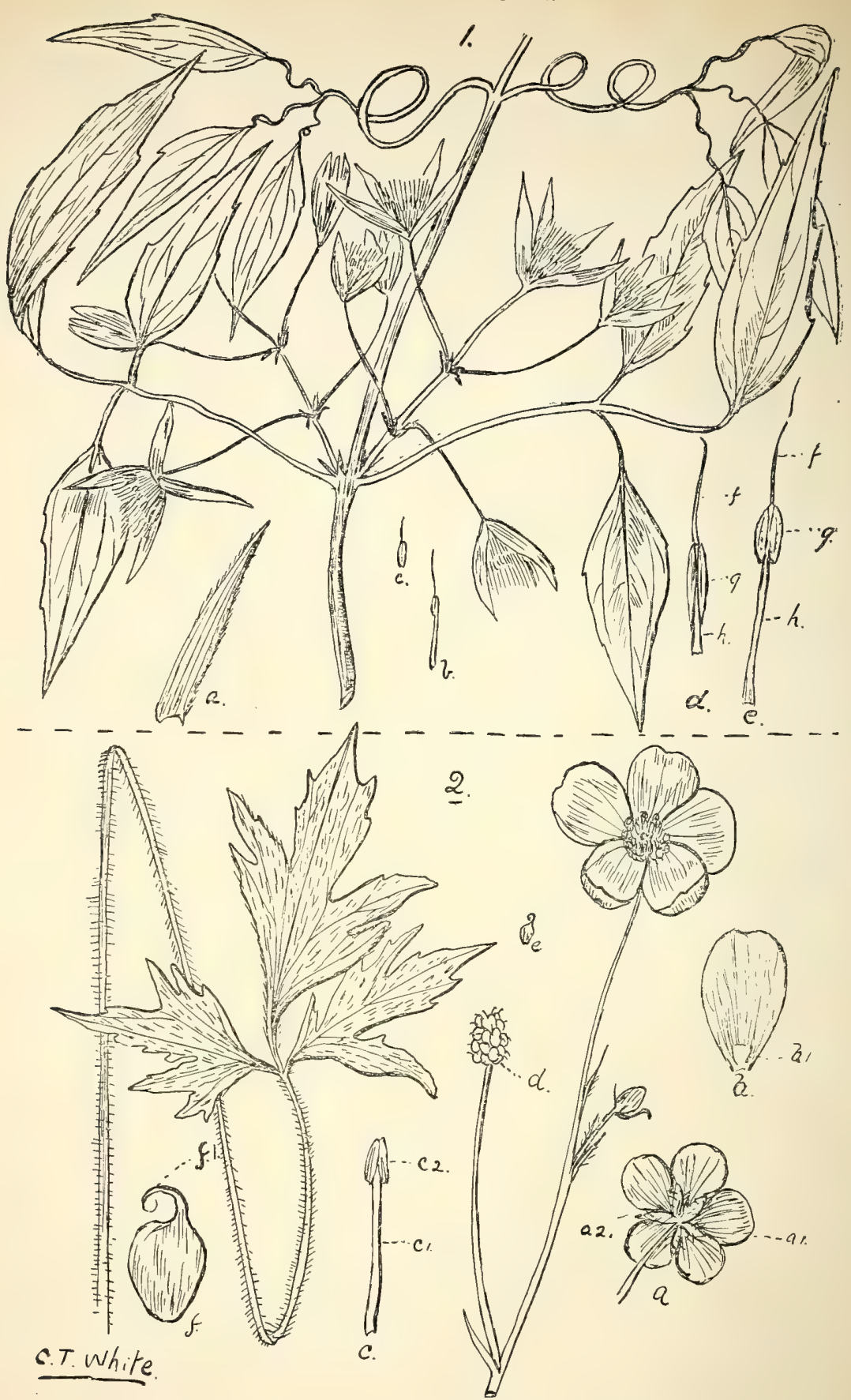

I. Clematis aristata, R. Br.; var. longiseta, Bail.

(a) Sepal, (b) outer stamen, (c) inner stamen, (d) inner stamen, (e) outer stamen, (f) anther appendage awn, (g) anther, (h) filament. (b) and (c) nat. size; (a), (d), (e), (f), (g), and (h) enl.

\section{Ranunculus lappaceus, $S m$.}

(a) Flower, back view, (a I) pet., (a 2) sep., (b) pet., (b I) glandular pit, (c) stamen, (c i) filament, (c 2) anther, (d) head of carpels, (e) single carpel, (f) carpel, (f I) style. (a), (b), (c), (d), (e), and (f) nat. size. 


\section{COMPREHENSIVE CATALOGUE}

\section{OF \\ QUEENSLAND PLANTS.}

[An asterisk denotes, that the plant is an introduction become more or less naturalised but not indigenous.]

\section{Class I.-DICOTYLEDONS.}

Subclass I.-POLYPETAL

SERIES I.-THALAMIFLOR㤅.

Alliance I.-RANALES.

\section{Order I. - RANUNCULACE E.}

Tribe I.-Clematides.

Clematis, Linn.-Virgin's Bower or Traveller's Joy.

aristata, $R . B r$.

var. longiseta, Bail. (Fig. I.)

glycinoides, $D C$.

var. submutica, Benth.

microphylla, $D C$.

var. colorata, Bair. Flowers a dull-purple.

var. Fawcettii, Bail. = C. Fawcetti, F. v. M.

Tribe II.-Anemoner.

*Adonis, Linn.

autumnalis, Linn.-Pheasant's Eye or Flos Adonis. Europe.

Myosurus, Linn.

minimus, Linn.-Mouse-tail.

B 
Tribe III.-Ranuncules.

Ranunculus, Linn.-Buttercup. As a help to those studying botany, a number of carpological names are given. Thus the fruit of this genus is termed an Etærio. I may remark, however, that botanists in describing plants seldom use these terms; but sometimes they are mentioned in examination papers, and being here inserted may assist pupils to answer a question or give an example.

lappaceus, Sm. (Fig. 2.)

var. pimpinellifolius, forma multiplex, Bail.-Double Buttercup, or Bachelor's Buttons.

plebeius, R. Br. = R. hirtus, Banks and Sol.

rivularis, $B$. et Sol.--River Buttercup.

var. major, Benth.

parviflorus, Limn., zar. australis, Benth.

*muricatus, Linn.-A weed of Europe, Asia, and America.

*arvensis, Limn.-A field weed of Europe.

\section{Order II.-DILLENIACEÆ.}

TRIBE I.-DELIME后.

Tetracera, Limn.

Dæmeliana, F. ข. $M$.

Nordtiana, F.v.M. (Fig. 3.)

Cowleyana, Bail._- “Teeweeree” of Barron River. (Fig. 3 bis.)

Wuthiana, $F \cdot v \cdot M$.

Tribe II.-Dilleniex.

Wormia, Rottb.

alata, R. Br.-A dark prettily marked cabinet-wood. (Plate II.)

\section{Tribe III.-Hibbertie.}

Hibbertia, Andr.

\section{Section I.-Hcmistemma.}

Banksii, Benth. (Fig. 4.)

dealbata, Benth.

Brownii, Benth.

candicans, Benth.

Millari, Bail. 
PLATE II.

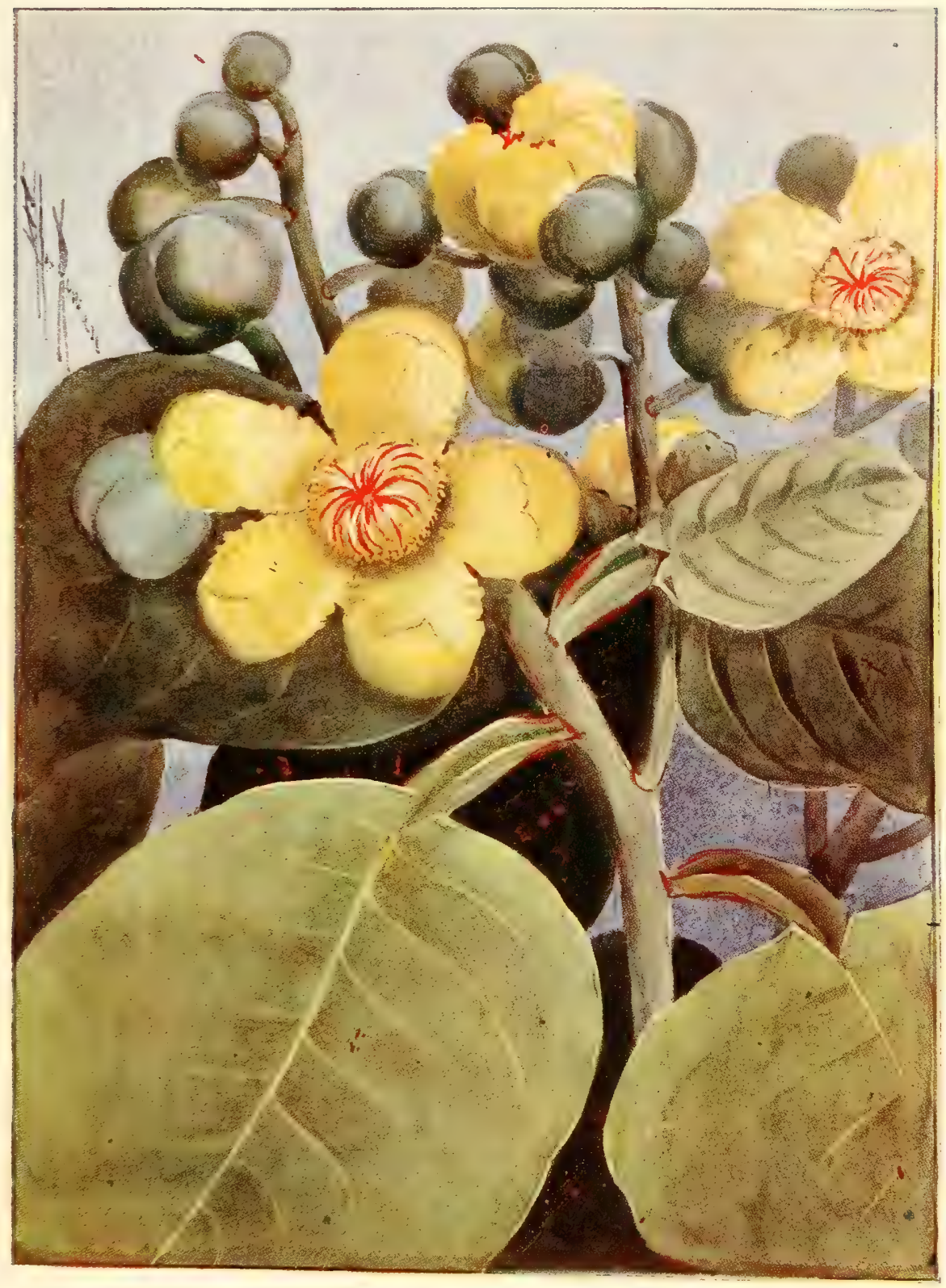

WORMIA ALATA, R. Br. 



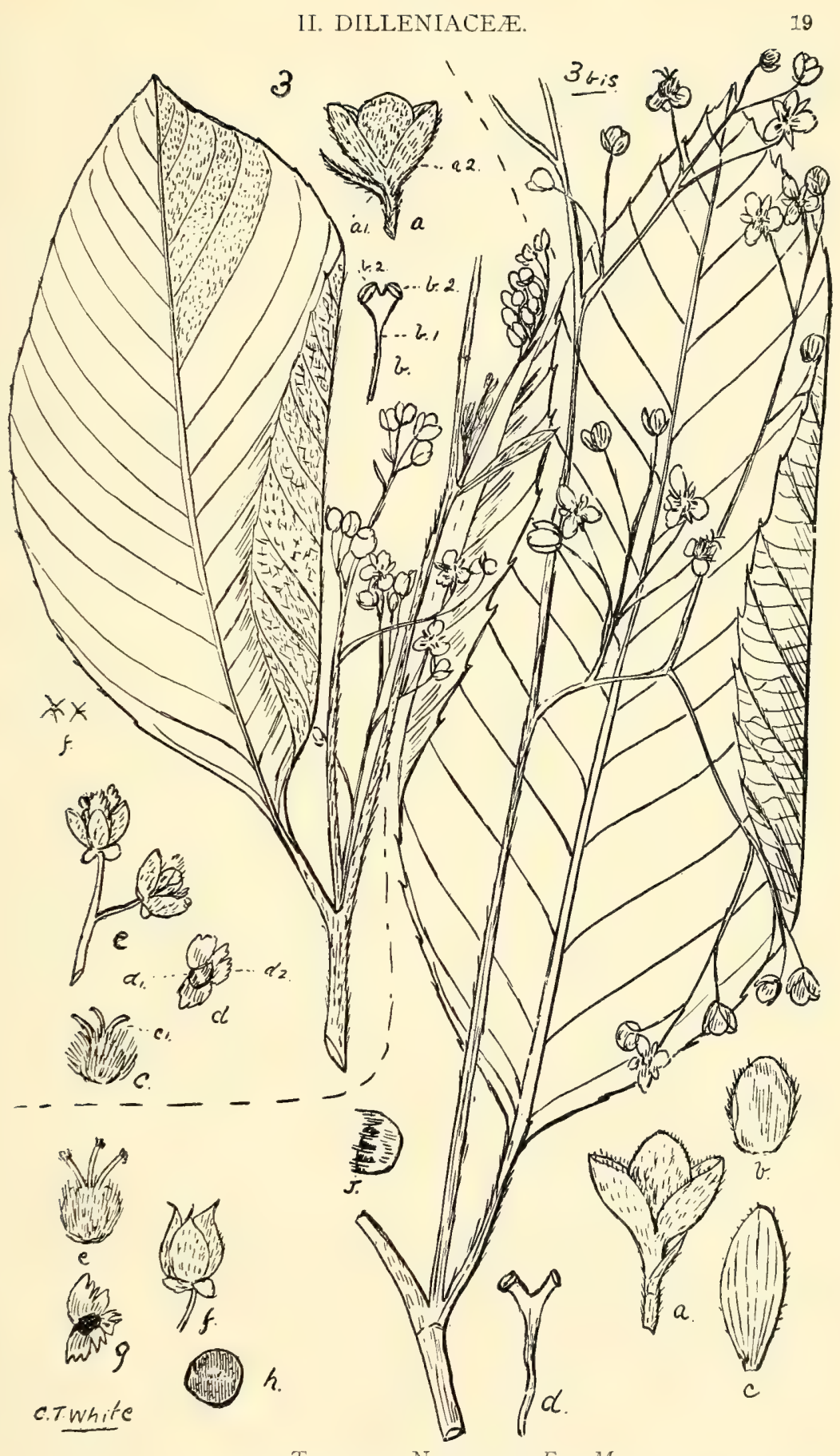

3. Tetracera Nordtiana, F. $\tau$. $M$.

(a) Fl. bud, (a r) bracteole, (a z) sepal, (b) stamen, (b. I) filament, (b a) anther cells, (c) pistil, (c I) styles, (d I) seed, (d z) arillus, (e) rife carpels. (a), (b), and (c) en1, ; (d) and (e) nat. size.

\section{3 bis. Tetracera Cowleyana, Bail.}

(a) F1. bud, (b) sep., (c) pet., (d) stamen, (e) pistil, (f) ripe carpels, (g) seed and its arillus, (h) seed, (j) side view seed enclosed in the arillus. (a), (b), (c), (d), (e), and (h) enl.; (f), (g), and (j) nat. size. 


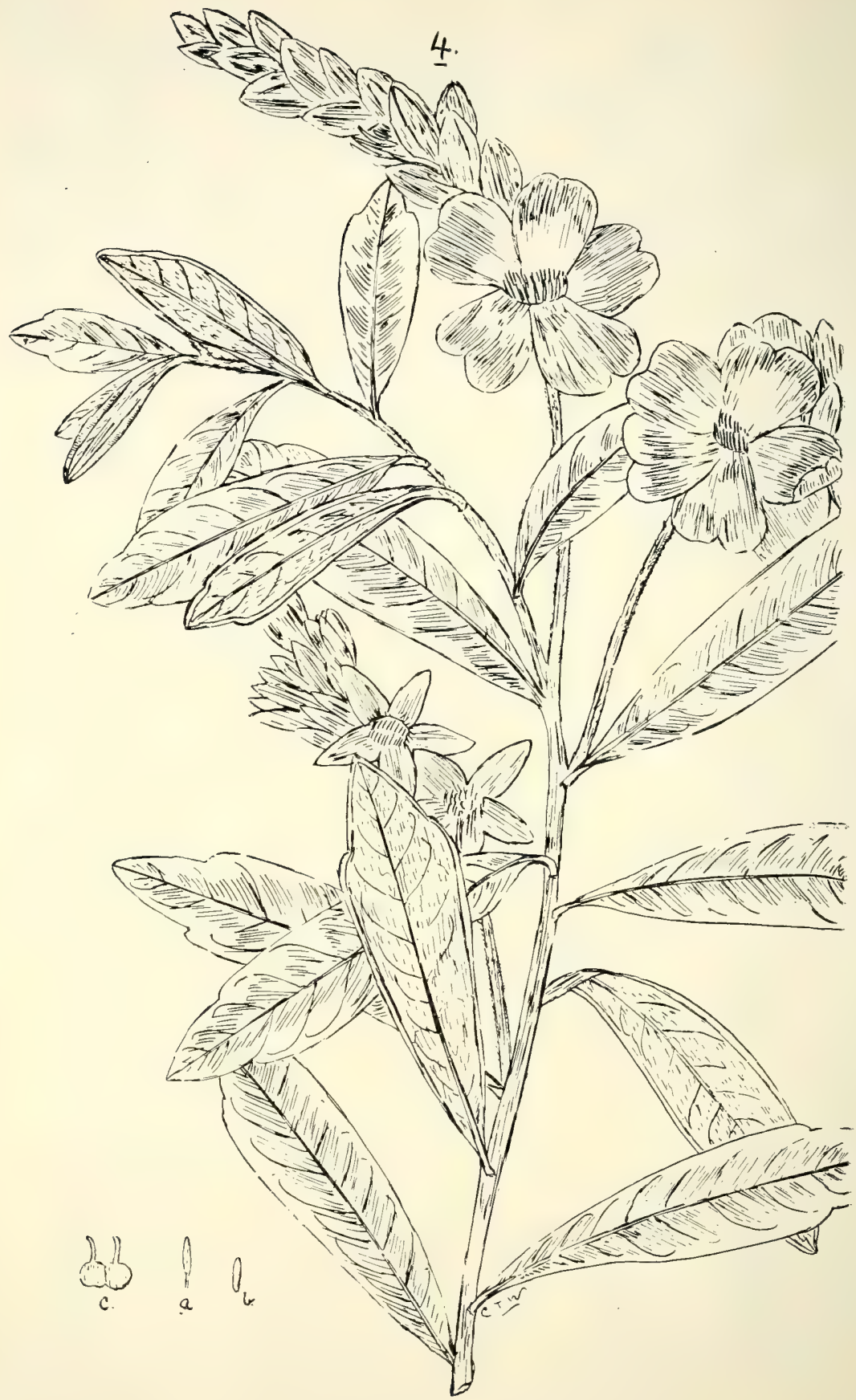

4. Hibbertia Banksii, Benth.

(a) Stamen, (b) staminode, (c) carpels. All nat. size. (After Banks and Solander.) 
Hibbertia-contd.

Section II.-Pleurandra.

synandra, F.v.M.

stricta, $R . B r$.

var. canescens, Benth.

z'ar. hirtiflora, Benth.

Billardieri, F.v.M.

acicularis, F. $M$.

concinna, Bail.

Section III.-Euñibbertia.

velutina, R. Br.

lepidota, R.Br. (Fig. 5.)

melhanoides, F.v. M.

vestita, A. Cumn.

var. thymifolia, Benth.

fasciculata, R. Br.

virgata, $R$. $B r$.

linearis, $R . B r$.

var. floribunda, $A$. Cunn.

var. obtusifolia, Benth.

diffusa, R. $B r$.

dentata, R. Br.

glaberrima, F.v.M. - Poisonous plant. "Gnanjeen" of

Walsh River natives.

volubilis, Andr.-Flower yellow, fetic.

longifolia, F.v.M.-A poisonous shrub, called "Arsenic plant."

cnotheroides, F.v.M.

Bennettii, Bail.-Arsenic plant

Adrastæa, DC. (Included in Hibbertia by F. v. Mueller.)

salicifolia, $D C$. (Fig. 6.)

\section{Order III.-MAGNOLIACEA.}

Tribe I.-Winteree.

Drimys, Forst.

dipetala, F.v.M. = Tasmannia insipida, R. Br. (Fig. 7.)

membranea, F.v.M.

semecarpoides, F.v.M. 
II. DILLENIACER-III. MAGNOLIACEN.
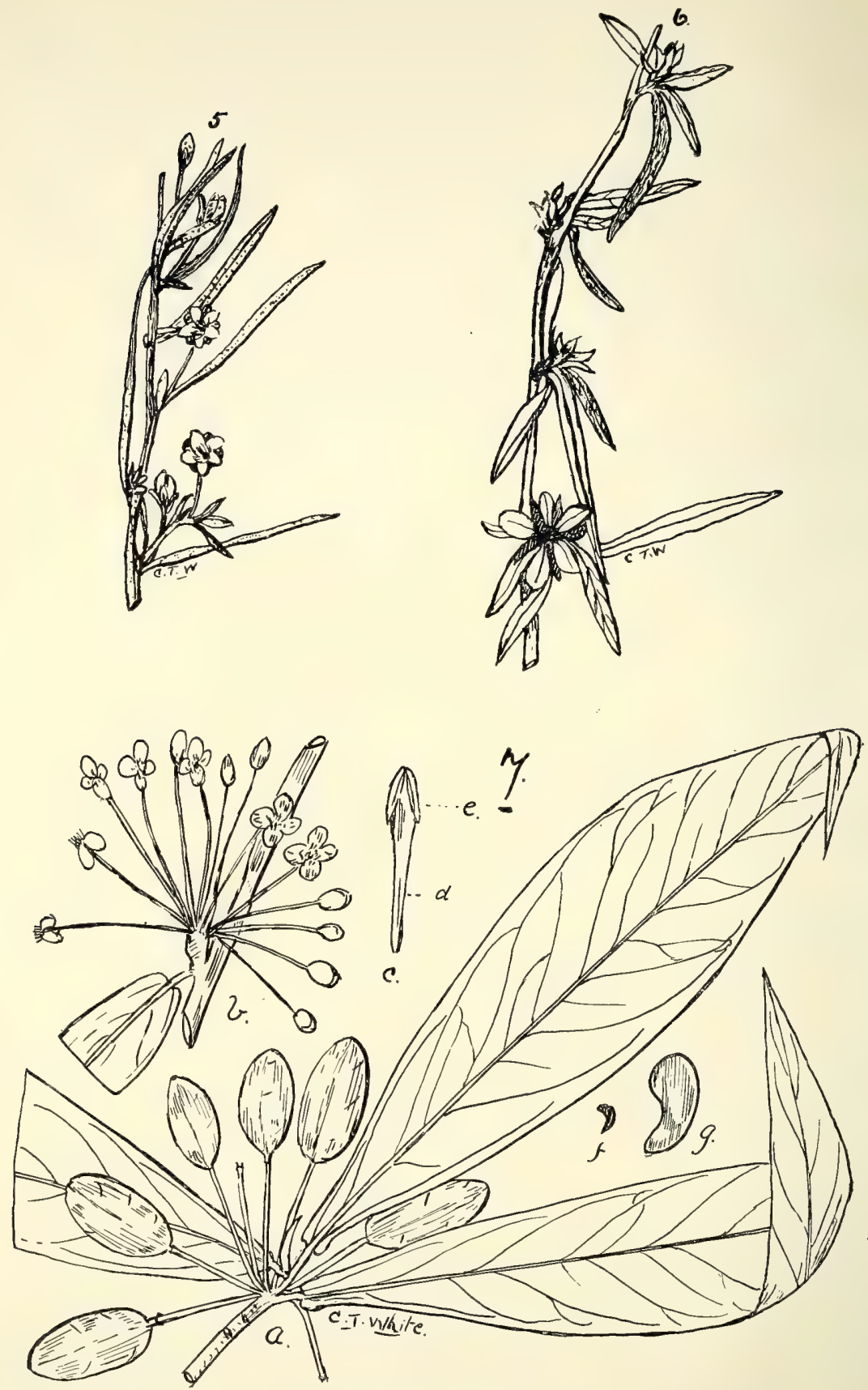

5. Hibeertia lepidota, $R$. $B r$.

6. Adrastea salicifolia, $D C$.

7. Drimys dipetala, F. v. $M$.

(a) Fruiting branchlet, (b) flowers, (c) stamen, (d) filament, (e) anther, (f) seed, (g) seed. (a), (b), and (f) nat. size; (c), (d), (e), and (g) enl. 
III. MAGNOLIACEA:.

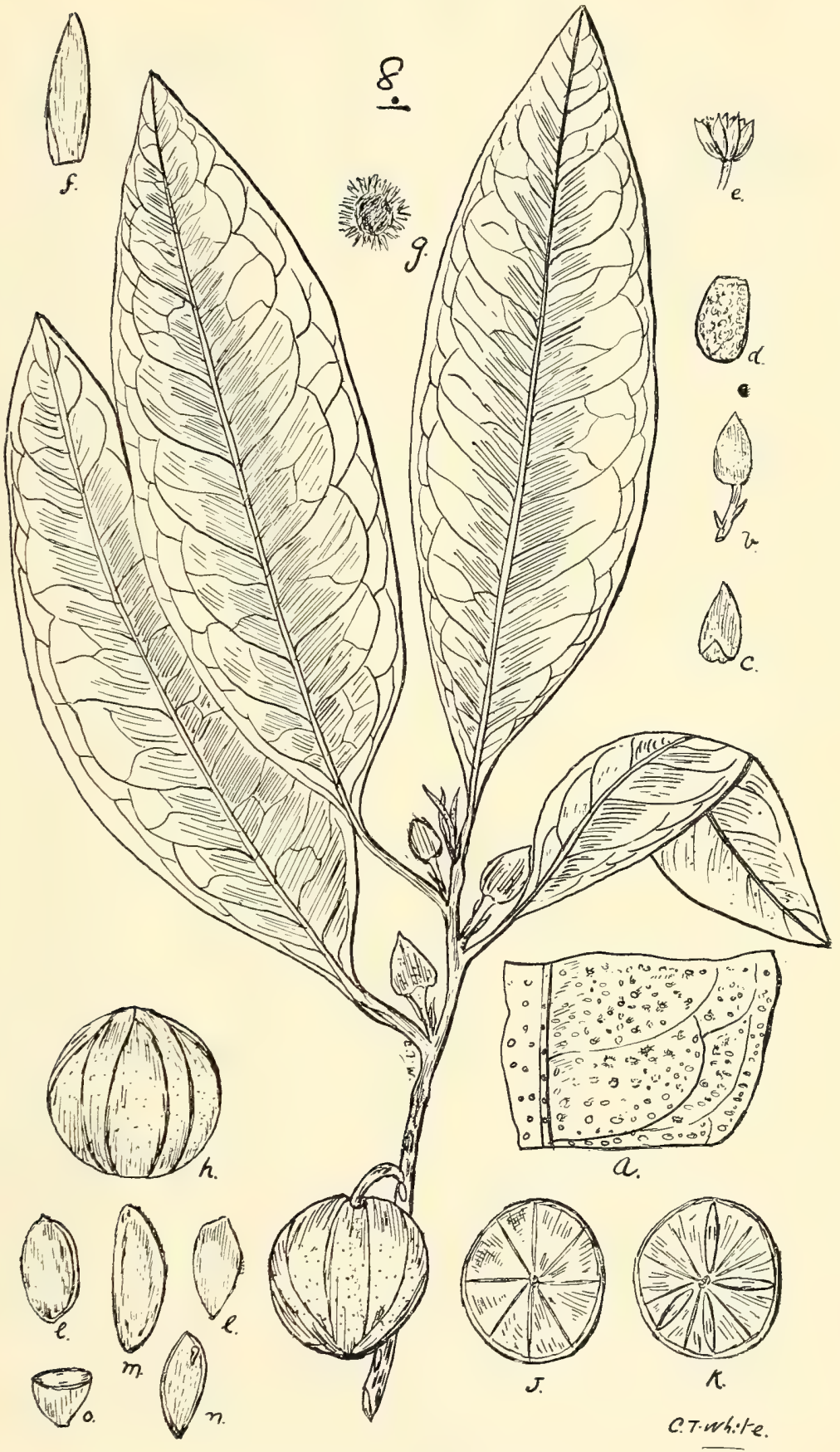

8. Galbulimima baccata, Bail.

(a) Port. under surface of a leaf, (b) fl. bud, (c) the outer sepal, (d) port. surface of a sep., (e) fl, bud, sepals removed, ( $f$ ) one of the outer staminodia ? petals, ( $g$ ) a scale, (h) a fruit, (i) sect. fruit containing no perfect seeds, (k) sect. fruit containing four perfect seeds, ( 1 ) seeds, (m) seed enclosed in its loose rugged coat, ( $n$ ) long. sect. seed, (o) trans. sect seed. (a), (d), (f), and (g) enl.; (b), (c), (e), (i) , and (o) nat. size. 


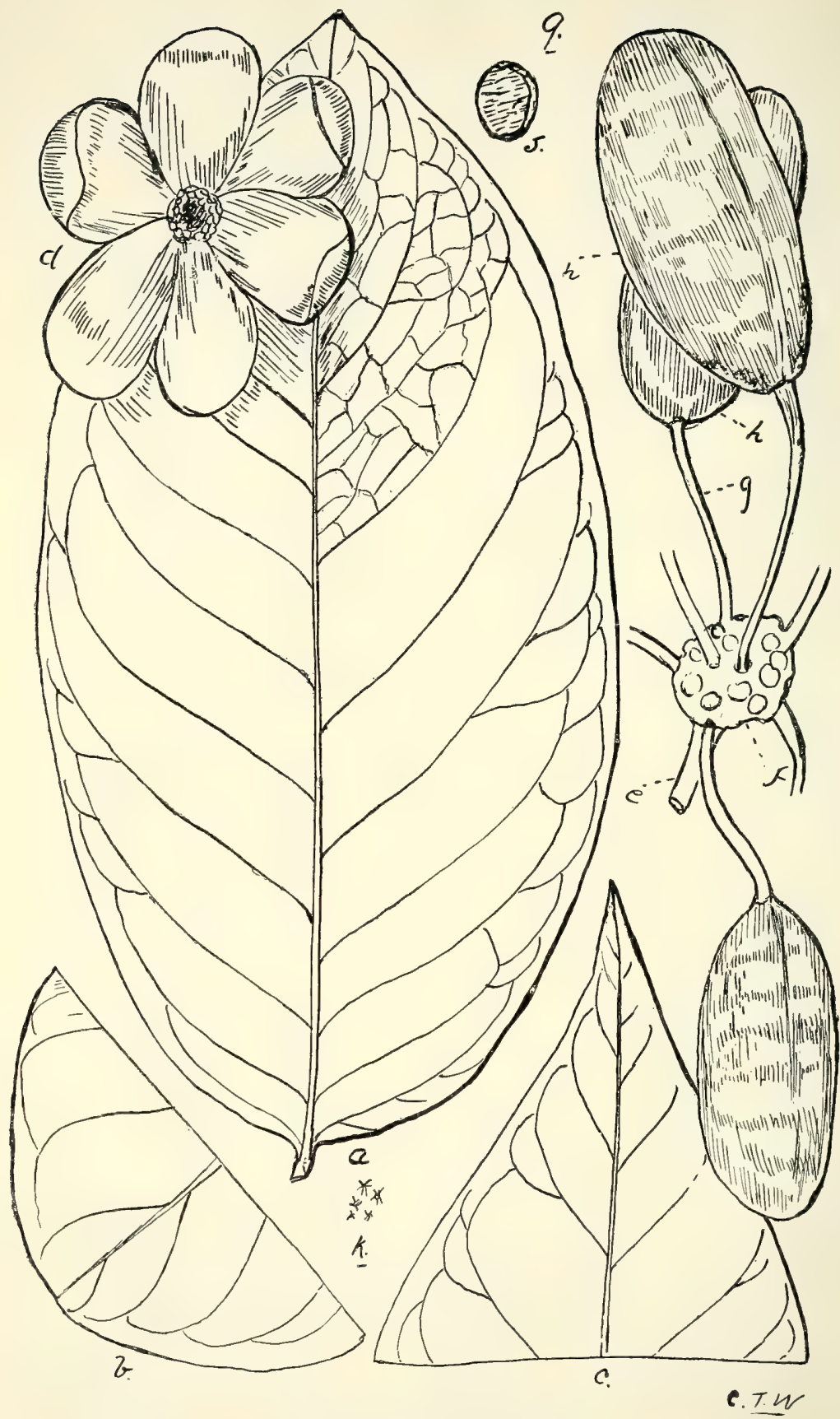

9. Uvaria membranacea, Benth

(a) Leaf, (b and c) apices of leaves, (d) flower, (e) peciuncte, (t) receptacle, (g) stipites, (h) ripe carpels, (j) seed, (k) stellate tomentum. (a) -(j) nat. size, (k) enl. 
Galbulimima, Bail.

baccata, Bail.-Wood soft. The fruits are free from tannin and prussic acid, yielding glucoside, but contain a pungent ethereal oil (resembling in odour juniper berries) and traces of alkaloid (J.C.Brïnnich). (Fig. 8.)

\section{Order IV.-ANONACEAE.}

Uvaria, Limn.

Tribe I.-UVARIE.e.

membranacea, Benth. (Fig. 9.)

Goezeana, F.v. $M$.

Fitzalania, F.v.M.

heteropetala, F.v.M.-? Uvaria heteropelala, F.v. M., Fl Austr. i. 5 I.

Tribe II.-Unones.

Cananga, Rumph.

odorata, H.f. et T.-Wood hard, grey.

Unonia, Limn.

Wardiana, Bail.-- "Niadoa" of Mapoon natives. (Fig. 9 bis.) Aricana, F. v. M.

stenopetala, F. v. M.

Polyalthia, Blume.

nitidissima, Benth.-- "Wo-a" of Thursday Island and "Manguru" of Batavia River natives. Wood nicely marked, with a spicy fragrance when freshly cut.

Armitiana, F.v. M.

Tribe III.-Mitrephorea.

Mitrephora, Blume.

Froggattii, F.v.M.

Haplostichanthus, F.v. M.

Johnsoni, F.v.M.

Melodorum, Dun.

Tribe IV.-Xylopiez.

Leichhardtii, Benth.-Fruit edible; forty years ago the Brisbane children called the fruit "Wild Bananas." Native name at Rockhampton, "Merangara." Bark yields a brownish-red dye.

Uhrii, F. $\approx \cdot M$. (Fig. IO.)

Maccreai, F.v.M. (Fig. Io bis.) 


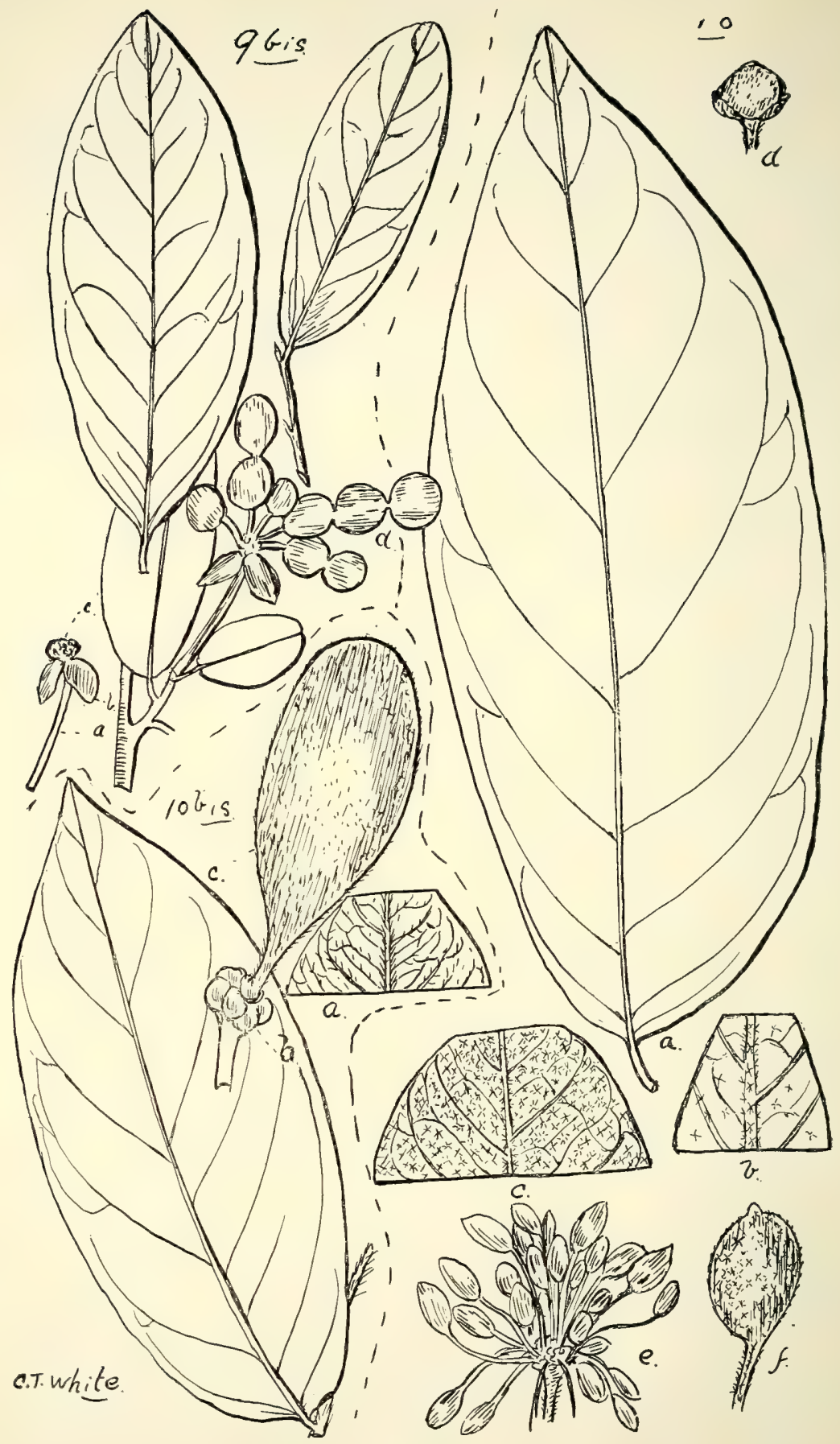

o bis. Unona Wardiana, Bail.

(a) Peduncle, (b) sepal, (c) receptacle, (d) carpels.

io. Melodorum Uhrit, $F . v . M$.

(a) Leaf, (b) port. under surface of a leaf, (c) port. upper surface of a leaf, (d) a fl. bud, (e) carpels, (f) a single carpel. (a), (d), and (e) nat. size; (b), (c), (f) en]. 
V. MENISPERMACEÆ.

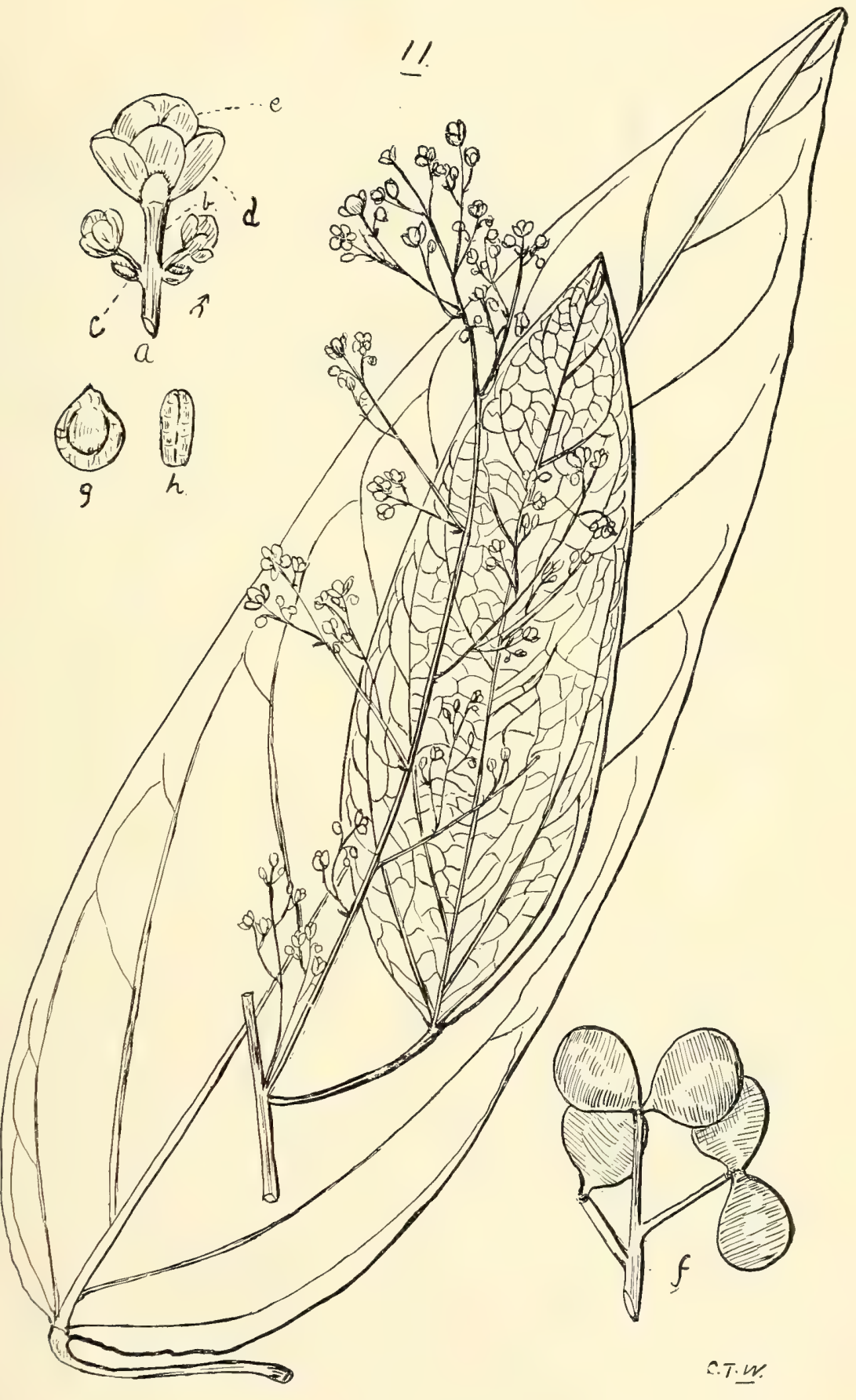

it. Limacia Selwynit, F.v. M.

(a) Male fl. buds, (b) pedicel of flower, (c) bract, (d) sepal, (e) petal, (f) drupaceous carpels, (g) putamen, (h) putamen seen on its edge. (a)-.(e) enl., (f)(h) nat. size. 

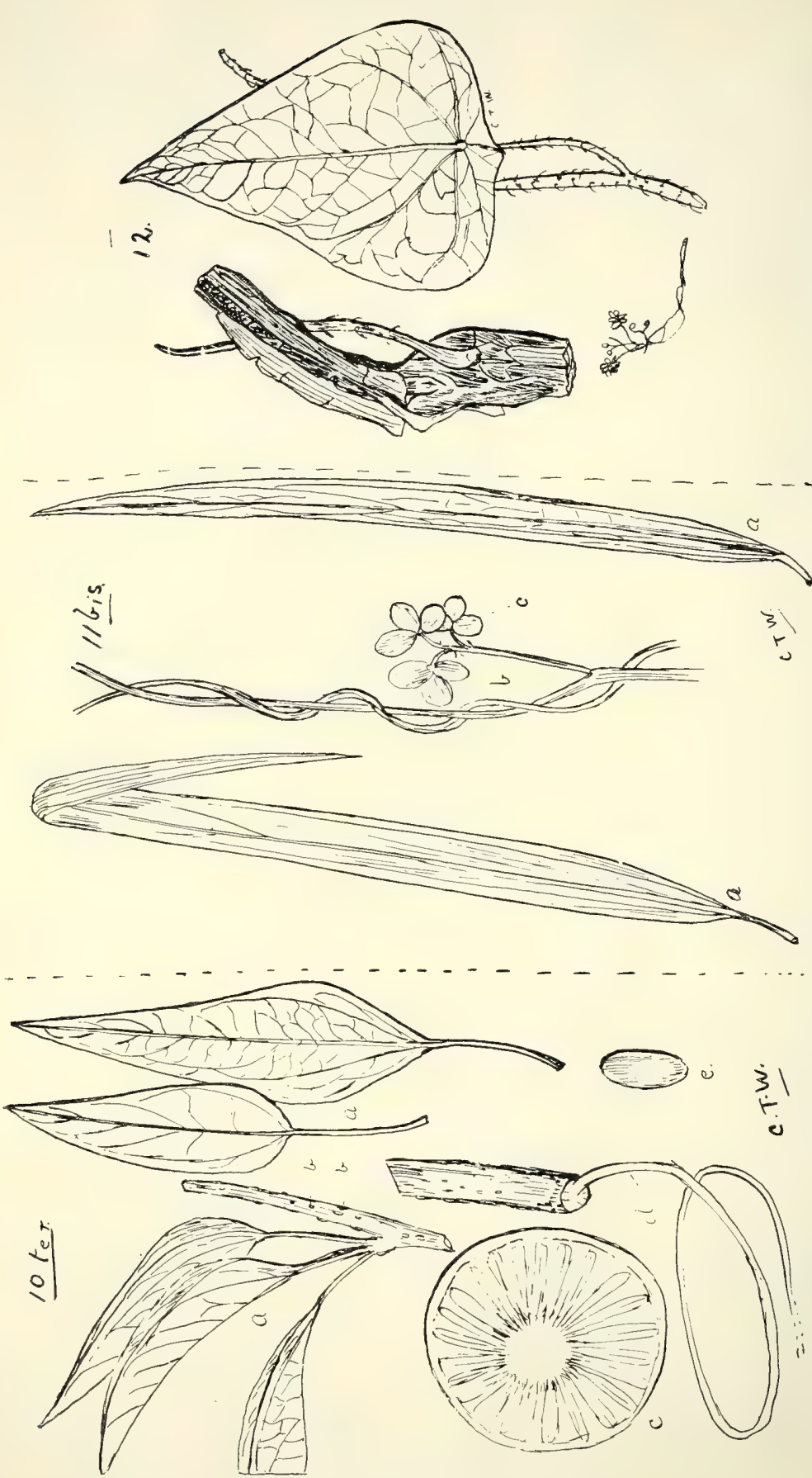

突 
Saccopetalum, Benth.

\section{Tribe V.-Miliusede.}

Bidwilli, Benth.

Brahei, F. v. M.

Eupomatia, R. Br.

Bennettii, F.v.M.

laurina, R.Br. Wood of a light colour and prettily marked.

\section{Order V.-MENISPERMACE $Æ$.}

Tribe I.-TinosPore. 2 .

Tinospora, Miers.

smilacina, Benth.

Berneyi, Bail.-Yam vine, of Hughenden. (Fig. Io ter.)

Tribe II.-Coccule $\pi$.

Legnephora, Miers.

Moorei, Miers $=$ Cocculus Moorei, F. v. M.

Pericampylus (Miers).

incanus (Miers), Benth., F1. Austr.

Limacia, Lour.

Selwyni, F.v.M. = Hypserpa Selwyni, F.v.M. (Fig. II.) esiangkara, Bail.-Native name at Mapoon. (Fig. II bis.)

Tristichocalyx, F.v. M.

pubescens, F.v. M.

diffusus, Miers.

Tribe III.-Ciss.ampelide.

Sarcopetalum, F.v. M.

Harveyanum, F.v. $M$.

Leichhardtia, F.v.M.

clamboides, F.v.M.

Stephania, Lour.

hernandiæfolia, $W$ alp.-Root poisonous.

aculeata, Bail.-Root poisonous. (Fig. I2.)

Bancroftii, Bail.-Poisonous. (Fig. I3.)

Cissampelos, Linn.

Pareira, Linn.-Spurious Pareira brava plant. The Pareira brava of commerce is yielded by Chondrodendron tomentosum, Ruiz et Pavon.

Pleogyne, Miers.

TRIBE IV.-PAChyGONE.E.

Cunninghami, Miers. 


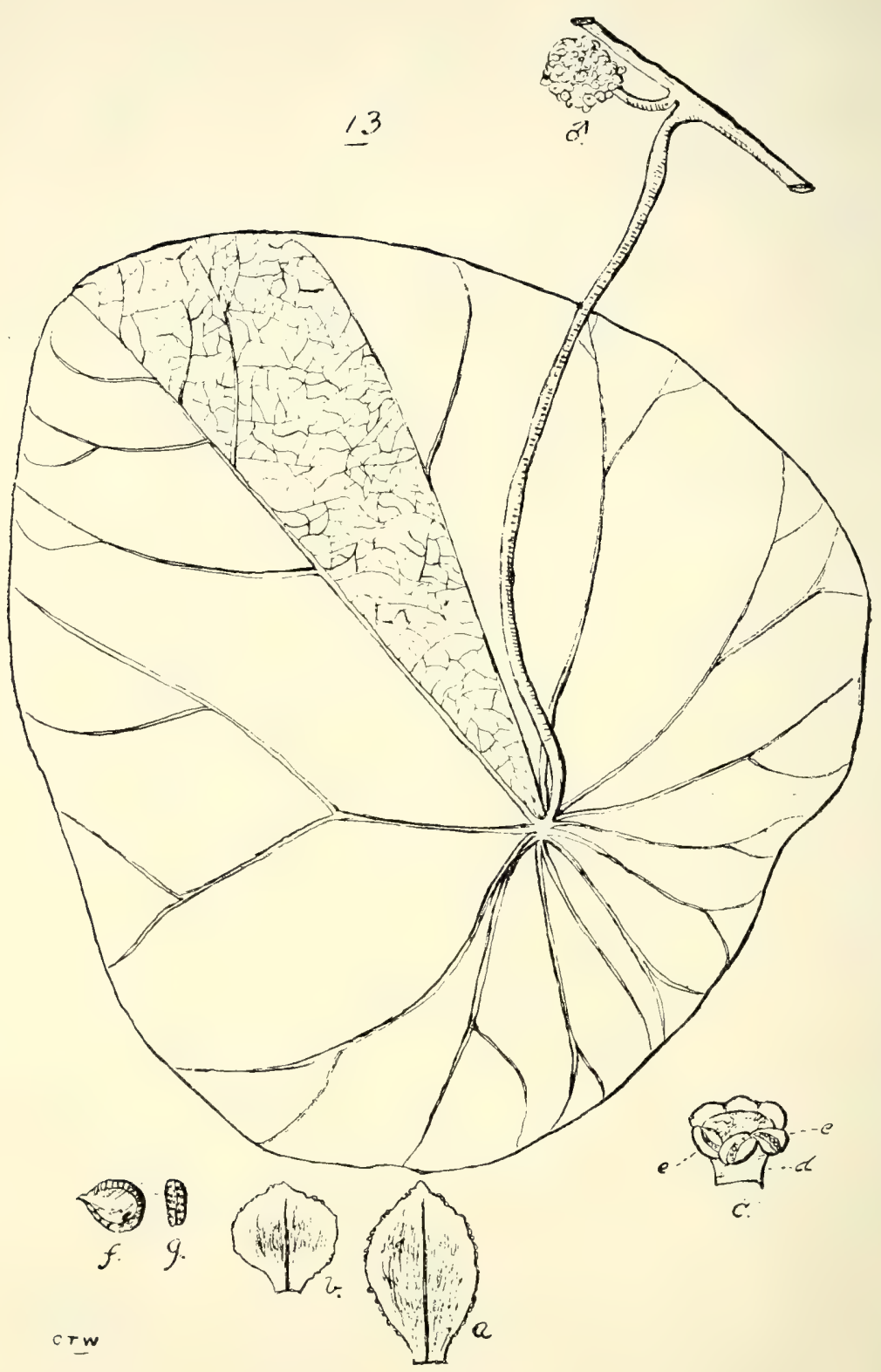

i3. Stephania Bancroftit, Bail.

(a) Sepal, (b) petal, (c) stamen, (d) staminal column, (e) anthers, (f) putamen, (g) putamen seen on its elge. (a)--(e) enl.; (f) and (g) nat. size. 
VI. NYMPHÆACEÆ.

31

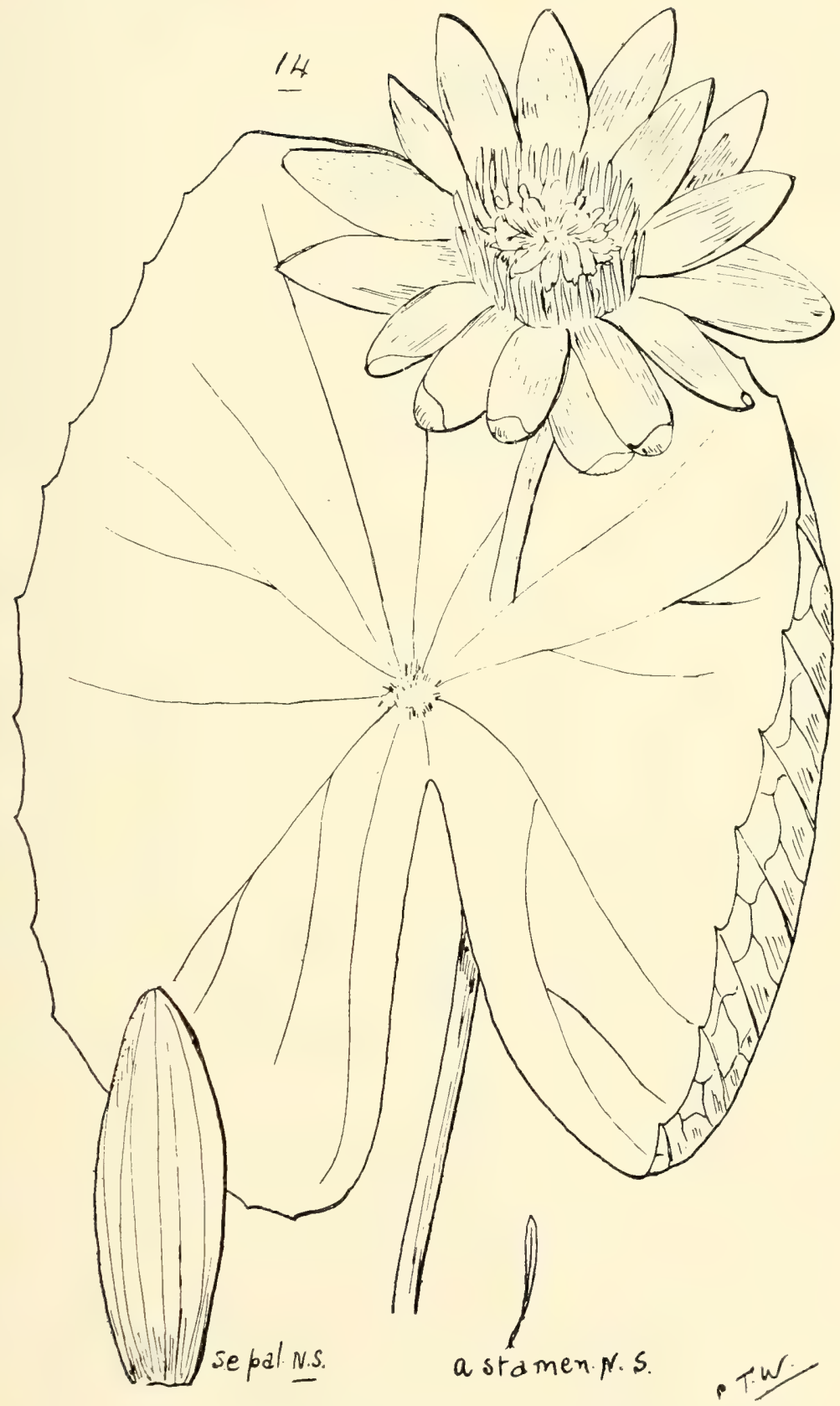

14. Nymfh.ea Lotus, Linn, var. australia, Bail. 
Microclisia, Benth. australis, Benth.

Adeliopsis, Benth. decumbens, Benth.

Husemannia, $F . v \cdot M$. protensa, F.v.M.

Pycnarrhena, Miers. australiana, F.v.M.

Pachygone, Miers. Hullsii, F.v.M. longifolia, Bail.

\section{Order VI.-NYMPH无ACE㞋.}

Suborder I.-CABOMBE正.

Brasenia, Schreb.

peltata, Pursh. $=$ Cabomba peltata, F. v. M.-Water Shield.

\section{Suborder II.-NYMIPHAE.}

Nymphæa, Limn.

gigantea, Hook.-Large Blue Water-Lily. "Kaooroo" of Cleveland Bay natives; rhizome and seed eaten.

Lotus, Linn., var. australis, Bail.-White Water-Lily. (Fig. I4.)

Brownii, Bail.-Small Blue Water-Lily. tetragona, Georgi.-Small Bluish Water-Lily. *flava, Leit.-Yellow Water-Lily. Florida.

\section{Suborder III.-NELUMBONEE.}

Nelumbium, Juss.-Nelumbo, Tournf.

speciosum, Willd.--Pink Water-Lily. Sacred Lotus;

"Pooma" of Cardwell natives (R.B.H.). Seeds eaten by the natives.

Alliance II.-PARIETALES.

\section{Order VII.-PAPAVERACEÆ.}

\section{Suborder PAPAVEREAE.}

Papaver, Linn.

\section{Tribe Eupapavere压.}

horridum, DC.-Native Poppy. (Fig. I5.) *hybridum, Linn.-Rough Poppy. Europe. *rhæas, Linn.-Field Poppy. Europe. 
*Argemone, Linn.

mexicana, Lim.-Prickly Poppy; a noxious weed. Flowers dark yellow. Seeds said to yield by ordinary pressure about 37 per cent. of oil, the acridity of which when applied to wood is supposed to repel the attacks of white ants and borers; also used for burning purposes and as a purge; poisonous if taken in quantity.

var. ochroleuca, Bail. - The kind with cream-coloured flowers; also a bad weed.

\section{Suborder FUMARIEZE.}

*Fumaria, Limn.

parviflora, Lam.-Small-flowered Fumitory. Europe.

officinalis, Limn.-The Common Fumitory. Medicinal; said to be laxative and diuretic.

\section{Order VIII.-CRUCIFERE.}

TRIBE I.-ARABIDEe.

Nasturtium, R. Br.-Fruit a Siliqua.

*officinale, $R . B r$.-Common Water-cress.

palustre, DC.-Marsh Water-cress. This plant was much used by the pioneers of South Australia as a salad, especially by those suffering from scurvy, so prevalent in those days.

Cardamine, Linn.

stylosa, $D C$.

hirsuta, Linn.-Bitter Cress.

eustylis, F.v.M. (Fig. I5 bis.)

heterophylla, Hook.

Tribe II.-AlyssineÆ.

Alyssum, Linn.-Madwort.

linifolium, Steph.

TRIBE III.-SISYMBRIEÆ.

*Sisymbrium, Linn.

officinale, Scop.-Hedge Mustard. Europe.

Iris, Linn.-London Rocket. Europe.

*Conringia, Reichb.

orientalis, Dum.-Hare's-ear Treacle Mustard. Europe.

C 


\section{Tribe IV.-Camelineæ.}

Blennodia, R. Br.

trisecta, Benth. (Fig. I6.)

eremigera, Benth.

lasiocarpa, F.v.M.- “Woombun-woombun” of St. George natives.

Cunninghamii, Benth.

cardaminoides, F. v. M.

canescens, $R . B r$.

nasturtioides, Benth.

Geococcus, J. Drumm.

pusillus, J. Drumm.

Stenopetalum, R. Br.

velutinum, F.v.M. (Fig. I7.)

lineare, $R$. $B r$.

nutans, $F . v . M$.

Tribe V.-Brassice在.

*Brassica, Linn.

nigra, Boiss.-Black Mustard. Europe.

campestris, Linn.

subspecies Napus, Linn.-The Turnip.

subspecies Rapa.-The Rape.

\section{Tribe VI.-Lepidinex.}

Capsella, Monch.

*Bursa-pastoris, Moench.-Shepherd's-purse. Europe.

Andræana, F.v.M.

humistrata, F. v. M.

Senebiera, Poir.

*didyma, Pers.-Wart Cress. Europe. Imparts a bad flavour to milk.

integrifolia, $D C$.

var. scaber, Bail.

Lepidium, Linn.-Fruit a Silicule.

strongylophyllum, F. v. M.

*ruderale, Linn.-Narrow-leaved Pepperwort. Europe.

foliosum, Desv. 
VII. PAPAVERACEA.-VIII. CRUCIFERÆ.
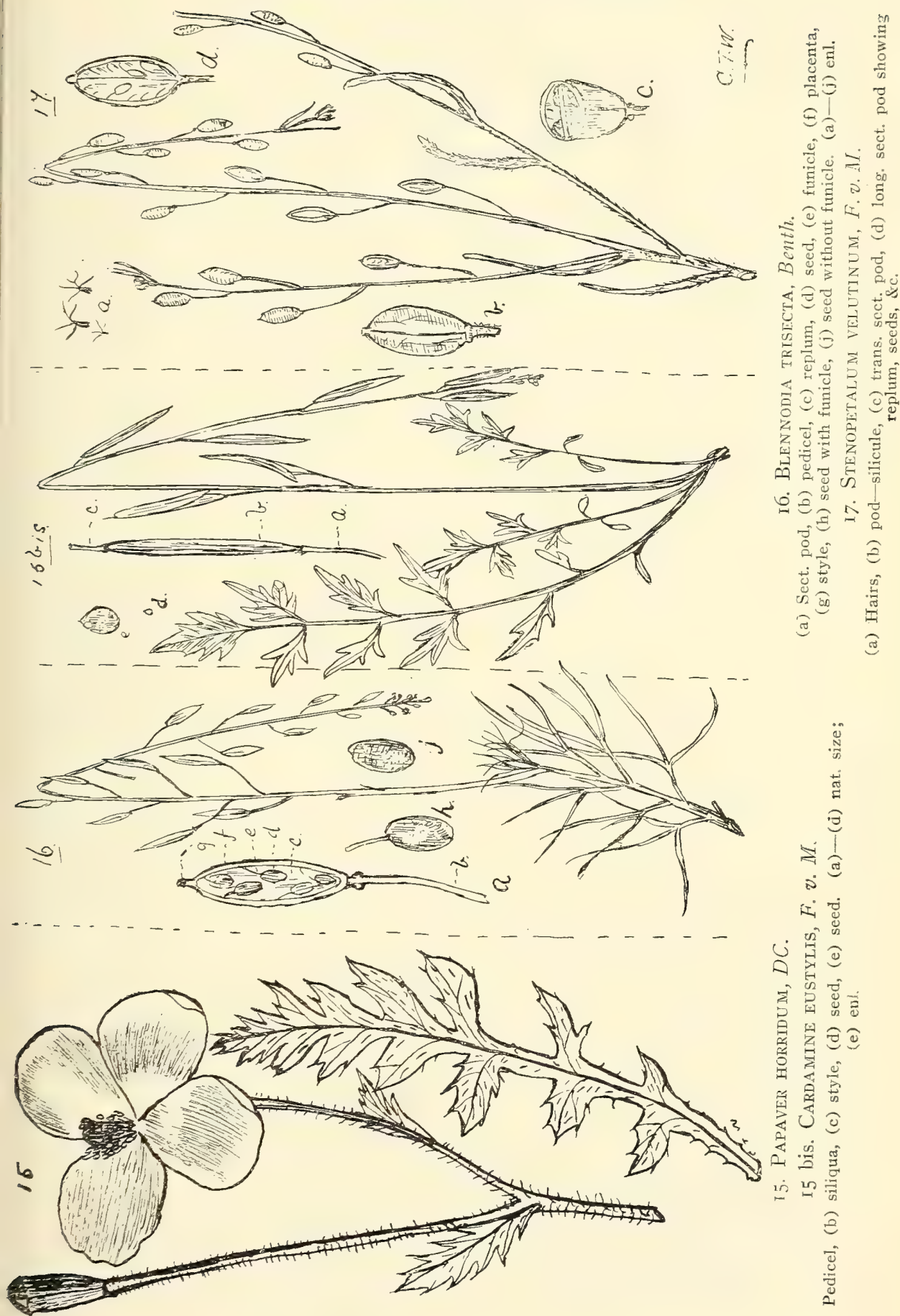

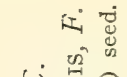

仓気き

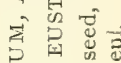

뵉으

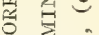

开我

舲

दे

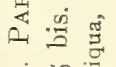

in 늠

ఏ

d 
Lepidium-contd.

monoplocoides, F. v. M.

papillosum, $F . v . M$.

*virginicum, Linn.-North America.

Thlaspi, Linn.

Tribe VII.-Thlaspidex.

cochlearinum, F.v.M.-Australian Pennycress.

Tribe VIII.-Cakilineæ.

*Rapistrum, Desv.

rugosum, Allioni--European weed introduced with birdseed.

Tribe IX.-Raphaneж.

*Raphanus, Linn.

Raphanistrum, Linn.-Jointed Charlock or Wild Radish Europe.

\section{Order IX.-CAPPARIDEA.}

Tribe I.-Cleomeæ.

Cleome, Linn.

oxalidea, $F \cdot v \cdot M$.

tet:andra, Banks.

grandiflora, $F \cdot v \cdot M$.

*pungens, Willd. - Spider-flower; a wide-spread Northern. weed. South America.

Polanisia, Rafin.

viscosa, $D C .=$ Cleome viscosa, Linn.-A troublesome tropical weed. In parts of India known as "Dog's Mustard." Used in diseases of the ear in Bombay.-Dr. Dymock. var. grandiflora, Benth.

Gynandropsis, $D C$.

pentaphylla, $D C$.

Muelleri, Benth. = Raperia cleomoides, F. v. M.

Tribe II.-CAPPAREe.

Capparis, Linn.-Most inland species considered good stock food, and those forming trees yield excellent cabinet wood.

Section Eucapparis.

umbellata, $R \cdot B r$.

lasiantha, R. Br.- "Wyjeelah" or "Thulla-kurbin" of Flinders River and Cloncurry natives. Fruit eaten by the settlers, known under the name of "Nipang."

quiniflora, $D C$. 


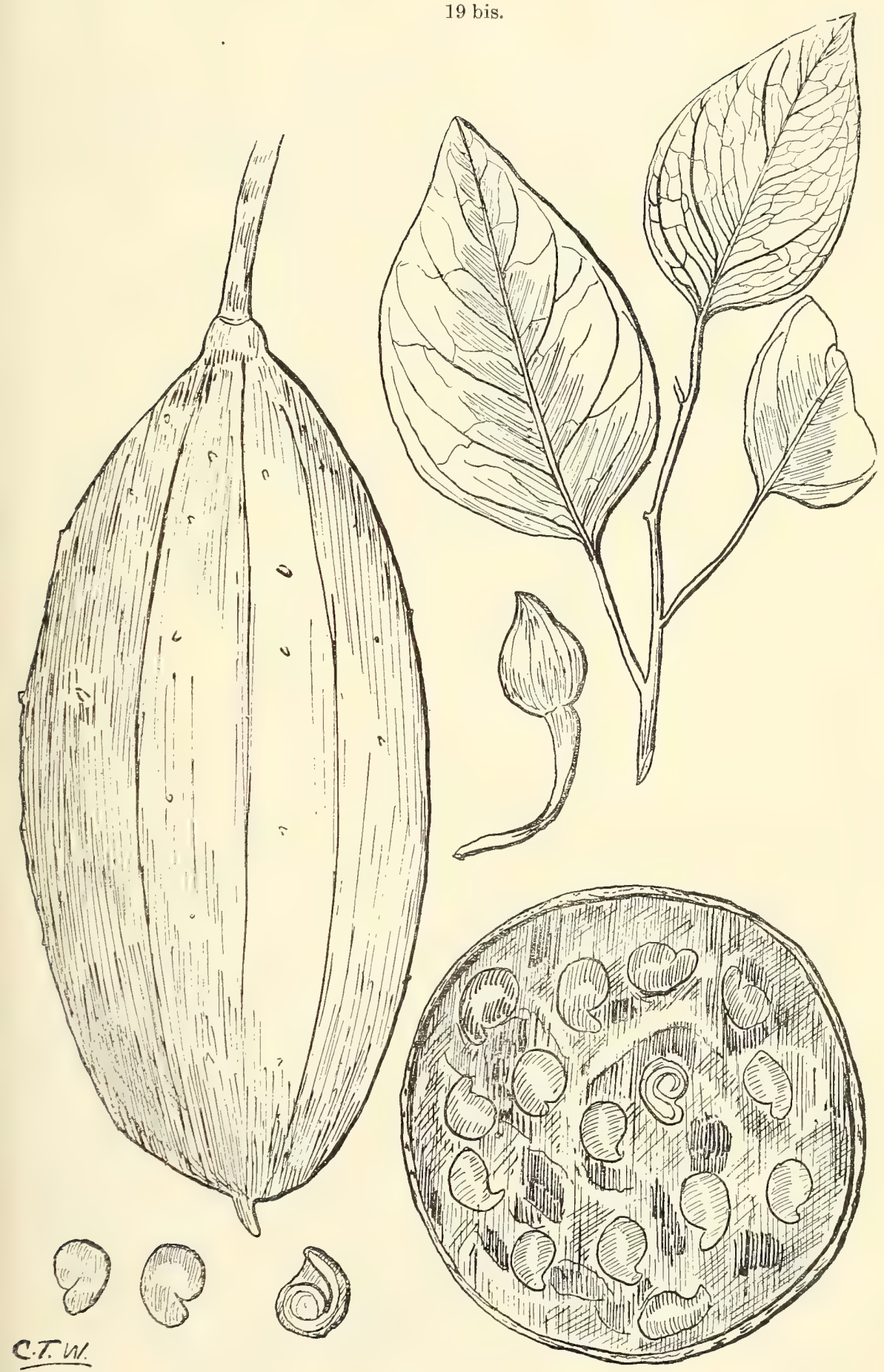

ig bis. Capparis ornans, $F, v, M$. 


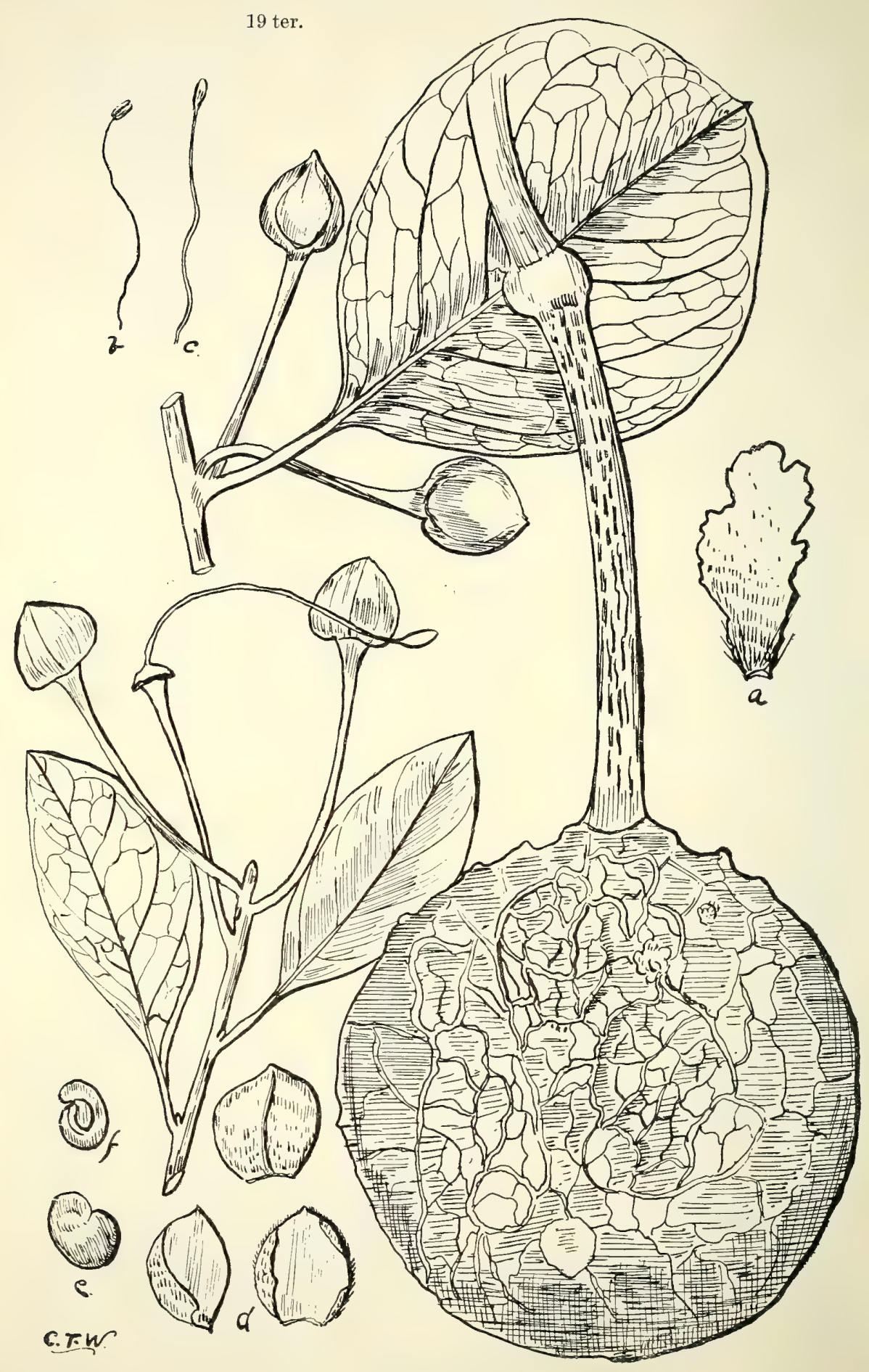

ig ter. Capparis areolata, Bail.

(a) Petal, (b) stamen, (c) stipes and ovary, (d) front, back, and side view of a calyx valve, (e) seed, (f) section of seed. 

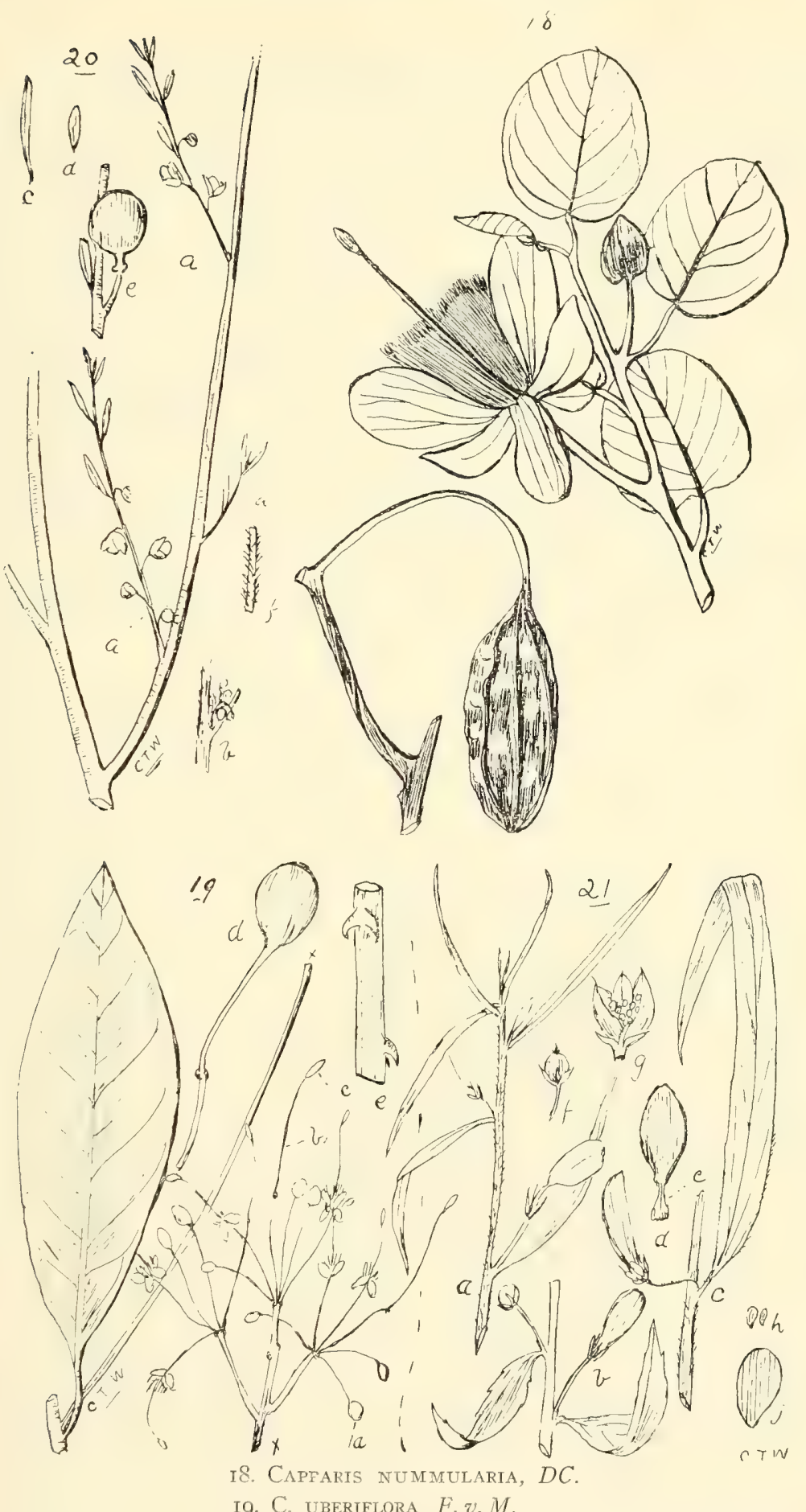

(a) F1. buds, (b) stipes, (c) ovary, (d) berry, (e) port. of a branch with stipular spines. (a)-(c) about $1 / 4$ nat. size; ((d) and (e) nat. size.

20. Apophyllum anomalum, $F$. v. $M$.

(a) Young shoots bearing leaves and flowers, (b) fls. in a cluster, (c) and (d) leaves, (e) fruit, (f) port. young shoot. (a)-(e) nat. size; (f) enl.

21. Ionidium suffruticosum, Ging.

(a), (b), and (c) forms, (d) lowest pet., (e) claw, (f) capsule, (g) capsule dehiscing, (h) seeds. (a) - (f) and (h) nat. size; (g) enl. 
Capparis-contd.

nummularia, DC.-_ Longullah" and "Mijah" of Cloncurry natives. (Fig. I8.)

uberiflora, F.v.M. (Fig. I9.)

sarmentosa, A. Cunn. Fruit edible. At Killarney, goes under the name of "Mulpup."

Section Busbeckia.

ornans, F.v.M.- "Manni " of Batavia River natives. (Fig. r9 bis.)

nobilis, F. v. M.-“Rarum" of North Queensland natives. Wood useful for engraving

canescens, Banks.-Native Pomegranate. "Mondoleir" of

North Queensland natives (the diminutive of "Mondo"). var. glauca, Benth.

areolata, Bail. (Fig. I9 ter.)

lucida, R. Br.- "Thoogeer" of Cloncurry and "Kurpuri”" of Cape Grafton natives.

Thozetiana, F.v.M.

Shanesiana, F.v. M.

Mitchelli, Lindl.-Darling Downs Pomegranate. "Mumble" and "Kam-doo-thal" of Cloncurry and "Mondo" of Rockhampton natives. The fruit, when ripe, supposed to resemble the heel of a native. It is also said that the township of Taroom is named after this plant, "Taroom" being its native name there. Wood excellent for carving. var. pubescens, Benth.

loranthifolia, Lindl.

umbonata, Lindl.

humistrata, F.v.M.- "Ko-par" of Cape Grafton ratives Apophyllum, F. v. M.

anomalum, F.v.M. (Fig. 20.)

\section{Order X.-VIOLARIEAE.}

Viola, Linn.

Tribe I.-Violex.

betonicæfolia, Sm.-The large-flowered Native Violet. var. alba, Bail.

hederacea, Labill.-Native Creeping Violet. 


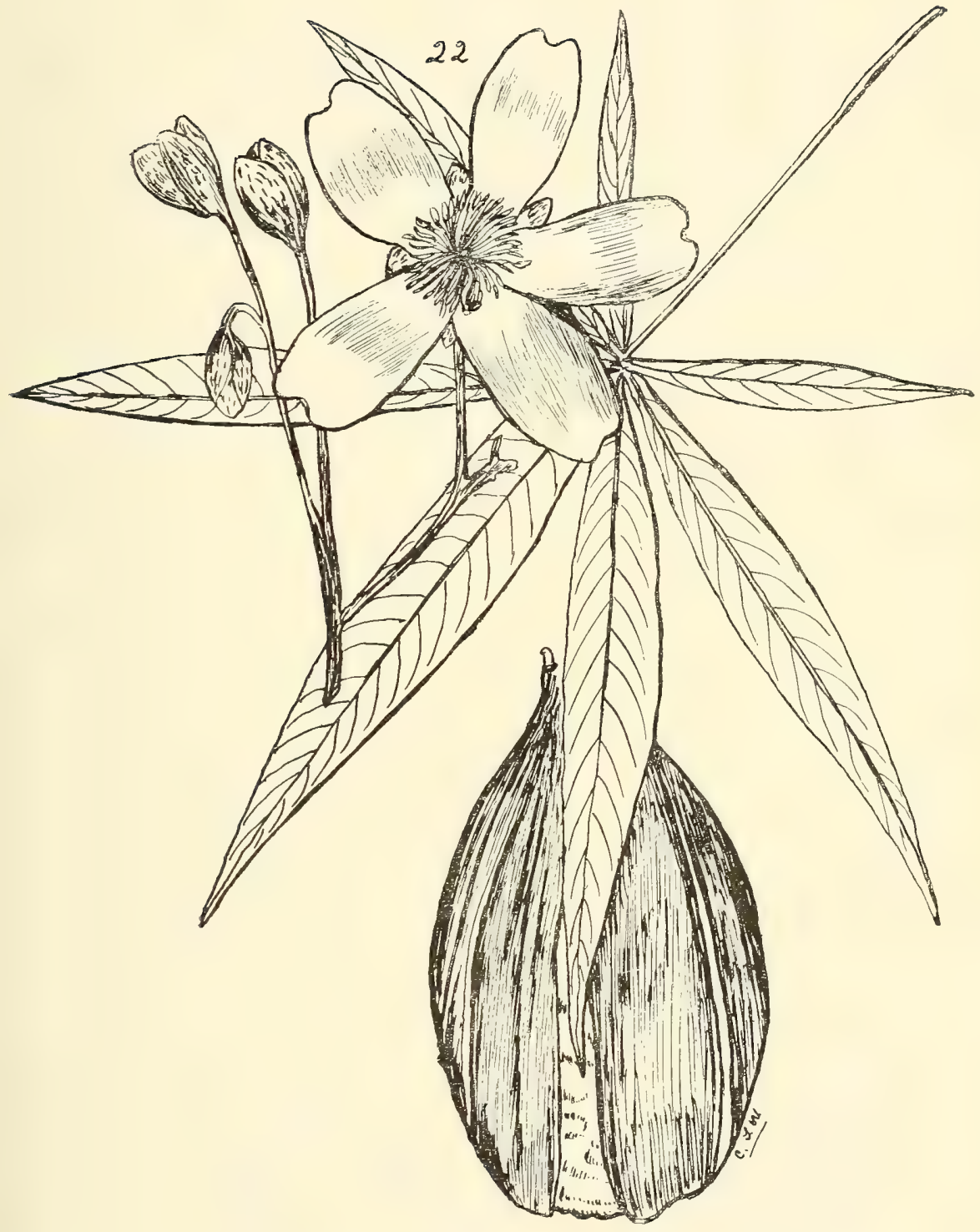

22. Cochlospermum Gregorit, $F, v . M$. 
Ionidium, Vent. $=$ Hybanthus (Jacq.), F. v. M.

suffruticosum, Ging.-Spade-flower. (Fig. 2I.)

aurantiacum, F.v.M.

Vernonii, $F, v \cdot M$.

filiforme, $F . v . M$.

\section{Order XI. - BIXINEÆ.}

Tribe I.--BixeÆ.

Cochlospermum, Kunth.

Gillivræi, Benth.-Yields a useful kapok.

Gregorii, F.v.M.-Yields a useful kapok. (Fig. 22.)

Tribe II.-Flacourtiex.

Scolopia, Schreb.

Brownii, F.v.M.-Wood pinkish, close-grained.

Xylosma, Forst.

ovatum, Benth.

Alliance III.-POLYGALINEE.

\section{Order XII.-PITTOSPOREE.}

Pittosporum, Banks.-The tree kinds furnish good cabinet woods, and the flowers of most are valuable for perfumery. Several species contain saponin.

rhombifolium, A. Cunn.-A good carving wood.

setigerum, Bail.

melanospermum, F.v.M.

var. ? lateralis, Benth.

undulatum, Vent.-Mock Orange; contains prussic acid and saponin.

ventulosum, $F \cdot v \cdot M$.

revolutum, Ait.

ferrugineum, Ait. $=$ P. ovatifolium, F. v. M.

Wingii, F.v.M.

rubiginosum, $A$. Cunn.

phillyræoides, $D C$.-Contains saponin. A slender-stemmed small tree yielding a close-grained wood.

Hymenosporum, $F . v . M$.

flavum, F.v.M.-Wood whitish, close in grain. Flowers abundant and very fragrant, suitable for perfumery. 
Bursaria, Cav.

spinosa, Cav.

incana, Lindl.-Wood suitable for engraving.

tenuifolia, Bail. (Fig. 23.)

Marianthus, Hueg.

Series Procumbentes.

procumbens, Benth.

Citriobatus, A. Cunn.

multiflora, A. Cunn.- " Kery" of Rockhampton natives

pauciflorus, A. Cunn.

lancifolius, Bail. (Fig. 24.)

Billardiera, $S m$.

scandens, Sm.-In some of the Australian States called "Apple Berry."

Cheiranthera, A. Cunn.

linearis, A. Cunn.

\section{Order XIII.-TREMANDREÆ.}

Tetratheca, Sm.

thymifolia, Sm. (Fig. 25.)

var. alba, Bail.

\section{Order XIV.-POLYGALEÆ.}

Salomonia, Lour.

oblongifolia, DC. (Fig. 26.)

Polygala, Limn.

japonica, Houtt. $=$ P. veronica, F. v. M.

leptalea, $D C$.

persicariæfolia, $D C$.

rhinanthoides, Sol.

arvensis, Linn.-Australian Milkwort.

stenoclada, Benth.

rar. stenosepala, Benth. 
Comesperma, Labill.

sphærocarpum, Steet:. (Fig. 28.)

volubile, Labill. $=$ C. gracile, Paxt., Mag. v. 145 .

var. album, Bail.

secundum, Banks.

retusum, Labill.

ericinum, $D C$.

var. patentifolium, Benth.

var. oblongatum, $R . B r$.

sylvestre, Lindl.

præcelsum, $F . v . M$.

defoliatum, $F, \tau . M$.

Xanthophyllum, Roxb.

Macintyrii, F.v.M.-Wood useful for cigar-boxes.

(Fig.

27.)

Alliance IV.C-CARYOPHYLLIN E.

\section{Order XV. - FRANKENIACEZE.}

Frankenia, Linn.

pauciflora, DC.-Australian Sea-heath. (Fig. 28 bis.)

var. serpyllifolia, Benth. (Fig. 29.)

var. thymoides, Benth.-A Saltbush. This small shrub furnishes the bushman with a plentiful supply of a nice ciean salt. Probably no other plant is so thickly encrusted with this useful material. (Fig. 30.)

\section{Order XVI.-CARYOPHYLLEÆE.}

\section{Tribe I.-SileneÆ.}

*Dianthus, Linn.

prolifer, Linn.-The Proliferous Pink. Europe.

Gypsophila, Linn.

tubulosa, Boiss.

*Saponaria, Linn.

vaccaria, Linn.-Cowherb. Europe.

*Lychnis, Linn.

githago, Lam.-Corn Cockle. Europe.

Silene, Linn.

gallica, Limn.-Catchfly. Europe. 

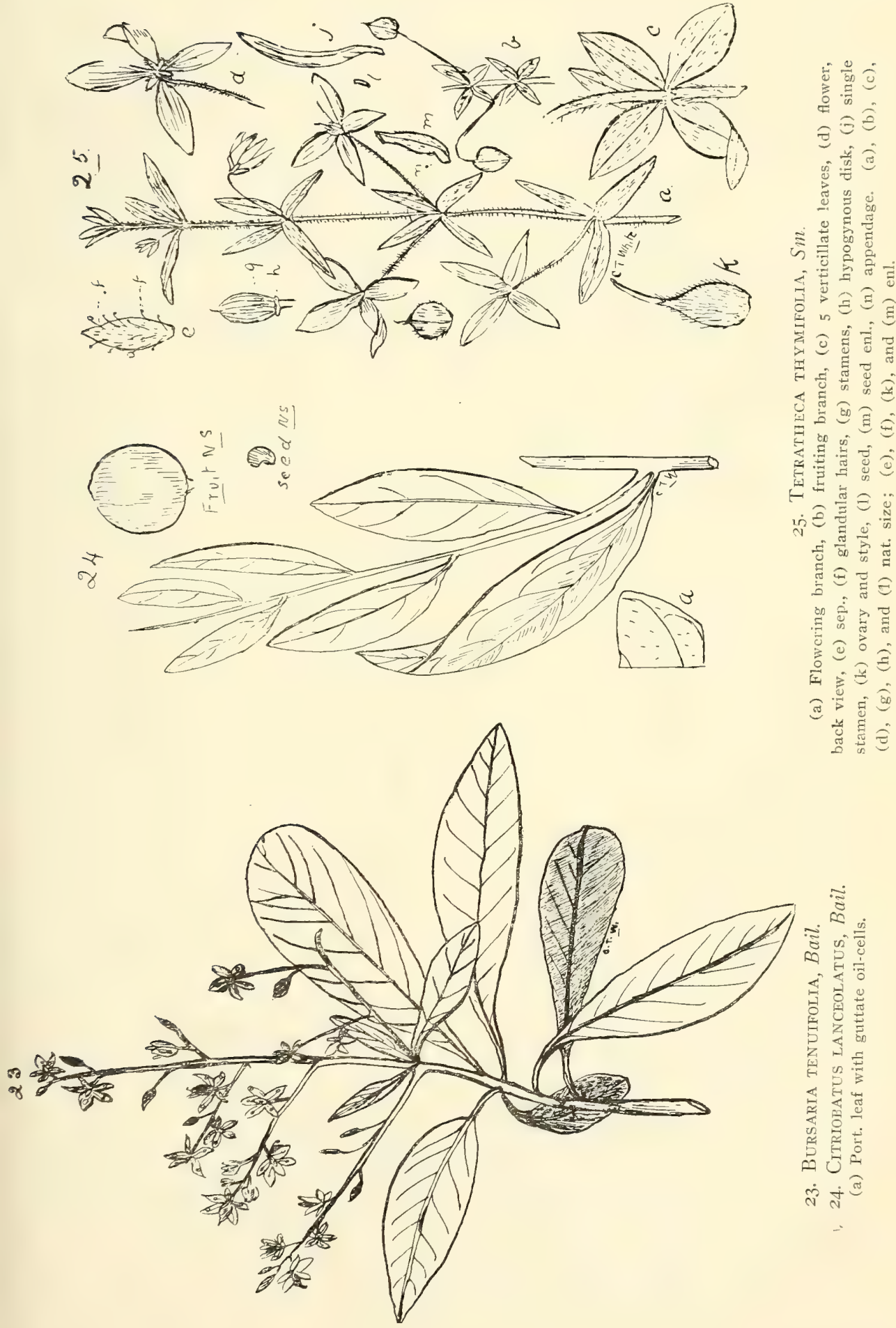


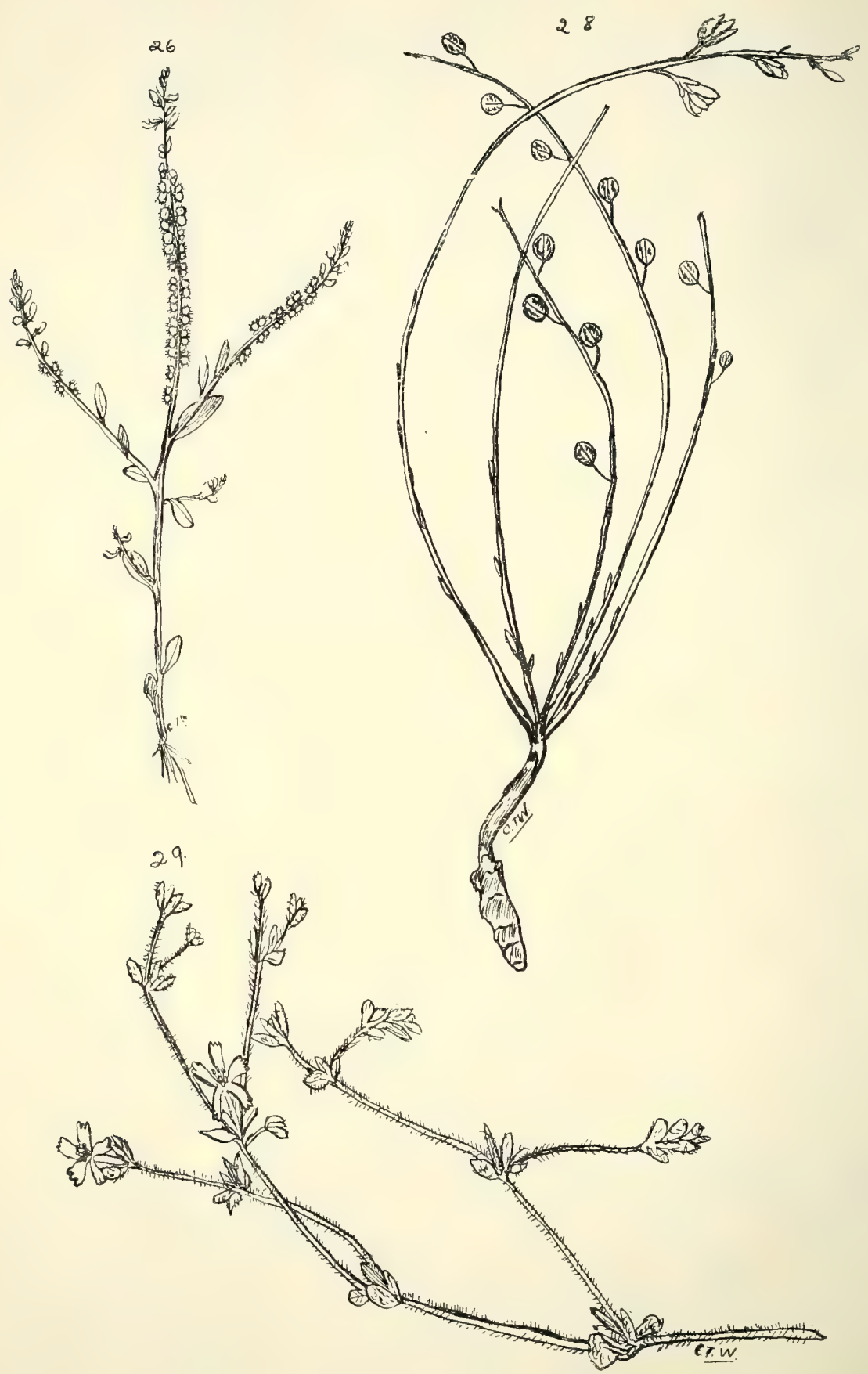

26. Salomonia oblongifolia, $D C$.

28. Comesperma spherocarpum, Steetz.

29. Frankenia pauciflora, $D C$., var. serpyllifolia, Benth. 
Cerastium, Linn.

Triee II.-Alsine艮.

vulgatum, Linn.-Mouse-ear Chickweed. Europe.

Stellaria, Linn.

pungens, Brongn.

glauca, Withering.

media, Linn.-Chickweed. Europe.

flaccida, Hook.

*Spergula, Limn.

arversis, Linn.-Corn Spurry; said to enrich the milk of cows fed on it in Europe.

* Spergularia, Pers.

rubra, Pers.-Common Sand Spurry. Europe. Sagina, Linn.

procumbens, Linn.-Pearlwort.

Tribe III.-Polycarpeæ.

Drymaria, Willd.

diandra, Blume.

Polycarpon, Linn.

tetraphyllum, Linn. $f$.

Polycarpæa, Lour.

Section Planchonia.

synandra, $F . v . M$.

longiflora, F. v. M.

var. leucantha, Benth.

Burtoni, Bail. (Fig. 3I.)

spirostyles, F.v.M.-Copper-plant of Herberton.

corymbosa, Lour.

Section Polycarpia.

breviflora, F.v.M. (Fig. 32.)

\section{Order XVII.-PORTULACEÆ.}

Portulaca, Linn.-Purslane plants. Fruit a Pyxidium.

oleracea, Linn.-Pigweed. "Thukouro" of Cloncurry natives.

The whole plant devoured by fowls; the seed used for

food by the natives; a splendid substitute for spinach.

var. grandiflora, Benth.

var. *sativa, DC.--A European salad-plant. Growth erect. 
Portulaca-contd. australis, Endl._."Me-mama” of Batavia River natives. Rootstock roasted and eaten.

napiformis, F.v.M. - "Karedilla" of Cloncurry natives.

Root tuberous, eaten; seeds eaten.

filifolia, F.v.M.-Root tuberous.

digyna, $F . v . M$.

Armitii, F. v. M.

bicolor, F.v.M.-Heart-plant. Root tuberous. (Fig. 33.)

oligosperma, F.v.M.

Calandrinia, H. B. et $K$.

uniflora, $F . v . M$.

balonensis, Lindl.

polyandra, Benth.

quadrivalvis, $F . v . M$.

pusilla, Lindl.

voiubilis, Benth.

calyptrata, Hook,

pumila, F. v. $M$.

pogonophora, $F . v . N_{\text {. }}$.

ptychosperma, $F . v . M$.

spergularina, F.v.M. (Fig. 34.)

pleiopetala, F. v. M.

*Talinum, Adans.

patens, Willd.-Garden weed introduced from South America.

Alliance V.-GUTTIFERALES.

\section{Order XVIII.-ELATINE $æ$.}

Elatine, Linn.-Waterwort.

americana, Arn., var. australiensis, Benth.

Bergia, Linn.

ammannioides, Roth. (Fig. 35.)

perennis, $F . v . M$.

\section{Order XIX.-HYPERICINEA.}

Hypericum, Linn.-St. John's Wort.

gramineum, Forst. (Fig. 36.)

japonicum, Thunb. (Fig. 37.)

D 

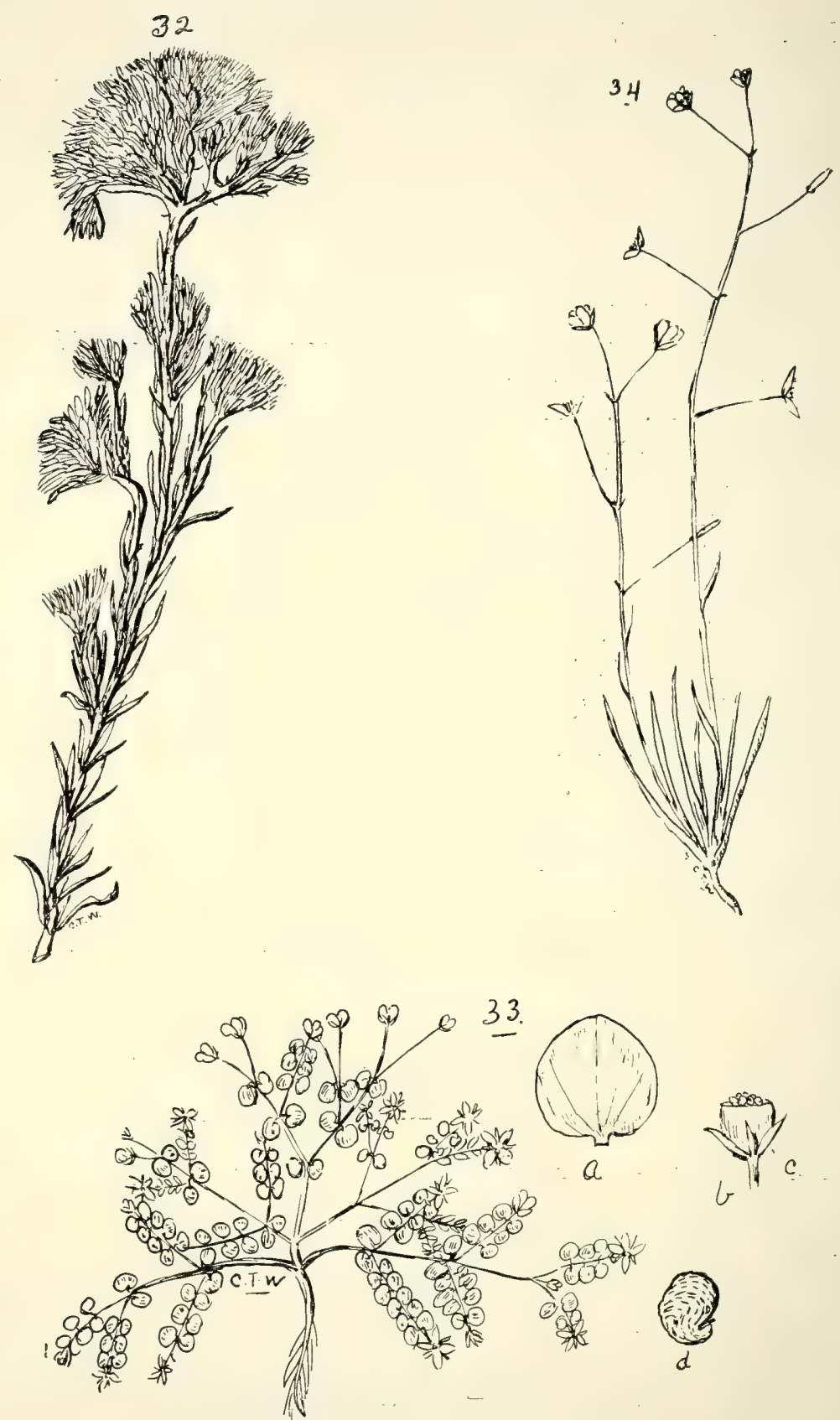

32. Polycarpeta breviflora, $F . v . M$.

33. Portulaca bicolor, $F$. $v$. $M$ :

(a) Leaf, (b) capsule, (c) floral leaves, (d) seed. All enl.

34. Calandrinia spergularina; $F . v . M$. 
XVIII. ELATINEÆ.-XIX. HYPERICINE王-XX. GUTTIFER杘. 51
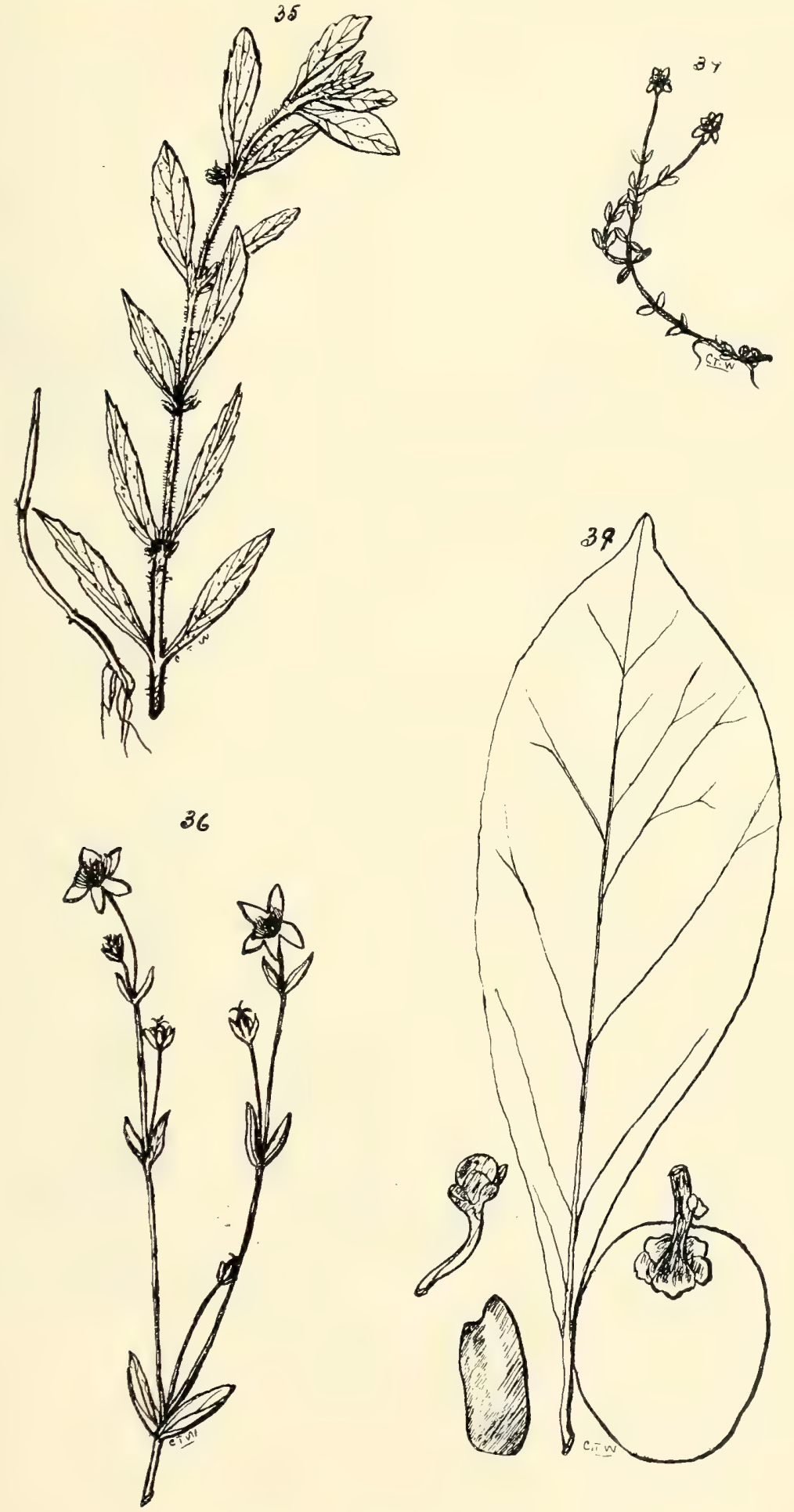

35. Bergia ammanNiotdes, Roth.

36. Hyiericum gramineum, Forst.

37. H. JAPONICUM, Thunb.

39. Garcinia Cherryi, Bail. 


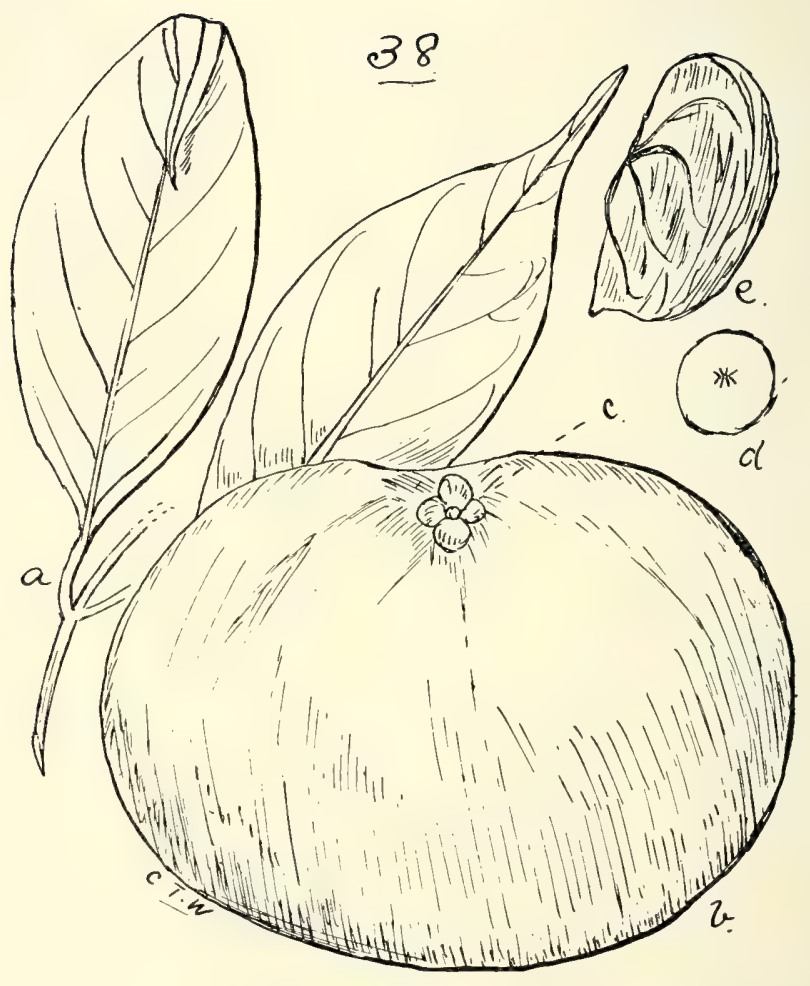

38. Garcinia Mestoni, Bail.

(a) Leaves, (b) fruit, (c) calyx, (d) the eight stigmatic lobes, (e) seed with its integument. All nat. size. 
40

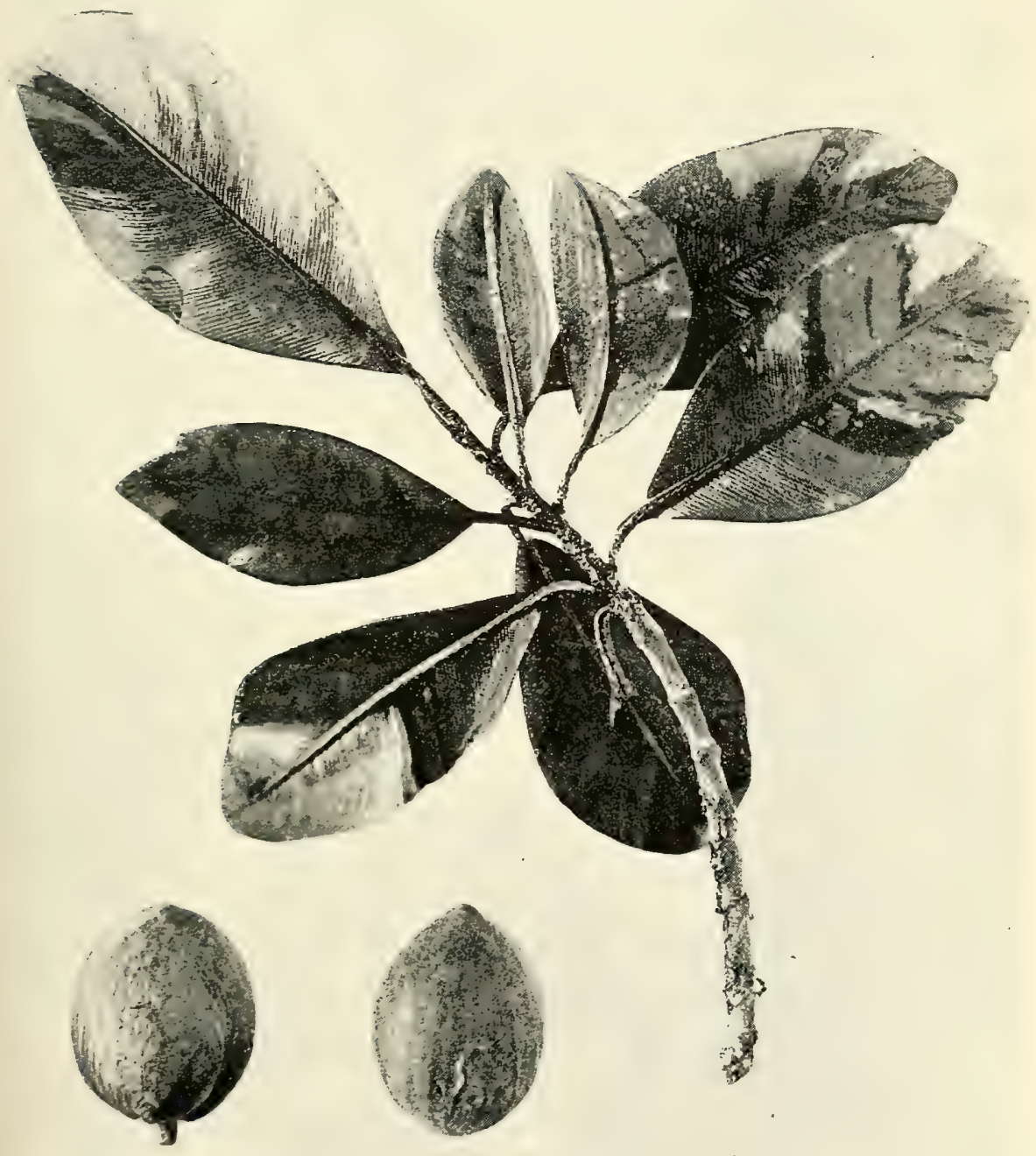

40. Calophylum costatum, Bail. 


\section{Order XX.-GUTTIFERA.}

Garcinia, Linn.

Tribe I.-Garcinieæ.

Mestoni, Bail.-Meston's Mangosteen; a useful fruit. (Fig. 3ช.)

Warrenii, F.v.M., Vict. Nat. Nov. I89ז; Bail., Q1. Agri. Journ. Mar. Igo6.

Cherryi, Bail. (Fig. 39.)

Kayea, $W$ all.

Tribe II.-CALOphyllex.

Larnachiana, $F . v: M$.

Calophyllum, Linn.

inophyllum, Linn.-Alexandrian Laurel, Tacamahac tree, Indian Doomba Oil tree. The seeds are said to contain 60 per cent. of a fixed oil. "Ulee-ree" of Dunk Island and "Wurri" of Cardwell natives. One of our most valuable coastal woods.

tomentosum, Wight.-Keena or Poon Spar tree.

costatum, Bail.-Evelyn Teak. Wood useful. (Fig. 40.)

sustralianum, F. v. M.

\section{Order XXI.-TERNSTRGEMIACEÆ.}

Tribe Saurauje⿸.

Saurauja, Willd.

Andreana, Oliver $=$ Dillenia Andreana, F.v. M., Fragm. v. 175. (Fig. 4r.)

Alliance VI.--MALV ALES.

\section{Order XXII.-MALVACEÆ.}

Many of this Order yield fibres in great quantity and valuable material suitable for paper-making:

Lavatera, Limn.

Tribe I.-Malvez.

plebia, Sims.-Tall Mallow. The thick roots of this plant were largely used for food by the Adelaide tribe of aborigines, after being cooked in their underground ovens.

* Malva, Limn.-The fruit is a carcerulus.

rotundifolia, Linn.-Mallow. Europe.

verticillata, Linn.-Mallow. Europe.

sylvestris, Linn.-Mallow. Europe.

parviflora, Linn.-Mallow. Europe. 


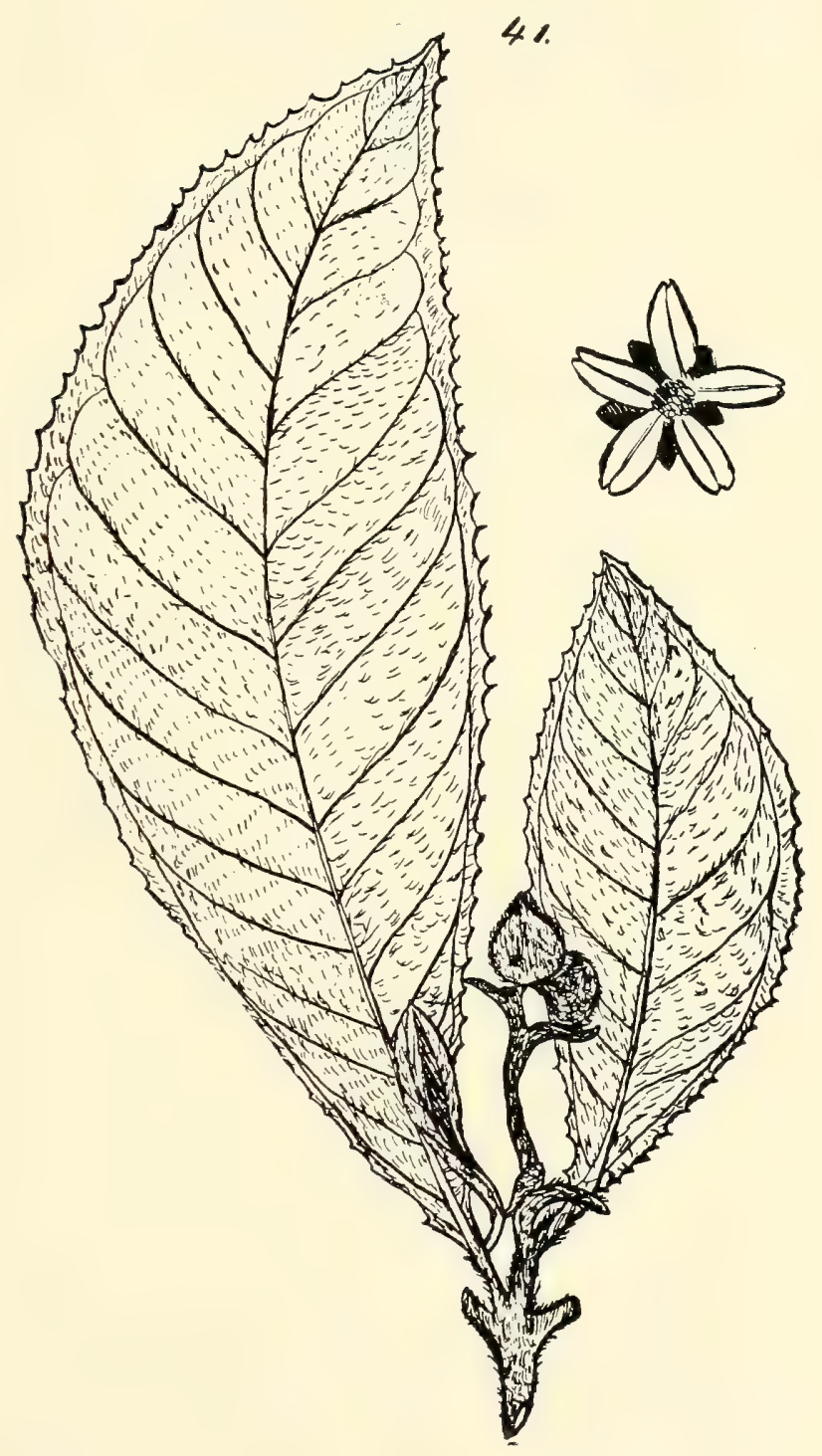

41. Saurauja Andreana, Oliver. 

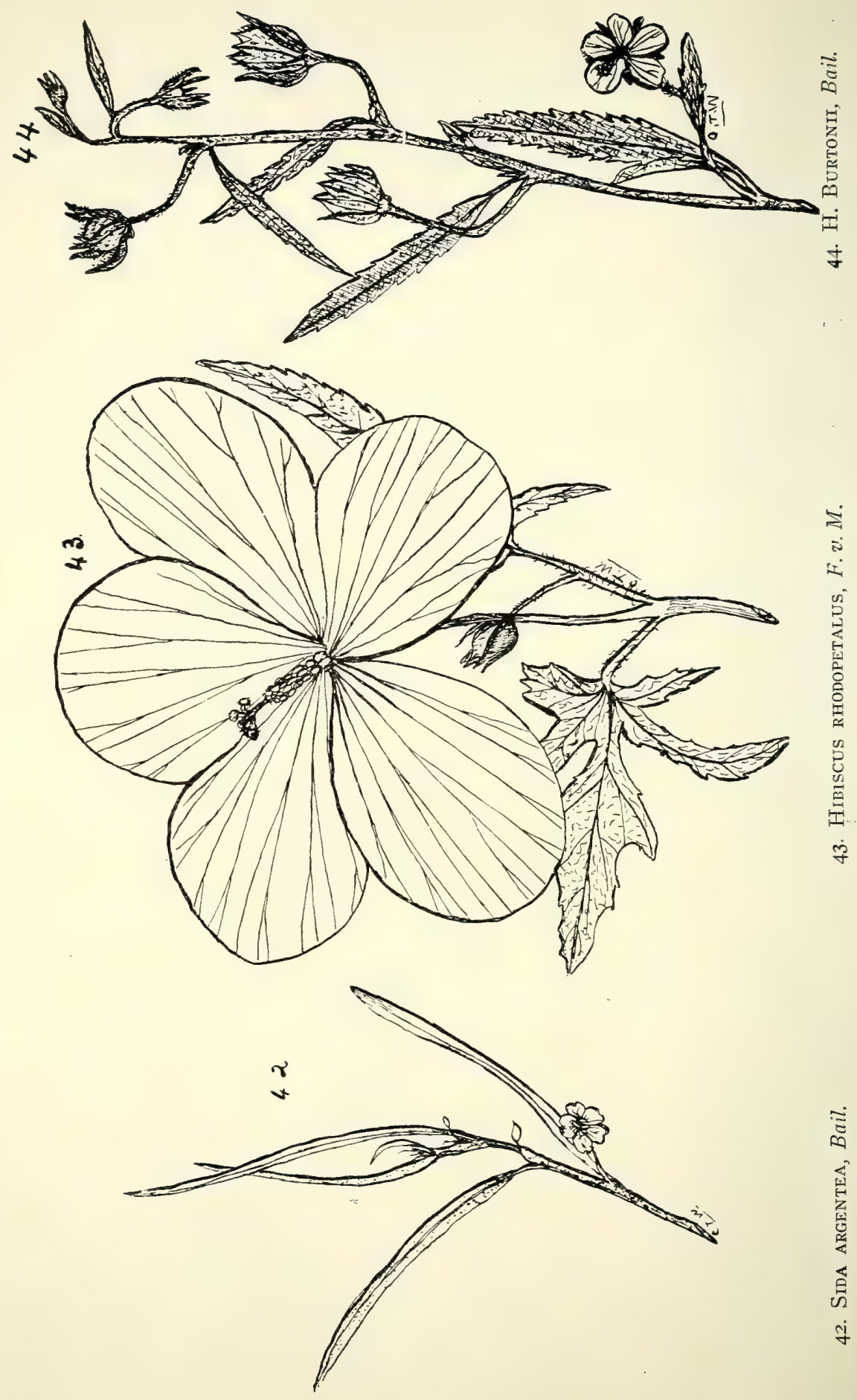
*Malvastrum, A. Gray. spicatum, A. Gray.

tricuspidatum, A. Groy.--Variegated forms of this plant are often met with.

Plagianthus, Forst.

glomeratus, Benth. = Laurencia glomerata, Hook.

microphyllus, F.v.M.

Sida, Limn.-All yield good fibre.

corrugata, Limn.

var. orbicularis, Benth.

var. ovata, Benth.

var. angustifolia, Benth.

var. trichopoda, Benth.

var. goniocarpa, F.v.M.

intricata, F. $\% . M$.

Spenceriana, F.v.M.

argentea, Bail. (Fig. 42.)

macropoda, F.v.M.

var. cardiophylla, F.v.M.

virgata, Hook.

petrophila, F.v.M.

cryphiopetala, $F \cdot v \cdot M$.

subspicata, F. $v . M$.

pleiantha, F.v.M.

spinosa, Linn.

rhombifolia, Lim.$=S$. retusa, Linn.-Sida-weed. The fruit has been thought injurious to young fowls, by the sharppointed carpels causing internal inflammation.

var. incana, Benth.

cordifolia, Linn.

var. mutica, Benth.

platycalyx, F. v. M.

inclusa, Benth.

Abutilon, Gartn.-Lantern-flower. All yield good fibre.

tubulosum, Hook.

var. breviflorum, Benth.

leucopetalum, F.v.M.

Mitchelli, Benth.

var. mollissima, Benth.

micropetalum, Benth.

otocarpum, F.v.M.

subviscosum, Benth.

graveolens, $W$. et Arn. 
Abutilon-contd.

oxycarpum, F.v. $M$.

var. malvæfolium, Benth.

muticum, G. Don.

Cunninghamii, Benth.

indicum, G. Don.

Fraseri, Hook.

var. halophilum, Benth.

auritum, G. Don.

*Modiola, Moench.

multifida, Monch. $=M$. caroliniana, Linn., of America.-A wide-spreading weed, first introduced into Queensland about 40 years ago in the packing around young fruit.trees from America.

Urena, Linn.

TRIBE II.-URENEÆ.

lobata, Linn.-A good fibre-plant.

var. grandiflora, Benth.

Armitiana, F,v. $M$.

Pavonia, Cav.

hastata, Cav.-A South American species, doubtful if really indigenous in Queensland.

Tribe III.-Hibisce..

Hibiscus, Linn.-All yield good fibre.

Bracteoles free.

ficulneus, Linn.- "Cooreenyan" of Cloncurry natives.

rhodopetalus, F.v.M.-A white flowering form of this has been met with at Mackay. (Fig. 43.)

Manihot, Limn.

Notho-Manihot, F. v. M.

trionum, Linn. $=H$. tridactylites, Lindl., $H$. africanus or Bladder Ketmia.

brachysiphonius, F. v. M.

microchlænus, F. v. $M$.

Burtonii, Bail. (Fig. 44.)

zonatus, $F . v . M$.

Elsworthii, F. ข. $M$.

radiatus, Cav._- Quootham" of Port Curtis natives.

divaricatus, Grah._- "Ngar-golly" of Cloncurry natives,

"Ithnee" of Mitchell district.

var. luteus, Hoch.$=H$. radiatus var. luteus, F. v. M.

Fitzgeraldi, F. v. M.

heterophyllus, Vent.-Native Rosella. Native name, "Batham," of North Queensland. This, Bentham says, is a form Cunningham named $H$. Margerice. 


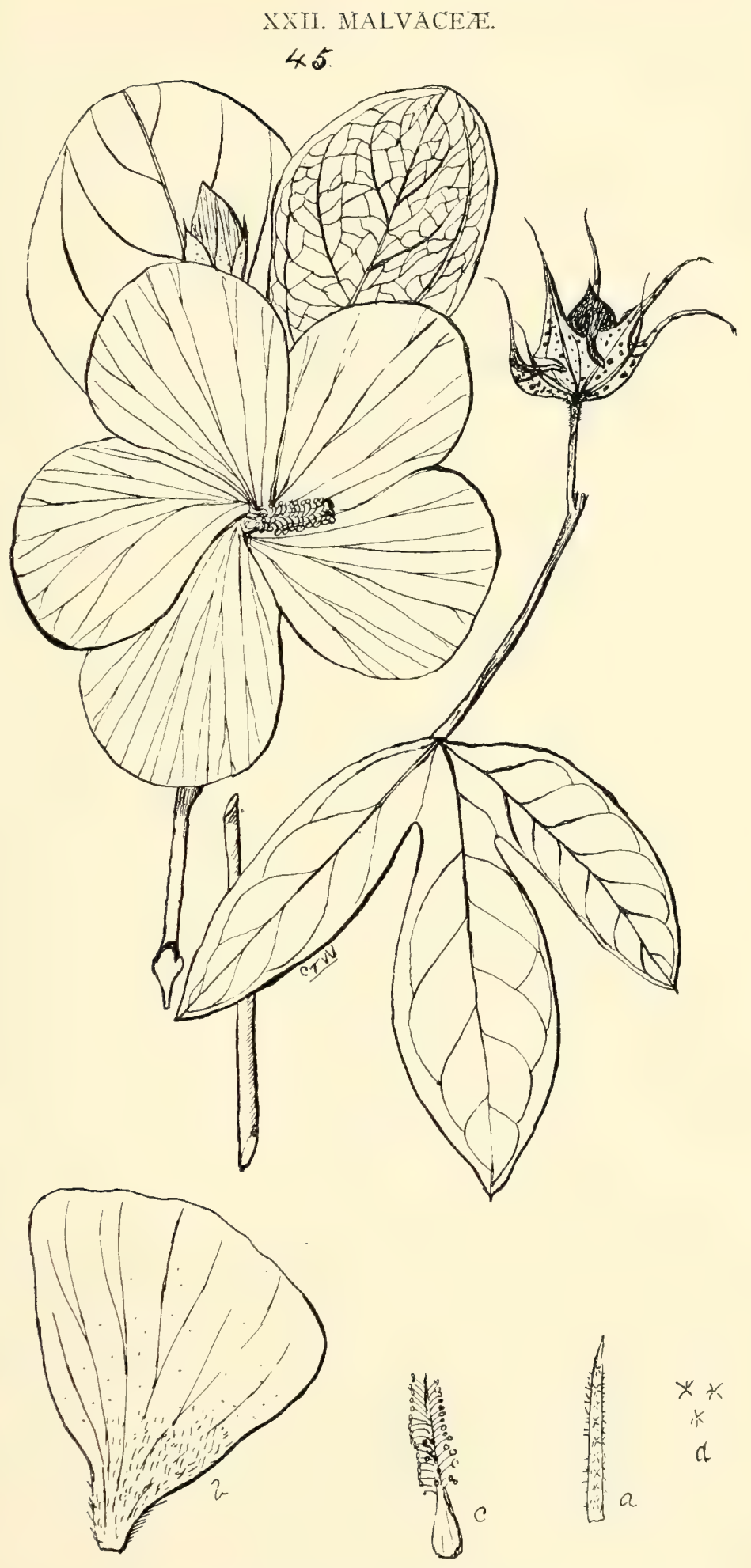


45 bis.

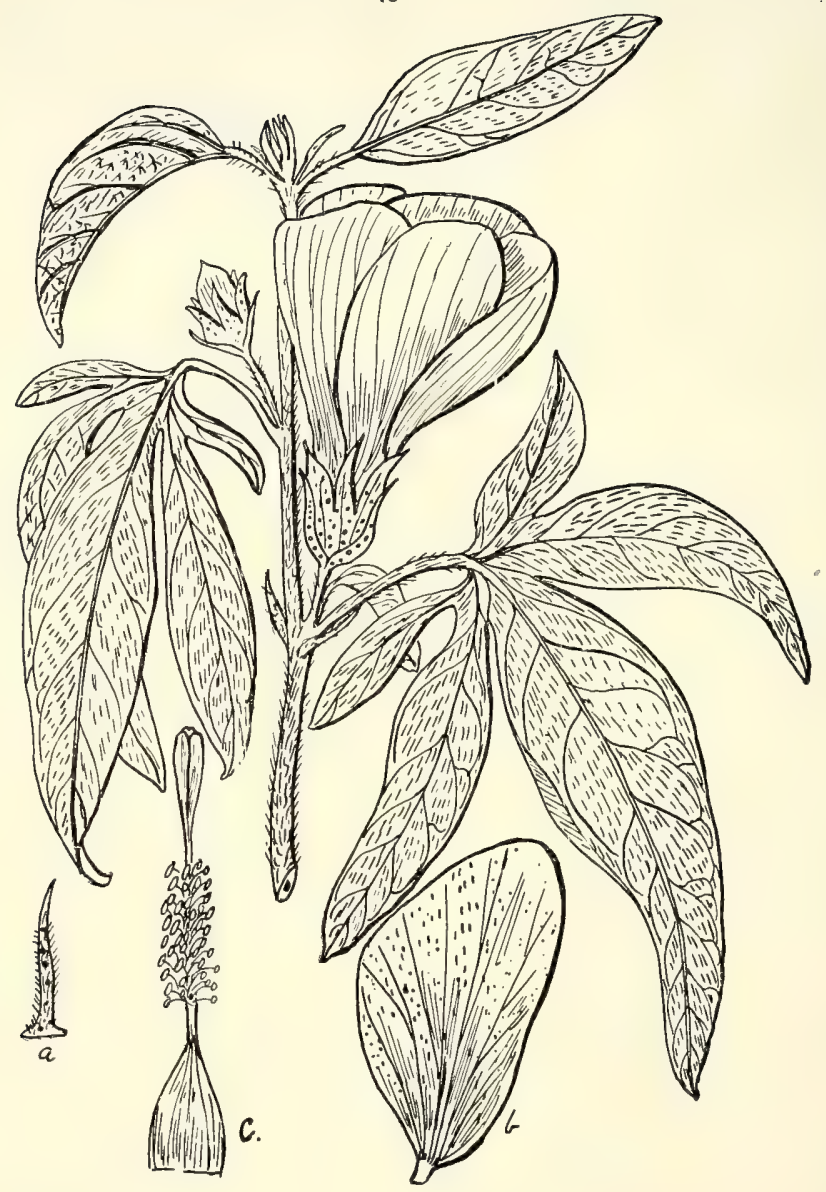

45 bis. Fugosia pedata, Bail.

(a) Bracteole, (b) petal, (c) staminal column. (a) and (c) enl.; (b) nat. size. 
Hibiscus-contd.

diversifolius, Jacq.

splendens, Fraser.

vitifolius, Limn.

panduriformis, Burm._- Bee-allo" of Mitchell River natives.

Normani, F. v. M.

Krichauffii, F. v. M.

phyllochlænus, F. v. M.

Bracteoles united.

Sturtii, Hook.

var. grandiflora, Benth.

var. Muelleri, Benth.

var. Sturtii, Benth.

var. campylochlamys, Benth.

var. platychlamys, Benth.

tiliaceus, Limn.-Cotton-tree. Plants bearing variegated foliage are sometimes seen. "Talwalpin" of Moreton Bay and "Man-djar" of Dunk Island natives. A beautifully marked cabinet-wood of an invisible green.

Lagunaria, G. Don.

Patersoni, Don., var. bracteata, Benth.-Wood whitish, stitable for cabinet-work.

Fugosia, Juss.

australis, Benth. = Gossypium australe, F. v. M. (Fig. 45.)

pedata, Bail. (Fig. 45 bis.)

Thespesia, Corr.

populnea, Corr.-Indian Tulip-tree. Said to contain, in bark and wood, tannin and a fine red colouring matter. The seed is also said to furnish a thick oil.

Gossypium, Linn.

Sturtii, F.v.M.-Australian Cotton.

*herbaceum, Linn.-This and other kinds of the "Cottonbush" are sometimes met with as strays from cultivation.

Bombax, Linn.

Tribe or Suborder IV.-Bombaceж.

malabaricum, DC.-Silk-cotton tree; deciduous. Yields kapok. On the Mitchell River the natives form their koolamons from the wood of this tree. True "kapok" is the product of Eriodendron anfractuosum, DC. 


\title{
Order XXIII.-STERCULIACEÆ.
}

\author{
Tribe I.-STerculie.
}

Sterculia, Linn. (Including Brachychiton, Schott.)

quadrifida, R. Br.-Seeds edible. "Ko-ral-ba " of Cooktown, "Gorarbar" of Cape Bedford, and "Ku-man" of Atherton natives. "Convavola" of some Northern natives. Fibre from bark used for making kangaroo nets (Roth). (Plate I., Frontispiece.)

laurifolia, F. v. M.

Garrawayæ, Bail._-“Morna” of Palmer River natives. ramiflora, Benth._- An-ji-ur” of Princess Chárlotte Bay.

vitifolia, Bail.

Bidwilli, Hook.

discolor, F. v. M.

lurida, F. v. $M$.

trichosiphon, Benth-Broad-leaved Bottle-tree. "Ketey" of Northern natives. Root of young trees eaten, also seeds. (Plate III.)

acerifolia, A. Cunn.-Flame-tree. The leaves of this deciduous tree are usually lobed, but at times they are seen quite entire, as shown in trees cultivated in Brisbane Botanic Gardens and in a beautiful painting by Mrs. F. C. Rowan made from Northern plants.

diversifolia, G. Don.-Kurrajong. “Kalan” of Palmer River natives; also, of other Northern natives, "Dewtie" and "Taromeo."

caudata, Hew.- "Kel-lan" of Princess Charlotte Bay natives. (Fig. 46.)

rupestris, Benth.-Narrow-leaved Bottle-tree. "Binkey" of natives, by whom it is largely used for food.

Tarrietia, Blume.

argyrodendron, F.v.M._- Boiong” of natives. Stavewood. var. grandiflora, Benth.

var. trifoliolata (F. v. M.), Bail.

var. angustifolia, Bail.-Wood useful for tool-handles.

var. peralata, Bail._- "Peirir" of Upper Barron River, Red Beech of Johnstone River, and Crow's-foot Elm of Atherton. Wood useful for cabinet-work; darkcoloured.

var. macrophylla, Bail.

actinophylla, Bail.-The wood has been considered excellent for making billiard-cues. 
PLATE III.

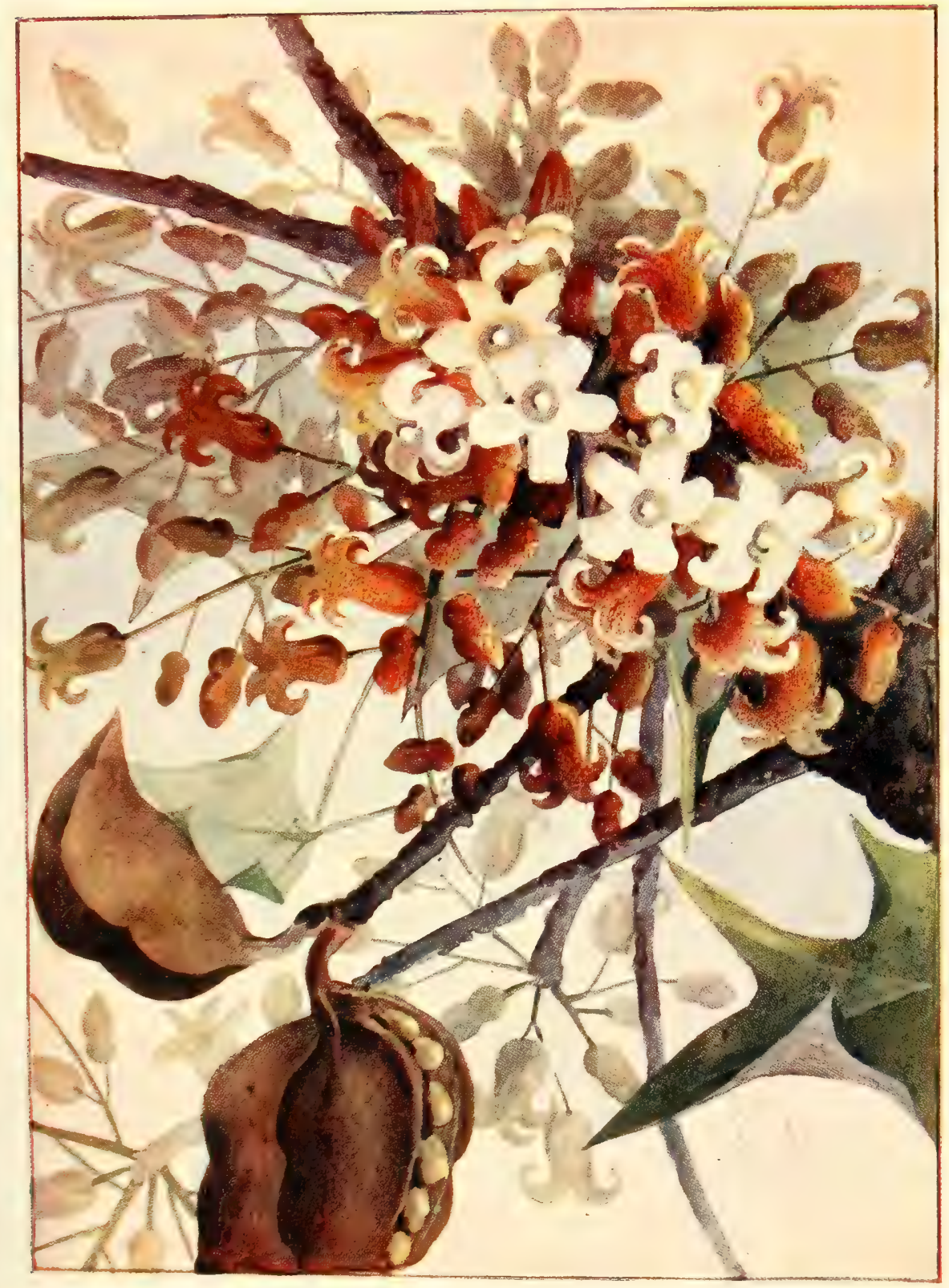

Sterculia trichosiphoN, Benth. 


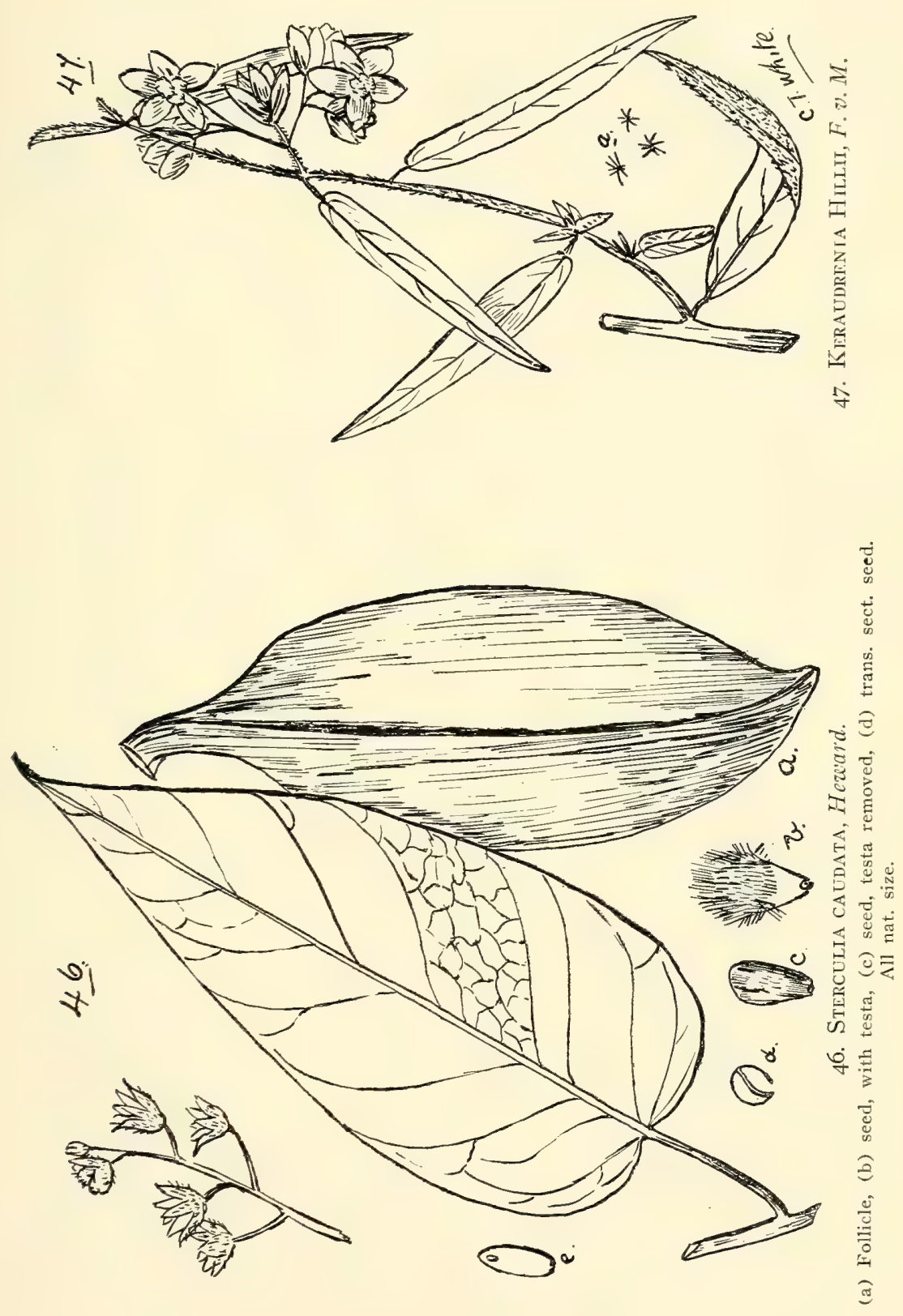


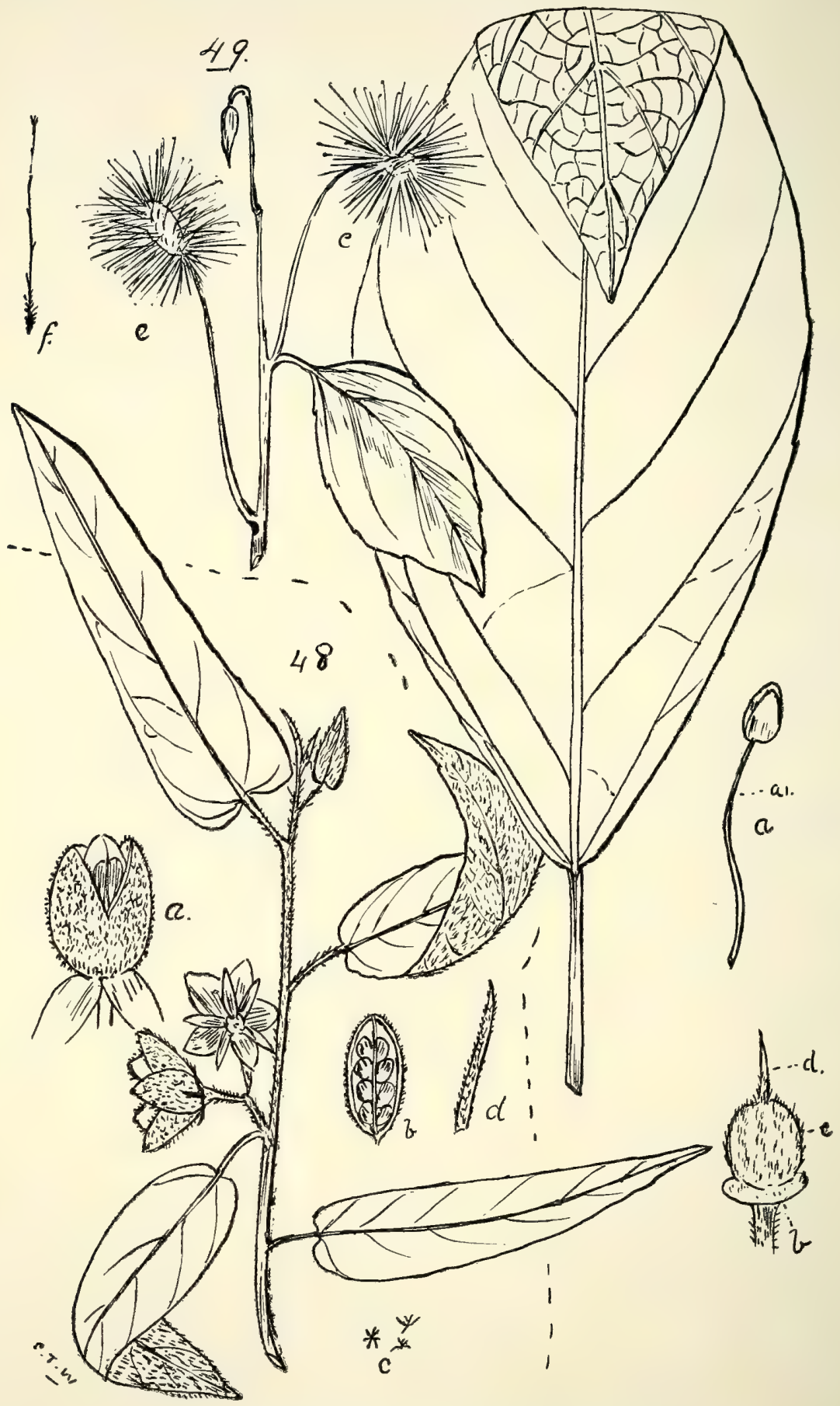

48. Hannafordia Shanesit, F. $v . M$.

(a) Capsule, (b) long. sect. capsule, (c) stellate hairs from capsule, (d) bracteole. (a) and (b) nat. size; (c) and (d) enl.

49. Sloanea Langit, $F . v . M$.

(a) Stamen, (a $\left.{ }^{1}\right)$ filament, (b) disk, (c) ovary, (d) style, (e) capsule, (f) a seta. (a), (b), (c), (d), and (f) enl.; (e) nat. size. 
Heritiera, Ait.

littoralis, Ait.-Looking-glass tree or Red Mangrove. A useful wood for boat-building and cabinet-work. The bark of this tree in East Africa is said to contain I4 per cent. of tannin.

Kleinhovia, Linn.

Tribe II.-HelictereE.

Hospita, Linn.

Helicteres, Linn.

semiglabra, F.v. M.

var. procumbens, Benth.

var. flagellaris, Benth.

spicata, Colebr.

Pterospermum, Schreb.

acerifolium, Willd.-Doubtful if indigenous. A tree in the Brisbane Botanic Gardens, I was told by Mr. Walter Hill, had been obtained in a South Queensland scrub.

Melhania, Forsk.

TRIBE III.-DOMBEYEA:

incana, Heyne.

abyssinica, A. Rich.

Melochia, Linn.

TRIBE IV.-HERMANNIE质.

pyramidata, Linn.

corchorifolia, Linn.

Waltheria, Linn.

americana, Linn.-A wide-spread weed of tropical countries.

Tribe V.-Buettineriez.

Abroma, Jacq.

fastuosa, R. Br.-A weed of tropics, but yields an excellent fibre.

Rulingia, R. Br. (Included in Commersonia by Baron von Mueller.)

salvifolia, Benth.

pannosa, R. Br.

rugosa, Steetz.

Commersonia, Forst.

Leichhardtii, Benth.

Fraseri, J. Gay.

echinata, Forst.-Brown Kurrajong. "I)im” of Maroochie natives. Yields a strong fibre used by the natives for fishing lines and nets.

var. Bancroftii.-The leaves beautifully variegated. 


\section{TRibe VI.-LASiopetale压.}

Seringia, J. Gay.

platyphylla, J. Gay.

Keraudrenia, J. Gay. (Included by F. von Mueller in Seringia.)

lanceolata, Benth.

Hillii, F.v.M. (Fig. 47.)

Hookeriana, Walp.

adenolasia, $F$. v. $M$.

Hannafordia, F. v. $M$.

Shanesii, F.v.M. (Fig. 48.)

\section{Order XXIV.-TILIACEÆ.}

Series A.-Holopetal 2 .

Tribe I.-Browlowieæ.

Berrya, Roxb.

Ammonilla, Roxb., var. rotundifolia, Benth.

Tribe II.-Grewiex.

Grewia, Limn.

orientalis, Lim.

var. latifolia, Benth.

multiflora, Juss.

latifolia, F. v. M.

polygama, Ro.rb.- "Koolin" of Cloncurry, "Pam-mo" of Cooktown, "Ouraie" of Cleveland Bay, and "Kou-nung" of Middle Morehead River natives. A valuable remedy in cases of dysentery.

pleiostigma, F.v.M.-Wood probably of use for making oars. scabrella, Benth.

Triumfetta, Linn.

procumbens, Forst.; Hemsl., Jl. Bot. I890, p. i., t. 293, fig. 2. subpalmata, Sol. ex Hemsl. in J1. Bot. I89o, p. i., t. 293, fig. I ; Britt., Bot. Cook's Voy. t. $23=$ T. procumbens, Benth. appendiculata, $F . v \cdot M$.

plumigera, $F . v . M$.

pilosa, Roth.

Winneckeana, F. v. M.

*rhomboidea, Jacq.-Chinese Burr; a troublesome weed. nigricans, Bail.-A troublesome burr-weed of our tropical parts. 
Corchorus, Limn.

TrIBE III.-TiLIEÆ.

Cunninghamii, F.v.M.-Plants bearing variegated foliage are at times met with.

hygrophilus, $A$. Cunn.

trilocularis, Limn.

acutangulus, Lam.

pumilio, R. Br.

sidoides, F. v. M.

tomentellus, F. v. M.

\section{Series B.-Heterofetal E.}

TRIBE IV.-SLOANIEA.

Sloanea, F.v.M.=Echinocarpus, Blume; Benth. in F1. Aust. i. 279.

Woollsii, F.v.M.- "Carrabean" of Tambourine Mountain.

Wood useful for flooring-boards; when newly cut has somewhat the scent of celery.

Langii, F.v.M. (Fig. 49.)

Macbrydei, F.v.M.-Also known as "Carrabean."

australis, F.v.M.-Maiden's-blush timber; pinkish; useful for inside work.

Tribe V.-ELAOCARPEÆ.

Aristotelia, L'Herit.

australasica, $F . v . M$.

megalosperma, F.v.M. (Fig. 50.)

Elæocarpus, Linn.

arnhemicus, F. v. M.

foveolatus, F.v. $M$.

sericopetalus, F. v. $M$.

Grahami, F. v. M.

ruminatus, F. v. M

obovatus, G. Don=E. parviflorus, A. Rich.- "Woolah" of Moreton Bay natives.

cyaneus, Ait. $=$ E. reticulatus, Sw.-Flowers white or rose coloui.

Kirtonii, F.v. M.-White Beech of Bunya Mountains. Wood somewhat resembling English sycamore.

eumundi, Bail. (Fig. 52.)

grandis, F.v.M.-"Quandong" of Brisbane and "Moorum" of Barron River natives. The nuts used for ornamental purposes. Fruit eaten. 

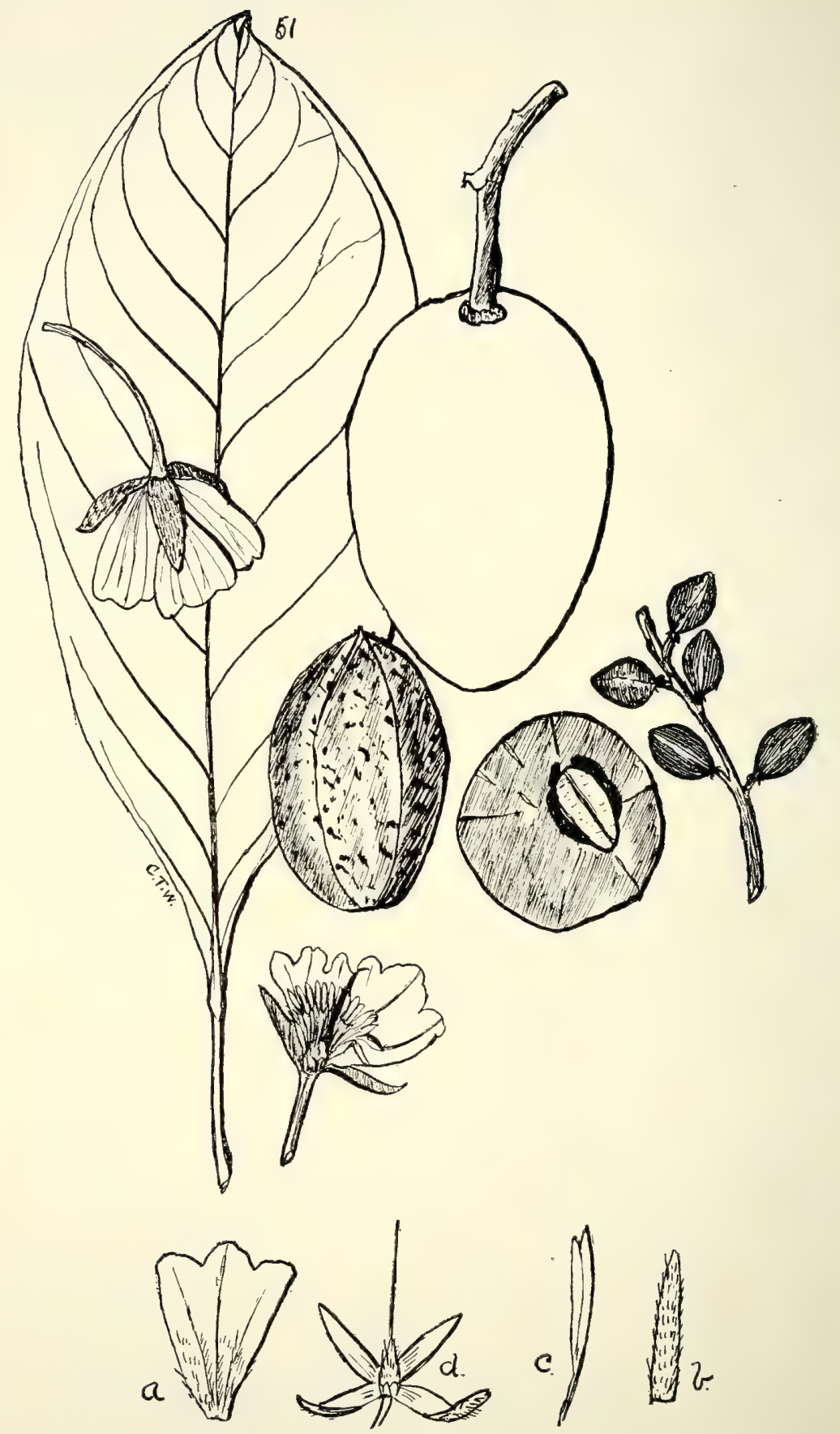

51. Eleocarpus Bancroftit, F. v. M. et Bail.

(a) Petal, (b) calyx-lobe, (c) stamen, (d) calyx and pistil. (a)--(d) enl. 
Elæocarpus-contd.

Bancroftii, F. v. M. et Bail.--Johnstone River Almond. Bonyheart or Ebony-heart of Cairns. The seed has an agreeable flavour and is eaten by the settlers. (Fig. 5I.)

$$
\text { SERIES II.-DISCIFLOR E. }
$$

Alliance VII.-GERANIALES.

\section{Order XXV.-LINEA.}

Linum, Linn.

Tribe I.-Eulinee.

*usitatissimum, Linn.-The common Flax; a native of Southeastern Europe and Asia Minor; contains prussic acid. marginale, A. Cumn.

suædæfolium, Planch.

*gallicum, Linn.-French or Yellow Flax. Mediterranean Regions.

"Reinwardtia, Dun.

trigyna, Planch. = Linum trigynum, Roxb.-East Indies.

Tribe II.-HugoniEA:

Hugonia, Linn.

Jenkinsii, F. $\varepsilon^{\prime} .1 I .=$ Durandea Jenkinsii, Staph.; Hk., Ic. P1. under Tab. 2822.- "Katakarkal" of Tully River natives, who use the hooked tendrils as fish-hooks. (Fig. 53.)

Tribe III.-ErythroxyleA.

Erythroxylon, Linn.

australe, F.v.M.- "Moolkellam" of Rockhampton natives. Bark yields a brownish-yellow dye. Leaves contain coca-tannic acid.

ellipticum, R.Br.-A valuable cabinet-wood, of a reddishbrown, close-grained and nicely marked.

\section{Order XXVI.-MALPIGHIACEA.}

Tribe I.-Banisterieæ.

Ryssopterys, Blume.

timorensis, Blume. (Fig. 55.)

Tribe II.-Hirex.

Tristellateia, Thouars.

attstralasica, A. Rich. 


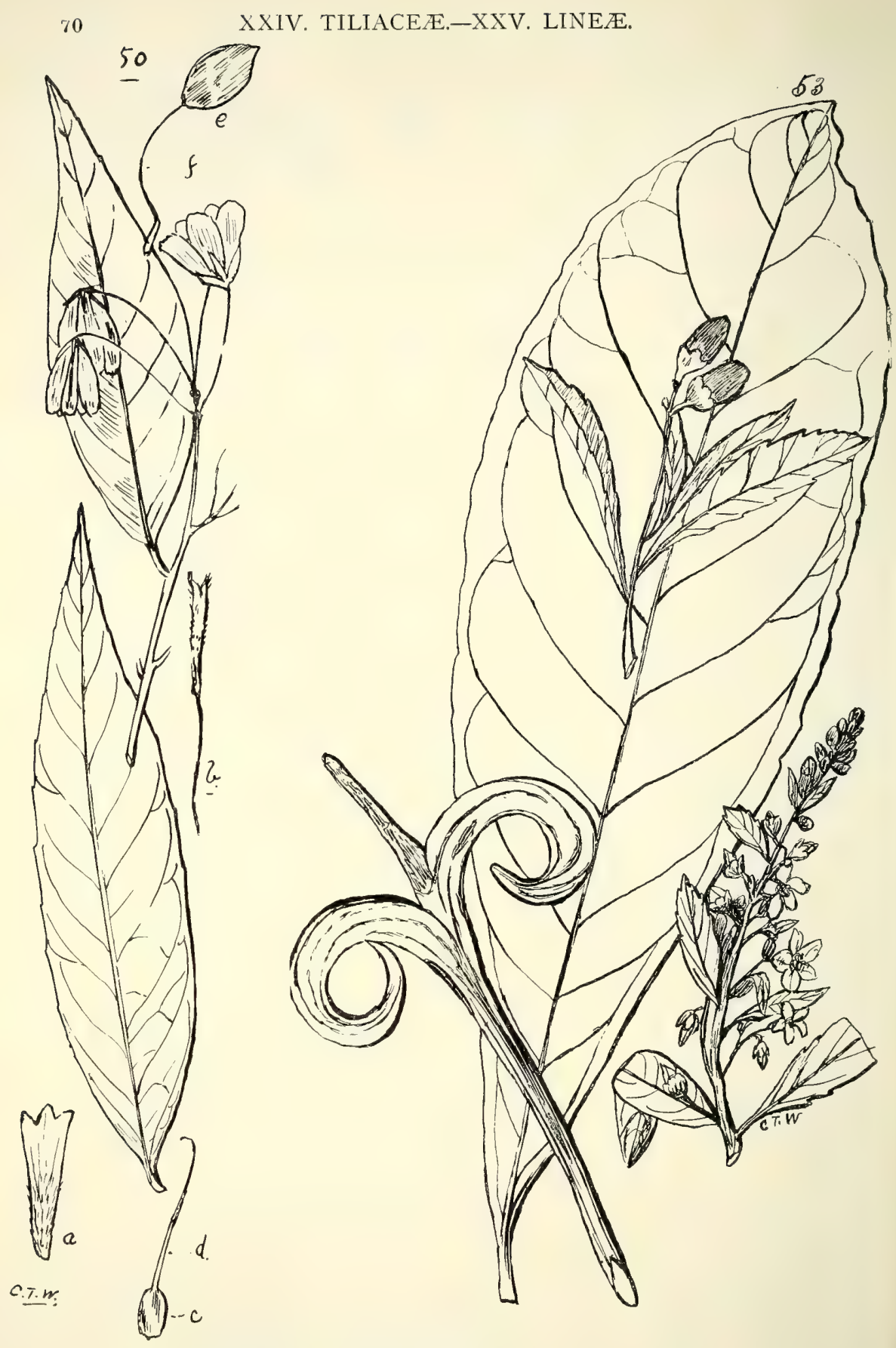

50. Aristotelta megalosperma, $F, v$. $M$.

(a) Petal, (b) stamen, (c) ovary, (d) style, (e) berry, (f) pedicel. (a)-(d) enl.; (e)-(f) nat. size.

53. Hugonia Jenininsis, $F . v \cdot M$. 
XXIV. TILIACEÆ.XXV. LINE压.
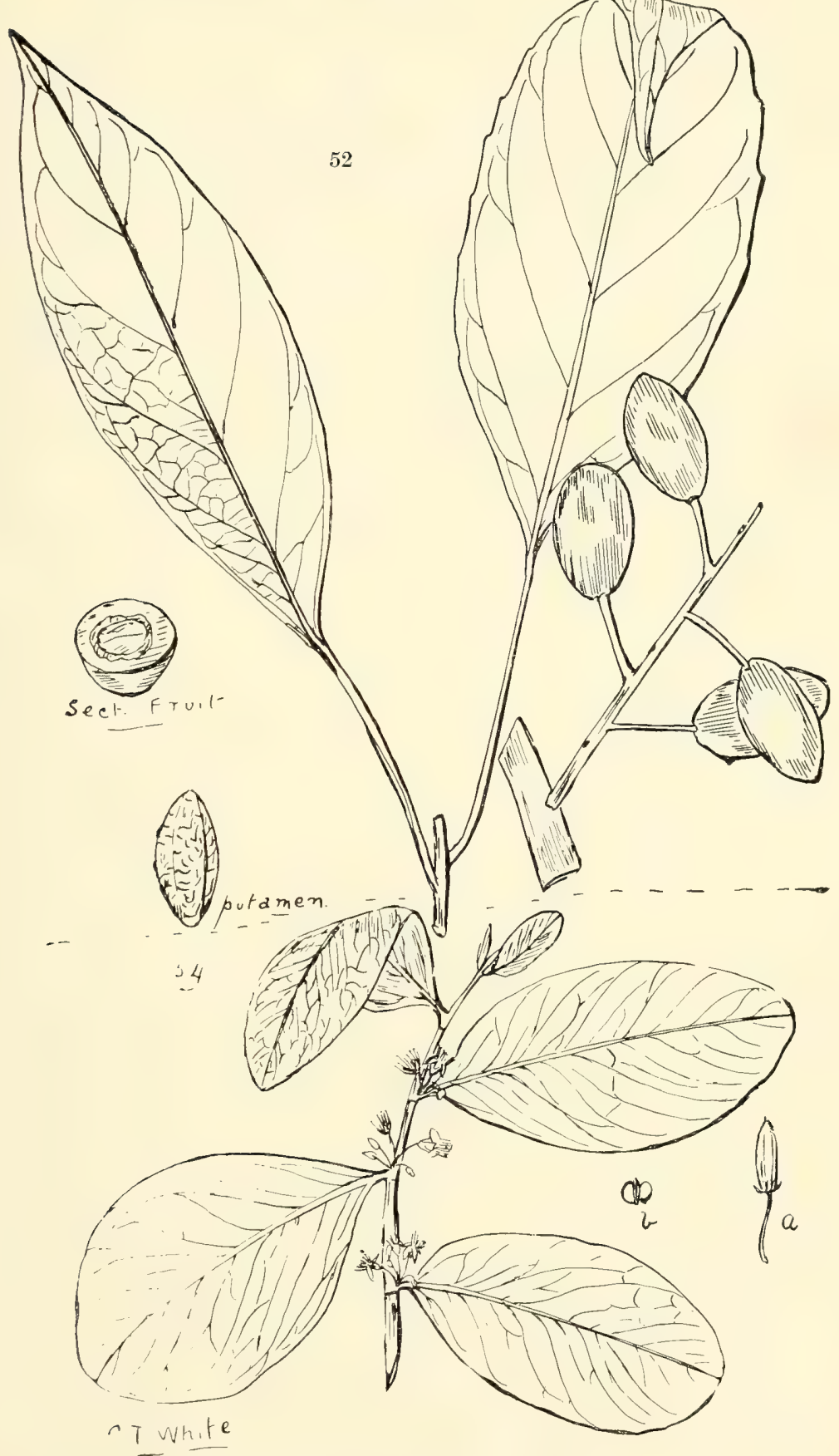

52. Elmocarpus eumund, Bail.

54. ERythroxylON ELLIPTICUM, $R$. $B r$.

(a) Drupe, (b) trans, sect. drupe, (a)-(b) nat. size. 


\section{Order XXVII.-ZYGOPHYLLEA.}

Tribulus, Linn.

terrestris, Linn.-Caltrops or Cat's-head. Fruit of this and other species have been known to injure the feet of sheep. cistoides, Linn._- "Dardatra” of Pennefather River natives. occidentalis, R. Br. (Fig. 56.)

hystrix, $R$. $B r$. pentandrus, Benth.

Solandri, F.v.M.- "Longarate" of Batavia River natives; the roots eaten roasted (Roth).

angustifolius, Benth.

leptophyllus, Bail. (Fig. 57.)

minutus, Leichh.

Nitraria, Limn.

Schoberi, Linn. = Zygophyllum australasicum, Miq.

Zygophyllum, Linn.-Bean Capers.

apiculatum, F. v. $M$.

glaucescens, $F . v$. $M$.

var. lobulatum, Benth.

iodocarpum, F. v. M.

prismatothecum, F. v. $M$.

Billardicri, $D C$.

fruticulosum, $D C$.

var. bilobum, Benth.

\section{Order XXVIII. - GERANIACE E}

Tribe I.-GERANIEA:.

Geranium, Linn.-Crane's-bill.

dissectum, Linn., var. australe, Benth.

forma pilosa, Benth.-Native Carrot. A useful fodder herb, the rootstock greatly relished by sheep, who in times of drought root it up out of the dry soil and devour it.

forma potentilloides, Benth.-The rootstock not so carrotlike as in the other form.

Erodium, L'Herit.

cygnorum, Nees.-Good fodder-herb.

cicutarium, L'Her.

TRIBE II.-PELARGONIEE.

Pelargonium, L'Herit.-Stork's.bill.

australe, Willd.-Generally found on rocks in Southern inland localities. 
TRIBE III.--OXALIDEA.

Oxalis, Limn.

* corymbosa, DC.-A troublesome garden weed; native of Brazil.

corniculata, Linn.-Wood sorrel; Sourgrass. Very variable as to size of foliage and flowers.

sessilis, Hamilt. $=$ O. Petersii, Klotz., Biophytum Apodiscias, Turcz.-Leaves sensitive to the touch. (Fig. 58.)

\section{Order XXIX.-RUTACEA.}

Tribe I.-Boroniex.

Zieria, Sm.

lævigata, $S m$.

aspalathoides, A. Cunn.

pilosa, Rudge.

obcordata, A. Cunn.

cytisoides, $S m$.

Smithii, Andr.-Yields a rich oil.

var. parvifolia, Benth.

var. macrophylla, Benth.

granulata, C. Moore.

Boronia, Sm.-The fragrant oil of many might be used in perfumery.

\section{Series Valvatce.}

artemisiæfolia, F. v. M.

eriantha, Lindl.

alulata, Sol. - "Bala-bal-balgal" of Endeavour River natives.

ledifolia, J. Gay.

var. rosmarinifolia, Benth.

lanceolata, $F . v . M$.

platyrrhachis, F.v.M.

Bowmani, F. v. M.

Series Pinnatie.

microphylla, Sieb.

pinnata, Sm.

var. alba, Bail. 

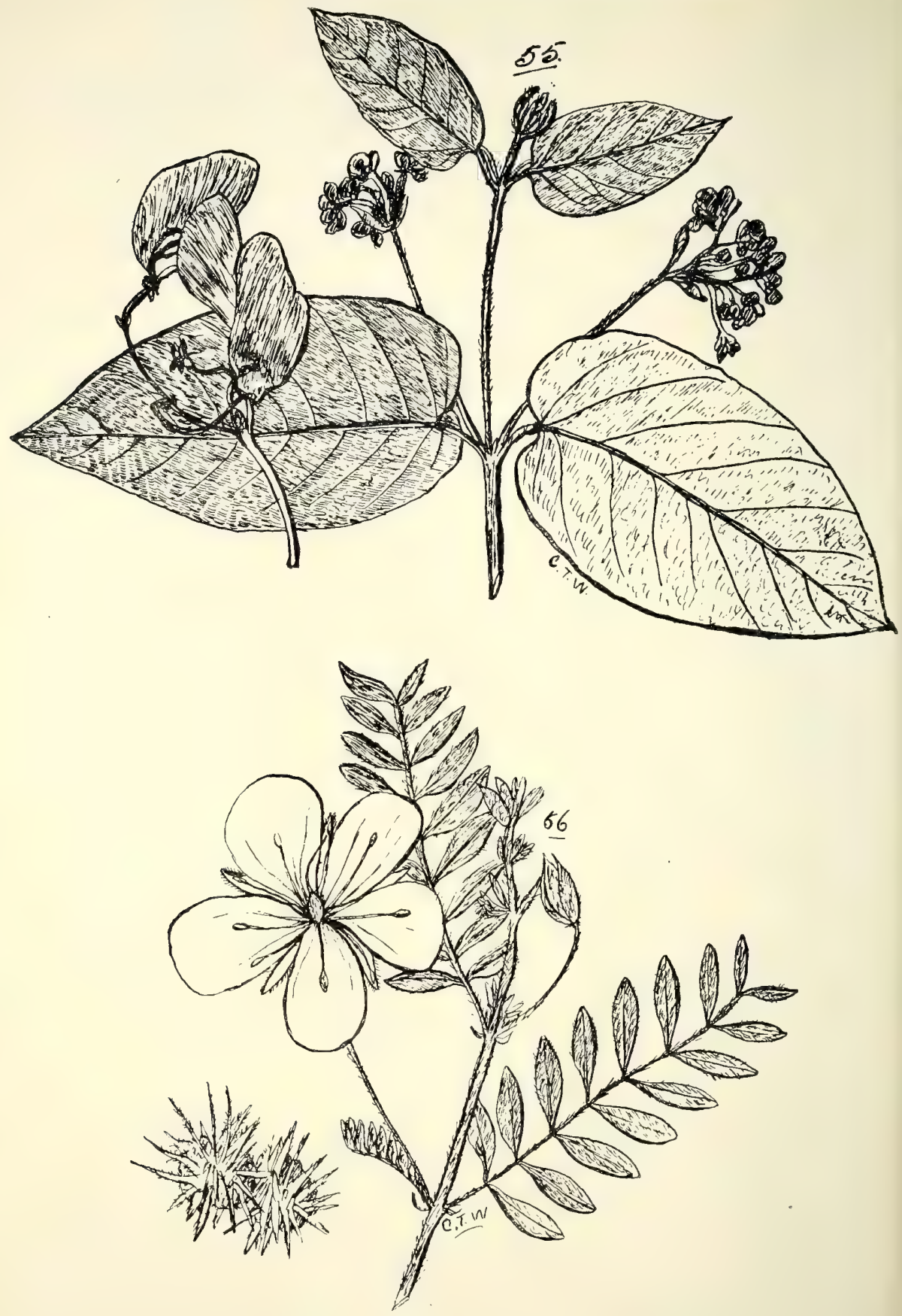

55. Ryssopterys timorensis, Blume. 56. Tribulus occidentalis, $B . B r$. 
XXVII. ZYGOPHYLLEÆ.-XXVIII. GERANIACEÆ.-XXIX. RUTACEÆ.
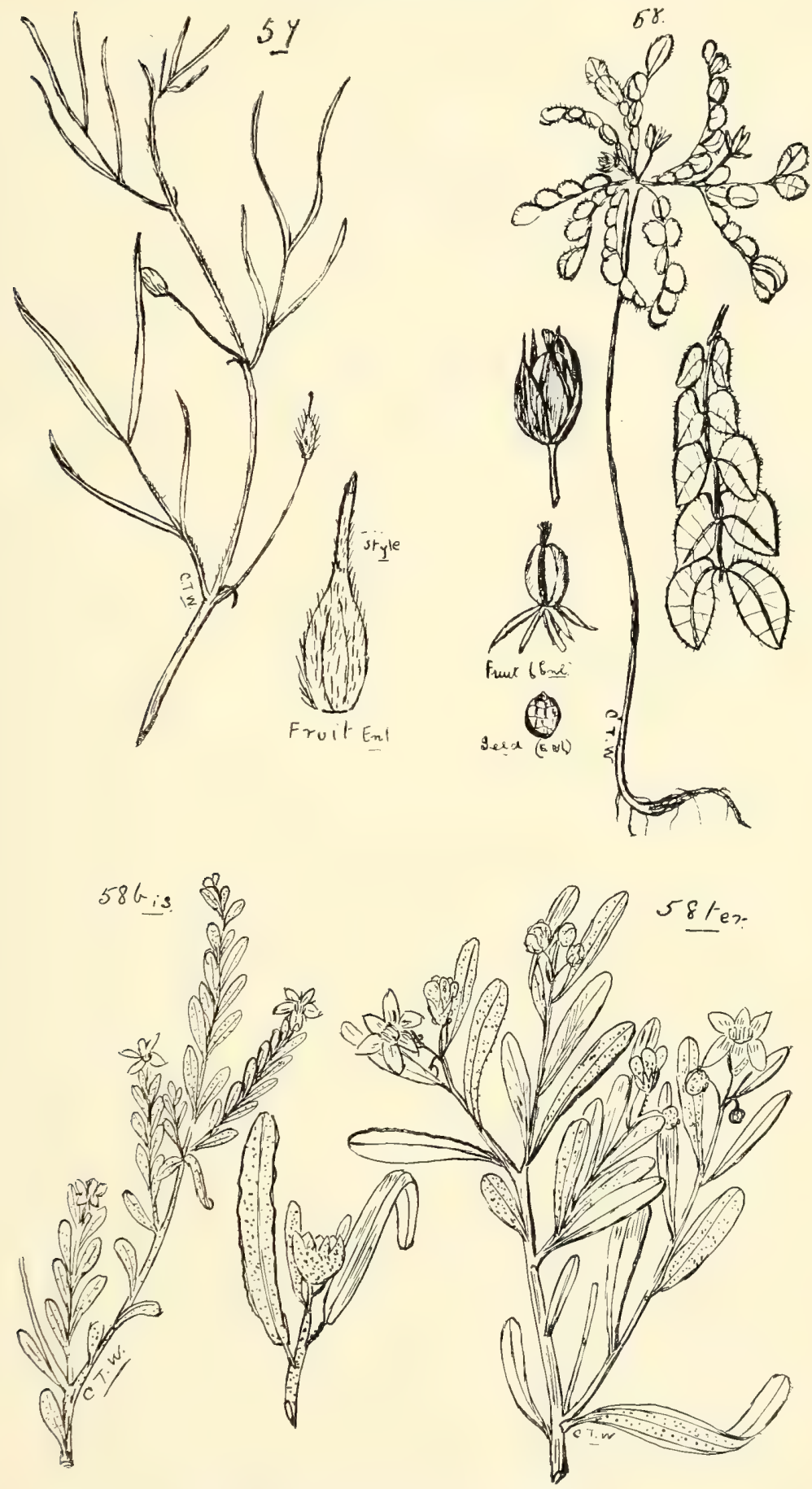

57. Tribulus leptophyllus, Bail.

58. Oxalis sessilis, Hamilt.

58 bis. ERiostemon parvifolius, $R$. $B r$.

58 ter. Phebalium Nottit, F. $v . M$. 
Boronia-contd.

polygalifolia, $S m$.

\section{Series Variabiles.}

var. robusta, Benth.

var. ? pubescens, Benth.-Pubescent; leaves 3-foliate; leaflets very small; flowers small.

Hab.: Stannary Hills (Dr. T. L. Bancroft).

anemonifolia, A. Cunn.

var. anethifolia, Benth. $=$ B. bipinnata, Lindl.

falcifolia, $A$. Cunn.-Leaves 3-5 foliate.

var. alba, Bail.

Series Terminales.

parviflora, $S m$.

Crowea, Sm. (Included by F. v. Mueller in Eriostemon.)

saligna, Andr.

Eriostemon, Sm.

salicifolius, $S m$.

Banksii, A. Cunn.

myoporoides, $D C$.

var. minor, Benth.

hispidulus, Sieb.

scaber, Paxi.

difformis, A. Cunn.

var. Smithianus, Benth.

parvifolius, R. Br. (Fig. 58 bis.)

Phebalium, A. Juss. (Included by F. v. Mueller in Eriostemon.)

elatius, Benth.

Section Leionema.

rotundifolium, Benth.

Section Euphebalium.

squamulosum, $V$ ent.

glandulosum, Hook.

Notii, F.v.M. (Fig. $5^{8}$ ter.)

Billardieri, A. Juss.

Philotheca, Rudge.

australis, Rudge.

var. intermedia, Bail.

var. parviflora, Benth.

calida, $F$. v. $M$.

Asterolasia, F.v. M. (Included by F. v. Mueller in Eriostemon.)

woombye, Bail.

var. parvifolia, Bail.

Correa, $\mathrm{Sm}$.

speciosa, Ait. 


\section{Tribe II.-ZanthoXyleE.}

Bosistoa, F. v. M.

sapindiformis, F.v.M.-“"Towra," native name at Nerang.

A very hard wood used for handspikes and levers.

Melicope, Forst. (Included by F. v. Mueller in Evodia.)

Fareana, F. v. M.

chooreechillum, Bail.

neurococca, Benth.=Bouchardatia neurococca, Baillon.Wood hard, close-grained, of a light-yellow colour.

erythrococca, Benth. _ "Thal-ango-thera" of Forest Hill natives. The bark possesses a most peculiar acrid pungency and promotes a great flow of saliva. This feature was recorded by Mr. H. Tryon many years ago, who thought the matter worthy the attention of the medical faculty.

Broadbentiana, Bail.

australasica, F.v.M.-The resin exuded by this tree largely used as a cement by the Tully River natives, by whom it is known as "Ngobi."

pubescens, Bail.-Fruit fleshy, of an agreeable acid. (Fig. 59.)

Evodia, Forst.

micrococca, $F \cdot v \cdot M$.-Wood close in grain, tough, light-yellow. accedens, Blume $=$ E. Elleryana, F.v. M.- "Boogoobi" of Herberton and "Arral" of Dunk Island natives. Wood. somewhat resembles that of the European Lime-tree (Tiliamopaa).

littoralis, Endl.

vitiflora, F. v. M.

xanthoxyloides, F.v.M.-Wood hard, yellowish.

alata, F. v. M.

Bonwickii, F. v. M.

Fareana, F. v. $M$.

haplophylla, F. v. M

Melicosma, Hook f. (Included by F. v. M. in Evodia.)

Cunninghamii, Hook. $f$ : = Acronychia Cunninghamii, Hook. $\mathrm{f}$. -Bone-wood or Pink-heart; wood a light-yellow, useful for cabinet-work.

Brombya, F. v. M. platynema, F.v.M. (Fig. 6o.)

Pagetia, F. v. $M$. medicinalis, $F \cdot v \cdot M$--Used medicinally by the natives. monostylis, Bail. (Fig. 6I.) 


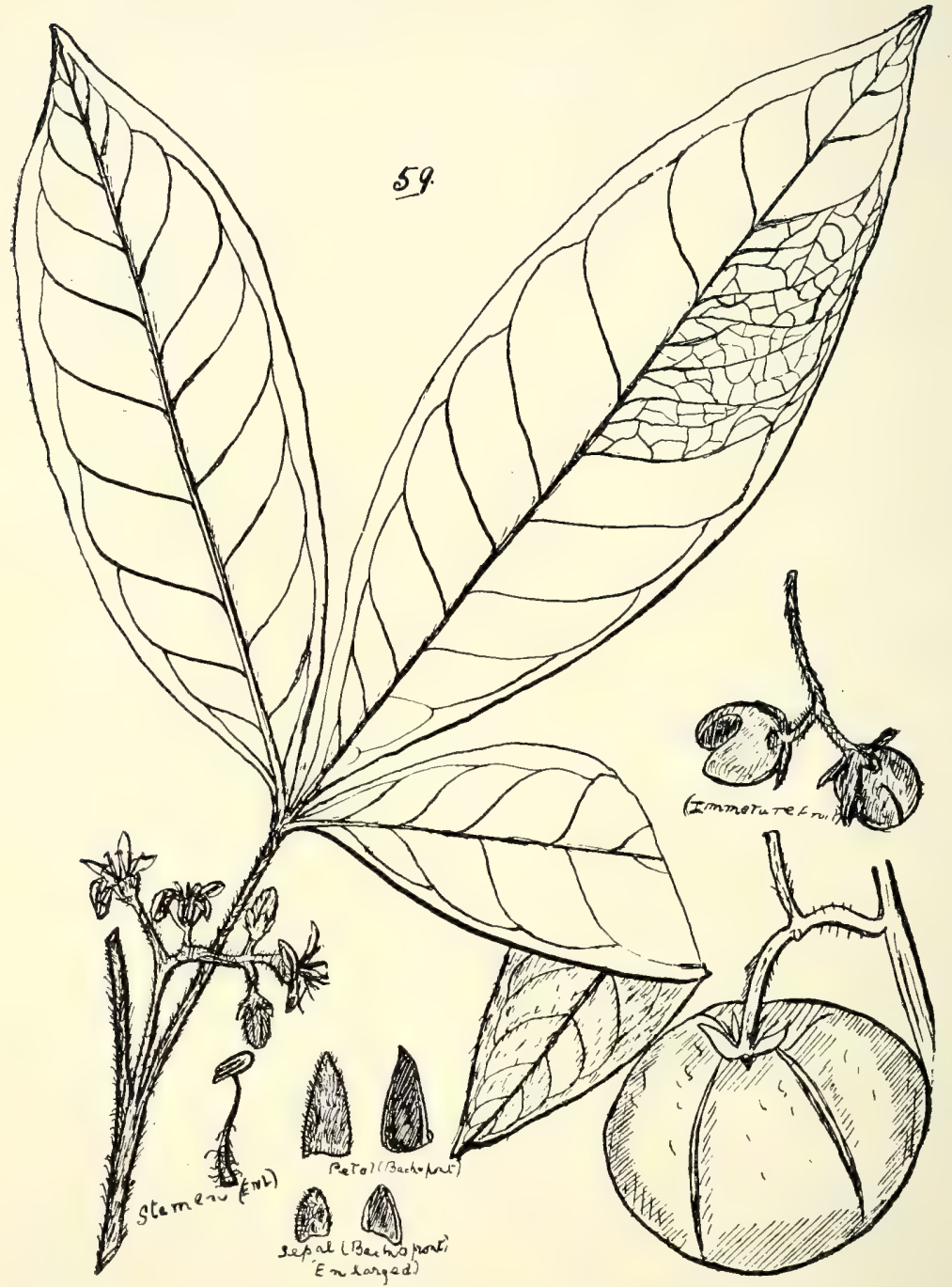

59. Melicope pubescens, Bail. 
XXIX,-RUTACE正.

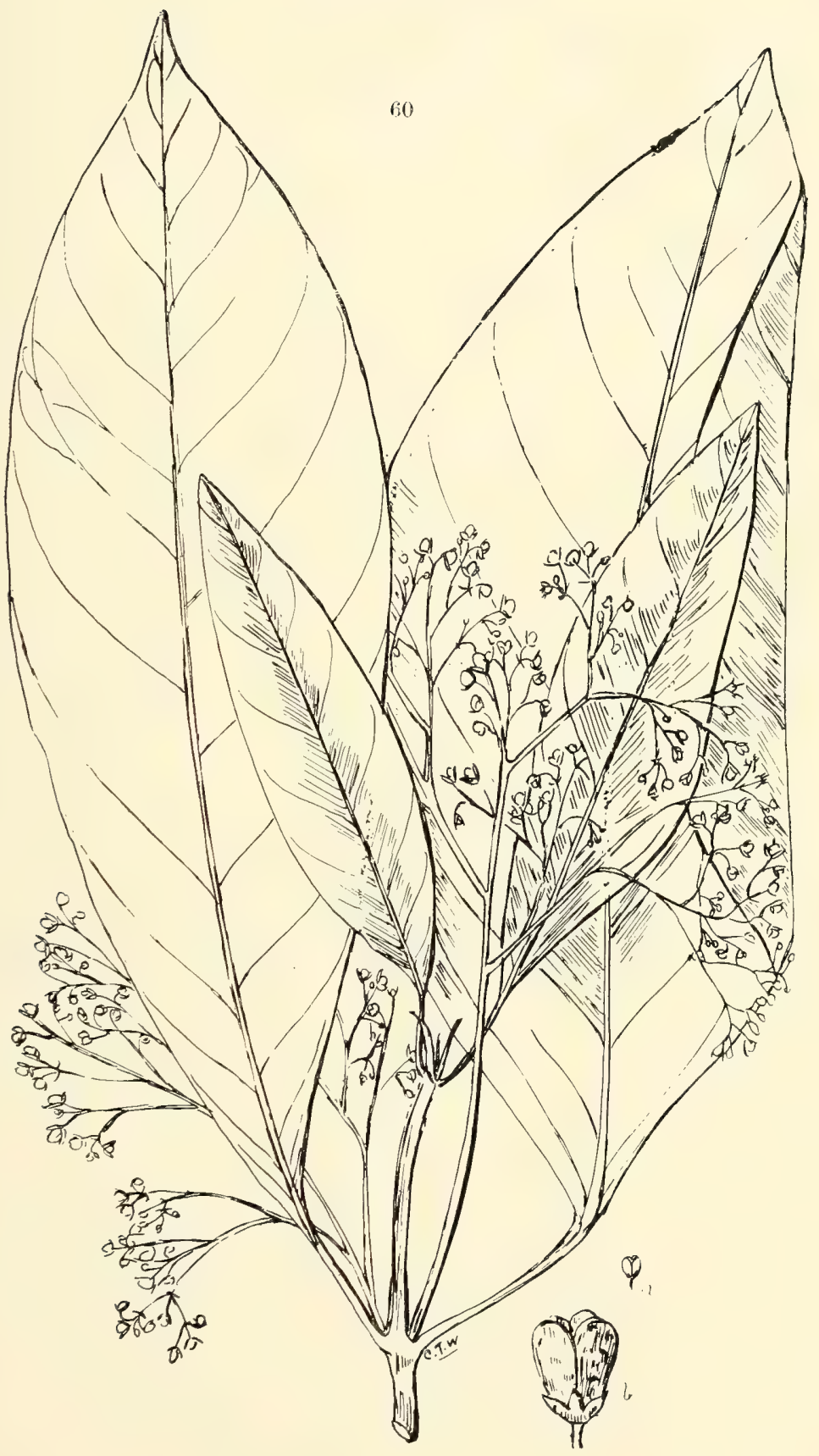

60. Brombya platynema, $F$. $v \cdot M$.

(a) Cocci nat. size; (b) cocci enl. 


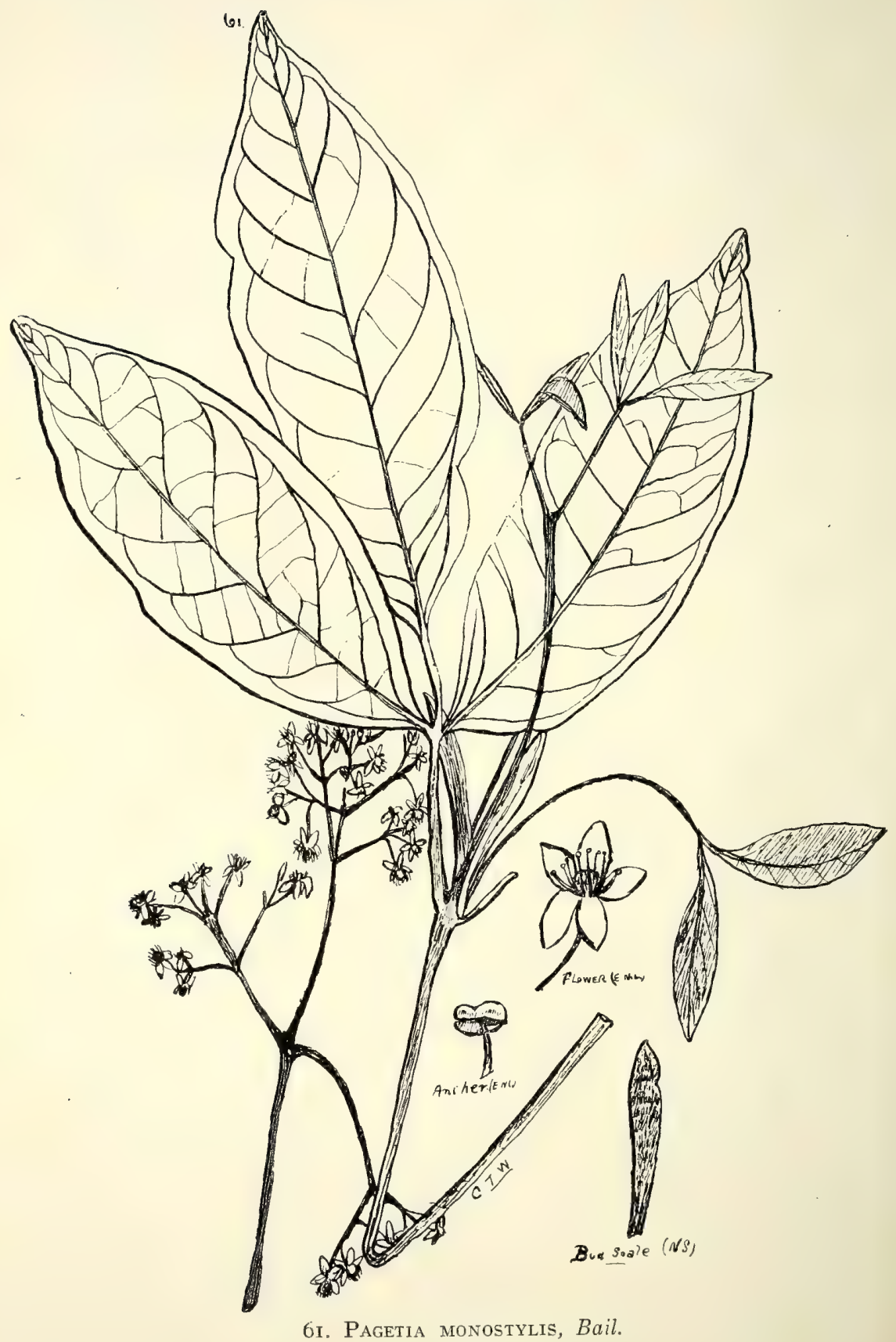


Zanthoxylum, Limn.

brachyacanthum, F.v.M.-Satinwood. Poisonous. Bark yields a brownish-yellow dye.

torvum, F.v.M.-Poisonous.

veneficum, Bail.-Poison-tree. Wood yellow.

parviflorum, Benth.

Geijera, Schott.

Helmsiæ, Bail.

Muelleri, Benth.-A nankeen dye wood; Qucensland Balsam of Capivi tree. A useful cabinet-wood, having a beautifully clouded heartwood.

salicifolia, Schott.

parviflora, Lindl.- "Wilga" of southern border.

Pentaceras, Hook. f.-Fruit a samara.

australis, Hook. f.= Ailanthus punctata, F. v. M.-Wood hard and light-coloured.

Tribe III.-Tondalie.e.

Acronychia, Forst.

Baueri, Schott.-Wood hard, yellow.

lævis, Forst.-Plants at times are met with with variegated foliage. Wood hard and light-coloured.

var. normalis, Bail.-Leaves small; fruit not prominently angled.

var. purpurea, Bail.-Fruit purplish, prominently angled.

var. leucocarpa, Bail.--Fruit white, angles blunt.

imperforata, F.v.M.-Frazer Island Apple. Wood hard, yellow.

melicopoides, F.v.M.-Wood hard and light-coloured.

Scortechinii, Bail.-Logan Apple. Fruit reddish, fleshy, of an agreeable acid.

vestita, F.v.M. = A. acidula, F.v. M.-Wood light-coloured, sofi.

tetrandra, F. v. M.

Pleiococca, F.v.M.

Wilcoxiana, F.v. M.

Halfordia, F. v. M.

drupifera, $F . v . M .-W o o d$ durable, yellowish.

scleroxyla, F. v. M.-Kerosene-tree, "Ghittoe" of Herberton natives. Wood hard, tough, and very inflammable even in a green state.

F 
Tribe IV.-Aurantiex:

Glycosmis, Corr.

pentaphylla, Corr.-In the Australian form, the leaves are I, 2 , or 3-foliate.

Micromelum, Blume.

pubescens, $B l .=M$. glabrescens, Benth. ; M. mimutum (Forst.) Seem.

Mirraya, Linn.

exotica, Lim.

var, ovatifoliolata, Engler.-This, our indigenous form, is of a more straggling habit with more numerous and larger oil-dots, and is often decidedly hirsute and tomentose, thus very distinct from the two Indian ones of our gardens. (Fig. 6I bis.)

crenulata, Oliv.

Clausena, Burm.

brevistyla, Oliv.

Atalantia, Corr.

glauca, Hook. f.-Native Kumquat or Downs Lime. "Wumbanya" of Boulia and "Kandertal" of Cloncurry natives. Fruit used for making preserves.

? recurva, Benth.

Citrus, Lim.-Fruit a hesperidium.

australis, Planch--Native Orange. The wood of this and other kinds useful for turnery work. (Fig. 62.)

australasica, F.v.M.-Finger Lime. The unripe fruit of some forms nearly black. (Fig. 63.)

var. sanguinea, Bail-_Red Finger Lime.

inodora, Bail.-Russell River Lime. (Fig. 64.)

Garrawayi, Bail., Q. Agri.-Journ. xv. 491.-Mount White Lime. (Fig. 65.)

\section{Order XXX.-SIMARUBE $\not$.}

Tribe I.-Simarube功.

Ailanthus, Desf.

imberbiflora, F.v.M. (Fig. 65 bis.)

var. Macartneyi, Bail.__ "Koorootha" of Forest Hill natives. The various forms of this species furnish a quantity of resin of which Dr. Lauterer says:- "Pure or mixed with wax and lard, makes a good ointment for chronic ulcers. Fresh cuts and sores, however, should 
XXIX. RUTACE压.

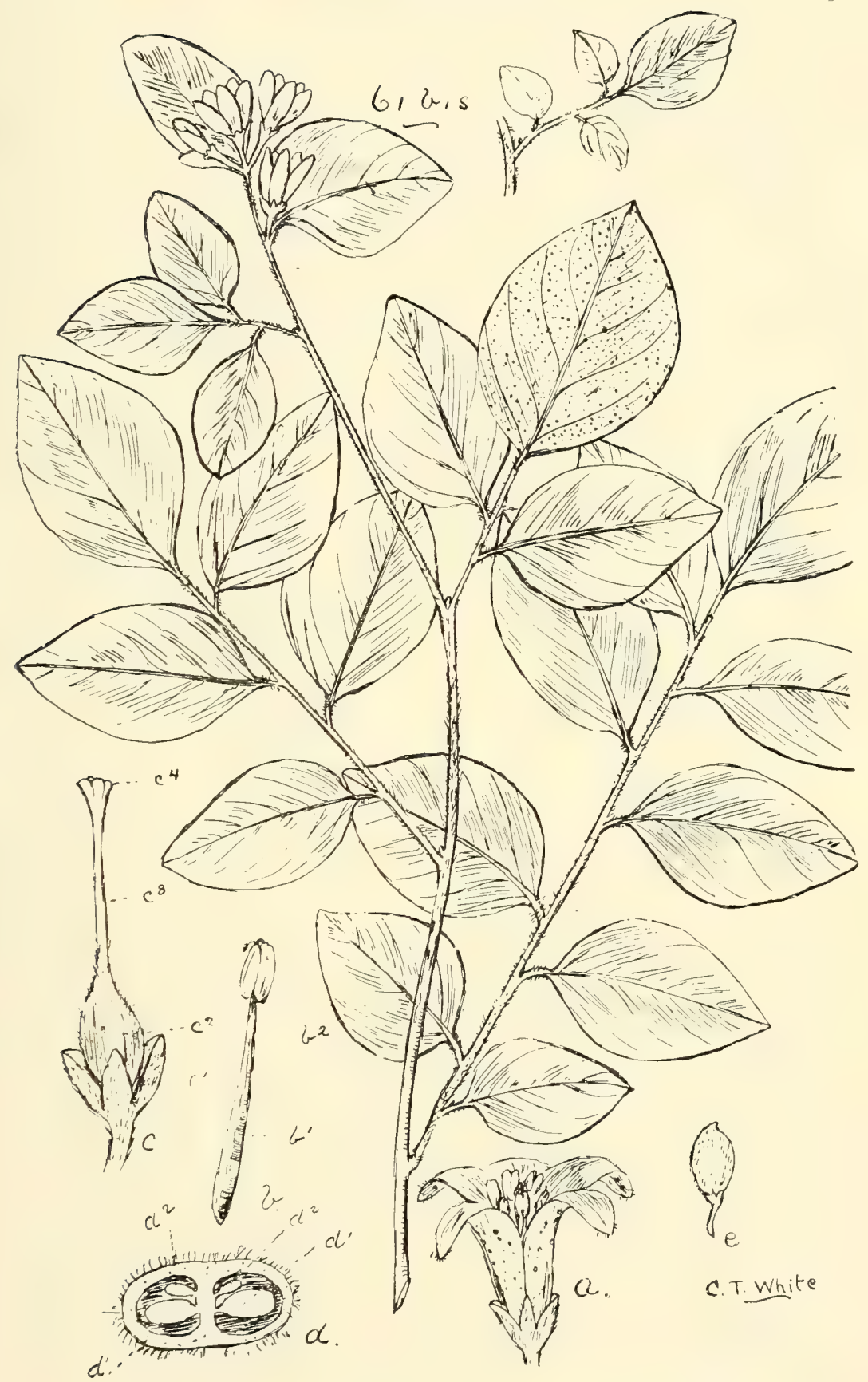
$d^{\prime}$,

6i. bis. Murraya exotica, Limn., var. ofatifoliolata, Engl.

(a) Single flower, (b) stamen, (b y) filament, (b z) anther, (c) calyx and pistil, (c I) calyx, (c 2) ovary, (c 3) style, (c 4) stigma, (d) trans. sect. ovary, (d I and (l 2) ovules, (e) berry: (a)-(d). enl.; (e) nat. size. 
XXIX. RUTACE王.

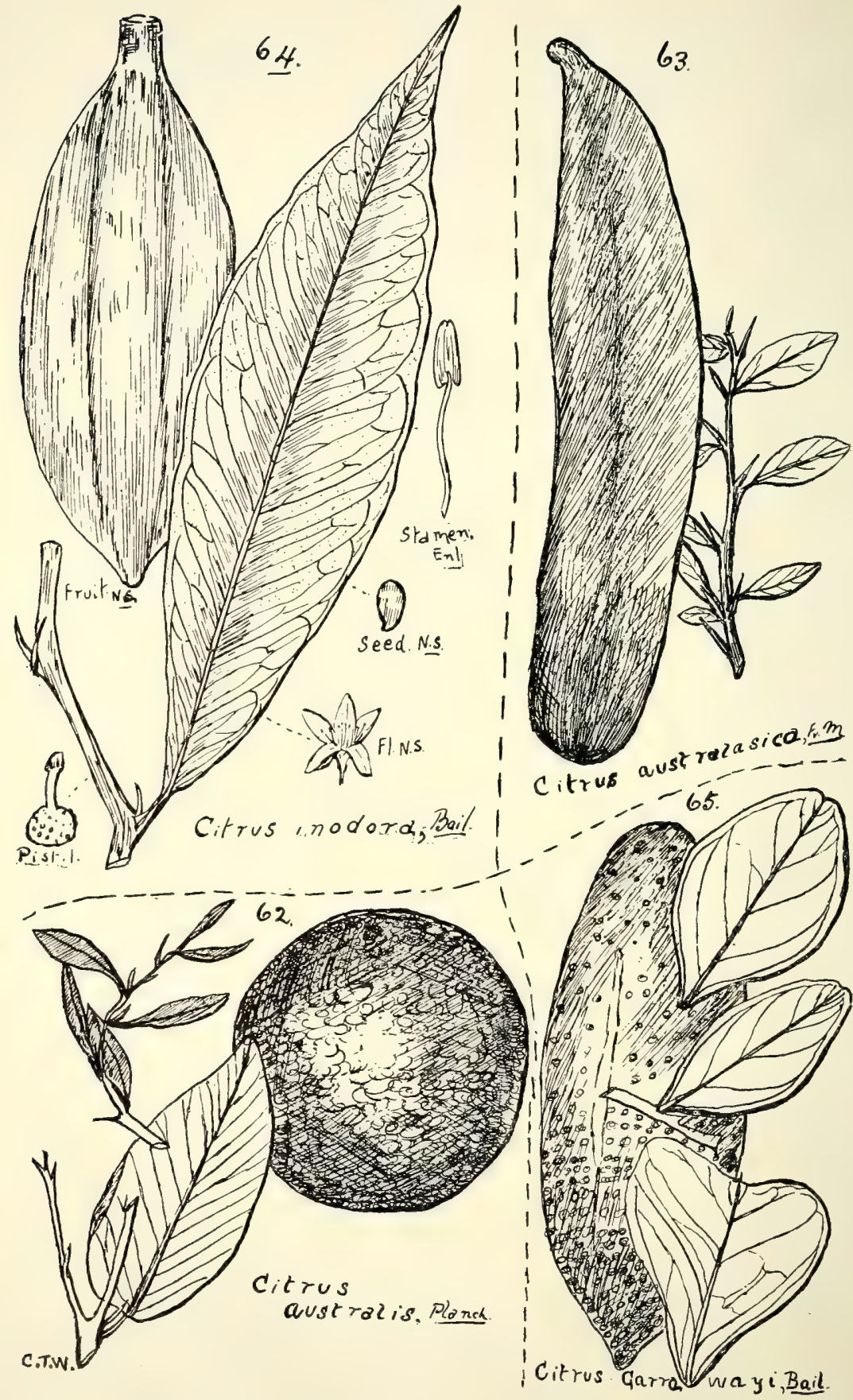

62. Citrus australis, Planch.

63. Citrus australasica, $F . v . M$.

64. Citrus inodora, Bail.

65. Citrus Garrawayi, Bail. 
not be treated with this resin, on account of the acrid oil. If mixed with wax and turpentine would make a good material for fixing paper labels on tin boxes." (Fig. 65 ter.)

*glandulosa, Desf.-Tree of Heaven, a native of China and Japan; said to contain a tanning matter (gallotannic acid) of about II per cent.

Brucea, Mill.

sumatrana, Roxb.-The seeds are called " Macassar kernels," and are very bitter, and used in Java to cure dysentery. (Fig. 66.)

Samadera, Goertn

Bidwillii, Oliv. = Hyptiandra Bidzvilli, Hook. f.

Baileyana, Oliv. $=H$. Bidrilli, var, grandiuscula, Bail, and F. v. M. (Fig. 67.)

Cadellia, F. v. M.

pentastylis, F.v. M. = Guilfoylea pentastylis, F. v. M.-Solidwood of Taroom. Wood durable; also yields a red dye.

monostylis, Benth. = G. monostylis, F. v. M.-Wood resembling some kinds of walnut and satinwood. (Fig. 68.)

var. minor, Bail., n. var. Fruit globose, black, three lines diam. (Fig. 69.)

Suriana, Linn.

Hab.: Killarney ( $J$. Wedd).

maritima, Linn.

Harrisonia, R. Br.

TRIBE II.--PICRAMNIEE.

Brownii, A. Juss.

\section{Order XXXI.-OCHNACE压.}

Tribe OCHNEF.

Brackenridgea, A. Grayy.

australiana, F.ข.M. (Fig. 7O.)

\section{Order XXXII, -BURSERACEÆ.}

Garuga, Roxb.

Tribe Bursere⿸.

floribunda, Dcne.-The wood close-grained and tough, of a grey colour.

Bursera, Linn.

australasica, Bail-C Carrotwood of Eumundi. Wood of a greasy nature; does not shrink or warp in drying. (Fig. 7I.) 
Canarium, $\operatorname{Linn}$.

australasicum, F.v.M._ "Kame" of Batavia River natives;

"Tchaln-ji" of Bloomfield River natives, who use the gum as a cement. Wood grey, dark towards the centre. Muelleri, Bail. - Elemi-tree. Of this Elemi Dr. Joseph Lauterer, in "Queensland's Gums and Resins," says:--"As to the commercial value of the resin, it must be stated that it is the best substitute for elemi, and it is a very good healing agent for cuts, sores, and chronic ulcers, as it has been proved by experiment." This resin is known to the natives of the Bloomfield River as "Yikari." Wood of a grey colour.

Ganophyllum, Blume.

falcatum, Blume.

\section{Order XXXIII.-MELIACEÆ.}

Tribe I.-Melie .

Turræa, Linn.

pubescens, Hellen.-Wood hard, outer yellow, centre dark. (Fig. 72.)

Melia, Linn.

composita, Willd. = M. asedarach, Linn., var. australasica, C. DC. ; M. australasica, Juss.-White Cedar. The gum is a good substitute for gum-arabic ( $D r$. Lauterer). Wood light-red, soft and light.

Tribe II.-TRICHILIE.T.

Dysoxylon, Blume.-The wood of most species good for indoor use.

latifolium, Benth.

ärourescens, $M$ iq.

Muelleri, Benth.-Kedgy-kedgy or Pencil Cedar. A useful cabinet wood.

rufum, Benth.

Klandieri, F. v. M. = ptychocarpum, Radlk.; Schleichera ptychocarpa, F. v. M.

var. glabrescens, Benth.--Wood straw-coloured. 


\section{5 bis.}
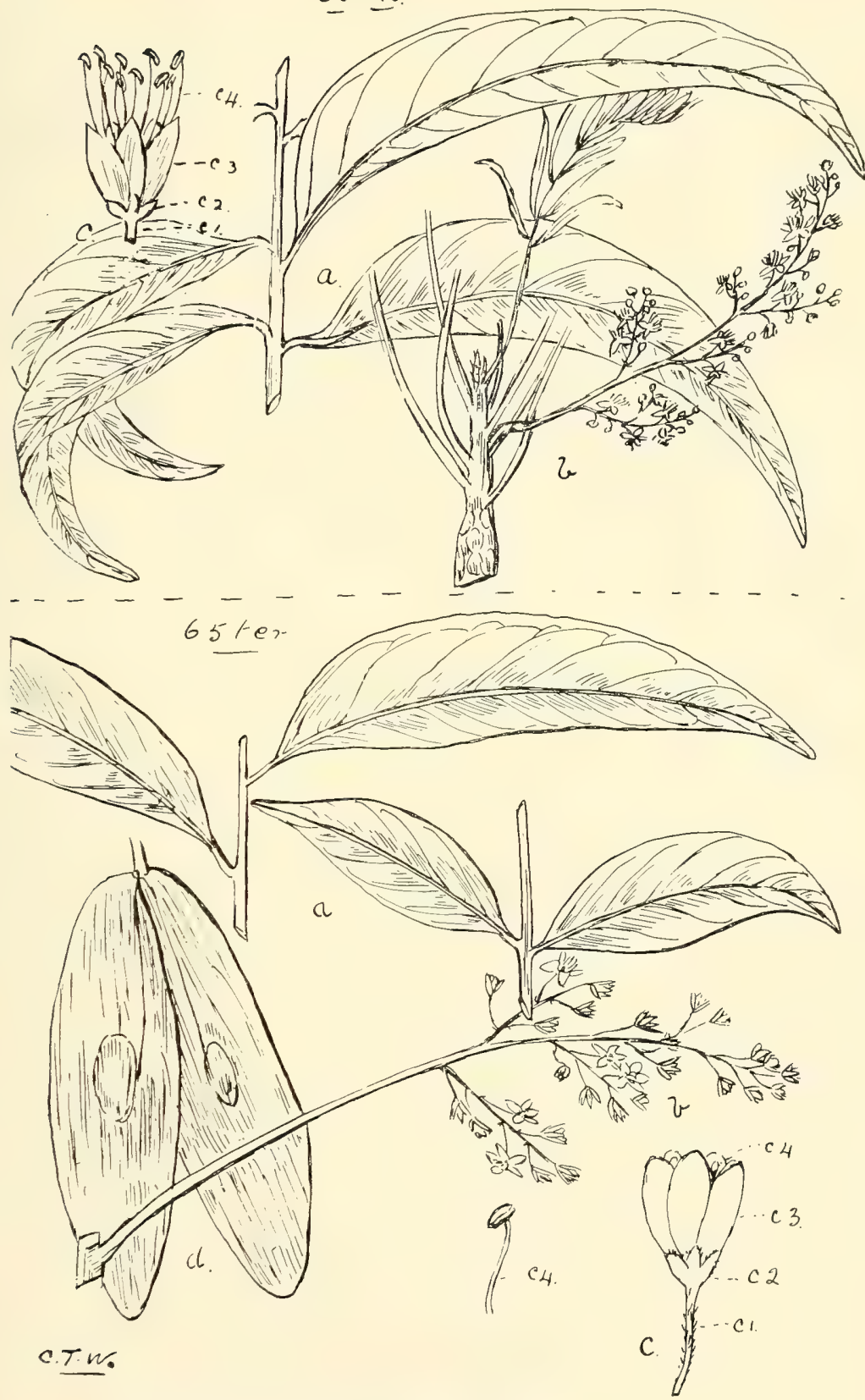

65 bis. Ailanthus imberbiflora, $F . v . M$.

65 ter. Ailaninus imberiflora, F. v. M., var. Macartneyi, Bail.

(a) Leaflets, (b) inflorescence, (c) flower, (c r) pedicel, (c 2) calyx, (c 3) petals, (c 4) stamens, (d) samaræ. (a), (b), and (d) nat. size; (c) enl. 
Dysoxylon-contd.

Pettigrewianum, Bail.-Cairns Satinwood; a useful cabinetwood.

Nernstii, F. थ. $M$.

Fraserianum, Benth. = Lessertianum, Benth.

Schiffneri, F.v. M.

oppositifolium, F.v.M.-A useful cabinet wood.

Becklerianum, $C . D C .=D$. Lessertianum, var. pubescens, Benth.

cerebriforme, Bail.-Brain-fruit.

Aglaia, Lour.

elæagnoidea, Benth.

Amoora, Roxb.

nitidula, Benth._- Jimmie Jimmie" of Herberton natives.

Wood light-coloured, hard and tough.

Synoum, A. Juss.

glandulosum, A. Juss.-Wood useful for indoor work; colour reddish.

Muelleri, C. DC.

Hearnia, F. $\tau^{\prime} . M$.

sapindina, F. v. M.- "Boodyarra" of Herberton natives. Wood of a grey colour, hard and tough.

Owenia, F. v. M.

acidula, F.v.M.-Emu Apple. "Gumin Gumin," “Bulloo," and "Dillyboolen" of inland natives; "Eldin" and "Uroka" of Cloncurry natives. Wood reddish, useful for cabinet work.

vernicosa, F. v. M.

var. (?) pubescens, Benth.

venosa, F. v. M.-Crow's Apple; a very hard wood.

cepiodora, F. v. M.-Onion-tree.

reticulata, F. v. M.

Carapa, Aubl.

moluccensis, Lam.-Cannon-ball tree. Apple Mangrove. "Boora" of Cardwell natives (R.B.H.). Wood very hard. Mr. Brünnich found the bark to contain 22.I5 per cent. of tannin. Bark said to contain about 35 per cent. of tannin in East Africa.

Tribe III.--CEDRELE .

Cedrela, Limn.

Toona, Roxb., var. australis, C. DC. $=$ C. australis, F. v. M.Red Cedar. "Manin" and "Mugurpul” of Brisbane "Woota" of Wide Bay, and "Wanga" of Atherton natives. 


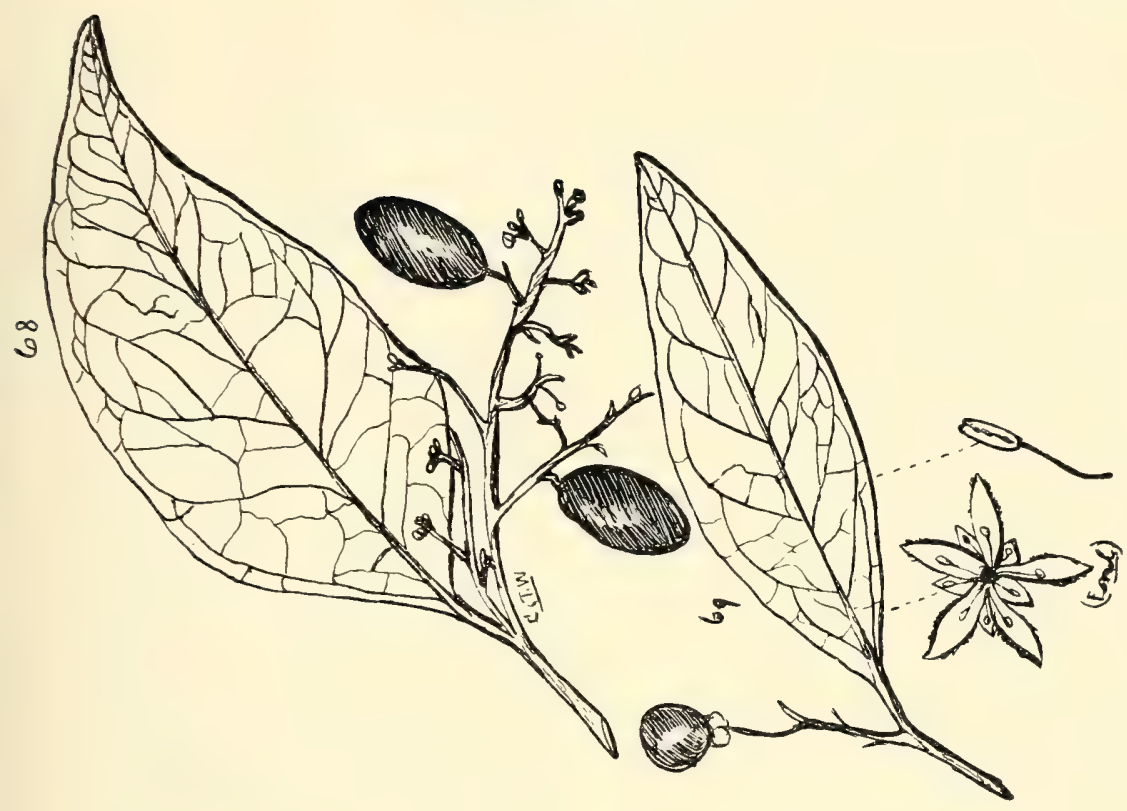

$\underset{\tilde{\Xi}}{n}$

芯菅

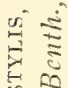

${ }^{\circ} w_{i}^{2}$

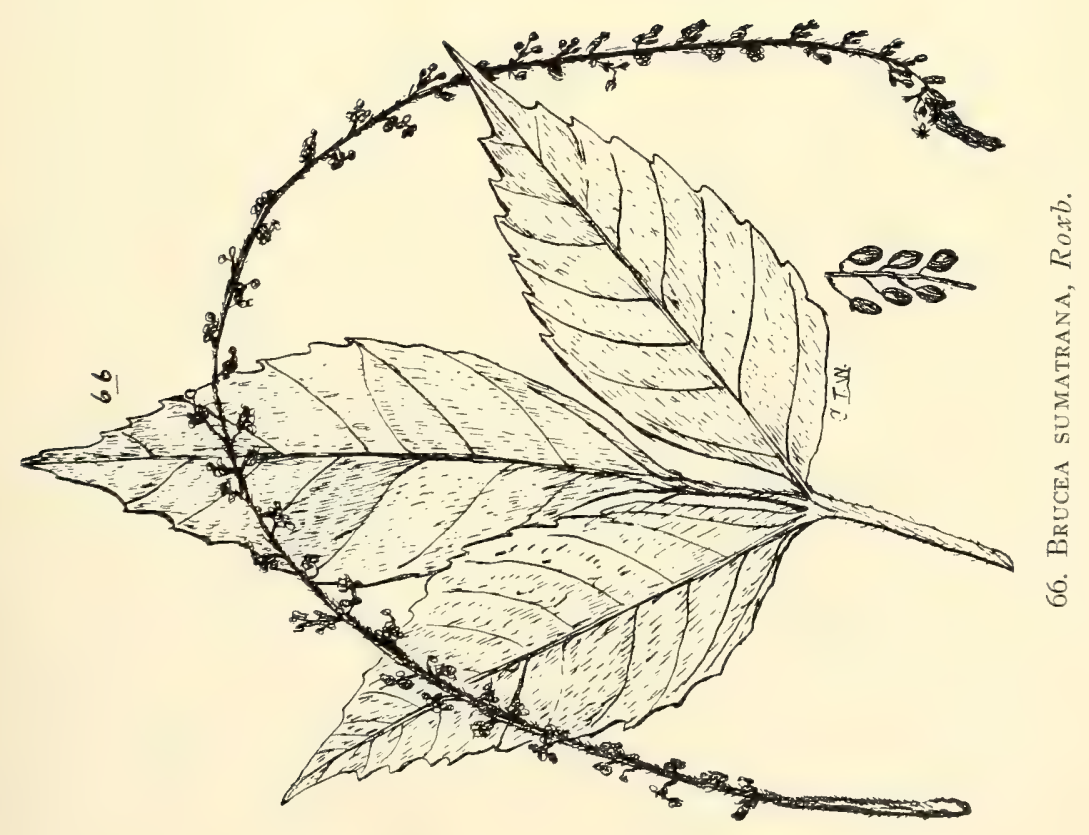




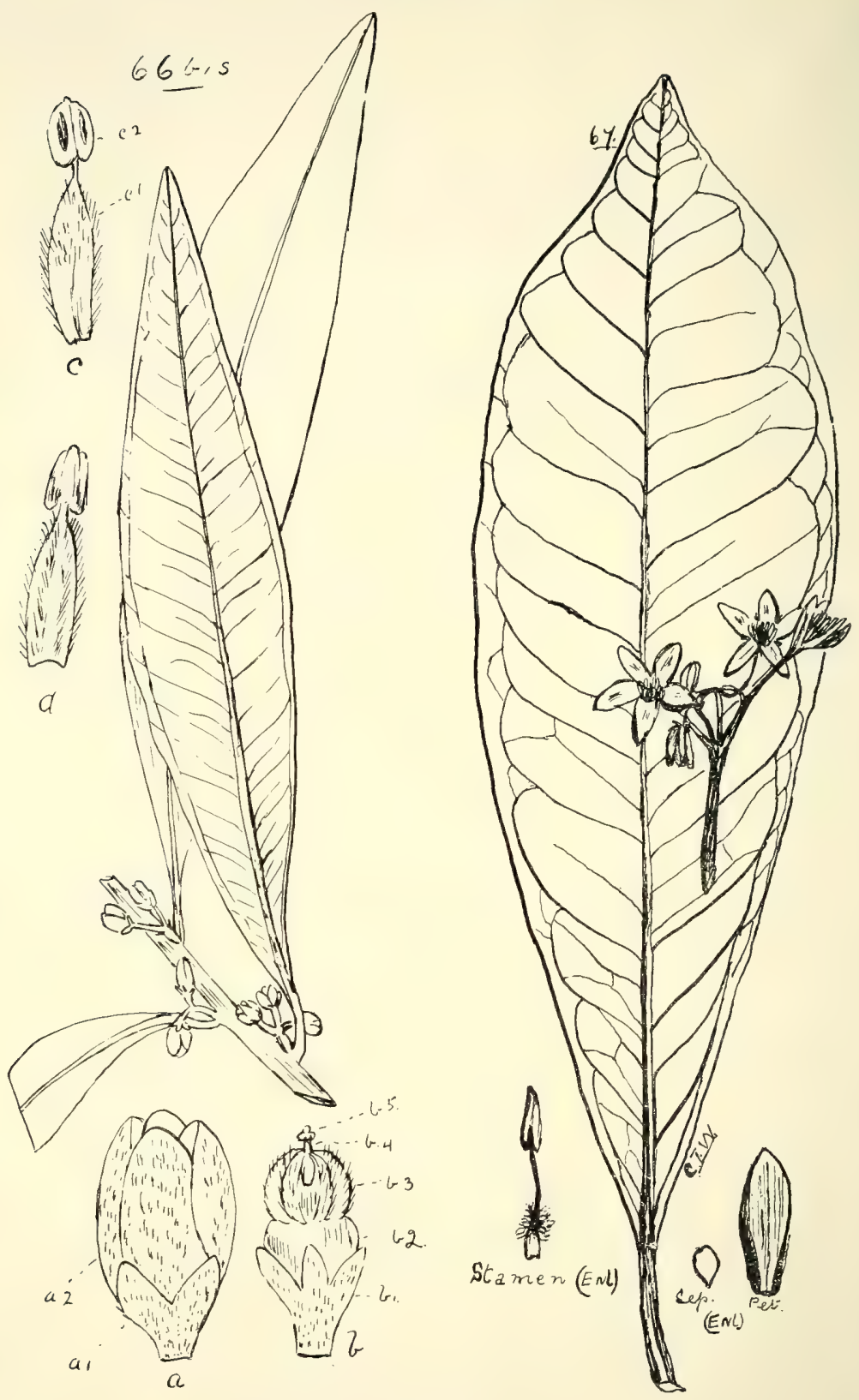

66 bis. Samadera Bidwilli, Oliver.

(a) Flower, (a I) calyx, (a 2) petals, (b) calyx and pistil, (b I) calyx, (b 2) disk, (b 3) ovary, (b 4) style, (b 5) stigma, (c) stamen, front view, (d) stamen, back view. (a) - (d) enl. (After Oliver, Hk. Ic. P1.).

67. Samadera Baileyana, Oliver. 

PLATE IV.

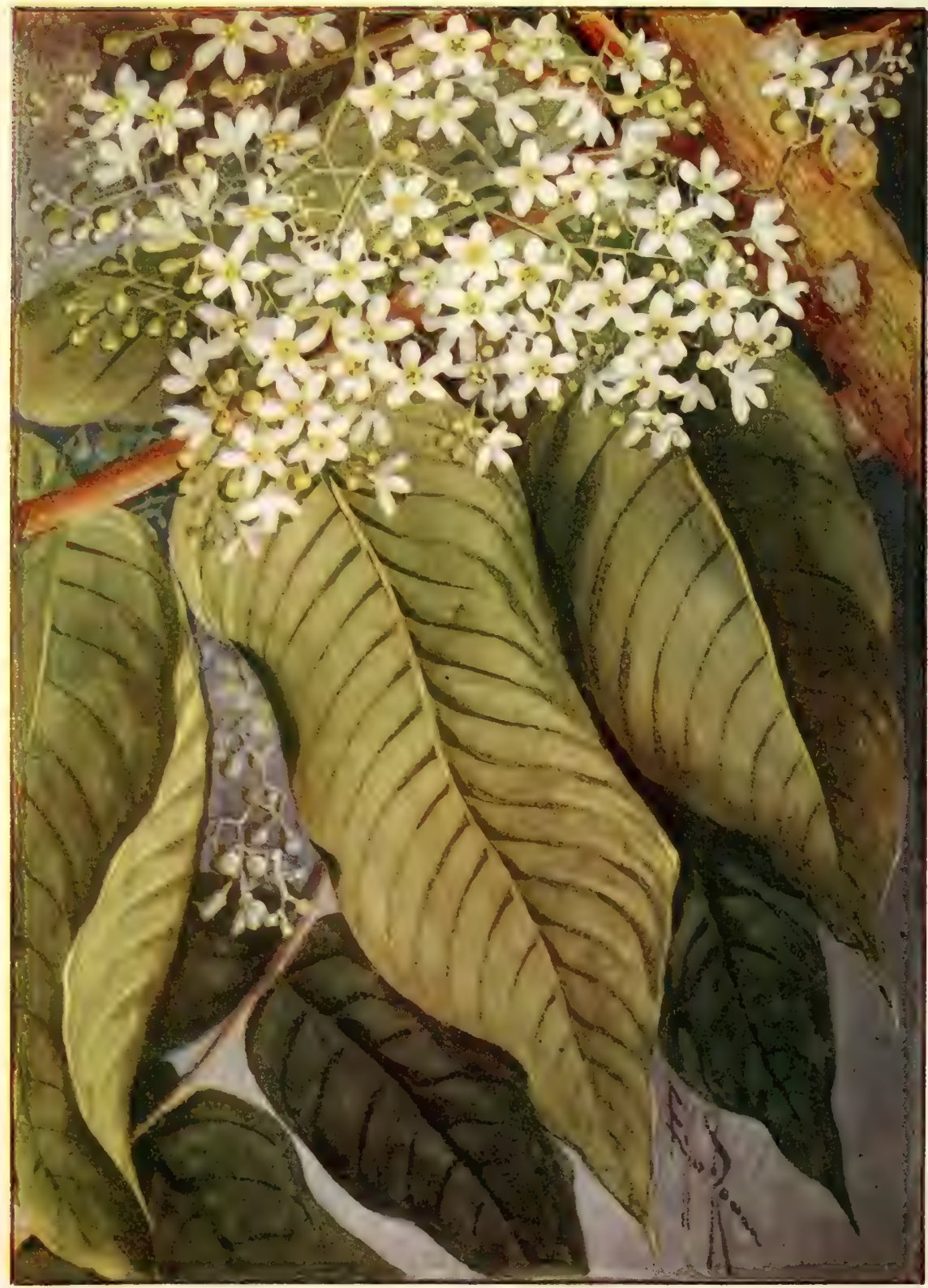

Flindersia pubescens, Bail. 
Flindersia, $R$. $B r$.-The aboriginal name for the genus at Cardwell is "Kangabberoo" (R.B.H.). The wood of all the kinds useful.

\section{Leaves pinnate.}

australis, R. Br.--Crow's Ash. Wood durable; said not to rust iron. The capsules used for ornamental purposes, pincushions, \&c.

Leichhardtii, C. $D C$.

Schottiana, F. v.M.--"Bunji-bunji" of Herberton natives. The bark said to contain a poisonous principle.

Oxleyana, F.v.M.-Yellow-wood. This excellent wood is considered suitable for buggy-shafts and hand-screws.

Bennettiana, F. v. M.-Bogum-bogum.

Chatawaiana, Bail.--Red Beech; Cardwell Maple; has also been called "Silkwood." "Narroo" of Atherton natives. Brayleyana, F.v.M.-Wood has been cut under the name of "Silkwood."

Ifflaiana, F.v.M.-Cairns Hickory.

Mazlini, Bail.

Pimenteliana, F. v. M.

Bourjotiana, F. v. M.

pubescens, Bail.-A very useful shade tree. (Plate IV.)

Tysoni, C. DC., Bull. de L'Herlier Boissier vi. ( I906).

collina, Bail_-Gum contains 83.5 per cent. of arabin (Lauterer).

Strzeleckiana, F.v.M.-Spotted-tree. (Fig. 73.)

Leaves simple.

maculosa, Lindl. = Eloodendron maculosum, Lindl. Leopard-tree.-Gum may be used as a substitute for gum-arabic; contains 8I.4 per cent. of arabin ( $\mathrm{Dr}$. Lauterer). (Fig. 73 bis.)

Alliance VIII.-OLACALES.

\section{Order XXXIV.-OLACINEA.}

Ximenia, Linn.

Tribe I.-Olace.e.

americana, Limn-Yellow Plum of Hammond Island; contains prussic acid. "Gotoobah" of Bellenden-Ker and "Bed-yew-rie" of Dunk Island natives. The wood resembles English Box. 


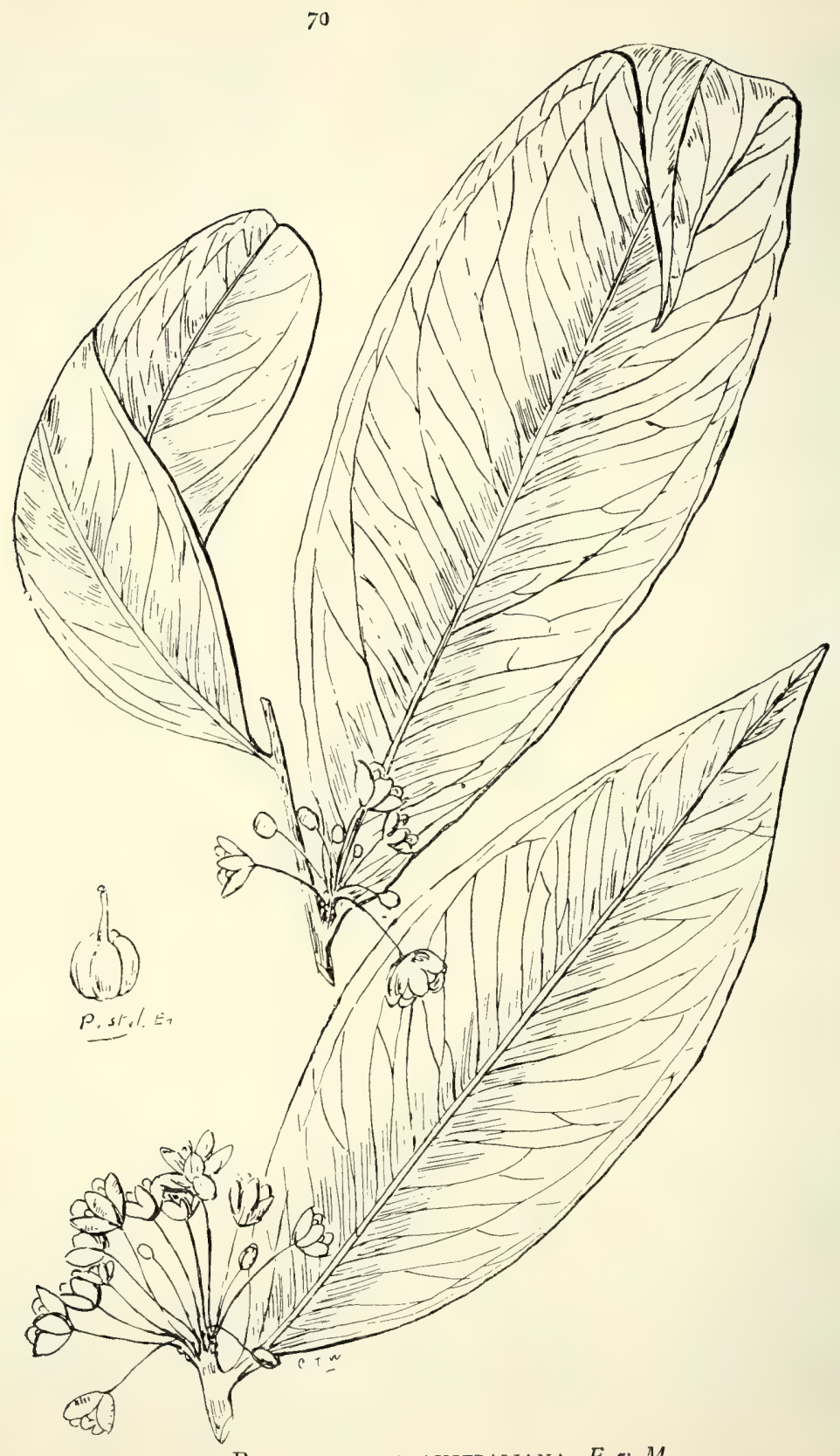

7o. Brackenridgea australiana, $F, v \cdot M$. 
Olax, Limn.

retusa, $F . v . M$.

stricta, R. $B r$.

aphylla, R.Br. (Fig. 74.)

Tribe II.-OpilieÆ.

Opilia, Roxb.

amentacea, Roxb. (Fig. 74 bis.)

Cansjera, Juss.

leptostachya, Benth.

Tribe III.-ICACINE 2.

Gomphandra, Wall.

australiana, $F . v . M$.

polymorpha, Wight., var. 6, Bail.-Wood of light colour and nicely marked.

Apodytes, E. Meyer.

brachystylis, F.v.M.-Wood hard, yellow; useful for cabinet-work. (Fig. 75.)

Pennantia, Forst.

Cunninghamii, Miers.

Phlebocalymna, Griff.

lobospora, F. $\cdot M$.

Villaresia, Ruiz et Pav.

Smythii, F.v. M.

Moorei, F. v. M.-Churnwood of Kin Kin. Wood lightcoloured and prettily marked.

Cardiopteris, Wall.

lobata, R.Br., var. moluccana, Masters. (Fig. 76.)

\section{Order XXXV.-ILICINEA.}

Ilex, Linn.

peduncularis, F.v.M. (Fig. 76 bis.) Figured from specimen out of the Muellerian herbarium, kindly given for the purpose by Professor A. J. Ewart.

Alliance IX.-CELASTRALES.

\section{Order XXXVI.-CELASTRINEAE.}

Tritie I.-Celastree.

Euonymus, Toumef.

australianus, $F$. ข. $M$.

Celastrus, Linn.

australis, Harv. et Muell. 


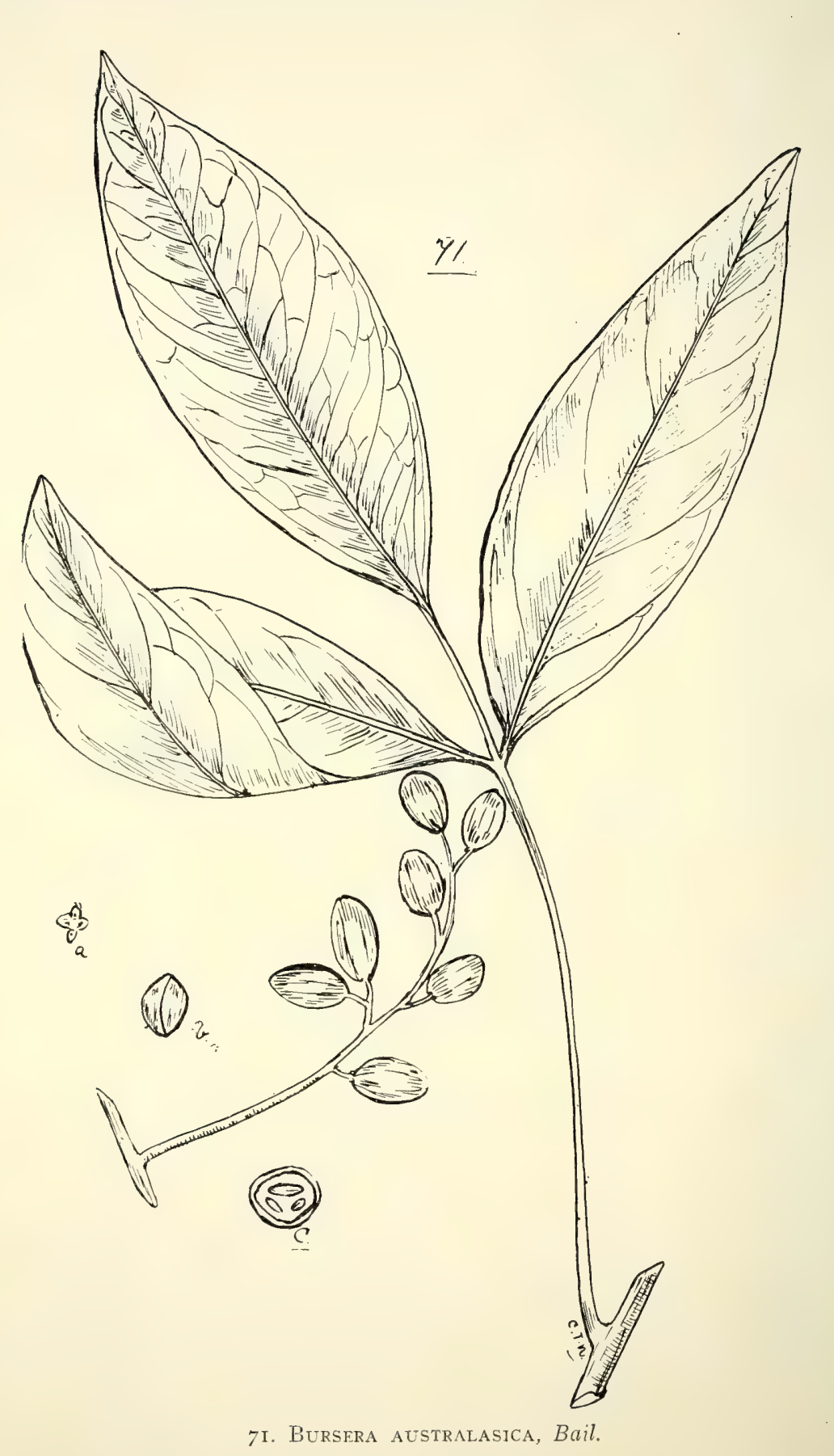


XXXIII. MELIACEÆ.-XXXIV. OLACINEÆ.

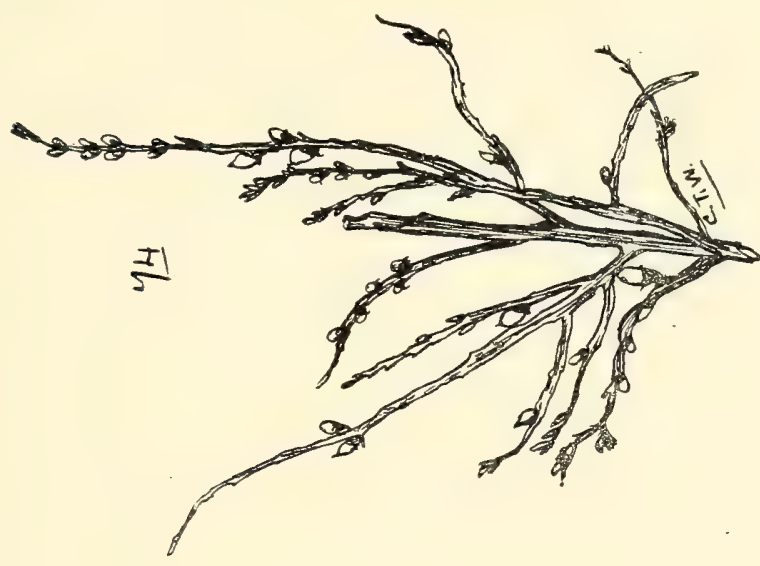

过

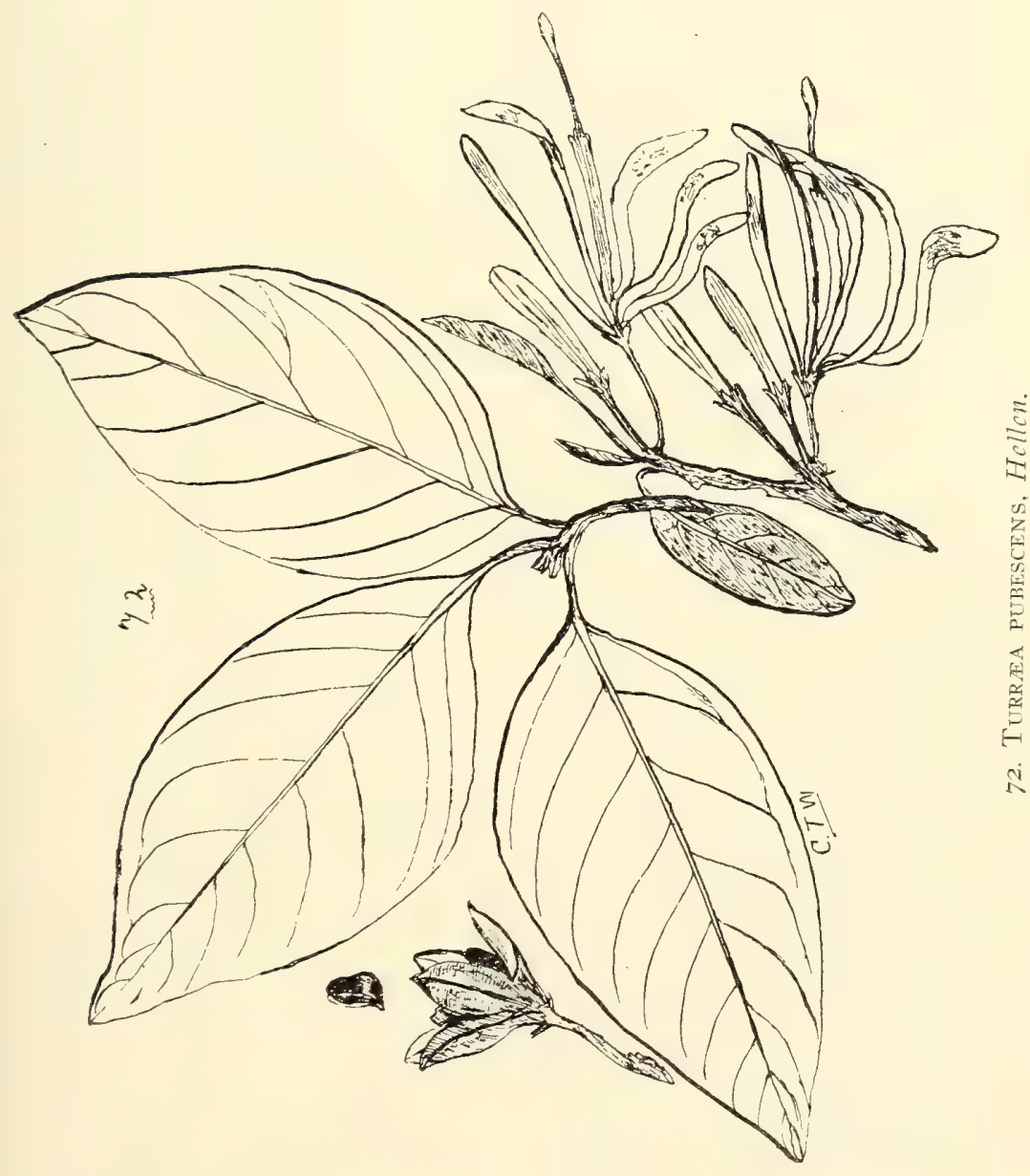


Celastrus-contd.

dispermus, F.v.M.-Wood light-coloured, prettily marked. bilocularis, F. v. M.-Wood light-grey, hard and tough.

Cunninghamii, F.v. M.

Gymnosporia, $W$. et Arn.

montana, $W$. et Arn.

Hypsophila, F. v. $M$.

Halleyana, F.v. M.

oppositifolia, F. v. M.

Hedraianthera, F. v. $M$.

porphyropetala, F.v.M.-Flowers small, dark-purple. (Fig.

$7 \%$.)

Denhamia, Meissn.

oleaster, F.v.M.

obscura, Meissn.- "Tchannan" of Pennefather River and

"Treine" of Mapoon natives.

pittosporoides, F. v. M.-Wood resembling the English Elder; suitable for pattern-making.

viridissima, Bail. et F.v.M. (Fig. 78.)

Caryospermum, Blume.

arborescens, F.v. M. (Fig. 79.)

Elæodendron, Jacq. $f$.

australe, Vent.--Fruit red. Wood warps a good deal in drying if cut up before being properly seasoned.

var. angustifolia, Benth.-Fruit red.

melanocarpum, F. v. M.-Fruit black.

TRIBE II.-HIPPOCRATEA.

Salacia, Linn.

prinoides, $D C$.

Siphonodon, Griff.

australe, Benth.-Ivory-wood, useful for carving; white.

var. Keysii, Bail. (Fig. 79 bis.)

pendulum, Bail.-_"Bambudal" of Cooktown and Cape Bedford, "Aguridal" of Palmer River, and " Ngarwowoya" of Princess Charlotte Bay natives. (Fig. 8o.)

membranaceum, Bail. (Fig. 80 bis.)

Hippocratea, Linn.

obtusifolia, Roxb., var. barbata, Benth. 


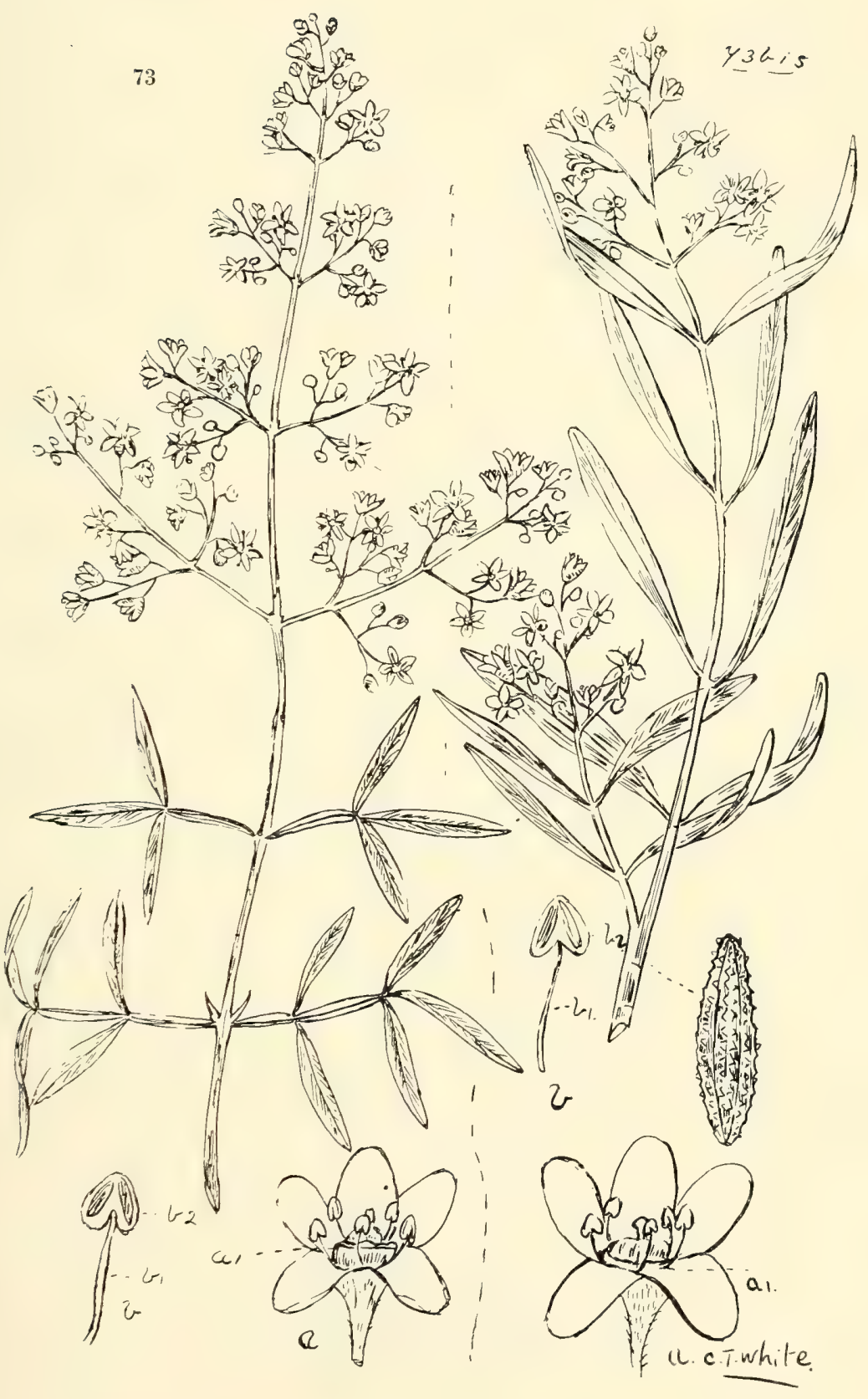

73. Findinersia Strzeleckiana, F.v. $M$.

73 bis. F. Maculosa, Lindl.

(a) Flower, (a r) disk, (b) stamen, (b r) filament, (b 2) anther. (a) and (b) enl. 
98

XXXIV. OLACINE压.
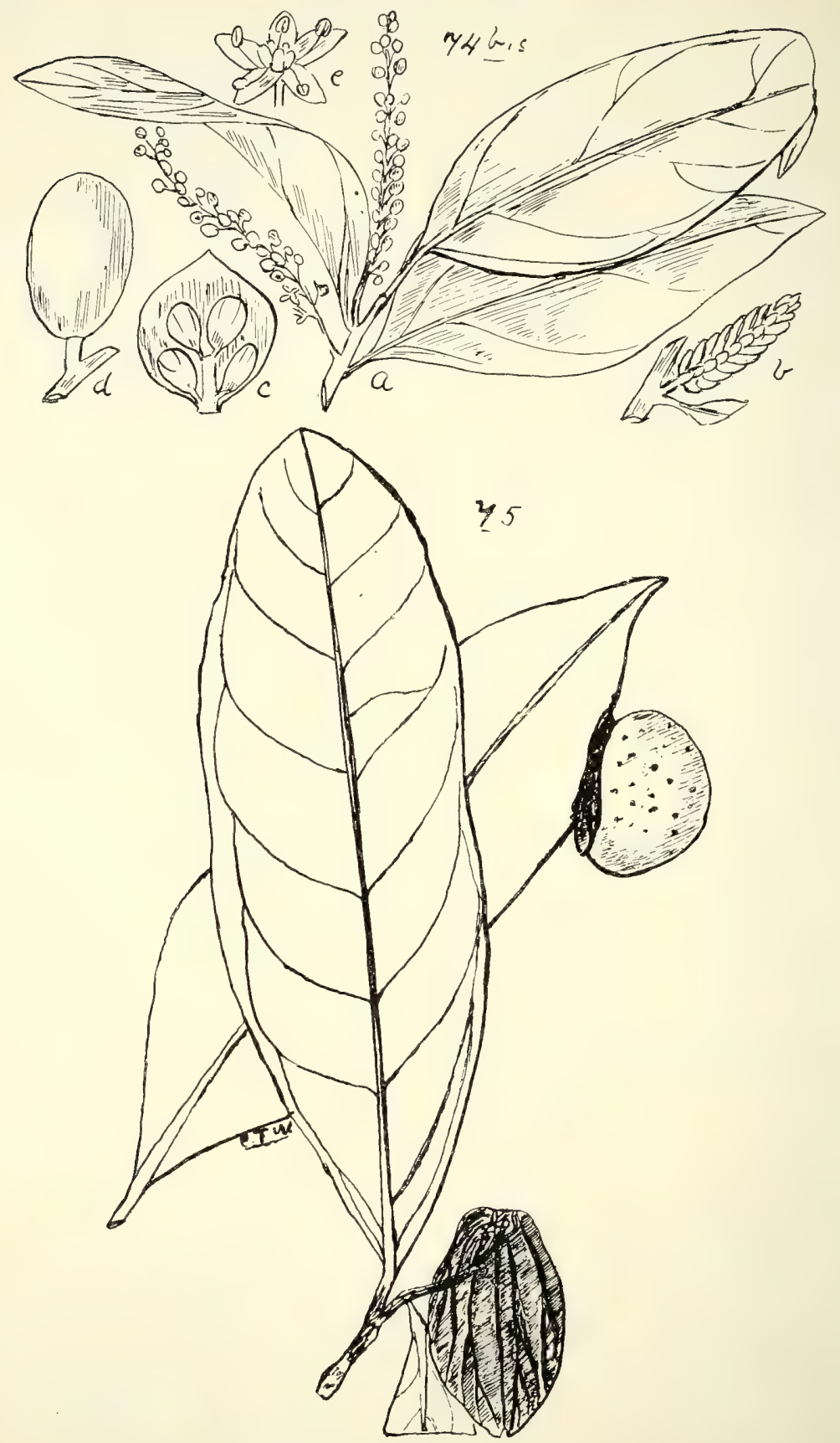

74 bis. Opilia amentacea.

(a) A flowering shoot, (b) early raceme (amentum), (c) a scale of the amentum with enclosed flower-buds, (d) fruit, (e) flower. (a) (b) and (d) nat. size; (c) and (c) enl. After Wight)

75. Apodytes BRAChystylis, F. $v . M$. 

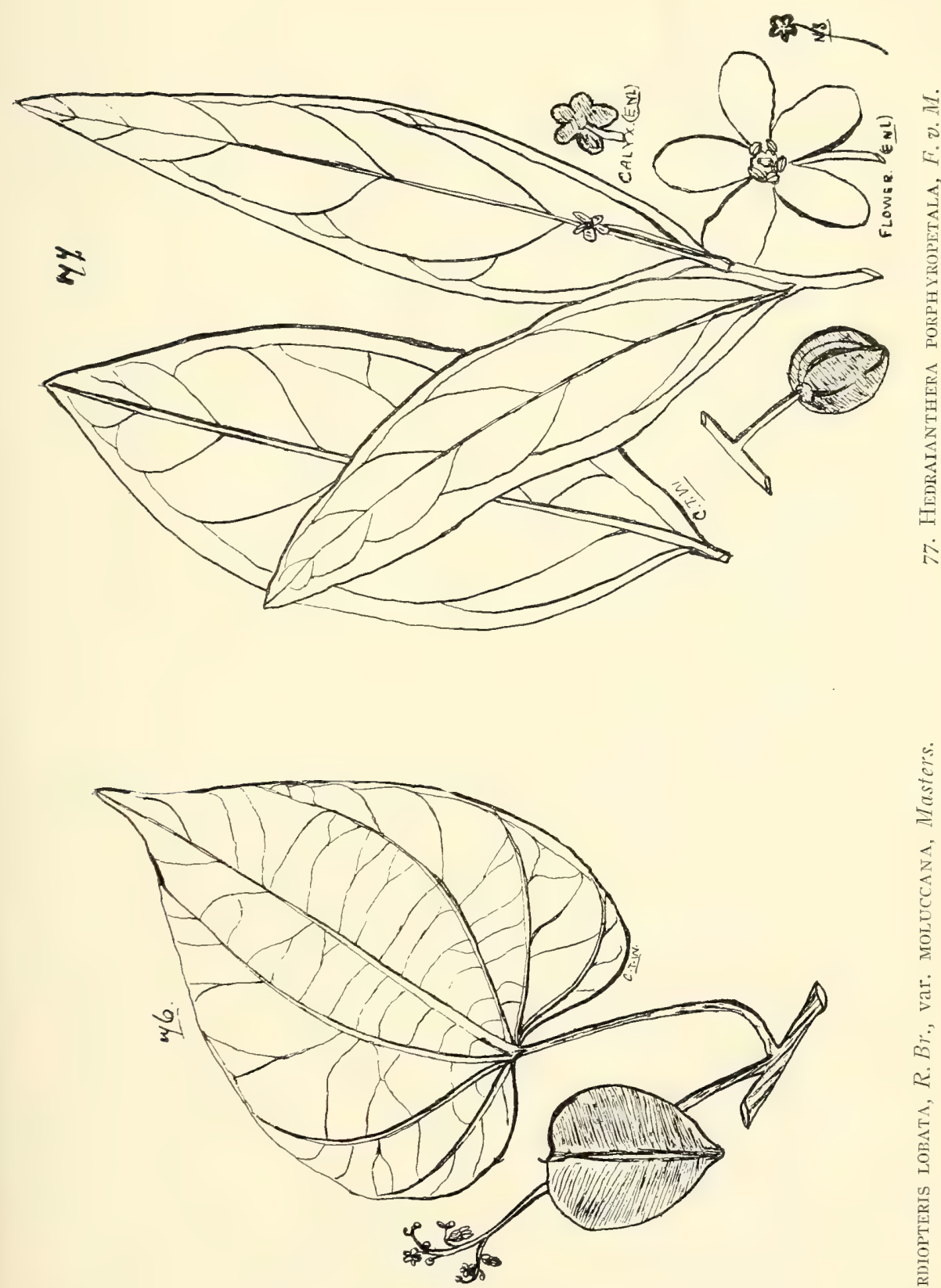


\section{Order XXXVII.-STACKHOUSIEÆ.}

Stackhousia, $S m$.

spathulata, Sieb.

monogyna, Labill.

muricata, Lindl.

intermedia, Bail. (Fig. 8I.)

viminea, $S m$.

var. elata.

Tryoni, Bail. (Fig. 82.)

Macgregoria, F. v. $M$.

racemigera, F.v.M. (Fig. 83.)

\section{Order XXXVIII.-RHAMNEÆ.}

Tribe I.-Ventilagineæ.

Ventilago, Gartn.

viminalis, Hook.- " Thandorah" of Cloncurry natives.

ecorollata, F.v.M. = Berchemia ecorollata, F.v. M. (Fig. 84.)

Zizyphus, Juss.

TRIBE II.-ZiZYPHE.尺.

Enoplia, Mill.

jujuba, Lam.-Common Jujube. A noxious weed in some Northern localities.

Tribe III.-RhAMNE.E.

*Rhamnus, Linn.

Alaternus, Limn.-Buckthorn. Europe.

Dallachya, F. v. M.

vitiensis, F.v.M. = Rhamnus vitiensis, Benth._" Murtilam" of natives. Bark yields a reddish-brown dye.

var. pubescens (Thozet), F.v.M.

Schistocarpæa, F. v. M.

Johnsoni, F.v.M. (Fig. 85.)

Colubrina, L. C. Rich.

asiatica, Brongn.

Alnhitonia, Reissek.

excelsa, Reissek.-Red Ash. The leaves, with water, rubbed on the hands by school-children to remove ink-stains. "Ane" of Batavia River and "Mee-a-mee" of Moreton Bay natives. 


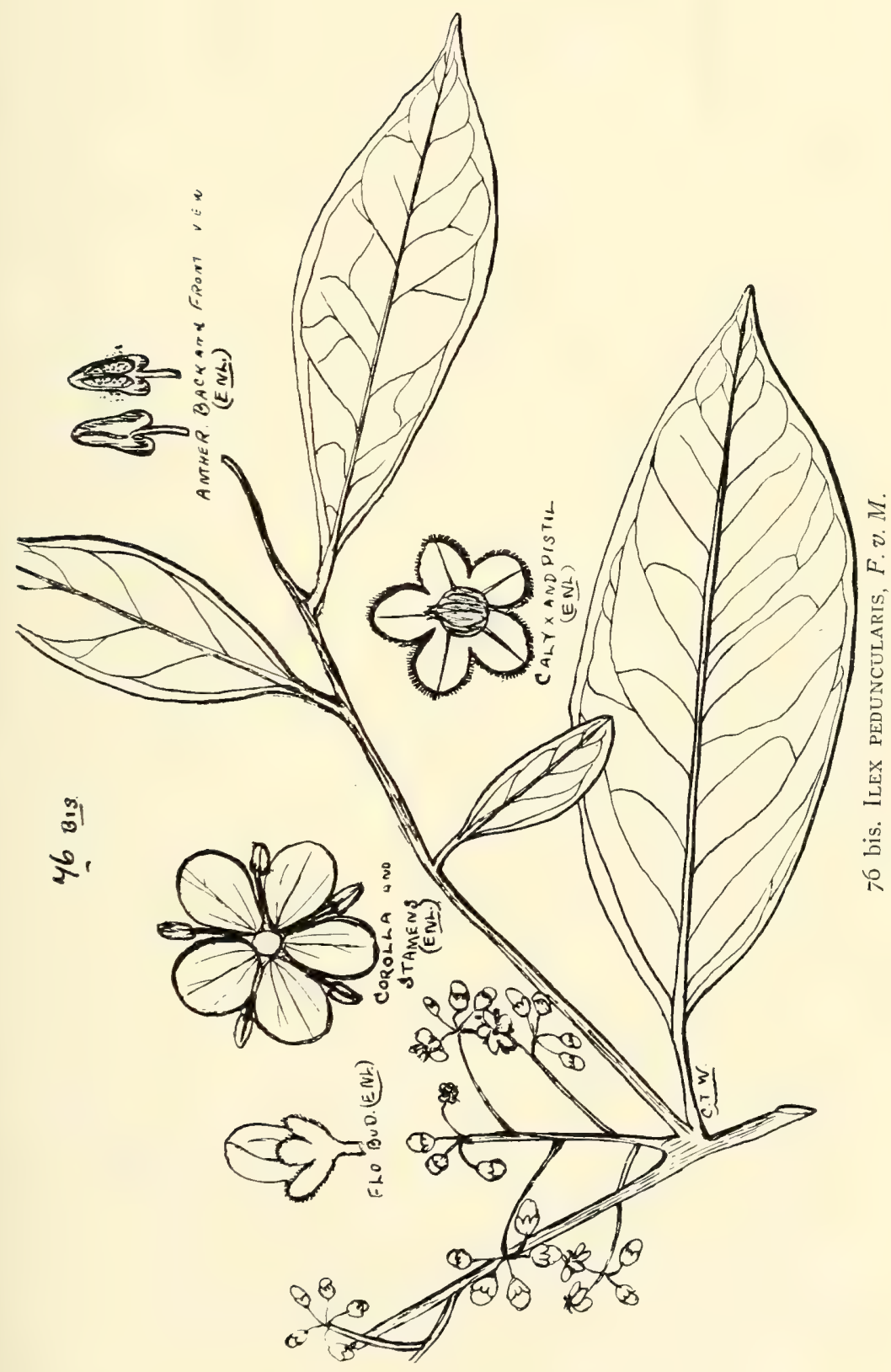



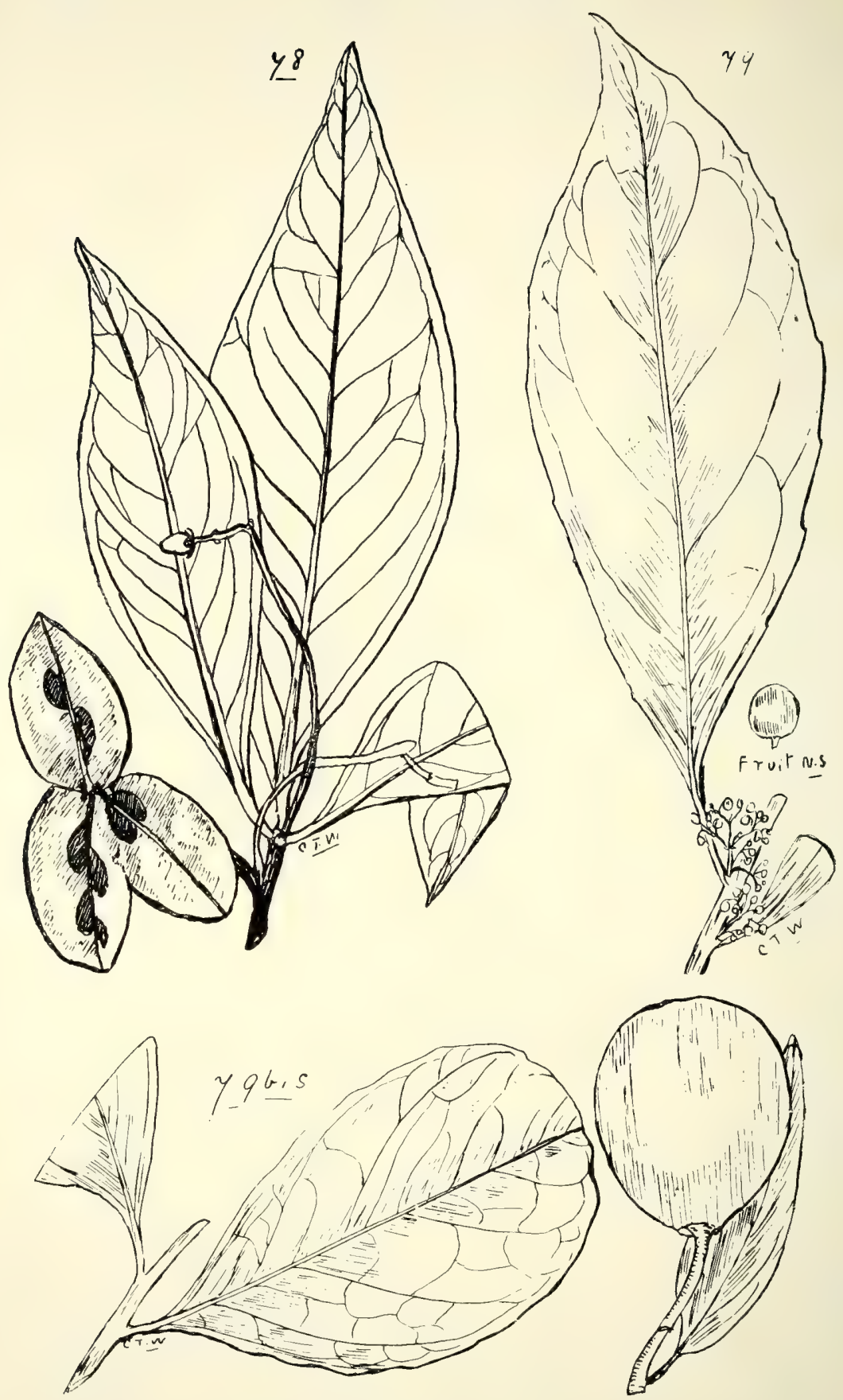

78. Denhamia viridissima, Bail. et F. v. $M$.

79. Caryospermum arborescens, F. v. $M$.

79 bis. Siphonodon australe, Benth, var. Keysir, Bail. 


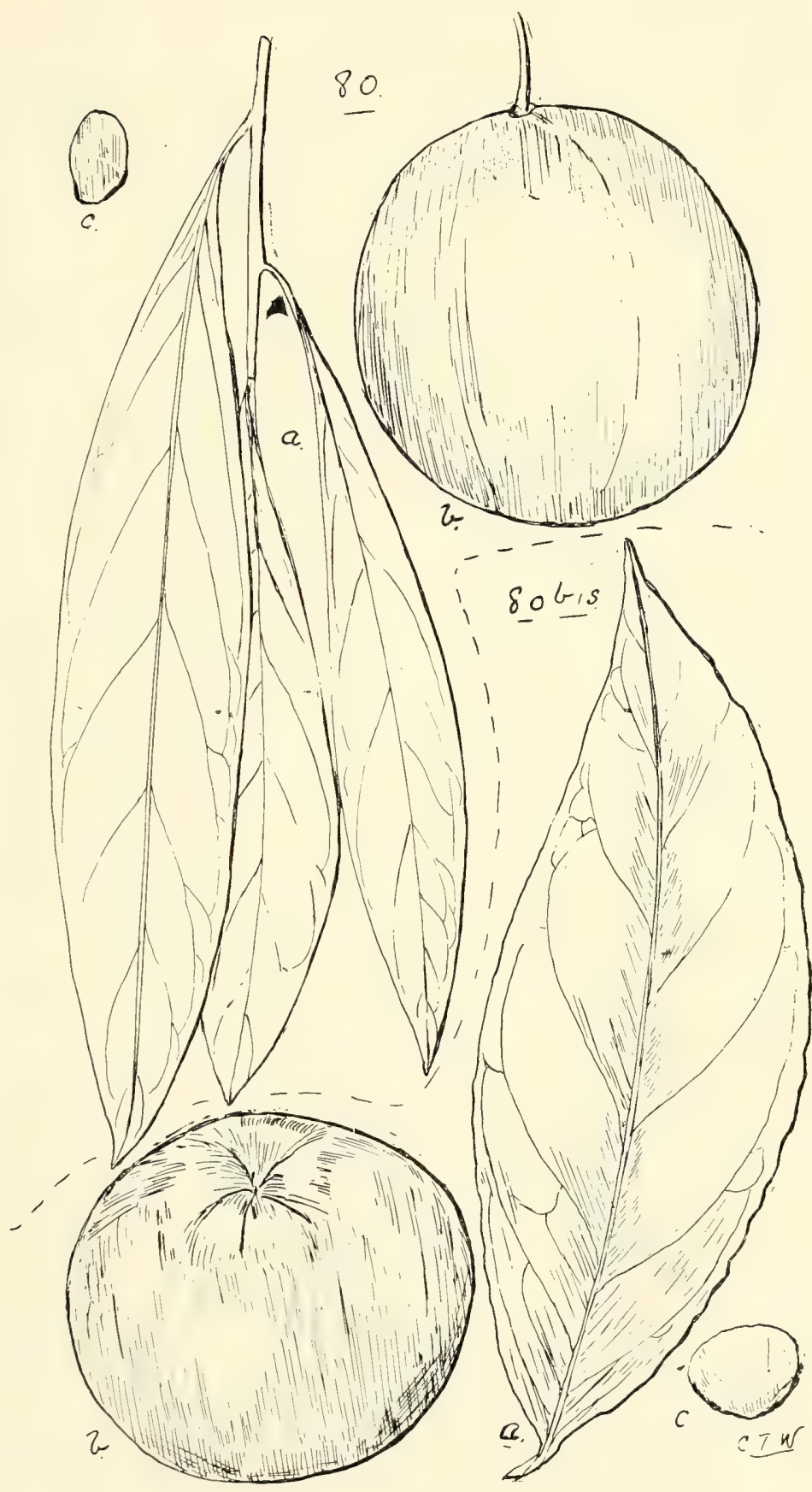

8c. Siphonodon pendulum, Bail.

80 bis. S. membranaceum, Bail.

(a) Leaf, (b) fruit, (c) seed. All nat. size. 


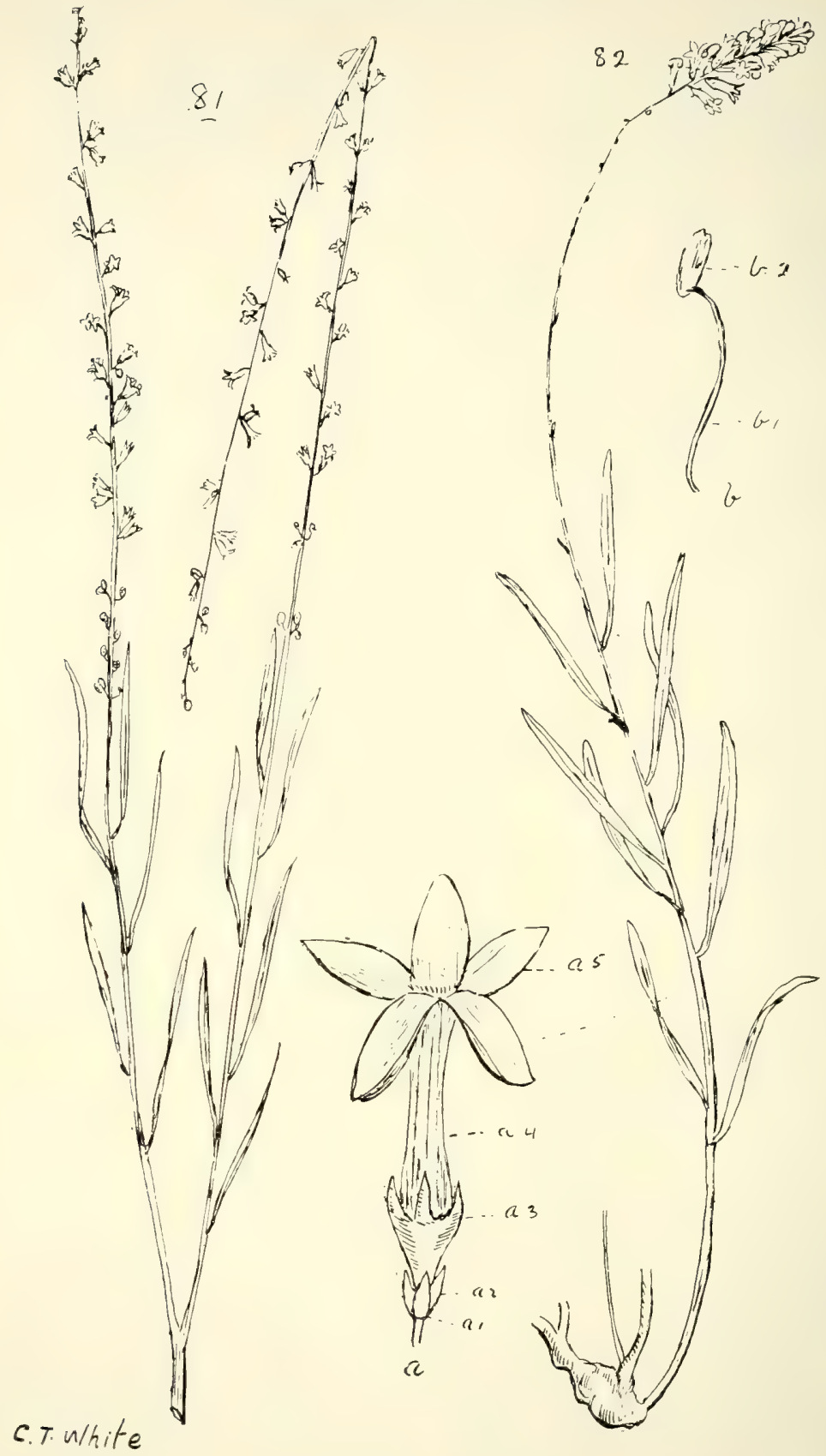

8I. Stackhousia intermedia, Bail.

82. S. TRYONT Bail.

(a) Flower enl., (a 1) bract, (a 2) bracteoles, (a 3) calyx lobes, (a 4) corolla tube, (a 5) corolla lobes. 


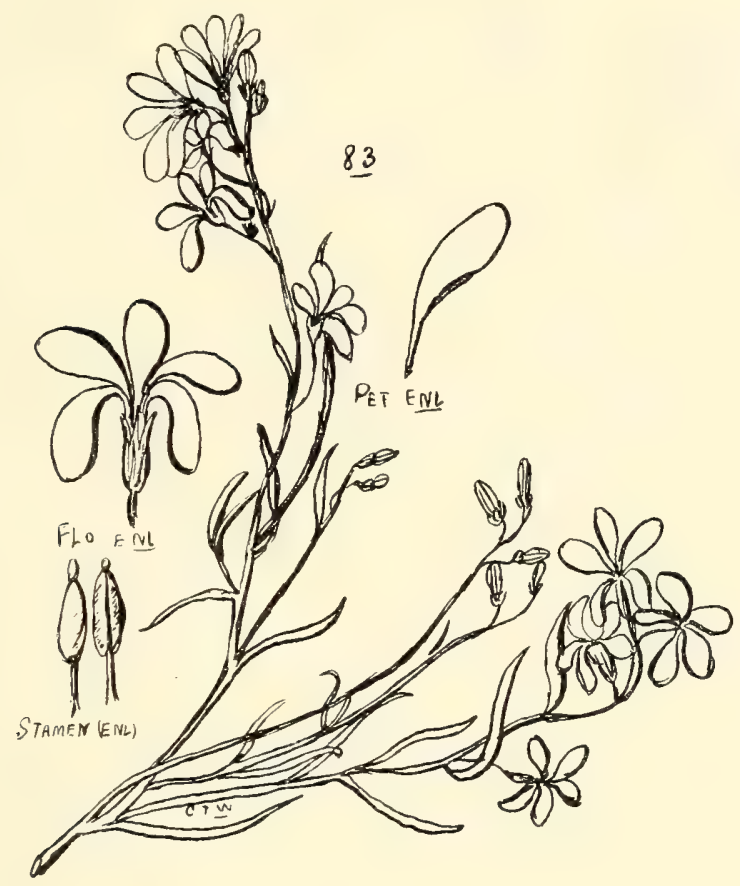

83. Macgregorta Racemigera, $F \cdot v . M$.

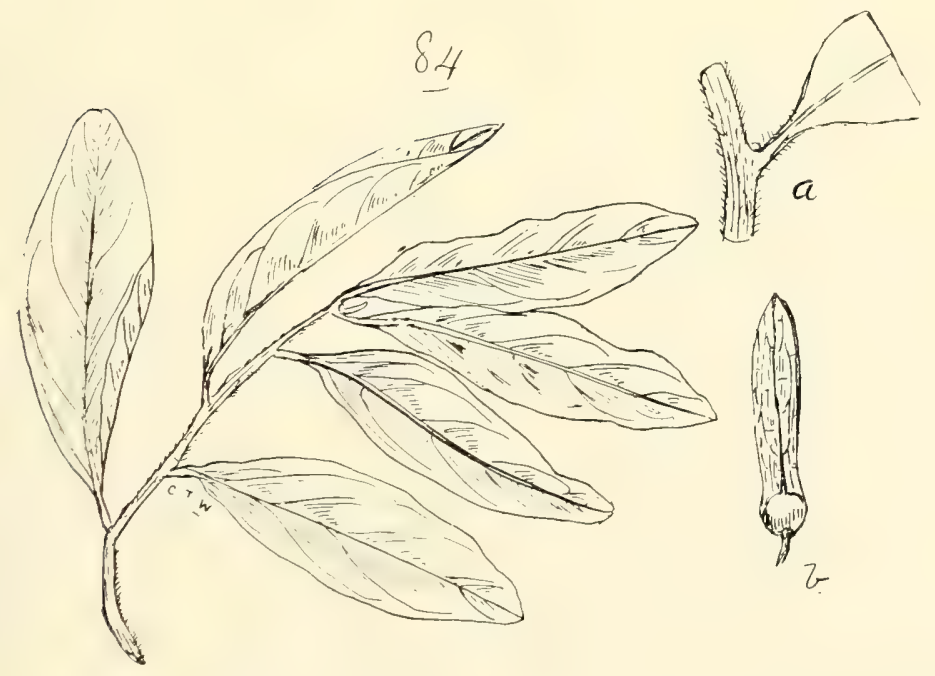

84. Ventilago ecorollata, $F$. ข. $M$.

(a) Portion of a branchlet enl., (b) nut with its appendage (wing). 
Emmenospermum, F. $v . M$.

alphitonioides, F. v. M._- Jingull" of Cairns natives.

Cunninghamii, Benth.

Pomaderris, Labill.

elliptica, Labill.

lanigera, Sims.

ferruginea, Sieb.

var. canescens, Benth.

phillyreoides, Sieb.

var. nitidula, Benth.

prunifolia, A. Cunn.

Cryptandra, $S m$.

ericifolia, $S m$.

spinescens, Sieb.

amara, $S m$.

longistaminea, $F . v . M$.

Stenanthemum, Reissek.

Scortechinii, F.v. M.

Tribe IV.-Colletieæ.

Discaria, Hook.

australis, Hook. = Colletia pubescens, Brongn.

Tribe V.-GouAnieж.

Gouania, Linn.

Hillii, F. v. M.

australiana, F.v.M. (Fig. 86.)

\section{Order XXXIX.-AMPELIDEÆ.}

Vitis, Linn. (including Cissus).-The fruits of several of these

Native Grapes are used for jam-making. Fruit a Nuculanitum.

antarctica, Benth.-Tuberous.

oblonga, Benth. (Fig. 87.)

cordata, Wall. (Fig. 88.)

adnata, Wall.

nitens, $F$. v. M.-The wood of this, $V$. hypoglauca, and others of the large-growing species is very handsome when seasoned and worked up into beading for shelves of bookcases, \&c., as the colouring is good and the veining beautiful.

saponaria, Seem.

acris, F. v. M. 
XXXVIII. RHAMNEÆ.

107

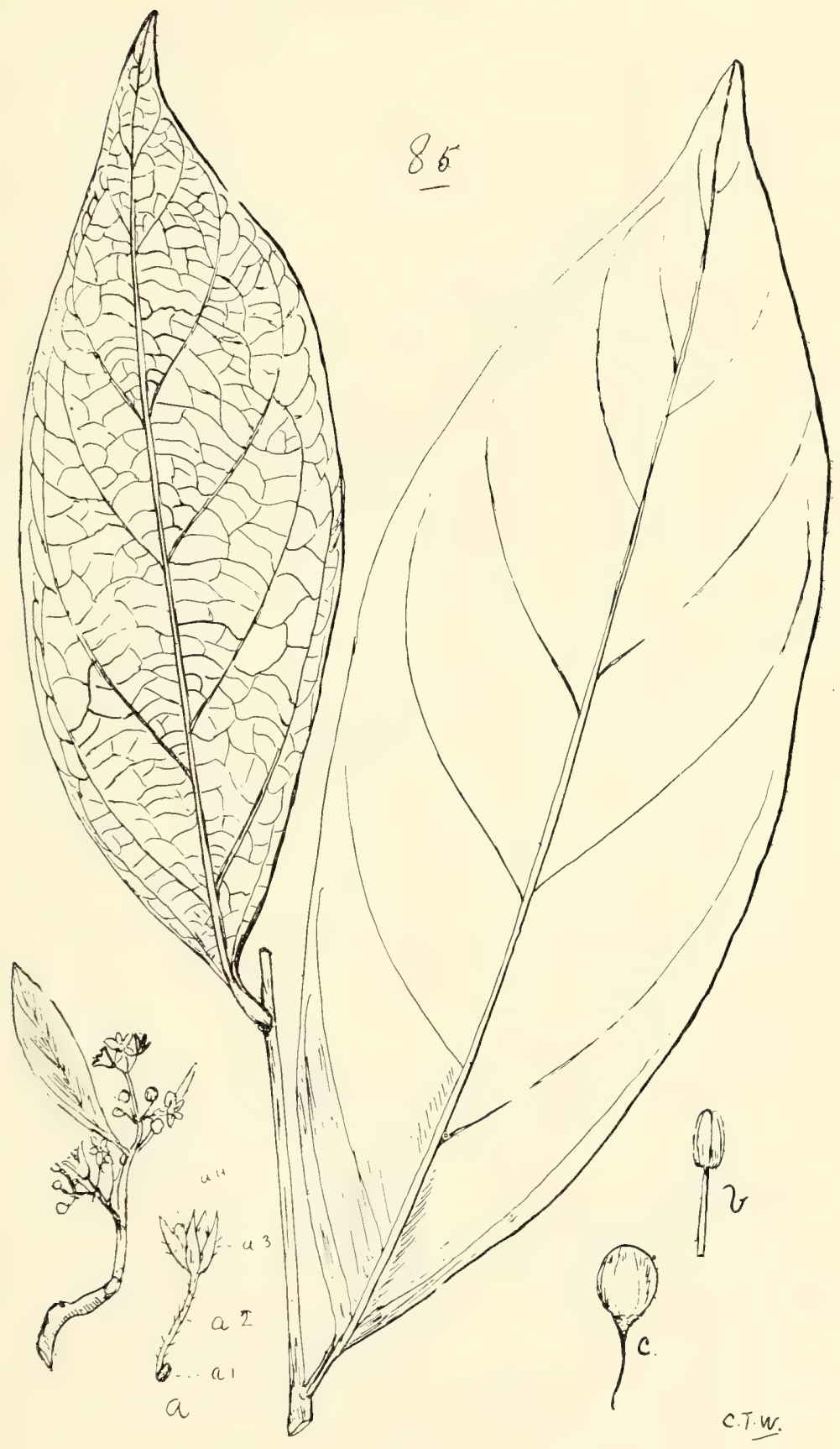

85. Schistocarpera Johnsoni, F.v. M.

(a) Flower, (a 1) bract, (a 2 ) pedicel, (a 3 ) calyx lobes, (a 4 ) petals, (b) stamen,

(c) fruit. (a) and (b) enl, ; (c) nat. size. 


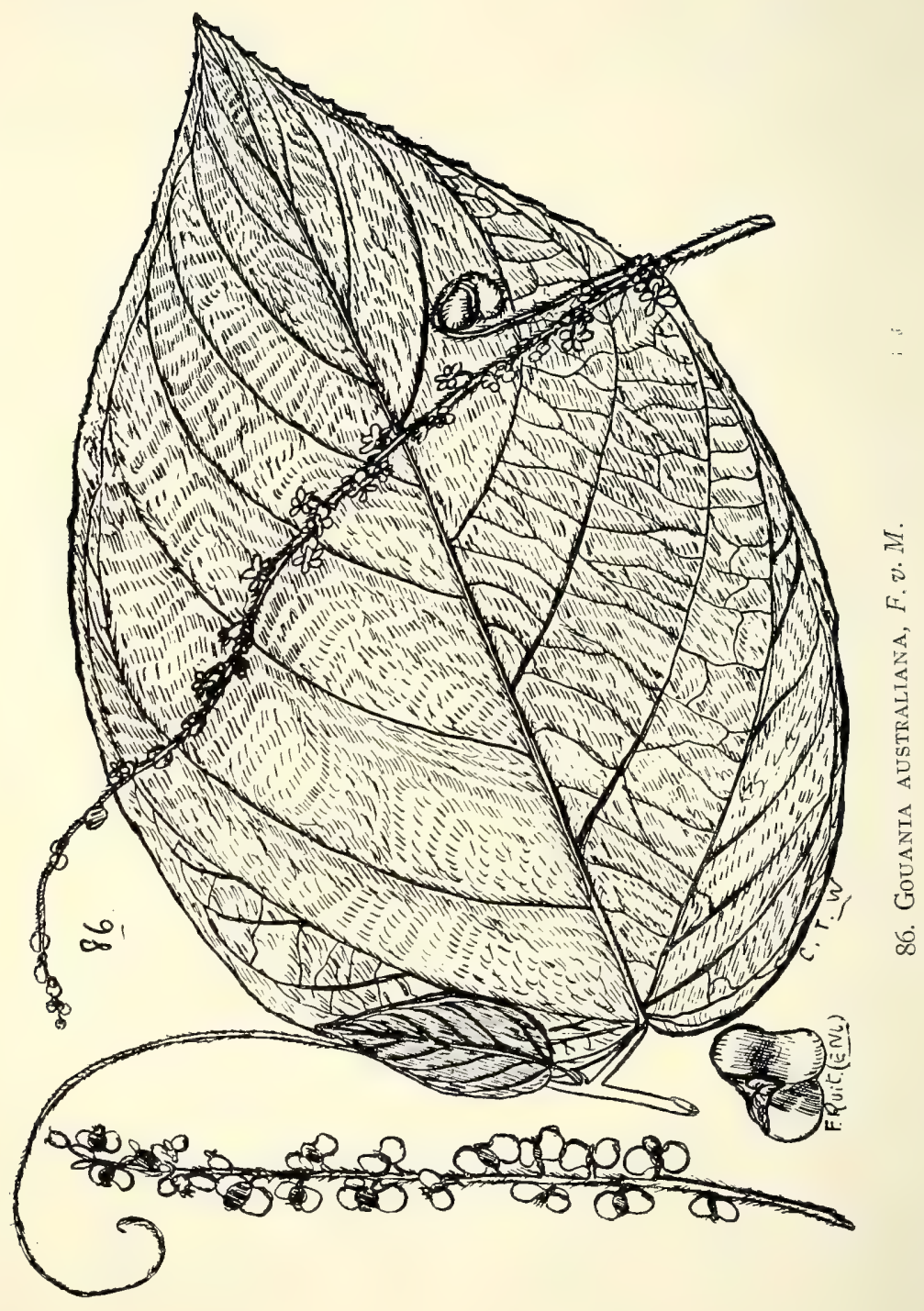




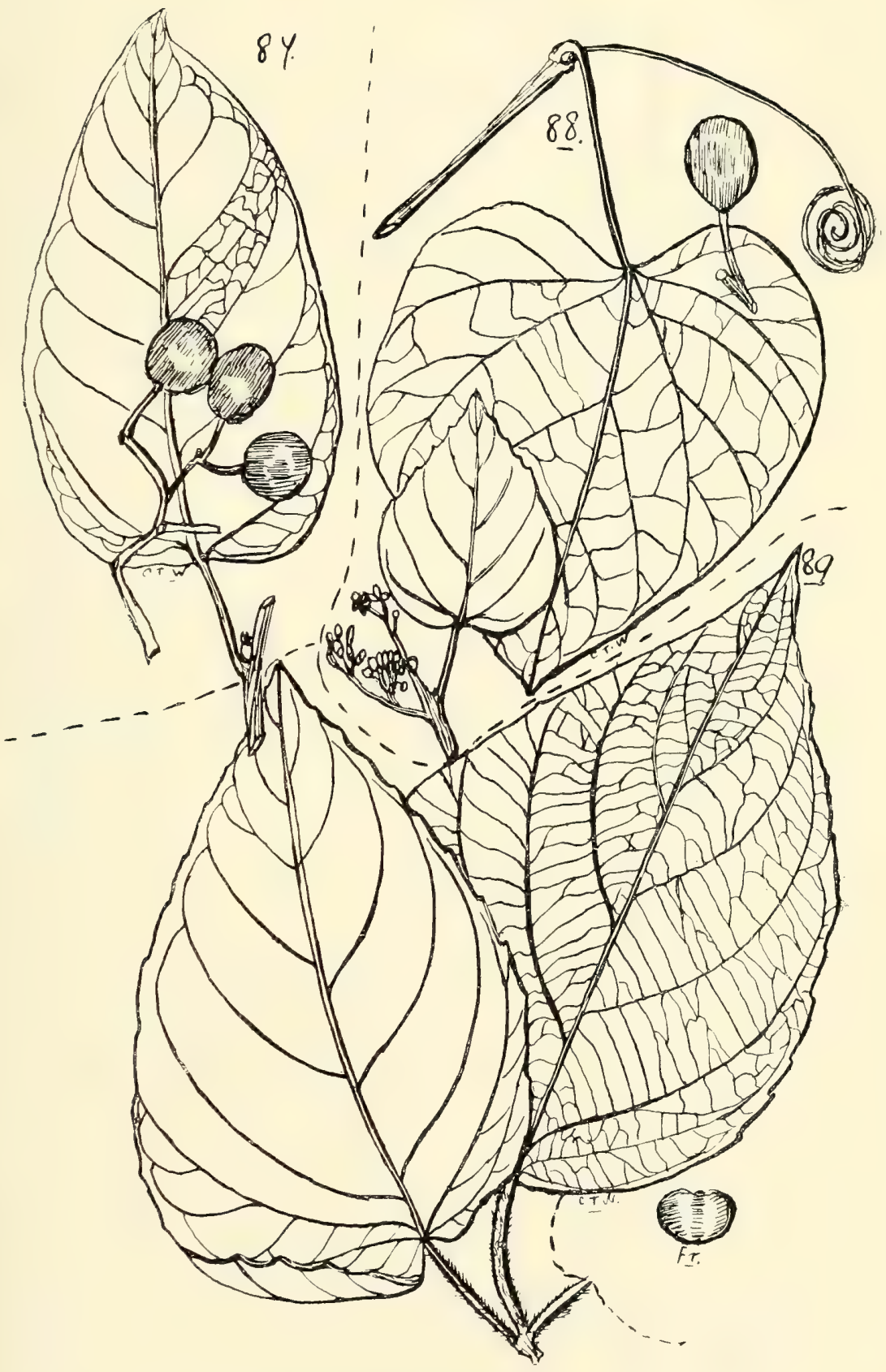

87. Vitis oblonga, Benth.

88. V. CORDATA, Wall.

E9. V. strigosa, Bail. 


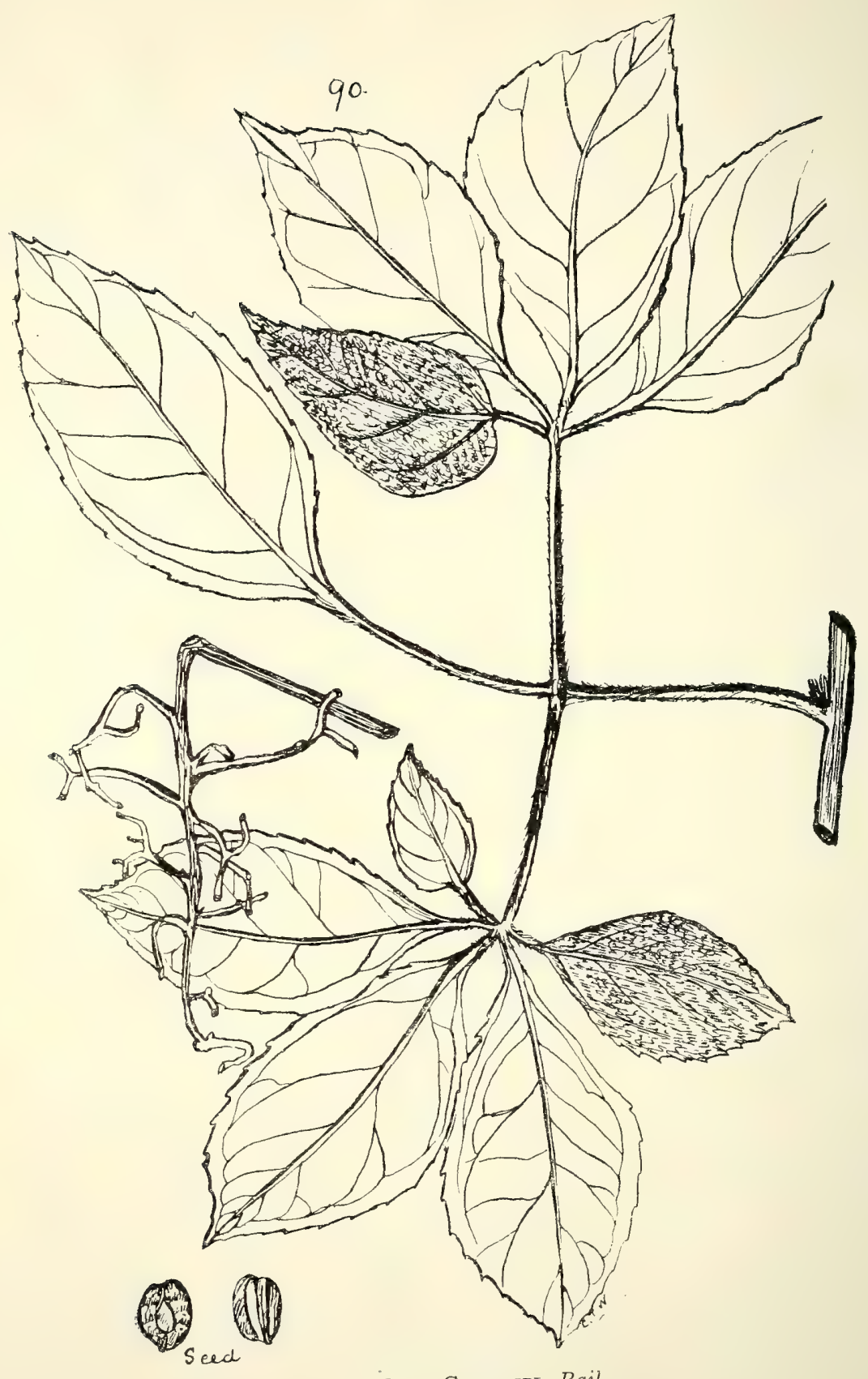

90. Vitis Gardneri, Bail. 
XL. SAPINDACER.

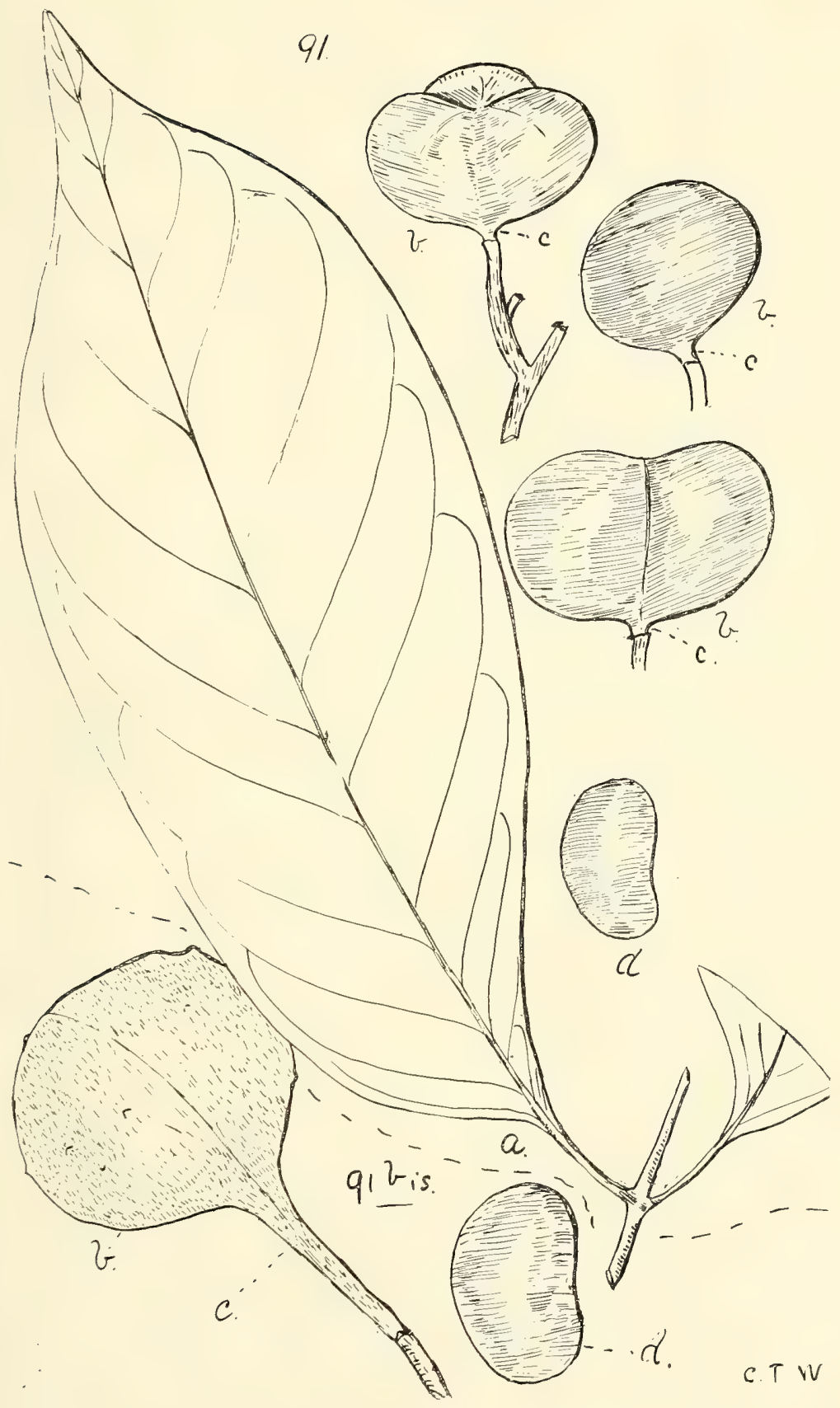

91. Castanospora Alphandi, $F, v . M$.

9I bis. C. Longistipitata, Bail.

(a) Leaflet, (b) capsule, (c) stipes, (d) seed. All nat. size. 
Vitis-contd.

trifolia, Linn.- "Lorwora" of Palmer River, "Takking” of Nassau and Staaten Rivers, "Tampara” and "Lenn” of Princess Charlotte Bay, and "Pulkun” of Butcher's Hiil natives. This and some other species form tubers at the roots which are used by the natives for food after being roasted $($ Roth). It has been stated that in some parts of India the juice of this plant has had the reputation of being an antidote to the bites of snakes. Our Cape York peninsula natives say the same of the juice of $V$.acetosa, even to the bite of the death-adder.

strigosa, Bail. (Fig. 89.)

brachypoda, F. v. M.

penninervis, $F . v . M$.

clematidea, F. v. M.-Tuberous. "Mor-bir" of Cooktown and "Bu-yan" of Cape Bedford natives.

japonica, Willd.

acetosa, F.v.M.- “Mbau-nu” of Batavia River natives.

hypoglauca, F.v. M.- "Billangai" of Barron River, "Kang-

ga" of Cooktown, "Gang-gu-gur" of Cape Bedford,

"Gang-a” of Bloomfield River, " Achealla” of Palmer

River, and "Nai-ya" of Princess Charlotte Bay natives. sterculifolia, F.v.M.-“Yaroong" of Moreton Bay natives.

opaca, F.v.M.-Pepper-vine. Tuberous. "Wappo-wappo" of Rockhampton natives.

Gardineri, Bail.- The largest bunches of fruit are said to be met with on limestone country near the Walsh River.

Leea, Linn.

sambucina, Willd.

Brunoniana, Clarke.

Alliance X.-SAPINDALES.

\section{Order XL.-SAPINDACE $Æ$.}

Suborder I.-SAPINDEF.

Cardiospermum, Linn.

Halicacabum, Limn.-Heart Pea or Balloon vine. It is said

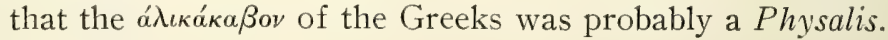

Diploglottis, Hook. $f$.

Cunninghamii, Hook. f.-Native Tamarind-tree. Edible fruit. var. Muelleri, Bail. = Cupania diphyllostegia, F.v. M. - Smaller Native Tamarind. Edible fruit. 
Schmidelia, Linn.

serrata, DC. = Allophyllus ternatus (Lour.), F. v. M.; Ornitrophe serrata, Roxb.

Castanospora, F. v. M.

Alphandi, F.v.M. (Fig. 9I.)

? longistipitata, Bail.-A doubtful species: no flowers seen, but when known the species may have to be placed in Meliace. (Fig. 9 I bis.)

Cupania, Limn.

Wadsworthii, F. v. M. = Harpullia Wadsworthii, F. v. M. (Fig. 91 ter.)

Shirleyana, Bail.- "Cooraloo" of Bundaberg natives. (Fig. 92.)

anacardioides, A. Rich._- "Tuckeroo" of Moreton Bay natives.

var. parvifolia, Bail.

serrata, F. v. M.

flagelliformis, Bail._- "Maraguigi" of Barron River natives.

curvidentata, Bail.

foveolata, F. v. M.

tomentella, F. v. M.

pseudorhus, A. Rich._ "Kilbugan" of Cardwell natives (R.B.H.). Wood suitable for pick-handles; and for ornamental planting this tree is one of the most beautiful we know.

xylocarpa, A. Cunn.

nervosa, F.v.M.-This and C. Bidwilli Baron Mueller places under C. xylocarpa.

Bidwilli, Benth.

Mortoniana, F. v. M.

Robertsonii, F..$M$.

erythrocarpa, F.v. M.

sericolignis, Bail.

Ratonia, DC. (Included in Cupania by F. v. Mueller.)

lachnocarpa, F.v. M.

grandissima, $F . v . M$.

pyriformis, Benth.-Wood suitable for axe-handles.

allodonta, Benth.

punctulata, F. ข. M.

Cordierii, F.v.M.

tenax, Benth.-Wood very tough.

O'Shanesiana, F. . M.

Nugentii, Bail._-"Chambin" of Barron River natives. 


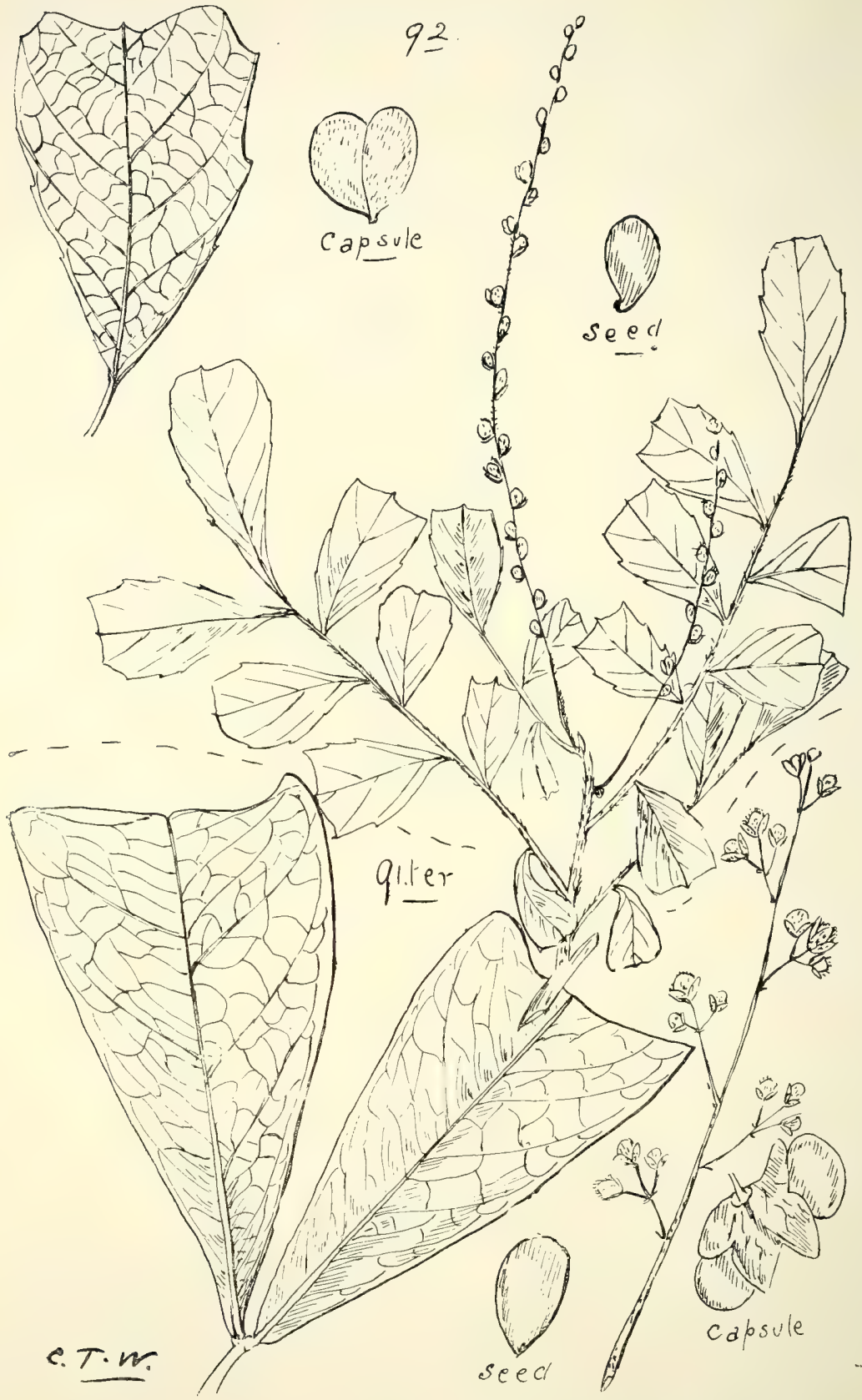

9i ter, Cupania Wadsworthi, $F, v, M$.

92. C. Shirleyana, Bail. 

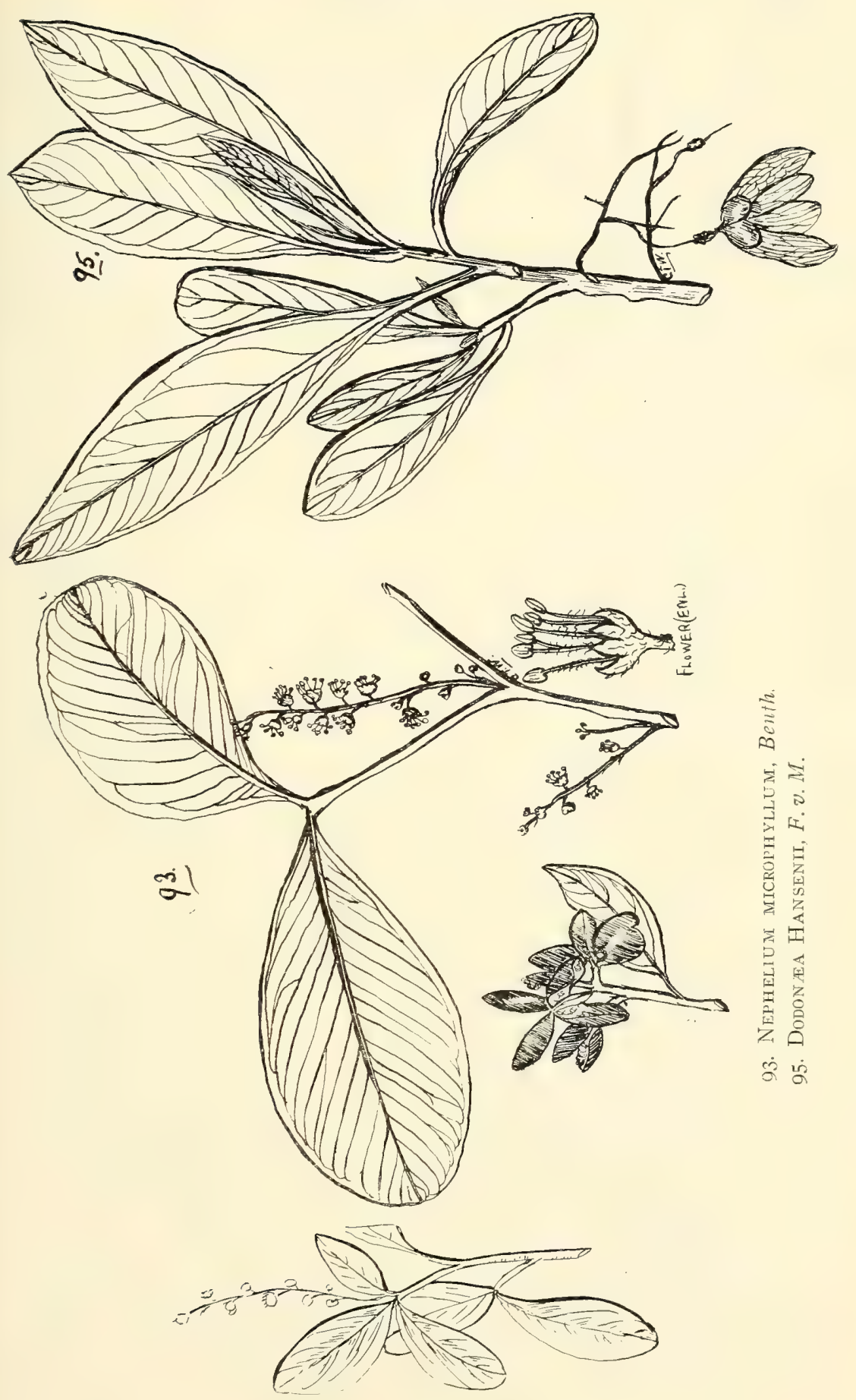
Ratonia-contd.

exangulata, F.v. M.

Lessertiana, Benth. and Hook._- "Murgon" of Cardwell natives (R.B.H.).

Dæmeliana, F.v. M.

Martyana, F.v. M.

Atalaya, Blume.

multiflora, Benth.

hemiglauca, F.v. M.-Whitewood; Cattle-bush. "Boorbal" of St. George natives. Yields a quantity of gum, often in lumps of Io or II oz.

variifolia, F.v. $M$.

Sapindus, Linn.

? australis, Benth.

Nephelium, Linn.

semiglaucum, F.v. M.

connatum, F.v. $M$.

subdentatum, F.v. M.

semicinereum, F.v. M.

tomentosum, F.v. M.-Anther large, red.

coriaceum, Benth.

foveolatum, F.v. M.

leiocarpum, F.v.M.

Lautererianum, Bail.-Edible fruit. (Fig. 94.)

divaricatum, F.v. $M$.

microphyllum, Benth. (Fig. 93.)

distyle, F.v. M. = Ratonia distylis, F.v. M.-In the Kin Kin district known as "Corduroy," from the appearance of the outer part of the stem next the bark having some resemblance to the clothing material so called.

callarrie, Bail. (the native name at Barron River).-Also met with at Kin Kin (W. D. Francis) and Blackall Range (J. Keys). The fruit of Northern tree unknown. Fruit of Kin Kin trees-Globose, pointed at each end, about I in. diam., indehiscent, of a deep pink with a peach-like down; seed solitary, depressed; mahogany colour; aril abortive. Probably when better known may have to be placed under Sapindus.

Euphoria, Juss.

Leichhardtii, Benth.

var. hebepetala, Benth.-Native name, "Nurrum-nurrum."

Heterodendron, Desf.

oleæfolium, Desf.-Wood very hard; useful in turnery.

diversifolium, F. v. $M$. 
XL. SAPINDACEA.

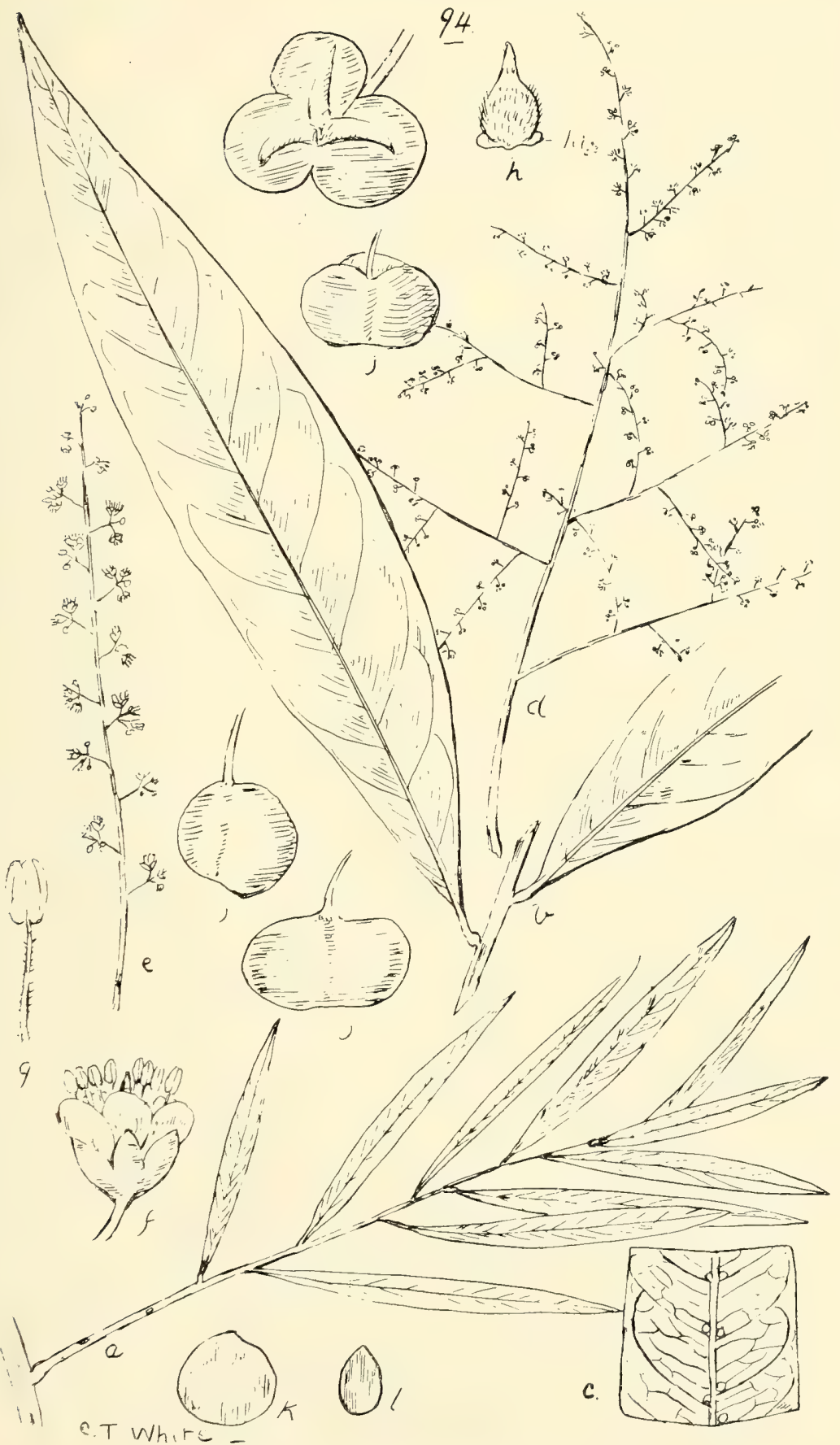

94. Nephelium Lauterekianum, Bail.

(a) Leaf, (b) leaflets, (c) portion under surface of leaf, (d) panicle, (e) a single branch of the panicle, (f) flower, (g) stamen, (h) ovary, (h I) disk, ( $\mathrm{j}$ ) capsules, (k) seed enclosed in the arillus, (1) seed. (a) and (d) reduced; (b), (e), (j), (k), 
Harpullia, Rorb. alata, F.v. M.

frutescens, Bail.

Hillii, F. v. $M$.

pendula, Planch.-Tulipwood; useful in turnery.

Akania, Hook. $f$.

Hillii, Hook. f.-Turnipwood.

\section{Suborder JI.-ACERINEA.}

Blepharocarya, F. v. M.

involucrigera, F. v. M.- "Chargir" of Herberton natives. (Fig. 94 bis.)

\section{Suborder III.-DODON EAE.}

Dodonæa, Linn.-Mostly known as "Hop-bushes."

\section{Series Cycloptere.}

triquetra, Andr. - "Kinginga kilamul" of Moreton Bay natives.

lanceolata, F.v. M.

petiolaris, F.v.M.

viscosa, Linn.-Hop-bush. "Tecan" of St. George natives. The leaves of a form of this tree are in India used as a febrifuge.

zar. vulgaris, Benth.

var. angustifolia, Benth.

var. spathulata, Benth.

attenuata, A. Cunn.

cuneata, Rudge.

peduncularis, Lindl.

filifolia, Hook.

var. lobulata.

Series Platyptere.

truncatiales, $F . v . M$.

Hansenii, F.v.M. (Fig 95.)

stenophylla, F.v. M.

Series Apterce.

triangularis, Lindl. 
Dodonæa-contd.

\section{Series Pinnater.}

megazyga, F.v. M.

physocarpa, F.v.M.

macrozyga, F. $v$. $M$.

vestita, Hook.

oxyptera, F.v. M.

adenophora, Miq. $=D$. tenuifolia, Lindl. (kept as a species by F. v. Muell.).

tenuifolia, Lindl.

Macrossanii, F. г. M. et Scortechini.

Distichostemon, F.v.M.

phyllopterus, F.v.M. = Dodonaa hispidula, Endl. (Fig. 96.)

\section{Order XLI.-ANACARDIACEÆ.}

TRIBE I.-AnACARDIE.⿻.

Rhus, Linn.

rufa, Teysm. et Binn. $=$ R. retusa, Zoll.; R. panaciformis, F. v. M. (Fig. 97.)

Rhodosphæra, Engl.

rhodanthema, Engl. = Trichilia scabra, A. Cunn. - Deep

Yellow-wood. One of the most beautiful of our cabinet woods.

*Mangifera, Limn.

indica, Linn.-Mango. The bark has been found to contain in Madras I6 per cent. of tannin.

Buchanania, Roxb.

Muelleri, Engl._- "Bandai" of Cape Bedford natives.

mangoides, $F . v$. M.

Semecarpus, Linn.

australiensis, Engl. - Marking-nut. "Jaln-ba” of Annan

River and "Eger" of Murray Island natives. It may be here pointed out that the enlarged growth of the peduncle beneath the fruit is called a hypocarpium. This is wholesome, either raw or cooked, but the oily pericarp is poisonous. (Fig. 98.)

Euroschinus, Hook. $f$.

falcatus, Hook. f.-Maiden's-blush wood. "Punburra" of

Moreton Bay and "Kokare" of Atherton natives. var. angustifolius, Benth. 
94 bis.

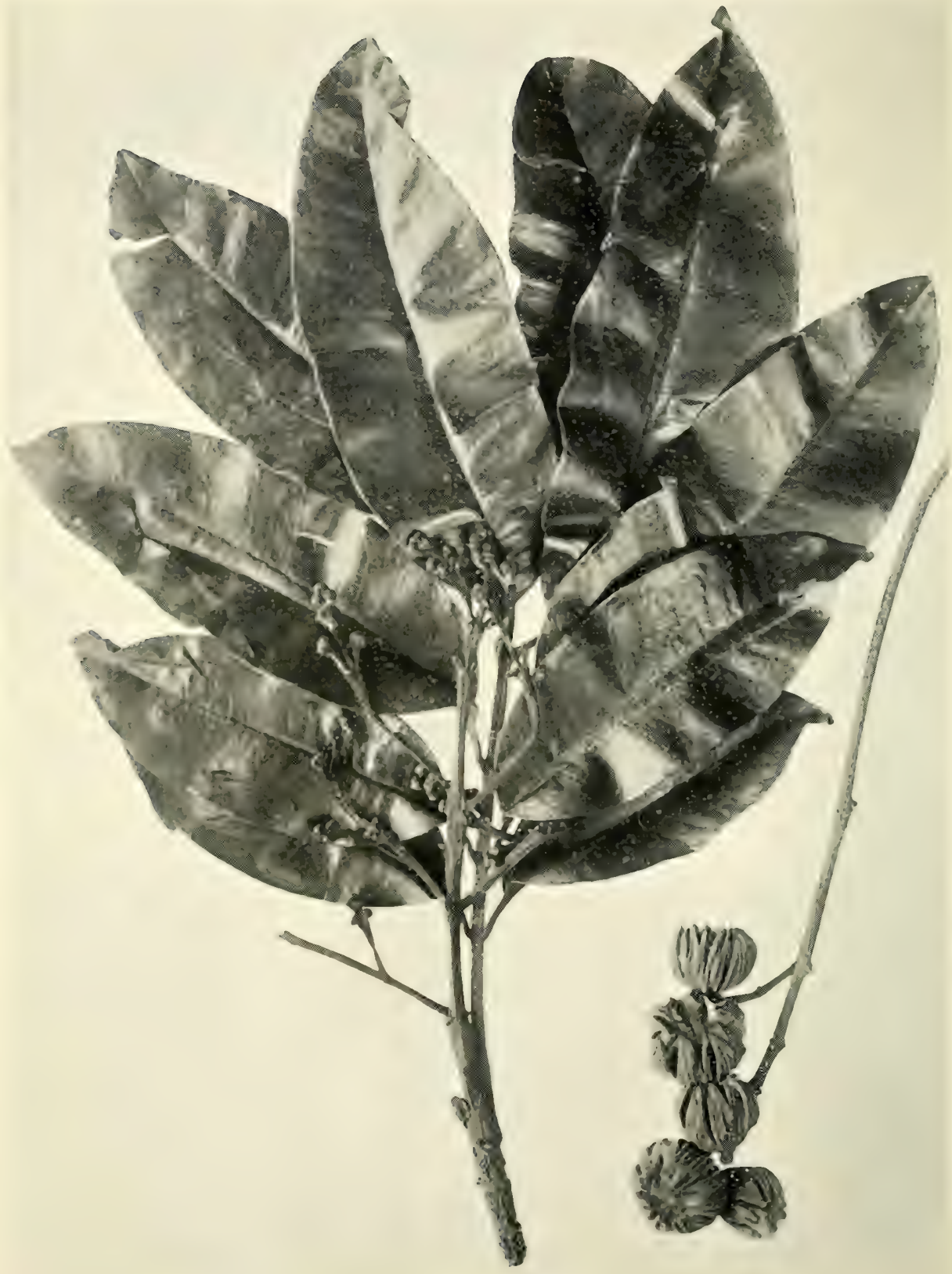

94 bis. Blepharocatya involucrigera, $F \cdot v \cdot M$. 


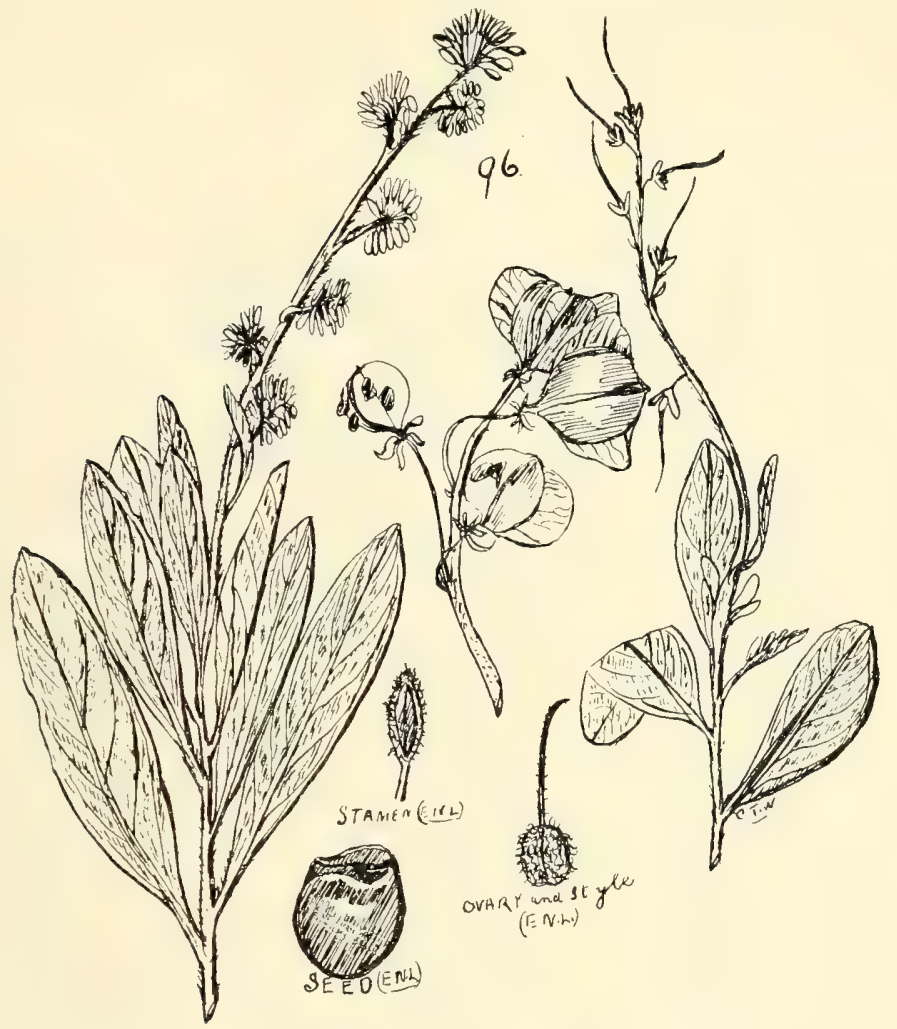

96. Distichostemon Phyllopterus, F.v. M.

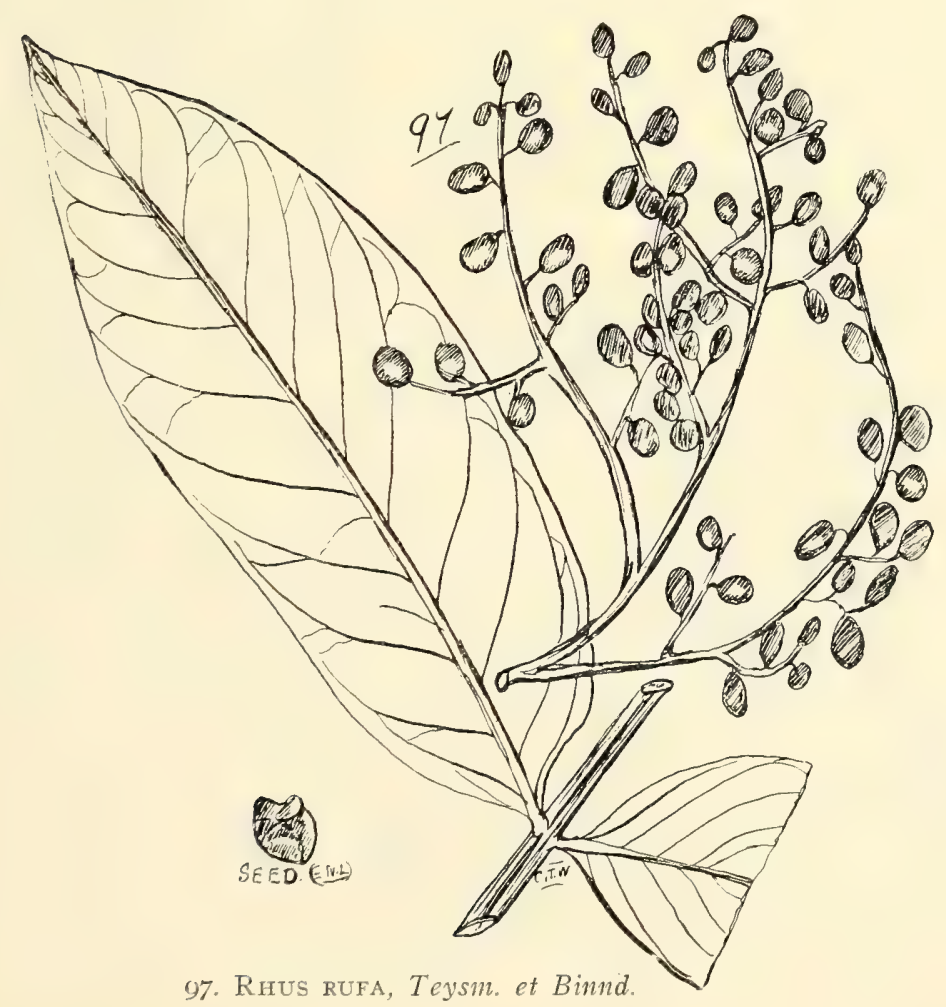




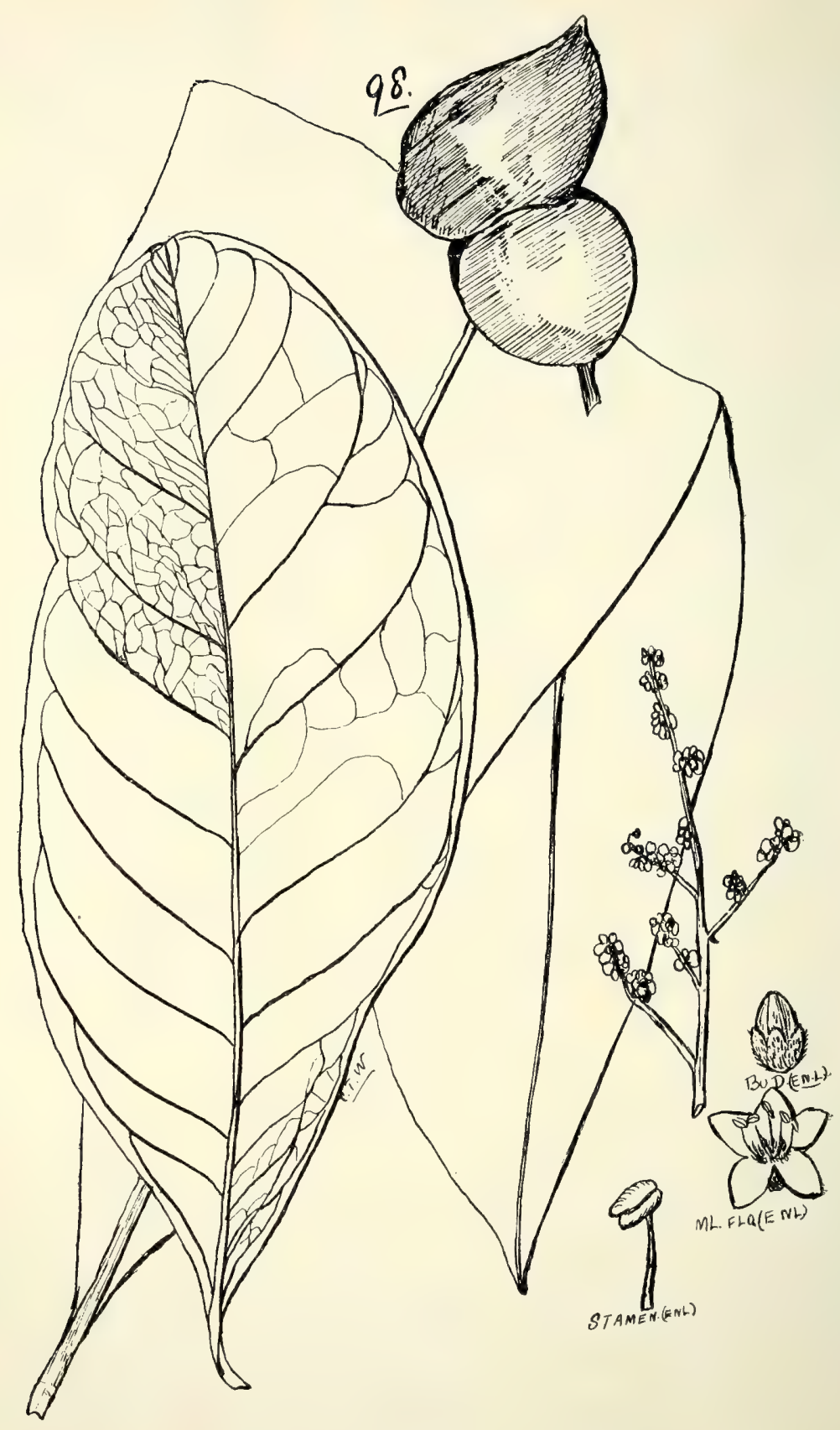

98. Semecarpus australiensis, Engl. 


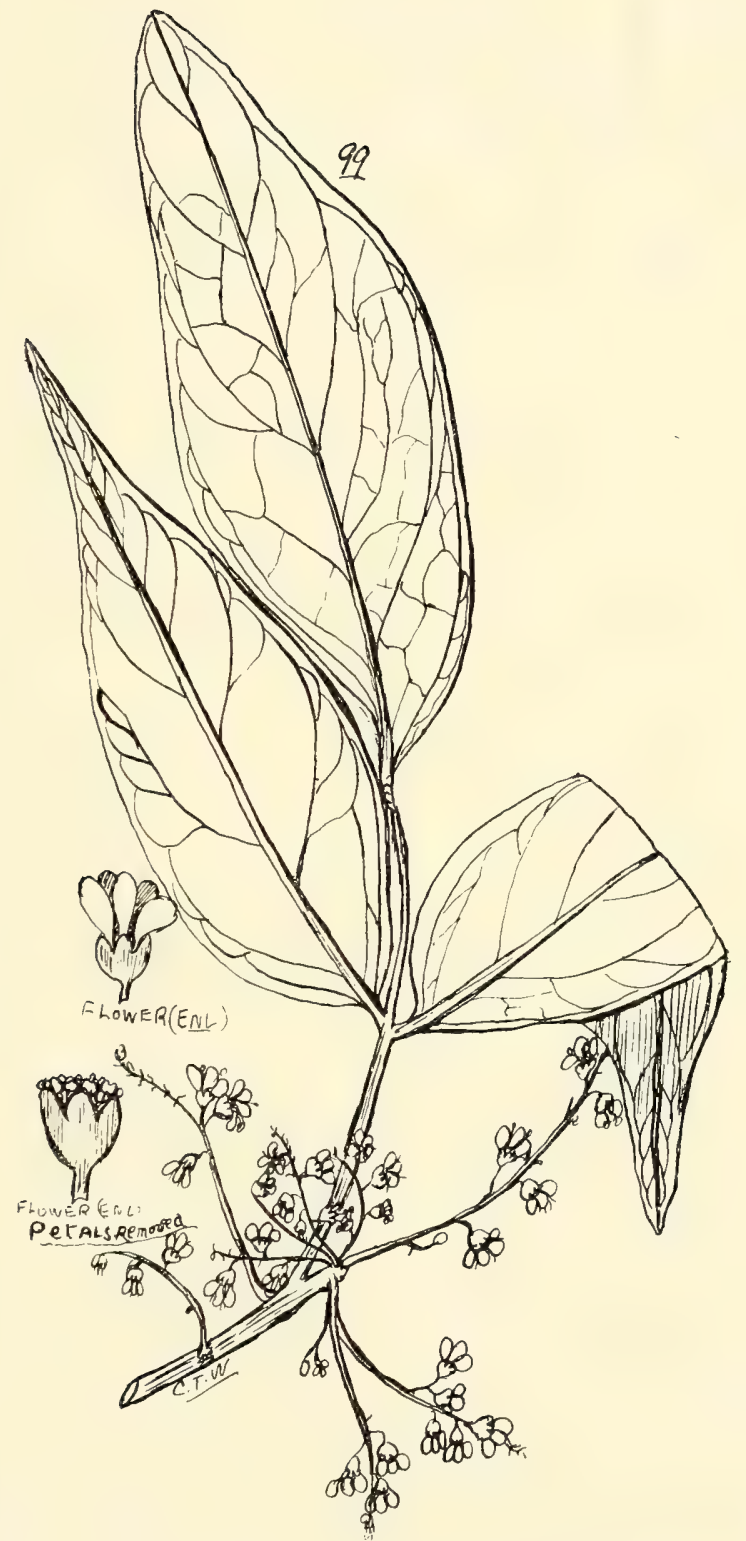

99. ROCREA BRACHYANDRA, F.v. $M$. 
Pleiogynium, Engl.

Tribe II.-Spondiex.

Solandri, Engl.-Sweet Plum or Burdekin Plum. "Noongi" of Port Curtis, "Bungya" of Bundaberg, "Rancooran" of Rockhampton, and "Toolbar" or "Doolbi" of Isis Scrub natives. This tree has been known botanically by the following names:-Spondias acida, Soland., S. Solandi, Benth., Ozvenia cerasifera, F. v. M., and Spondias pleigynia, F. v. M. The wood somewhat resembles American walnut; dark and heavy.

SerIes III.-CALYCIFLOR Æ.

Alliance XI.-ROSALES.

\section{Order XLII.-CONNARACEE.}

Tribe I.-Connarex.

Rourea, Aubl.

brachyandra, F.v.M. (Fig. 99.)

Tribe II.-CnestideE.

Tricholobus, Blume.

connaroides, F.v.M._-“" Na-bun-yu-ban" of Cardwell natives (R.B.H.). The seeds used in making hat-pins. (Fig. IOO.)

\section{Order XLIII.-LEGUMINOSÆ.}

Suborder I.-PAPILIONACEA.

Tribe I.-Podalyriez.

Brachysema, R. Br.

oxylobioides, Benth. $=$ Leptosema orylobioides, F. v. M. uniflorum, R. Br.

Oxylobium, Andr.

ellipticum, R. Br.

Series Callistachyce.

var. angustifolium, Benth.

Series Laxiflora.

scandens, Benth.

var. obovatum, Benth. 
Oxylobium--contd.

\section{Series Podolobiece.}

trilobatum, Benth. = Pultencea ilicifolia, Andr.

aciculiferum, Benth. = Podolobium aciculiferum, F.v.M. (Fig. IOI.)

staurophyllum, Benth. = Podolobium staurophyllum, DC:

Chorizema, Labill.

parviflorum, Benth.

Mirbelia, Sm.

reticulata, $S m$.

aotoides, F. v. M.

speciosa, Sieb.

pungens, A. Cunn.

oxyclada, F.v. M.

Ringrosei, Bail. (Fig. IO2.)

Isotropis, Benth.

filicaulis, Benth.

parviflora, Benth.

Gompholobium, Sm.

latifolium, $S m$.

virgatum, Sieb.

nitidum, Sol. (Fig. Io3.)

pinnatum, $S m$.

Burtonia, R. Br.

subulata, Benth.

foliolosa, Benth.

Jacksonia, R. Br.

\section{Series Phyllodincr}

dilatata, Benth.

Series Ramosissima.

ramosissima, Benth.

odontoclada, F. v. M.

vernicosa, $F . v . M$.

Series Scoparia.

rhadinoclona, $F . v . M$.

purpurascens, F..$M$.

Stackhousii, F. v. $M$.

thesioides, A. Cunn.

scoparia, R. Br.-Dogwood. "Mamboo" of Bundaberg ank

"Denna" of Brisbane River natives. 


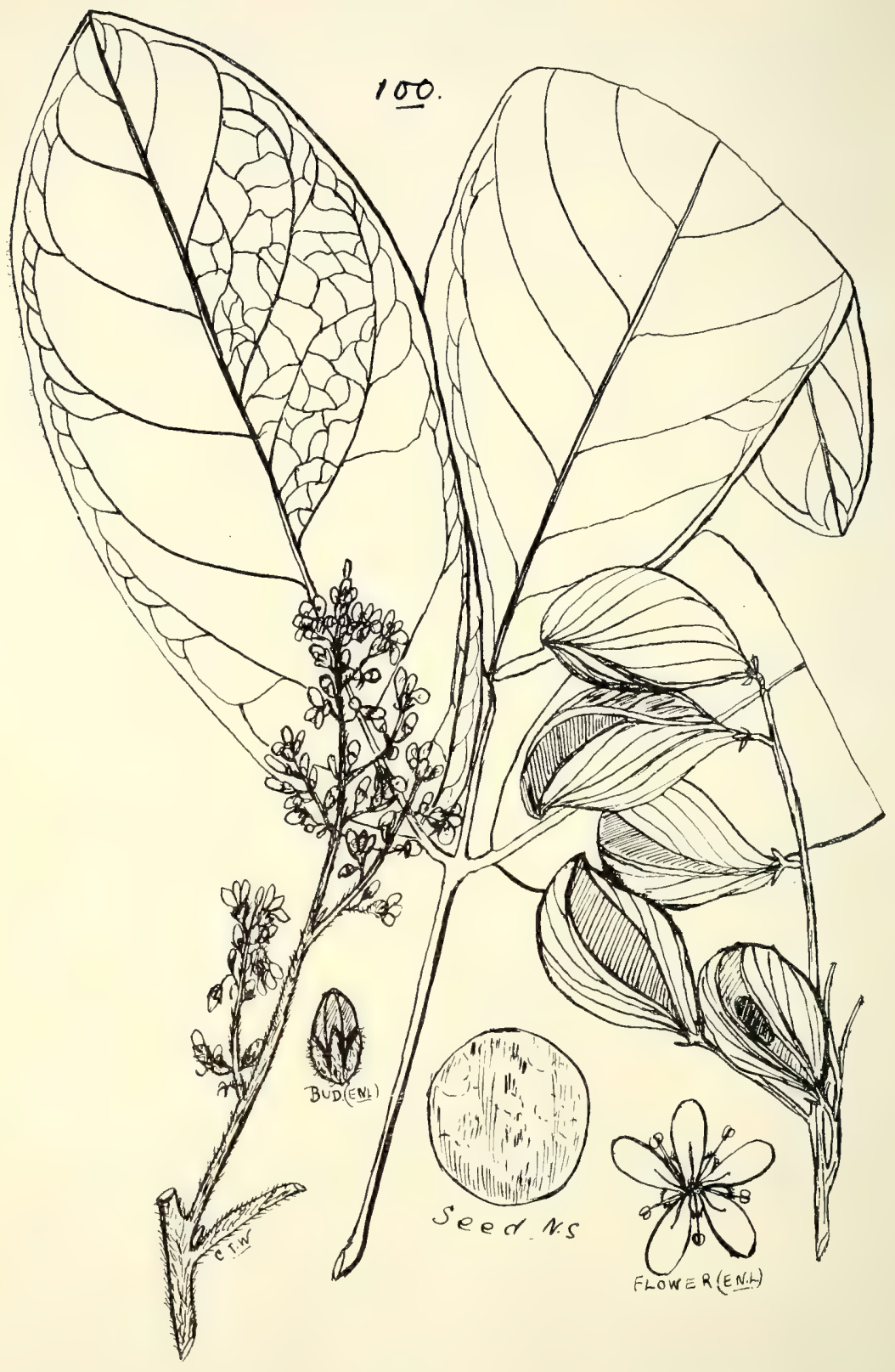

ioo. Tricholobus conjaroides, F. $v . M$. 

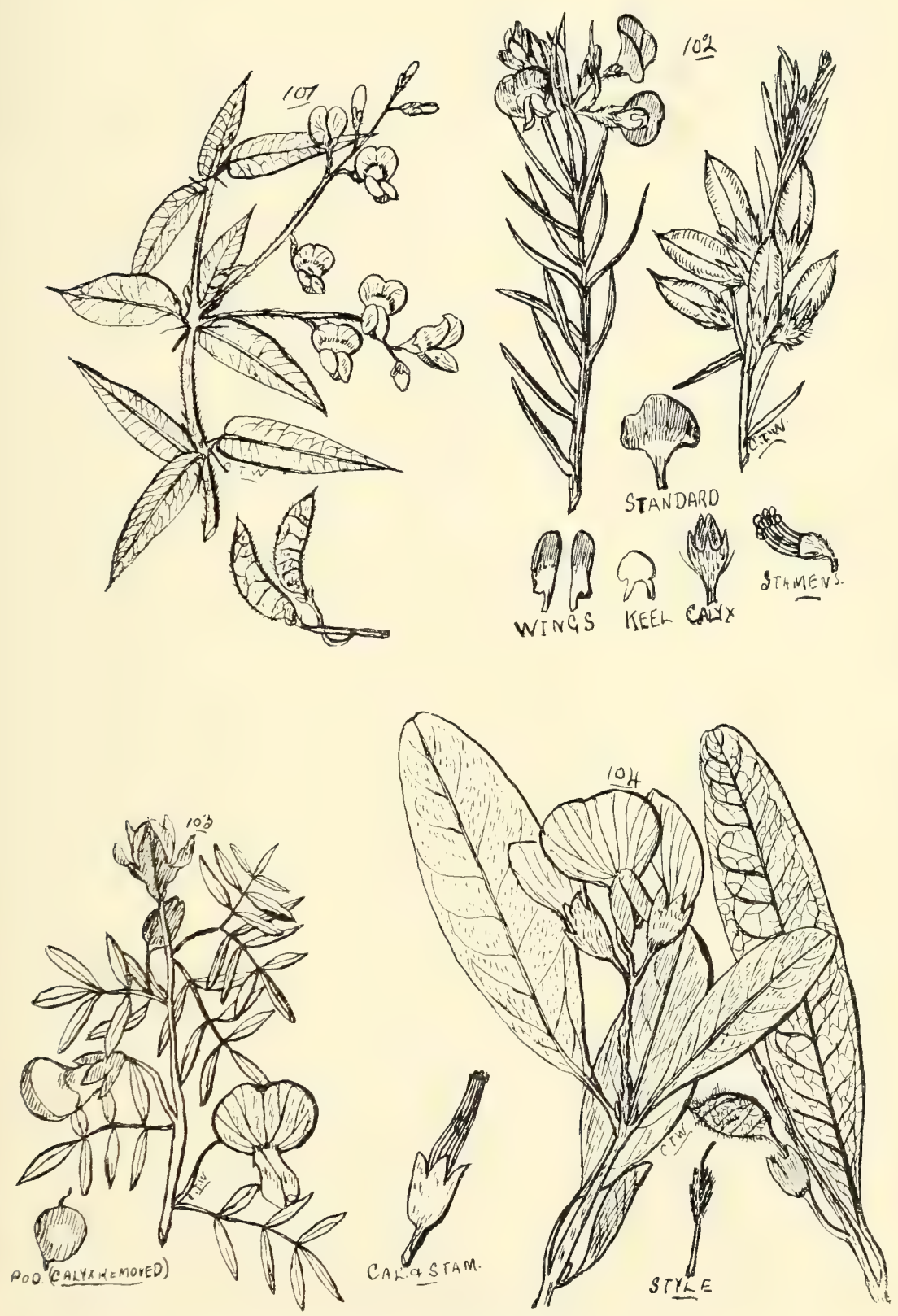

jor. Oxylobium aciculiferum, Bcnth.

102. Mirbelia Ringrosei, Bail.

ro3. Gompholobium nitidum, Sol.

I04. Gastrolobium Grandiflorum, F. v. $M$. 
Sphærolobium, Sm. vimineum, $S m$.

Viminaria, $S m$. denudata, Sm.-Rush Broom.

Daviesia, Sm.

\section{Series Umbellata}

concinna, R. Br.

Wyattiana, Bail.-Leaves very long, some reaching I2 in., and scarcely $\mathrm{T} / 4$ in. wide.

Series Racemosa.

umbellulata, $S m$.

corymbosa, $\mathrm{Sm}$. var. mimosoides, Benth.

arborea, $W$. Hill.-The wood useful for cabinet-work; fragrant when first cut.

filipes, Benth.

Series Fasciculata.

squarrosa, $S m$.

var. villifera, Benth.

ulicina, $S m$.

var, angustifolia, Benth.

acicularis, $S m$.

\section{Series Teretifolice}

genistifolia, A. Cunn.

Aotus, Sm.

villosa, $S m$.

mollis, Benth.

lanigera, $A$. Cunn.

Phyllota, $D C$.

phylicoides, Benth.

Gastrolobium, R. Br.

grandiflorum, F.v.M.-Wallfiower Poison-bush, Pea-flower Poison-bush, Desert Poison-bush, the Australian Poisonbush; a most dangerous plant to stock. As an antidote, the use of a solution of potassium permanganate (Condy's fluid) has been used with success (see Q'land Agric. J1., Aug. I908). (Fig. I04.) 
Pultenæa, Sm.

retusa, $S m$.

Section Eupultenaa

pycnocephala, $F . v . M$.

myrtoides, A. Cunn.

polifolia, A.Cunn.

petiolaris, A. Cunn.

paleacea, Willd.

microphylla, Sieb.

zar cuneata, Benth.

Hartmanni, F. v. $M$.

ternata, F. v. M.

Section Euchilus.

var. cuspidata, Benth.

var. pubescens, F.v. $M$.

echinula, Sieb.

Section Coelophyllum

parviflora, Sieb.

setulosa, Benth.

villosa, $W$ illd.

var. latifolia, Benth.

var. glabrescens, Benth.

enchila, $D C$.

flexilis, $S m$.

var. mucronata, Benth.

Millari, Bail. (Fig. IO5.)

Dillwynia, Sm.

ericifolia, $S m$.

Section Dillwymastrum.

var. normalis, Benth.

var. phylicoides, Benth.

var. parvifolia, Benth.

var. tenuifolia, Benth.

var peduncularis, Benth.

var. glaberrima, Benth.

floribunda, Sm.

Section Xeropetalum

juniperina, Sieb.

Tribe II.-Genis'Tis.

Platylobium, Sm.

formosum, Sm. 

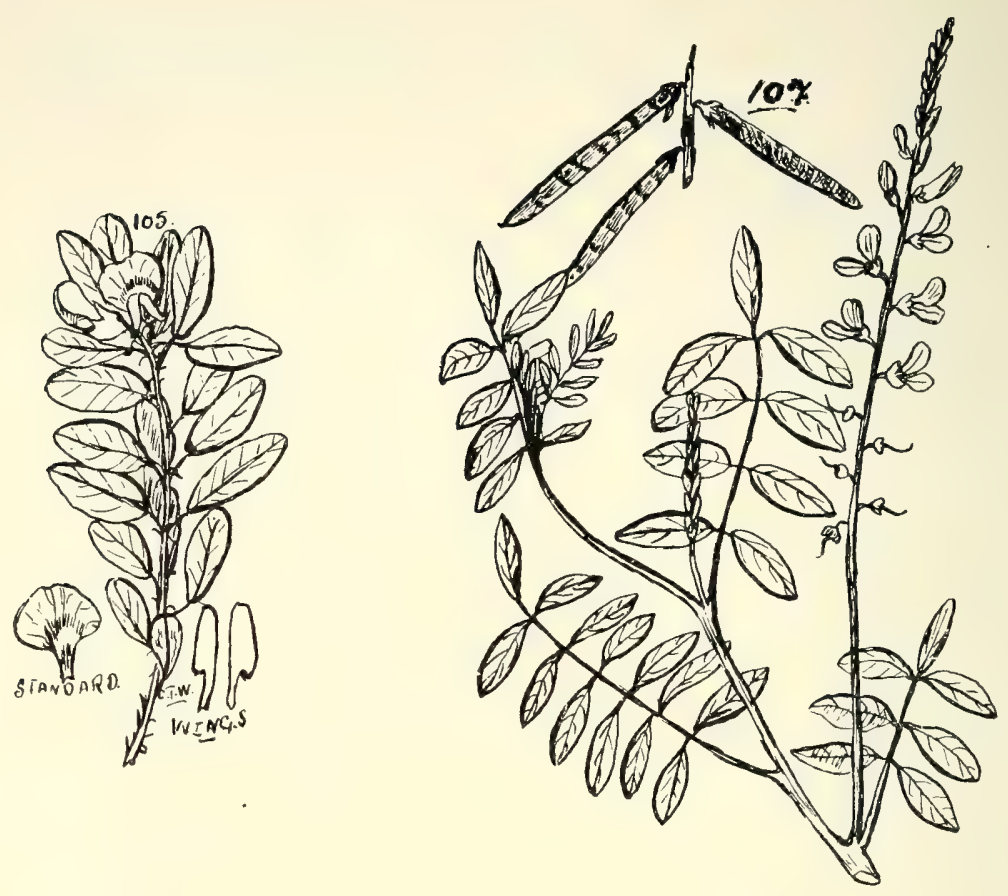

105. Pulten fea Millari, Bail.

107. Indigofera Baileyi, F.v. $M$.

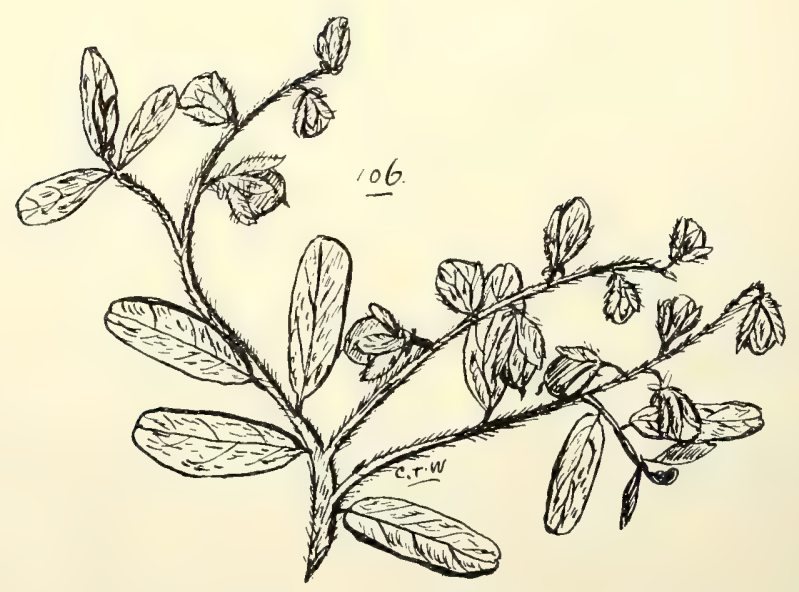

io6. Crotalaria crispata, $F, v, M$. 
XLIII. LEGUMINOSÆ.
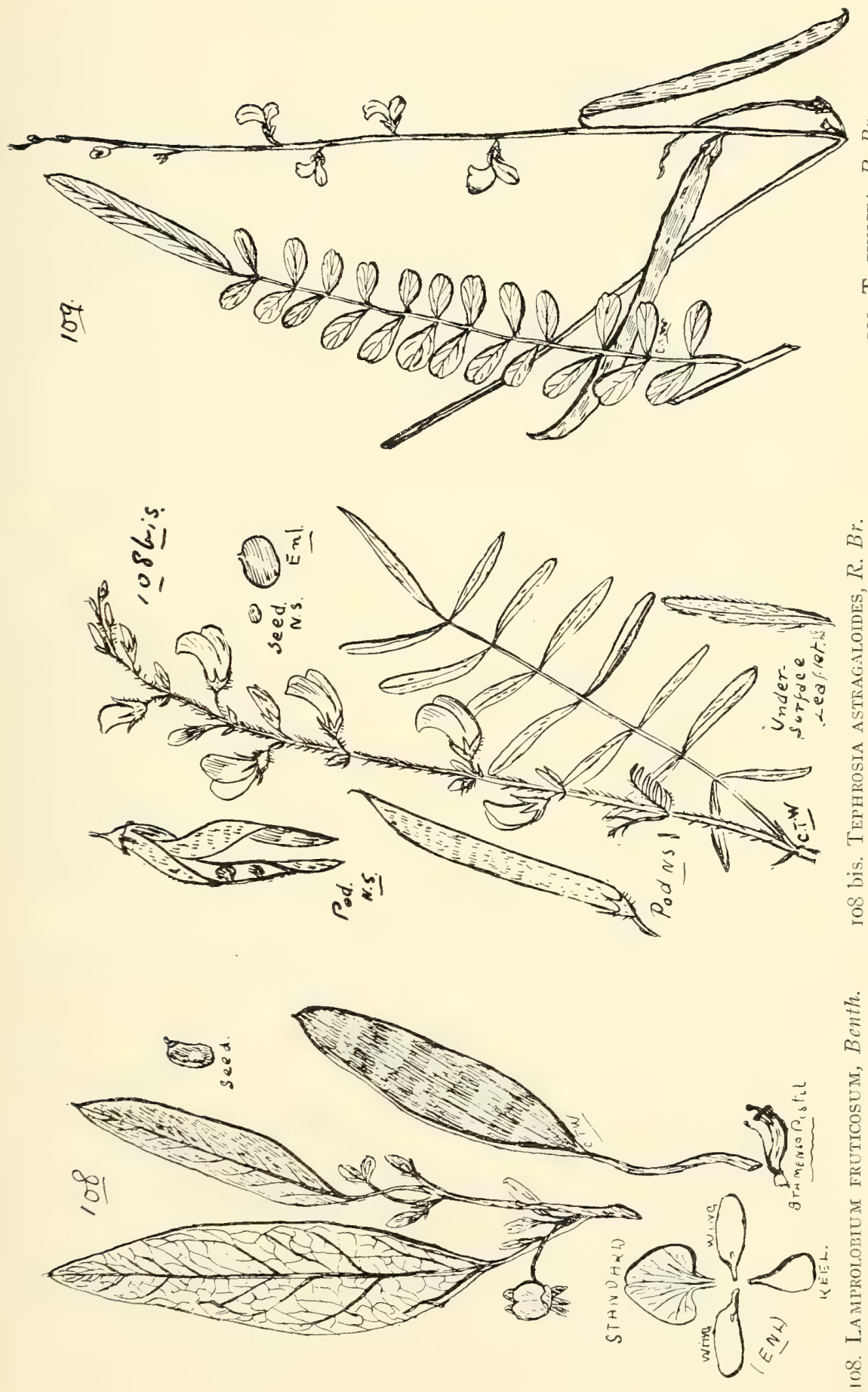
Bossiæa, Vent.

Series Normales.

Scortechinii, F. v. $M$.

carinalis, Benth.

rupicola, $A$. Cunn.

prostrata, R. $B r$.

buxifolia, $R . B r$.

Brownii, Benth.

rhombifolia, Sieb.

heterophylla, $V$ ent.

ensata, Sieb.

Armitii, F. v. $M$.

phylloclada, F. v. $M$.

Templetonia, R. Br.

Muelleri, Benth.

Hookeri, Benth.

egena, Benth.

Hovea, R. Br.

linearis, R. $B r$.

heterophylla, A. Cunn.

longifolia, $R$. $B r$.

var. normalis, Benth.

var. lanceolata, Benth.

var. pannosa, Benth.

acutifolia, $A$. Cunn.

longipes, Benth.-Port Curtis Yellow-wood; useful in turnery.

Goodia, Salisb.

latifolia, Salisb.

Crotalaria, Linn.-Rattlepods or "Pop-pods." All yield good fibre. Species of this genus have been, both here and elsewhere, considered injurious to stock.

Series Alatce.

*alata, Hamilt.-An Indian species.

verrucosa, $\operatorname{Linn}$.

\section{Series Simplicifolioe.}

crispata, F.v.M. (Fig. Io6.)

juncea, Linn.-Sun Hemp of commerce.

linifolia, Linn. $f$.

nana, Burm.

calycina, Schranch.

retusa, $\operatorname{Linn}$.

Mitchelli, Benth.

humifusa, Grah. 
Crotalaria-contd.

\section{Series Unifoliatre.}

novæ-hollandiæ, $D C$.

forma parviflora, Benth.

forma oblongifolia, Benth

forma lasiophylla, Benth.

crassipes, Hook. ? Bail., Queensland Flora, 375.

Cunninghamii, R. Br.

\section{Series Digitatce.}

trifoliastrum, Willa.

incana, Linn.

striata, $D C$.

dissitiflora, Benth.

var. eremæa, Benth

var. rugosa, Benth.

laburnifolia, Limn.-This has sometimes been supposed to have caused what is known as. "Chillagoe disease" among horses.

quinquefolia, Linn.

*Lupinus, Linn.

hirsutus, Linn.-Hairy Lupine. America. Met with as a stray from garden culture.

*Ulex, Limn.

europæus, Linn.-Furze-bush of Europe. A drug made from the seeds of this plant has been considered valuable in cases of dropsy (T. Christy).

Tribe III.-TRIFOL.IE.F.

*Medicago, Linn.

sativa, Limn.-Lucerne. Europe.

denticulata, Willd.-Medick Burr. Europe.

maculata, Willd.-Spotted Medick. Europe. Mostly found on city lawns.

orbicularis, All.-Europe.

Melilotus, Tournef.

parviflora, Desf. $=M$. indica, All.-Melilot, small-flowered. Hexham Scent.

*alba, Lam.-White Melilot. Etrope. 

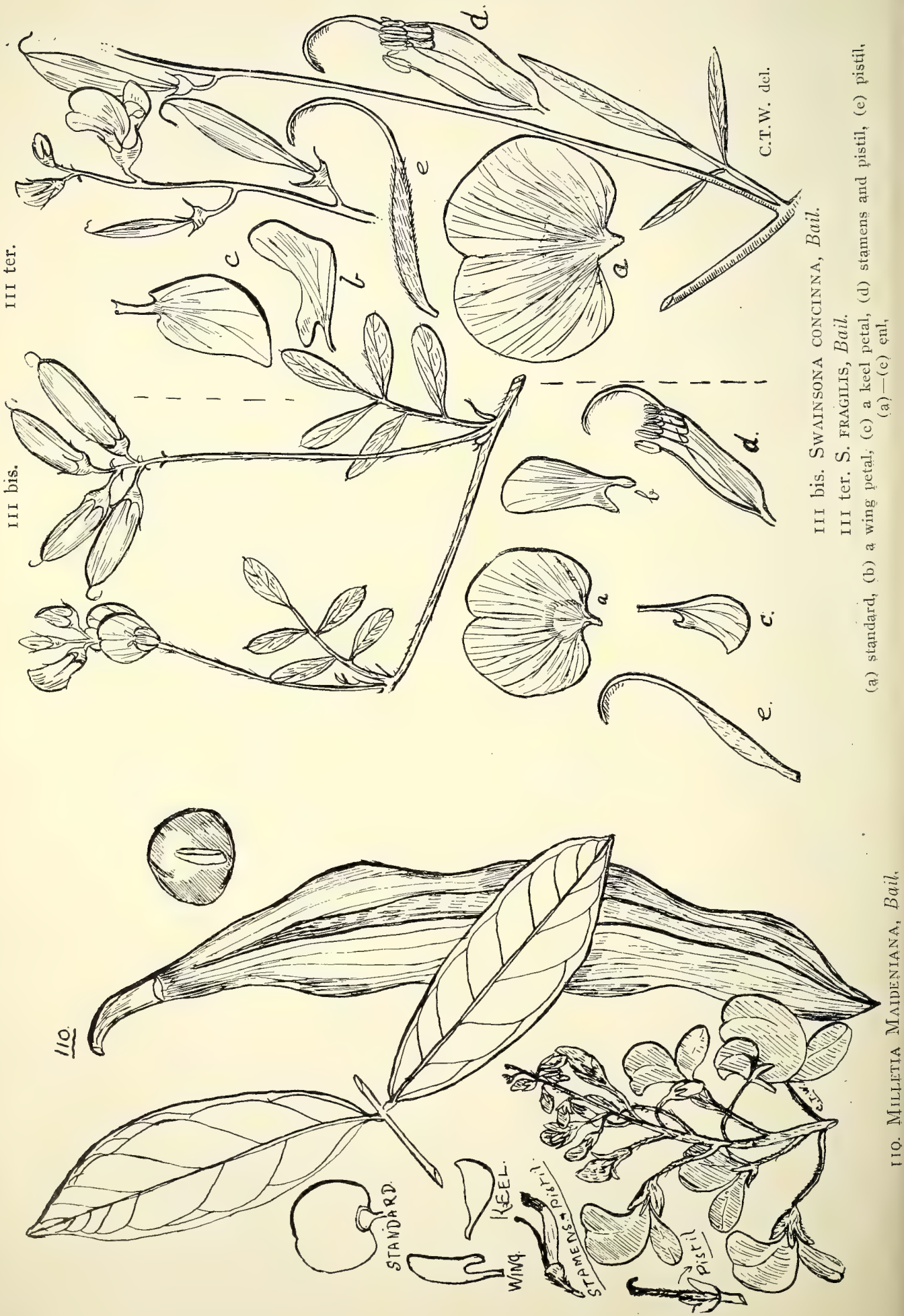


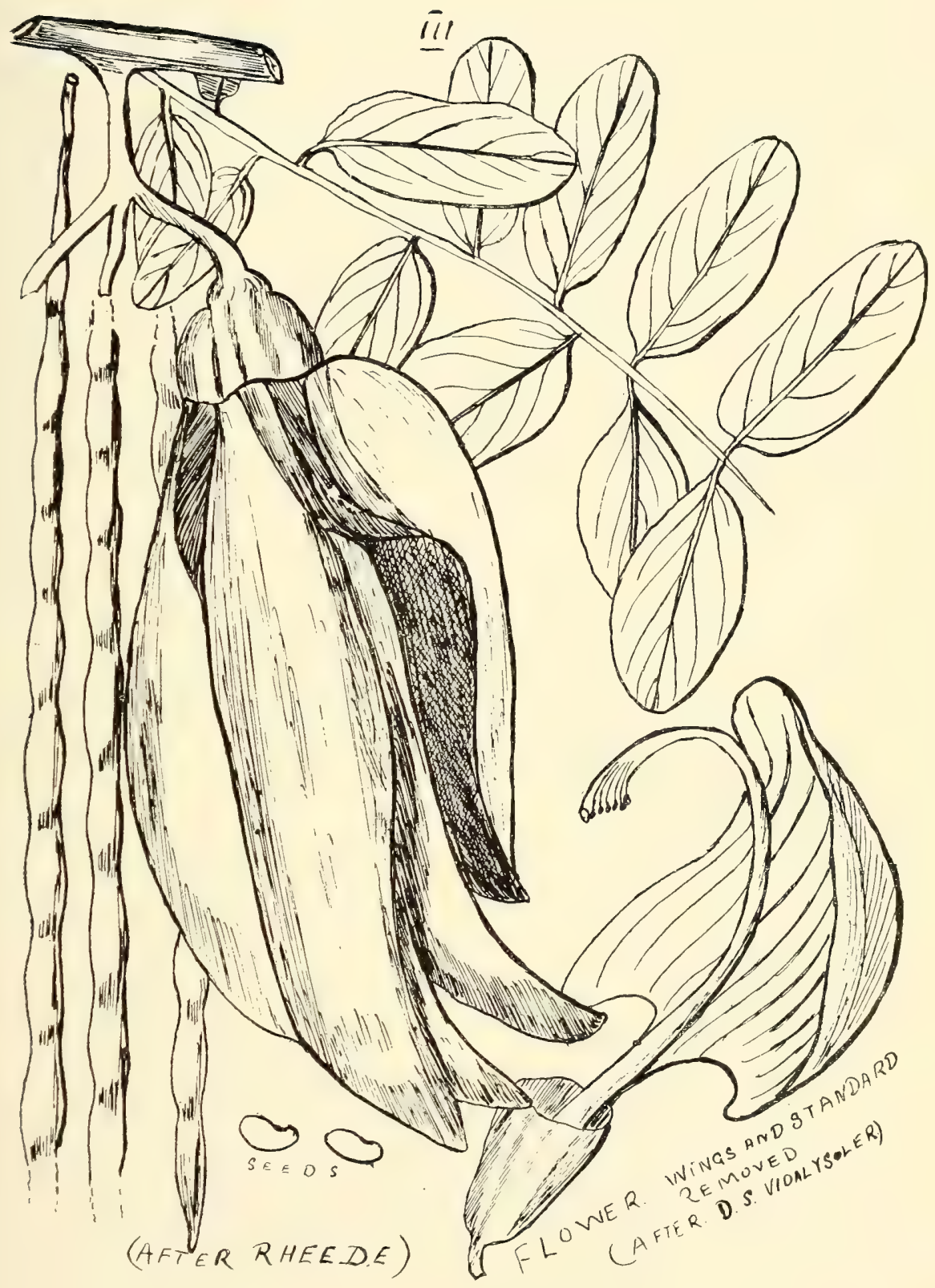


*Trifolium, Linn.

resupinata, Limn.-Europe.

procumbens, Linn.-Europe.

repens, Linn.-White Clover. Europe.

glomeratum, Linn.-Clustered Clover. Europe.

tomentosum, Linn.-Southern Europe.

Bonanni, Presl.-Europe.

agrarium, Linn.-European Hop Clover. Europe.

Trigonella, Linn.

suavissima, Lindl.-Sweet Fenugreek. Sir Thomas Mitchell speaks of this as being a useful vegetable.

Lotus, Limn.

\section{Tribe IV.-Lote}

australis, Andr.--Poisonous to stock; contains prussic acid.

var. parviflorus, Benth.

corniculatus, Linn.

\section{Tribe V.-GaLege $\pi$.}

Psoralea, Limn.-In other parts of the globe some species of this genus are considered poisonous to cattle.

badocana, Benth._. A-mega" and "Alpu-rara" of Princess Charlotte Bay natives, by whom the roots are used for food after being scraped and roasted.

Archeri, F.v.M.

plumosa, $F \cdot v \cdot M$.

pustulata, F.v.M.

cephalantha, F.v.M.

Testariæ, F.v.M.

eriantha, Benth.

patens, Lindl.

cinerea, Lindl.

leucantha, F.v.M.

tenax, Lindl.-Emu Grass; considered excellent fodder.

var. major, Benth.

Indigofera, Limn.-Most species considered poisonous to stock.

linifolia, Retz.

enneaphylla, Linn.

glandulosa, Willd.

haplophylla, F.v.M.

trifoliata, Linn.

trita, Linn. $f$.

parviflora, Heyne. 
Indigofera-contd.

viscosa, Lam.

hirsuta, Linn.

*argentea, Linn.-An Indigo-plant. India.

var. cærulea, Baker $=$ I. carulea, Roxb. India.

*tinctoria, Limn.-The Indigo-plant, a stray from cultivation

It may be well to bear in mind that it is considered that bright sunshine favours the development of the dye principle. A native of India.

*Anil, Linn.-An Indigo-plant. West Indies.

pratensis, F.v.M.

saxicola, F.v.M.

Baileyi, F.v.M. (Fig. IO7.)

*decora, Lindl.-A Chinese species.

australis, Wiild.-Poisonous to stock.

var. angulata, Benth.

var. gracilis, $D C$.

var. minor, Benth

var. signata, $F . v \cdot M$.

var. platypoda, Benth.

brevidens, Benth.

var. uncinata, Benth.

var. ? galegoides, $R$. $B r$.

Lamprolobium, Benth.

fruticosum, Benth.-Supposed poisonous to stock. (Fig. 108.)

*Galega, Linn.

officinalis, Lim.-Goat's Rue. Europe.

Tephrosia, Pers.

flanmea, F.v.M.

reticulata, $R . B r$.

crocea, R. Br.

oblongata, $R . B r$.

porrecta, R. Br.

polyzga, $R . B r$.

leptoclada, Benth.

astragaloides, $R \cdot B r$. (Fig. 108 bis.)

var. ? macrostachya, Benth.

juncea, $R \cdot B r$. (Fig. Iog.)

filipes, Benth.

var. latifolia.

oligophylla, Benth.

purpurea, Pers.

var. brevidens, Benth. 
Tephrosia-contd.

purpurea-contd.

var. rufescens, Benth.

var. longifolia, Benth.

var. sericea, Benth.

var. ? laxa, Benth.

Bidwilli, Benth .

var. ? densa, Benth.

rosea, F.v.M.-Poisonous.

var. ? angustifolia, Benth.

Millettia, $W$. et Arn.

megasperma, F.v.M.-Native Wistaria. The gum contains 75 per cent. of tannin.

Maideniana, Bail.-The seeds useful for ornamental purposes.

(Fig. IIO.)

pilipes, Bail.

Sesbania, Pers.

grandiflora, Pers. $=$ Agati grandiflora, Desv.-In India the flowers are cooked and eaten as a vegetable. (Fig. ri I.) ægyptiaca, Pers.- "Ngean-jerry" of Cloncurry natives. The green pods, as well as the seeds, nutritious ( $T$. Gulliver). aculeata, Pers.-Pea-bush. In some parts a troublesome weed; yields a rope fibre worth $£_{3} 0$ to $£_{4} 0$ per ton (Mueller).

var. sericea, Benth.

var. ? erubescens, Benth.

Clianthus, Sol.

Dampieri, A. Cunn. = Sturt's Pea. (Colutea nove-hotlandice, Woodw. in Dampier's Voy. vol. iii. t. 4.)

Swainsona, Salisb.

Greyana, Lindl.

galegifolia, R.Br.-Darling Pea. Poison-plant.

var. coronillæfolia, Salisb. (as a species).

z'ar. albiflora, G. Don. (as a species).

brachycarpa, Benth.

phacoides, Benth.

var. parviflora, Benth.

var. grandiflora, Benth.

campylantha, F.v. $M$.

procumbens, $F . v \cdot M$.

var. ? minor, Benth.

canescens, $F \cdot v \cdot M .=$ Cyclogyne canescens, Benth

phacifolia, $F . v . M$. 
Sivainsona-contd.

oroboides, F.v. M.

concinna, Bail. (Fig. I I I bis.)

fragilis, Bail. (Fig. I I ter.)

monticola, A. Cunn.

luteola, $F . \tau \cdot M$.

parviflora, Benth.

microphylla, A. Gray.

Fraseri, Benth.

laxa, $B . B r$.

Tribe VI.-Hedysarer.

*Scorpiurus, Linn.

subvillosa, Linn.-Scorpion or Caterpillar pod.

Ormocarpum, Beauv.

sennoides, $D C$.

Eschynomene, Linn.

indica, Linn.

falcata, DC., var. paucijuga, Benth.

americana, Linn.

Smithia, Ait.

sensitiva (Ait.), DC.-Leaves sensitive. (Fig. II2.)

geminiflora (Roth.), DC., var. conferta, J. G. Baker in Hook.

F1. Brit. Ind.-Leaves sensitive. (Fig. I13.)

*Arachis, Linn.

hypogæa, Linn.-Pea-nut; here and there a stray from culti vation. A native of Africa.

Zornia, Gmel.

diphylla, Pers.

var. vulgaris, Benth.

var. zeylonensis, Benth.

var. gracilis, Benth.

var. filifolia, Bail. (Fig. II4.)

var. Stirlingi, Bail. (Fig. II5.)

Desmodium, Desv.-Fruit a lomentum.

dependens, Blume.- "Wai-gen" of Barron River natives

pulchellum, Benth.

umbellatum, DC.-Supposed by some to cause the "Chillagoe disease" of horses.

biarticulatum, F.v. M.

gangetictim, $D C$.

brachypodum, A. Gray.--Solomon Islands Indigo.

varians, Endl. 
Desmodium-contd.

rhytidophyllum, F.v. $M$.

campylocaulon, $F . v . M$.

nemorosum, F.v.M.-Under the name "Wild Pea," this is reputed poisonous to stock.

trichostachyum, Benth. (Fig. I I 5 bis.)

polycarpum, $D C$.

trichocaulon, $D C$.

Muelleri, Benth.

reniforme, $D C$.

parvifolium, $D C$.

acanthocladum, F. v. $M$.

triquetrum, $D C$.

*gyrans, DC.-Moving-plant; sometimes seen as a garden weed. The lateral leaflets moving by jerks make it an interesting object. A native of India and Malay Islands.

Pycnospora, R. Br.

hedysaroides, R. Br.

Uraria, Desv.

picta, Desv.

cylindracea, Benth. (Fig. I15 ter.)

lagopoides, $D C$.

Alysicarpus, Neck.

vaginalis, $D C$.

rugosus, $D C$.

Lespedeza, Mich.

cuneata, G. Don.

*Vicia, Linn.

Tribe VII. VICIEA.

sativa, Limn. - Common Vetch; contains prussic acid. Europe.

hirsuta, Koch. - Hairy Vetch; contains prussic acid. Europe.

Abrus, Linn.

precatorius, Lim.---Seeds called "Crab's-eyes"; also "Je. querity." "Do-anjin-jin" of Batavia River and "Pundirpundir" of Cooktown natives. "Boan" of Cardwell natives (R.B.H.). Seeds considered poisonous. The seed said by some writers to be the unit of weight employed by the Burmese. 
XLIII. LEGUMINOS Æ.
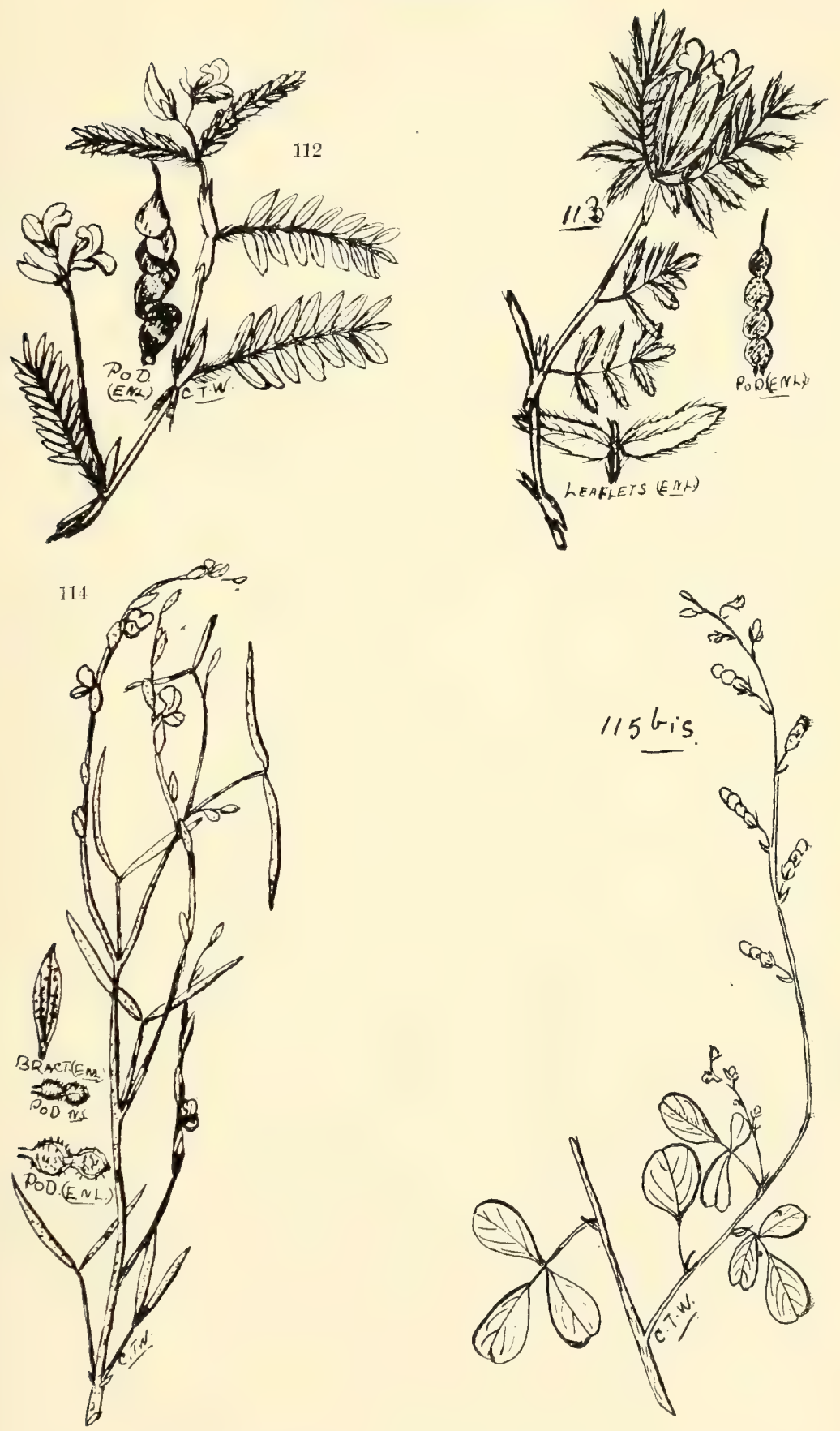

ii2. Smithia sensitiva (Ait.), $D C$.

II3. S. gEMINIFLoRA (Roth.), DC., var. CONFERTA, Bakcr. ii4. Zornia diphylla, Pers., var. filifolia, Bail.

iis bis. Desmodium trichostachyum, Benth. 

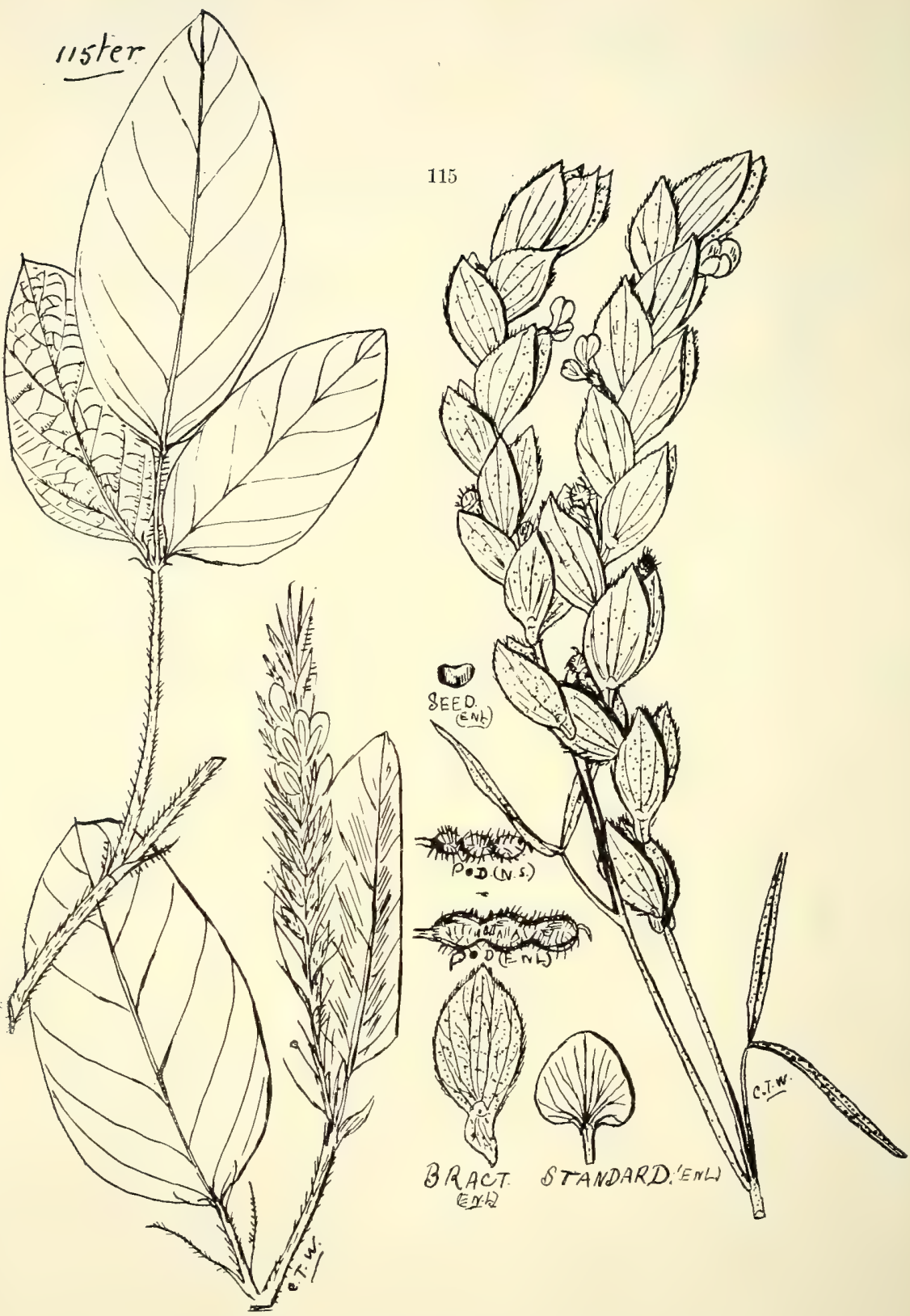

I15. Zornia diphylia, Pers, var. Stirlingi, Bail. it 5 ter. Uraria cylindracea, Benth. 
PLATE V.

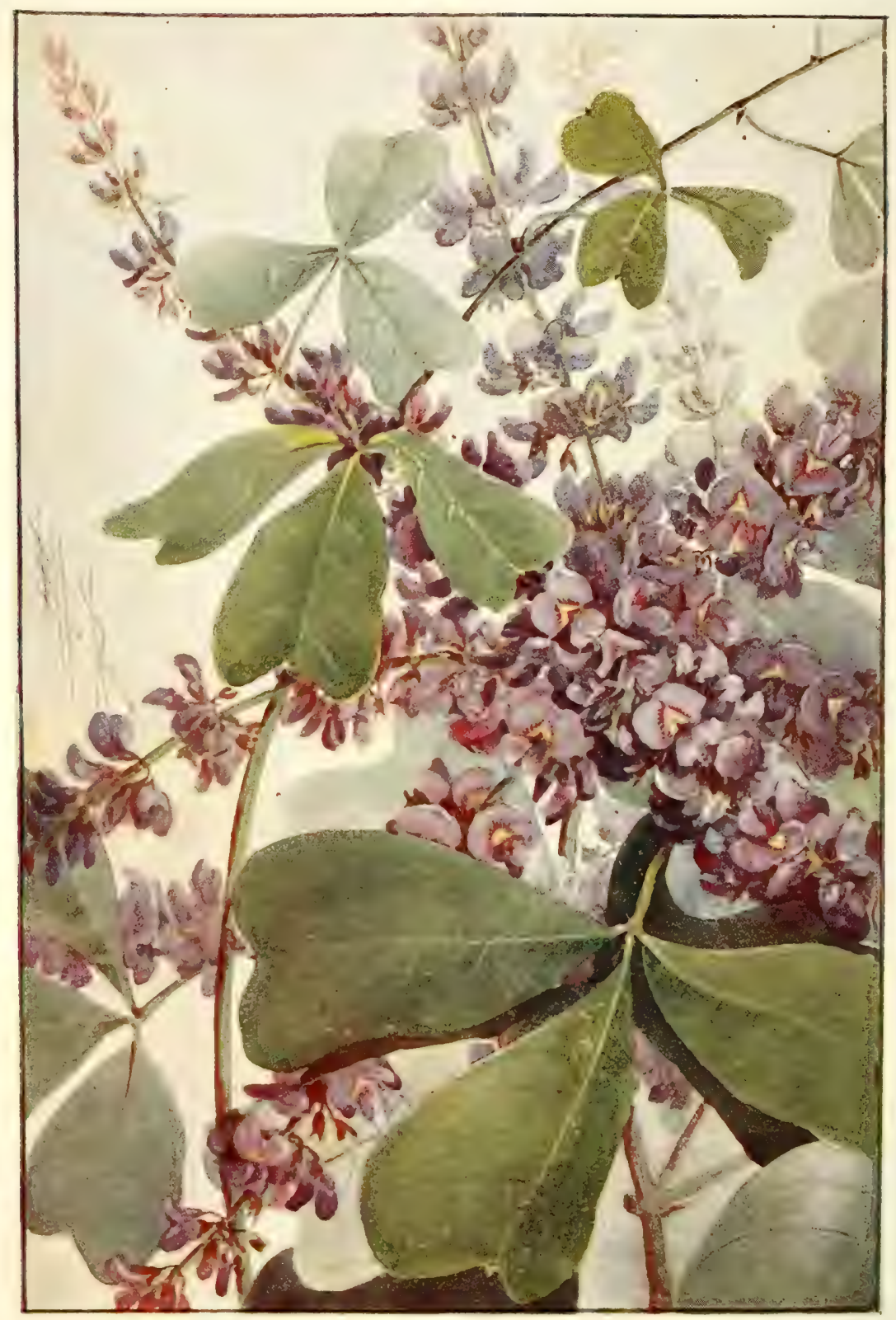

HARDENBERgia RETUSA, Benth. 
Tribe VIII.-Phaseole.e.

Clitoria, Limi.

australis, Benth.

*Ternatea, Linn.-Molucca. In parts of India the flowers are called "Ears of Vishnu." The root is said by Roxburgh to equal jalap as a purgative.

Glycine, Linn.

falcata, Benth.

clandestina, $W$ endl.

var. sericea, Benth.

tabacina, Benth.

var. uncinata, Benth.

var. latifolia, Benth.

sericea, Benth.

tomentosa, Benth.

Hardenbergia, Benth.

monophylla, Benth.-The Bushman's Sarsaparilla.

var. ovata, Bail.

var. longiracemosa, Bail. = Kennedya longiracemosa, Lodd., Bot. Cat. t. I940.

retusa, Benth._ "Kong-an" of Cooktown and "Ru" of Batavia River natives. (Plate V.)

Kennedya, $V$ ent.

rubicunda, $V$ ent.

procurrens, Benth.

prostrata, R. Br.

? exaltata, Bail.

Erythrina, Limn.-The red seeds of many kinds used for ornamental purposes.

vespertilio, Benth.-Bat's-wing Cork-tree. "Goomurrie" of Nanango, "Aranyi" of Batavia River, and "Kuntan" of Brisbane River natives. Roots eaten raw; flowers worn as a sign of mourning by the Northern aborigines (Roth).

indica, Lam.-Coral-tree. Probably, from its lightness and other properties, the wood of this tree, which is abundant both in Northern Queensland and New Guinea, may prove suitable in the manufacture of aeroplanes.

insularis, Bail.

phlebocarpa, Bail.

Strongylodon, Vogel.

ruber, $V$ og. 
Mucuna, Adons.

gigantea, DC.--The Black Bean; has been used for ornamental purposes. "Na-go" of Cardwell natives (R.B.H.).

Galactia, R. Br.

tenuiflora, Willd.

Muelleri, Benth

varians, Bail.-A cure for rheumatism; has been tested and found to be efficacious in some cases. The root, which is the part used, should be obtained and carefully tested. "Mornimi-an" of the Morehead River natives.

Canavalia, $D C$.-Fruit a legume.

obtusifolia, DC.-McKenzie Bean; not considered wholesome.

"Yugam" of Prisbane River natives.

*ensiformis, $D C .=C$. gladiata, DC.-Sword Bean. May be used as a vegetable, but is very coarse.

Phaseolus, Limn.

*vulgaris, Limn.-French Bean, a well-known vegetable.

truxillensis, H.B. et $K .=P$. adenanthus, Meyer, and $P$. rostratus, Wall.

Mungo, Linn._- Komin" and "Kerdolo" of Rockhampton and Cleveland Bay natives.

*semierectus, Linn. = P. psoraleoides, W. and A., Prod. 244.

Vigna, Savi.

vexillata, Benth.- "To-e" of Cloncurry natives. The pods lave been used as a substitute for French beans. var. Youngiana, Bail.

lutea, A. Gray.- "Andan-ga" and "Ara-ra" of Princess Charlotte Bay and "Balcha” of Bloomfield River natives. luteola, Benth.

lanceolata, Benth._- Malaga” of Cloncurry natives. var. filiformis, Benth.

*Catjang, Walp. $=V$. sinensis, Endl.-Cowpea; a stray from cultivation.

Dolichos, Limn.

*Lablab, Linn.-The seeds of this plant, so long used as a food in India and elsewhere, vary much in form and colour.

*lignosus, Bot. Mag. 380 .

biflorus, Linn._- Mal-kan" of Cape Bedford and "Tandaji" of Bloomfield River natives. The seeds are esculent and known as "Madras Gram" or "Horse Gran " in India. 

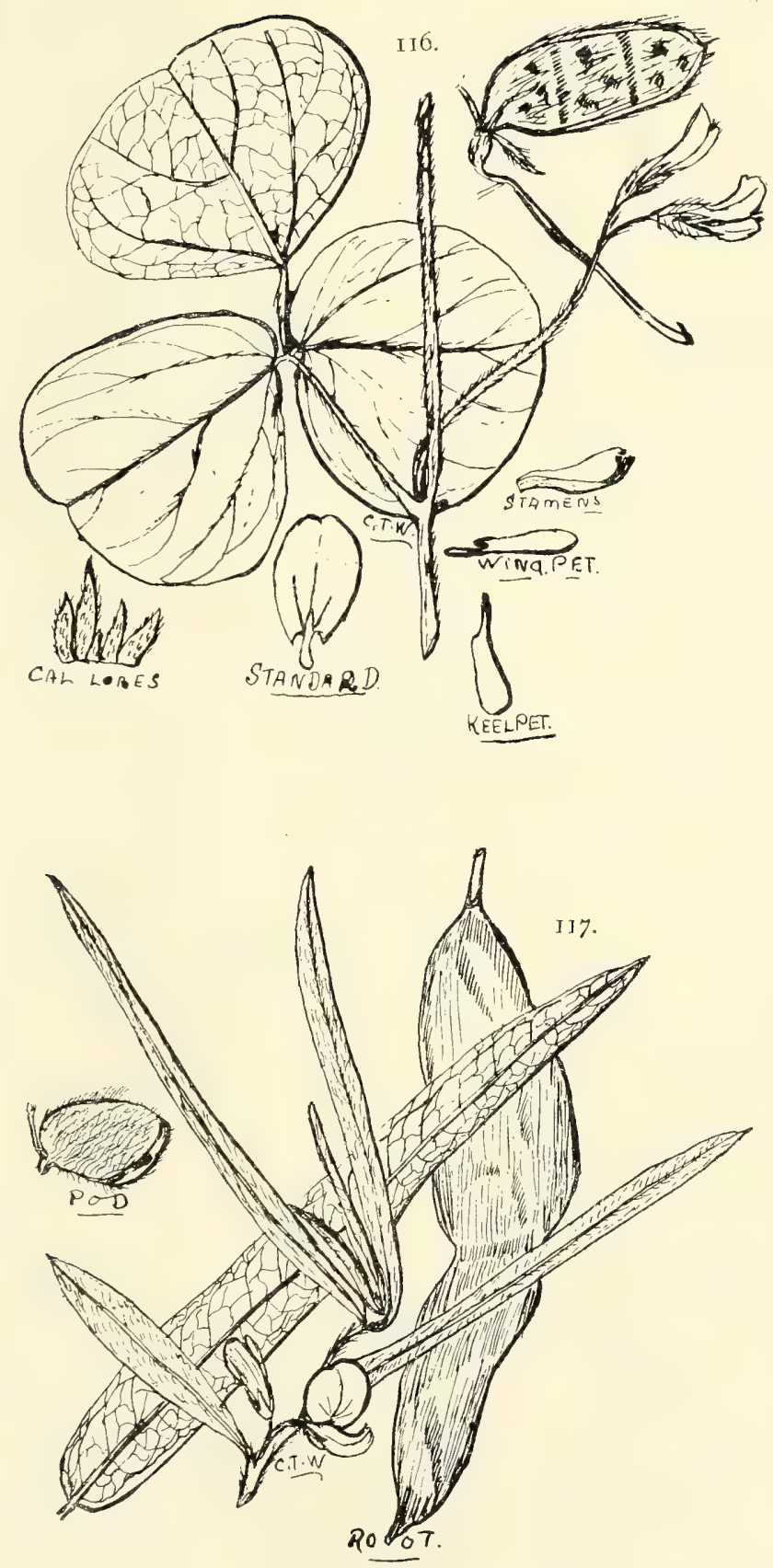

116. Atylosia marmorata, Benth.

I7 Eriosema chinense, $V o g$. 

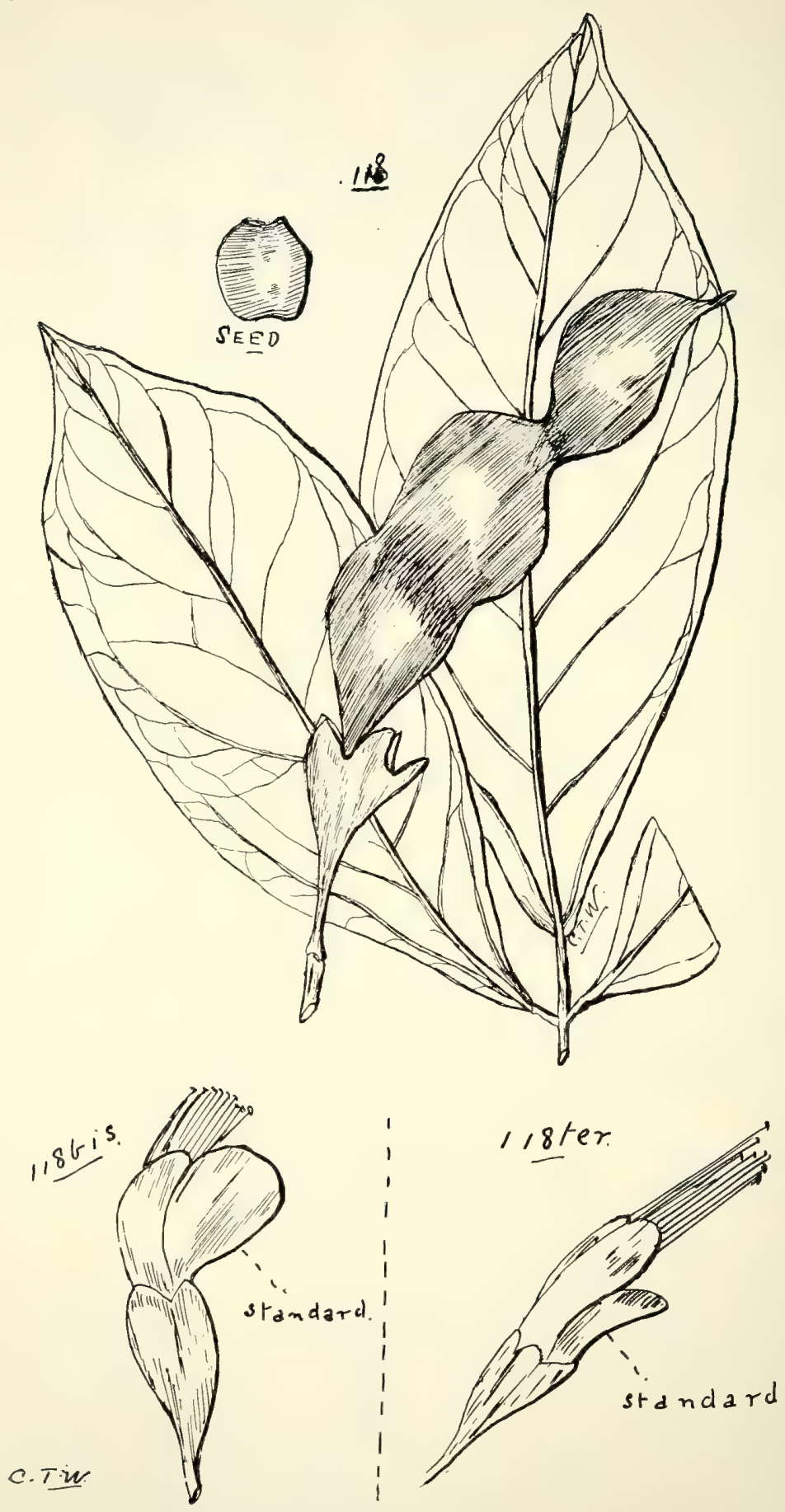

ii8. Podopetalum Ormondi, Bail.

i 8 bis. Castanospermum australe, $A$. Cunn.

i 8 ter. C. australe, $A$. Cunn., var. brevivexillum, Bail. 
Dumbaria, $W$. et $A$. conspersa, Benth.

Atylosia, $W$. et $A$.

marmorata, Benth. = Glycine marmorata, R. Br. (Fig. II6.) scarabæoides, Benth.

reticulata, Benth.- "Korlbun" of Cooktown natives, by whom the roots are eaten after roasting and hammering.

grandifolia, F.v. M.

pluriflora, F.v. M.

Rhynchosia, Lour.

acutifolia, F.v. M.

Cunninghamii, Benth-Blue Bean. Would be useful for ornamental purposes, but the seed does not retain its beautiful blue colour for any length of time.

minima, $D C$.

australis, Benth.

Eriosema, DC.

chinense, Vog._. Torakal" of Palmer River, "Pand-ja” of Cooktown, "Kal-lar" of Bloomfield River, and "Nargul " of Middle Morehead River natives. Roots roasted and skinned before eating by the natives. (Fig. I77.)

Flemingia, Roxb.

lineata, Rorb.

pauciflora, Benth.

parviflora, Benth.

involucrata, Benth.

TRIBE IX.-DALBERGIEA.

Dalbergia, Limn.

densa, Benth.

Lonchocarpus, H. B. et $K$.

Blackii, Benth--Bloodbark; exudes a blood-red juice, which on exposure dries to a brownish gum containing arabin 3.8 per cent., resin 1.4 per cent., tannic acid 74.2 per cent., and water 20.6 per cent.

nesiotes, Bail.

Jerris. Lour.

koolgibberah, Bail.

scandens, Benth.- "Pogg-ara” of Dunk Island natives.

uliginosa, Benth.- "Mo-kor-ja” of Cooktown, "Mara" of Tully River, and "Eycyabba” of Herbert River natives. Used by them in catching fish, like most other tanninbearing plants. Accorling to Trimen, the roots of the 
plant are used for the same purpose by the natives of Ceylon, who also use the strong fibre of the stem in making ropes and fishing-lines.

Pongamia, Vent.

glabra, Vent.-Karum or Poona Oil tree. "Napum-napum" of Cardwell natives (R.B.H.). Oil of seed is a cure for skin diseases. All parts of plant emetic. Extract of bark Dr. T. L. Bancroft found poisonous to frogs. In India, oil from the seeds has long been used as an application in scabies, herpes, and other cutaneous diseases. var. minor, Benth.

Sophora, Linn.

tomentosa, Linn.-Sea-coast Laburnum.

Fraseri, Benth.

Podopetalum, F.v.M.

Ormondi, F.v.M._-"Belgo-belgo" of Barron River natives.

Wood of a pinkish-grey colour; the red seeds useful for ornamental purposes. (Fig. II8.)

Castanospermum, A. Cunn.

australe, A. Cunn.-Moreton Bay Chestnut or Bean-tree. "Mai" and "Mi” of Brisbane River, "Ko-par" of Cooktown, "Wac-kay" of Barron River, "Yinyin" of Burnett River, and "Tinda-burra" of Dunk Island natives. Contains saponin. Largely used for food by the aborigines, after preparation. Considered poisonous to horses. Wood excellent for cabinet and other ornamental work. (Fig. 118 bis.-flower only.)

var. brevivexillum, Bail. (Fig. I 88 ter.-flower only.)

Barklya, F.v.M.

syringifolia, F.थ.M.-Wood blackish-grey, very tough; suitable for tool-handles.

var. tortuosa, Bail.

\section{Suborder II.-CAESALPINIEE.}

Tribe XI.-E.uc.esalpinie住.

Cæsalpinia, Linn.

Bonducella, Flem.-Kernels of the seeds are the "Nickernuts" of India, used in medicine. The prickly pods and blue or slate-coloured seeds are useful for ornamental purposes. 


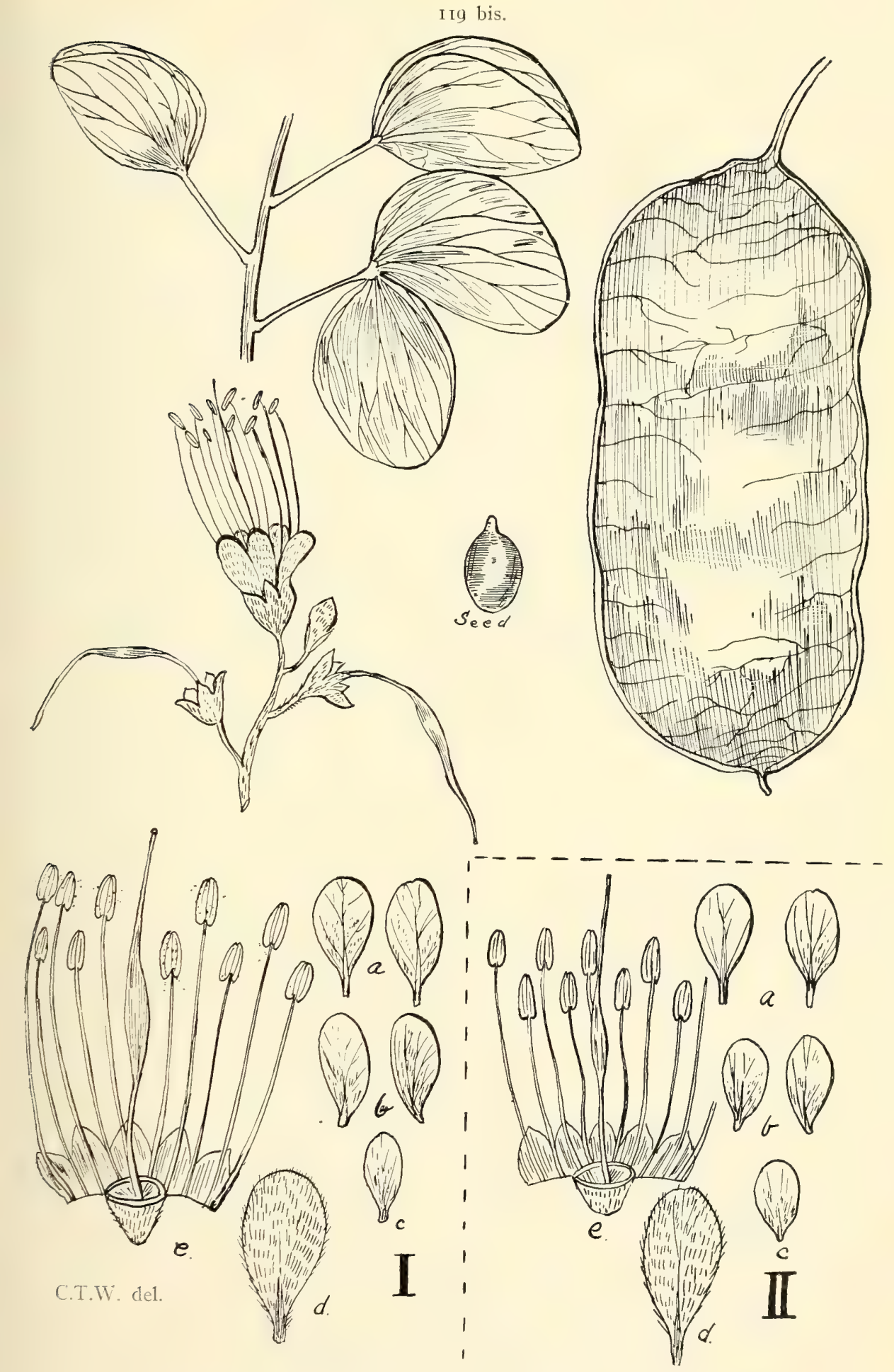

itg bis. Bauhinia Cunninghamit, Benth.

I. forma rosea, Bail. n. form.; II. forma gilva, Bail. n. form.

(a) 2 outer lower petals, (b) 2 lateral petals, (c) uppermost inner petal, (d) back

view of a petal to show tomentum, (e) calyx laid open to show 

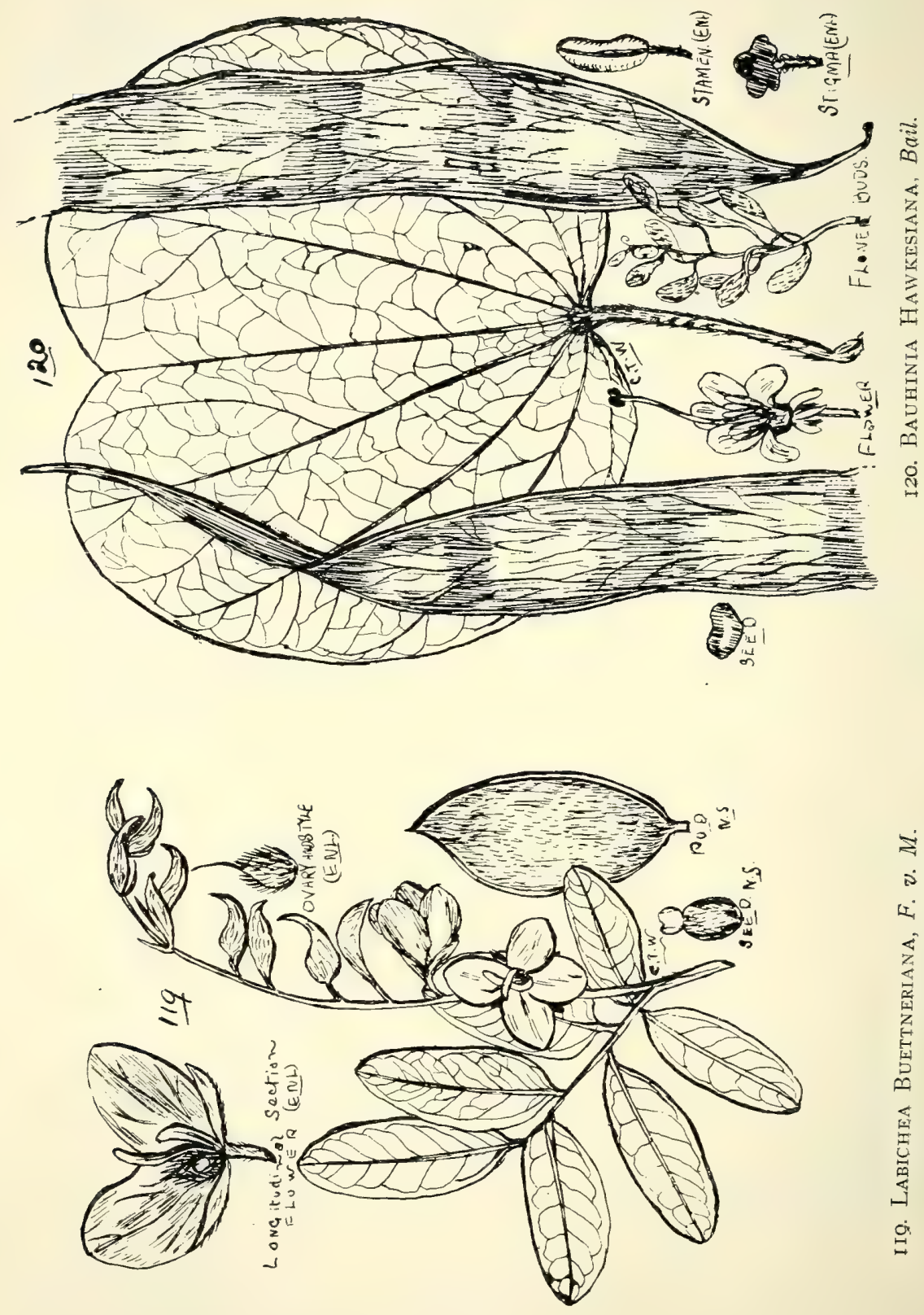


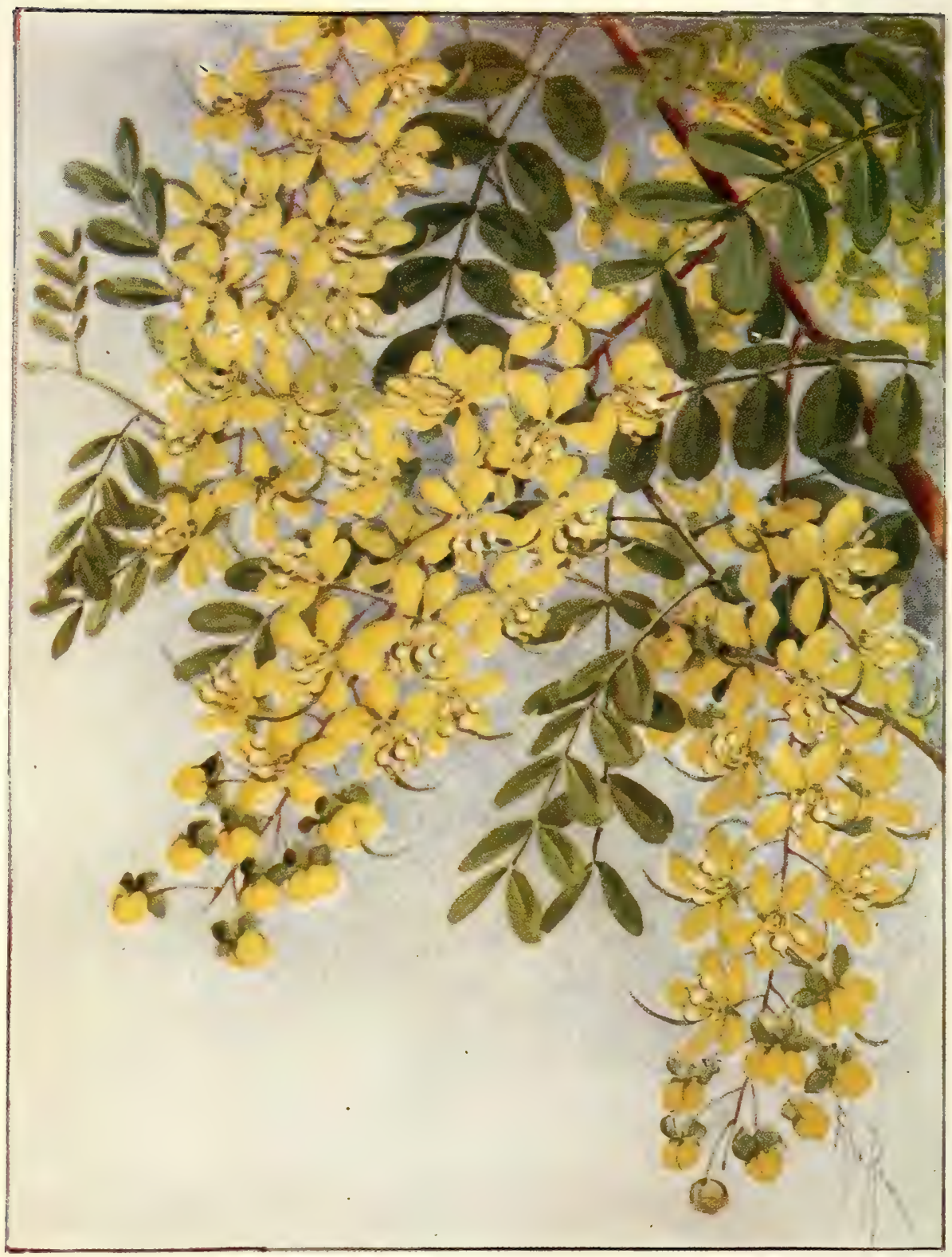

CAssia Brewsteri, F. v. M., VAR. SYlvestris, Bail. 
Casalpinia-contd.

nuga, Ait.

*sepiaria, Roxb.-Mysore Thorn; a noxious weed. Thorny hedge-plant; in Brisbane called Thorny Acacia. India.

Mezoneurum, Desf.

brachycarpum, Benth.-Cork-vine.

Scortechinii, F.v.M.--Flat Blue-bean. The seeds useful for ornamental purposes.

Fterolobium, R. Br.

nitens, $F . v \cdot M$.

Peltophorum, $V o g$.

ferrugineum, Benth.

*Parkinsonia, Linn.

aculeata, Linn.-Jerusalem Thorn of West Indies; a noxious weed in some northern parts of Queensland. South America.

\section{TRIBE XII.-CASSIEE.}

Cassia, Linn.

Subgenus I.-Fistula (Cathartocarpus).

Brewsteri, F.v. M.

war. tomentella, Benth-Bean-tree of Obum Obum. Furnishes a good cabinet-wood.

var. sylvestris, Bail._ "Moweii" of Cardwell natives

(R.B.H.). Furnishes a good cabinet-wood, and would be excellent for bitter-cups. (Plate VI.)

var. Marksiana, Bail.-Furnishes a good cabinet-wood.

\section{Subgenus II.-Senna.}

Section Chamcfistula.

*lævigata, Willd--Tropical America.

*tomentosa, Limn.-Spreading in some localities, and may become a pest. South America.

Sophera, Linn., var. schinifolia, Benth.-A troublesome weed. var. pubescens, Benth.

*ocidentalis, Linn.-A troublesome weed; the whole plant purgative. Africa.

*alata, Lim.-Ringworm Shrub; at Port Douglas called "Yellow-top"; a troublesome weed. Eczema is said to be cured by bathing the affected parts with a decoction of the bark, leaves, and flower of this plant. India. 
Cresalpinia-contd.

Section Chamasenna.

magnifolia, F. v. M.

venusta, F. v. M.

notabilis, F.v.M.

pleurocarpa, F. . M.

Subgenus IV.-Psilorhegma.

glanca, Lam.

retusa, Sol.

australis, Sims.-A troublesome weed.

var. revoluta, Benth. $=$ C. revoluta, F. v. M.

Chatelainiana, Gaud.

pruinosa, F.v. M.-Bark yields a red dye.

circinata, Benth.

phyllodinea, R. Br.

eremophila, A. Cunn.

var. platypoda, Benth. = C. platypoda, R. Br.

var. zygophylla, Benth.

artemisioides, Gand.

Sturtii, R. $B r$.

desolata, F.v. M.

oligophylla, F.v.M.-Turkey-bush.

oligoclada, F.v.M.

leptoclada, Benth.

Subgenus $V$.-Absus.

Absus, Linn.-Chichm or Cismatan; used in medicine in various parts of Asia.

Subgenus VI.-Chamcerista.

pumila, Lam.

concinna, Benth.

mimosoides, Linn.

Petalostyles, R. Br.

labicheoides, R. Br.

var. cassioides, Benth.

Labichea, Gaudich.

Buettneriana, F.v.M. (Fig. II9.)

nitida, Benth.

rupestris, Benth. 
Tribe NIII.-Baulinie.

Bathinia, Limn.-The inland species considered good food for stock.

Carronii, F. v. M.- "Pergunny" and "Thalmera" of Cloncurry natives. Wood light-brown, darker near the centre, suitable for cabinet-work.

Hookeri, F. $\% \cdot M$.-Queensland Ebony. Furnishes a heavy, dark wood useful for many purposes. Gum contains arabin I 2 per cent, metarabin .67 per cent,, water 2I per cent.

var. puberula, Benth.

acuminata, Linn.

monandra, Kurs.

Cunninghamii, Benth.-Furnishes a heavy, dark wood useful for many purposes. (Fig. II

forma rosea, Bail.

forma gilva, Bail.

Hawkesiana, Bail. (Fig. I20.)

\section{Tribe XIV.-Amherstieæ.}

Afzelia, Sm

australis, Bail.-Johnstone River Teak. Wood yields a purplish dye. (Fig. I2I.)

Cynometra, Linn.

Tribe XV.-Cynometrex.

ramiflora, Linn., var. bijuga, Benth.-Wood yields a purplish dye.

Tribe XVI.-DimorPhANDRE飞.

Erythrophlœum, Afzel.

Laboucherii, F.v.M.-Poison-tree or Ironwood. "Ah-pill" of Mitchell River, "Arriga" of Palmer River, and "Naumuta" of Batavia River natives. According to Mueller, contains erythrophlœin, the active principle of $E$. grimeense. Wood very heavy and dark; used by the natives for woomerahs and spear-points.

\section{Suborder III.-MIMOSEAE.}

Tribe XVII.-Adenanthere.

Entada, Adams.

scandens, Benth-Watchbox Bean; Gogo-vine. "Barbaddah" of Cleveland Bay, "Na-gobar" of Cardwell, and "Parpangata" of Batavia River natives (R.B.H.). 
The Queensland aborigines, according to E. Palmer, use, after preparation, the seeds for food. The roasted seeds, we are told, are also eaten in some parts of India. Seeds contain saponin. "The use made of the mashed bark of this climber is well known throughout the Philippines. Cut in strips and beaten thoroughly between stones, it is sold under the name of 'gogo'; it is macerated in water, to which it imparts a reddish colour, and forms a substitute for soap. The Filipinos use this preparation for bathing, especially the hair, for which purpose there is no more useful or simple preparation." - T. H. Pardo de Tavera.-J. M. Hillier in "Medicinal Plants of the Philippines," Kew Bull. (I9II), 475.

Adenanthera, Lour.

abrosperma, F. v. M.- "Oon-doo" of Mitchell River and

"Rokowara" of Palmer River natives, who use the seeds for food after roasting. (Fig. 122.)

var. falcata, Bail., n. var. (Fig. I23.)

Hab.: Koorboora, near Chillagoe (W. A. Waddell).

pavonina, Limn.-Red Sandalwood. The seeds used for weights by the Indian jewellers; the seeds of the Queensland form are much smaller than the Indian.

Neptunia, Lour.

gracilis, Benth.-Native Sensitive-plant; leaves sensitive.

var. major, Benth. (Fig. I24.)

var. villosula, Benth. (Fig. I25.)

monosperma, F.v. M. (Fig. I26.)

Trine XVIII.-Eumimose..

*Mimosa, Linn.

pudica, Linn.-The Common Sensitive-plant of Tropical

America. Leaves sensitive; seeds said to be poisonous in India; a troublesome weed. The roots of this weed said to contain about Io per cent. of tannin.

\section{TrIBE XIX.-ACACIE}

Acacia, Willd.-The bark of many used in tanning; and many also, at certain periods of their growth, produce a saponin poisonous to stock browsing upon them at that time. 


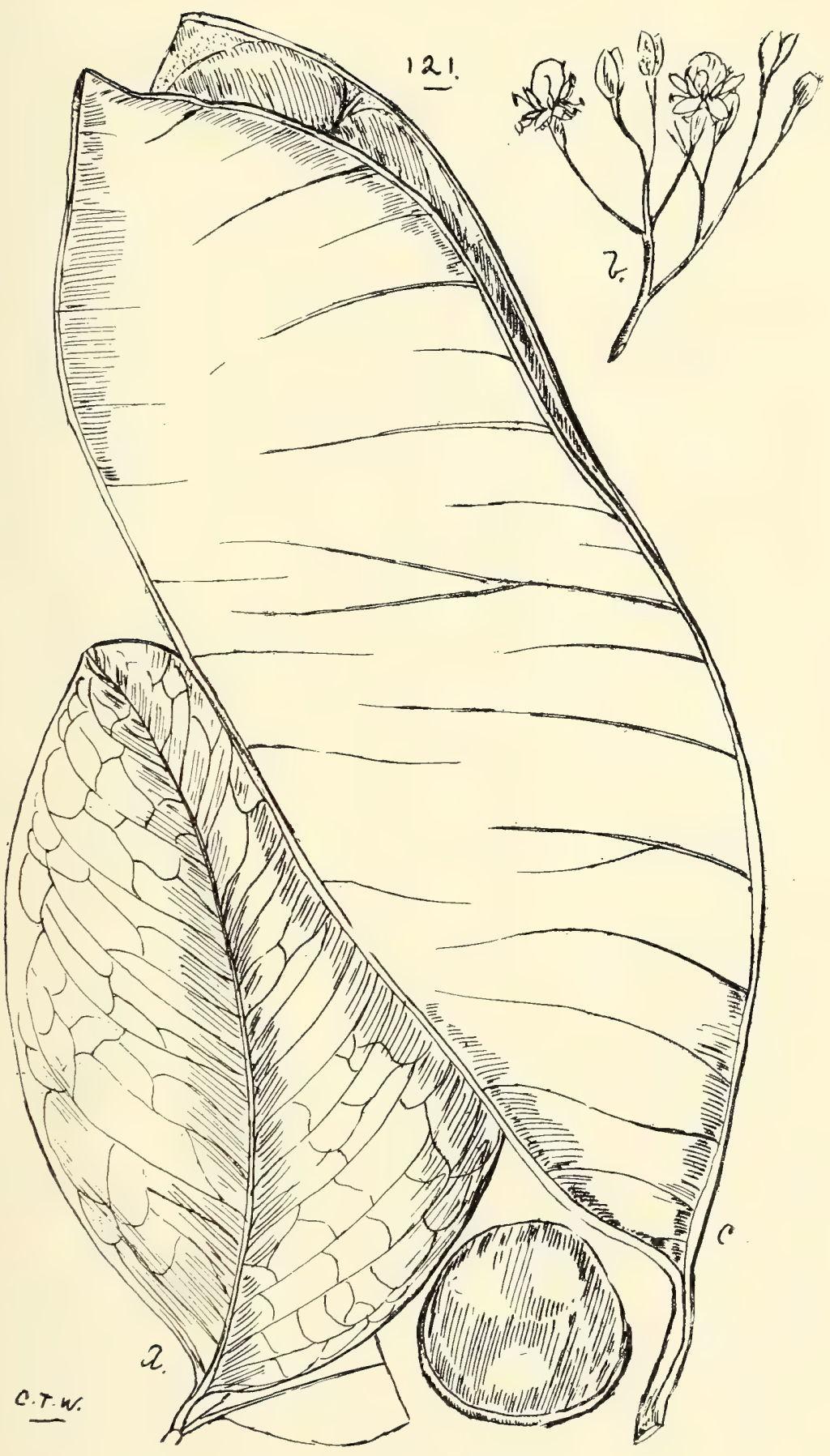

121. Afzetia australis, Bult. 


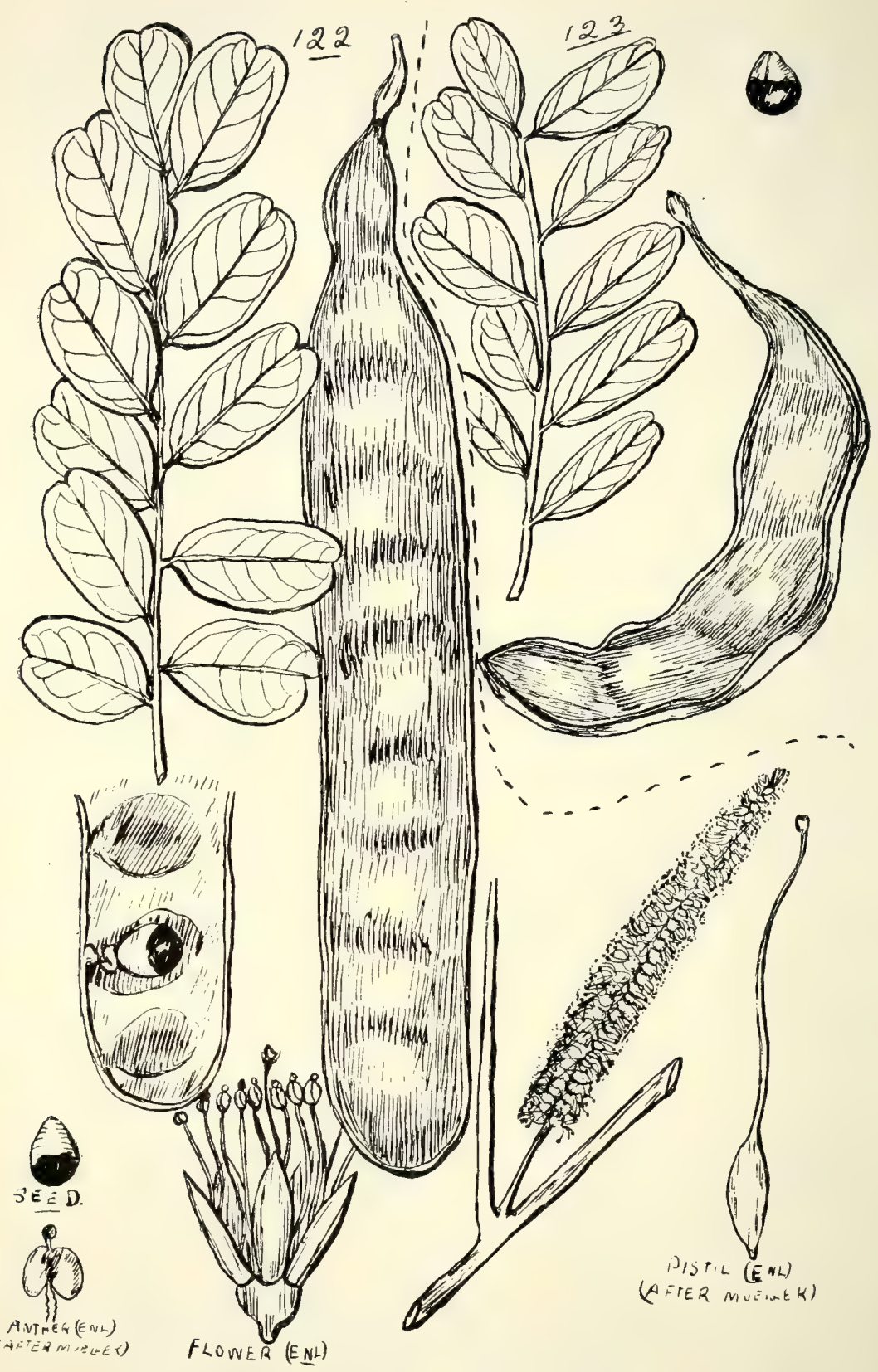

122. Adenanthera abrosperma, $F . v \cdot M$.

I23. A. abrosperma, $F$. $\imath^{\prime}$. M., var, falcata, Bail. 
Acacia-contd.

\section{Division I.-Phyllodinece.}

Series Continuæ.

Pence, F. v. M.--Ironwood or Pine Acacia. Wood heavy and hard; pods flat and broad. (Fig. I27.) triptera, Benth.

\section{Series Pungentes.}

lanigera, A. Cunn.

var. gracilipes, Benth.

phlebocarpa, F.v. M.

tetragonophylla, F.v. $M$.

juniperina, Willd.

var. Brownei, Benth.

Series Calamiformes.

Bynoeana, Benth.

pugioniformis, Wendl.

juncifolia, Benth.

var. planifolia, Benth.

calamifolia, Srveet.

Series Brunioideæ.

hippuroides, Heward. (Fig. I28.)

lycopodifolia, A. Cunn.

var. glabrescens, Benth.

spondylophylla, F. v. M.

galioides, Benth.

Baueri, Benth.

subternata, Bail.

bruniades, A. Cunn.

conferta, A. Cunn.

\section{Series Uninerves.}

armata, R. Br.-Not very abundant in Queensland. One of the first plants used in Australian hedge-making, and known in South Australia as "Kangaroo Island Prickly Acacia."

var. angustifolia, Benth.

plagiophylla, F.v.M. = A. undulifolia, var. humilis, Benth. lineata, A. Cunn. = A. runciformis, A. Cunn.

undulifolia, A. Cunn.

hispidula, Willd.

Gnidium, Benth. 
Acacia-contd.

sentis, F. v. M.- “" Gundey-bluey," St. George. "Narran” of St. George natives.

fasciculifera, F.v.M.-Wood red.

falcata, Willd.-Wood light-brown and yellow; useful for cabinet-work. The bark contains 27.17 per cent. of tannin (J.C.Brïnnich).

macradenia, Benth.-Wood close-grained and tough.

penninervis, Sieb.-The foliage of this species differs in shape and size very considerably; it is at times met with 7 in. long and 3 in. broad, bearing $I$ or 2 teeth-like lobes bearing at their points the usual marginal glands of the species. Trees when large yield a useful cabinet-wood. var. falciformis, Benth.

neriifolia, A. Cunn.-Bark found to contain I3.91 per cent. of tannin.

accola, Maid. and Betche.-Near Stanthorpe.

rubida, A. Cunn.

salicina, Lindl._- "Baka" of Rockhampton natives. A useful dark-coloured cabinet-wood.

var. varians, Benth.

amœna, Wendl.

hakeoides, $A$. Cumn.

suaveolens, Willd.

Dietrichiana, F. v. M.

Murrayana, F. v. M.

linifolia, Willd.-Wood light-coloured, red towards centre, close-grained and tough.

Leichhardtii, Benth.

crassiuscula, $W$ endl.

decora, Reichb.-Gum eaten by Cloncurry natives (Palmer). buxifolia, A. Cunn.

lunata, Sieb.

podalyriæfolia, A. Cunn.--Silver Wattle of Queensland. Bark contains I2.40 per cent. of tannin.

uncifera, Benth.

cultriformis, A. Cunn.

myrtifolia, Willd.

purpureapetala, Bail. (Fig. I29.)

Series Plurinerves.

sublanata, Benth.

amblygona, A. Cunn.

translucens, $A$. Cunn.

impressa, F. v. M. 
Acacia-contd.

elongata, Sieb.

Simsii, A. Cunn.

var. multisiliqua, Benth.

homalophylla, A. Cunn.-- Gidgee. "Wong-arrah" of Cloncurry natives. Gum eaten. Wood prettily marked, of a dark colour; used for boomerangs and spears. Odour of plant very objectionable.

georginæ, Bail.-Georgina Gidgee; considered poisonous to stock.

pendula, A. Cumn-Weeping Myall. "Boree" of some. Bark contains 3.80 per cent. of tannin (J.C. Brïnnich). Wood fragrant, excellent for cabinet-work.

Oswaldi, F.v.M.

coriacea, $D C$.

stenophylla, A. Cunn.-Dalby Nyall; a good cabinet-wood. The bark contains 8.30 per cent. of tannin $(J . C$. Brünnich).

viscidula, $A$. Cunn.

rar. angustifolia, Benth.

ixiophylla, Benth.

dictyophleba, F. v. M.

venulosa, Benth.

melanoxylon, R. Br.-Blackwood of the Southern States.

implexa, Benth.-A useful cabinet-wood.

harpophylla, F. v. M.-Brigalow. "Ogarah" of St. George natives. Bark contains $\mathrm{I} 3.34$ per cent: of tannin $(J$. C. Brïnnich); yields a reddish-brown dye. A useful timber; yields a light or dark brown gum of a sour, astringent taste, entirely soluble in water, and forming a mucilage as sticky as arabic-gum (Lauterer).

excelsa, Benth.-Ironwood Wattle. A large tree, furnishing a useful dark timber. "Tooloo" of St. George, "Darreen" of Eidsvold natives. Bark contains Io.88 per cent. of tannin ( J.C. Brïnnich).

homaloclada, $F, v . M$.

complanata, A Cunn.

binervata, $D C$.

Bakeri, Maid.-Furnishes a useful timber.

Rothii, Bail._"Lar" of Batavia River natives.

dineura, F. v. M.

sericata, A. Cunn.

oraria, F.v.M.-A tropical beach-tree.

flavescens, A. Cunn.- "Dun-dul" of Cape Bedford and

"Tun-jol" of Cooktown natives. Wood prettily marked.

Bark contains .56 per cent. of tannin (J.C. Brïnnich). 


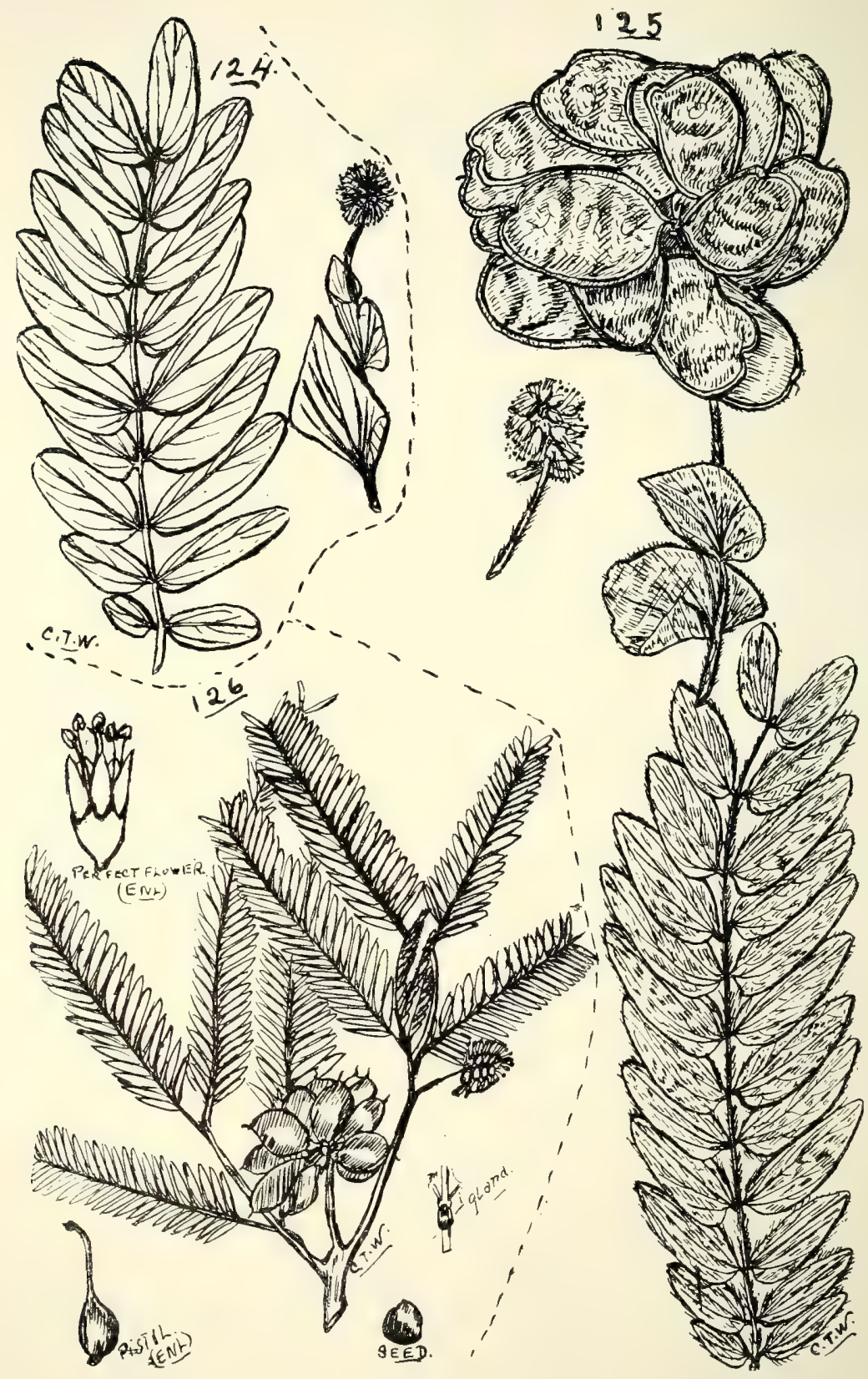

I24. Neftunia gracilis, Benth, var. major, Benth. 125. N. GRACILIS, var. villosula, Benth. I26. N. MONOSPERMA, $F$. v. $M$. 


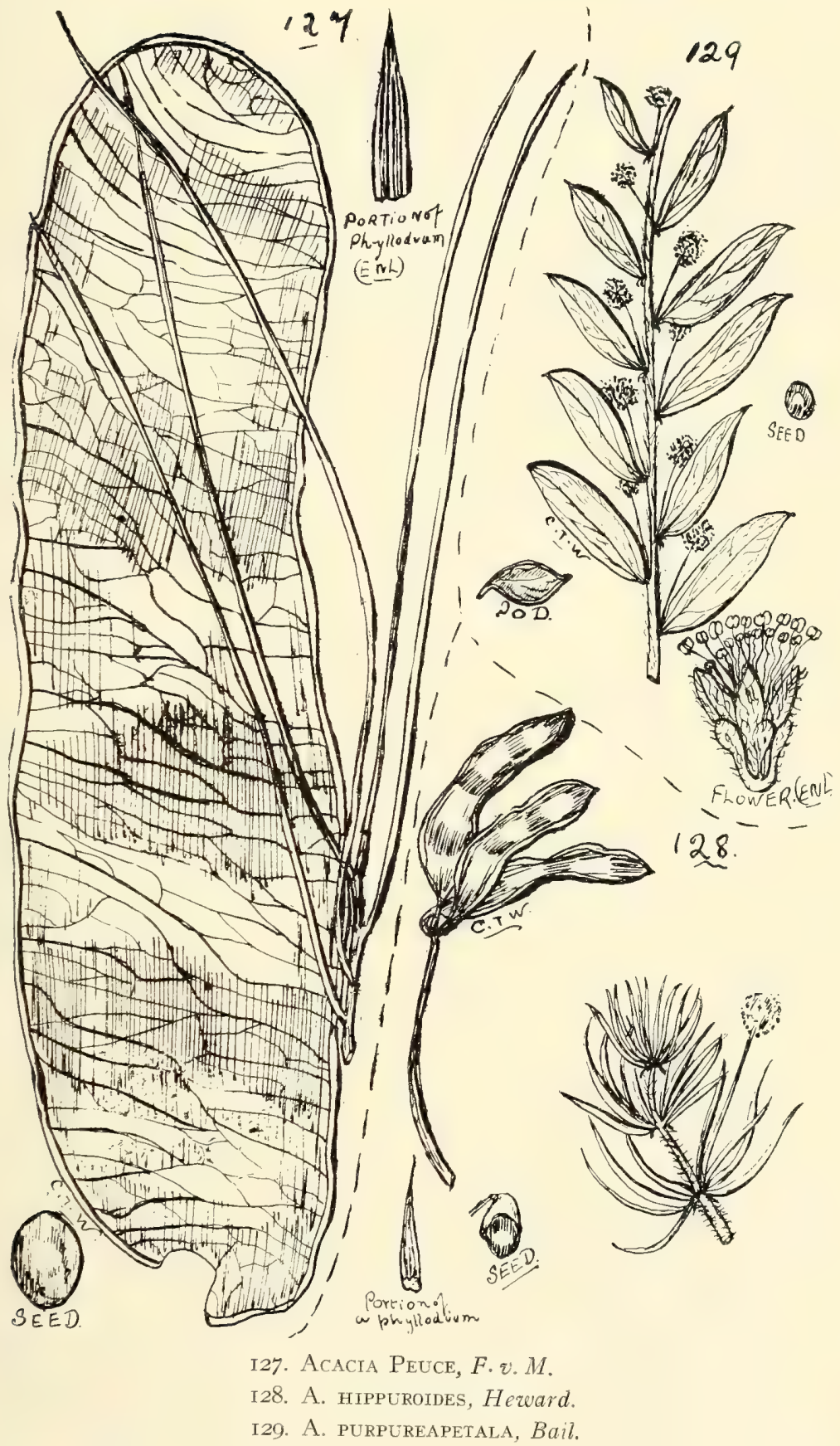


Acacia--contd.

Wickhami, Benth.

Series Julifloræ.

lysiphleea, F.v. M.- "Urr tee" of Batavia River natives.

linarioides, Benth.

Chisholmi, Bail.

stipuligera, F. v. M.

umbeilata, $A$. Cunn.

brevifolia, Benth.

gonoclada, F. v. M.

longifolia, Willd.-Bark yields about $\mathrm{x} 2.67$ per cent. of tannin.

var. Sophoræ, F. v. M.

var. typica, Benth.

var. floribunda, $F, v . M$.

linearis, Sims.

cyperophylla, F. v. M.

pityoides, $F . v . M$.

aneura, F.v.M.-Mulga. Foliage largely used as food for stock in times of drought.

cibaria, F. v. M.

Kempeana, F.v. M.

xylocarpa, A. Cunn.

var. tenuissima, Benth.

gonocarpa, F. v. M.

drepanocarpa, F.v.M.

conspersa, F. v. M.

doratoxylon, A. Cunn.--Spearwood of Allan Cunningham in I8I7. Lancewood at Rockhampton. Splinters of the wood said to be as venomous as a snake-bite. A useful timber.

delibrata, A.Cunn.-Contains saponin.

torulosa, Benth.

julifera, Benth.

Solandri, Benth.

leptostachya, Benth.

glaucescens, Willd--Rosewood; also called Lancewood at Mount Morgan. This tall, upright tree has one of the thinnest barks known. Furnishes a useful timber; when of a twisted growth the wood is known as "Ringy Rosewood."

Maideni, F. v. M.

Cunninghamii, Hook. - "Tchilgar" of Bunclaberg and "Kowarkull" of Stradbroke Island natives. Contains saponin. Bark contains 9.I3 per cent. of tannin (Staiger). Gum contains Io per cent. of arabin and 72.5 per cent. of metarabin (Lauterer). Unripe pods contain saponin. 
Acacia-contd.

Cunninghamii-contd.

var. longispicata, Benth.

leptocarpa, A. Cumn.- " I-wa-wal" of Palmer River natives.

The bark contains 2.59) per cent, of tannin ( $J . C$. Brïnich).

polystachya, A. Cunn.-Yields about 7.59 per cent. of tannin. holcocarpa, Benth.

plectocarpa, A. Cunn.

aulacocarpa, A. Cunn.-Hickory Wattle. "Carr-lee" of Du1k

Island, "Dilka" of Port Curtis, and "Boorgun" of

Nanango natives. A useful cabinet-wood.

var. ? nacrocarpa, Benth.

calyculata, A. Cunn.

crassicarpa, A.Cunn.-Lancewood of Cairns. "Mon-jin" of

Butcher's Hill and "Tara” of Morehead River natives.

The bark contains . I7 per cent of tannin (J.C. Brïnnich). atriculiformis, $A$. Cunn.

Mangitm, Willd.

cincinnata, F.v.M.-Mr. W. Macartney, of Forest Hill,

states that the timber of this tree resists the attacks of

the teredo. Wood tough, useful in forming umbrellahandles, \&c.

holosericea, A. Cunn.-- "War-roon" of Cooktown natives.

var. pubescens, F.v. M.

latifolia, Benth._" Yoo-a-bal" of Princess Charlotte Bay natives.

dimidiata, Benth.

liumifusa, A.Cunn. (Fig. I30.)

\section{Division II.-Bipinnata.}

Series Botryocephalæ.

spectabilis, A. Cunn.

var. ? Stuartii, Benth.

polybotrya, Benth.

var. foliolosa.

decurrens, Willd.-Green Wattle. Furnishes the usual tanning bark. Bark contains I $_{5} .08$ per cent. tannin (Staiger).

zar. normalis, Benth.

var. mollis, Lindl.

var. pauciglandulosa, F. v. $M$.

dealbata, Link.

Arundelliana, Bail.-A handsome, small tree or tall shrub, very distinct from all its allies. (Fig. I3I.)

ieptoclada, A. Cunn. 
Acacia-contd.

\section{Series Gummiferæ.}

farnesiana, Willd., var. lenticellata (F.v.M.), Bail.-"Bunkerman" of Cloncurry natives; pods roasted and eaten. Flowers of the normal form are used in the manufacture of the well-known "Cussie pomade" of France.

Bidwilli, Benth.- "Yadthor" of Cloncurry and "Warrew" of Rockhampton natives. Sometimes found useful as food for stock. Roots of young trees roasted, after peeling, by the natives.

var. ? major, Benth.

Sutherlandi, F.v.M. = Albizzia Sutherlandi, F. v. M.; Acacia melaleucoides, Bail.

\section{Tribe XX.-InGeæ.}

Albizzia, Durazz.

basaltica, Benth.-Dead Finish; a beautiful cabinet-wood.

Thozetiana, F.v.M.-Wood reddish, very tough. canescens, Benth._- "Thow-i-ee" of Mackay natives.

"Lebbeck, Benth.-Bark said to contain about II.33 per cent. of tannin.

procera, Benth.-Wood resembling walnut.

Toona, Bail-_Acacia Cedar; a valuable wood for many purposes. "Carrabbe" of Barron River natives., Gum contains arabin 56.4 per cent., metarabin 25 per cent., water I8.6 per cent. (Lauterer). (Fig. I32.)

Pithecolobium, Mart. (Included by Mueller in Albizzia.)

pruinosum, Benth.-Wood light-yellow.

ramiflorum, F.v. M.

grandiforum, Benth. $=$ Albizzia Tozeri, F.v. M.-Wood yellow.

Lovellæ, Bail. (Fig. I33.)

Hendersoni, $F . v . M$.

moniliferum, Benth.- "Mullar" of Cloncurry natives; young. pods roasted and eaten by them.

Archidendron, F. v. M. (Included by Mueller in Albizzia.)

Vaillantii, F.v.M.-Wood red, strong and durable. The bark is said by Dr. T. L. Bancroft to be more poisonous than the pods or leaves. (Plate VII.)

Lucyi, $F . v . M$. 
PLATE VII.

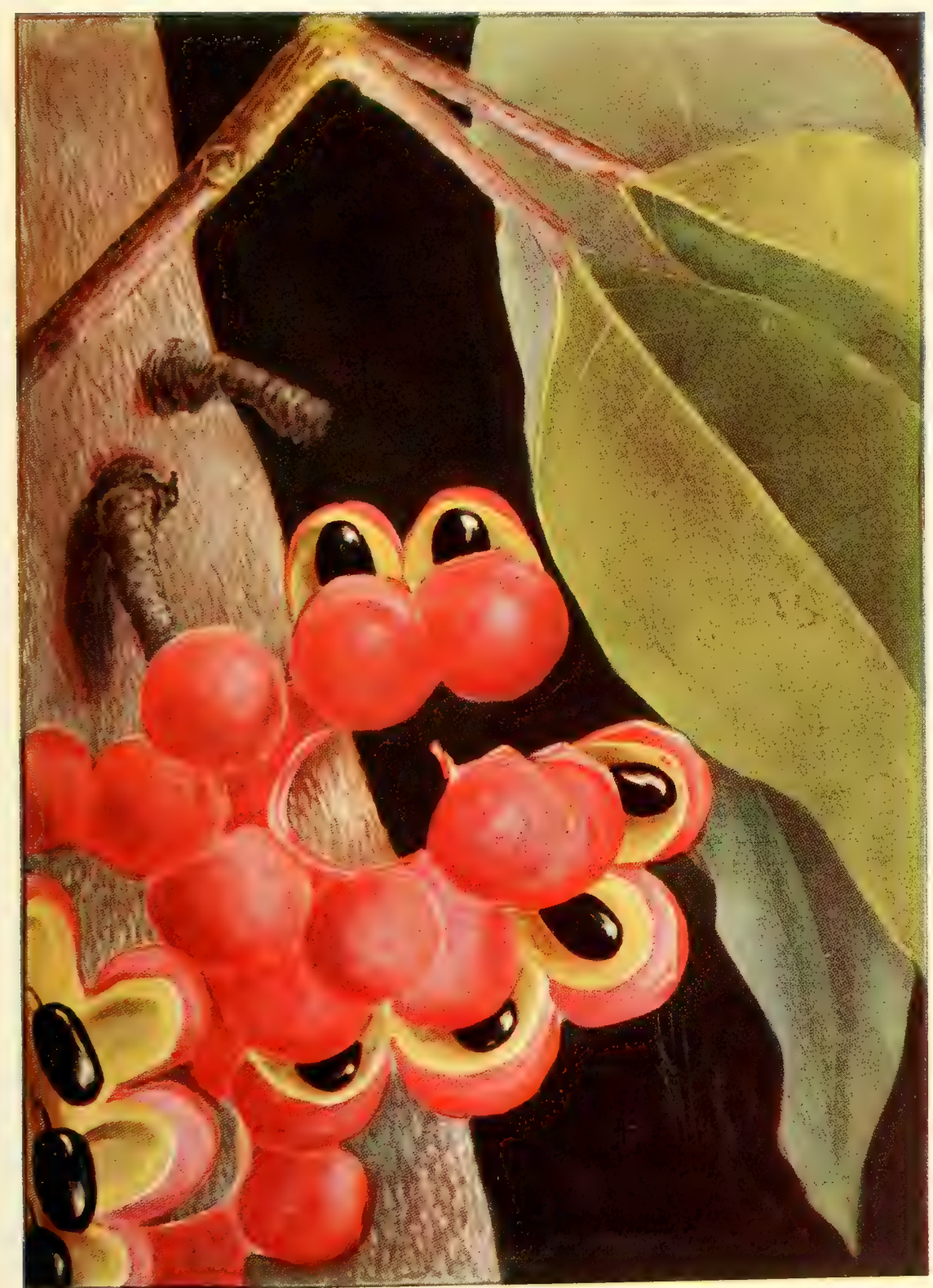

Archidendron Vaillantil, F. v. M. 


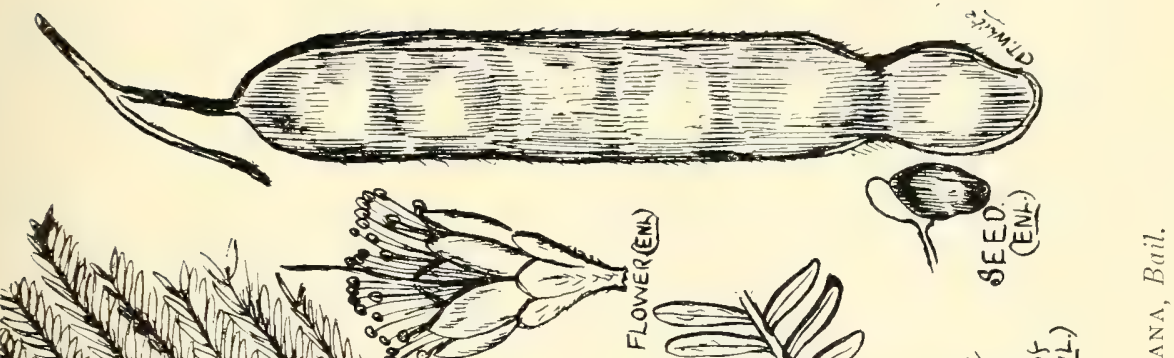

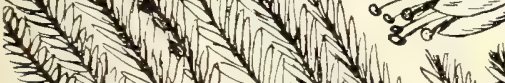

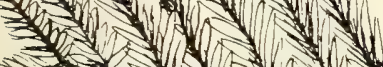

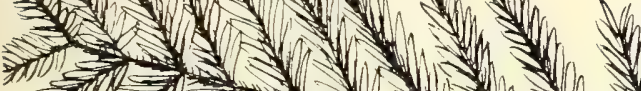

2)

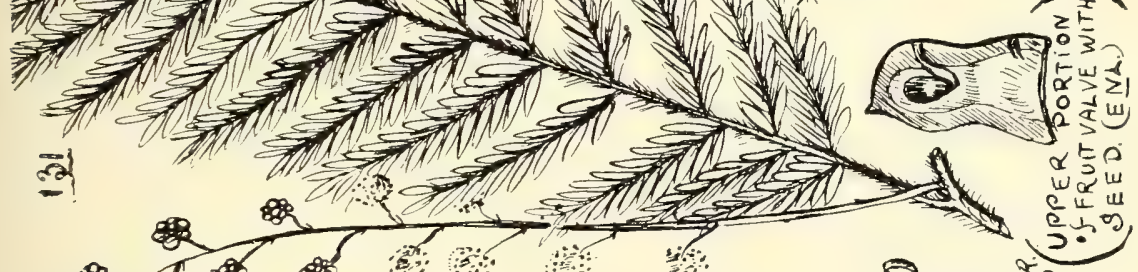

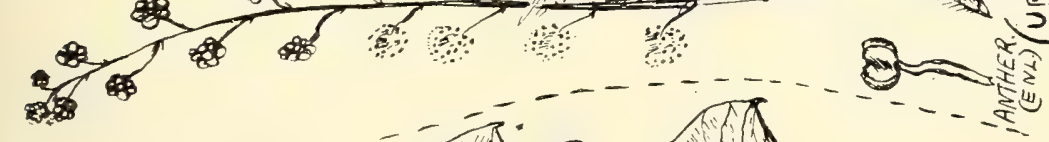

$\Longrightarrow 2 \div 24$

要

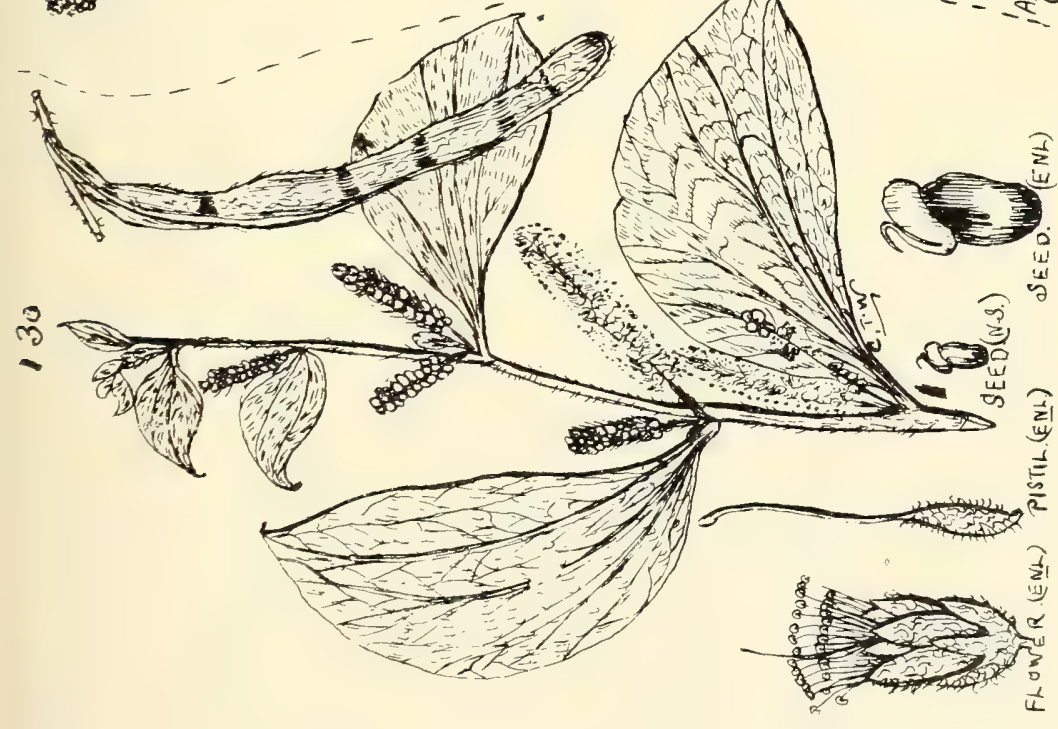

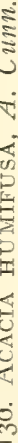




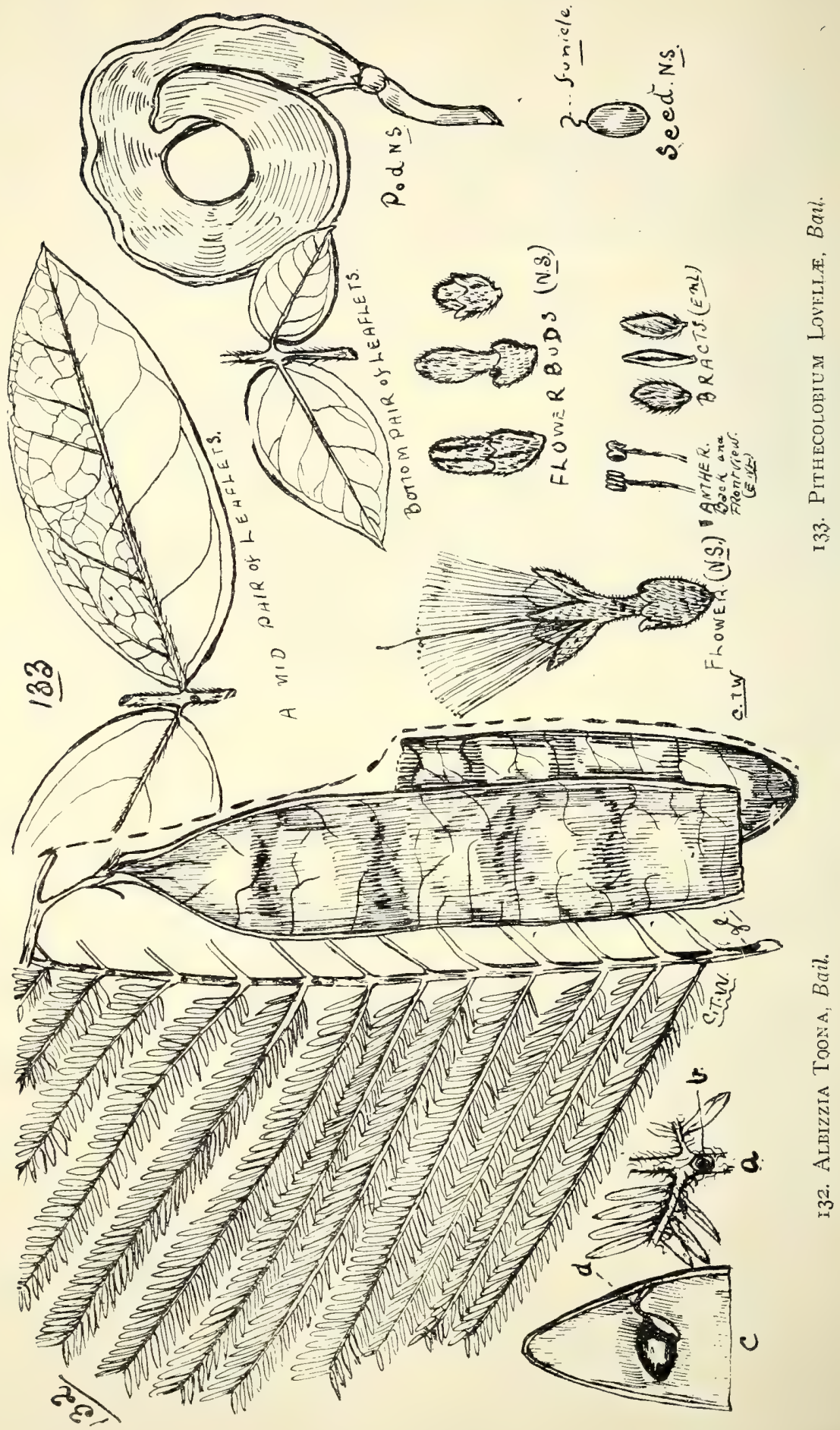




\section{Order XLIV.-ROSACEA.}

Tribe I.-Chrysobalaneæ.

Parinarium, Juss.

Nonda, F.v.M.-_" Ranna" of Morehead River, "Wo-in-ya" of Cooktown, and "Wiepa" of Mabuiag Island natives (R.B.H.).

Griffithianum, Benth. = Grymania salicifolia, Presl.

laurinum, A. Gray.-A fruit of this tree was picked up on the beach at Masthead Island in 1907, by Dr. F. Hamilton Kenny, so probably the tree is growing on some island off our coast or on the mainland. It is known at Fiji by the name of "Makita." The tree is also a native of the Samoan Islands.

Tribe II.-PRUNE.T.

*Prunus, Limn--Fruit a Drupe.

persica, Benth. et Hook.-Peach; contains prussic acid.

Pygeum, Gartn.

Turnerianum, Bail._- " Abill" of Barron River and "Joon¿a”" of Bloomfield River natives. Wood useful for cabinet and turnery work. The nuts useful for ornamental purposes.

TRIBE III.-RUBE.E.

Rubus, Limn.--Fruit an Etærio.

*ellipticus, Smith $=R$. flavus, Ham. - The yellow-fruited Bramble; an Indian species.

moluccanus, Linn.-The common Queensland Bramble;

"Native Raspberry" of some.

parvifolius, Linn.- " Neram" of North Queensland natives.

*fruticosus, Linn.-British Blackberry; a noxious weed.

rosæfolius, Sm.-Native Raspberry. "Panga-panga" of

Dunk Island and "Neram" of Rockhampton natives.

Plants may at times be seen with variegated foliage.

Muelleri, Bail.-Helidon Blackberry. (Fig. I34.)

Moorei, F.v. M.-Native Blackberry.

TRIBE IV.-POMEA.

The following genera of this tribe may be sometimes met with as strays from cultivation:-

*Pyrus.-The Quince. The fruit a Pome.

*Cratægus.--The Hawthorn.

*Photinia.-The Loquat.

*Rhaphiolepis.-The Indian Hawthorn. 
Tribe V.-Potentillede.

*Fragaria, Linn.-Fruit an Etærio.

indica, Andr.-India. A stray from garden culture.

Acæna, Linn.

Tribe VI.-PoterieÆ.

ovina, A. Cunn.-Fruit a prickly burr.

sanguisorbæ, $V a h l$.-Fruit a prickly burr.

TRIBE VII.-RoseÆ.

*Rosa, Linn.-Frtuit a Cynarrhodum.

rubiginosa, Limn.-Sweet Briar; a noxious weed of Europe.

So far only met with in the most southern parts of the

State.

\section{Order XLV. SAXIFRAGEA.}

TRIBE I.-ESCALLONIEA.

Argophyllum, Forst.

Lejourdanii, F. v. $M$.

var. cryptophleba, F.v.M.

nitidum, Forst.

var. fulva, Bail.

Abrophyllum, Hook. f.

ornans, Hook. $f$.

var. microcarpum, Bail.

Cuttsia. F.v. M.

viburnea, F.v. $M$.

Balls-Headleya, F.v. M. (inedited)

stipellosa, F. v. $M$.

Quintinia, A. DC.

Sieberi, $A, D C$.

Quatrefagesii, F.थ.M. (Fig. I35.)

Verdonii, F. v. $M$.

Fawkneri, F.v.M. (Fig. 136.)

Polyosma, Blume.

rigidiuscula, F. v. M. et Bail.

reducta, F.v. M. (Fig. I37.)

Cunninghamii, J. J. Benn.

alangiacea, $F$. v. $M$.

Anopterus, Labill.

Macleayanus, F.v. $M$. 
Callicoma, Andr.

serratifolia, Andr. = ferruginea, D. Don.-Contains saponin.

Stutzeri, F..$M$.

Spiræanthemum, A. Gray.

Davidsonii, F. $\cdot$. $M$.

Aphanopetalum, Endl.

resinosum, Endl.

Gillbeea, F.v. M.

adenopetala, F.v.M. (Fig. I38.)

Ceratopetalum, $S m$.

Virchowii, F.थ.M. (Fig. I39.)

Schizomeria, D. Don.

ovata, D.Don.-Fruit white, edible; has been used for jam. Davidsonia, F. v. M.

pruriens, F.v.M.-Davidsonian Plum. "Ooray" of Tully River natives. Fruit purple, edible. Wood useful for tool-handles.

rar. Jerseyana, Bail. = D. Jerseyana, F.v. M. Fruit much smaller than the Northern form.

Ackama, A. Cumn.

Muelleri, Benth.

Weinmannia, Linn.

Benthami, Bail. = Geissois Benthamii, F. v. M.

Biagiana, F. v. $M$.

lachnocarpa, F.v. M.-Scrub Rosewood and Scrub Redwcod. "Merrany" or "Marara" of Nerang. Wood light-pink; useful for making planes, mallets, and chisel-handles. (Fig. I39 bis.)

war. parvifolia, Bail. (Fig. I 39 ter.)

rubifolia, $F$. ข. $M$.

apetala, Bail.

Bauera, Banks.

rubioicles, Andr.

capitata, Ser. (Fig. I40.)

\section{Order XLVI. CRASSULACE.E.}

Tillæa, Linn.

verticillaris, $D C$. (Fig. I4I.)

purpurata, Hook.f. (Fig. I42.)

recurva, Hook. (Fig. I43.) 


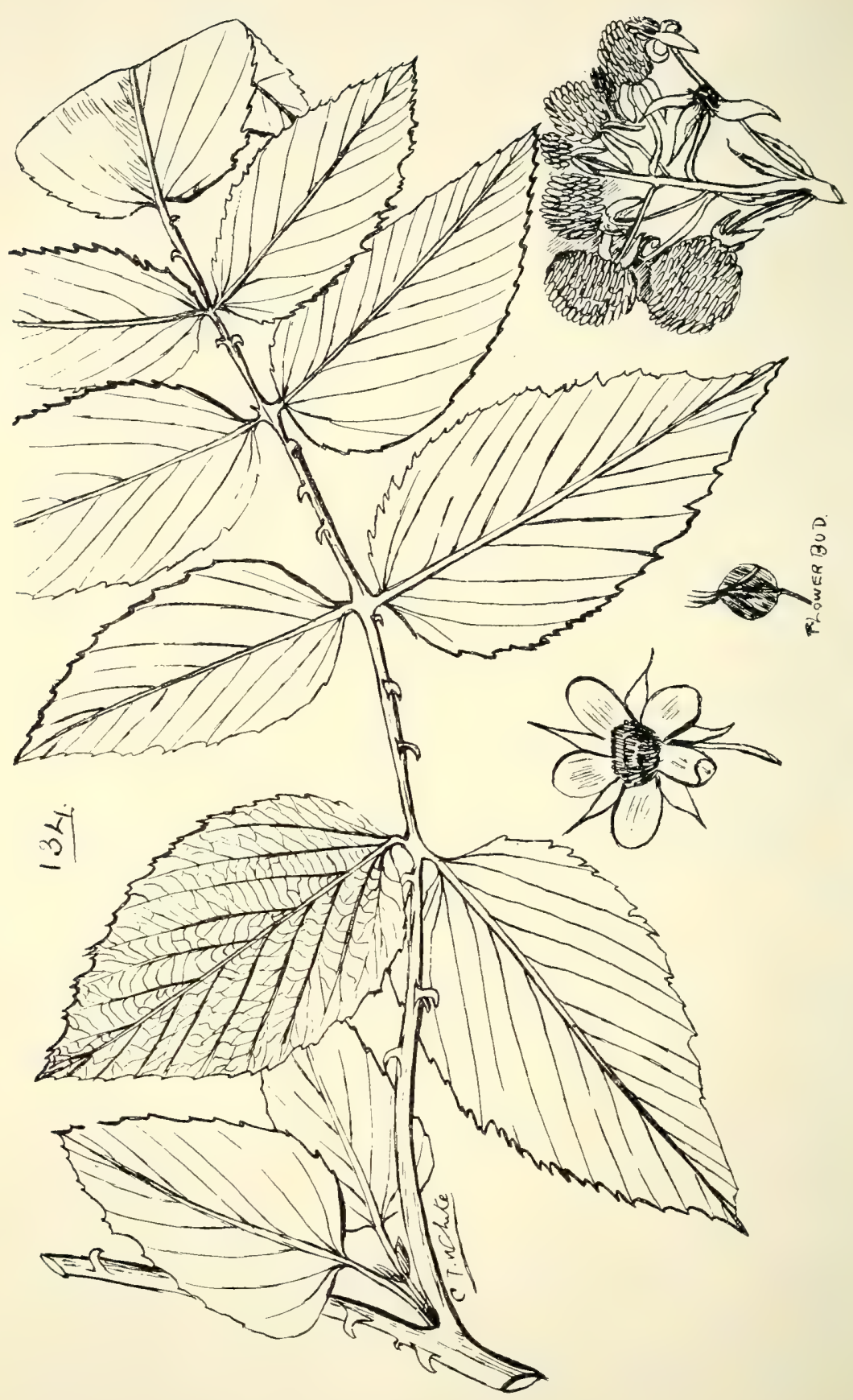

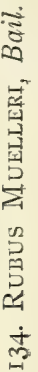




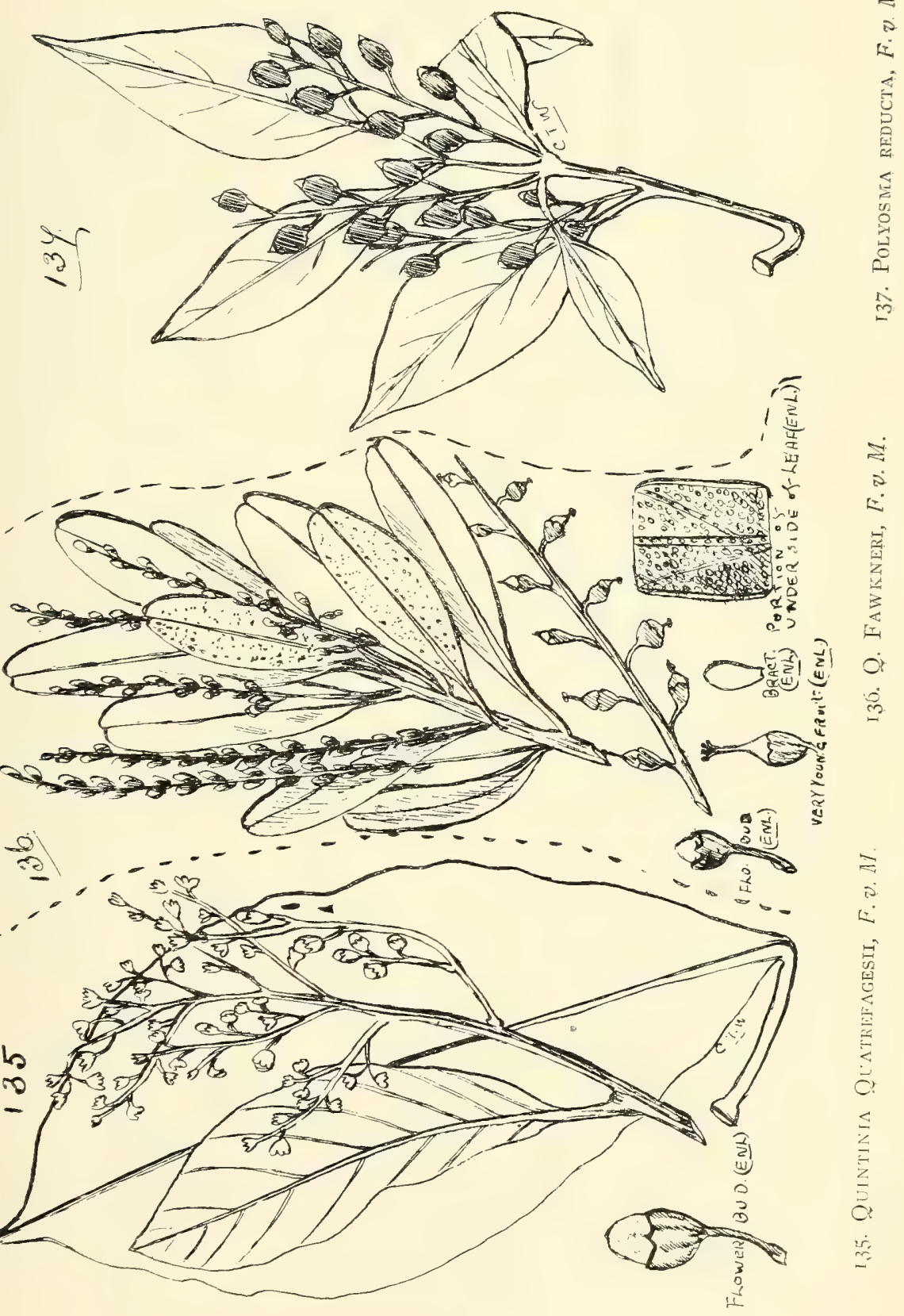




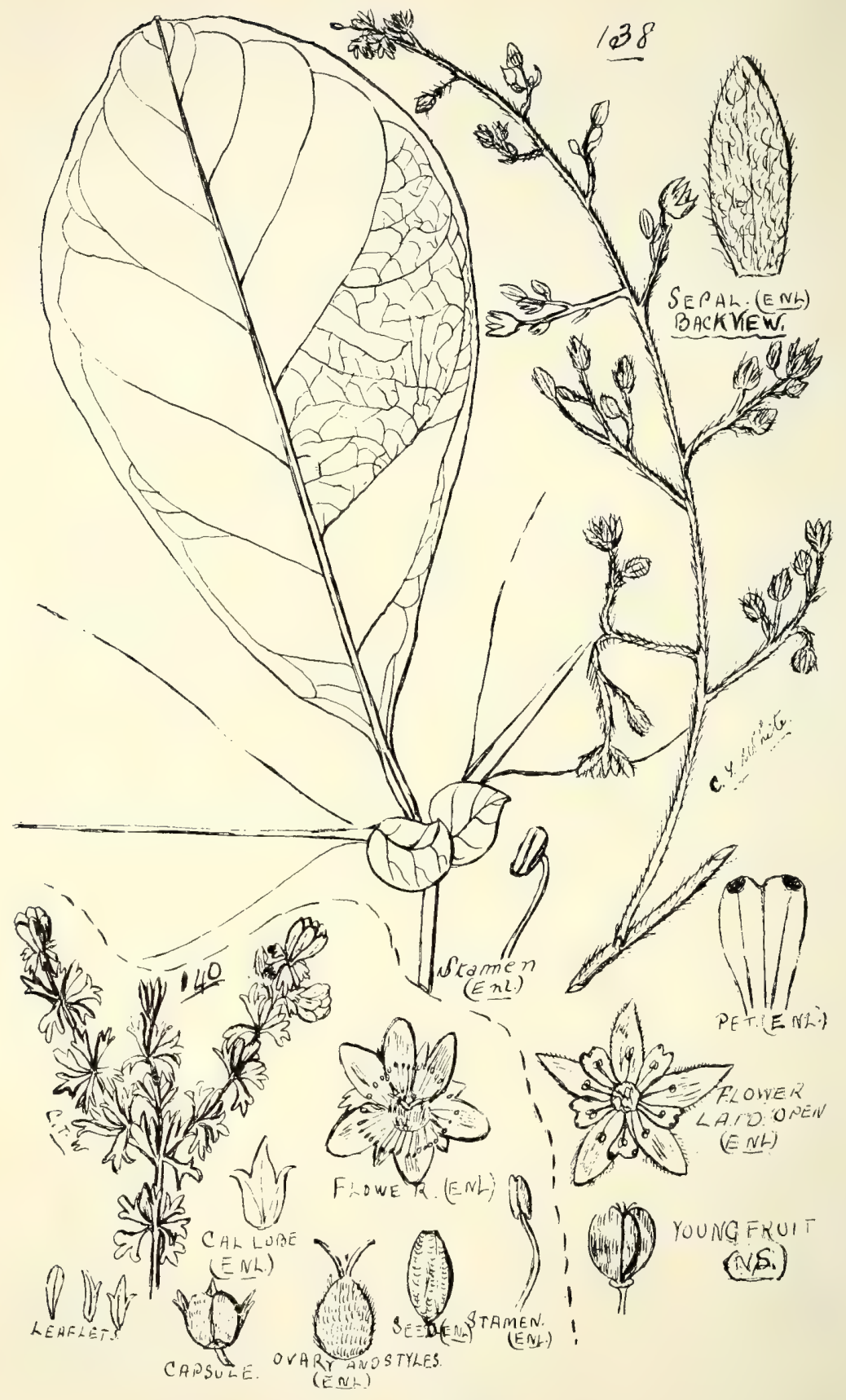

138. Gilleeea adenopetala, $F$. $v . M$.

I40. Bauera capitata, Ser. 


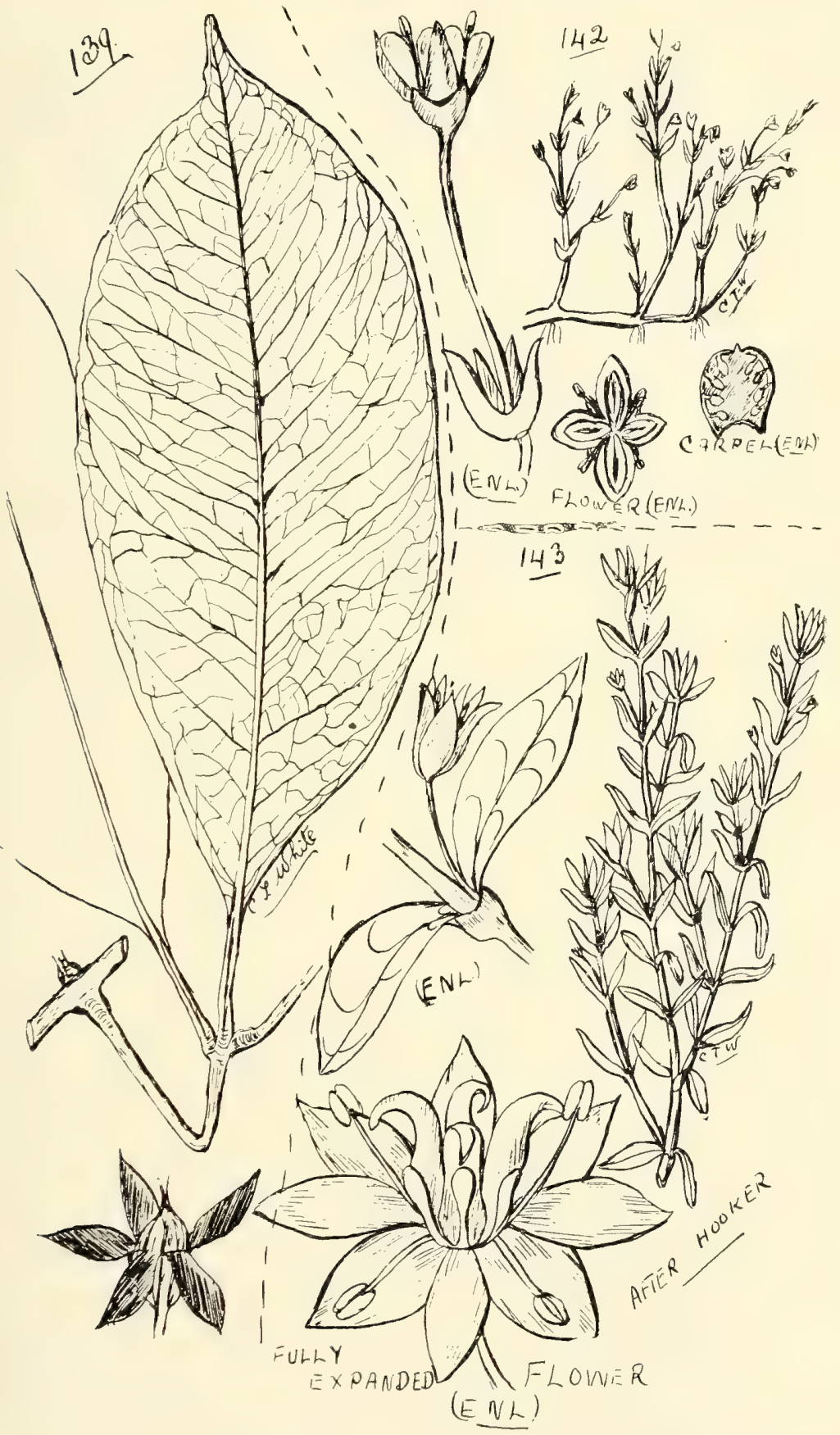

I39. Ceratopetalum Virchowit, $F, v, M$.

I42. Tillea purpurata, Hook. $f$.

r43. T. RECURVA, Hook. 
*Bryophyllum, Salisb.

calycinum, Salisb.-An African plant. The leaves, slightly toasted, are used in India as an application to contused wounds and swellings, and is said to prevent discolouration of bruises.

\section{Order XLVII.-DROSERACEA.}

Aldrovanda, Limn.

vesiculosa, Linn.

Drosera, Linn.--Sundews.

Section I.-Rorella.

Adelæ, F. v. $M$.

indica, Limn.

pygmæa, $D C$.

Lovellæ, Bail. (Fig. I44.)

Burmanni, Vahl.

spathulata, Labill.

petiolaris, $R$. Br.

binata, Labill.-Contains prussic acid.

Section II.-Ergaleium.

auriculata, Backh.

peltata, Sm.-Supposed harmful to cattle.

var. gracilis, Benth.

var. foliosa, Benth.

Banksii, R. Br.

Byblis, Salisb.

liniflora, Salisb. (Fig. I45.)

\section{Order XLVIII. - HALORAGEÆ.}

Haloragis, Forst.

elata, A. Cunn.

Gossei, F. v. M.

ceratophylla, Endl.

alata, Jacq.

micrantha, R.Br. (Fig. I46.)

heterophylla, Brongn.

tetragyna, Hook.

var. glabrescens, Bail.

var. micrantha, Benth.

var. hispida, Benth.

stricta, R. Br. (Fig. I47.)

acanthocarpa, Brongn. 
Haloragis-contd.

tencrioides, A. Gray.

depressa, $W$ alp.

Bæuerlenii, F.v. M.

Myriophyllum, Linn.-Water Milfoils.

variæfolium, Hook.

verrucosum, Labill.

latifolium, $F . v$. $M$.

gracile, Benth. (Fig. I48.)

Callitriche, Linn.

verna. Limn.--Water Starwort.

Alliance XII.--MYRTALES.

\section{Order XLIX.--RHIZOPHOREA}

The bark of many useful in tanning. Honey of the native bees, when obtained near mangrove swamps, is considered poisonous by some of the natives.

TRIBE I.-RHizophorez.

Rhizophora, Lim.-Mangrove.

mucronata, Lam.-Black Mangrove. "Binaroley" of the natives of Forest Hill. Bark contains 28.85 per cent. of tannin, air-clried (Staiger) ; 27.05 per cent. (Brïnnich). (Fig. I49.)

Ceriops, Arn.

Candolleana, Arn.-Grey Mangrove. "Larchanama" of Batavia River natives. In samples of the bark from different localities, Mr. Brïnnich found the yield of tannin to be $26.20,2 \mathrm{I} .04$, and 25.95 per cent. (Fig. I 5O.)

Bruguiera, Lam.

Rheedii, Blume.--Red or Orange Mangrove. " Kowinka" of Stradbroke Island, "Biree" of Bundaberg, "Bambir" of Cooktown. and "Tcherda" of Batavia River natives. The bark has been found to contain from I8 to I9 per cent. of tannin (Staiger); I3.3I per cent. (Brünnich). grmnorhiza, Lam.-The bark is said to contain I3 to I7 per cent. of tannin.

caryophylloides, Blume.

parviflora, $W$. et Arn.-Red Mangrove. Mr. Brünnich found the bark to yield Io.i6 per cent. of tannin. (Fig. I5I.)

Tribe II.-LEGNotide A.

Carallia, Roxb.

integerrima, DC._-"Mel-joor-ang" and "Bokor" of Cooktown and "Bil-be-a" of Musgrave natives. Samples of the bark examined in India afforded only I.5 per cent. of tannin. 


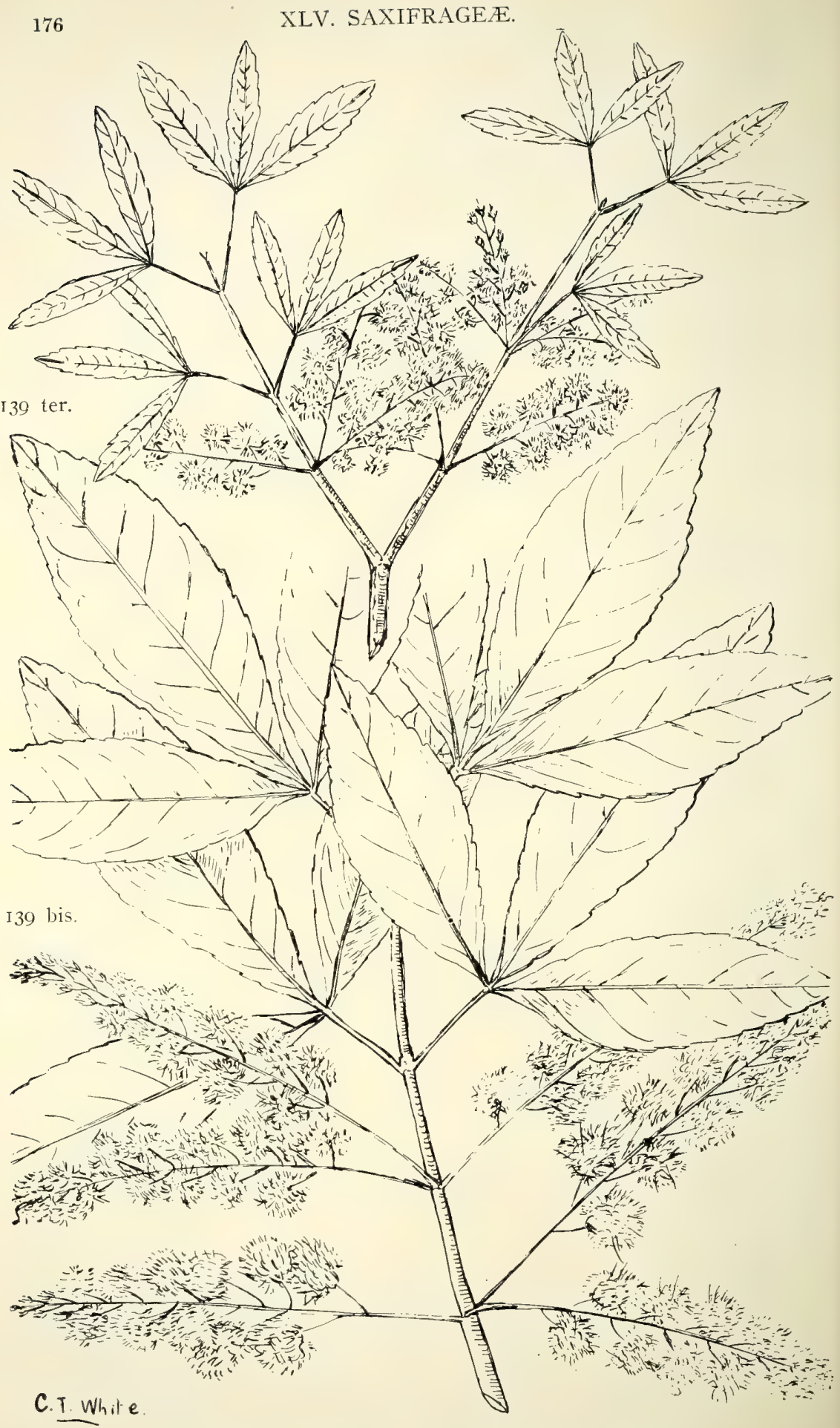

I39 bis. Wein mannia lach nocarpa, $F \cdot v \cdot M$. I39 ter. W. LAChNOCARPa, var. PARvifolia, Bail. 


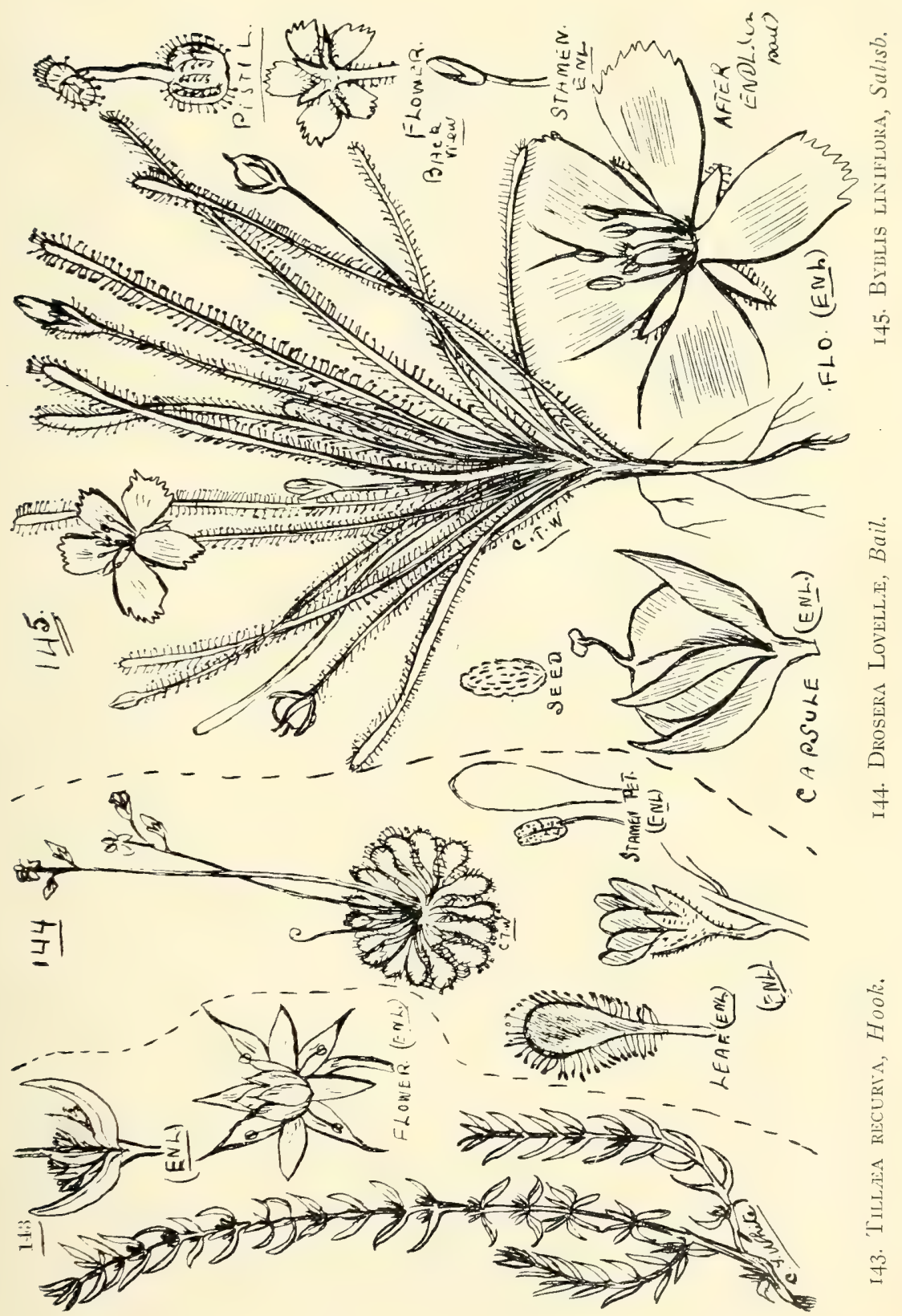




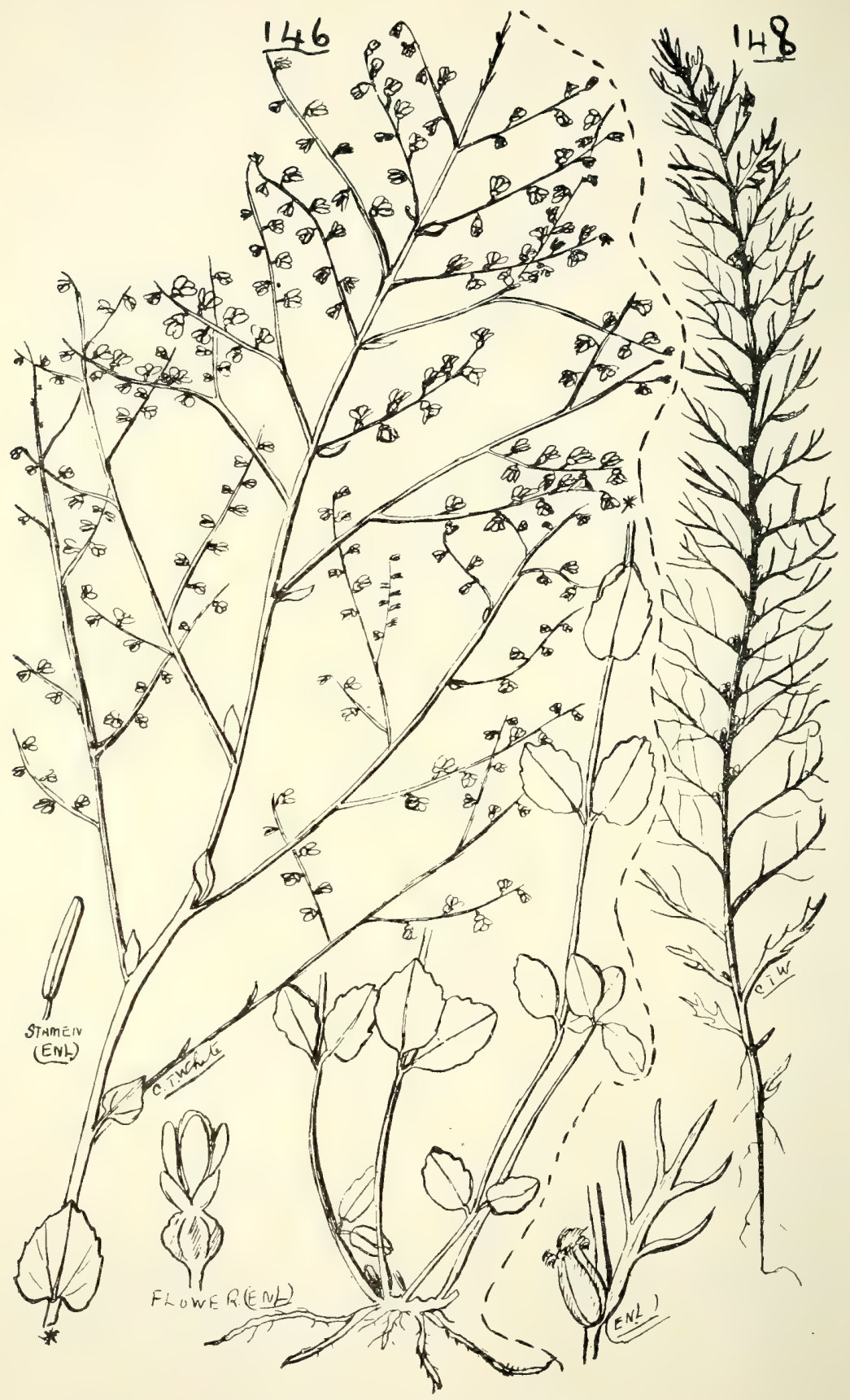

146. Haloragis micrantha, $B . B r$.

I48. Myriophyllum gracile, Benth. 
XLVIII. HALORAGE无-XLIX. RHIZOPHORE无.
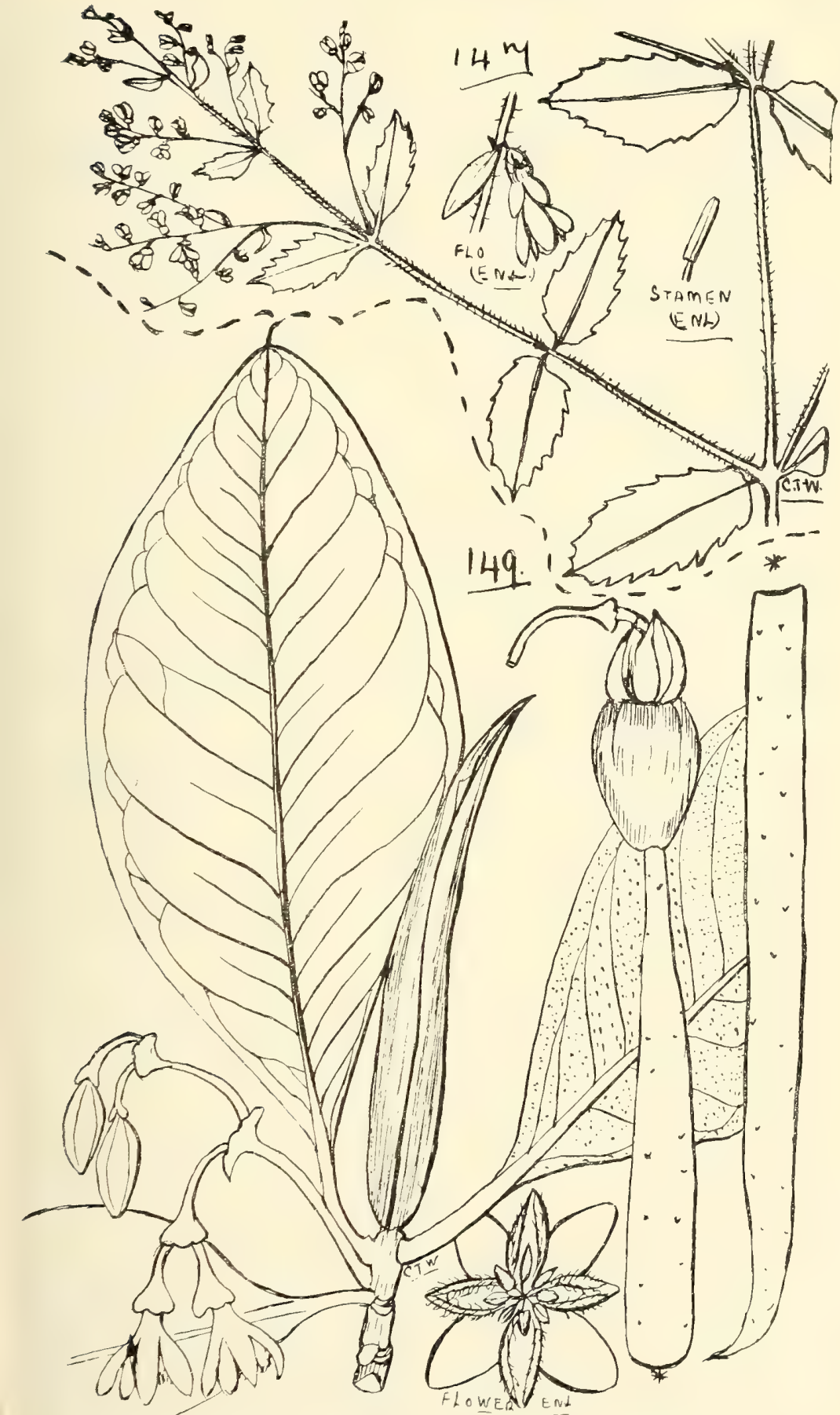

I47. HALORAGIS STRICTA, R. Br.

I49. Rhizophora MUCRONATA, Lam. 


\section{Order L. - COMBRETACE正.}

Terminalia, Linn.

\section{Suborder I.-COMBRETEXE.}

catappa, Linn.--Indian or Country Almond. "Tom-min" of

Cooktown natives. The bark is said to contain about

9 per cent. of tannin.

platyptera, F.v.M. (Fig. I52.)

var. glabrata, Benth.

volucris, Herb. R. Br.

z'ar. ? coriacea, Benth.

oblongata, F. v. M.- “Tananolen" of North Queensland natives. (Fig. I53.)

bursarina, F.v.M.-A useful cabinet-wood. (Fig. I54.)

circumalata, F. v. M.

pterocarpa, F.v.M. (Fig. r55.)

Thozetii, F. v. M.

melanocarpa, F.v.M.- “Moo-jee" of Dunk Island natives.

seriocarpa, F.v.M.-." Ngo-go-ro" of Cape Bedford, "Ngoku-ru" of Cooktown, and " Jinjalga" of Bloomfield River natives. Wood suitable for chair-making.

Muelleri, Benth._."Eandi" of Batavia River natives. Wood suitable for axe-handles.

var. minor, Benth.

porphyocarpa, F.v. M.-Wood suitable for building and cabinet-work.

var. ? eriantha, Benth.

platyphylla, F.v.M.- "Durin" of Flinders River natives. microcarpa, Dcne.- " Draiputo" of Pennefather River natives. grandiflora, Benth.

Lumnitzera, Willd.

coccinea, W. et Arn.

racemosa, Willd.-Wood of a pinkish grey, close-grained.

Macropteranthes, $F, v . M$.

montana, F.v.M. (Fig. I56.)

Leichhardtii, F.v. M.

Fitzalani, F.v.M.-A useful cabinet and turnery wood. (Fig. I 57.)

Gyrocarpus, Jacq.

\section{Suborder II.-GYROCARPEE.}

Jacquini, Roxtb. G. americanus, Jacq. Many botanists now reverse the position of these specific names, giving Jacquini as the synonym. In India the wood is used to make boxes and toys, and is preferred to all others for catamarans; the seeds made into rosaries and necklaces. (Fig. $\left.{ }^{5} 5.\right)$ 


\section{Order LI.-MYRTACEXE.}

Many of this Order are useful bee-plants. Dr. Joseph Latuterer remarks that the tan-resin gums of MrrtaceÆ are entirely endemic in Australia, no plants of other countries yielding an exudation similar to them in chemical composition.

\section{Tribe I.-Cham ÆLaucieæ.}

Subtribe I.-Euchamcelanciex.

Darwinia, Rudge.

fascicularis, Rudge.

Thomasii, Benth.

Homoranthus, A. Cumn. virgatus, $A$. Cumn.

Verticordia, $D C$.

Cunninghamii, Schau.

Subtribe II-Calythricece.

Calythrix, Labill.

microphy1la, A. Cunn.

longiflora, F. ข. $M$.

leptophylla, Benth. (Fig. I59.)

tetragona, Labili.

laricina, $R . B r$.

Homalocalyx, $F$. v. $M$.

ericæus, $F, v . M$.

polyandrus, $F . v \cdot M$.

Thryptomene, Endl.

oligandra, F.v.M. (Fig. I6o.)

var. parviflora, F. v. M.

Micromyrtus, Benth.

microphylla, Benth.

leptocalyx, Benth. (Fig. I6I.)

Tribe II.-Leptosperme. .

Bæckea, Linn.

Subtribe I.-Backace.

Section Schidiomyrtus.

crenulata, $D C$.

var. tenella, Benth.

diosmifolia, Rudge.

linifolia, Rudge.

stenophylla, $F \cdot v \cdot M$. - Dry foliage yielded $4 \frac{\mathrm{T}}{2}$ oz. oil per $\mathrm{c} W^{t}$.

(Fig. I62.) 


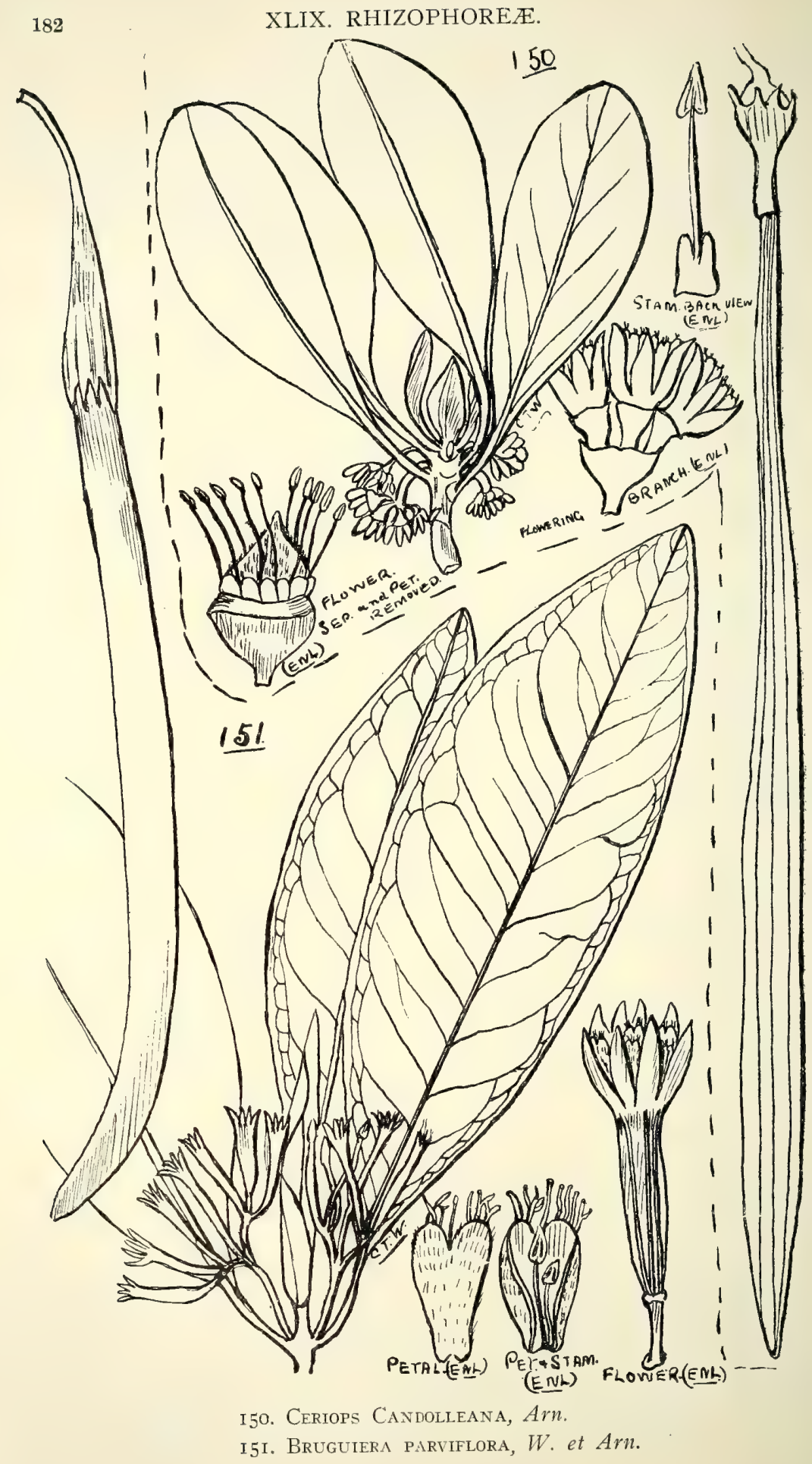


L. COMBRETACEÆ.
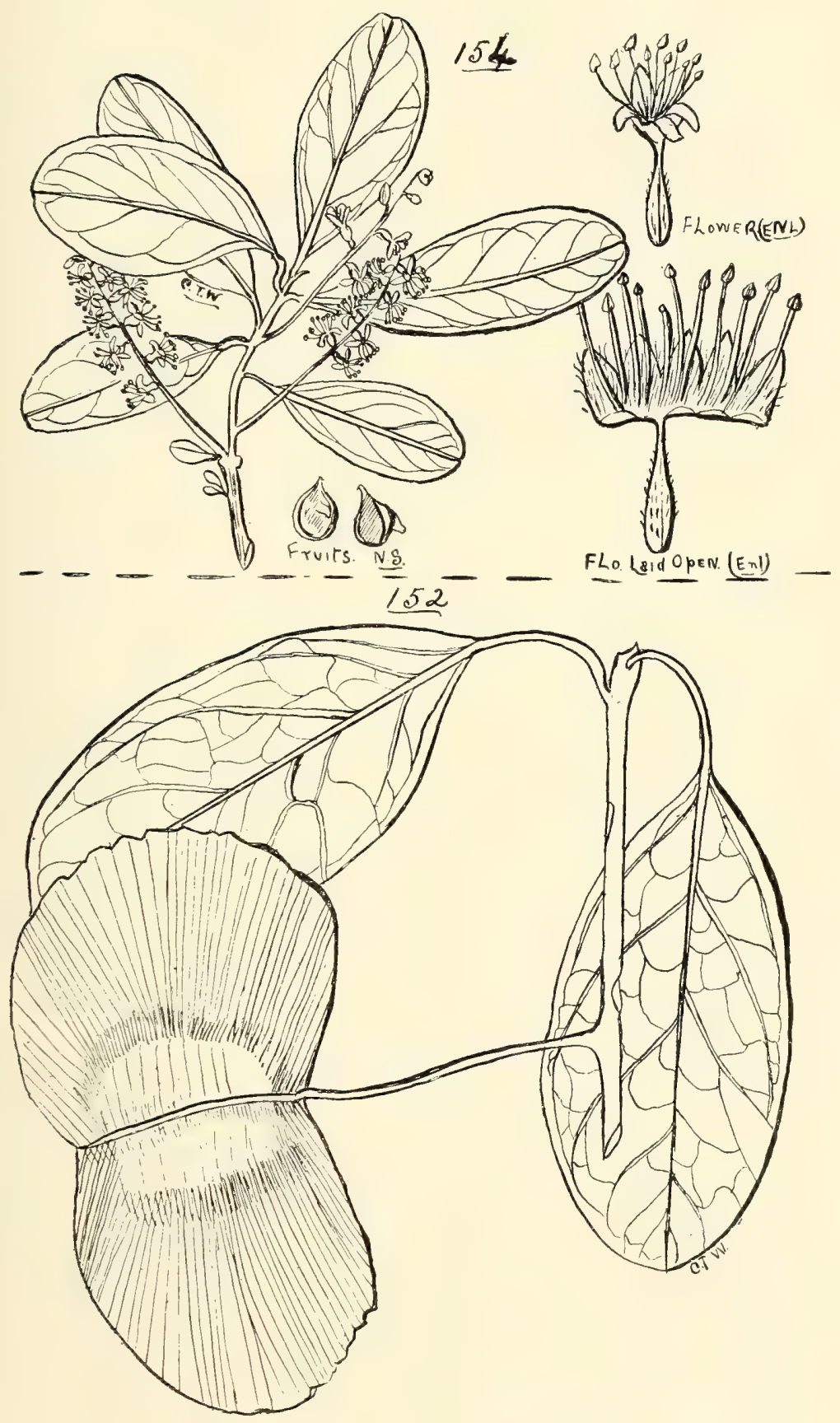

I52. Terminalia platyptera, $F, v \cdot M$.

I54. T. BURSARINA. F. v. M. 


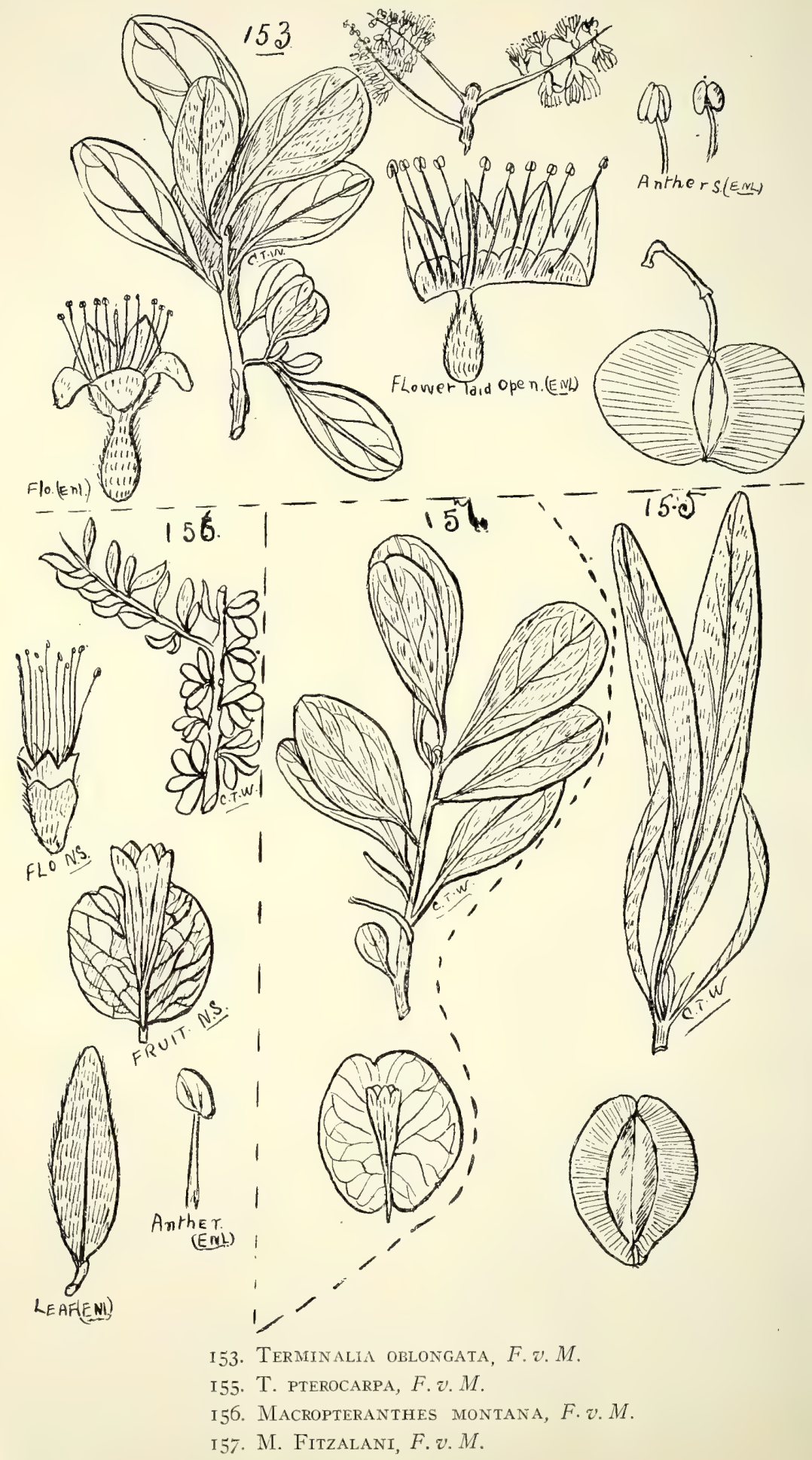




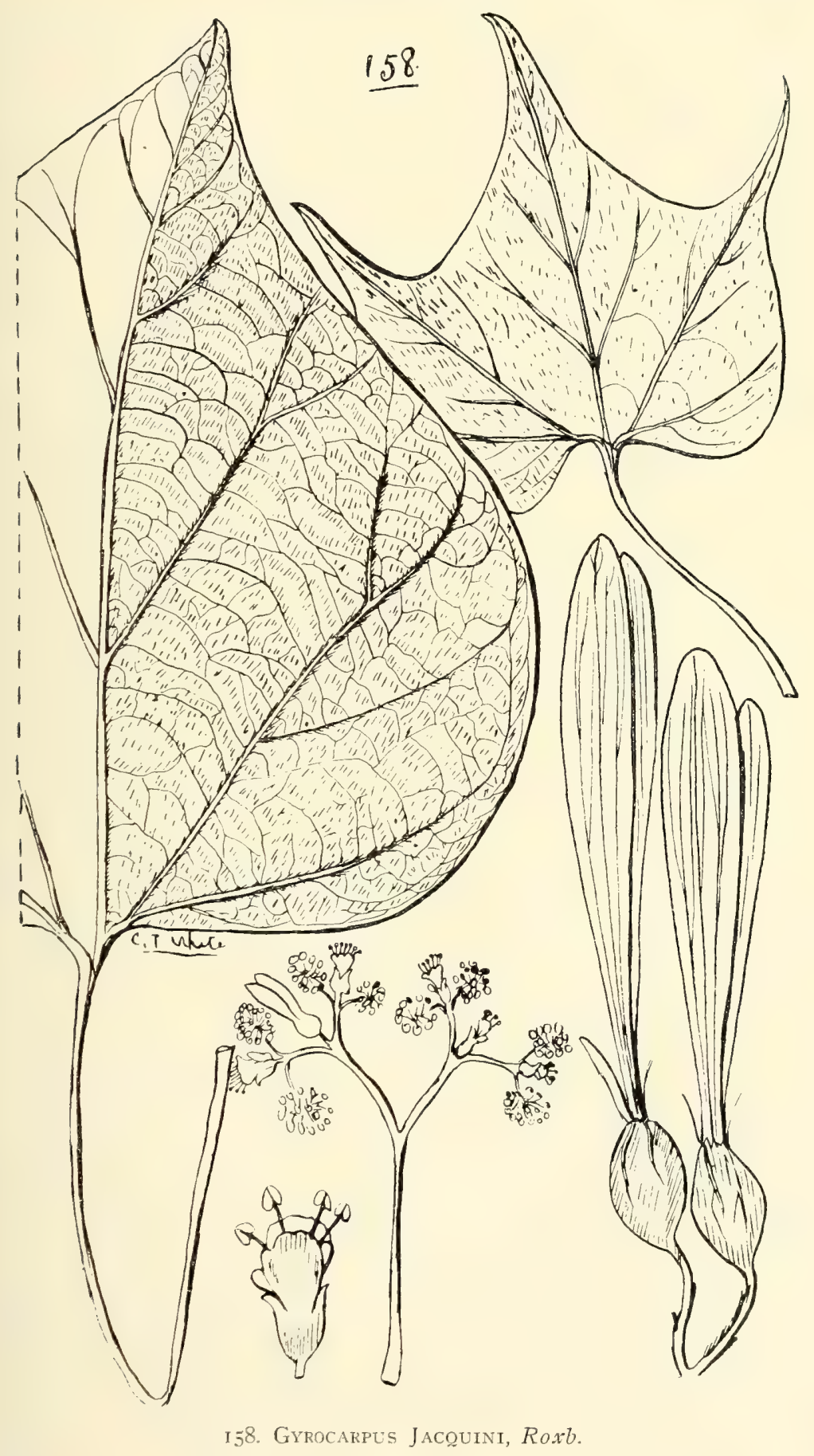


Bæckea-contd .

virgata, Andr.

\section{Section Harmogia.}

var. parvula, Benth.

densifolia, $S m$.

Subtribe II.-Euleptospermea.

Agonis, $D C$.

Section Ataxandria.

Scortechiniana, F.v. M. (Fig. I63.)

lysicephala, F.v. M. et Bail.-Kennedy's Heath.

Leptospermum, Forst.-The fruit is a Diplotegia. The common name for the genus is "Tea-tree." It may here be pointed out that the word should be spelt "tea," not "ti" as so commonly used. The latter word is the Maori name for Cordyline, a Liliaceous genus. Dr. William Anderson, who accompanied Captain Cook in his third voyage as surgeon of the "Resolution," used the leaves of Leptospermum scoparium as a substitute for Chinese tea, and drank the infusion, finding it of pleasant taste and smell. Dr. Lauterer is of opinion that the young shoots and leaves of all the species of this genus are of the same service to the urinary organs, and against gravel, as those of the Uva-ursi (Arctostaphylos Uvaursi.

Fabricia, Benth.

Section I.-Fabricia.

Section II.-Euleptospermum.

flavescens, Sm.- "Tantoon" of Bundaberg natives.

var. commune, Benth.

var. obovatum, F. v. M.

var. citriodorum, Bail.-This might be worthy of cultivation for its citron-scented oil. (Fig. I64.)

var. microphyllum, Benth.

var. grandiflorum, Benth.

var. minutifolium, Benth.

scoparium, Forst.-Captain Cook's Tea-tree.

arachnoideum, $S m$.

lanigerum, $S m$.

stellatum, Cav.

var. grandiflorum, Benth.

attenuatum, Sm._- Booah” of Bundaberg natives.

myrtifolium, Sieb.

abnorme, F. v. $M$. 
Leptospermum-contd.

wooroonooran, Bail. (Fig. I65.)

Luehmanni, Bail.-This tree sheds its bark in long, thin, brown strips, often somewhat curled.

Petersoni, Bail.

Kunzea, Reichb.

peduncularis, $F . v . M$.

calida, F. v. $M$.

Callistemon, R. Br.-Flowers of some yellowish, of most darkish crimson.

speciosus, DC.-Swamp Bottle-brush.

lanceolatus, DC.-Red Bottle-brush. "Narum” of Nerang natives. "Warringin" of Cardwell natives (R.B.H.). Wood useful in wheelwrights' work.

Polandii, Bail.

rigidus, $R$. $B r$.

coccineus, F. v. $M$.

salignus, $D C$.-Wood strong and durable, bark papery.

var. angustifolia, Benth.

pityoides, Miq. (Fig. I66.)

Melaleuca, Linn.-The common name for the genus is Tea-tree.

\section{Series Callistemoneæ.}

hypericifolia, Sm.-The Queensland habitat given is on the authority of the late Mr. W. Hill, who recorded that he obtained a plant near Ipswich in I860.

Series Decussatæ.

thymifolia, $S m$.

Series Laterales.

acacioides, F. v. M.

Series Circumscissæ.

symphyocarpa, F. v. M.

angustifolia, Gartn.

\section{Series Spicifloræ.}

linariifolia, Sm._" Poorga" of Bundaberg and "Woollerwooller" of St. George natives. Yield of oil from dry foliage, $28 \mathrm{I} / 2$ oz. per cwt. Wood very durable, bark papery.

var. trichostachya, Benth.- "Tee-doo" of Dunk Island natives. 
Melaleuca-contd.

leucadendron, Limn.-Broad-leaved Tea-tree. The leaves of all the kinds yield an oil of commercial value, and which was considered by Dr. Joseph Lauterer as a valuable antiseptic. Wood useful for underground work and in water. "Oodgeroo" of Stradbroke Island, "Mor-ngi" of Palmer River, "Kyenbooree" of Mackay, "Bichuma" of Forest Hill, and "Atchoourgo" of Mitchell River natives. (Plate VIII.)

var. lancifolia, Bail.

var. saligna, Bail.-Weeping Tea-tree. A Northern tree; wood very durable, useful for wharves and underground work.

var. Cunninghamii, Bail. (Fig. I67.)

lasiandra, F. v. M.

genistifolia, Sm.- "Moonah" of Bundaberg natives. Bark hard and blackish.

Preissiana, Schau.

iar. leiostachya, Benth. $=$ M. parriflora, Lindl.

armillaris, $S m$.

styphelioides, $S m$.

nodosa, $\mathrm{Sm}$.

Series Capitatæ.

Subseries Pallidifloræ.

ericifolia, $\mathrm{Sm}$.

var. erubescens, Benth.

uncinata, $R . B r$.

Subseries Longifoliæ.

hakeoides, F.v.M.

minutifolia, F.v.M.

Series Peltatæ.

foliolosa, A. Cunn. (Fig. I68.)

tamariscina, Hook. (Fig. I69.)

Subtribe III.-Eucalyptece.

Angophora, Cav.

subvelutina, Cav--Apple-tree. Wood used by wheelwrights. intermedia, DC.-Apple-tree. "Bu-groo" of Brisbane River natives.

var. Woodsiana, Bail.--The wood often contains hollows full of liquid red gum.

lanceolata, Cav.-Rusty Gum, Sugar Gum, and Cabbage Gum, in different localities. Very like in growth a Spotted Gum tree, but wood only suitable for indoor work. 


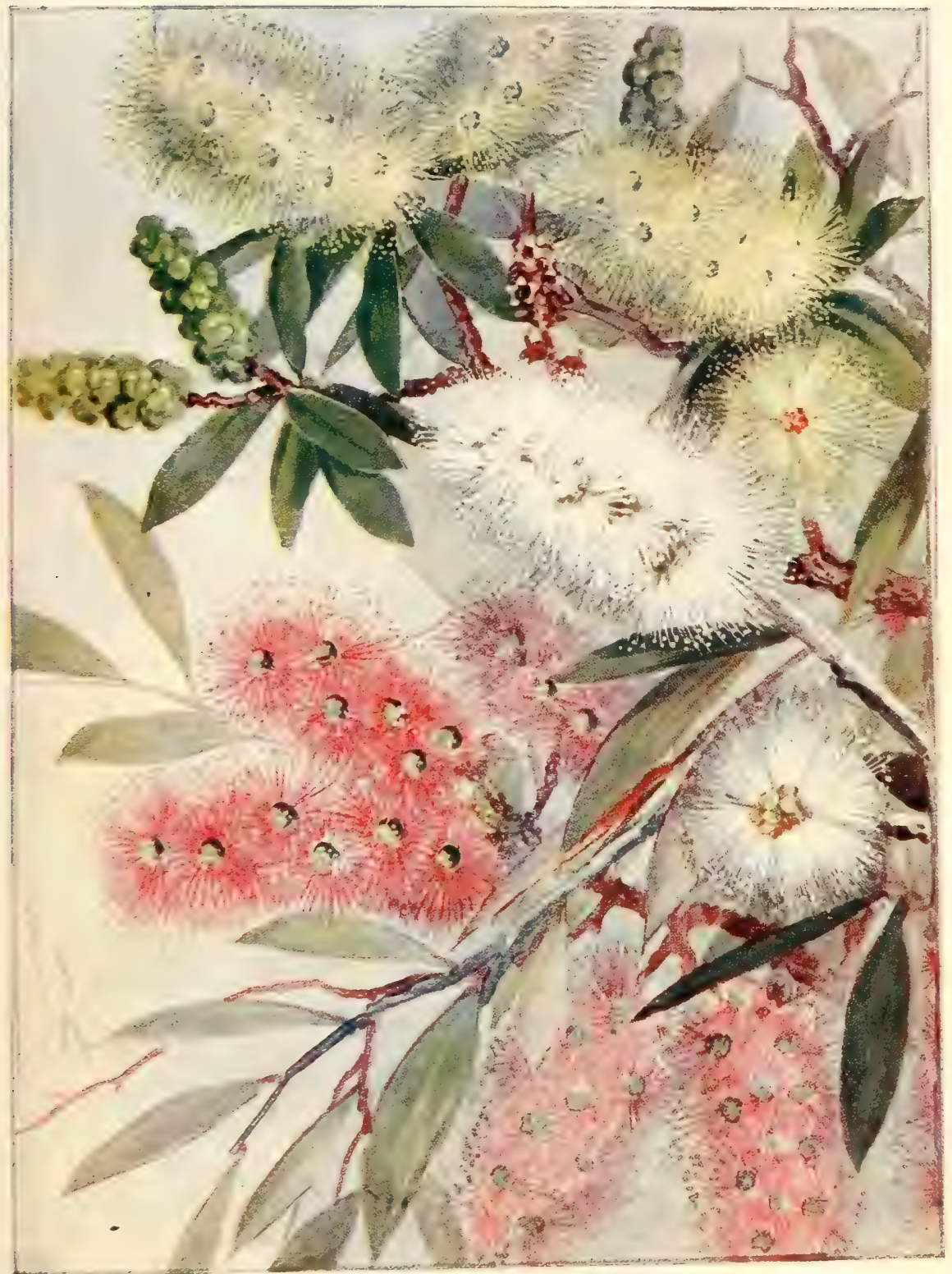

Three Forms of MELALEUCA LeUCADENDRON, Linn. 

L1. MYRTACEA:
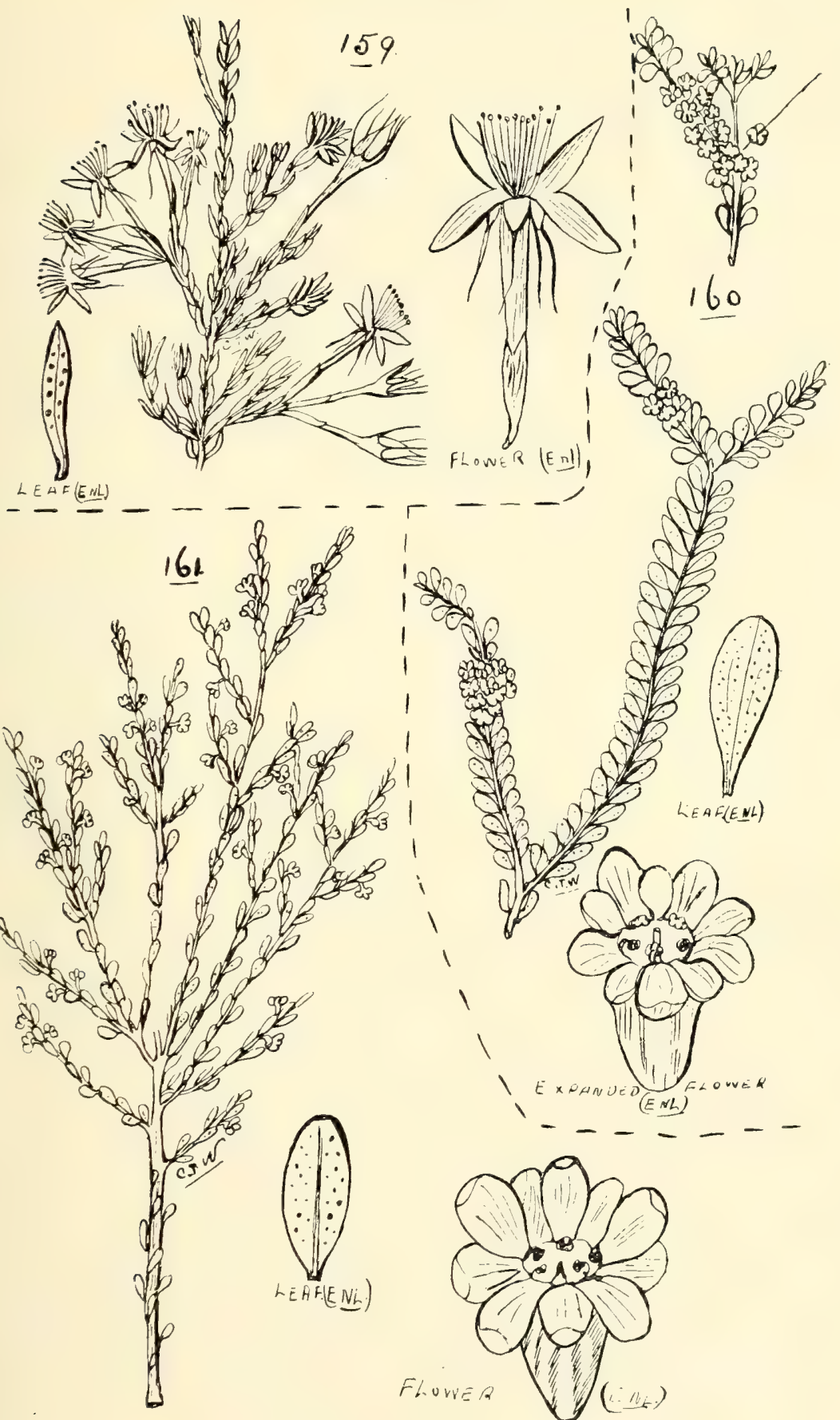


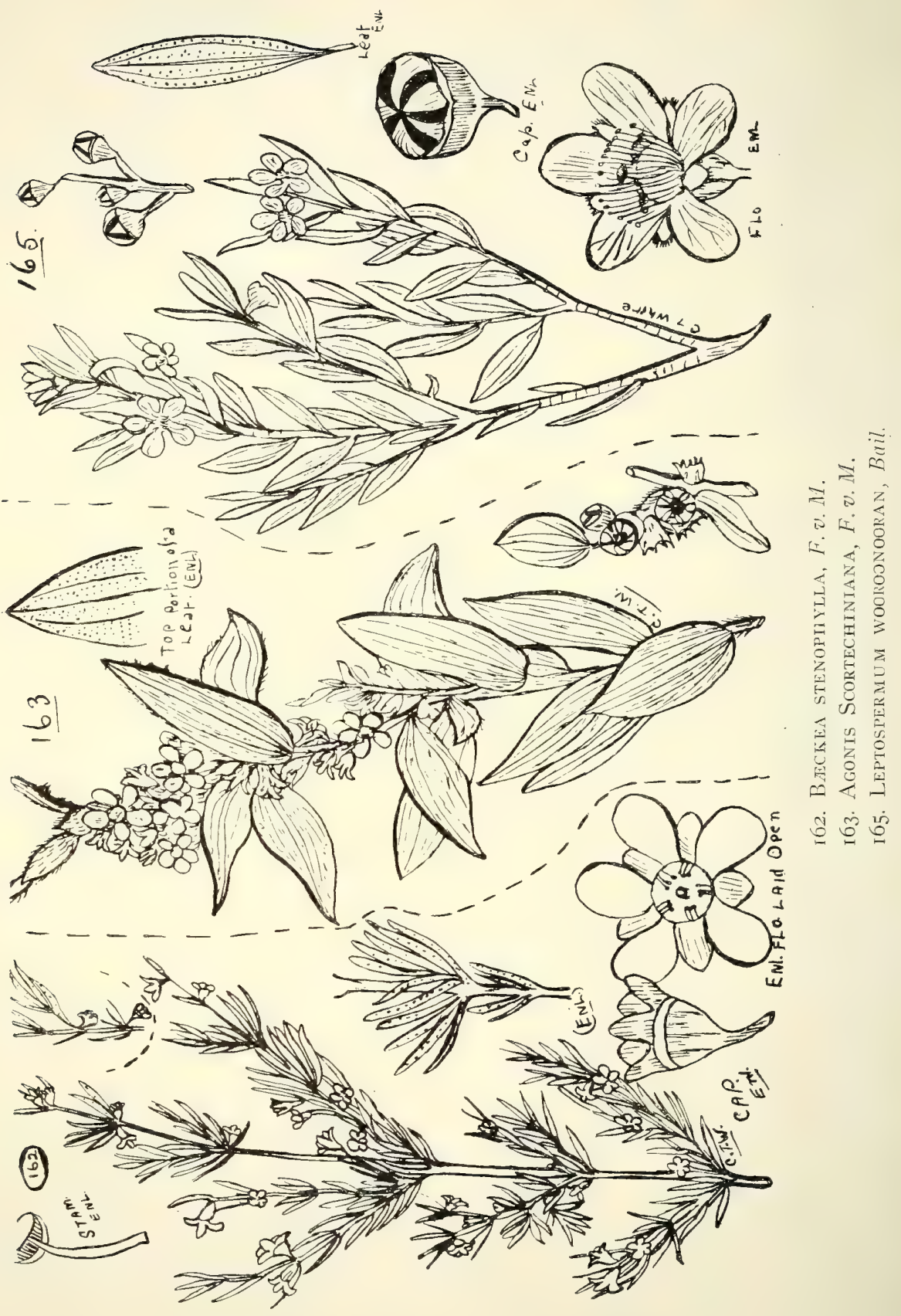



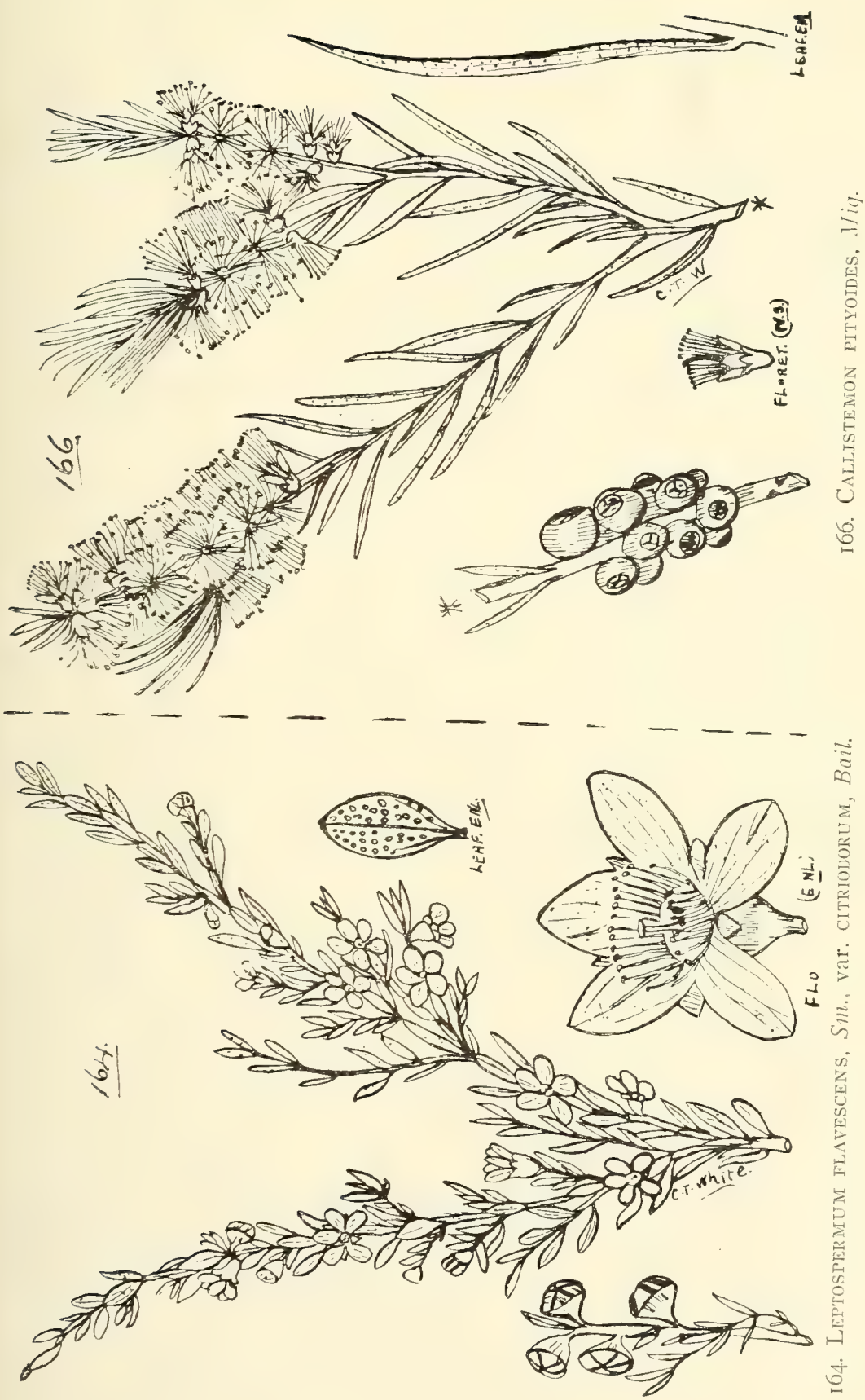
192

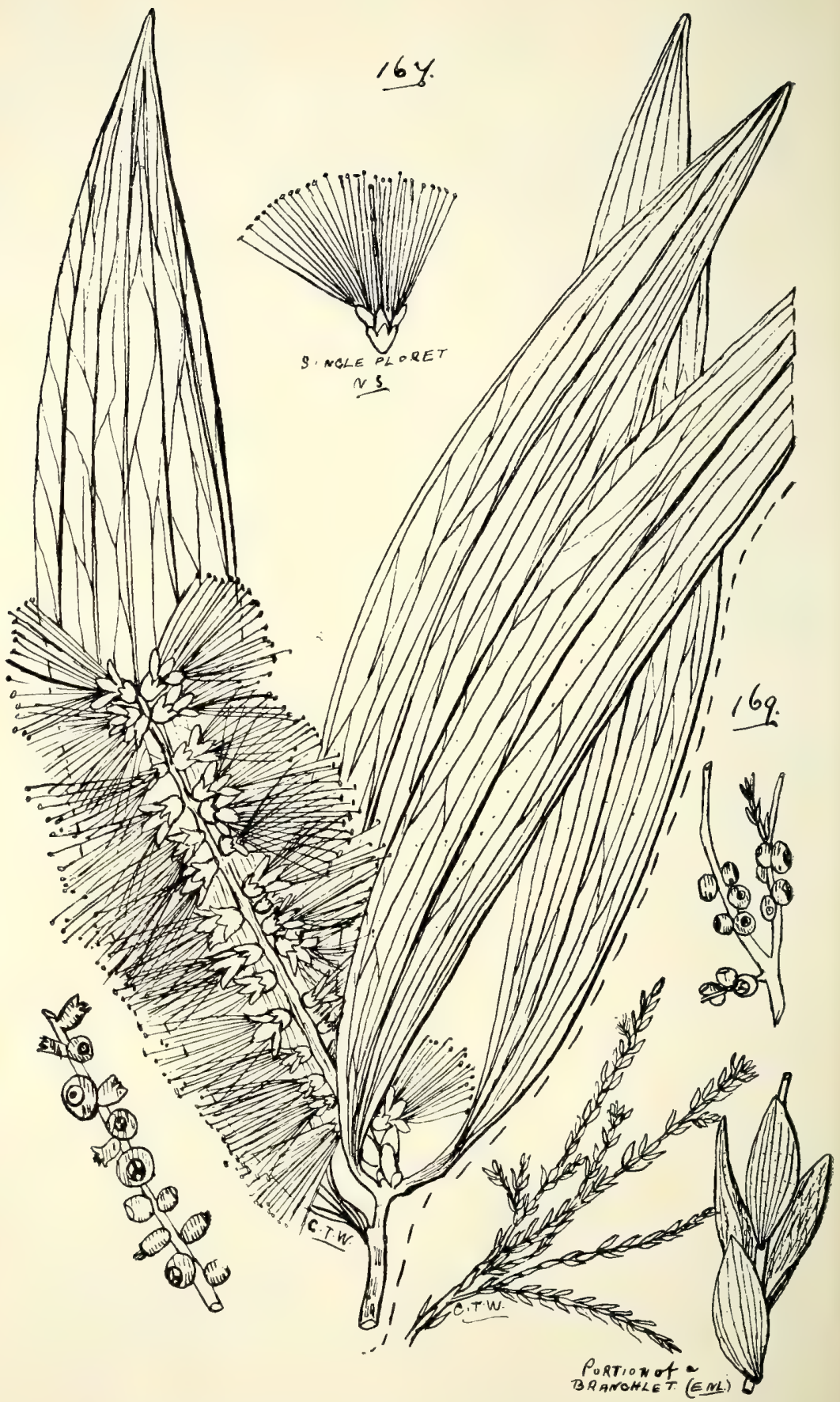

i67. Melaleuca leucadendron, Linn., var. Cunninghami, Bail. i69. Melaleuca tamariscina, Hook. 
Eucalyptus, L'Her.-The gum of any of these trees is useful in cases of diarrhœa and dysentery, but perhaps the gum of E. hamastoma is the best.

\section{Series Renantheræ.}

Planchoniana, F.v.M.-Wood grey, hard, and heavy. Gum contains 68 per cent. of tannin and 6.4 per cent. of metarabin (Lautcrer).

Baileyana, F. ข.M.-Rough Stringybark. A ton of the fresh leaves yields $322 \mathrm{oz}$. of oil. Wood suitable for toolhandles.

capitella, Sm.--See note, Bail. Q1. F1. ii. 6r3.

piperita.-The Queensland tree probably only E.eugenioides, Sieb. Gum contains 66 per cent. tannin, I I per cent. metarabin (Lauterer).

eugenioides, Sieb.-White Stringybark. Gum contains 65 per cent. tannin, 7 per cent. metarabin (Lauterer).

acmeniodies, Schau.-Broad-leaved Stringybark. Yellow Stringybark of some places. "Joora" of the Brisbane natives. Gum contains $6_{5}$ per cent. tannin, 7 per cent. metarabin (Lauterer).

pilularis, Sm. - Blackbutt. "Tcheergun" of Stradbroke Island and "Toi" of Brisbane River natives. A good building timber. Gum contains 65 per cent tannin, 5.7 per cent. metarabin (Lauterer).

\section{Series Heterostemones.}

$\left.\begin{array}{l}\text { leucoxylon, F. v. M. } \\ \text { sideroxylon, A. Cunn. }\end{array}\right\}$ See note Q1. Ag. J1. xv. (1905) 898. melliodora, A. Cunn. gracilis, F.v.M.-Red or White Mallee.

var. Thozetii, F.v.M. paniculata, $S m$.

hæmastoma, Sm.-Scribbly Gum. "Curgura” of Brisbane

River natives. The gum of this tree is valuable in cases of diarrhœa, and has been known to cure calves bad with "scours." One ton of fresh leaves yields $672 \mathrm{oz}$. of oil (Staiger). Gum contains tannic acid 66.3 per cent., metarabin 8 per cent. (Lauterer).

var. micrantha, Benth.

microcorys, F.v.M.-Tallow-wood. "Tee" of the Maroochie natives. One of our most valuable hardwoods. Fresh leaves yield $375 \mathrm{oz}$. of oil to the ton. Gum contains 62.5 per cent. tannin, 6.4 per cent. tan resin, 7.4 per cent. arabin and metarabin (Lauterer). 
Eucalyptus-contd.

\section{Series Porantheræ.}

pruinosa, Schau.- "Kullingal" of Cloncurry natives, by whom the inside bark is stripped, damped, and wound round the chest and body for pains, rheumatism, \&c. polyanthemos, Schau.-Poplar Box. populifolia, Hook. (includes E: bicolor, var. parviflora, F. v. M.).-Poplar Box. "Mullet" of St. George natives. ochrophloia, F. v. M.-Yellow-jacket. "Yapunyah" of Bulloo River. (Fig. I70.)

largiflorens, F. $v \cdot M .=$ ? E. bicolor, A. Cunn., Benth. in F1.

Austr. Seed a staple article of diet of the Boulia and other inland natives.

hemiphloia, F.v.M.-Gum-topped Box. "Woorgun" of

Nanango natives. Wood very tough and elastic; dry leaves yield I2 oz. of oil to the cwt. (J. F. Bailey). zar. ? parviflora, Benth.--Leichhardt's Box; Fitzalan's Ironbark. Mount Elliott. Mueller, Eucalyptographia, Dec. 5, places this under E. drepanophylla.

\section{Series Micrantheræ.}

Bowmani, F.v. M.

Stoneana, Bail.-Bastard Gum-leaved Box. (Fig. I7o bis.) siderophloia, Benth.-Black Ironbark. "Biggar" of Brisbane

River and "Wooroola" of Bundaberg natives. Wood useful where strength and durability are required. Gum contains (air-dry state) tannic acid 28.5 per cent., arabin 42.4 per cent., metarabin 3 per cent. (Lauterer).

zar. rostrata, Benth.-Large-leaved Ironbark. Wood deep red, operculum long, giving a well-marked distinction to the variety.

forma decorticans, Bail., Q1. Agric. J1. xxvi. (I9II) I27.A large tree, the branches from a diameter of 4 in. shedding the bark in thin red strips, leaving them of a yellowish colour (see photos. on pp. Io and I2).

melanophloia, F.v.M.--Silver-leaved Ironbark. "Oombah" of St. George and "Gaygar" of Nanango natives. Wood durable, outer greyish, centre red.

drepanophylla, F.v.M.-Messmate.

leptophleba, $F, v \cdot M$.

crebra, F.v.M.-Narrow-leaved Ironbark; Grey Ironbark. "Tandoor" of Brisbane River natives. Gum contains 30 per cent. tannin, 42 per cent. arabin (Lauterer).

Staigeriana, F.v.M. inedit.-Lemon-scented Ironbark. A ton of the dry leaves yields I,290 oz. of oil (Staiger). 
Eucalyptus-contd.

Howettiana, F.v.M.

Cloeziana, F. v. M.

Raveretiana, F.v. M.-Thozet's Box or Iron Gum tree.

microtheca, F.v.M. (Bentham unites this with E. brachy'poda, Turcz.)—Black Box. "Coolibar," "Jimbul," "Kurleah," and "Moolar" of inland localities. Leaves and branches used as a fish-poison by the natives in the North-West-Central districts, and the inner bark beaten uip and used as a poultice for snake-bite.

Series Normales.

Subseries Robustæ.

miniata, A. Cunn---Tobacco-pipe Gum-tree. The large fruits might be used for making tobacco-pipes. (See Q1. Ag. J1. xix. (I907) 34I, P1. xxxiı.)

robusta, Sm.- "Grorpin" of Stradbroke Island and "Gu1gong" of Dunk Island natives. Gum contains tannin 30 per cent., arabin 4I per cent. (Lauterer).

botryoides, Sm.-Woollybutt; also called Blue Gum in some places. A very large tree. Yield of oil about $6 \mathrm{oz}$. per cwt. of dry leaves (J.F. Bailey).

\section{Subseries Exsertæ.}

pallidifolia, $F . v . M$.

insulana, Bail. (Fig. I7I.)

pachyphylla, $F \cdot v \cdot M$.

dealbata, A. Cunn.

rostrata, Schlecht.-Stanthorpe Yellow-jacket. "Yarra" of Moonie River natives.

exserta, F. v. M.-."Bendo" of Burnett River natives.

tereticornis, Sm.-Blue Gum and Red Gum. "Mungal" of

Brisbane River natives. The dry foliage yields about 28 oz. of oil per cwt. (J. F. Bailey). Gum contains 62 per cent. tannin, 7 per cent. metarabin (Lauterer).

var. brachycorys, Benth.

Subseries Subexsertæ.

platyphylla, F. v. M.-Broad-leaved Poplar Gum. "Wongoola" of Mackay natives.

alba, Reinze.

Stuartiana, F.v.M.-Stanthorpe Box; Messmate. 


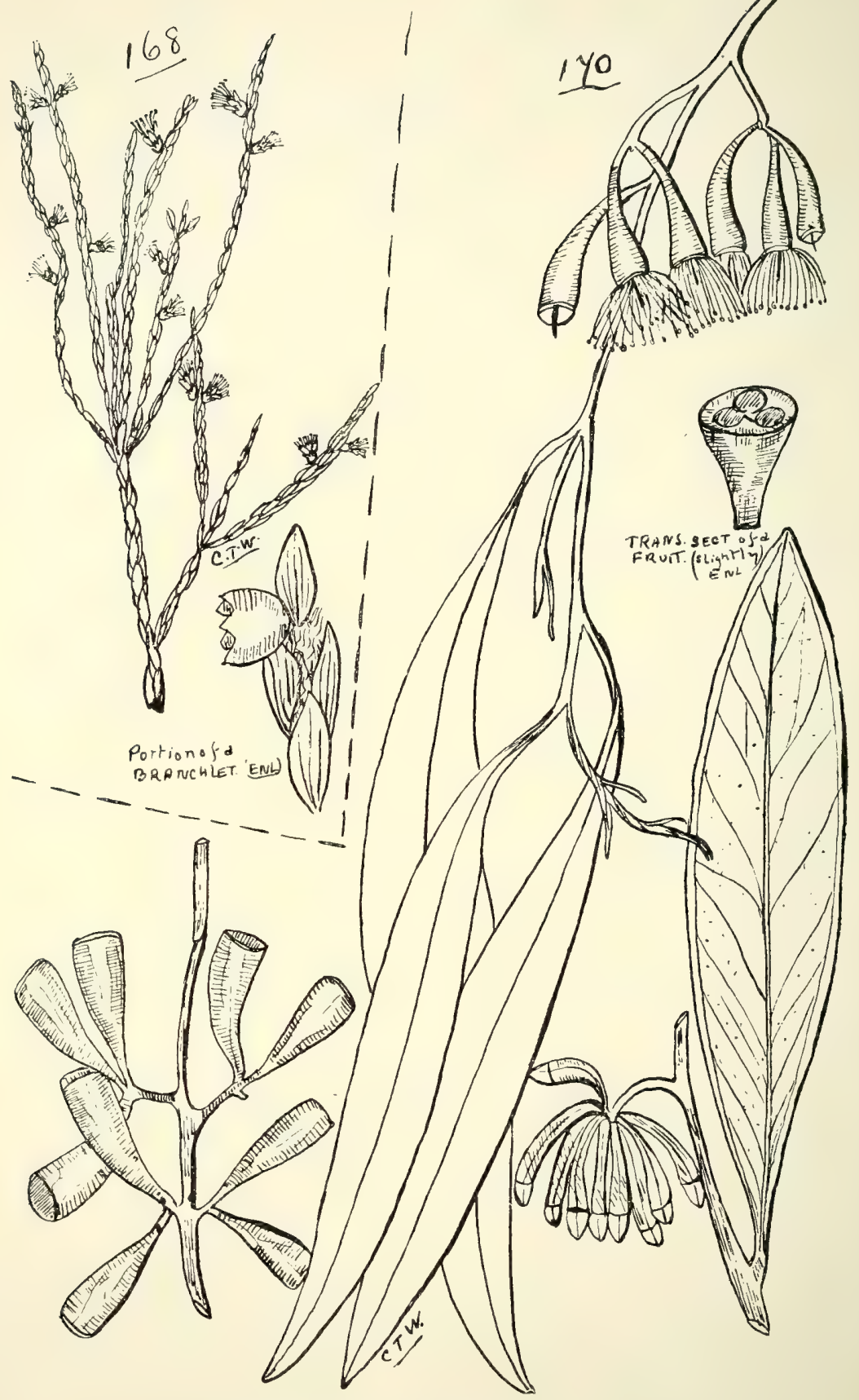

i68. Mrlaleuca foliolosa, A. Cunn.

izo. Eucalyptus ochrophloia, F.v. $M$. 


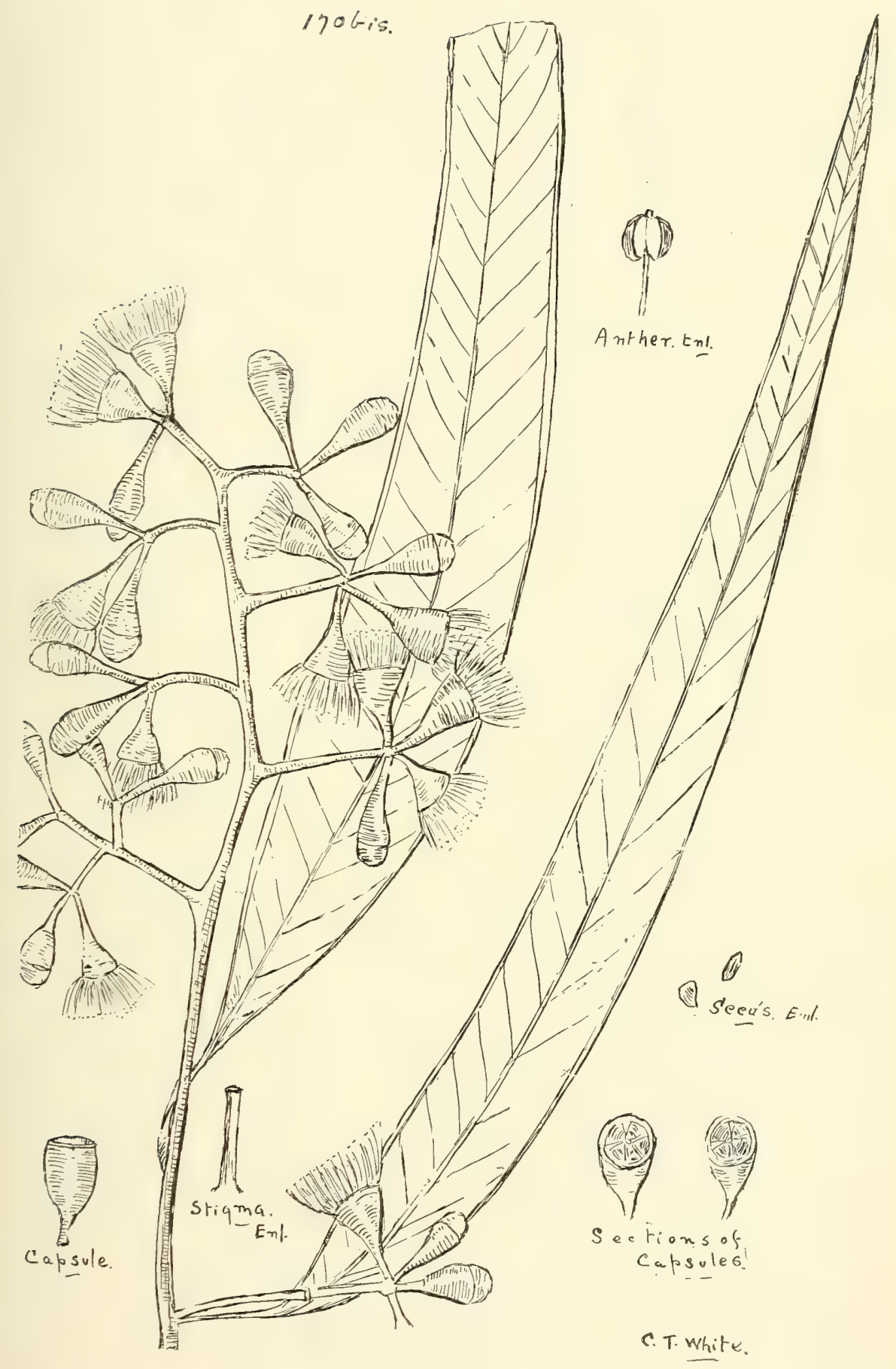

170 bis. Eucai.yptus Stoneana, Bail. 


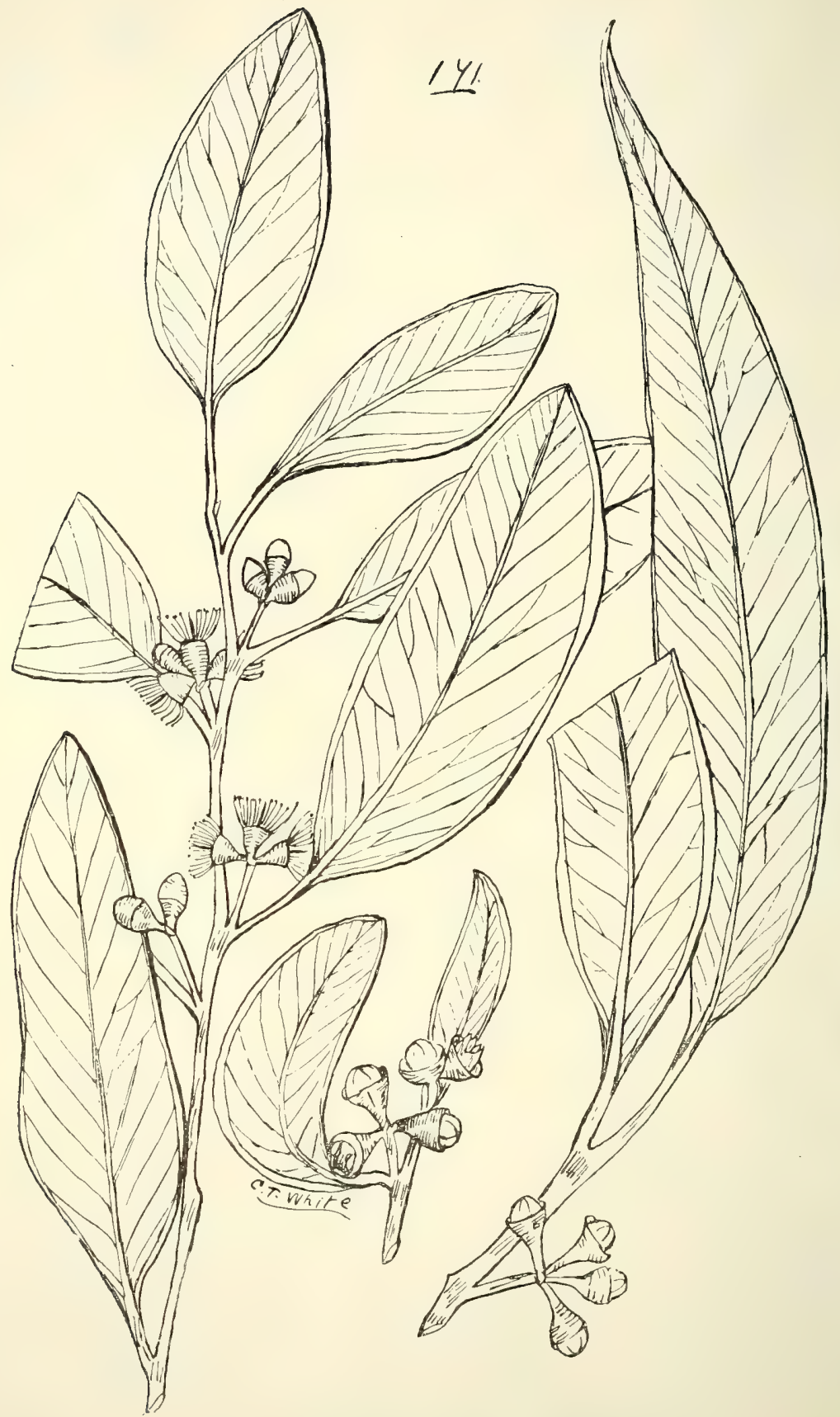

i7. Eucalyptus insulana, Bail. 


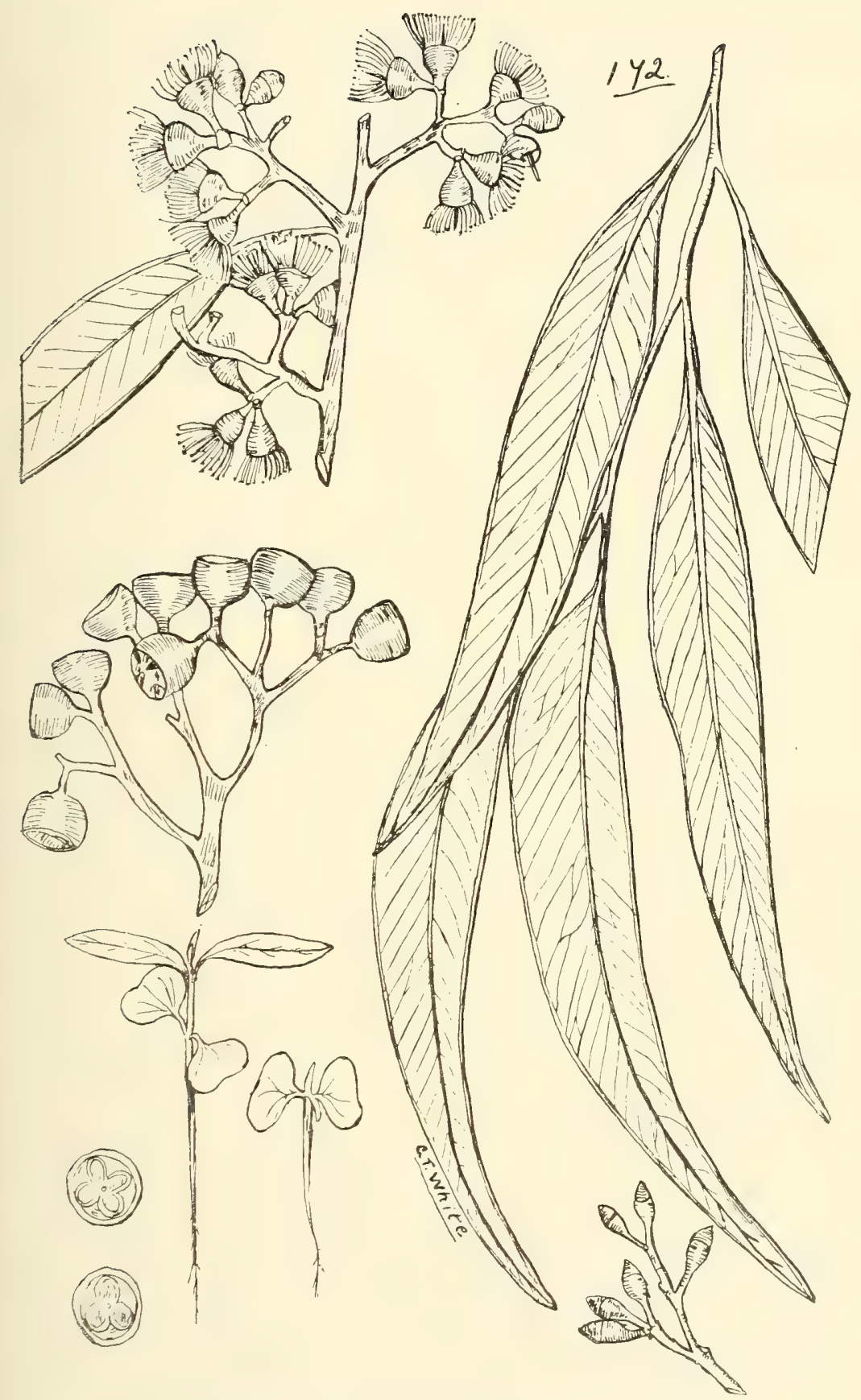

I72. Eucalyptus stannariensis, Bail. 
Eucalyptus-contd.

saligna, Sm.-Grey Gum. A large tree, timber very durable. Yield of oil from dry leaves, I8 oz. per cwt. $(J$. F . Bailey). Gum contains 28.4 per cent. tannin, 42 per cent. arabin (Loewenberg's method) (Lauterer).

resinifera, Sm.-Red Stringybark, Jimmy Low, and Foresi Mahogany. "Roangga" of Palmer natives. Gum contains tannic acid 26 per cent, arabin 48.4 per cent (Lauterer). Wood useful for piles, fencing-posts, and similar work.

var. grandiflora, Benth .

pellita, F.v.M.

Subseries Inclusæ.

phonicea, F.v.M.

clavigera, A. Cunn.--The wood dark, hard, and durable. grandifolia, R. Br.

stannariensis, Bail.-Dead Finish of Stannary Hills. (Fig. I72.)

tessellaris, F. v. M.-Moreton Bay Ash. "Carbeen" and "Ori" of St. George, "Woonara" of Nanango, "Urrgula" of Palmer River, "Tchunba" of Bundaberg, "Wonkara" of Port Curtis, and "Algoori" of Mackay natives. Wood tough and durable. Gum contains tannin 52.3 per cent., arabin and metarabin 5.5 per cent. (Lauterer).

var. Dallachiana, F.v.M.-Cabbage Gum and Pudding Gum.

Subseries Corymbosæ.

setosa, Schau.

peltata, Benth.

latifolia, F.v. M.

corymbosa, Sm.-Bloodwood. "Ambool" of St. George, "Gooden" of Nanango, "Boona" of Brisbane River and Stradbroke Island, and "Jingil" of Cooktown natives. The gum will quickly cure ringworm (W. Macartney). terminalis, F. v. M.-Bloodwood. "Narm-boon-bong" of Cloncurry, "Kulcha" of Gilbert River, "Gaja" of Palmer River, and "Arangmill" of Rockhampton natives.

var. carnosa, Bail., Q1., Agric. J1. xv. (I905) 898.-An inland tree; fruit unustially fleshy. 
Eucalyptus-contd.

dichromophloia, F. v. M.

maculata, Hook.-Spotted Gum. "Urar" of Brisbane River natives.

var. citriodora, Bail and F.v.M.-Citron-scented Gumtree. "Boabo" of Rockhampton natives. Schimmer and Co. give as the yield of oil I to I.5 per cent. from the fresh leaves, and 3 to 4 per cent. from dry.

Leichhardtii, Bail.-A close ally of E. eximia, Schat. (Fig. I73.)

Subseries Eudesmieæ.

tetradonta, F.v. M.

Torelliana, F. ข. M.

Abergiana, F.v. M. (Albergiana, Q1. F1.).

trachyphloia, F.v. M.-White Bloodwood.

var. fruticosus, Bail.

Watsoniana, F.v.M.- "Olm-bah" of Mitchell River natives.

Tristania, R. Br.

Subtribe IV.-Metrosiderea.

\section{Section Lophostemon.}

straveolens, Sm.-Swamp Mahogany. "Boolarchoo" of Stradbroke Island and "Bujir" of Cooktown natives. A splendid wood for piles and underground work; resembles Spanish mahogany.

var. glabrescens, Bail.

conferta, R. Br.-Brisbane Box-tree. "Tubill-pulla" of

Stradbroke Island and Brisbane River natives. The young shoots of this tree have a milky sap. Wood when kept dry very durable, shrinks much in drying. var. fibrosa, Bail.-Perhaps only a peculiar growth.

\section{Section Eutristania.}

exiliflora, F.v.M.-Wood tough and elastic; useful for toolhandles.

laurina, R. Br.-Water Gum of most Southern localities; a most valuable timber for tool-handles.

longivalvis, F.v.M.-Buttercup-tree. (Fig. I74.)

lactiflua, $F$. г' $M$.

Brownii, S. Moore in Britt. Journ. Bot. x1. (I902) 25. 
Syncarpia, Ten.-The fruit a Syncarp. laurifolia, Ten.-Turpentine-tree.

Hillii, Bail.-Peebeen. Besides a useful timber this tree yields a resin which can be put to the same services as the Strasburg turpentine, and can be used as a healing agent on chronic ulcers and sores like the best Hamburg plaster (Dr. Joseph Lauterer). The bark contains 768 per cent. of tannin.

leptopetala, F.v.M. = Metrosideros glomulifera, $\mathrm{Sm}$.

Lysicarpus, F. v. M.

ternifolius, F.v.M.-Tom Russell's Mahogany. A usefu1 cabinet-wood, heavy and elastic. (Fig. I75.)

Metrosideros, Banks.

tetrapetala, F. v. M.

Xanthostemon, F. v. M.

chrysanthus, F. v. M.- "Choolo-choolo" of Barron River and "Currijello" of Johnstone River natives.

pachyspermus, F. v. M.-Yellow-wood of Johnstone River. oppositifolia, Bail.-Luya's Hardwood; Penda at Noosa. (Fig. I76.)

Backhousia, Hook, et Harv. myrtifolia, Hook. et Harv.

angustifolia, $F \cdot v \cdot M$.-Yields a good essential oil. sciadophora, $F . v \cdot M$.

citriodora, F.v.M.-The Sweet Verbena tree; oil of commercial value.

Bancroftii, Bail. et F. v. M.-Johnstone River Hardwood or Langdon's Hardwood. (Fig. I77.)

Osbornia, F. z. M.

octodonta, F.v.M. (Fig. I78.)

Tribe III.-Myrteæ.

*Psidium, Limn.

Guajava, Linn.-White Guava. Tropical America. A stray from cultivation. The bark is said to contain about I9 per cent. of tannin. The leaves and bark are used in India in cases of diarrhœea; Dr. Waitz recommends $3 / 2 \mathrm{oz}$. of the root-bark to be boiled down in $6 \mathrm{oz}$. of water to $3 \mathrm{oz}$. as an external application in cases of Prolapsus ani of children (Dymock).

Araca, Raddi $=P$. guinensi, Sw.-Green Guava. Tropical America.

littorale, Raddi.-Shore Guava. Tropical America. 


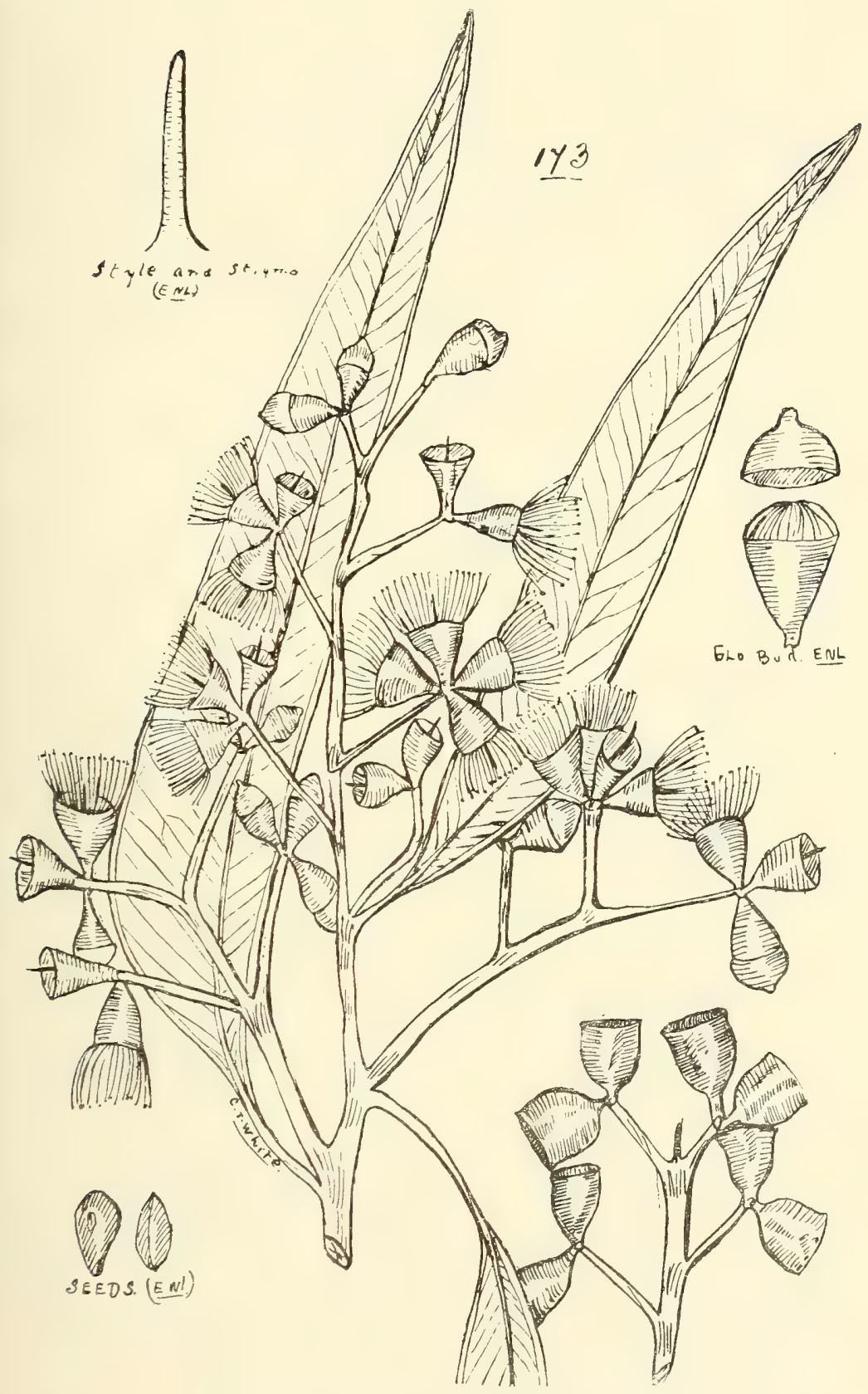

I73. Eucalyptus Leichhardtit, Bail. 


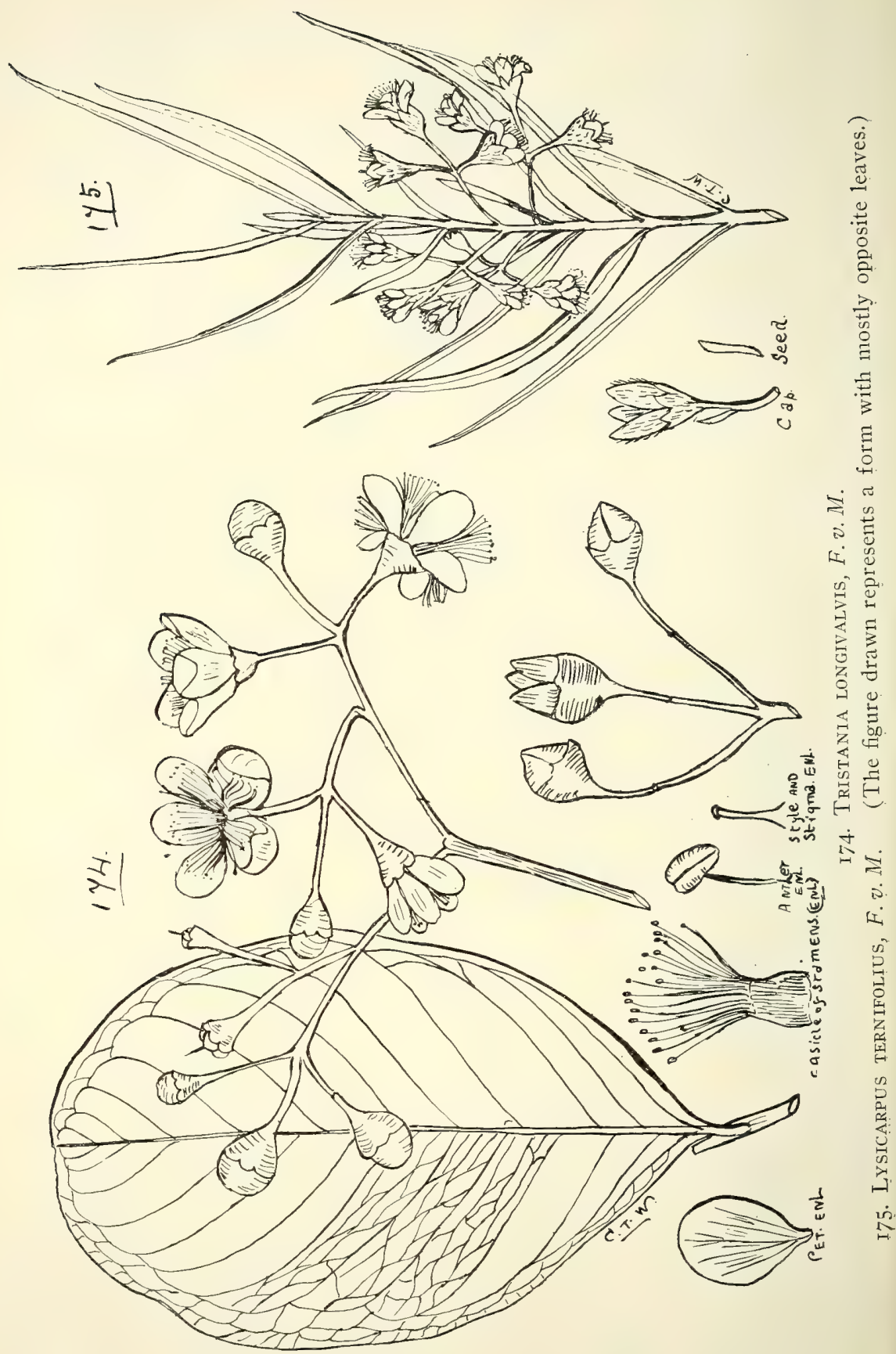


LI. MYRTACEE.

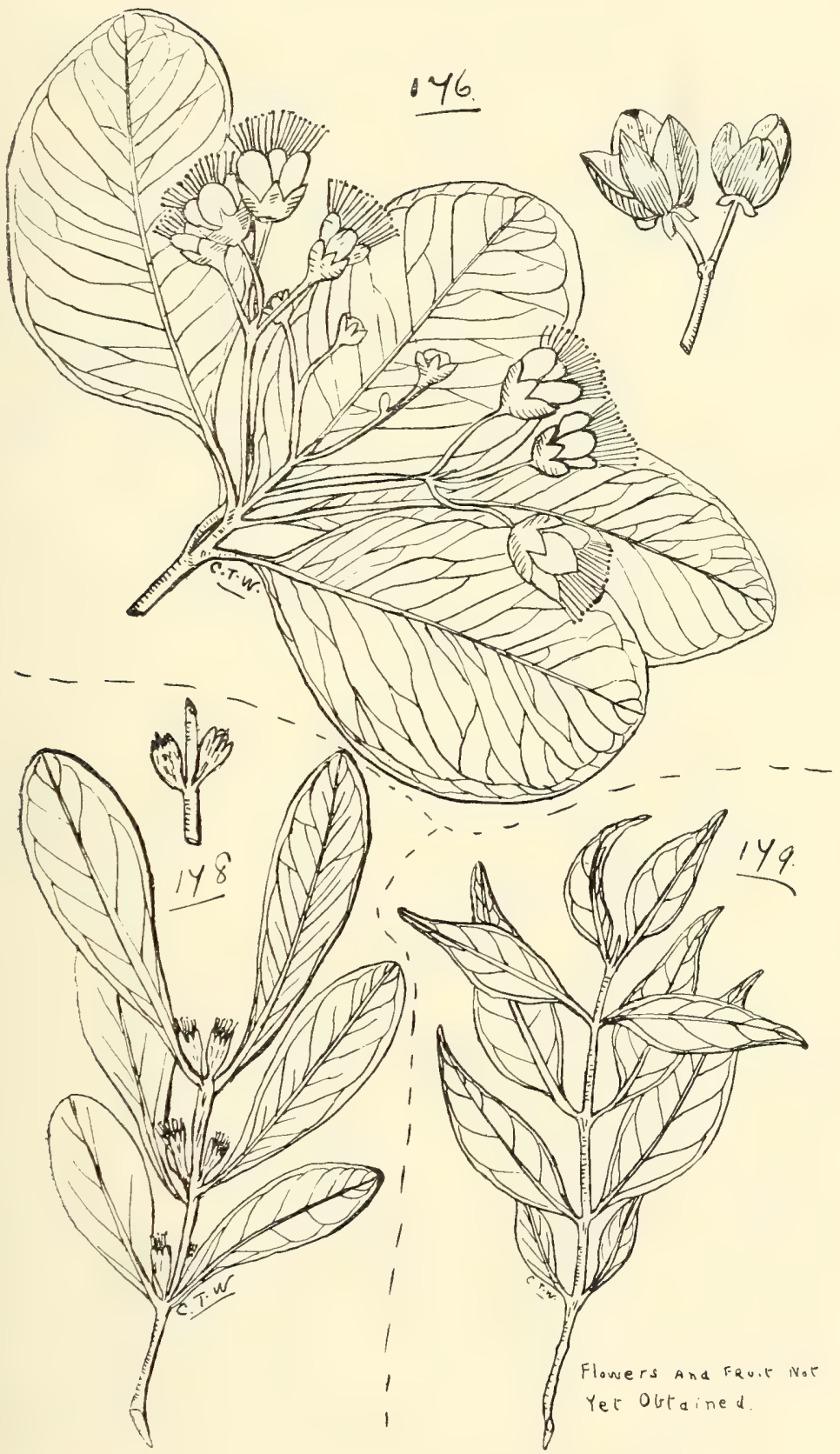

176. Xanthostenon oppositifolites, Bail.

ij8. Osmornia octodonta, $l i v \cdot . M$.

I79. Myrtis metrosideros, Bail. 


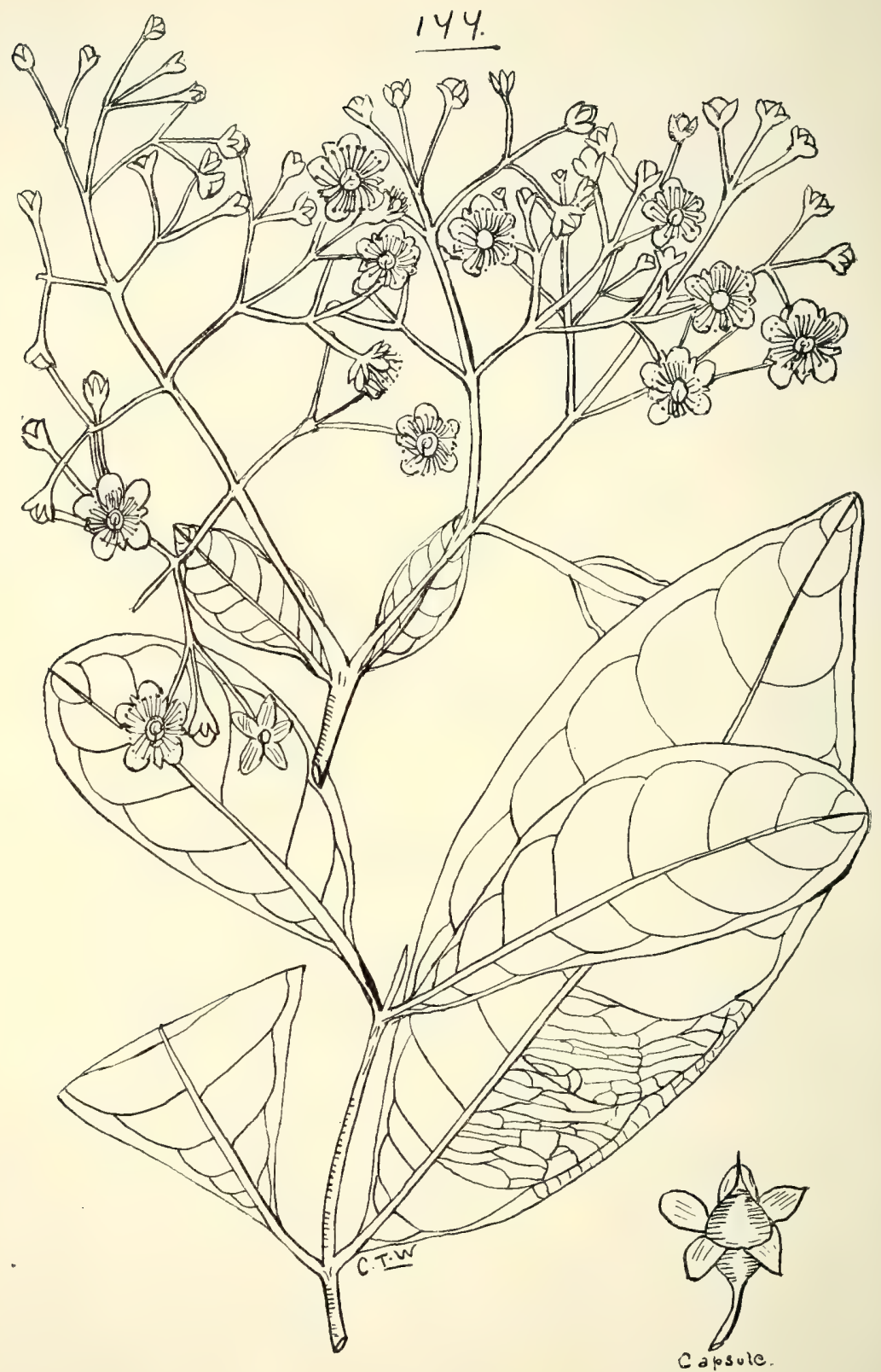

177. Backhousia Bancrofti, Bail. et F.v. M. 
Rhodomyrtus, $D C$.

psidioides, Benth. = Myrtus Tozerii, F.v. M.

trineura, $F . v . M$.

cymiflora, $F, v \cdot M$.

macrocarpa, Benth-Cooktown Loquat. The diseased fruit supposed to cause blindness (myopia) and death (see Q1. Bot. Bu1l. x. (I895) 37). "Wannakai" of Cape Bedford, "Kalburadji" of Bloomfield River, and "Poolboo-nong" of Dunk Island natives.

Myrtus, Linn.

rhytisperma, F. v. M.

var. grandifolia, Benth.

teniifolia, Sm._- Midyim" of Brisbane River natives.

gonoclada, F.v. M.

lasioclada, $F, v \cdot M$.

Shepherdi, F.v.M.

Hillii, Benth.-Scrub Ironwood. "Kalaara" and "Mangoor" of Barron River natives.

Becklerii, F. $v \cdot M$.

Bidwillii, Benth.

racemulosa, Benth.

var. conferta, Benth.

acmenioides, F.v.M.

fragrantissima, F.v.M.

nitida, J. F. Gmel.

exaltata, Bail.-A large tree. Fruit used for jam-making (E. Cowley).

metrosideros, Bail. (Fig. I79.)

Rhodamnia, Jack.

sessiliflora, Benth._- "Koorka-bidgan" of Barron River natives.

trinervia, Blume.

var. spongiosa, Bail._- "Din-din” of Barron River natives. argentea, Benth-Furnishes a pretty wavy-marked wood,. useful for cabinet-work.

Blairiana, F.v.M. (Fig. I8o.)

Fenzlia, Endl.

obtusa, Endl.

var. microphylla, Benth.

retusa, Endl.

Decaspermum, Forst.

paniculatum, Baill. = Nelitris paniculata, Benth.

var. laxiflora, Benth. 
Eugenia, Linn.-The fruits of many useful for cooking.

Section I.-Euengenia.

carissoides, F.v.M.- "Esyu-ro" of Batavia River natives. (Fig. I8I.)

*uniflora, Linn.-Brazilian Cherry. Tropical South America. Section II.-Syzıgium.

Smithii, Poir.-"Lilly-Pilly." Bark yields I7 per cent. of tannin $(F . v . M$.$) .$

Francisii, Bail.-Water Gum of Kin Kin; so called from the large quantity of liquid contained in its stem. A very large tree with thin bark and close-grained pinkish wood: The bark contains Io per cent. of tannin; will give a light colour to leather (J. C. Brünnich): (Fig. I8I bis; photo. on p. 2.)

hemilampra, F. v. M.

kuranda, Bail.-Native name in the Atherton district, "Wanchan."

gustavioides, Bail.

Ventenatii, Benth:-Water Gum of Brisbane River. leptantha, Wight._-“"Kai-go” of Cape Grafton natives.

var. parviflora, Bail.

Jambolana, Lam.-Seeds used as a remedy against diabetes. corynantha, F.v.M.

Section III.-Jambosa.

cormiflora, F.v.M.-A White Apple. "Moorool" of Barron River natives.

Hislopii, Bail.- "Walkaran" of Cooktown natives. I have never seen a good specimen of this tree, but Mrs. F. C. Rowan's painting (Plate IX.) shows it as one of the most beautiful of the genus.

Hodgkinsoniæ, F.v.M.=E. odoratissima, Bail.; E. Fitzgeraldi, F.v. M. and Bail.

Tierneyana, F. v. M.

grandis, Wight.-White Apple. "Waargoon-waargoon” of Herberton natives.

Johnsoni, $F . v \cdot M$.

suborbicularis, Benth.— "Pudginjacker" of Bloomfield River, "É-sie" of Batavia River, and "Murl-kue-kee" of Dunk Island natives.

Wilsonii, F:v.M.-The fruit used for jam-making in the early days of the colony.

fibrosa, Bail.

Banksii, Britt. and S. Moore, Journ. Bot. (x1.) I902, 26; I1l. Bot. Cook's Voy. iii. App. t. I22. 
PLATE IX.

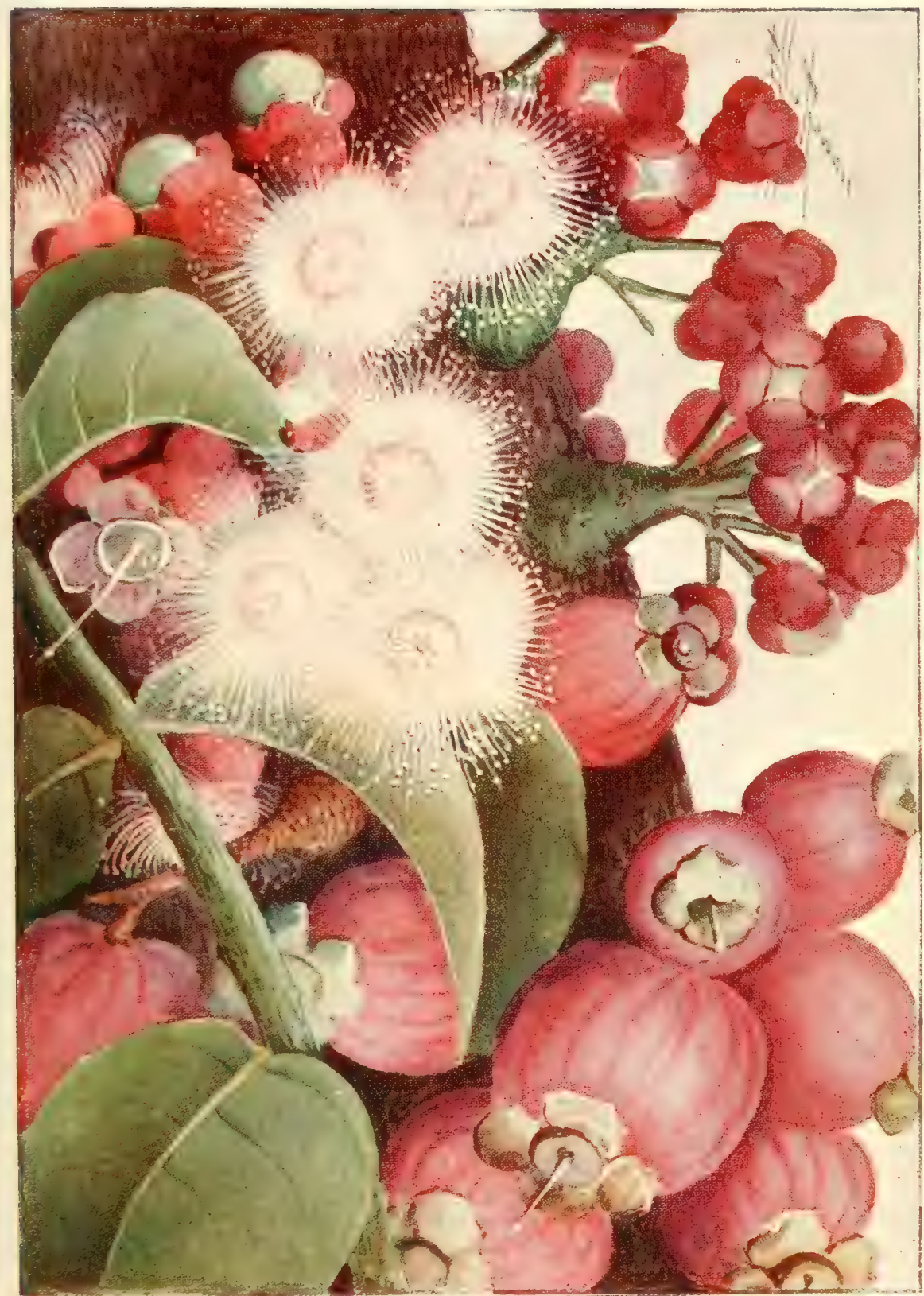

Eugenia Hislopil, Bail. 

Eugenia-contd.

Bungadinnia, Bail.-A native food.

eucalyptoides, F.v.M.-Fruit used for jam-making under the name of Native Pear (see Q1. Ag. J1. ii. (I898) 47I, P1. 37).

Simmondsæ, Bail. (Fig. I8I ter.)

paniculata, Banks and Sol.

punctulata, Bail.-Red Apple of Tambotirine Mountains. Sour Cherry of North Coast line.

sordida, Bail.

macrooai, Bail.

Leuhmanni, $F . v \cdot M$.

angophoroides, F. v. $M$.

oleosa, F.v.M. (Fig. I82.)

apodophylla, F. v. $M$.

hedraiophylla, F. v. M.

cryptophlebia, F. v. M.

Dallachiana, F.v.M. (Fig. I83.)

subopposita, Bail_- "Tarbugai" of Tully River natives.

\section{TRIBE IV.-LECYTHIDEÆ.}

Subtribe Barringtoniea.

Barringtonia, Forst.

speciosa, Limn. $f$.

racemosa, Gaudich.

acutangula, Gortn.

edulis, Seem., F1. Vit. 82; Bailey, Q1. Ag. J1. (I907) I25, P1. XI.

calyptrata, R. Br.

Careya, Roxb.

australis, F. v. M.- "Go-onje" of Cloncurry, "Gunthamarrah" of Mitchell River, "Barror" of Rockhampton, "Kuiperi" of Batavia River, and "Karoo" of Dunk Island natives. The pulped leaves are considered a sure and safe cure for ulcers. (Fig. I84.)

\section{Order LII.-MELASTOMACEÆ.}

Tribe I.-OSBEckieæ.

Osbeckia, Linn.

chinensis, Limn.

Otanthera, Blume.

bracteata, Korth. (Fig. I85.) 


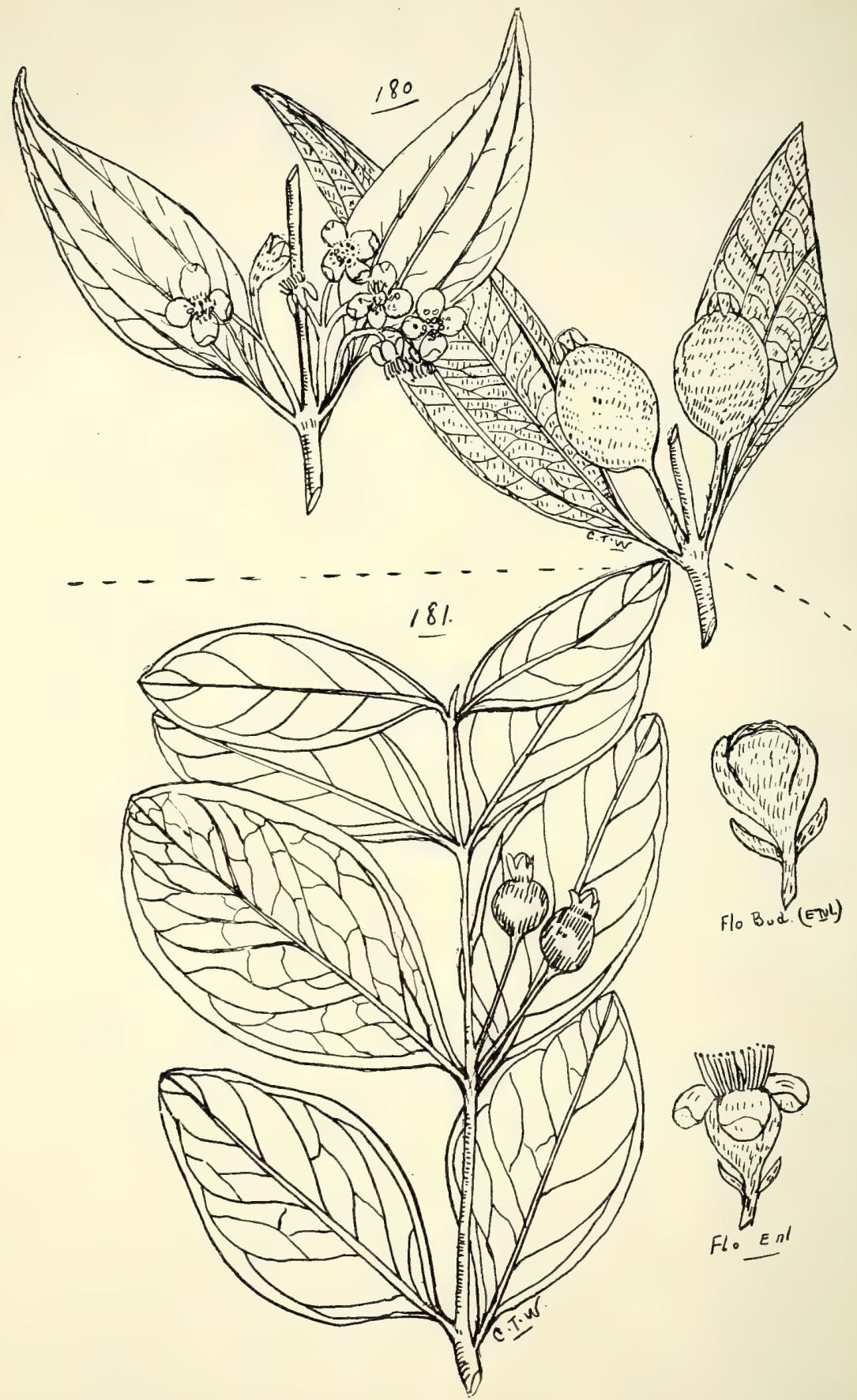

iso. Rhojamnia Blairiana, $F \cdot v \cdot M$. 18I. Elgenia carissoides, $F, \tau^{\prime} \cdot M$. 


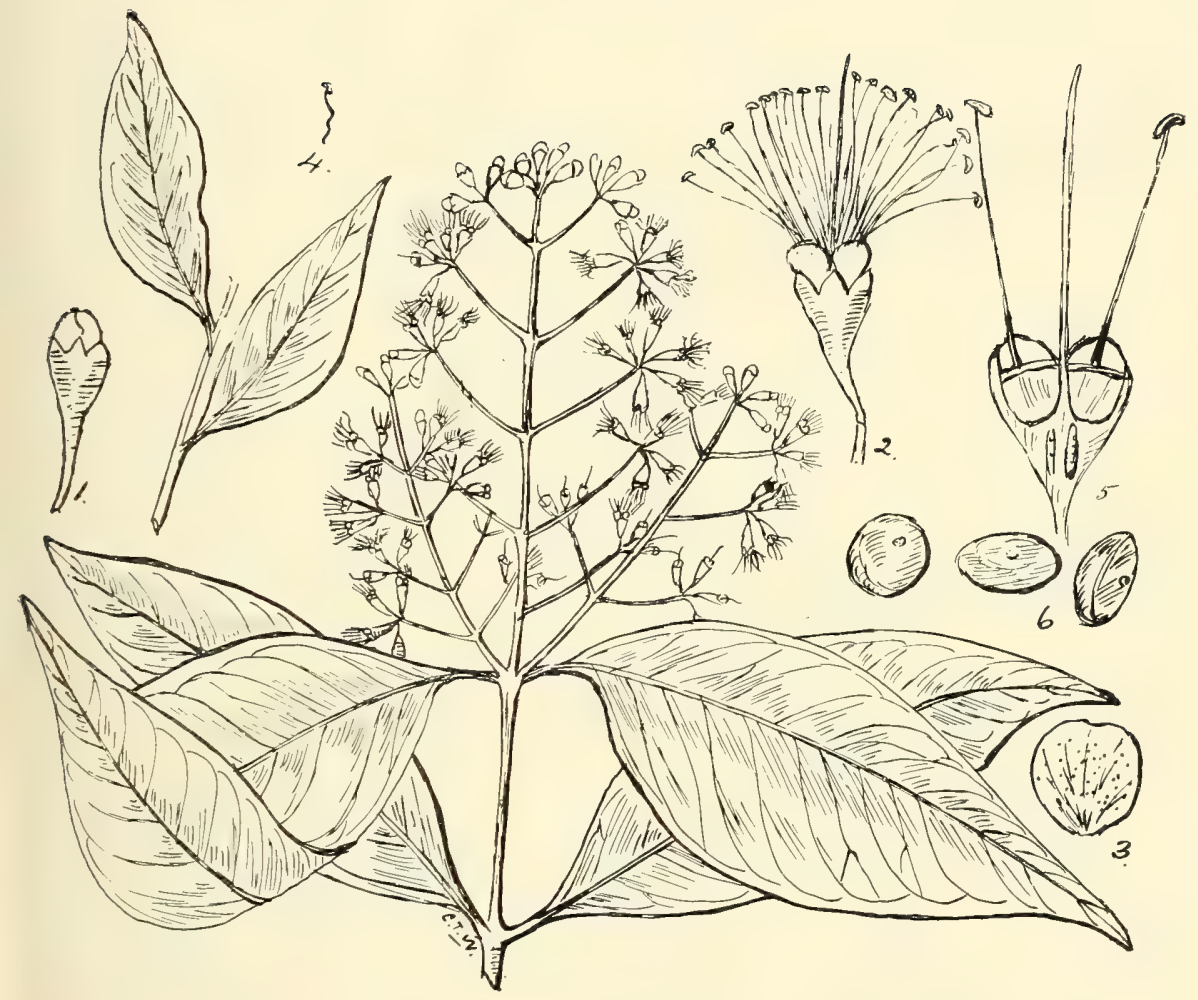

I8I bis. Eugenia firancisis, Bail.

(1) Flower bud, (2) fiower, (3) petal, (4) anther, (5) long. sect. flower petal and all the stamens except two removed, (6) fruit. (I), (2), (3), and (5) enl.: (4) and (6) nat. size. 
LI. MYRTACEE.

.8I ter.

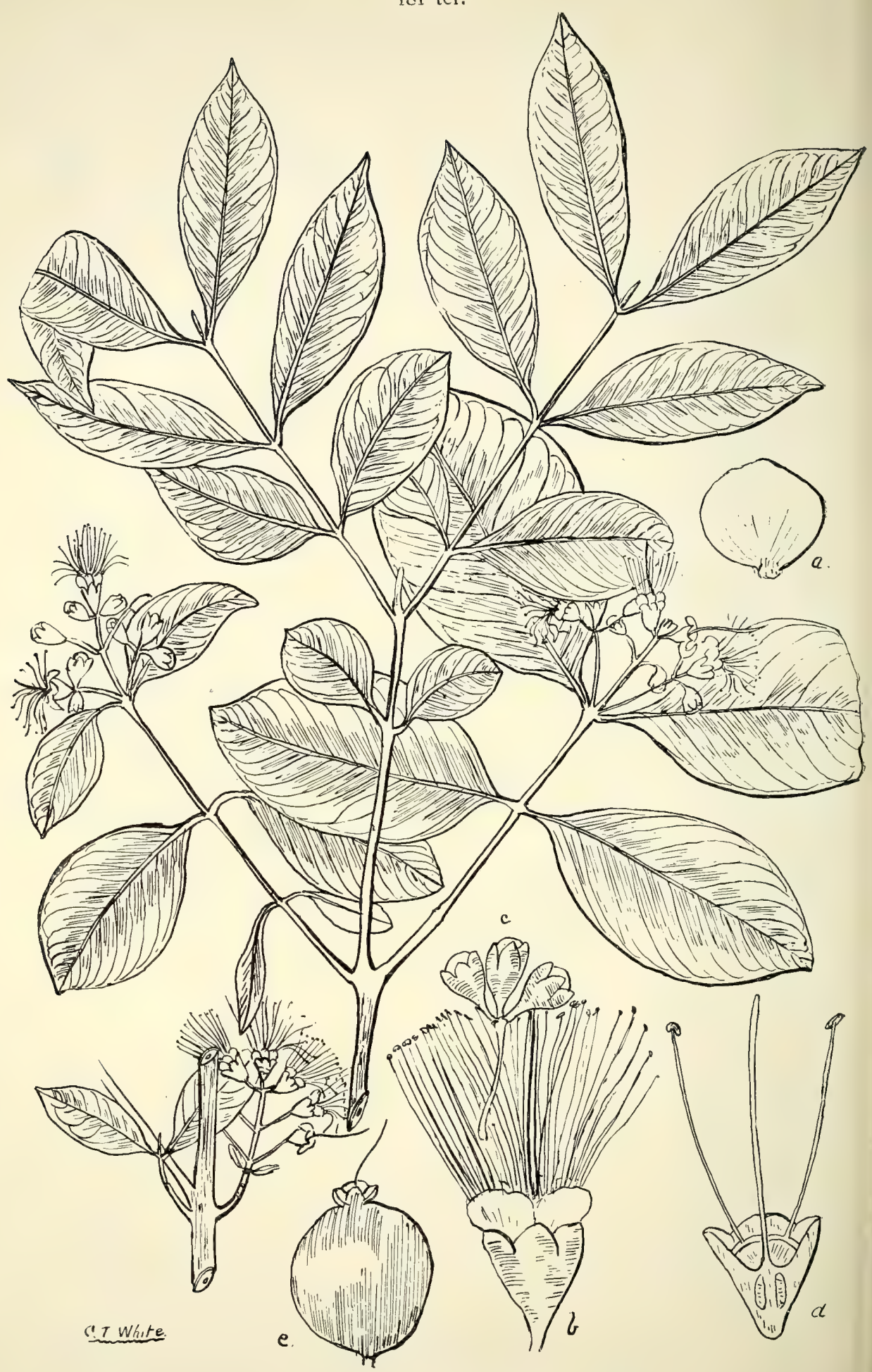

i8t ter. Eugenia Simmondse, Bail.

(a) A petal, (b) flower, (c) flower buds, (d) longitudinal section of a flower, pet and all the stamens except two removed, (e) fruit (nat. size). 
LI. MYRTACEF.

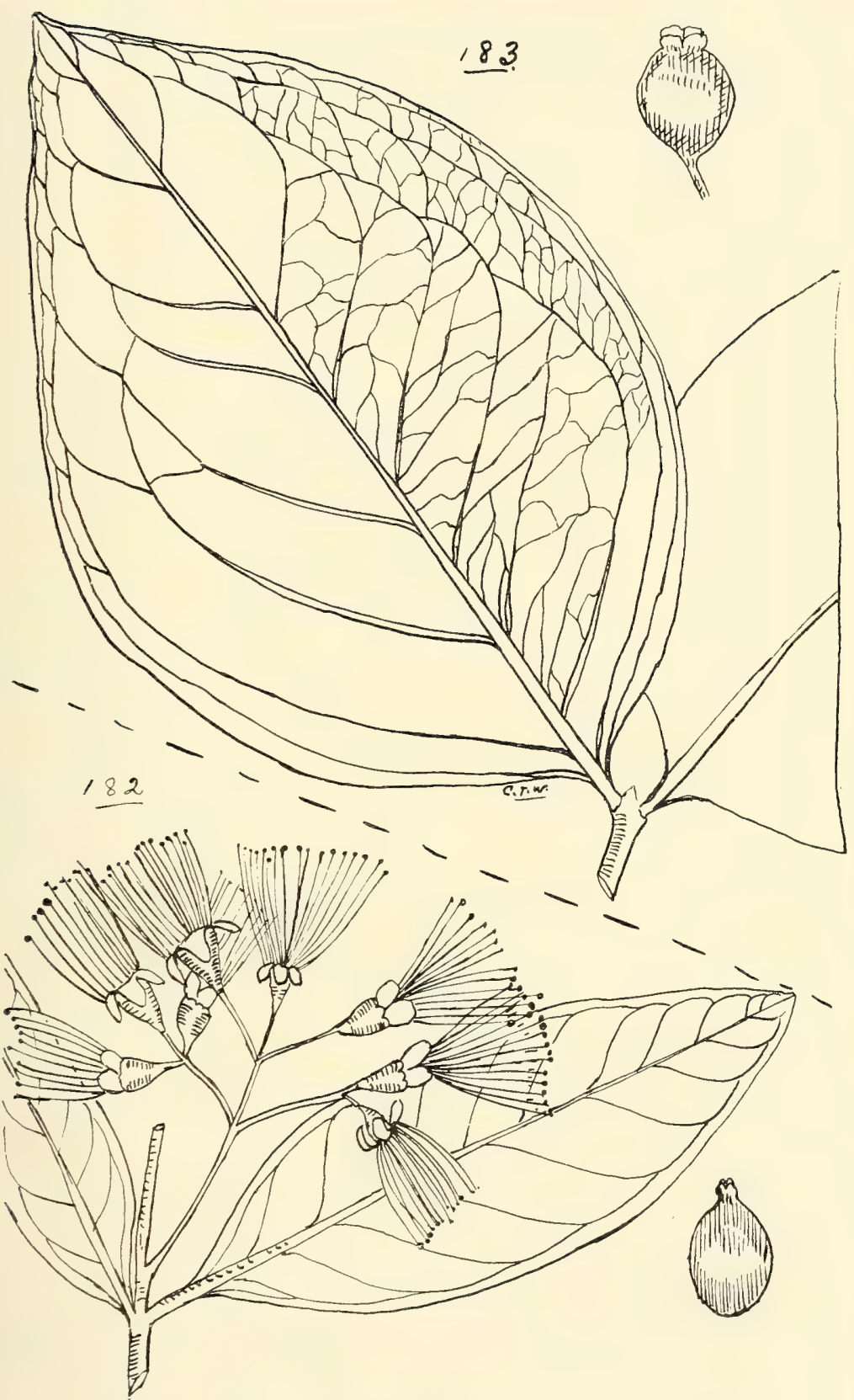

182. ELGENIA OLEOSA, $I . \tau, M$.

I83. E. Dillachiana, $J^{\prime}$ i'. 1 . 
Melastoma, Linn.

malabathricum, Linn., var. polyanthum, Benth.--.. "Di-eni" of Cape Bedford natives.

var. nanum, Bail.

Medinilla, Gaud.

TRIBE II.-MEDINILLEA.

Balls-Headleyi, F. v. M.

Memecylon, Linn.

TRIBE III.--MraecyleA.

umbellatum, Burm.

\section{Order LIII.-LYTHRARIEAE.}

Tribe I.-Ammannie.e.

Ammannia, Limn.--Seeds of many used for food by the natives.

pentandra, Roxb.

var. decussata, Benth.

occultiflora, Kohne.

diandra, F.v. M.

triflora, R.Br. (Fig. I86.)

indica, Lam.

auriculata, Willd.

multiflora, Roxb.

*Peplis, Linn.

portula, Linn.-Water Purslane. Europe.

TRIBE II.-LYTHREA.

Lythrum, Linn.

Salicaria, Linn.-Loosestrife.

hyssopifolium, Linn.

paradoxum, Koehne in Engl. Yahrb. xxiii., Beibl. lvii.

Nesæa, Comm.

Robertsii, F. $\cdot$. M. (Fig. I87.)

*salicifolia, H.B. and K.-Tropical America.

Pemphis, Forst.

acidula, Forst.

Lagerstrœmia, Limn.

*Flos-Reginæe, Retz-India. Has been given by some as naturalised, but perhaps only because found in old, deserted plantations, \&c.

*indica, Linn.-India.

Archeriana, Bail.

var. glabrescens, Bail. 
Sonneratia, Linn. f.

alba, $S m .=S$. acida, Benth. (non Linn. f.), F1. Aust. iii. 30r. The thick, horizontal roots are furnished with large, erect breathing processes. Bark contains 7.40 per cent. tannin (Brïnnich).

Anomalous Genus.

Punica, Linn.-Fruit a balausta.

Granatum, Linn.-Pomegranate. Asia. Bark said to contain about 25 per cent. tannin.

\section{Order LIV.—ONAGRARIE E.}

*Enothera, Linn.

rosea, Willd-America.

biennis, Linn.-Evening Primrose. Europe.

elata, H.B. et K. = E. salicifolia, Desf.-South America.

longiflora, Jacq.-A native of Buenos Ayres.

sinuata, Linn., forma.-America.

Epilobium, Linn.-Willow-wort.

junceum, Forst.

Billardierianum, Ser.

Jussiæa, Linn.

repens, Limn.-The Australian plant considered by Baron

Mueller to be $J$. diffusa, Forst., because of its yellow

flowers, while $J$. repens proper has white flowers.

suffruticosa, Limn.

iar. angustifolia, Bail. = J. angustifolia, Lam.

var. villosa, Bail. =J. villosa, W. et Arn.

Ludwigia, Limn.

parvifiora, Roxb. (Fig. 188.)

\section{Alliance XIII.-PASSIFLORALES.}

\section{Order LV.-SAMYDACEÆ.}

Casearia, Lmm.

Tribe I.-Casearieae.

esculenta, Roxb.

Dallachii, F. v. M.-C. B. Clarke places this under $C$. tomentosa, Roxb.

Homalium, Jacq.

TRIBE II.-HOM ALIE..

vitiense, Benth. (Fig. I89.)

circumpinnatum, Bail.-Shuttlecock plant. "Darrinjar" of Cairns natives.

brachybotrys, F. $\cdot M$.-Dr. Thos. L. Bancroft found this to contain a poison. (Fig. I9o.) 


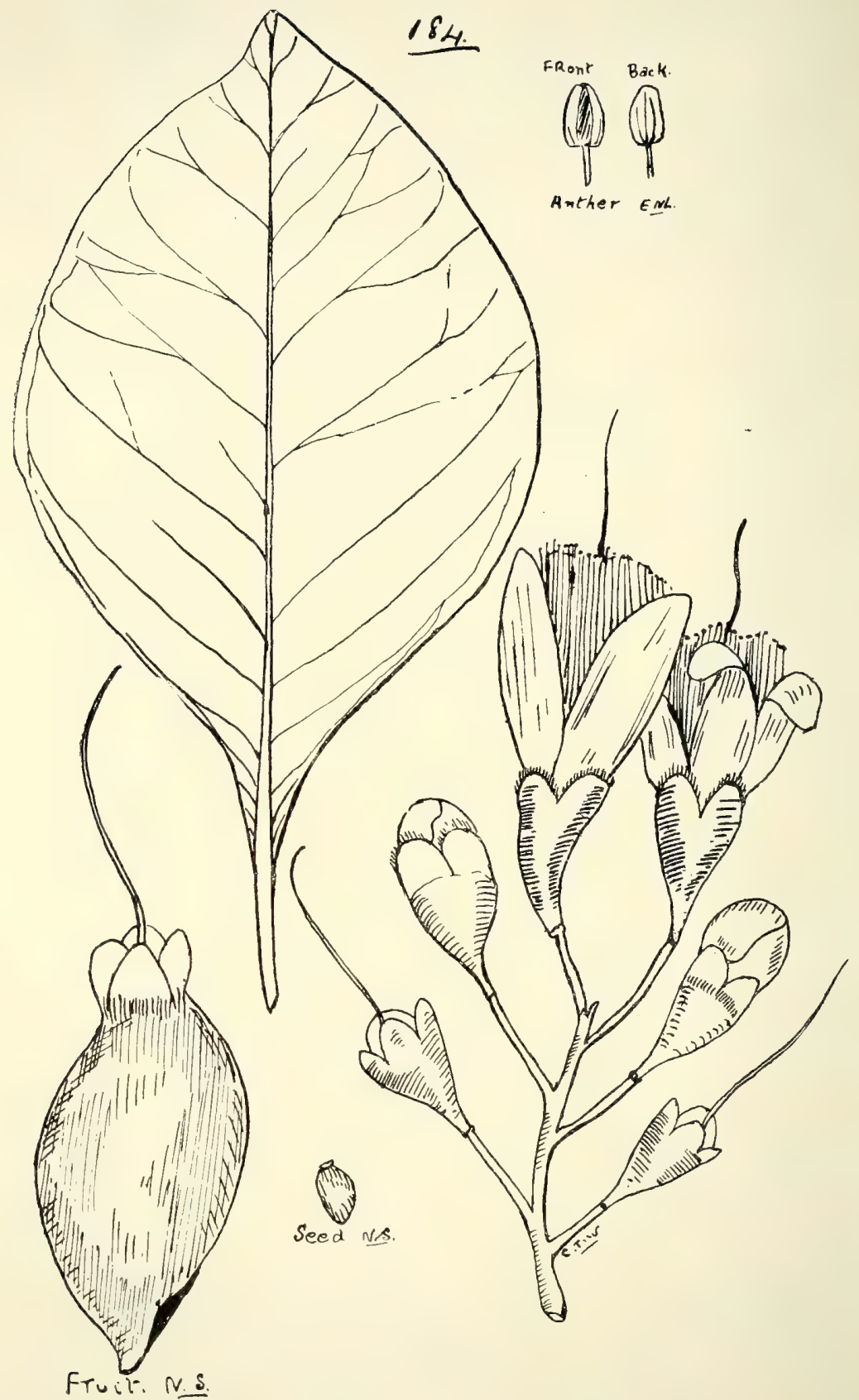

i84. Cakeya australis, F. $v . M$. 

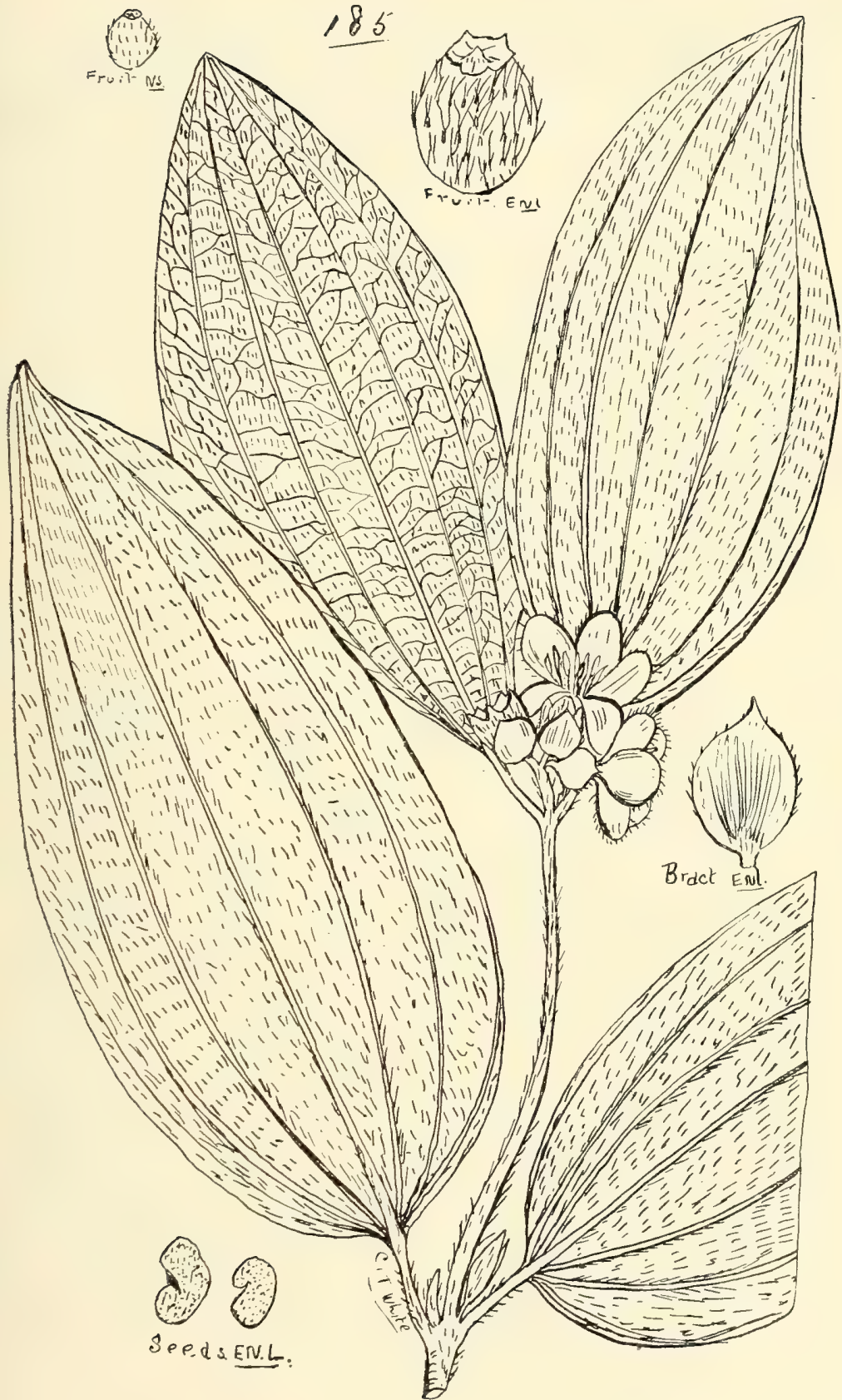

185. Otanthera bracteata, Korth. 


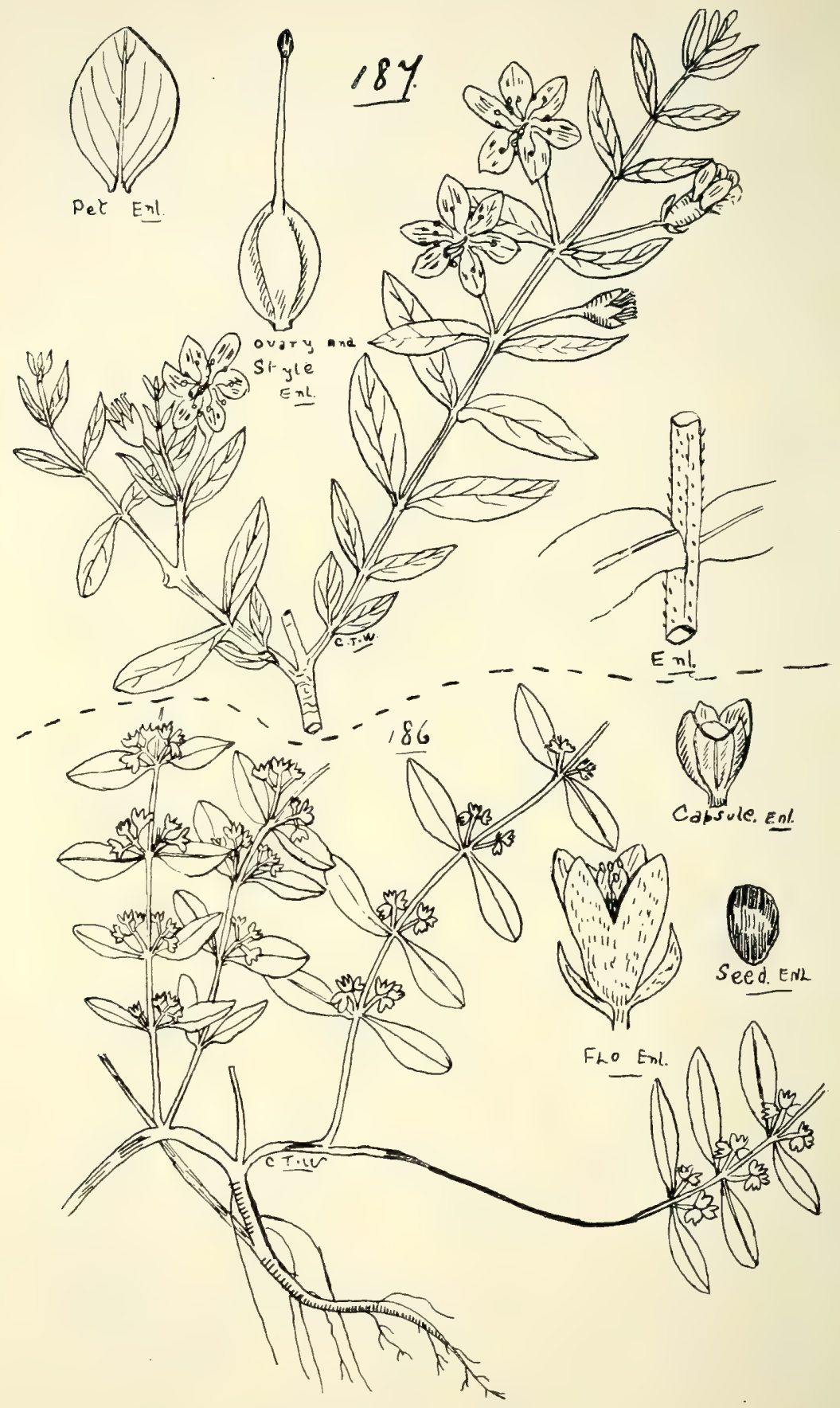

I86. Ammannia triflora, $R . B r$. 187. Nesea Robertsit, F. $v . M$. 


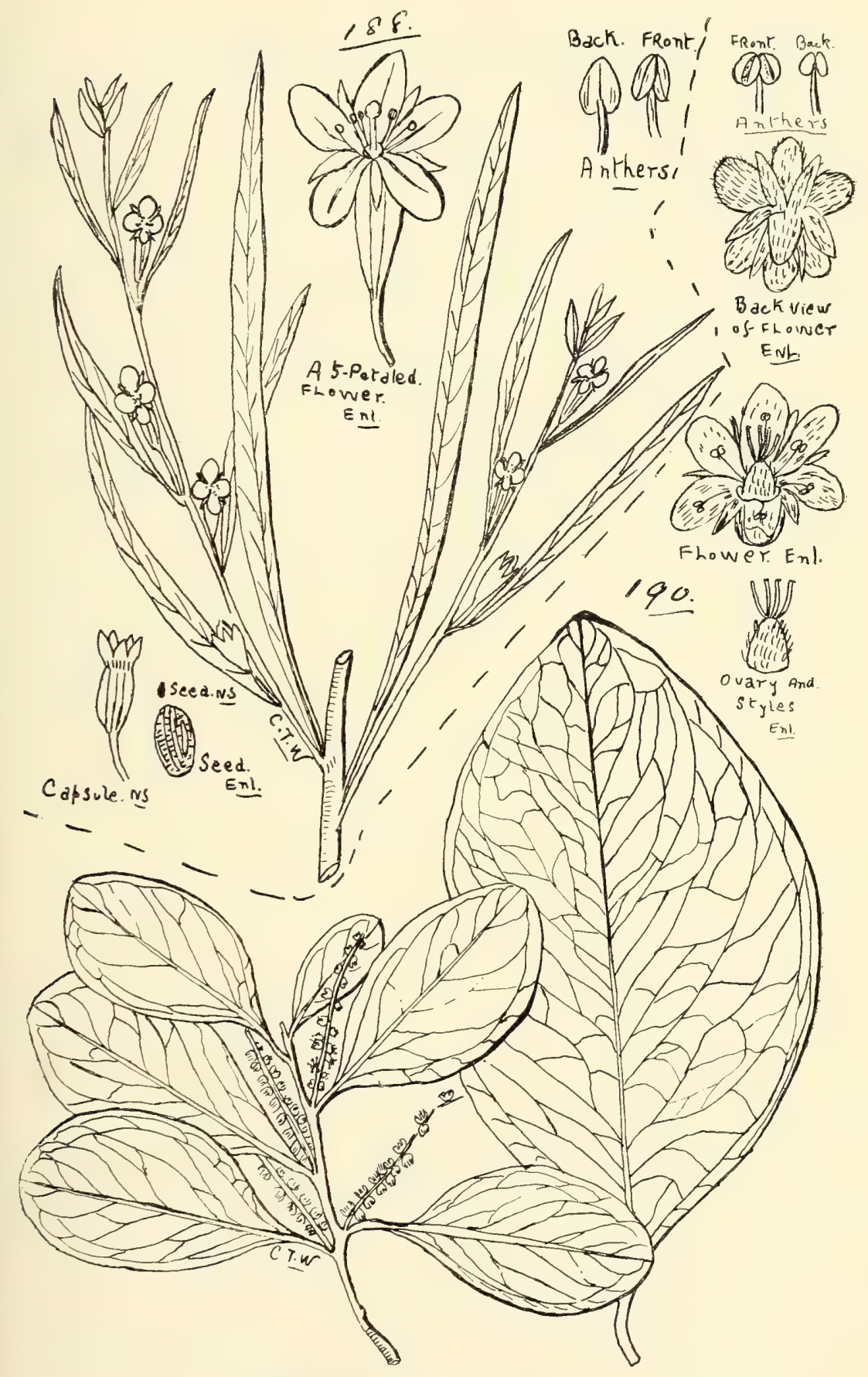

I8S. LEDWIGIA PARTIFLOR, Roxb.

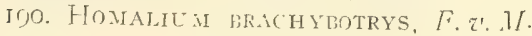




\title{
Order LVI.--PASSIFLOREAE.
}

\author{
Tribe I.-Passifloreæ.
}

Passiflora, Linn.

Herbertiana, Lindl.

aurantia, Forst.

var. Banksii, Bail.-Glabrous; petiolary glands present. (Fig. I9I.)

zar. pubescens, Bail.-Pubescent; petiolary glands absent.

(Fig. 19I bis.)

brachystephana, $F . v \cdot M$.

*fœtida, Linn.-." Nor-po" of Cooktown natives; apparently a name given to any climber (Roth). A noxious weed. Brazil.

*edulis, Sims.-Passion-fruit. Brazil.

*alba, Link et Otto.-Brazil. Has poisoned stock (see Q1. Agri. Jl. xxiv. (IgIO) 88.

*suberosa, Linn., var. minima, Jacq.-Brazil. Supposed to have poisoned cows.

*quadrangularis, Linn.-Granadilla; contains prussic acid. South America.

Tribe II.-Modecce压.

Modecca, Lam.

australis, R. Br. (Fig. 192.)

populifolia, Blume. (Fig. I93.)

TRibe III.-PAPAyACE.

*Carica, Linn.-A South American plant.

Papaya, Linn.-Papaw; an excellent fruit. Remarkable medicinal virtues are ascribed to the milky sap of this plant. According to Dr. M. Greshoff, carpaine occurs in the leaves in a crystalline form, and might be used as a substitute for digitalis. Another writer regards it as the only substitute for digitalis that can be used hypodermically without occasioning irritation or abscesses. The leaves may be used as a substitute for soap (Secman). Now naturalised in Northern scrubs. 


\section{Order LVII.-CUCURBITACEÆ.}

Tribe I.-Cucumerine.

Trichosanthes, Linn.--Fruit a Pepo.

pentaphylla, F.v.M.

cucumerina, Limn.

palmata, Roxb.-Plant and fruit considered medicinal in India, and in Bombay the leaves are sometimes smoked as a remedy for asthma (Dy'mock).

Hearnii, F. v. M.

nervifolia, Linn.-Root tıberous.

subvelutina, F. v. M.

Lagenaria, Ser.

vulgaris, Ser.-Bottle-gourd of the tropics. Seed contains saponin.

Luffa, Cav.

regyptiaca, Mill.-Native Towel-gourd.

var. peramara, Bail.

Benincasa, Savi.

vacua, $F, v . M$.

*Momordica, Linn.

Balsamica, Linn.-Balsam Apple of Asia and Africa; botl species.

Charantia, I imn.- The fruit of both kinds is bitter but wholesome; it requires, however, to be steeped in salt water before being cooked (Dymock).

Cucumis, Linn.

trigonus, Ro.rb.

*myriocarpus, Naud:-Gooseberry Cucumber. Supposed to cause blindness in horses. Africa.

*Citrullus, Schrad.

vulgaris, Schrad-Pie Melon. The Pie Melon and Water Melon are met with as strays from cultivation. Tropical Africa, probably, the original habitat.

*Ecballium, A. Rich.

Elaterium, A. Rich.-Squirting Cucumber. Asia.

*Cucurbita, Linn.

Pepo, Linn.-Pumpkin. Met with as a stray from cultivation. Native country doubtful: probably Tropical America.

Bryonia, Linn.

laciniosa, Limn.- The whole plant in India supposed to possess medicinal virtues. It is bitter and aperient. 
Melothria, Linn.

Cunninghamii, F.v.M. (Fig. I95.)

Muelleri, Benth.

Zehneria, Endl.

ejecta, Bail.-The Queensland Squirting Cucumber. (See Q1. F1. p. 699.)

Mukia. Arn.

scabrella, Arn. (Fig. I94.)

TRIBE II.-SICYOIDEA:

Sicyos, Limn.

angulata, Limn.

Tribe III.-ZANONIEA.

Alsomitra, B. et $H$.

capricornica, F. $M$.

suberosa, Bail.

Hookeri, F. v. M.

Stephensiana, Cogn.

Alliance XIT'-FICOID.ALES.

\section{Order LVIII.-CACTEÆ.}

Tribe I.-EChinocacte 2.

*Cereus, Har'.

triangularis, Haw.--Strawberry Pear. Brazil.

Tribe II.-OPUntie E.

*Opuntia, Mill.-All American.

vulgaris.--Prickly-pear. Stamens sensitive. Noxious weed.

This is a great pest. The specific name here chosen is that which has been used mostly when speaking of it as a noxious weed, but systematically it is most probably a form of O. Dillenii, Haw.- the Cactus indicus of Roxburgh.

Tuna, Mill.-Noxious weed.

ferox, Haw.-Noxious weed. The plant naturalised in Queensland is a large form of this species.

monacantha, Haw.-Noxious weed; sometimes met with round about Brisbane.

$\because$ Pereskia, Mill. Dict.

aculeata, Mill. Dict.-Barbadoes Gooseberry; a useful plant for rearing the cochineal insect upon; a stray from hedges. 


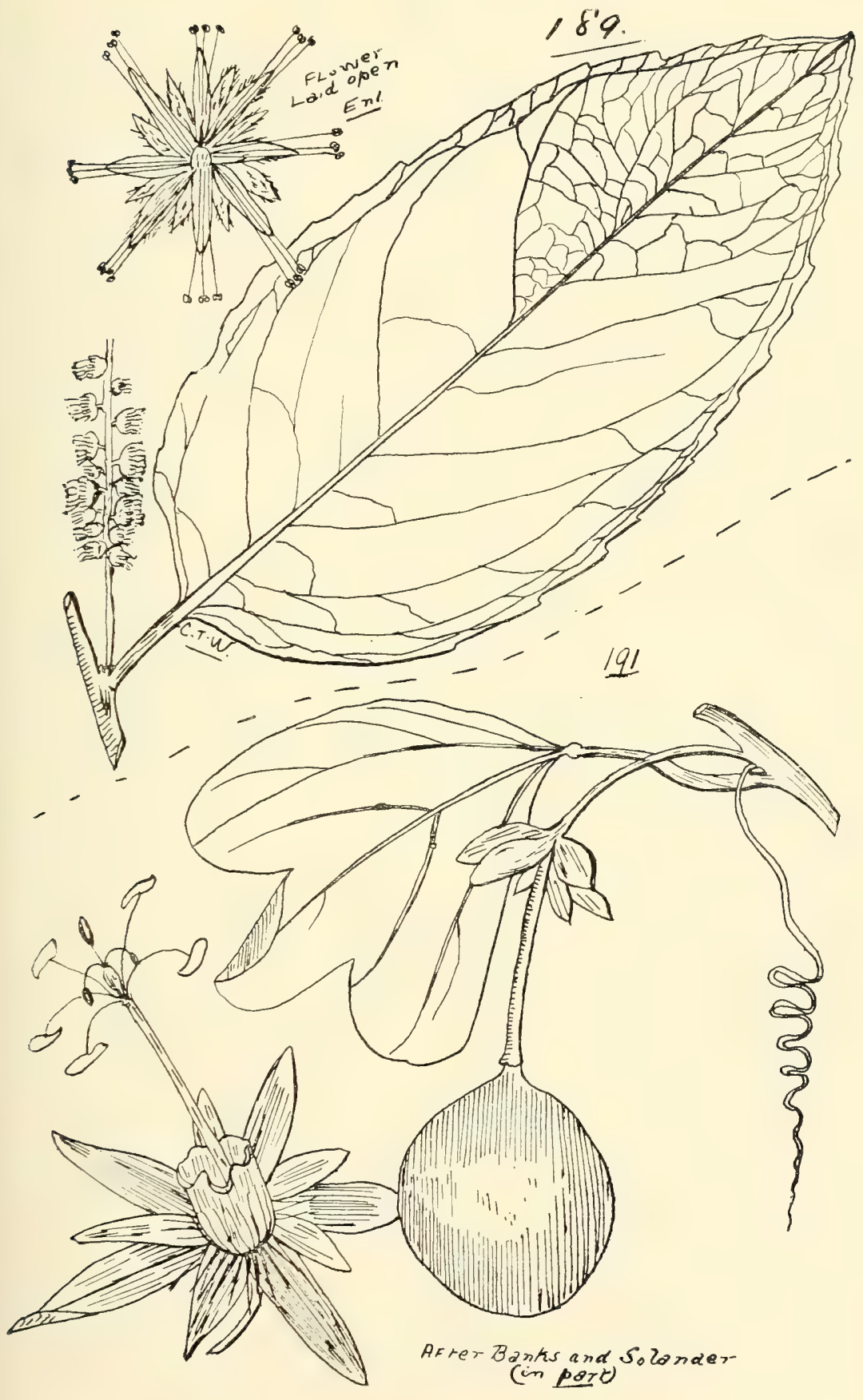

ieg. Homalita vitiense, Benlh.

igr. Passiflora alrantia, Forst., var. Banksir, Buil. 
I9I bis.

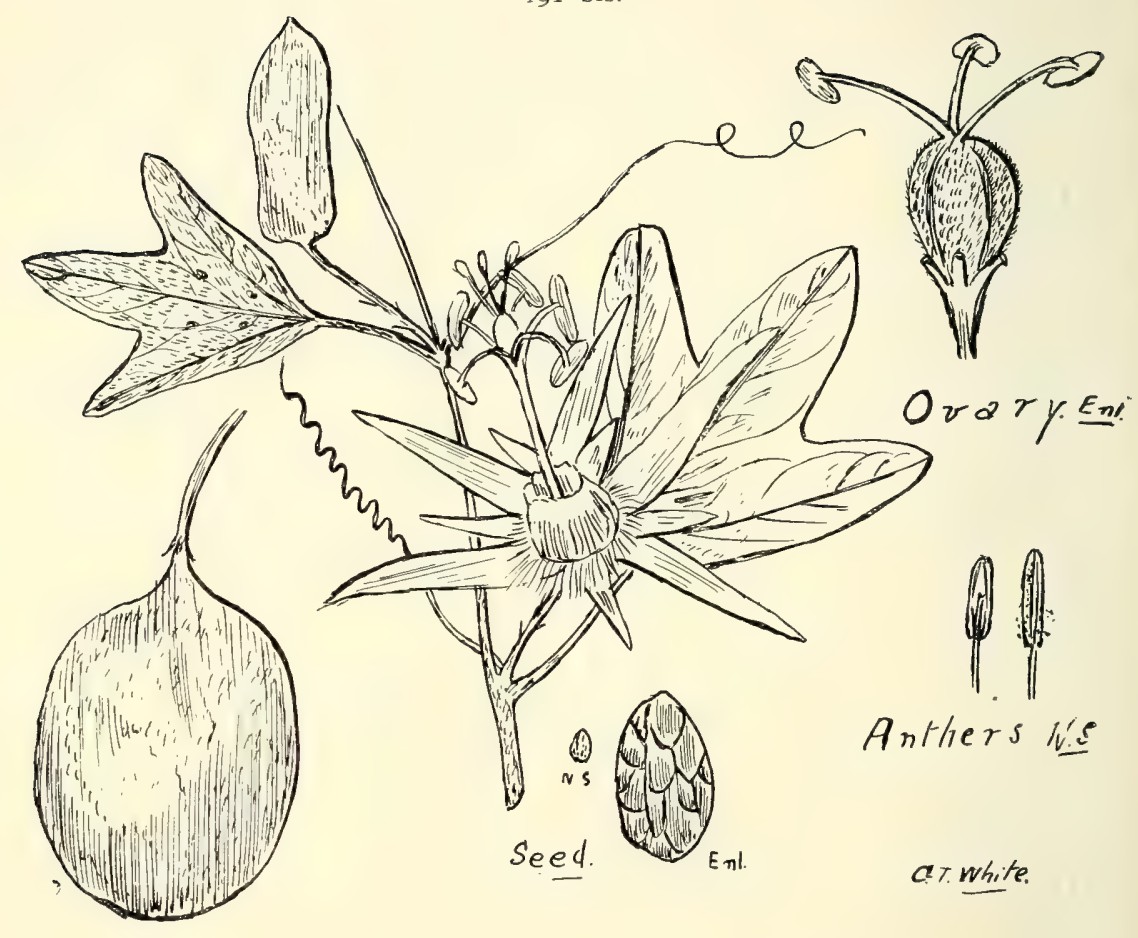

igi bis. Passiflora aurantia, Forst, var. pubescens, Bail. 


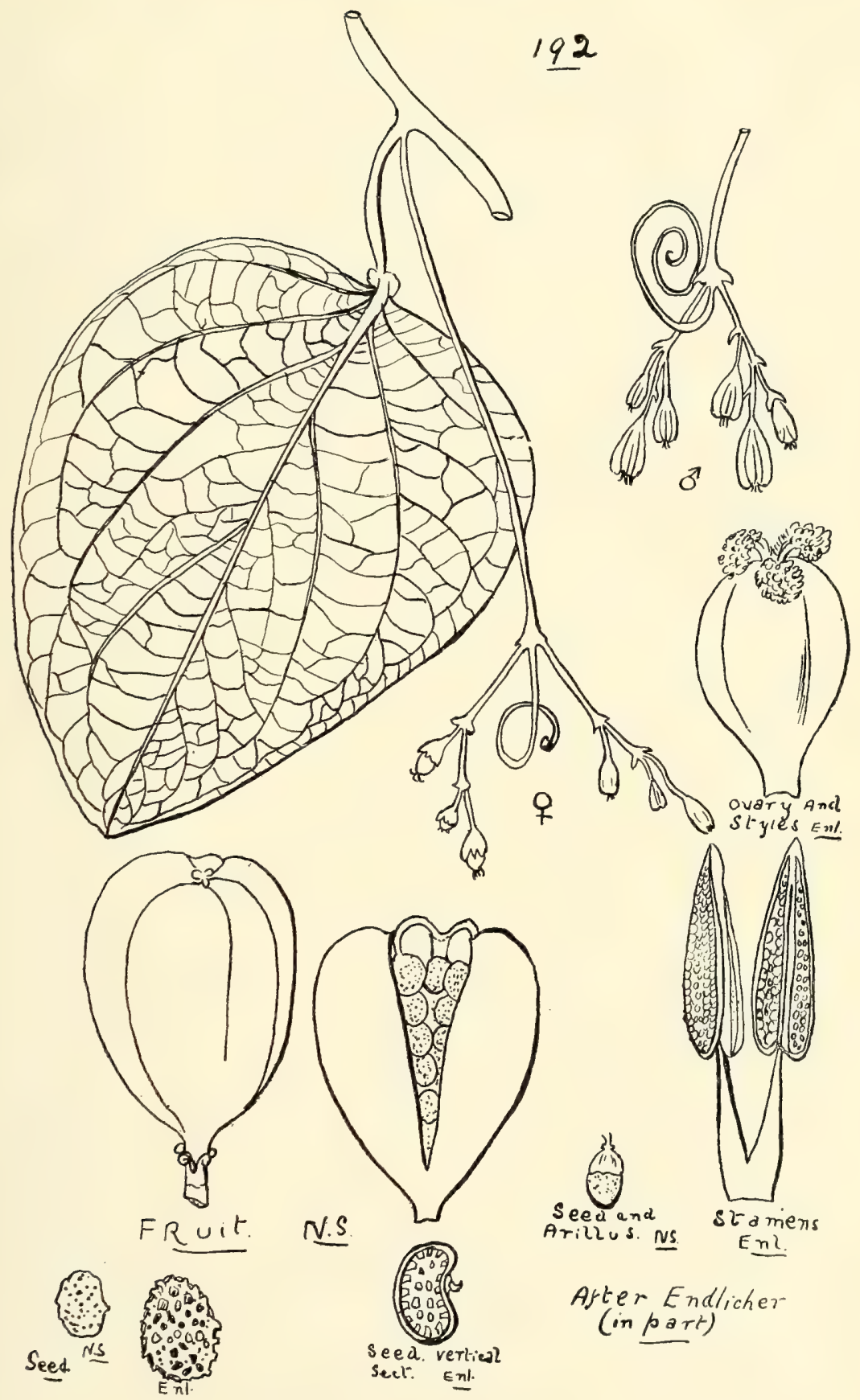

I92. Modecca atstralis, $R \cdot B r$. 


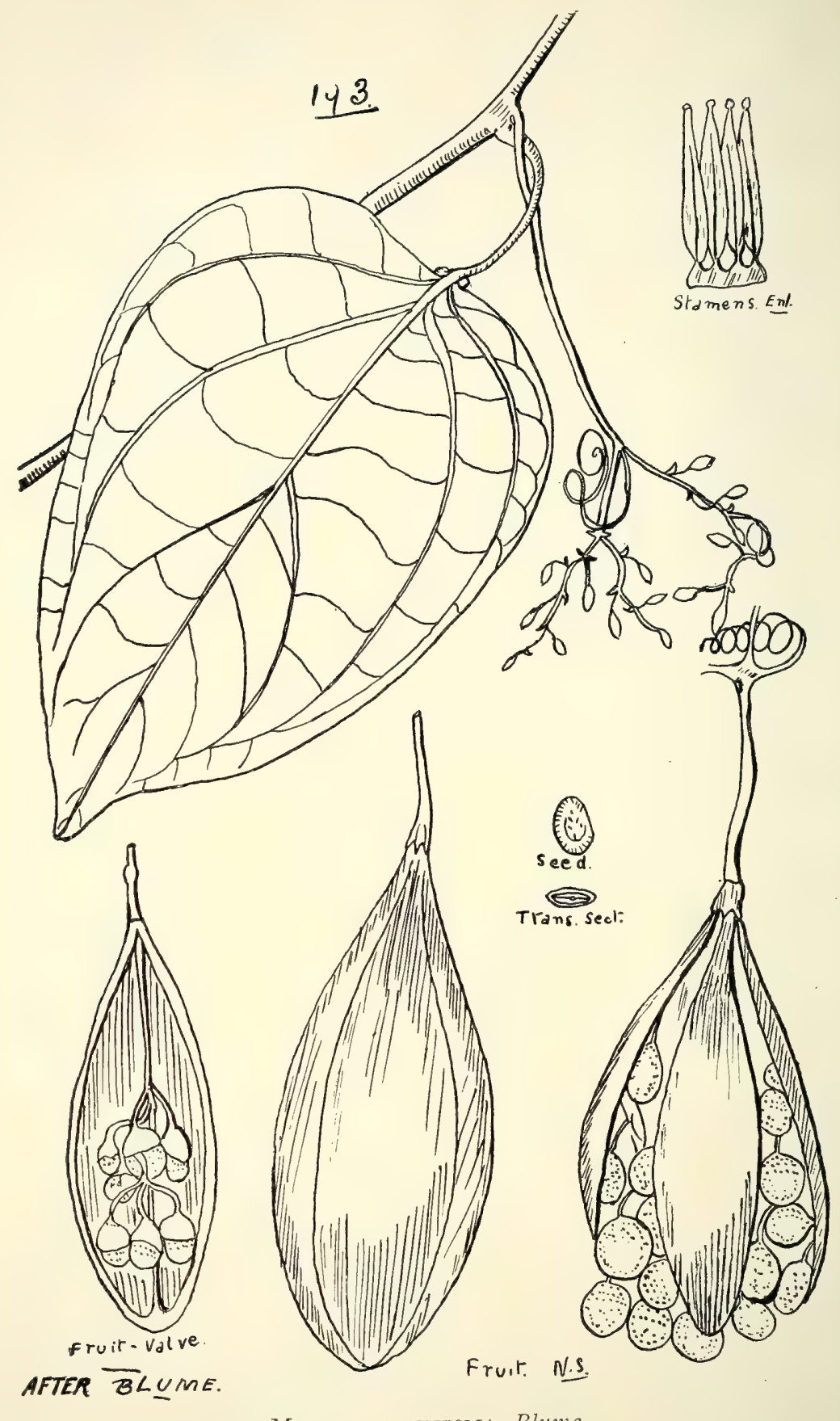

193. Modecca populifolia, Blume. 


\section{Order LIX.-.FICOIDEÆ.}

\section{Tribe I.-Mesembryez.}

Mesembryanthemum, Linn.

æquilaterale, Haw.-Pig's-face.

australe, Sol.

Tetragonia, Lim.

expansa, Murr.-New Zealand Spinach. The shoots of this plant contain saponin, but not the seeds (Greshoff).

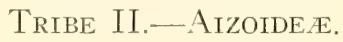

Aizoon, Limn.

quadrifidum, F.v. M.

zygophylloides, F. v. M.

Gunnia, F. v. M.

septifraga, F.v.M.

Sesuvium, Linn.

portulacastrum, Linn.

Trianthema, Linn.

decandra, Limn. = Zaleya decandra, Burm.-A very common succulent garden weed, abundant in the hot wet months. turgidifolia, F. v. M.

crystallina, $T$ ahl.

pilosa, F. ข.M. (Fig. I96.)

rhynchocalyptra, F.v. M.

cypseloides, Benth.

Tribe III.-MOLLUGINEA.

Macarthuria, Hueg.

neocambrica, F.v. $M$.

Mollugo, Linn.

Section I.-Glinus.

Glinus, A. Rich.

Spergula, Linn.

orygioides, F.v.M.

Cerviana, Ser.

stricta, Linn. 
Alliance XV.-UMBELLALES.

\section{Order LX. - UMBELLIFERÆ.}

Series I.-Heterosciadie.

Tribe I.-Hydrocotyleж.

Hydrocotyle, Linn.-Fruit a Cremocarpium.

Section I.-Euhydrocotyle.

vulgaris, Limn.-Whiterot or Pennywort. hirta, $R . B r$.

var. acutiloba, F.v. M.

var. pusilla, Benth.

laxiflora, $D C$.

var. minor, Benth.

pedicellosa, F.v.M.

tripartita, $R . B r$.

Section II.-Centella.

asiatica, Linn.-Indian Pennywort. The juice of this plant is found useful in skin diseases.

Trachymene, $R$ udge $=$ Didiscus, DC.

cyanopetala, Benth

Dusenii, Domin (under Didiscus).

australis, Benth.-Native Parsnip. To the Newcastle Range plant, Dr. K. Domin, in his Monographie der Gattung Didiscus (DC.), gives the name D. Benthami, Dom., var. bivestitus, Dom.

glaucifolia, Benth.

glandulosa, Benth.

incisa, Rudge.

var. pilosa, Benth.

procumbens, Benth

var. Hookeri, Domin, Monogr. Gattung Didicus (I908) 55. geraniifolia, Bail. (Fig. 197.)

Siebera, Reichb. $=$ Trachymene, DC.

valida, Benth. (Fig. I98.)

ericoides, Benth.

z'ar. thymifolia, A. Cunn. 
Siebera-contd.

linearifolia, Benth.

Billardieri, Benth.

var. crassifolia, Benth.

Xanthosia, Rudge.

pilosa, Rudge.

Tribe II.-SAniculeæ.

Actinotus, Labill.

Helianthi, Labill.-Flannel-flower.

minor, $D C$.

Gibbonsii, F.v.M.

Eryngium, Linn.

rostratum, Cav.

var. subdecumbens, Benth.

vesiculosum, Labill.

plantagineum, F.v.M.

expansum, $F . v \cdot M$.

\section{Series II.-Haplozygie}

Tribe III.-AmMine..

*Bupleurum, Linn.

rotundifolitm, Linn-Common Hare's-ear or Thorow-wax. Europe.

aristatum, Benth.-Narrow-leaved Hare's-ear. Europe

Apium, Linn.

australe, Thou.

leptophyllum, F.v.M.

*Ammi, Linn.

majus, Linn.-Bishop's weed. Europe.

Sium, Linn.

latifolium, Linn.-Water Parsnip.

*Scandix, Limn.

Pecten-Veneris, Linn.-Shepherd's-needle or Venus's-comb.

Britain.

\section{Tribe IV.-Seseline A.}

*Fœniculum, Adans.

vulgare, Mill., Gard. Dict.-Common Fennel. A plant of the Mediterranean Regions, used as a condiment and in medicine.

Enanthe, Linn.-Water Dropwort. stolonifera, $W$ all.

Crantzia, Nutt.

lineata, Nutt. 


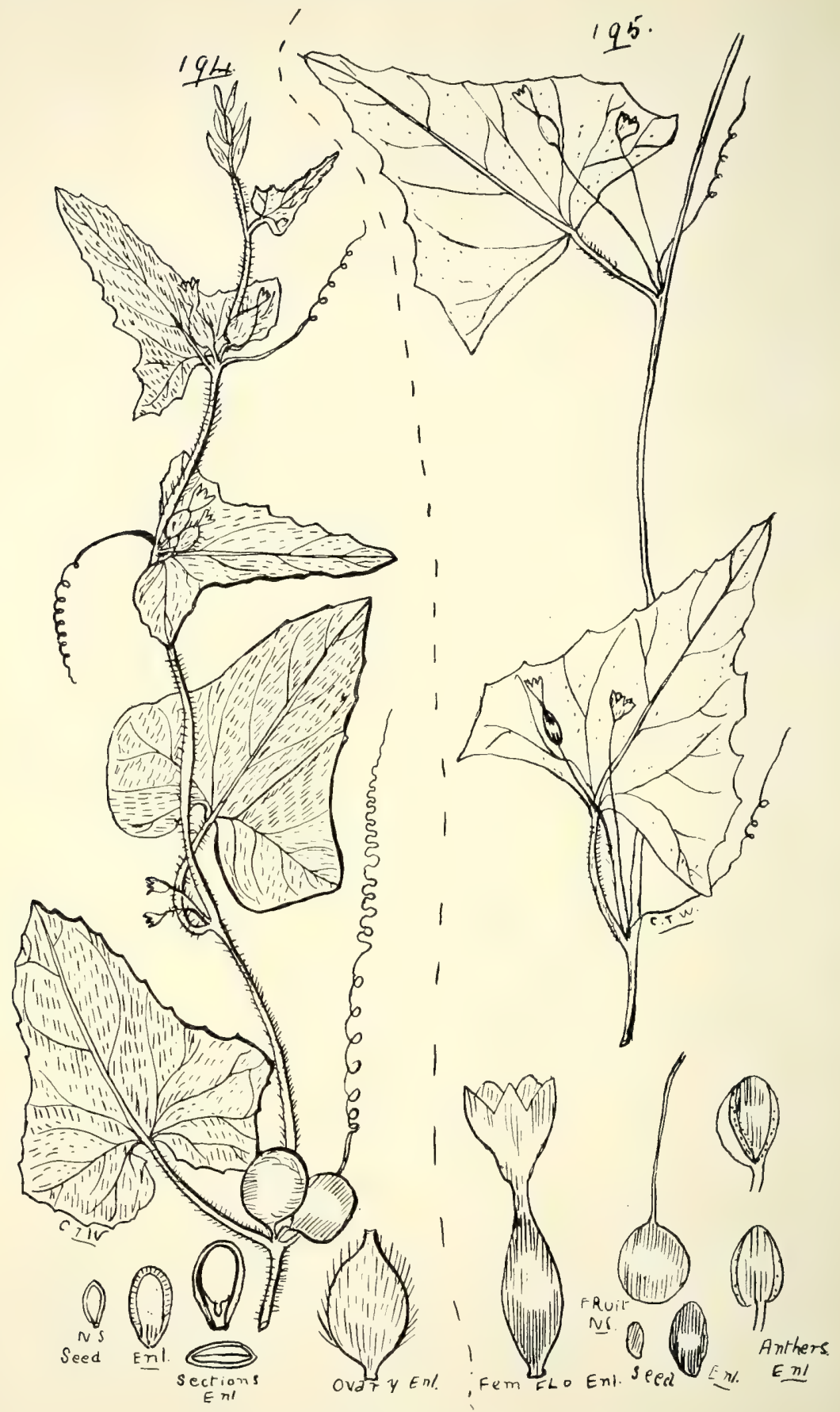

194. Mukia scabrella, Arn.

195. Melothria Cunninghamit, F. v. M. 


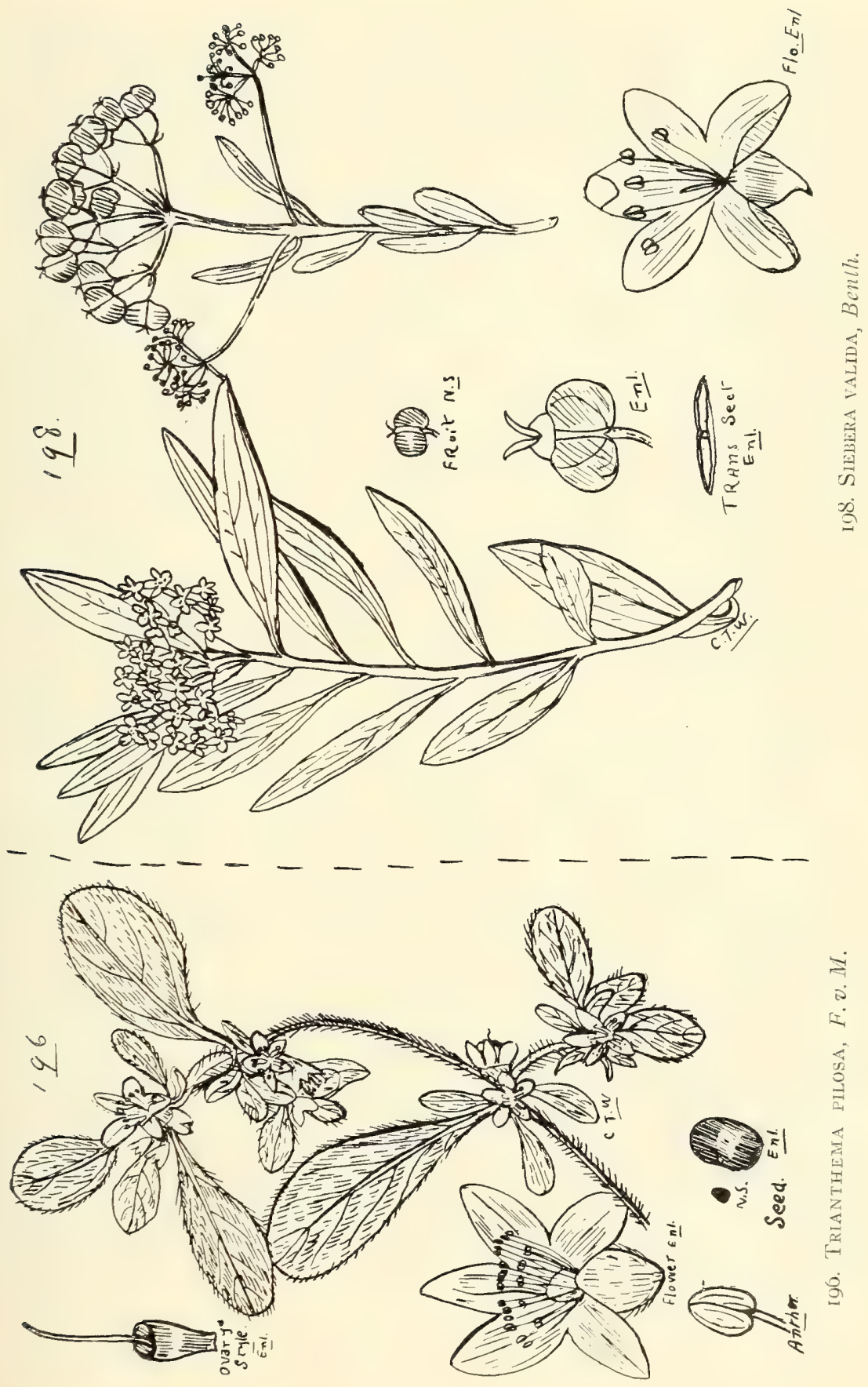


Series III.-DiplozygiÆ.

Tribe V.-Caucaline存.

*Coriandrum, Limn.

sativum, Linn.-Coriander. Europe. The seeds of this plant have been used as a spice for many years.

Daucus, Linn.-Carrot.

brachiatus, Linn.

\section{Order LXI. - ARALIACEF.}

Series I.-Aralie.e.

Delarbrea, Vicii . = Porospermum, F. v. M.

Michieana, F.v.M. (Plate X.)

Aralia, Linn.

Macdowalli, F.v. M.

Pentapanax, Seem.

Willmottii, F.v.M. (Fig. I99.)

bellenden-kerensis, Bail. (Fig. 200.)

Series II.-Mackinlayiez.

Mackinlaya, F.v. M.

macrosciadum, F.v.M.

confusa, Hemsl.

Series III.-Panacex.

Astrotricha, $D C$.

pterocarpa, Benth. (Fig. 20I.)

floccosa, $D C$.

var. brevifolia, F.v. M.

longifolia, Benth.

var. glabrescens, Bail.

ledifolia, $D C$.

var. glabriflora, F.v. M.

Biddulphiana, F.v.M. (Fig. 202.)

Motherwellia, F.v.M.

haplosciadea, $F . v . M$.

Panax, Linn.

Murrayi, F.v.M.- "Koorgarrie" of Herberton natives.

Wood useful for lining-boards.

mollis, Benth.

Macgillivræi, Benth.

sambucifolius, Sieb. 
PLATE $X$.

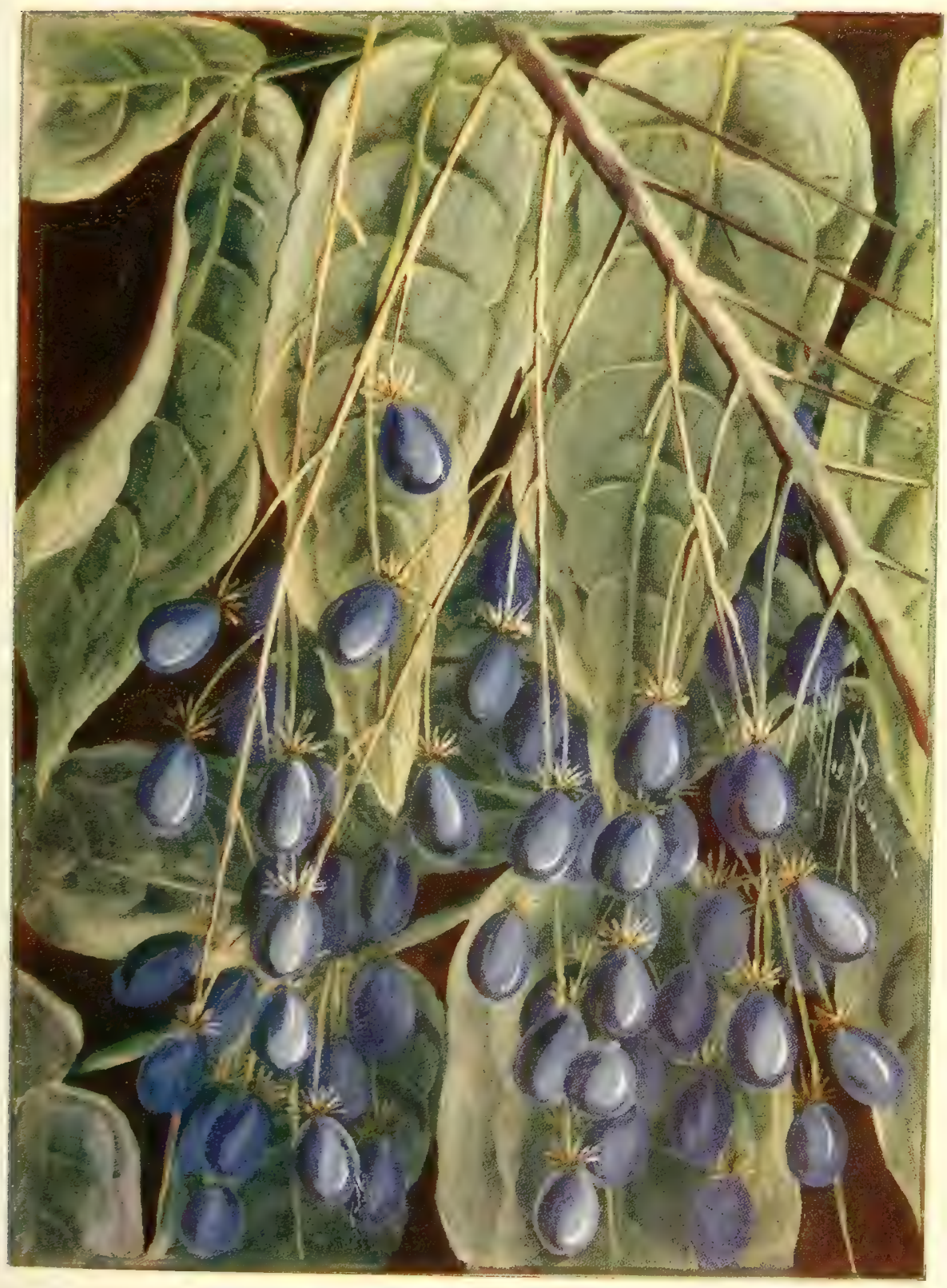

Delabrea Michieana, F. v. M. 

LX. UMBELLIFERÆ.

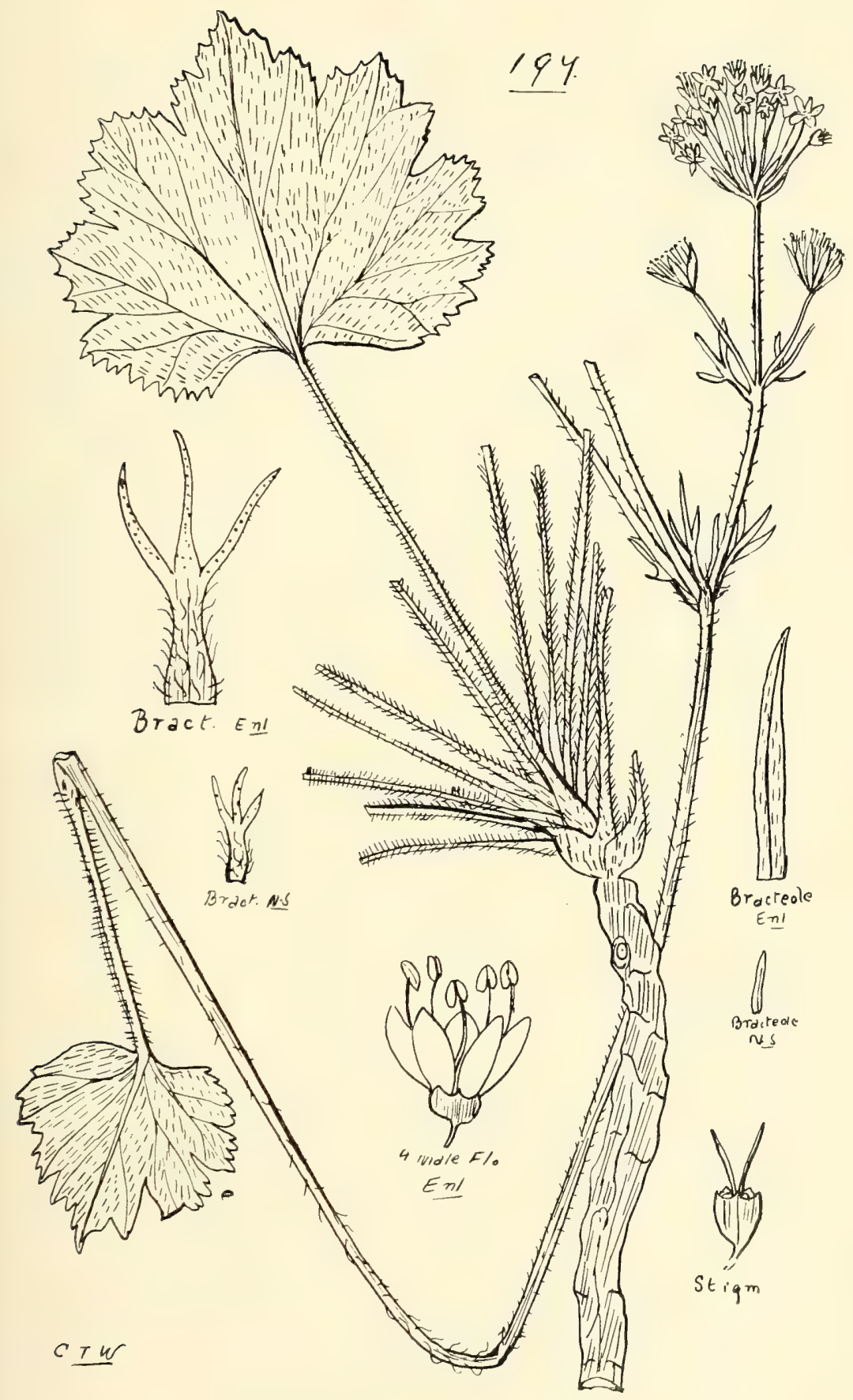

197. Trachymene geraniffolia, Bail. 


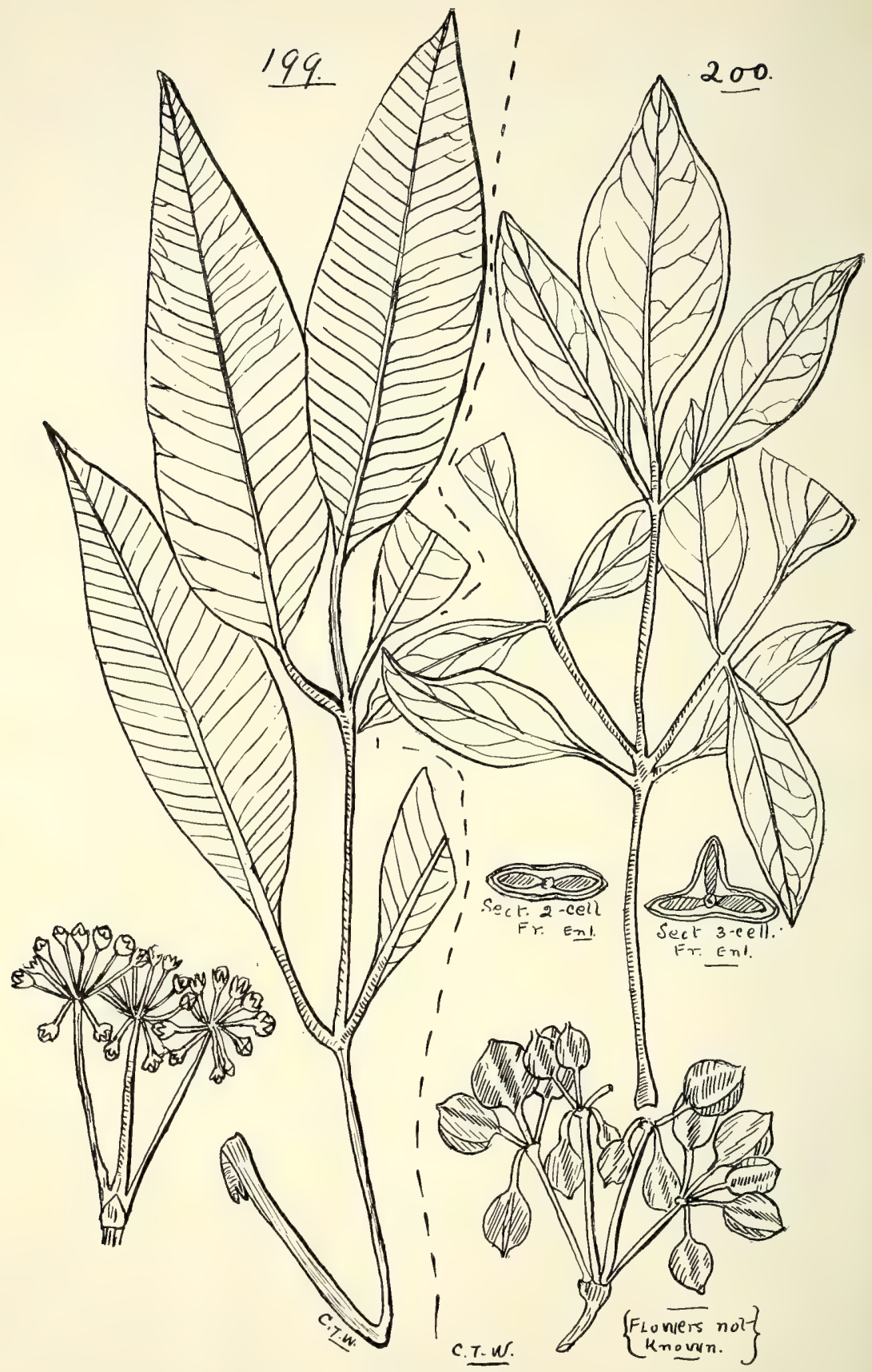

I99. Pentapanax Willmottit, F. v. M.

200. P. Bellenden-kerensis, Bail. 


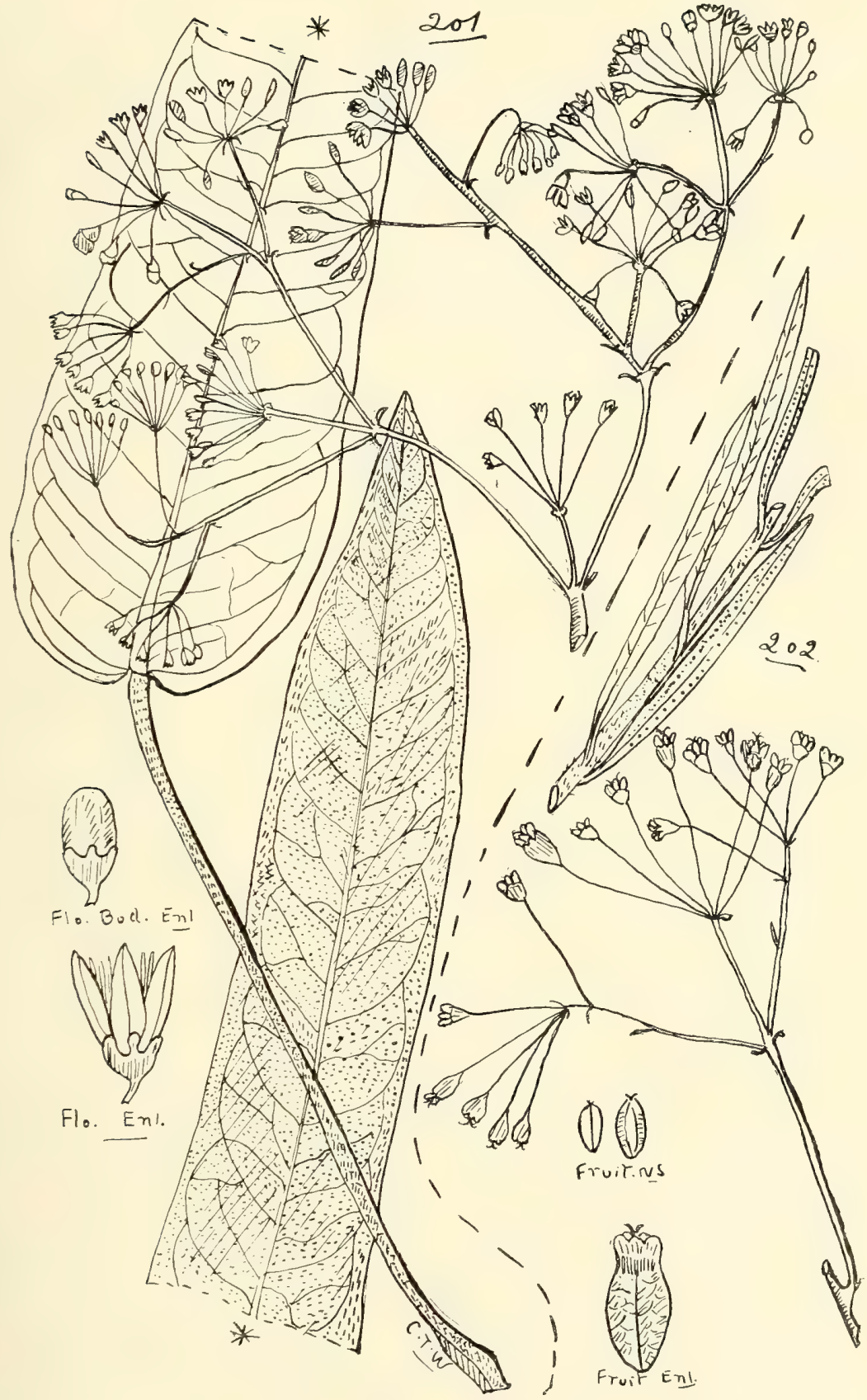

20I. Astrotricha Pterocarpa, Benth.

202. A. Biddulphiana, $F . v \cdot M$. 
Panax-contd.

cephalobotrys, $F \cdot \tau \cdot M$.

elegans, F.v.M.-Mowbulan Whitewood. "Greyanger" of Bunya Mountain natives. The wood, Mr. C. Madsen (who supplied the practical notes for my "Catalogue of Queensland Woods") considered, might be useful in the manufacture of musical instruments.

*Fatsia, Dcne.

papyrifera, Benth. and Hook., Gen. Pl.-Rice-paper plant, run out in several places near Brisbane; a native of the island of Formosa.

Heptapleurum, Gartn.

venulosum, Seem.-The leaves contain saponin.

Brassaia, Endl.

actinophylla, Endl.--Umbrella-tree.

Series IV.-Hedereæ.

Hedera, Limn.

australiana, F.v.M.

\section{Order LXII.-CORNACEÆE.}

Marlea, Roxb.

vitiensis, Benth-Muskwood of W. Hill.

rar. tomentosa, Benth. = Pseudalongium polyosmoides, F. v. M.-Bark considered poisonous by Dr. T. L. Bancroft. "Cartalogoor" of Herberton natives. (Fig. 203.)

Subclass II.-GamopetalÆ. Series I.-InFER Alliance I.-RUBIALES.

\section{Order LXIII.-CAPRIFOLIACE 2 .}

Tribe I.-Sambucee.

Sambucus, Linn.

xanthocarpa, F.v.M.=Tripetelus australasicus, Lindl.Yellow Elderberry. (Fig. 204.)

Gaudichaudiana, DC. (Fig. 205.)

Tribe II.-LONICEREÆ.

*Lonicera, Limn.-Honeysuckle.

confusa, $D C$.- Gold and Silver Flower; a native of China and Japan. 


\section{Order LXIV.-RUBIACE无.}

\section{SERIES A.}

Ovules numerous in each cell (rarely a few in Hedyotideæ).

Sarcocephalus, Afz.

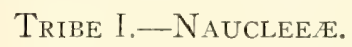

cordatus, Miq.-Leichhardt-tree or Canary-wood. "Oolpanje" of Mitchell River, "Coobiaby" of Cloncurry, "Toka" of Rockhampton, and "Koo-badg-aroo" of Dunk Island natives. "Bul-boora" of Cardwell natives (R.B.H.). Bark yields a canary-yellow dye. A good cabinet-wood. Bartlingii, Miq.-Some of the aboriginal names given under $S$. cordatus probably belong to this species.

Nauclea, Limn.

Gordoniana, Bail.-A useful wood for inside work. (Fig. 206.)

Uncaria, Schreb.

ferrea, $D C$.-Native Gambier or Terra Japonica; a powerful astringent.

Tribe II.-RondeletieE.

Wendlandia, Bartl.

basistaminea, F. v. M. (Fig. 207.)

psychotrioides, F.v.M. = Hedyotis psychotrioides, F. v. M.

Tribe III.-Hedyotide $\mathbf{E}$.

Oldenlandia, Linn.

corymbosa, Limn.

paniculata, Limn.

polyclada, F. ข.M. = Hedyotis polyclada, F. v. M.

cærulescens, F.v.M. = Hedyotis carulescens, F. v. M.

Hedyotis, Linn.

auricularia, Linn.-Has been used as a cure for deafness.

(Fig. 208.)

mitrasacmoides, F.v. M.

galioides, F.v.M. (Fig. 209.)

trachymenioides, F.v.M. (Fig. 2I0.)

Synaptantha, Hook.

tillæacea, F.v.M. = Hedyotis tilloeacea, F.v. M.

Dentella, Forst.

repens, Forst.

Ophiorthiza, Linn.

áustraliana, Benth.-Australian Snakeroot. 


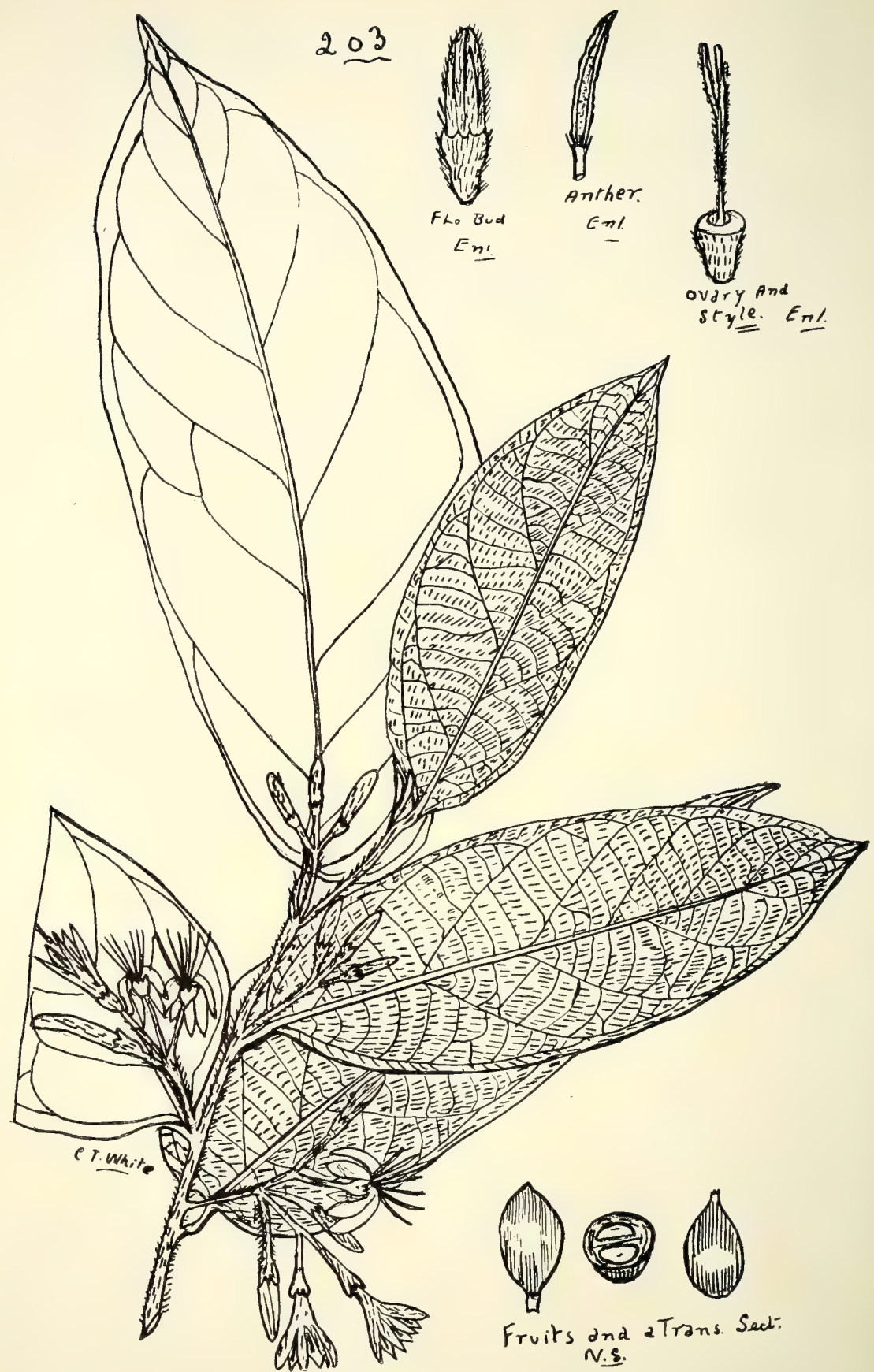

203. Marlea vitiensis, Bcnth., var. tomentosa, Benth 

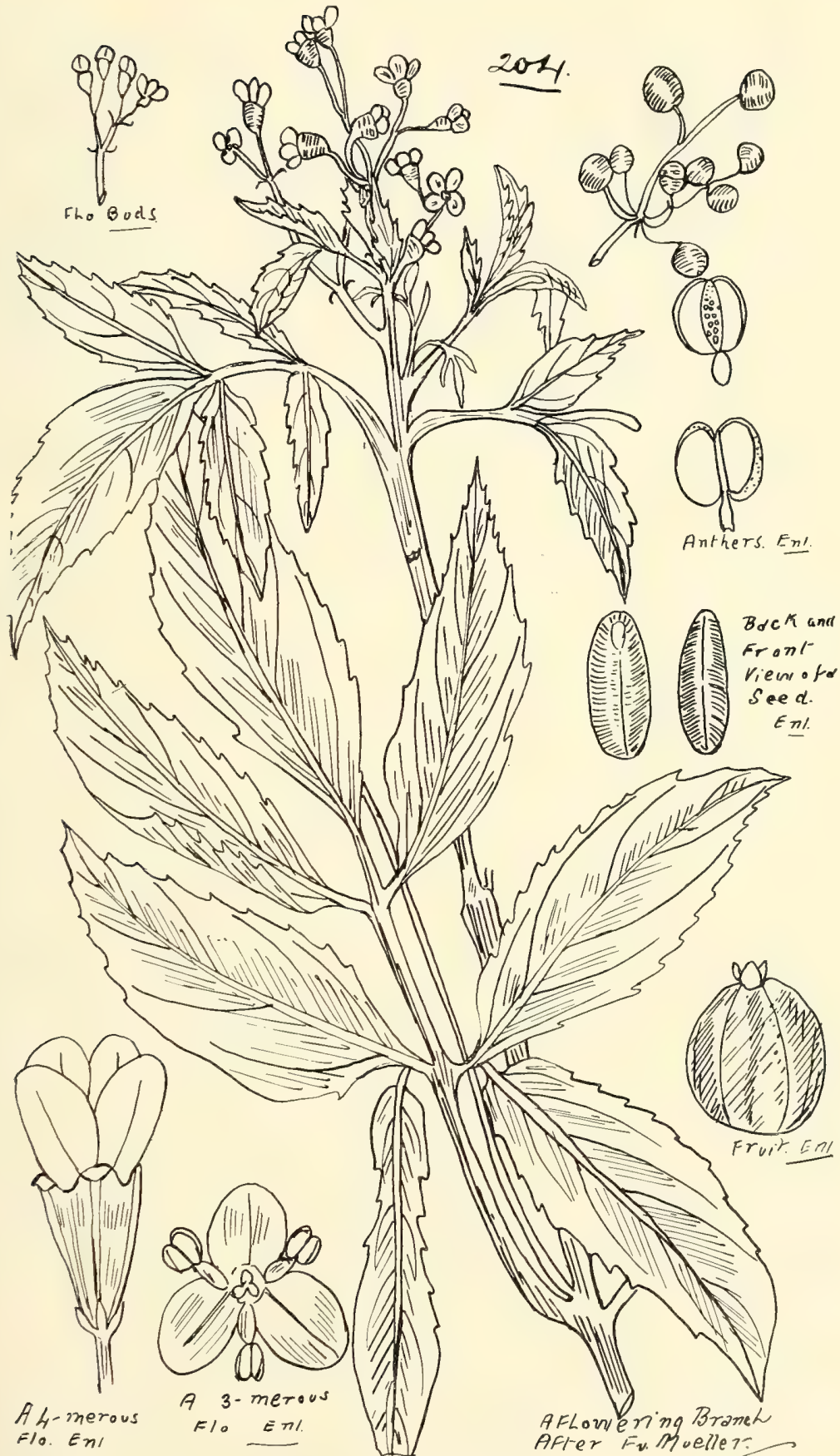

204. Sambecles xanthocarpa, F. v. $M$ 


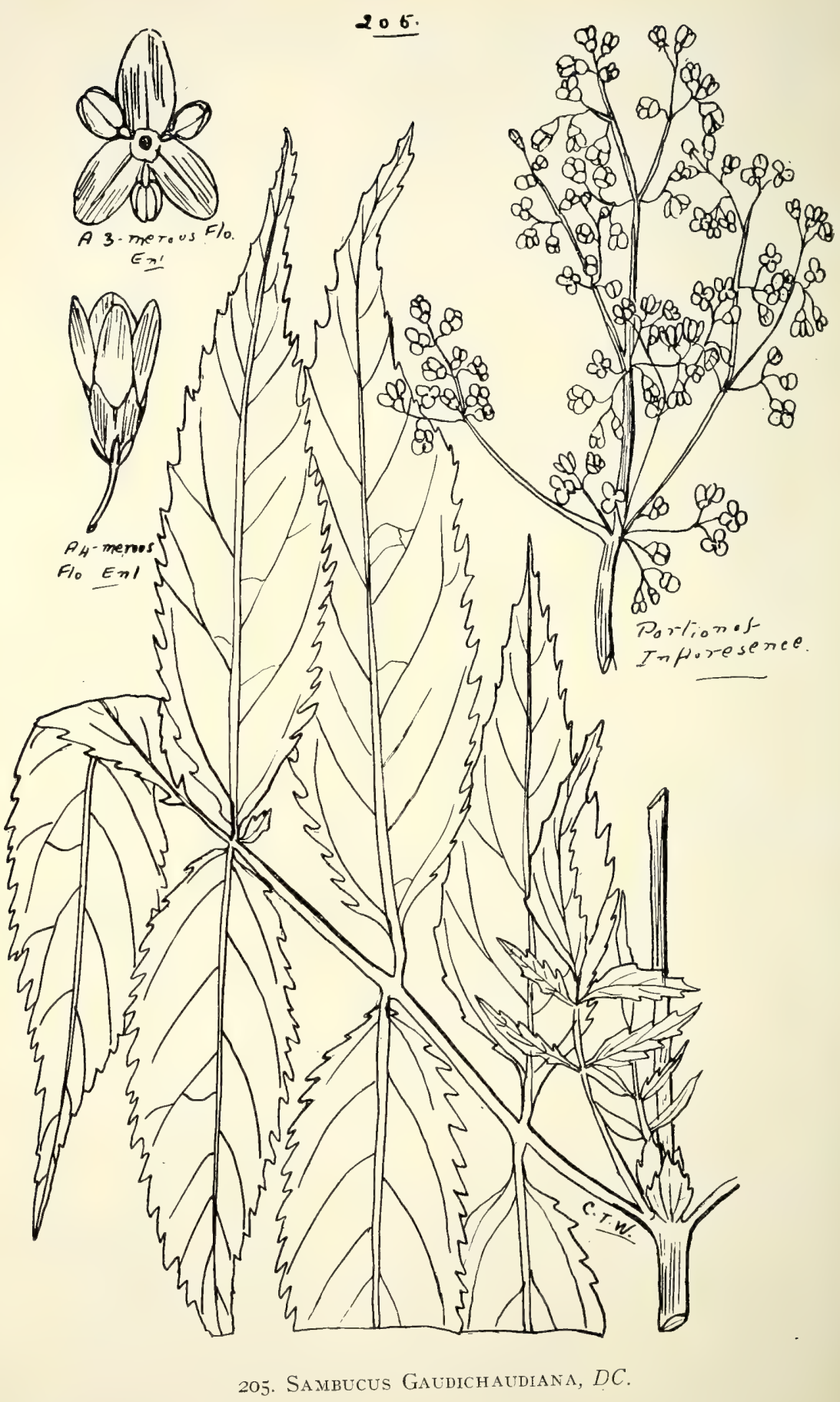


Tribe IV.-MUSSANDEX.

Abbottia, F.v. M.

singularis, F.v. $M$.

Tribe V.-Gardenieæ.

Webera, Schreb.

Dallachiana, F.v.M. (Fig. 2II.)

Randia, Linn.

hirta, F.v. M.

sessilis, F.v.M. (Fig. 2I2.)

chartacea, F.v. M. = Gardenia chartacea, F. v. M. " Whalebone," used for fishing-rods.

Moorei, F.v.M.

Fitzalani, F.v.M._- "Papajarin" of Mount Cook and "Kumar" of Bloomfield River natives. (Fig. 213.)

densiflora, Benth.

Benthamiana, F.v.M.

tuberculosa, Bail. = R. spinuligera, F. v. M. (Fig. 2r4.)

Gardenia, Limn.

edulis, F.v. $M$.

merikin, Bail._" Merikin" of Atherton natives.

megasperma, F.v. $M$.

Macgillivræi, Benth.

ochreata, F.v.M. (Fig. 215.)

suffruticosa, R. $B r$.

fucata, R. Br.

Jardinei, F.v. M.

ovularis, Bail.

Diplospora, $D C$.

australis, Benth. (Fig. 216.)

ixoroides, F.v.M. (Fig. 217.)

\section{SERIES B.}

Ovules 2 in each cell.

TRIBE VI.-Retiniphyllę.

Scyphiphora, Gertn. (Genus of uncertain affinity.)

hydrophylacea, Gartn._- Yar-ro-kal " of Cooktown natives.

Sertes C.

Ovules solitary in each cell.

Guettarda, Linn. speciosa, Limn. 


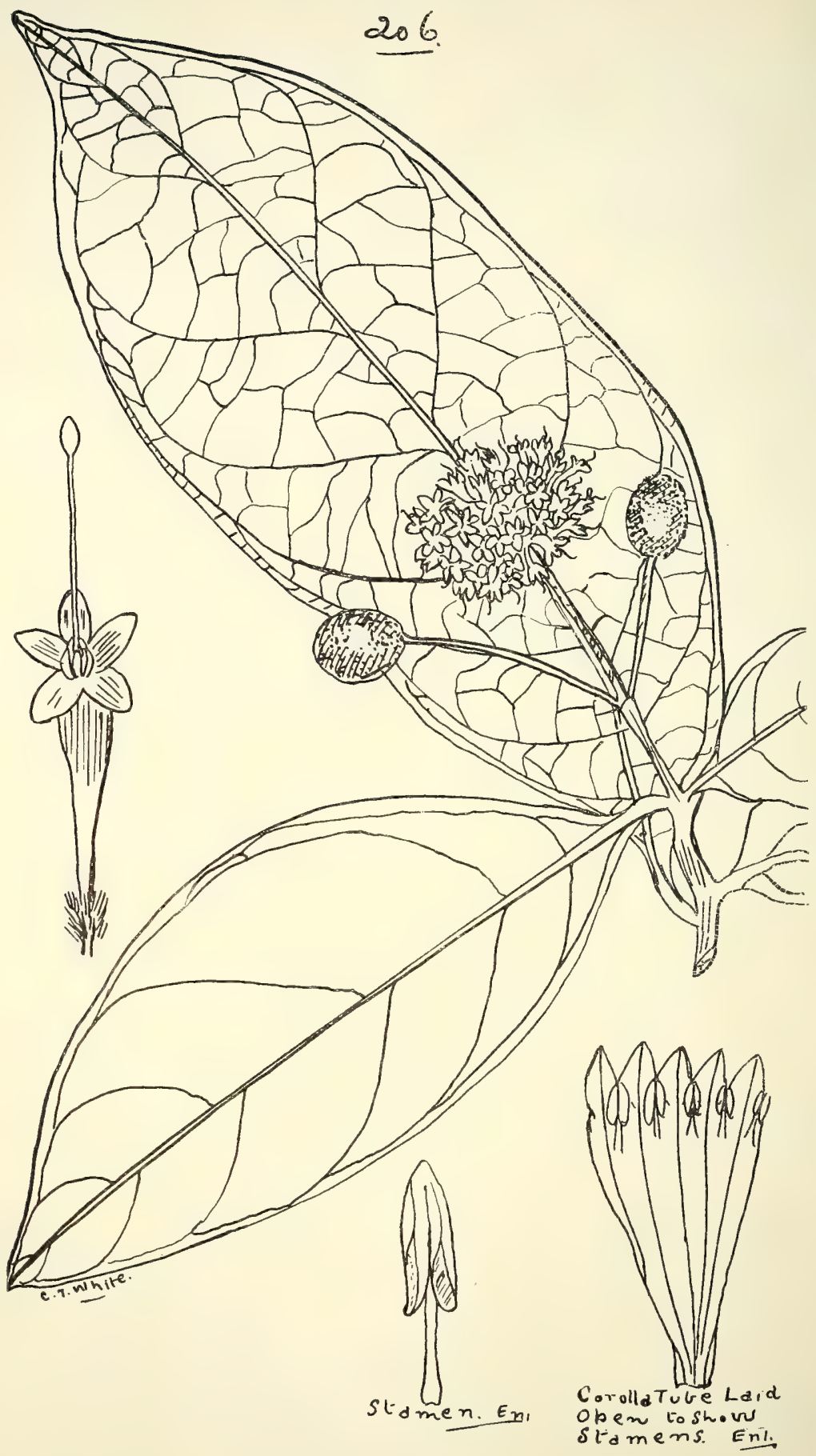

206. Nauclea Gordoniana, Bail. 


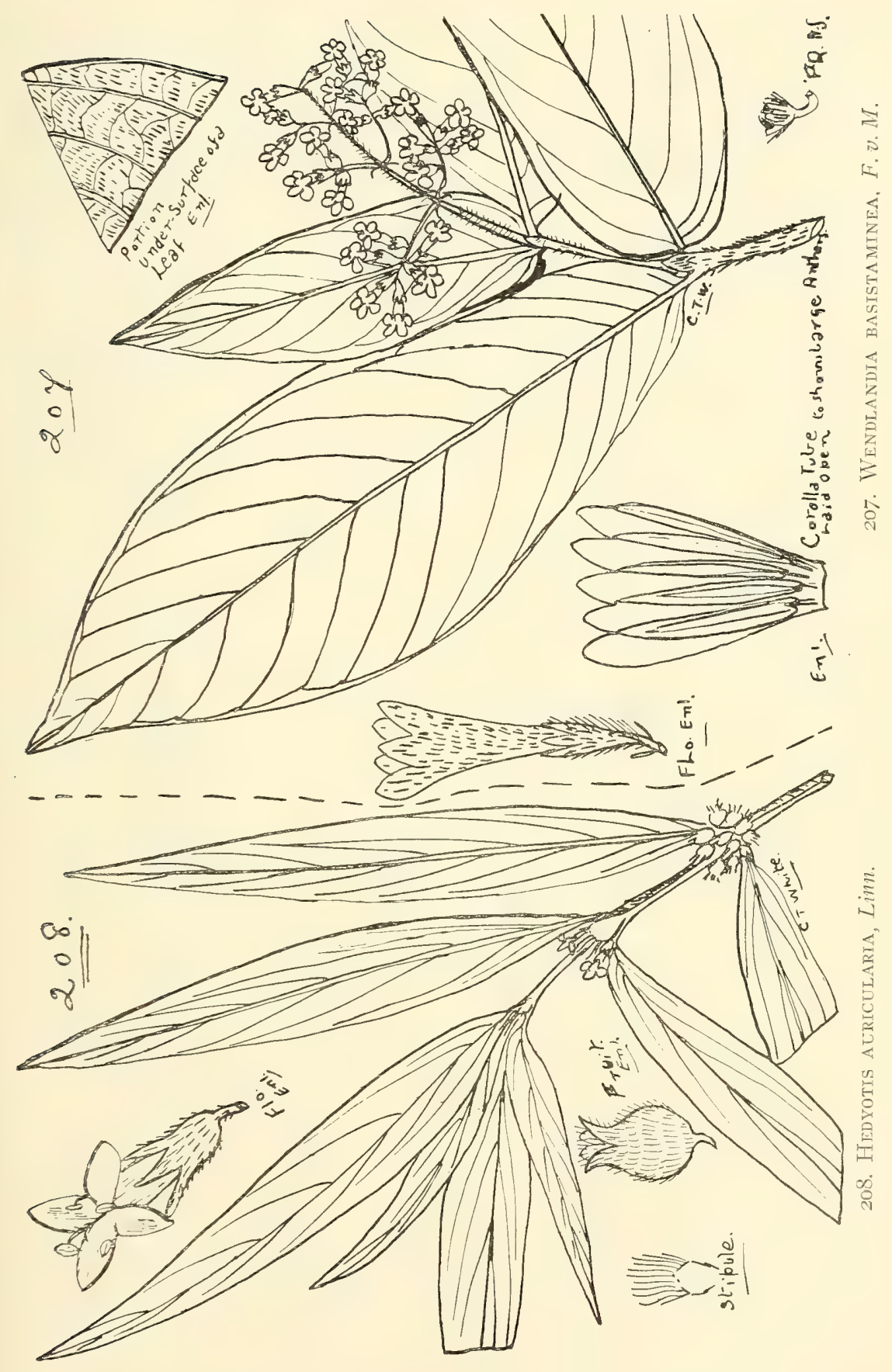




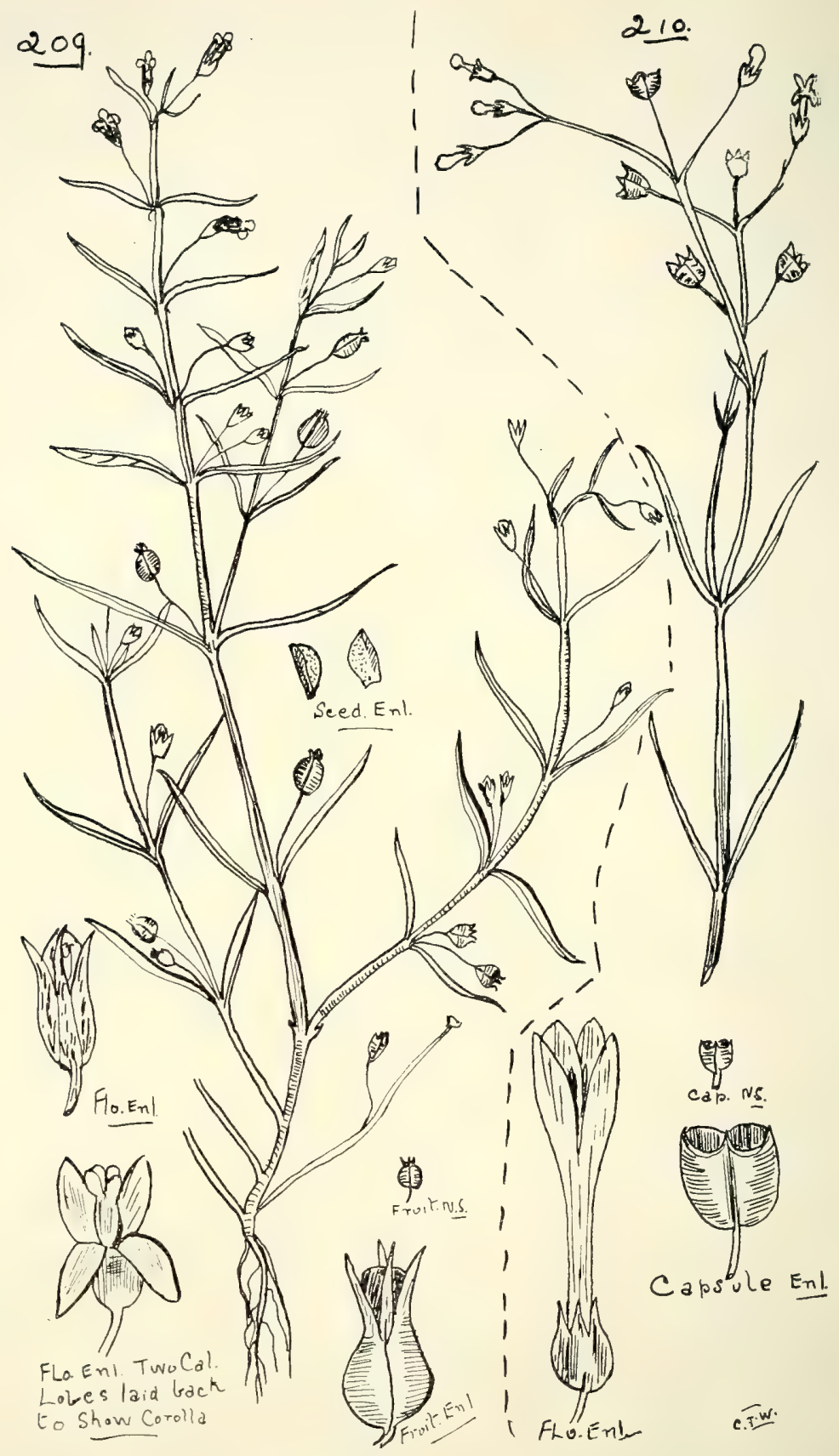

209. Hedyotis galioides, $F . v \cdot M$. 210. H. tRachy Menioides, $F$. v. $M$. 
Antirrhæa, Juss.

Tribe VII.-Guettarde.

tenuiflora, F.v.M. = Guettarda temuiflora, F.v. M.

putaminosa, Benth. and Hook. = Guettarda putaminosa,

F. v. M. Bark yields a brownish-yellow dye; wood equal to Box. (Fig. 218.)

myrtoides, F.v.M. = Guettarda myrtoides, F. v. M.

Timonius, Rumph.

Rumphii, DC.- "Kavor-kavor" of Rockhampton natives.

The wood of this tree resembles English Sycamore.

Tribe VIII.-KNoXiex.

Knoxia, Linn.

corymbosa, Willd.

Tribe IX.-Chiococceæ.

Hodgkinsonia, F.v. M.

ovatiflora, F.v.M.-Wood white, close-grained, and firm.

TRIBE X.-VANGUERIEÆ.

Canthium, Lam. = Plectronia, Linn.

latifolium, F.v.M.

attenuatum, R. Br._- "Dun-jo" of Cooktown natives. (Fig. 219.)

lucidum, Hook. and Arn.- "Warra-anji" of Batavia River natives.

oleifolium, F.v.M._ "Boorgoolbean" of St. George natives. buxifolium, Benth.

didymum, Roxb.

vaccinifolium, F.v. M.

coprosmoides, F.v.M.- "Kooni" of Bundaberg, "Bil-loobal " of Mount Cook, and “ Mar-ko" of Bloomfield River natives. Wood useful for turnery and cabinet-work.

Ixora, Linn.

TRIBE XI.-IXORE.æ.

timorensis, Dcne._- Goombargne" of Cape Grafton natives. Becklerii, Benth.

\section{Section Pentadium.}

pentamera, Benth.

Pavetta, Linn.

indica, Limn. = Ixora Pavetta, Roxb.

tomentosa, Sm. =Ixora tomentosa, Roxb.

Coffea, Linn.

benghalensis, Roxb. 


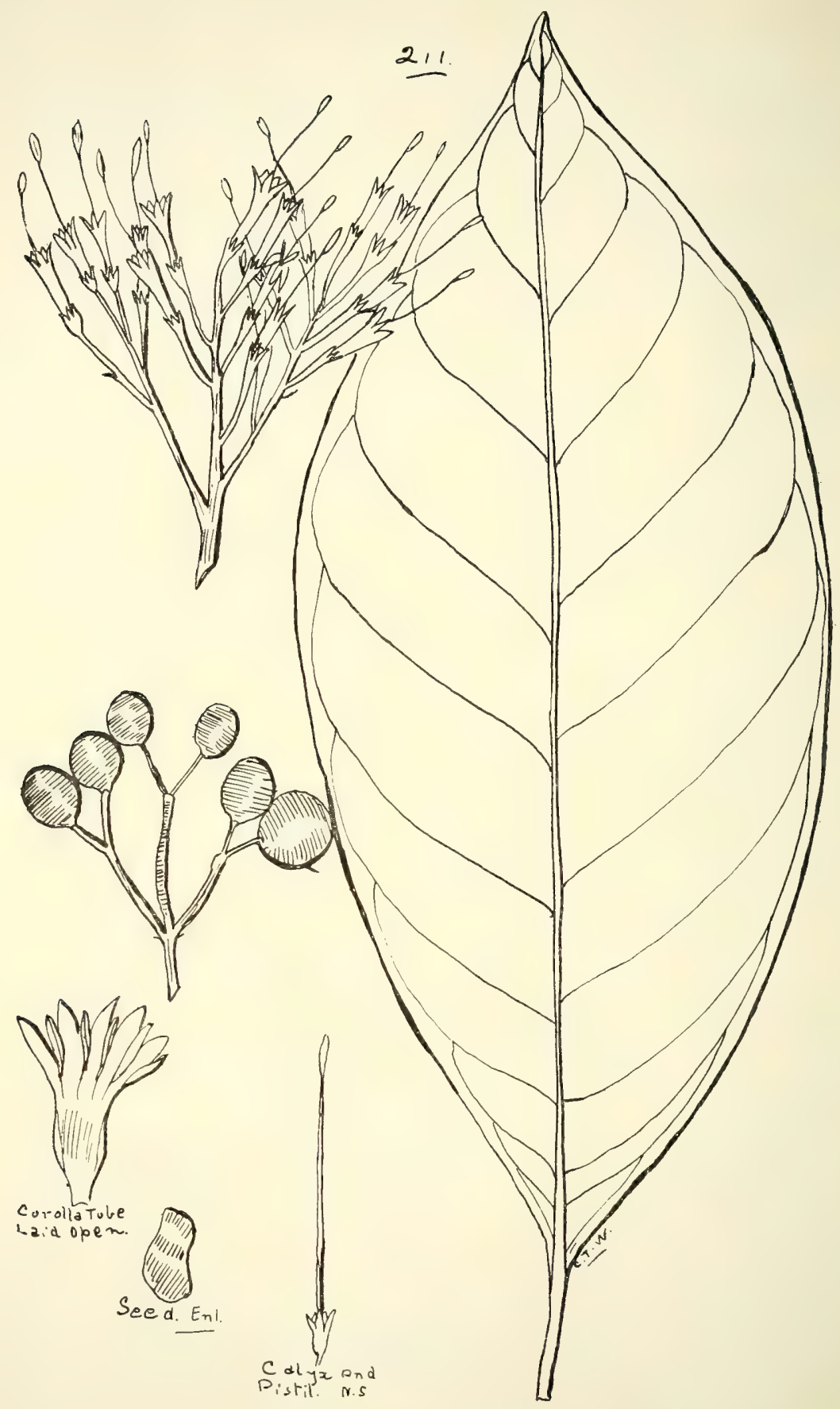

2it. Webera Dallachiana, $F, v, M$. 
LXIV. RUBIACEÆE.

247

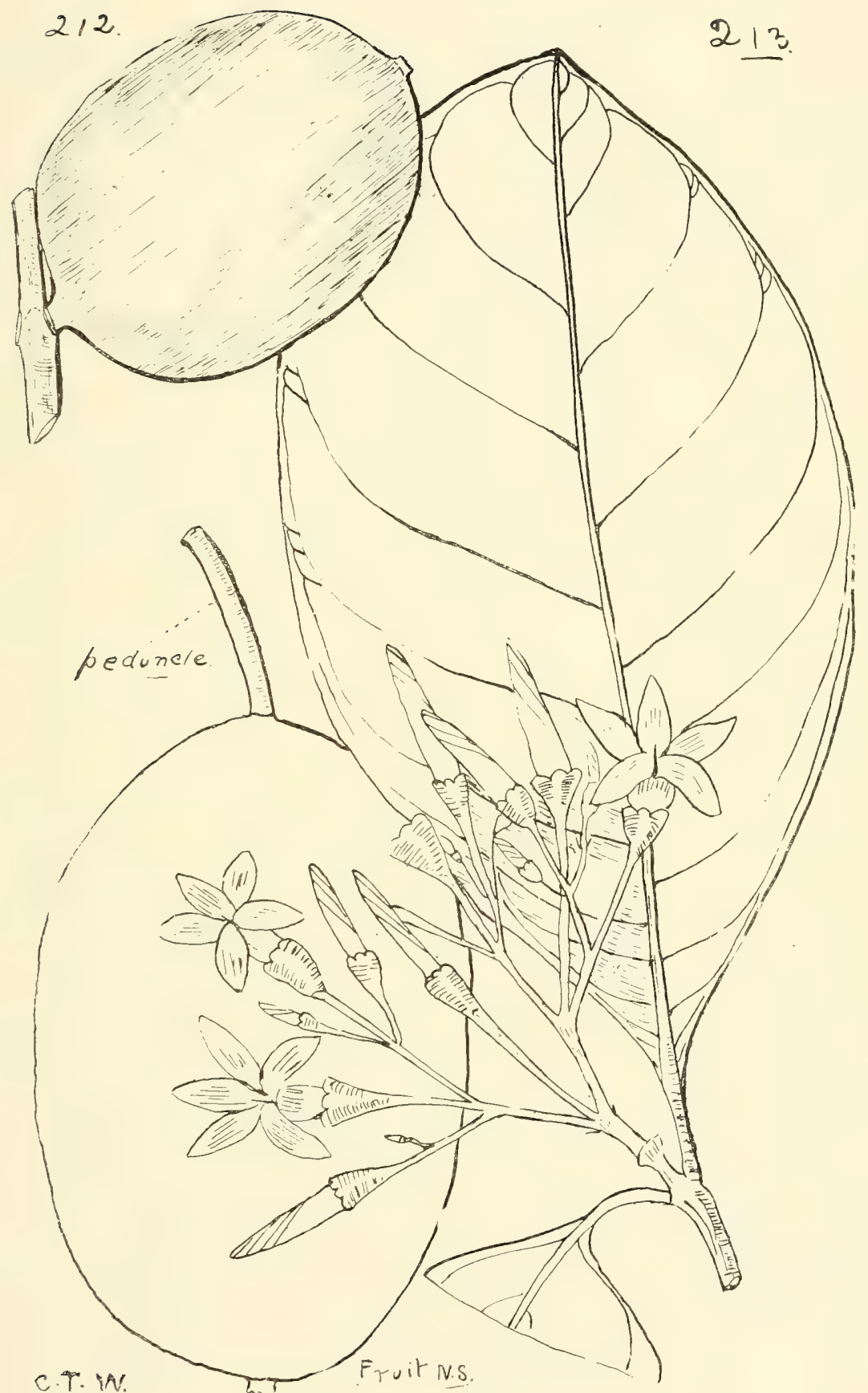

212. Randia sessilis, $F . v \cdot M$.

(Fruit only, for comparison.)

2I3. R. Fitzalani, $F, v \cdot M$. 


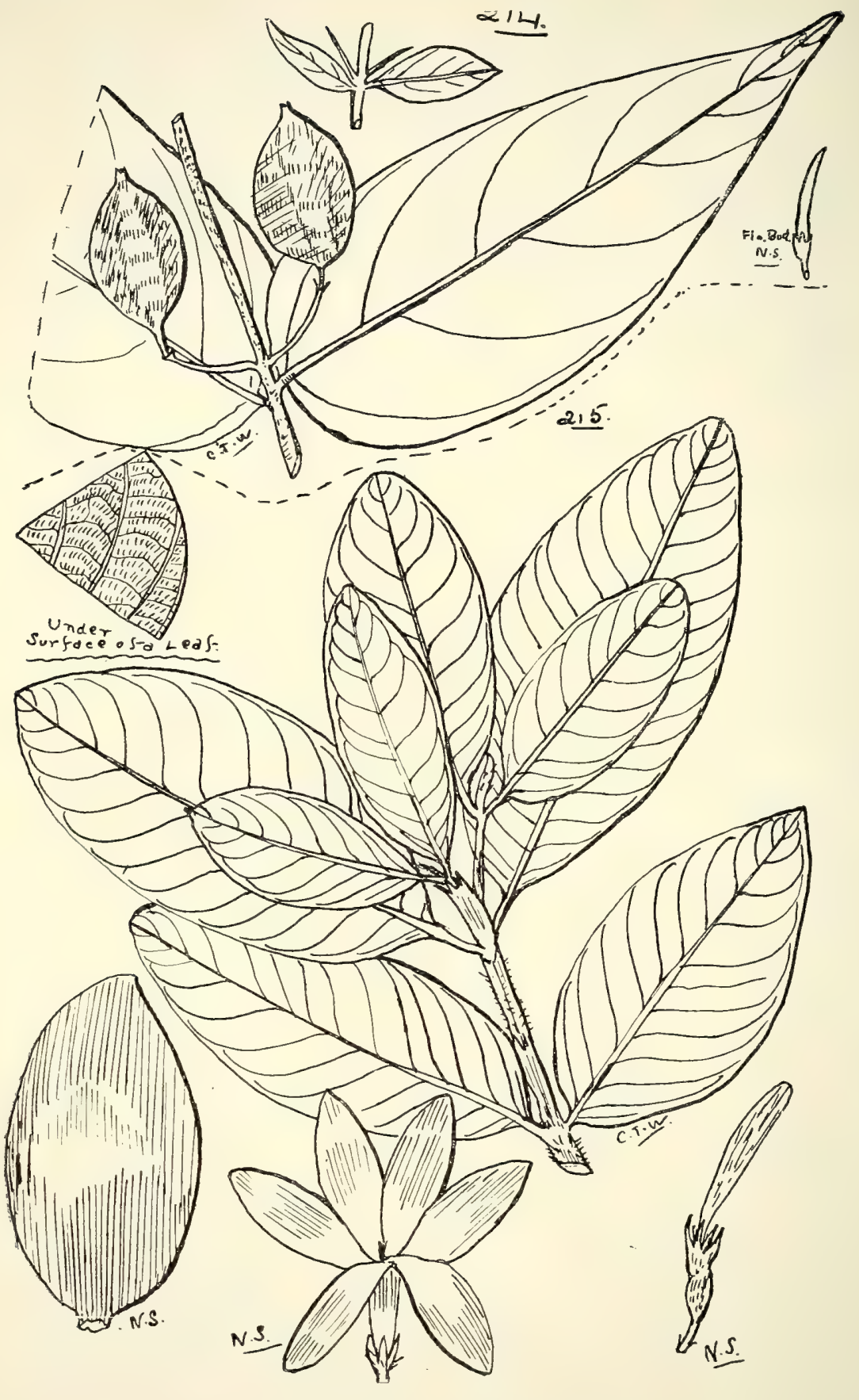

2T4. Randia tuberculosa, Bail.

215. Gardenia ochrenta, F. v. $M$. 


\section{TRIBE XII.-MorindeE.}

Morinda, Limn.

citrifolia, Limn. - "Ko-on-je-rung" of Morehead River natives.

var. bracteata, Hook.

jasminoides, A. Cunn.-Wood yellow, prettily marked.

hypotephra, F.v. M.

umbellata, Linn.

reticulata, Benth.- "Ada-a" of Mapoon natives. A showy plant from its large white leafy bracts; the yellow colouring matter from the roots is used by the natives to stain their apron-belts (Roth).

acutifolia, F.v. M.

CColospermum, Blume.

paniculatum, F.v.M._- Boorom" of Tully River natives.

(Fig. 220.)

reticulatum, Benth._-"Lu-luka" of Red Island natives.

Tribe XIII.-Psychotriem.

Psychotria, Linn.

Section I.-Grumilia.

nesophila, F.v. M.

Dallachiana, Benth._- "Yedgengen” of Barron River natives.

(Fig. 22r.)

Section II.-Mapourea.

loniceroides, Sieb.

var. angustifolia, Benth.

daphnoides, A. Cunn.

var. angustifolia, Benth.

var. pubescens, Bail., n. var.-All parts more or less pubescent.

Hab.: Nanango district (C. T. White).

poliostemma, Benth.

Fitzalani, Benth.

nematopoda, F. r. M.

Simmondsiana, Bail._- Bulbon" and "Bolbona" of Atherton natives. (Fig. 222.)

var. glabrescens, Bail.

i'ur. ? exigua, Bail. (Fig. 223.)

colospermum, Bail.

Geophila, Don.

reniformis, D. Don.-Ground Cherry.

Lasianthus, Jack.

strigosus, Wight.

graciliflorus, Bail. (Fig. 224.) 


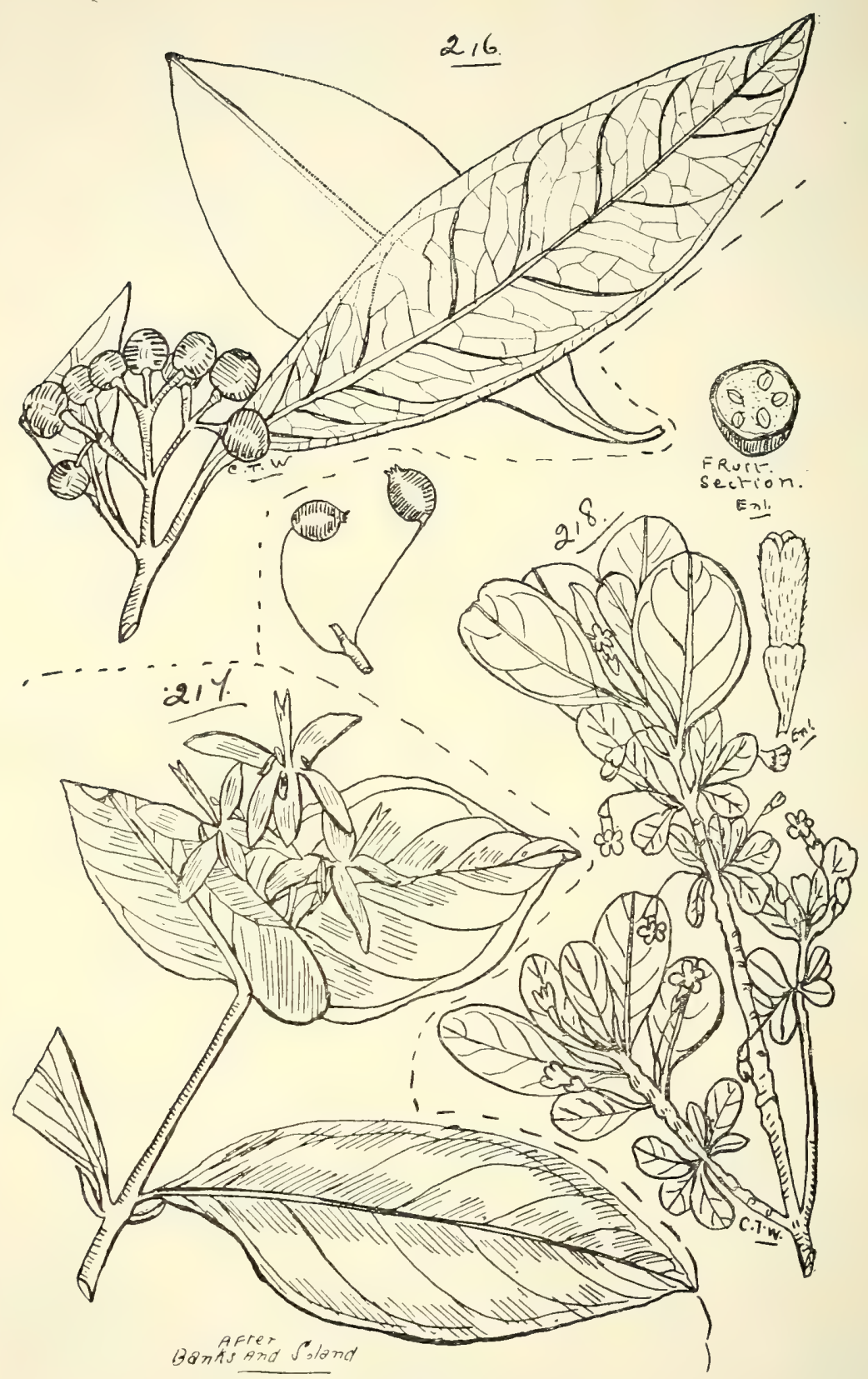

2i6. Diplosfora alstralis, Benth.

$21 \%$. D. ІхокоIDEs, F. $2 . M$.

2i8. Antirri, Ea putaminosa, Benth. et Hook. 


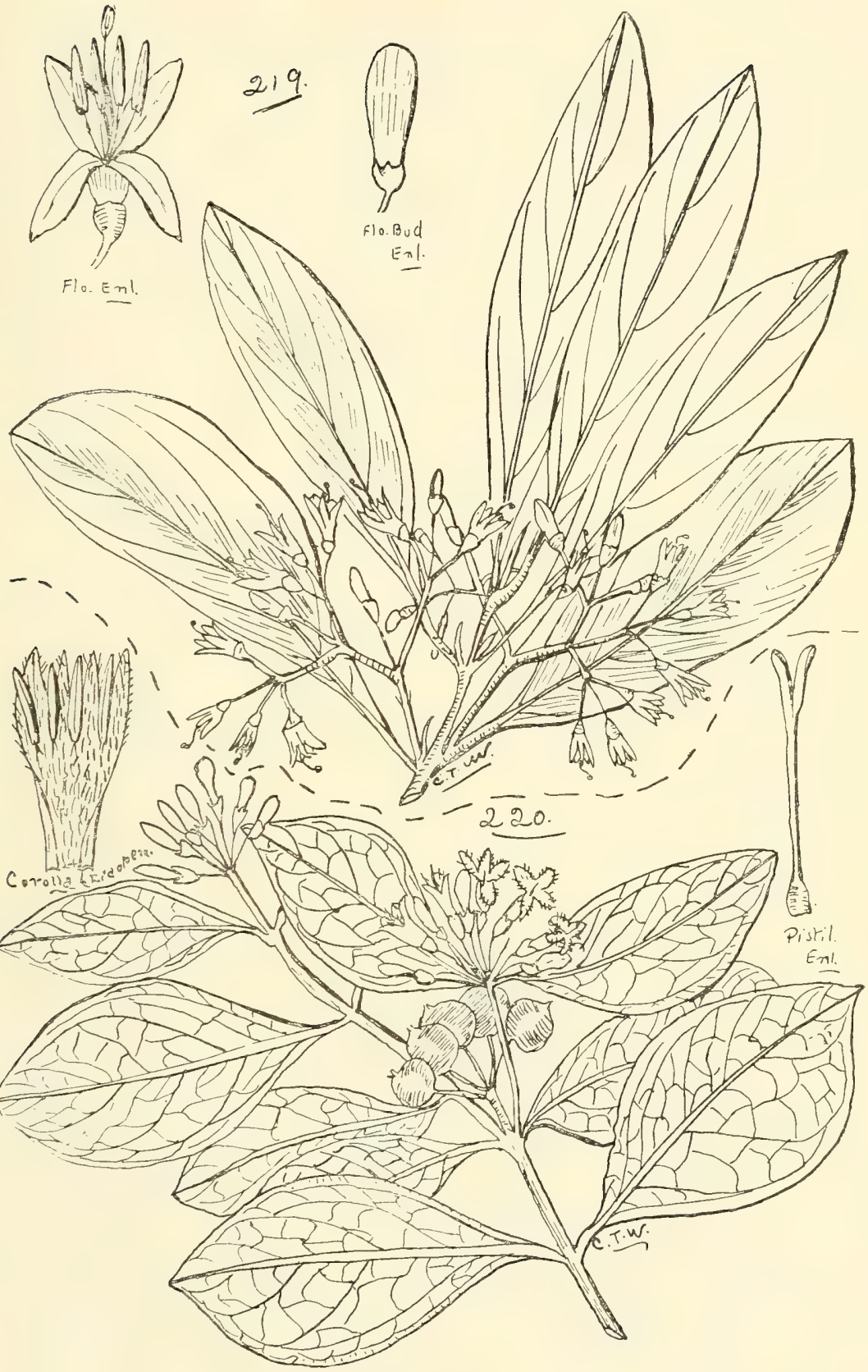

2ig. Canthium attenuatum, $R$. $B r$.

220. Ceelospermum paniculatum, $F . v \cdot M$. 


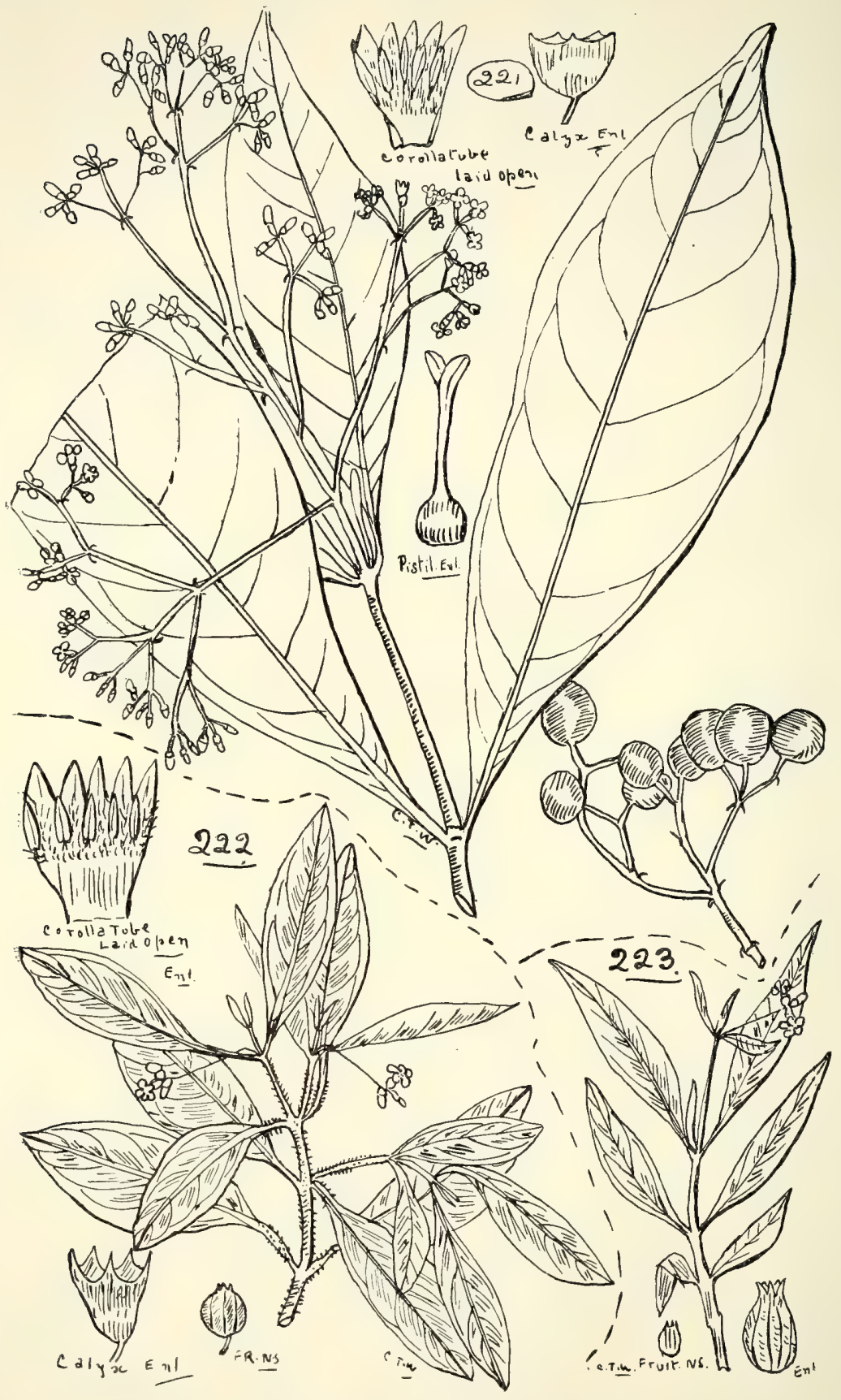

221. Psychotria Dallachiana, Benth.

222. P. Simmondsiana, Bail.

223. P. Simmondiana, var. ? exigua, Bail. 
Hydnophytum, Jack.

formicarum, Jack.-Ant-house. The stems are often hollow, and occupied by ants; the hollows, however, are not formed by these insects.

Myrmecodia, Jack.-The stems often hollow and occupied by ants, as in Hydnophytum.

Antonii, Becc.-Ant-house. (Fig. 224 bis.)

Muelleri, Becc.-Ant-house.

Beccarii, Hook.-Ant-house.

Tribe XIV.-Anthosperme..

Opercularia, Gertn.

aspera, Gartn.

var. ligustrifolia, Benth.

var. hyssopifolia, Benth.

diphylla, Gartn.

varia, Hook. f.

Pomax, Soland.

umbellata, Sol.

Tribe XV.-Spermacocez.

Spermacoce, Limn.

brachystema, $R . B r$.

pogostoma, Benth.

var. hispida, Bail.

uniseta, Bail. (Fig. 225.)

marginata, Benth.

multicaulis, Benth.

Jacobsoni, Bail. (Fig. 226.)

var. glabrescens, Bail., $n$. var.-Plant glabrescent.

Hab.: Herberton (Dr. F. H. Kenny).

exserta, Benth.

membranacea, $R . B r$. (Fig. 22\%.)

debilis, Benth.

stenophylla, F.v.M. (Fig. 228.)

lævigata, F.v.M. (Fig. 229.)

var. ? hispida, Benth.

var. ? dilatata, Benth.

breviflora, $F, v . M$.

suffruticosa, R. Br.

*Richardsonia, Kunth.

scabra, Limn--Tropical America. The roots of this weedi have been used in medicine under the name of "White Ipecacuanha," but their effect is said to be less certain. stellaris, Cham.-Tropical America. 


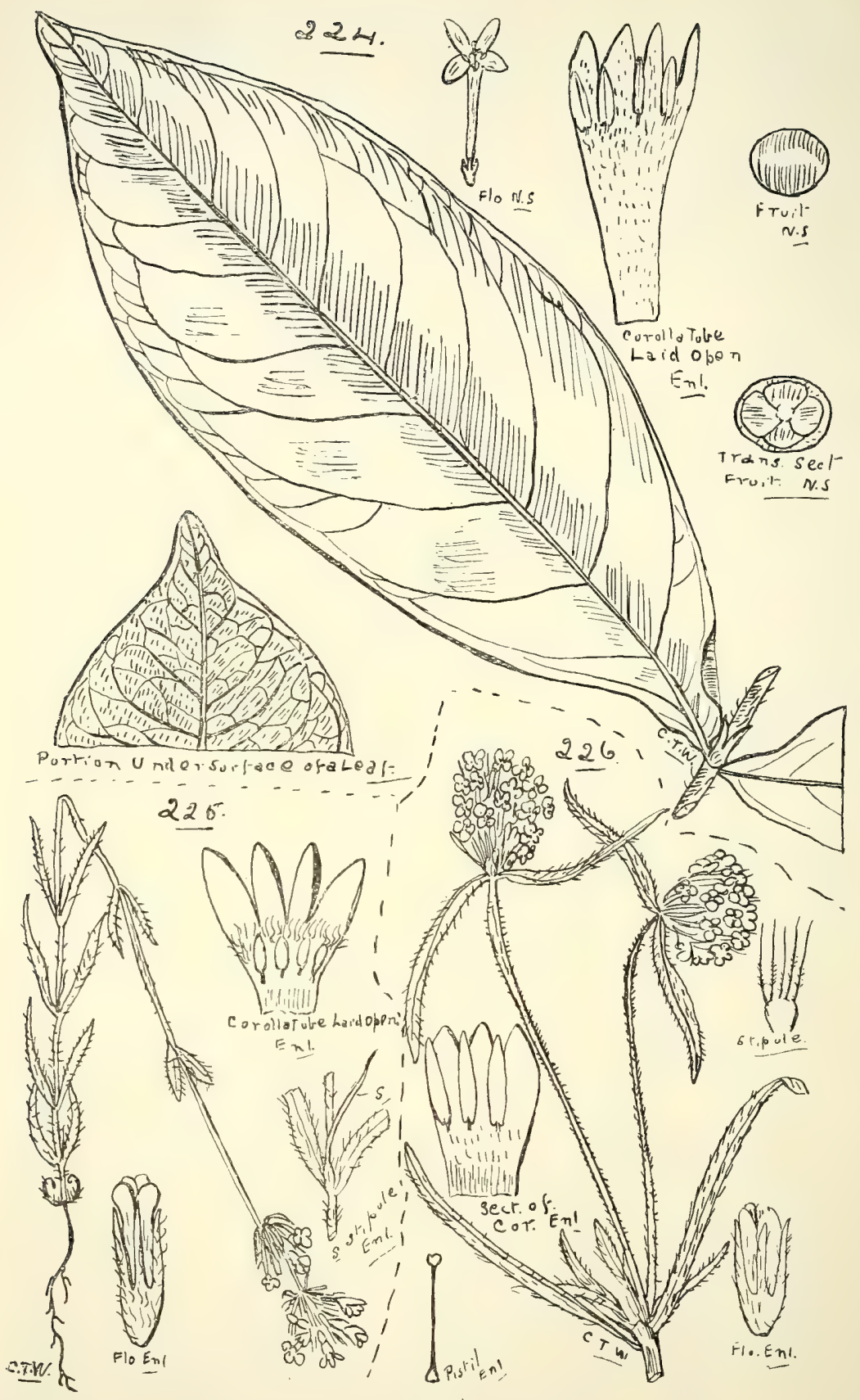

224. Lasianthus graciliflorus, Bail.

225. Spermacoce uniseta, Bail.

226. S. Jacobsont, Bail. 
224 bis.

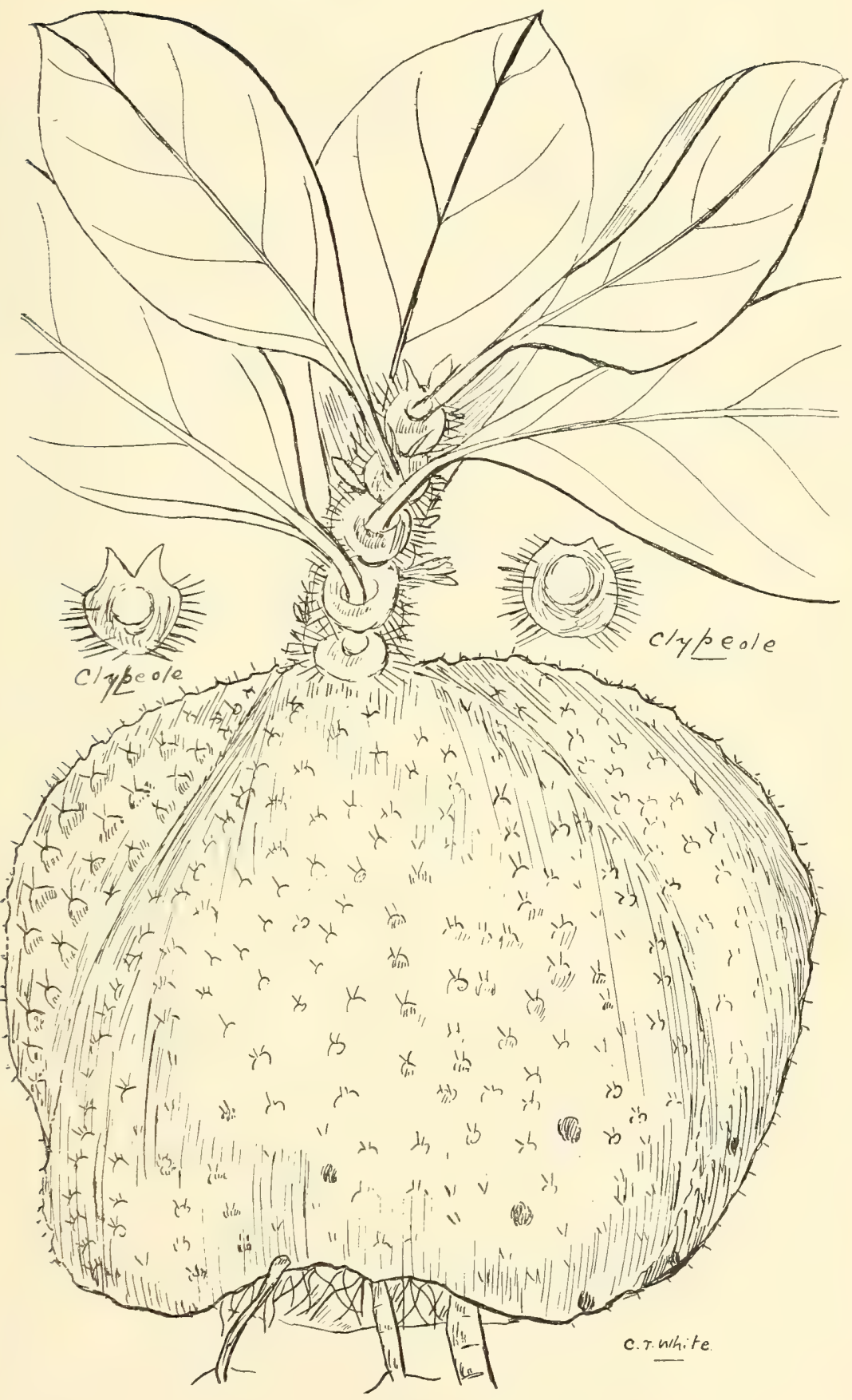

224 bis. Myrmecodia Antonit, Becc. 


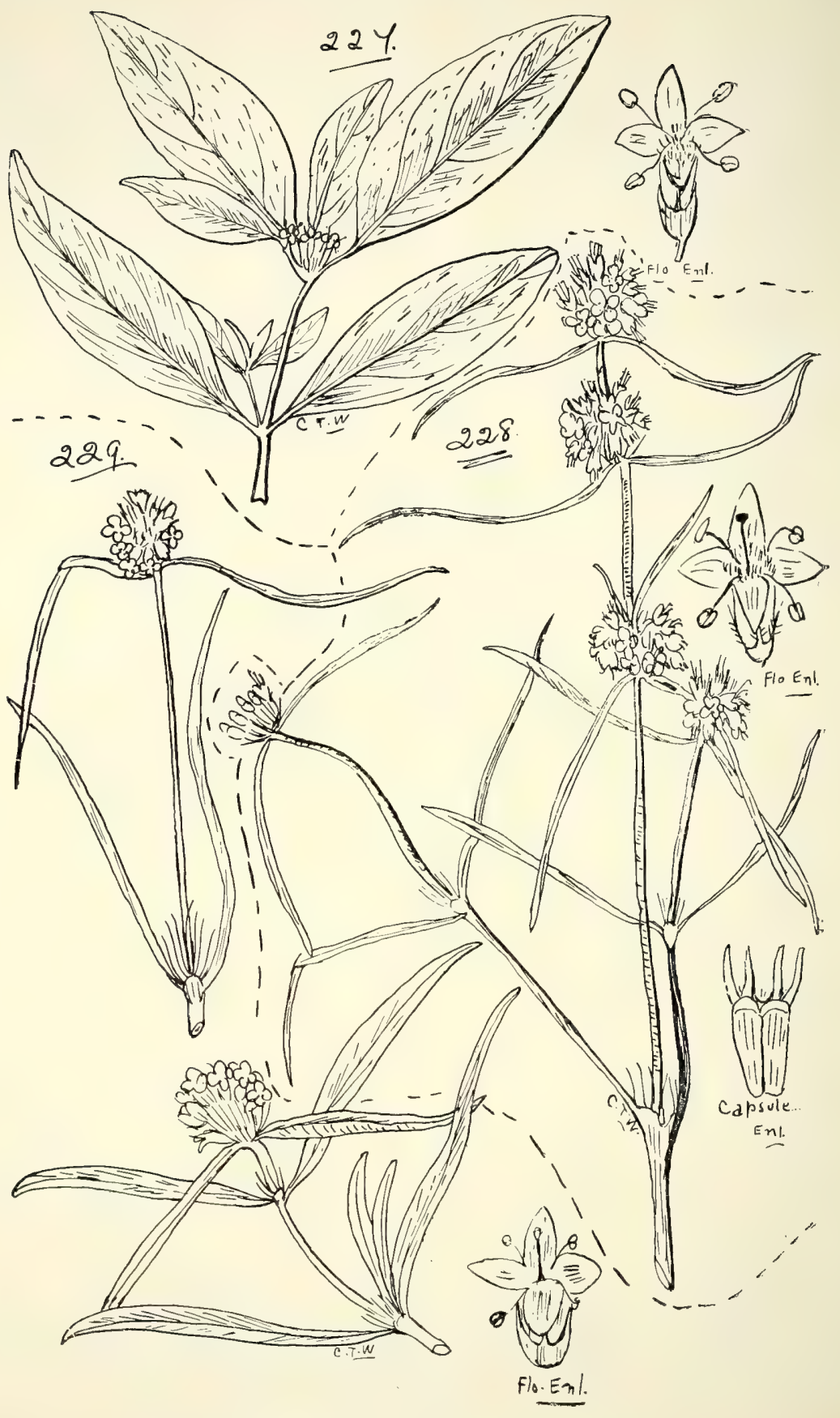

227. Spermacoce membranacea, Benth.

228. S. STENOPHyLla, $F . v . M$.

229. S. LevigatA, $F . v . M$. 
Galium, Linn .

\section{Tribe XVI.-Galiez.}

Gaudichaudii, $D C$.

var. glabrescens, Benth.

var. muriculatum, Benth.

australe, $D C$.-Australian Bedstraw.

*aparine, Linn., war. minor, Benth.-Cleavers or Goose-grass,

Europe.

Asperula, Linn.

geminifolia, F.v.M.

scoparia, Hook.

conferta, Hook.

var. elongata. Benth.

Alliance II.-ASTERALES.

\section{Order LXV.-COMPOSITA.}

The fruits of this Order are called by Mirbel and Lindley

"Cypsela"; most other botanists use the word "Achene."

TrIbe I.-VERNONIACEæ.

Ethulia, Linn.

conyzoides, Limn. $f$.

Centratherum, Cass.

muticum, Less.

Pleurocarpæa, Benth.

denticulata, Benth.

Vernonia, Schreber.

cinerea, Less.-There are two principal varieties in Australia, one either nearly glabrous or rarely hirsute, with short rigid hairs, the other very hoary-tomentose or woolly, with softer more obtuse leaves, and usually smaller flower-heads; the corolla-lobes also appear shorter. The flowers very purple or white, but almost always purple in the more glabrous form.-Bentham, Flora Austr. iii. 460.

These forms are probably among my Queensland specimens, but such have never been good enough to fully describe the distinctions (F.M.B.).

scandens, $D C$.

Elephantopus, Linn.

scaber, Linn. 
Tribe II.-Eupatoriace.玉.

Adenostemma, Forst.

viscosum, Forst.

Eupatorium, Linn.

cannabinum, Linn.--Hemp Agrimony.

Ageratum, Linn.

conyzoides, Linn-Billygoat plant. South America. The foliage at times variegated. In India the juice is consiclered a good remedy in cases of Prolapsus ani, if freely applied, and the parts replaced (Dymock). The plant is held in great esteem in South America as a remedy for metrorrhagia (Pharm. Journ. June I9Io)

var. mexicana, Hemsl.

var. album.

Dichrocephala, $D C$.

TRIBE III.-ASTEROIDEE.

latifolia, $D C$.

Lagenophora, Cass.

Billardieri, Cass.

forma microcephala, Benth.

forma normalis, Benth.

Solenogyne, F. v. M.

Emphysopus, Hook. f.

Brachycome, Cass.

Section 1.--Brachystephium.

diversifolia, Fisch. et Meyer.

melanocarpa, Sond. et F. $\%$.

pachyptera, Turc:.

Section 11--Paquerina.

microcarpa, F.v.M.-Brisbane Daisy.

Stuartii, Benth.

scapigera, $D C$.

graminea, F.v. $M$.

basaltica, F.v.M. (Fig. 230.)

var. gracilis, Benth.

trachycarpa, F..$M$.

Section III.-Brachycome.

decipiens, Hook.-Downs Daisy.

chrysoglossa, F. ซ. M.

discolor. C. Stuart.

multifida, $D C$.

ciliaris, Less

scapiformis, $D C$.

marginata, Benth. 
Minuria, $D C$.

leptophylla, $D C$.

var. ? hispida, Benth.

integerrima, Benth.

denticulata, Benth.

Cunninghamii, Benth.

suredifolia, F.v.M.

Calotis, R. Br.-Many of the species are noxious weeds, but it would be impossible to get rid of them by Act of Parliament.

Section Eucalotis.

dentex, R. Br.-White Burr Daisy.

cuneifolia, $R, B r$.

Section Cymbaria.

cymbacantha, F.v.M.

var. pumila, Benth.

erinacea, Steetz.

Section Acantharia.

scabiosifolia, Sond. et Muell.--"Bindii" of inland natives.

var. lasiocarpa, F.v.M.

var. pubescens, F.v.M.

var. integrifolia, F.v. M.

var. elongata, Benth.

var. ? cuneata, F.v.M.

scapigera, Hook.

lappulacea, Benth.-Yellow Burr Daisy.

microcephala, Berk.

breviseta, Benth.

plumulifera, F.v.M.

porphyroglossa, F. v.M.--"Bindii." This name is used by bushmen for many kinds of burr-weeds.

pterosperma, R. $B r$.

Section Cheiroloma.

hispidula, F.v.M.

Olearia, Møench.

stellulata, $D C$.

Section Asterotriche.

var. canescens, Benth.

Nernstii, F.v.M.

hygrophila, Benth. 


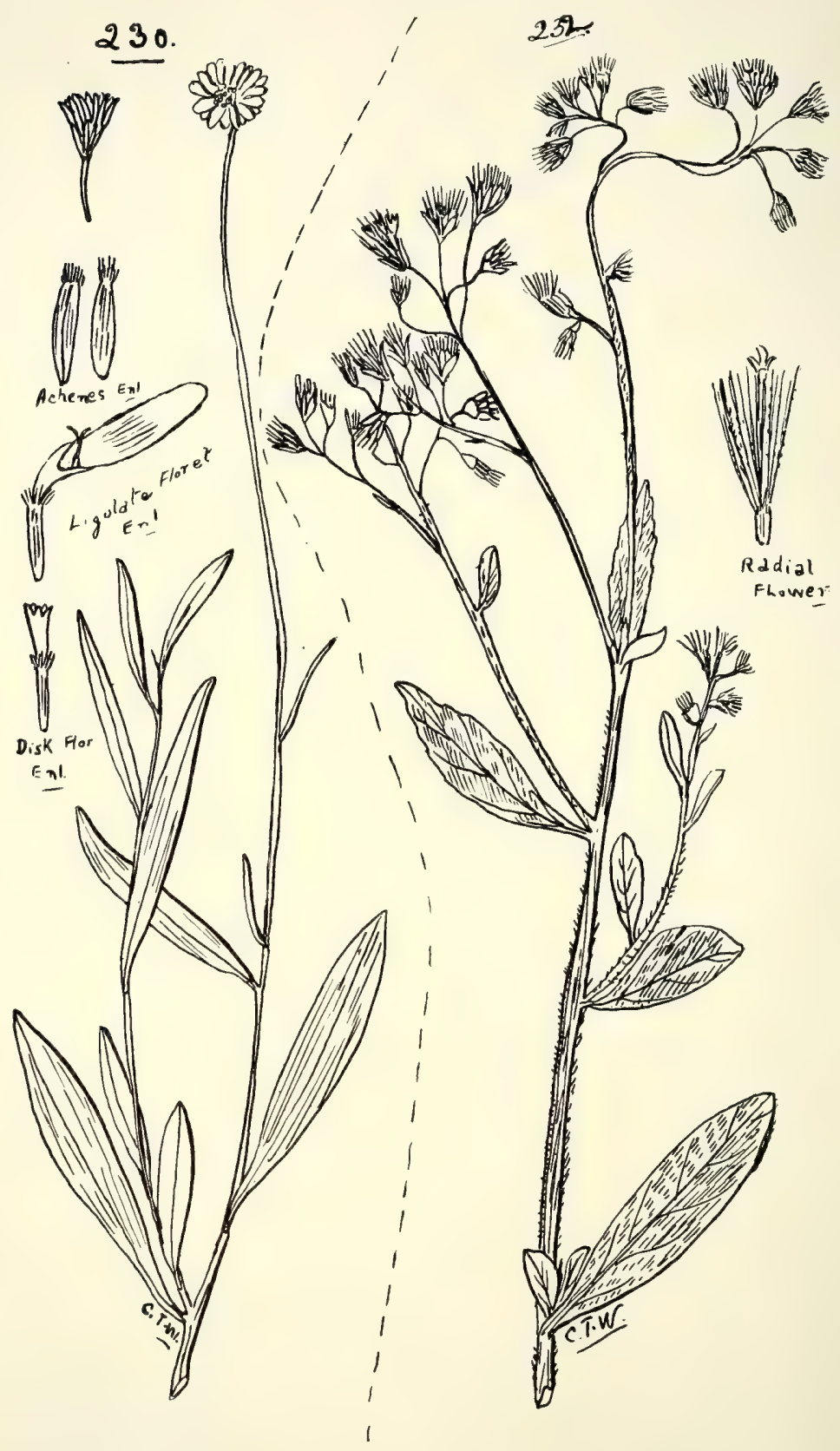

230. Brachycome basaltica, $F \cdot v \cdot M$.

232. Conyza egyptiaca, Ait. 


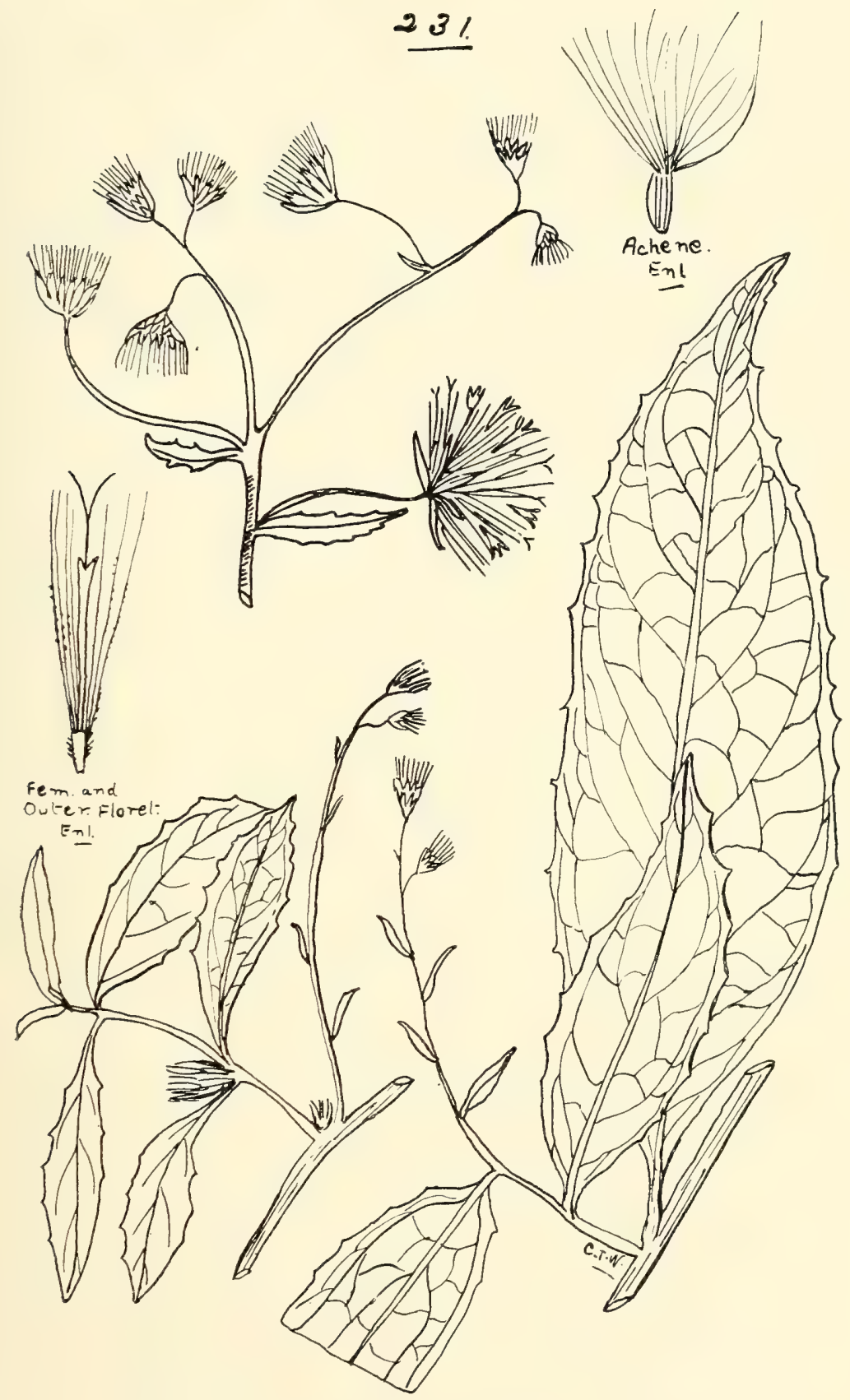

23i. Conyza elata, Bail. 
Olearia-contd.

Section Eriotriche.

ramulosa, Benth.

var. microphylla, Benth.

var. communis, Benth.

subspicata, Benth.

ramosissima, Benth.

pimeleoides, Benth.

Section Adenotriche.

magniflora, F. v. M.

elliptica, $D C$.

Section Merismotriche.

xerophila, F.v. M.-Warrego Spinifex-bush.

rudis, F.v. M.

arguta, Benth.

Podocoma, Less.

cuneifolia, R. Br.

Erigeron, $\operatorname{Linn}$.

Section Euerigeron.

ambiguus, F.v. M.

*canadensis, Linn.-Canadian Flea-bane.

Section Canotus.

linifolius, Willd.-Cobbler's-pegs; Rag-weed.

Vittadinia, A. Rich.

Section Vittadinia vera.

brachycomoides, $F, v, M$.

var. ? latifolia, Benth.

australis, A. Ruch.

var. dissecta, Benth.

zar. tenuissima, Benth.

scabra, $D C$.

Section Eurybiopsis.

macrorrhiza, A. Gray.

Conyza, Linn.

elata, Bail. (Fig. 23r.)

viscidula, $W$ all.

xgyptiaca, Ait. (Fig. 232.)

*Baccharis, Linn.

halimifolia, Linn.-Groundsel-tree. America. 
Tribe IV.-INULOIDEA.

Blumea, DC.

glandulosa, $D C$.

var. minor, Benth.

amplectens, $D C$.

integrifolia, $D C$.

diffusa, R. Br.

hieracifolia, $D C$.

var. holosericea, Benth.

Cunninghamii, $D C$.

lacera, $D C$.

acutata, $D C$

Pluchea, Less.

Section Pluchea.

Dioscoridis, $D C$.

indica, Less.--" Onogona" of Mapoon natives (J. F. Bailey). baccharoides, $F . \tau \cdot M$.

tetranthera, F.v.M. (Fig. 233.)

var. tomentosa, Benth.-Whole plant closely but softly tomentose. Clermont (H. Salmon). (Fig. 234).

Section Eyrea.

Eyrea, F.v.M. = Eyrea rubelliflora, F.v. M.

dentex, R. Br. (Fig. 235.)

Pterigeron, $D C$.

liatroides, Benth.

odorus, Benth.

macrocephalus, Benth. (Fig. 236.)

microglossus, Benth.

adscendens, Benth.

Thespidium, F.v. M.

basiflorum, F.v.M.

Coleocoma, F.v.M.

centaurea, F.v.M. (Fig. 237.)

Epaltes, Cass.

Cunninghamii, Benth.

australis, Less.

Harrisii, F.v.M.

Sphæranthus, Willd.

hirtus, Willd.

microcephalus, Willd. 


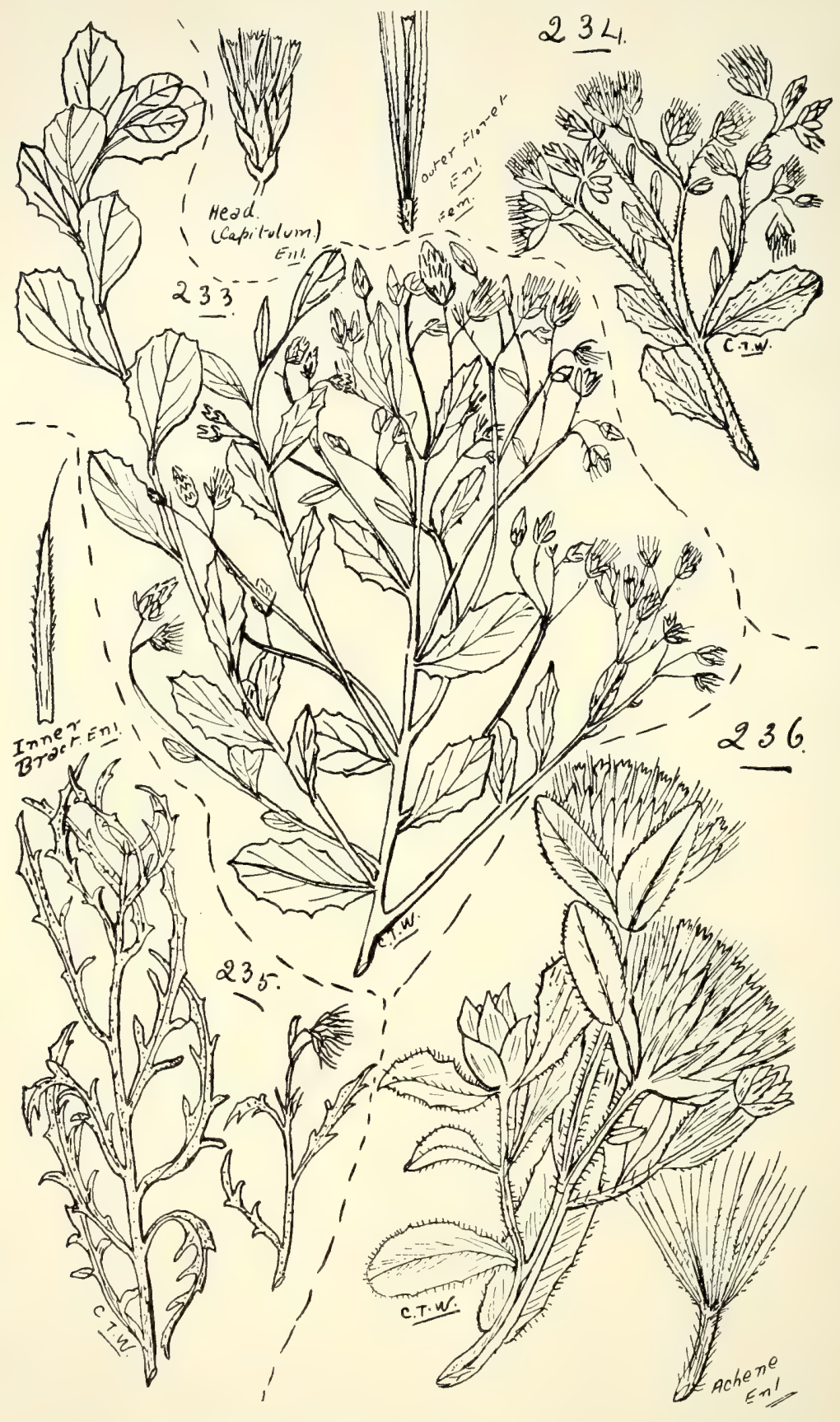

233. Pluchea tetranthera, $F$. v. $M$.

234. P. tetranthera, $F . v . M$., var. tomentosa, Benth.

235. P. DENTEX, R. Br.

236. Pterigeron macrocephalus, Benth. 


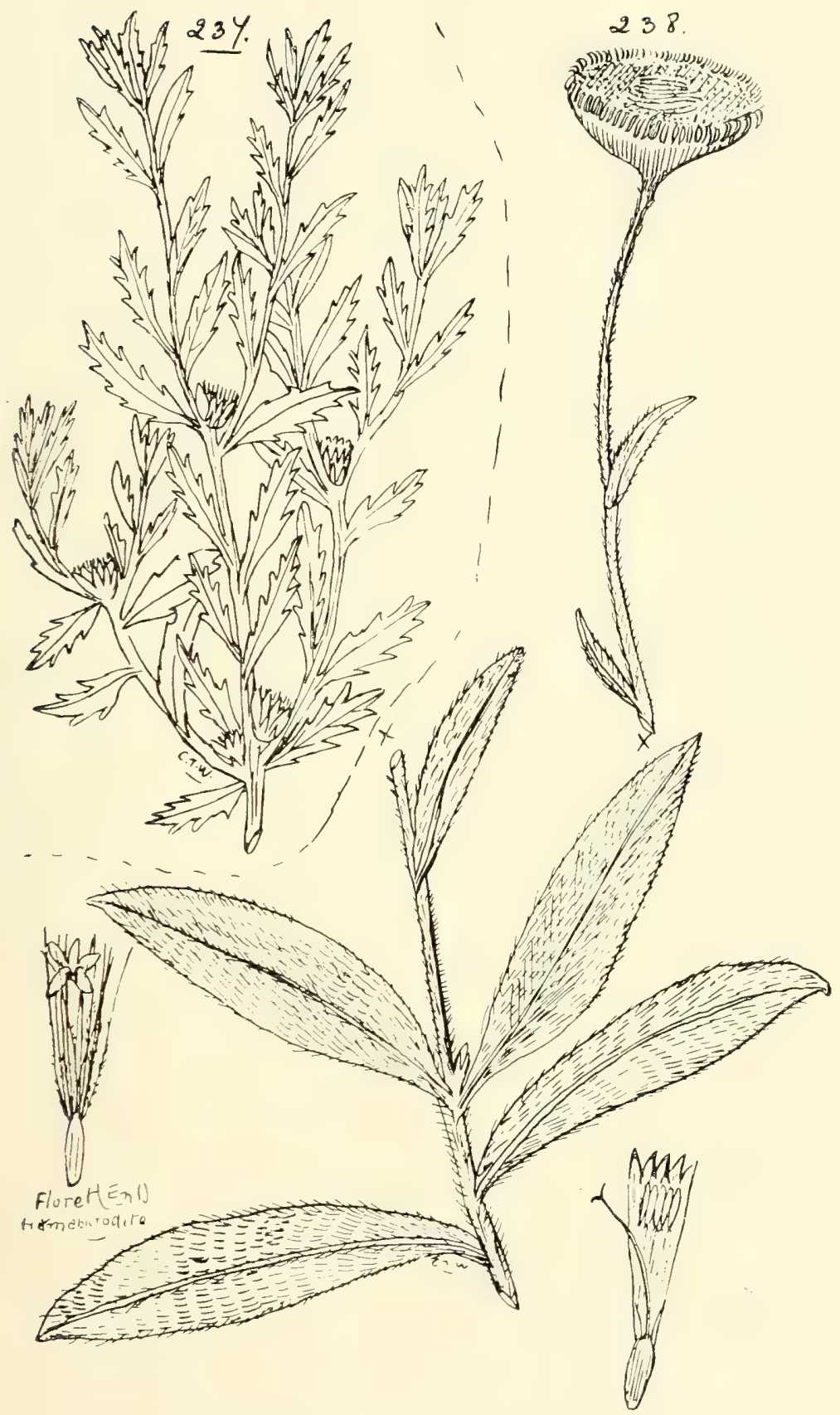

237. Coleocoma centaurea, $F . v . M$.

238. Helichrysum rupicola, $D C$. 
Pterocaulon, Ell.

verbascifolium, Benth. et Hook. = Monenteles verbascifolius, F. v. M.

cylindrostachyum, C. B. Clarke = Monenteles spicatus, DC.

sphacelatum, Benth. et Hook. = Monenteles sphacelatus, Labill.

glandulosum, Benth. et Hook. = Monenteles glandulosus, F. v. M.- "Alworm-angka-ina" of Palmer River natives.

Stuartiana, Sond.

Muelleri, Sond.

Gnaphalium, Linn.-Cudweed.

luteo-album, Linn._-“Karkar” of Mitchell River natives, by whom it is used medicinally as a drink in general sickness. luteo-album, Linn._- “ Karkar” of Mitchell River natives. japonicum, Thunb.

collinum, Labill.

purpureum, Linn. indicum, Linn.

indutum, Hook. $f$.

Leptorhynchus, Less.

squamatus, Less.

Baileyi, F.v.M.

pulchellus, $F . \sigma \cdot M$.

Waitzia, Wendl.

corymbosa, Wendl.

Helipterum, $D C$.

Section Euhelipterum.

anthemoides, $D C$.

polygalifolium, $D C$.

floribundum, $D C$.

var. Stuartianum, Benth.

incanum, $D C$.

Cotula, $D C$.

hyalospermum, F. v. M.

Section Pteropogon.

polyphyllum, F.v.M.

corymbiflorum, Schlecht.

pygmæum, Benth.

strictum, Benth.

Section Monencyanthes.

moschatum, Benth.

Dimorpholepis, Benth. 
Helichrysum, Vaill.

Section Lawrencella.

semifertile, F.v.M.

Section Xerochlana.

scorpioides, Labill.

rutidolepis, $D C$.

bracteatum, Willd.

elatum, $A$. Cunn.

var. Fraseri, Benth.

glutinosum, Hook.

oxylepis, F.v.M.

Section Orylepis.

collinum, $D C$.

rupicola, DC. (Fig. 238.)

podolepideum, F.v.M.

Section Chrysocephalum.

apiculatum, $D C$.

var. minor, Benth.

semipapposum, $D C$.

Bicwillii, Benth.

Section Ozothamnus.

Becklerii, F..$M$.

diotophylhım, F. ש., M.

diosmifolium, Less.

cinereum. F.v. M.

cassinivides, Benth.

Cassinia, R. Br.

longifolia, R. Br.

var. straminea, Benth.

compacta, F.v.M.

denticulata, $R \cdot B r$.

lævis, $R$. Br.-Rosemary shrub; a beautiful wood for veneers in cabinet-work.

quinquefaria, $R$. Br.-Rosemary shrub.

subtropica, F.v.M.

Ixiolæna, Benth.

brevicompta, F.v.M.

leptolepis, Benth.

tomentosa, Sond. et F.v.M

Millotia, Cass.

Greevesii, F.v.M. 
Rutidosis, $D C$.

helichrysoides, $D C$.

leucantha, F. v. $M$.

Murchisonii, F.v. M. (Fig. 239.)

Ammobium, R. Br.

alatum, $R . B r$.

Eriochlamys, Sond, et F.v. M.

Behrii, $S$. et $M$.

Phacellothrix, F..$M$.

cladochæta, F.v.M. = Rutidosis Brownii, Benth.; Helichrysum cladochatum, F. v. M.

Acomis, F.v.M.

Rutidosis, F.,$M$.

macra, F.v.M. (Fig. 240.)

Lesteri, Burkill in Kew Bull. (I90I) I40.

Myriocephalus, Benth.

rhizocephalus, Benth.

Rudallii, Benth.

Stuartii, Benth.

Angianthus, Wendl.

brachypappus, F.v. M.

pusillus, Benth.

Gnephosis, Cass.

arachnoidea, Turcz.

cyathopappa, Benth.

eriocarpa, Benth.

Calocephalus, R. Br.

platycephalus, Benth.

citreus, Less.

Sonderi, F.v. M.

Dittrichii, F.v.M.

Gnaphalodes, A. Gray.

t1liginosum, A. Gray.

Craspedia, Forst.

Richea, Cass.

pleiocephala, F.v.M.

chrysantha, Benth.

globosa, Benth.

Podolepis, Labill.

rhytidochlamys, F.v. M.

longipedata, A. Cunn.

acuminata, $R$. $B r$.

canescens, A. Cunn.

Siemssenia, F.v.M.

Lessoni, Benth. 


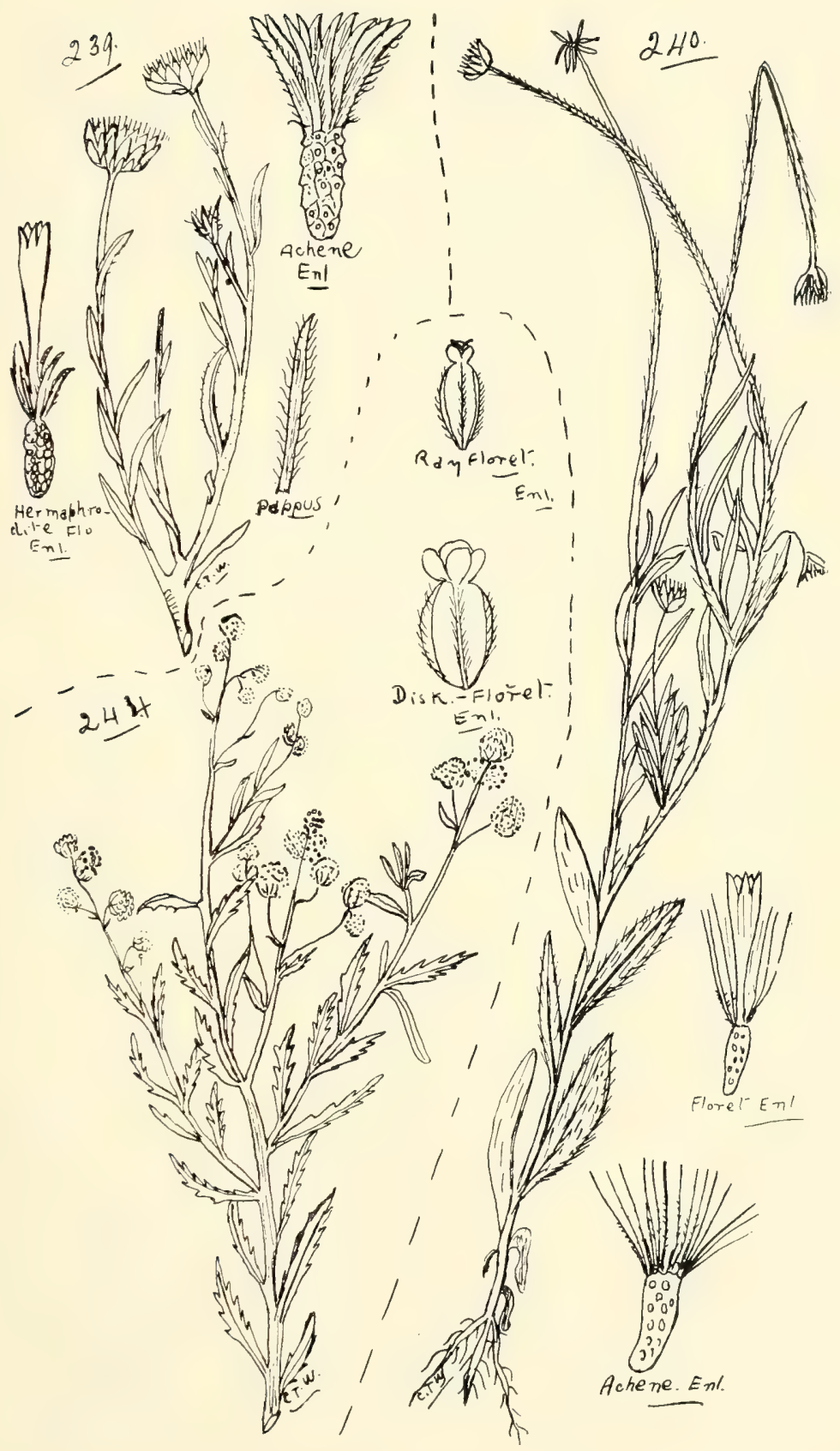

239. Rutidosis Murchisonit, F. v. $M$.

240. Acomis macra, F.v. $M$.

244. Centipeda racemosa, Hook. 


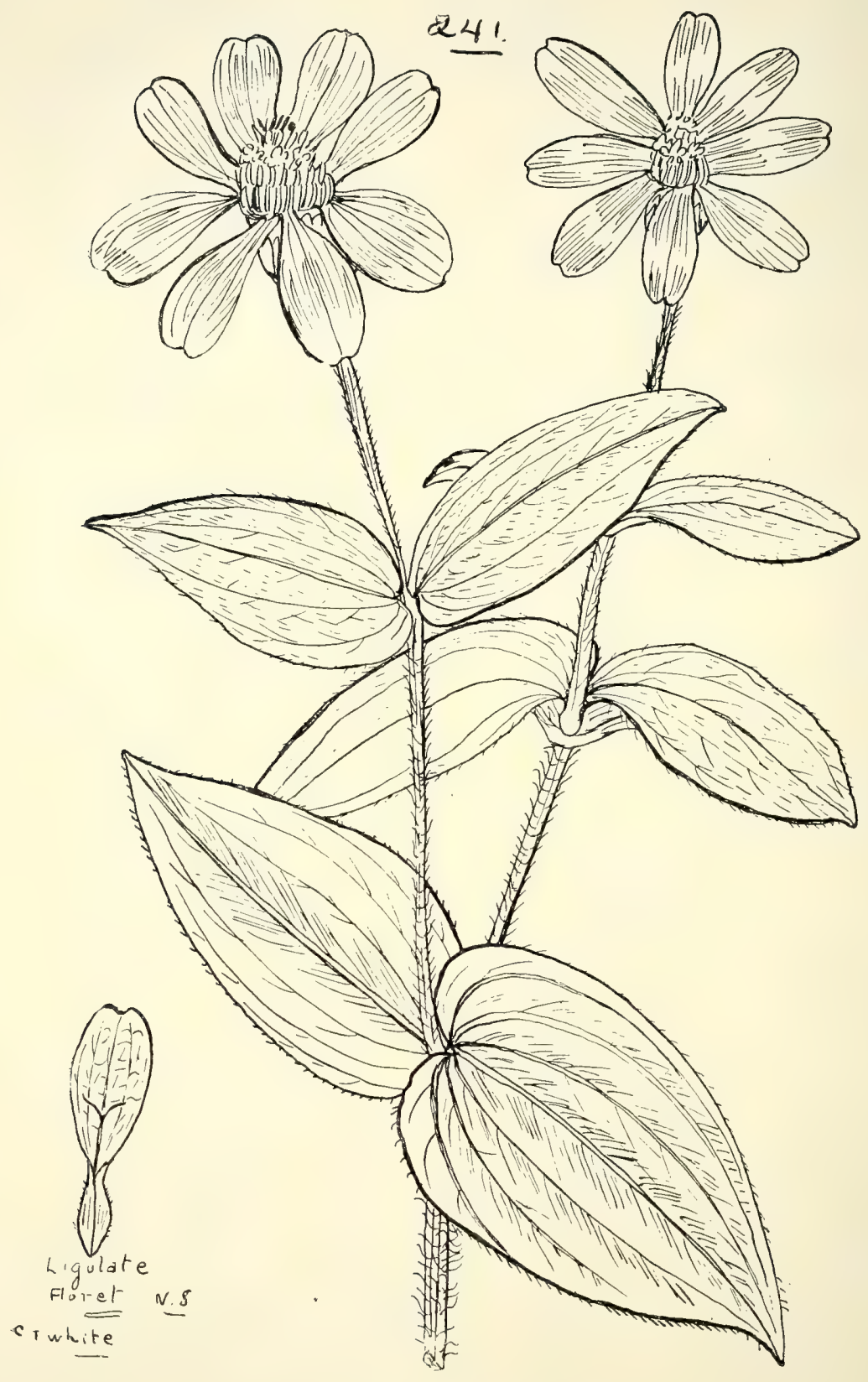

24I. Zinnia australis, Bail. 
Chthonocephalus, Stectz.

pseudoevax, Stect:

Carpesium, Limn.

cernutum, Linn.

TRIBE T.-HELIANTHOIDE.T.

*Acanthospermum, Schrank.

hispidum, $D C$.- Star Burr. A noxious weed, now known by some as "Bindii" (see note, p. 259).

*Ambrosia, Linn.

artemisizefolia, Limn.-In America known as Ragweed, Hayweed, Oxtail, Bitter-weed, and Roman Wormwood, and considered a troublesome weed; has also put in an appearance in some Queensland paddocks.

maritima, Linn.-A Burr-plant; a noxious weed. Africa.

*Xanthium, Linn.

spinosum, Limn.-Bathurst Burr; a noxious weed. Mediterranean Regions.

strumarium, Linn.-Noogoora Burr; a noxious weed. Mediterranean Regions.

Zinnia, Linn.

australis, Bail., Q1. Bot. Bull. ii. I4. (Fig. 24I.)

*pauciflora, Limn. = Z. multiflora, Linn.-America.

Siegesbeckia, Linn.

orientalis, Limn.-A cure for ringworm ( T. Christy).

Enhydra, Lour.

paludosa, $D C$.

Eclipta, Linn.

alba, Hassk.-Yields a black dye. Used in Ceylon as an alterative, as having the power to change a morbid state of the system to a healthy one without disturbance.

platyglossa, F.v.M.

Blainvillea, Cass.

latifolia, $D C$.

Wedelia, Jacq.

calendulacea, Less.-Considered useful as an alterative.

urticifolia, $D C$.

spilanthoides, F.v. M.

biflora, $D C$.

asperrima, Benth.

*hispida, H.B. and K.-Mexico.

*Verbesina, Linn. = Ximenesia, Cav.

encelioides, Benth. and Hook.-Dogweed of America. An abundant weed on some of the Downs farms (see Q1. Agric. Jl. xxviii. ( I912) 357, P1. 358). 
Spilanthes, Linn.

grandiflora, Turcz.

var. calva, Benth.

anactina, F.v.M.

*Coreopsis, Linn.

tinctoria, Nutt.--America.

*Cosmos, Cav.

bipinnatus, Cav.-South America.

Bidens, Linn.

tripartita, Linn.

Section 1.-Platycarpaca.

Section II.-Psilocarpaca.

pilosa, Linn.-Cobbler's-pegs. At times the foliage variegated.

bipinnata, Linn.

Glossogyne, Cass.

tenuifolia, Cass.

retroflexa, F.v.M. (Fig. 242.)

orthochrta, F.v.M.

*Galinsoga, Cav.

parviflora, Cav.-Yellow-weed. Foliage sometimes variegated. South America.

Tribe VI.-Helenioide不.

Flaveria, Juss.

australasica, Hook.- "Bogabri" of Flinders River natives.

Tagetes, Linn.

$$
\text { (Fig. 243.) }
$$

glandulifera, Schrank.-Stinking Rodger.

*Achillea, Linn.

Tribe VII.-AnThemidex.

tanacetifolia, All.-S. Europe.

*Anthemis, Linn.

Cotula, Linn.-Mediterranean Region.

Cotula, Linn.

coronopifolia, Limn.

australis, Hook.

Section Strongylosperma.

alpina, Hook.

reptans, Benth.

Section Leptinella.

var. major, Benth.

Centipeda, Lour. = Myriogyne, Less.

orbicularis, Lour.-Snuff-plant. 


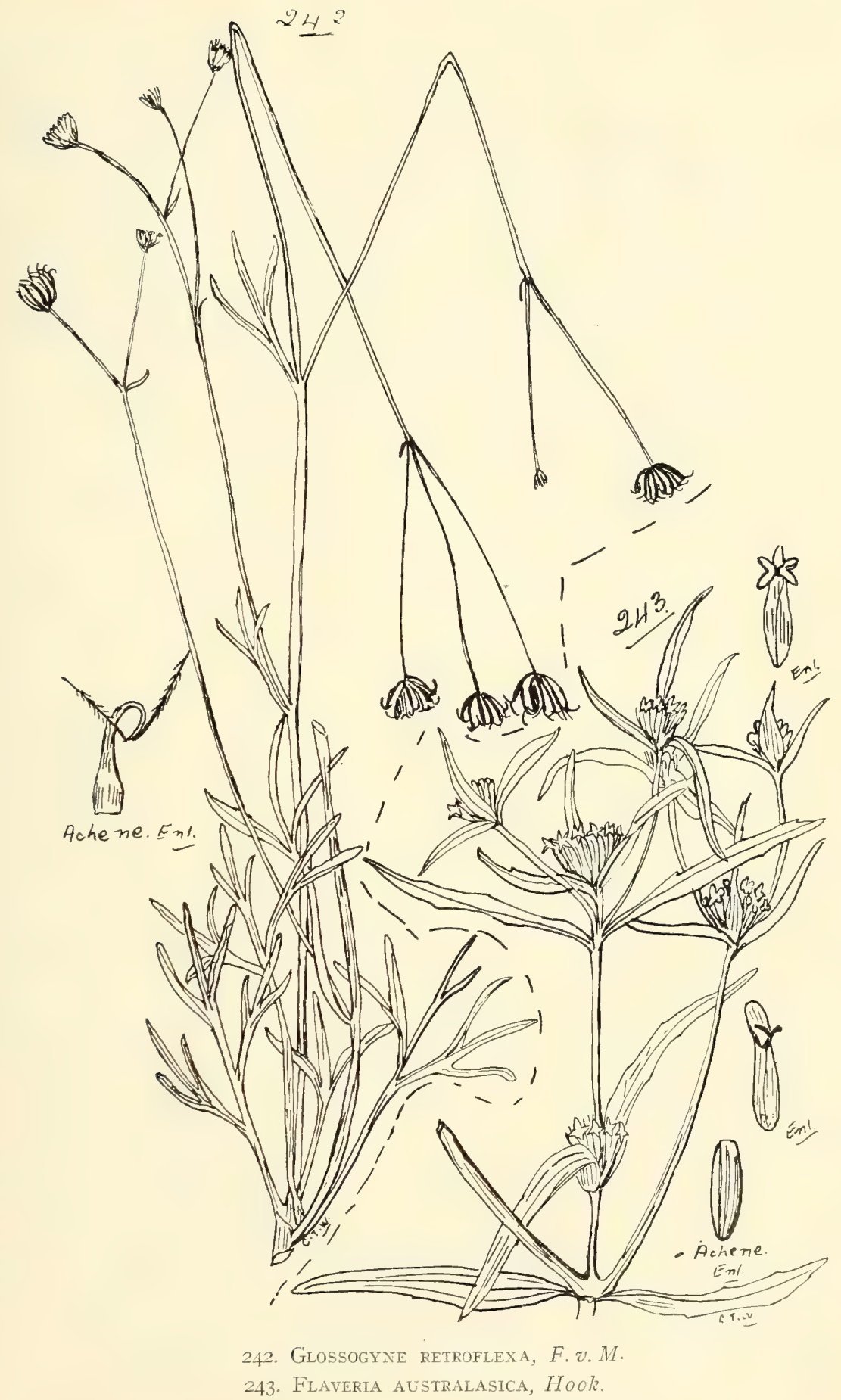




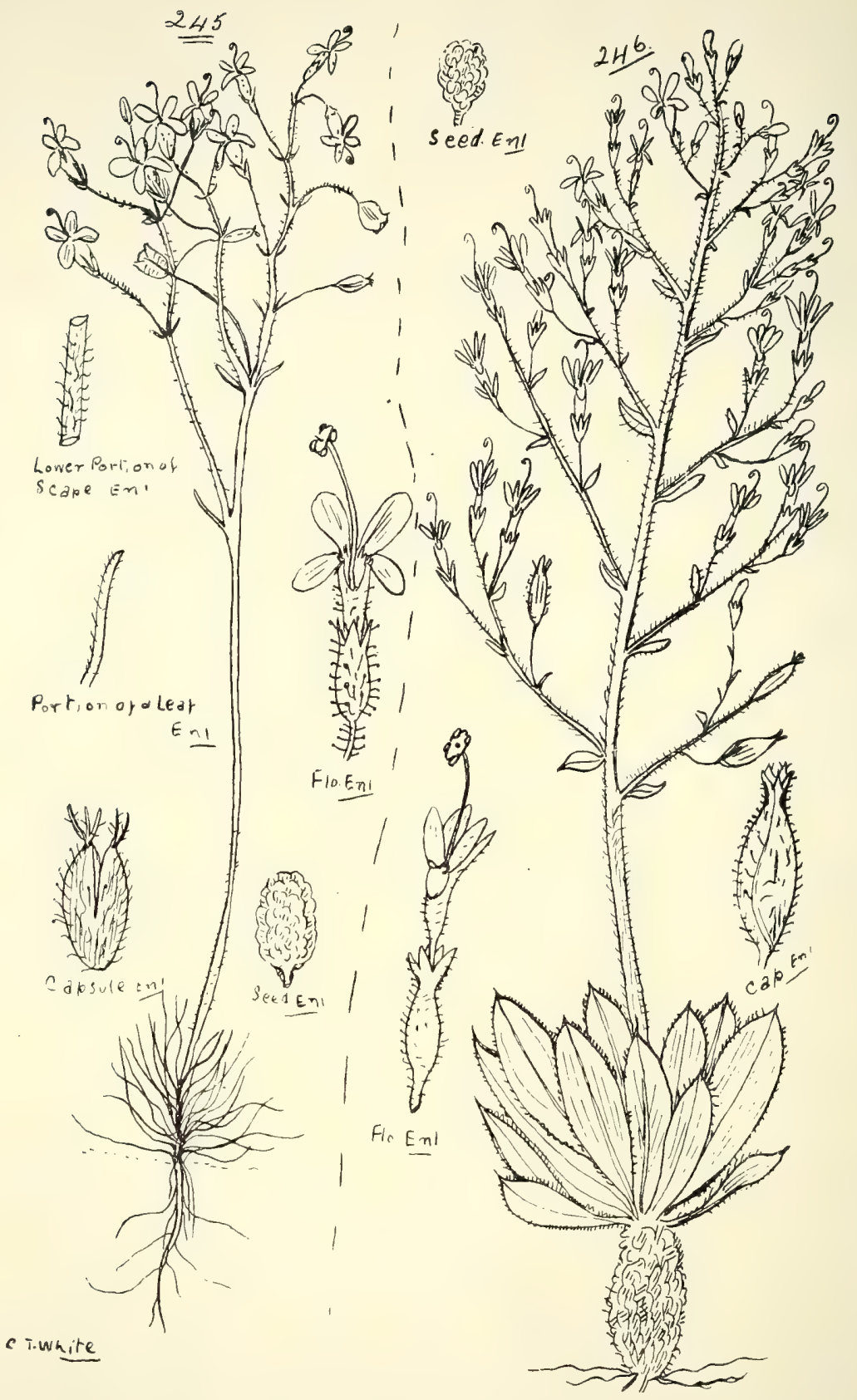

245. Stylidium Floodis, $F$. v. $M$.

246. S. eRIorhizum, F.v. $M$. 
Centipeda-contd.

orbicularis-contd.

var. minuta, Bail.

var. sternutatoria, Bail.

var. lanuginosa, Bail.

racemosa, Hook.-Plant glabrous. (Fig. 244.)

var. lanata, Bail.-Plant woolly.

Cunninghamii, F.v. M.

thespidioides, F.v. $M$.

Soliva, Ruiz et Pav.

anthemifolia, R. Br.-This is a favourite food of the emu.

Isoetopsis, Turc:.

graminifolia, Turcz.

Ceratogyne, Turcz.

obionoides, Turcz.

\section{Tribe VIII.-SEnECIONIDEA.}

Erechthites, Rafin.

Atkinsoniæ, F.v.M.

arguta, $D C$.

quadridentata, $D C$.

*valerianæfolia, DC.-Federal weed. South America.

Gynura, Cass.

pseudochina, $D C$.

Emilia, Cass.

sonchifolia, DC.-The juice of this plant has been used in

India in inflammation of the eyes, and said to be as cooling as rose-water.

Senecio, Linn.

$$
\text { Series I.-Radiati. }
$$

Gregorii, F.r. $M$.

platylepis, $D C$.

Daltoni, F.v.M.

spathulatus, R. Rich.-A maritime species.

lautus, Forst.

velleioides, $A$. Cunn.

brachyglossus, F. v. M.

amygdalifolius, F. v. M.

Series Discoidei.

odoratus, Hornem.

Cunninghamii, $D C$.

anethifolius, A. Cunn.

*vulgaris, Linn.-Common English Groundsel. 
Cymbonotus, Cass.

\section{TRIBE 1X.-ARCTOTIDEX.}

Lawsonianus, Gaudich.

*Cryptostemma, R. Br.

calendulacea, R. Br.-Cape-weed. Africa.

*Arctium, Linn.

Lappa, Limn.-Common Burdock; a noxious weed. Europe.

Tribe X.-Cynaroide.

*Carduus, Linn.

pycnocephalus, Linn.-Slender Thistle; a noxious weed. Europe.

nutans, Linn.-A noxious weed. Europe.

*Cnicus, Limn.

lanceolatus, Linn.-The Spear or Plumed Thistle; a noxious weed. Commonly known in Queensland as "Scotch Thistle"; the true Scotch Thistle is, however, Onopordon acanthium. Europe.

*Silybum, Gartn.

Marianum, Gartu.-Virgin Mary's Thistle; a noxious weed. Europe.

Saussurea, $D C$.

carthamoides, Benth

Centaurea, Linn.

australis, Benth. et Hook., Gen. P1. = Leusea australis, Gaud. *cyanus, Linn.-Corn-flower. Europe.

*melitensis, Limn.-Star Thistle; a noxious weed. Europe.

*solstitialis, Linn.-St. Barnaby's Thistle; a noxious weed. Europe.

*Carthamus, Linn.

lanatus, Limn. $=$ Kentrophyllum lanatum, DC.-Yellow Distaff Thistle; a noxious weed. Europe.

Tribe XI.-Cichoriaces.

Microseris, Don.

Forsteri, Hook.-Rootstock, uncooked, used as a food by the natives in South Australia.

Picris, Linn.-Ox-tongue.

hieracioides, Linn.-Hawk-weed.

var. squarrosa, Benth.

Crepis, Limn.-Hawk's-beard.

japonica, Benth.

Hypochæris, Linn.-Cat's-ear.

glabra, Linn.

*radicata, Limn.-Long-rooted Cat's-ear. Europe. 
*Taraxacum, Hall.

officinale, Wigg.-Dandelion. Europe. Root medicinal.

*Lactuca, Limn.

Scariola, Linn.-Prickly Lettuce. Europe. The milky juice, when inspissated, has been used as a substitute for opium (Lindley).

Sonchus, Linn.

oleraceus, Limn.-Sow Thistle. Europe.

There are two forms. In forma normalis the achenes are marked with transverse asperities; in forma asper the ribs of the achenes are smooth.

arvensis, Lim.-Corn Sow-thistle. Europe.

maritimus, Linn.-Europe.

*Tragopogon, Linn.-Goat's-beard.

porrifolium, Linn.-Salsify; Star of Jerusalem. Europe.

*Scorzonera, Limn

hispanica, Willd.-A native of Spain; sometimes met with as a garden weed in South Queensland.

Alliance III.-CAMPANALES.

\section{Order LXVI.-STYLIDIE}

(CANDOLLEACEE, F. v. M.)

Stylidium, Sw-Hair-trigger or Spring-back plant.

(All placed by Mueller under Candollea, Labill.)

\section{Section I.-Polypangium.}

Series Lineares.

graminifoliım, Sw. var. album, Bail.

lineare, $S w$.

Floodii, F. v. M. -(Fig. 245.)

Series Spathulatæ.

eriorhizum, R.Br. (Fig. 246.)

debile, F.v. M.

floribundum, $R . B r$.

leptorhizum, F.v.M.

Series Sparsifolix.

laricifolium, Rich. 


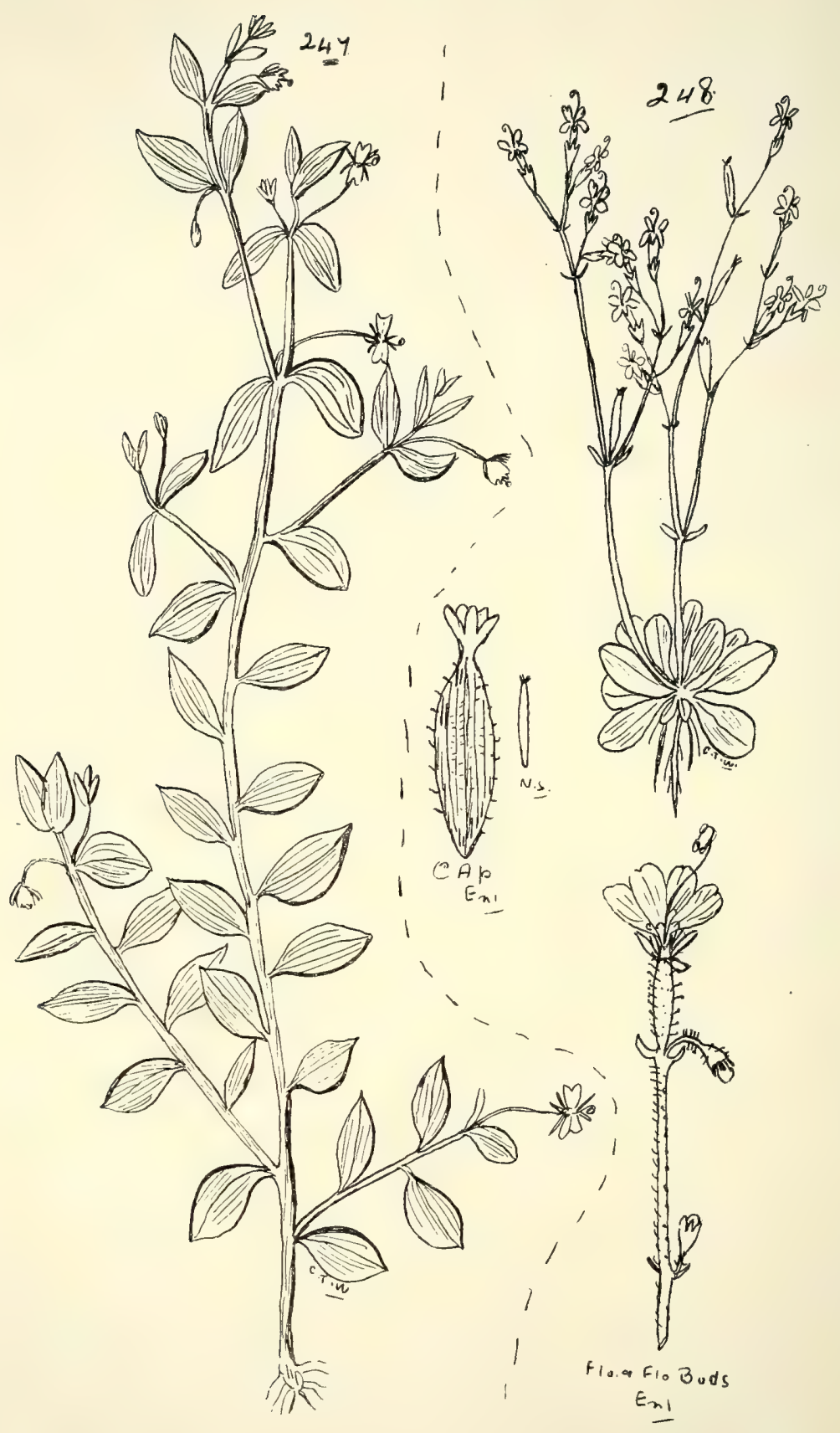

247. Stylidium alsinoides, $R$. $B r$.

248. S. Capillare, $R$. $B r$. 
LXVI. STYLIDIEÆ.-LXVII. GOODENOVIEÆ.
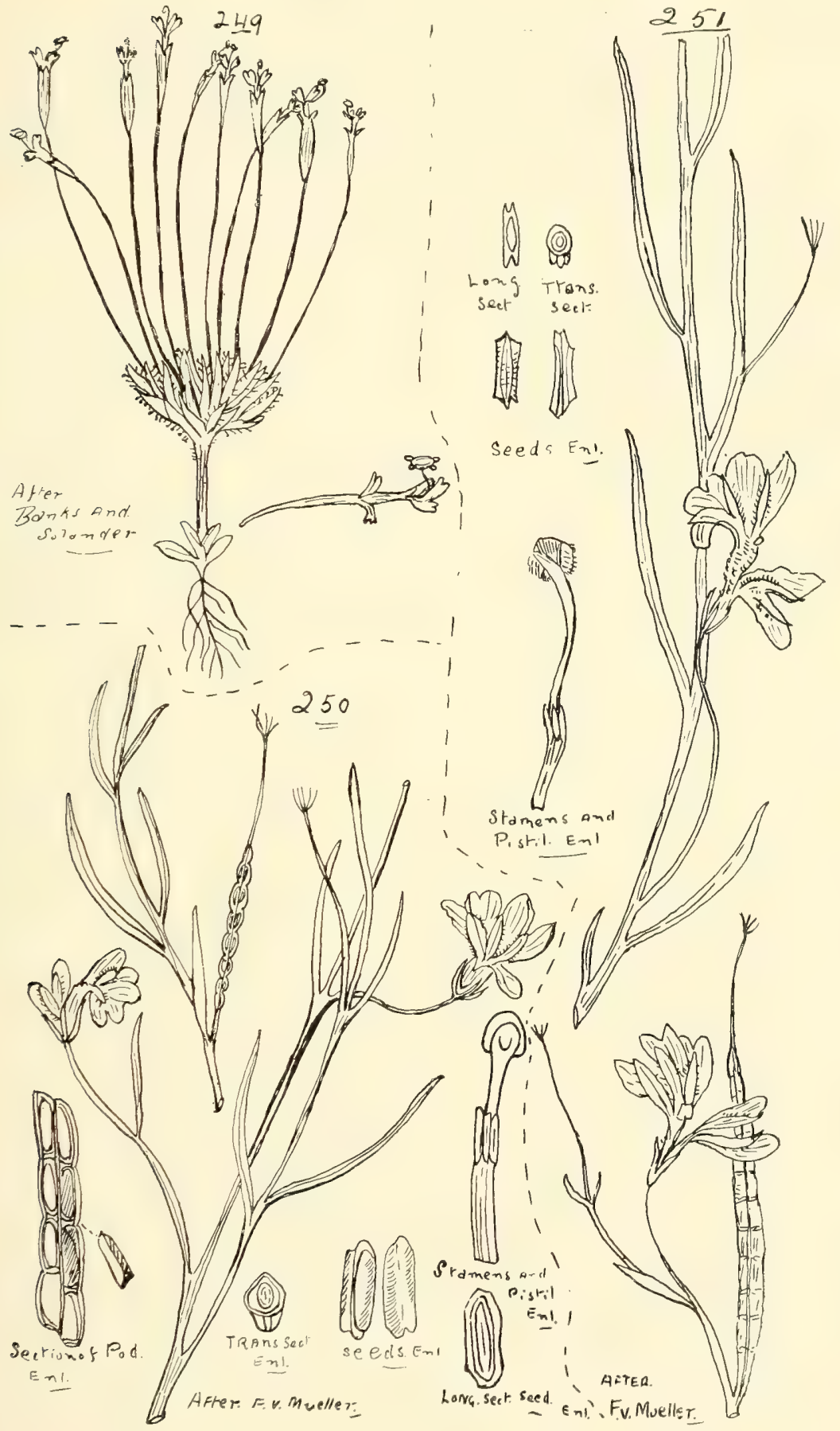

249. Stylidium pedunculatum, $R . B r$.

250. Leschenaultia filiformis, $R . B r$.

25I. L. Agrostophylia, F. v. $M$. 


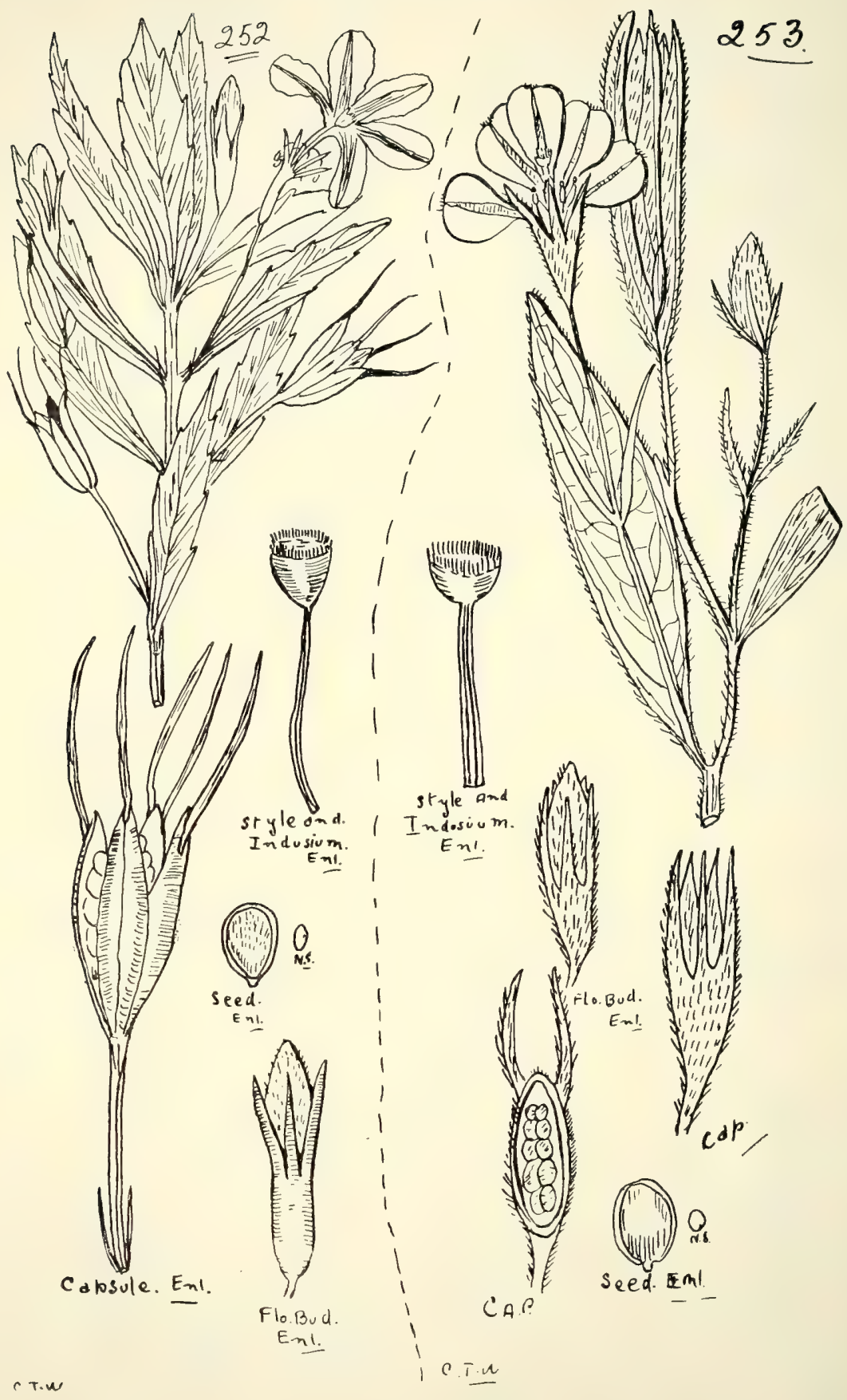

252. Goodenia Stirlingit, Bail.

253. G. Strangfordin, F. v. $M$. 


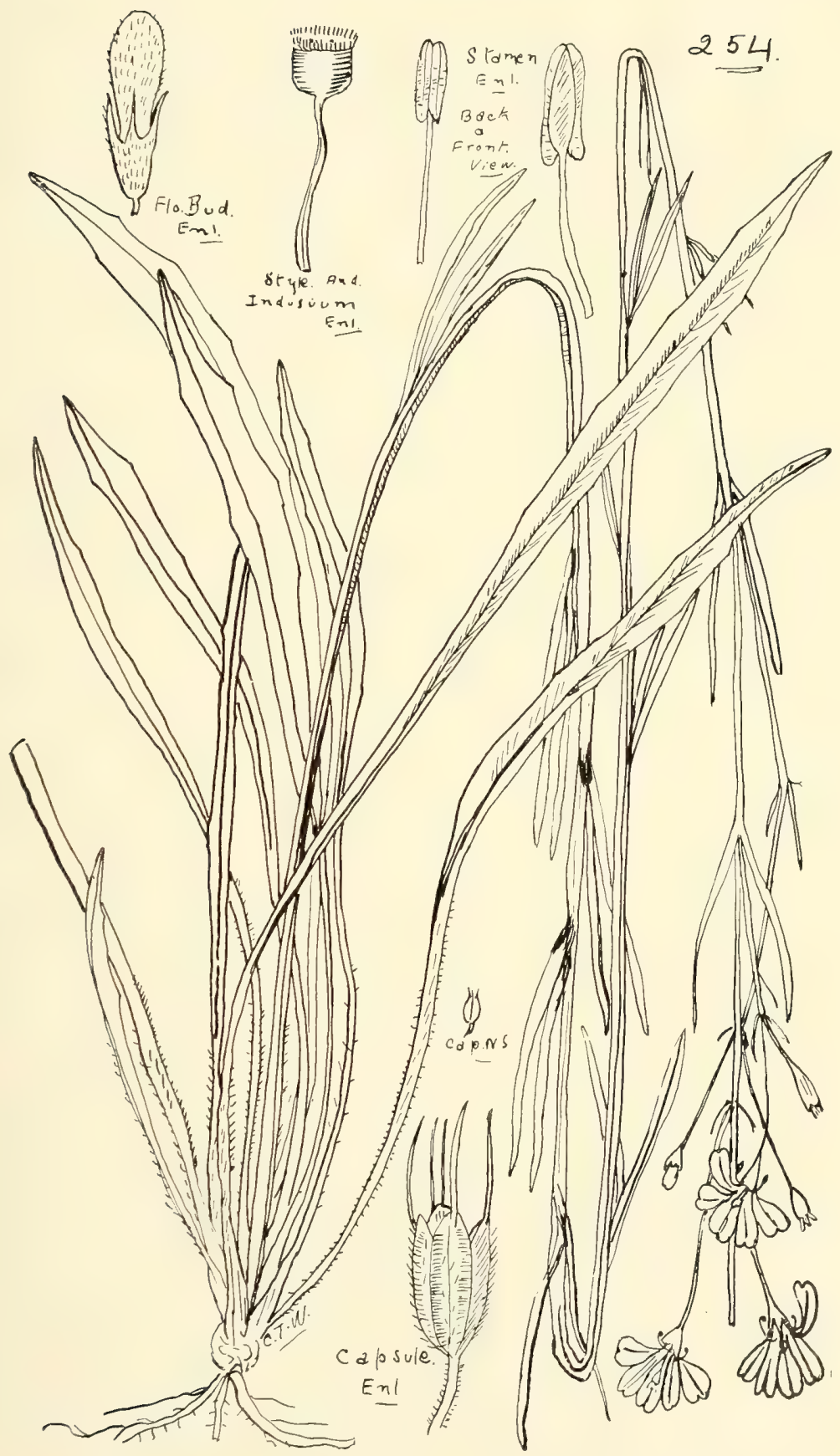

254. Goodenia plrptrascexs, R. Br. 
Stylidiun-contd.

\section{Section II.-Nitrangium.}

Series Tenellæ.

trichopodum, F.v. $M$.

diffusum, R. Br.

alsinoides, R.Br. (Fig. 247.)

Series Corymbulosæ.

capillare, R.Br. (Fig. 248.)

rotundifolium, $R$. Br.

uliginosum, Sze.

pedunculatum, R.Br. (Fig. 249.)

Series Thyrsiformes.

eglandulosum, F.v.M.

\section{Order LXVII.--GOODENOVIEAE.}

Leschenaultia, R. $B r$.

\section{Section Latouria.}

divaricata, F.v.M.

filiformis, R. Br. - (Fig. 250.)

agrostophylla, F.v.M. (Fig. 25I.)

Velleia, Sm.

connata, F.v.M.

perfoliata, R. Br.

paradoxa, R. $B r$.

var. stenoptera, F.v. M.

macrocalyx, De Vriese.

pubescens, R. Br.

spathulata, R. Br.

Goodenia, $S m$.-Probably some of this or allied genera possess an active medicinal property, as a fragment of what appeared a species of Goodenia was sent to the late Dr. Joseph Bancroft, with a note stating that the aboriginal women gave it to their babies to cause them to sleep when on long journeys. The writer, however, gave no information as to how the herb was administered.

\section{Section Eugoodenia.}

Series Racemosæ.

racemosa, F.v.M.

bellidifolia, $S m$.

stelligera, R. $\mathrm{Br}$. 
Goodenia-contd.

Series Bracteolate.

ovata, $S m$.

disperma, F.v.M.

geniculata, $R . B r$.

hederacea, $S m$.

heterophylla, $S m$.

glabra, R. Br.

strongylophylla, $F, \tau, M$.

rotundifolia, $R$. Br.

Series Foliosæ.

calcarata. F. ש. .1\%

grandiflora, Sims.

var. mollis, Benth.

Chambersii, F.v.M.

Stirlingi, Bail. (Fig. 252.)

Strangfordii, F.v.M. (Fig. 253.)

var. grandiflora, Benth.

Mitchellii, Benth.

heterochila, F.v.M.

hispida, R. Br.

Series Pedicellosæ.

mollissima, F. v. M.

cycloptera, R. Br.

pinnatifida, Schlecht.

pusilliflora, F.v. M.

coronopifolia, R. Br.

heteromera, F.v.M.

glauca, F.v. M.

Armitiana, F.v.M.

Section Amphichila.

paniculata, Sm.

purpurascens, R. Br. (Fig. 254.)

gracilis, R. $\mathrm{Br}$.

lamprosperma, F.v. M.

pumilio, R. Br.

Calogyne, R. Br.

heteroptera, F.v. M. (Fig. 255.)

pilosa, R. Br.

Catosperma, Benth.

Nivelleri, Benth. 


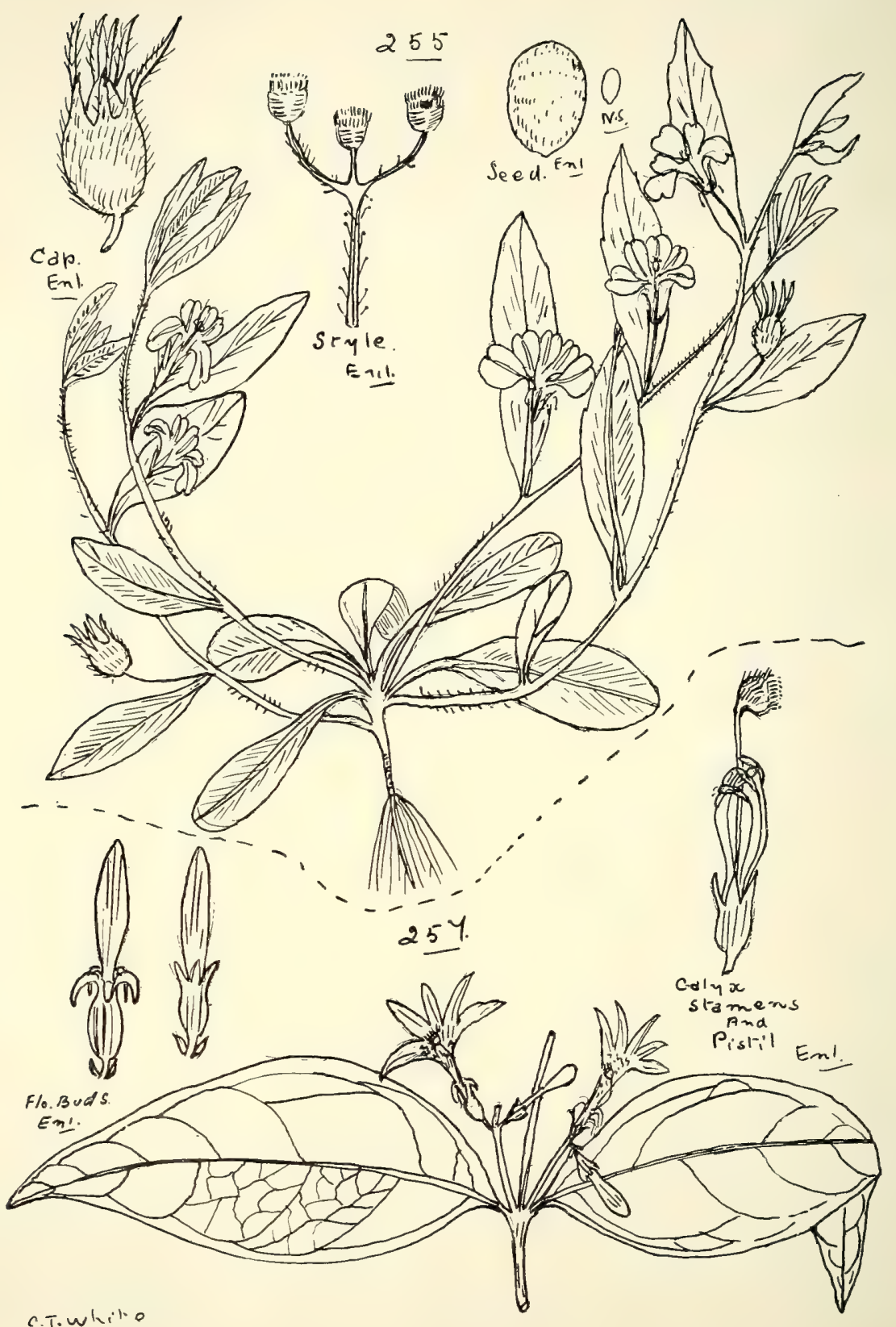

255. Calogyne heteroptera, F. v. $M$.

257. Sc.evola enantophylla, F.v. $M$ 


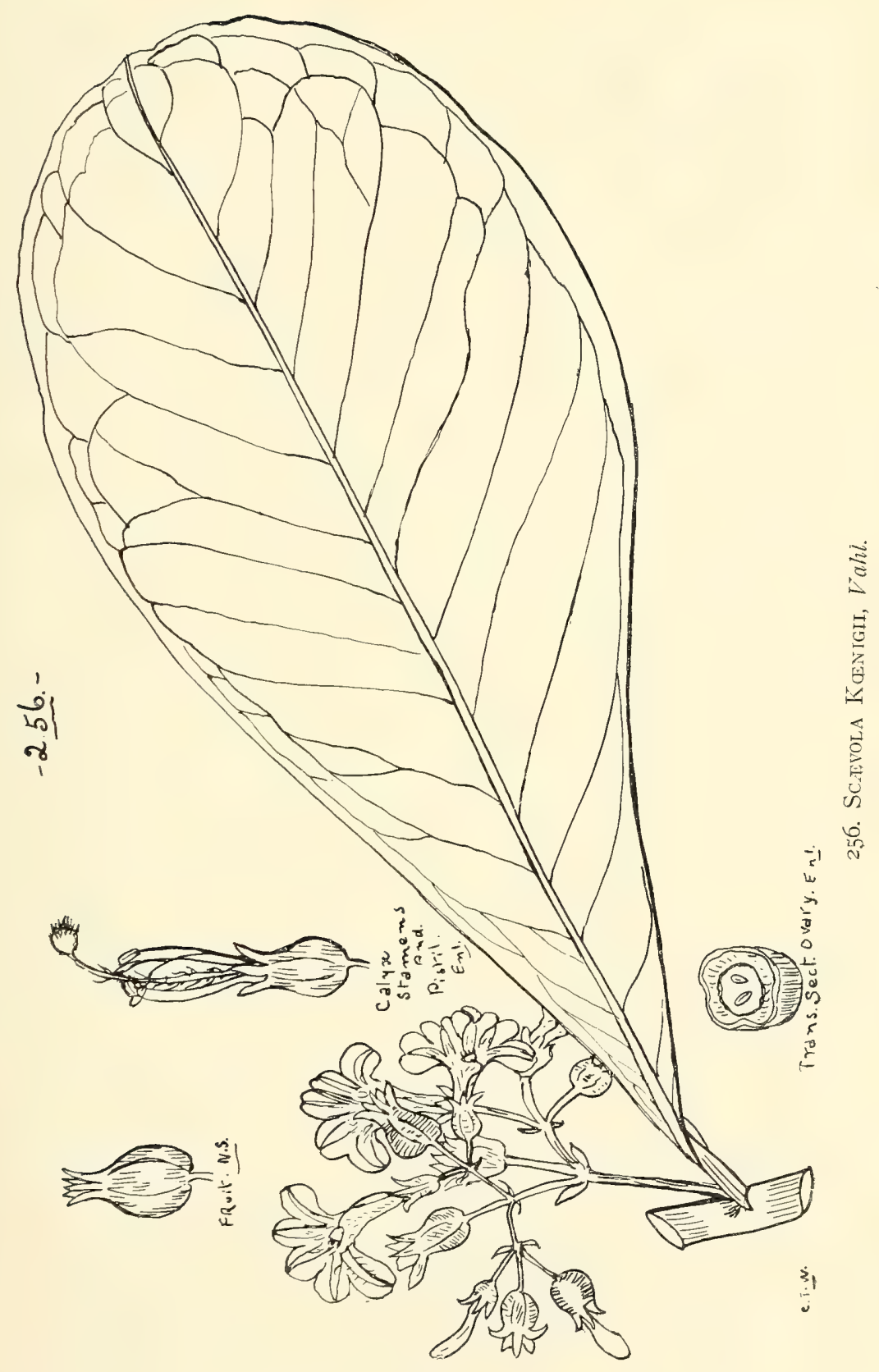




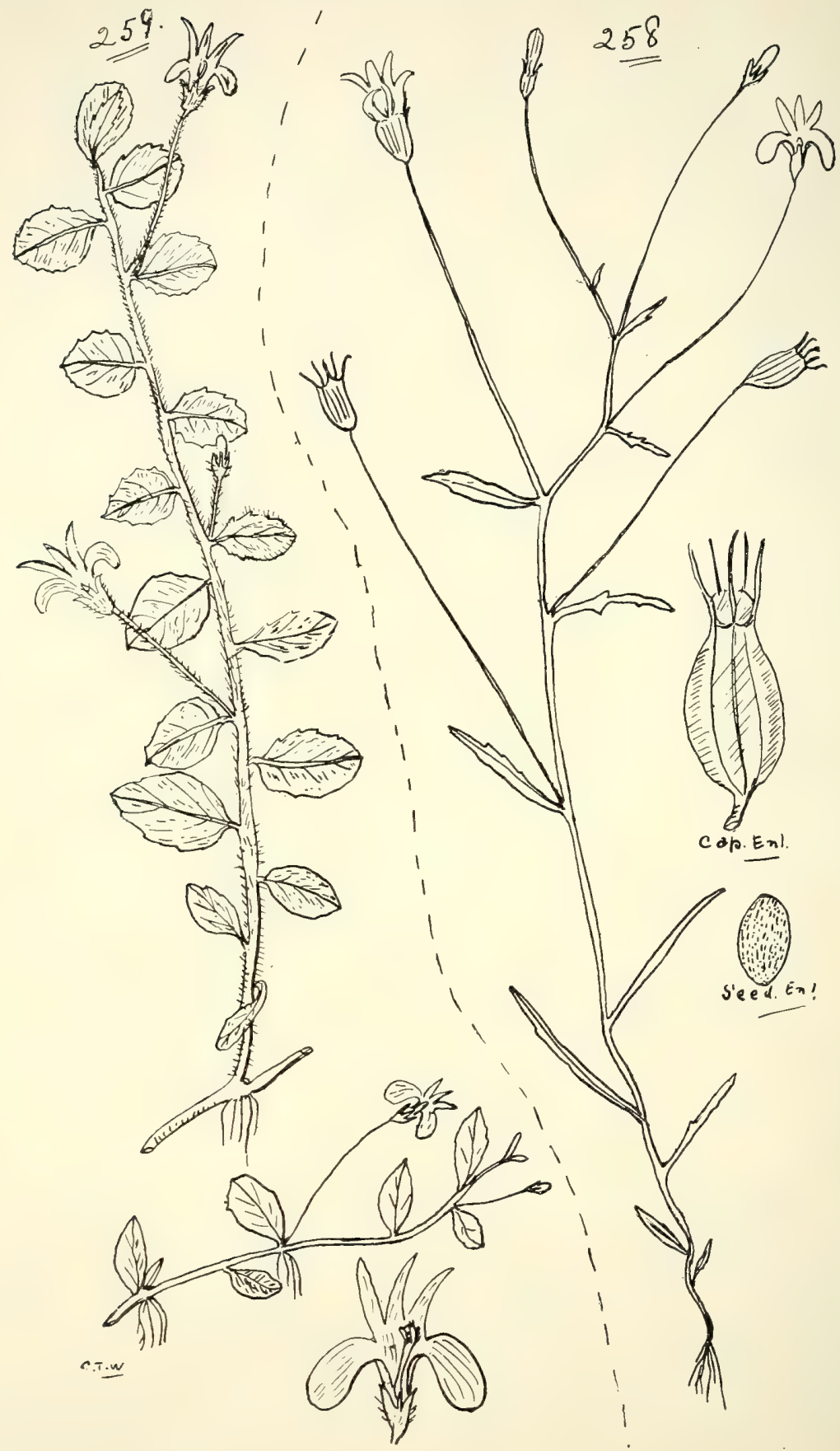

258. Lobelia Douglasiana, Bail.

259. L. humistrata, F. v. $M$. 
Scævola, Lmm.

Section 1.-Sarcocarpoea.

Konigii, Vahl. — Called "Native Cabbage" at Cardwell. "Ko-po" of the Cardwell natives (R.B.H.). Pith of stems used in Asia in the manufacture of artificial flowers, small figures, \&c. (Fig. 256.)

enantophylla, F.v.M. (Fig. 257.)

scandens, Bail.

Section II.-Crossotoma.

spinescens, R. Br.

Section III.-Pogonanthera.

hispida, Cav.

parvifolia, F.v. $M$.

Section IV.-Xerocarpaa.

Series I:-Globuliferæ.

angulata, $R \cdot B r$.

suaveolens, R. Br.

Series II.-Macrostachyz.

revoluta, $R . B r$.

ovalifolia, R. Br.

Series III.-Pogogynex.

æmula, R. Br.

amblyanthera, F.v.M.

Series IV.-Monospermæ.

laciniata, Bail.

microcarpa, Cav.

Dampiera, R. Br.

Section Linschotenia.

Linschotenii, F. $v . M$.

Section Eudampiera.

ferruginea, R. Br.

Brownii, F.v. $M$.

stricta, R. Br.

var. oblongata, Benth.-Several cows were supposed to have been killed a few years ago from eating this plant at Mount Cotton.

adpressa, A. Cunn.

diversifolia, $D e V r$.

Brunonia, Sm.

australis, $S m$. 


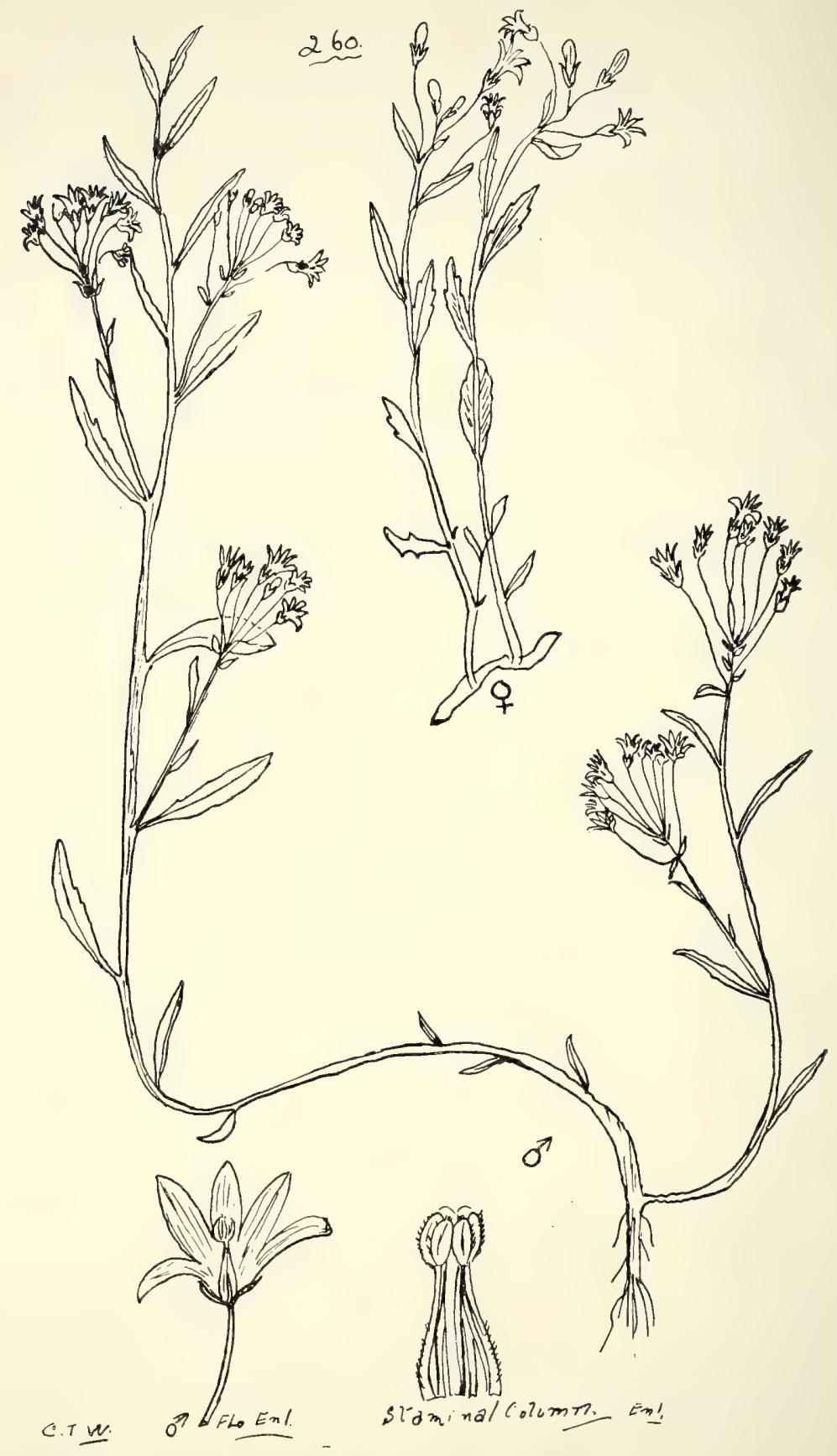

260. LoBelia dioicA, R. $\mathrm{Br}$. 


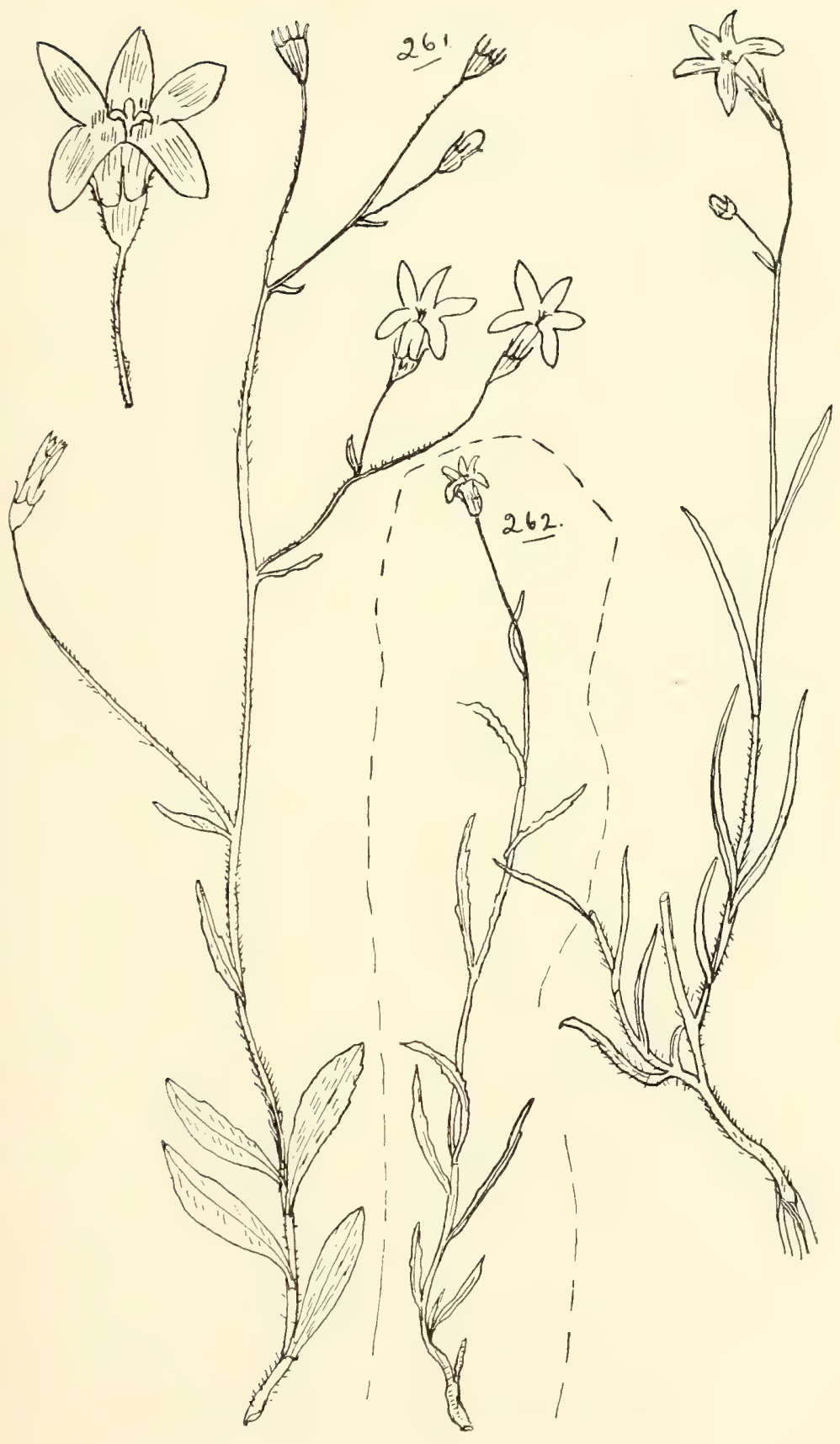

26r. Isostoma AXILLARIS, Lindl.

262. Wamlenbergia gracilis, $A . D C$., var. minutiflora, Bail. 


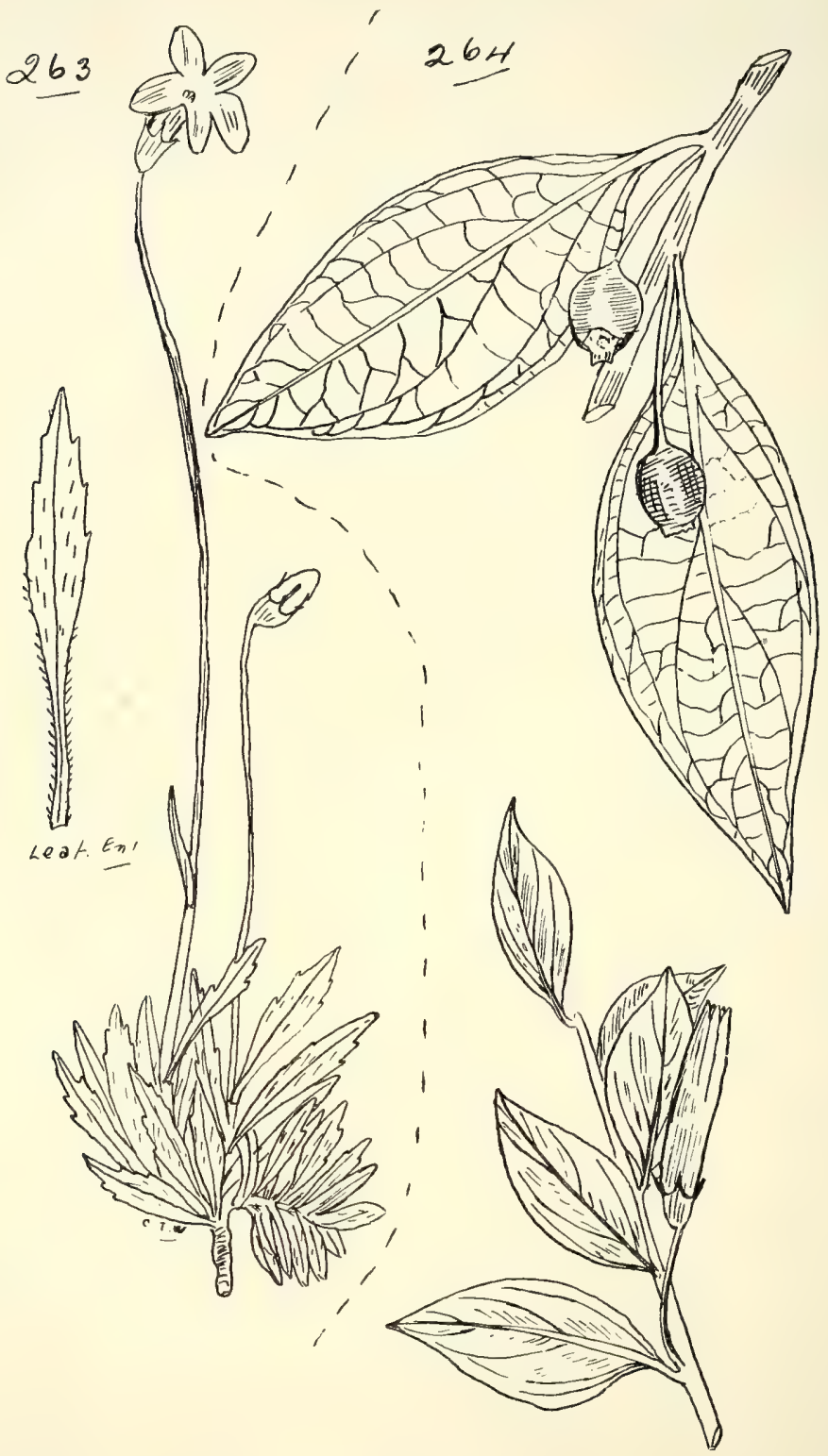

263. Wahlenbergia saxicoli, $A . D C$. 264. Agapetes Meiniana, $F . v . M$. 


\title{
Order LXVIII. -CAMPANULACEÆE.
}

Lobelia, Linn.

\author{
Tribe I.-LobeLiex.
}

Section I.-Holopogon.

gibbosa, Lindl.

var. microsperma, Bail.

forma alba, Bail.

var. Browniana, Bail.

var. simplicicaulis, Bail.

dentata, Car.

gracilis, Andr.

var. major.

trigonocaulis, F.v.M.

Douglasiana, Bail. (Fig. 258.)

anceps, Thunb.

Section II.-Hemipogon.

membranacea, $R \cdot B r$.

stenophylla, F.v.M.

quadrangularis, $R . B r .=$ humistrata, F. v. M. (Fig. 259.)

dioica, R. Br. (Fig. 260.)

purpurascens, R.Br. - A troublesome weed, poisonous to stock.

Pratia, Gaudich.

erecta, Gaudich.-A troublesome weed, poisonous to stock.

puberula, Benth.

pedunculata, Benth.

Isotoma, Labill.

axillaris, Lindl.-Poisonous to stock. (Fig. 26r.)

petræa, F.v.M.

fluviatilis, $F . v \cdot M$.

Gulliverii, F.v. M.

Tribe II.-Campanulee.

Wahlenbergia, Schrad. Fruit a Diplotegia.

gracilis, A. DC.-Native Bluebell.

var. minutiflora, Bail. (Fig. 262.)

saxicola, A.DC. (Fig. 263.)

Series II.-HETEROMER E.

Alliance IV.-ERICALES.

\section{Order IXIX.—VACCINIACE $\$$.}

Trime Thibaudieж.

Agapetes, D. Don

Meiniana, F.v.M. (Fig. 264.) 


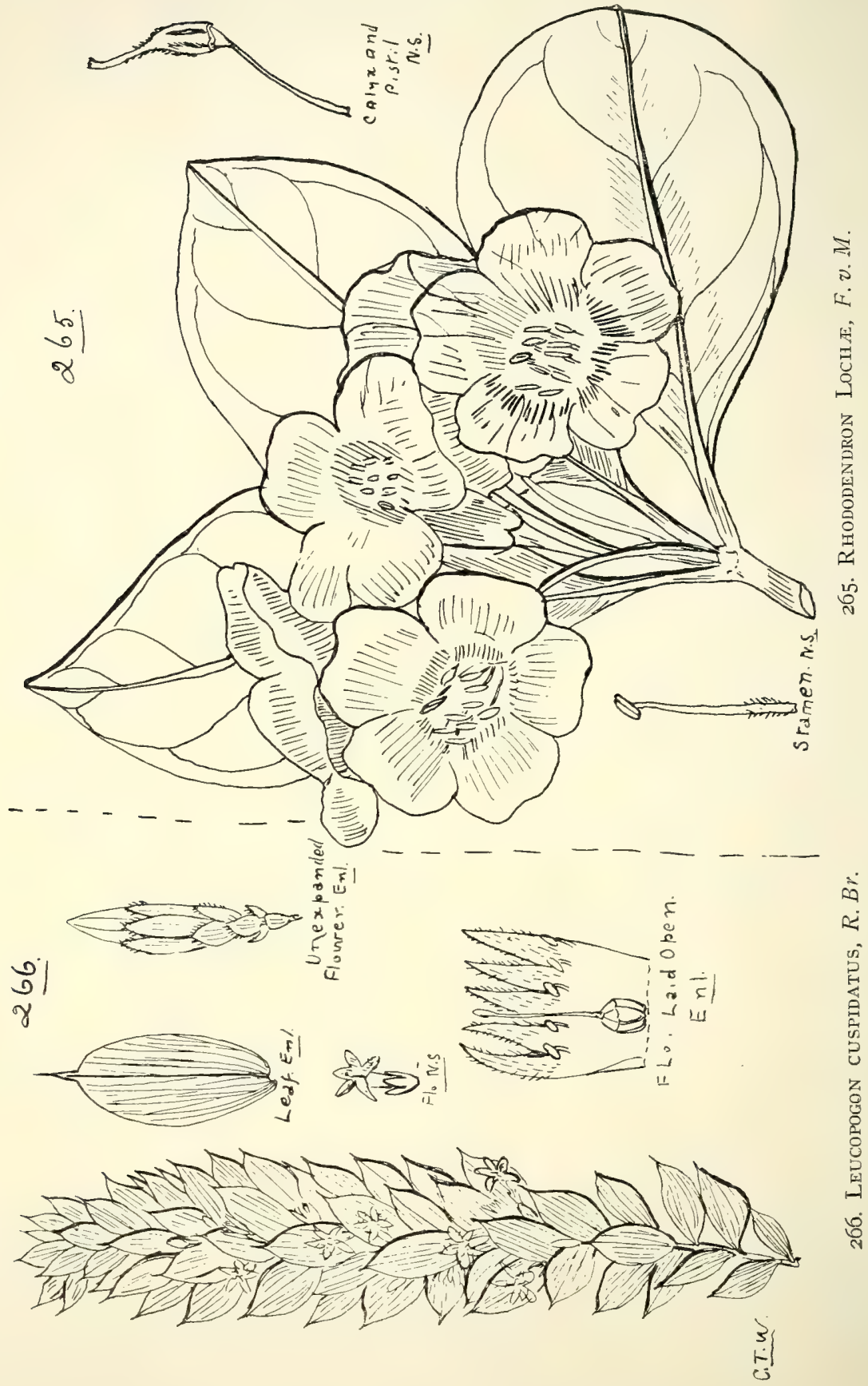




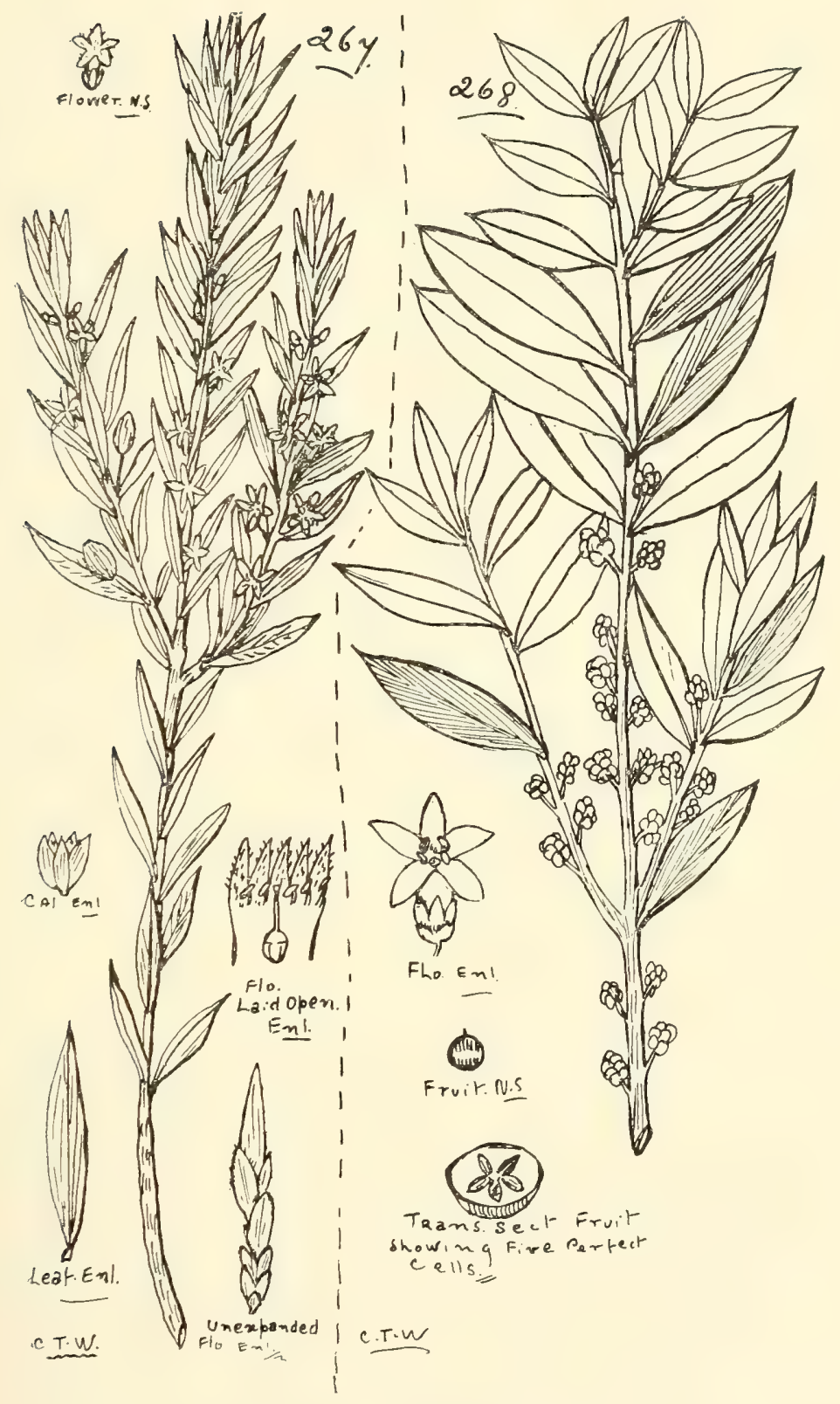

267. Leucopogon Leptospermoides, $R$. $B r$. 268. Monotoca lineata, $R . B r$. 


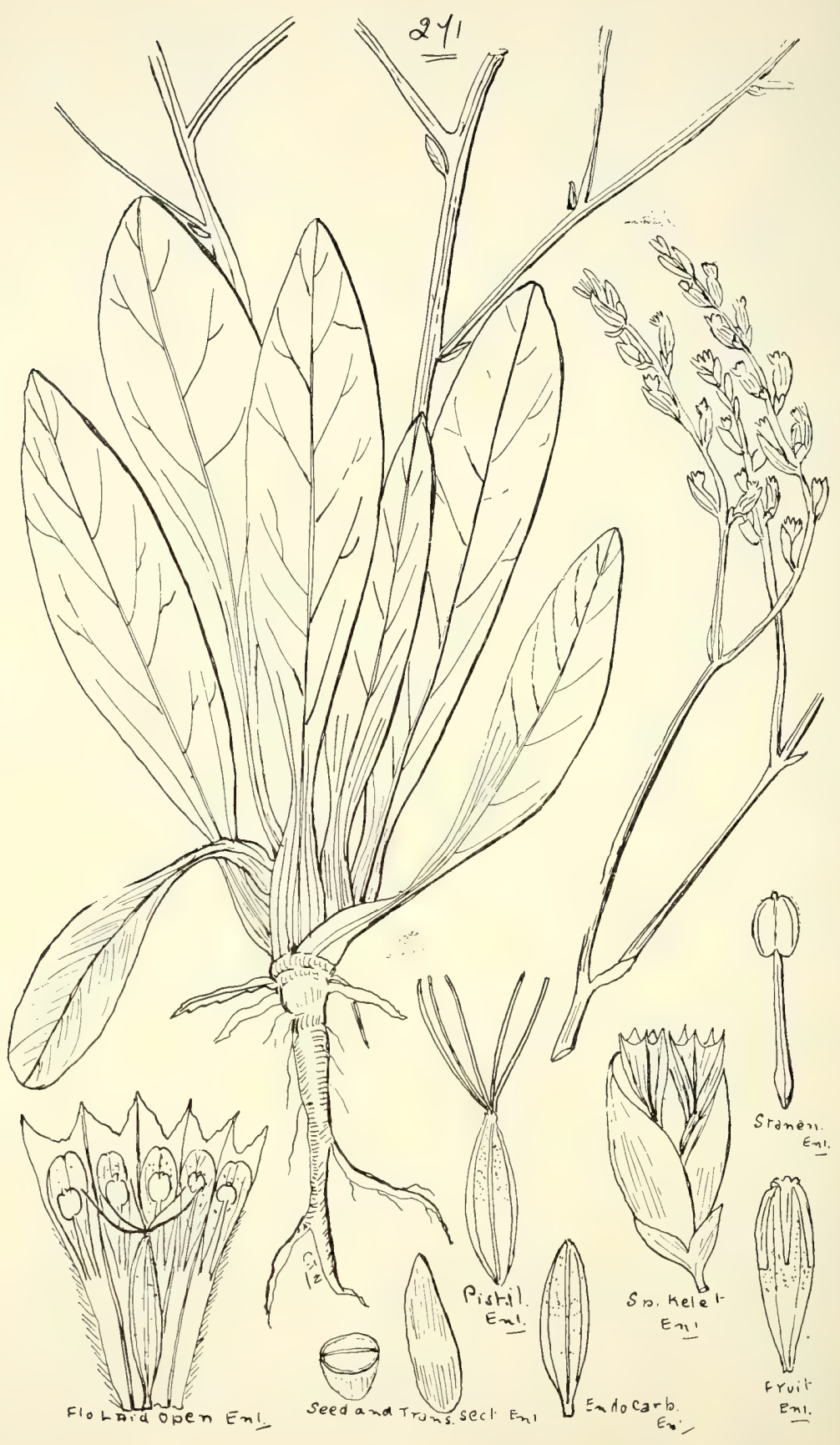

27i. Statice australis, Spreng. 


\section{Order LXX.-ERICACEÆ.}

Tribe Rhodorez.

Rhododendron, Lim.-Fruit a Capsule.

Lochæ, F.v.M. (Fig. 265.)

\section{Order LXXI. - EPACRIDEÆE.}

Tribe I.-STyPhelie玉.

Styphelia, Sm.

viridis, Andr.

var. breviflora, Benth.

triftora, $A n d r$.

Melichrus, $R \cdot B r$. (Included by F. v. Mueller in Styphelia.)

rotatus, $R . B r$.

urceolatus, $R$. $B r$.

Trochocarpa, R. Br.

laurina, $R \cdot B r$-Wood pinkish, nicely marked, useful in turnery.

Brachyloma, Sol.

Section I.-Lobopogon.

Scortechinii, F. ข. M.

Section II.-Lissanthoides.

daphnoides, Benth.

Lissanthe, R. Br. (Included by F. v. Mueller in Styphelia.)

strigosa, R. Br.

Leucopogon, R.Br. (Included by F. v. Mueller in Styphelia.)

Section I.-Perojoa.

Series Psilostachyæ.

lanceolatus, $R . B r$.

var. gracilis, Benth.

Richei, R. Br.

Series Australes.

Series Collinæ.

microphyllus, R. Br.

virgatus, $R$. Br.

Series Virgatæ.

Section II.-Heteranthesis.

Hookeri, Sond.

melalezcoides, A. Cunn.

pluriloculatus, F.v. $M$.

pleiospermus, F.v.M. 
Leucopogon-contd.

\section{Section III.-Pleuranthus.}

Series Ericoideæ.

ericoides, R. Br.

Series Micranthæ.

margarodes, $R \cdot B r$.

Series Planifoliæ.

ruscifolius, R. $B r$.

imbricatus, R. Br.

cuspidatus, R. Br. (Fig. 266.)

leptospermoides, R.Br. (Fig. 267.)

flexifolius, $R . B r$.

biflorus, R. Br.

Mitchellii, Benth.

juniperinus, $R$. $B r$.

Series Concavæ.

deformis, R. Br.

neoanglicus, F.v. $M$.

Acrotriche, R.Br. (Included by F. v. Mueller in Styphelia.)

divaricata, R. $B r$.

aggregata, R. $B r$.

Monotoca, R. Br. (Included by F. v. Mueller in Styphelia.)

elliptica, R. Br.

lineata, R.Br. (Fig. 268.)

scoparia, R.Br.

Tribe II.-EPACREÆ.

Epacris, Cav.-Anstralian Heaths.

obtusifolia, Sm.

microphylla, R. Br.

pulchella, Cav.

Lysinema, R. Br.

pungens, R.Br. $=$ Woollsia pungens, F. v. M.

Sprengelia, Sm.

Ponceletia, F.v.M.

Dracophyllum, Labill.

Sayeri, F. v.M. (Fig. 269.) -There are two forms of this plant on Bellenden-Ker, which may be distinguished thus:-Var. normale, Bail.: Corolla-tube white, lobes rose-coloured, more or less erect. $V$ ar. reflexım, Bail.: Flowers all white, corolla-lobes closely reflexed. 


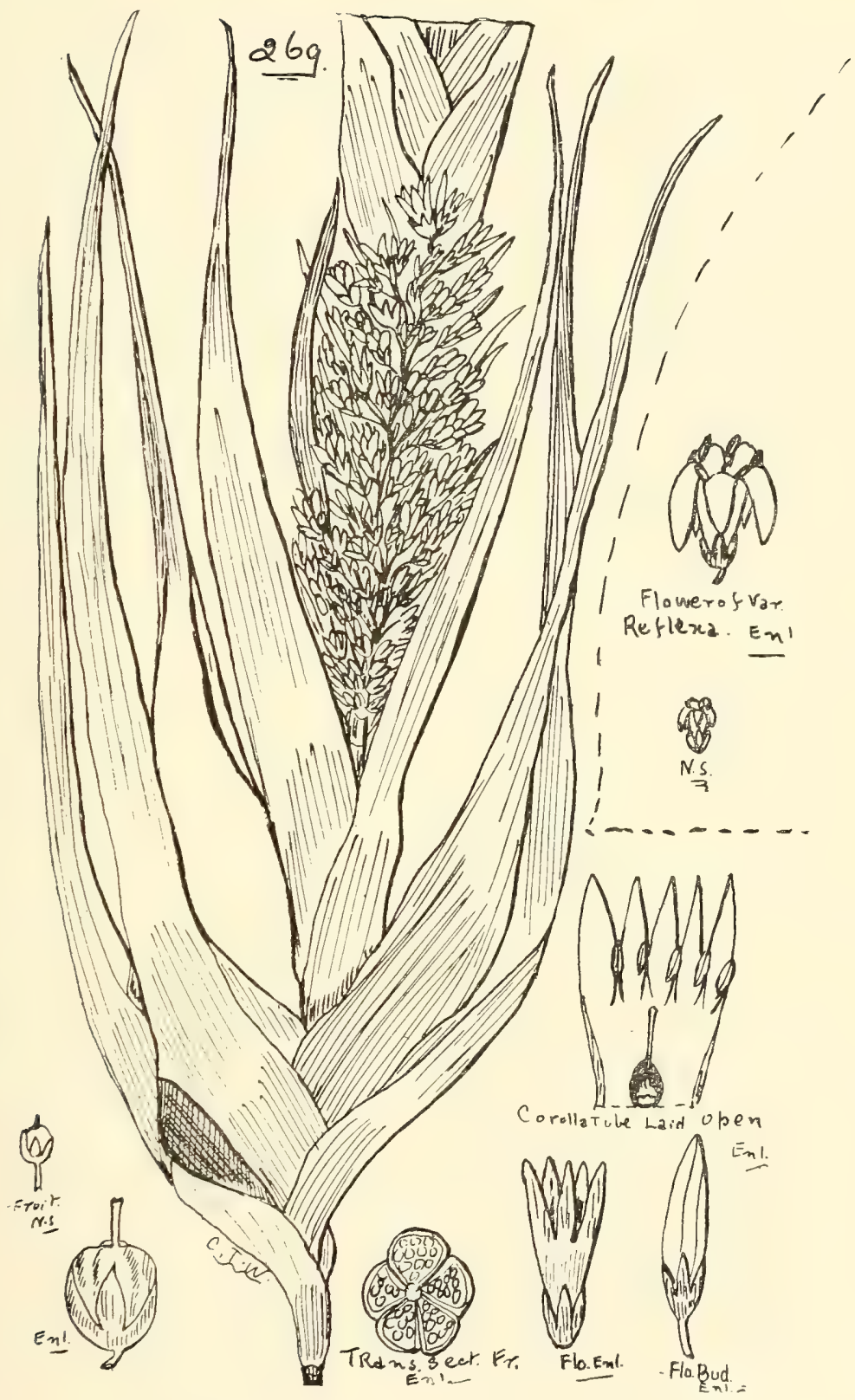

269. Dracophyllum Sayeri, $F, v, M$. 


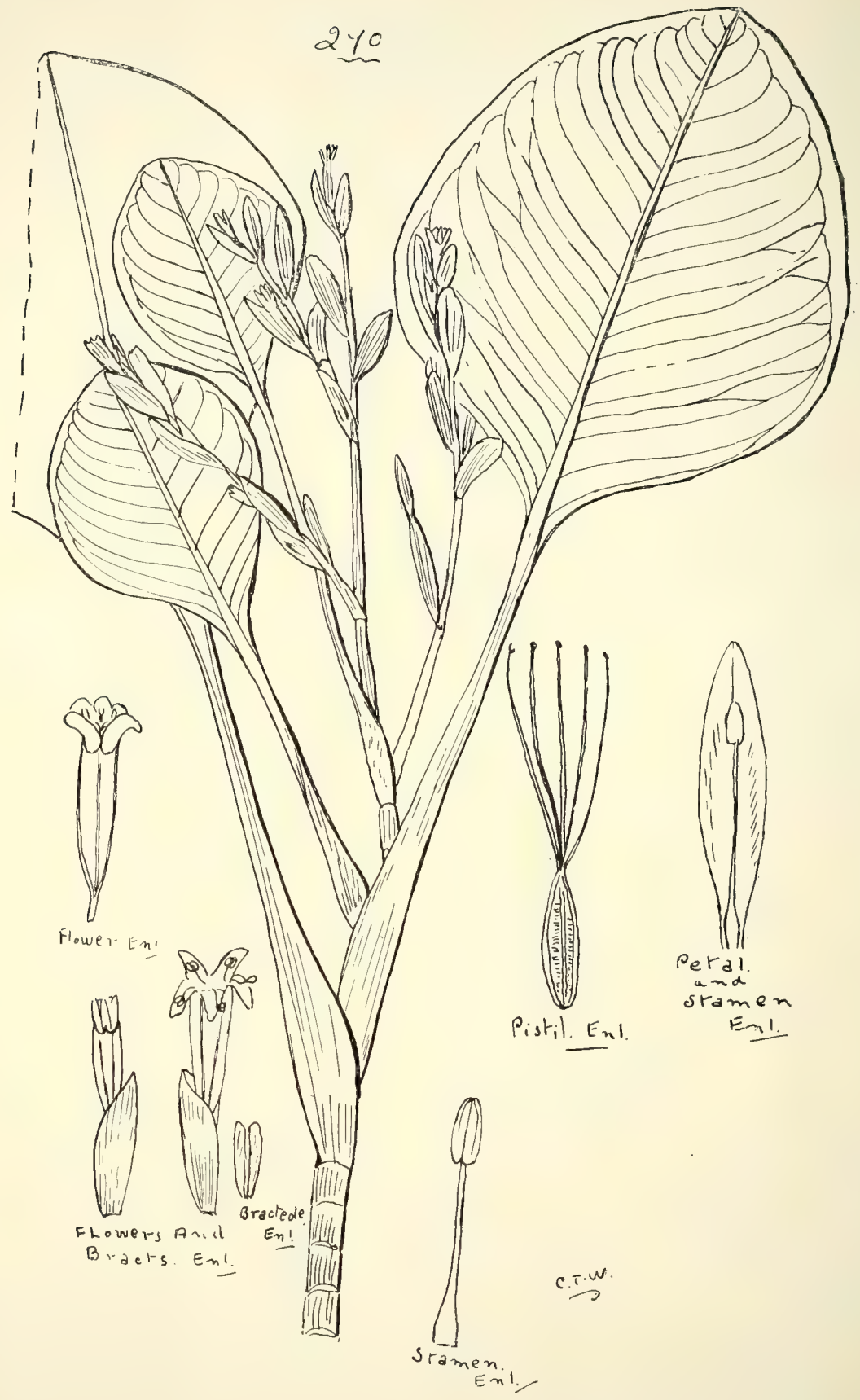

270. Egralitis annulata, $R$. $B r$. 


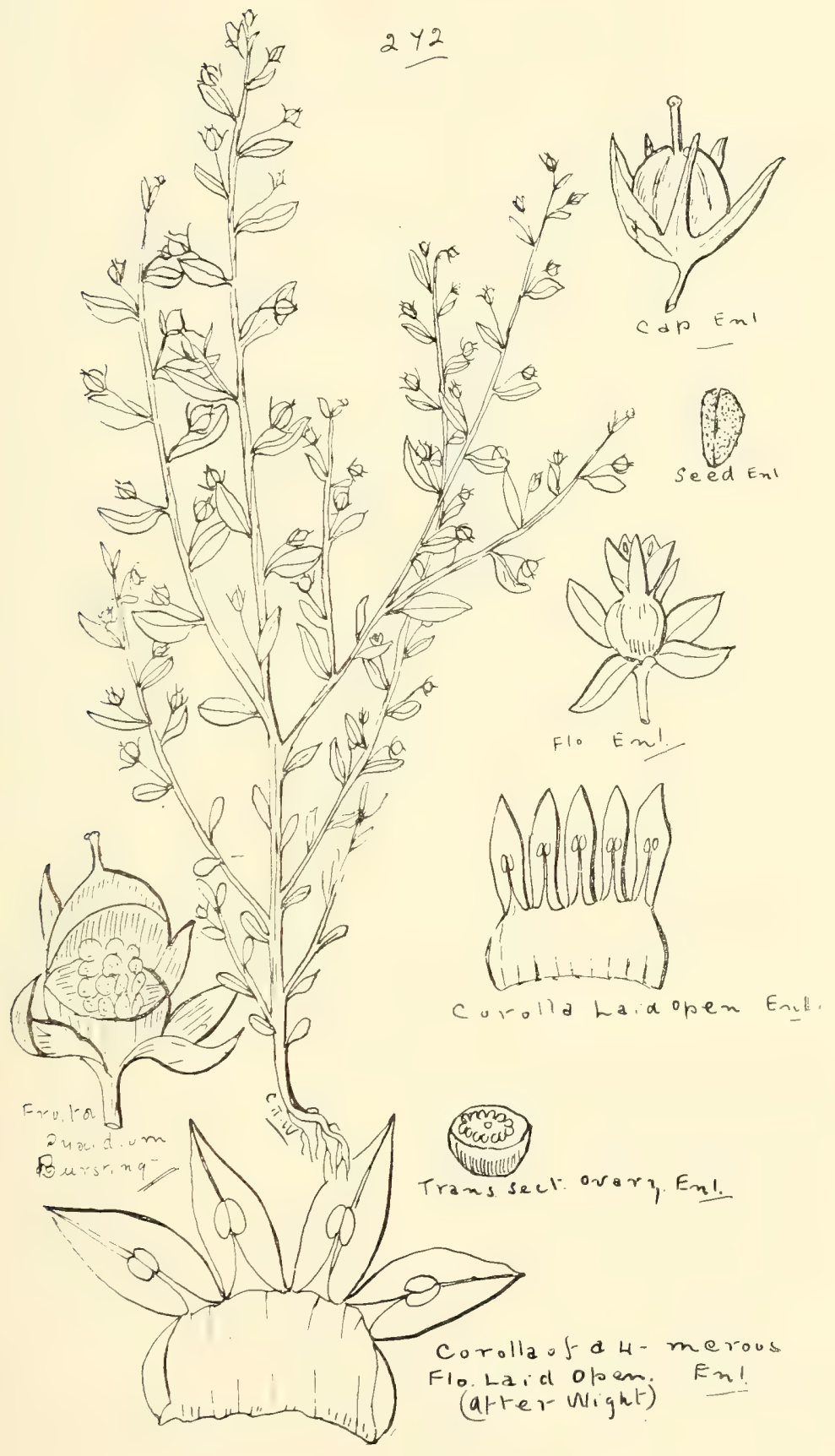

272. Anagallis pUMita, Sze. 


\section{Order LXXII.-PLUMBAGINEA.}

Tribe I.-STAticer.

Egialitis, R. Br.

annulata, R.Br. (Fig. 27o.)

Statice, Linn.

australis, Spreng.-Australian Sea Lavender. (Fig. 27I.)

Plumbago, Linn.

Tribe II.-Plumbage..

zeylanica, Limn.-The flowers of this Australian form vary from white to a lovely blue resembling P. capensis, Thunb. The roots of this and some other species are considered a dangerous irritant poison.

\section{Order LXXIII.-PRIMULACEÆE.}

Tribe I.—Lysimachie

Anagallis, Linn.-Fruit a Pyxidium.

*arvensis, Linn.-Pimpernel; poisonous. Europe. Both the red and blue flowering kinds are common in Queensland. pumila, Sw. (Fig. 272.)

Samolus, Linn.

Tribe II.-SAmoleæ.

Valerandi, Linn.-Brook-weed.

repens, Pers.

\section{Order LXXIV.-MYRSINEAE.}

Mæsa, Forsk.

TRibe I.-M Maser.

dependens, $F \cdot v \cdot M$.

var. pubescens, F.v.M. (Fig. 273.)

haplobotrys, F.v.M. (Fig. 274.)

Myrsine, Linn.

Tribe II.-Eumyrsinee.

urceolata, $R \cdot B r$.

crassifolia, R. Br.- "Marada" of Cape Grafton natives.

variabilis, R. Br.

achradifolia, $F . v \cdot M$.

campanulata, F.v.M. (Fig. 275.)

Embelia, Burm. = Samara, Linn.

australiana, Benth. and Hook.

Flueckigeri, F.v.M. 


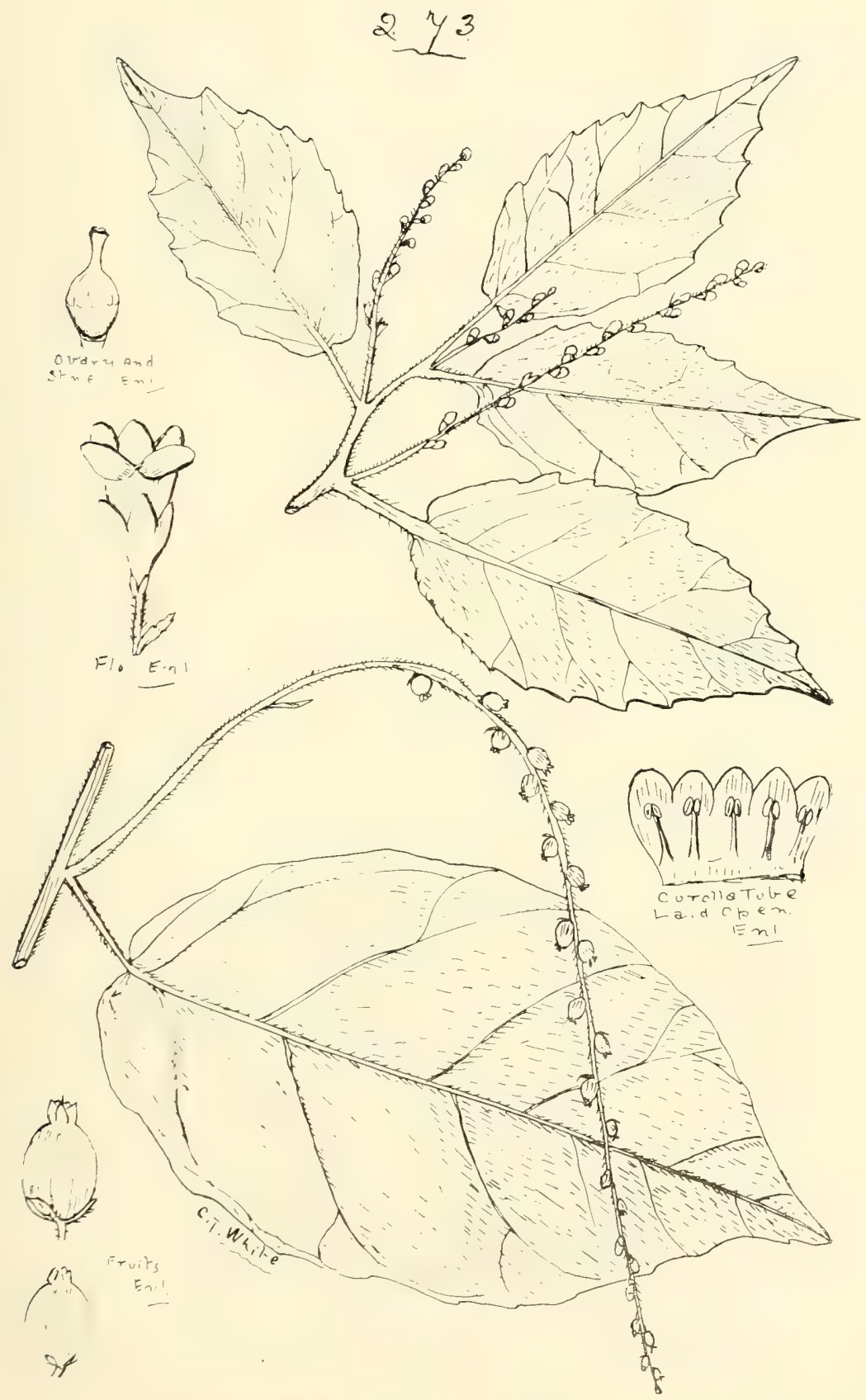

273. Mresa dependens, F. $v$. M., var. pubescens, F. v. $M$. 


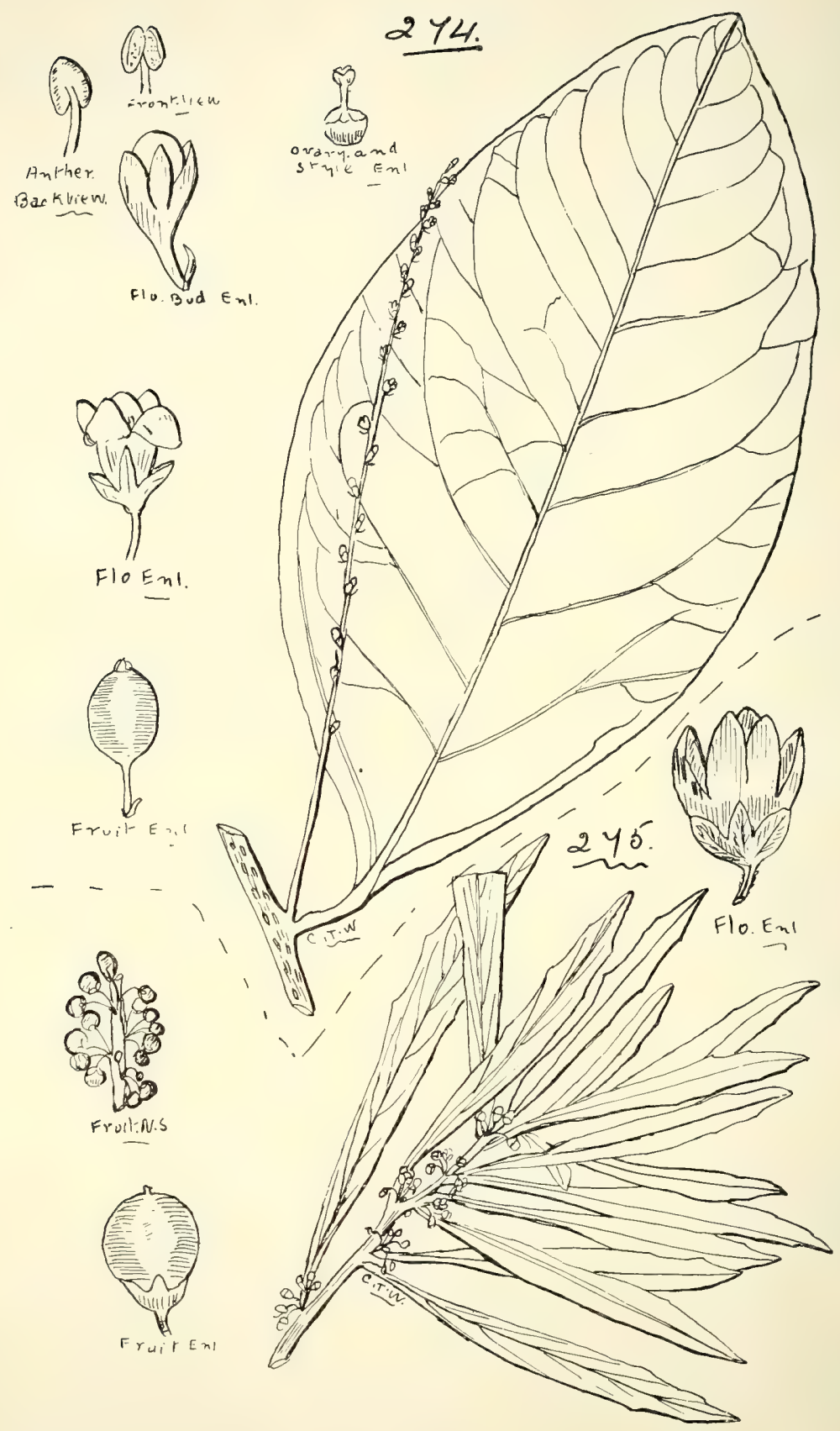

274. Masa haplobotrys, $F . v \cdot M$.

275. Myrsine campanulata, F. v. $M$. 


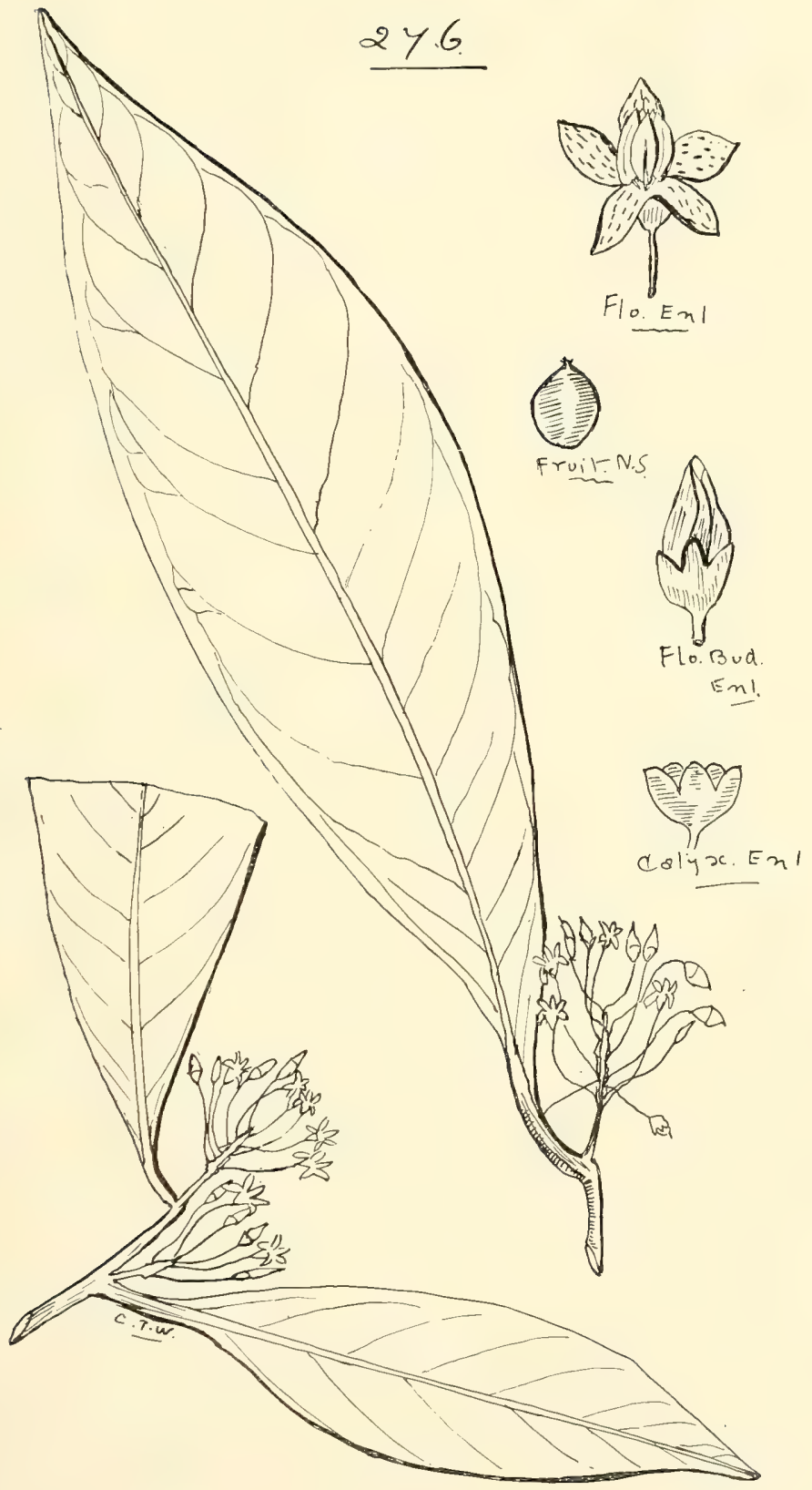

276. Ardisia brevipedata, $F \cdot v \cdot M$. 


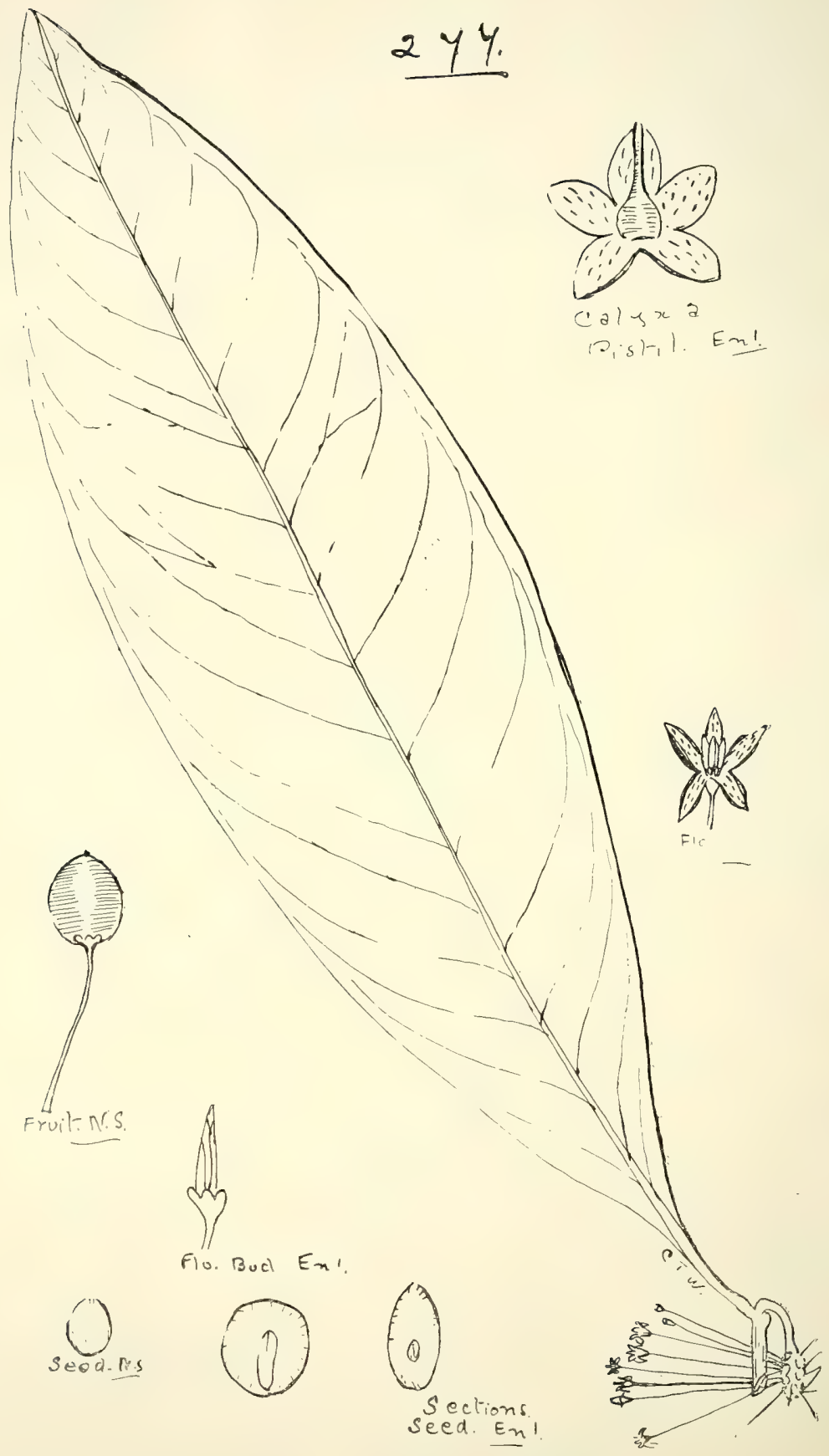

277. Ardisia pachyrRhachis, $F, v, M$. 
Ardisia, Lim.

pseudojambosa, F.v.M.

brevipedata, F.v.M. (Fig. 276.)

pachyrrhachis, F.v.M. (Fig. 277.)

Egiceras, Gcertn.

majus, Gertn.-River Mangrove. The bark contains a large quantity of saponin (Dr.T. L. Bancroft).

Alliance VI.-EBENALES.

\section{Order LXXV.-SAPOTACEE.}

Many plants of this Order yield Caoutchouc.

Chrysophyllum, Linn.

pruniferum, F.v.M.-Wood useful for bentwork.

Lucuma, Juss.

Unmackiana, Bail., Q1. Bot. Bu11. iv. (I891) I2.

(Sersalisia) sericea, Benth. et Hook.- "Mongo" of Mount Cook natives. A useful cabinet-wood.

(Sersalisia) galactoxylon, Benth. et Hook. =S. galacto.rylon,

F. v. M.; Bassia galactoxylon, F. v. M.-“"Murdarka”;

"Ngorbai" of Cape Grafton natives. Pencil Cedar of

Cairns; an excellent cabinet-wood. (Fig. 278.)

Amorphospermum, Benth. et Hook. = Amorphospermum antilogum, F. v. M.

chartacea, Bail.

obpyriformis, Bail.

Sideroxylon, Linn. (Including Achras.)

arnhemicum, Bcnth. et Hook. (Fig. 279.)

Pohlmanianum, F.v.M.- "Arlian" of Princess Charlotte

Bay natives. One of the best woods for engraving work. xerocarpum, F.v.M.

chartaceum, F.v.M.-- "Moiary" of Barron River natives. euphlebium, F.v. M. (Fig. 280.)

Brownlessianum, F.v.M.- "Wanakan" of Bloomfield River natives.

laurifolium, F.v.M.-Local name at Kin Kin, "Coondoo," from the Coondoo Mountain. The sweet astringent bark of this tree might be useful in throat diseases (Dr. Joseph Bancroft).

australis, Benth. et Hook.-Scrub Crab-apple; Black Apple.

A useful building wood.

argenteum, Spreng. 
Sideroxylon-contd.

eerwah, Bail. (Fig. 28I.)

dugulla, Bail. (Native name at Barron River.) (Fig. 282.)

myrsinoides, $A$. Cunn.

Hormogyne, A. DC.

cotinifolia, A. DC.-A useful cabinet-wood.

Mimusops, Limn.

parvifolia, R. Br.-“ Ngoru" of Batavia River natives. (Fig. 283.)

Browniana, Benth.- "Undoour" of Princess Charlotte Bay natives. (Fig. 284.)

\section{Order LXXVI.--EBENACEÆ.}

Maba, Forst.

laurina, R. Br.

sericocarpa, F. v. M.-Black Myrtle of Kin Kin. The Northern and Southern plants differ slightly in the calyx. rufa, Labill.

hemicycloides, F. $v, M$.

compacta, R. Br.

laxiflora, Benth.

fasciculosa, F. v. M.

reticulata, R.Br. (Fig. 285.)

geminata, $R$. Br.-An ebony wood.

humilis, R. Br.-Ebony-wood of Queensland. "Athea" of Batavia River and "Thankoin" of Mitchell River natives. (Fig. 286.)

Diospyros, Linn. (Including Cargillia.)

hebecarpa, A. Cunn.- "Kolin" of Cooktown, "Ngam-boi" of Bloomfield River, and "Kana-wara" of Barron River natives. Suspected of poisoning stock at Somerset. (Fig. 287.)

maritima, Blume $=$ Cargillia lara, R. Br.

mabacea, F.v.M. $=$ C. mabacea. F. v. M.

australis, R. Br.

australis, R.Br.= C. australis, R. Br.

pentamera, F.v.M. $=$ C. pentamera, F. v. M..-Black Myrtle.

\section{Order LXXVII.-STYRACE}

Symplocos, Linn.

spicata, Rorb., var. australis, Benth.-Wood like Box.

Thwaitesii, F.v.M.

paucistamineus, F. $\%$.M. et Bail. (Fig: 288.) 


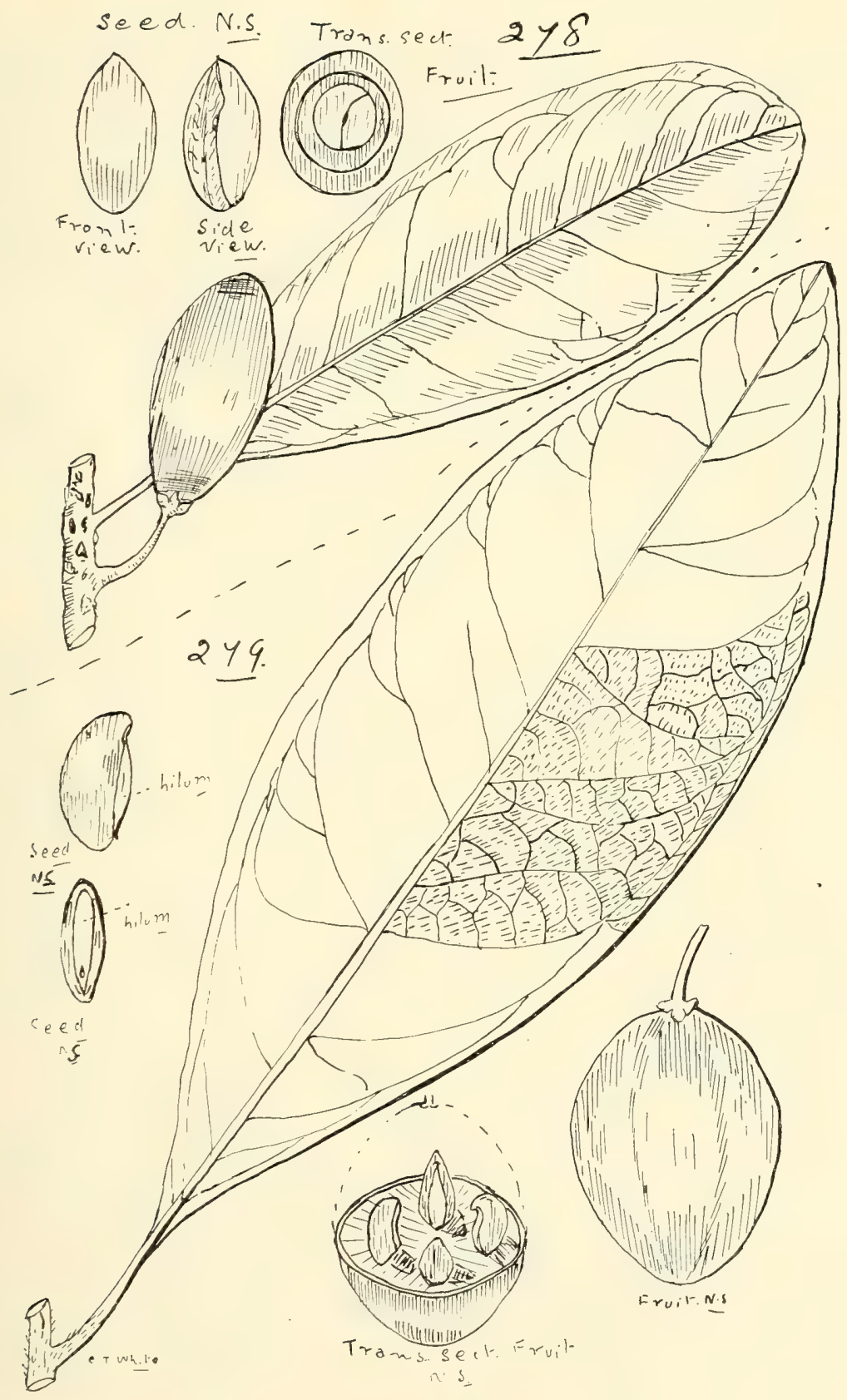

278. LuCU MA Galactoxylon, $F . v \cdot M$.

279. Sideroxylon arnhemicum, Benth. et Hook. 


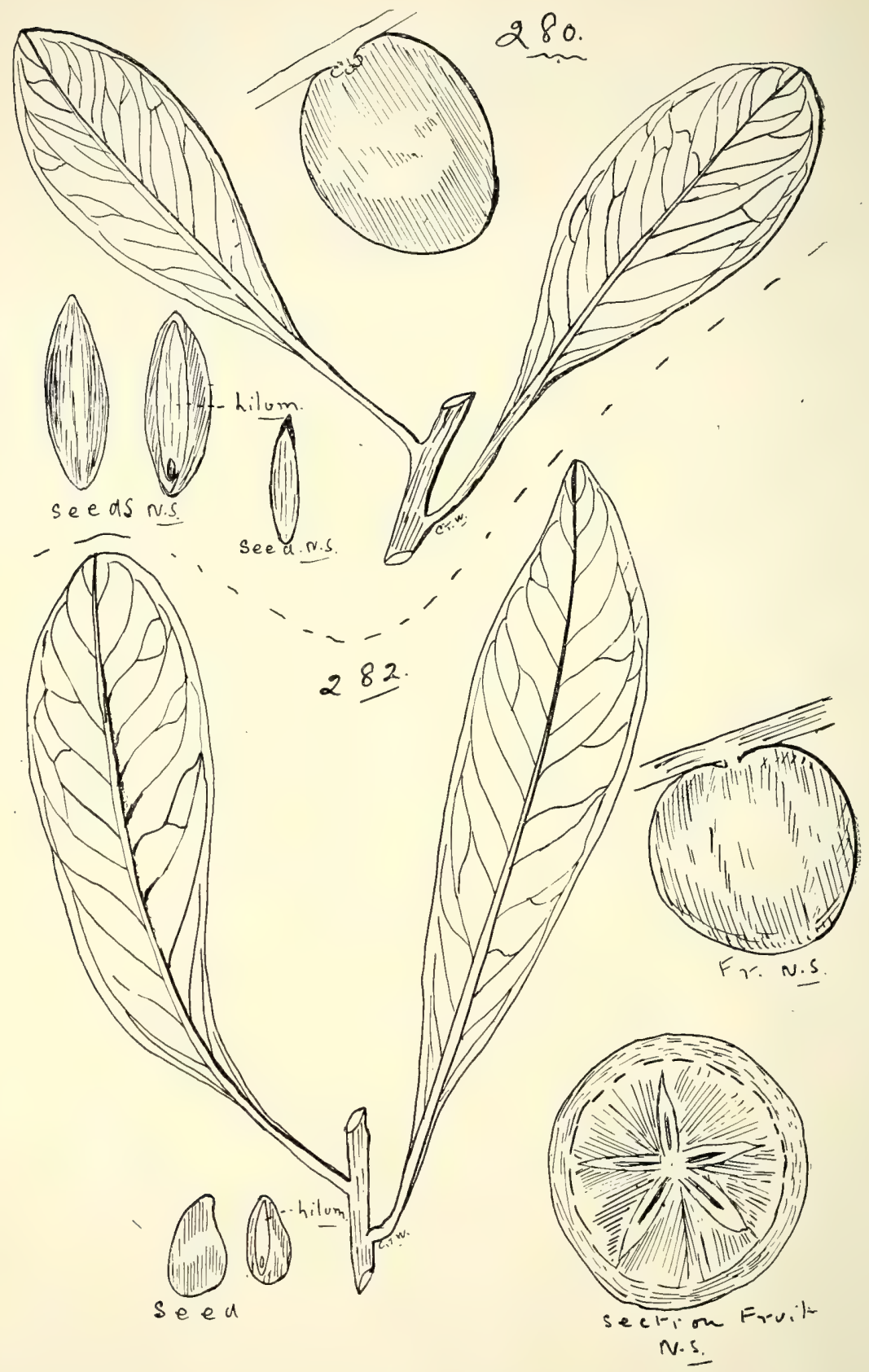

280. Sideroxylon euphlebium, $F, v \cdot M$.

282. S. dugulla, Bail. 



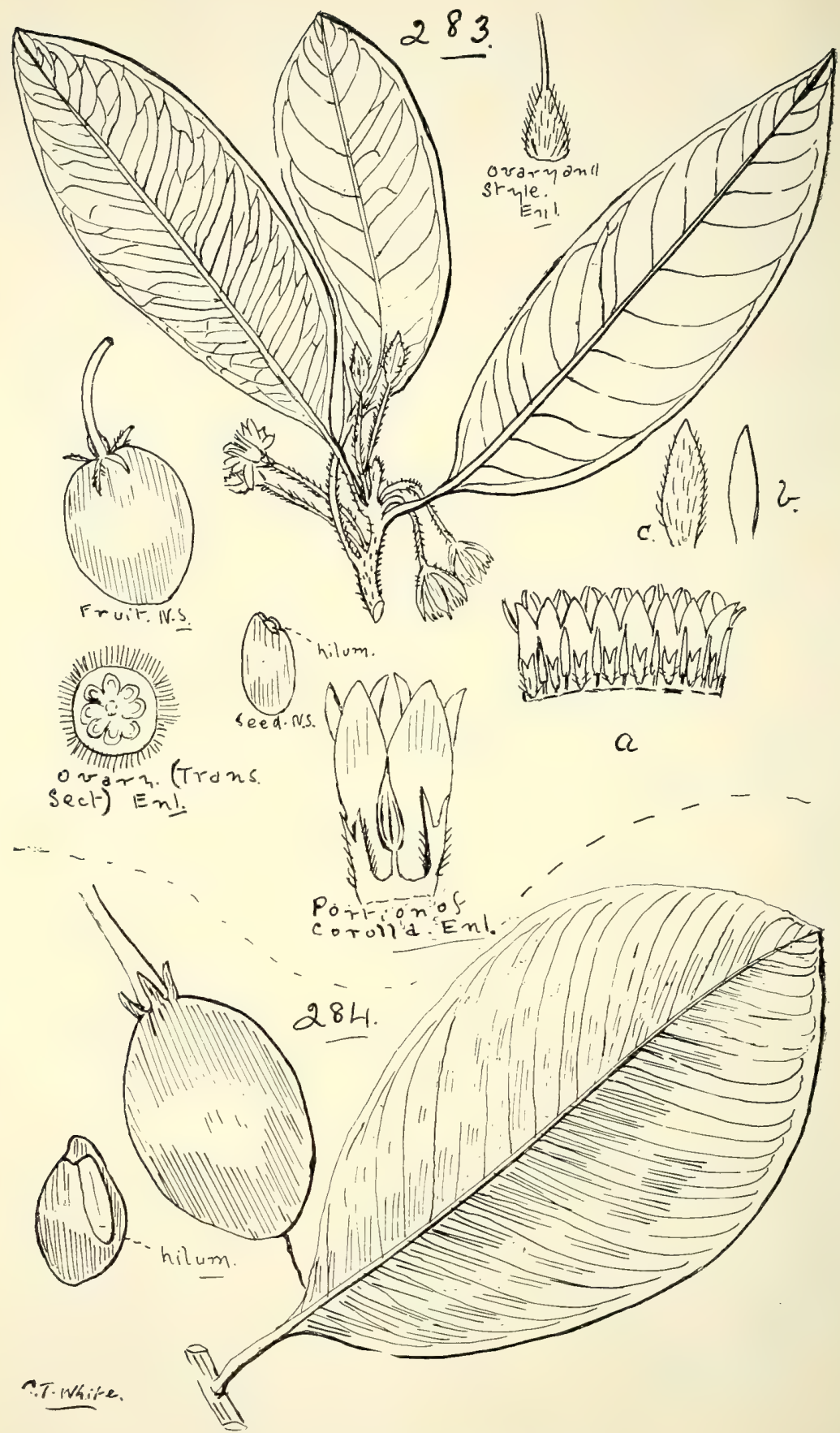

283. Mimusops pakvifolia, $R$. $B r$.

(a) Corolla laid open, (b) inner calyx segment, (c) outer calyx segment. (a) -(c) enl.

284. M. Browniana, Benth. 
SERIES III.-BICARPELLATÆ.

Alliance VII.-GENTIANALES.

\section{Order LXXVIII.-OLEACE\#.}

Tribe I.-Jasmineæ.

Jasminum, Limn.

Leaves all or almost all 3-foliolate.

didymum, Forst.

var. pubescens, Benth.

racemosum, $F \cdot v \cdot M$.

lineare, R. Br.- "Urooh" of Bundaberg natives.

singuliflorum, Bail. et F. v. M. (Fig. 290.)

Leaves simple (unifoliolate).

simplicifolium, Forst.

var. molle, Benth.

æmulum, R. Br. (Fig. 289.)

suavissimum, Lindl.

Tribe II.-Oleiner.

Linociera, Sw. = Chionanthus, Gærtn.; Mayepea, Aubl. ramiflora, $D C .=$ C. ramifiora, Roxb.

picrophloia, F.v.M. $=$ C. picrophloia, F.v. M.-Bark yields a brownish-yellow dye.

axillaris, $R . B r .=$ M. a.rillaris, F. v. M. (Fig. 29I.)

Notelæa, Vent.

ovata, R. Br.

longifolia, Vent. (Fig. 292.)

var. velutina, Bail., $n$. var.-Leaves velvety pubescent.

This variety is met with at Killarney. (Fig. 293.)

punctata, R. Br.

microcarpa, R. Br.

linearis, Benth.

Olea, Linn.

paniculata, R.Br.-Native Olive. "Billan-billan" of Bunya

Mountain natives. The wood of this tree when newly cut has a rose fragrance, and in some parts is known as "Pigeonberry Ash."

Ligustrum, Linn.

australianum, F.v. M. 


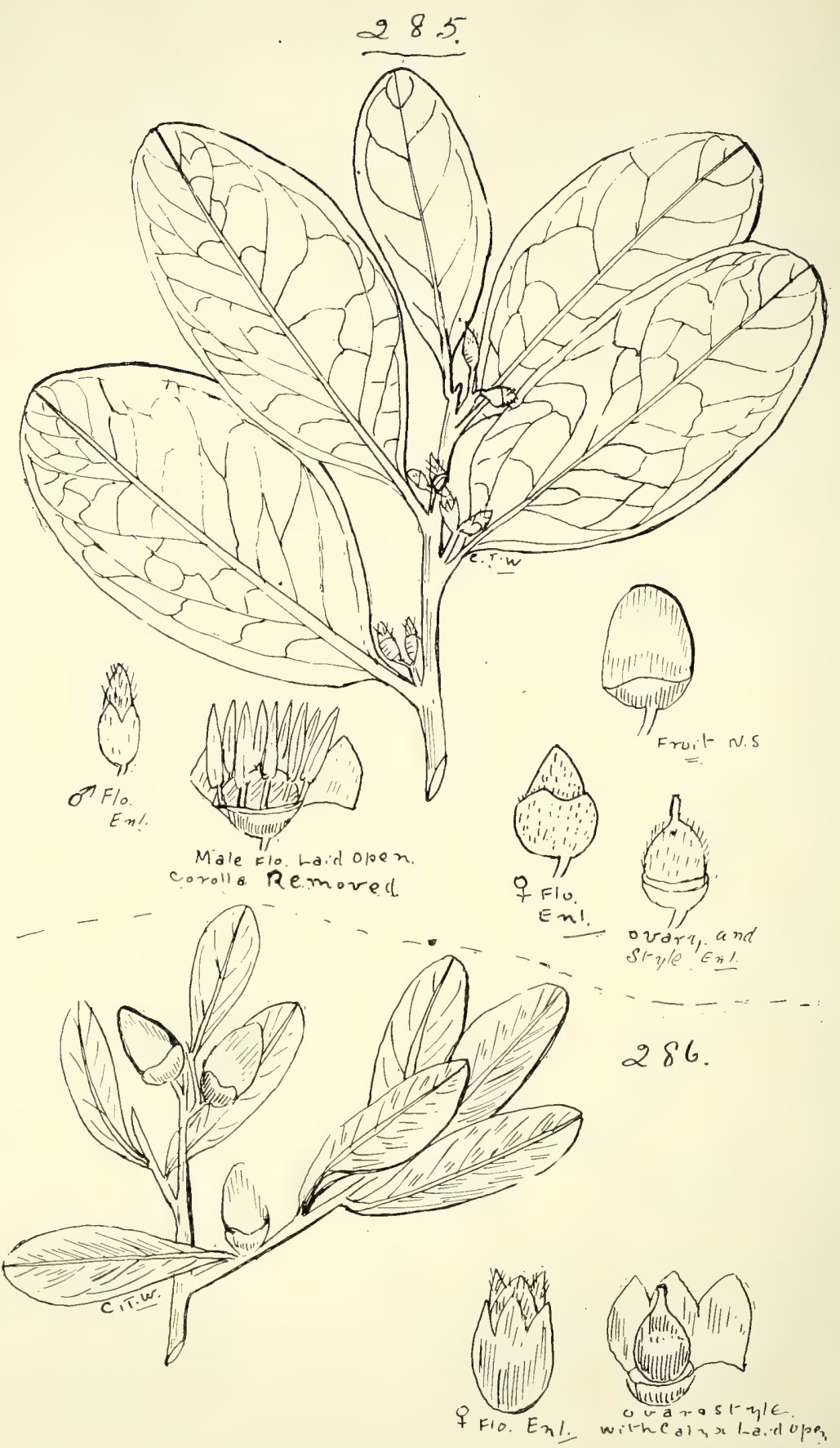

285. Maba reticulata, $R$. $B r$.

286. M. humilis, R. $B r$. 


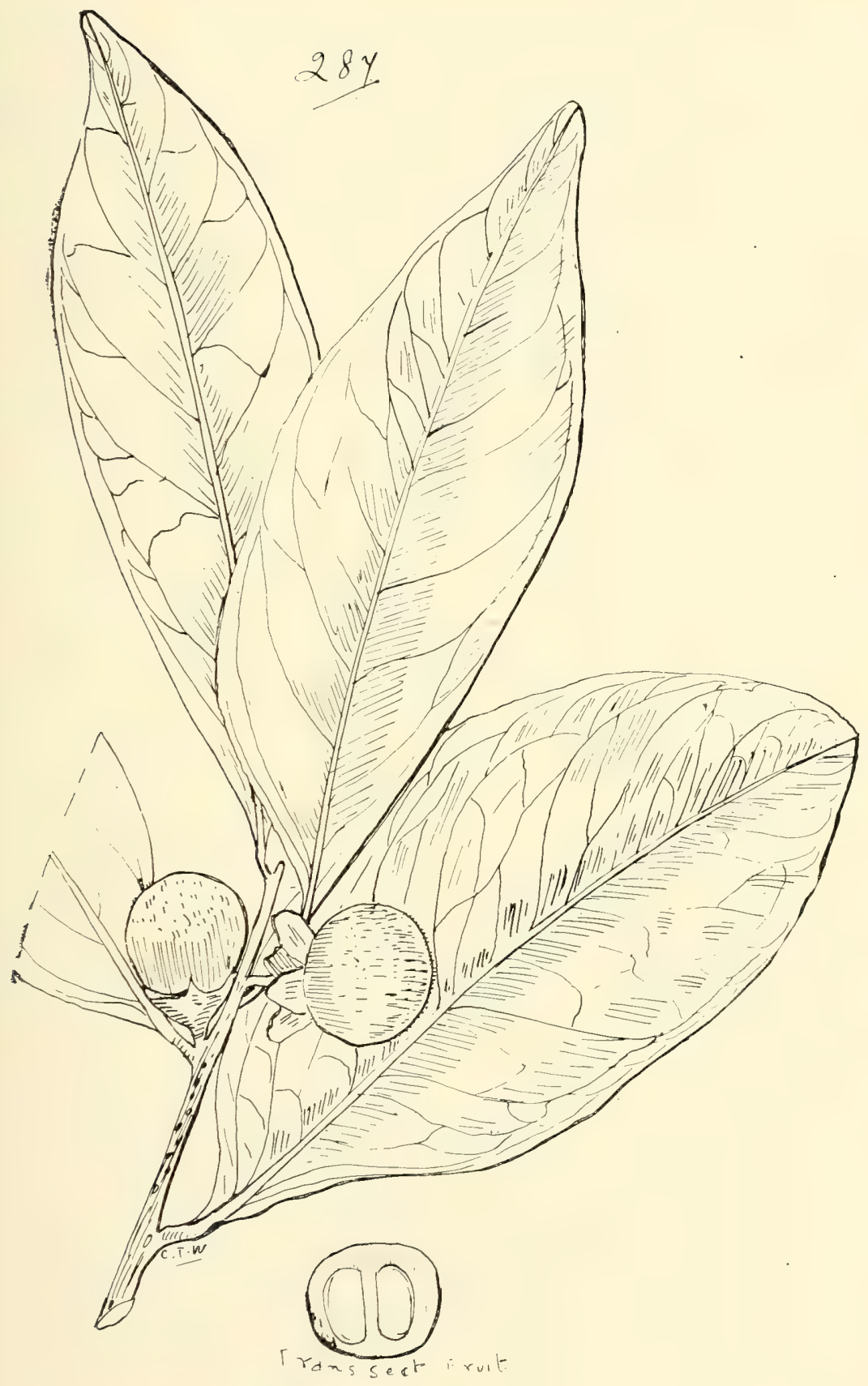

287. Diospyros hebecarpa, A. Cunn. 


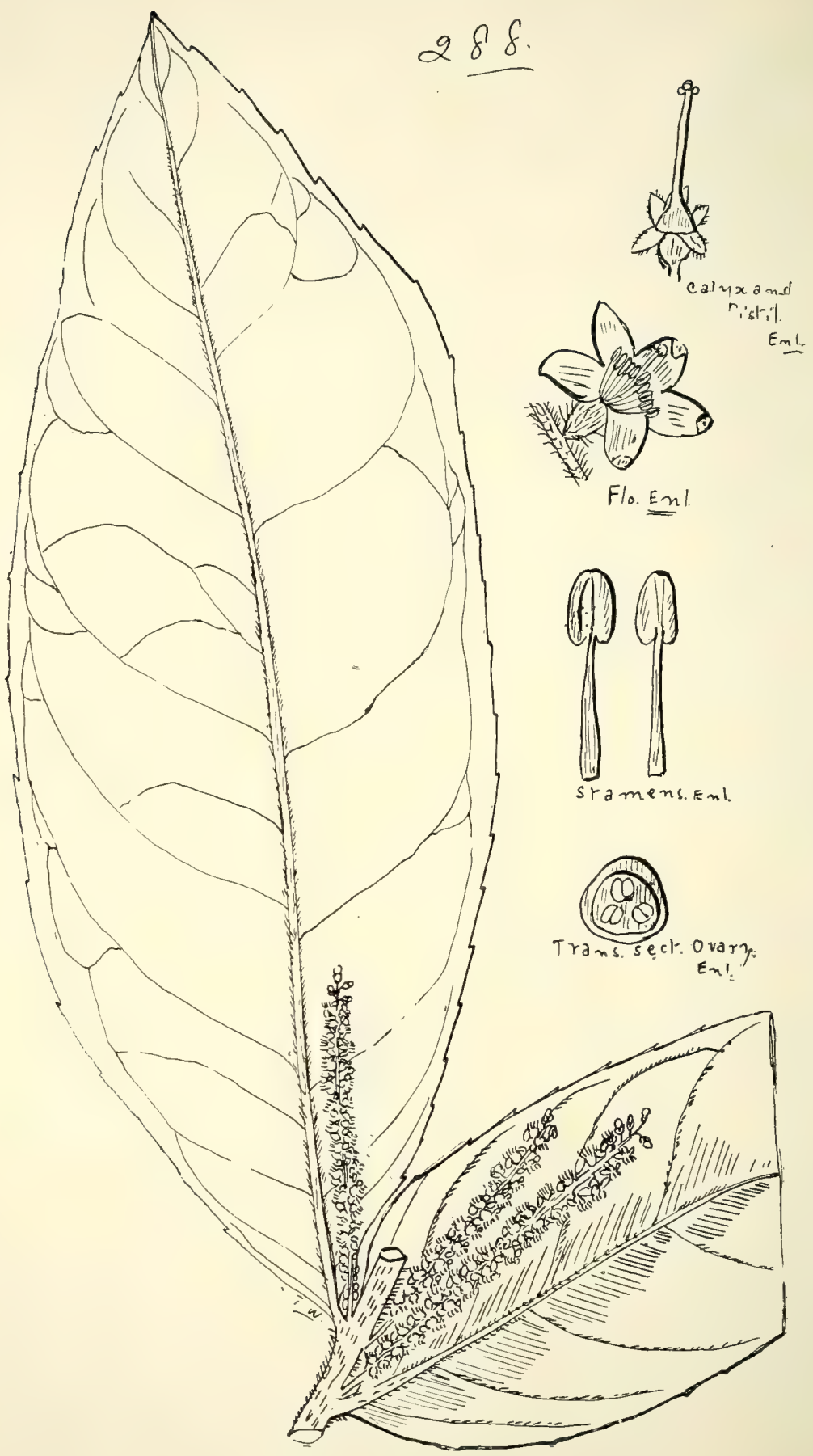

288. Symplocos paucistamineus, F. v. M. et Bail. 


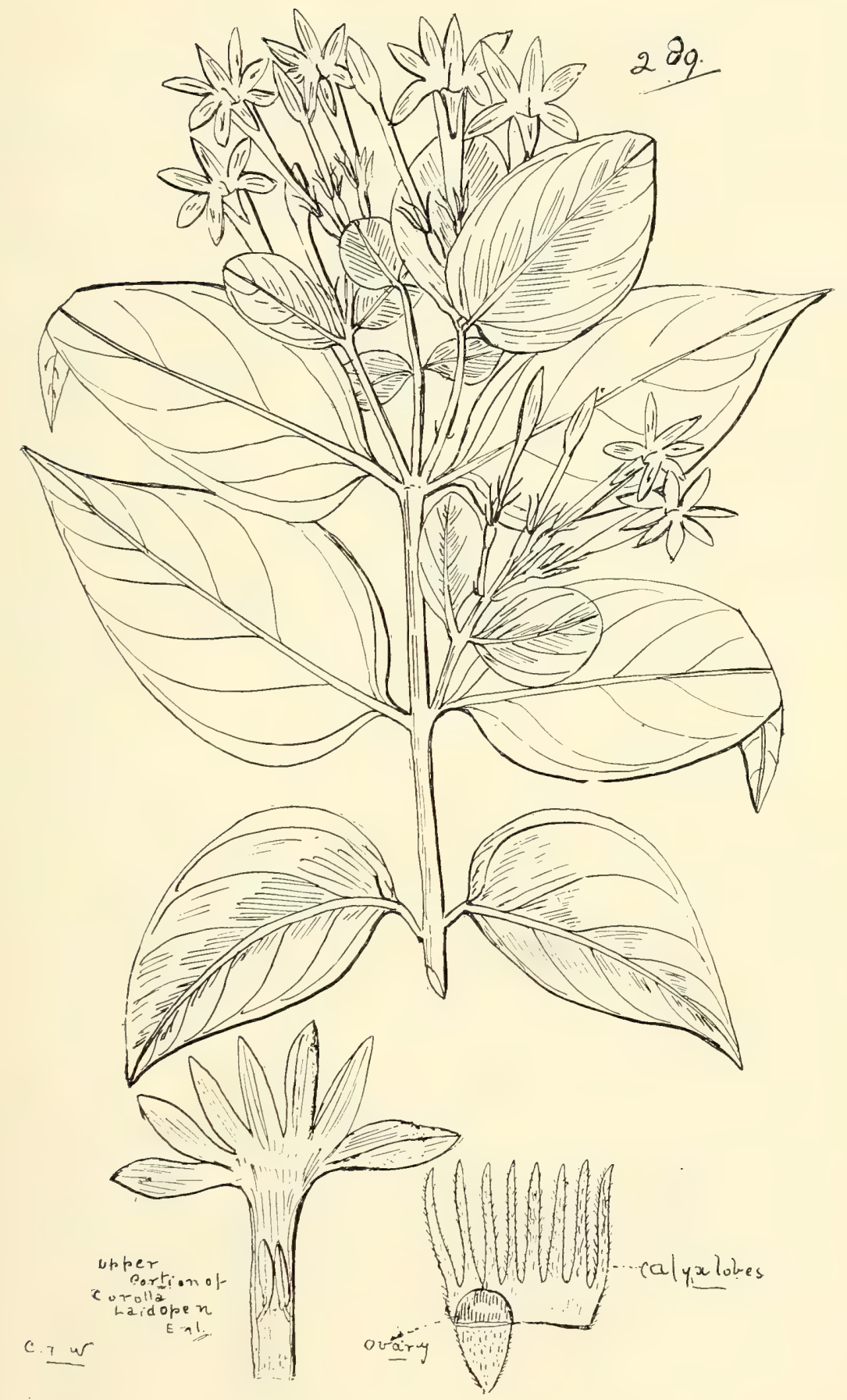

289. Jasminum emulum, $R$. $B r$. 

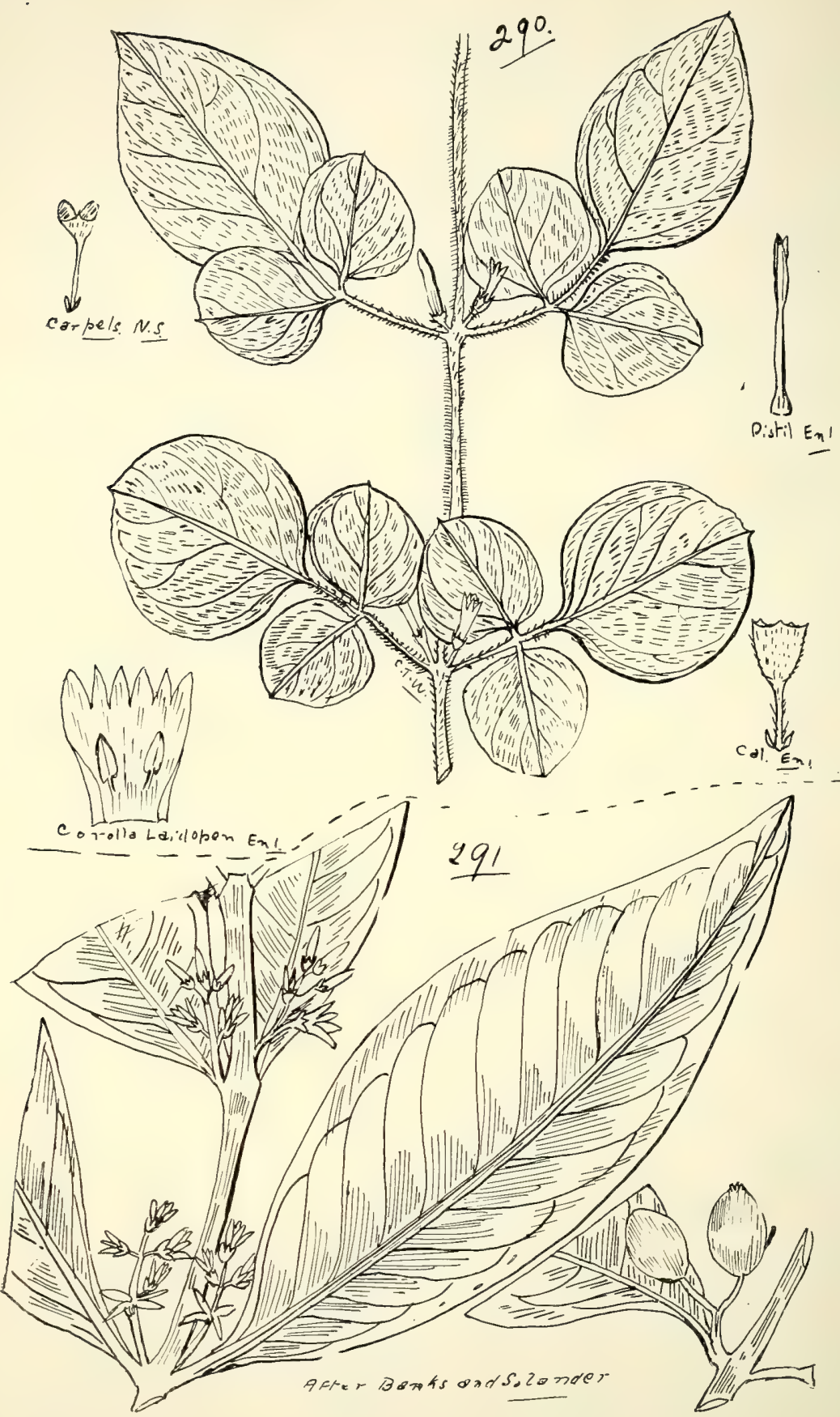

290. JASminum singuliflorum, Bail. et F. v. M. 291. Linociera axillaris, R. $B r$. 


\section{Order LXXIX.-APOCYNACEAE.}

Many plants of this Order yield Caoutchouc.

Tribe I.-CARISSE.E.

Chilocarpus, $B l$.

australis, F.v.M.- "Paiamara" of Tully River and "Wongali" of Barron River natives.

Melodinus, Forst.

acutiflorus, $F . v . M$.

Guilfoylei, F.v.M.-_ Byamurra” of Tully River natives.

murpe, Bail.-_"Murpe" of Russell River natives. (Fig. 294.)

Carissa, Linn.

laxiflora, Benth.

ovata, R.Br.- "Kunkerberry" of Cloncurry, "Karey" of Rockhampton, and "Ulorin" of Cleveland Bay natives. var. stolonifera, Bail.-Poisonous. (Fig. 295.)

var. pubescens, Bail, n. var.-Charters Towers (C. F.

Plant). Branchlets and leaves pubescent (especially on

the under surface). (Fig. 296.)

scabra, R. Br.

lanceolata, R.Br. (Fig. 297.)

Tribe II.-Pluneriete.

Alyxia, R. Br.-Chain-fruit.

ruscifolia, R.Br.- "Moonya” of Bundaberg natives.

var. pugioniformis, Bail.

var. ulicina, Bail.

magnifolia, Bail., Q1. Agri. Journ. xxv. Io. (Fig. 298.)

ilicifolia, F.v. M. (Fig. 299.)

obtusifolia, R. Br. (Fig. 30c.)

spicata, R. Br.

thrysiflora, Benth.

Thozetii, F.v. M.

Cerbera, Linn.

Odollam, Gartn.-Poisonous.

var. Mugfordi, Bair.

Ochrosia, Juss.

elliptica, Labill.

Newelliana, Bail.

Poweri, Bail.

Cowleyi, Bail.

Kilneri, F.v.M.

Moorei, F. v. M. 
*Vinca, Linn.

rosea, Limn.- Old Maid Flower. A weed of many tropical countries; common in Queensland, especially on oụr tropical coast.

var. roseus, G. Don.

var. albus, G. Don.

var. occellatus, G. Don.

Alstonia, R. $B r$.

scholaris, R.Br.- "Birrba" of Forest Hill and "Koorool" of Barron River natives. A valuable tonic in cases of fever. The wood is in some localities known as "White Pine."

verticillosa, F.v.M.- "Moranggal" of Cooktown natives.

villosa, Blume.

constricta, F.v.M.-Bancroft's Tonic-bark; Quinine or

Bitter-bark. "Biner canary-yellow dye. (Fig. 300 bis.)

var. mollis, Bail. (Under. Fig. 300 bis.)

var. montmariensis, Bail. (Under Fig. 300 bis.)

somersetensis, Bait.-May likely prove a valuable rubber-tree.

(Fig. 3OI.)

Tabernæmontana, Linn.

orientalis, R.Br.

var. angustisepala, Benth.

var. angustifolia, Benth.-Sometimes seen with pretty variegated foliage.

pubescens, R. Br.

TRIBE III.-ECHITIDE

Subtribe Parsonsica.

Lyonsia, R. Br.

lilacina, F. \% M.

straminea, R. Br.

reticulata, F.v.M.

Langiand, $F \cdot \tau \cdot M$.

largiflorens, F. .M. F. Fig. 30I bis.)

latifolia, Benth.

oblongifolia, Benth.

eucalyptifolia, F.v.M.-“Wedeely" of St. George natives.

Parsonsia, R. Br.

lanceolata, R. Br.

velutina, $R . B r$.

var. glabrescens, Benth.

nesophila, Bail. (Fig. 302.)

Leichhardtii, F.v. M.

ventricosa, $F . v, M$. 


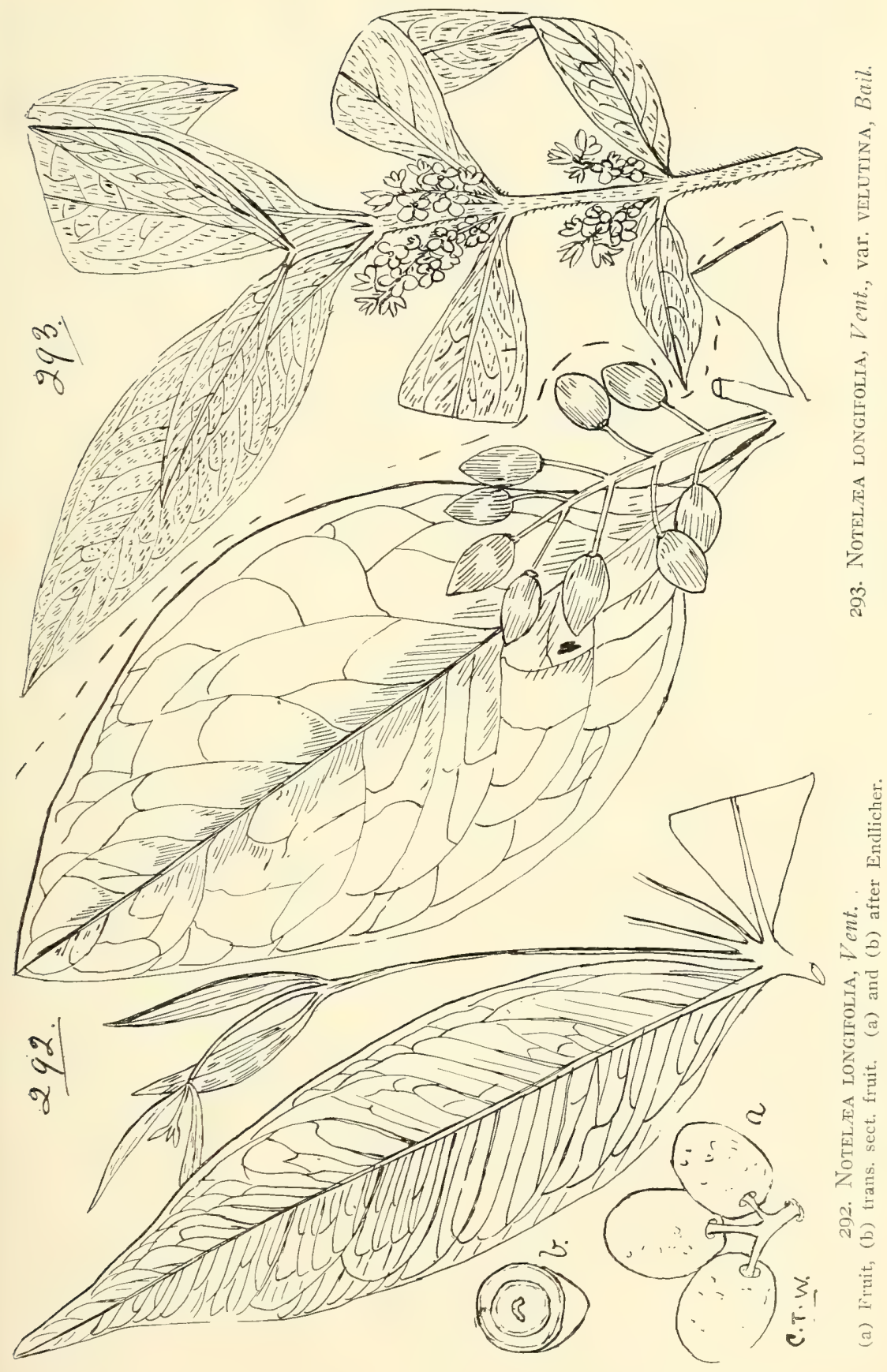




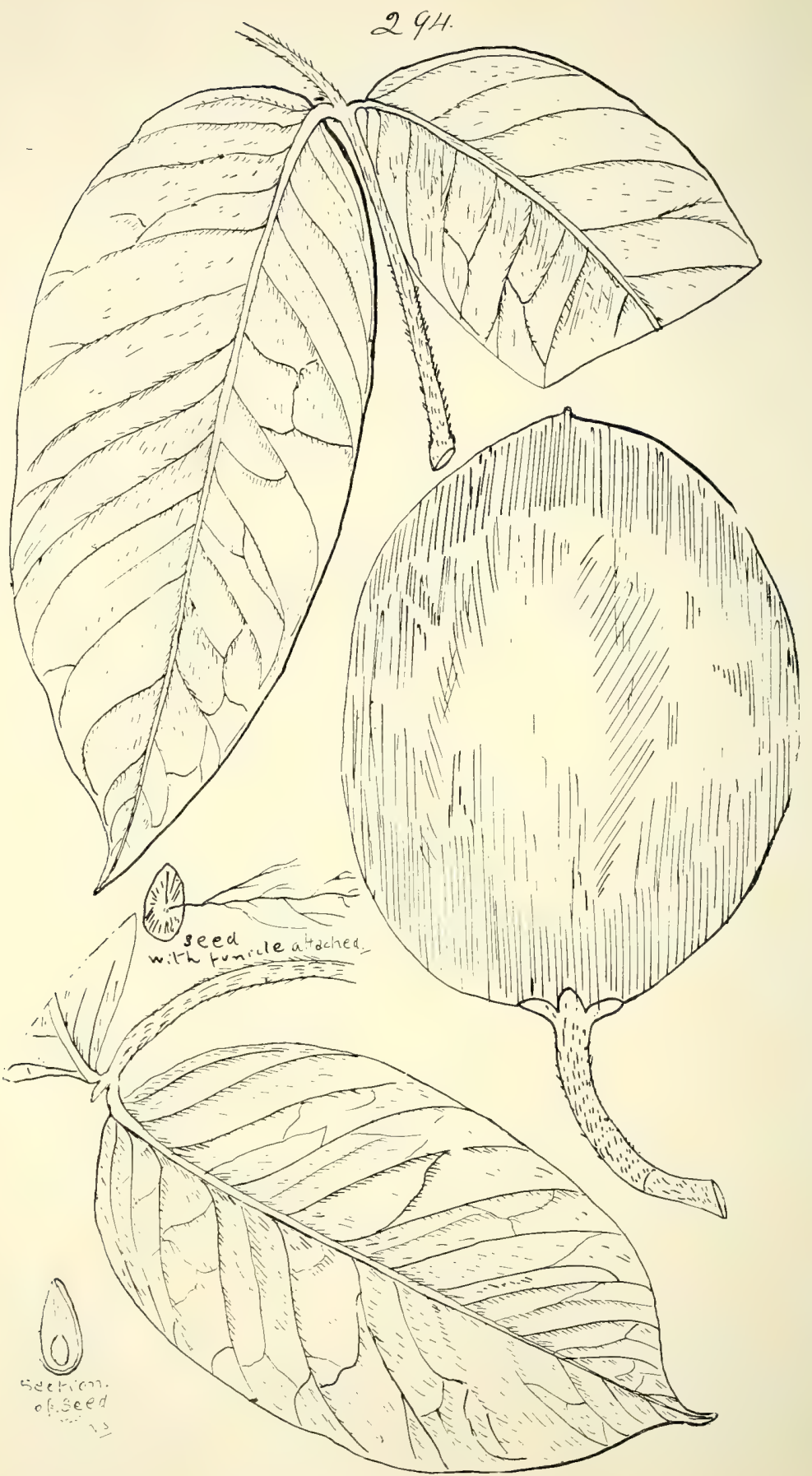

294. Melodinus murpe, Bail. 


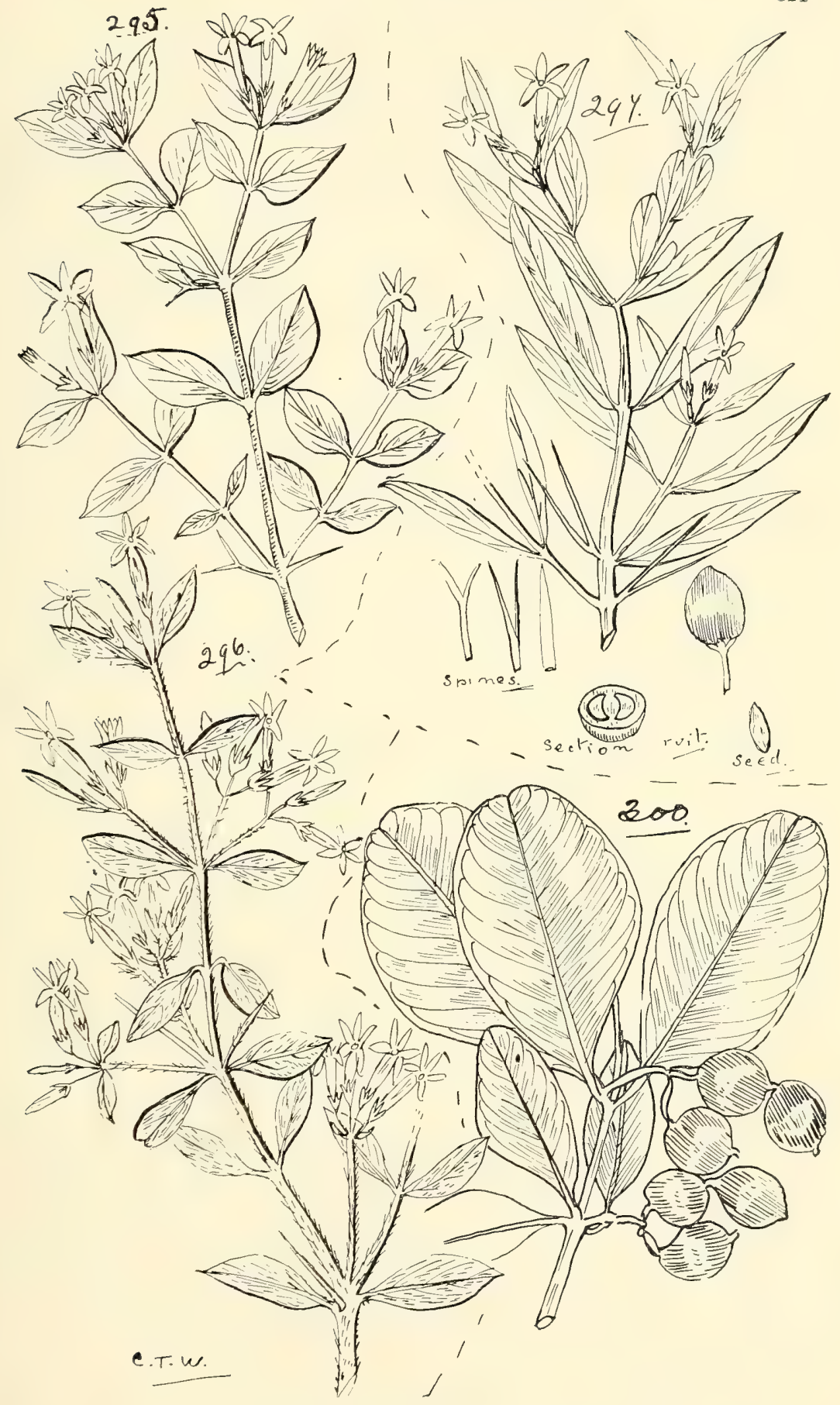

295. Carissa ovata, R. Br., var. stolonifera, Bail. 296. C. ovata, $R$. $B r$., var. pUbescens, $B a i l$.

297. C. Lanceolata, R. Br.

30G. Alyxia obtusifolia, $R$. $B r$. 


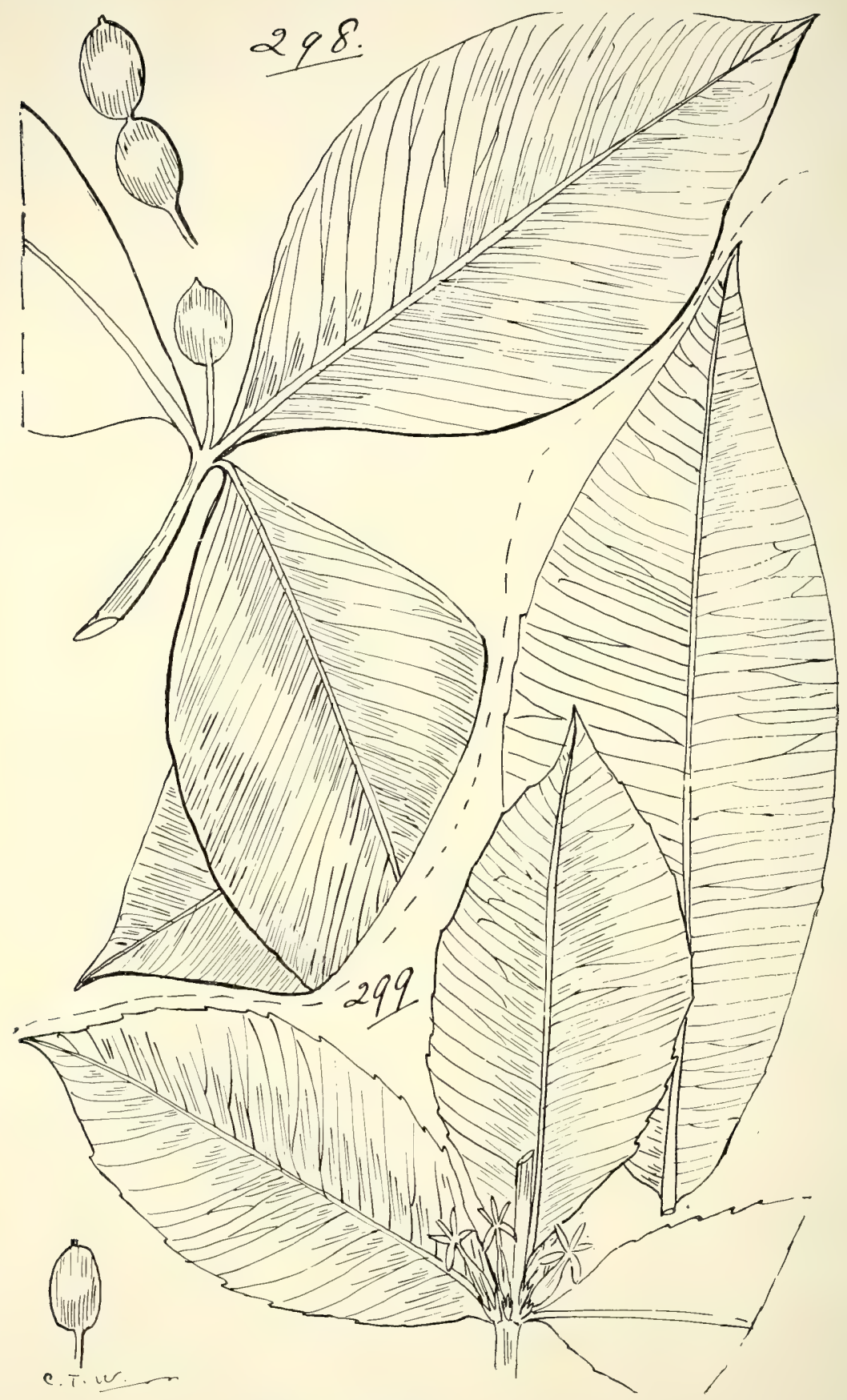

298. Alyxia magnifolia, Bail.

299. A. Ilicifolia, Bail. 


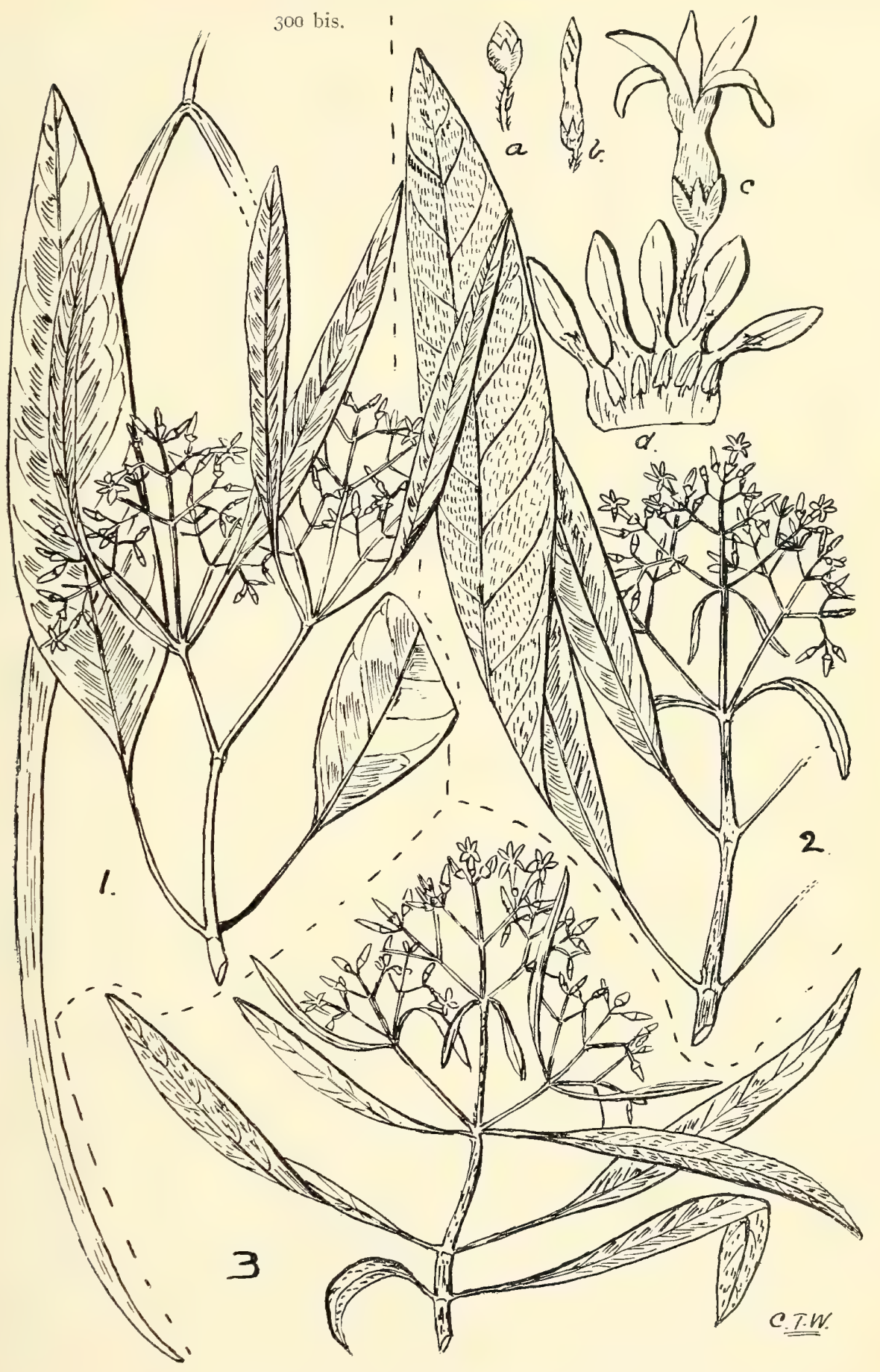

300 bis. Alstonia constricta, F. v. M.

I. Normal form.

2. A. CONSTRICtA, var. mollis, Bail.

(a) (b) (c) Flower in various stages, (d) top portion of corolla laid open at end.

3. A. Constricta, var. montmariensis, Bail. 


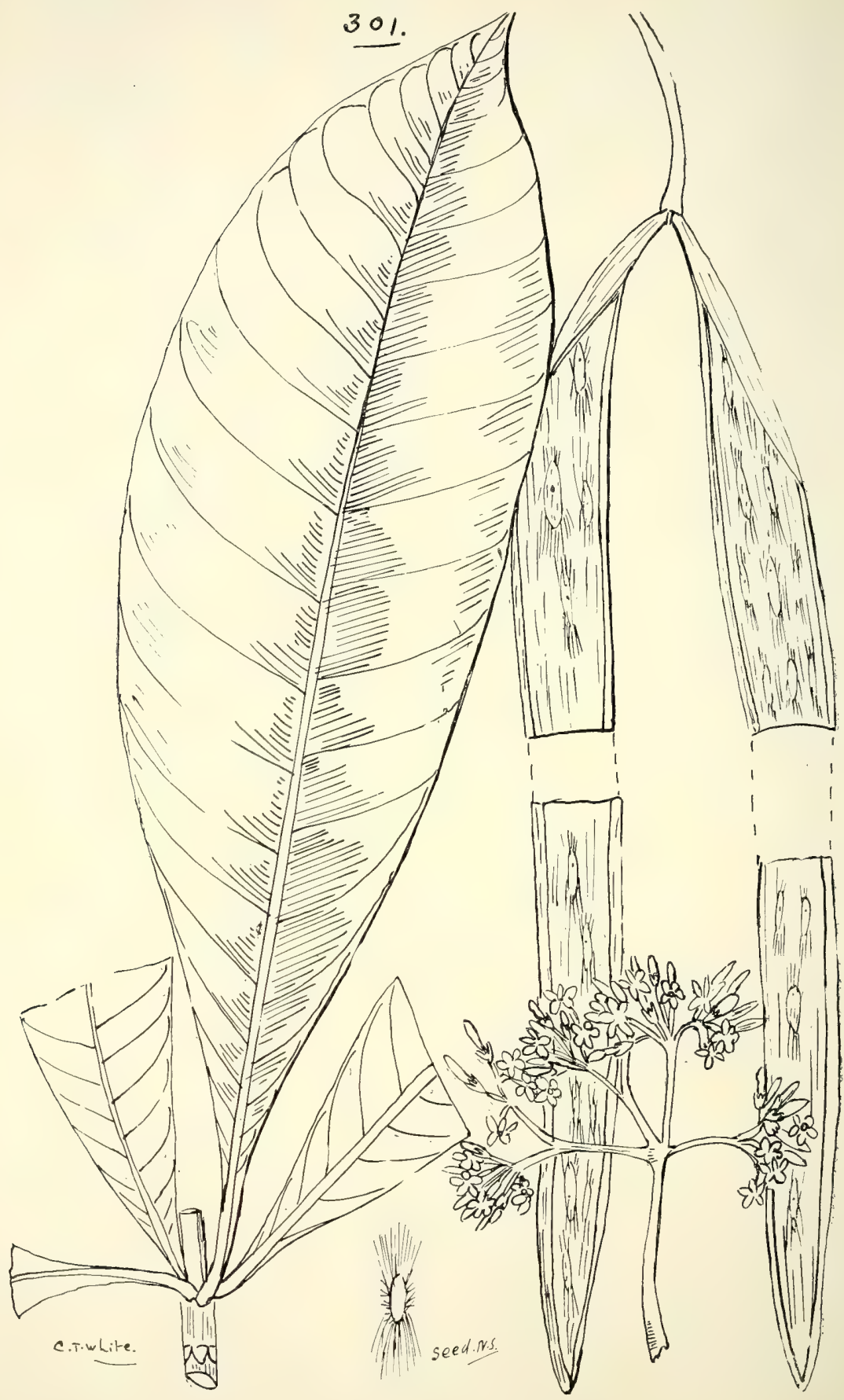

30i. Alstonia somersetensis, Bail. 
Wrightia, R. Br.

saligna, F. v. M.-Milk-bush. Wood resembles English Elder; useful for engraving and carving.

millgar, Bail. (Fig. 303.)

Bacelliana, F. .M. (Fig. 304.)

pubescens, R. Br.

rar. penicillata, Bail._- Cherite" of Mapoon natives.

*Nerium, Linn.

Subtribe Neriea.

odortum, Soland., var. ?-Oleander. The active principles of these plants are powerful heart-poisons. In South Africa the foliage is considered fatal to cattle and horses.

Ichnocarpus, R. Br.

Subtribe Ichnocarpea.

frutescens, R. Br.

\section{Order LXXX.-ASCLEPIADEZE.}

Plants of this Order yield Caoutchouc. The stems (but not the seed-pods) of many yield good fibre.

\section{Suborder I.-PERIPLOCE E.}

Tribe I.-Periploce.t.

*Cryptostegia, R. Br.

grandiflora, R.Br.-A Rubber-plant of Tropical Africa.

Gymnanthera, R.Br.

nitida, R. Br. (Fig. 305.)

\section{Suborder II.-EUASCLEPIADEAE.}

Tribe II.-SECAMONE

Secamone, R. Br.

elliptica, $R \cdot B r$.

ovata, R. Br.

TRIBE III.-CYNANCHE.E.

*Araujia, Brot.

albens, G. Don. = Physianthus albens, Mart. Peru.

*Gomphocarpus, R. Br.

brasiliensis, Fournier.-A noxious weed of Brazil.

*Asclepias, Linn.-Fruit a Conceptacle (conceptaculum), or double follicule.

curassavica, Limn.--Redhead; a noxious weed, poisonous to stock. 
Vincetoxicum, Monch.

ovatum, Benth.

elegans, Benth.

carnosum, Benth.

leptolepis, Benth.

pachylepis, Bail. (Fig. 306.)

Cynanchum, Linn.

erubescens, R. Br.

floribundum, R. Br.- "Thooromia" of Cloncurry natives.

pedunculatum, $R \cdot B r$.

Sarcostemma, R. Br.

australe, $R$. Br.-Caustic-vine; a noxious weed, poisonous to stock.

Pentatropis, $R \cdot B r .=$ Damia (R. Br.), F.v.M. atropurpurea, Benth.

Tribe IV.-Marsdenier.

Gymnema, R. Br.

Section I.-Gymnema.

sylvestre, $R . B r$.

pleiadenium, $F$. v. $M$.

Section II.-Bidaria.

brevifolium, Benth.

trinerve, R. $B r$.

Gongronema, Dcne.

micradenia, Benth.

Tylophora, R. Br.

erecta, $F \cdot v \cdot M$.

grandiflora, R. Br.

floribunda, Benth.

calcarata, Benth.

paniculata, R. Br.

flexuosa, R. Br.

Marsdenia, R. Br.

cinerascens, R. Br.

flavescens, $A$. Cunn.--Roots edible.

cymulosa, Benth.

velutina, R. $B r$.

araujacea, $F . v . M$.

rostrata, $R . B r$.

Fraseri, Benth.

longiloba, Benth.

leptophylla, F.v.M. 
LXXIX, APOCYNACE无.

\section{$30, \mathrm{~b}$ is}

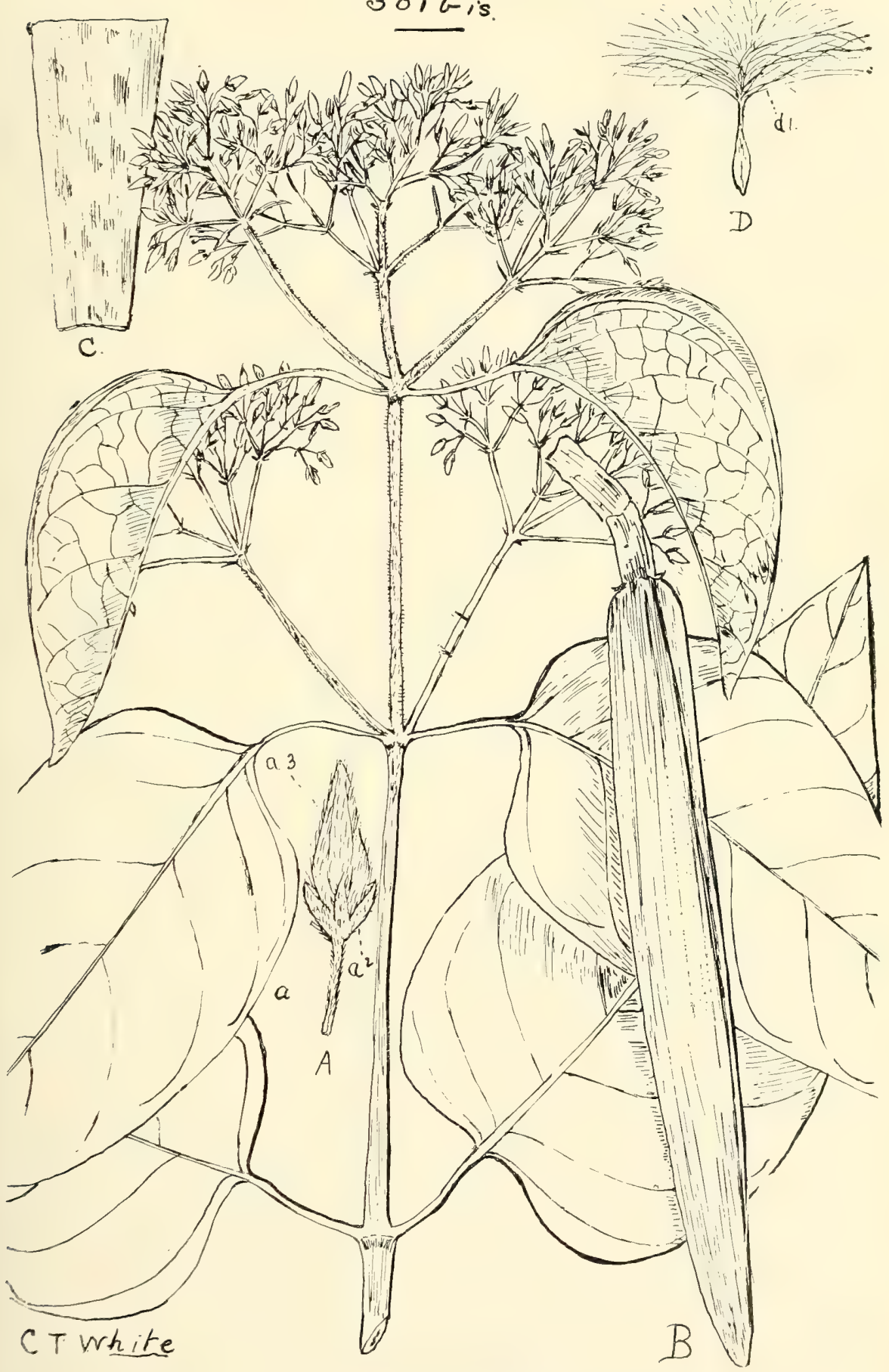

30 i bis. Lyonsta LARgiflorens, $F, v, M$.

(A) Flower, (ar) pedicel, (a2) calyx lobes, (a3) Corolla lobes, (B) follicle,

(C) portion of placenta, (D) seed. (A) enl.; (B)--(D) nat. size. 


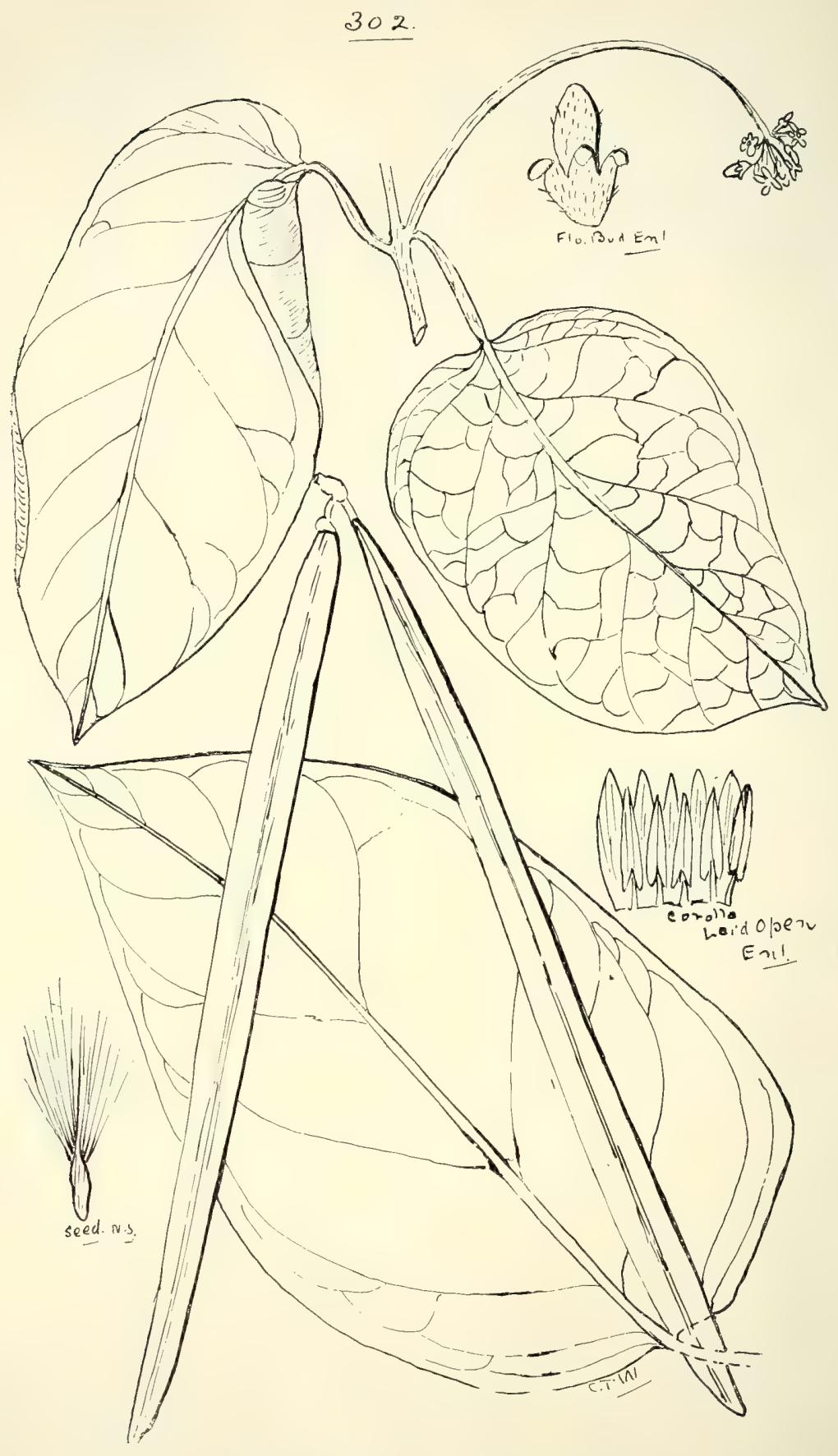

302. Parsonsia nesophila, Bail. 


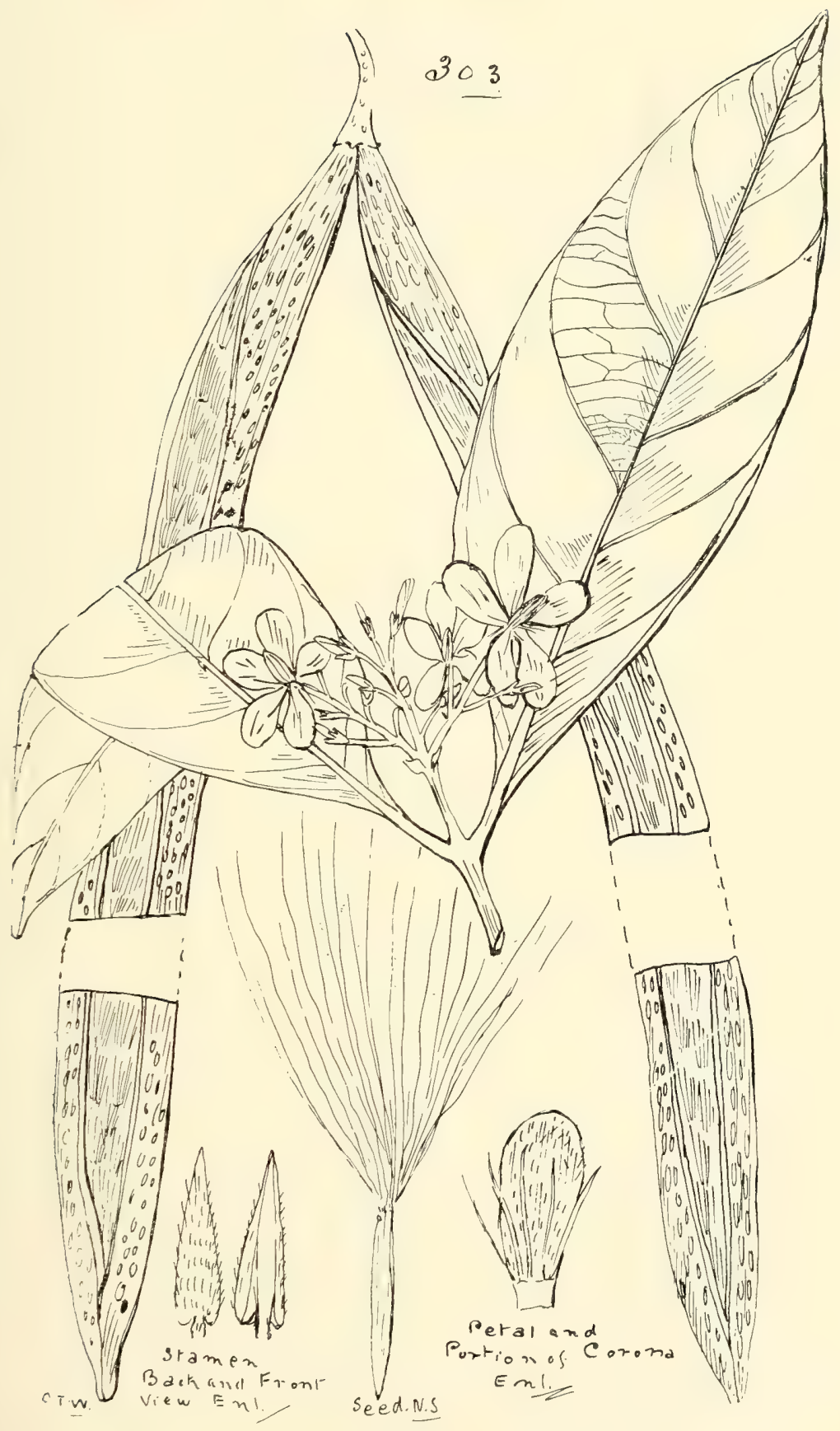

303. Wrightia millgar, Bail. 
LXXIX. APOCYNACEÆ.LXXX. ASCLEPIADE无.

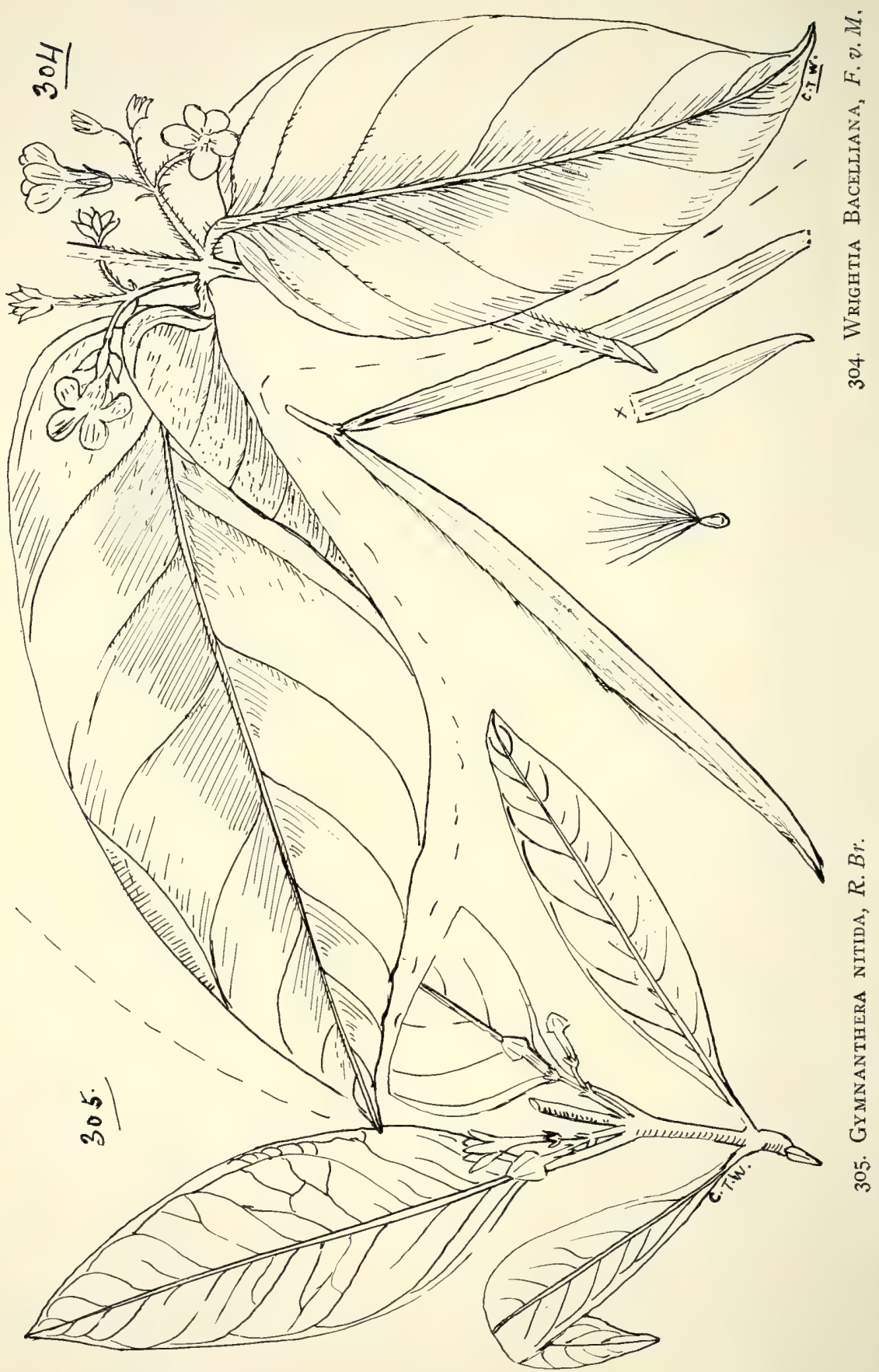




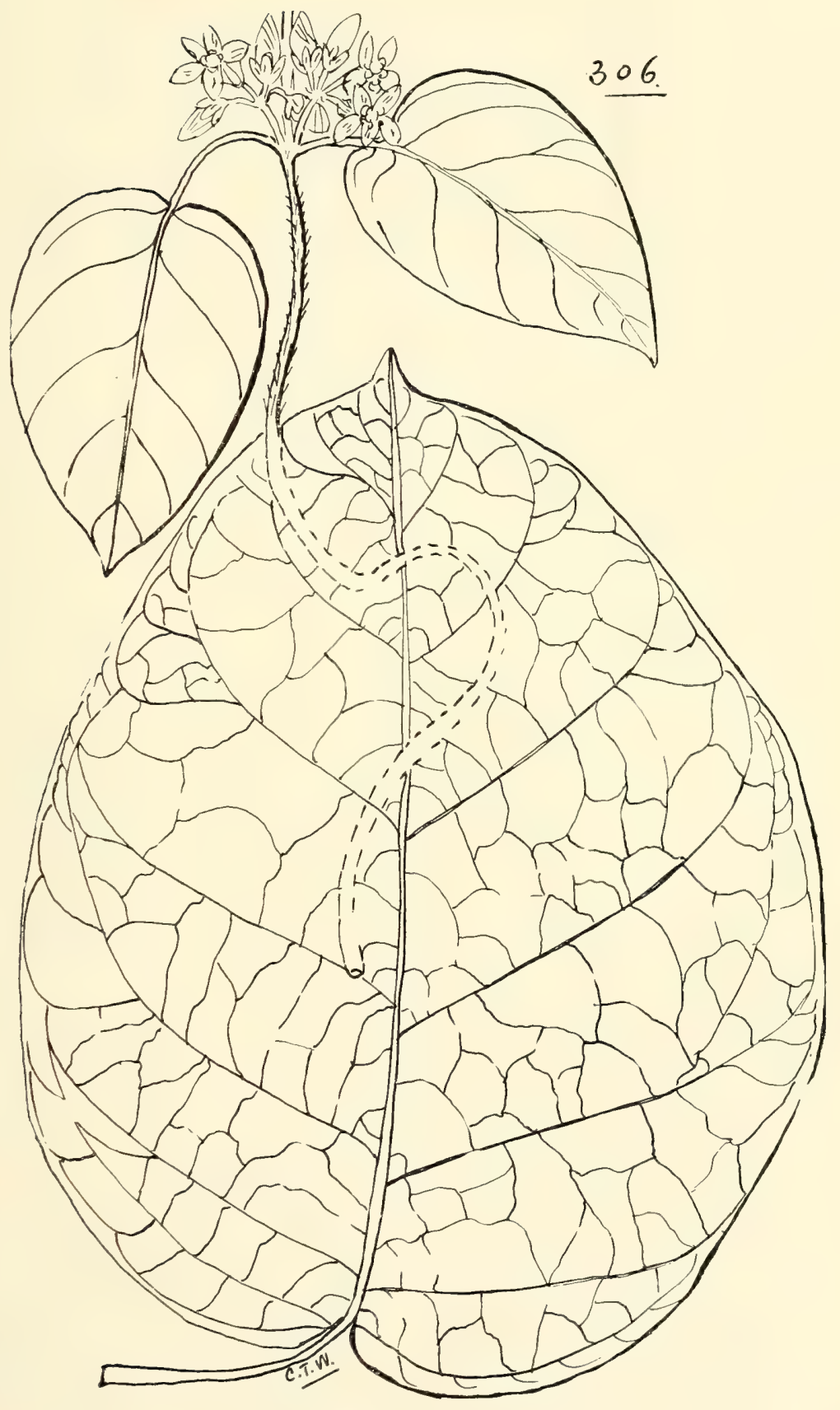

306. Vincetoxicu a fachylepis, Bail. 
Marsdenia-contd.

L.cichhardtiana, F.v.M.-Pods eaten by natives under the name of "Doubalı." (Fig. 307.)

viridiflora, R. Br.

coronata, Benth.

microlepis, Benth.

rhyncholepis, F.v. $M$.

Thozetia, F.v. M.

racemosa, F. v. $M$.

Hoya, R. Br.-Waxflower.

Sana, Bail.-This probably is the one referred to in Flora Australiensis as H. camosa. (Fig. 308.)

Keysii, Bail.-I consider the plant figured on Plate 203, Illustration of the Botany of Cook's Voyage, to be this species. (Fig. 309.)

australis, $R$. Br.-This in my opinion had better be kept to the plant figured in Bot. Mag. 5820, which is sometimes more or less hairy. Sheep poison. (Fig. 3IO.)

Nicholsoniæ, F.v.M. (Fig. 311.)

Dischidia, R. Br.

nummularia, R. Br.-Button Orchid of children.

timorensis, Dcne.

Rafflesiana, Wall.-Pitcher-bearer.

ovata, Benth. = D. picta, Blume. The upper surface of leaves marked with light-coloured veins.

Tribe V.-Ceropegieæ.

Microstemma, R. Br.

tuberosum, R.Br._-"Warbooga" of Cape Bedford natives. (Fig. 3I2.)

Ceropegia, $\operatorname{Linn}$.

Cumingiana, Dcne. - Yam. "Anareata" of Batavia River natives.

\section{Order LXXXI.-LOGANIACEA.}

Mitrasacme, Labill.

Tribe Euloganiere.

Section I.-Mitragyne

paludosa, R. $B r$.

alsinoides, R. Br.

polymorpha, R. Br.

longiflora, F.v. M.

elata, R. Br. (Fig. 3I3.)

ambigua, R. $\mathrm{Br}$. 


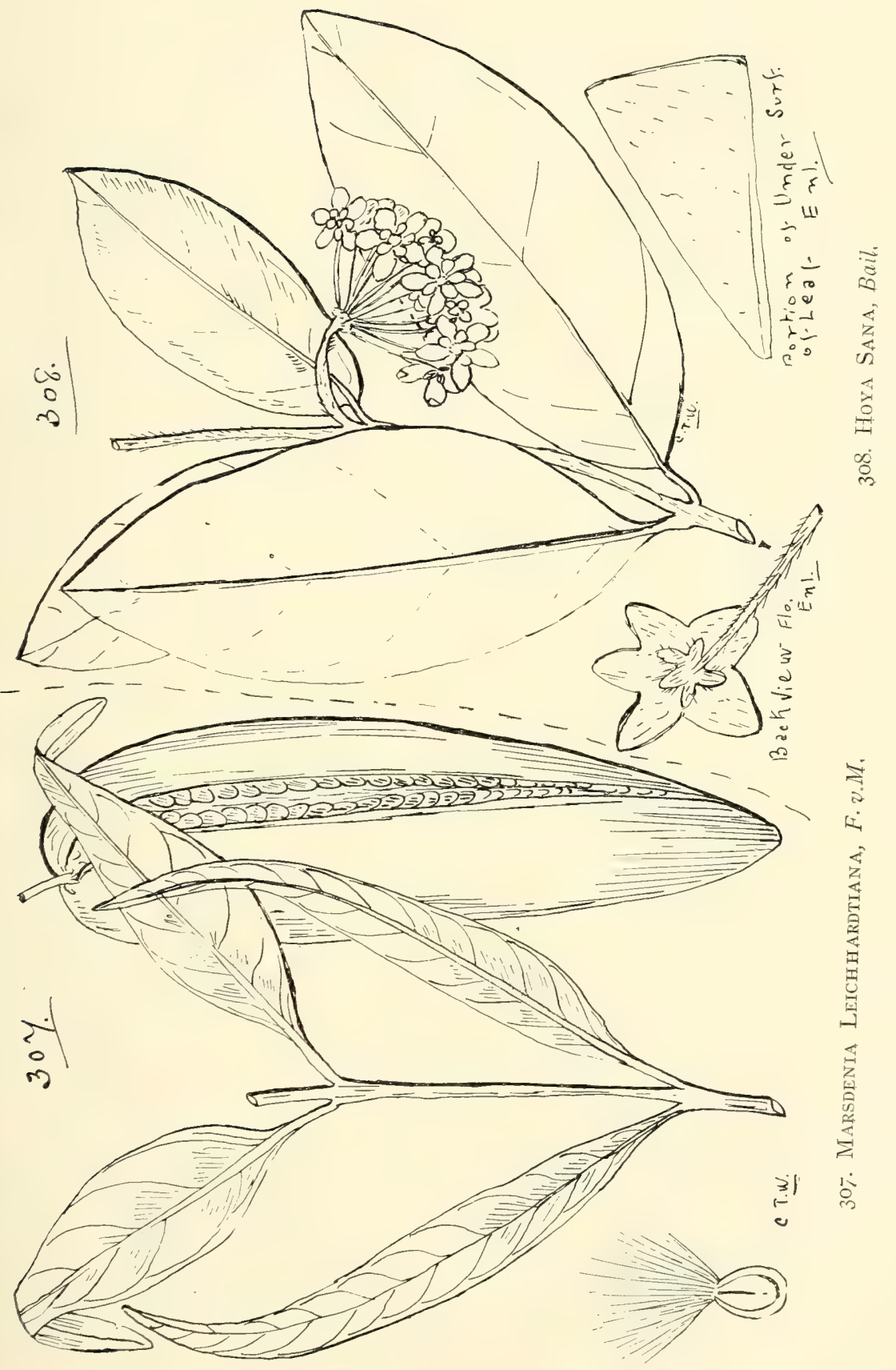




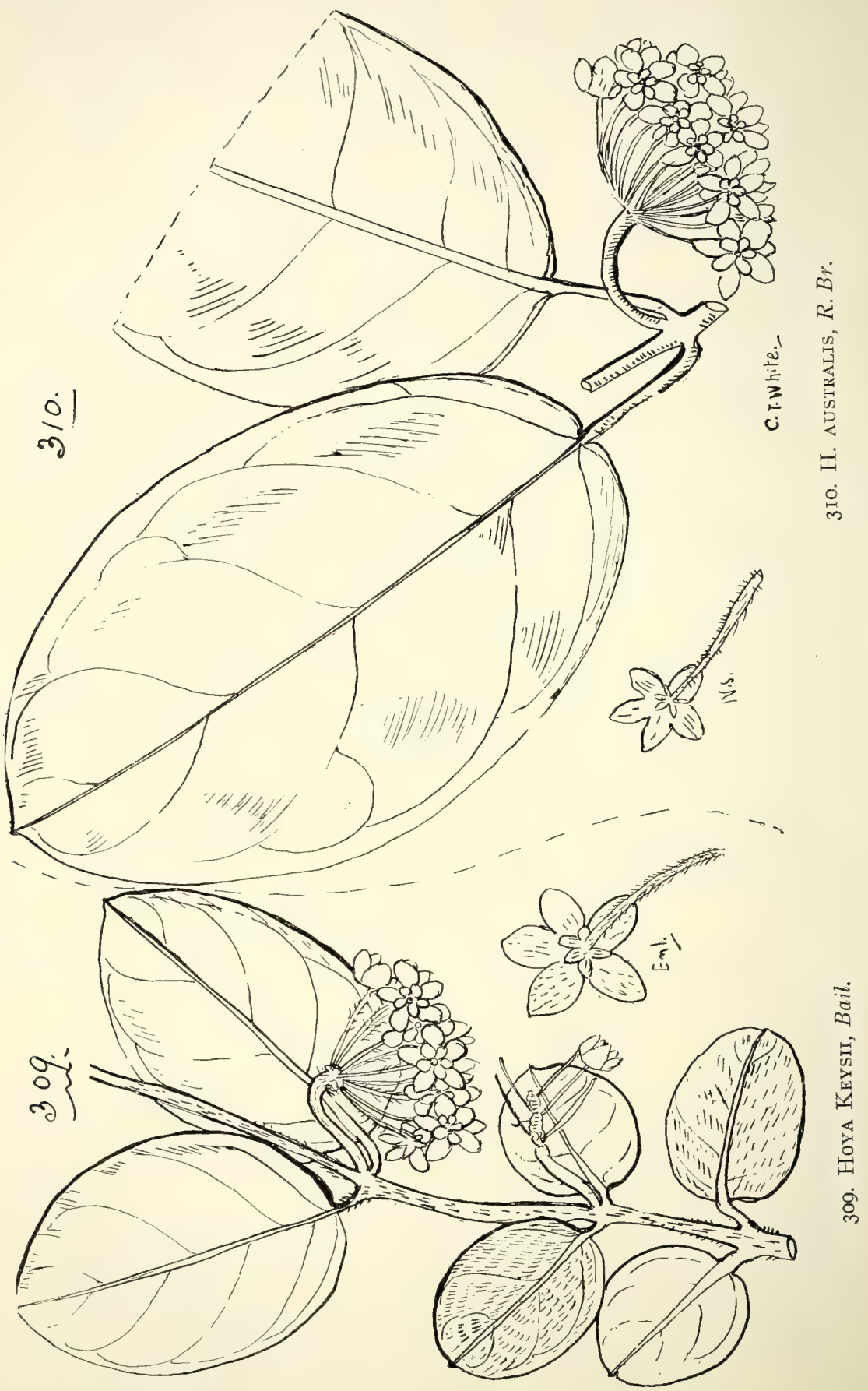


LXXX. ASCLEPIADEÆ.

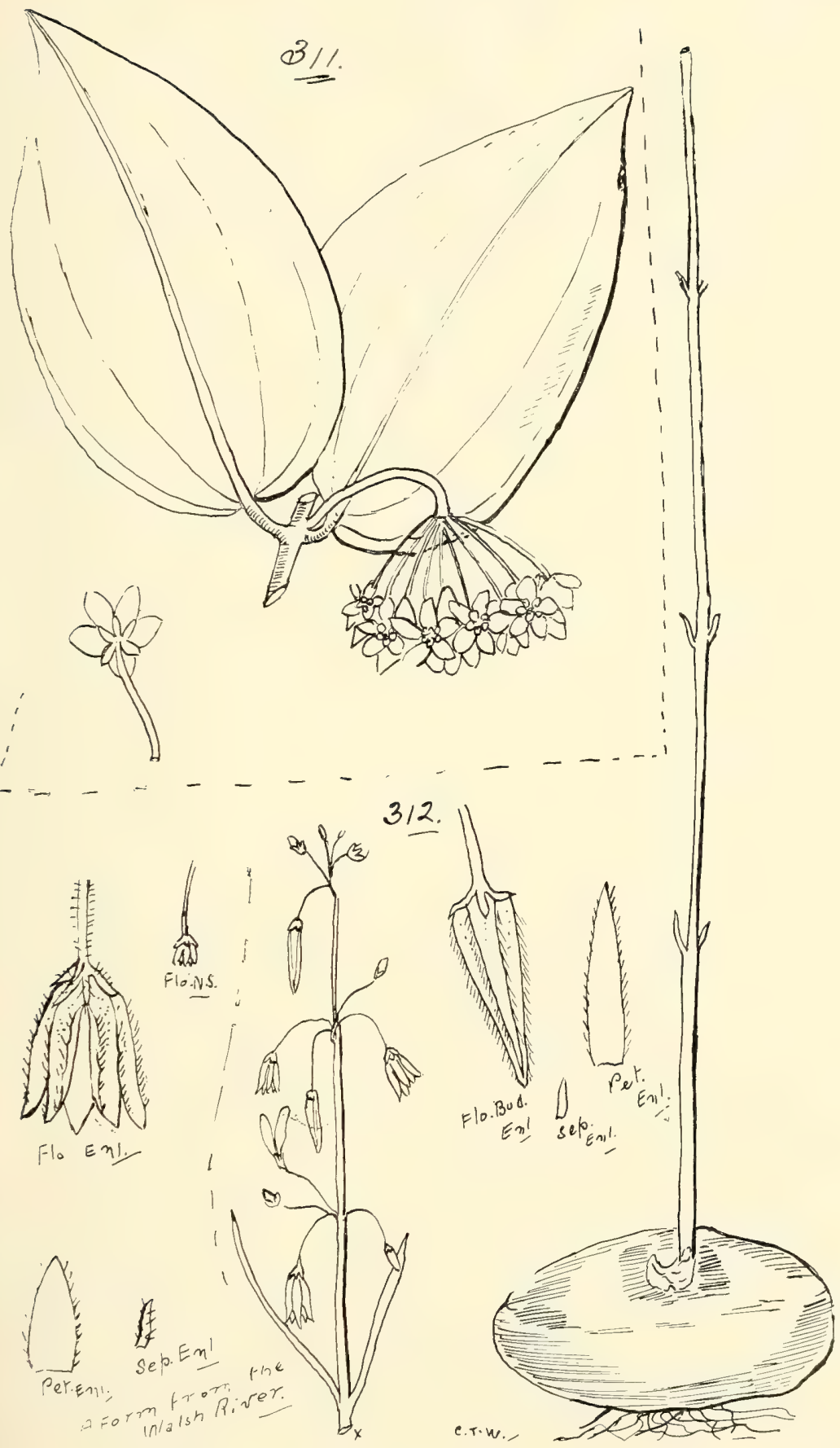

3i. Hoya Nicholsonie, F.v. M.

312. Microstemma tuberosum, $R$. $B r$; ; and forma, Walsh River, T. Barclay Millar. 


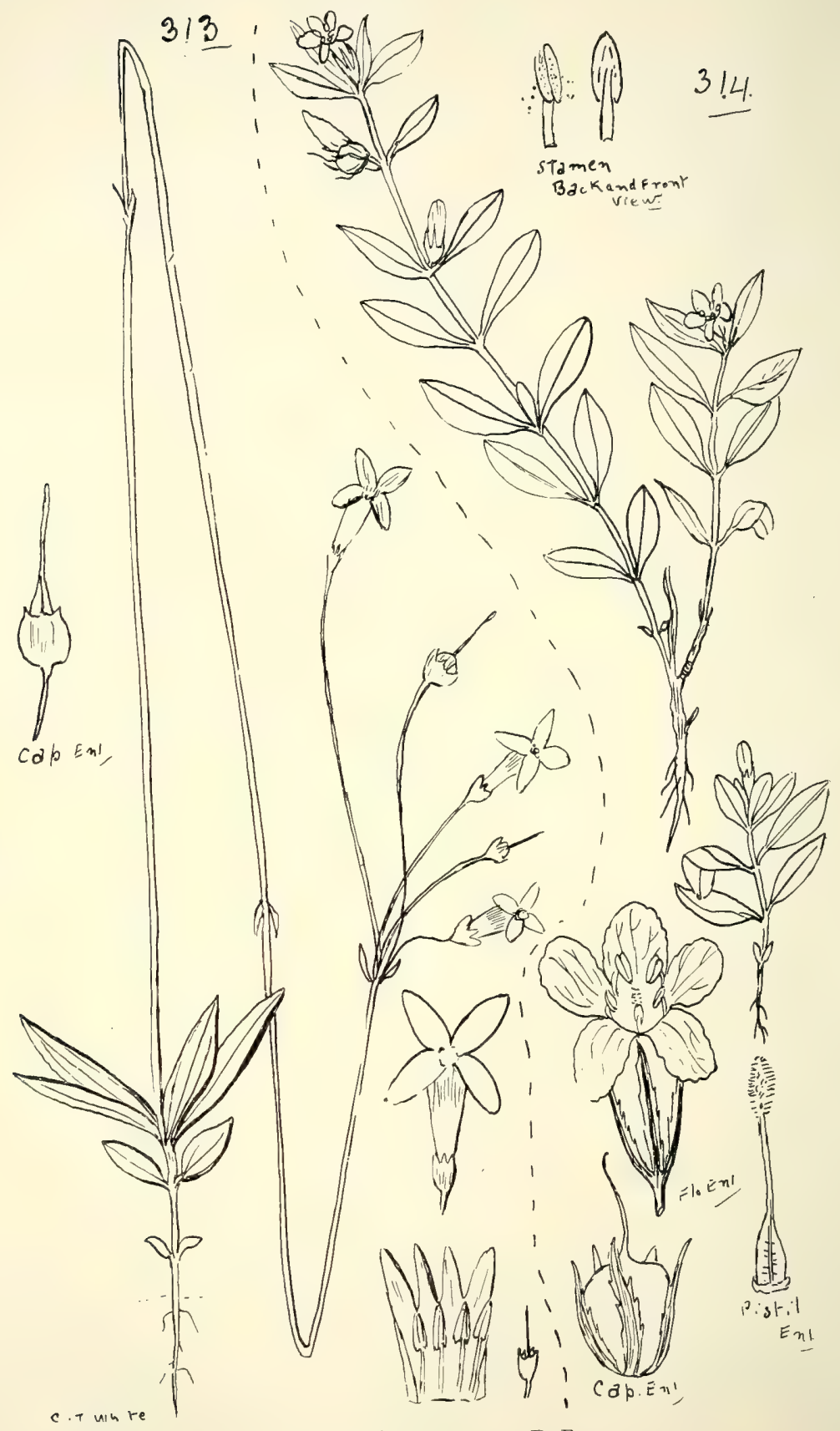

3i3. Mitrasacme elata, $R$. $B r$.

3I4. Logania pusilla, R. $B r$. 
Mitrasacme-contd.

connata, R. Br.

indica, Wight.

stellata, R. Br.

Cunninghamii, Benth.

pygmæa, R. $B r$.

multicaulis, $R . B r$.

laricifolia, $R . B r$.

prolifera, $R$. $B r$.

var. ? major, Benth.

phascoides, R. Br.

Logania, R. $B r$.

Section I.-Eulogania.

floribunda, R. Br.

cordifolia, Hook.

Section II.-Stomandra.

pusilla, R. Br. (Fig. 3I4.)

Geniostoma, Forst.

australianum, F.v.M.

Fagræa, Thunb.

Muelleri, Benth.

racemosa, Jack.— "Mucki" of Cardwell natives (R.B.H.).

Strychnos, Linn.-The Australian species have not been found to contain any poisonous substance.

lucida, R. Brr. (Fig. 3I5.)

psilosperma, F.v.M.-Northern form a weak, straggling tree; the Southern form an erect-stemmed tree $60 \mathrm{ft}$. or more in height. Wood hard and tough, with longitudinal white streaks. (Fig. 3I6.)

Bancroftiana, Bail.-Snakewood. A climbing species having strong tendrils. Fig. 3I6 bis.)

\section{Order LXXXII.-GENTIANEÆ.}

Sebæa, R. Br.

Tribe I.-Exaces.

ovata, R. Br.-A useful tonic herb.

Tribe II.-Chironieæ.

Erythræa, Pers.

australis, R.Br.-Australian Centaury. Tonic-herb. (Fig. 3I7.)

Canscora, Lam.

diffusa, R.Br. (Fig. 318.) 
Villarsia, Vent.

$$
\text { Tribe III.-Menyantheæ. }
$$

reniformis, R. Br.

Limnanthemum, Gmel. indicum, Thw.-Fringed Water-Lily (white). parvifolium, Griseb., var. Moonii, C. B. Clarke. crenatum, F.v.M.-Fringed Water-Lily (yellow). geminatum, Griseb.- "Murite" of Batavia River natives. hydrocharoides, F.v. M. exiliflorum, $F$. v. $M$.

Alliance VIII.-POLEMONIALES.

\section{Order LXXXIII.-HYDROPHYLLACEÆE.}

Hydrolea, Lim.

Tribe HydroleE $A$.

zeylanica, $V$ ahl. (Fig. 3I9.)

spinosa, Limn. (Fig. 320.)

\section{Order LXXXIV.-BORAGINEÆ.}

Cordia, Limn.

Tribe I.-CORDIEE.

stibcordata, Lam. (Fig. 321.)

aspera, Forst.

var. inciso-dentata, Benth.

Myxa, Linn.-Sebastan or Sapistan tree. "Bpa-Burry" of Forest Hill natives. The kernels of the nuts are eaten in India, and are said to taste like filberts.

Ehretia, Linn.

Tribe II.-Ehretie

\section{Section I.-Euehretia.}

acuminata, R. Br.-The wood resembles English Elm. var. laxiflora, Benth.

pilosula, F. v. M.

Section II.-Bourreria.

saligna, R.Br.-Wood useful in turnery. (Fig. 322.) membranifolia, R. $B r$.

lævis, Roxb.

Coldenia, Linn. procumbens, Linn.

Halgania, Gaudich. strigosa, Schlecht. 


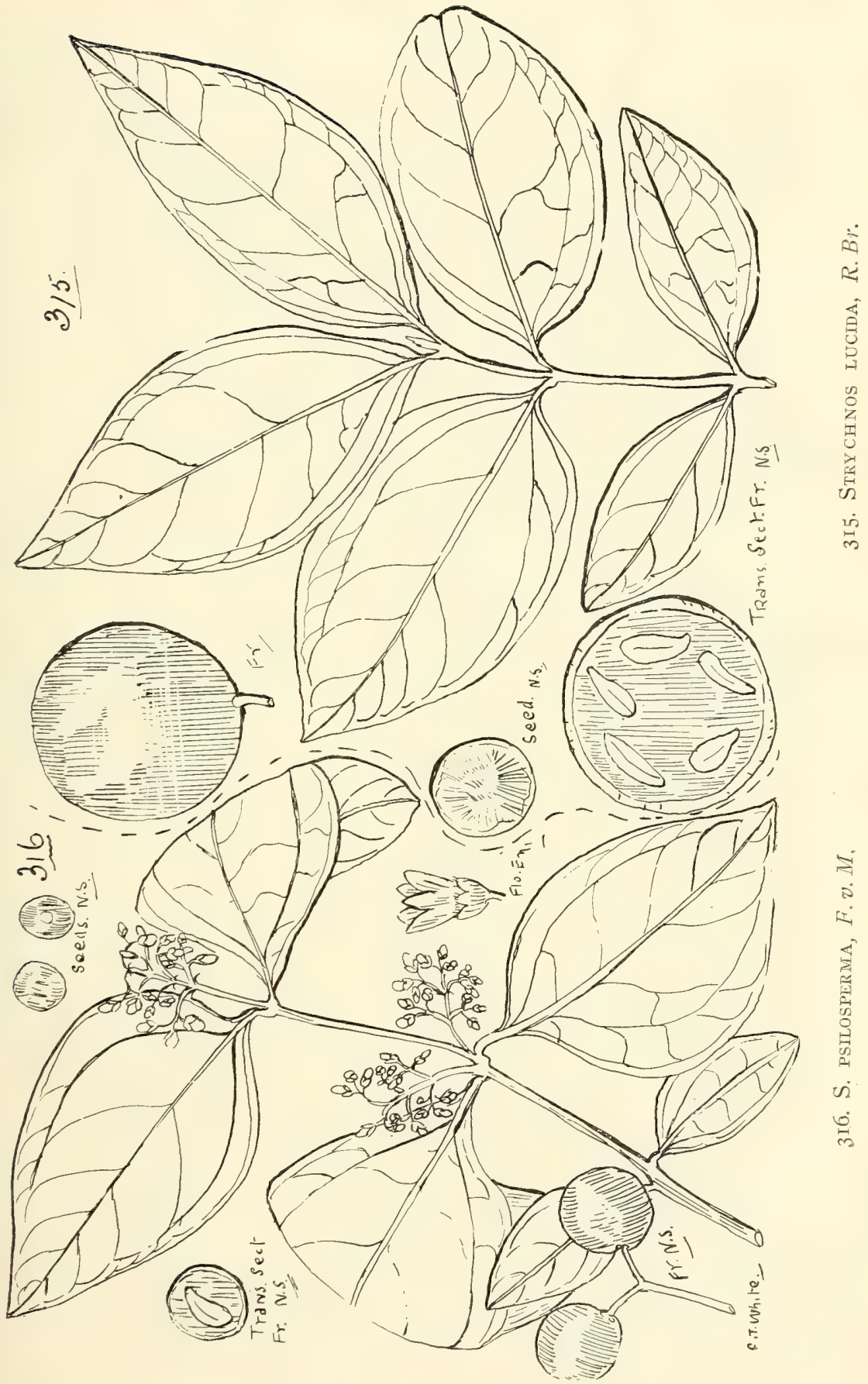


316 bis.

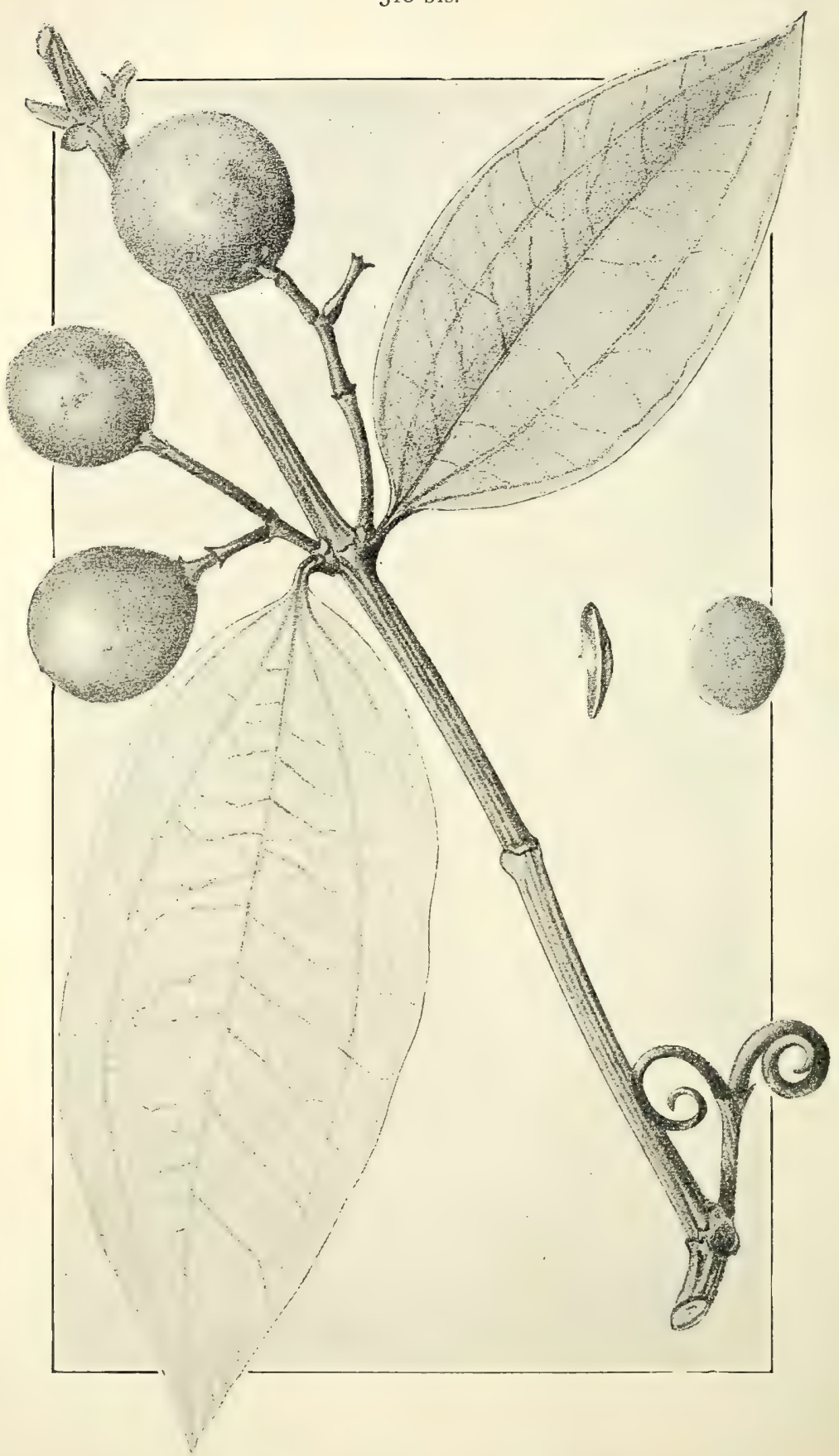

3 i6 bis. Strychnos Bancroftiana, Bail.

(From a drawing by F. C. Wills in "The Queensland Flora.") 


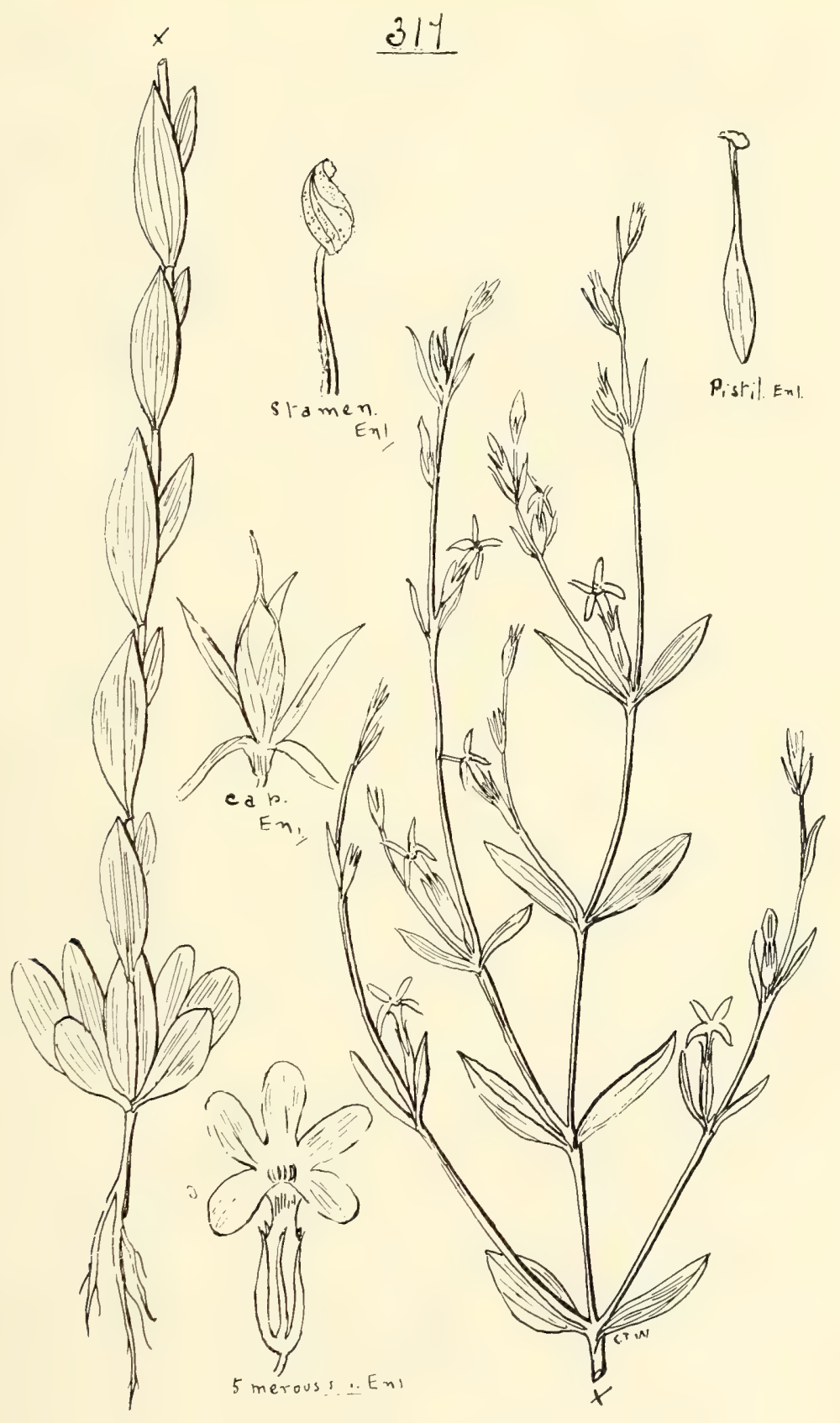

3I7. ERYTHREA AUSTRAITS, R. $B r$. 


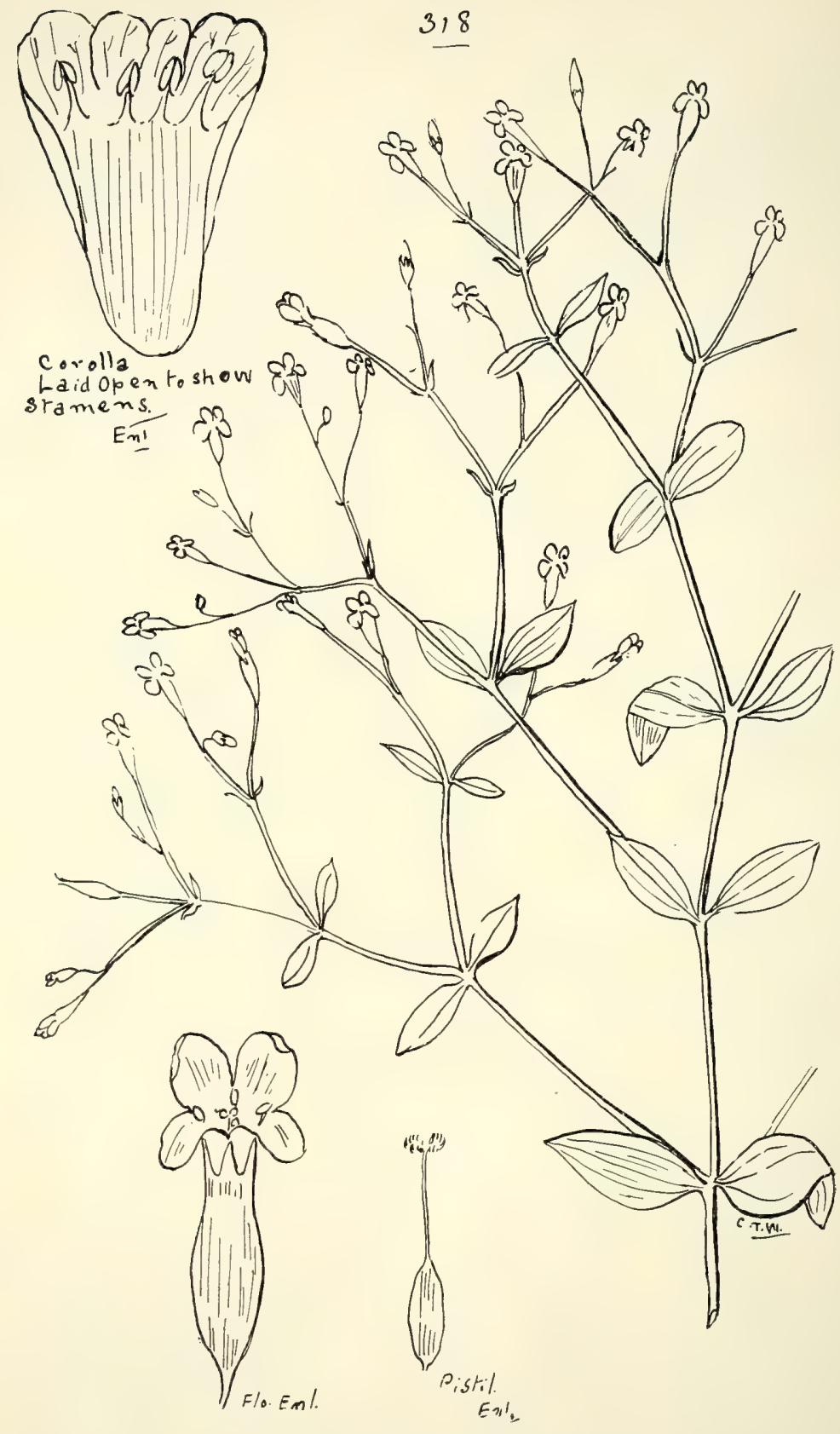

3i8. Canscora diffusa, $R$. $B r$. 


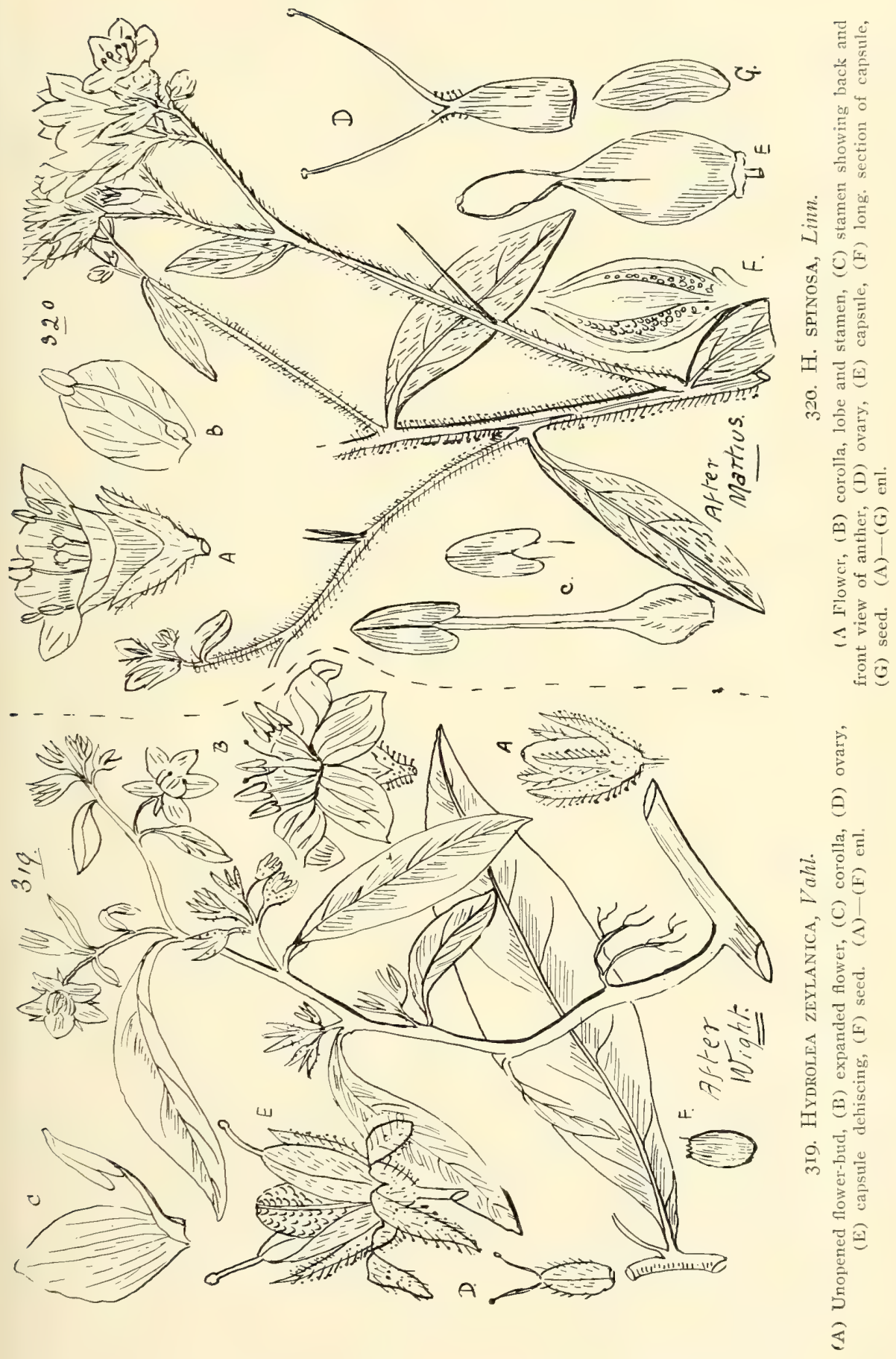




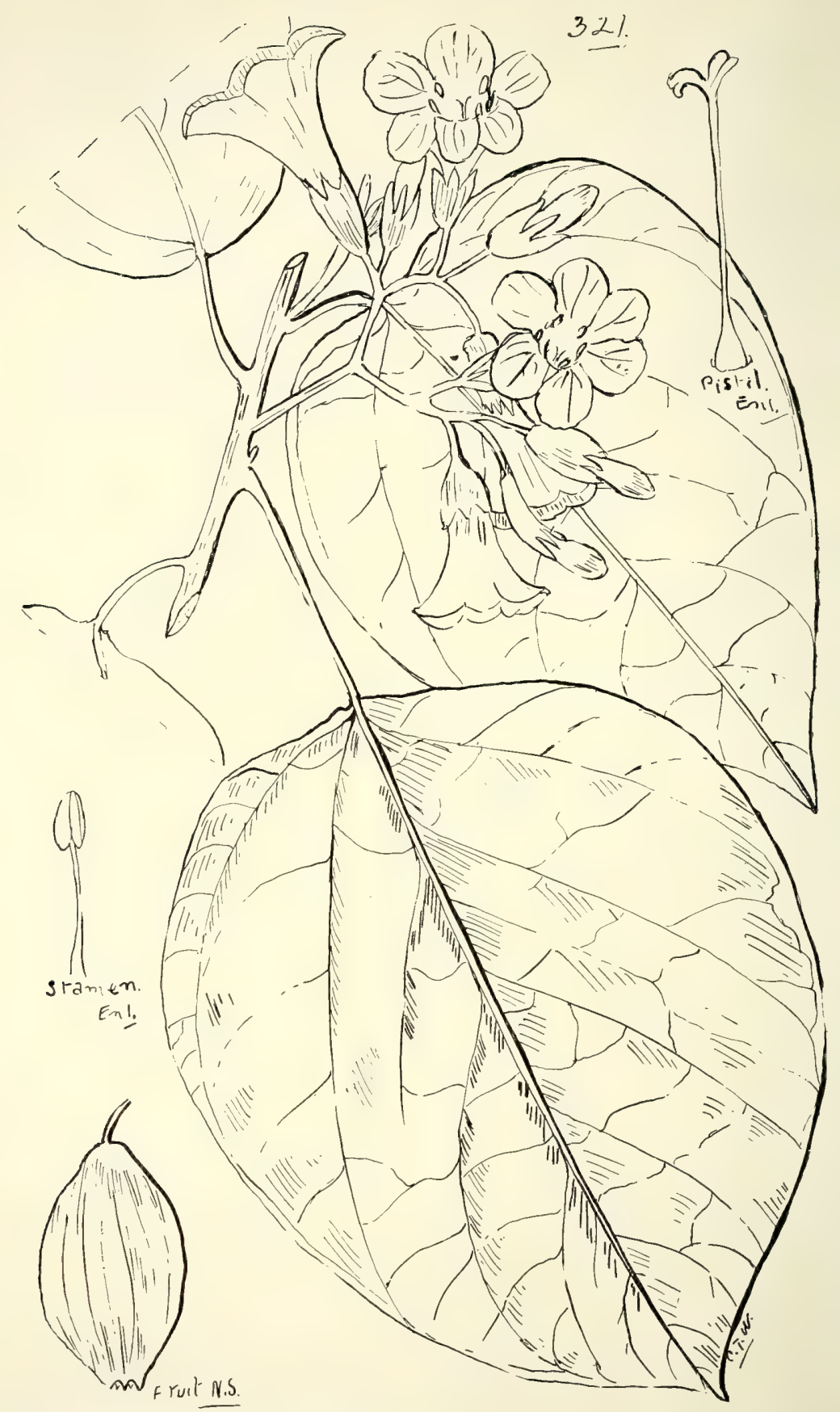

32i. Cordia subcordata, Lam. 


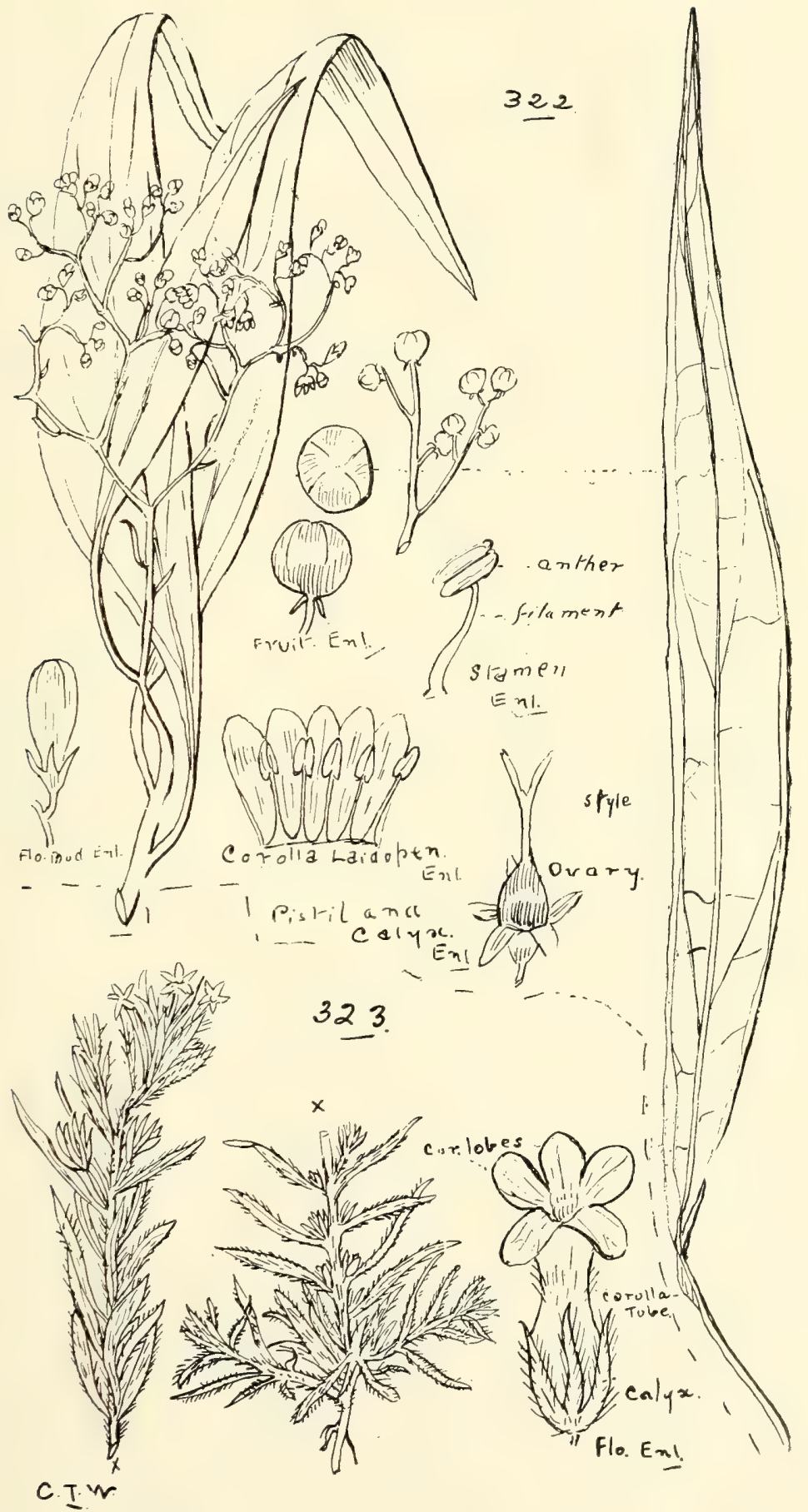

322. Ehretia saligna, $R$. $B r$.

323. Heliotropium ventricosum, $R$. $B r$. 
Tournefortia, Linn.

Tribe III.-Heliotropieas.

argentea, Linn.

mollis, F.v.M.

sarmentosa, Lam.

Heliotropium, Linn.

Section I.-Platygyne.

curassavicum, Linn.

Section II.-Euheliotropium.

asperrimum, $R \cdot B r$.

fasciculatum, $R . B r$.

brachygyne, Benth.

Section III.-Schleidenia.

ovalifolium, Forst.

var. oblongifolium, $D C$.

strigosum, Willd.

brachygyne, Benth.

prostratum, R. Br.

bracteatum, R. Br.

var. leptostachyum, Benth.

pauciflorum, R. Br.

filaginoides, Benth.

ventricosum, R. $B r$. (Fig. 323.)

tenuifolium, R. Br. (Fig. 324.)

Section Heliophytum.

indicum, Linn.-In Bombay the plant is used as a local application to boils, sores, and the stings of insects and reptiles (Dymock).

*anchusæfolium, Poir.-Buenos Ayres and Brazil.

Tribe IV.-Borage ex.

Subtribe I.-Cynoglossea.

Trichodesma, R. Br.

zeylanicum, $R \cdot B r$.-A favourite camel fodder.

var. latisepalum, F.v. M.

var. sericeum, Benth.

Cynoglossum, Linn.-Hound's-tongue.

latifolium, $R . B r$.

suaveolens, R. $B r$.

australe, R. $B r$.

Drummondii, Benth.

$$
\text { Subtribe II.-Eritrichiea. }
$$

Fchinospermum, Szo.

concavum, F.v.M. = Lapula concava, F. v. M. 
Eritrichium, Schrad.

australasicum, $A . D C$.

Rochelia, Reichenb.

Maccoya, F.v.M.

Myosotis, Linn.

Subtribe III.-Lithospermex.

australis, R. Br.-Australian Forget-me-not.

Lithospermum, Linn.-Lindley and Mirbel call the fruit of this and allied plants by the name of Achænium. arvense, Limn.-Corn Groundsel of Europe.

\section{Order LXXXV.-CONVOLVULACE E.}

Erycibe, Rox $x^{\circ}$.

Tribe I.-Convolvulex.

paniculata, Roxb.

var. coccinea, Bail. (Fig. 325.)

*Argyreia, Lour.

speciosa, Sweet.

Lettsomia, Roxb.

Soutteri, Bail.

Ipomøa, Linn.

digitata, Linn.

Series I.-Digitatce.

*Batatas, Lam.-Sweet Potato ; contains prussic acid.

var. leucorrhiza, Griseb.-Tubers white.

var. porphyrorhiza, Griseb.--Tubers purplish.

palmata, Forsk.

quinata, R. Br.

diversifolia, $R . B r$.

dissecta, Willd.-Contains prussic acid. The seeds used in making Noyau.

sinuata, Ortega.

Series II.-Pharbitides.

hederacea, Jacq.-The seeds in India considered a safe and valuable cathartic.

var. limbata, Hook. $f$.

congesta, R. Br.

*purpurea, Roth--Morning Glory. America.

rubro-cærulea, $R$. Br.-Mexico.

*Learii, Paxt.--Buenos Ayres.

peltata, Chois.

Series III.-Calycince.

Calobra, Hill et Muell.- "Calobra" of Barcoo and "Weir" of Balonne River natives. Tuberous.

alata, $R . B r$. (Fig. 326.) 
348 LXXXIV. BORAGINE无-LXXXV. CONVOLVULACEÆ.

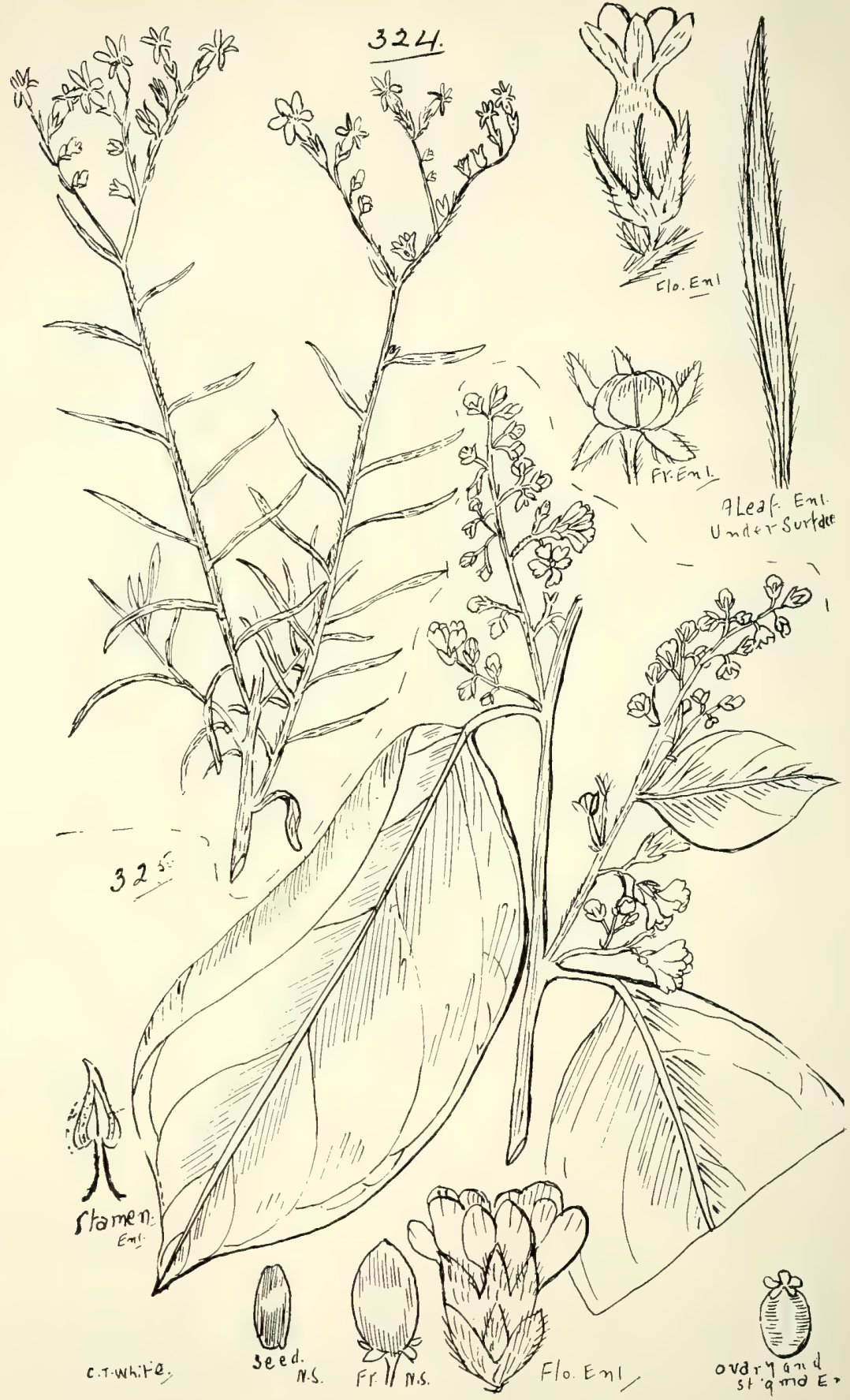

324. Heliotropium tenuifolium, $R$. $B r$.

325. Erycibe paniculata, Roxb., var. Coccines, Bail. 


\section{Ipomca-contd.}

Turpethum, R. Br._-"Kar-kor" of Cloncurry natives. Said to be used by the Cingalese (the root) as a cathartic. grandiflora, Lam.

*bona-nox, Linn. = Calonyction speciosum, Chois.-Moonflower.

Series IV.-Speciosa.

Pes-capræ, Roth-_."Walno-jo" of Mount Cook and "Endabari" of Cape Grafton natives.

carnosa, $R . B r$.

reptans, Poir.- Said to be cultivated as a vegetable by the Cingalese.

graminea, $R . B r$.

velutina, $R . B r$.

abrupta, R.Br. (Fig. 327.)

denticulata, Chois.

gracilis, R. Br.- "Almor-ira" of Palmer River natives.

sepiaria, Kon.

Muelleri, Benth.

Series V.-Campanulatce.

cymosa, Roem. et Schult.

linifolia, Blume.

chryseides, Ker.

flava, F. v. M.

obscura, Ker.

incisa, $R . B r$.

uniflora, Rom. et Schult.- "Kon-yara” of Palmer River natives.

angustifolia, Jacq.- " Kal-boo-roon-ga" of Cooktown natives. At times forms long tubers.

plebeia, $R . B r$.

eriocarpa, R.Br.- "Kand-ja" of Cooktown natives.

heterophylla, $R, B r$.

erecta, R. Br.

\section{Series VI.-Hypocrateriformes.}

*Quamoclit, Limn.-India. Cupid's Flower; but mostly in Queensland known as the Star of Bethlehem.

*coccinea, Linn.-South America.

cataractæ, Endl.

Lepistemon, $B l$.

urceolatus, F.v.M.

Lucæ, F. ข. M.

Calystegia, R. Br.-Hooded Bindweed.

marginata, R. Br. 


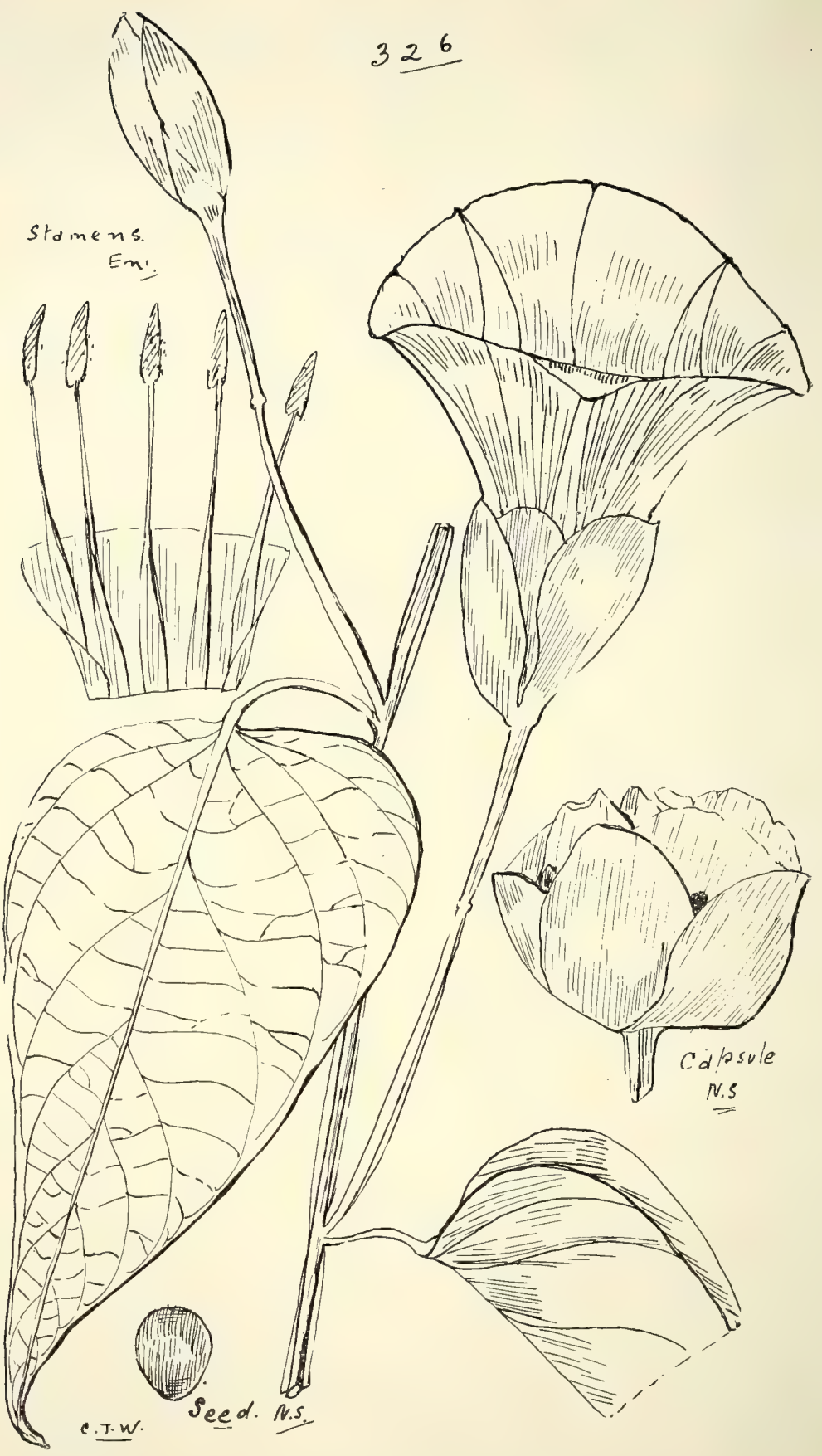

326. Iтомеa alata, R. Br. 


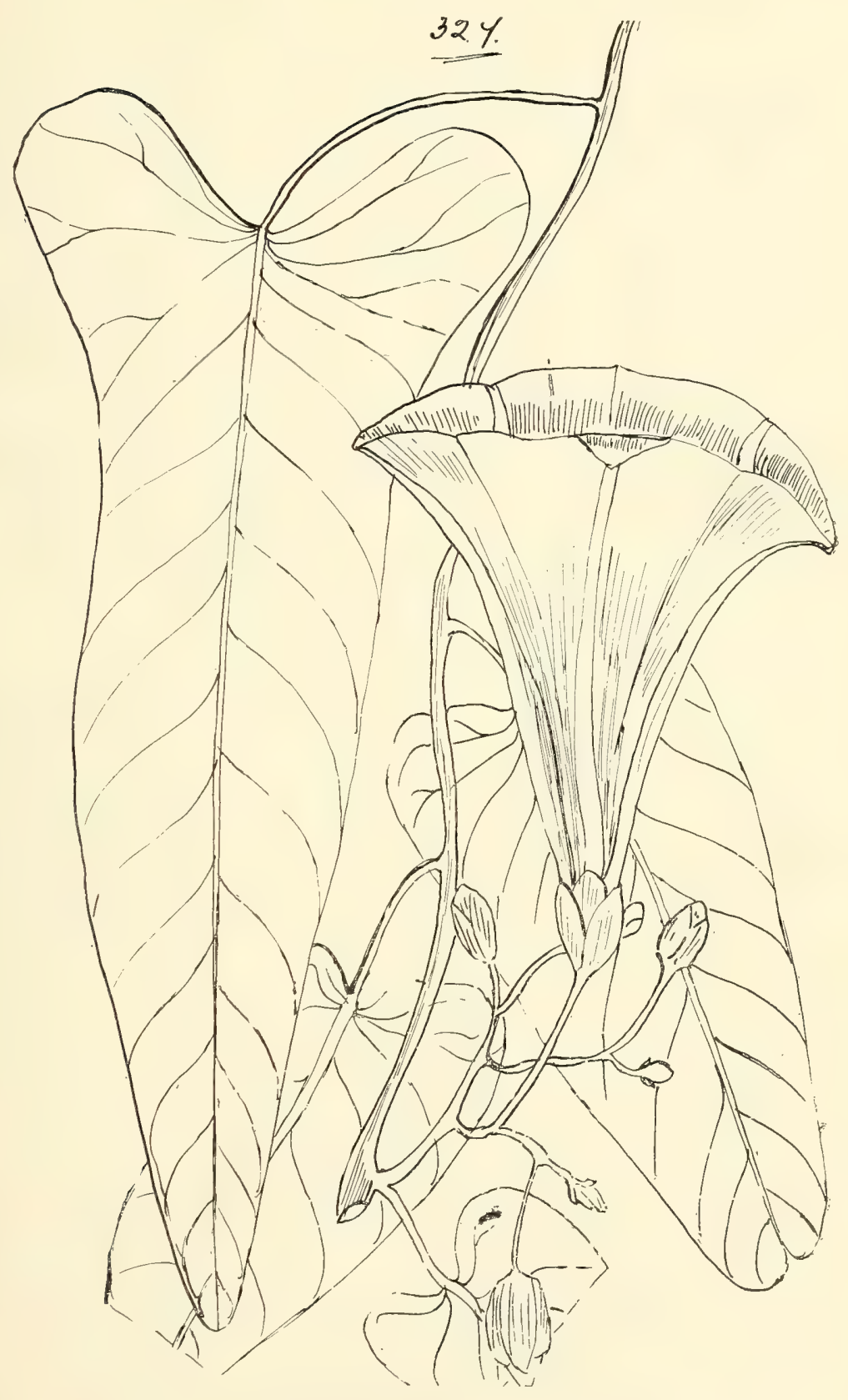

327. IPOMEA A BRUPta, R. Br. 


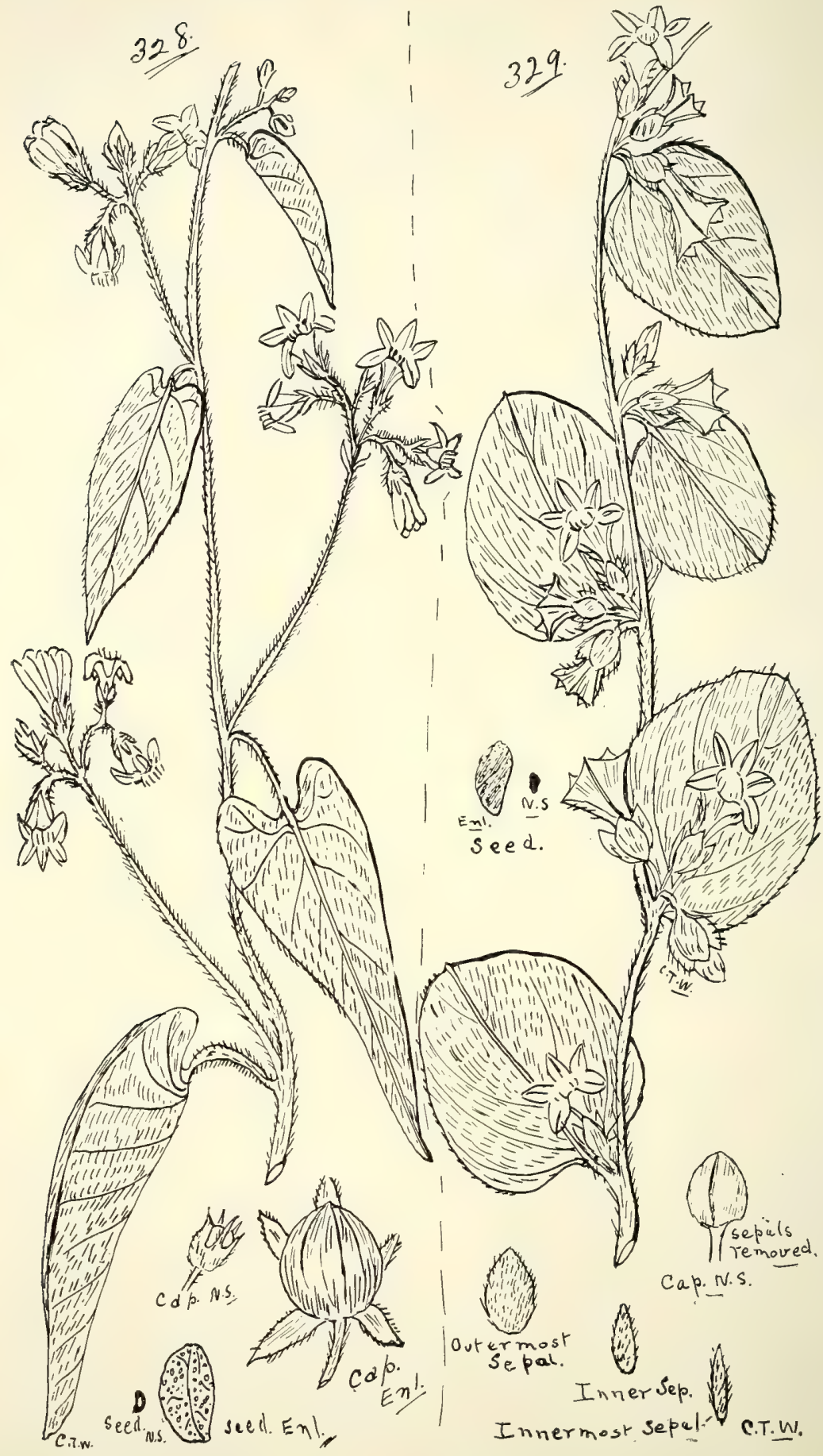

328. Convolvelus multivalvis, $R$. Br.

329. Breweria pannosa, $R . B r$. 
Calystegia-contd.

sepium, R. Br.

Soldanella, R. Br.

Convolvulus, Linn.-Bindweed.

erubescens, Sims.

multivalvis, R. Br. (Fig. 328.)

parviflorus, Vahl.

Polymeria, R. Br.

marginata, Benth.

longifolia, Lindl.

calycina, $R . B r$.

ambigua, $R . B r$.

pusilla, R. Br.

Evolvulus, Limn.

alsinoides, Linn.

var. sericets, Benth.

Breweria, R. Br.

linearis, R. Br.

media, $R . B r$.

pannosa, R.Br. (Fig. 329.)

TRIBE II.-DICHONDREæ.

Dichondra, Forst.

repens, Forst.

TRIBE III.-CRESSE.E.

Cressa, Limn.

cretica, Linn.

TRIBE IV.-Cuscute.

Cuscuta, Linn.--Dodders; all troublesome weeds. By treating the soil with 2 per cent. solution of ammonium nitrate or I per cent. solution of formalin, the growth of dodders on crops is entirely prevented.-G. d'Ippolito in Staz. Sper. Agrar. Ital., 44 (IgII) 30 I ex Pharm. Journ. and Pharmacist, 87 (Aug. I9II), 298.

chinensis, Lam.

australis, R. Br. (Fig. 33o.)

*europæa, Limn.-A pest on Lucerne. The Greater Dodder. Europe.

*epithymum, Murr.-The Lesser Dodder.

\section{Order LXXXVI.--SOLANACEÆ.}

TRIBE I.-SOLANE瓜.

*I.ycopersicum, Mill.

esculentum, Mill. = L. cerasiforme, Dunal--Tomato, a culinary fruit; the common one of our scrubs. South America. 
Solanum, Linn.-These plants are mostly of a poisonous character. Fruit a berry (Bacca).

I. Unarmed species.

nigrum, Linn.-Black Nightshade; poisonous to stock.

Blackberry of Brisbane children, and sometimes eaten by them without ill effects, at other times causing illness.

var. humile, Bail.-Probably more poisonous than the normal form.

aviculare, Forst.

simile, F.v. M.

*pseudo-capsicum, Linn.-False Capsicum of tropical countries; origin uncertain.

Shanesii, F.v.M.

2. Unarmed species, with stellate hairs.

viride, R.Br._ "Boolally" of Barron River natives. (Fig.

33I.)

tetrandrum, R. Br.

var. ? floribundum, Benth.

verbascifolium, Ait.-Flowers whitish. Fruit not used in Queensland, but said to be used in curries in India.

*auriculatum, Ait.-Flowers purplish. America.

3. Prickles often numerous, not on the calyxes. Pubescence stellate.

macrooai, Bail.-Fruit yellow. Summit of south peak, Bellenden-Ker.

discolor, R. Br.

stelligerum, $S m$.

var. lucorum, F. v. M.

var. ? magnifolitum, Benth.

parvifolium, R. Br.

ferocissimum, $L$ indl.

sporadotrichum, F.v.M.

defensum, F. $v \cdot M$.

violacetum, R. Br.

amblymerum, Dun

tetrathecum, F.v.M.

elachophyllum, F.v. M.

orbiculatum, Dun. $=S$. oligacanthum, F.v. M. (Fig. 332.)

esuriale, Lindl._- "Oondoroo" of Cloncurry natives.

chenopodium, F. \%. M.

Sturtianum, F.v. M.

furfuraceum, $R . B r$.

dianthophorum, Dun.

Dallachii. Benth._- Koori" of Bundaberg natives.

densevestitum, F.v. M. 


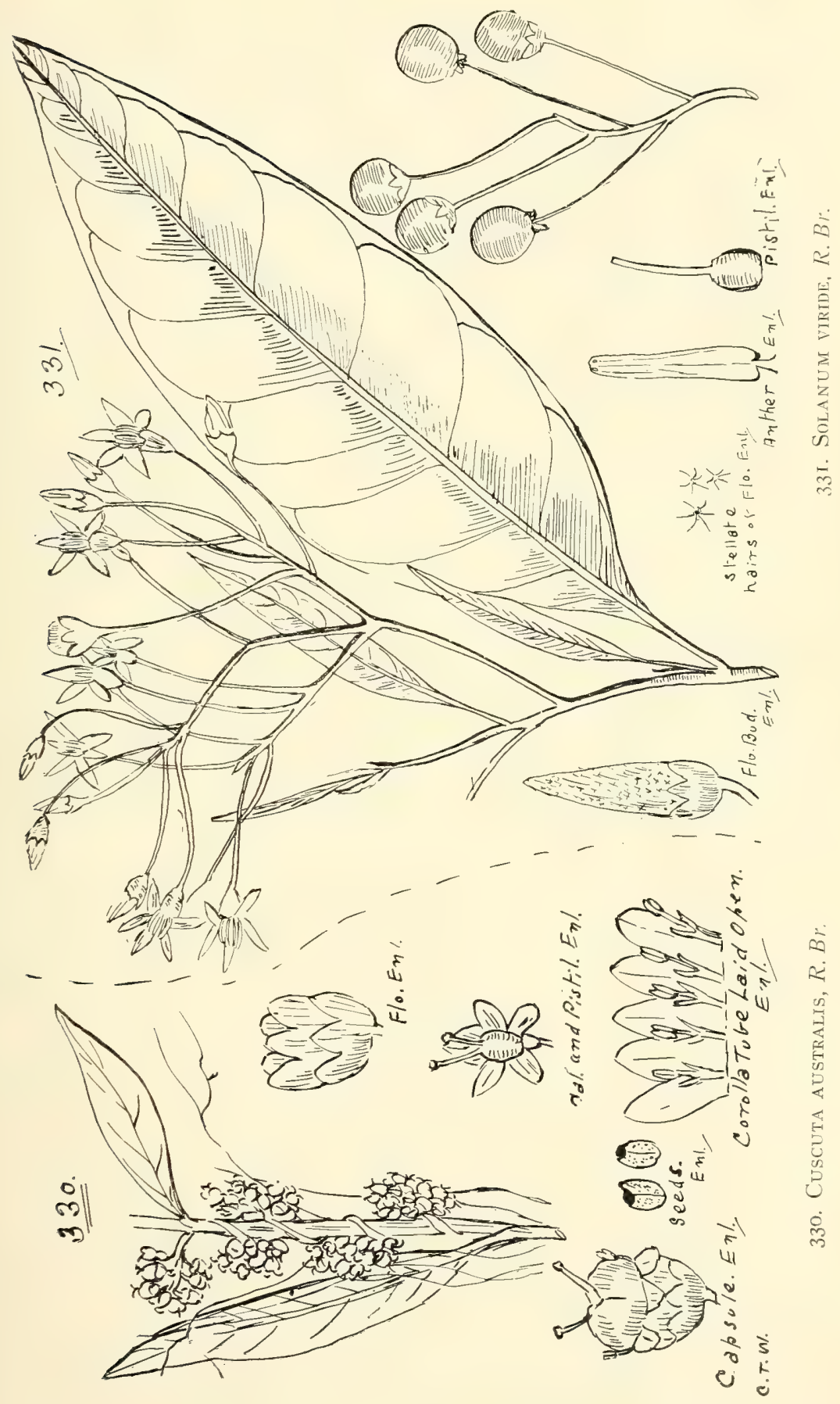




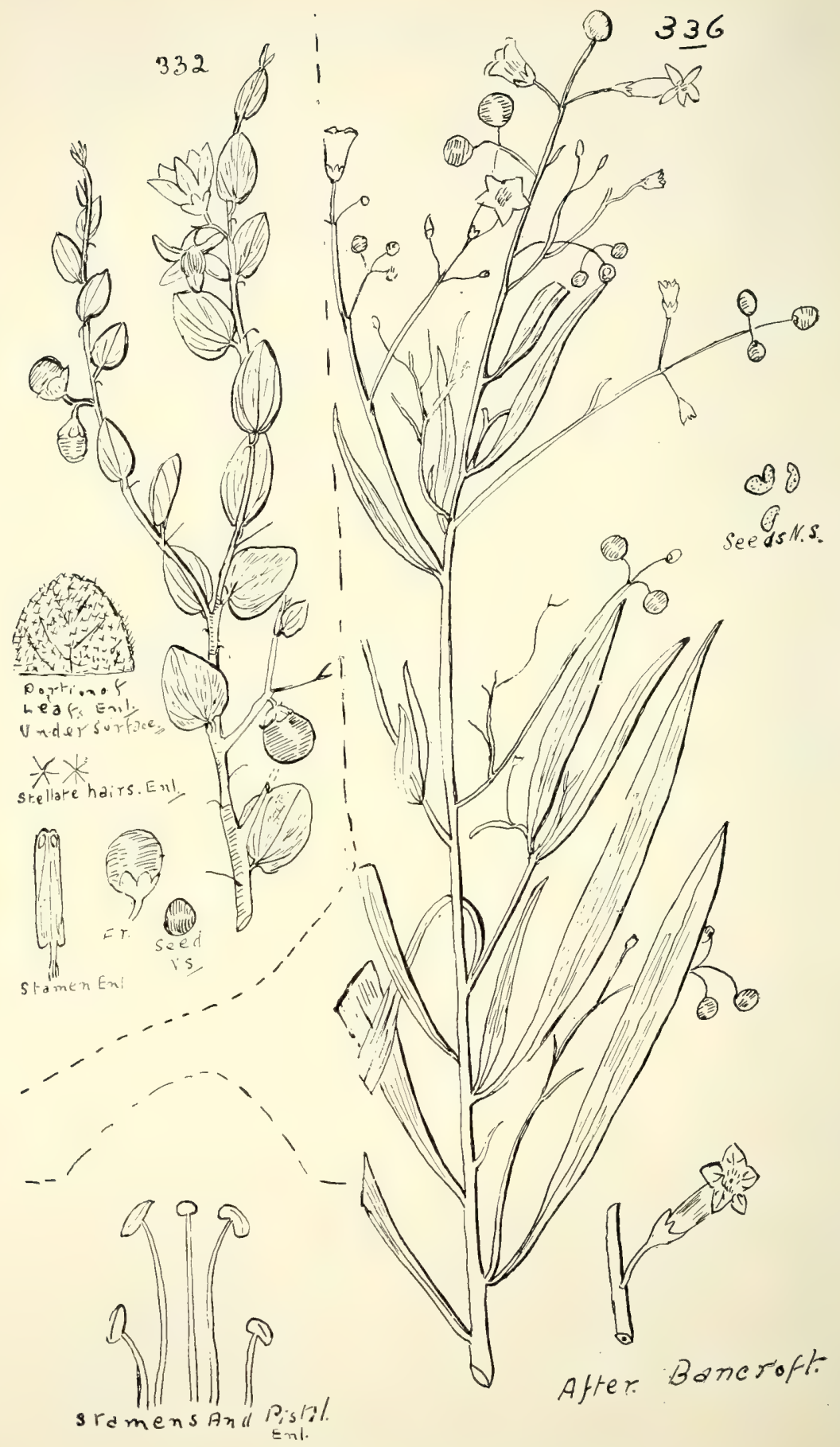

332. Solanum orbtculatum, Dun. 336. Duboisia Hopwoodi, F. v. $M$. 
Solantum-contd.

nemophilum, F. v.M.

semiarmatum, $F$. $r^{\prime} . M$.

4. Prickles on the caly.xes also. Pubescence various.

*pyracanthum, Lam.-Madagascar.

*sodomæum, Linn.-Devil's Apple; a troublesome weed.

South Africa.

*aculeatissimum, Jacq. = S. ciliatum, Lam.-Scarlet-fruited

Devil's Apple; a troublesome weed. South America.

*aculeastrum, Dun.-A troublesome weed. South Africa.

xanthocarpum, Schrad. and Wend.-A troublesome weed.

eremophilum, F.v.M.

pungetium, $R . B r$.

campanulatum, R. Br.

adenophorum, $F . v . M$.

cinereum, R. Br.

carduiforme, $F . v . M$.

echinatum, R. Br.

ellipticum, $R \cdot B r$.

quadriloculatum, F.v.M.

*rostratum, Dun. Sol.-Buffalo Burr; a troublesome weed. America.

*Cyphomandra, Sendtn.

betacea, Sendtn.-Tree Tomato. Mexico.

Physalis, Linn.

*peruviana, Linn.-Cape Gooseberry; a useful fruit. Peru.

minima, Linn.- "Neen" of Cloncurry natives.

var. indica, C. B. Clarke.

*virginiana, Mill., Gard. Dict.-A troublesome weed from America.

*Capsicum, Limn.

fastigiatum, Blume.-Chilli. The powder is the principal source of cayenne pepper. America.

*Nicandra, Gartn.

physaloides, Gartn.-Peru.

Tribe Il.-Atrope.e.

Lycium, Limn.-Box-thorns.

*afrum, Linn.-A useful hedge plant, but may become a troublesome weed. Africa.

*chinense, Mill.-China.

*europæum, Linn.-Europe.

australe, F.v. M. 
Datura, Linn.

$$
\text { Tribe III.-Hyoscyamez. }
$$

Leichhardtii, F.v.M.-Poisonous to stock.

*Stramonium, Linn.-Thorn or Mad Apple; poisonous. Used in cases of asthma.

var. Tatula, Hook.-Poisonous; useful in cases of asthma.

*fastuosa, Mill.-Poisonous.

Metel, Linn.--Poisonous. The properties of Stramonium and allied plants are anodyne and antispasmodic, and in overdoses they are very poisonous.

*Cestrum, Linn.

Tribe IV.-CestrineE.

nocturnum, Limn.-Poison-berry. A common shrubby weed on vacant places about towns; known as a bad poison weed in South Africa, especially to cattle.

Nicotiana, Limn.-Fruit a Capsule.

suaveolens, Lehm.-Native Tobacco; poisonous to stock.

var. parviflora, Benth. (Fig. 333.)

var. longiflora, Benth.-Six-o'clock; Wild Tobacco. (Fig. 334.)

var. Debneyi, Bail. (Fig. 335.)

*Tabacum, Limn.-Tobacco-plant. America.

*glauca, Grah.-Tobacco-tree. Buenos Ayres.

*Petunia, Juss.

Tribe V.-SAlpiglosside.

nyctaginiflora, Juss.-Stray from gardens.

Duboisia, R. Br.

myoporoides, R.Br.-Dr. Joseph Bancroft's Eye-plant; said to possess the properties of Belladonna, but is three times as powerful, and has other advantages.

Hopwoodii, F.v.M.-Pitury. The leaves of this plant are chewed by the natives as the white man chews tobacco. (Fig. 336.)

Leichhardtii, F.v.M.-Corkwood of the Nanango district.

Alliance IX.-PERSONALES.

\section{Order LXXXVII.-SCROPHULARINEÆ.}

Series Pseudosolaneæ.

*Verbascum, Linn.

Tribe I.-Verbasce压.

Blattaria, Linn.-Moth Mullein. Europe. 


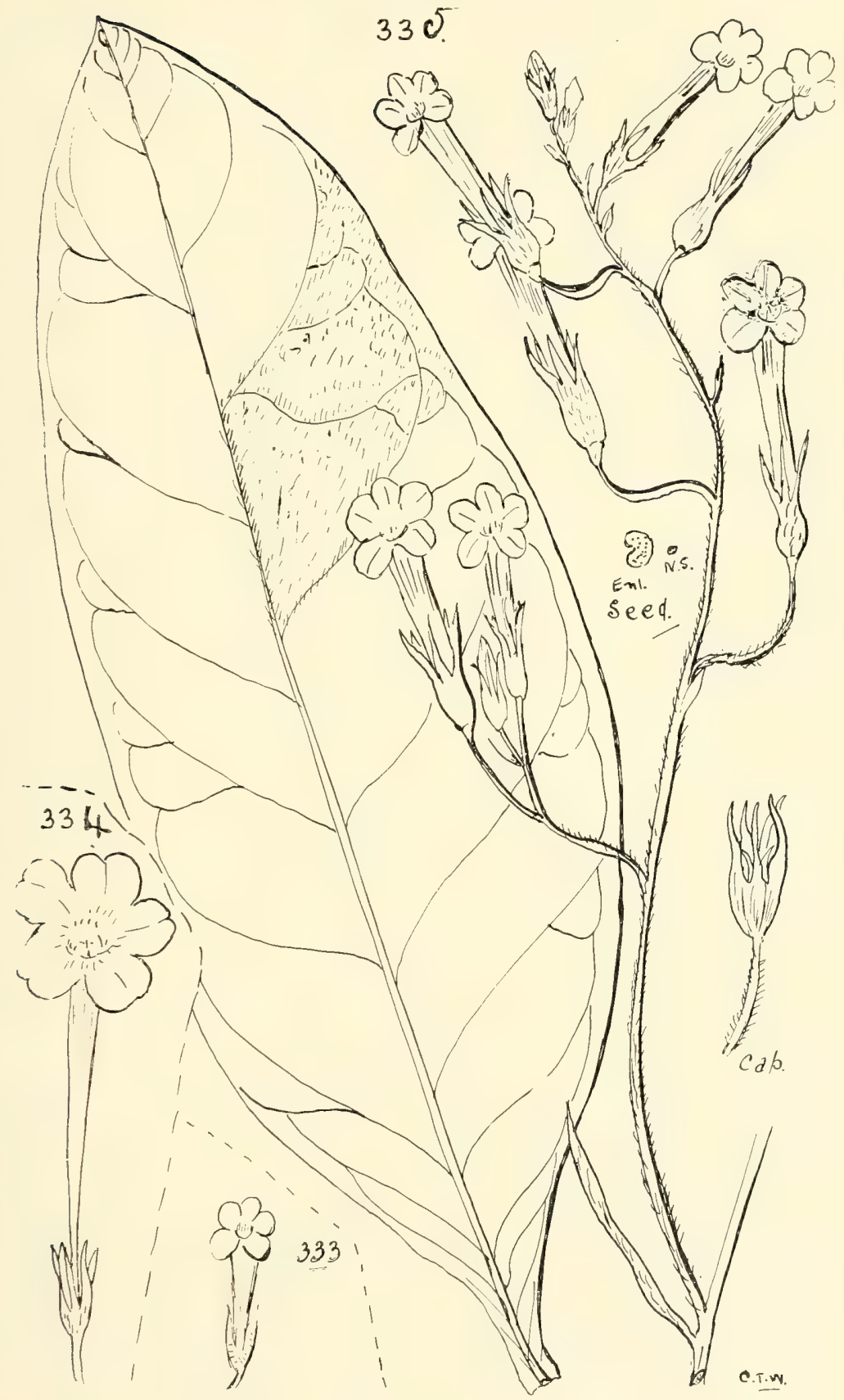

333. Nicotiana suaveolens, Lehm., var. parviflora, Benth. 334. N. suaveolens, Lehm., var. Longiflora, Benth. 335. N. suaveolens, Lehm., var. Debnfyi, Bail. 
*Celsia, Linn.

cretica, Linn.-Europe.

*Linaria, Juss.-Toad Flax.

bipartita, Willd.-North and West Africa,

\section{Series Antirrifinide}

Tribe II.-Antirrhined.

*Antirrhintm, Linn.

orontium, Linn.-Calf's-snout. Europe and Africa.

Tribe III.-Calceolarieł.

*Calceolaria, Limn.

scabiosæfolia, Roem. et Schultes.-Lady's Slipper plant. Peru.

Tribe IV.-GRATIOLEÆ.

Mimulus, Limn.

Subtribe I.-Mimulea.

Uvedalix, Benth. (Fig. 337.)

gracilis, $R . B r$.

repens, $R . B r$.

prostratus, Benth.

Mazus, Lour.

pumilio, R.Br-Maroochie is the only part of Queensland in which I have met with this plant.

Subtribe II.-Stemodiece.

Adenosma, R. Br.

cæruleum, R. Br.

Stemodia, Limn.

lythrifolia, $F \cdot$.'. $M$.

viscosa, Roxb.

Morgania, R. Br.

floribunda, Benth.-This plant has been suspected of poisoning stock near Boulia.

pubescens, R. Br.

glabra, $R . B r$.

Limnophila, R. Br.

gratioloides, R. $B r$.

punctata, Blume.

Subtribe III.-Herpestidea.

Herpestis, Goertn.

floribunda, R. Br.

Monnieria, H. B. et K.-Said to be employed in Ceylon as a mild purgative in fevers. 
Gratiola, Linn.

pedunculata, R.Br.-Medicinal; used in liver complaints. peruviana, Linn.

Dopatrium, Hamilt.

junceum. Hamilt.

Subtribe IV:-Vandeliece.

Artanema, Don.

fimbriatum, Don. = Torenia scabra, Grah.

var. album, Bail.

Vandellia, Limn.

crustacea, Benth.

alsinoides, Benth. (Fig. 338.)

scapigera, Benth.

subulata, Benth. (Fig. 339.)

Bonnaya, Link et Otto.

veronicæfolia, Spreng.

brachiata, Link et Otto.

Subtribe V.-Limosellece.

Microcarpæa, R. Br.

muscosa, R. Br.

Pepliditum, Delile.

humifusum, Delile.

Muelleri, Benth.

Glossostigma, Arn.

spathulatum, Arn.

Drummondii, Benth.

Limosella, Limn.

Curdieana, F.v.M.-Australian Mudwort

Tribe V.-.Digitalem.

Subtribe I,-Sibthorpiex.

Scoparia. Linn.

dulcis, Linn.-A common tropical weed, supposed of American origin.

Subtribe II.-Veronicea.

Veronica, Linn.-Australian Speedwells.

Derwentia, $A n d r$.

$$
\text { Section I.-Hebe. }
$$

Section II.-Chamædrys.

calycina, $R \cdot B r$.

plebeia, R. Br.

notabilis. F. v, M. 
LXXXVII. SCROPHULARINEA.

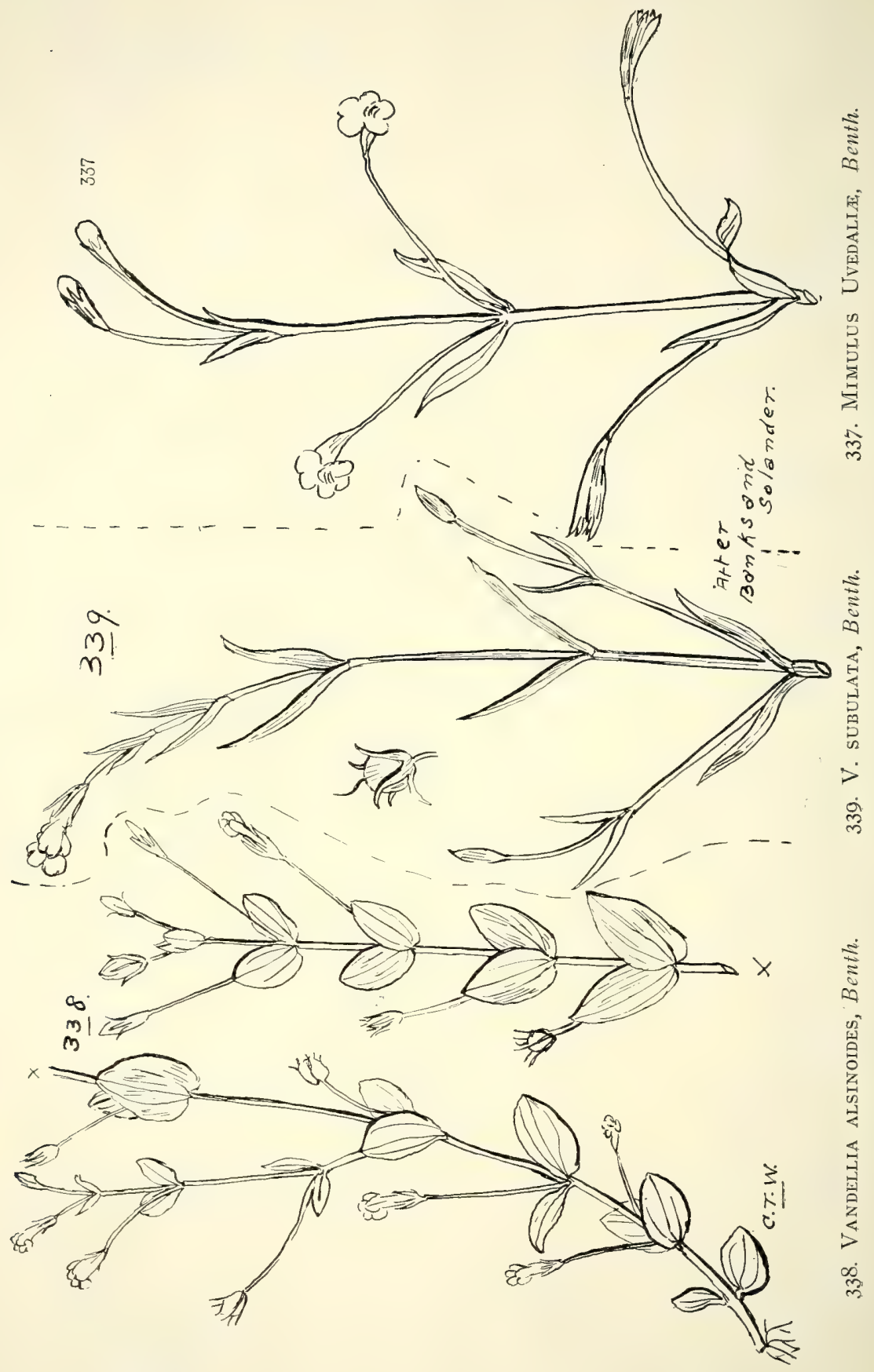


Veronica-contd.

Section III.-Veronicastrum.

serpyllifolia, Linn.

peregrina, Limn.

Buchnera. Linn.

Tribe VI.-Gerardiez.

Subtribe I.-Buchnerece.

tetragona, R. Br. (Fig. 340.)

urticifolia, R. Br.

linearis, R. $B r$.

var. asperata, Benth.

tenella, R. Br.

gracilis, R. Br.

ramosissima, R. Br.

var. parviflora, Benth.

Striga, Lour.

hirsuta, Benth.

parviflora, Benth.

curviflora, Benth.

Rhamphicarpa, Benth.

longiflora, Benth.

Subtribe II.-Eugerardiea.

Centranthera, R. Br.

hispida, $R . B r$.

Sopubia, Hamilt.

trifida, Hamilt.-Every leaf has in its axil a very short leafy branch which remains undeveloped; hence the "fascicled" leaves described in books.

Tribe VII.-Euphrasię.

Euphrasia, Linn.-Eye-bright.

collina, R. Br.

scabra, R. Br.

\section{Order LXXXVIII.-LENTIBULARIEÆE.}

Utricularia, Linn.-Bladderworts.

Sect. I.-Natantes. Stems floating.

stellaris, Linn.

flexuosa, R. $B r$.

exoleta, $R \cdot B r$.

tubulata, F. z. M.

Sect. 2.-Limosa. Plants erect.

albiflora, $R . B r$.

pygmæa, $R . B r$.

chrysantha, R.Br. (Fig. 341.) 
Utricularia-contd.

flava, Banks and Sol. (Fig. 342.)

barbata, R.Br. (Fig. 343.)

bifida, Lam.

cyanea, R. Br.

var. alba, Benth.

lateriflora, $R . B r$.

dichotoma, Labill.

var. uniflora, Benth.

biloba, R. $B r$.

limosa, R. Br.

Baneri, R. Br.

\section{Order LXXXIX.OROBANCHACEZE.}

Orobanche, Linn.-Broom Rapes.

cernua, Lœfl., var.-I have seen plants of this genus several times on cultivated plants, but never on the indigenous ones, so cannot say if native in Queensland.

\section{Order XC. - GESNERACEÆ.}

Tribe Cyrtandre E.

Cyrtandra, Forst.

Baileyi, F.v.M.

Didymocarpus, $W$ all.

Kinnearii, F.v.M. = Ro:tlera Kinnearii, F. v. M.

Bæa, Commers.

hygroscopica, F.v.M.-The Queensland Rose of Jericho. (Fig. 343 bis.)

\section{Order XCI.-BIGNONIACEÆ.}

Tribe I.-Tecomex.

Tecoma, Juss.

australis, $R . B r$.

var. Pandorea, Bail.-_"Dead Hen"; the flowers of bad odour.

var. meonantha, Bail.-Flowers fragrant.

var. linearis, Bail.

jasminoides, Lindl.

Hillii, F.v. M.

Baileyana, Maid. et Bak.

Dolichandrone, Fenal.

alternifolia, Benth. et Hook. = Spathodea alternifolia, R. Br.

heterophylla, F. v. M. = Spathodea heterophvlla, R. Br. (Fig. 344.) 


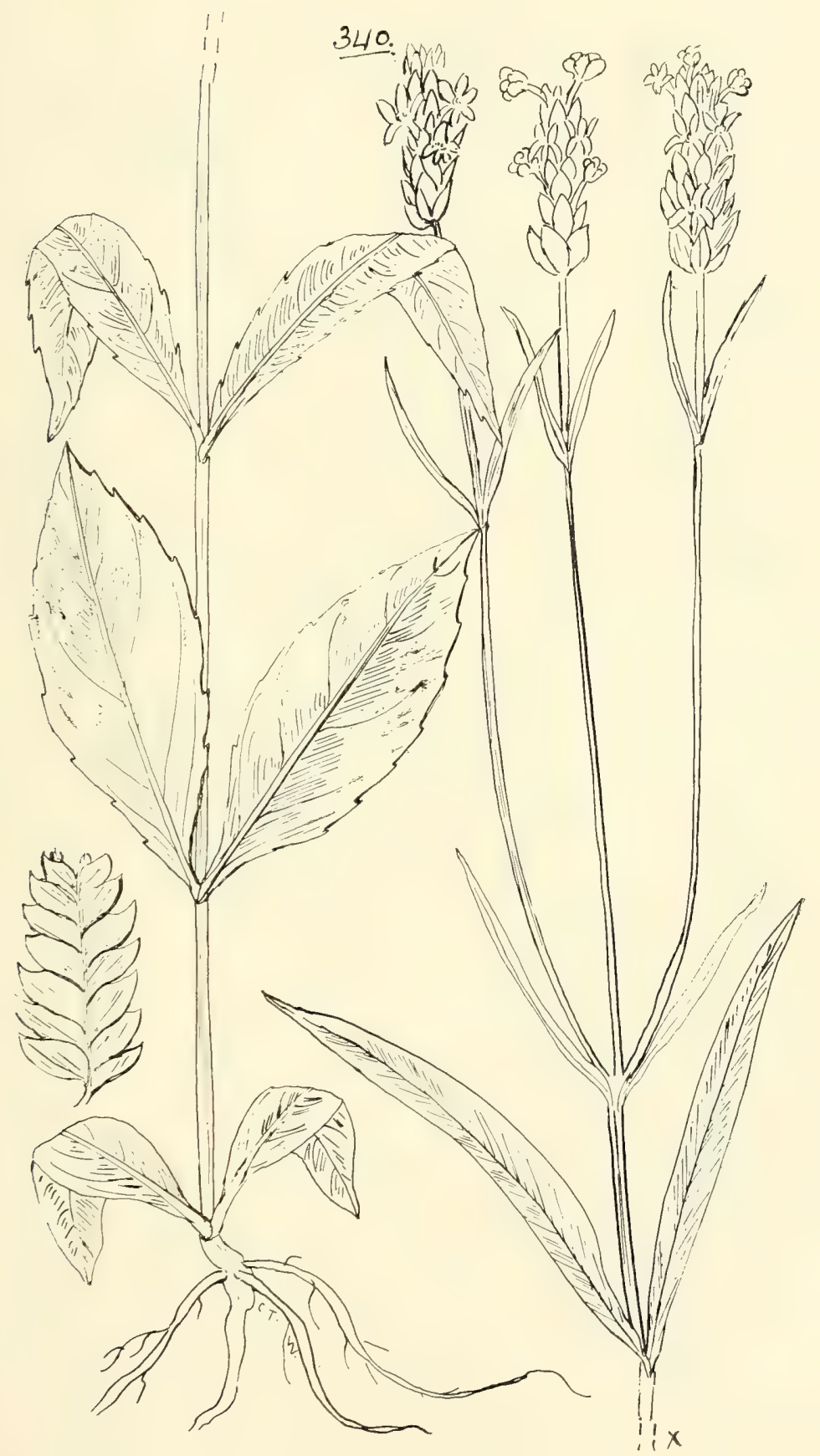

340. Buchnera tetragona, $R$. Br. 


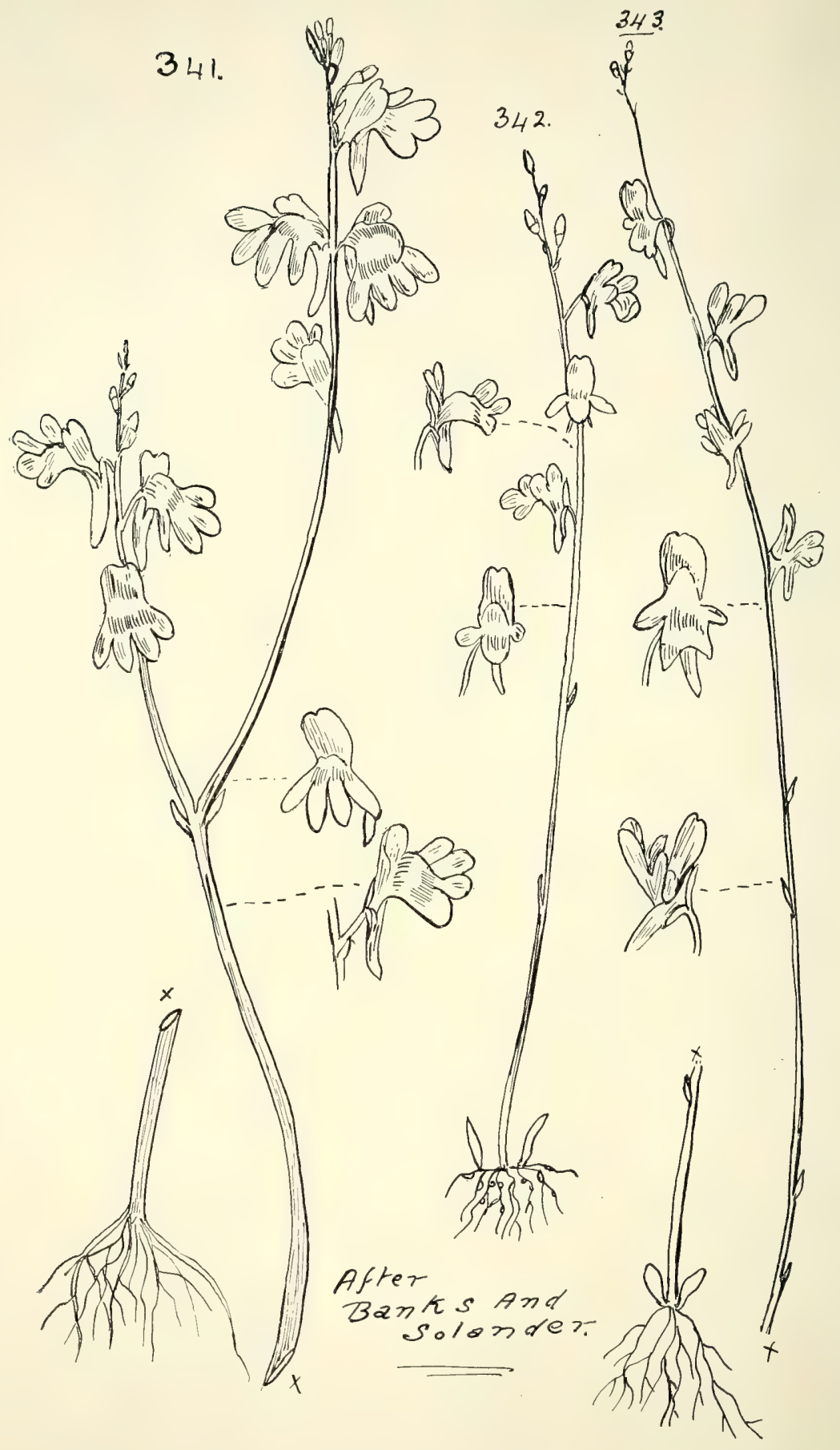

34I. Utricularia chrysantha, R. $B r$.

342. U. FlAVA, Banks and Sol.

343. U. BARBATA, $R . B r$. 


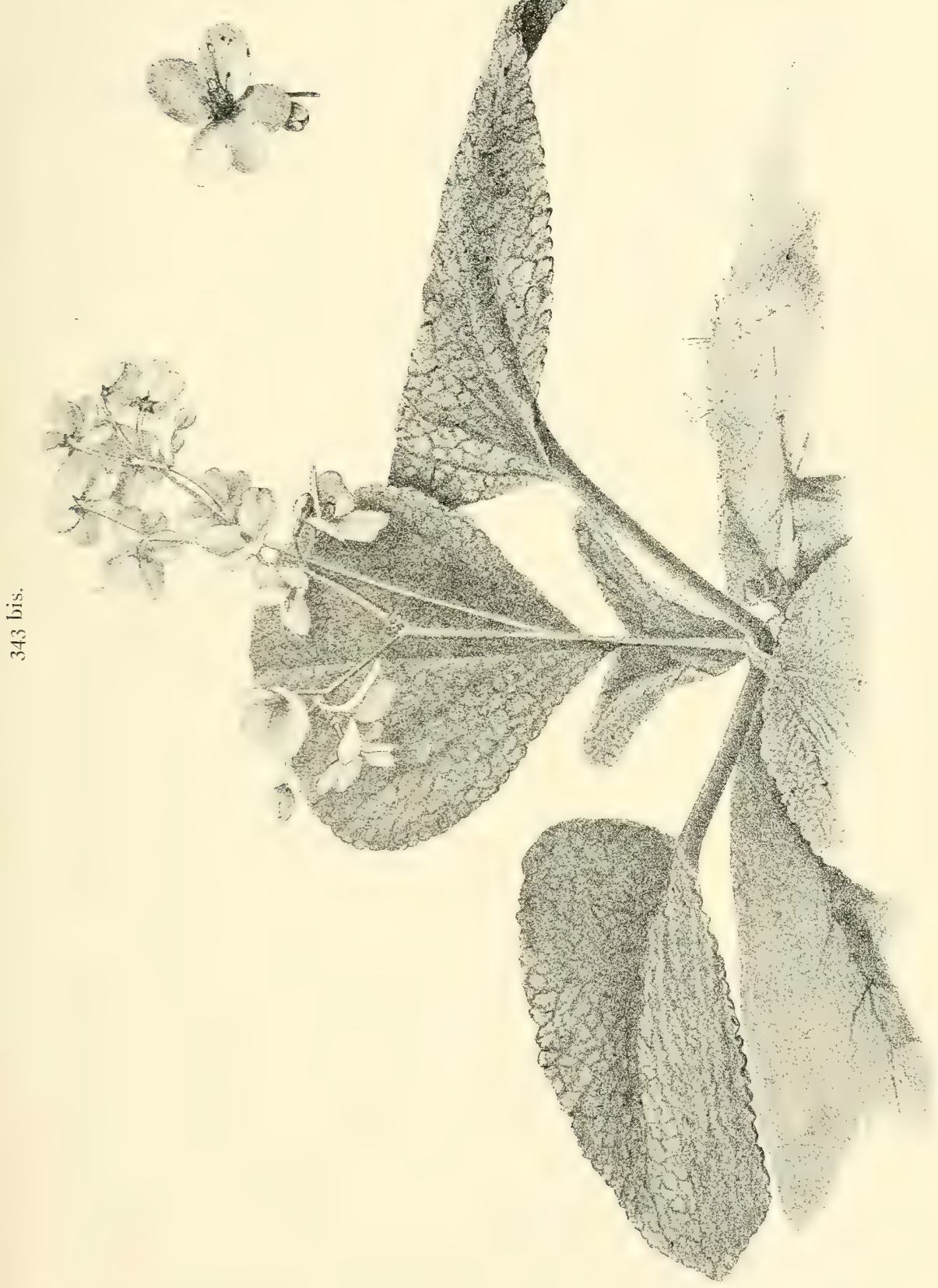


Haussmannia, F. v. M.

jucunda, F.v.M.-(For full description see Q1. Fl. p. I I36.) (Fig. 345.)

Diplanthera, R. Br.

tetraphylla, R. Br.-A large tree; wood at one time cut under the name of "Whitewood."

hirsuta, Bail.

\section{Order XCII.-PEDALINEÆ.}

Tribe MartynieÆ.

*Martynia, Linn.

proboscidea, Glox.-Unicorn fruit. Mexico.

diandra, Glox.-Tiger's-claw. South America.

lutea, Lindl. Brazil.

fragrans, Lindl.-Mexico.

*Sesamum, Linn.

Tribe Sesamez.

indicum, DC.-Til or Gingelly Oil plant. At one time a common weed along the Brisbane River. Asia.

Tribe Pretree

Josephinia, Vent.-Fruit a prickly burr.

grandiflora, R. Br. (Fig. 346.)

Eugeniæ, F.v.M. (Fig. 347.)

\section{Order XCIII.-ACANTHACEÆ.}

Tribe I.-Thunbergieæ.

*Thunbergia, Linn.

alata, Boj-Black-eyed Susan. Africa.

fragrans, Roxb.-So far as I can detect, T. Porvelli, F. v. M.s is identical with $T$. fragrans, Roxb.-so named from scent of the plant, not of the flower ( see note, Q1. Flora, p. II 42 ).

Nelsonia, R. Br.

T'ribe II.-NeLsoniex.

campestris, $R . B r$.

Ebermaiera, Nees.

glauca, Nees.

Tribe III.-Ruellieæ.

Hygrophila, R. $B r$. salicifolia, Nees. 
XCI. BIGNONIACEÆ.

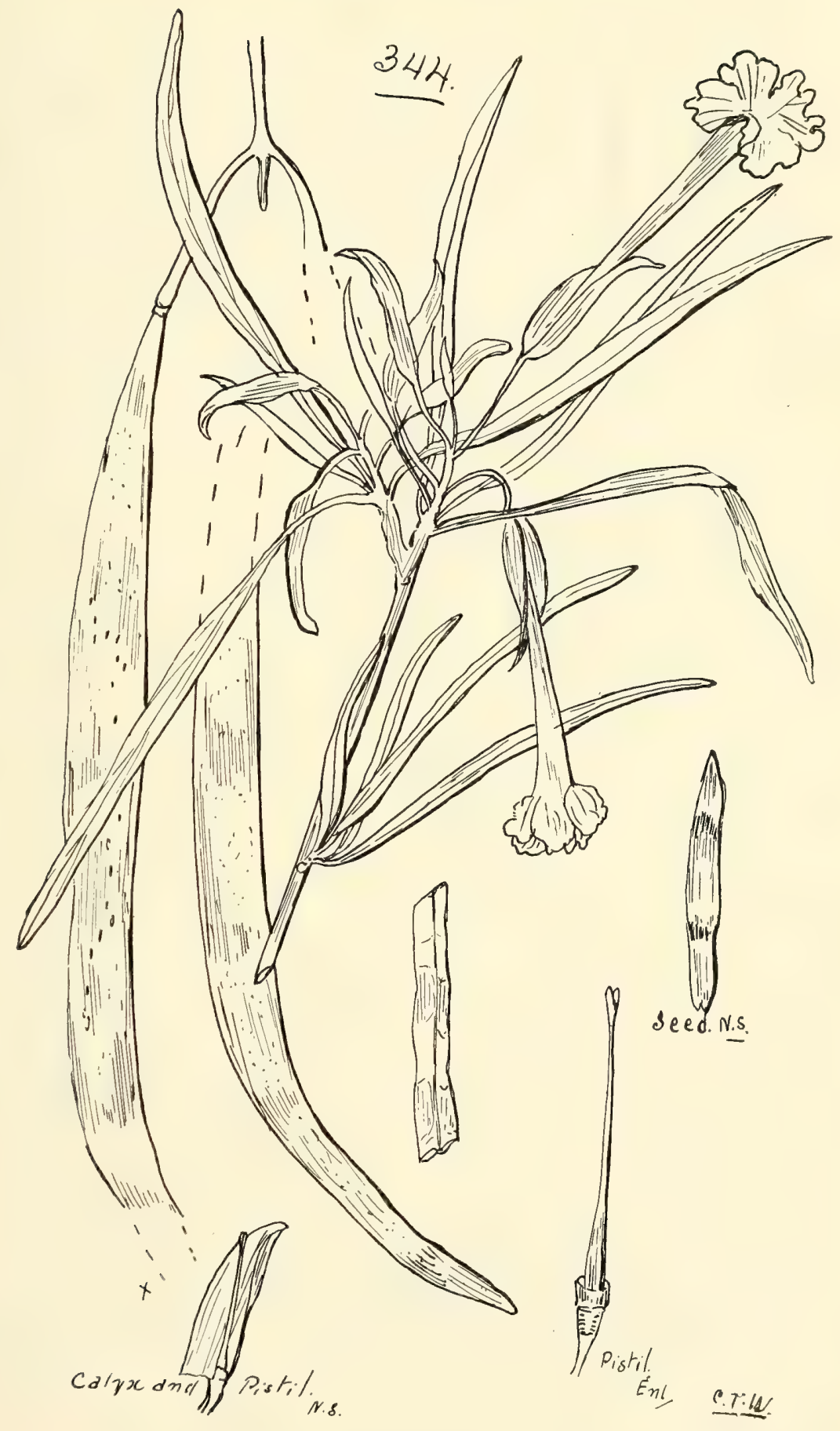

344. Dolichandrone heterophylla, $F . v . M$ 


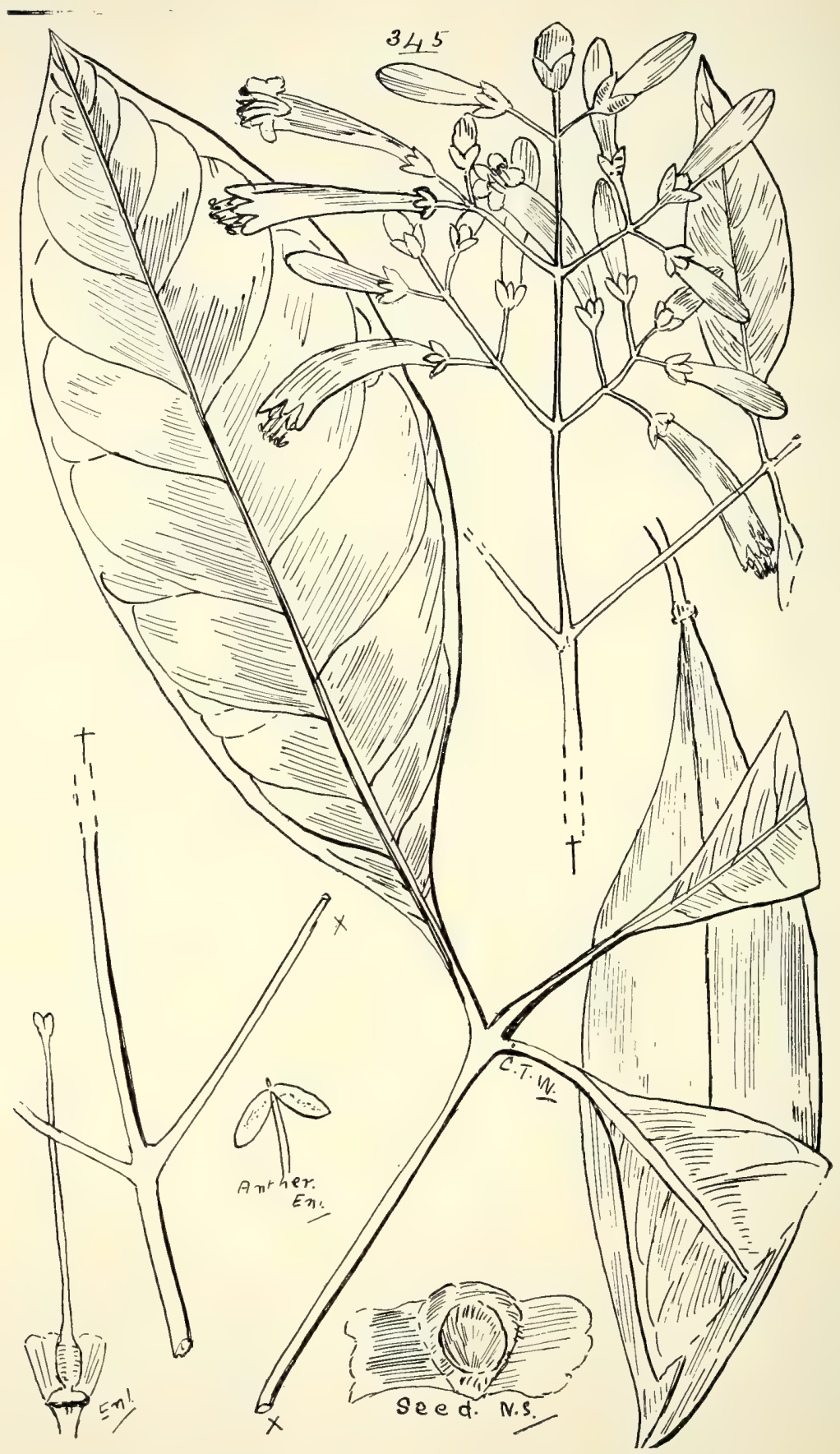

345. Haussmannia jucunda, F.v. M. 


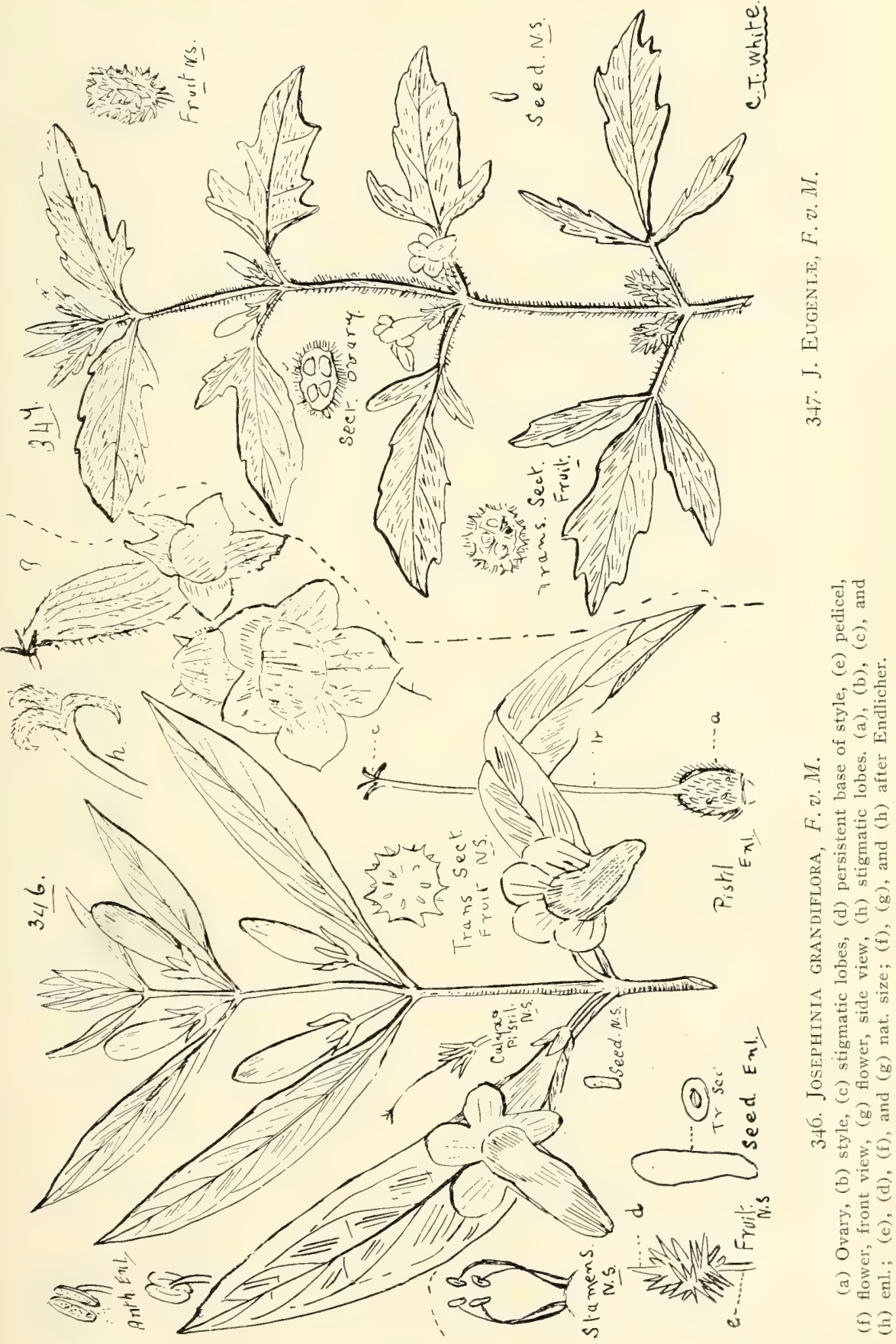




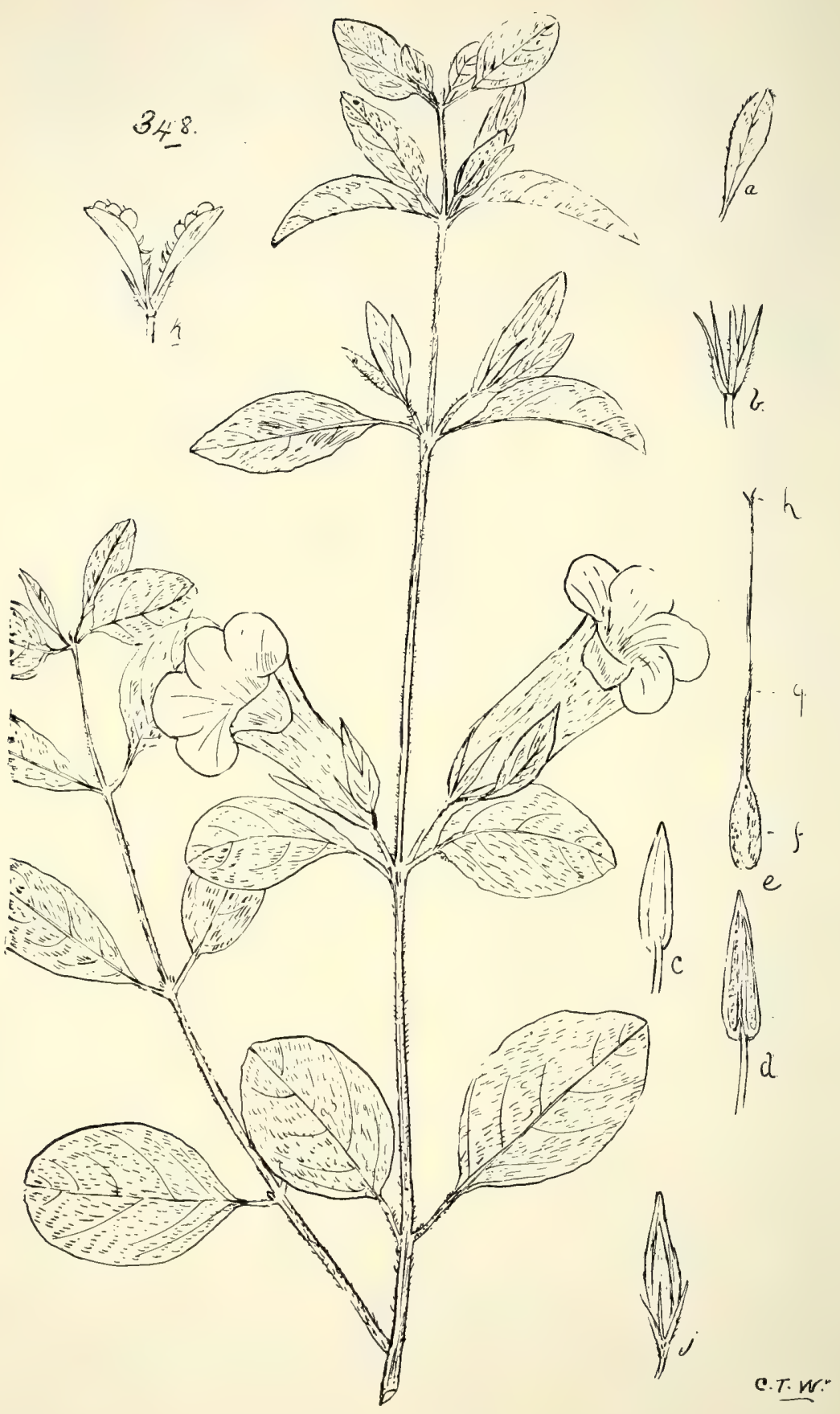

348. Ruellia primelacea, F. v. $M$.

(a) Bracteole, (b) calyx, (c) anther, back view, (d) anther, front view, (e) pistil, (f) ovary, (g) style, (h) stigma, (j) capsule, (k) capsule opening. (a), (b), (j), and (k) nat. size; (c)-(h) enl. 


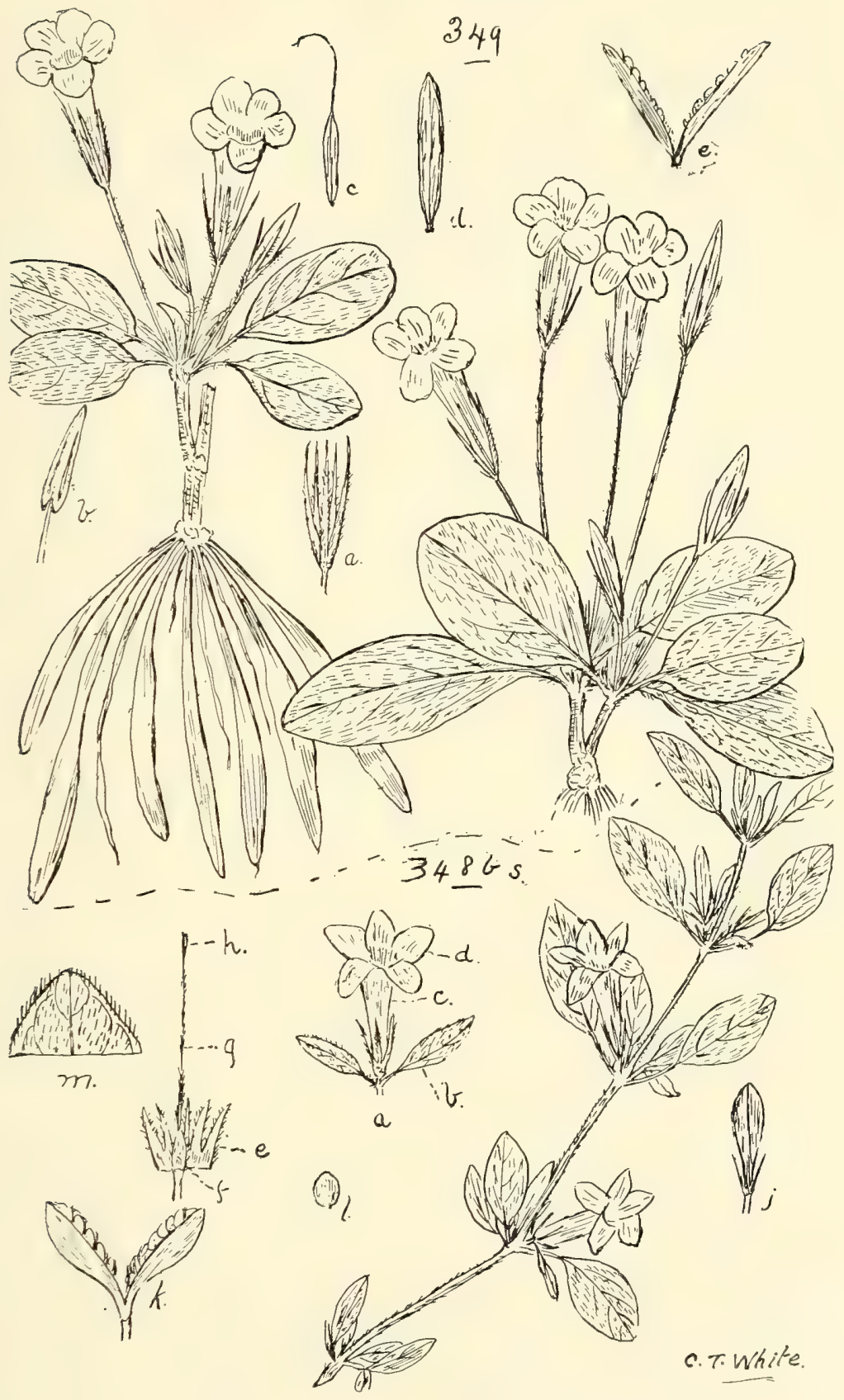

348 bis. Ruellia corynotheca, $F . v . M$.

(a) Flower, (b) bracteoles, (c) corolla-tube, (d) corolla-lobes, (e) calyx laid open, (f) ovary, (g) style, (h) stigma, (j) capsule, (k) opening capsule, (1) seed, (m) portion of leaf. (a)-(1) nat. size; (m) enl.

349. Ruellia acaulis, R. Br.

(a) Calyx, (b) anther, (c) immature capsule, (d) capsule, (e) opening capsule. (a), (c), (d), and (e) nat. size; (b) enl. 
Ruellia, Linn.

Section I.-Dipteracanthus.

bracteata, R. Br.

primulacea, F.v.M.-A shade-loving undershrub; will thrive under the shade of trees. Mr. J. Coghlan says, a valuable plant for stock, especially horses. (Fig. 348.)

corynotheca, F.v.M. (Fig. 348 bis.)

Section II.-Cryphiacanthus.

australis, R. Br.

var. scabra, Benth.

var. pumila, Benth.

spiciflora, $F . v . M$.

acaulis, $R$. Br.-Flowers a deep blue; the tuberous roots used as food. (Fig. 349.)

Acanthus, Linn.

Tribe IV.-Acantheæ.

ilicifolius, Linn.-Bear's-breech.

Tribe V.-Justiciex.

Subtribe I.-Asystasiece.

Asystasia, Blume.

australasica, Bail.

Eranthemum, Linn.

variabile. $R . B r$.

var. molle, Benth.

var. lineare, Benth.

tenellum, Benth.

Subtribe II.-Eujusticieae.

Hypoestes, R. Br.

densiflora, Benth.

paniculata, Benth.

floribunda, R. Br.

var. canescens, Benth.

var. pubescens, Benth.

Dicliptera, Juss.

spicata, Dcne. (Fig. 350.)

glabra, Dcne.

Leonotis, Dalz.

*Rungia, Nees.

latior, Nees.-India.

Justicia, Linn.

procumbens, Linn.

var. peploides, Bail. $=J$. peploides, T. Anders. 
Justicia-contd.

notha. Clarke.

hygrophiloides, F.v.M. (Fig. 35I.)

cavernarım, F.v.M.

eranthemoides, F.v. M.

Graptophyllum, Nees.

Earlii, F.v.M. (Fig. 352.)

ilicifolium, F.v.M. (Fig. 353.)

spinigerum, F.v.M. (Fig. 354.)

\section{Alliance X.-LAMIALES.}

\section{Order XCIV.-MYOPORINEA.}

Myoporum, Banks et Sol.

Section I.-Eumyoporum.

acuminatum, R. Br. - "Mee-mee" of Stradbroke Island natives.

var. ellipticum, Benth.

var. acuminatum, Benth.

var. parviflorum, Benth.

var. angustifolium, Benth.

deserti, A. Cunn.-Ellangowan Poison-bush; poisonous to stock.

deserti, A. Cunn.--Poisonous to stock.

laxiflorum, Benth. (Fig. 355.)

platycarpum, R. Br.

Section II.-Disoon.

Section III.-Chamapogonia.

debile, R. Br.- "Amula" of Rockhampton natives.

Pholidia, R. Br. (Included by F. v. Mueller in Eremophila.)

Dalyana, F.v. $M$.

divaricata, $F . v \cdot M$.

Bickii, Bail.

santalina, F.v.M. (Fig. 356.)

Eremophila, $R$. Br.-Several species supposed to be poisonous to stock.

Section I.-Eriocalyx.

Bowmanni, F.v.M.

strongylophylla, F.v.M.

Section II.-Eremocosmos.

rotundifolia, F. v. M.-Wood close-grained and nicely marked.

Sturtii, R. Br.

Mitchelli, Benth--Bastard Sandalwood. Wood fragrant when newly cut. "Baloora" of the Rockhampton natives. Latrobei, F.v.M. 


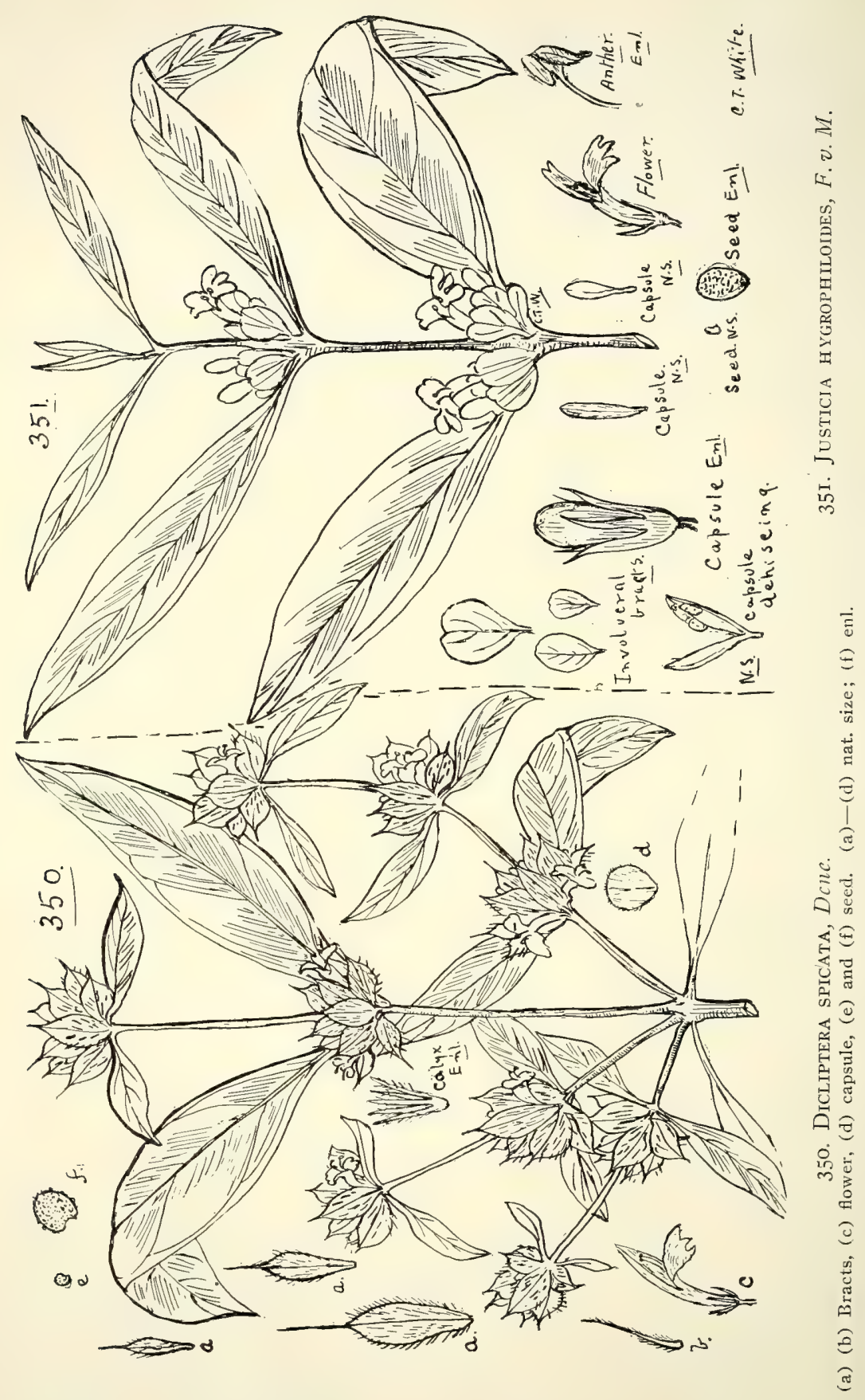




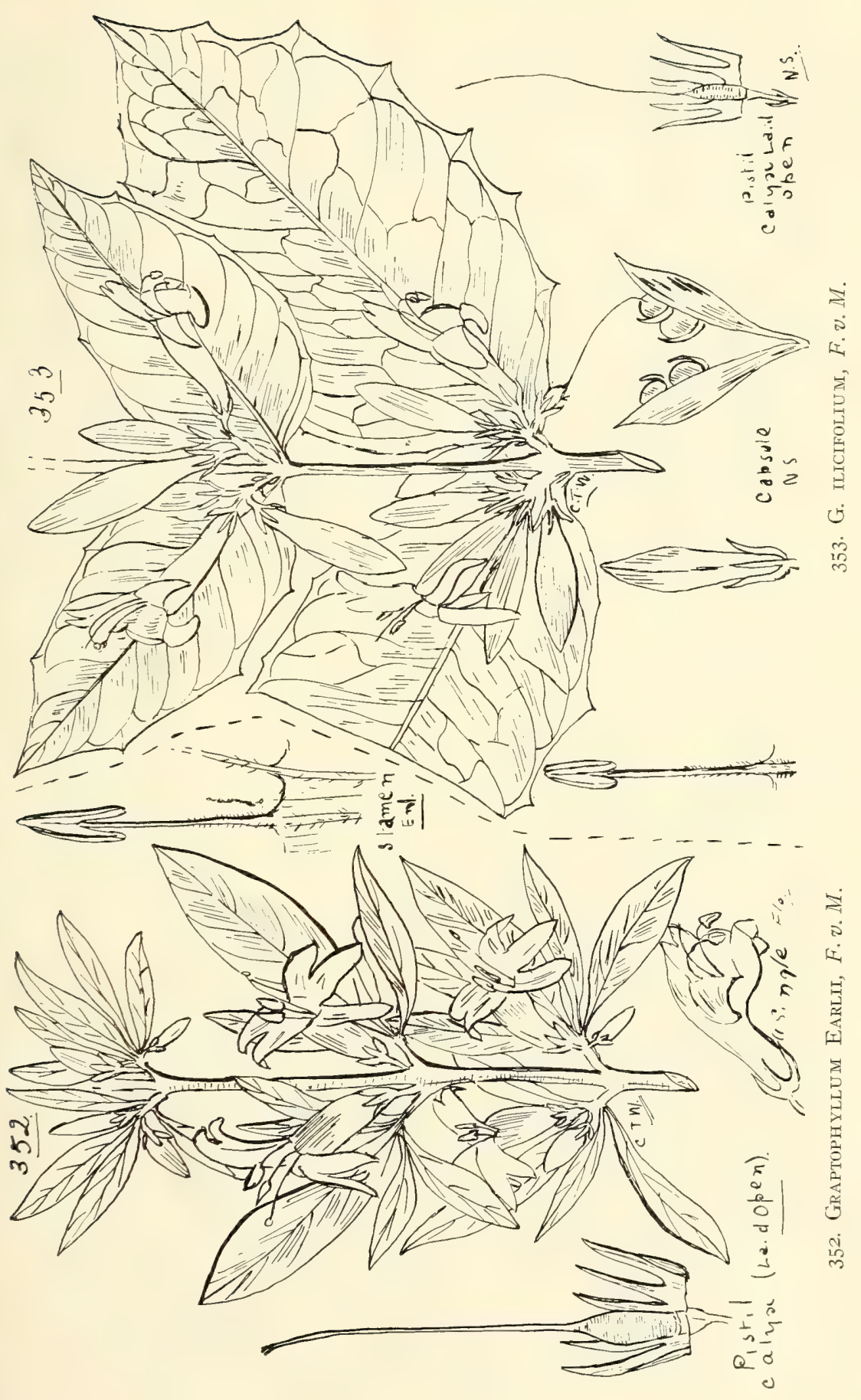




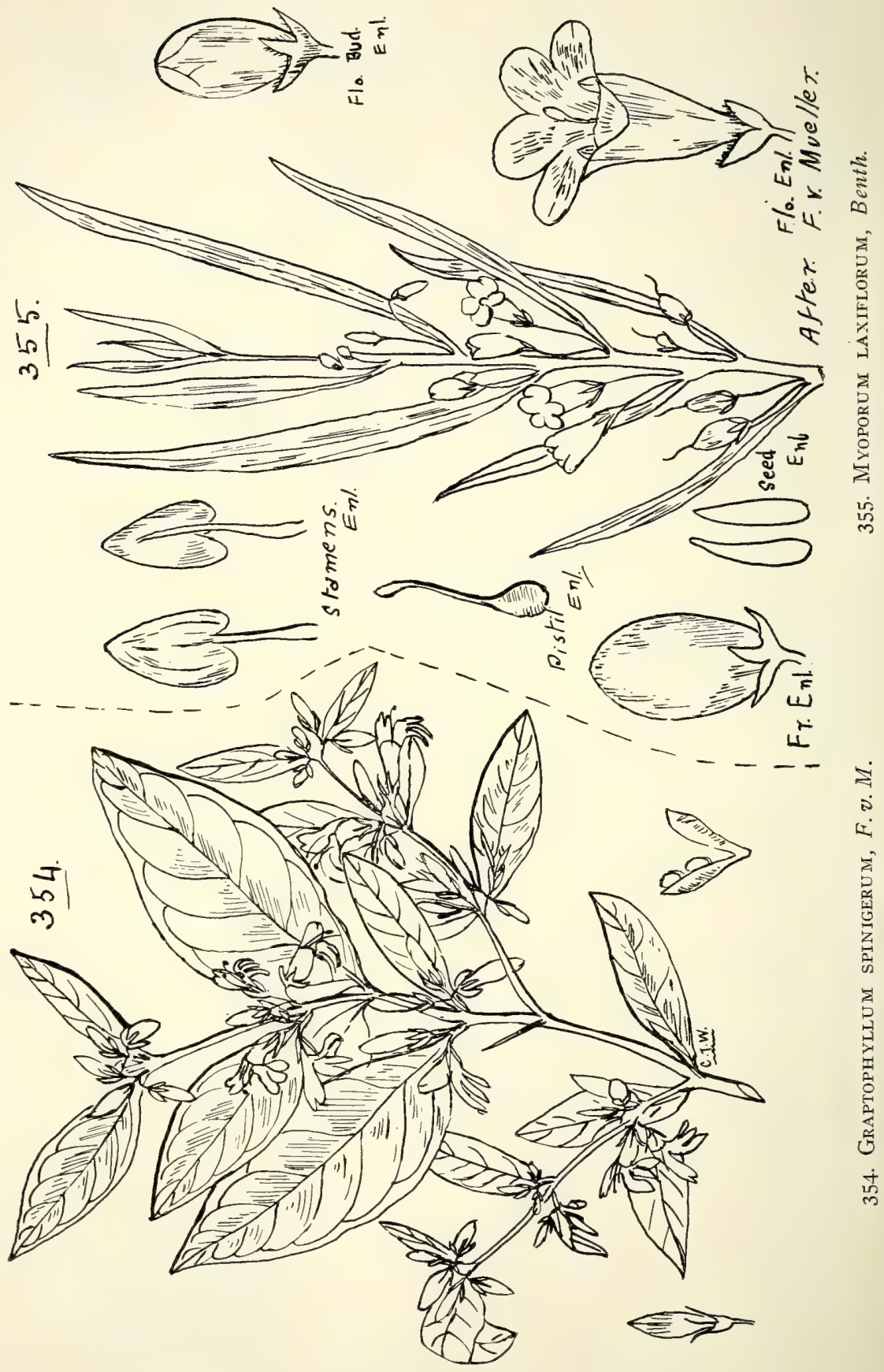



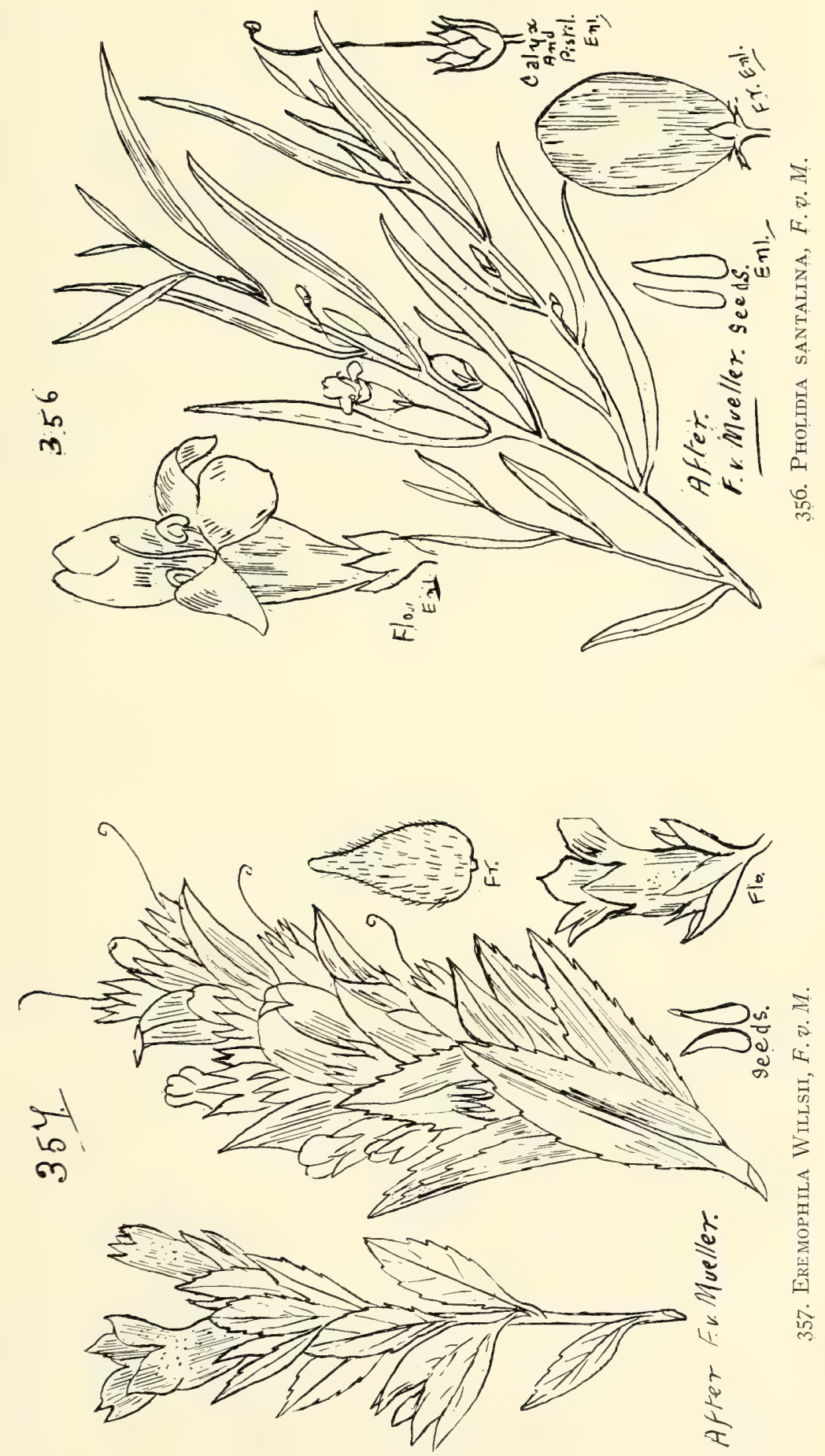


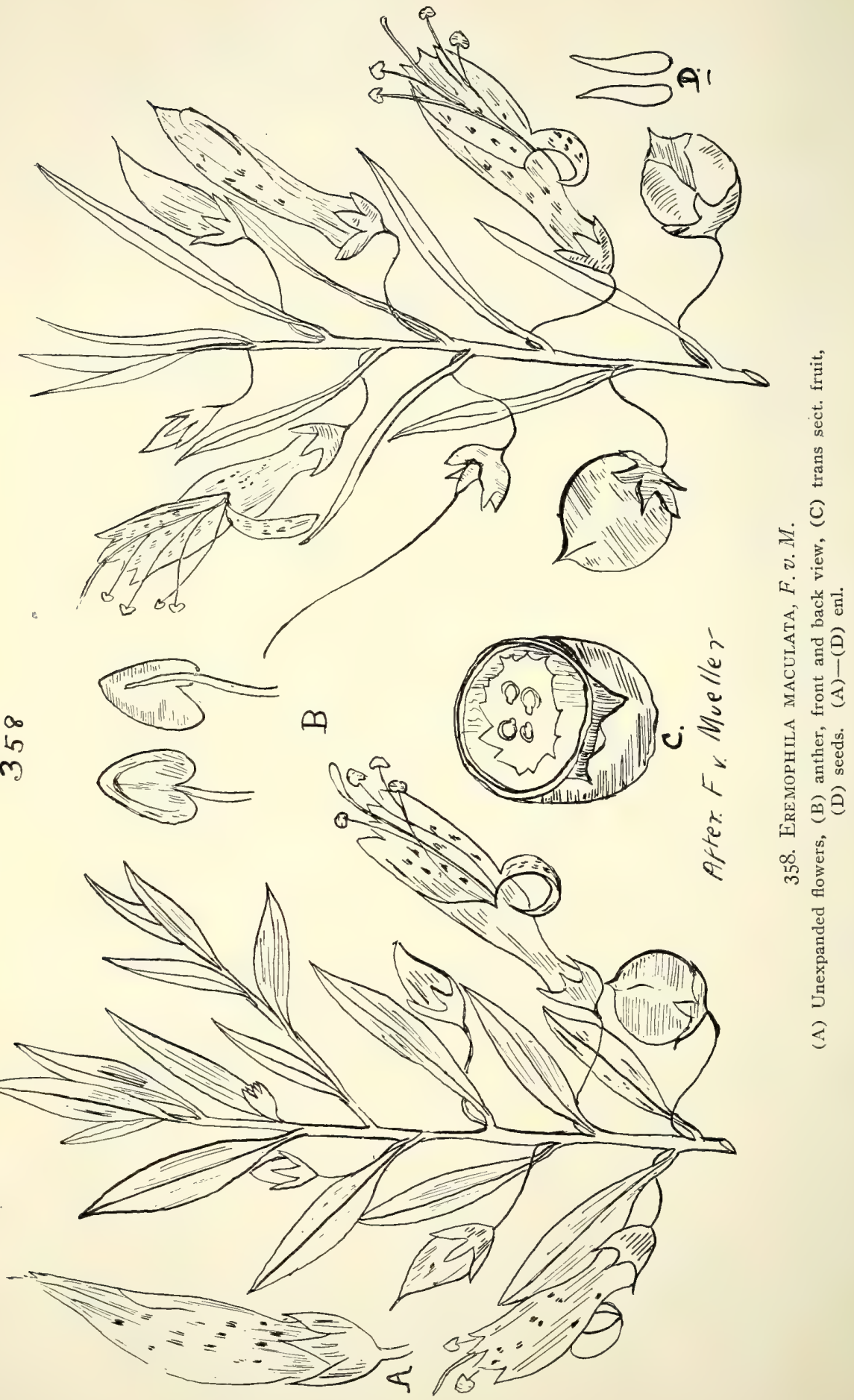


Eremophila-contd.

Section III.-Platycalyx.

Macdonnelli, F.v.M.

\section{Section IV.-Platychilus.}

longifolia, $F . v . M$.

polyclada, F.v.M.-Lignum. This name is also given to Muehlenbeckia Cunninghamii.

bignoniæflora, F.v.M.- "Quirramurrah" of Bulloo River natives. Wood fragrant, useful for cabinet-work.

Freelingii, F.v.M.

Goodwinii, F.v.M.

Willsii, F.v.M. (Fig. 357.)

\section{Section V.-Stenochilus.}

Brownii, F.v. M.--Poisonous to stock.

Duttoni, F. v. M.

maculata, F.v.M.-Native Fuchsia; a poison-bush. Contains a large quantity of prussic acid (J. C. Brïnnich). Mr. Brünnich says that if the leaves or the fruits were eaten by stock separately or at different times no harm would occur, but when taken together a violent poison is produced which quickly causes death (see Q1. Agric. J1. xxv. (I9I0) 293). (Fig. 358.)

var. flava, Bail.

\section{Order XCV.-DERBENACE $Æ$.}

Tribe. I.-Chloanthe.e.

Newcastlia, F.v.M.

cladotricha, F.v.M.

cephalantha, F.v.M. (Fig. 359.)

Dicrastyles, Drumm.

Costelloi, Bail.

Weddii, Bail. (Fig. 36o.)

Doranii, F.v. M.

Lewellini, F.v. M.

Chloanthes, R. $\mathrm{Br}$.

stœechadis, R. Br.

var. parviflora, Benth

parviflora, Walp.

Pityrodia, R. Br.

salvifolia, R. Br.

Spartothamnus, A.Cunn.

junceus, A. Cunn.

var. puberulus, Bait. $=S$. puberulus, Bail. 
Nesogenes, A.DC.

euphrasioides, A.DC.

*Lantana, Linn.

TRIBE II.-VERBENEÆ.

Camara, Linn.-A pest or noxious weed. This plant is considered to cause the disease in horses called "Pink-nose." Some plants may at times be seen with pretty variegated foliage. Tropical America.

crocea, Iacq.-Tropical America. This is not a troublesome weed, although it has run out in a few localities, where it has been used for hedge-making; the flowers are showy and of various colours.

Sellowiana, Link.-South America.

Lippia, Linn.

nodiflora, Rich.

geminata, Kunth.

* Stachytarpheta, $T a h l$.

dichotoma, $T$ ahl.-Tropical America.

mutabilis, $Y$ ahl.--Tropical America.

*Duranta, Limn.

Plumieri, Jacq.-This common and excellent hedge-plant has run out into the pasture very little, but should the fruit at any time become the food of some animal the plant will become a great pest, as it bears fruit so abundantly. The fruit has already caused the death of children near Brisbane. The genus belongs to South America. The following varieties may be distinguished:-

var. alba.-Flowers white, plant nearly or quite thornless.

var. Ellisia.-Flowers dark blue with darker lines. The best for hedge-making, as it makes a strong growth, and stands dry weather better than the other varieties. forma variegata.

var. normalis.-Flowers light blue.

Verbena, Limn.

officinalis, Lim.-Common Vervain.

z'ar. macrostachya, Benth.

*bonariensis, Linn.-Purpletop. Buenos Ayres. A troublesome weed.

*venosa, Gill et Hook.-Buenos Ayres.

Callicarpa, Linn.

Tribe III.-VITICE压.

cana, Linn.

pedunculata, R. Br. 


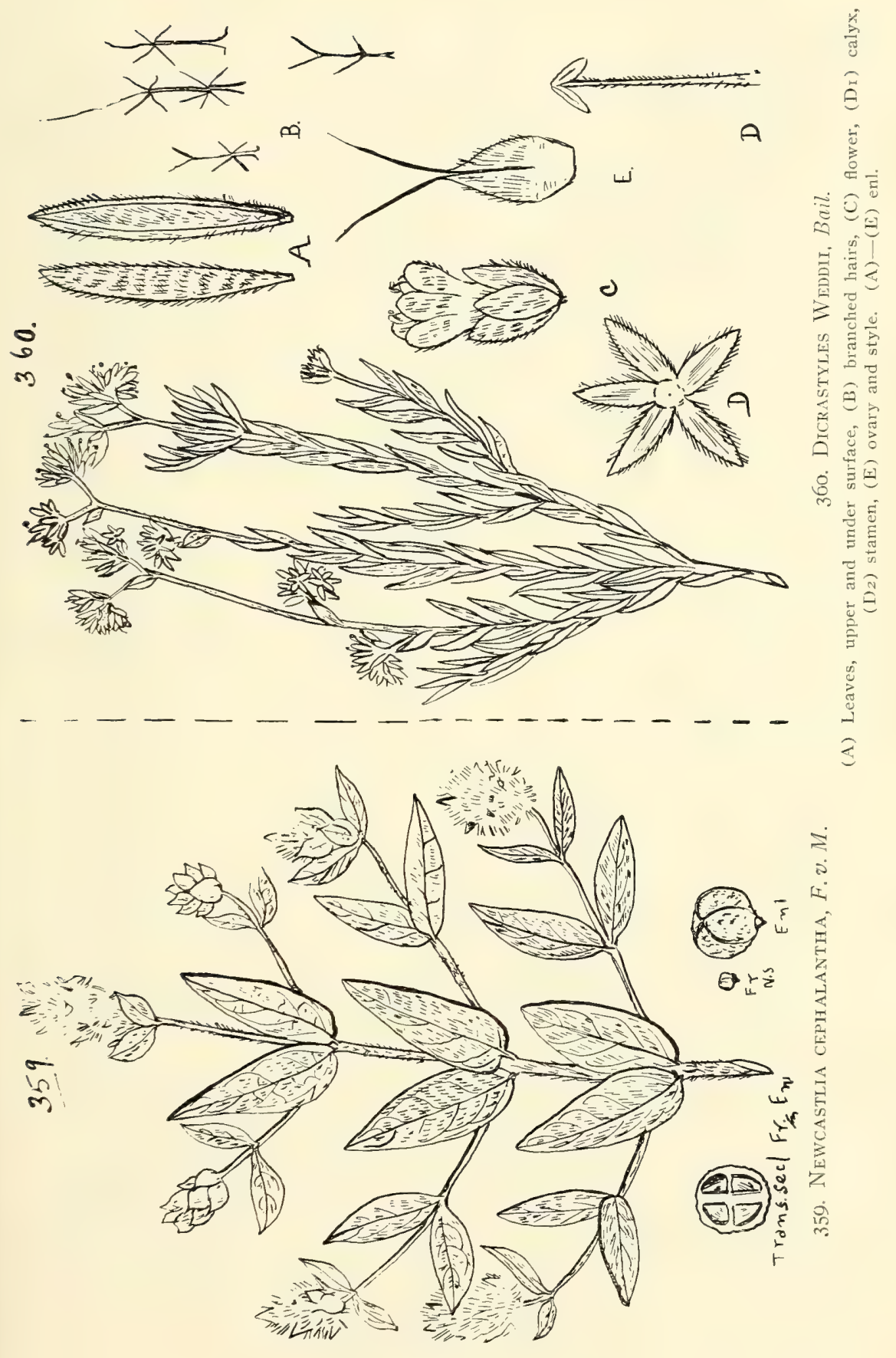




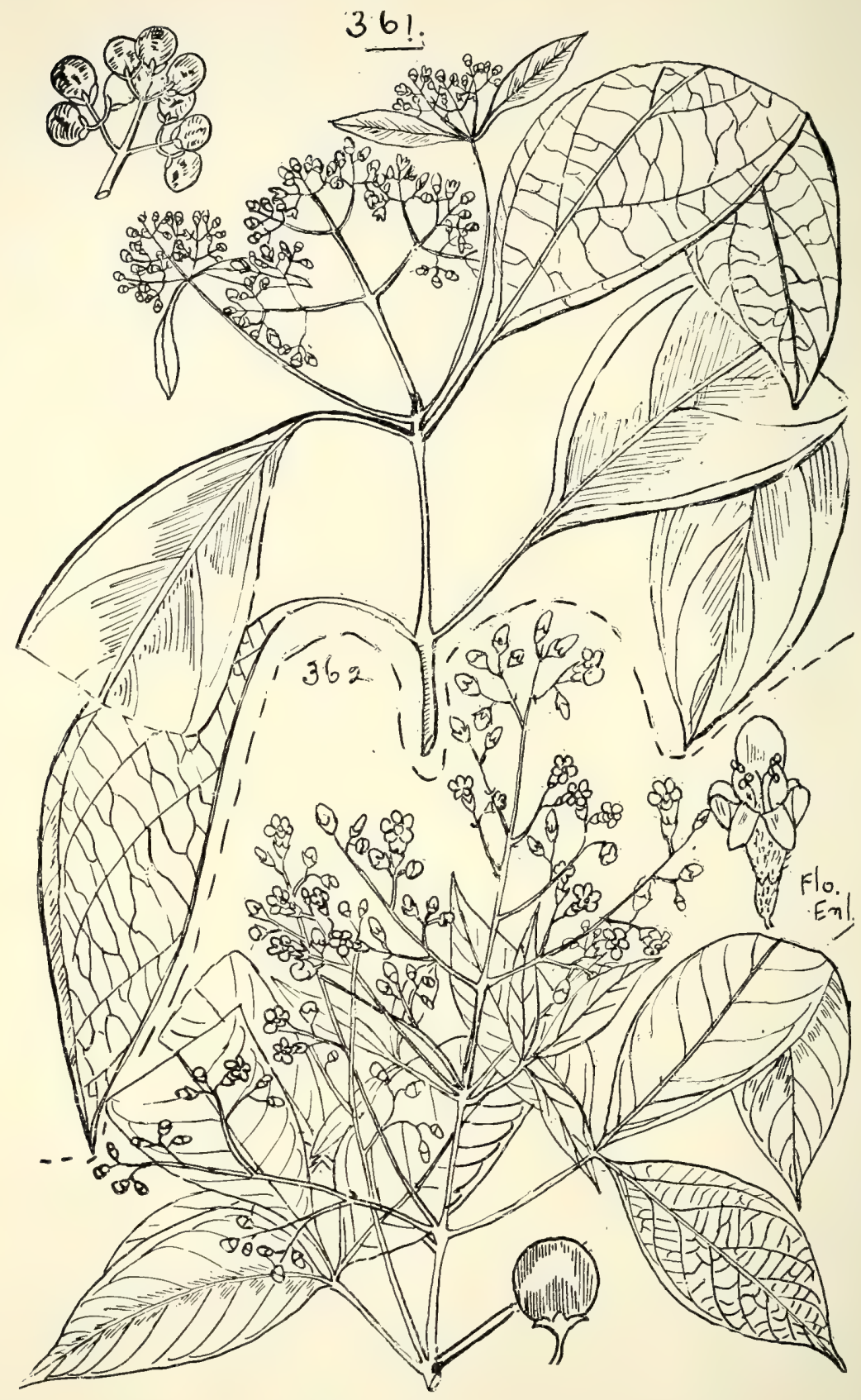

36r. Premna Tateana, Bail

362. Vitex acuminata, $R . B r$. 


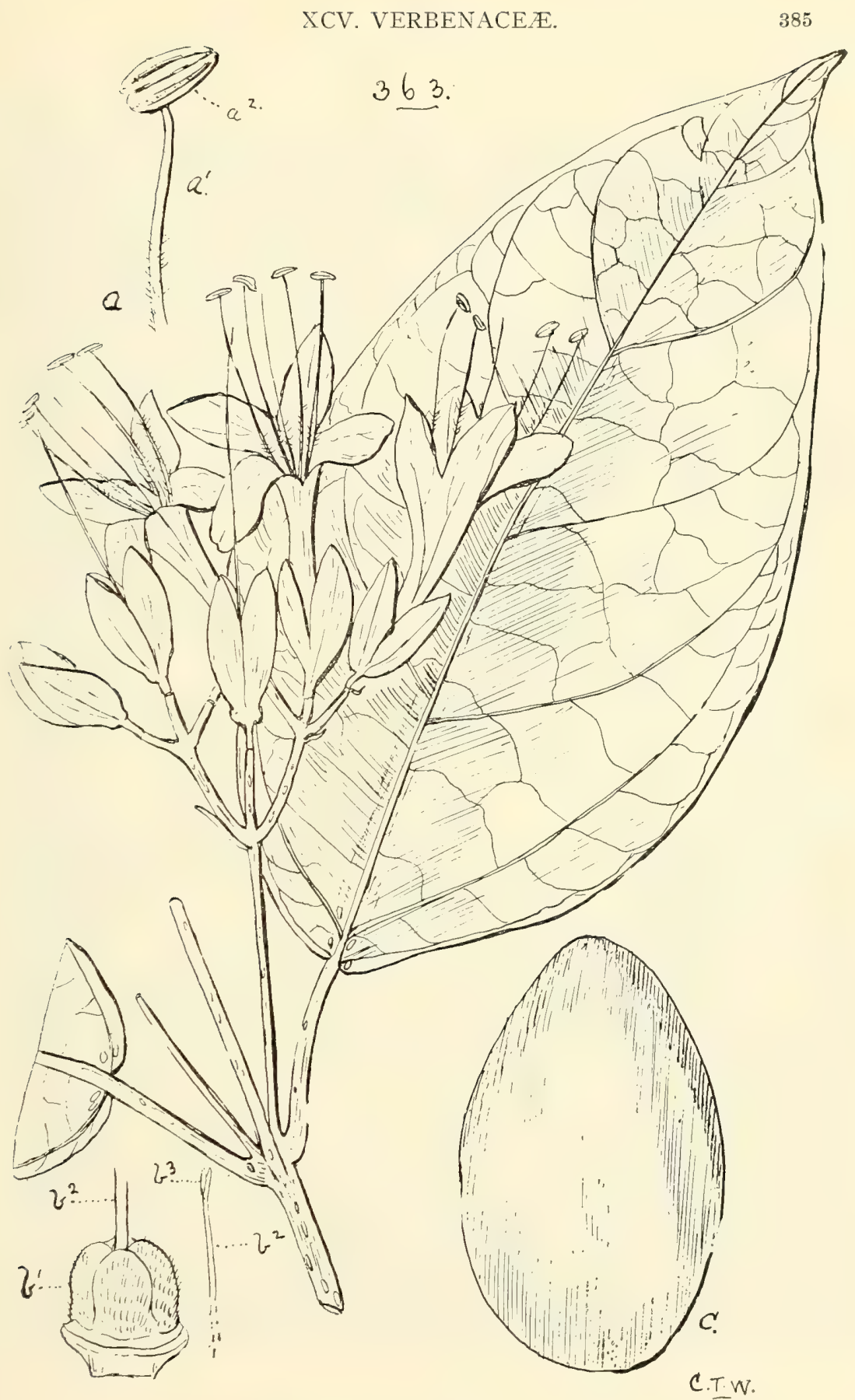

363. Faraday splendid, $F . v \cdot M$.

(a) Stamen, (ar) filament, (az) anther, (br) ovary, (ba) style, (by) stigma, (c) fruit. (a) and (b) enl.; (c) nat. size.

$2 \mathrm{~A}$ 
Callicarpa-contd.

longifolia, Lam._. "Chukin" of Cairns natives. The bark used by the Japanese on the Johnstone River as a substitute for the Betel leaf, when chewing the Areca nut with lime (Dr. T. G. White).

Premna, Linn.

obtusifolia, R.Br._-“Ngaeki” of Tully River natives. var. ? velutina, Benth.

integrifolia, Linn., ? var.; Benth., Fl. Austr. i. 59.

limbata, Benth.

Dallachyana, Benth.

Tateana, Bail. (Fig. 36r.)

acuminata, R. Br.

Gmelina, Limn.

macrophylla, Benth.-Queensland Beech.

fasciculiflora, Benth.- "Toeah" of Barron River natives; also White Beech.

Leichhardtii, F.v.M.-Queensland Beech. "Cullouen" of Mooloolah natives. Wood useful for veranda floors.

Vitex, Linn.

trifolia, Linn.-In Egypt called " The Hand of Mary.”

var. obovata, Benth.

var. acutifolia, Benth.

var. parviflora, Benth.

lignum-vitæ, A.Cunn.-Wood called "Lignum-vitæ."

acuminata, R.Br.-Wood useful for cabinet-work. 362.)

(Fig.

glabrata, R. Br.- "Ko-na-ru” of Cooktown natives.

Faradaya, $F \cdot v \cdot M$.

splendida, F.v.M.- "Buku” of Tully River and "Koie-yan" of Dunk Island natives. (Fig. 363.)

Clerodendron, Linn.

inerme, $R$. Br.- "Ta-anji " of Batavia River natives.

Tracyanum, $F . v . M$.

tomentosum, R. Br.

var. ? mollissima, Benth.

lanceolatum, F.v.M. (Fig. 364.)

floribundum, R. Br._." Thurkoo" of Cloncurry natives.

Cunninghamii, Benth. (Plate XI.)

costatum, R. Br.

TRIBE IV.-CARYOPTERIDE

Glossocarya, Wall.

hemiderma, Benth. and Hook. =Clerodendron hemiderma, F. v. M. (Fig. 365.) 
PLATE XI.

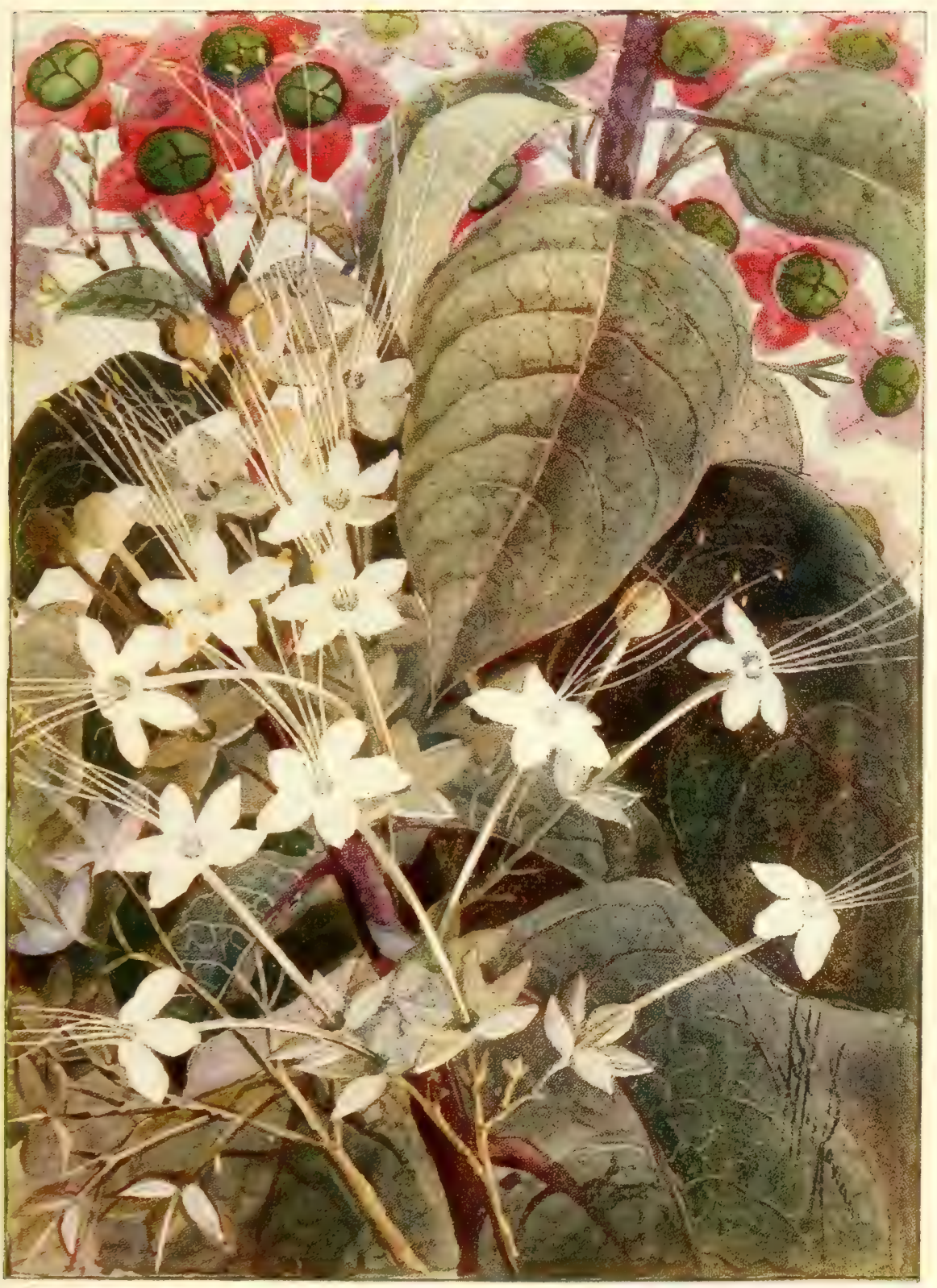

Clerodendron Cunninghamil, Benth. 



\section{Tribe V.-AvicennieÆ.}

Avicennia, Linn.

officinalis, Linn.-White Mangrove. The Malacca Bean of Nuttall. "Egaie" of Cleveland Bay, "Tagon-tagon" of Rockhampton, "E-pumer-an” of Red Island, "Rdai-ite" of Batavia River, "Parpoon" of Cooktown, "Tchuntchee" of Stradbroke Island, "Bootharoo" of Forest Hill, and "Kum-moo-roo" of Dunk Island natives. Furnishes a very tough, durable wood. The horizontal roots, as in species of Sonneratia, are furnished with erect breathing processes.

\section{Order XCVI.-LABIATÆ.}

\section{TRIBE I.-OCIMOIDEE.}

Ocimum, Linn.

sanctum, Limn. - Sacred Balm. "Mooda" of Cloncurry natives. This, like many other plants, is recommended by several writers as a mosquito-fuge, but, while it is probable that the essential oils of these plants may have this effect, it is doubtful if the growing plants act in a like manner.

var. angustifolium.

Moschosma, Reichb.

polystachyum, $D C$.

australe, Benth.

Orthosiphon, Benth.

stamineus, Benth.-Cat's moustache. (Fig. 366.)

Plectranthus, L'Her.

longicornis, F. v. M.-Flowers deep-purple; leaves few, mostly at the base.

parviflorus, Willd.

var. minor, Bail. (Fig. 367.)

var. major, Bail. (Fig. 368.)

congestus, R. Br._ "Kar-kar" of Mitchell River natives.

foetidus, Benth.

Coleus, Lour.

scutellarioides, Benth.

Tribe II.-SAtureineE.

Dysophylla, Blume.

verticillata, Benth. 
Mentha, Linn.

grandiflora, Benth.

australis, R.Br.-Australian Mint; yields pepperment oil. This herb is excellent for making a wholesome drink, for which it was used by the South Australian pioneers.

satureioides, R. Br. - Brisbane Pennyroyal; yields a large percentage of peppermint oil.

Lycopus, Limn.

australis, R. Br.-Australian Water Horehound.

Tribe III.--MonardeÆ.

Salvia, Limn.

plebeia, R. Br.

*coccinea, Linn.-South America.

*Monarda, Linn.

aristata, Benth.-North America.

TRIBE IV.-STACHYDEE.

Scutellaria, Linn.

humilis, R.Br.-Queensland Skullcap.

Brunella, Limn.

vulgaris, Linn.-Self-heal. Europe.

*Marrubium, Linn.

vulgare, Linn.-White Horehound. Europe. A common weed on our Downs country.

Anisomeles, R. Br.

salvifolia, R. Br.

*Stachys, Linn.

arvensis, Limn.-Queensland Stagger-weed; Corn Woundwort or Hedge Nettle of England. In Queensland, reputed poisonous to working stock (see Q1. Agric. J1. iv. (1899) 49, P1. Lxxix.).

*Lamium, Linn.

amplexicaule, Linn.-Henbit Nettle. Europe.

*Moluccella, Linn.

spinosa, Linn.-Molucca Balm. Mediterranean regions.

Leucas, R. Br.

flaccida, $R . B r$.

var. petiolaris, Benth.

*linifolia, Spreng.

Leonotis, R. Br.

nepetæefolia, R. $B r$. 


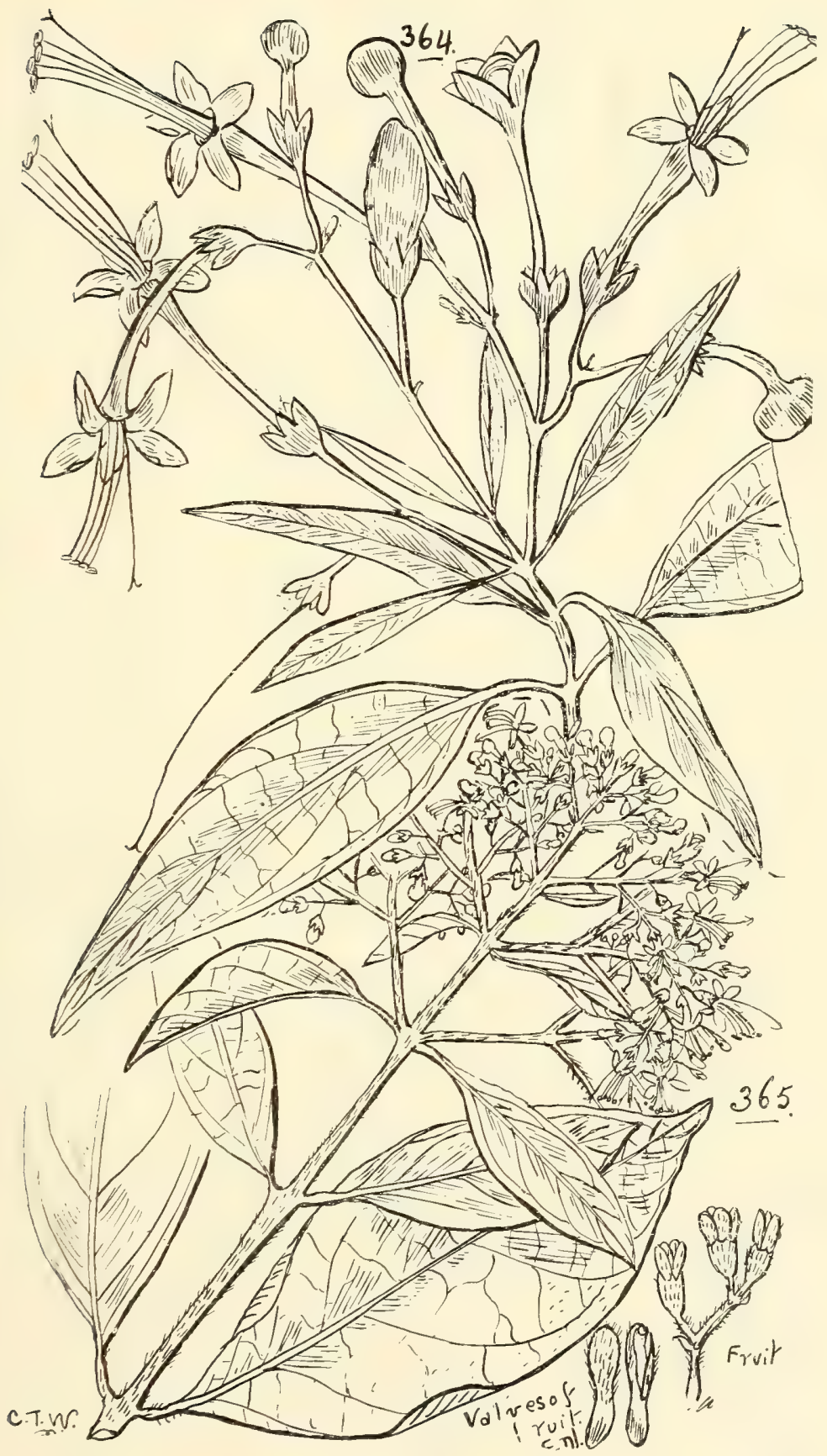

364. Clerodendron lanceolatum, $F, v, M$. 365. Glossocarya hemiderma, Benth. et Hook. 


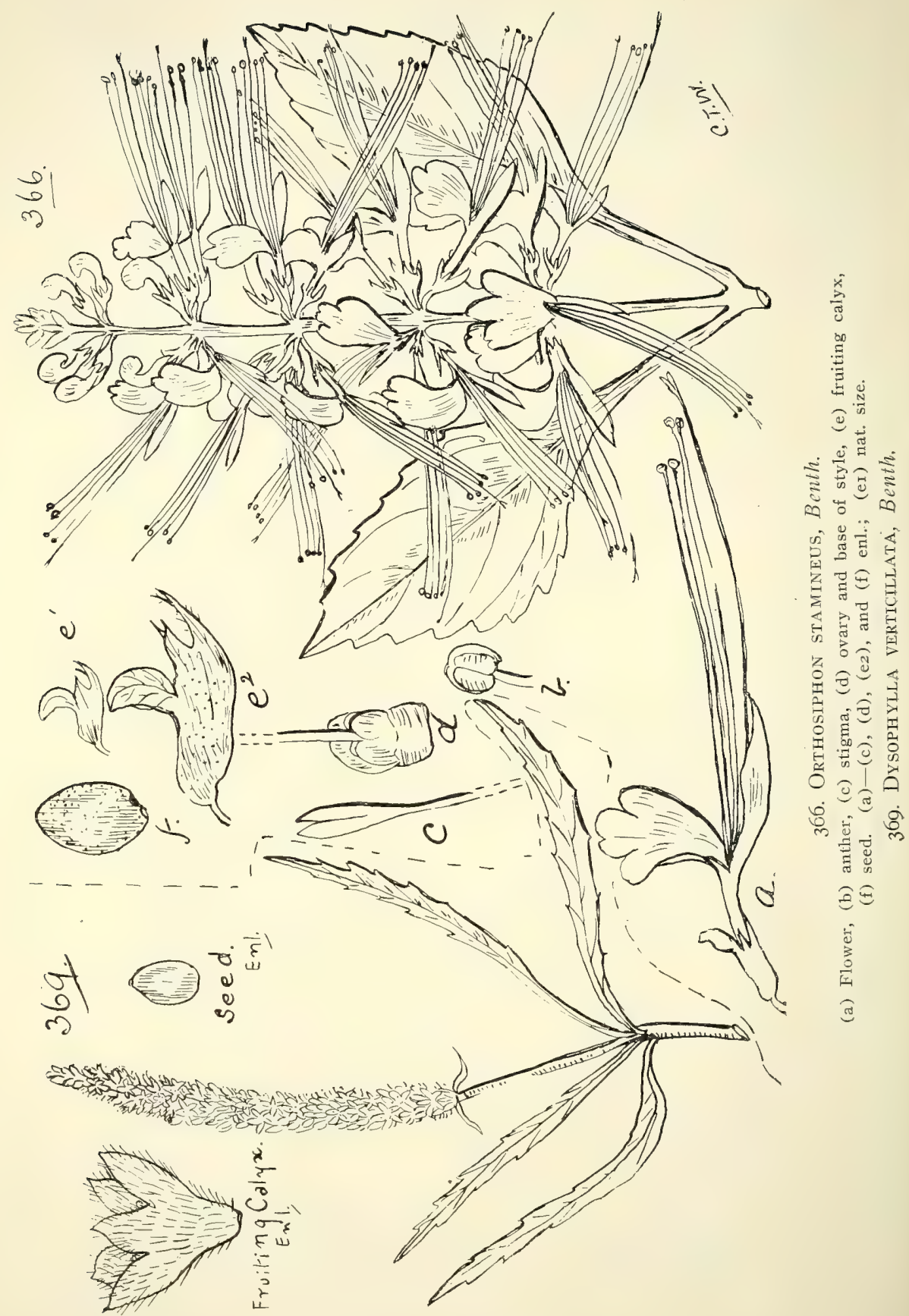




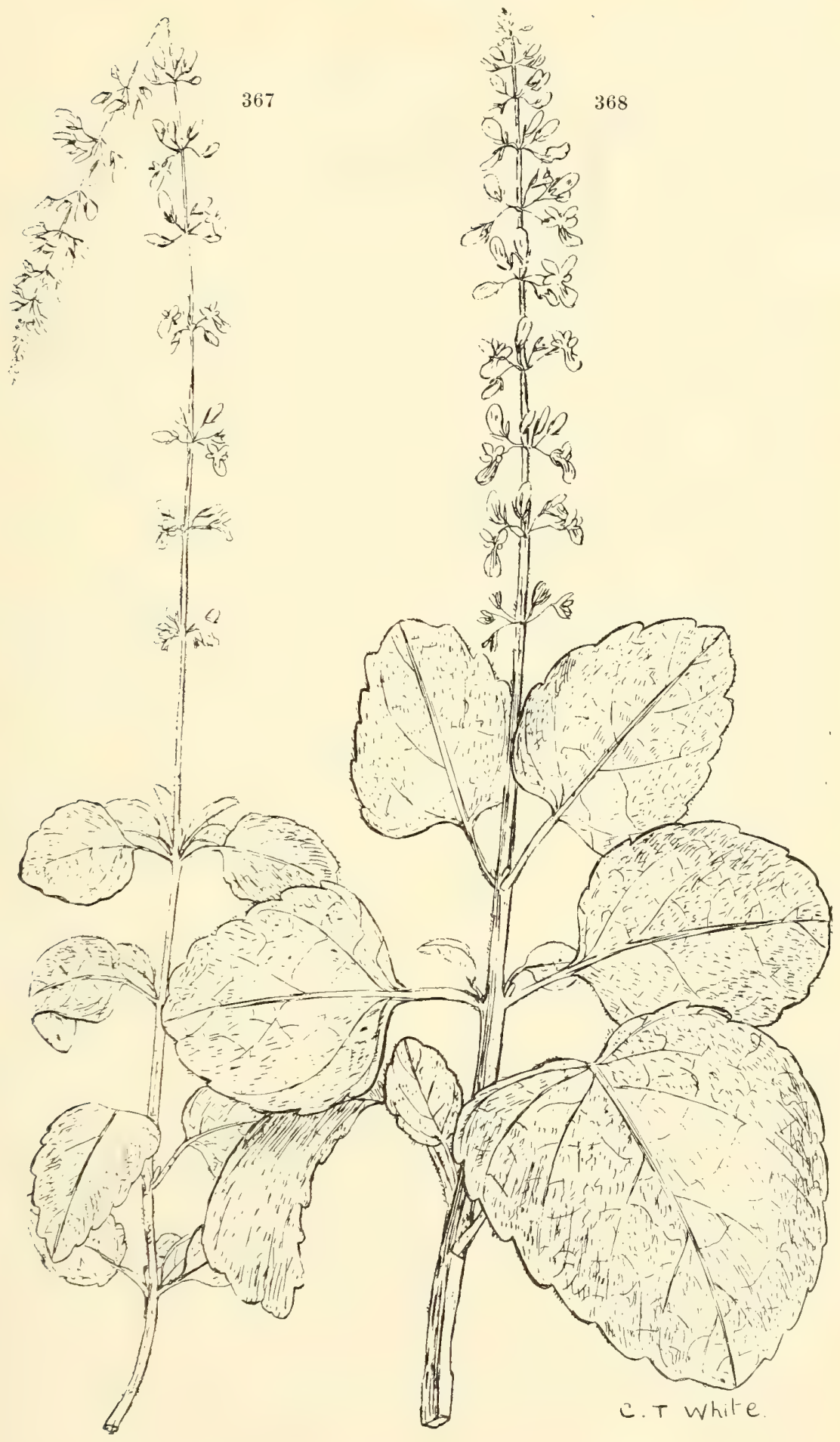

367. Plectranthus parviflorus, Willd., var. minor, Bail. 368. P. parviflorus, Willd., var. Major, Bail. 
Tribe V.-Prostanthered.

Prostanthera, R. Br.

Section I.-Euprostanthera.

Series I.-Racemosæ.

lasianthos, Labill.

ovalif́lia, R. Br.

var. ? latifolia, Benth.

incisa, R. Br.

linearis, $R . B r$.

Series II.-Convexæ.

phylicifolia, F.v.M.

Series III.-Subconcavæ.

lithospermoides, F.v.M.

atroviolacea, Bail. (Fig. 37o.)

nivea, A. Cunn.

striatiflora, F.v.M.

saxicola, R. Br.

var. major, Benth.

odoratissima, Benth.

euphrasioides, Benth.

ringens, Benth.

Section II.-Klanderia.

Leichhardtii, Benth.

microphylla, A. Cunn.

Hemigenia, R. Br.

Biddulphiana, F.v. $M$.

Clotteniana, Bail. (Fig. 37I.)

Westringia, $S m$.

grandifolia, F.v.M.

rosmariniformis, $S \mathrm{Sm}$.

rigida, $R$. $B r$.

eremicola, A. Cunn.

glabra, R. Br.

Tribe VI.-Ajugoide.

Teucrium, Limn.-Germander.

racemosum, $R . B r$.

integrifolium, F.v.M.

corymbosum, R. Br.

var. hirsutum, Benth.

argutum, R. Br.

var. incisa, Benth.

ajugaceum, Bail. et F.v.M. (Fig. 372.)

Ajuga, Linn.

australis, R.Br.-Australian Bugle. 


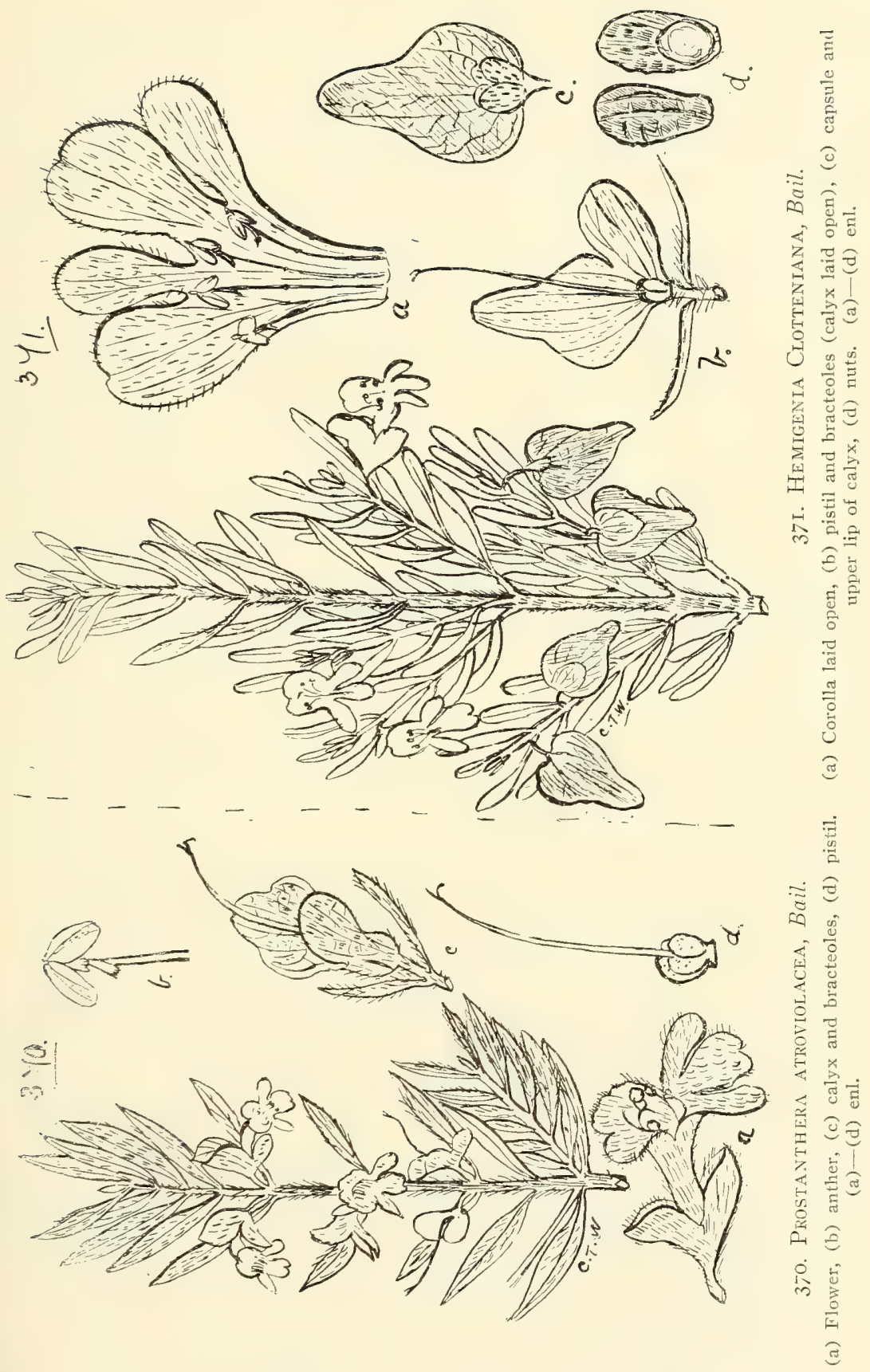




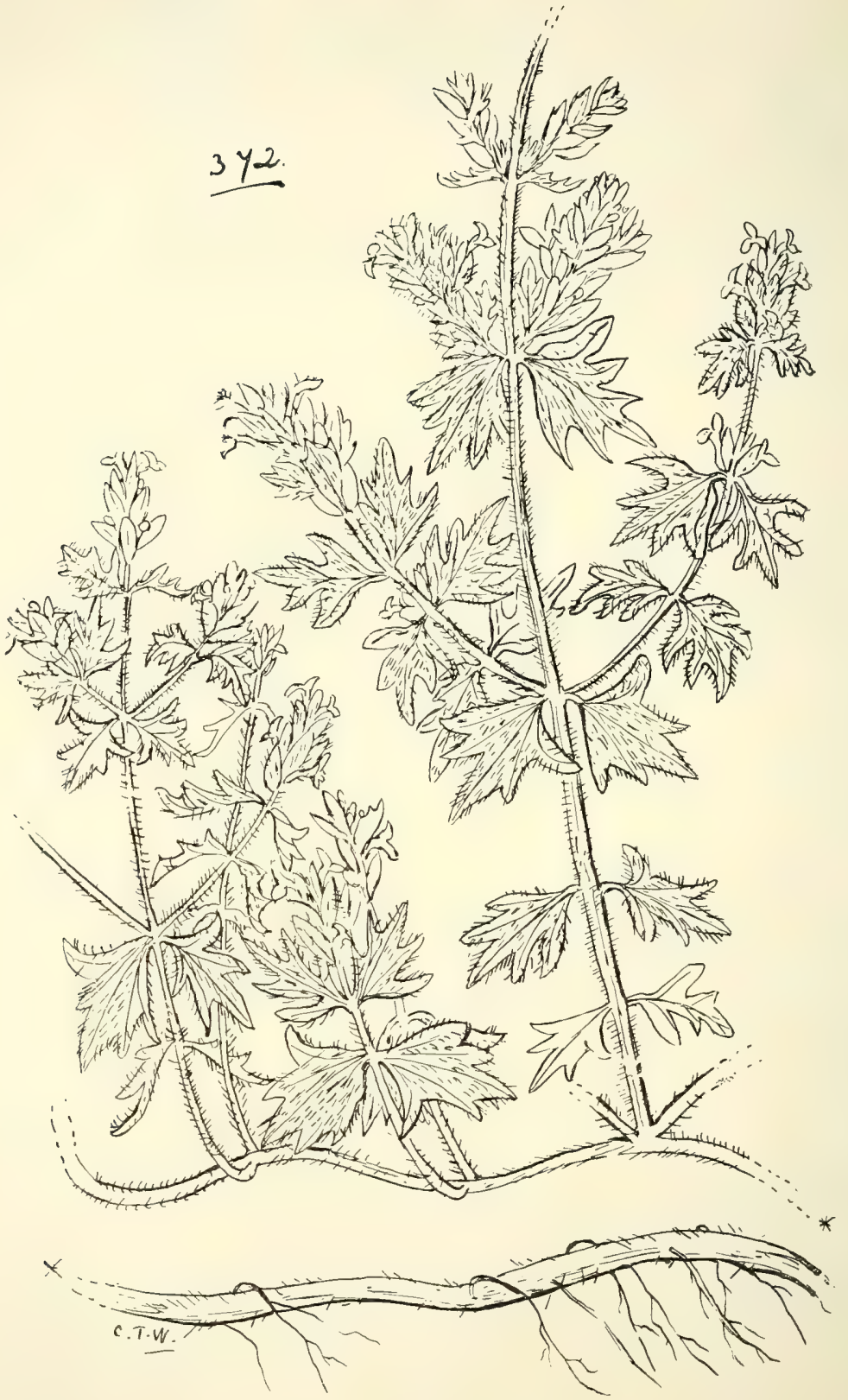

372. Teucrium ajugaceum, Bail. et F. $v . M$. 


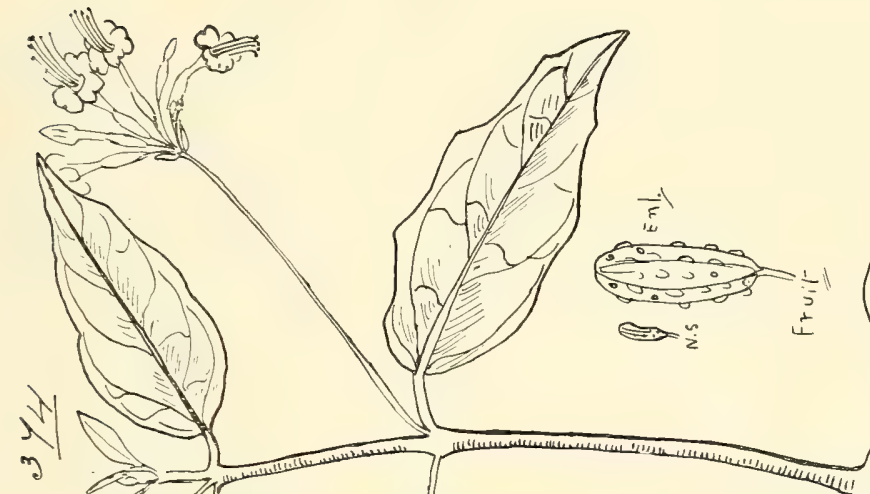

"ैh

$\lambda$
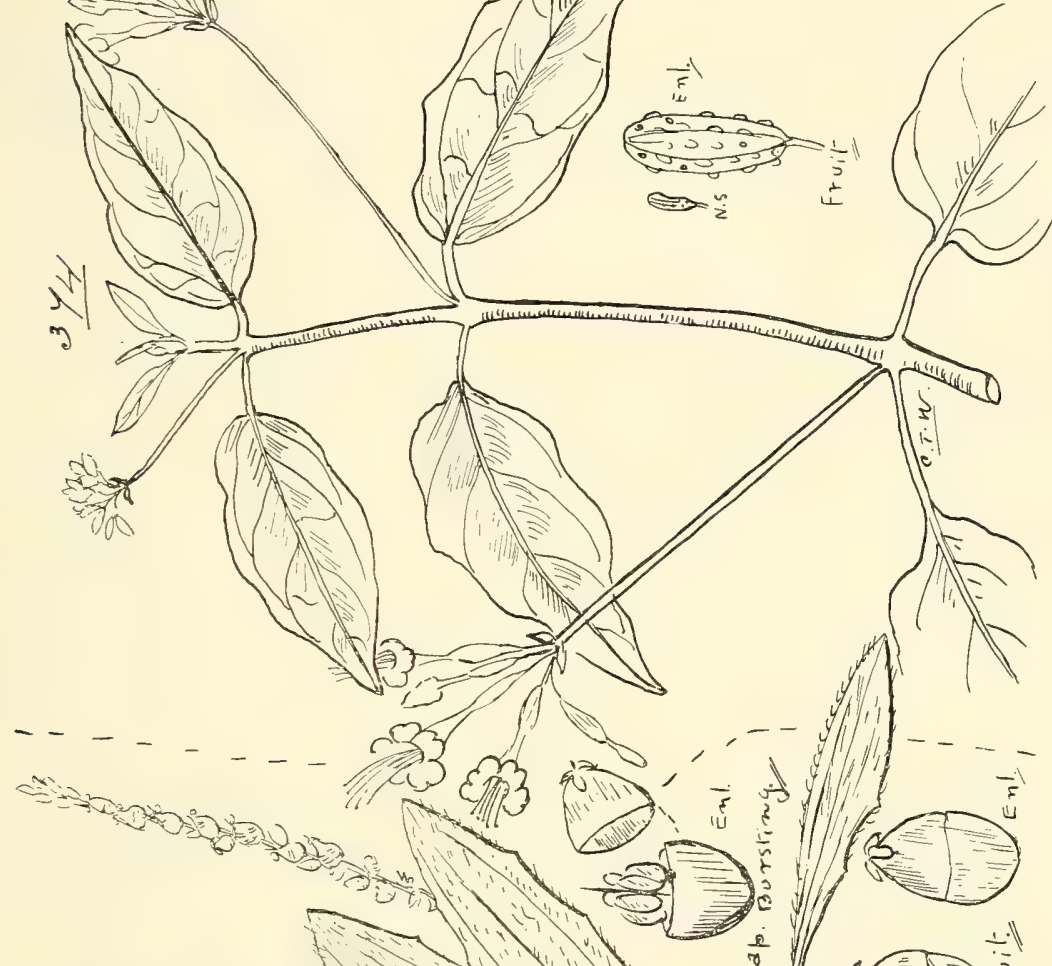

1

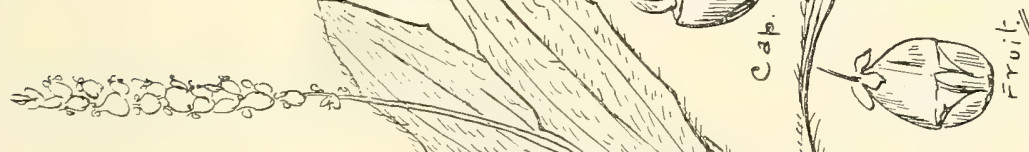

$\lambda^{m}$

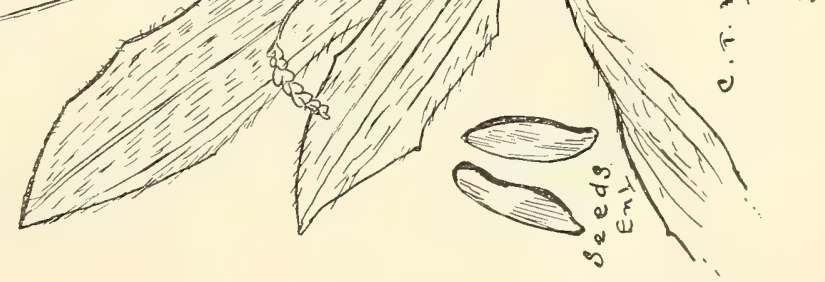




\section{Anomalous Order.}

\section{Order XCVII.-PLANTAGINEÆ.}

Plantago, Limn.

*major, Linn.-Plantain of Europe. Seeds much in favour for bird-seed, and both leaves and seeds said to be used in India for dysentery. Our native kinds equally useful. *lanceolata, Linn.-Rib-grass of Europe. debilis, R. Br.

varia, R.Br. (Fig. 373.)

Subclass III.-MONOCHLAMYDE王.

Series I.--CURVEMBRYEÆ.

\section{Order XCVIII.-NYCTAGINEÆE.}

Tribe I.-Mirabilię.

*Mirabilis, Limn.-Fruit a Diclesium.

jalapa, Linn.-Marvel of Peru or Fonr-o'clock. Tropical America.

Boerhaavia, Linn.

diffusa, Linn. - Hogweed. "Koo-joo" and "Goitcho" of Cloncurry and "A-mi" of Batavia River natives. May be used as an expectorant in cases of asthnta.

repanda, Willd. (Fig. 374.)

Tribe II.-PISONIEA.

Pisonia, Linn.-The sticky fruits of plants of this genus often catch and cause the death of a large number of small birds, insects, \&c., in a similar manner to bird-lime.

aculeata, Linn.

inermis, Forst.

Brunoniana, Endl._- Ahm-noo" of Dunk Island natives. (Fig. 375.)

\section{Order XCIX.-ILLECEBRACE E.}

Dysphania, R. Br.

Tribe I.-Pollichiex.

myriocephala, Benth. (Fig. 376.)

plantaginella, F. $\cdot$. M. (Fig. 377.)

littoralis, R. $B r$.

Scleranthus, Linn.

Tribe II.-SclerantheÆ.

biflorus, Hook. 


\section{Order C.-AMARANTACEÆ.}

*Celosia, Linn.

Tribe I.-Celosies.

argentea, Linn.

var. cristata, Benth. - Cock's-comb. The native habitat uncertain.

Deeringia, R. $B r$.

celosioides, $R . B r$.

aitissima, $F, v . M$.

Tribe II.-Amarante.

Amarantus, Limn.-Fruit a Utriculus.

Section I.-Euamarantus.

paniculatus, Linn.-The foliage of this weed is sometimes seen variegated.

*spinosus, Limn-A troublesome weed. At the Mauritius the leaves and root are considered diuretic, and in India the root has been found of great service in the treatment of eczema (Dymock).

*caudatus, Limn. - Love-lies-bleeding. The native habitat uncertain.

leptostachyus.

pallidiflorus, F.v.M.

Section II.-Euxolus.

Mitchellii, Benth.

interruptus, R. Br. (Fig. 378.)

viridis, Linn. = Euxolus viridis, Moq. (Fig. 379.)

macrocarpus, Benth.

var. pallida, Benth.

Ptilotus, R. Br.

conicus, R. Br.

corymbosus, R. Br.

spicatus, F.v. M. (Fig. 380.)

var. leianthus, Benth.

Murrayi, F.v.M.

latifolius, $R . B r$.

Trichinium, R. Br.

Series I.-Astrotricha.

obovatum, Gaudich.

parviflorum, Lindl.

dissitiflorum, F.v.M. 


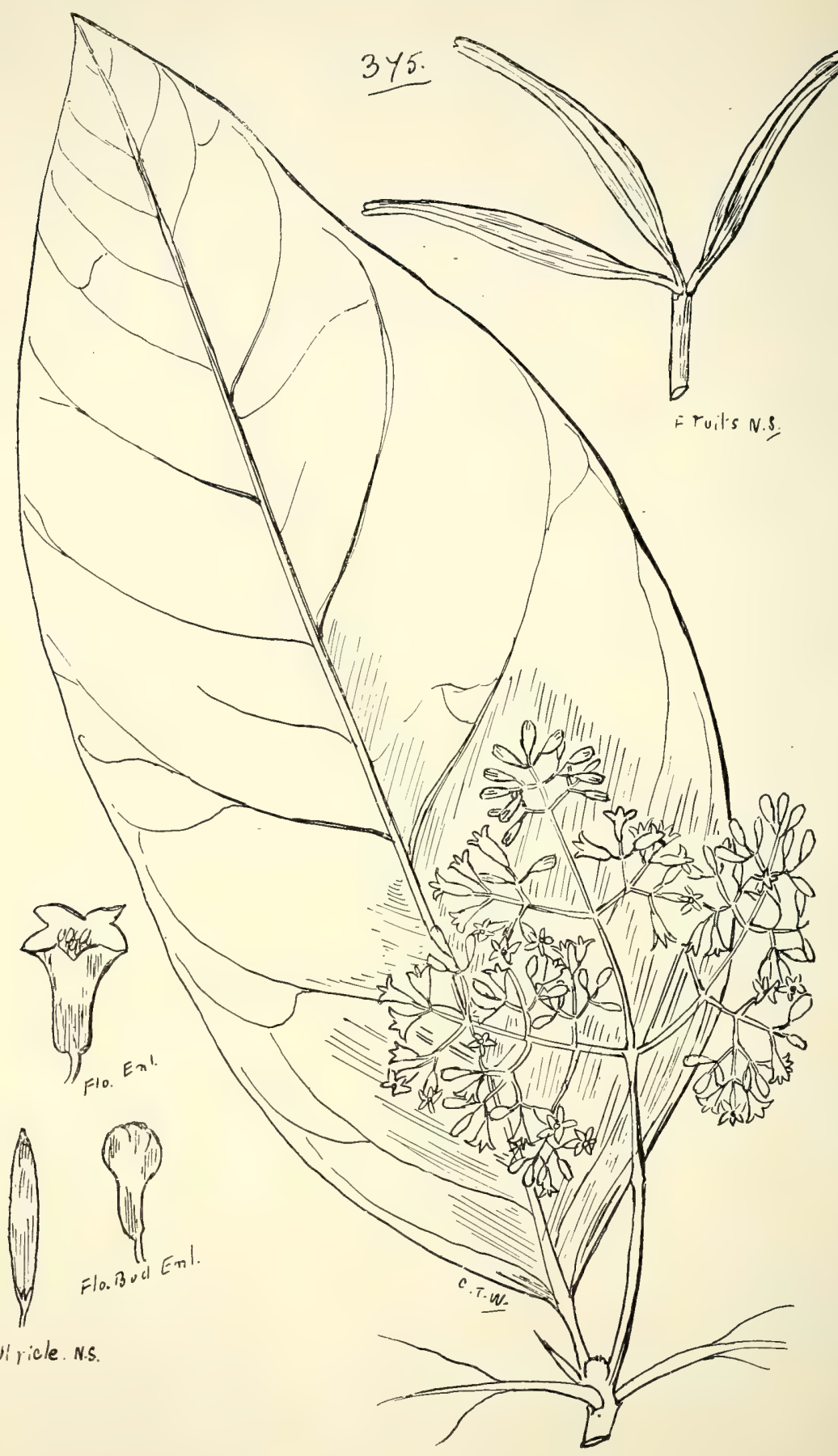

375. Pisonia Brunoniana, Endl. 


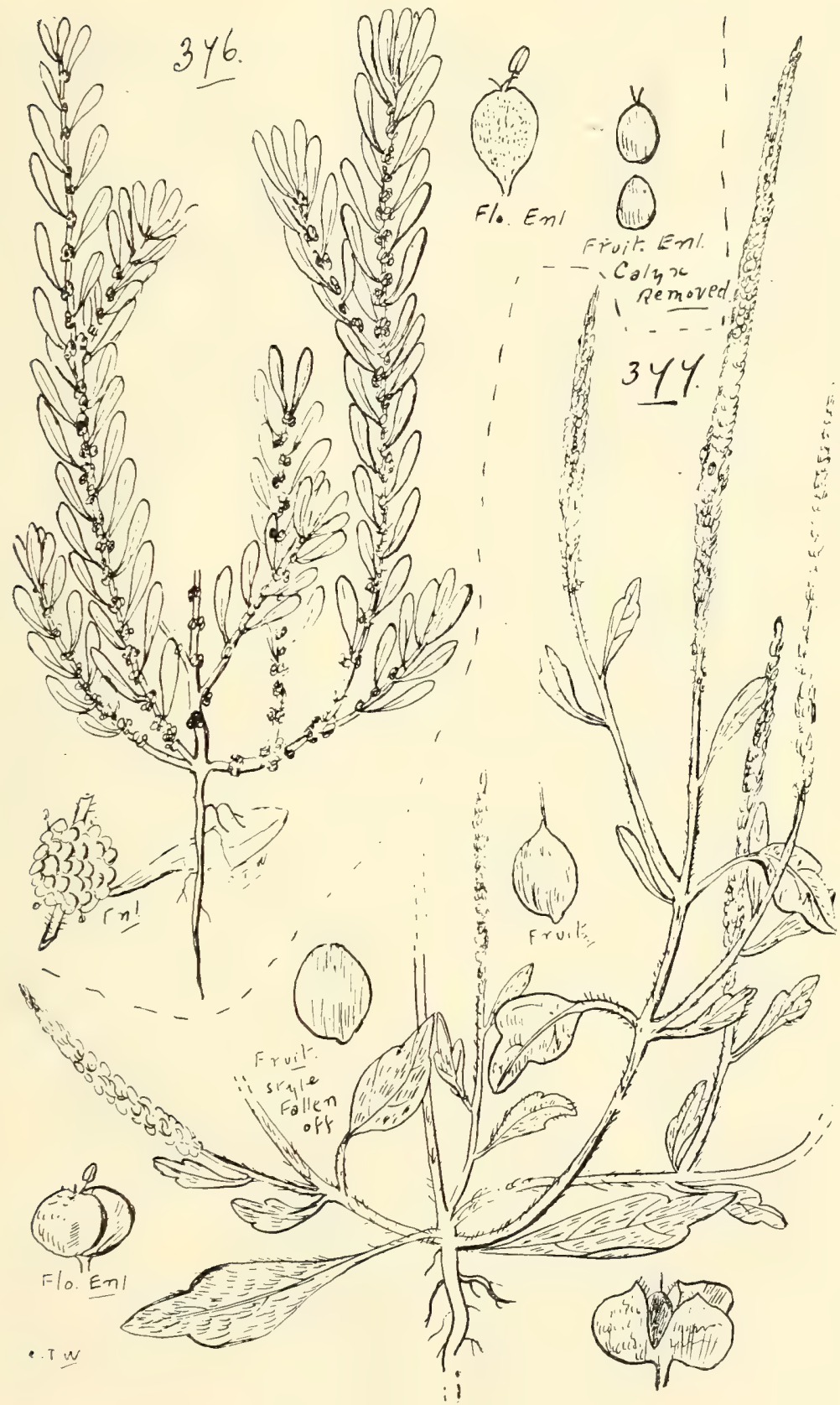

376. Dysphania myriocephala, Benth.

377. D. plantaginelia, F. v. $M$. 


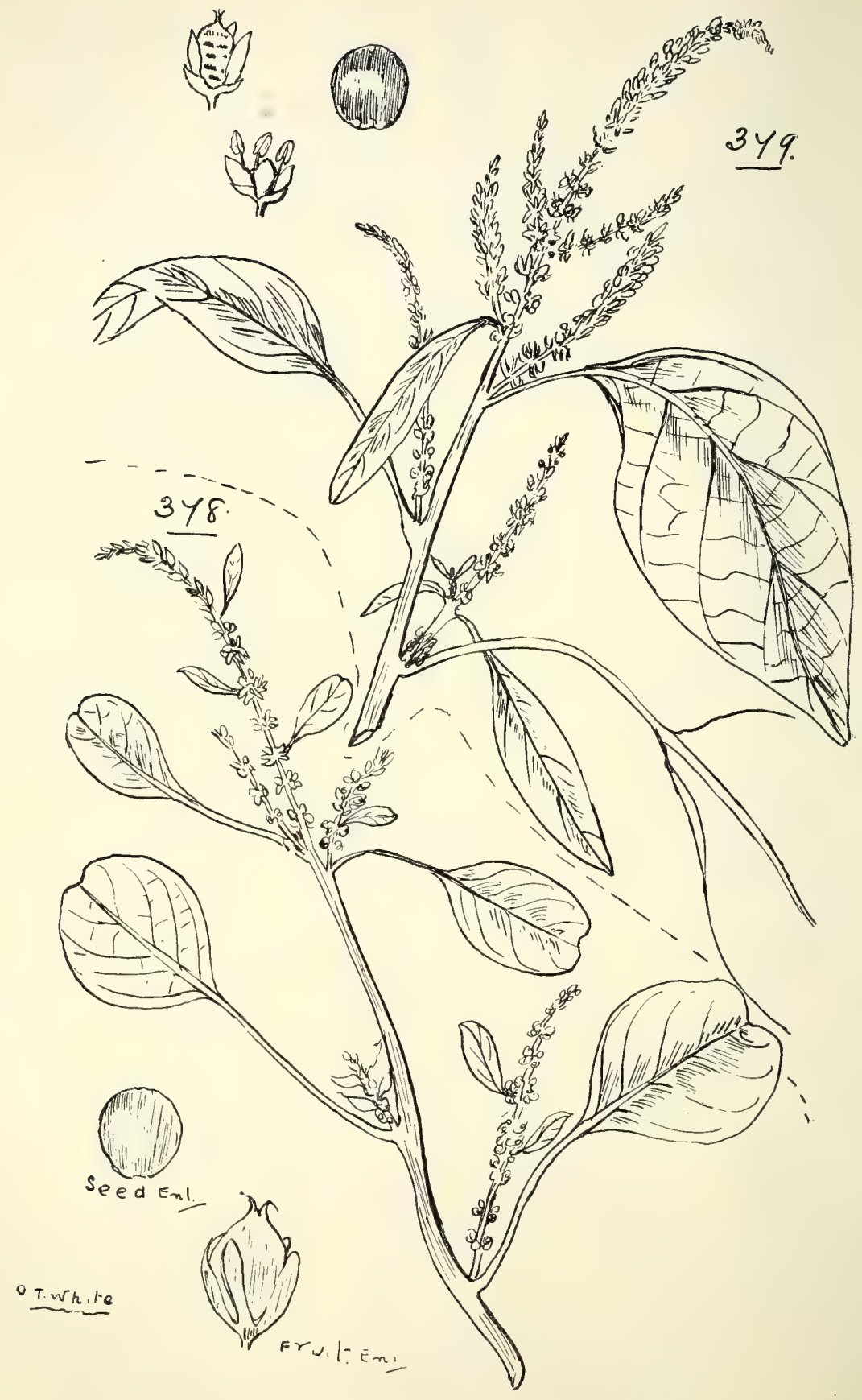

378. Amarantus interruptus, $R$. $B r$. 379. A. vIRIDIS, Linn. 
C. AMARANTACEÆ.

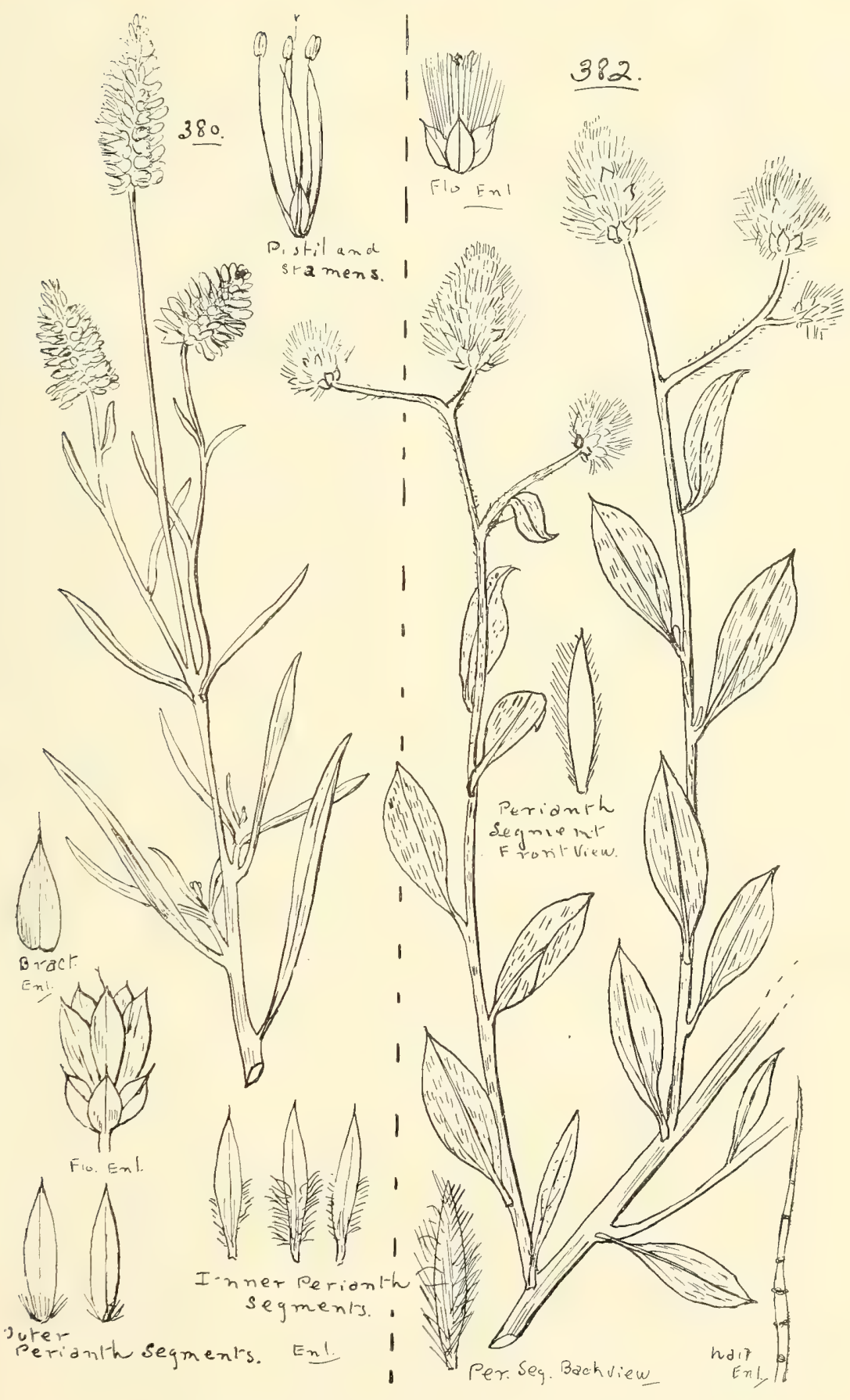

380. Ptilotus spicatus, F. v. $M$.

382. Trichinium errvoides, $F . v V$. 


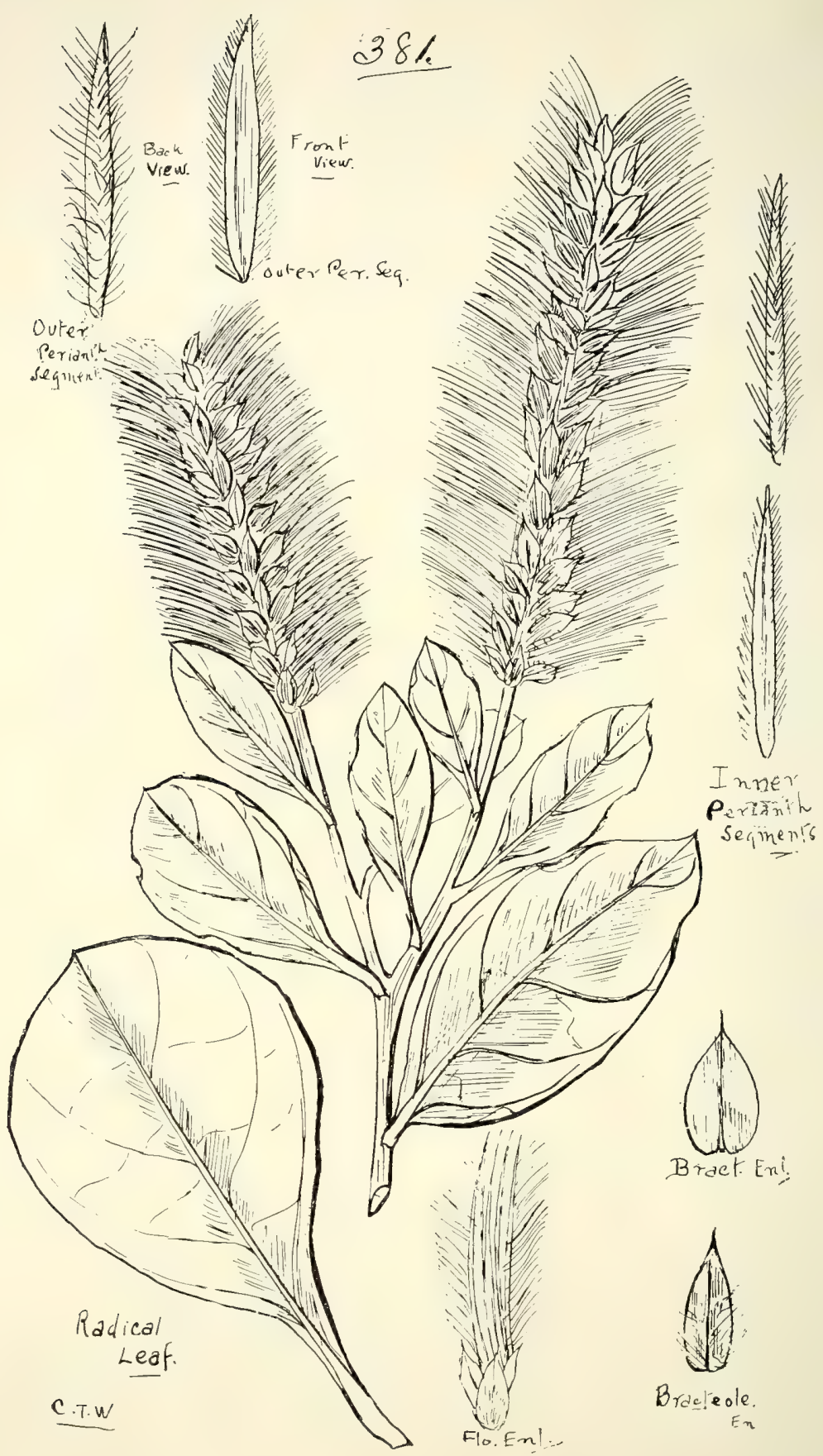

38i. Trichinium nervosum, Bail. 
Trichinium-contd.

Series II.-Straminea.

distans, R. Br.

alopecuroideum, Lindl.

nobile, Lindl.

nervosum, Bail. (Fig. 38r.)

macrocephalum, R. Br.

corymbosum, Gandich.

var. parviflora, Benth.

Series III.-Rhodostachya.

exaltatum, Benth.

semilanatum, Lindl.

Burtonii, Bail.

Series IV.-Polycephala.

fusiforme, $R . B r$.

gracile, R. Br.

Series $V$.-Squamigera.

calostachyum, F. v. M.

Series VI.-Parviflora.

ærvoides, F.v.M. (Fig. 382.)

leucocoma, $M o q$.

Cyathula, Lour.

prostrata, Blume.

Achyranthes, Linn.

aspera, Linn.-Washerman's plant, of India.

var. canescens, Benth.

Nyssanthes, R. Br.

erecta, R. Br.-This spinous plant is a common scrub pest in Queensland.

diffusa, R. Br.

TRIBE III.-GOMPHRENEE.

Alternanthera, R. Br.

nodiflora, R. $\mathrm{Br}$.

denticulata, R. Br.

var. ? micrantha, Benth.

nana, R. Br.

var. major, Benth.

angustifolia, R. Br.

decipiens, Benth. (Fig. 383.)

*Achyrantha, R.Br., var. leiantha, Seubert. Tropical America. 
Gomphrena, Linn.

*globosa, Linn.-Bachelors' Buttons. America. canescens, R. Br. (Fig. 384.)

flaccida, $R . B r$.

humilis, R. Br.

Brownii, Moq.

conica, Spreng.

conferta, Benth.

diffusa, Spreng.

\section{Order CI.-CHENOPODIACEZ.}

[Genera marked thus † are given in Baron von Mueller's works under Bassia, Allioni.]

\section{Suborder I.-CHENOPODIEÆ.}

Series I.-Cyclobez.

Tribe I.-EucheNopodie必.

Rhagodia, R. Br.-Saltbushes.

Billardieri, R. $B r$.

parabolica, R.Br. (Fig. 385.)

crassifolia, R. Br.

spinescens, R. Br.

hastata, R. Br.-Fruit red.

nutans, $R . B r$.-Fruit red.

linifolia, R.Br.-Fruit red.

Chenopodium, Linn.

Section I.-Rhagodioides.

nitrariaceum, F.v. $M$.

Section II.-Chenopodiastrum.

auricomum, Lindl.-Bluebush. (Fig. 386.)

*album, Linn.-Fat-hen. Europe.

*murale, Limn.-Europe.

triangulare, R. Br.

Section III.-Ambrina.

*ambrosioides, Linn.

*Botrys, Willd--Jerusalem Oak. Europe, Asia, and North America.

Section IV.-Orthosporum.

carinatum, R. Br.

cristatum, F.v. $M$.

simulans, F.v. M. 


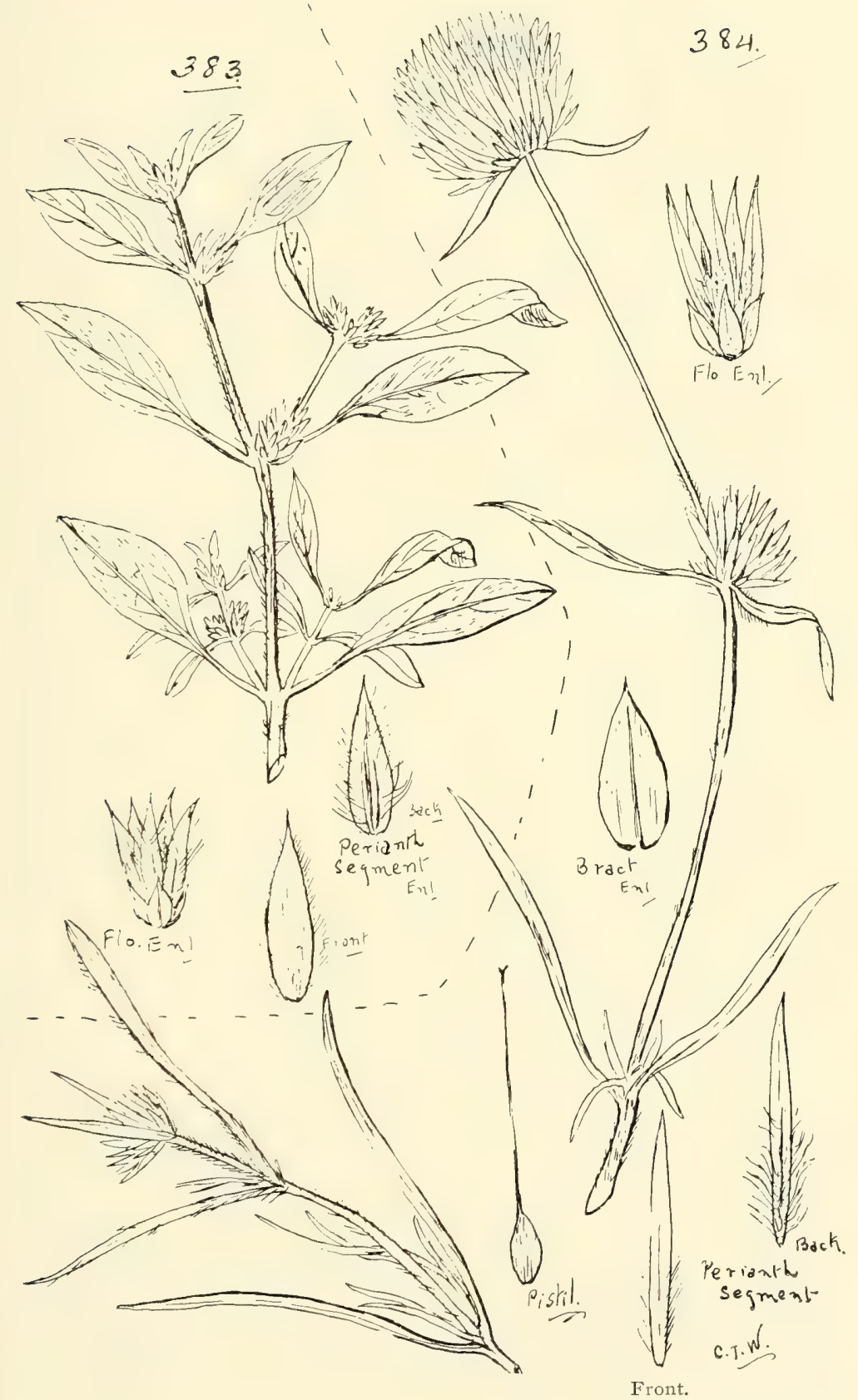

383. Alternanthera decipiens, Benth. 384. Gomphrena canescens, $R, B r$. 


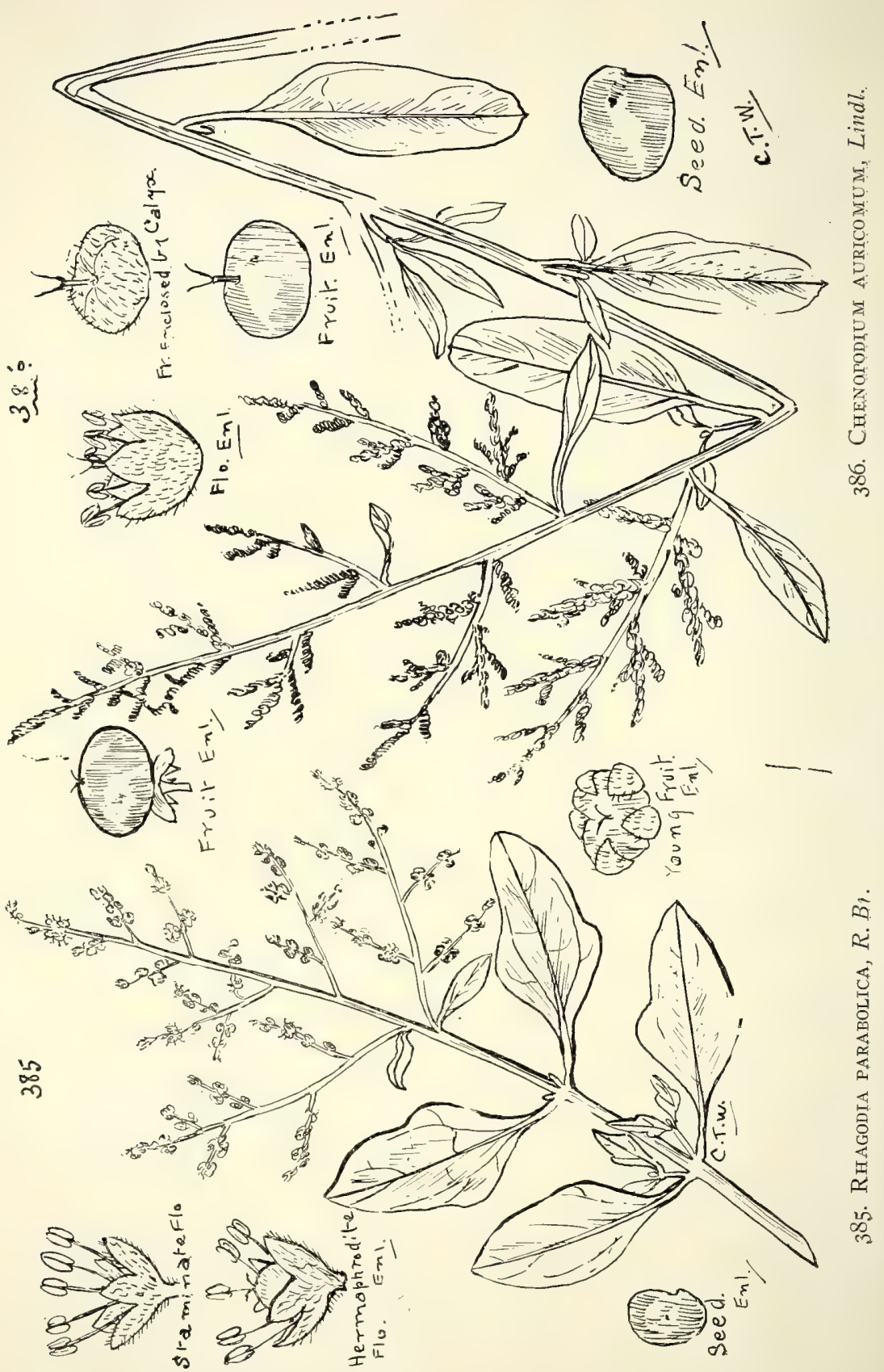




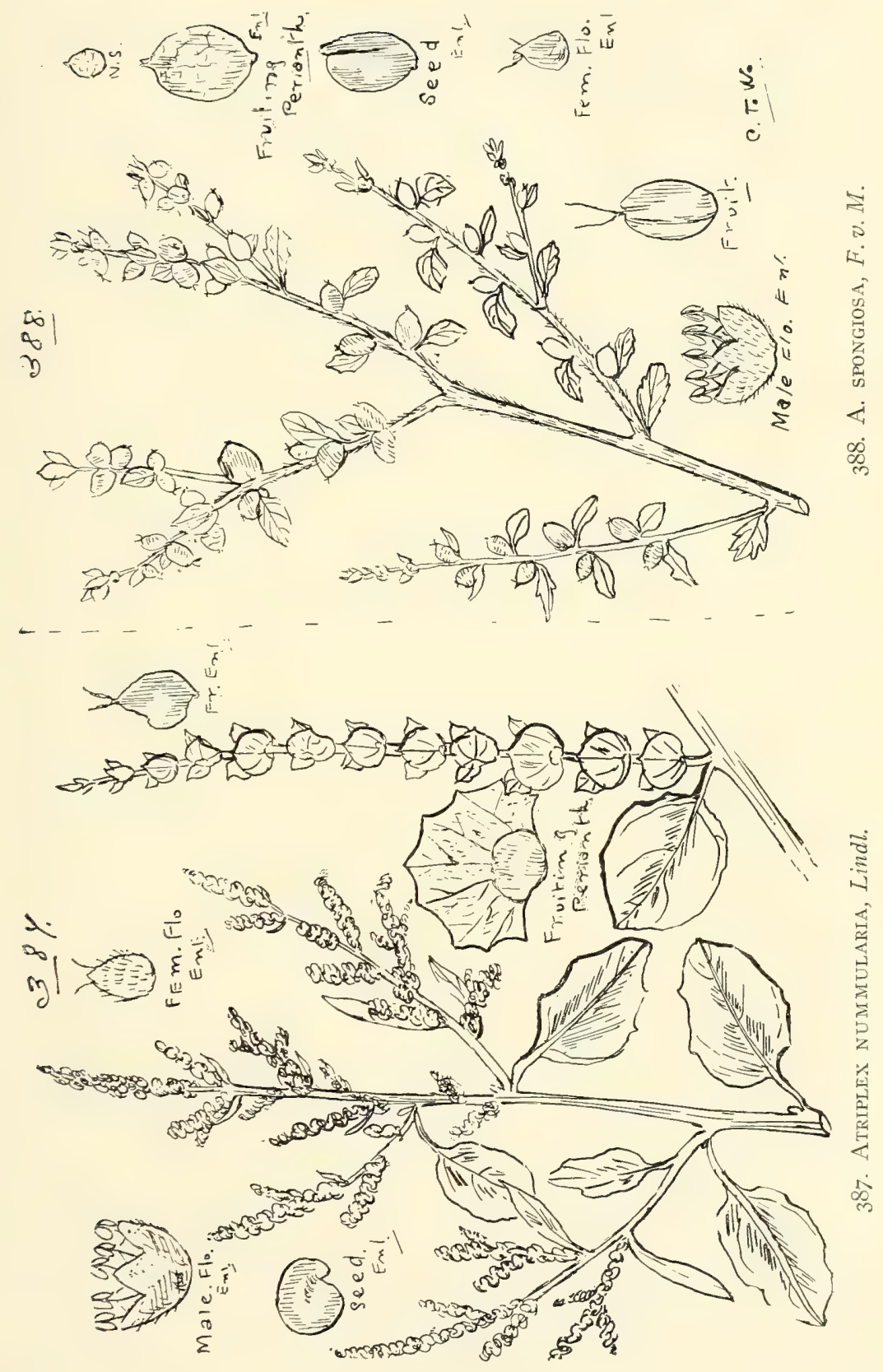


Tribe II.-Atriplice

Atriplex, Linn.-Saltbushes.

stipitata, Benth.

Series 1.-Paniculata.

Quinii, F.v. M.

nummularia, Lindl.-Old-man Saltbush. (Fig. 387.)

cinerea, Poir.

Series II.-Vesicarice.

vesicaria, Hezvard.-Bladder Saltbush; contains saponin.

patula, Linn.

Series III.-Oleracee.

Series IV.-Glomeratce.

humilis, F.v. M.

angulata, Benth.

semibaccata, R. Br.

Muelleri, Benth.

var. lobaticarpa, F.v.M.

campanulata, Benth.

fissivalvis, $F . v$. $M$.

Series V.-Parviloba.

leptocarpa, F.v.M.

limbata, Benth.

halimoides, Lindl.

holocarpa, F.v.M.

spongiosa, F.ข.M. (Fig. 388.)

Tribe III.-ChenoleE E.

+ Chenolea, Thunb.

sclerolænoides, F.v. M.

Kochia, Schrad.

lanosa, Linn.

triptera, Benth.

brevifolia, R. Br.-Cotton-bush. (Fig. 389.)

villosa, $L$ indl.

var. tenuifolia, F.v.M.

planifolia, F.v. $M$.

aphylla, R. Br.-Cotton-bush.

dicoptera, F.v.M. (Fig. 390.)

brachyptera, $F . v . M$.

sedifolia, F.v. M.

spongiocarpa, F.v. $M$.

stelligera, F.v. $M$. 
Enchylæna, R. Br.

microphylla, Moq. = Kochia microphylla, F. v. M.

tomentosa, R.Br._ "Kooloo-loomoo" of Cloncurry natives.

var. ? leptophylla, Benth.

var. glabra, Benth.

villosa, $F$. ข. $M$.

Babbagia, F. v. M.

dipterocarpa, F.v. $M$.

scleroptera, F.v. M.

acroptera, F.v. $M$.

Sclerolena, B. Br.

diacantha, Benth.

lanicuspsis, F.v.M.

bicornis, Lindl.

biflora, R. Br.

paradoxa, R. Br.

+ Anisacantha, R. Br.

muricata, Moq.-Roley-poley.

var. villosa, Benth.

divaricata, R. Br.

bicuspis, F. v. M.

echinopsila, F. ข. $M$.

Birchii, F.v. M.

Threlkeldia, R. Br.

brevicuspis, F. ข. $M$.

proceriflora, F.v. M.

Tribe IV.-SALiCORNIEA.

Tecticornia, Hook. f.

cinerea, Benth. et Hook.

Arthrocnemum, Moq.

arbusculum, Moq.

Salicornia, Linn.-Glasswort.

leiostachys, Benth.

tenuis, Benth.

australis, Sol.

Series II.-Spirolobez.

Sureda, Forsk.

Tribe V.-Suedeæ.

maritima, Dumort.-Sea Blite. 


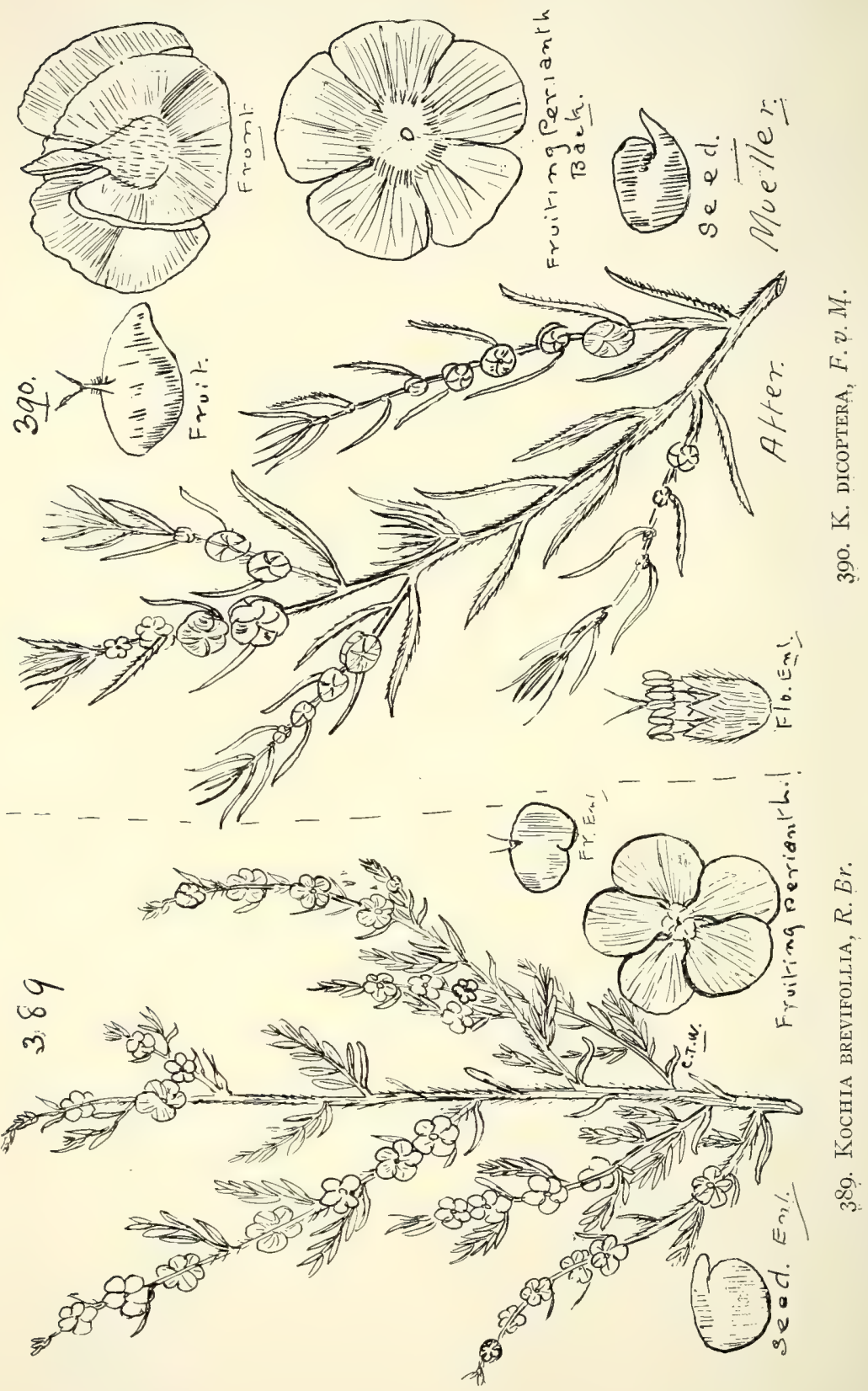


Salsola, Linn.

Tribe VI.-Salsoleæ.

Kali, Linn.-Russian Thistle or Prickly Saltwort.

var. leptophylla, Benth.

var. strobilifera, Benth.

var. brachypteris, Benth.

\section{Suborder II.-BASELLEÆE.}

Tribe VII.-Boussingaultie E.

*Boussingaultia, H. B. et $K$.

baselloides, H. B. et K.-Lambs'-tails. South America.

\section{Order CII.-PHYTOLACCACEÆ.}

*Rivina, Limn.

TRIBE I.-Rivine..

lævis, Limn.-South America. Plant used as a febrifuge in Ceylon. (Trimen). Rogots said to be poisonous.

Monococcus, F. v.M.

echinophorus, F.v.M.-The strong odour of the foliage of this shrub is said to taint the milk of cows which may feed upon it. Found generally in the scrubs of brackish rivers. (Fig. 39I.)

\section{Tribe II.-Euphiytolacce在.}

*Phytolacca, Linn.

octandra, Limn.--Red Ink plant, a noxious weed of America; contains saponin. Flowers nearly sessile.

decandra, Limn.-America. Contains saponin. Flowers distinctly pedicellate.

dioica, Linn. = Pircunia dioica, Moq.-Bella Sombra; a South American tree common about towns in Queensland. Cows will devour the stems of this tree when cut down, as they do our Bottle-tree. Seeds and roots contain saponin.

Tribe III.-Gyrostemoneæ.

Codonocarpus, A. Cunn.

australis, A.Cunn.-Bell-fruit. (Fig. 392.)

cotinifolius, F.v.M. (Fig. 393.) 


\title{
Order CIII.-POLYGONACEA.
}

Polygonum, Linn.

\author{
Tribe I.-Eupolygone 2.
}

\section{Section I.-Avicularia.}

aviculare, Linn.-Wire-grass, Knot-grass, or Hog-weed. Yields a dye like indigo. A vulnerary and astringent herb used to stop bleeding from the nose, \&c. (Dymock). The systematic name is said to have been given on account of the seeds being fed to singing-birds.

var. erectum, Roth. $=P$. erectum, Linn.--This principally differs from the normal form in its upright growth and larger leaves.

plebeium, R. Br.

orientale.

Section II.-Amblygonon.

attenuatum, R.Br. (Fig. 394.)

Section III.-Persicaria.

prostratum, R. $B r$.

hydropiper, Linn.- “ Tang-gul " of Brisbane River natives. minus, Huds.

subsessile, R. Br.

barbatum, Limn.-Said to produce a good dark-blue colour. (Fig. 395.)

articulatum, R. Br.

lapathifolium, Limn.-Smart-weed of America.

lanigerum, R. Br.

Section IV.-Echinocaulon.

strigosum, R. Br.

Section $V$.-Tiniaria.

*convolvulus, Linn.-Climbing Buckwheat; a native of Asia, Europe, and Africa.

*Fagopyrum, Tournef.

esculentum, Monch.-Buckwheat. Often met with as a stray from cultivation. A native of Europe and Northern Asia, and in many countries cultivated as a bread-corn.

Tribe II.-Rumiceæ.

Rumex, Limn.-Docks; all bad weeds in cultivation.

"hymenosepalus, Torrey.- "Canaigre." A stray from cultivation, said to contain 20 to 25 per cent. of tannin. S.W. United States and Mexico. crispus, Limn.-Curled Dock. 
CII. PHYTOLACCACEÆ.

413

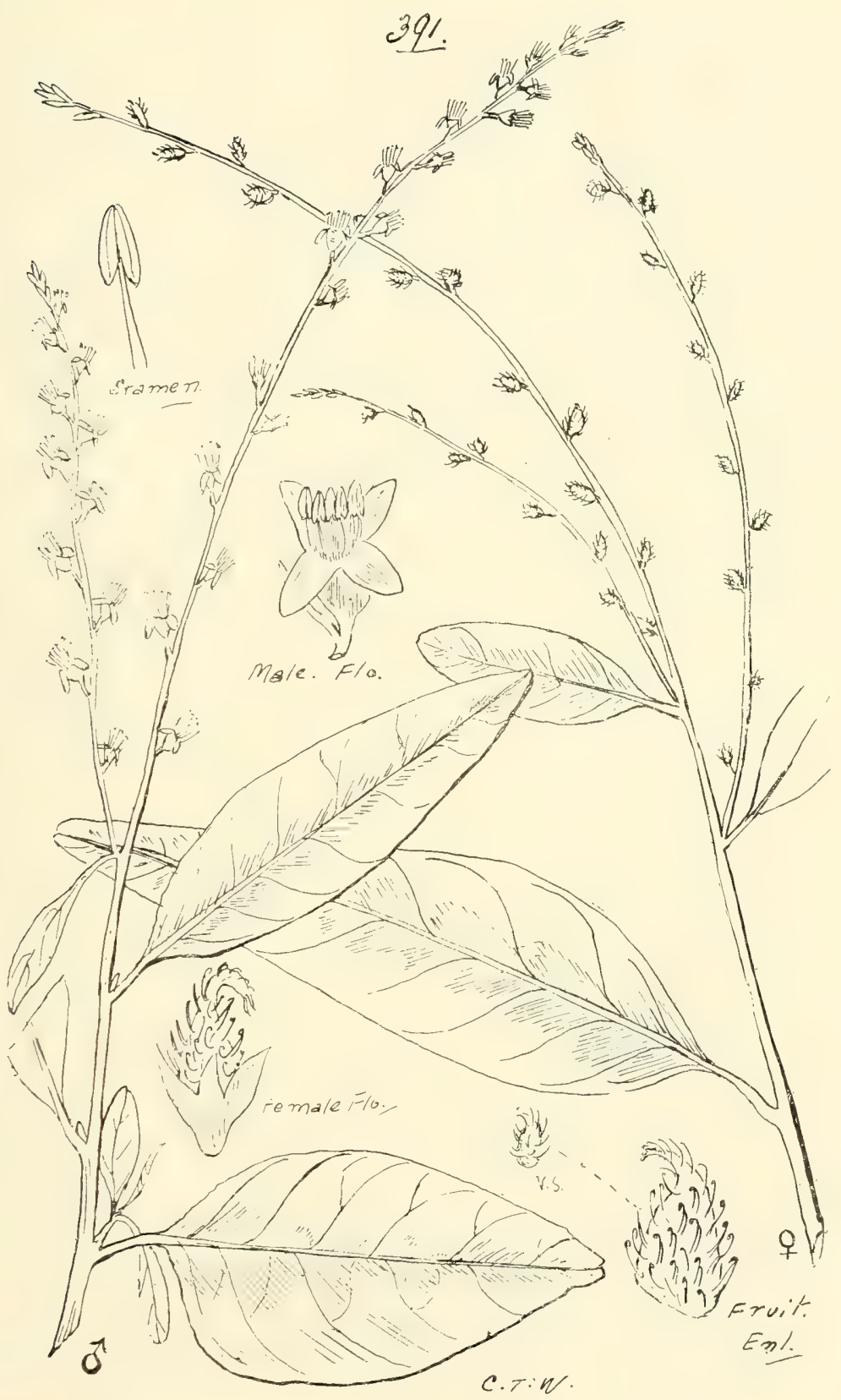

39i. Monococcus echinophorus, F. v. M. 


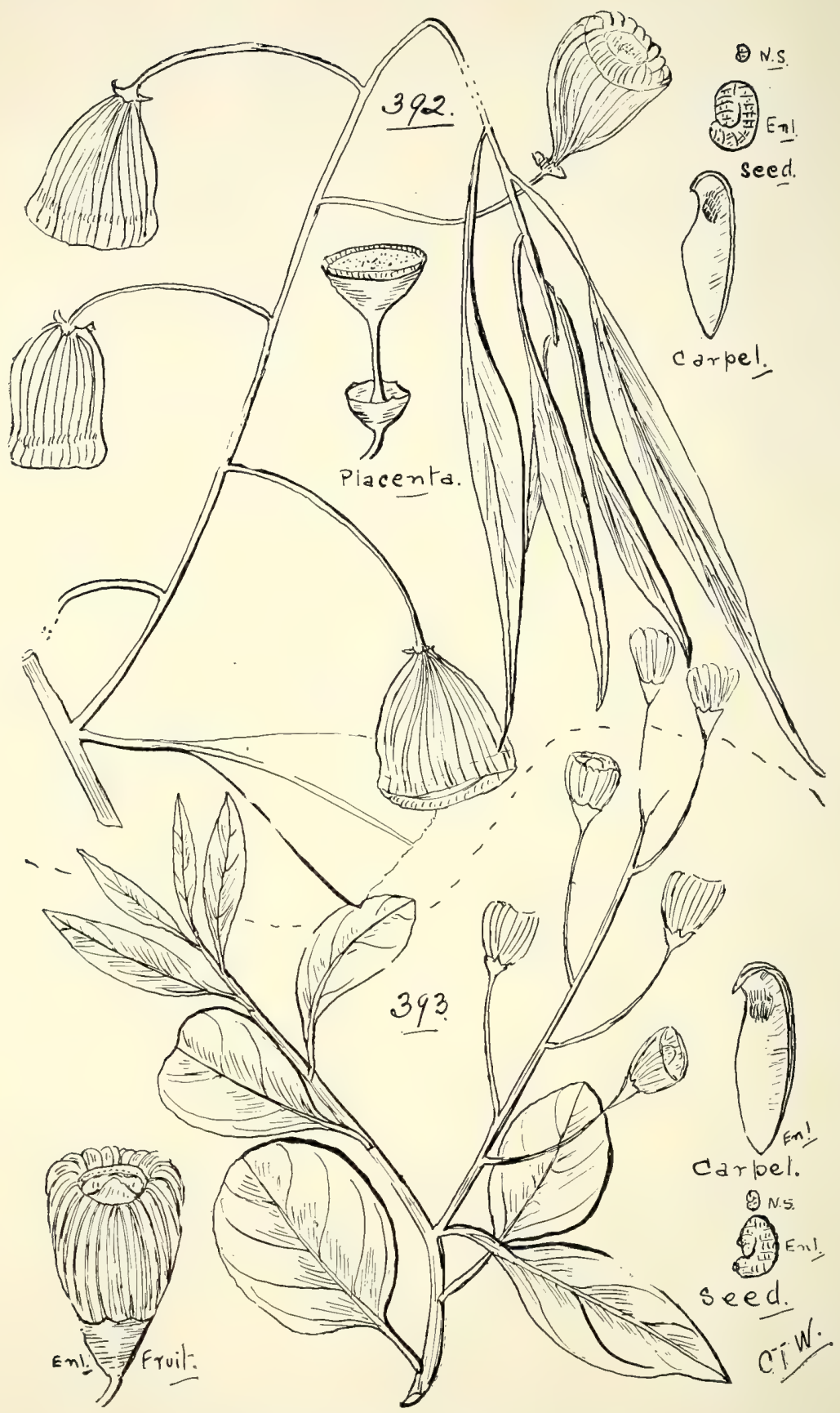

392. Codonocarpus australis, $A$. Cunn.

393. C. cotinifolius, F. . $M$. 


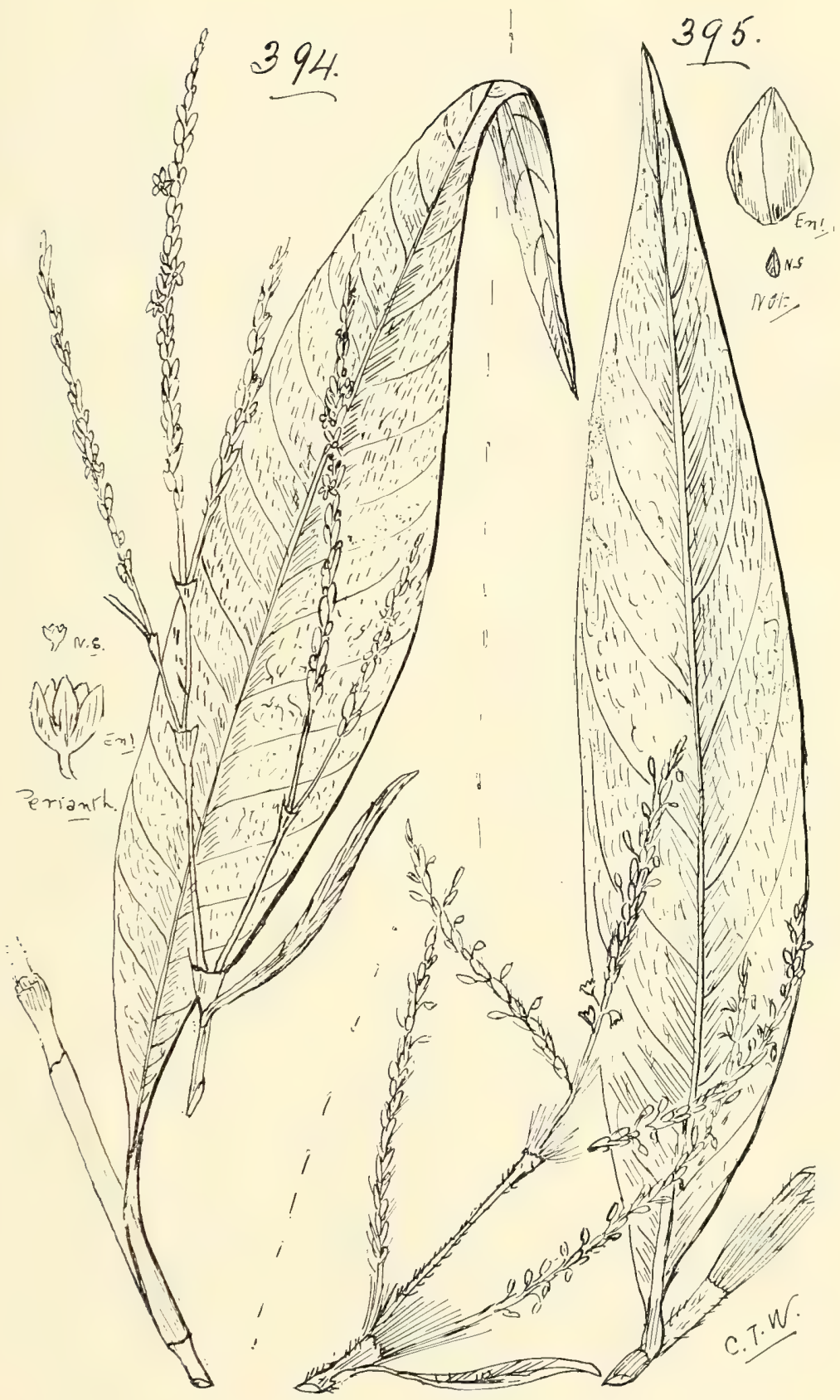

394. Polygonum attenuatum, $R$. $B r$. 395. P. Barbatum, Linn. 


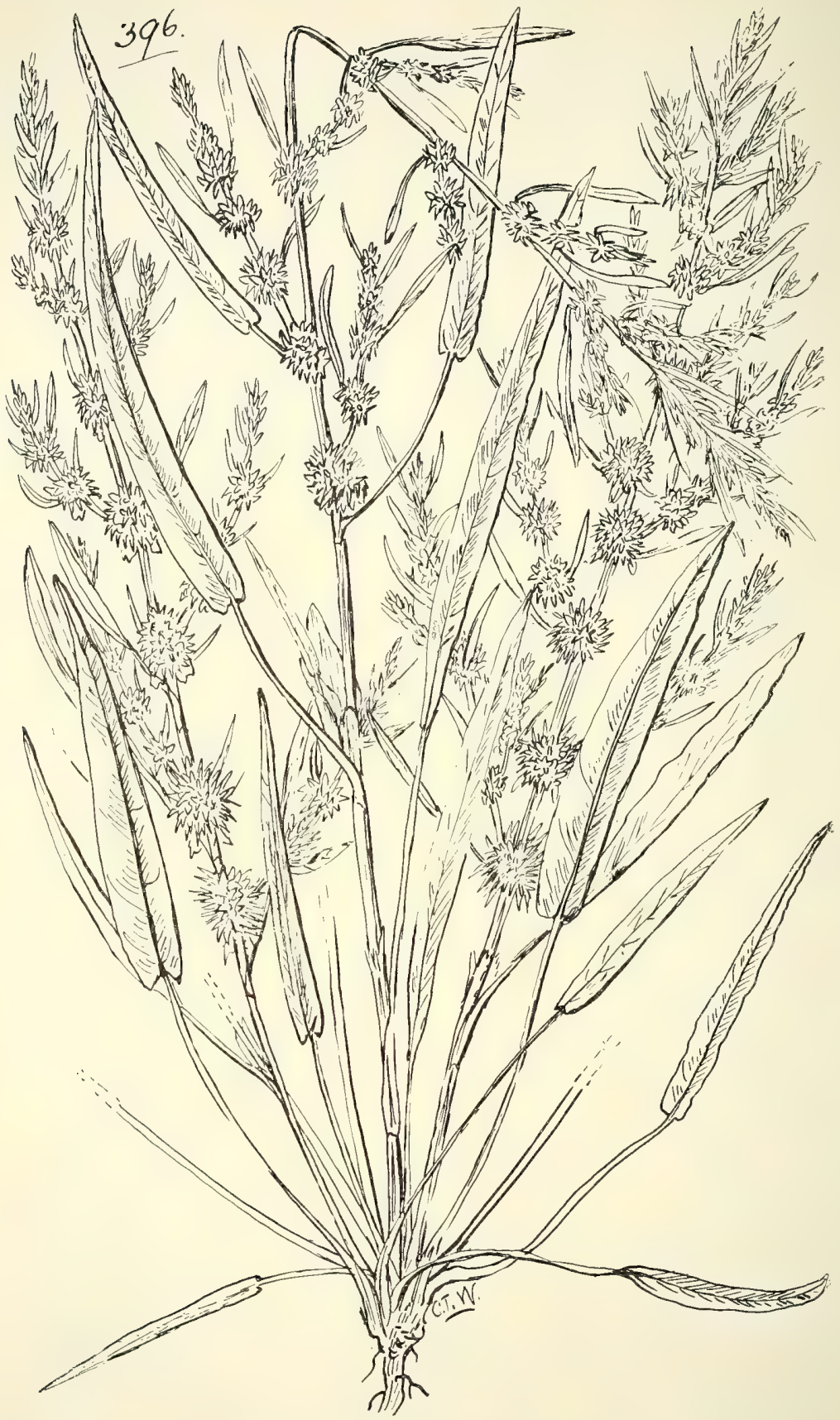

396. Rumex halophilus, $F . v . M$. 
Rumex-contd.

conglomeratus, Murr.-Sharp Dock.

Brownii, Camp.

†pulcher, Linn.--Fiddle Dock. Europe. Not an uncommon weed on lawns about Brisbane.

*obtusifolius, Limn--Broad-leaved Dock.

halophilus, F.v.M. (Fig. 396.)

bidens, $R . B r$.

acetosella, Linn.-Sheep's Sorrel; a troublesome weed.

Emex, Necker.

australis, Steinheil.-Devil's Thorn. A troublesome weed; like its allies, furnishes a mild purgative.

Tribe III.-Coccolobeæ.

Muehlenbeckia, Meissn.

gracillima, Meissn.

rhyticarya, F.v.M.

Cunninghamii, F.v.M.-Lignum of some.

Series II.-MULTIOVULAT王 AQUATIC王.

(Representatives undetermined.)

\section{Order CIV.-PODOSTEMONACEÆ.}

Baron von Mueller records in Cen. Austr. P1. that plants of this Order have been met with in Queensland, but that the genera and species had not been determined. The authority rests, so far as at present known, upon specimens collected by Mr. Walter Hill at the Johnstone River in 1873 .

Series III.-MULTIOVULATE TERRESTRES.

\section{Order CV.-NEPENTHACEÆ.}

Nepenthes, Limn.-Pitcher-plants. The beauty of our indigenous species is lost in the dried specimens; when fresh, many show beautiful markings.

Kennedyana, F.v.M.

Bernaysii, Bail.

albo-lineata, Bail.

Moorei, Bail. (Fig. 397.)

Jardinei, Bail.

Rowanæ, Bail.

Alicæ, Bail.

Cholmondeleyi, Bail.

pascoensis, Bail.

Armbrusteæ, Bail.

Garrawayæ, Bail.

$2 \mathrm{C}$ 


\section{Order CVI.-ARISTOLOCHIACEÆ.}

Aristolochia, Linn.-Birthwort. Fruit a Capsule. E. W. Armit mentions species of this genus having been used by the aborigines in cases of midwifery.

deltantha, F.v.M. (Fig. 398.)

var. Laheyana, Bail.

prævenosa, F. v. M.

pubera, R.Br. (Fig. 399.)

Thozetii, F.v.M. (Fig. 40o.)

var. angustissima, Benth.

indica, Linn., var. ? magna, F.v. M.-The roots and stems of the normal form are in India considered as useful in medicine as those of $A$. serpentaria, the Serpentary or Snake-root of commerce.

\section{SERIES IV.-MICREMBRYE压.}

\section{Order CVII.-PIPERACE王。}

Tribe Pipereæ.

Piper, Linn.

subpeltatum, Willd.

novæ-hollandiæ, Miq.-Australian Pepper-vine. Dr. Joseph

Bancroft considered this to be a powerful stimulant tonic to the mucous membrane.

var. mollis, Bail.-Only so far known from the Burpengary scrubs.

Banksii, Miq. (Fig. 40т.)

triandrum, F.v. $M$.

(Chavica) Mestoni, Bail.-Long Pepper. (Fig. 402.)

(Chavica) Rothiana, Bail._- "Chib-bi" of Atherton natives. (Fig. 403.) (Plate XII.)

Peperomia, Rui et Pav.

leptostachya, Hook. et Arn.

affinis, Domin. (Fig. 404.)

reflexa, A. Dietr. (Fig. 405.)

enervis, C. DC. and F.v. M. (Fig. 406.)

Johnsonii, C. DC. 
PLATE XII.

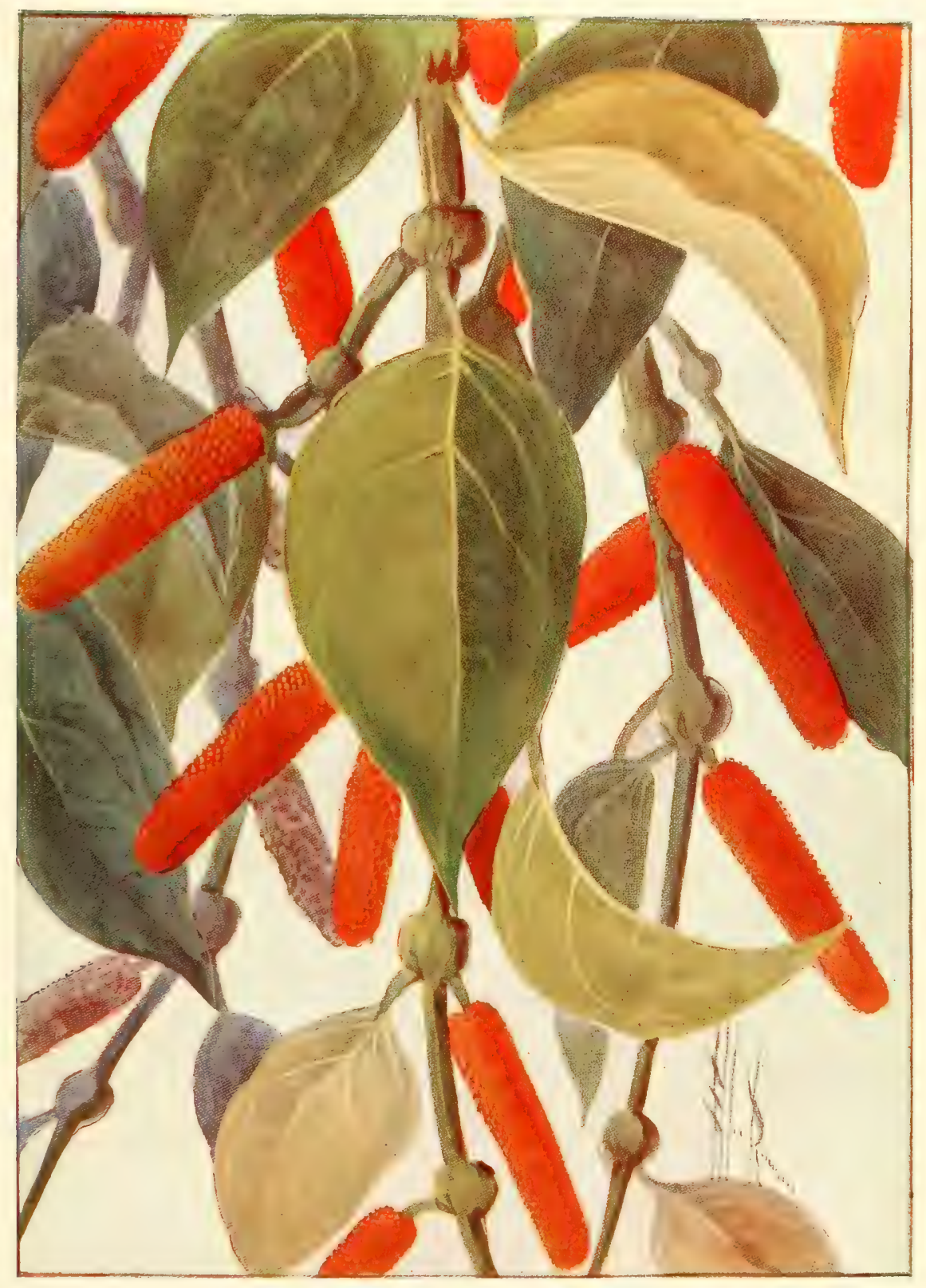

Piper Mestoni, Bail. 



\section{Order CVIII.-MYRISTICEÆE.}

Myristica, Limn.

insipida, $R$. Br. $=M$. cimicifera, R. Br.-Queensland Nutmeg. "Kurroonbah" of Barron River and "Gooroombah" of Tully River natives. Wood of a pinkish-grey, easily worked.

Dr. Warburg (Monographie der Myristicaceen) divides the Queensland species of Myristica as follows:cimicifera, R. Br.

var. typica, Warb. (Fig. 407.)

var. insipida, Warb. (Fig. 408.)

Muelleri, Warb. (Fig. 409.)

\section{Order CIX.-MONIMIACE王.}

Palmeria, F.v.M.

Tribe I.-Monimieæ.

scandens, F.v.M.

Mollinedia, Ruiz et Pav.

Huegeiiana, Tul.-Wood light-coloured, hard and tough.

angustifolia, Bail. (Fig. 4IO.)

Wardellii, F.v.M. (Fig. 4II.)

macrooraia, Bail. (Fig. 4I2.)

loxocarya, Benth.

acuminata, F.v.M.

subternata, Bail.- "Wonda" of Tully River natives. (Fig. 4I3.)

Kibara, Endl.

macrophylla, Benth.-Wood nicely marked.

longipes, Benth.-Wood straw-coloured, nicely marked. (Fig. 4I4.)

laxiflora, Benth.

pubescens, Benth.

Hedycarya, Forst.

angustifolia, A. Cunn.

Piptocalyx, Oliv.

Moorei, Oliv.

TRIBE II.-ATHEROSPERMEX.

Atherosperma, Labill.

moschata, Labill.-Australian Sassafras; is used as a diaphoretic and diuretic in asthma, and in some forms of heart disease.

Doryphora, Endl.

sassafras, Endl.-The Queensland tree, so far as known, only met with near the border of New South Wales. 


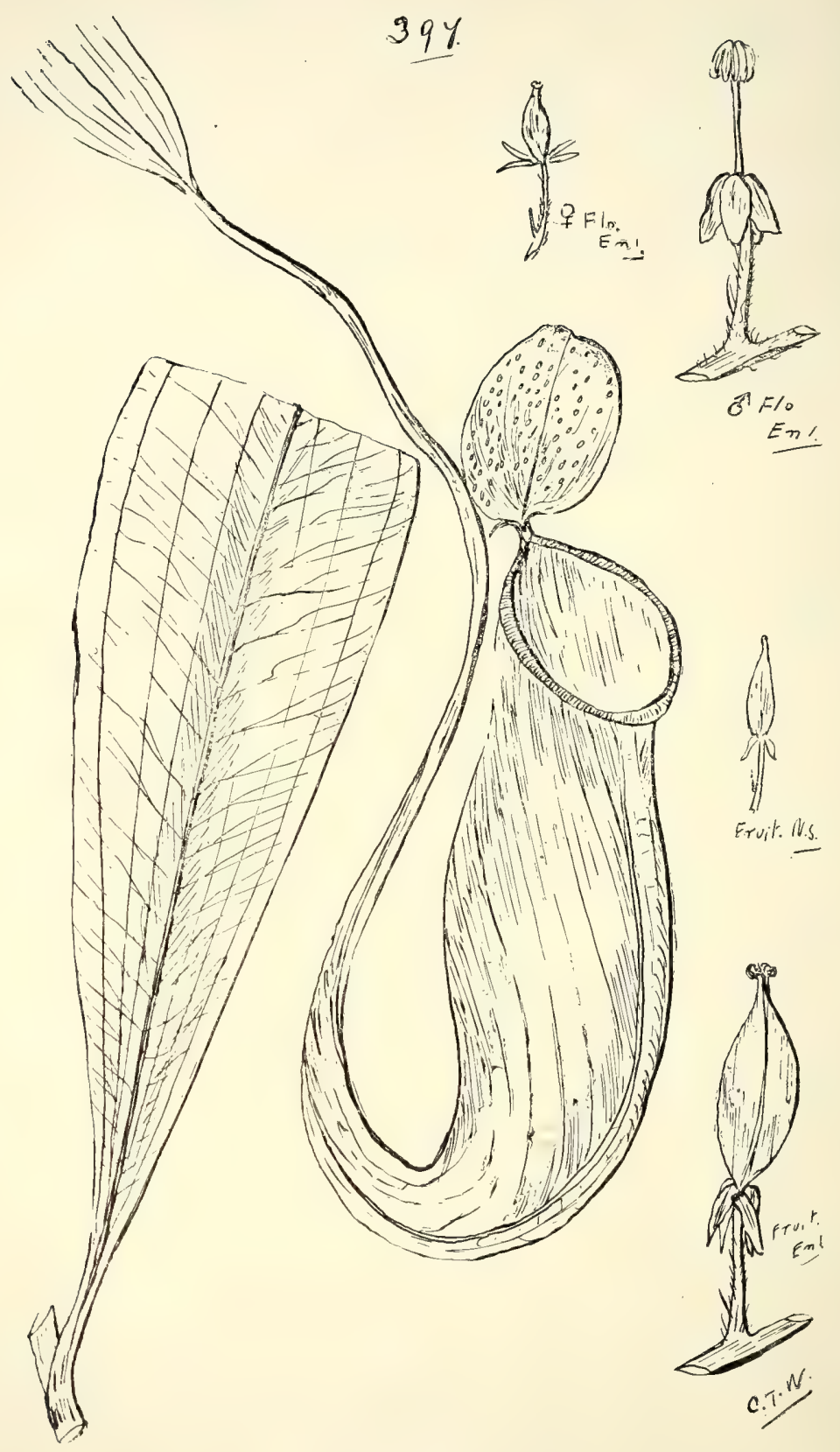

397. Nepenthes Mooret, Bail. 


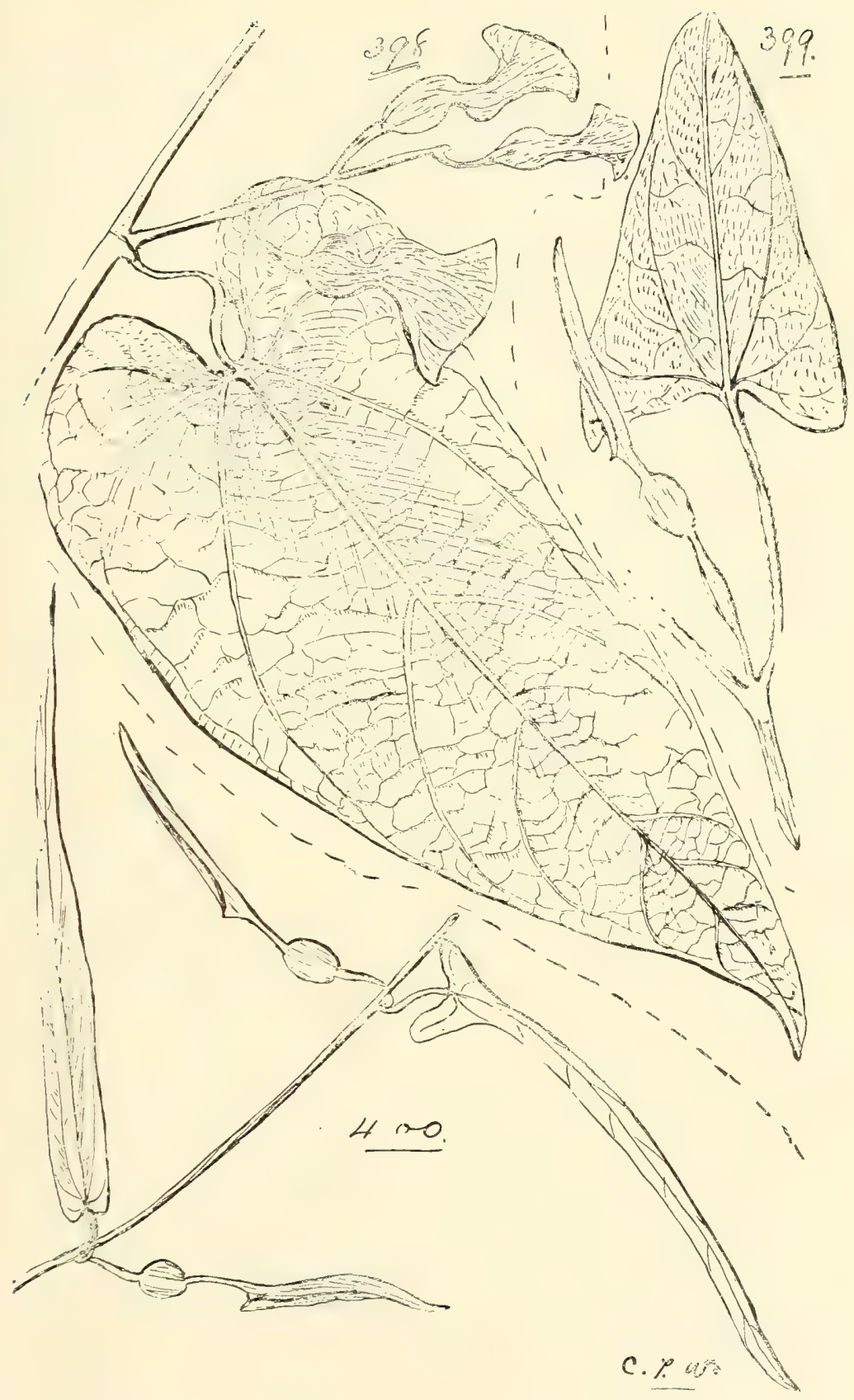

398. Aristolochia diltantha, F.v. $M$.

399. A. PUBeRA, R. $B r$.

400. A. Thozetil, F. v. M. 


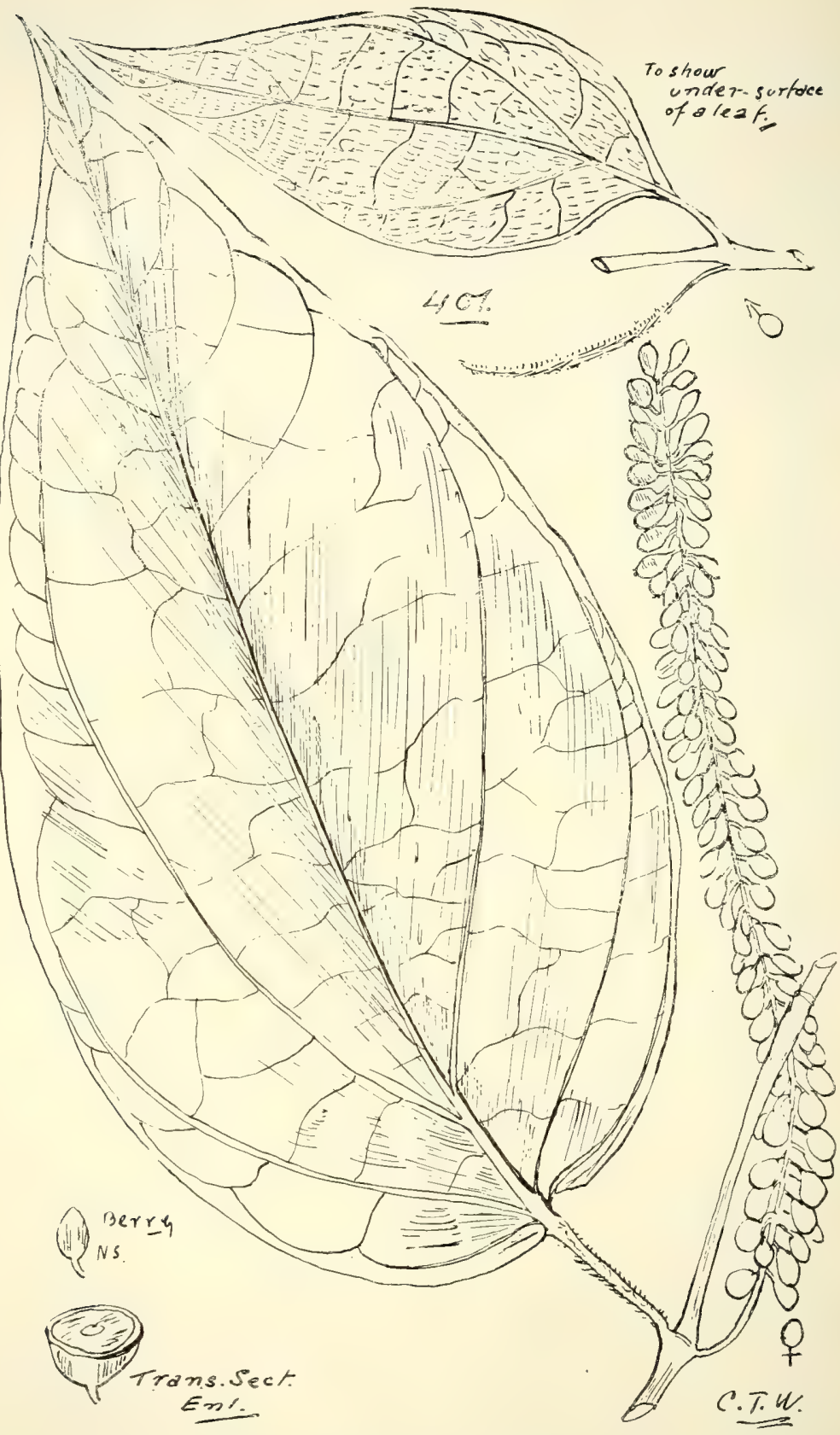

40I. Piper Banksit, Miq. 


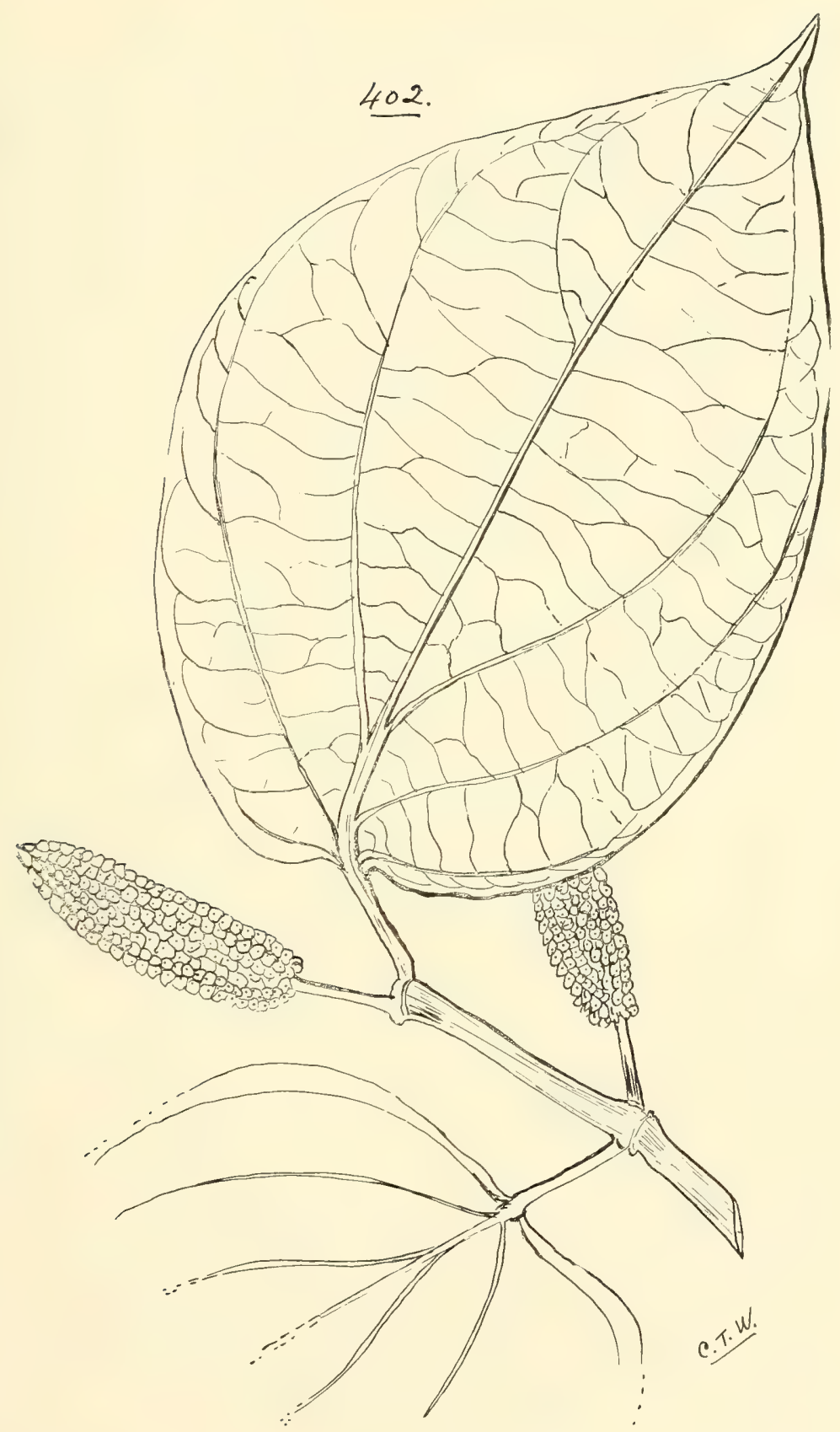

402. Piper Mestoni, Bail. 


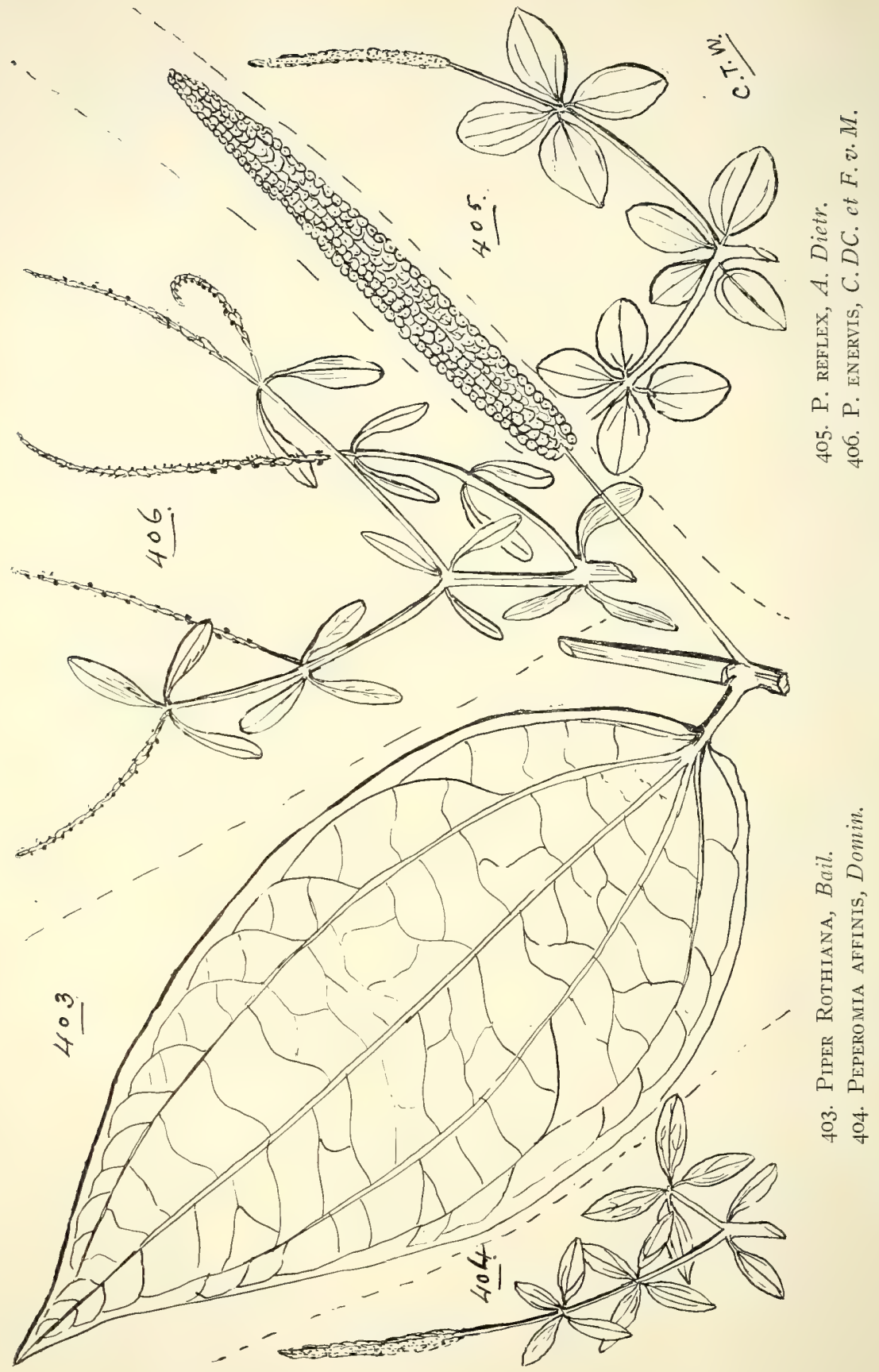


CVIII. MYRISTICEÆ.

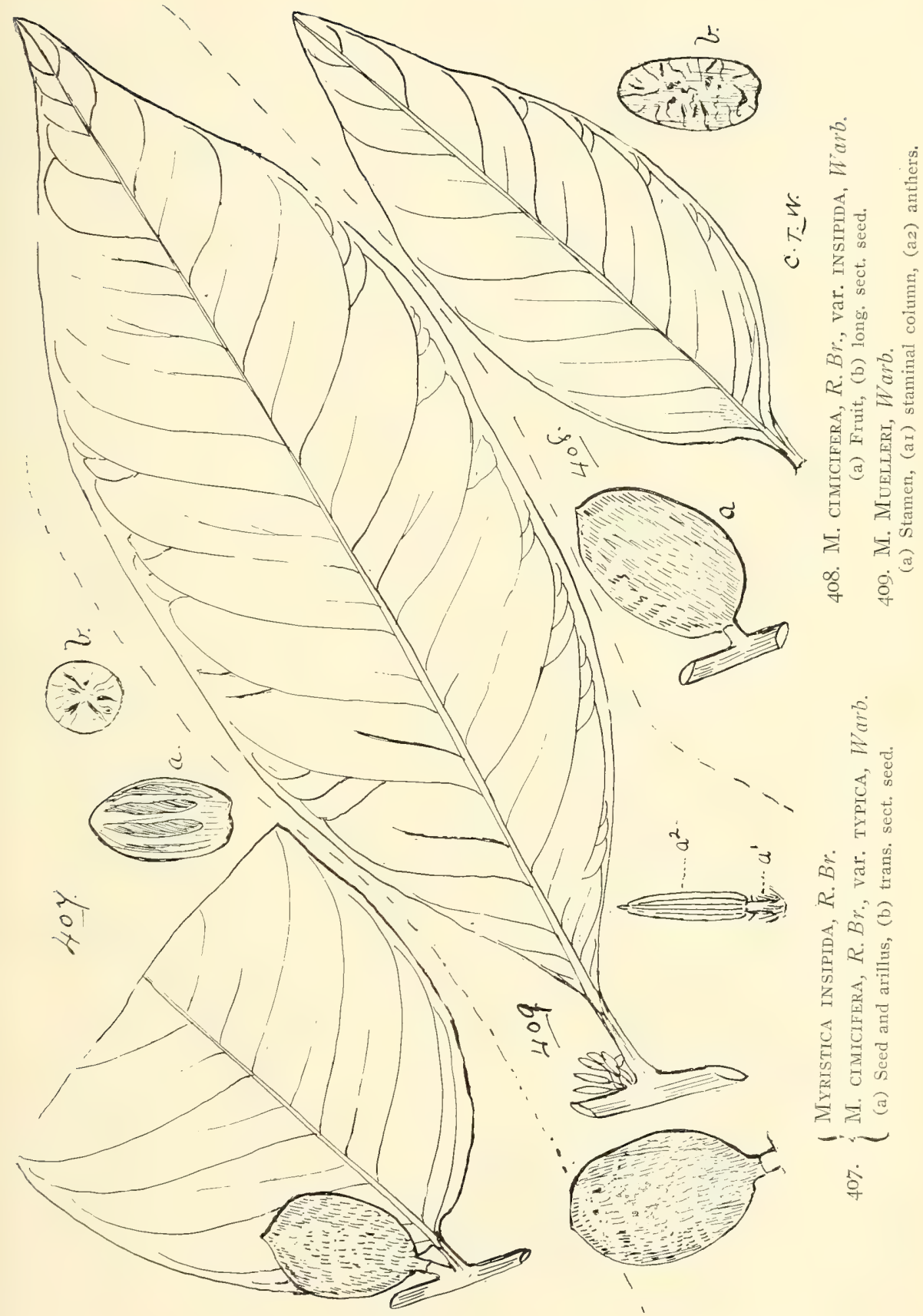




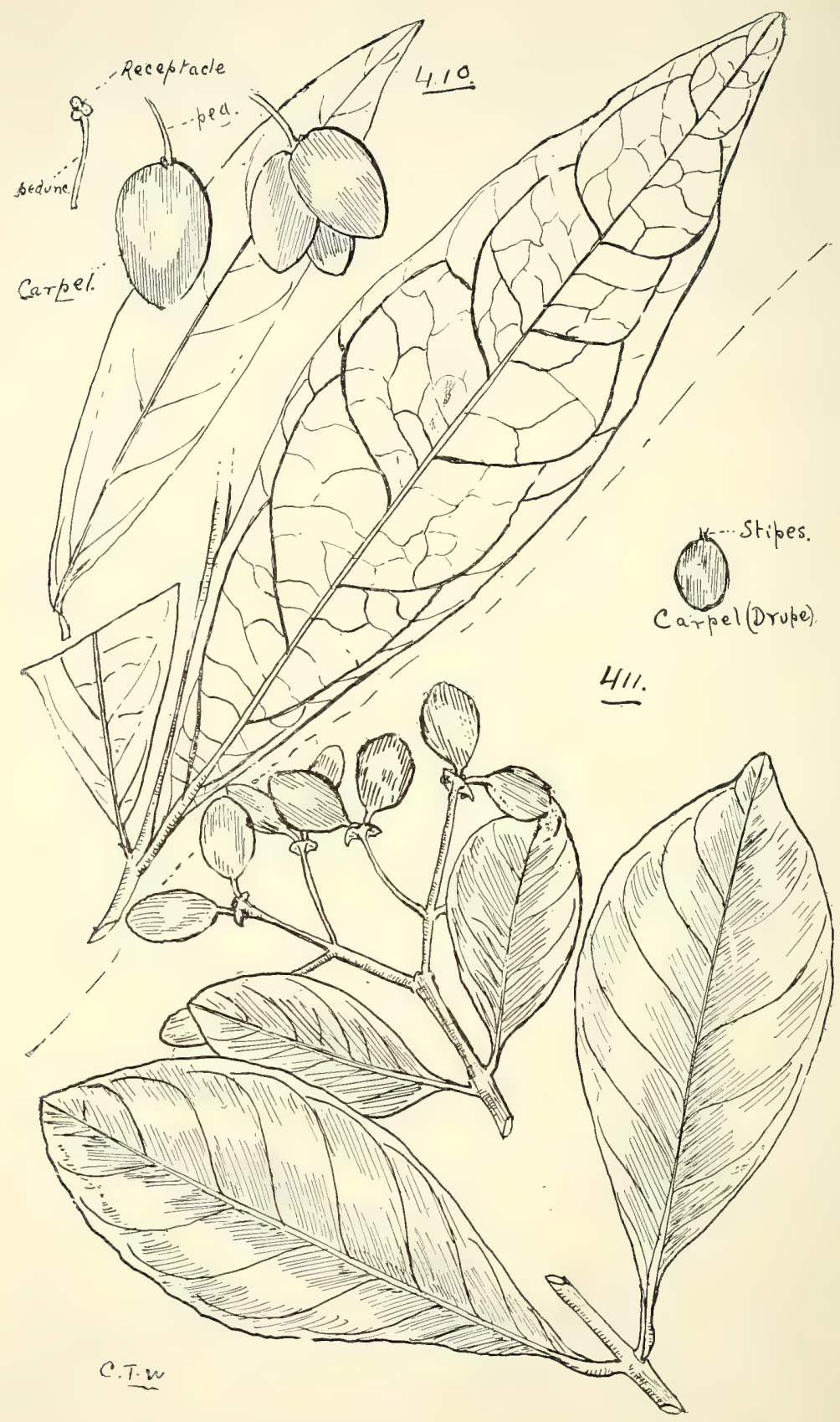

4io. Mollinedia angustifolia, Bail. 4II. M. Wardellit, F.v. $M$. 


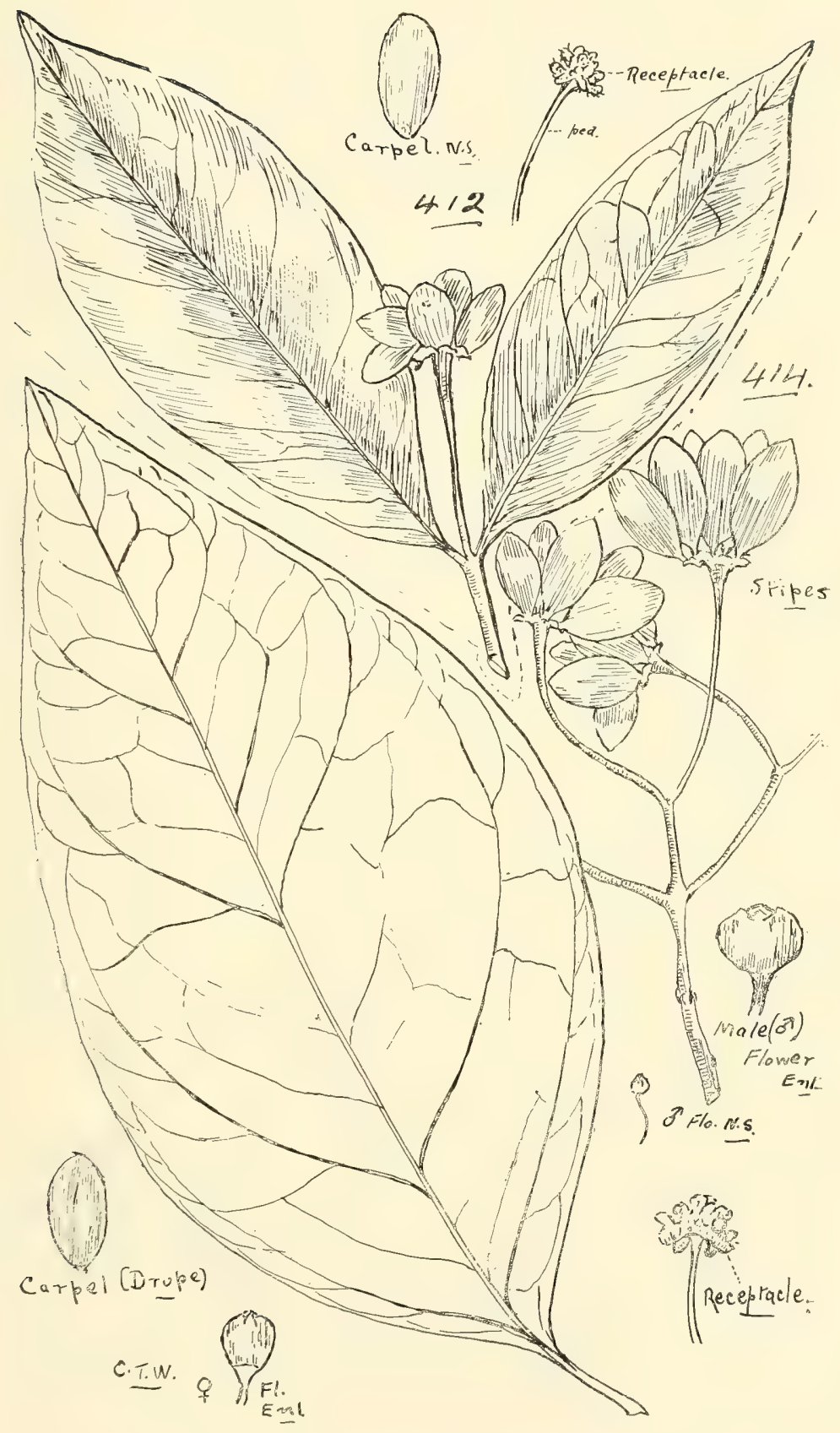

412. Mollinedta macroorata, Bail. 4I4. Kibara longites, Benth. 


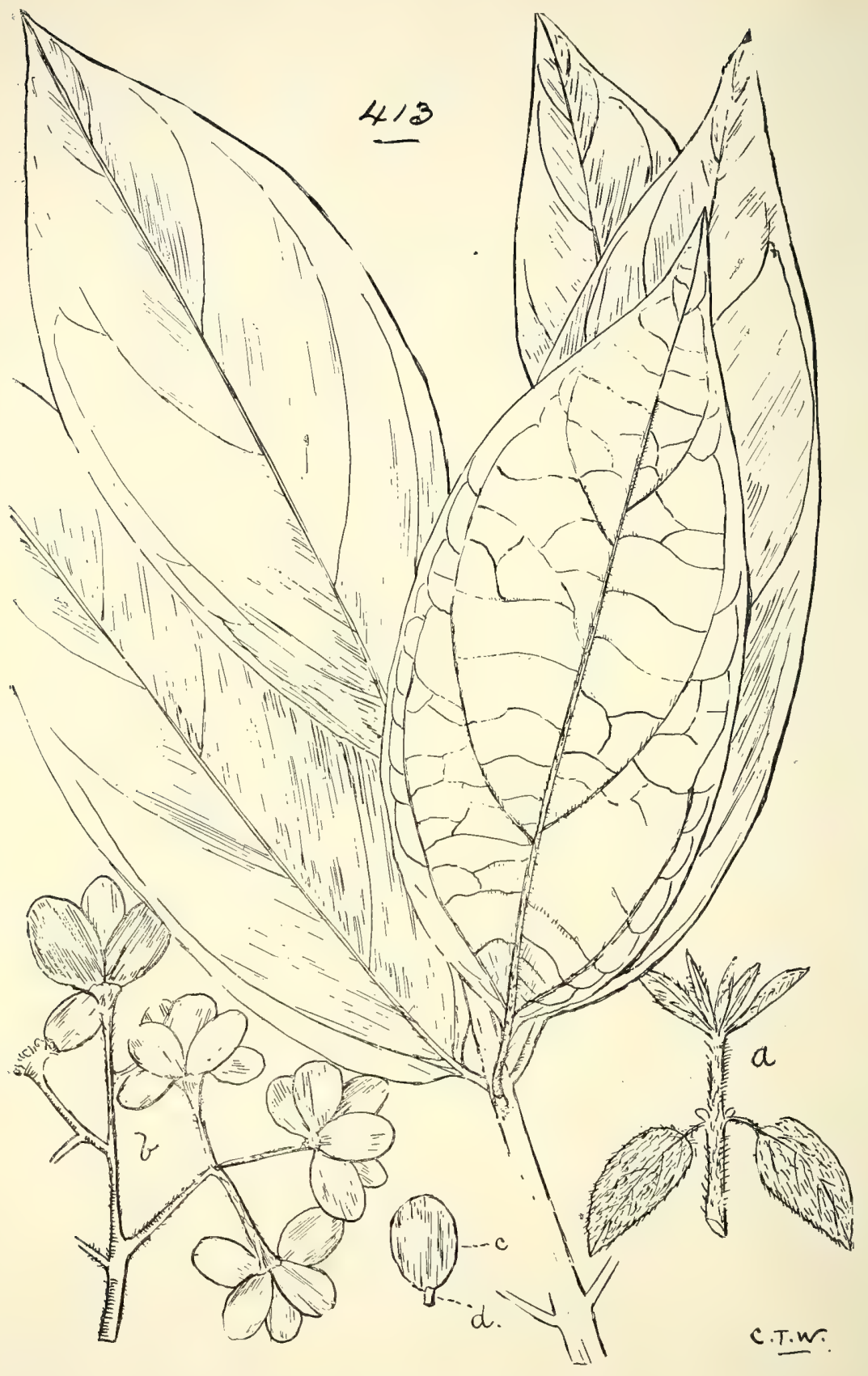

4i3. Mollinedia subternata, Bail.

(a) Young growth, (b) carpels, (c) a single carpel, (d) stipes. All nat. size. 


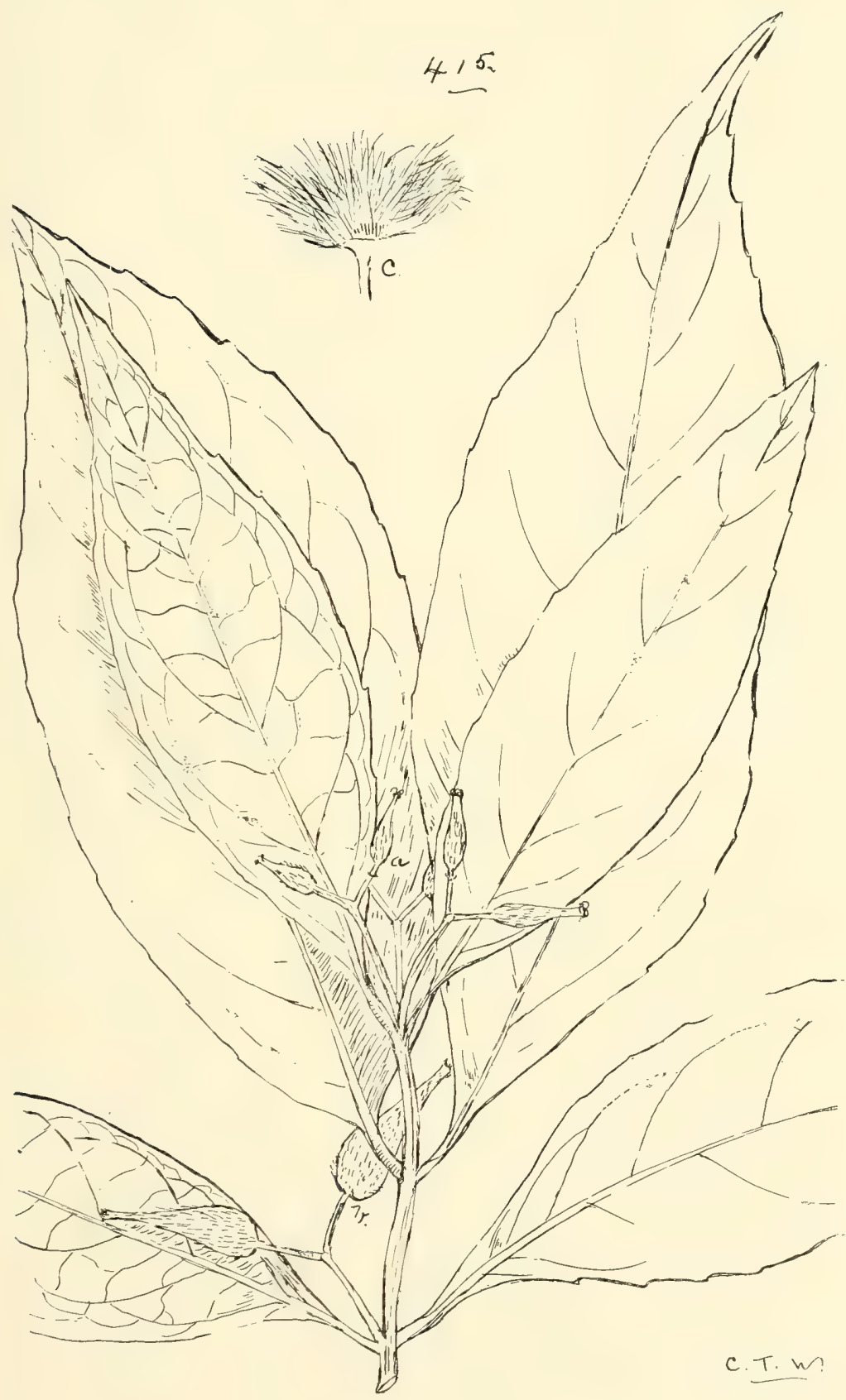

415. Daphnandra aromatica, Bail.

(a) Flowers, (b) fruiting perianth, (c) carpels. All nat, size. 
Daphnandra, Benth.-The bark of all the species contains a cardiac poison, according to Dr. T. L. Bancroft.

micrantha, Benth.-A good cabinet-wood, of a glossy yellow colour; stands well in seasoning.

repandula, F. v. M.-Wood resembles English Holly. aromatica, Bail._A spice-bark. "Cheed-ingnan" of Barron River natives. Wood resembling Pine. (Fig. 4I5.)

\title{
SERIEs V.-DAPHNALES.
}

\section{Order CX.-LAURINEÆ.}

Cryptocarya, R. Br.

\author{
Tribe I.-Perseace $x$.
}

Murrayi, F.v.M.-Wood close-grained and of dark colour. (Fig. 4I6.)

Mackinnoniana, F.v.M. - "Koonjoongaroo" of Barron River natives. Wood of a grey colour, close-grained. (Fig. 4I7.)

patentinervis, F. v. M.-Wood light-coloured with dark streaks.

obovata, R. Br.-Long Tom.

var. tropica, Bail.

glaucescens, R. Br.

var. reticulata, Meissn.

var. coriacea, Benth.

var. nitida, Benth.

triplinervis, R. Br.-The foliage of this tree is said to be poisonous to pigs; and Dr. T. L. Bancroft found it to contain a poisonous alkaloid closely allied to "Curarine." cinnamomifolia, Benth.

var. parvifolia, Bail.

Meissneri, F.v.M.

australis, Benth. - "Gellmerra" of Barron River natives.

Two forms of this plant, differing in the fruit, are met with-one bearing red and the other yellow fruit. oblata, Bail.

graveolens, Bail.- "Gir-irjal” of Johnstone River natives.

Palmerstoni, Bail._Black Walnut. "Koi-ie" of Barron River and "Pal-la-ga" of Atherton natives.

Bancrofti, Bail. - Red Walnut. "Bara" of Tully River and "Wanga" of Cardwell natives.

Beilschmiedia, Nees obtusifolia, Benth

lachnostemonea, F. v. M. 
Endiandra, R.Br.

glauca, R. Br.

hypotephra, F.v.M.

discolor, Benth.

Sieberi, Nees._"Till" of Moreton Bay natives. Wood suitable for tool-handles.

virens, F.v. M.

Muelleri, Meissn.

pubens, Meissn.

Cowleyana, Bail.

dichrophylla, F.v. M.

exostemonea, F.v. $M$.

Lowiana, Bail.

Sankeyana, Bail.-_" Goolaway" of Barron River natives.

insignis, Bail.-_"Boomban" of Barron River and "Kooroong-gi" of Atherton natives.

tooram, Bail.

Cinnamomum, Burm.

Oliveri, Bail.-Sassafras. The dry bark contains $770 \mathrm{oz}$. of oil to the ton. It also contains a tannin similar to cinchona tannin (K. T. Staiger). (Fig. 4r8.)

propinquum, Bail. (Fig. 4I9.)

Tamala, Th. Nees.-Wood very fragrant.

Persea, Gartn.

Baileyana, F.v.M., ex Bail., 2nd Suppl. Syn. Q1. F1. 51.Candlewood. Wood dark grey; slightly fragrant. (Fig. 420.)

Litsea, Lam.

Tribe II.--Litseace $\pi$.

chinensis, Lam.

zeylanica, Nees.-Bark contains 7 per cent. of tannin.

dealbata, Nees._- Marragiddie" of Barron River natives.

Wood yellowish with short brown streaks.

var. rufa, Benth.

Bindoniana, F.v. M.

ferruginea, R. Br.

var. lanceolata, Meissn.

reticulata, Benth. et Hook. f.-Bally Gum; a cooper's wood. (Fig. 42 I.)

TRIBE III.-CAssythe玉.

Cassytha, Linn.-Laurel Dodders. All these are destructive to other plants:

glabella, R. Br. (Fig. 422.)

pubescens, R.Br. (Fig. 423.) 
CX. LAURINEA:

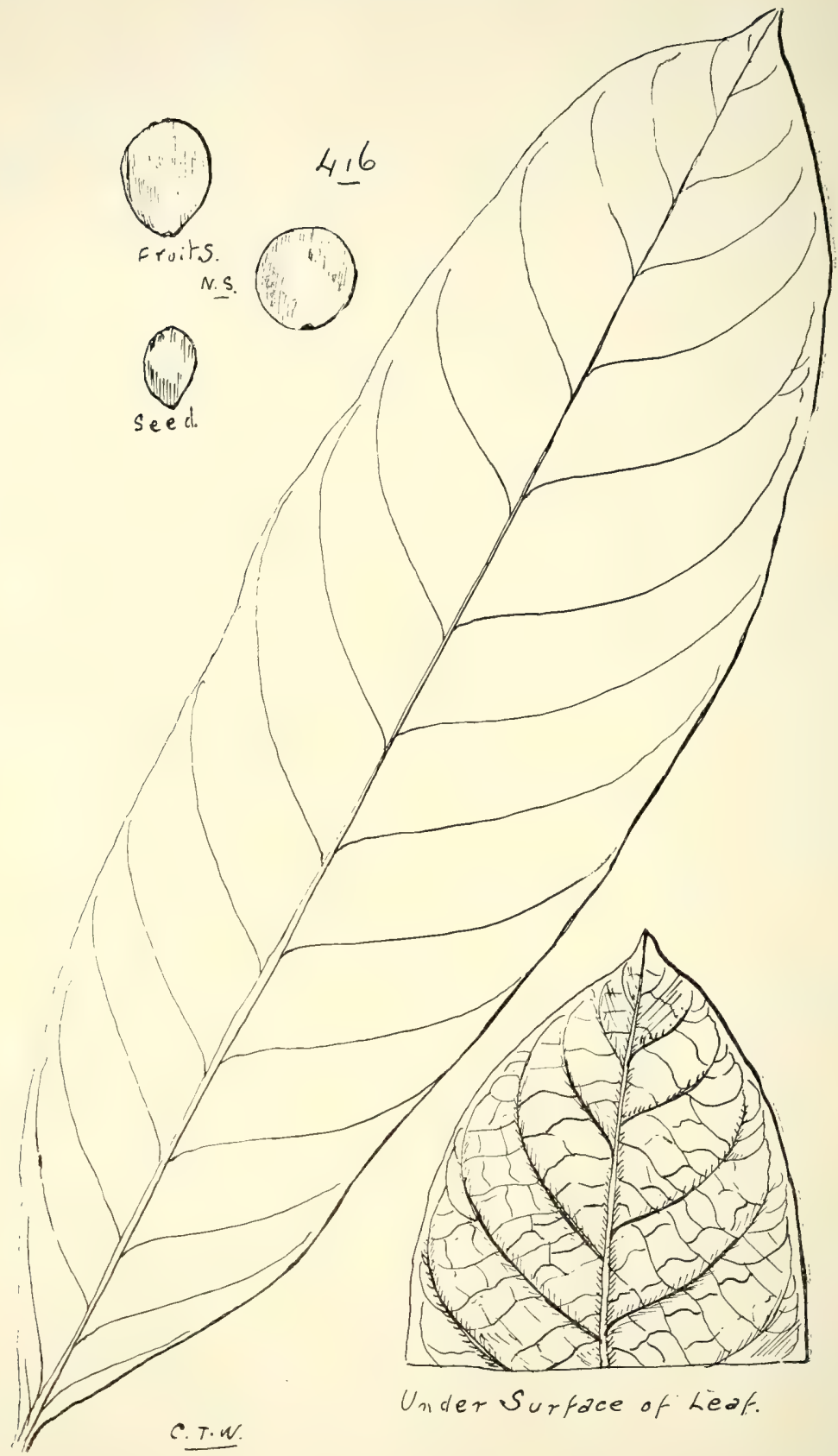

4i6. Cryptocarya Murrayi, $F . v \cdot M$. 


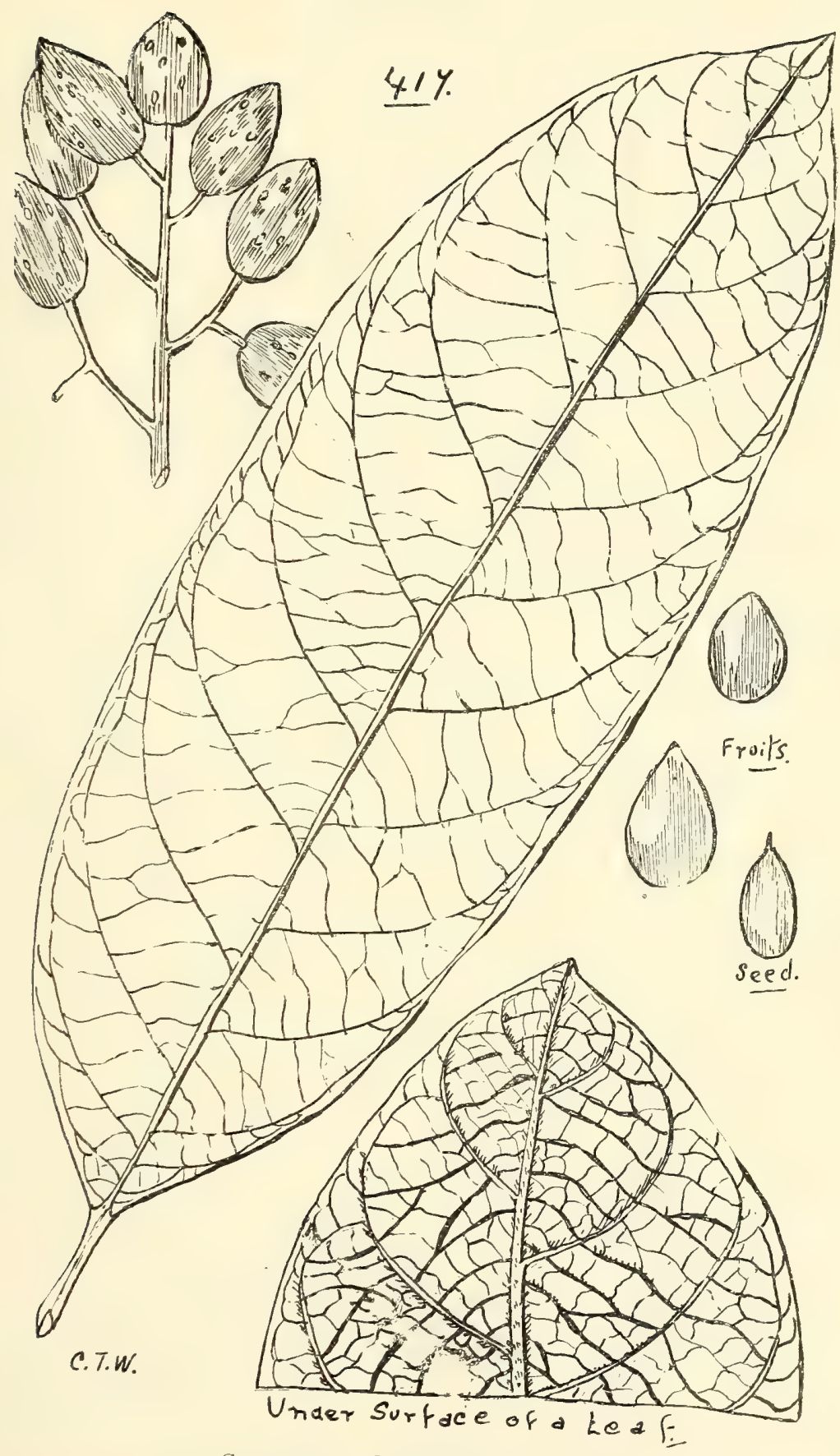

4T. Critotocarta Mackinioniana, F. v. M. 


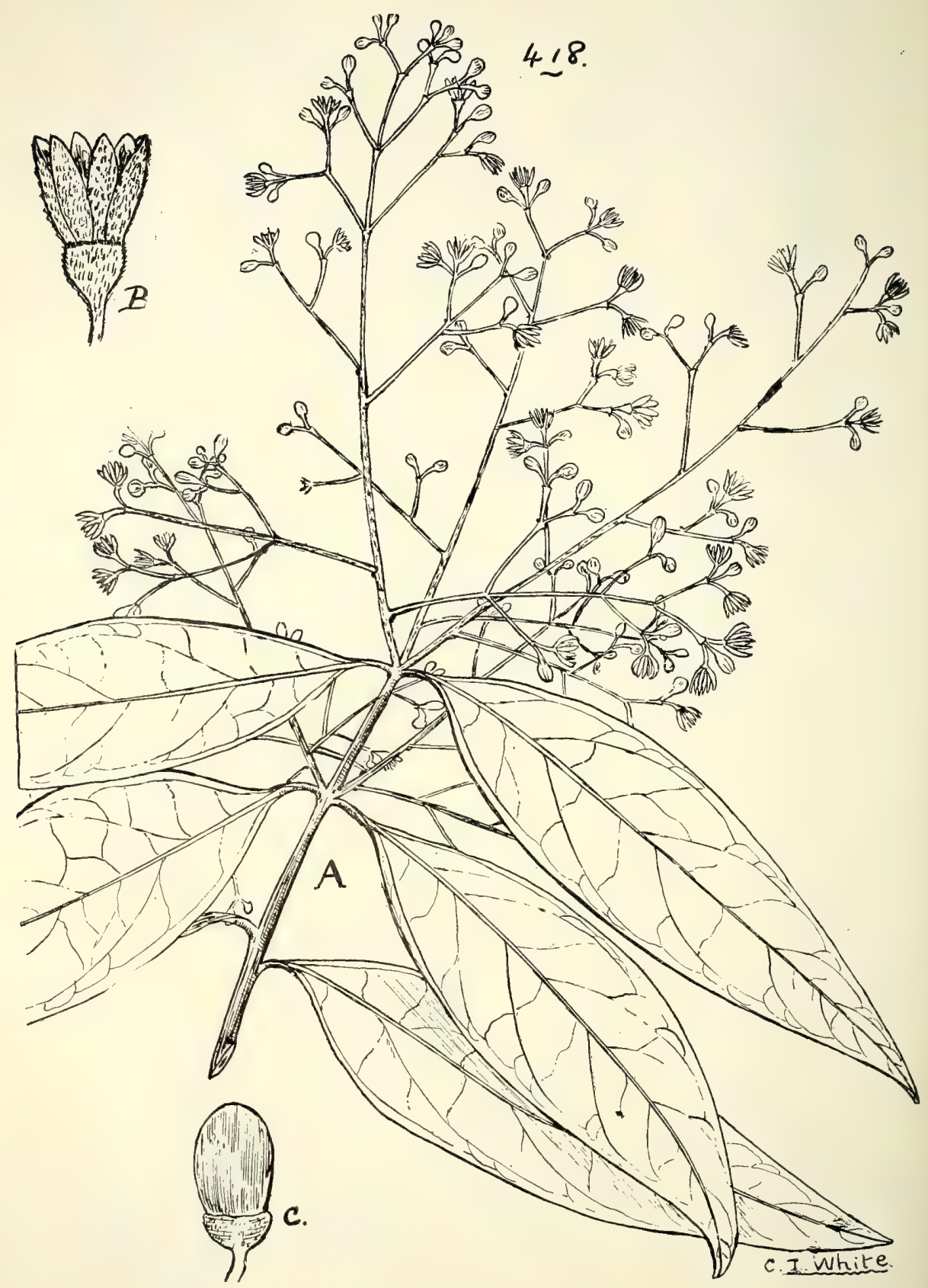

4i8. Cinnamomum Oliveri, Bail.

(A) A shoot, reduced, (B) a flower, enl., (C) a fruit, nat. size. 


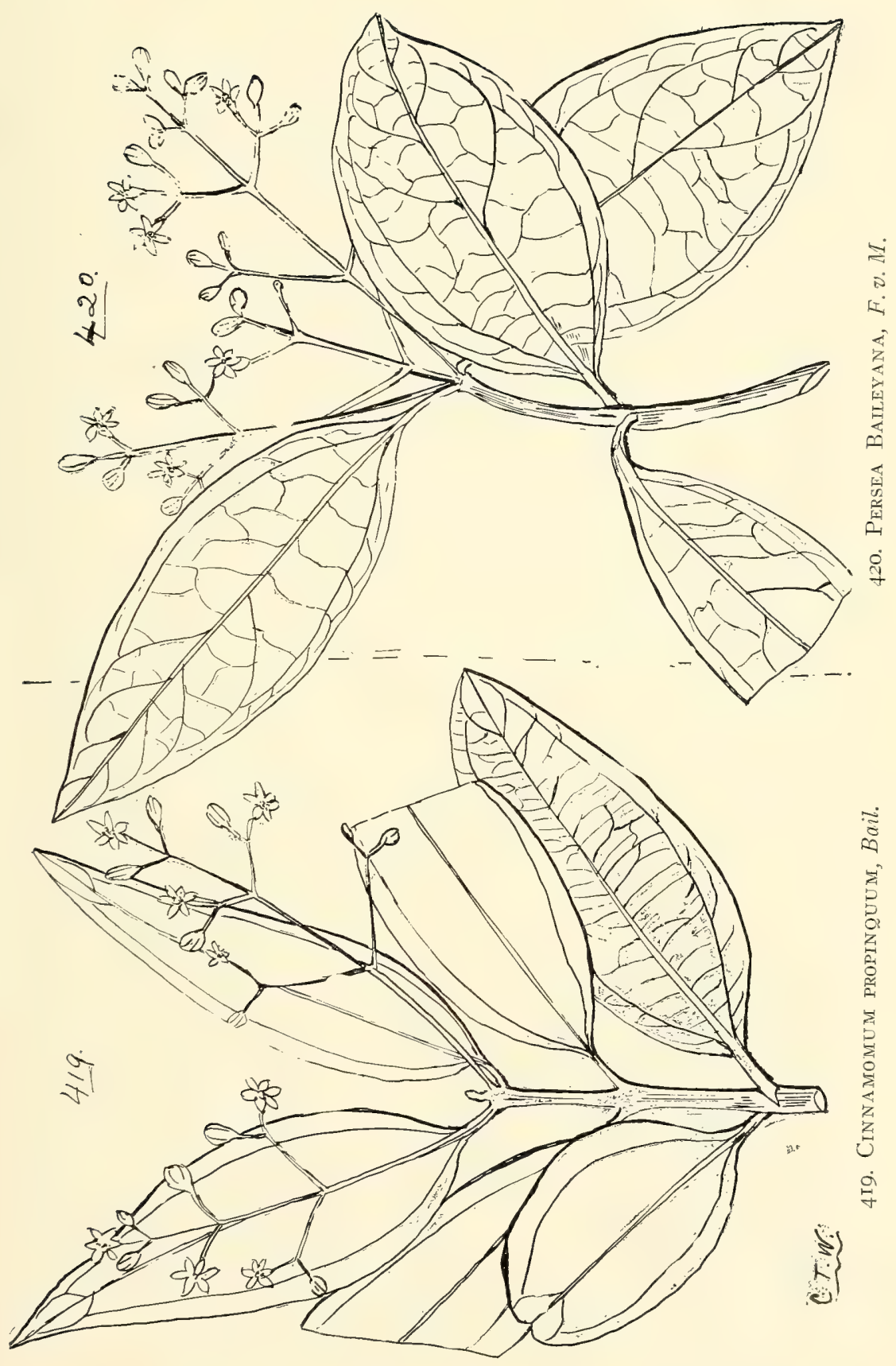


436

CX. LAURINE王.

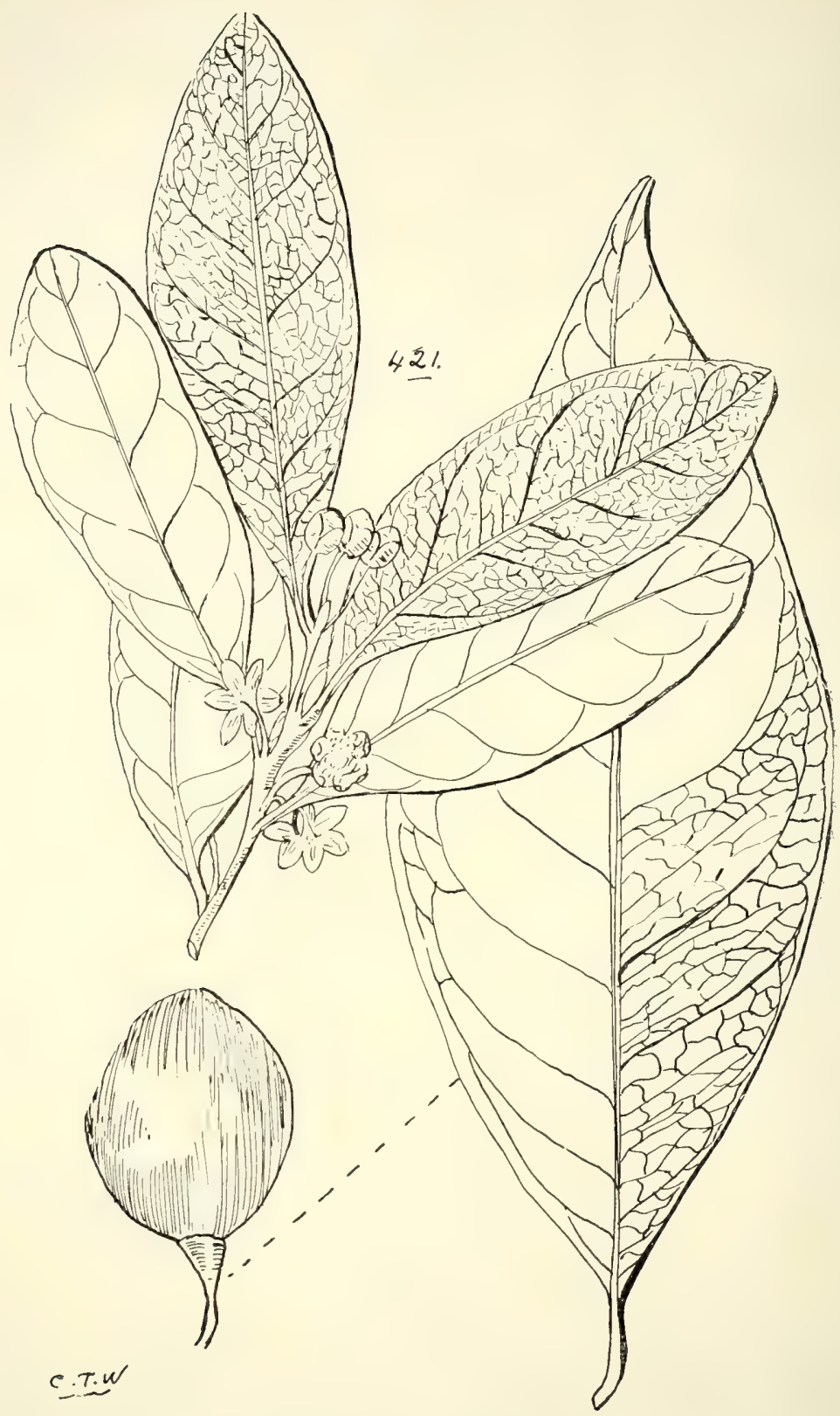

421. Litsea reticulata, Benth. et Hook. 


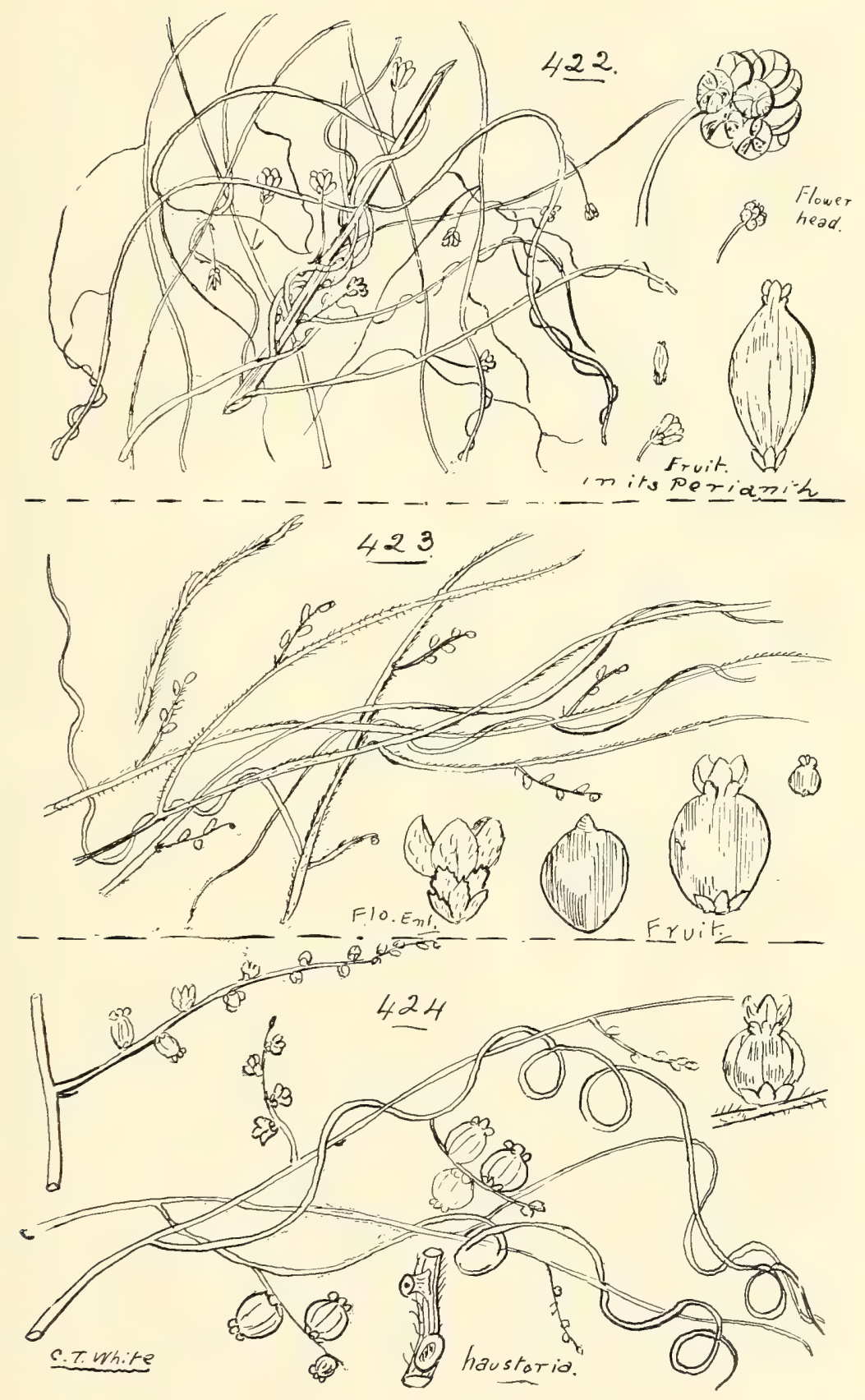

422. Cassytha glabella, $R$. $B r$.

423. C. PUbescens, R. $B r$.

424. C. FILIFORMIS, R. $B r$. 


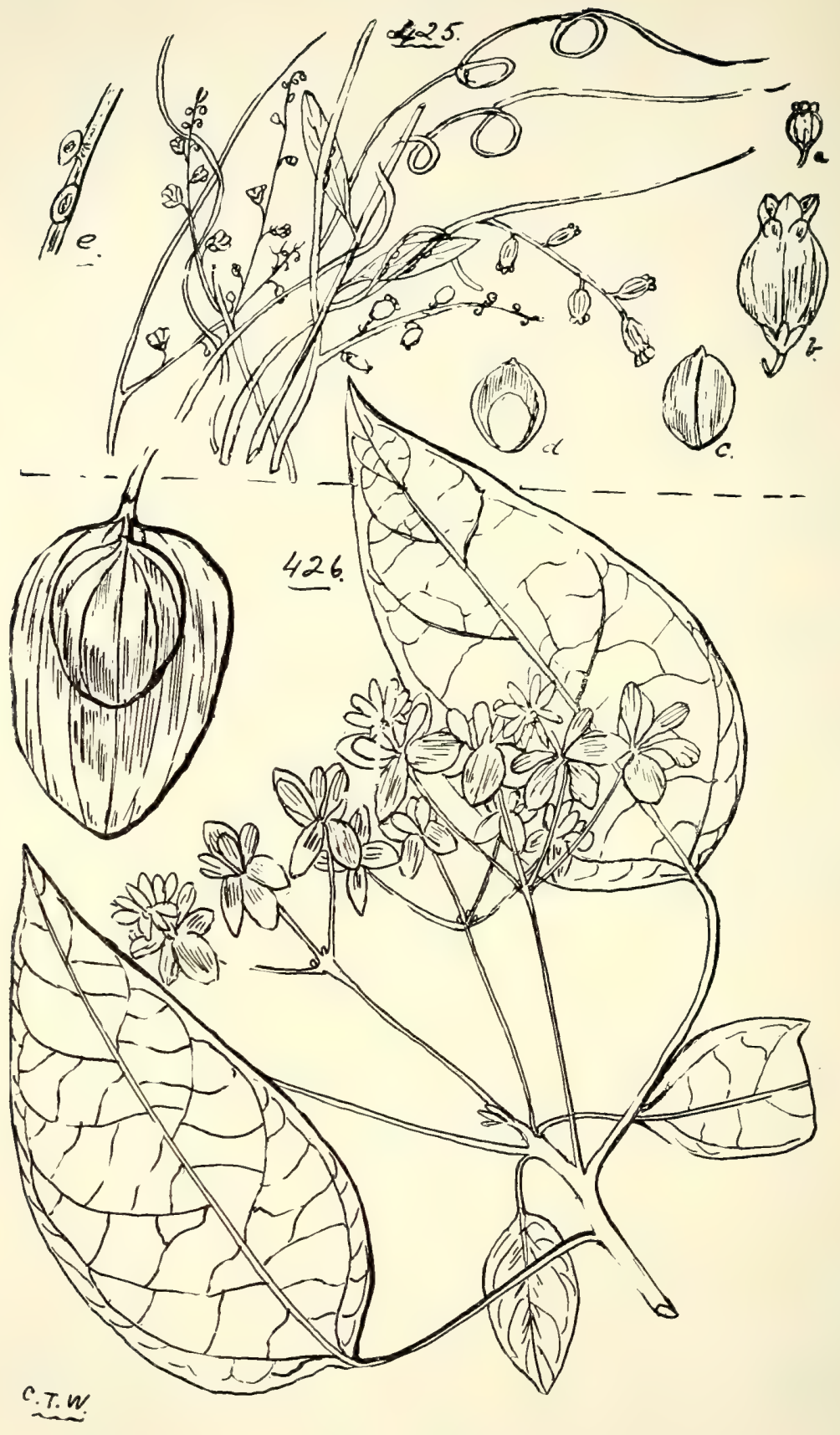

425. Cassytha racemosa, Nees.

(a) and (b) Fruits enclosed in the calyx, (c) fruits, calyx removed, (d) section fruit, (e) haustoria. (a) nat. size; (b)-(d) enl.

426. Hernandia Bivalvis, Benth.

(Fruit shown with one of the valves of the involucel removed.) 

PLATE XIII,

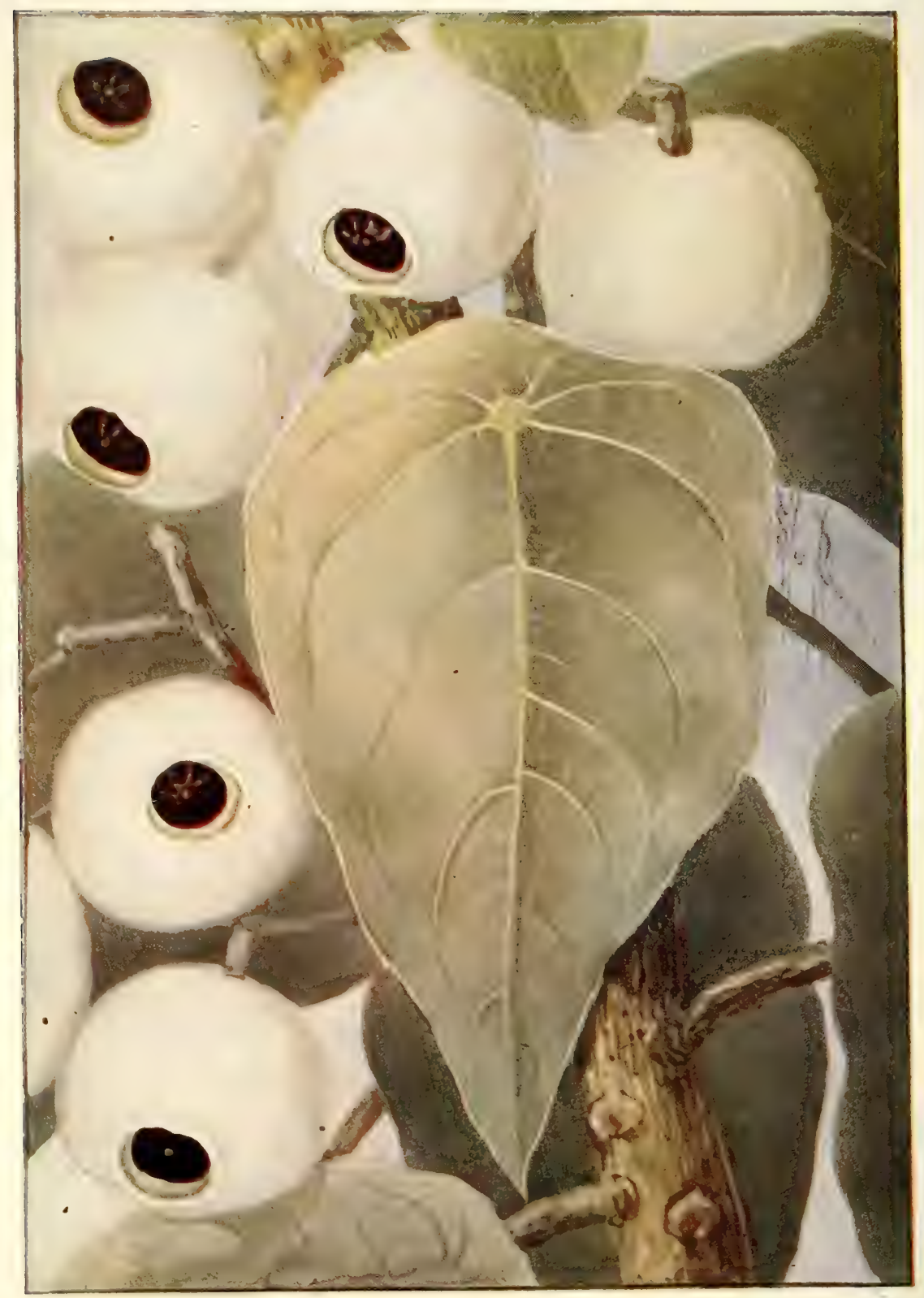

Hernandia peltata, Meissn. 
Cassytha-contd.

paniculata, R. Br.

var. remotiflora, Benth.

filiformis, Linn. (Fig. 424.)

melantha, R. Br.

racemosa, Nees. (Fig. 425.)

TRIBE IV.-HERNANDIEA.

Hernandia, Linn.

peltata, Meissn.-A sea-coast tree. (Plate XIII.)

bivalvis, Benth. - Cudgerie or Grease-nut. The kernel contains 64.8 per cent. of oil similar to laurel oil (K.T. Staiger). (Fig. 426.)

\section{Order CXI.-PROTEACEA.}

The trees of this Order furnish very handsome cabinet and building woods.

\section{Series I.-Nucamentacez.}

Petrophila, R. Br.

Tribe I.-Protee...

pedunculata, R. $\mathrm{Br}$.

Section Petrophyle.

Shirleyæ, Bail.

pulchella, $R . B r$.

sessilis, Sieb.

Isopogon, R. $\mathrm{Br}$.

Section Eustrobilus.

petiolaris, A. Cunn.

anemonifolius, Knight.

Tribe II.-Conosperme.e.

Conospermum, Sm.

Section Euconospernum.

sphacelatum, Hook.

taxifolium, Sm.

var. linifolium, Benth.

Persoonia, Sm.

Tribe III.-Persooniez.

Section I.-Pycnostylis.

falcata, R. Br.- "Tarpoon" of Annan River and "Nanchee" and "Booral" of Mitchell River natives. Wood hard and close-grained. (Fig. 427.) 
Persoonia-contd.

Section II.-Amblyanthera.

media, R. Br. - Geebung. "Koom-barra" of Stradbroke Island natives. Wood somewhat resembling English Beech.

cornifolia, A. Cunn.

sericea, A. Cunn.

Mitchelli, Meissn.

fastigiata, $R . B r$.

prostrata, R. Br.

lanceolata, $A n d r$.

linearis, $A n d r$.

virgata, $R . B r$.

tenuifolia, R. Br. (Fig. 428.)

\section{Series II.-Folliculares.}

Tribe IV.-Grevillee $\pi$.

Macadamia, F.v.M.

ternifolia, F.v.M.-Queensland Nut. Edible; good cabinetwood. The leaves of this tree contain prussic acid. Nos. 429, 430, and 43I represent forms met with in Queensland which, though apparently distinct, do not in my opinion differ enough to bear names even as forms or varieties.

Lowii, Bail., Q1. Agric. J1. xxvi. (I9I I) г27 $=$ M. ternifolia forma, Bail., Rept. Aus. Assoc. Advanc. Sci., vol. vi. (I895) 374, Bot. Bull. xii. I5.-Maroochie Nut. (Fig. 432.)

minor, Bail.-Smaller Queensland Nut; very similar to M. ternifolia, but much smaller in all its parts. It is a small tree or large shrub sending up several stems from the base. (Fig. 433.)

Whelani, Bail.-Nuts contain a large amount of prussic acid; eaten after preparation by the aborigines.

prealta, Bail. = Helicia praalta, F.v. M.-Wood useful for cabinet-work, also for tool-handles and other purposes.

Xylomelum, $S m$.

pyriforme, Knight.-Wooden Pear. Leaves contain saponin. Southern localities.

salicinum, A. Cunn.-Wooden Pear. Northern and Southern localities. (Fig. 434.)

Scottianum, F.v.M.-Wooden Pear. Northern localities. (Fig. 435.) 


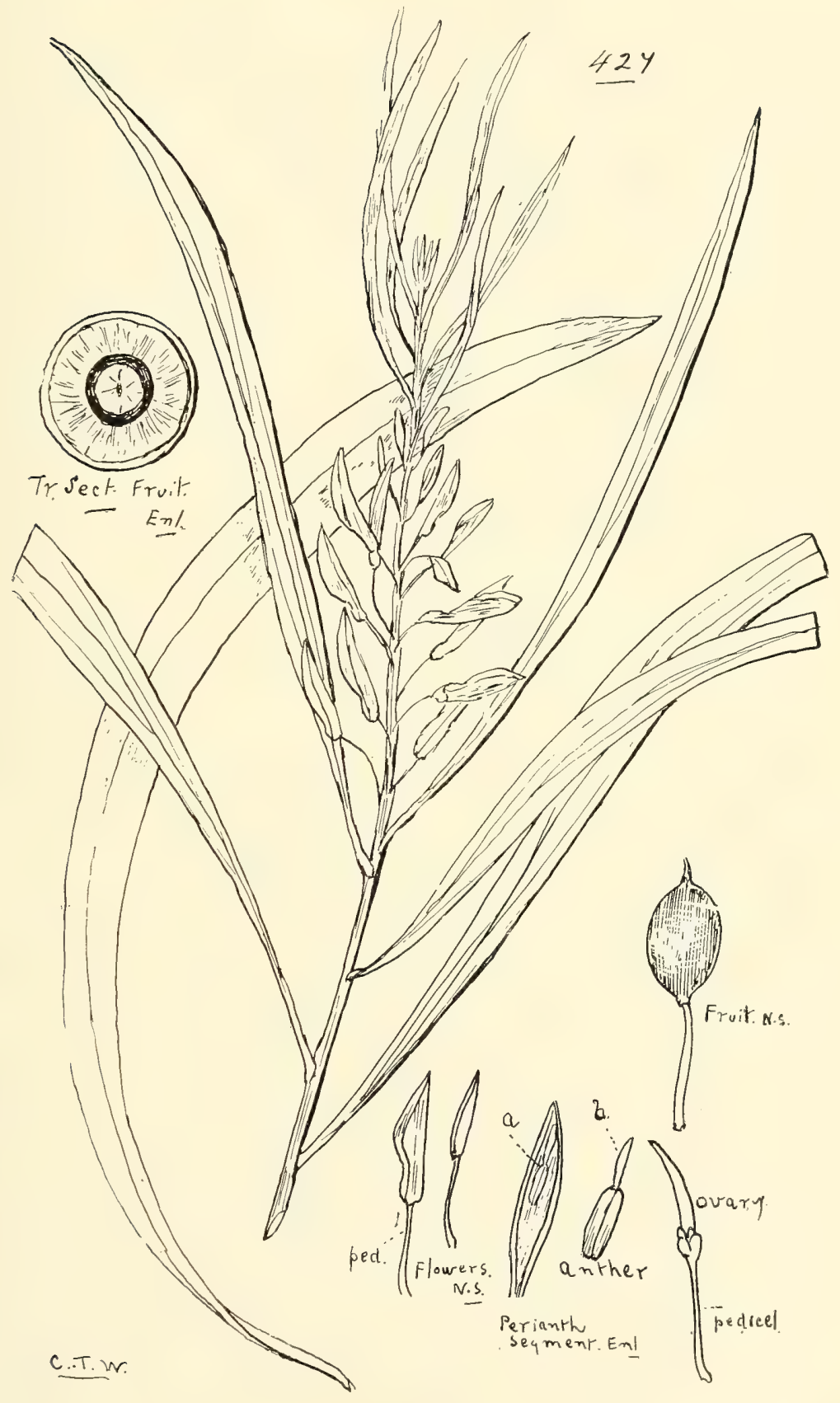

427. Persoonia falcata, $R$. $B r$.

(a) Anther, (b) connective produced into a point. 


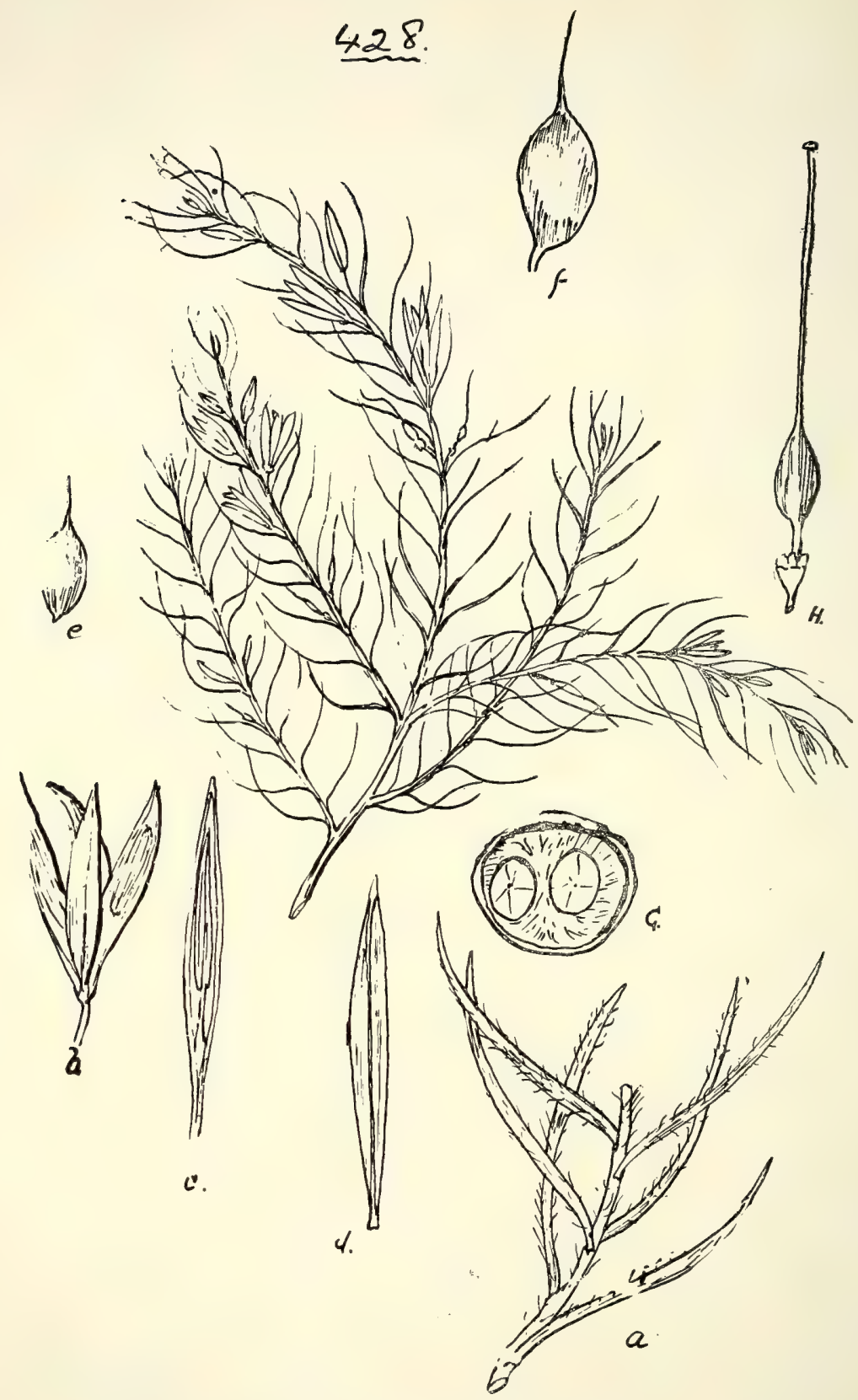

428. Persoonia tenuifolia, $R$. Br.

(a) Portion of branchlet with leaves, (b) a flower, (c) frontal view of a perianth segment, (d) dorsal view do., (e) young fruit, (f) mature do., (g) sect. do., (h) pistil. (a), (h), (c), (d), (g), and (h) enl.; (e) and (f) nat. size. 
CXI. PROTEACEÆ.

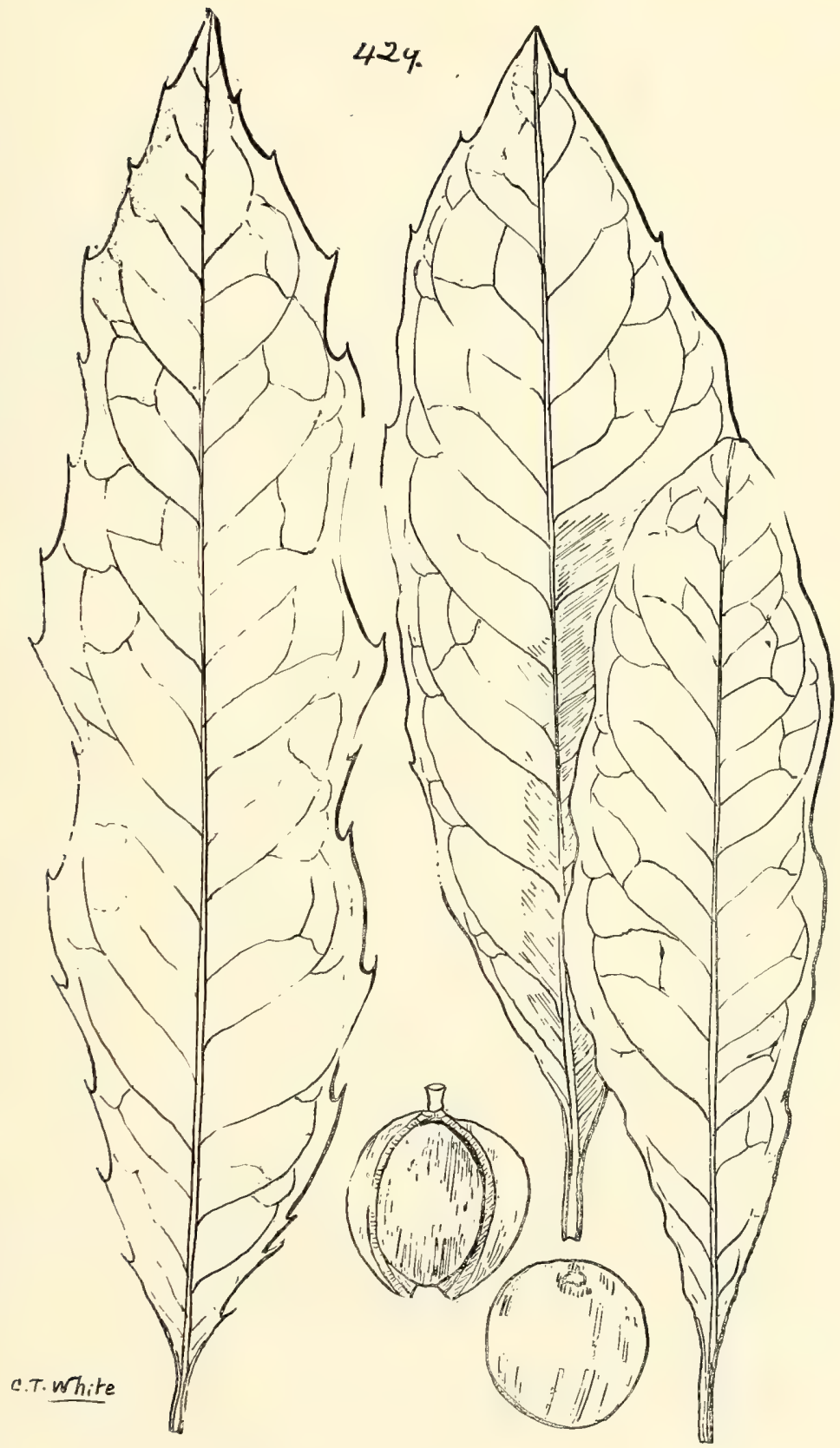

429. Macadamia ternifolia, $F . v . M$. 


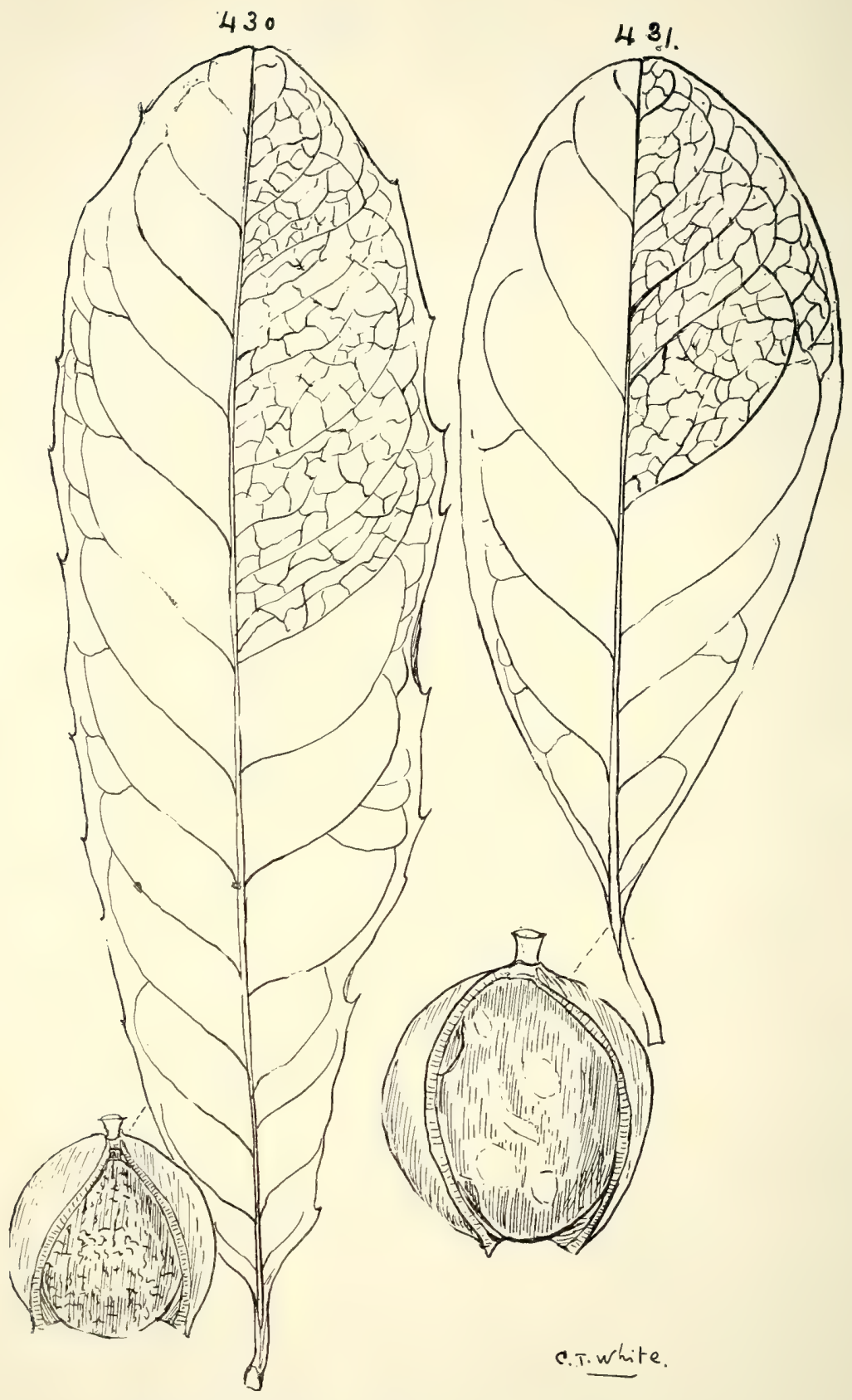

430 and 43 I. Forms of Macadamia ternifolia, F. $v . M$. 


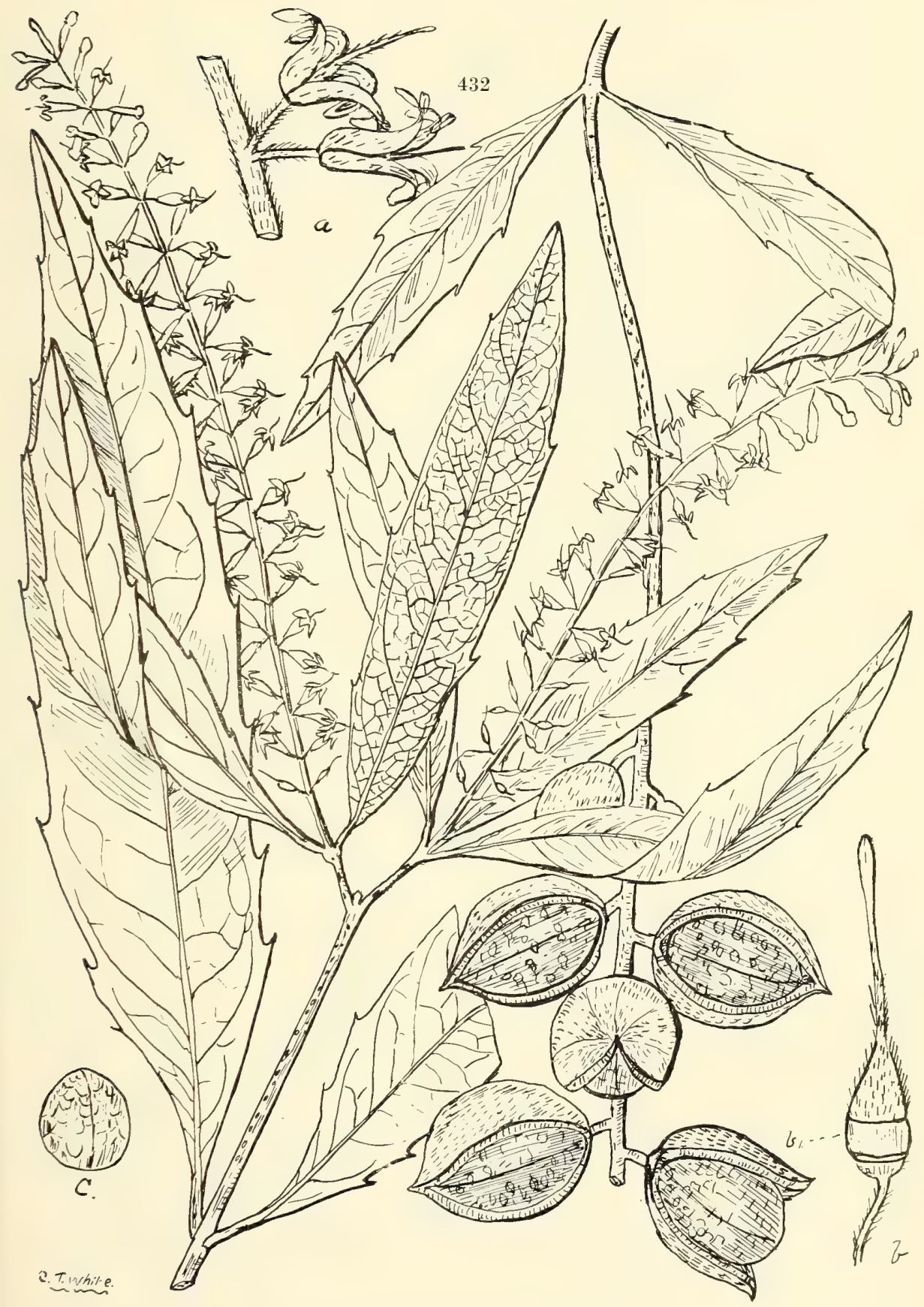

432. Macauamia Lowit, Bail.

(a) Two flowers on the rhachis, (b) pistil, (bI) hypogynous glands united in a ring, 


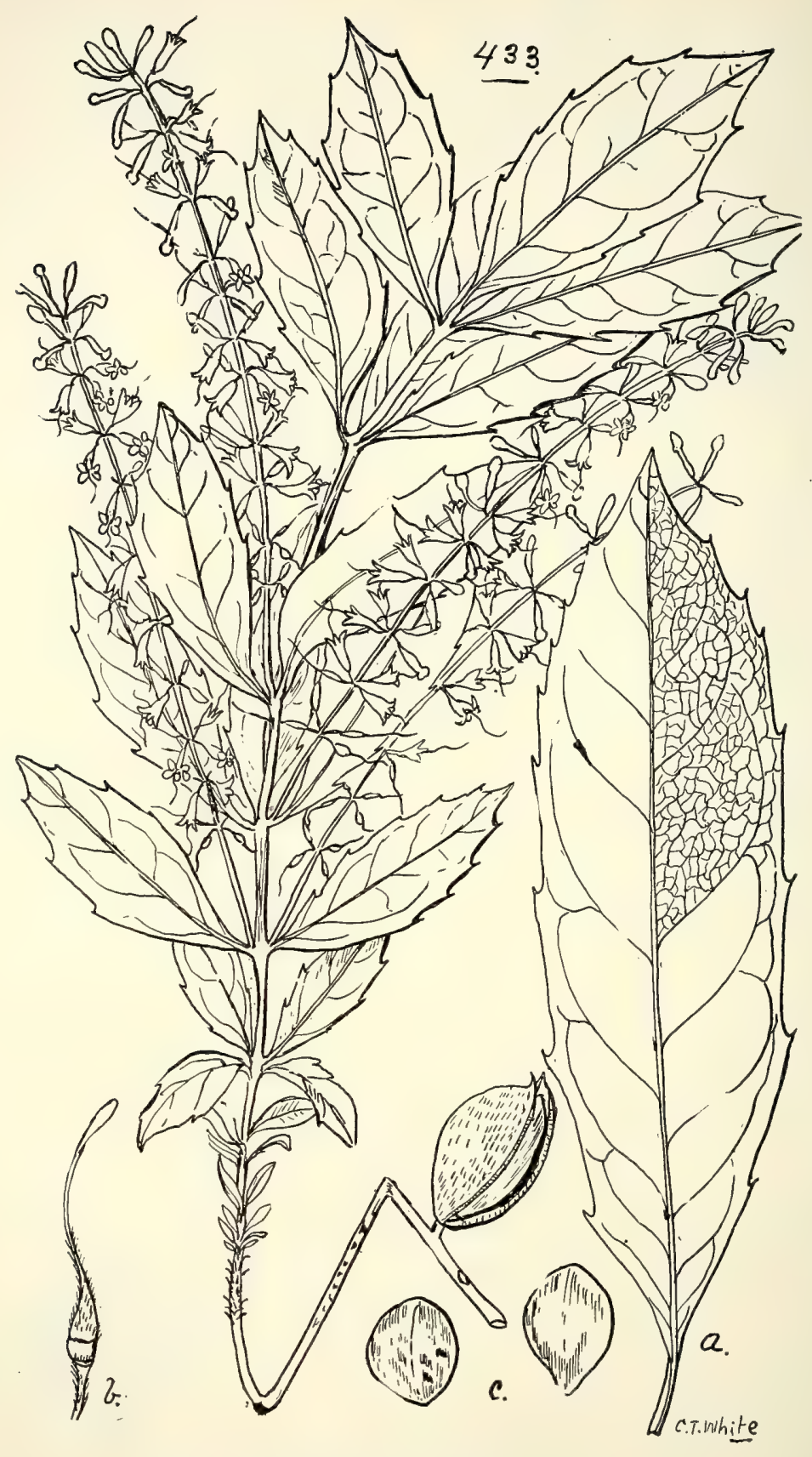

433. Macadamia minor, Bail.

(a) Leâ̂ from barren branchlet, (b) pistil, (c) nut showing putamen. (a) and (c) nat. size; (b) enl. 


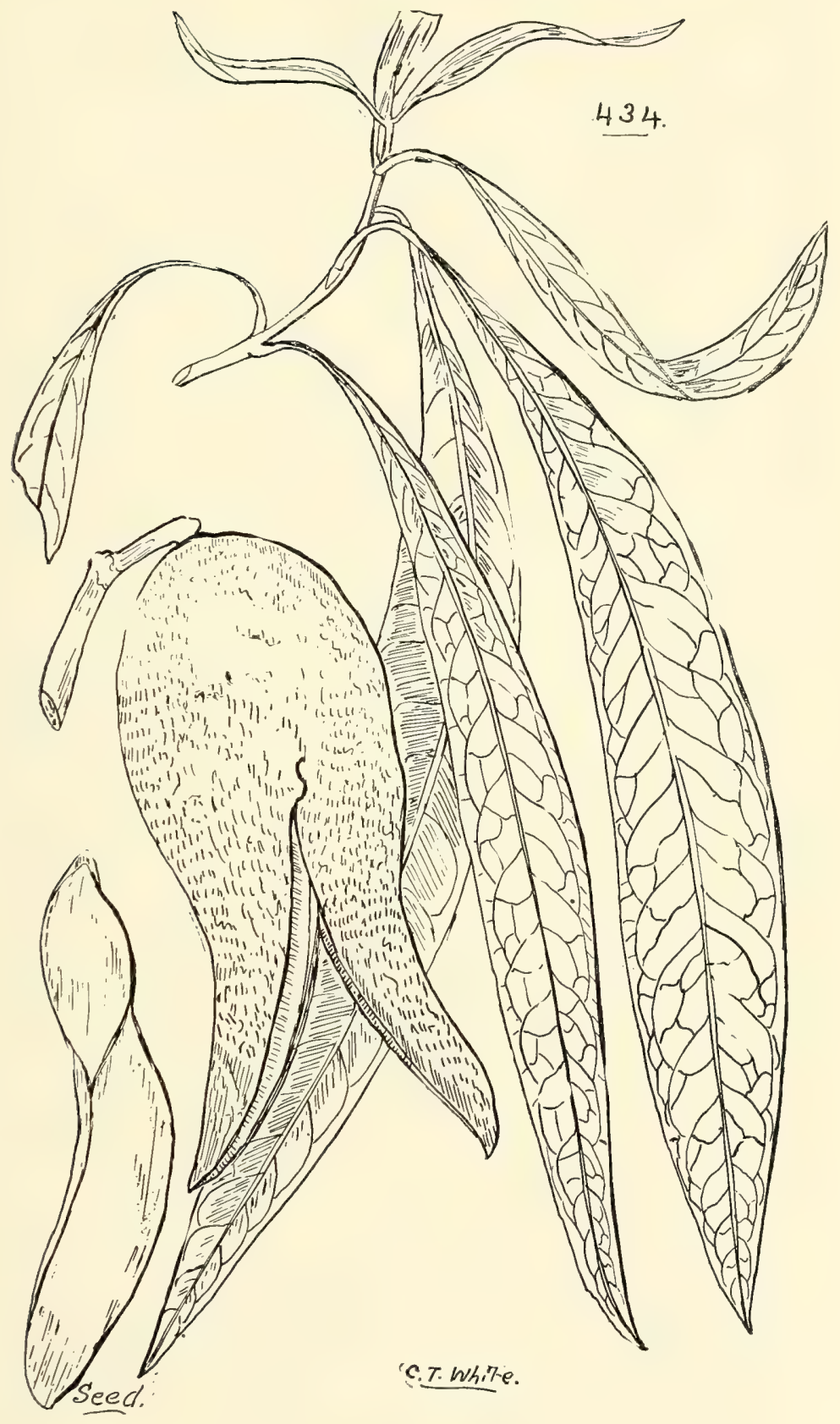

434. Xylomelum salicinum, $A . C u n n$. 


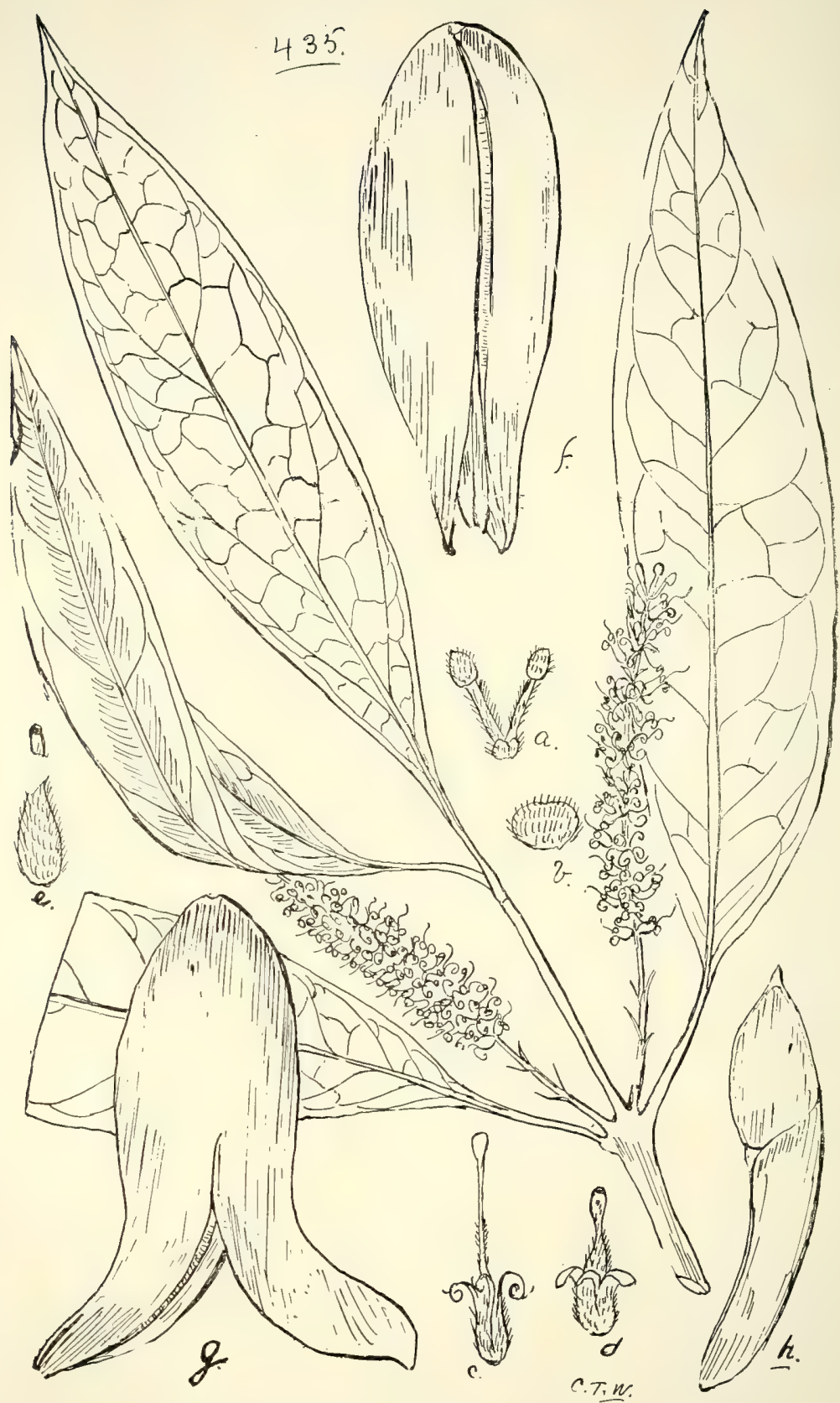

435. Xylomelum Scottianum, F. v. $M$.

(a) Flower-buds, (b) bract, (c) flower, (d) flower more advanced, (e) ovary, (f) fruit, (g) fruit opening, (h) seed. (a)--(e) enl.; (f)-(h) nat. size. 


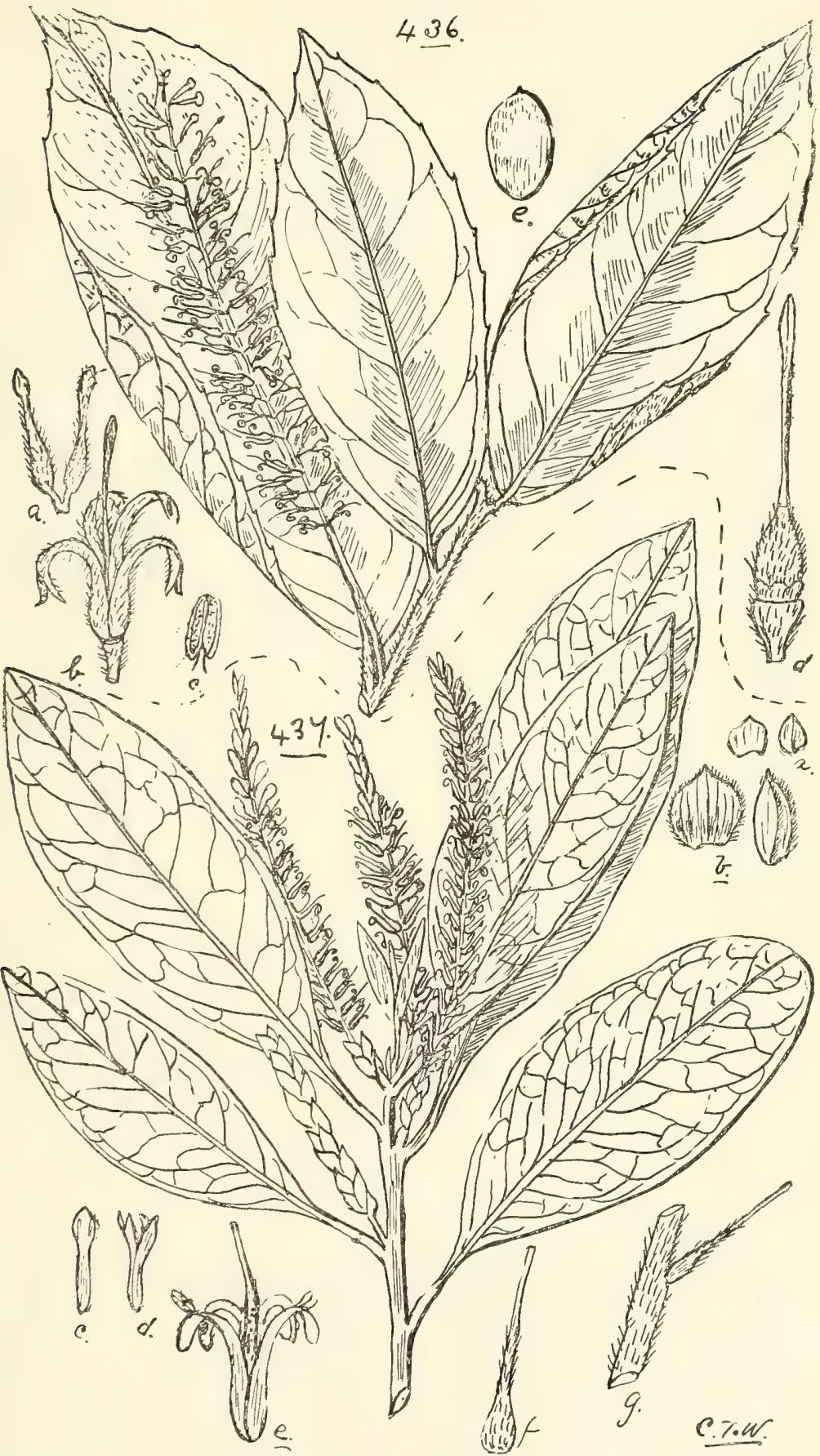

436. Helicia Nortoniana, Bail.

(a) Pair of flower-buts, (b) flower, (c) anther, (d) pistil, (e) fruit. (a)-(d) enI. ; (e) nat. size. 


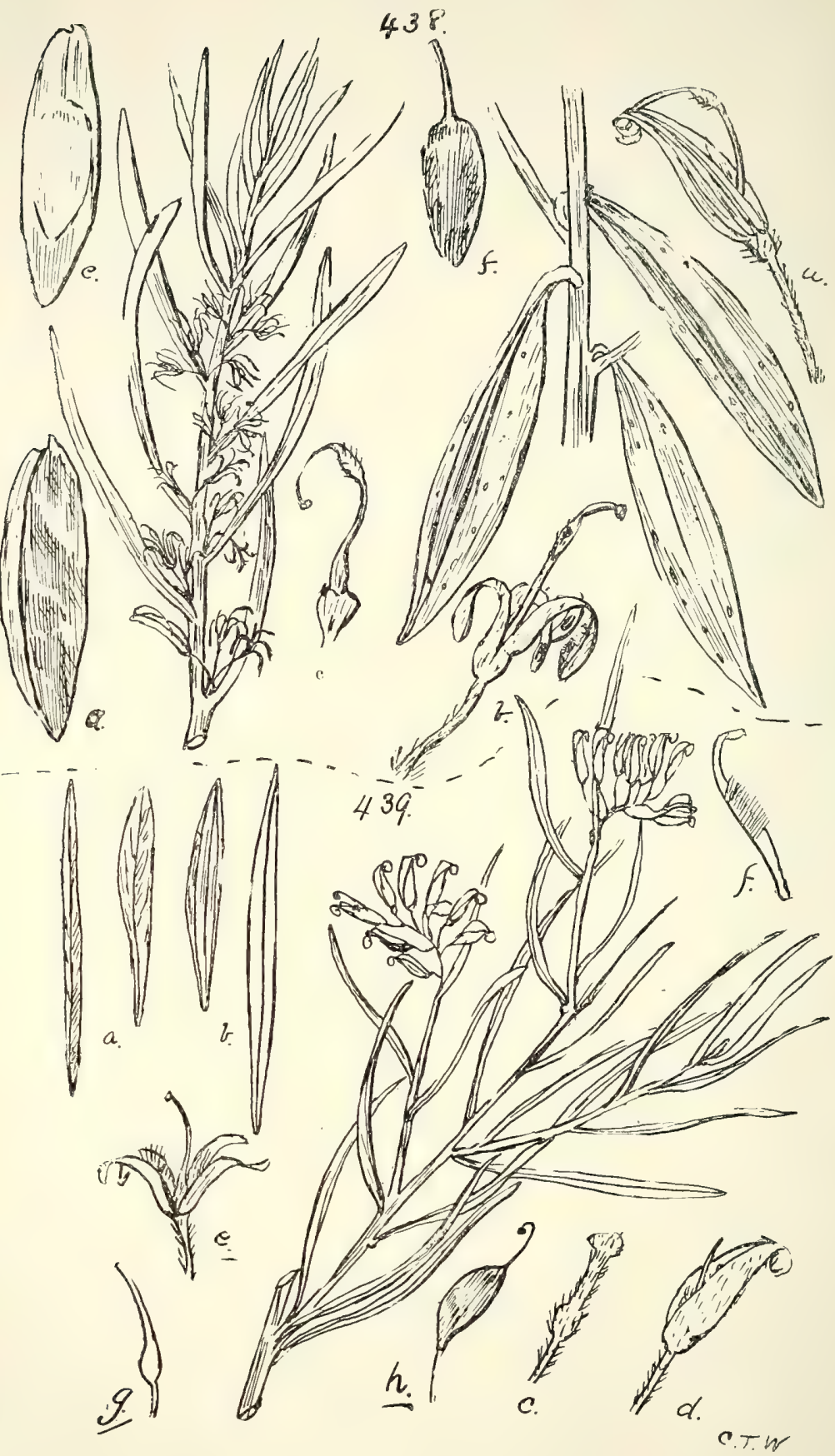

438. Strangea linfaris, Meissn.

(a) Young flower, (b) flower, (c) pistil, (d) exocarp, (e) endocarp, (f) pendulous seed. (a)-(c) enl.; (d)-(i) nat. size.

439. Grevillea leiophylla, $F . v . M$.

(a) Leaves, upper surface, (b) leaves, under surface, (c) flower-bud, (d) unopened flower, (e) flower, (f) a perianth segment, (g) pistil, (h) fruit. (a), (b), and (h) nat. size. 


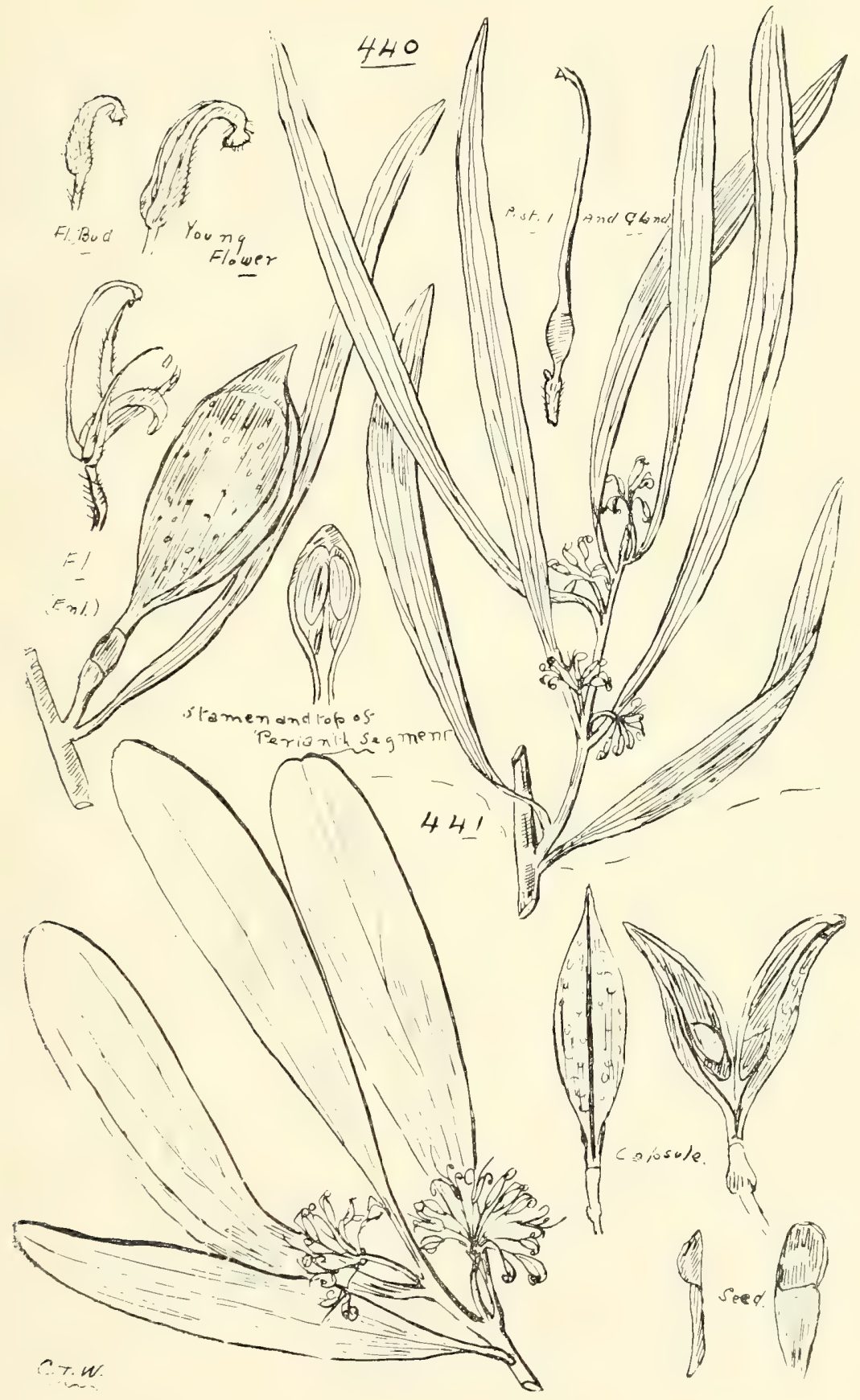

440. Hakea arborescens, $R$. $B r$.

441. H. pedunculata, F. $v \cdot M$. 
Helicia, Lour. = Cyanocarpus, Bail.

Youngiana, C. Moore and F.v. M. = Macadamia Youngiana, F. v. M.

Cribbiana, Bail.

glabriflora, F.v. M.

ferruginea, F.v. M.

var. tropica, Bail., n. var. = H. ferruginea, var., Bail., Q1.

Woods (I886), No. 33IA; later editions, No. 33IB.

Wood pinkish; useful for cabinet-work.

Nortoniana, Bail. = Cyanocarpus Nortoniana, Bail. (Fig. 436.)

australasica, F. $\cdot M$.

Heyana, Bail.

Orites, R. Br.

excelsa, R. Br.-A good cabinet-wood.

fragrans, Bail. (Fig. 437.)

Kermadecia, Brongn.

Bleasdalii, Benth.

Hicksbeachia, F.v. M.

pinnatifolia, F.v.M.-Nut edible.

Strangea, Meissn.

linearis, Meissn. = Grevillea Strangea, Benth. (Fig. 438.)

Grevillea, R. Br.

Section I.-Eugrevillea.

Series Hebegyne.

chrysodendron, R. Br._- Abergubber" of St. George natives Banksii, R. Br. cirsiifolia, Meissn.

ilicifolia, R.Br., var. Scortechinii, Bail.

Section II.--Ptychocarpa.

floribunda, R. Br.

Helmsiæ, Bail.

Section III.-Plagiopoda.

Goodlii, R. Br.

venusta, $R . B r$.

longistyla, Hook.

juncifolia, Hook.

Kennedyana, F.v.M.

singuliflora, F. $\%$.

Section IV.-Calothyrsus.

Wickhamii, Meissn.

heliosperma, $R$. Br. 
Grevillea-contd.

refracta, $R . B r$.

var. ceratophylla, Benth.

Dryandri, R. Br.

stenobotrya, F. v. M.

polystachya, R. Br.

var. hebestachya, Benth.

robusta, A. Cunn.-Silky Oak; the original tree so called.

"Tuggan Tuggan" of Brisbane River natives. Exudes

a gum which may be used as a substitute for gum-arabic

(Dr. Lauterer).

Section V.-Cycloptera.

striata, R. Br.-Beefwood. "Willer" of St. George, "Arroo-in" of Princess Charlotte Bay, and "Arrongg" of Palmer River natives. Exudes a resin which may be used medicinally as a substitute for pitch (Dr. Lauterer). The foliage considered good food for stock in time of drought. Wood useful for staves and cabinet-work.

mimosoides, R. Br.

Hilliana, F.v.M.-Wood prettily marked.

pinnatifida, Bail. = Kermadecia pinnatifida, Bail--Brown

Silky Oak. Wood useful for cabinet-work and cooper's work.

gibbosa, R.Br.- "Nalgo" of Cardwell natives (R.B.H.).

Wood dark-brown, of a greasy nature.

Section VI.-Lissostylis.

Series I.-Sericeæ.

leiophylla, F.v.M. (Fig. 439.)

linearis, R. Br.

Hakea, Schrad.

Section I.-Grevilleoides.

chordophylla, F.v.M.

lorea, R. Br.-Cat-o' nine-tails.

Ivoryi, Bail.

macrocarpa, A. Cunn.

arborescens, R.Br. (Fig. 440.)

Persiehana, F.v.M.

trineura, F.v.M.- "Miera" of Cardwell natives (R.B.H.).

Section II.-Euhakea.

eriantha, $R \cdot B r$.

Series I.-Pubifloræ.

pugioniformis, Cav.

vittata, $R . B r$. 
Hakea-contd.

Series II.-Glabrifloræ.

pedunculata, F.v.M.-Wood dark-brown, nicely marked. (Fig. 44I.)

saligna, Knight.

purpurea, Hook.

leucoptera, R. Br.

gibbosa, $C a v$.

microcarpa, R. Br.

Section III.-Conogynoides.

plurinervia, F.v. M.

Series Nervosæ.

dactyloides, Cav.

Carnarvonia, $F . v \cdot M$.

araliæfolia, F.v.M.-Red Oak. "Niah" of natives. Evelyn Scrub. Wood useful for coopers' and cabinet work.

Buckinghamia, F.v.M.

celsissima, F.v.M.-Flowers white, very fragrant.

Darlingia, $F \cdot v \cdot M$

spectatissima, F.v.M.- "Chalagar" of Barron River natives.

The wood useful to coopers and cabinet-makers.

? ferruginea, Bail., ex J. F. Bailey, Rept. Timb. Trees, Her-

berton District, Q1. Agric. J1. v. (I899) 402.-Brown Silky Oak.

Musgravea, F.v.M.

stenostachya, F.v.M.-An excellent cabinet-wood.

Hollandæa, F.v.M.

Sayeri, F.i'M.

Lamingtoniana, Bail.

Cardwellia, F.v.M.

sublimis, F.v.M.-Gold-spangled wood or Northern Silky

Oak. "Oongaary" of Tully River natives.

Stenocarpus, R. Br.

sinuatus, Endl--Tulip-flower or Firewheel; White Silky

Oak at Barron River; a good cabinet-wood.

salignus, R. Br.

var. Moorei, Benth.

var. concolor, Benth.

Cunninghamii, R. Br. (Fig. 442.)

Lomatia, R. Br.

fraxinifolia, F.v.M.

ilicifolia, R. Br.

silaifolia, R.Br.-An extremely variable plant. 
Lomatia-contd.

var. induta, $F, v \cdot M$.-Specimens from Woombye and Buderim Mountain (North Coast line) pubescent in all parts; under surface of leaves densely pubescent, upper surface sprinkled with scattered hairs.

Tribe V.-Embothrie飞.

Embothrium, Forst.

Wickhami, F.v.M.-Red Silky Oak; a cabinet-wood.

Tribe VI.-Banksiex.

Banksia, Linn.f.-- Chinana," Cardwell name for the genus (R.B.H.).

Section I:-Oncostylis.

ericifolia, Lim.

collina, R. Br.-Golden Candlestick.

Section II.-Eubanksia.

integrifolia, Limn. f.-Honeysuckle. "Pomera" and "Tchoomeroo" of Stradbroke Island and "Burthargong" of Bundaberg natives. A useful cabinet-wood.

var. paludosa, Benth.-Coastal localities in South Queensland.

var. compar, Bail. = B. compar, R. Br. (Fig. 443.)

dentata, Linn. f.- "Kabir" of Cape Bedford natives. Wood prettily marked; suitable for cabinet-work.

Section III.-Orthostylis.

latifolia, R.Br.-."Bumbar" of Stradbroke Island natives. The branchlets and main nerves of leaf sometimes a blood-red.

æmula, R.Br.-" Mintie" of Stradbroke Island and "Wallum" of Wide Bay natives. A useful cabinet-wood.

\section{Order CXII.-THYMELÆACE $Æ$.}

Tribe I.-Euthymel שeE.

Pimelea, B. et Sol.

Section I.-Thecanthes.

cornucopiæ, Vahl. sanguinea, $F, v \cdot M$.

Section II.-Calyptrostegia.

glauca, R. Br.

Subsection I.-Calyptridium. collina, R. Br. 


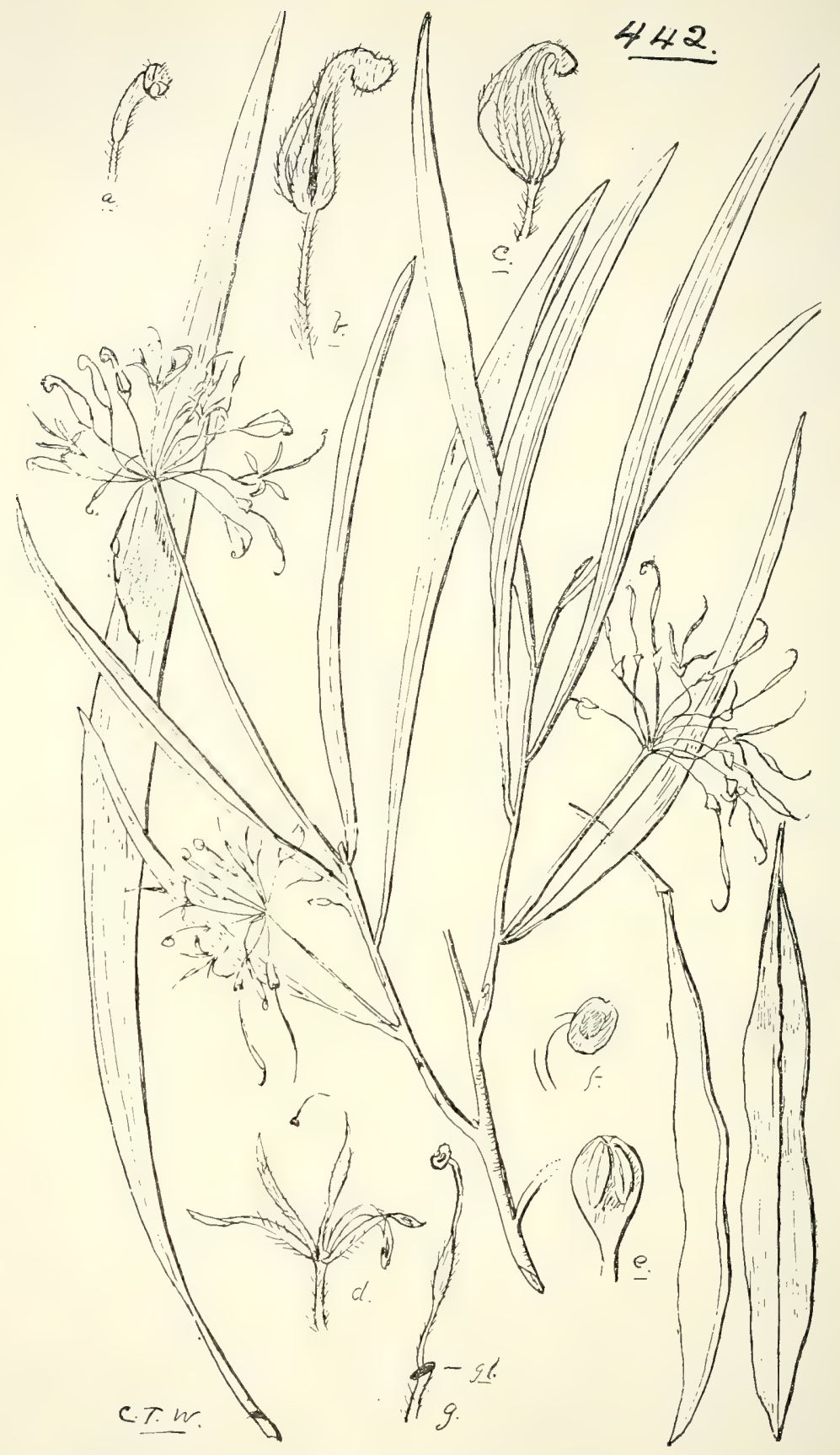

442. Stenocarpus Cunninghamit, $R$. $B r$.

(a) -(d) Flower in various stages, (e) top of perianth segment with anther, (f) stigma, (g) pistil, (gl) gland. (a)-(d) nat. size; (e)-(g) eril. 


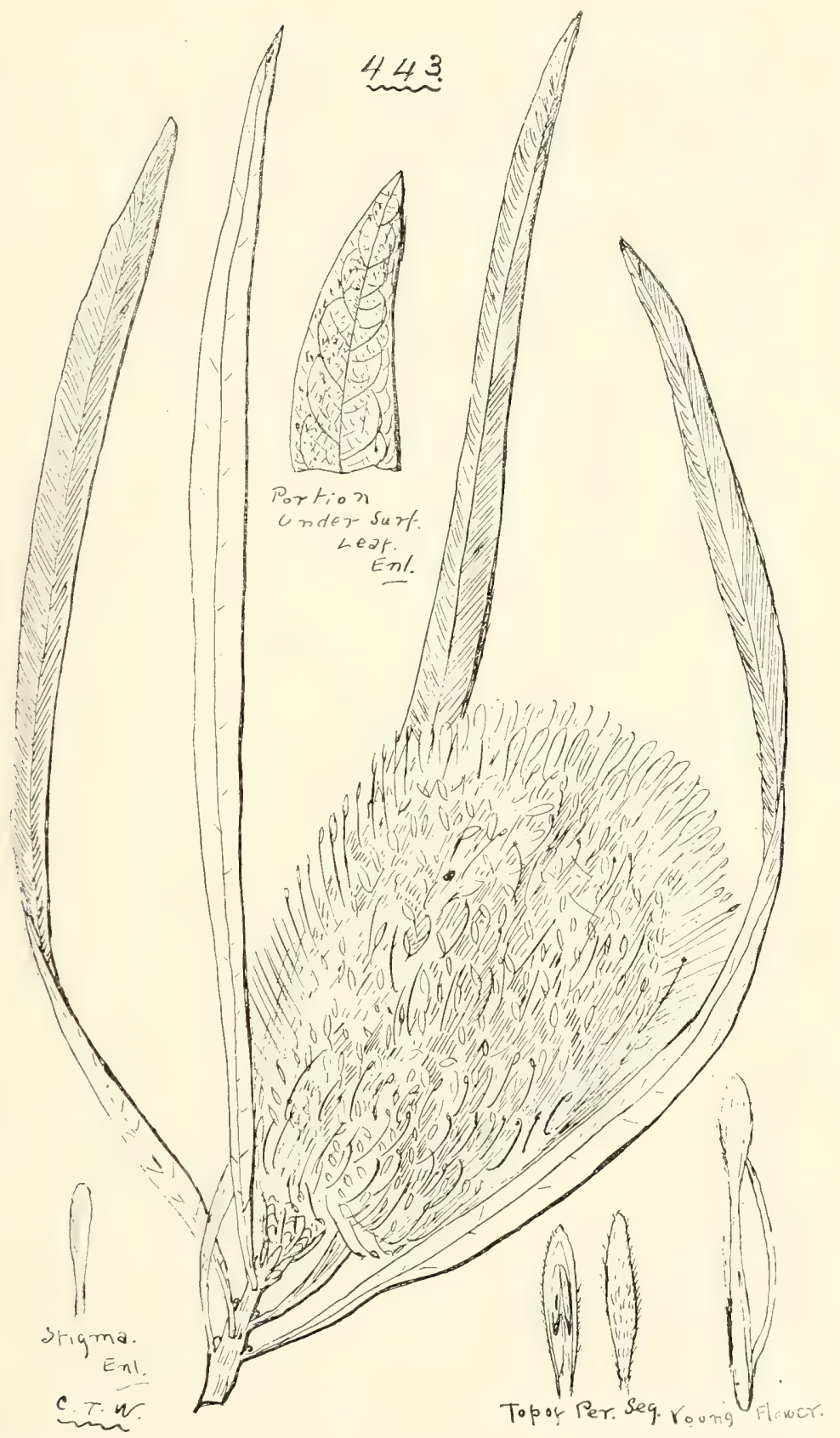

443. Banimsia integrifolia, Limn. f., var. compar, Bail. (B. compar, R. Br.). 


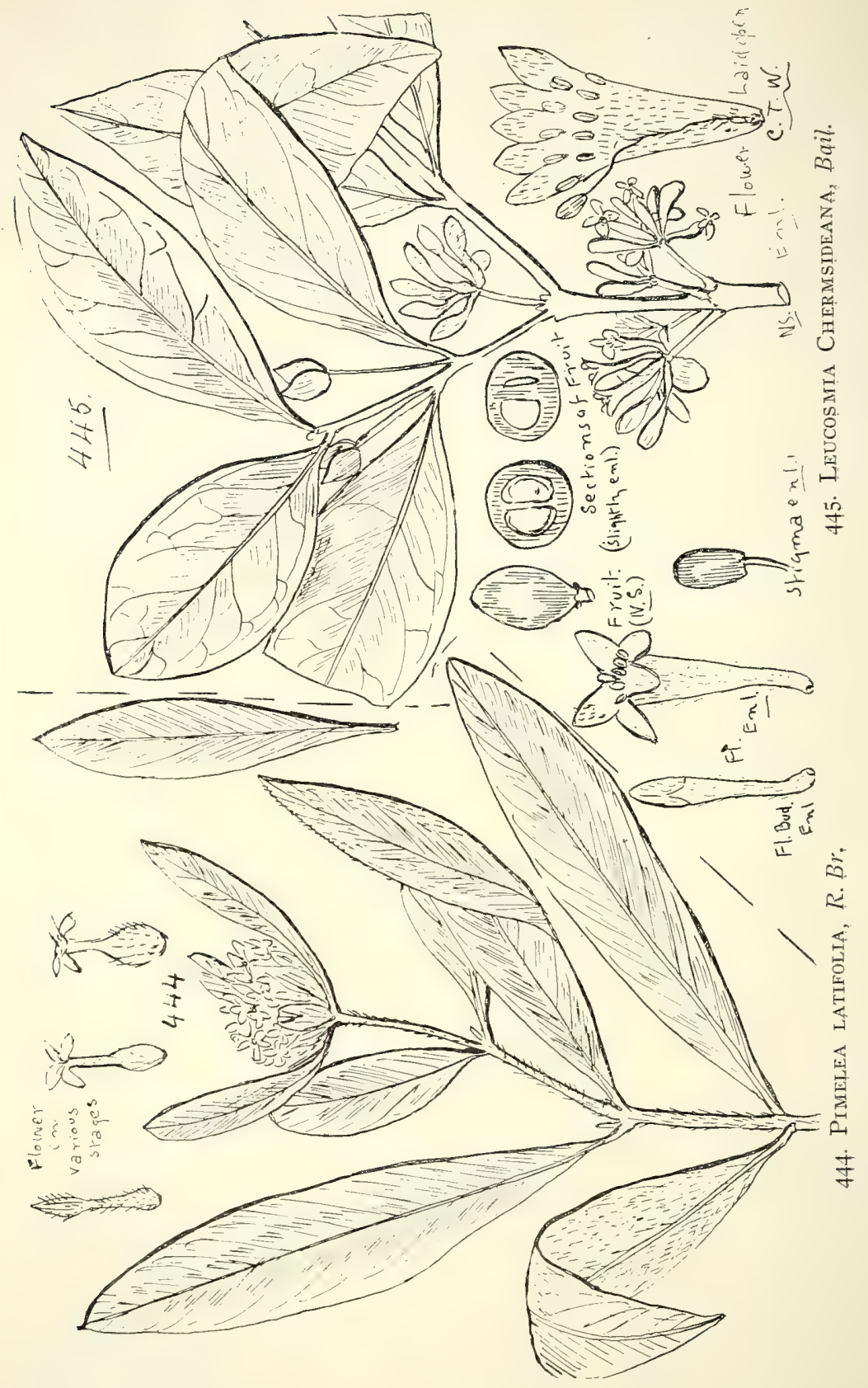



PLATE XIV。

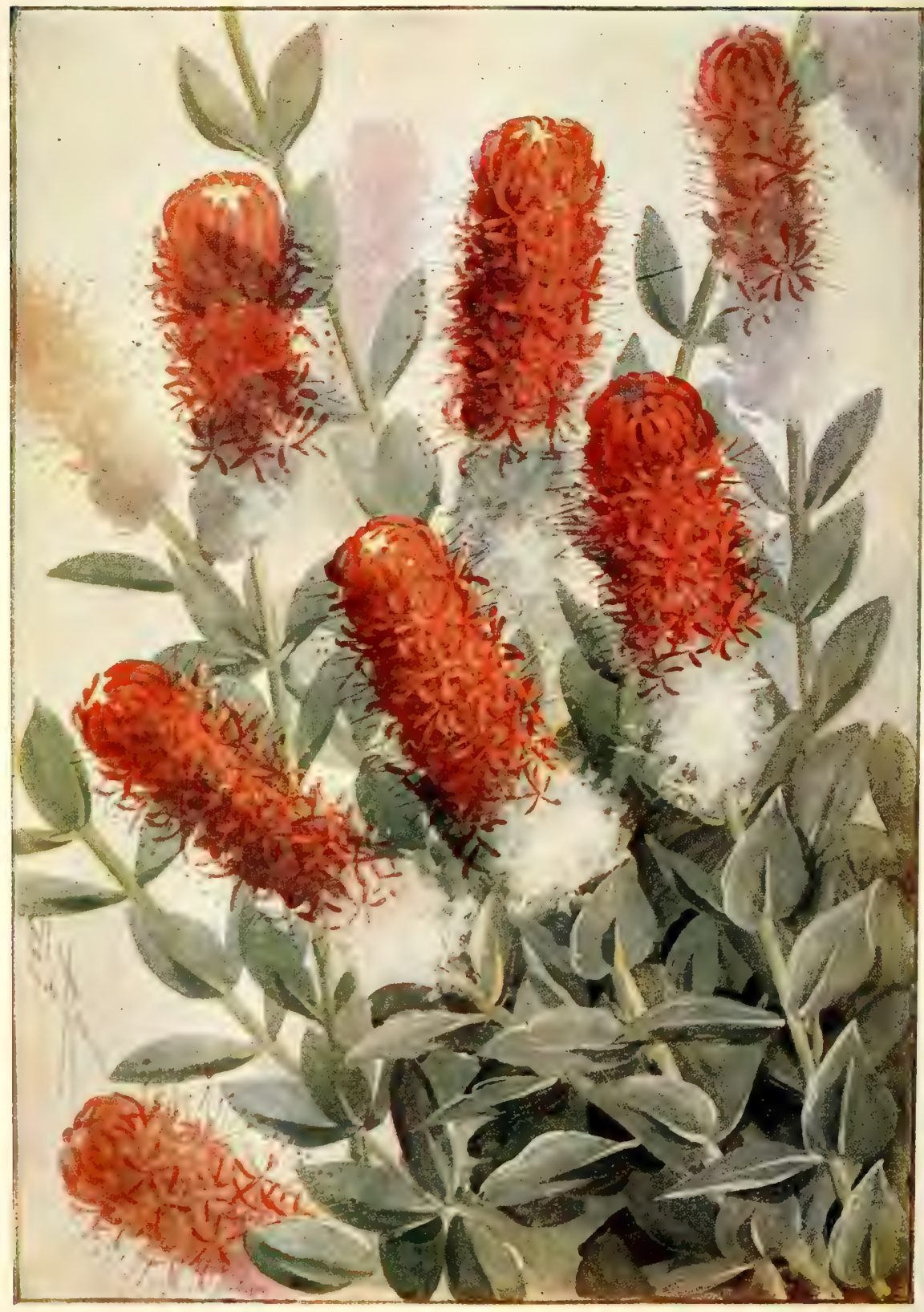

Pimelea hematostachya, F. v. M. 
Pimelea-contd.

linifolia, $S m$.

ligustrina, Labill.

Subsection II.-Choristachys.

hrematostachya, F.v.M.--Poisonous to stock. (Plate XIV.) latifolia, R.Br. (Fig. 444.)

simplex, F.v.M.

sericostachya, F.v.M.

var. parvifolia, Benth.

trichostachya, Lindl.

leptostachya, Benth.

Section III.-Dithalamia.

leptospermoides, F.v.M.

microcephala, R. Br._ "Yackahber" of St. George natives.

pauciflora, R.Br.-This has been considered poisonous to stock.

flava, R. Br.

Bowmanni, F.v. M.

Section IV.-Epallage.

penicillaris, $F \cdot v \cdot M$.

curviflora, R. Br.

var. sericea, Benth.

var. pedunculata, Benth.

altior, F.v.M.

Wikstromia, Endl.

indica, C. A. Mey.-Poisonous to stock.

Tribe II.-Phalerier.

Leucosmia, Benth.

Chermsideana, Bail. (Fig. 445.)

Phaleria, Jack.

Blumei, Benth.

Neumanni, F.v.M. (Fig. 446.)

clerodendron, F.v.M.

\section{Order CXIII.-ELÆAGNACEÆ.}

Elæagnus, Linn.

latifolia, Linn._-"Millai-millai" of Atherton natives. Wood yellow. At Kin Kin the fruit is eaten and is said to be pleasant to the taste. (Fig. 447.) 


\section{SERIES VI.-ACHLAMYDOSPOREÆ.}

\section{Order CXIV. - LORANTHACE $\nexists$.}

TRIBE I.-EulORANTHE.

Loranthus, Linn.-Mistletoes. I may here remark that, while collectors frequently note the trees or shrubs upon which they find the species growing, yet these mistletoes are by no means particular, as they will thrive on any tree or shrub upon which you place the fruit.

Anthers versatile, oblong; petals free.

celastroides, Sieb.

myrtifolius, A. Cunn.

Bidwillii, Benth.

Anthers adnate, linear; petals united by the middle or higher up.

longiflorus, Desr.- The bark of this parasite is said to contain Io per cent. of tannin.

Beauverdiana, Bail., Q1. Agric. J1. xxi. $294=$ L. tenuifolius, Bail., Q1. Bot. Bull. xvi. (Fig. 449.)

dictyophlebus, F. v. M. - "Durandool" of Barron River natives.

alyxifolius, F.v. M.

odontocalyx, F.v.M.

linearifolius, Hook.

Exocarpi, Behr.- "Thappin" of Cloncurry natives.

Anthers adnate, linear; petals free.

signatus, F.v. $M$.

Maytenifolius, Gray.

sanguineus, $F . v . M$.

bifurcatus, Benth.

linophyllus, Fenzl.

pendulus, Sieb.

var. parviflorus, Benth.

Quandang, Lindl.

var. amplexifolius, Benth.

var. Bancroftii, Bail. (Fig. 448.)

conspicuus, Bail. (Fig. 448 bis.)

grandibracteus, F.v. M.

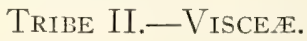

Viscum, Linn.-Mistletoes.

orientale, Willd. (Fig. 450.)

angulatum, Heyne. (Fig. 45I.) 


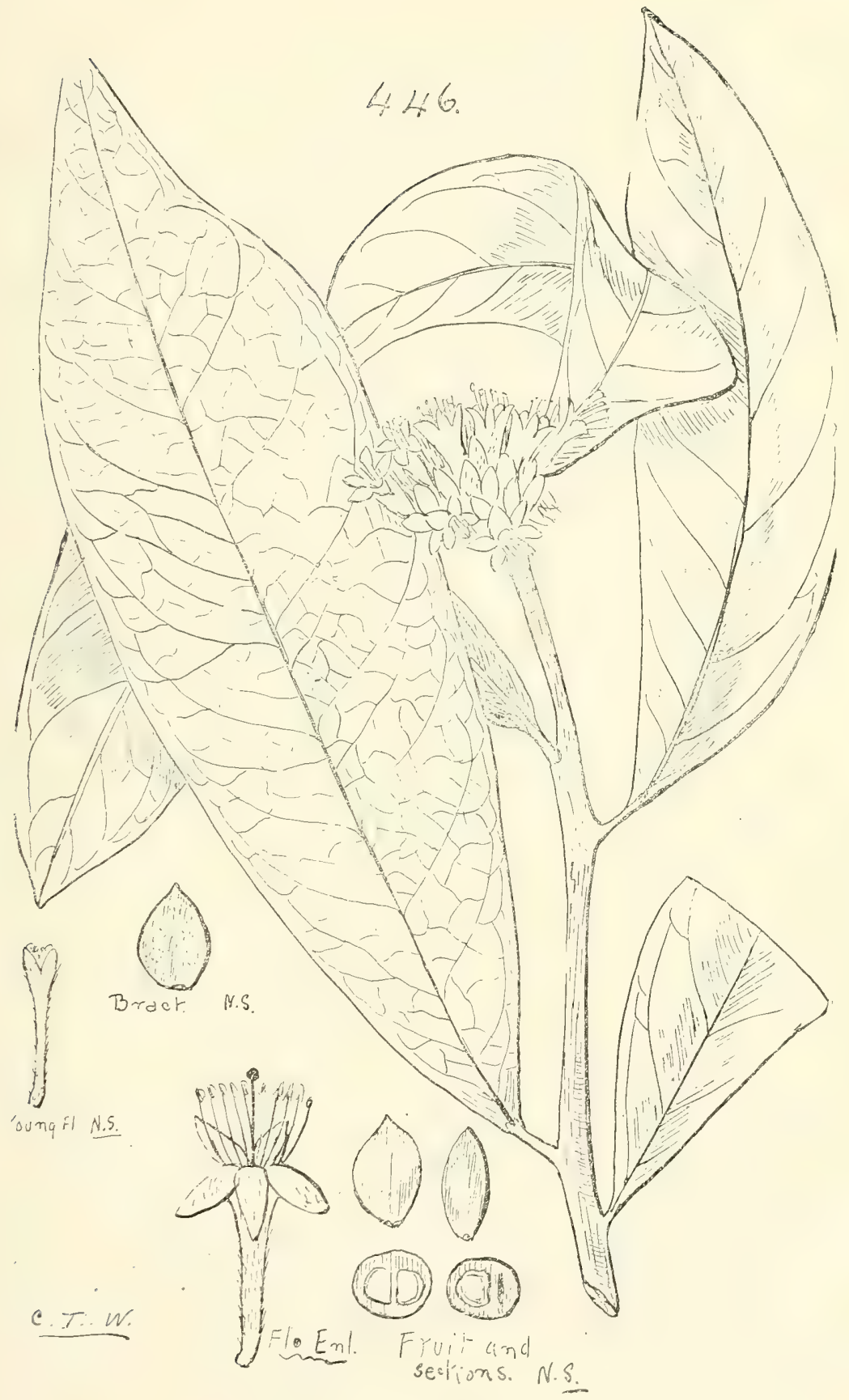

446. Phaleria Neumanni, F. v. M. 


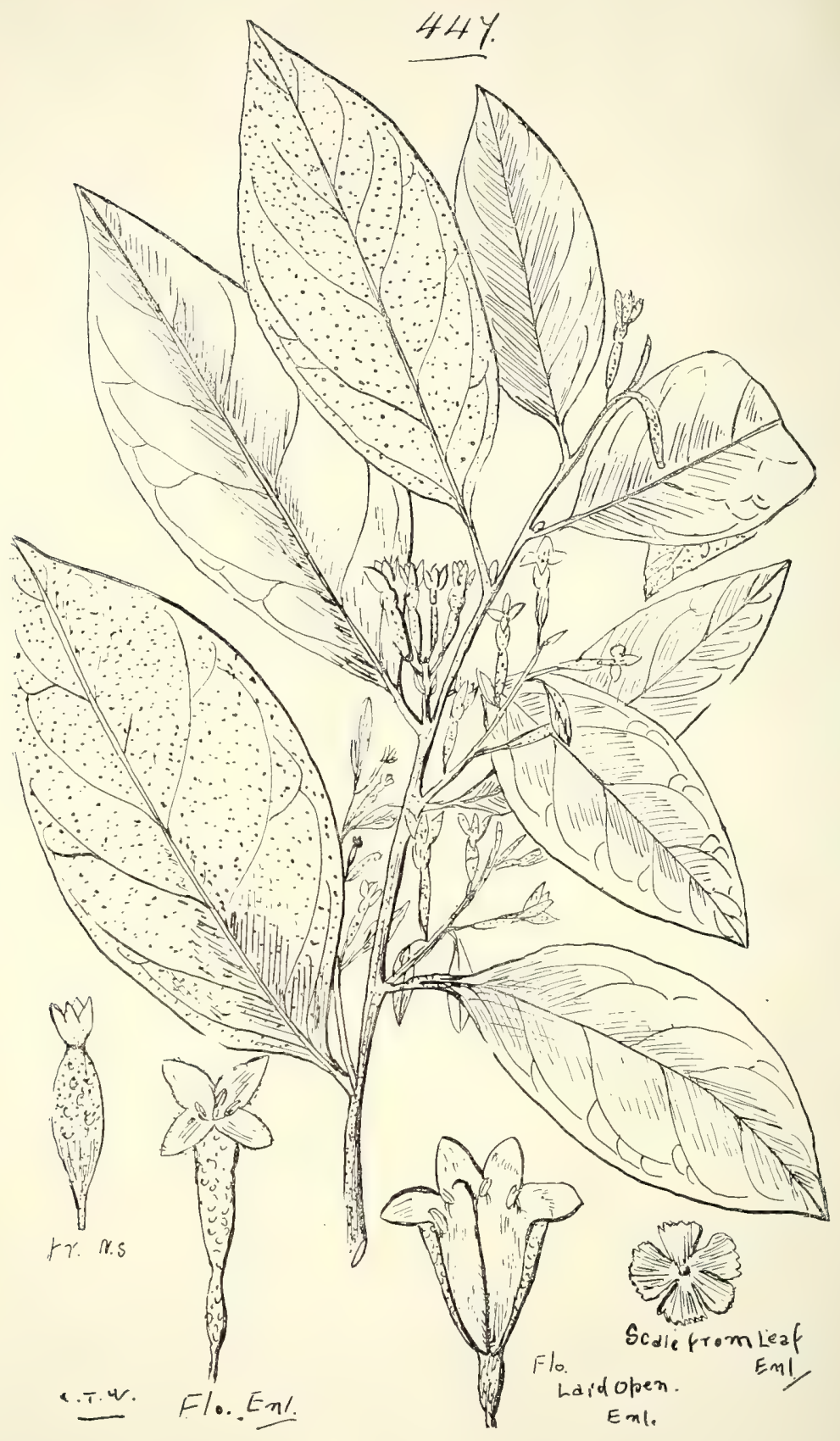

447. Eleaginus latifolia, Linn. 


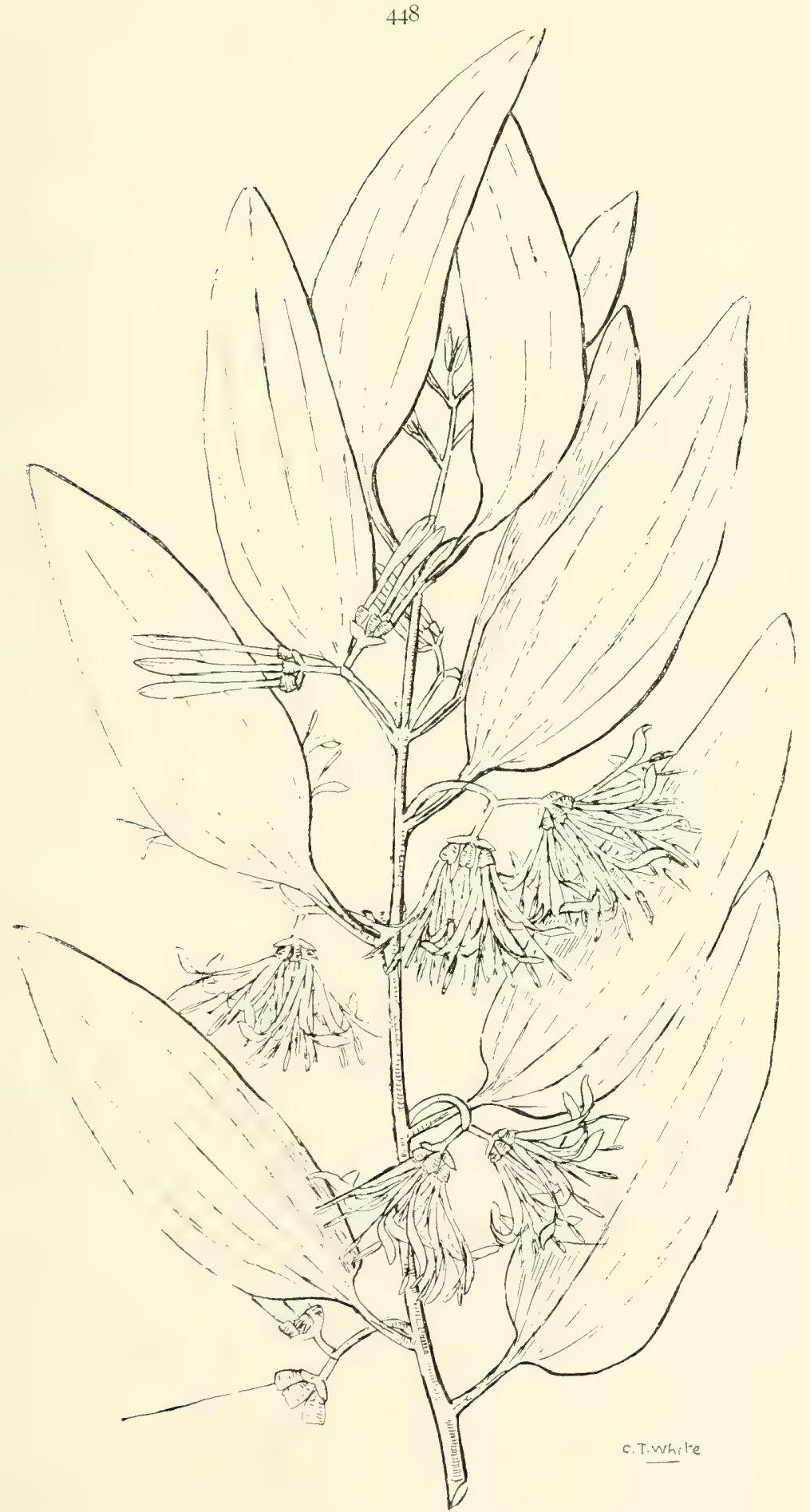

448. Loranthus QuANdang, Lindl, var. Bancroftit, Bail. n. var. 


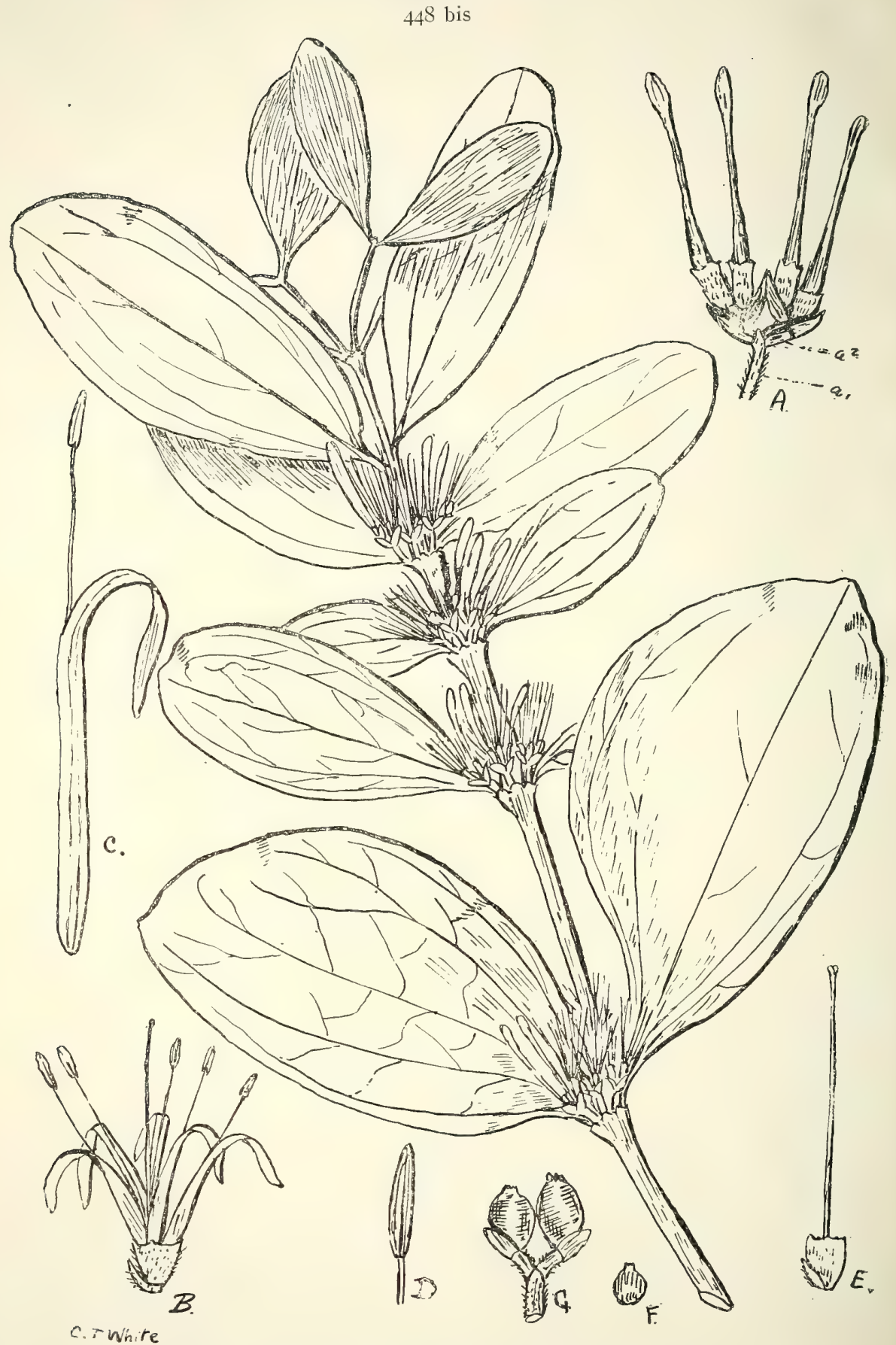

448 bis. Loranthus conspicuus, Bail., n. $s p$.

(A) Unopened flowers, (aI) peduncle, (a2) bract, (B) open flower, (C) petal and stamen, (D) an anther, (E) pistil, (F) fruit, n.s., (G) fruit from Broadsound, slightly enlarged. 


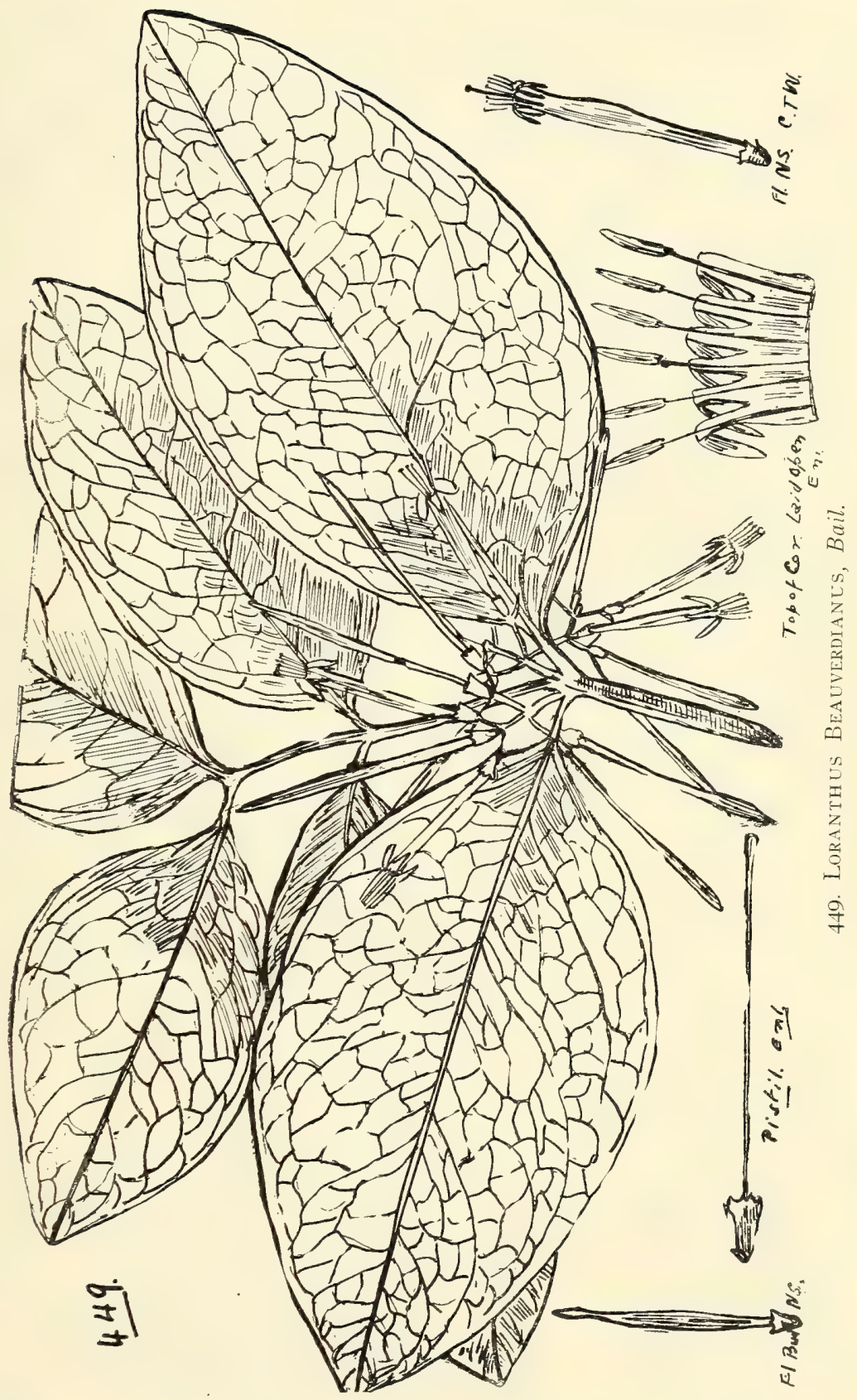




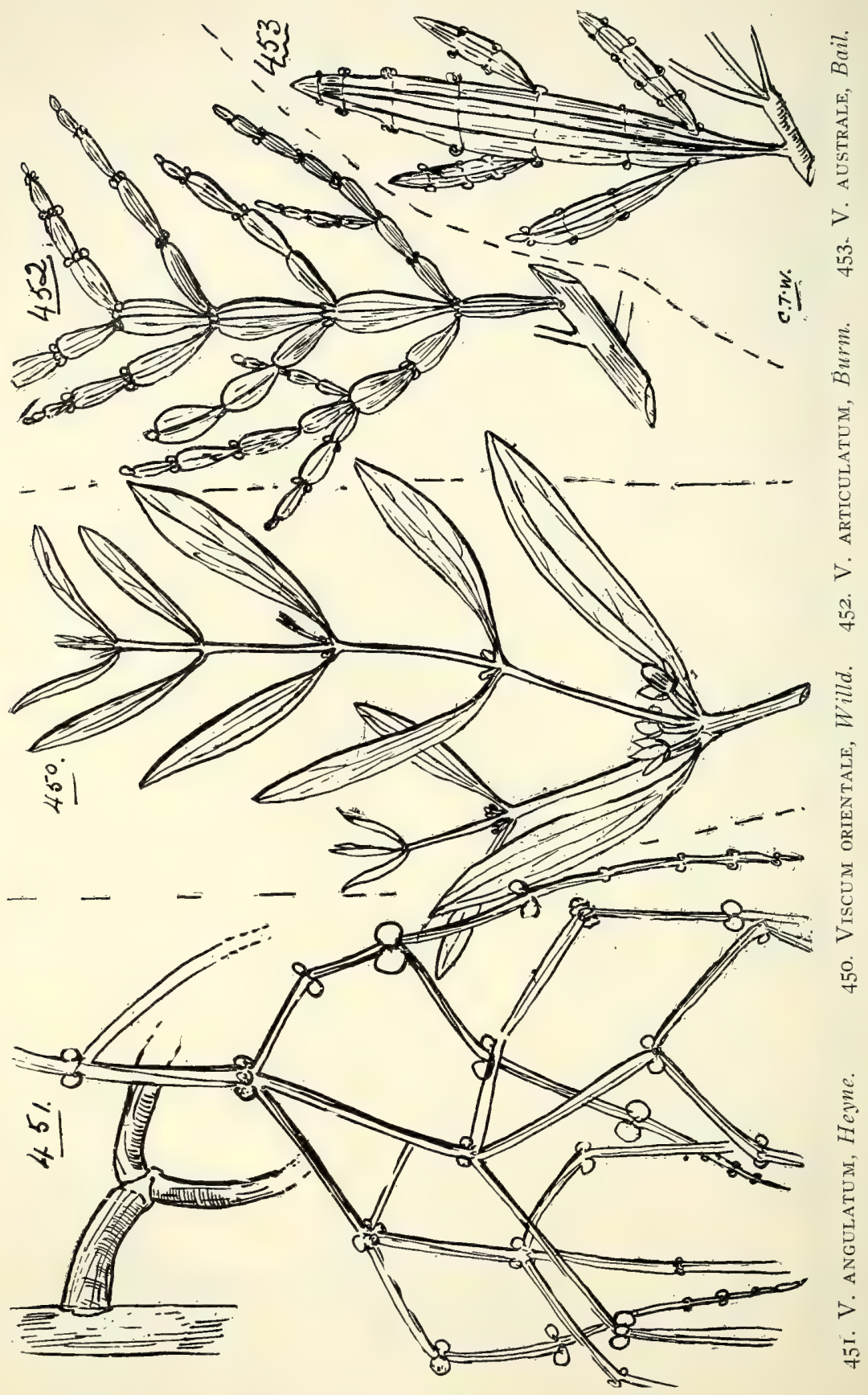


CXV. SANTALACE仺.

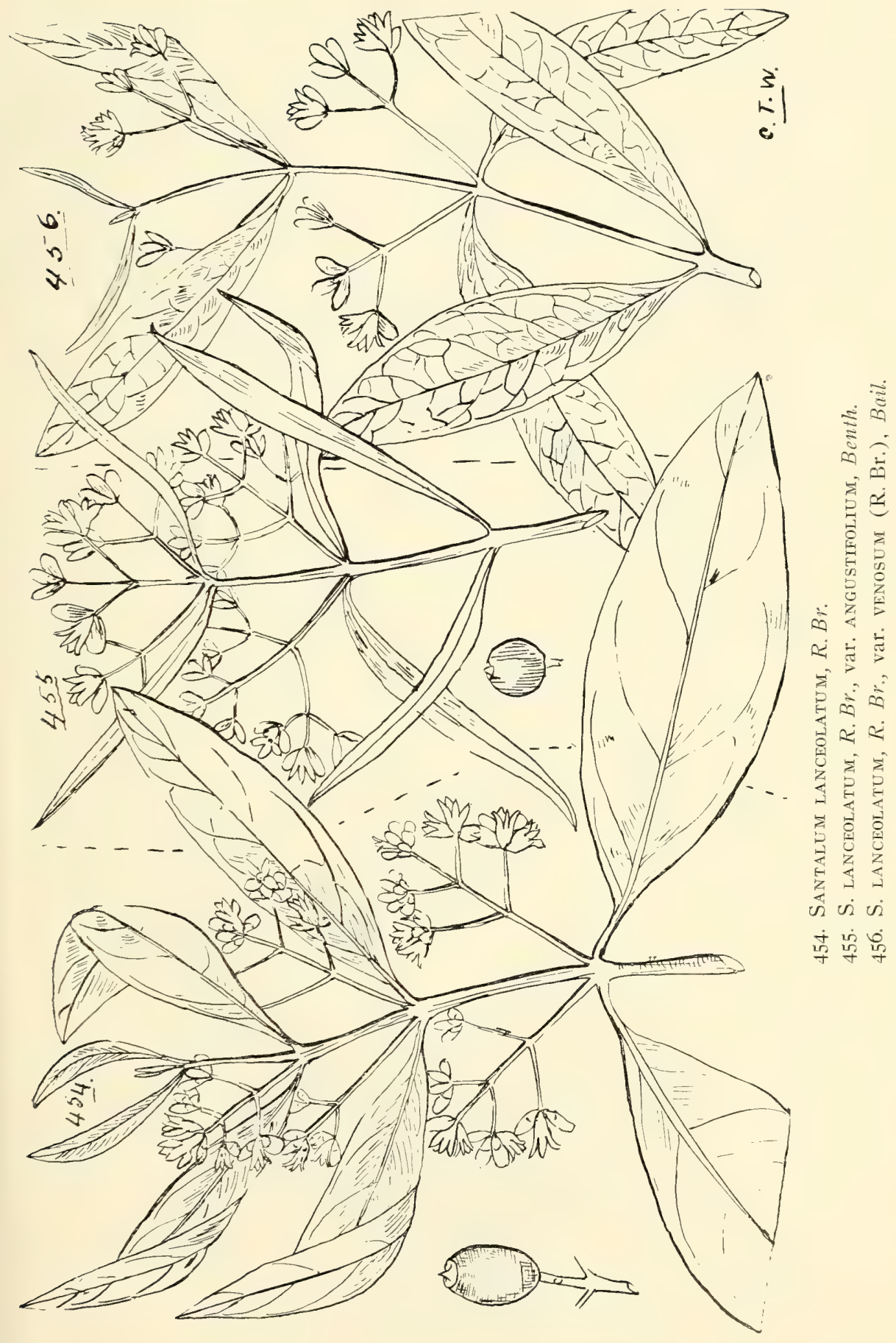




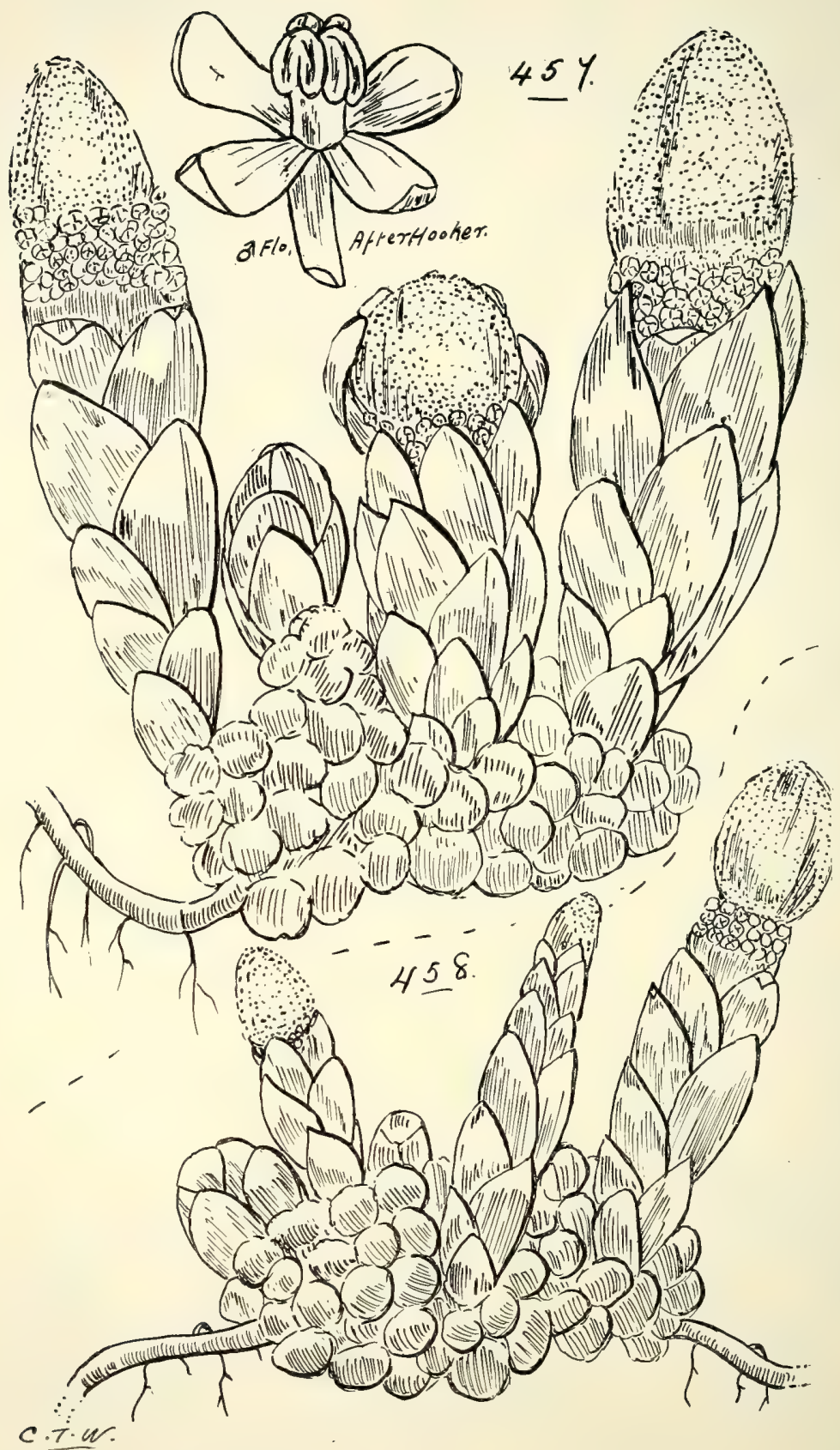

457. Batanophora fingosa, Forst.

458. B. Fungosa, Forst., forma extratropica, Bail : 
Tiscum-contd.

articulatum, Burm.-Jointed Mistletoe. This, Sir J. D.

Hooker says, in F1. Brit. Ind., is rather V. japonicum,

Thunb. (Fig. 452.)

australe, Bail., Q1. Agric. J1. xxvi. (I9I I) I99. (Fig. 453.)

Notothixos, Oliv.

incana, Oliv.

cornifolius, Oliv.

z'ar. subaureus. Bail.-Golden Mistletoe.

\section{Order CXV.-SANTALACEÆ.}

Thesium, Linn.

Tribe I.-ThesieÆ.

australe, $R . B r$.

Tribe II.-OSYridex.

Santalum, Linn.

lanceolatum, R. Br._- "Bolan" of Palmer River and "Tharragibberah" of Cloncurry natives. (Fig. 454.)

var. angustifolium, Benth. (Fig. 455.)

var. venosum, Bail. =S. venosum, R. Br.-Wood fragrant; known as Sandalwood, and has been an article of export for some time. (Fig. 456.)

obtusifolium, R. Br.

Fusanus, Linn.

acuminatus, R. Br.

persicarius, F.v.M.-Native Peach. "Tangber" of St.

George natives.

Choretrum, R. Br.

glomeratum, R. Br.

lateriflorum, R. Br.

Candollei, F.v.M.

Leptomeria, R. Br.

acida, R. Br.-Frazer Island Currant.

Billardieri, R. Br.

Anthobolus, R. Br.

Tribe III.-Anthobolede.

filifolius, $R \cdot B r$.

triqueter, R. Br.

leptomerioides, F.v.M.

Exocarpus, Labill.

latifolia, R. Br.- "Oringorin" of Rockhampton natives. A fragrant wood suitable for cabinet-work. 
Exocarpus-contd.

cupressiformis, Labill.-Cypress Cherry. "Por-nu-poy” of

Mount Cook and "Tchimmin-dillen" of Stradbroke

Island natives.

spartea, $R . B r$.

aphylla, R. Br.

\section{Order CXVI.-BALANOPHOREA.}

Tribe Eubalanophoreæ.

Balanophora, Forst.

fungosa, Forst. (Fig. 457.)

forma extratropica, Bail., n. form.-A more slender form than the typical plant not uncommonly met with in the tropical portions of Queensland. (Fig. 458.)

Hab: Parasitic on roots of trees, Mt. Eerwah, N.C. Line (H. Andreson).

Series VII.-UNISEXUALES.

\section{Order CXVII.-EUPHORBIACEÆ.}

Many of this Order yield Caoutchouc.

Tribe I.--Euphorbiex.

Euphorbia, Linn.-Fruit a Regma.

$$
\text { Section I.-Anisophyllum. }
$$

atoto, Forst.

Mitchelliana, Boiss.

var. glauca, Benth.

schizolepis, F.v.M.

var. ? glabra, Benth.

Schultzii, Benth.

australis, Boiss.-Mr. G. F. Leney, of Allora, writes me that

a decoction of this plant cured a very bad case of sciatica.

var. erythrantha, Benth.-Poisonous to sheep.

Drummondii, Boiss.-Caustic creeper; a well-known sheeppoison. Used by the natives in cases of snake-bite. The fresh milky sap possesses great healing properties, and is in constant use by bushmen.

var. rubescens, Benth.

Muelleri, Boiss.

alsinæflora, Baill.

Wheeleri, Baill.

carissoides, Baill. (Fig. 459.)

micradenia, Boiss. 


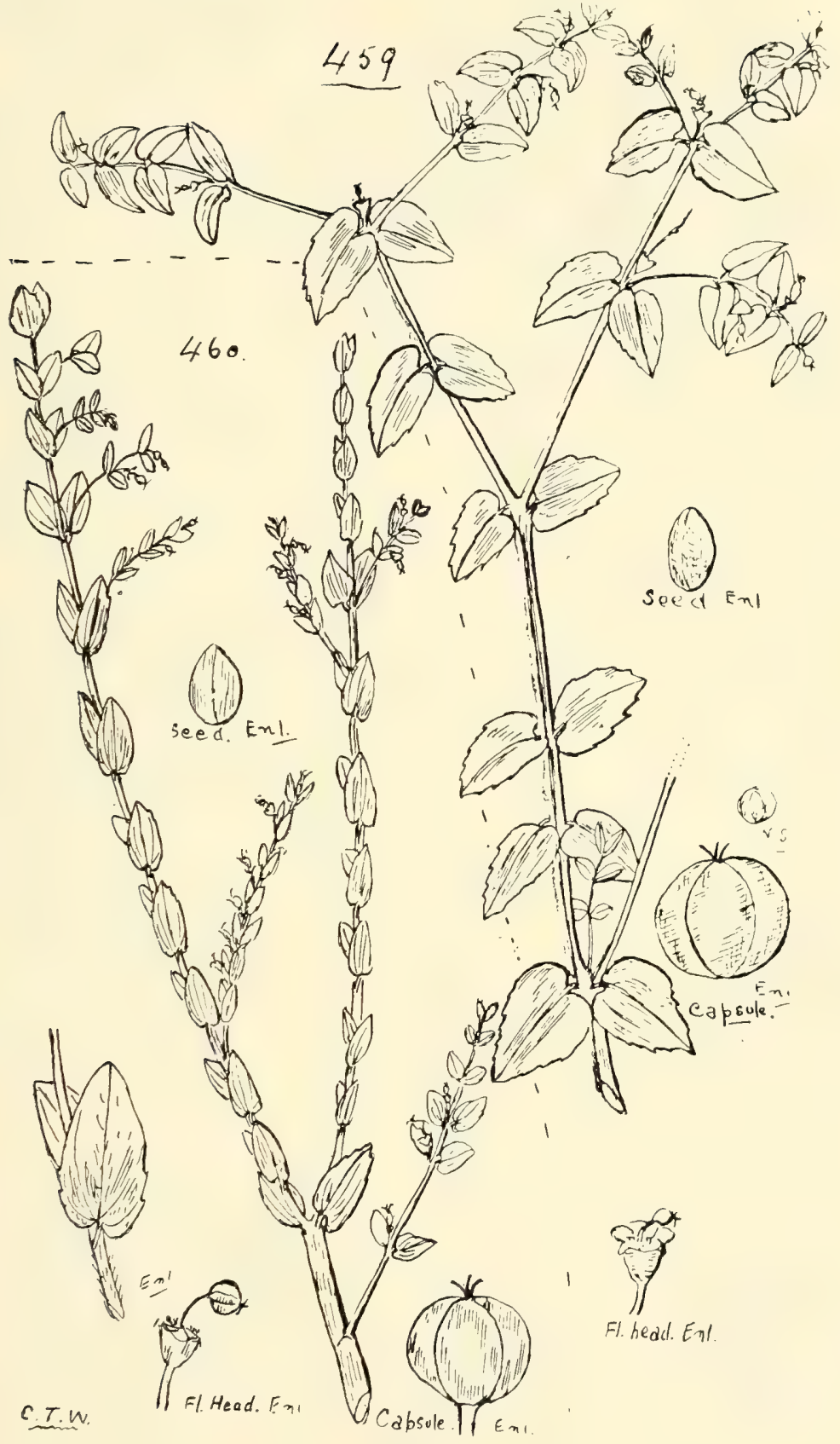

459. Euphorbia carissoides, Bail. 46o. E. Coghlani, Bail. 


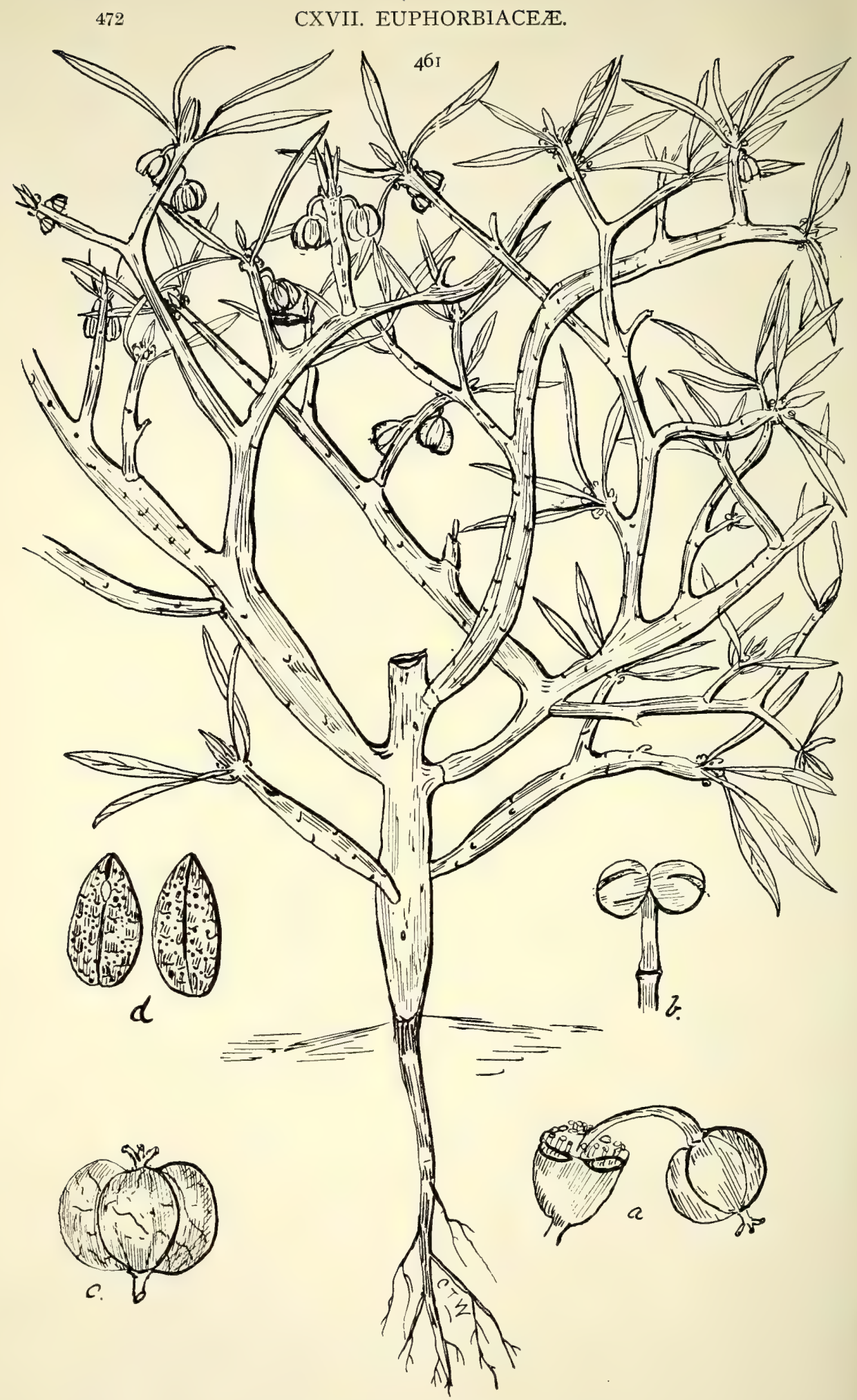

46i. Euphorbia Stevenir, Bail, sp. nov.

(a) Head of flowers, (b) anther, (c) capsule, (d) seeds. (a)-(d) enl. 
Euphorbia-contd.

Macgillivrayi, Boiss.

serrulata, Reinw'.

filipes, Benth.

Coghlani, Bail. (Fig. 46o.)

pilulifera, Linn.-An infusion of this plant is used as a cure for asthma and bronchitis.

Stevenii, Bail.-Steven's Caustic plant. (Fig. 461.)

Section II.-Eremophila.

eremophila, A. Cunn.-Sheep-poison or Caustic plant.

Section III.-Euphorbium.

corynocladia, F.v. M.

Section IV.-Tithymalus.

*Peplus, Linn.-Europe.

*Helioscopia, Linn.-Sun Spurge.

Section $V$.-Poinsettia.

*heterophylla, Linn.-South America.

Tribe II.-STENolobie E.

Poranthera, Rudge. microphylla, Brongn.

Micrantheum, Desf. ericoides, Desf.

Pseudanthus, Sieb.

pimeleoides, Sieb.

ovalifolius, F.v.M.

orientalis, F.v.M.

Beyeria, Miq.

viscosa, Miq.

Section I.-Eubeyeria.

opaca, F.v.M.- "Gundur-gundur" of the Endeavour River natives.

Section II.-Oxygyne.

tristigma, F.v. M.

Ricinocarpus, Desf.

pinifolius, Desf.

Bowmanni, F. r. $M$.

ledifolius, F.v. M.

speciosus, Muell. Arg.

Bertya, Planch.

pinifolia, Planch.

rosmarinifolia, Planch. 
Bertya-contd.

oppositifolia, F. $\%$.

oleæfolia, Planch.

pedicellata, F.v. M.

Monotaxis, Brongn.

macrophylla, Benth

linifolia, Brongn.

Amperea, A. Juss.

spartioides, Brongn.

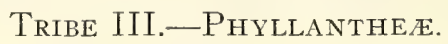

Bridelia, Willd.

exaltata, F.v.M.-Wood resembles walnut, and is useful for cabinet-work.

tomentosa, Blume.

var. ovoidea, Benth.

faginea, $F . v . M$.

Cleistanthus. Hook.

Cunninghamii, Muell. Arg.

apodus, Benth.

Dallachyanus, Baill.

semiopacus, F.v M.

Actephila, Blume.

grandifolia, Baill.

Mooreana, Baill.

latifolia, Benth.

petiolaris, Benth.

sessilifolia, Benth.

Andrachne, Linn.-A species of this genus has been known to poison cattle in India.

Decaisnei, Benth.

Phyllanthus, Linn.

Section I.-Synostemon.

thesioides, Benth.

hirtellus, Muell. Arg.

rigens, Muell. Arg.

ramosissimus, Muell. Arg.

albiflorus, F.v.M. (Fig. 462.)

elachophyllus, F.v.M.

Section II.-Kirganelia.

novæ-hollandiæ, Muell. Arg.

brisbanicus, Bail.-A common weed of Brisbane bush-houses.

(Fig. 463.) 


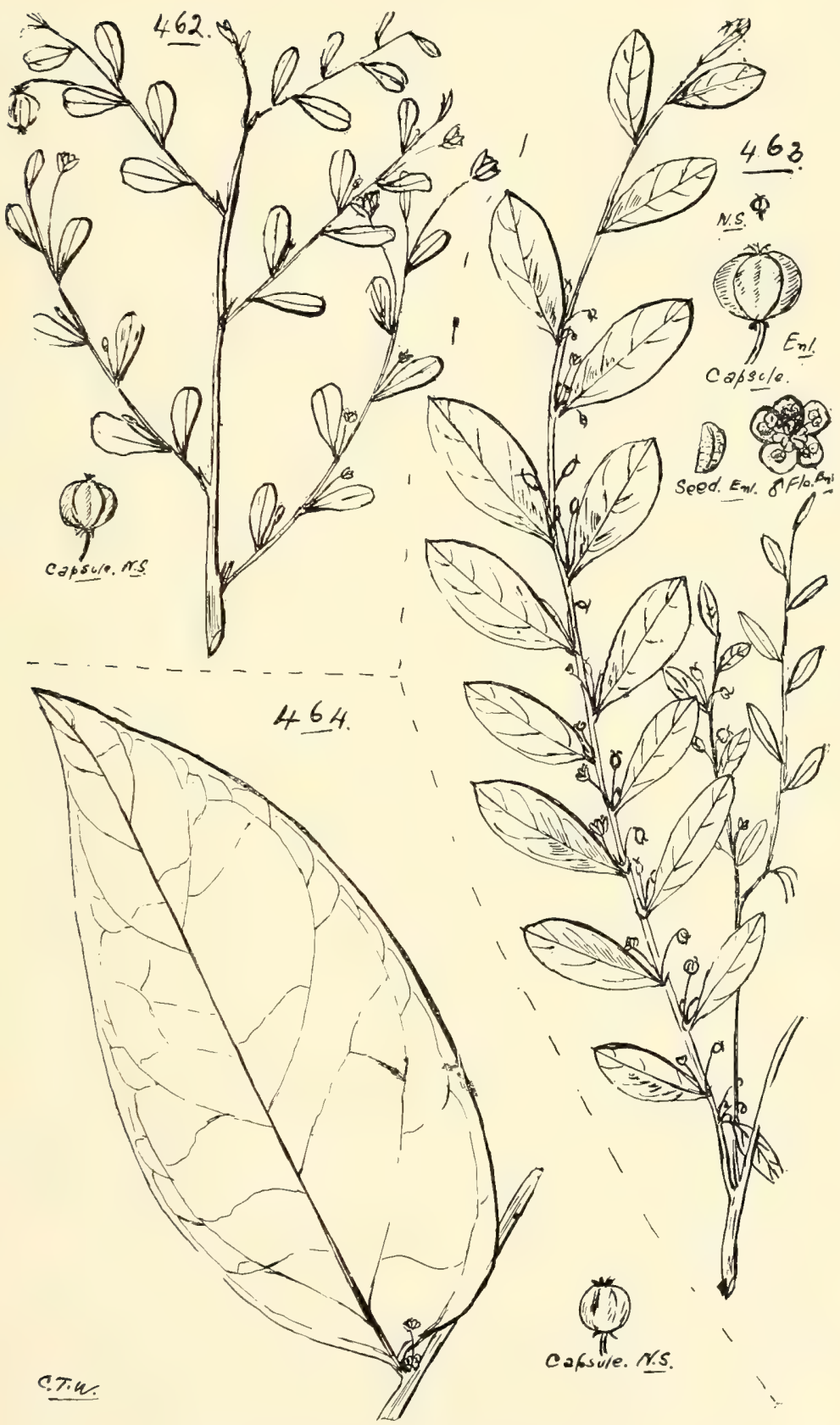

462. Phyllanthus albiflorus, $F . v . M$.

463. P. bRiseanicus, Bail.

464. P. Hypsopodius, F. $v . M$. 
Phyllanthus-contd.

Section III.-Paraphyllanthus.

urinaria, Linn.- “ Te-mo” of Mapoon natives.

maderaspatanus, Linn.

Mitchelli, Benth.

Gasstrœmii, Muell. Arg.

Dallachyanus, Benth.

subcrenulatus, F.v. M.

similis, Muell. Arg.

microcladus, Muell. Arg.

Fuernrohrii, F.v. M.

hebecarpus, Benth.-Said to be refused by rabbits, therefore considered poisonous.

lacunarius, $F . v . M$.

trachyspermus, F.v. M.

thymoides, Sieb.

cuscutæflorus, S. Moore in Journ. Bot. xliii. ( I905) 148.

Section IV.-Euphyllanthus.

simplex, Retz.

var. leiospermus, Benth.

filicaulis, Benth.

minutiflorus, F.v. M.

var. gracillimus, Benth.

hypsopodius, F.v.M. (Fig. 464.)

buxifolius, Rein.

Glochidion, Forst.

Ferdinandi, Muell. Arg. = Phyllanthus Ferdinandi, Muell. Arg.- "Jow-war" of Stradbroke Island natives. The wood warps much in drying.

var. minor, Benth.

var. supra-axillaris, Benth.

var. mollis, Benth.

lobocarpus, Benth. =P. lobocarpus, Benth.--Wood tough and elastic, of a pinkish colour, useful for tool-handles.

Neorœepra, Muell. Arg.

buxifolia, Muell. Arg.

Banksii, Benth.

Flueggea, Willd.

microcarpa, Blume = Securinega obovata, Muell. Arg."Tharginyah" of Cloncurry, "Arrimby" of Mitchell River, "Koy-ka” of Cooktown, and " Eramba” of Palmer River natives.

Leucopyrus, Willd.-Bark poisonous. 
Breynia, Forst.

cernua, Muell. Arg.

oblongifolia, Muell. Arg.-_"Willgar" of Bundaberg natives.

stipitata, Muell. Arg.

Petalostigma, F.v. $M$.

Banksii, Britten and S. Moore. (Fig. 465.)

quadriloculare, F.v.M.-Bitter Crab. "Ungwe" of Bundaberg, "Muntenpin" of Stradbroke Island, "Oolgar" of St. George, and "Tronganongan" of Port Curtis natives. Bark yields a brownish-yellow dye. Mr. E. W. Armit states the ripe fruit is a valuable vermifuge for horses. (Fig. 466.)

var. glabrescens, Benth. (Fig. 467.)

Hemicyclia, $W$. et Arn.

sepiaria, $W$. et $A .=$ ? oblongifolia, Benth.

australasica, Muell. Arg.

Dissiliaria, F.v.M.

baloghioides, F.v.M. - Teak. "Currumgul" of Nerang natives, and used by them for nullahs. Wood resembles somewhat that of the English Apple. (Fig. 468.)

Muelleri, Baill.

tricornis, Benth.

Bischoffia, Blume.

javanica, $B l .=B$. trifoliata, Hook.

Aporosa, Blume.

australiana, F.v. M.

Antidesma, Limn.

Ghæsembilla, Gortn.

Dallachyanum, Baill.-Herbert River Cherry. "Top-kie” of Dunk Island and "Je-jo" of Cape Grafton natives. (Fig. 4óg.)

Bunius, Spreng. - "Moi-kin" of Tully River and "Chunka" of Bloomfield River natives.

parvifolium, F.v.M.-Currant-tree of Gilbert and Barron

Rivers.

erostre, F.v.M.-Currant-tree of Cairns.

sinuatum, Benth.-Currant-tree of Cairns.

Tribe IV.-Crotone玉.

Subtribe I.-Jatrophea.

*Jatropha, Linn.

multifida, Linn.-South America. 
Jatropha-contd.

Curcas, Linn.-Physic or Purging Nut; become naturalised at Charters Towers. Fruit poisonous. The Pinksen Oil is the product of the seeds of this shrub (Q1. Agric. J1. (I900) 382, tab. cxcv.). Coromandel Coast.

gossypifolia, Linn.-Cotton-leaved Physic Nut. Brazilian.

Aleurites, Forst.

moluccana, Willd. = Jatropha moluccana, Linn.; Aleurites triloba, Forst.-Candle Nut. "Tar-kal" of Cooktown, "Nappalla" of the Barron River natives. The nuts of this tree may sometimes be eaten without any ill effects, while at other times they have been known to cause dangerous illness.

Croton, Linn.

Subtribe II.-Eucrotonea.

insularis, Baill. - Queensland Cascarilla Bark. The bark yields a reddish-brown dye.

phebalioides, F.v.M.

var. hirsuta, Bail.

opponens, F.v.M.

Verreauxii, Baill.

acronychioides, $F . v . M$.

triacros, F.v.M.

arnhemicus, Muell. Arg.

var. urenæfolius, Baill.

Subtribe III.-Chrozophorca.

Codiæum, Rumph.-The so-called Crotons of our gardens belong to this genus.

variegatum, Bl.; var. moluccana, Muell. Arg.

Baloghia, Endl.

lucida, Endl.-Scrub Bloodwood. Wood suitable for engraving and cabinet-work. The red blood-like sap hardens into a red gum.

Fontainea, Heckel.

Pancheri, Heckel. = Baloghia Pancheri, Baill. - Fruit poisonous.

Subtribe IV.-Adrianea.

Adriana, Gaudich.

acerifolia, Hook.-Sometimes called Cotton-bush.

var. glabrata, Benth.

Subtribe V.-Acalyphea.

Claoxylon, A. Juss.

angustifolium, Muell. Arg. 
Claoxylon-contd.

tenerifolium, F. v. $M$.

australe, Baill.

var. latifolia, Benth.

var. laxiflora, Benth.

Hillii, Benth.

Acalypha, Limn.-It is likely, where a quantity of these plants is growing, that the pasture may prove dangerous to stock, as some Indian species possess a like property to that of Ipecacuanha.

indica, Linn., var. australis, Bail., Q1. Bot. Bull. iii. I6.

nemorum, F.v. M.

eremorum, Muell. Arg.

capillipes, F.v.M.

Alchornea, Sw.

ilicifolia, Muell. Arg. = Colebogyne ilicifolia, J. Sm."Mai" of Bundaberg natives.

Thozetiana, Baill. = Cladodes Thozetiana, Baill.

var. longifolia, Benth.

Mallotus, Lour.

ricinoides, Muell. Arg.— "Barrinya" of Cairns natives.

paniculatus, Muell. Arg.

claoxyloides, Muell. Arg.-It is this tall shrub which causes

that peculiar odour so noticeable in all Queensland scrubs.

var. ficifolia, Benth.

var. macrophylla, Benth.

zar. angustifolia, Bail.

philippinensis, Muell. Arg. - Kamela-tree. "Poodgeepoodgee" of some natives. The bark is used for tanning in India, and the red powder surrounding the capsules gives a red dye.

angustifolius, Benth.

polyadenus, F.v.M.-Wood suitable for mallets and chiselhandles.

repandus, Muell. Arg.

nesophilus, F.v.M.

discolor, F.v.M.-The capsules give a bright-yellow dye.

Macaranga, Thouars.

Dallachyi, F.v.M.

subdentata, Benth.

inamœena, $F \cdot v \cdot M$.

involucrata, Baill.

Tanarius, Muell. Arg.- " Tumkullum” of Stradbroke Island and "Do-ang-al" of Morehead River natives. var. tomentosa, Muell. Arg. 
*Ricinus, Limu.

communis, Linn.-Castor Oil plant; contains prussic acid. Africa. The leaves, naturally applied, of this plant form a valuable galactopoietic (see Dr. W. Woolls, Cont. to F1. of Austr.). Among the naturalised plants are several forms or varieties.

Tragia, Linn.

Subtribe VI.-Plukenetiea.

novæ-hollandiæ, Muell. Arg.-Twining Nettle; clothed with stinging hairs.

Subtribe VII.-Hippomanea.

Homalanthus, A. Juss.

populifolius, Grah--Poisonous to stock (Mueller).

stillingiæfolius, F.v. M.

Omphalea, Linn.

queenslandiæ, Bail.

Sebastiania, Spreng.

chamelæa, Muell. Arg.

Excæcaria, Linn.-The barks yield caoutchouc in abundance.

Agallocha, Linn.-Milky Mangrove or River Poison-tree; also Blinding-tree. "Coongera" of Forest Hill and "Me-laba” of Cooktown natives. The bark contains I3-I5 per cent. of tannin (Brïnnich).

Dallachyana, Baill.-Scrub Poison-tree. Wood very tough, suitable for tool-handles.

parvifolia, Muell. Arg.-Gutta-percha tree. Yields a very handsome cabinet-wood. (Fig. 470.)

\section{Order CXVIII.-BALANOPSEÆ.}

Balanops, Baill.

australiana, F.v.M.-Figured from specimens kindly given for the occasion, from the Muellerian herbarium, by Professor A. J. Ewart. (Fig. 47I.)

\section{Order CXIX.-URTICACEÆ.}

(Many plants of this Order yield Caoutchouc, many valuable fibres, and material suitable for paper-making.)

Celtis, Linn.

Tribe I.-Celtideæ.

philippinensis, Blanco.

paniculata, Planch.-Investigator-tree. Gulf of Carpentaria. 


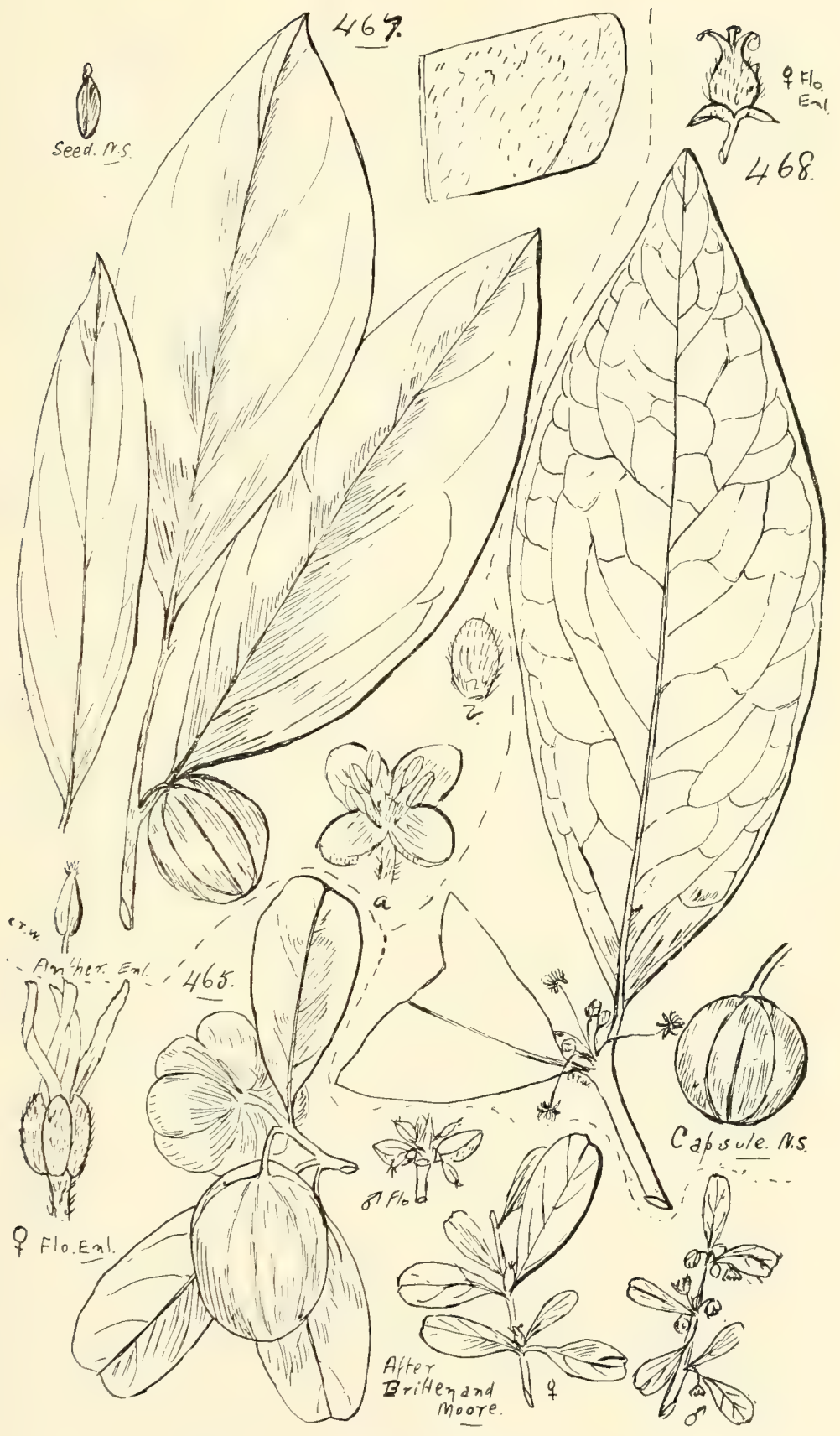

465. Petalostigma Banksin, Britt. et S. Moore.

467. P. quajriloculare, F. v. M., var. glabrescens, Bail. 468. Dissiliaria baloghiomes, $F . v . M$. 


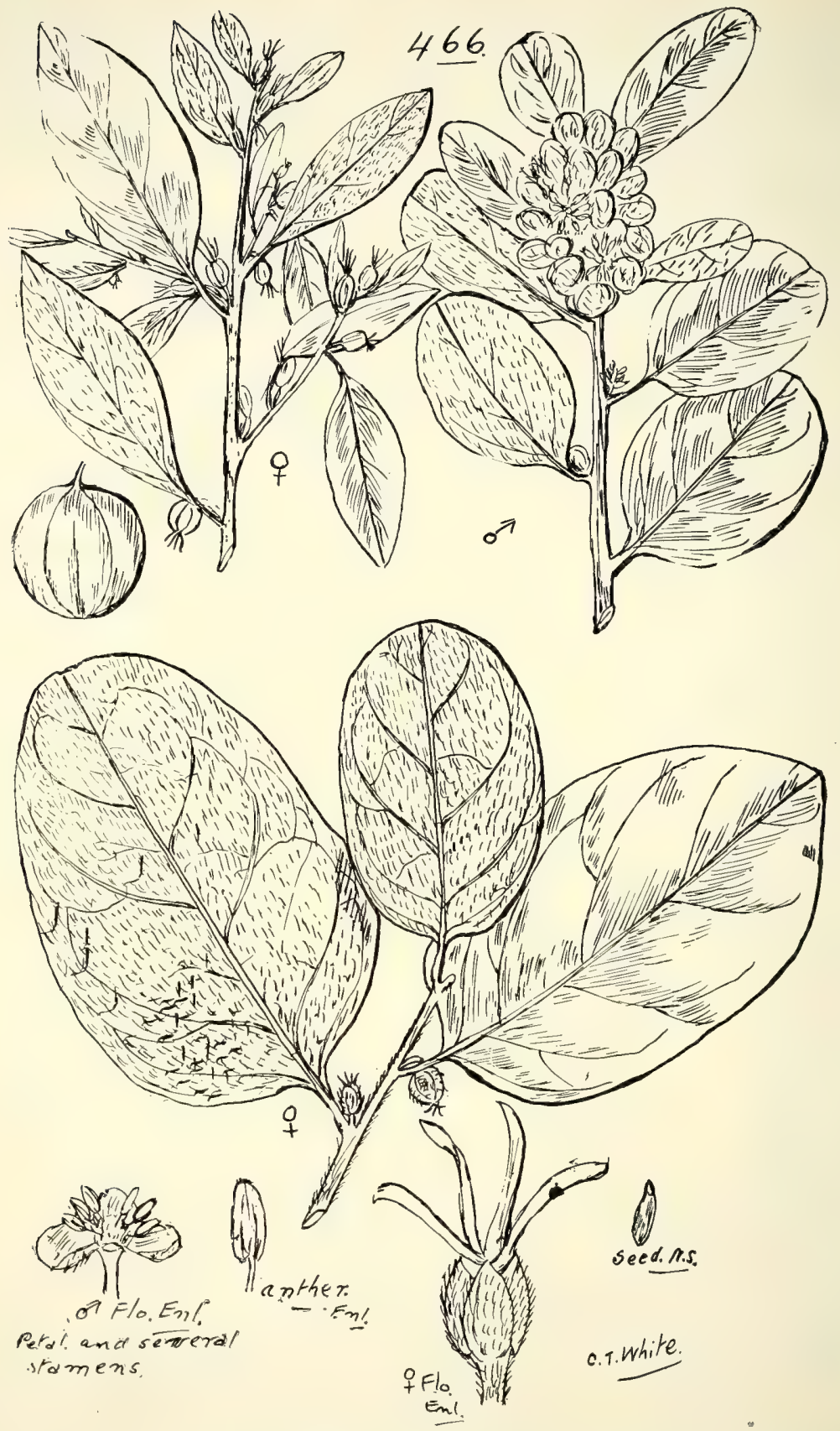

466. Petalostigma quadriloculare, $F . v \cdot M$. 


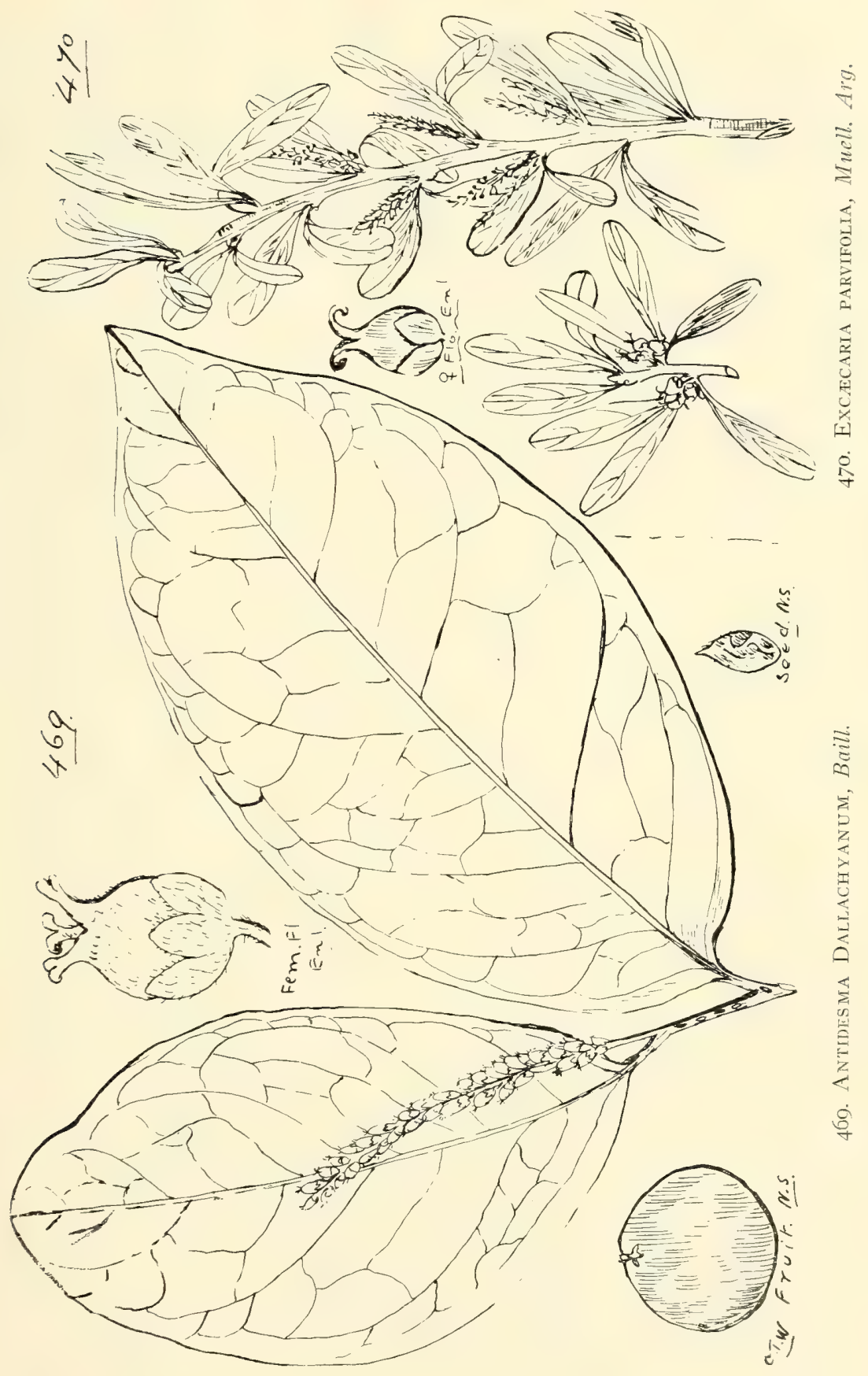




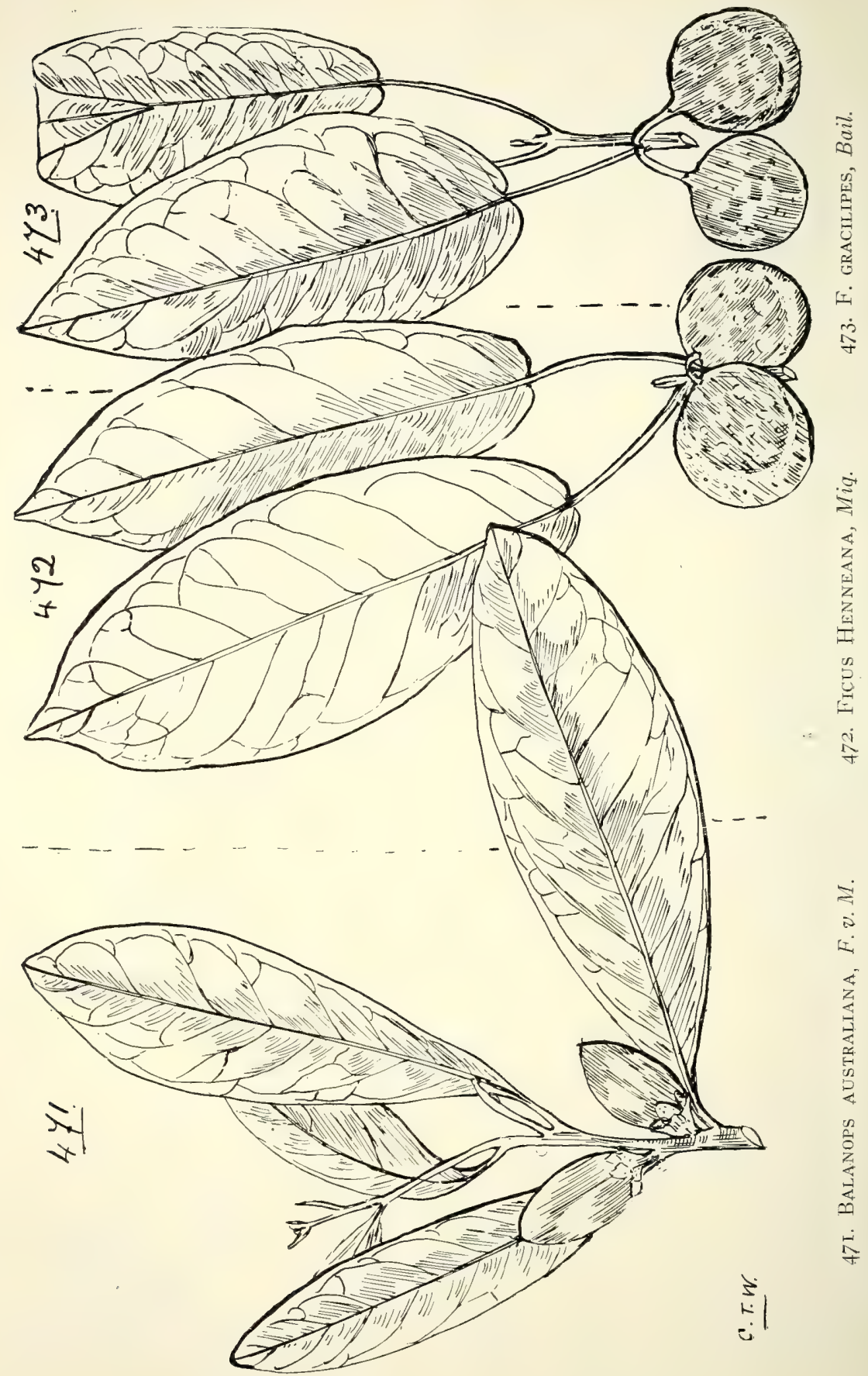


Trema, Lour.-All the Queensland species considered injurious to stock.

aspera, Blume.-Peach-leaved Poison-tree. A pretty variegated form of this plant is sometimes met with.

var. viridis, Benth.

orientalis, Blume-A charcoal tree of India; wood resembles Red Cedar.

amboinensis, Blume.- "Birula" of Forest Hill natives.

Aphananthe, Planch.

philippinensis, Planch.- "Mallban" of Barron River natives.

Wood might be useful for stamps.

TRIBE II.-Moree.

Subtribe I.-Broussonetiea.

Malaisia. Blanco.

tortuosa, Blanco.- "Deng-ul-ka" of Morehead River, "Kaiinuna" of Mapoon and "Nannan" of Brisbane natives. Wood hard and very tough.

Subtribe II.-Eumorece.

Pseudomorus, Bureau.-Fruit a small Sorosis.

Brunoniana, Bureau.-Wood yellow, close-grained, hard and tough.

Subtribe III.-Dorsteniece.

*Dorstenia, Linn.

arifolia, Lam. Brazil.

Tribe III.-ArtocarpeÆ.

Subtribe I.-Ficea.

Ficus, Linn.-The milky sap of many yields Caoutchouc. Fruit a Syconus.

Section I.-Urostigma.

colossea, F.v.M.-“"Ban-na-bool-ka" or "Ko-ko-ya" of Cooktown natives. A deciduous tree of considerable size. pilosa, Reinw.-A deciduous tree; "Burrowa," Cape Grafton native name.

glabella, Bl., var. papuana, King, Ann. Roy. Bot. Gard. Calc. i. (I887) $50=F$. nesophila, Müll. MS., Miq. in Ann. Mus. Lugd. Bot. iii. 286.

Parkinsoni, Hiern., Journ. Bot. 39 (I9OI) i. tab. 4I7.

Henneana, Miq.-Tree sometimes deciduous; yields a fruit suitable for preserving. (Fig. 472.)

gracilipes, Bail.-Sometimes deciduous; a good fruit for preserving. Perhaps only a variety of F. Henneana. (Fig. 473.)

virginea, Banks and Sol., ex Hiern., Journ. Bot. 39 (Igor) 2. 
Ficus-contd.

infectoria, Roxb. = Cunninghamii, Miq.- "Mooleeah" of Bundaberg and "Bene" of Mapoon natives. Tree sometimes deciduous.

var. Lambertiana (Miq.), King.-A common Queensland form.

subulata, Blume.

validinervis, $F \cdot v \cdot M$.

retusa, Linn.- " Tunduli " of Cape Grafton and "Dortalama" of Batavia River natives.

var. nitida (Thunb.), King $=F$. nitida, Thunb.-Forms a tree with very wide-spreading head, as may be seen from two handsome trees in the Brisbane Botanic Gardens.

Thynneana, Bail.-Queensland Banyan. Bears columnar stems from adventitious roots (see photo. Q1. Agric. J1. i. (I897) 23I). "Dorndorli" of Cape Grafton natives. (Fig. 474.)

eugenioides, F.v.M.- "Tilgul" of Atherton natives. (Fig. 474 bis.)

var. puberula, Benth.

Hillii, Bail.-Hill's Weeping Fig. (Fig. 475.)

Tryoni, Bail. (Fig. 476.)

leucotricha, Miq. $=$ F. lanata, F. v. M.-The receptacles borne by South Queensland trees are roughly ribbed and nearly sessile. (Fig. 476 bis.)

rubiginosa, Desf. $=F$. australis, Willd.-Forming stems from adventitious roots. The foliage not so coriaceous, and receptacles less warted, than in the more southern parts of Australia. Fig. 477 is a form from Percy Islands (H. Tryon).

var. glabrescens, Bail.; $n$. var.-The foliage smaller and plant nearly or quite glabrous. (Fig. 477 bis.)

Hab.: Eidsvold (Dr. T. L. Bancroft).

platypoda, A. Cunn.

var. minor, Miq.-A large tree with small fruit. Brisbane River scrubs. (Fig. 478.)

var. petiolaris, Benth.-Receptacles sometimes on peduncles of 4 lines (see Fig. 479 bis). "Kunnen" of Stradbroke Island natives. (Fig. 479.)

var. mollis, Benth. (Fig. 480 .)

var. subacuminata, Benth. (Fig. 48r.)

Muelleri, Miq., Bail., Q1. Agric. J1. 26 (I9I I), 316, tab. xxxii.

(Fig. 482.)

dictyophleba, $F, v \cdot M$. 
Ficus-contd.

macrophylla, Desf.-Moreton Bay Fig. "Peemith" of Bundaberg natives. Always glabrous or nearly so in the normal form. The basal bracts of receptacle 2, emarginate, slightly downy, about 5 lines long, deciduous. (Fig. 483.)

zar. pubescens, Bail., Q1. Agric. J1. 26 (I9II), 3I6, tab. xxxiii. This tree has been long confused with the normal form, and is similar to it in habit and form, but differs considerably in fruit besides the pubescence. The basal bracts of receptacle pubescent, 3 or 4 , often 4 or 5 , lines long, with ciliate margins, very deciduous. I have received specimens of what $\mathrm{I}$ consider this variety from the Coomera River. (Fig. 484.)

Watkinsiana, Bail.--One of the best kinds for affording shade. (Fig. 485.)

Simmondsii, Bail., Q1. Agric. J1. 25 (I910), 234, tab. xix. (Fig. 486.)

Section II.-Eusyce.

magnifolia, F.v.M.

ehretioides, F.v.M.- "Magura" of Tully River natives. This species fruits on compact excrescent-like formations upon the thick branches of the tree. (Fig. 487.)

*pumila, Linn.-This Chinese species may sometimes be met with in deserted garden-plots; thus it has been spoken of as indigenous. An Ivy-like climber.

scandens, Roxb., var. australis, Bail. (Fig. 488.)

leptoclada, Benth.

depressa, Benth.

phillippinensis, Miq.

mollior, F.v.M.

Pinkiana, F.v.M. (Fig. 489.)

stenocarpa, F.v.M.- "Ke-ril" of Cape Grafton natives. scabra, G. Forst. $=$ F. aspera, Forst. Prod.-Rough or Purple

Fig. "Noomaie" of Rockhampton and "Balemo" of Cleveland Bay natives.

orbicularis, A. Cunn.-- "Mó-i" of Batavia River natives.

aculeata, A.Cunn.

var. micracantha (Miq.), Benth.

subglabra, Benth.-The fruit has a disagreeable odour. Receptacles unisexual. (Fig. 490.)

opposita, Miq.- " Murn-tyul" of Morehead River and "Moinjal" of Palmer River natives. F. radula, Banks's Journ. (non Willd.), ex Hiern. J1. Bot. xxxix. (I9OI) 4. Native name "De-poor," Solander MS.

scobina, Benth. 


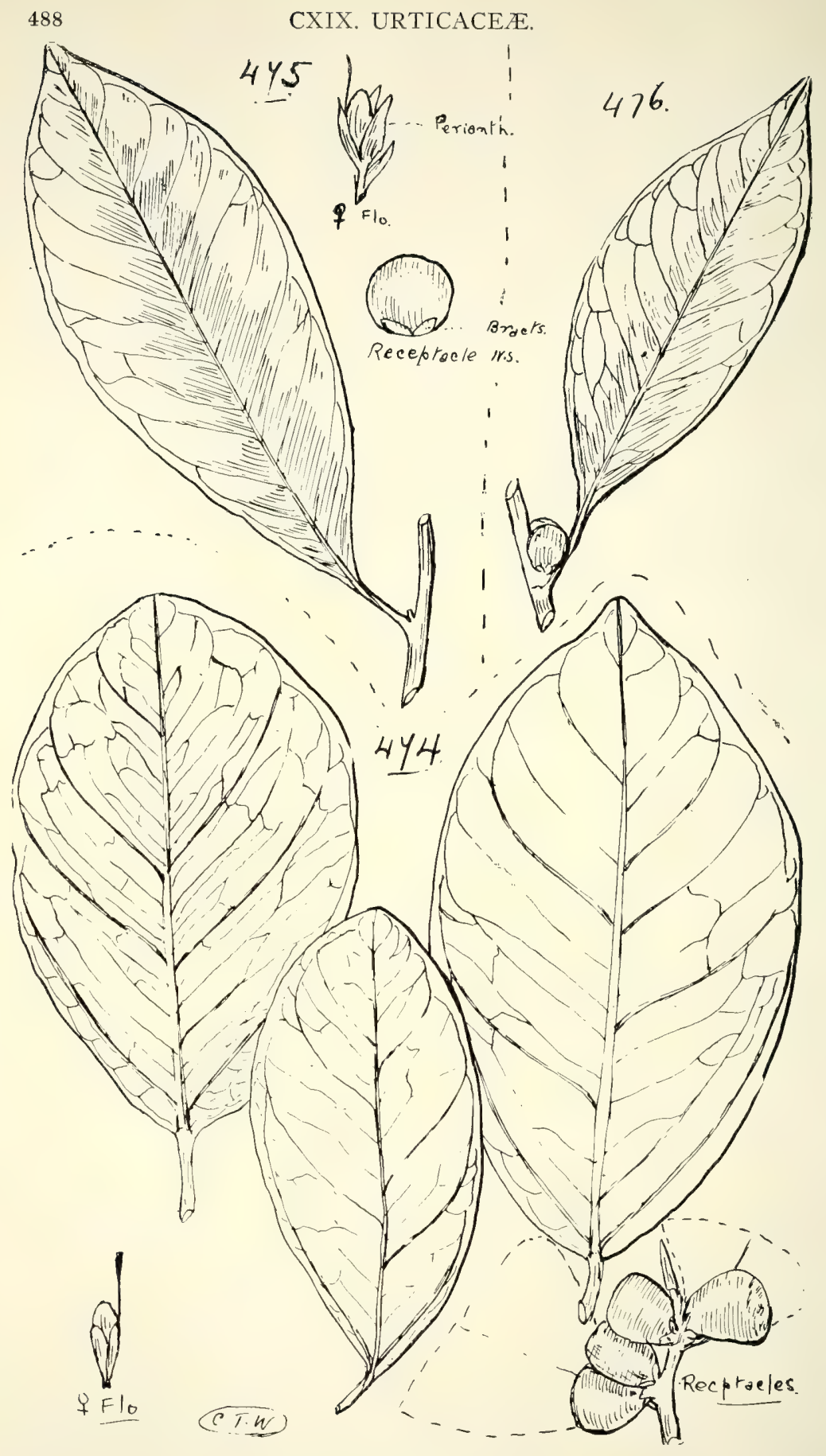

474. Ficus Thynneana, Bail.

475. F. HilliI, Bail.

476. F. Tryoni, Bail. 


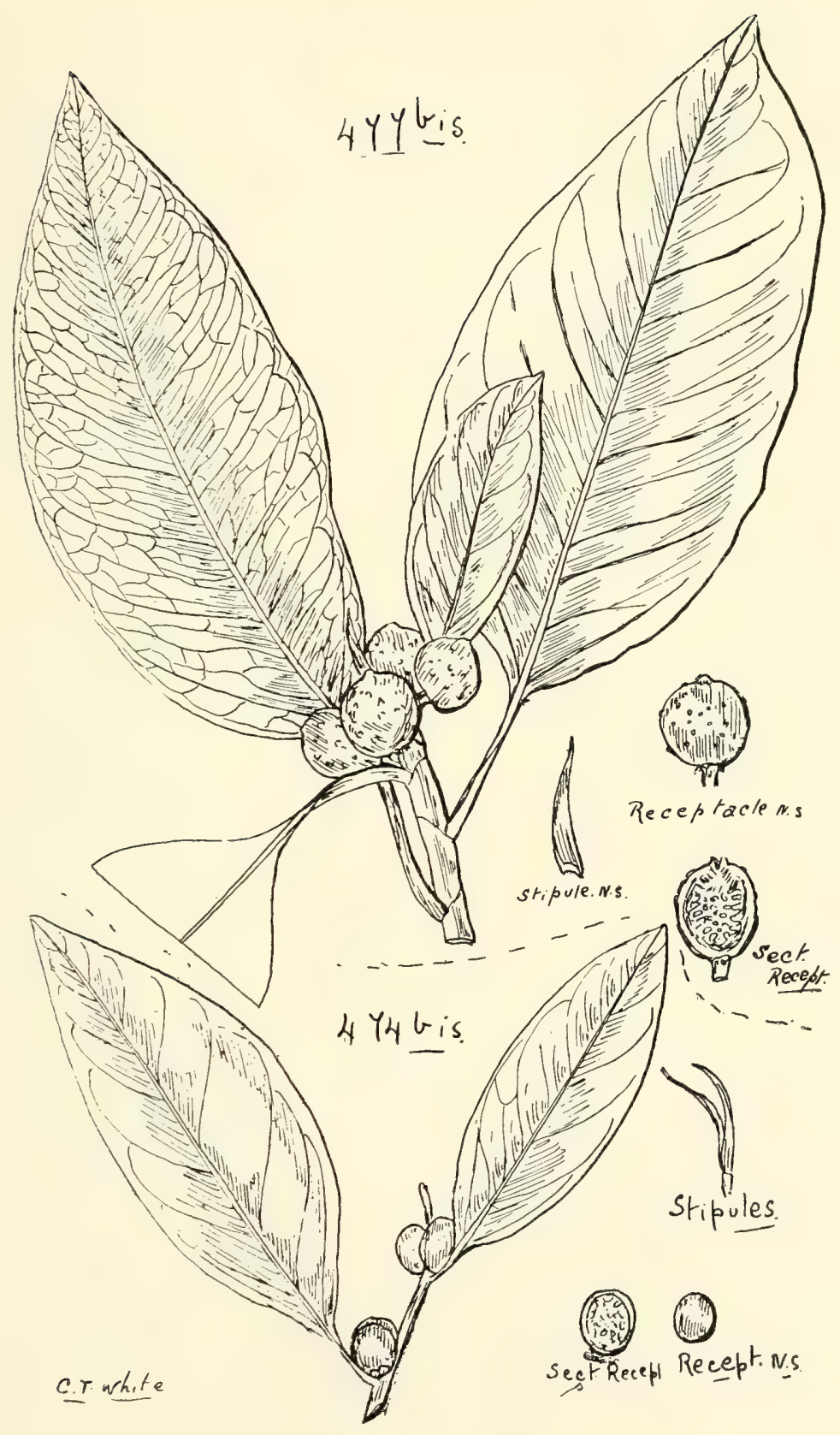

474 bis. Ficus elgeniordes, $F$. $v . M$.

477 bis. F. Rubiginosa, Desf., var. glabrescens, Bail. 


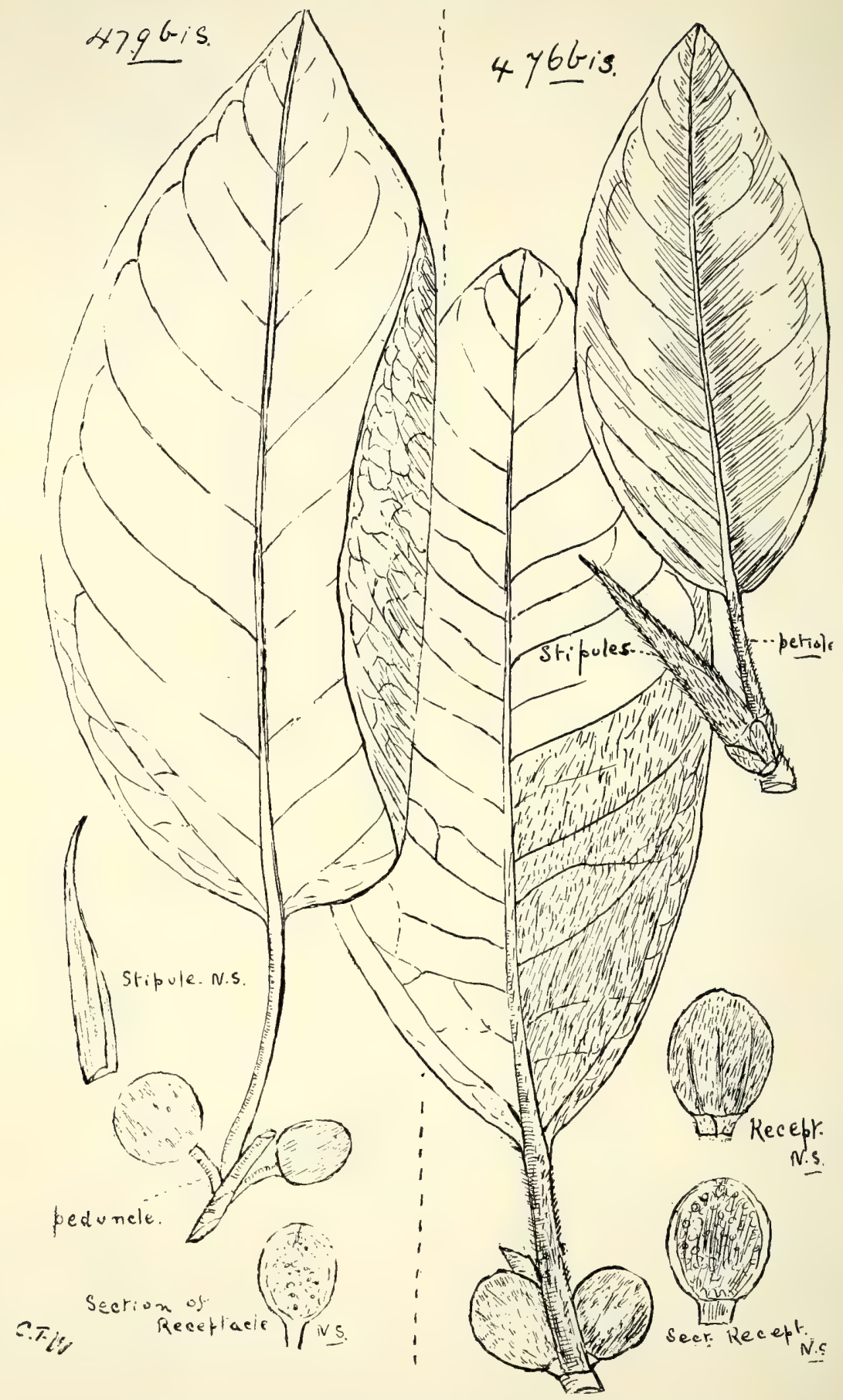

476 bis. Ficus leucotricha, Miq.

479 bis. F. Platypoda, A. Cunn., var. pettolaris, Benth.

(A form with long peduncles.) 


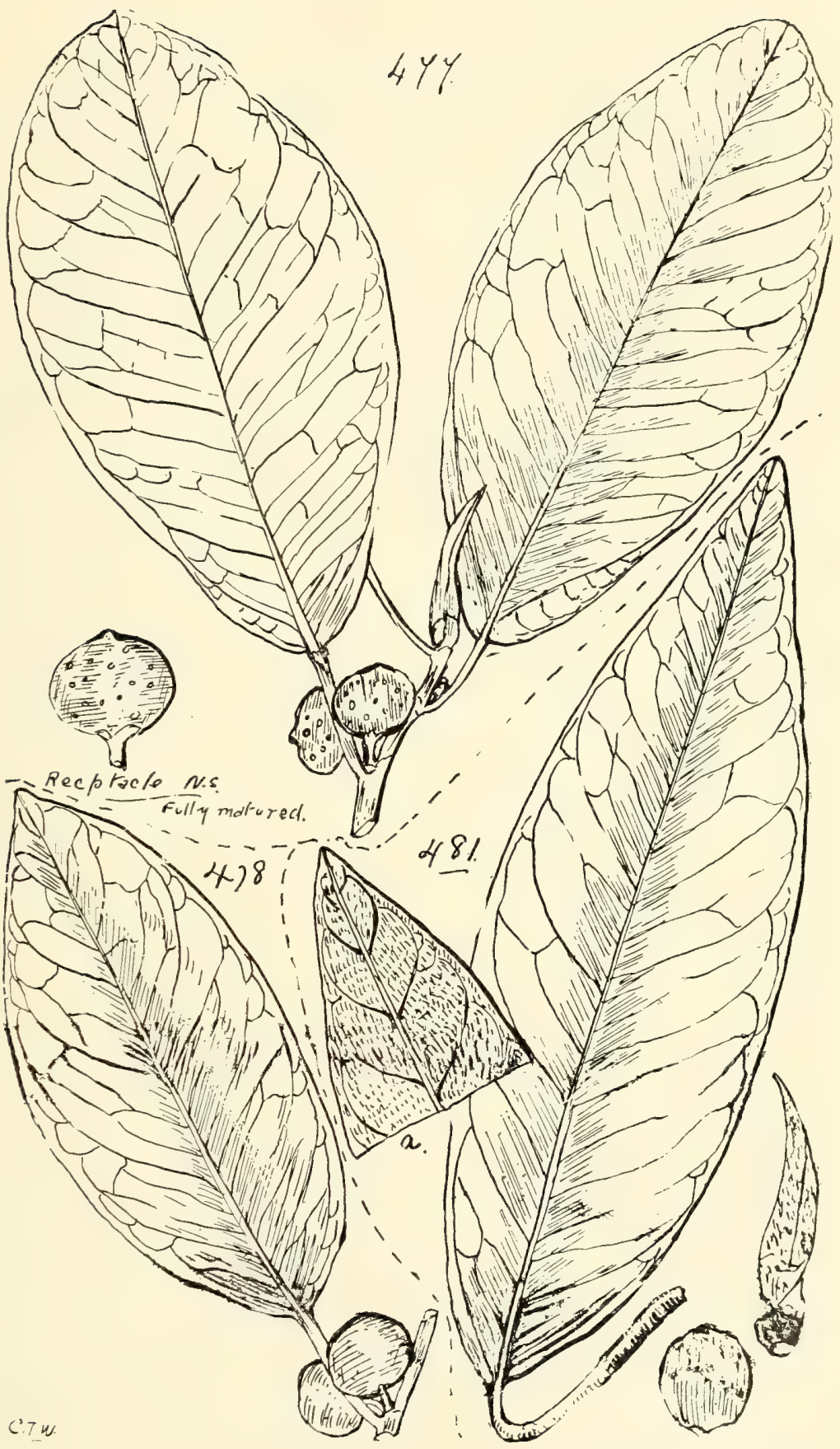

477. Ficus rubiginosa, Desf., forma.

478. F. platypoda, A. Cunn., var. minor, Miq.

48r. F. platy poda, A. Cunn., var. subacuminata, Benth. 


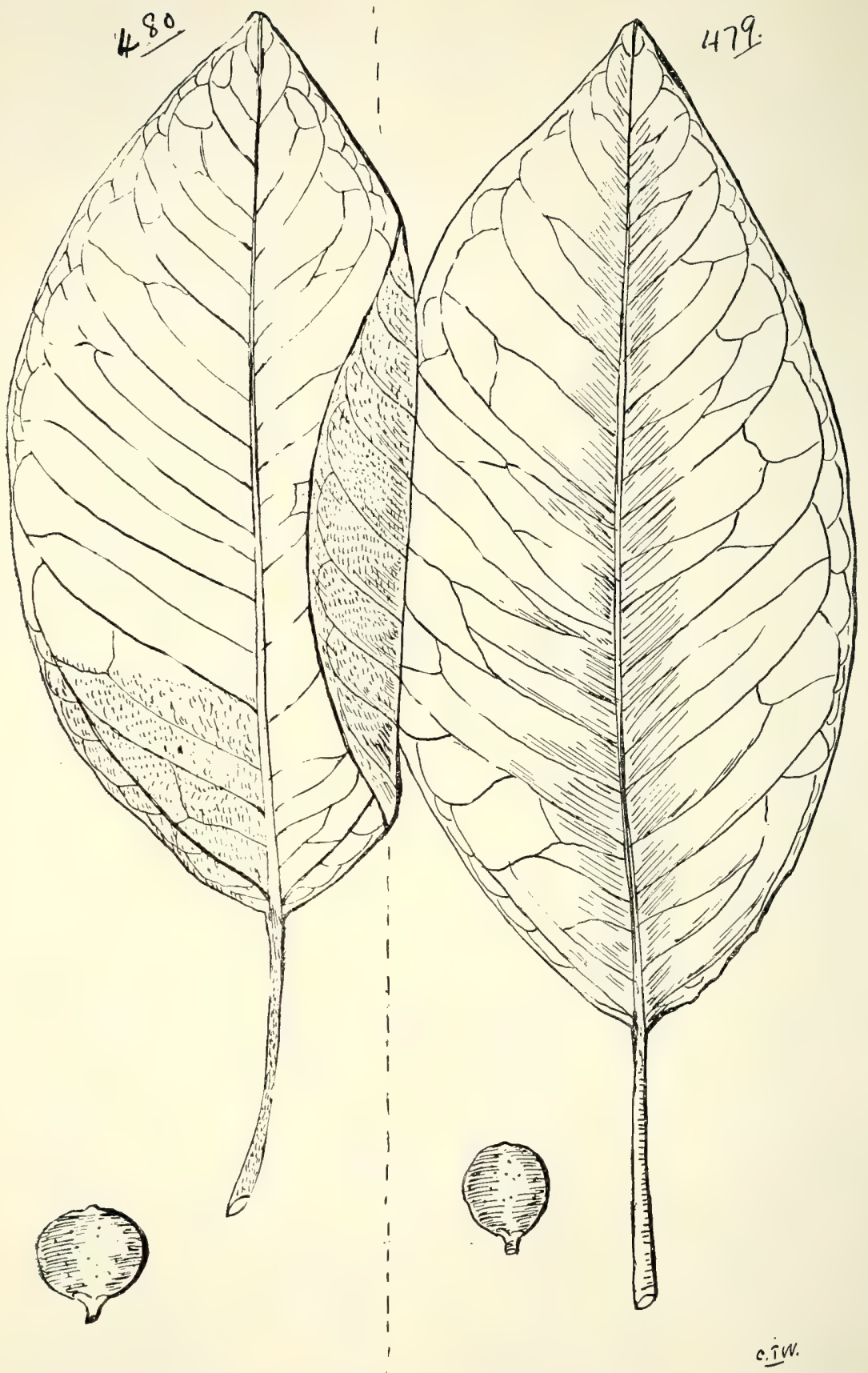

479. Ficus platypoda, A. Cunn., var. Petiolakis, Benth. 48o. F. platypoda, A. Cunn., var. mollis, Benth. 


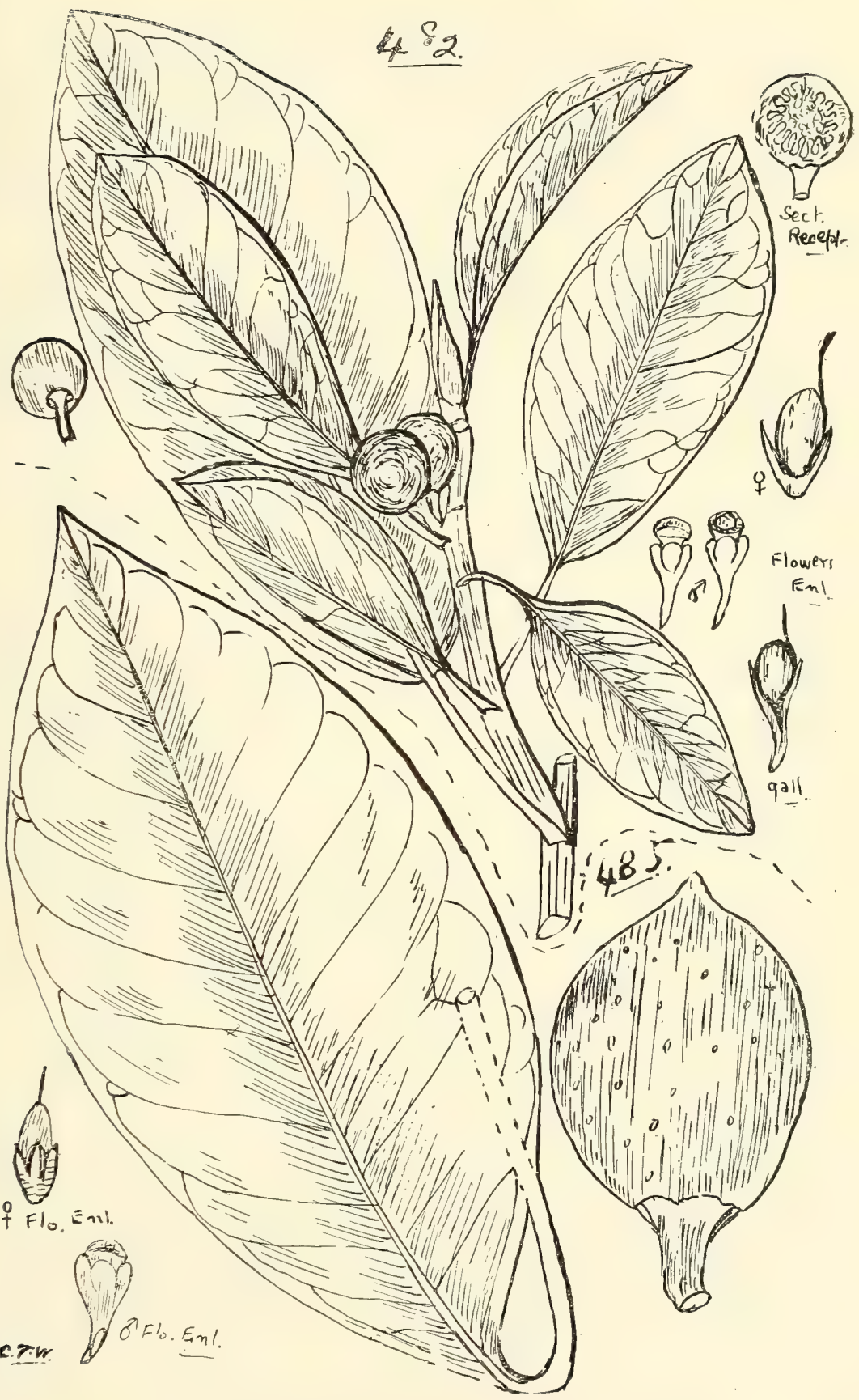

482. Ficls Muelleri, Miq.

485. F. Watkinsiana, Bail. 


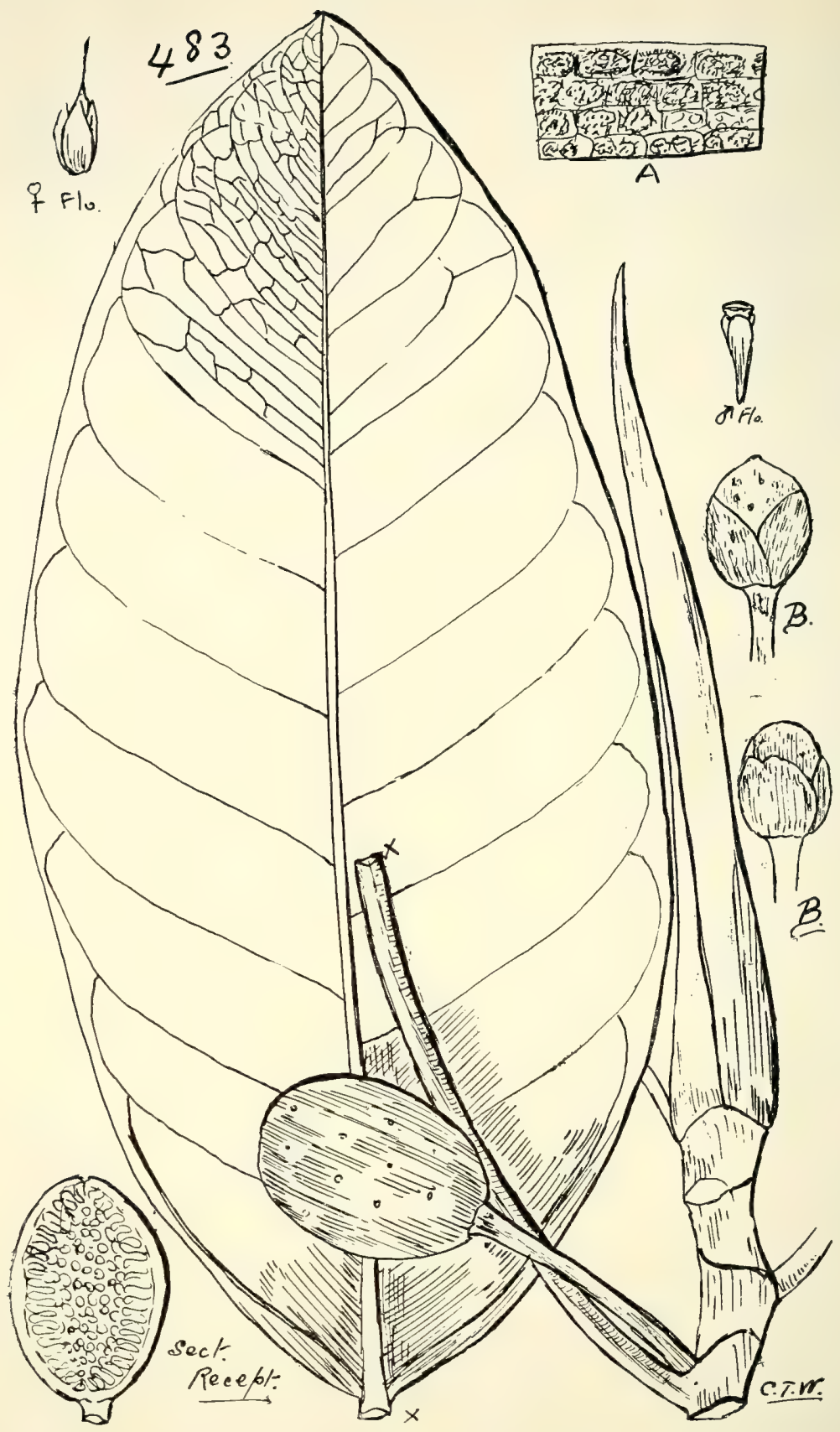

483. Ficus macrophylla, Desf.

(A) Portion of under surface of a leaf (en1.), (B) young receptacles with bracts (nat. size). 


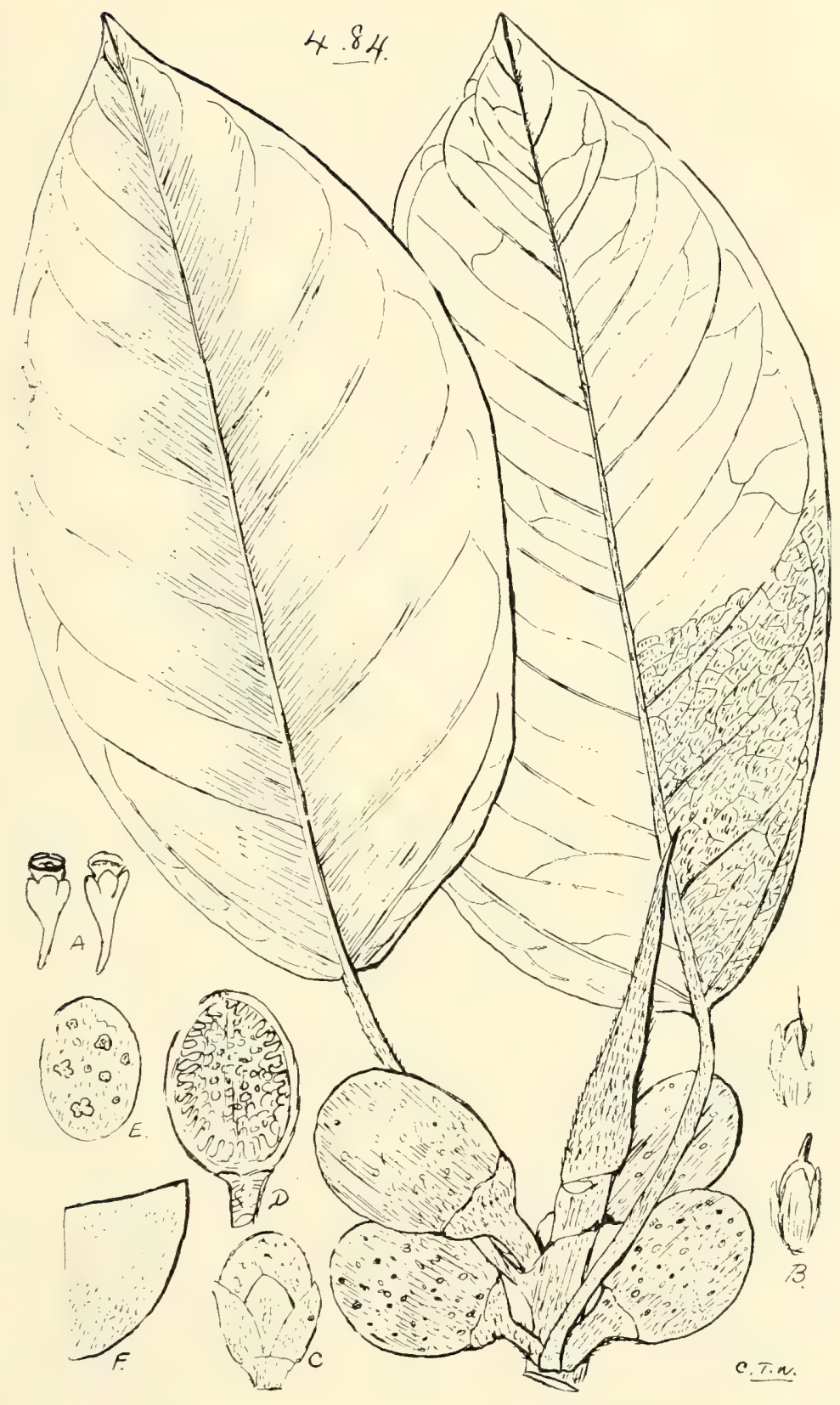

484. Ficus macrophylla, Desf., var. pubescens, Bail.

(A) Male flowers, (B) female flowers, (C) young receptacle arid bracts, (D) sect. receptacle, (E) portion of surface of receptacle, (F) portion upper surface of a leaf. $(A),(B),(E)$, and (F) enl.; (C) and (D) nat. size. 


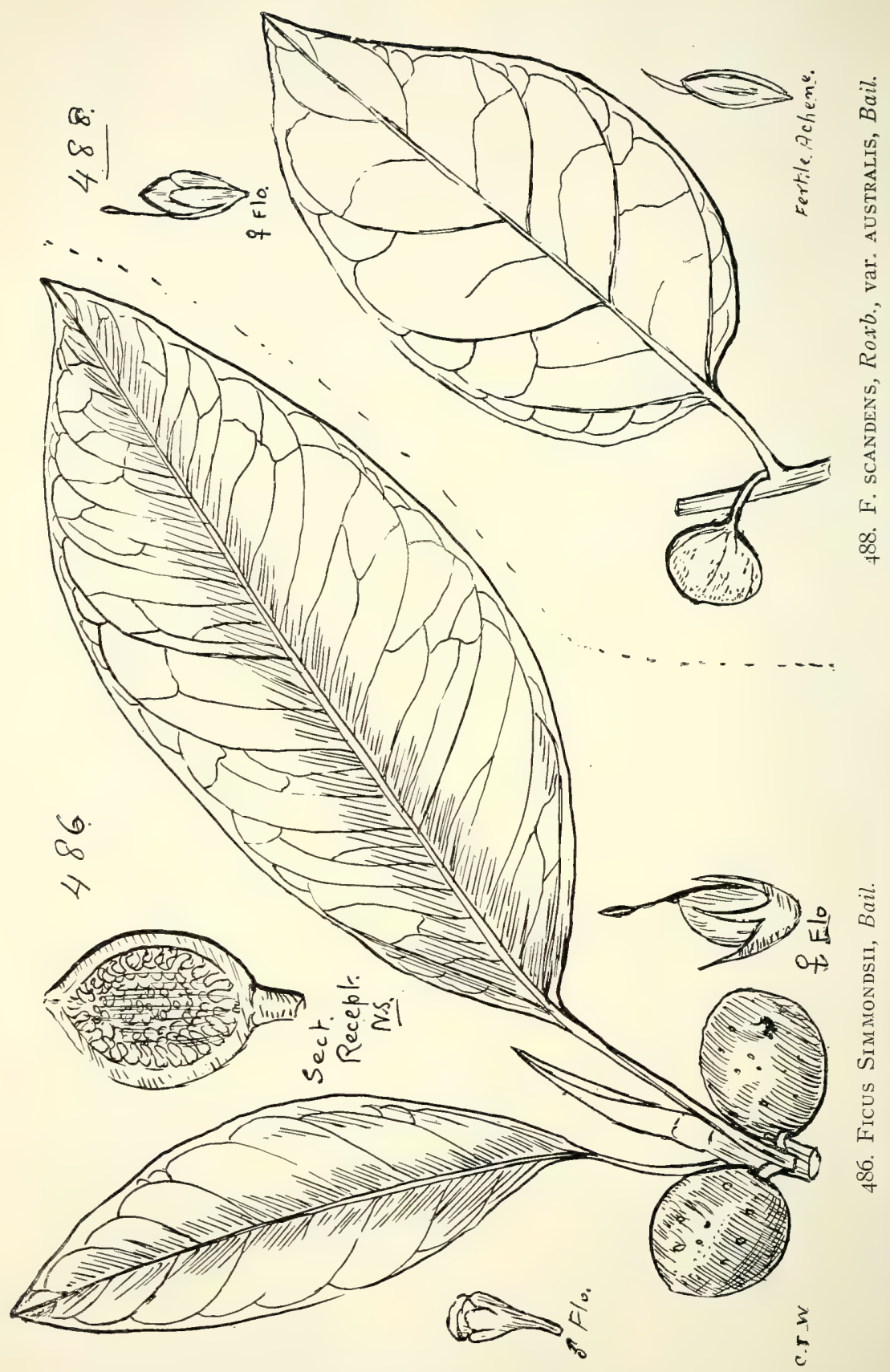




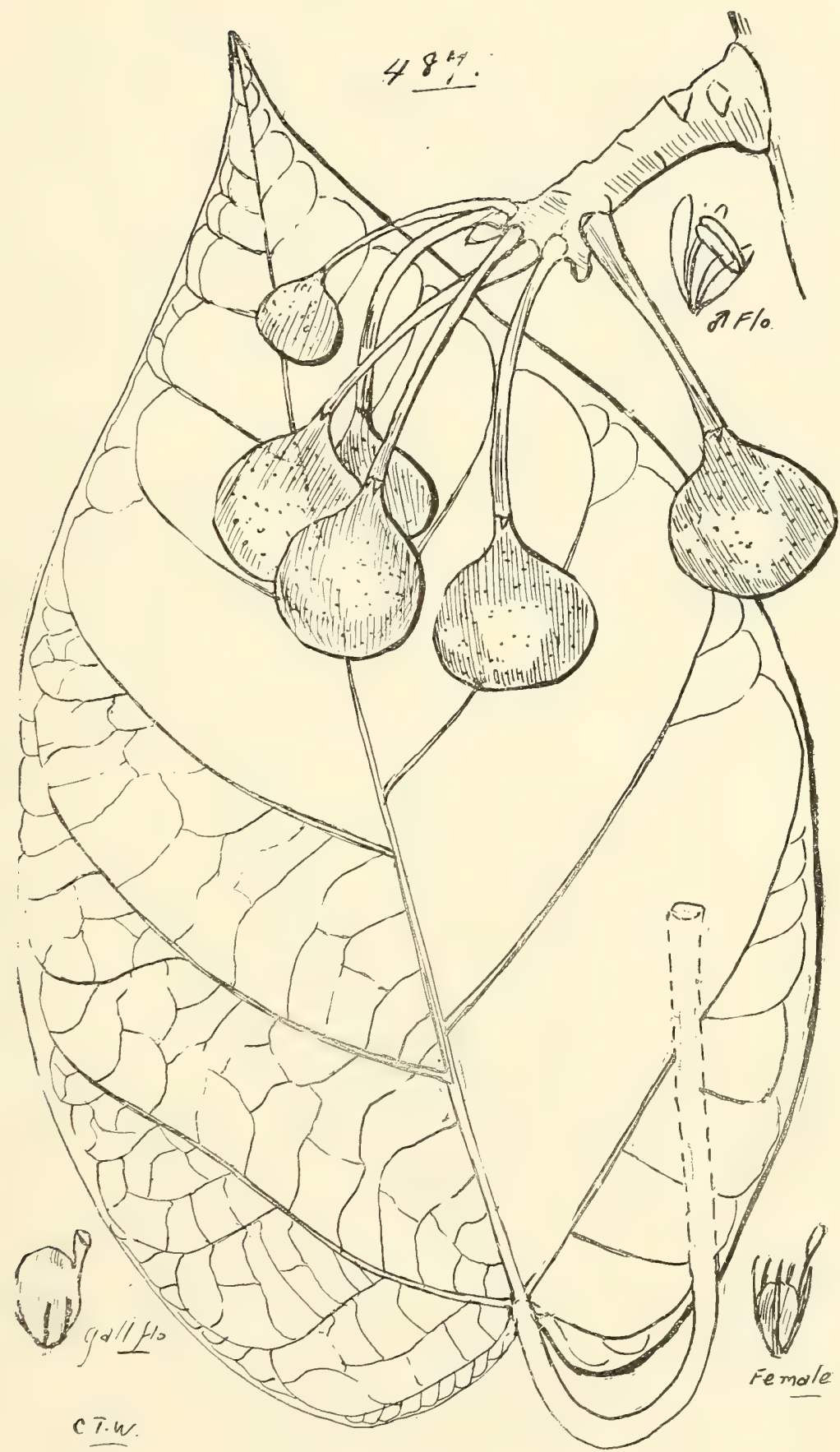

487. Ficus eHRetiordes, F. $v . M$. 


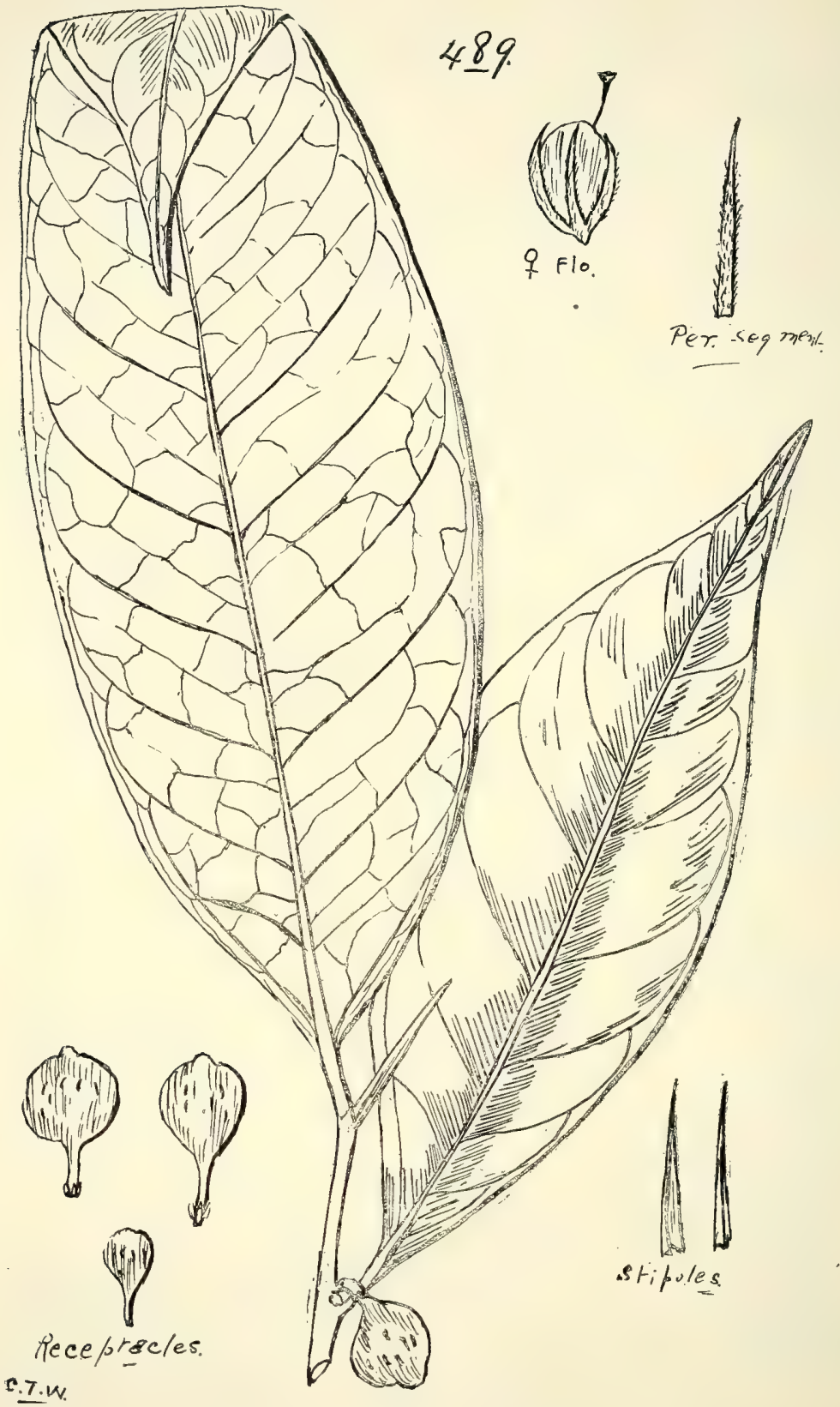

489. Ficus Pinkiana, F. v. $M$. 


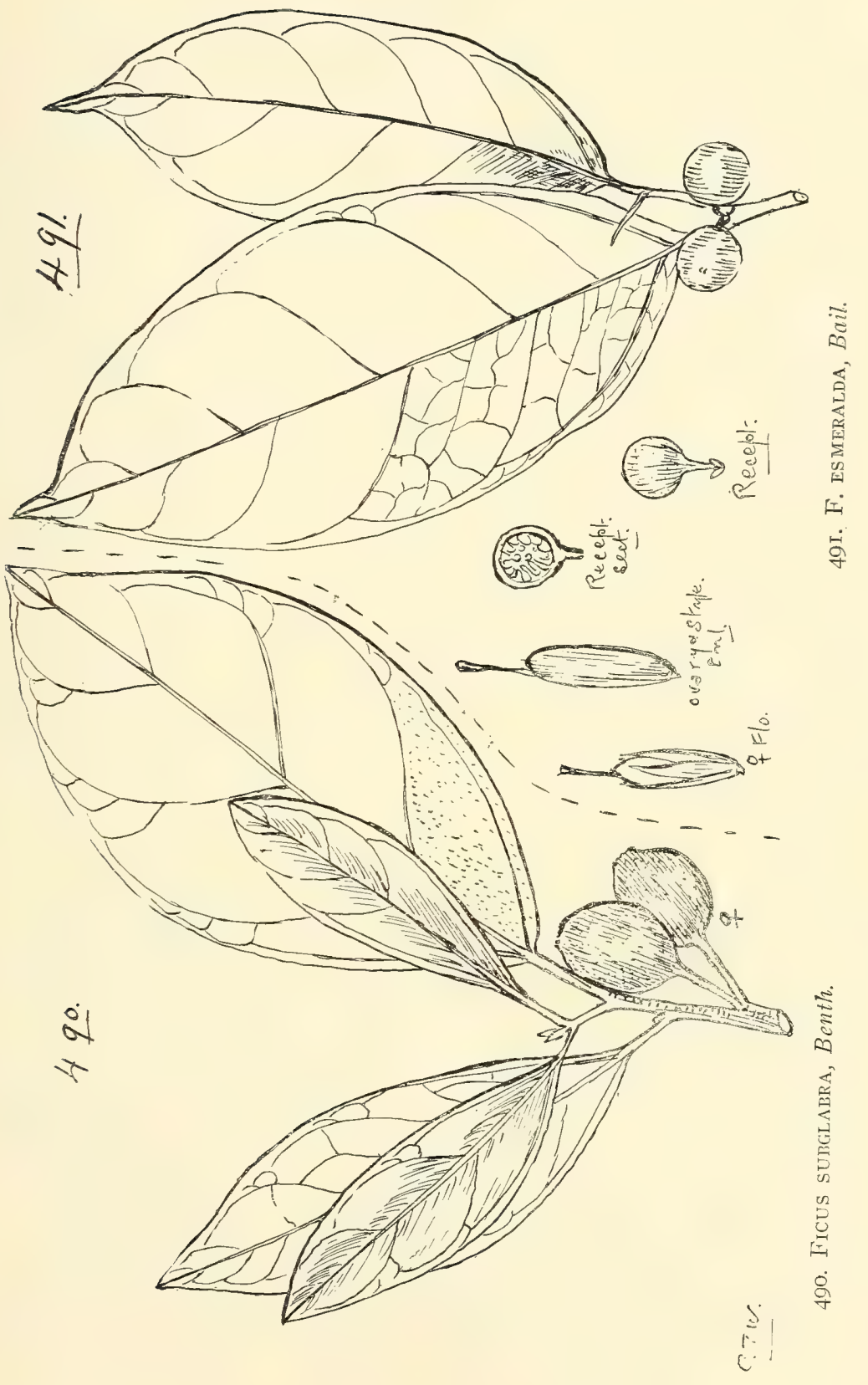


CXIX. URTICACEÆ:
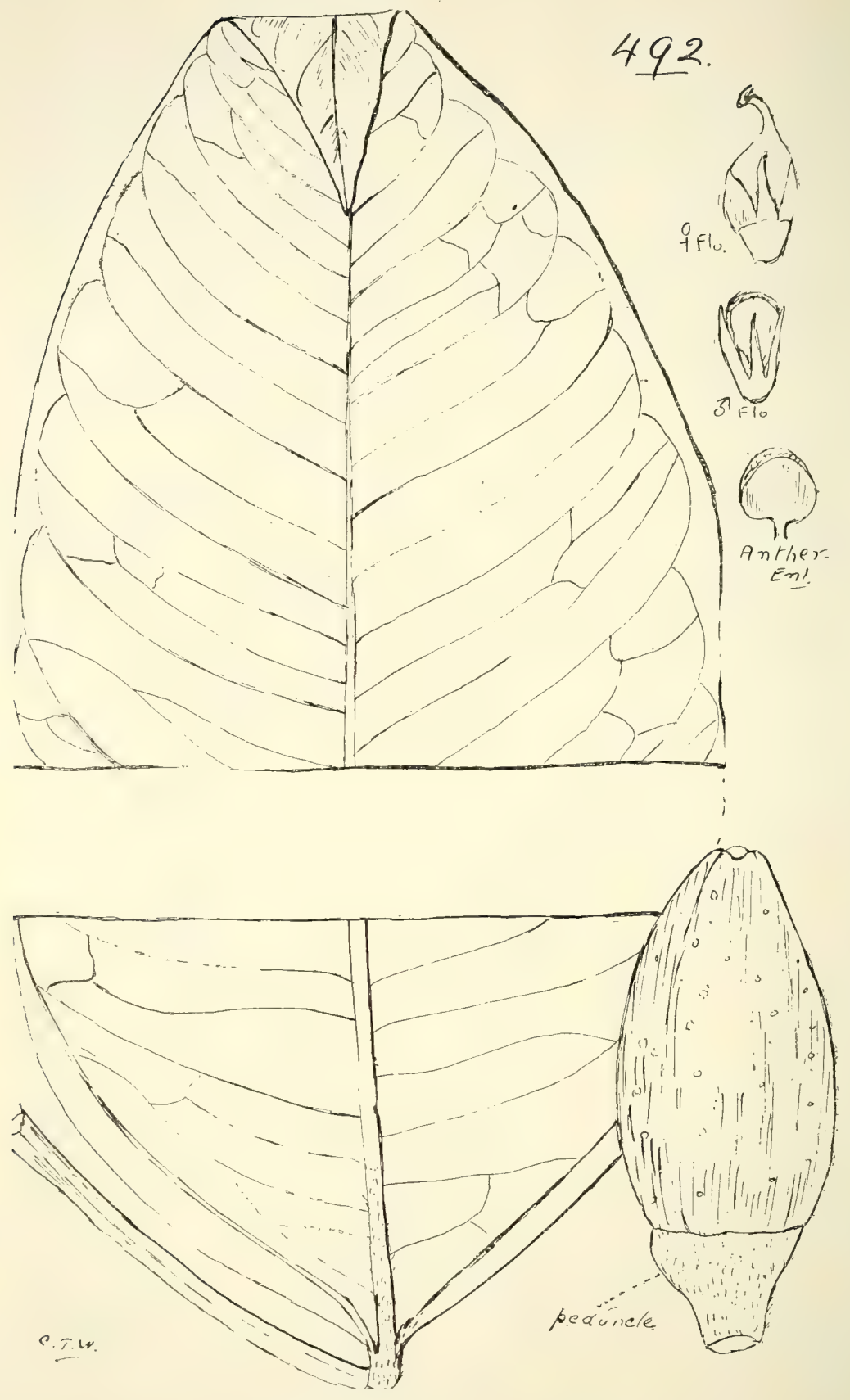

492. Ficus pleurocarp $\Lambda, F . v . M$. 
CXIX. URTICACEÆ.

501

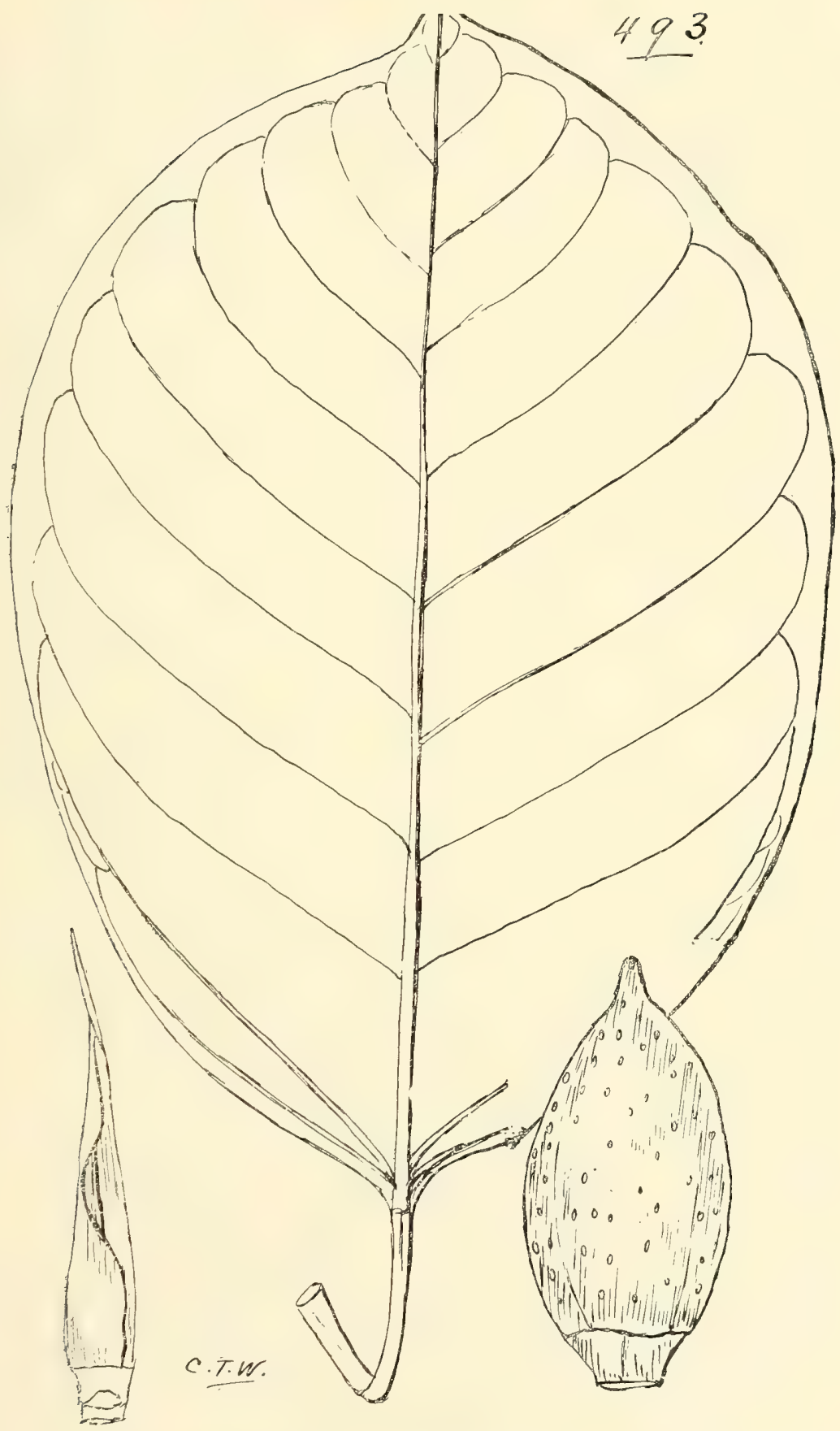

493. Ficus crassipes, Bail. 


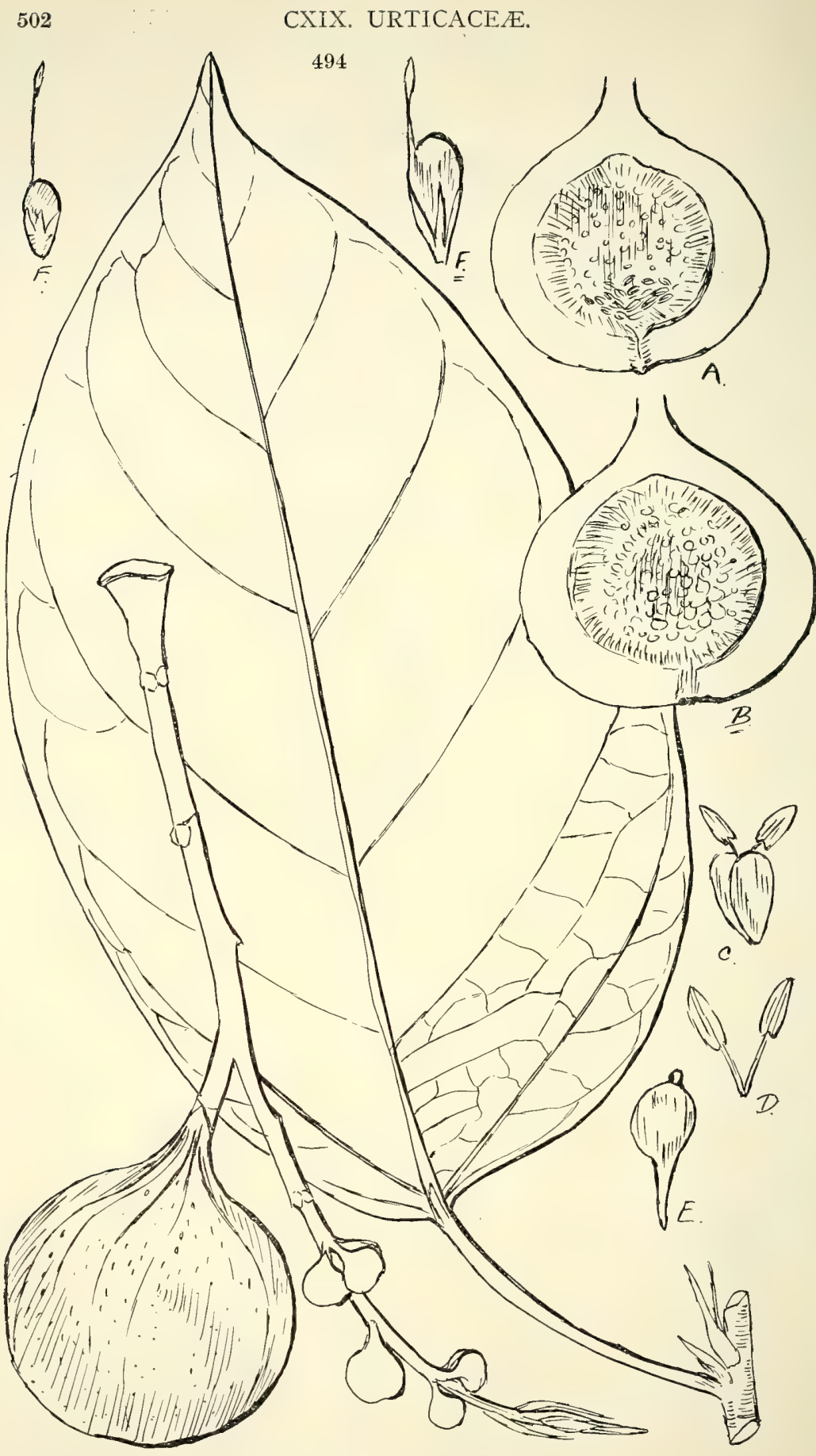

494. Ficus semicostata, Bail., sp. nov.

(A) Sect. recept. containing male and gall fowers, (B) sect. recept. containing female flowers, (C) male flower, (D) anthers, (E) gall flower, (F) female flowers. 


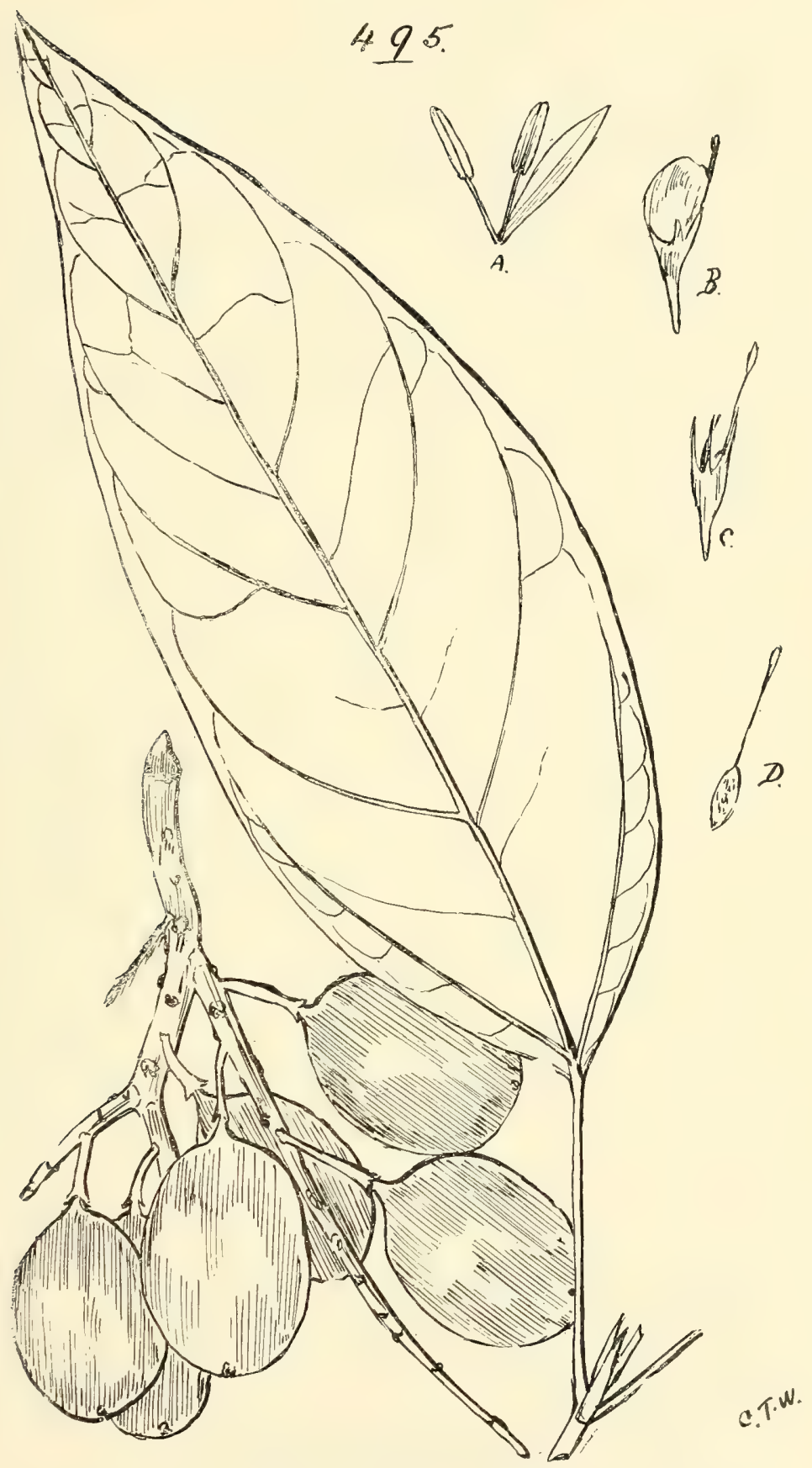

495. Ficus glomerata, Willd.

(A) Stamens, (B) neuter flower, (C) female flowers and perianth segments, (D) female achene. 
Ficus-contd.

Section III.-Covellia.

hispida, Limn. f.- "Wo-o" of Tully River and "Boombil" of Johnstone River natives.

fasciculata, F.v.M.- "Berdi-ga" of Starcke River natives.

var.? subopposita, Benth.

mourilyanensis, Bail.

esmeralda, Bail._-"Pandara" of Cape Grafton natives. (Fig. 49I.)

casearia, F.v.M.

pleurocarpa, F.v.M.- "Kar-pe" of Atherton natives. The fruit is prominently ribbed in a dry but not in a fresh state. ' (Fig. 492.)

crassipes, Bail. (Fig. 493.)

semicostata, Bail.-A very prolific fruit-bearer; receptacles I-2 inches diameter, striped with red. The fruit, which it bears in great abundance, is a useful food for many of our native animals when kept in confinement.

glomerata, Willd. $=$ F. vesca, F.v. M.; F. caudiciflora, Sol. MSS. and Banks's Journal (Hiern. J1. Bot. xxxix. (IgOr) 5).-Cluster Fig. "Moochai" of Cooktown and "Parpa" of Rockhampton natives. In Bombay the sap is a popular remedy which is locally applied to mumps and other inflammatory glandular enlargements. Another species of Fig was used by the Greeks, and is still in use in Egypt, for the same purpose. (Fig. 495.)

Cudrania, Trécul.

Subtribe II.-Euartocarpece.

javanensis, Trécul.-Cockspur Thorn. "Barra” of Brisbane natives. Wood and bark yield a yellow dye. A useful cabinet-wood.

var. Bancroftii, Bail.-Foliage variegated.

\section{Tribe IV.-URTICEÆ.}

\section{Subtribe 1.-Urerea.}

Urtica, Limn.

incisa, Poir.- "Cood-mo-ro" of Forest Hill natives.

*urens, Linn.-Common English Nettle. Europe.

Fleurya, Gaudich.

interrupta, Gaudich.

Laportea Gaudich.-The formic acid contained in the stinging hairs of these plants has been used by the natives of various parts where these trees abound to cure rheumatism, often by only stinging the locality affected. 
Laportea-contd.

Section Sarcopus.

gigas, Wedd.-Large Stinging-tree. "Braggain” of Brisbane natives. Should be useful for paper-making. Have met with trees having trunks over Io ft. in diameter. The dried leaves cause violent sneezing.

photiniphylla, Wedd.-Shiny-leaved Stinging-tree.

moroides, Wedd.-Stinging-tree. "Gympie" of natives.

Pilea, Lindl.

Subtribe II.-Procridece.

muscosa, Lindl.-Gunpowder-plant.

Elatostemma, Forst.

reticulatum, $W e d d$.

stipitatum, Wedd.

Bohmeria, Jacq.

Subtribe III.-Boehmeriece.

nivea, Hook. and Am.-_ Jalcan-jalcan" of Johnstone River natives. A useful fibre plant.

platyphylla, Don.-A useful fibre plant.

Pouzolzia, Gaudich.

indica, Gaudich.

Section I.-Pouzolzia.

Section II.-Memorialis.

quinquenervis, Benn.

Pipturus, $W e d d$.

argenteus, Wedd.--"Thil-la-wo" of Forest Hill, "Koomerookoomeroo" of Stradbroke Island, and "Kongangn" of Rockhampton natives.

Subtribe IV.-Parietariece.

Parietaria, Linn.-Pellitory. A European species is used for placing in corn to keep it free of weevils.

debilis, Forst.

Australina, Gaudich.

Subtribe V.-Forskaleece.

Muelleri, Wedd.

\section{Order CXX.-CASUARINEÆE.}

Casuarina, Linn.-Queensland Oaks. The word "Oak" is used in Queensland for all species of this genus; " Shea-oaks" in the Southern States. It is derived from the sound made by the passing wind through the slender branchlets of the trees, and has nothing to do with the well-known Oak-tree. 


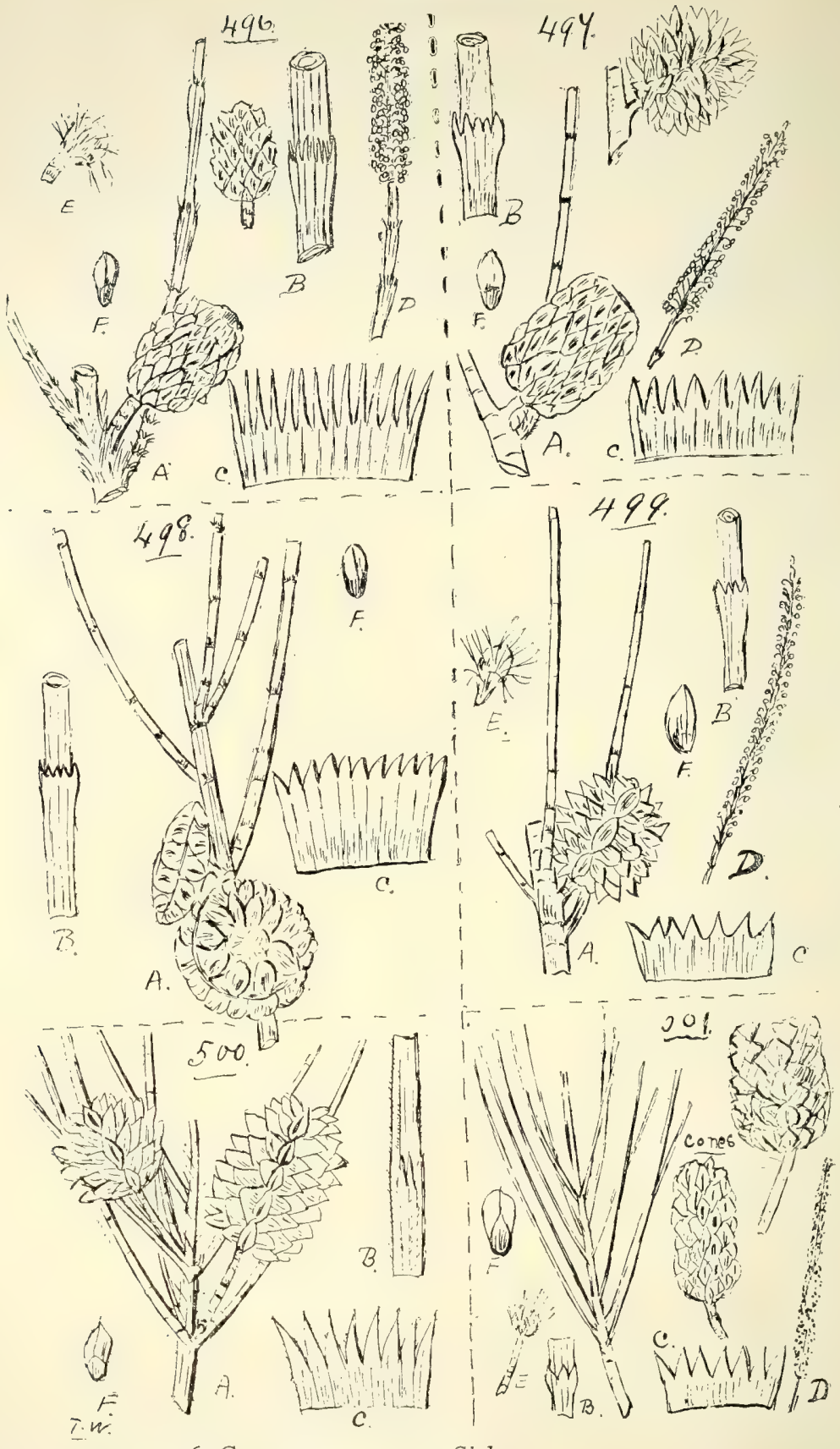

496. Casuarina glauca, Sieb.

497. C. lepidophloia, $F . v . M$.

498. C. Lueh Manni, $R . T$. Baker.

499. C. equisetifolta, Forst.

500. C. equisetifolia, Forst., var. incana, Benth.

501. C. suberosa, Ott. et Dietr.

(A) Fruiting cone, (B) portion of branchlet, (C) whorled scales opened out, (D) spikeof maie flowers, (E) inflorescence of female flowers, (F) seed. 


\section{2}
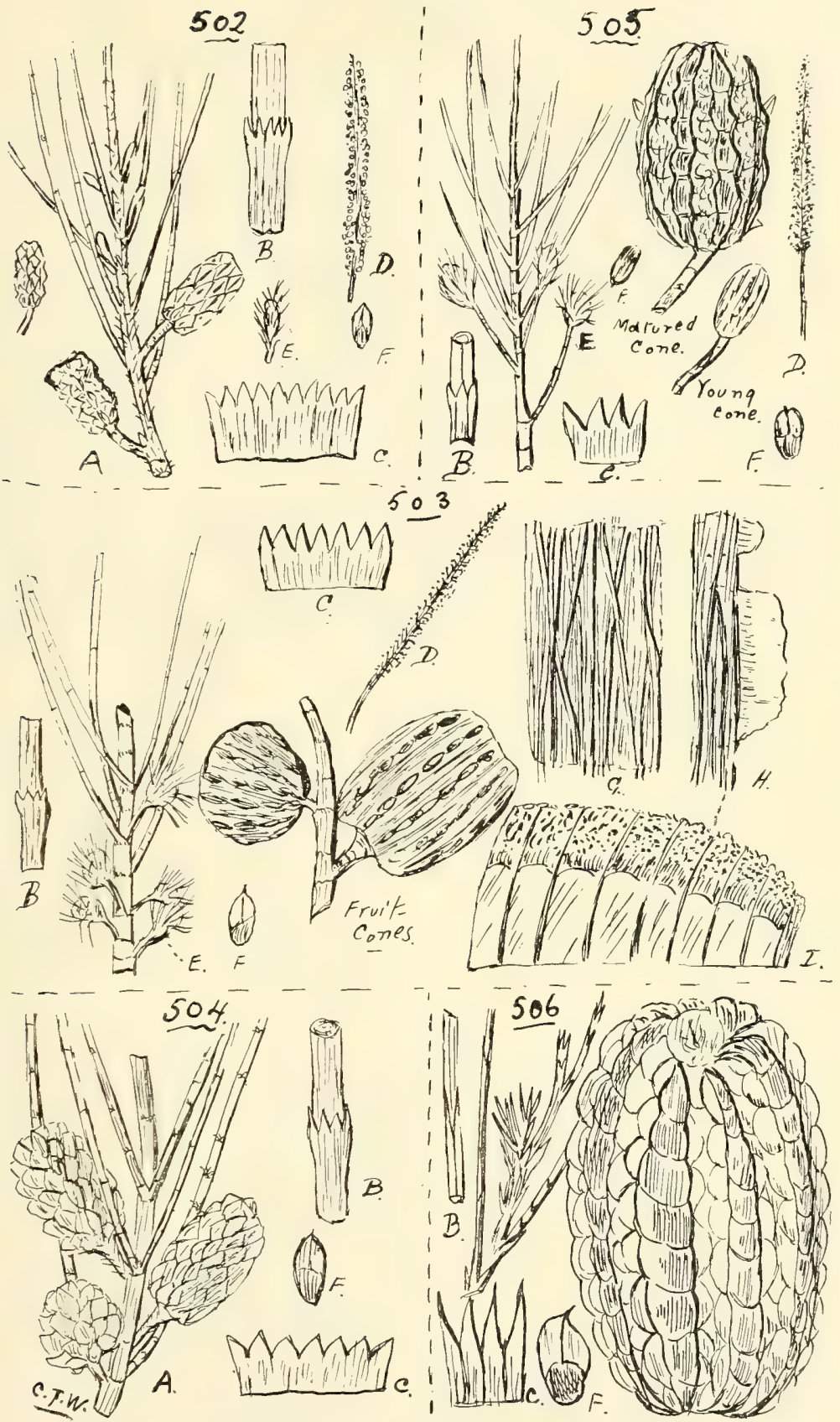

502. Casuarina Cunninghamit, Miq.

503. C. inophlota, F. v. M. et Bail.

504. C. DISTYLA, Vent.

505. C. torulosa, Ait.

506. C. Decaisneana, $F, v, M$.

(A) Cone, (B) portion of branchlet with leaf-teeth, (C) whorled scales (leaf-teeth). opened out, (D) male inflorescence, (E) head of female flowers, (F) seed, (G) the bark, (H) side-view of bark and medullary rays, (I) cross-section of timber showing: medullary rays. 
Casuarina-contd.

Section I.-Leiopity's.

glauca, Sieb.-Swamp Oak. "Billa" and "Wunna Wunnarumpin" of Stradbroke Island and "Woongul" of St. George natives. (Fig. 496.)

lepidophloia, F.v.M., Fragm. x. I 8. (Fig. 497.)

Luehmanni, R. T. Baker, Proc. Linn. Soc. N.S.W. xxiv. (i899) 608, tab. 47. (Fig. 498.)

equisetifolia, Forst.-The bark, according to Dr. Gibson, is an excellent astringent, and may be used with advantage in chronic diarrhœa and dysentery (Dymock). It is also used in tanning. (Fig. 499.)

var. incana, Benth. (Fig. 500.)

suberosa, Ott. et Dietr. - The C. leptoclada, Miq., placed by most authors as a synonym, is the monœcious form, of which the only Queensland specimens I have seen were some collected near Mooloolah Heads, in April, I9II. (Fig. 50I.)

Cunninghamiana, Miq. (Fig. 502.)

inophloia, F.v.M. et Bail.-Stringybark Oak. The hard, dark-coloured plates of the medullary rays form a pretty feature in the wood when used in cabinet-work. (Fig. 503.

distyla, Vent. (Fig. 504.)

Section II.-Trachypitys.

torulosa, Ait.-Forest Oak. "Boorooda" of Brisbane River and "Koondeeba" of Stradbroke Island natives. (Fig. 505.)

Decaisneana, F.v.M. (Fig. 506.) Figured from specimen kindly lent for the purpose from the Muellerian Herbarium, by Professor A. J. Ewart.

\section{Order CXXI.-CUPULIFER E.}

Fagus, Linn .

Moorei, F. $v . M_{0}=F$. Carronii, C. Moore. - Negro-head Beech of N. S. Wales. In Queensland of little value as a timber, as the stems are so often pipy. (Fig. 507.)

\section{Serifs ViII.-Order of Doubtful Afrinity.}

\section{Order CXXII.-CERATOPHYLLEÆ.}

Ceratophyllum, Linn.

demersum, Linn.-Hornwort; a troublesome pond-weed. (Fig. 508.) 
CXXI. CUPULIFERÆ_-CXXII. CERATOPHÝLLE仺.

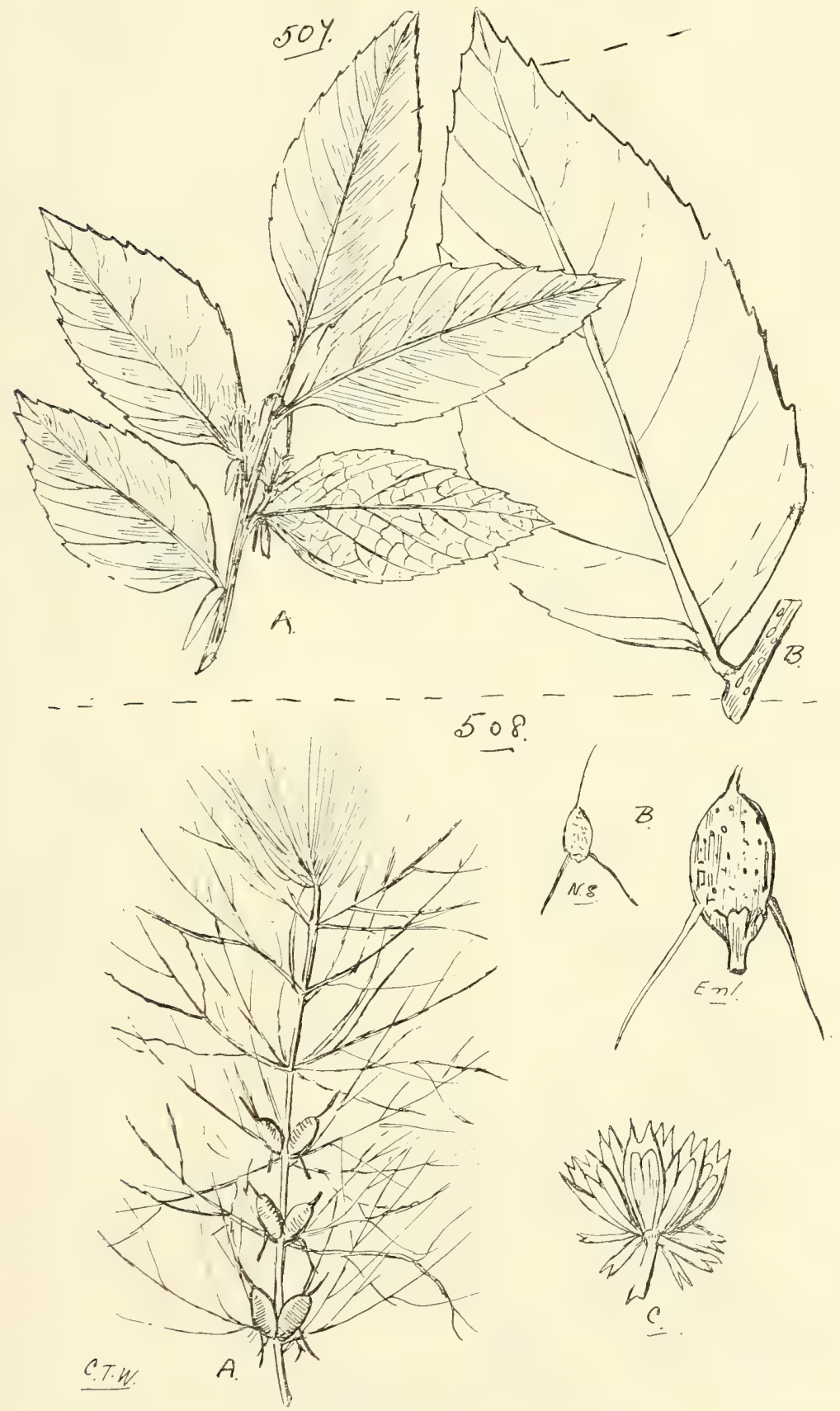

507. Fiaus Mooret, F. v. $M$.

(A) Flowering branchlet, (B) leaf from a sterile branchlet.

508. Ceratophyllum demersum, Lim.

(A) Portion of plant in fruit, nat. size, (B) fruit with prickles, (C) male flower, enl.. 


\section{Class II.-GYMNOSPERMEAE.}

\section{Order CXXIII.-CONIFER}

Tribe I.-Cupressinez.

Callitris, Vent. =Octoclinus, F. v. M. ; Frenela, Mirb. Cypress Pine is the commonest vernacular name applied to these trees.

Macleayana, F.v.M. $=C$. Parlatorei, F. v. M.-Stringybark Pine. (Fig. 509.)

robusta, R. Br.-Cypress Pine. This species is frequently met with inland. (Fig. 5Io.)

columeliaris, F.v.M. =F. Moorei, Parl__ "Buragari" and "Pooragri" of Brisbane River and "Coolooli" of Wide Bay natives. (Fig. 5II.)

forma glauca, Bail.

Hab.: Rosewood Scrub and a few coastal localities; not very abundant in Queensland.

verrucosa. R. Br. (Fig. 512.)

Muelleri, Parlat. (Fig. 5I3.)

cupressiformis, Vent.- "Brorogorre" of Stradbroke Island natives., (Fig. 5I4.)

calcarata, $R . B r .=F$. rhomboidea, Endl.-The twigs are said to be used to expel worms in horses. (Fig. 5I5.)

TRIBE II.-PODOCARPEE.

Podocarpus, L'Her. = Nageia, Gærtn.

elata, R. Br.-She-Pine. "Kidneywallum" of Mooloolah and "Daalgaal" of Barron River natives.

pedunculata, Bail.-Black Pine. "Chupolla" of Atherton natives.

Ladei, Bail., Q1. Agric. J1. xv. (I905) 899, tab. 22.-The Mount Spurgeon Black Kauri Pine. (Fig. 5I6.)

Tribe III.-Araucaries.

Agathis, Salisb. = Dammara, Rumph.-Fruit a Strobilus.

robusta, C. Moore.-Queensland Kauri Pine, Dundathu Pine, or "Dundarum" of the Brisbane River natives (Petrie); "Ki-gunno" of Cardwell natives (R.B.H.).

Palmerstoni, F.v.M.- "Togoi" of Barron River natives. Araucaria, Juss.

Cunninghamii, Ait.-Hoop Pine; at one time better known as Moreton Bay Pine. "Koonya" of Bundaberg, "Thookooroo" of Forest Hill, and "Coonam" and "Kumbartcho" of Wide Bay natives. The bark yields 2.56 per cent. of tannin (Brïnnich). 
CXXIII. CONIFER死.

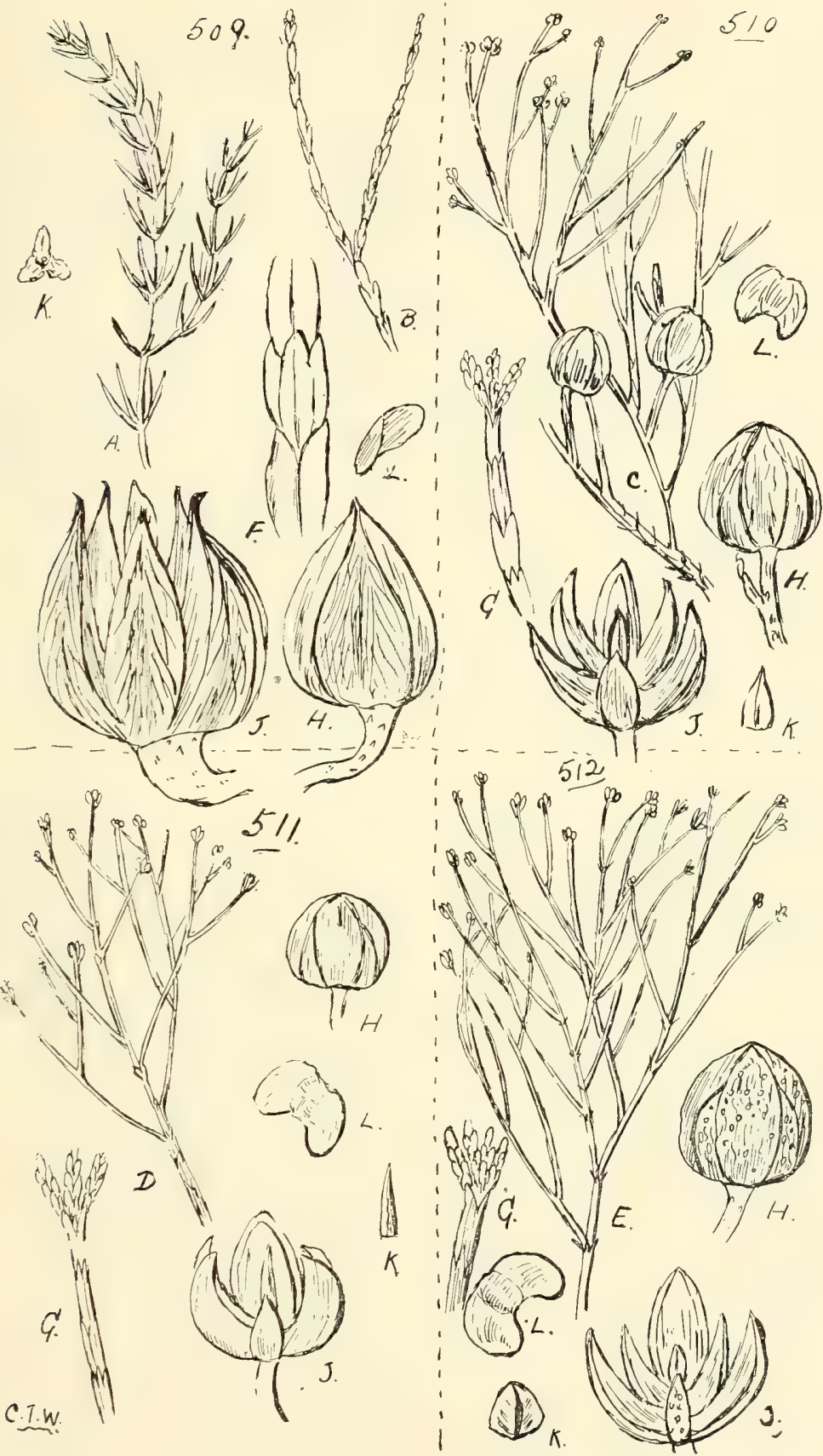

509. Callitris Macleayana, $F, v \cdot M$.

5IO. C. ROBUSTA, R. Br.

5II. C. COLU Mellaris, $F . v . M$.

5I2. C. verRuCOSA, R. Br.

(A) Branchlet with leaves from a young tree, nat. size, (B) branchlets, nat. size, (C) branchlet bearing male flower and cones, nat. size, (D) and (E) branchlets bearing male flowers, nat. size, (F) portion of branchlet, enl., $(G)$ portion of branchlets bearing male flowers, enl., (H) cone, nat. size, (J) opened cone, nat. size, (K) columella, nat. size. 

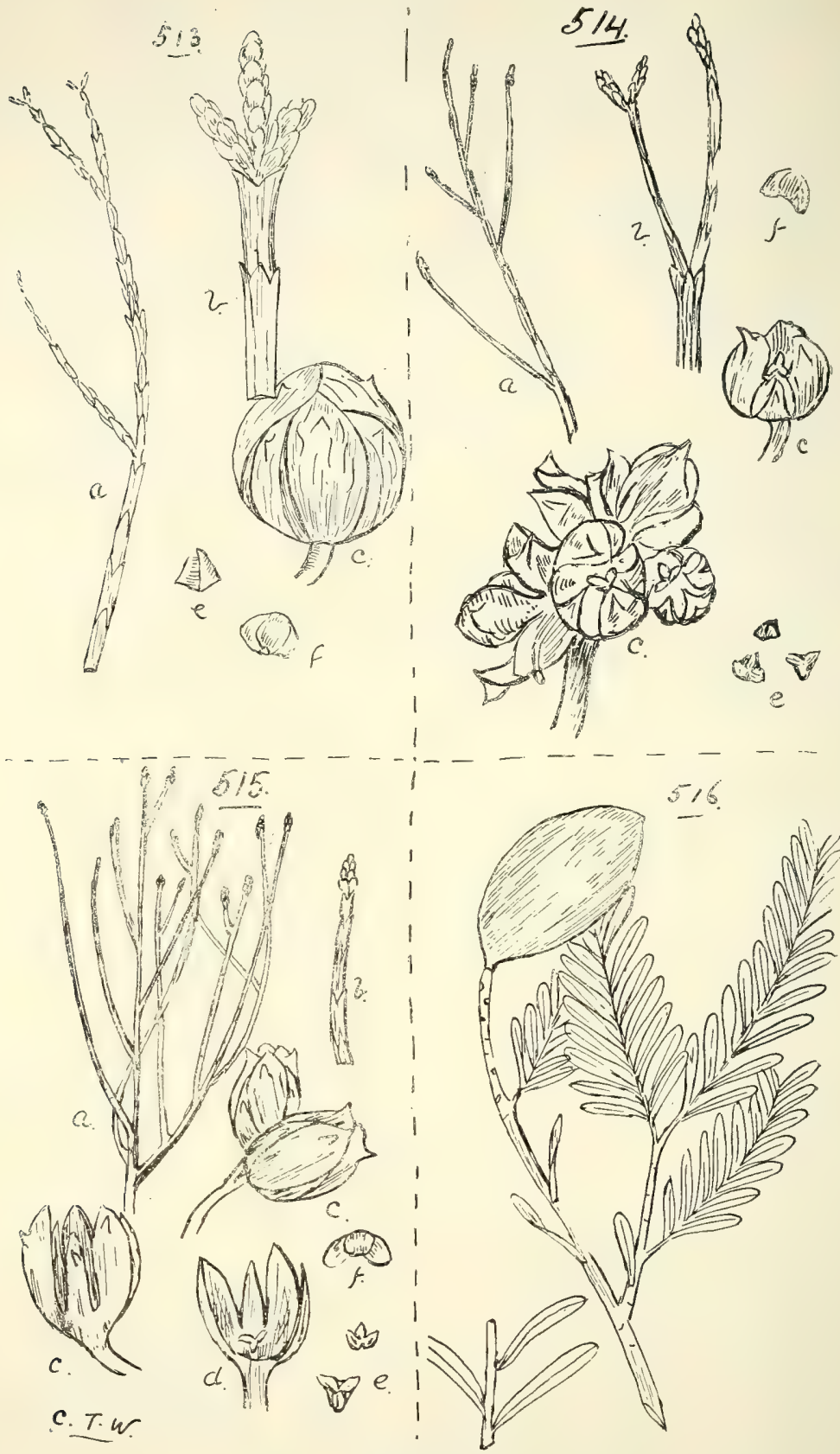

513. Callitris Muelleri, Parl.

5I4. C. Cupressiformis, Vent.

5I5. C. calcarata, R. $B r$.

(a) Branchlets, nat. size, (b) portion of branchlet bearing male flowers, enl., (c) cone, nat size, (d) sect. cone, nat. size, (e) columella, nat. size, (f) seed, nat. size. 5i6. Podocarpus Ladei, Bail. 
Aratucaria-contd.

Cunninghamii-contd.

forma glanca, Bail. $=$ A. glauca, Antoine.-A native of the islands off our N.E. coast; differs from the normal tree in its sometimes glaucous colour.

Bidwilli, Hook.-Bunya-bunya Pine; from the native name "Bon-ye," "Bon-yer," or “Bon-yee." "Ki-gera” of Cardwell natives (R.B.H.). This grand Conifer was originally discovered by Mr. Andrew Petrie.

\section{Order CXXIV.-CYCADACE\#.}

(The roots of plants of this Order are gatherers of nitrogen.)

Cycas, Linn.

\section{Tribe I.-Cycades.}

media, R. Br. - "Kammama" of Atherton, "Ma-ra" of Bloomfield, "Bodell" of Cairns, "Nijar" of Cape Grafton, "Baven" of Rockhampton, "Kimalo" of Tully River, and "Ba-dur" of Cooktown natives. "Bun-jin-oo" of Cardwell natives (R.B.H.).

Normanbyana, F.v.M.-Curly Pine Palm.

Cairnsiana, F. ז'. 1 .

Kennedyana, F.v.M.

Rumphii, Miq. =C. Scratchleyana, F.v. M.-The Director, Royal Botanic Gardens, Kew, England, informs me that specimens of this plant collected at Cape York in I864 were forwarded to the Kew Herbarium by Mr. Walter Hill in I88I.

Tribe II.-EnCEPHALARTEÆ.

Macrozamia, Miq. = Encephalartos, Lehm.- "Rickets" plants, all supposed poisonous to stock (see Bail., Q1. Agric. J1. ii. (I898), I3I, tab. xv.; Lauterer, Proc. Roy. Soc. Q. xiv. ( $(899), 5)$.

platyrhachis, Bail.-This was said by Mr. H. C. BrockHollingshead not to produce "rickets" in stock; but during the present year Mr. G. D. Logan sends me word that many stock suffer with the disease from eating this plant. (Fig. 5I7.)

spiralis, Miq.-Native Pineapple.

Paulo-Gulielmi, F.v. M.

Miquelii, F.v.M. = M. Mackenzii, Hort. Gard. Chron. I877 (I), 665, Fig. Io9. "Banga " of Rockhampton natives. Under the name of "Banga," the wool or dense down covering the bottom of the petioles is used for stuffing pillows. 


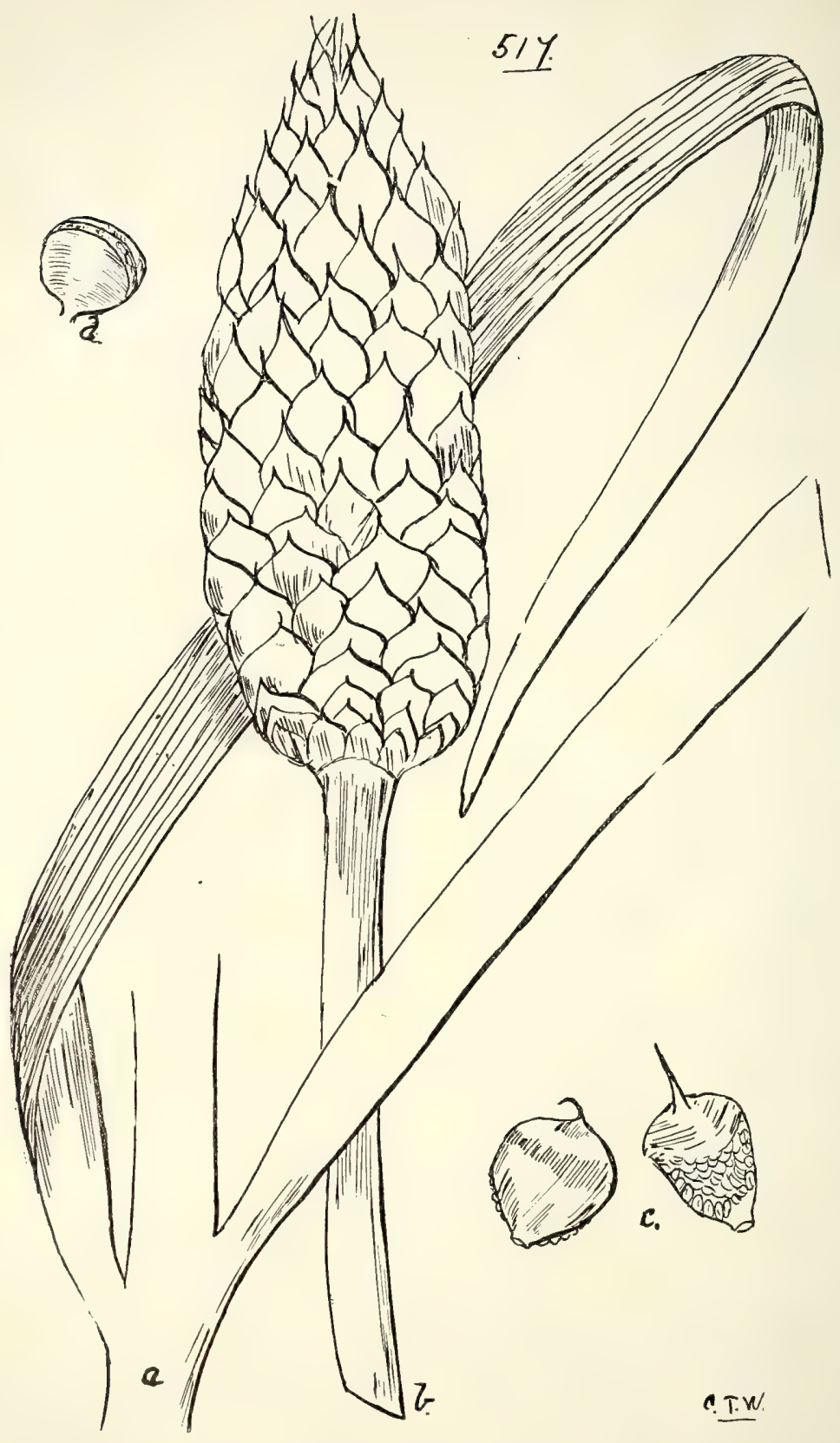

5I7. Macrozamia platyrhachis, Bail.

(a) Rhachis and a pair of pinnæ, (b) male cone, (c) antheriferous scales, (d) anther. (a)-(c) nat. size; (d) enl. 


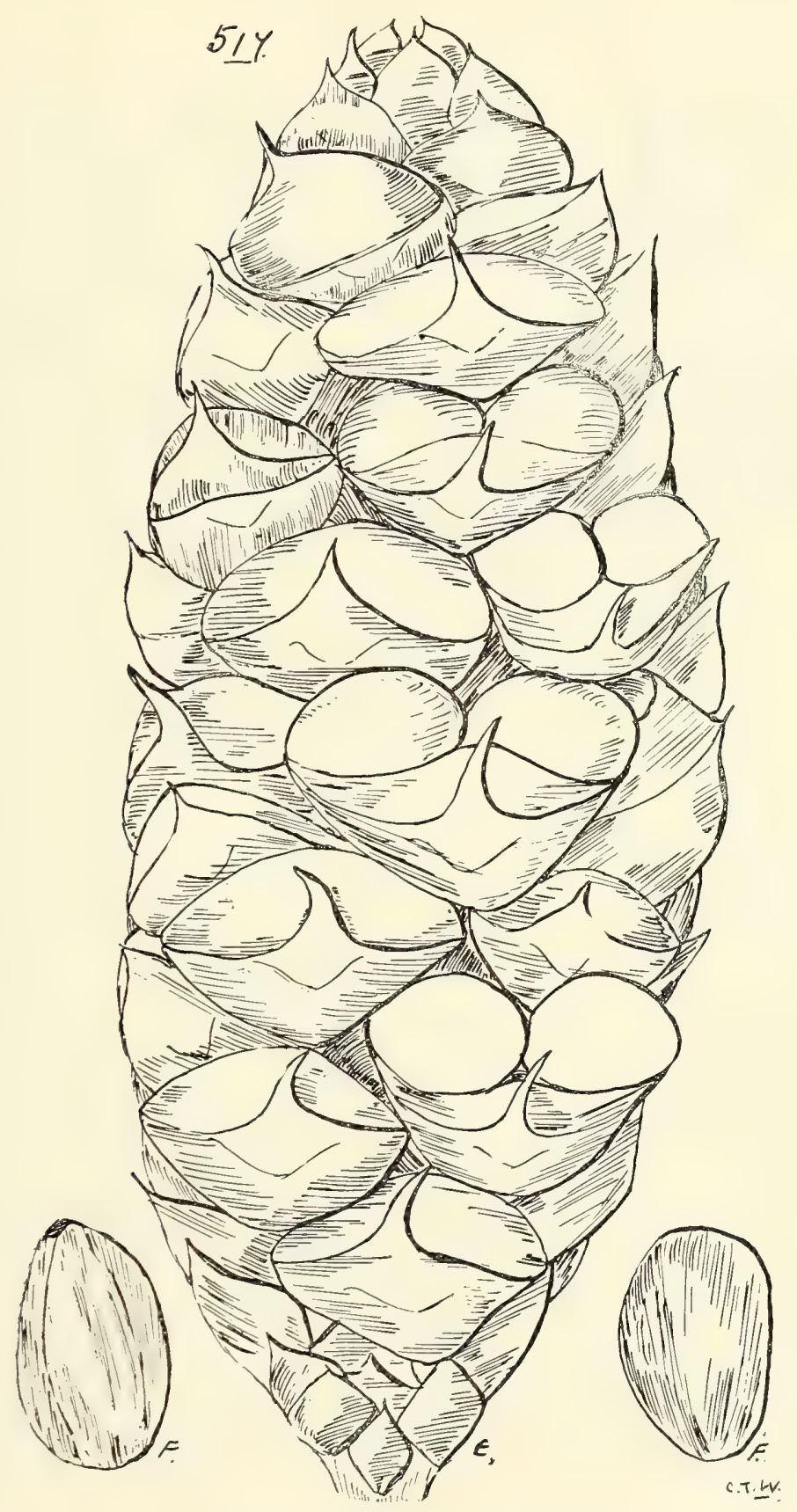

517. Macrozamia platyrhachis, Bail.

(e) Female cone, (f) seeds. (e) and (f) nat. size 
$5 \mathrm{I} 7 \mathrm{~b}$ is

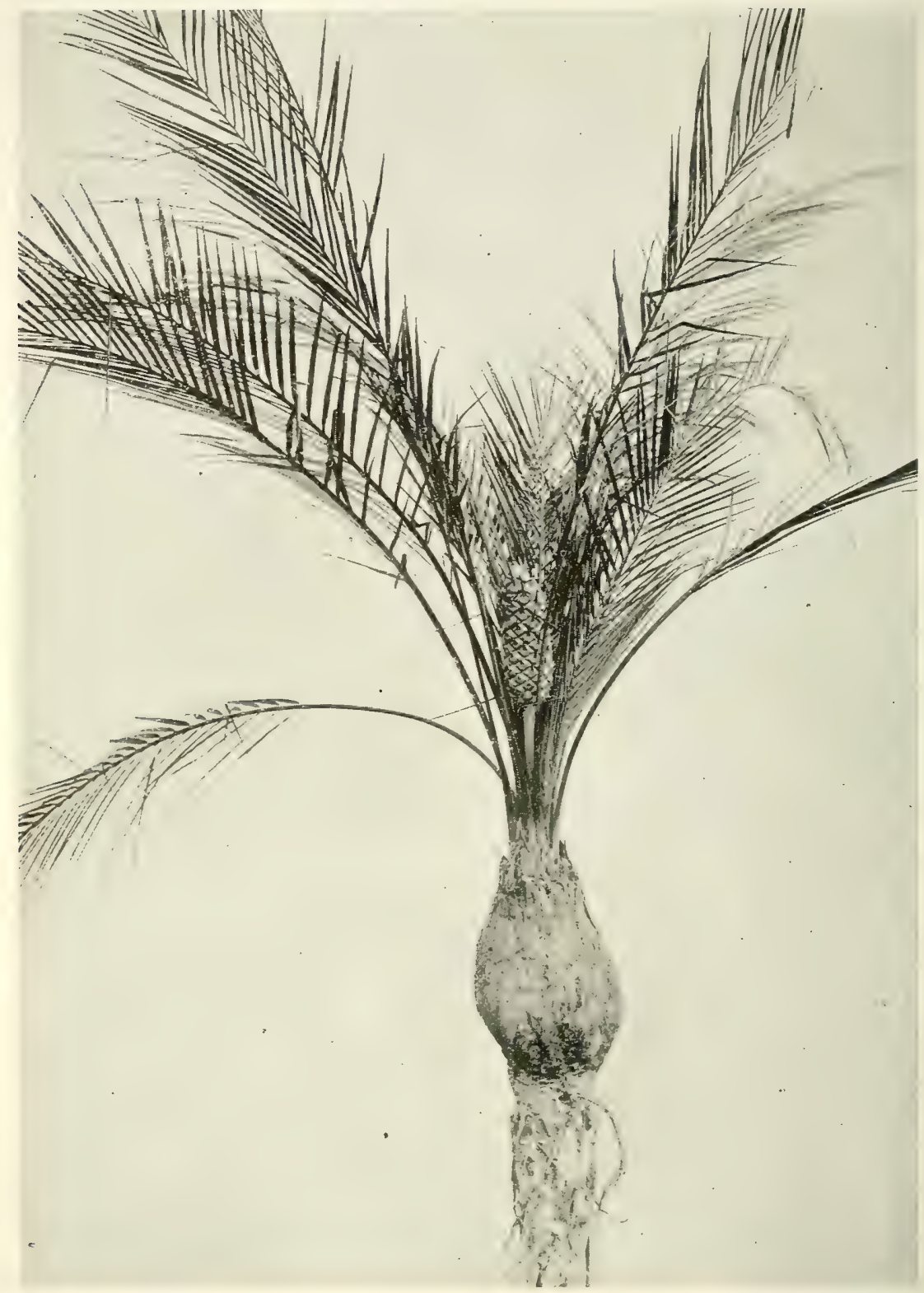

5it bis. A Female Plant of Macrozamia mountperriensis, Bail. 


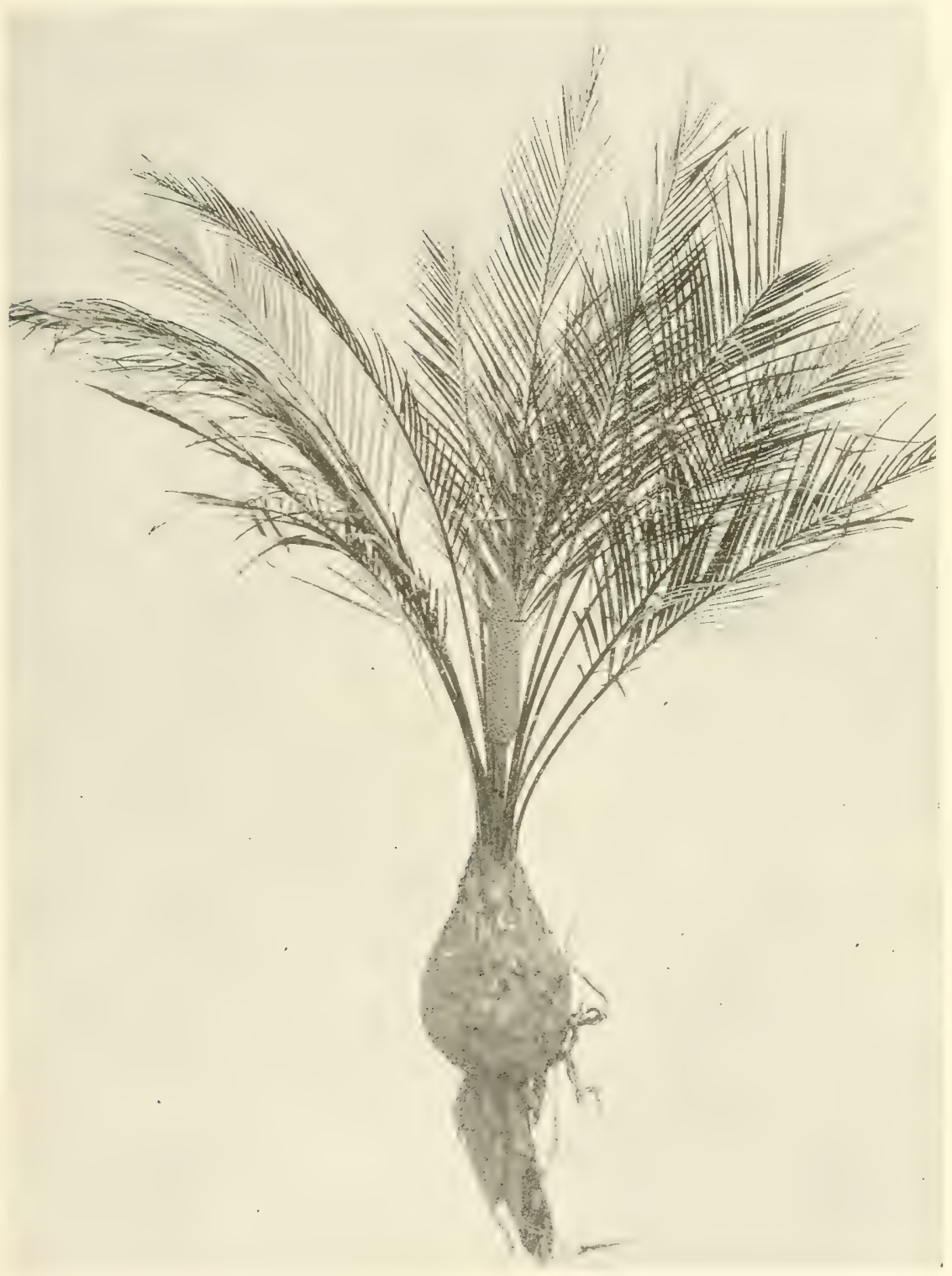

5I7 bis. A Male Plant of Macrozamia mountperriensis, Bail. 
Macrozamia-contd.

mountperriensis, Bail.-_" Tchalli" of Mount Perry natives. (Fig. 517 bis.)

Douglasii, W. Hill.-_Coobine" or "Coobyn" of Fraser Island natives.

Moorei, F.v.M.

Denisonii, C. Moore $=$ M. Perowskiana, Miq.

Hopei, W. Hill = Catakidozamia Hopei, W. Hill._-"Arımba” of Cairns natives.

Bowenia, Hook.

spectabilis, Hook._- "Ju1-bin" of Mount Cook natives; " moonah," the root or yam. "Gunyoo" of Cardwell natives. var. serrata, Bail. (F. Muell., Fragm. Phytog. viii. 279.)

\section{Class III.-MONOCOTYLEDONS.}

Series I.-MICROSPERMÆ.

\section{Order CXXV.-HYDROCHARIDEÆ.}

Series I.-Glycydræ.

Tribe I.-Hydrilleæ.

Hydrilla, Rich.

verticillata, Casp.-A bad water-weed.

Tribe II.-VAlLISNERIEæ.

Vallisneria, Linn.

spiralis, Linn.-Tape-grass. The growth of this. plant is so dense in some of our rivers that it impedes the progress of small boats; and in bathing persons should avoid approaching too near to where it is seen growing, for even good swimmers have become entangled in its leaves and been drowned.

gracilis, Bail. (Fig. 5I8.)

caulescens, Bail. et F.v.M. (Fig. 5I9.)

Blyxa, Thouars.

Roxburghii, Rich.

Tribe III.-Stratioteie.

Hydrocharis, Limn.

morsus-ranæ, Lim.-Frogbit.

Ottelia, Pers.

alismoides, Pers.

ovalifolia, L.C. Rich. 
Series II.-Marinæ.

Tribe IV.-Thalassie

Enhalus, L. C. Rich.

Kœnigii, Rich.—“Wa-pan" of Cape Bedford natives. (Fig. 520.)

Halophila, Thour.

ovalis, Hook.

spinulosa, Benth.

\section{Order CXXVI.-BURMANNIACEE.}

Burmannia, Linn.

disticha, Linn. (Fig. 521.)

juncea, Solander.-This plant has only, so far as I know, been met with in the two following localities: Endeavour River, N.Q. (Banks and Sol.); Logan River, S.Q. (Scortechini).

\section{Order CXXVII.-ORCHIDEÆ.}

Tribe I.-EPIDENDRE 2 .

Microstylis, Nutt.

Subtribe I.-Malaxed.

congesta, Reichb.f. = M.Bernaysii, F. v. M.

Subtribe II.-Liparidea.

Oberonia, Lindl.

iridifolia, Lindl.

palmicola, F.v.M.

pusilla, Bail. = Phreatia pusilla, Rolfe. (Fig. 523.)

Liparis, Rich. = Sturmia, Endl.

Nugentæ, Bail.

reflexa, Lindl.

cuneilabris, F.v.M. =S. angustilabris, F.v. M. (Fig. 524.)

cœlogynoides, F.v.M.

mowbulana, Bail., Q1. Agric. J1. ii. (I898) I97, tab. ı6.

habenarina, F.v.M.

Simmondsii, Bail.

Swenssonii, Bail., Q1. Agric. J1. I6 (I906), 564.

Subtribe III.-Dendrobiea.

Dendrobium, Sw.

Section I.-Stachyobium.

Muellerianum, Schlechter in Fedde. Repert. iii. (I907) 3I6.

Section II.-Dendrocoryne.

bigibbum, Lindl._"Zula” of Batavia River natives. var. album, Bail. 

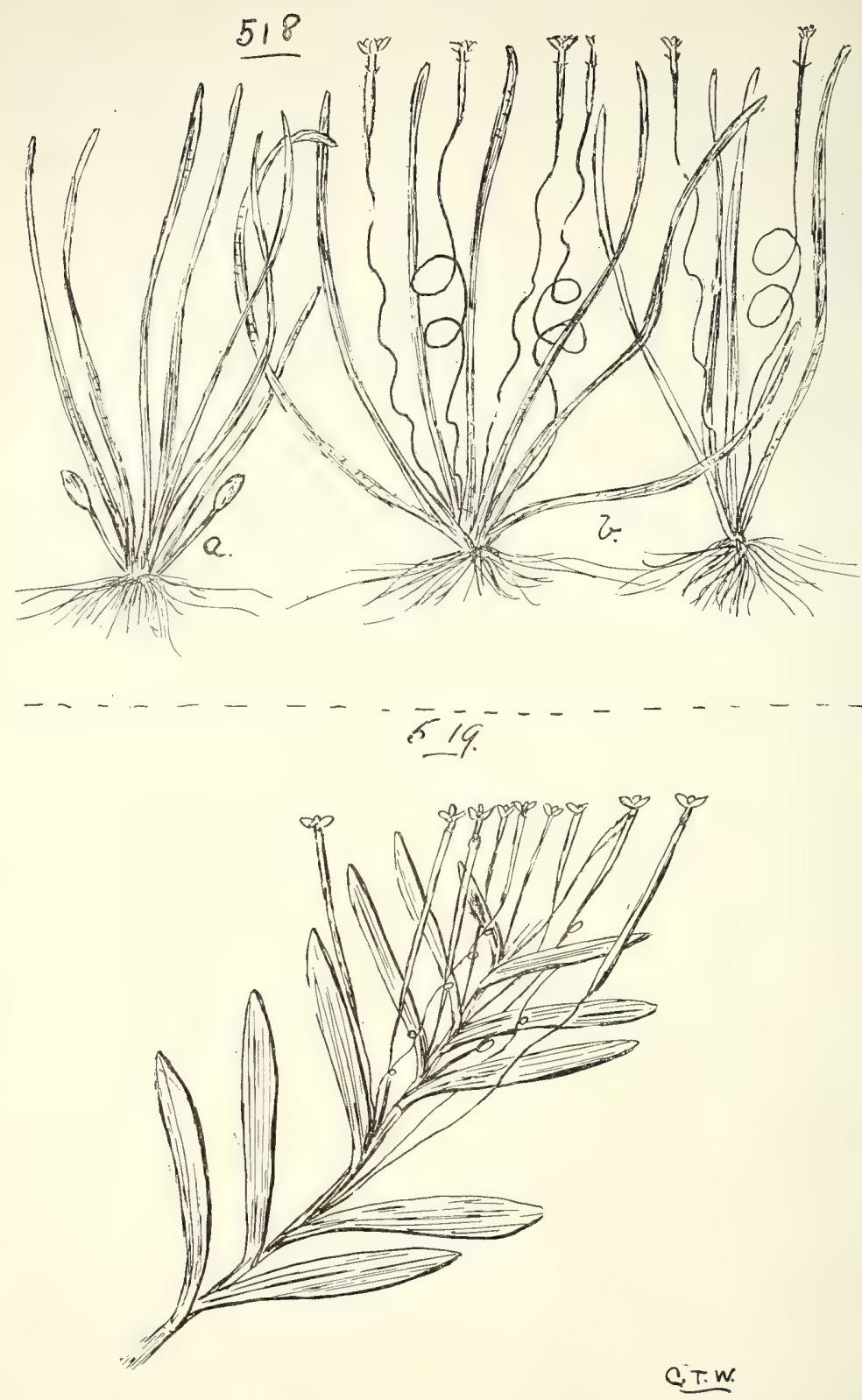

5I8. Vallisneria gracilis, Bail.

5I9. V. Caulescens, Bail. et F. v. M. 


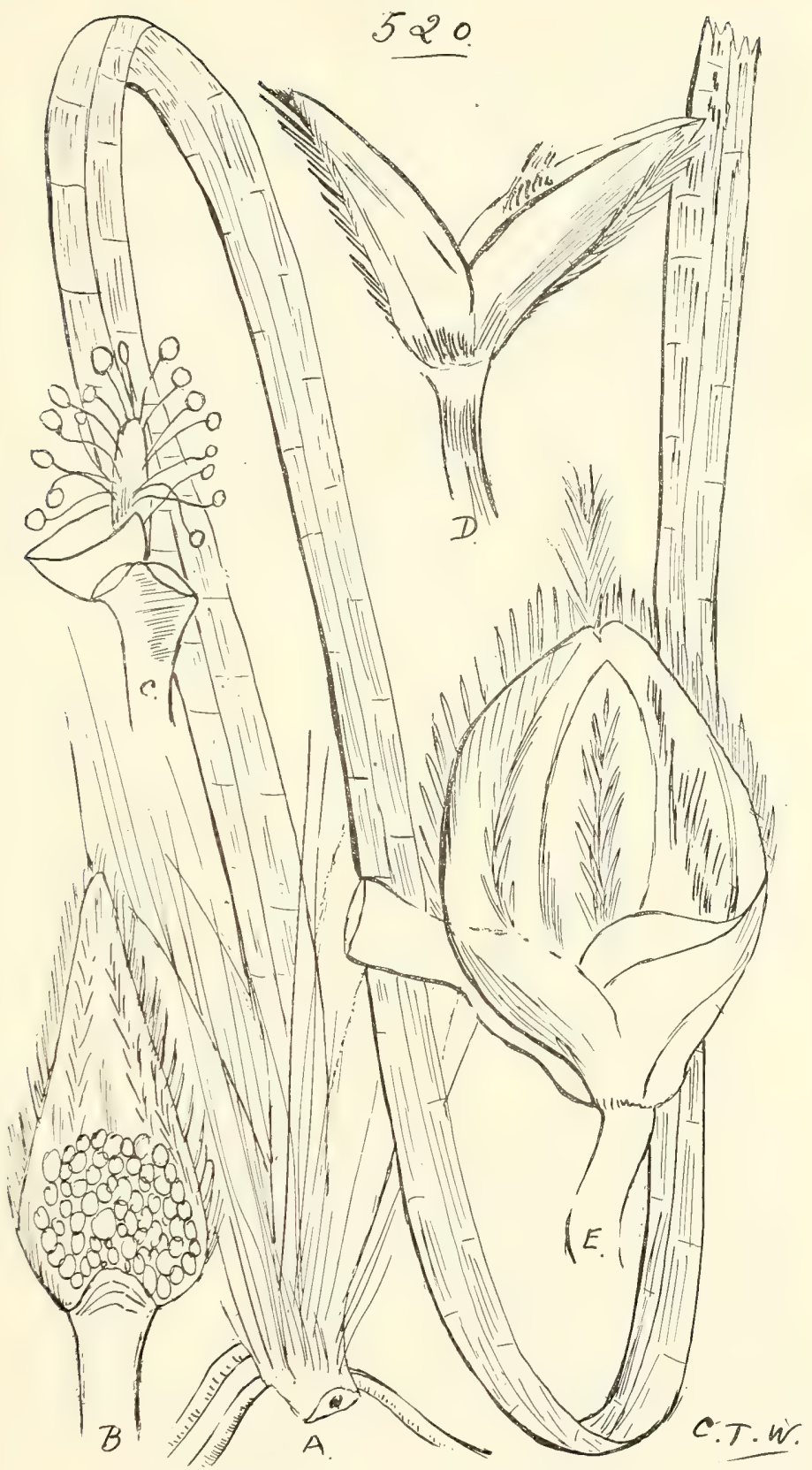

520. Enhalus Kenigit, Rich.

(A) Portion of plant, (B) male flower-buds, one valve of spathe removed, (C) a few pedicelled male flowers on the short spadix, (D) the two valves of the male spathe, (E) female spathe. All about nat. size. (B)-(E) after Griffith. 


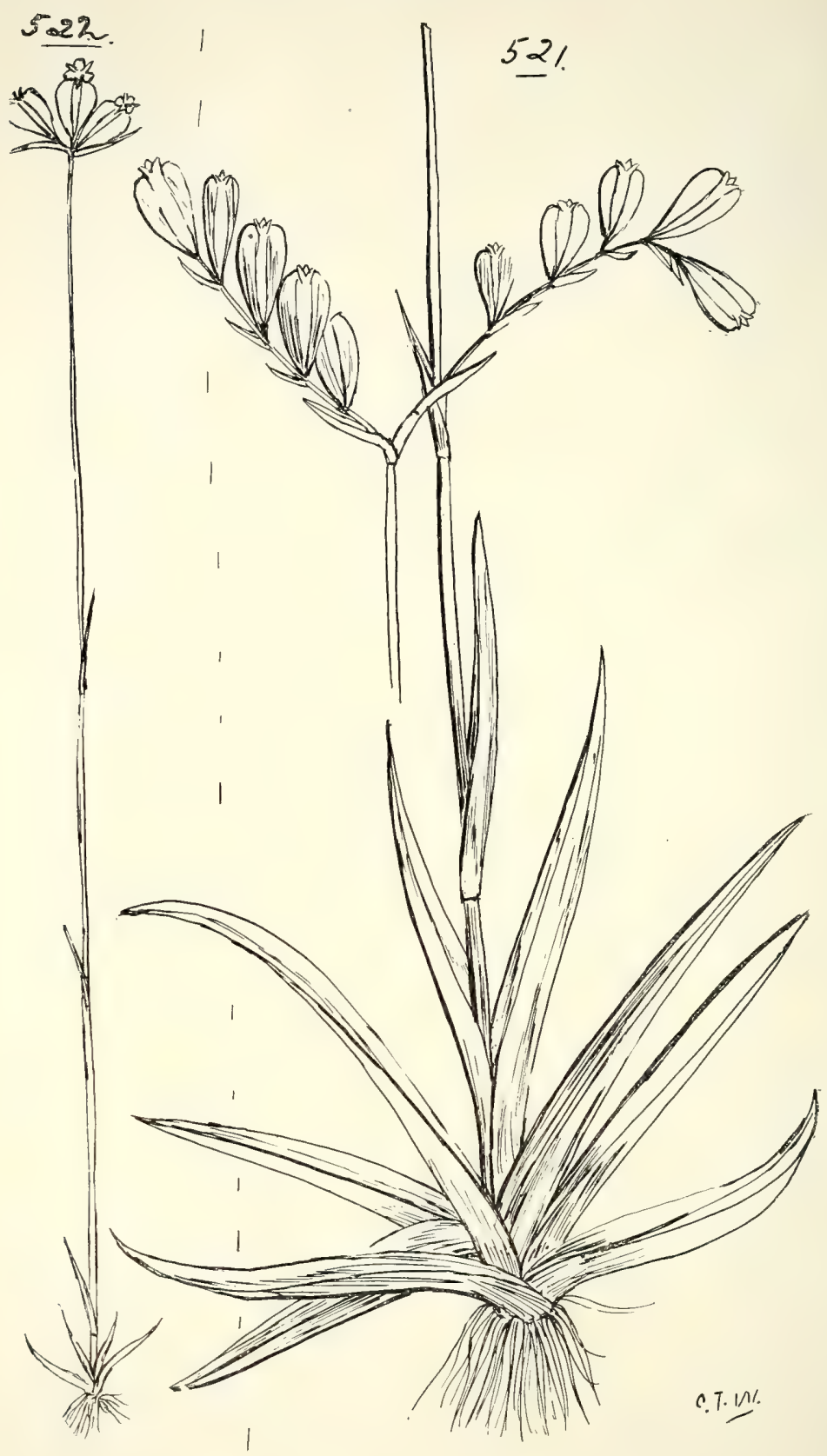

521. Burmannia disticha, Limn.

522. B. JunceA, Sol. 


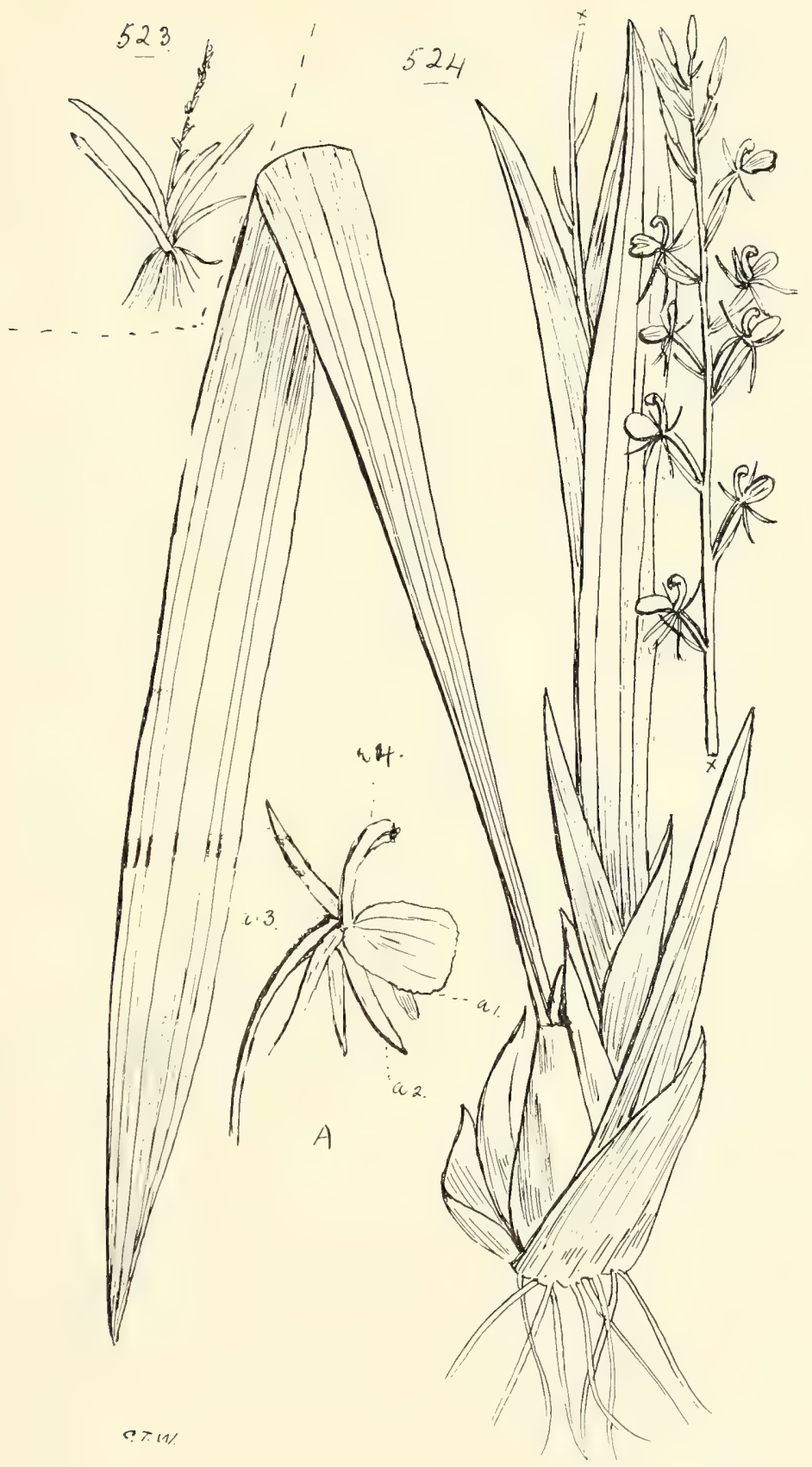

523. Oberonia pusilla, Bail.

524. Liparis cuneilabris, $F . v \cdot M$.

(A) Flower, enl., (аг) labellum, (a2) sepal, (a3) petal, (a4) column. 


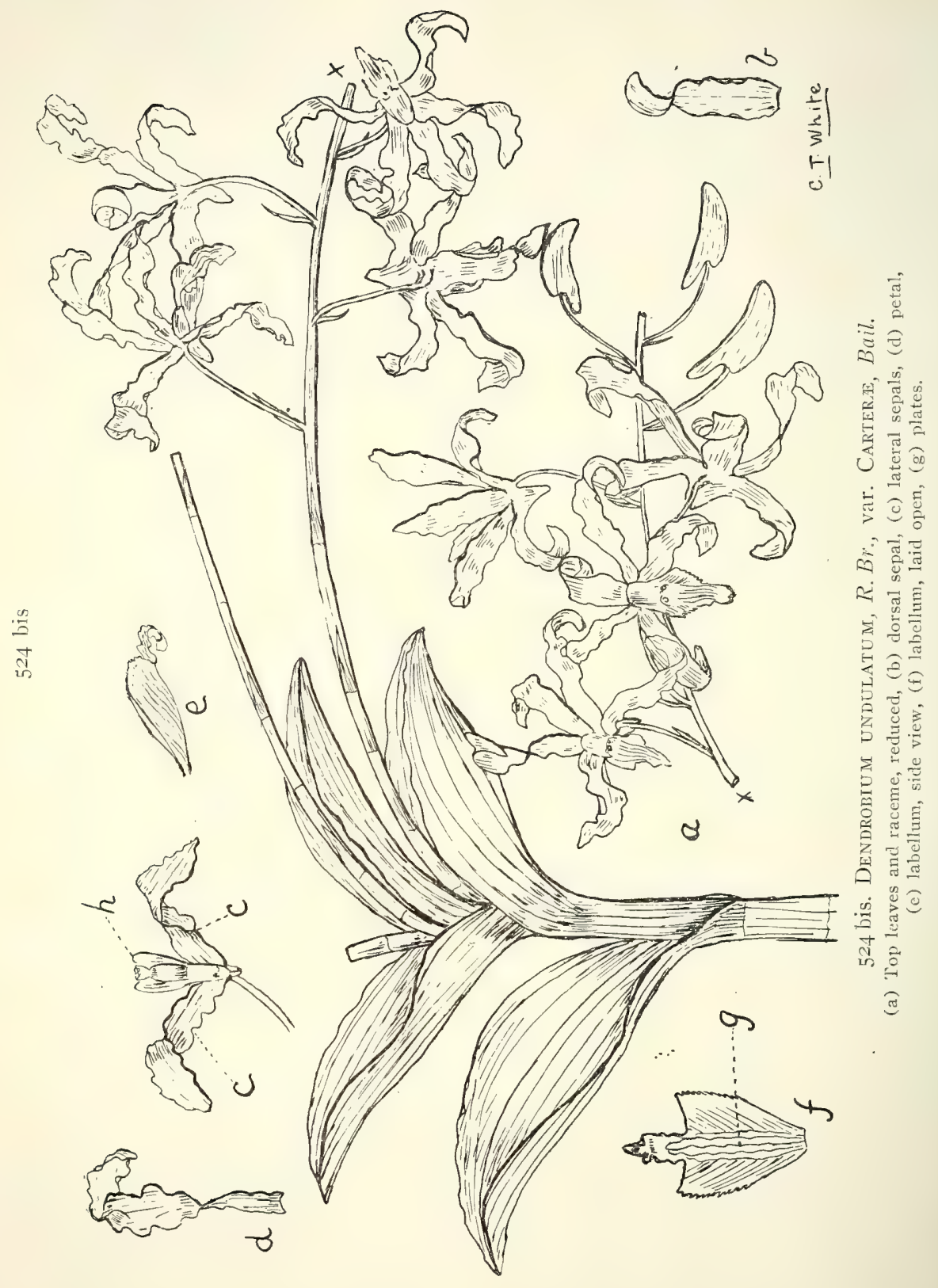


Dendrobium-contd.

bigibbum-contd. var. albomarginatum, Bail. war. Phalænopsis, Bail. forma venosum, Bail.

\section{- superbiens, G. Reichb.}

Fitzgeraldi, F.v.M.

Summeri, F.v.M.

u1idulatum, R. Br.

war. Broomfieldii, Fitzg.

var. Carteræ, Bail. (Fig. 524 bis.)

Gouldii, Reichb., var. acutum, Reichb. f.

Hab.: Thursday Island (J. J. Smith).

Tofftii, Bail.-Flowers stained with violet.

fuscum, Fitzger.

Johannis, Reichb. f.-Flowers vary much in size and colouring.

speciosum, $S m$.

var. Hillii, F. v. M.

forma grandiflorum, Bail.

var. nitidum, Bail.

var. curvicaule, Bail.; Kranzlin in Engl. Pflanz. (Orchid Mon.), pt. ii. p. 27 I.

fusiforme, Bail.

delicatum, Bail. = D. speciosum, var. Bancroftianum, Reichb. $\mathrm{f}$.

tetragonum, A. Cunn.-Size and colouring of flowers variable.

æmulum, R. Br.

Bairdianum, Bail.

Kingianum, Bidw.

var. pallidum, Bail.

var. Silcockii, Bail.

var. Aldersonæ, Bail.

gracilicaule, F.v.M.-Colouring and marking of flowers variable.

Adæ, Bail.-Very fragrant.

Stuartii, Bail.

agrostophyllum, F.v.M.-Flowers few, yellow.

Baileyi, F. . M.

Keffordii, Bail.

canaliculatum, $R . B r .=D$. Tattonianum, Batem.-The

Onion Orchid of Cairns. "Yamberin" of the natives.

Jonesii, Rendle in Journ. Bot. xxxix. (I90I) I97. 
Dendrobium-contd.

Section III.-Pedilonium.

Smillix, F.v.M. = Celandria Smillice, Fitzg.

Hollrungii, Kränzl., var. australiense, Rendle in Journ. Bot. 37 ( I899), 339.

ophioglossum, H. G. Reichb.

Section IV.-Monophyllæa.

monophyllum, F.v. M.

Schneideræ, Bail.

hispidum, A. Rich.

var. Taylori, Bail. = D. Taylori, Fitzg.; D. uniflos, Bail.;

Bulbophyllum Taylori, F.v. M.

Section V.-Rhizobium.

cucumerinum, Lindl.-Gherkin Orchid.

pugioniforme, A. Cunn. =D. pungentifolium, F.v. M.

rigidum, $R . B r$.

linguiforme, $S w$.

var. Nugentii, Bail.

Section VI.-Strongyle.

teretifolium, R.Br.-Pencil Orchid.

var. Fairfaxii, F.v.M.

var. aureum, Bail.

Beckleri, F.v. M.

Mortii, F.v. M.

Bowmanii, Benth.

eriæoides, Bail. = D. Erie, F. v. M.

Palmerstoniæ, Schlechter, Fedde. Repert. iii. (I907) 3I7.

bifalce, Lindl. $=D$. breviracemosum, Bail.

Hab.: Saibi Island (Hartmann), Kranzlin.

Johnsoniæ, F.v. M.

Hab.: Cape York Peninsula (Kranzlin).

Bulbophyllum, Thouars.

lichenastrum, $F . v . M$.

nematopodum, F.v. M.

Baileyi, F.v.M.-Flowers large, beautifully spotted with purple.

radicans, Bail.

Shepherdi, F.v.M.-Wheat Orchid; leaves resembling in form a grain of wheat.

intermedium, Bail.

aurantiacum, F.v. M.

Prenticei, F.v. $M$.

exiguum, $F$. v. $M$. 

PLATE XV.

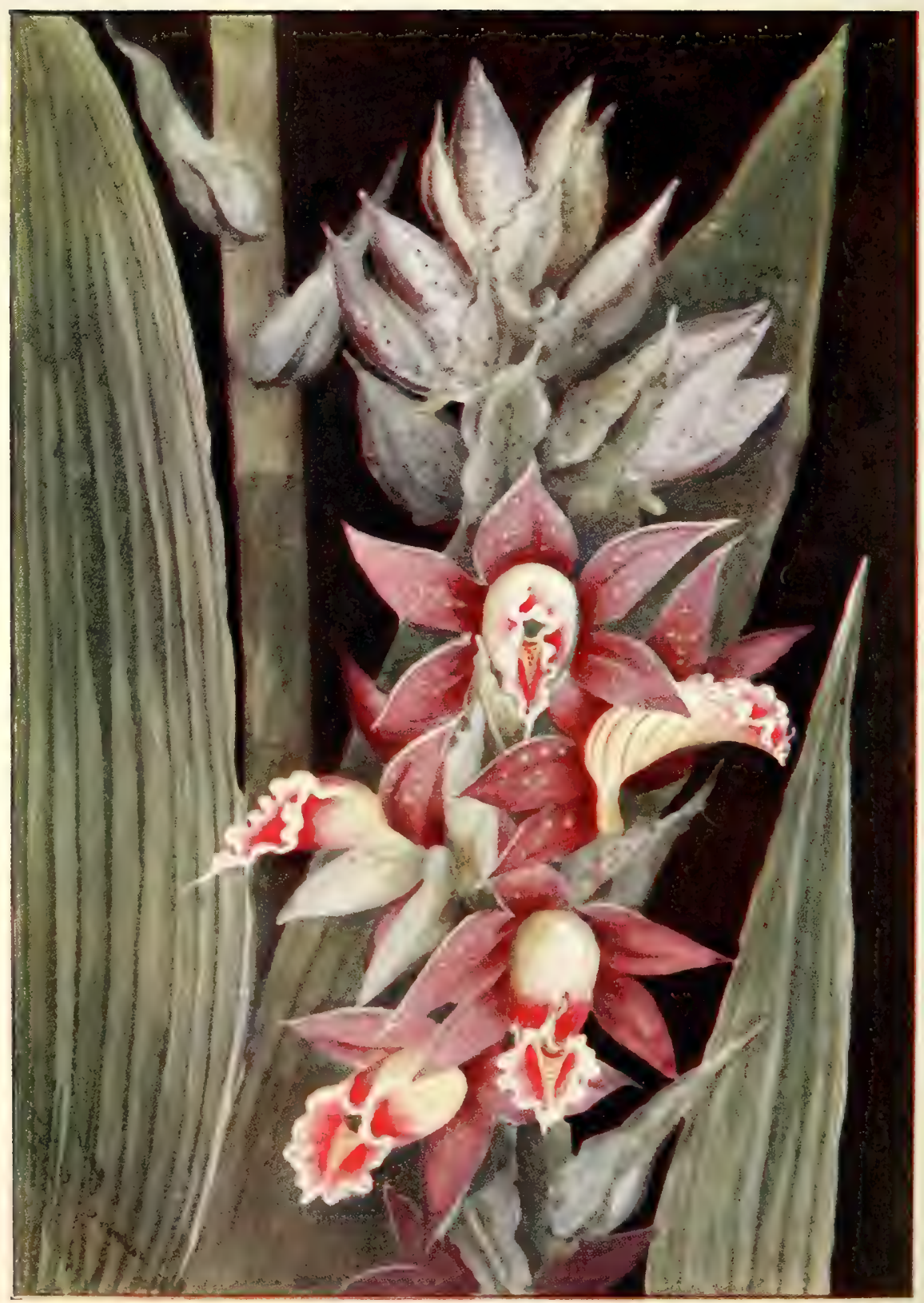

Phaius grandifolius, Lour., var. Rowanae, Bail. 
Eulbophyllum-contd.

exiguum-contd.

var. Dallachyi, Benth.-Flowers white.

minutissimum, F.v. M.

Toressæ, Bail.

bracteatum, Bail. = ? Adelopetalum bracteatum, Fitzg.

punctatum, Fitzg.

Elisæ, F.v.M.

Bowkettæ, Bail.

lageniforme, Bail.

Cirrhopetalum, Lindl.

clavigerum, Fitzg.

Osyricera, Blume.

purpurascens, Deane. $=$ Bulbophyllum purpurascens, Bail.

Subtribe IV.-Eriea.

Eria, Lindl.

Fitzalani, F.v.M. (Fig. 525.)

australiensis, Bail.-Kranzlin (Engl. Pflanz. (Orchid. Mon.) pt. ii. p. I66) considers this a species of Podochilus.

Phreatia, Lindl.

limenophylax, Reichb. $f$.

Spathoglottis, Blume.

Paulinæ, F.v.M. = Bletia Pauline, F. v. M.

Soutteriana, Bail.

Subtribe $V$.-Bletiea.

Phaius, Lour.

grandifolius, Lour.

var. Bernaysii, Bail.

forma Soutteri, Bail.

forma Idæ, Bail.

var. Rowanæ, Bail., Q1. Agric. J1. xxviii. (I9I2) 74. The Spotted Lily of the Murray River, N.Q. (Plate XV.)

Subtribe VI.-Cologynea.

Pholidota, Lindl.

imbricata, Lindl.

Calanthe, R. Br.

veratrifolia, $R . B r$.

var. Kennyi, Bail. (Fig. 525 ter.) 


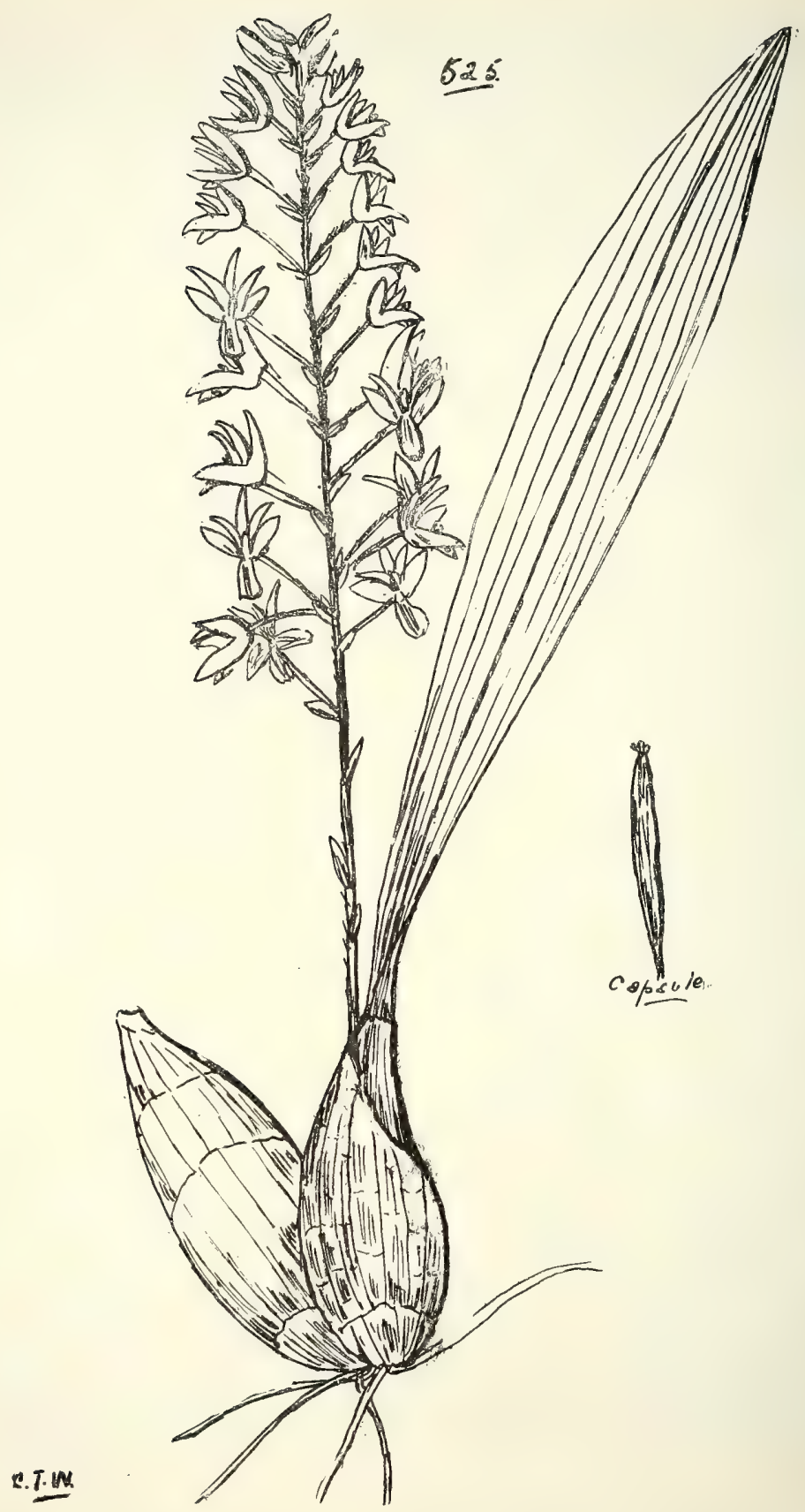

525. Eria Fitzalani, F.v. M. 


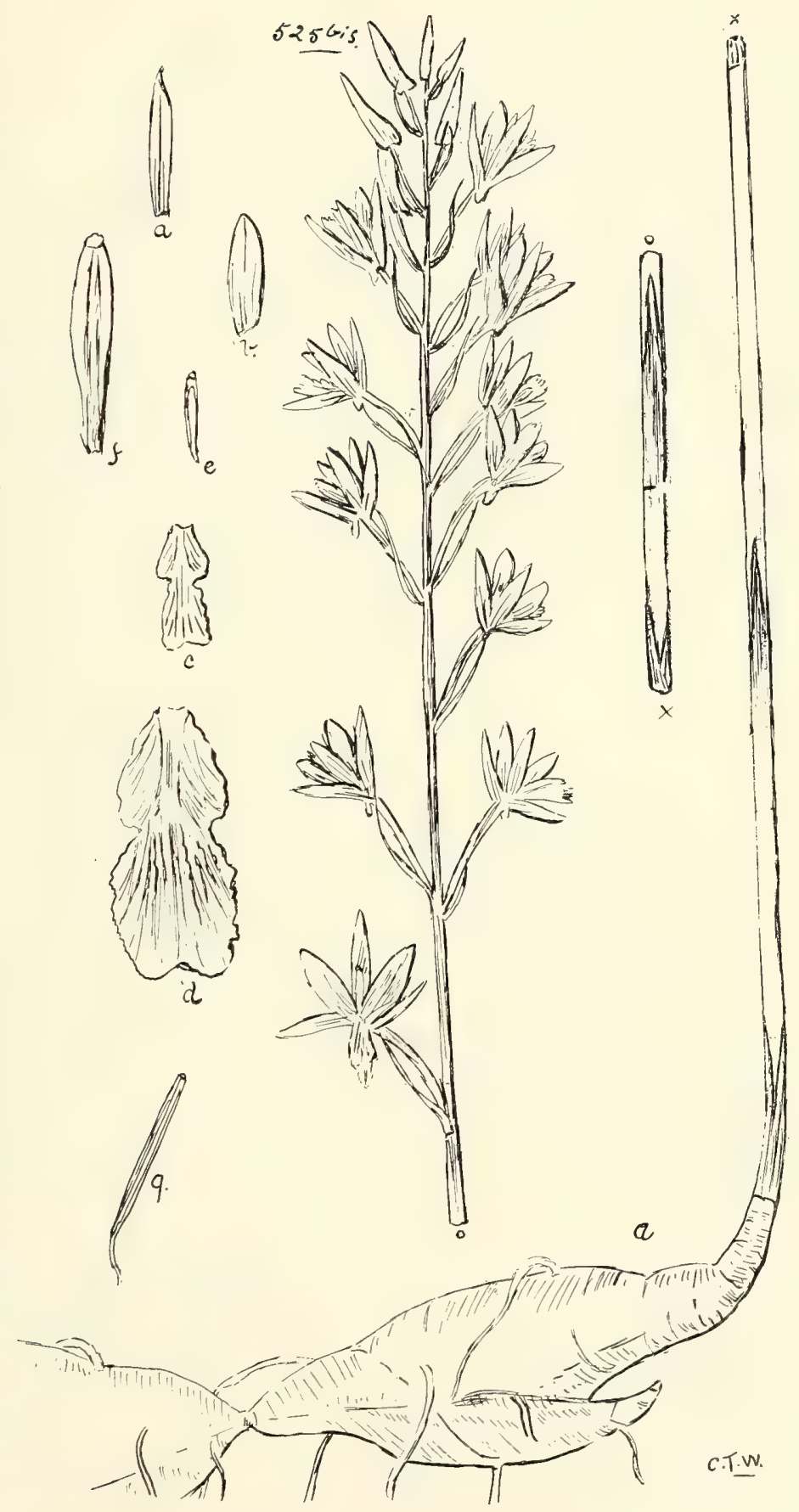

5 I5 bis. Eulophia venosa, Reichb.

(a) Rhizome, (a) sepal, (b) petal, (c) and (d) labellum, (e) and (f) column

(g) immature capsule. (a), (b), (c), (e), and (g) nat. size; (d) and (f) enl. 


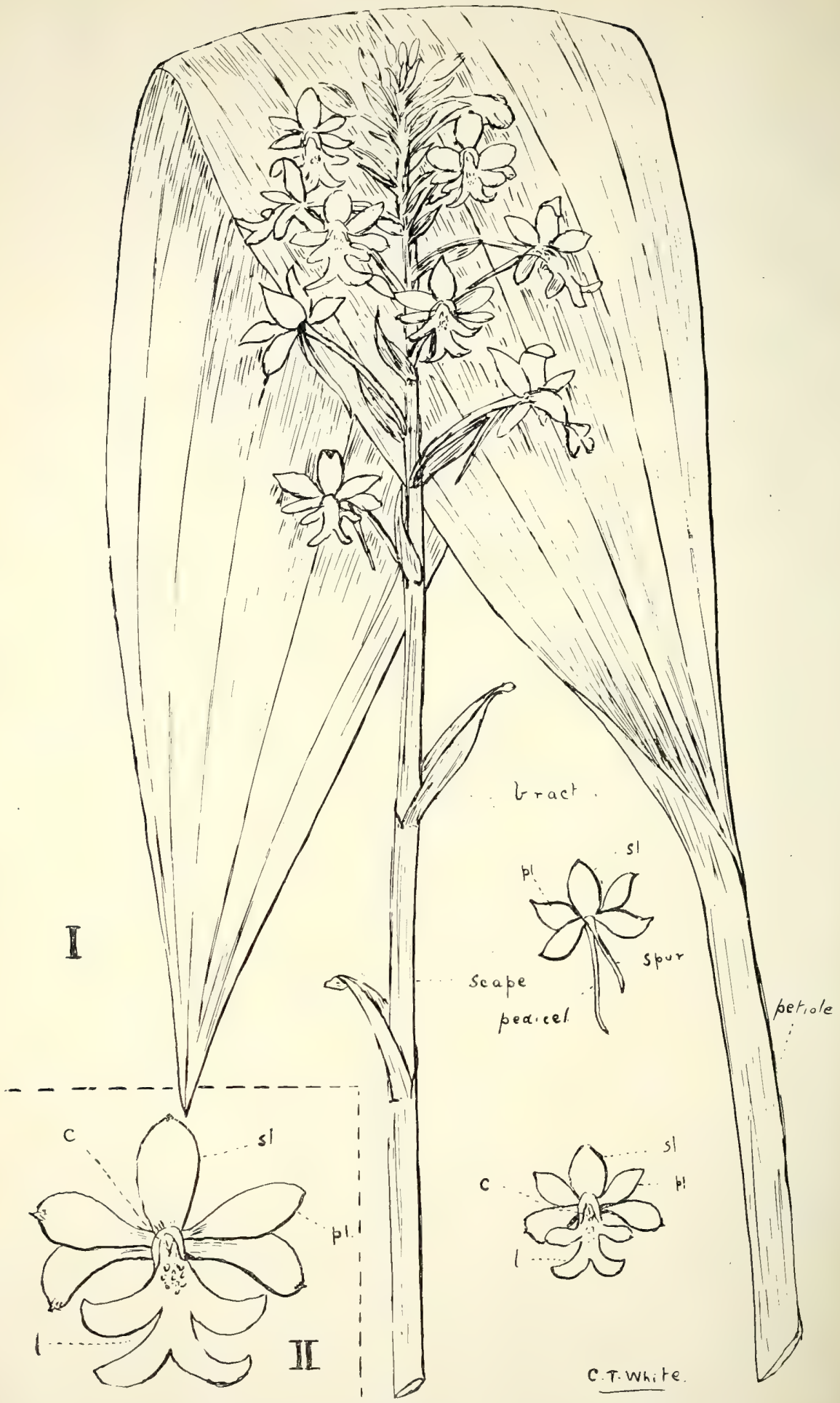

525 ter. I. Calanthe veratrifolia, $R$. Br., var. Kennyi, Bail. $n$. var. (sl) Sepal, (pl) petal, (1) labellum, (c) column; all nat. size.

II. Flower of normal form, nat. size. 


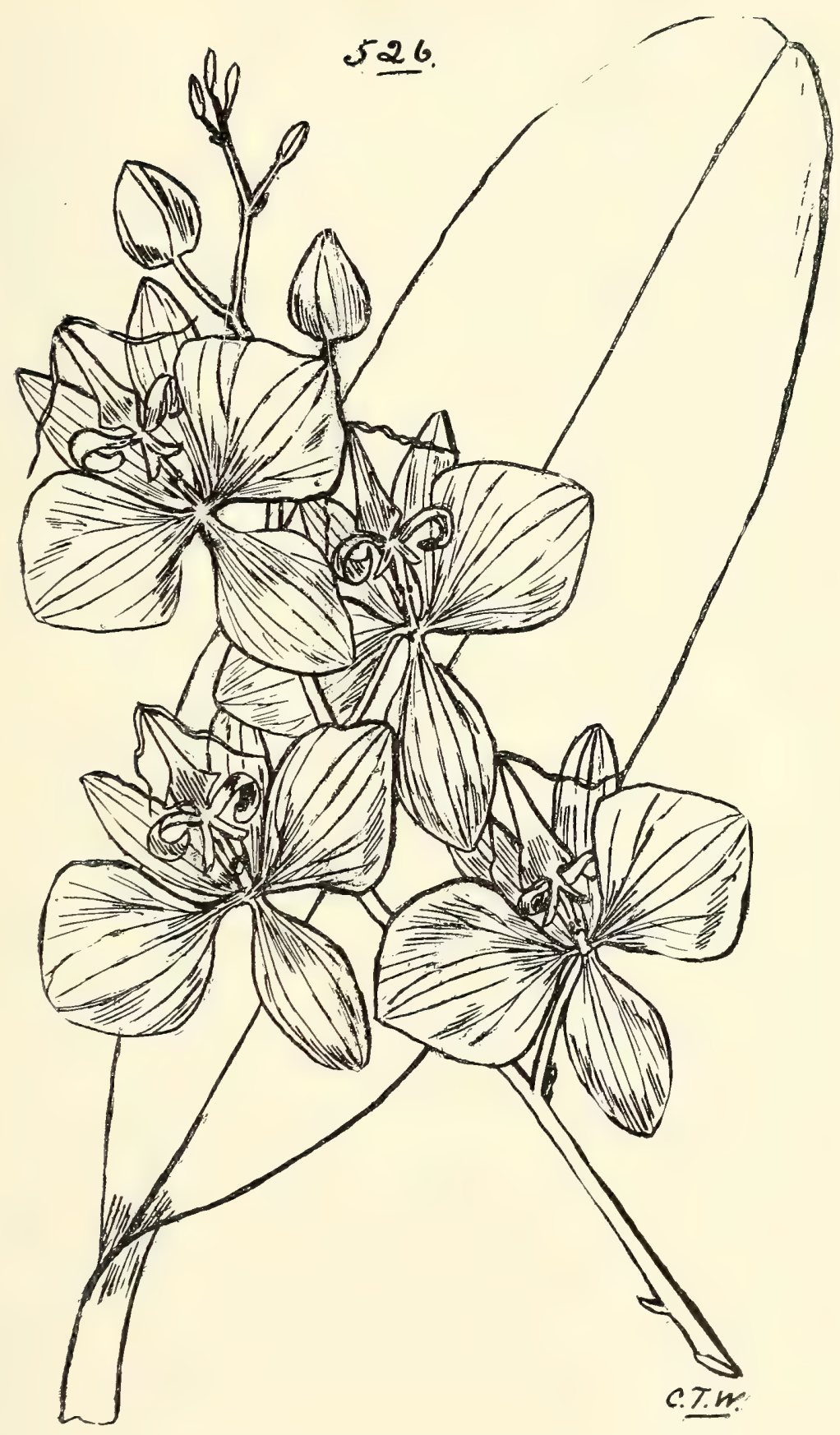

526. Phalemopsis Rosenstromit, Bail. 


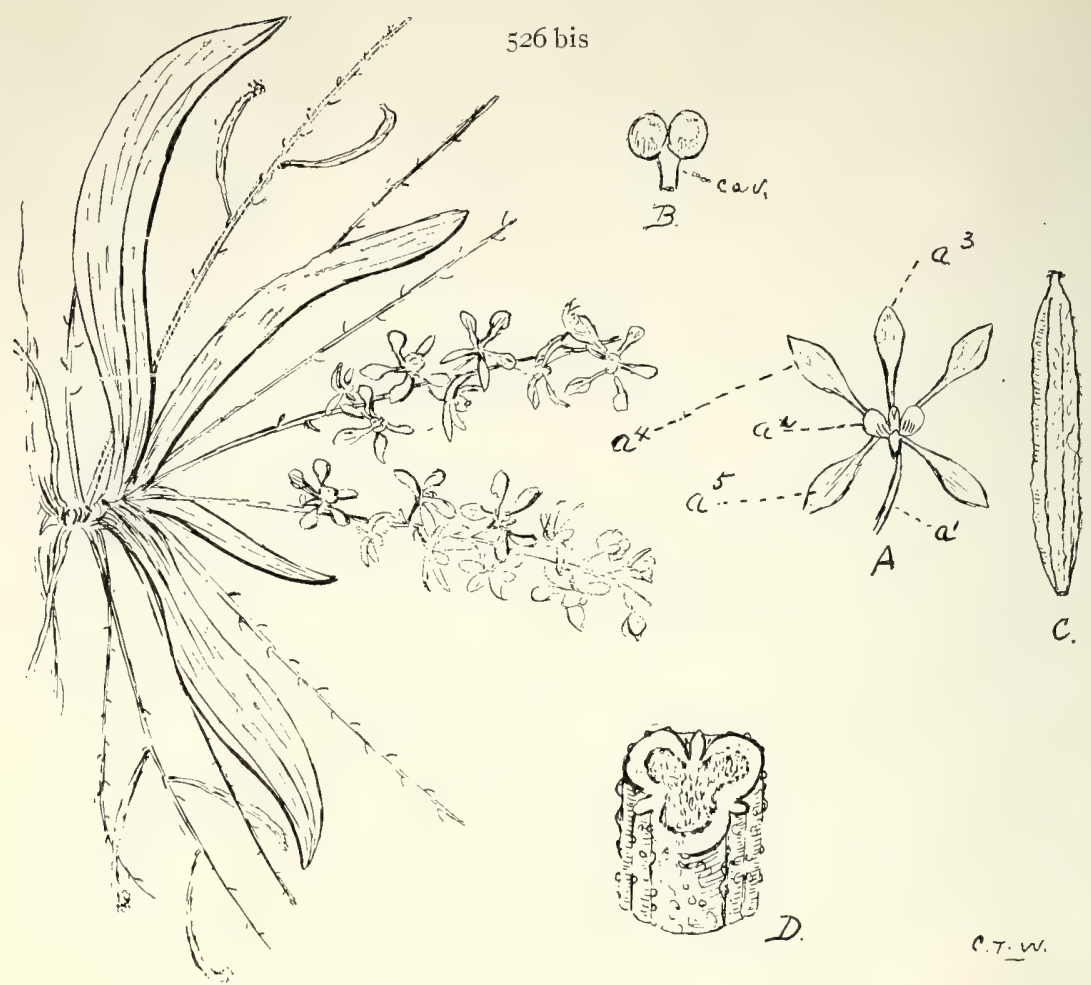

526 bis. Sarcochilus Bancroftit, Bail.

(A) Flower, enl., (aI) pedicel, (az) labellum, (a3) dorsal sel.al, (a4) petals, (a.5) lateral sepals, (B) pollen-masses, enl., (C) capsule, nat. size, (D) sect. capsule, enl.

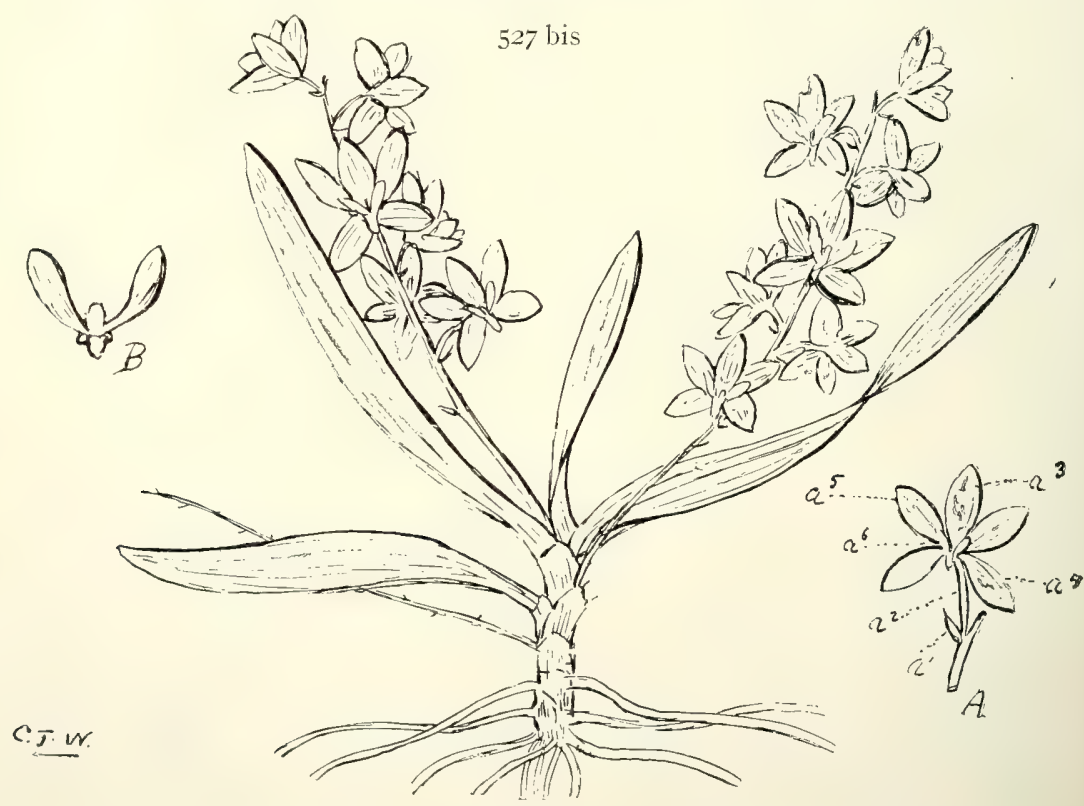

527 bis. Sarcochilus Longmanit, Bail.

(A) Flower, nat. size, (ar) bract, (a2) pedicel, (a3) dorsal sepal, (a4) lateral sepals, (a5) petals, (a6) labellum, (B) labellum, enl. 
CXXVII. ORCHIDEÆ.
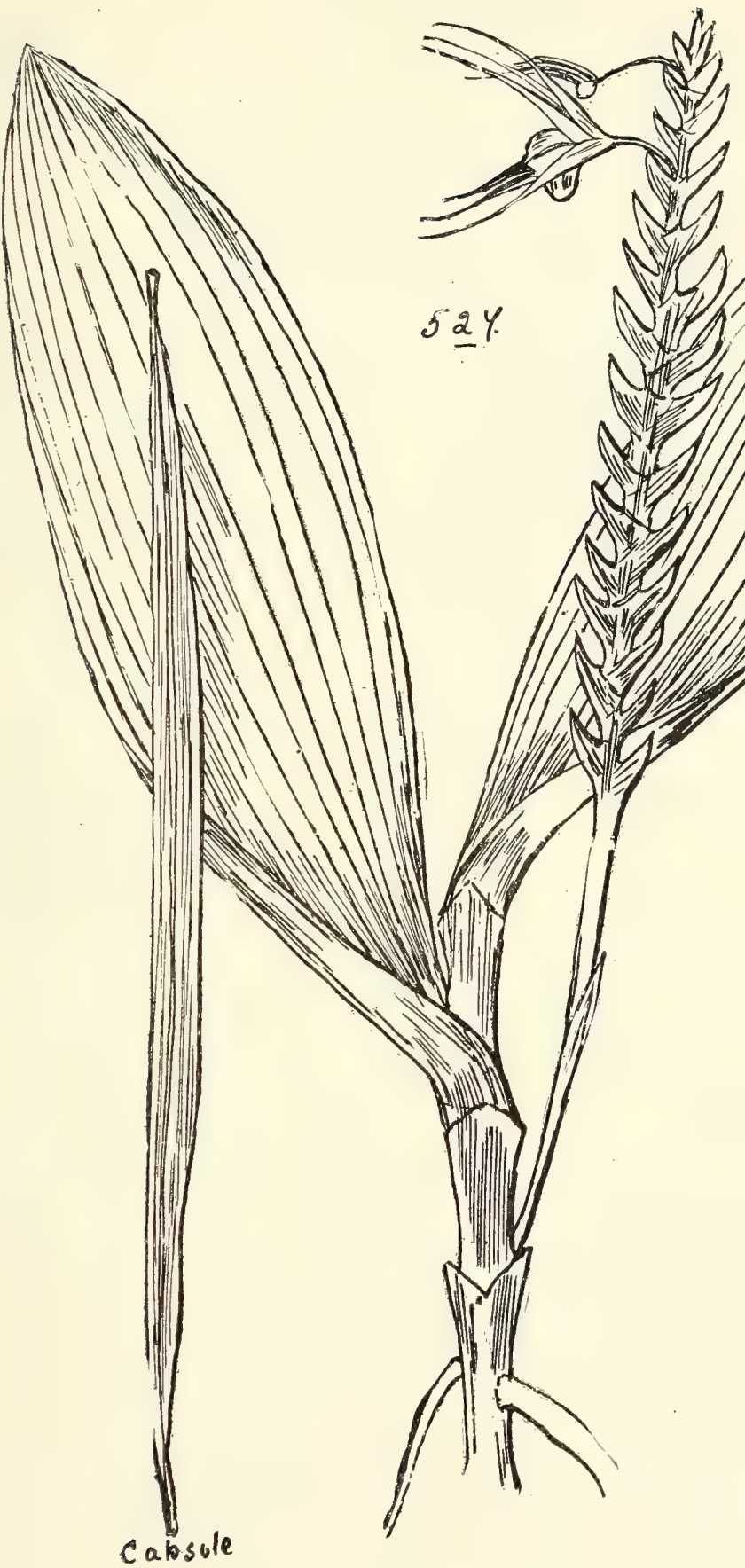

$52 \%$

(1)

II

I

II

(1)

11

1)

. 111

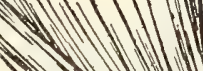

1... I.

1

N. 


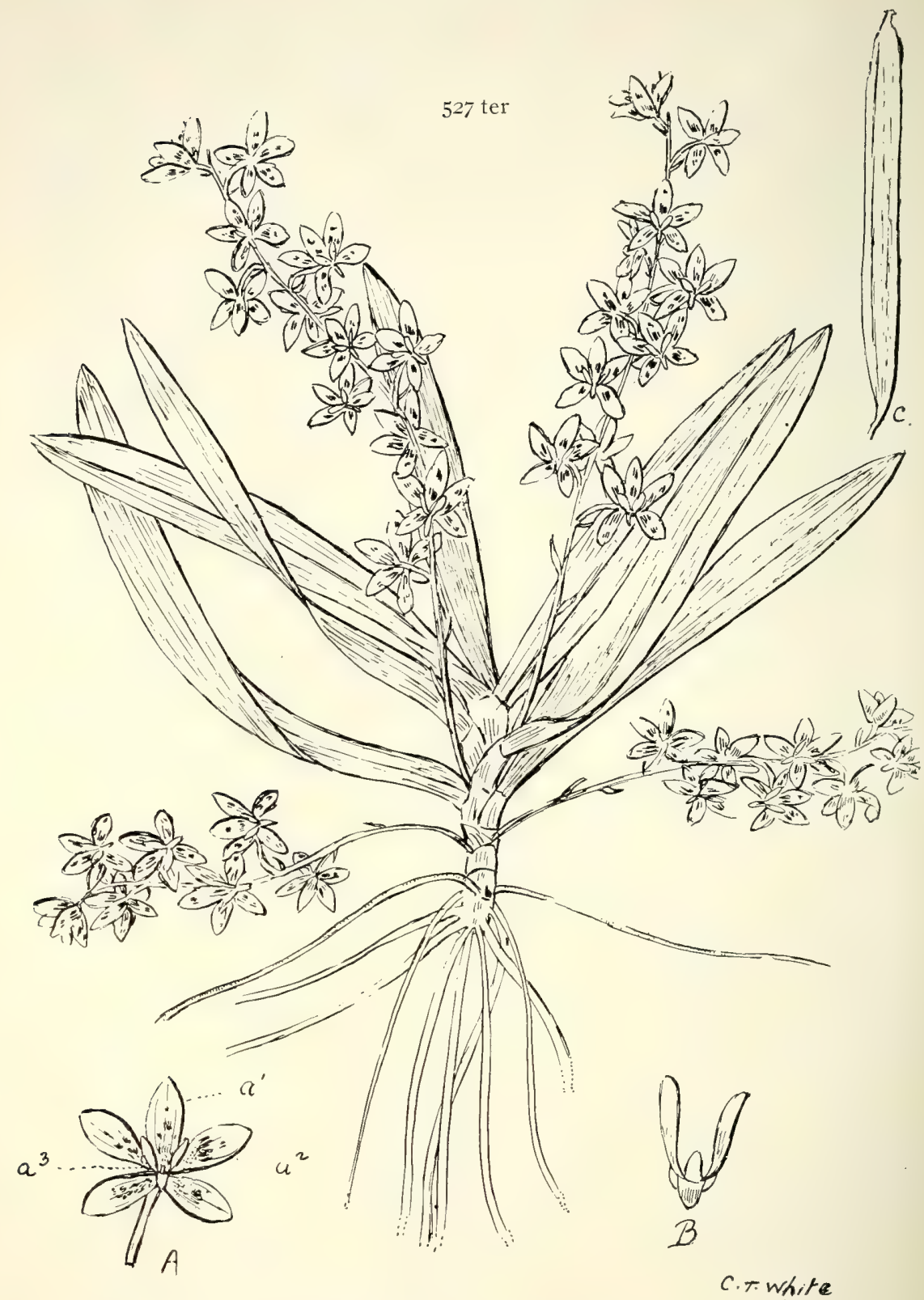

527 ter. Sarcochilus Weinthalis, Bail.

(A) Flower, enl., (ar) dorsal sepal, (a2) petals, (аз) labellum, (B) labellum, enl., (C) capsule, nat. size. 
Tribe II.-VAndex.

Eulophia, R. Br.

Subtribe I.-Eulophiece.

venosa, Reichb. = Dipodium venosum, F.v. M.-Strong plants of this tropical orchid, from which the present plate was made, were collected at Eumundi by Mr. J. Staer, Oct. I9I I. (Fig. 525 bis.)

Fitzalani, F.v.M.

Cymbidium, Sw.

Subtribe II.-Cymbidiece.

canaliculatum, R. Br. =C. Sparkesii, Rendle; C. Leai, Rendle.

Hillii, F. v. M.

albuciflorum, F.v.M. = C. madidum, Lind1.-"Dampyampy" of Port Curtis and "Bungkiam" of Tully River natives. Pseudobulb stewed for dysentery (Roth). suave, $R . B r$.

Geodorum, Jackson.

pictum, Lindl.-_" Yeenga" of Gladstone and "Uine" of Rockhampton natives.

dilatatum, R. Br., var., Bail., Q1. Bot. Bu1l. xvi. (I903) I.

Dipodium, R. $B r$.

punctatum, R. Br.

var. Hamiltonianum, Bail.

var. album, Bail.

ensifolium, $F \cdot v \cdot M$.

Subtribe III.-Sarcanthece.

Luisia, Gaudich.

teretifolia, Gandich.

Phalænopsis, Blume.

Rosenstromii, Bail. (Fig. 526.)

Sarcochilus, $R . B r .=$ Thrixspermum, Lour.

divitiflorus, F.v.M.-Roots flattened, rough like a rasp.

falcatus, R. Br.

var. montanus, Bail. $=S$. montanus, Fitzg.

Fitzgeraldi, F.v. M.

parviflorus, Lindl.

Bancroftii, Bail. (Fig. 526 bis.)

Hartmanni, F.v.M. = S. rubricentrum, Fitzg.

olivaceus, Lindl.

Cecilix, F.v.M.

Hillii, F.v. $M$.

phyllorrhizus, F.v.M. 
Sarcochilus-contd.

platystachys, Bail. (Fig. 527.)

Longmanii, Bail. (Fig. 527 bis.)

Weinthalii, Bail. (Fig. 527 ter.)

Newportii, Bail.

Cleisostoma, $\mathrm{Bl}$.

tridentatum, Lindl. = Saccolabium calcaratum, IF. v. M.

Nugentii, Bail.

congestum, Bail.

Keffordii, Bail.

Armitii, F.v.M.= Sarcochilus Armitii, F.v. M.

Macphersonii, F.v. M.

brevilabre, F.v. M.

Ornithochilus, Wall.

Hillii, F.v.M. = Saccolabium Hilli, IF. v. M.

Tæniophyllum, Blume.

Muelleri, Lindl. = Sarcochilus Baileyi, F. v. M.-A minute leafless Orchid.

Tribe III.-NeOtTIE A:.

Subtribe I.-Vanillew.

Galeola, Lour. = Ledgeria, F. v. M.

cassythoides, Reichb.-A climbing leafless Orchid; a saprophyte, or, a plant which lives upon dead organic matter.

foliata, F.v.M. = G. Ledgerii, F. v. M.-A climbing leafless Orchid; a saprophyte.

Subtribe II.-Corymbiece.

Corymbis, Thouars.

veratrifolia, Reichb. = Corymborchis veratrifolia, Plume.

Subtribe III.-Spiranthece.

Anoectochilus, Blume.

Yatesæ, Bail., Q1. Agric. J1. xix. (1907) I48.

Spiranthes, Rich.

australis, Lindl.

Listera, R. Br.

amplexicaulis, Bail. = Microstylis amplexicantis, Bail.

Goodyera, R. Br.

viridiflora, Blume.

polygonoides, F.v. M.

Hetæria, Blume.

tenuis, Lindl. = Ramphidia tenuis, Lindl. 
Subtribe IV.-Diuridece.

Thelymitra, Forst.

\section{Section Cucullaria.}

ixioides, Sm.-Native Hyacinth, Tasmania (Rodway).

longifolia, Forst.

Jiuris, $S m$.

alba, $R \cdot B r$.

punctata, $S m .=D$. elongata, Sw., and D. lilacina, F. v. M.

aurea, $S m$.

maculata, Sm.-Tiger Orchid of Tasmania.

pedunculata, R. Br.

abbreviata, F.v.M.

secundiflora, Fitzg.

pallens, Benth.

sulphurea, R. $B r$.

Cryptostylis, R. Br.

longiflora, R.Br.-Duck Orchid, Tasmania (Rodway).

Prasophyllum, R. Br.

Section I.-Euprasophyllum.

australe, R. Br.

flavum, R. Br.

brevilabre, Hook.

patens, $R$. Br.

fuscum, R. Br.-Fly Orchid, Tasmania (Rodrway).

Section II.-Genoplesium.

nigricans, $R . B r$.

rufum, $R . B r$.

Microtis, R. Br.

porrifolia, Spreng.

parviflora, $R . B r$.

Corysanthes, R. Br.

pruinosa, R. Br.-Little Hood, Tasmania (Rodway).

bicalcarata, R. Br.-Little Hood, Tasmania (Rodway).

fimbriata, R. Br.

Pterostylis, R. Br.-Nun-flower, or Parson-in-the-pulpit.

Section I.--Antennæa.

Series I.-Grandifloræ.

ophioglossa, R. Br.

concinna, $R$. $B r$.

curta, R. Br.

var. ? grandiflora, Benth.

acuminata, R. Br.

Baptistii, Fitzg. 
Pterostylis-contd.

nutans, $R$. $B r$.

grandiflora, $R . B r$.

reflexa, R. $B r$.

obtusa, R. Br.

parviflora, R. $B r$.

Series II.-Parviflora.

Section II.-Catochilus.

mutica, R. Br.

rufa, $R . B r$.

longifolia, R. Br.

depauperata, Bail.

Whitei, Bail.

Daintreana, F.v. $M$.

Caleana, $R$. Br.-Flowers sensitive.

major, $R . B r$.

minor, R. Br.

Drakæa, Lindl.-Flowers sensitive.

irritabilis, Reichb. = Arthrochilus irritabilis, F.v. M.

Acianthus, R. Br.

fornicatus, R. Br.

exsertus, R. $B r$.

Eriochilus, R. Br.

autumnalis, $R . B r .=$ Epipactis circulata, Labill.

Cyrtostylis, R. Br.

reniformis, $R . B r$.

Caladenia, R. Br.

Section I.-Calonema.

Patersoni, R. Br.-Spider Orchid, Tasmania (Rodrway). var. dilatata, Benth.

Section II.-Eucaladenia.

suaveolens, Reichb. = Leptoceras sulphurea, Lindl.

latifolia, R. Br.

carnea, R. Br._-"Chingum " of Brisbane River natives.

var. alba, Benth.

cærulea, R. Br.

Glossodia, R. Br.

major, R. Br.

Section Euglossodia.

minor, R. Br.

var. alba, Bail.

Calochilus, R. $\mathrm{Br}$.

campestris, R.Br.-Longbeard, Tasmania (Rodway). var. grandiflora, Benth.

paludosus, $R . B r$. 
Chiloglottis, R. Br.

diphylla, R. Br.-Beetle Orchid.

trilabra, Fitzg.

Pogonia, Juss.

Subtribe Arethusec.

uniflora, F.v. M.

holochila, F.v.M.

Dallachyana, F.v. $M$.

pachystomoides, F.v. $M$.

Gastrodia, R. Br.

sesamoides, R. Br.

ovata, Bail.

Epipogum, Gmel.

nutans, Lindl._-"Maapa" of Barron River natives.

TRIBE IV.-OPHRYDEÆ.

Subtribe Habenariea.

Habenaria, Willd.

elongata, $R \cdot B r$.

graminea, Lindl.

Millari, Bail.

xanthantha, F.v. M.

Banfieldii, Bail. (Fig. 528.)

Tribe V.-CyPRIPEDIE.E.

Apostasia, Blume.

stylidioides, Reichb.

Series II.-EPIGYNÆ.

\section{Order CXXVIII.-SCITAMINEÆ.}

Tribe I.-ZingibereÆ.

Curcuma, Linn.

australasica, Hook. f.- "Kumbiji" of Cooktown and "Andan” of Starcke River natives.

Zingiber, Adans.

officinale, Rosc., var. Cholmondeleyi, Bail. $=Z$. Cholmondeleyi, K. Schum.-This yields a marketable ginger.

Zerumbet, Sm., forma australiana, Bail.

Amomum, Linn.

Daliachyi, F.ข.M.-."To-choon" or "Tor-chun" of Mount Cook, "Dor-churn" of Cape Bedford, and "Jun-jun" of Bloomfield River natives. (Fig. 529.) 


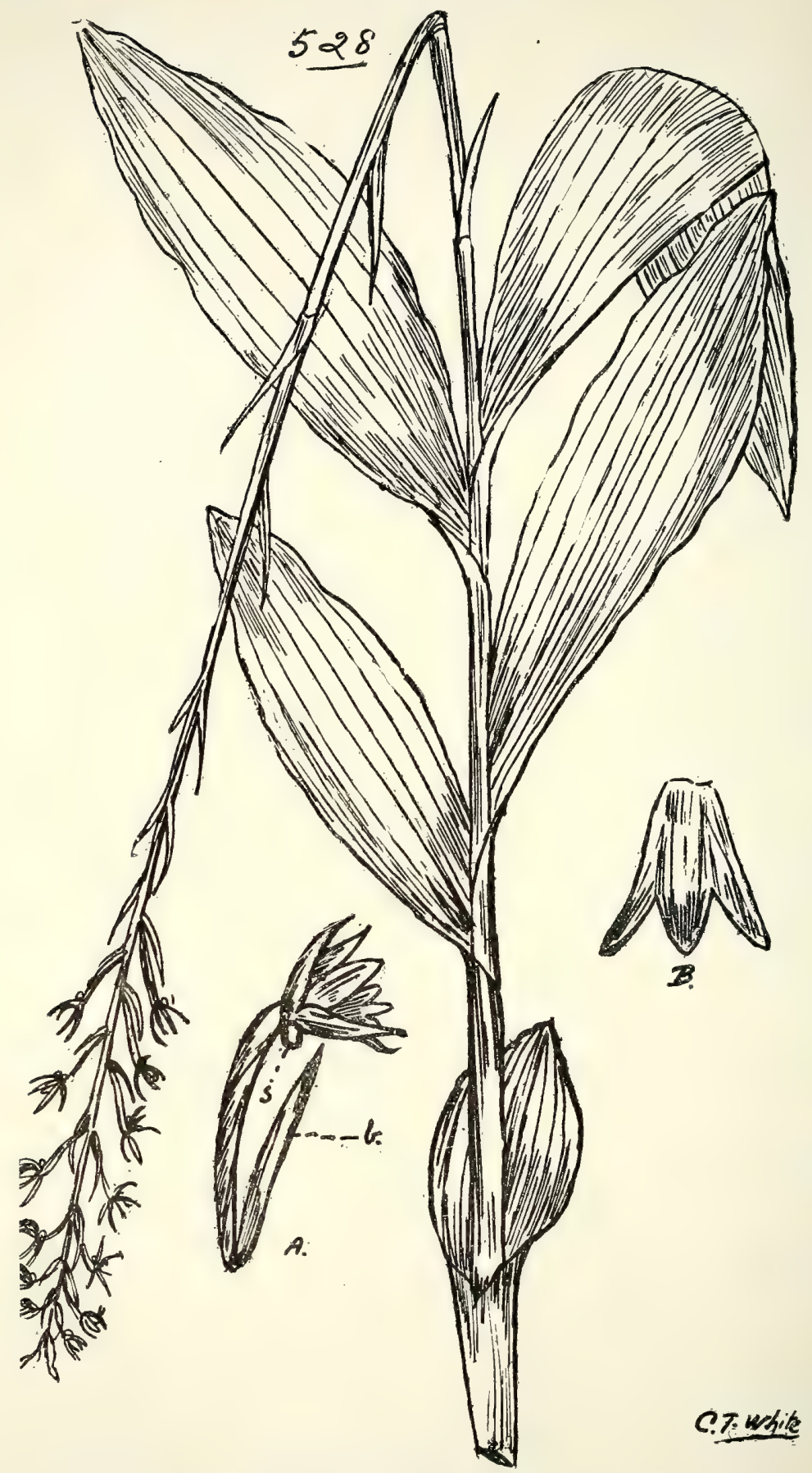




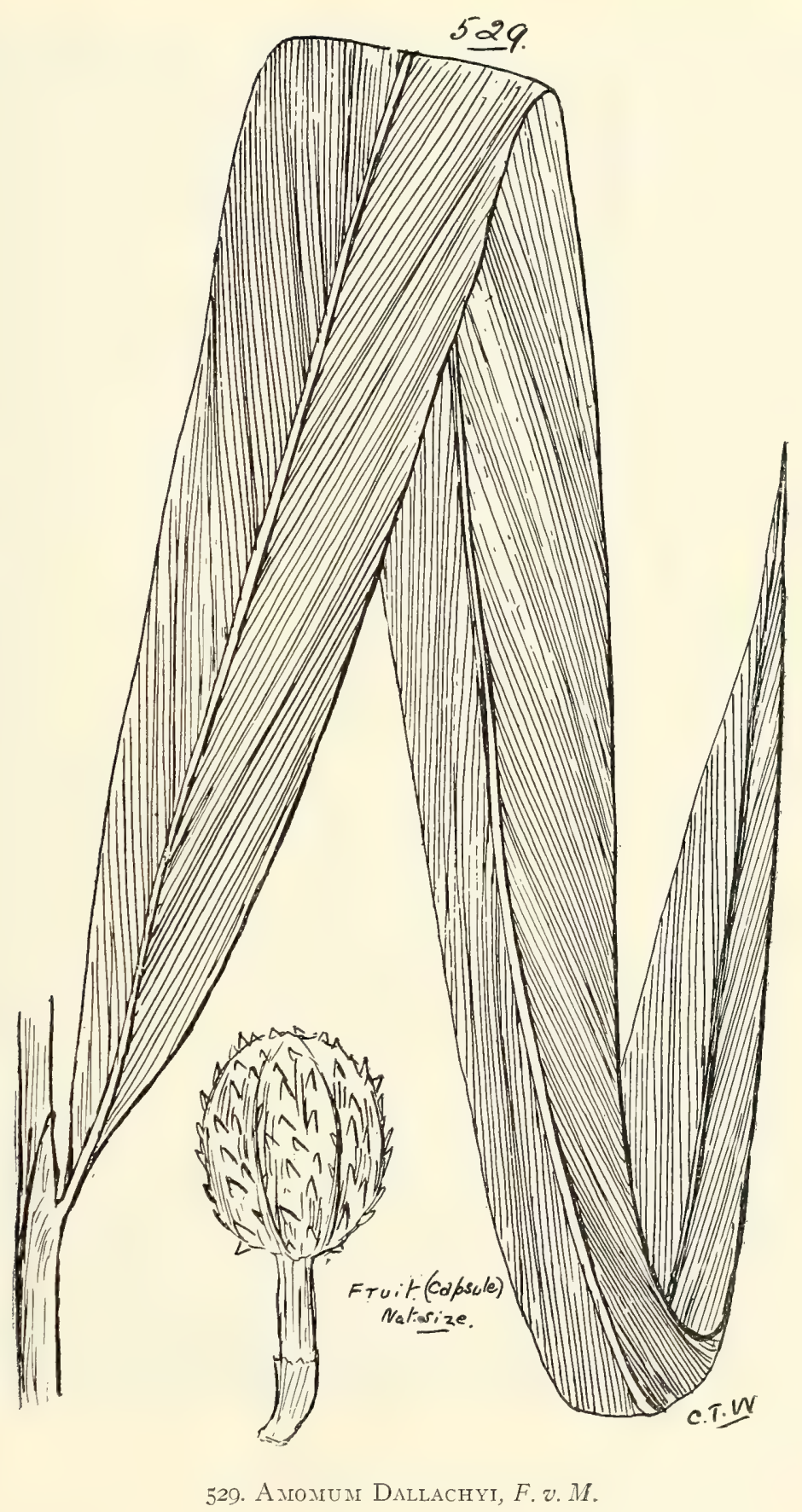


Elettaria, White.

Scottiana, F.v.M. - "Jiddo" of Bloomfield and "Bija" of Johnstone River natives.

Alpinia, Linn. = Helienia, Willd.

racemigera, $F$. v. $M$.

cærulea, Benth.—“Ool-pun” of Dunk Island natives.

"Moregi" or "Fida" of Cardwell natives (R.B.H.).

var. Arundelliana, Bail. $=$ A. Arundelliana, K. Schum.

arctiflora, F. v. M.- “Booroogun” of Johnstone River natives. (Plate XVI.)

modesta, F.v.M.ex Schum.

Costus, Linn.

Potieræ, F.v. M.

Tapeinocheilos, Miq.

pungens, Miq., var. queenslandiæ, Bail. =T. queenslandia, K. Schum.

TRIBE II.-MUSEÆ.

Musa, Limn.-Bananas.

Banksii, F. v. M. - "Morgogaba" of Cleveland Bay and

"Boo-gar-oo" of Dunk Island natives. This and the other two species might furnish good fibre, but are useless for fruit, as the seeds are so numerous.

Hillii, F.v. M.

Fitzalani, F.v.M.

\section{Order CXXIX.-HÆMODORACEÆ.}

TRIBE I.-EUH EMODORE.

Hæmodorum, Sm.

planifolium, R. Br.

coccineum, R.Br._- On-tho" of Mitchell River, "Tandi" of Cooktown, and "Anto" of Palmer River natives. Has been reputed as poisonous to horses (see Q1. Agric. J1. v. ( I899) 4I, pl. II8).

ensifolium, F. v. $M$.

tenuifolium, A. Cunn.

Tribe II.-OphiopogoneÆ.

*Ophiopogon, Ker.

japonicus, Ker.

\section{Order CXXX.-IRIDACE}

Subtribe I.-Sisyrinchiea.

Libertia, Spreng.

paniculata, Spreng.

Sisyrinchium, Linn.

micranthum, Cav.-Scour-weed. 
PLATE XVI.

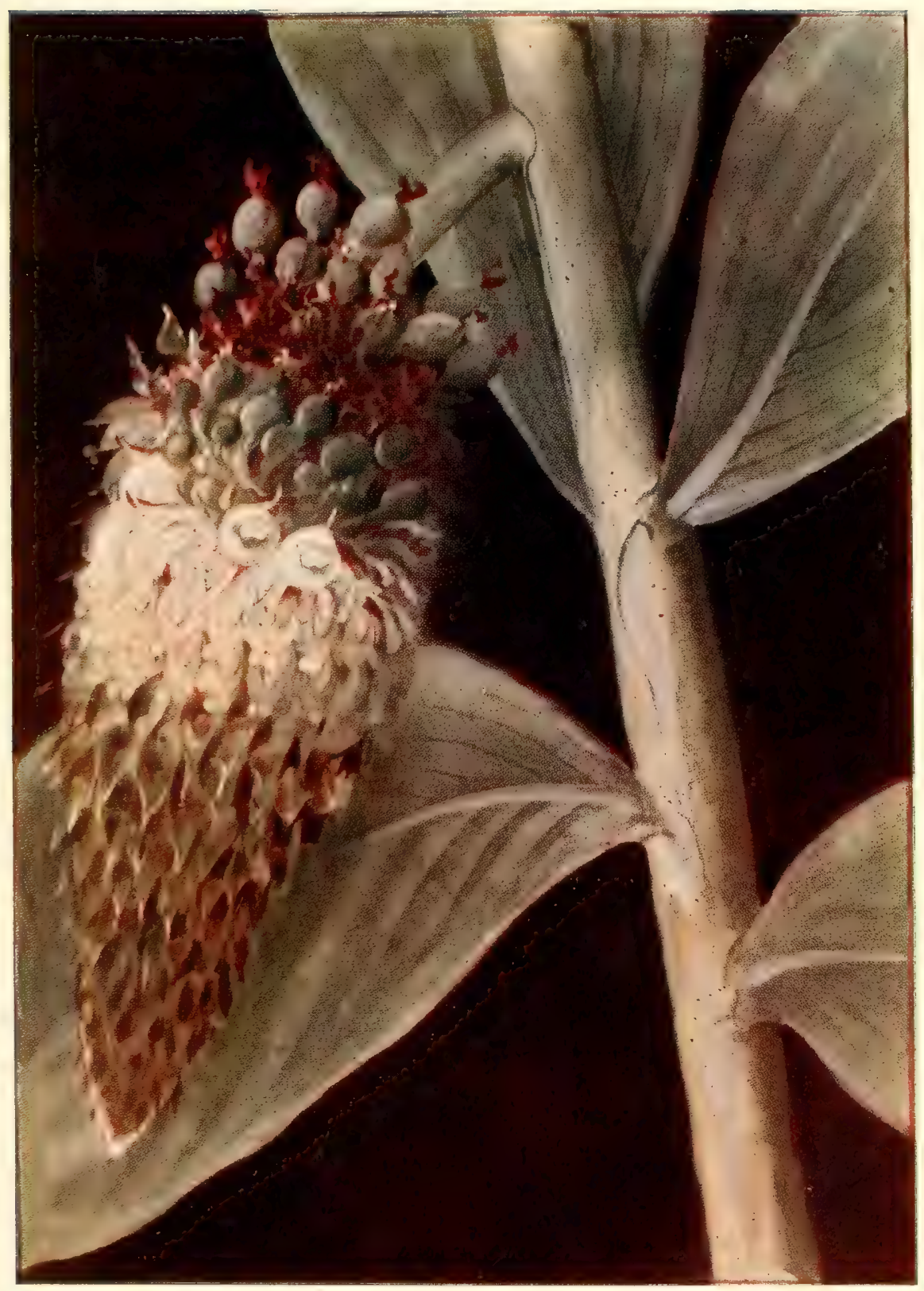

Alpinia ARCtiflora, F. v. M. 

Subtribe II.-Aristec.

Patersonia, R. Br. = Genosiris, Labill.

glauca, R. $B r$.

sericea, $R . B r$.

glabrata, R. Br.

\section{Order CXXXI.-AMARYLLIDEÆ.}

Tribe I.-Hypoxideæ.

Hypoxis, Linn.

hygrometrica, Labill.

var. pratensis, Benth.

var. elongata, Benth.

glabella, R. Br.

marginata, R. Br.

Curculigo, Gcertn.

recurvata, Ait.

ensifolia, R. Br. - “Harpea” of Dunk Island, "Yuara" of Palmer River, "Jool-lun” of Butcher's Hill, "U-o-ba” of Morehead River, "Un-dor-a" of Musgrave River, and "Kom-mol" of Cooktown natives.

Tribe II.-Amarylle.

Crinum, Linn.-Likely all more or less poisonous to stock.

Douglasii, Bail.

venosum, R.Br. (Fig. 530.)

brachyandrum, Herb.

brevistylum, Bail.

angustifolium.

var. confertum, Baker.

var. blandum, Rom.

uniflorum, F.v.M. (Fig. 53I.)

pestilentis, Bail.-Poisonous.

brisbanicum, Bail.

flaccidum, Herb.

pedunculatum, R. Br.

Eurycles, Salisb.

amboinensis, Loud.- "Bitamon" of the Cardwell natives (R.B.H.).

Cunninghamii, Ait. var. Whittlei, Bail.

Calostemma, R. Br.

luteum, Sims.-Poisonous to stock.

Scott-Sellickiana, Bail. (Fig. 532.)

album, R. Br. (Fig. 535.) 


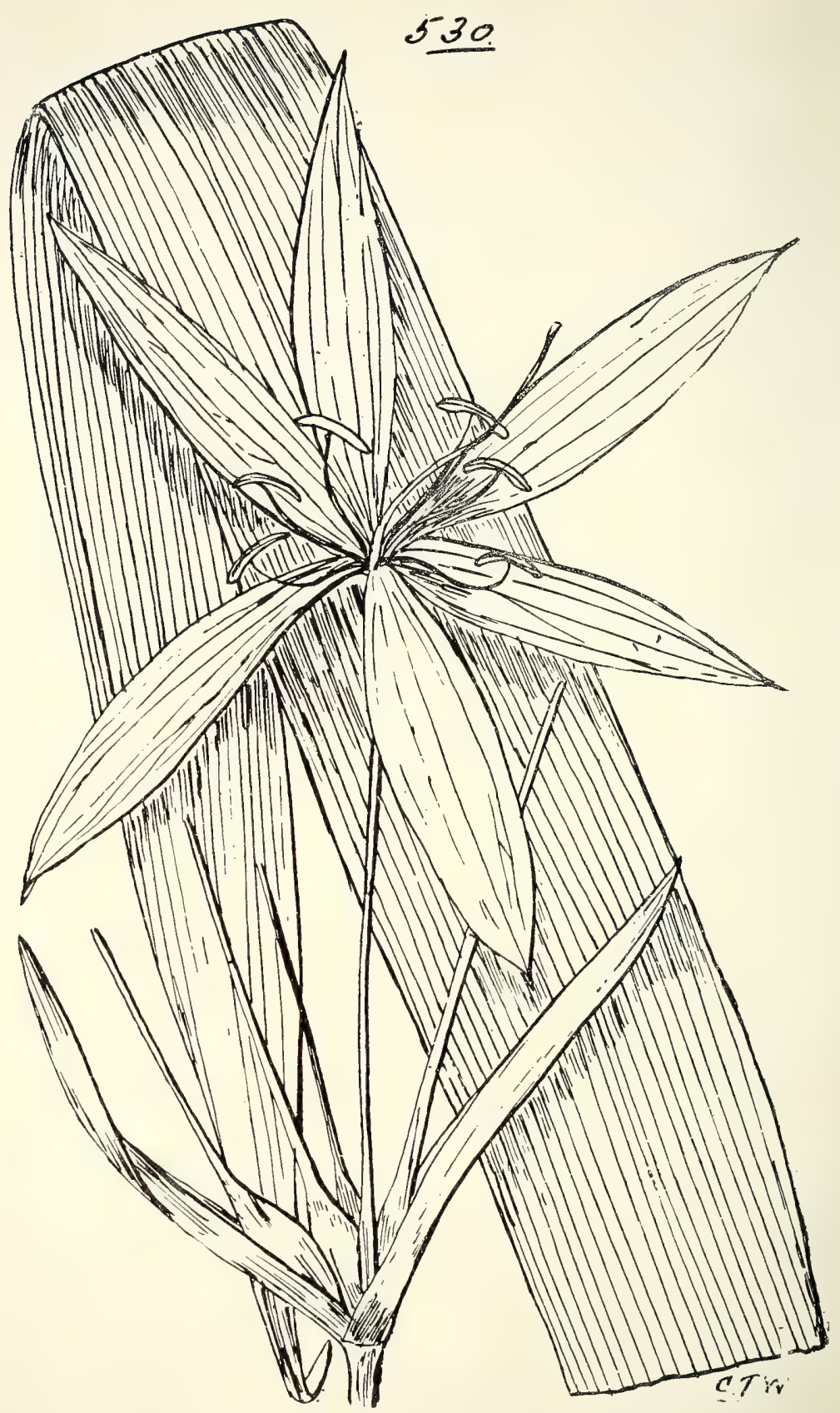

530. Crinum venosum, $R$. $B r$. 


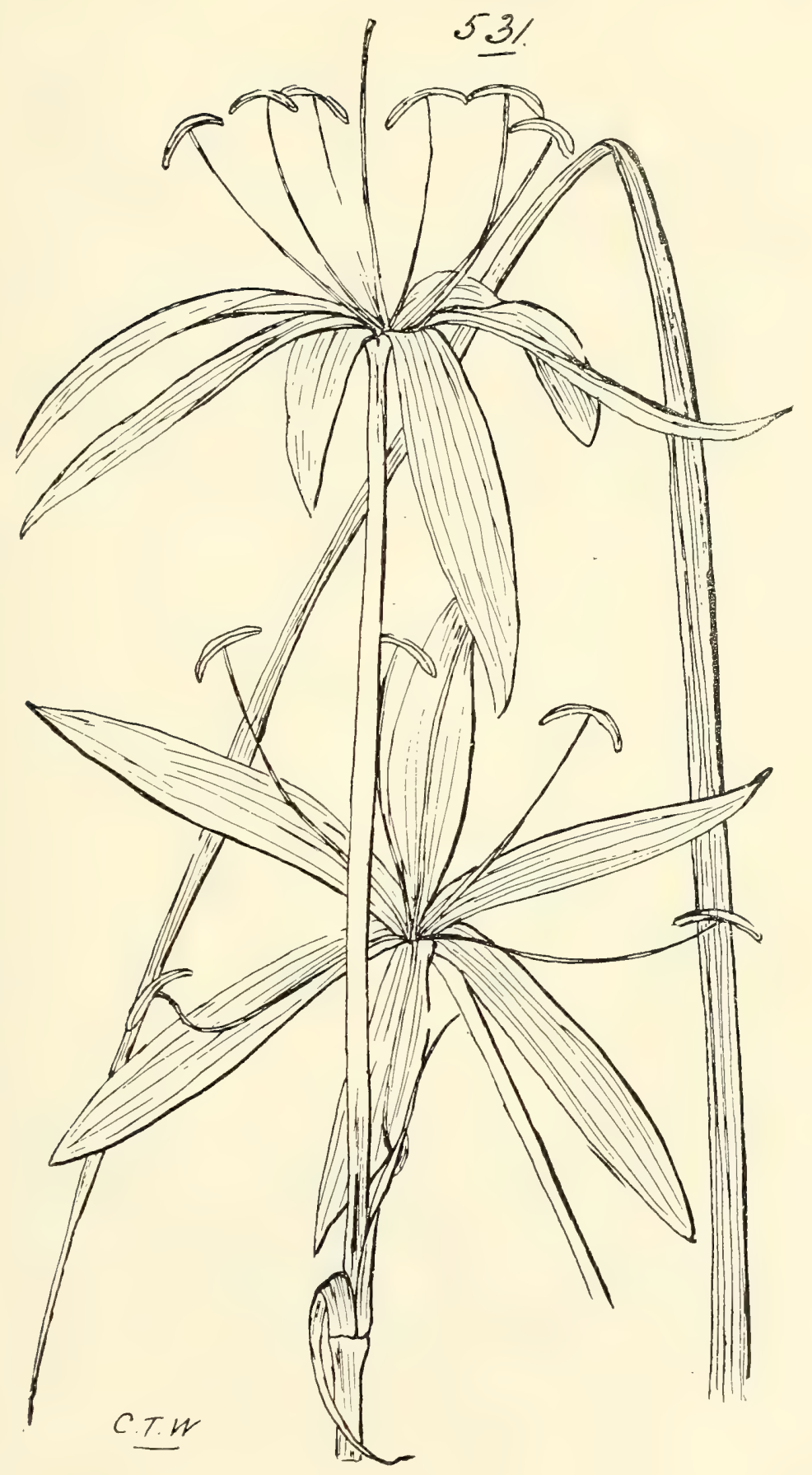

$2 \mathrm{~L}$

53i. Crinum uniflorum, $F . v . M$. 


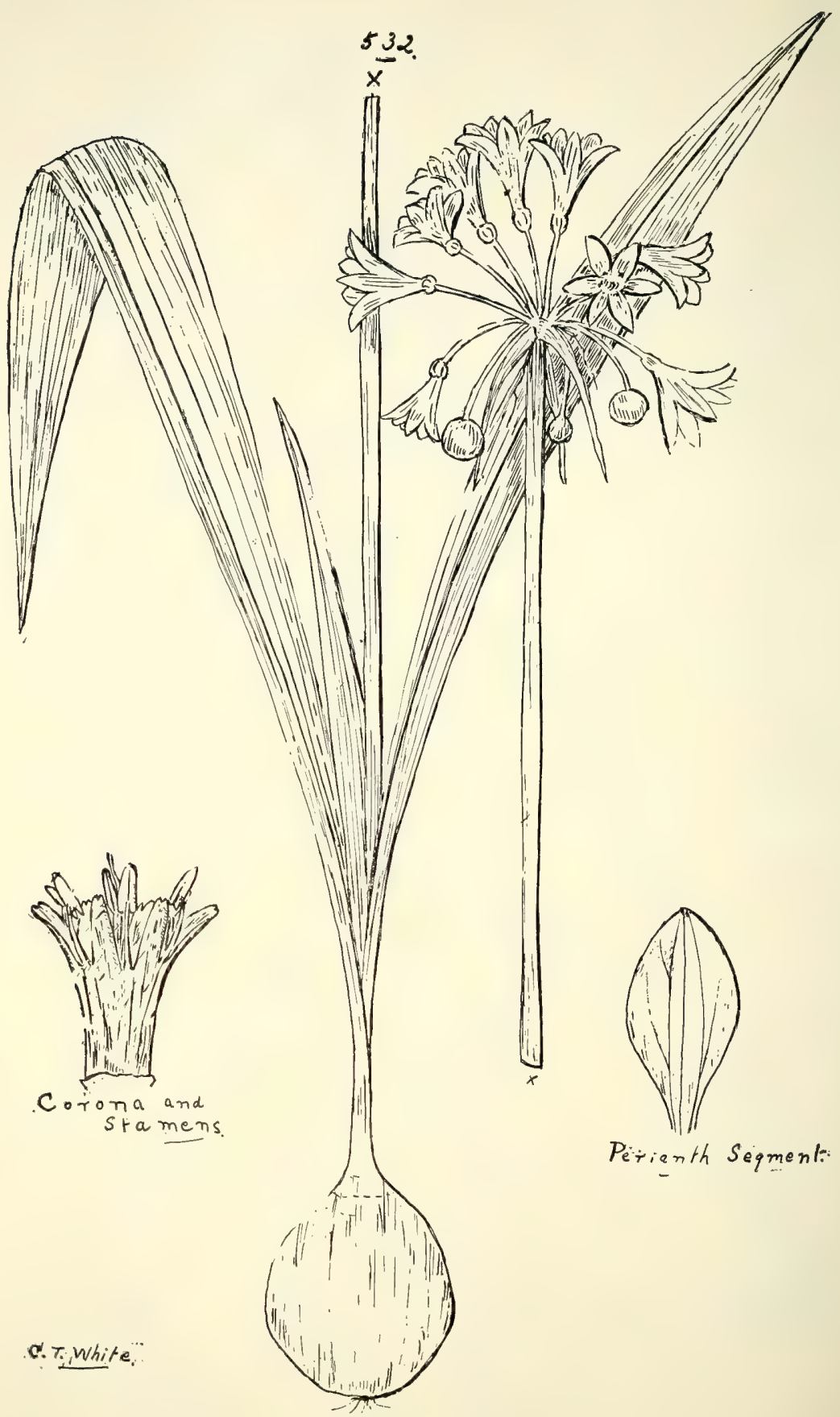

532. Calostemma Scott-Sellickiana, Bail. 


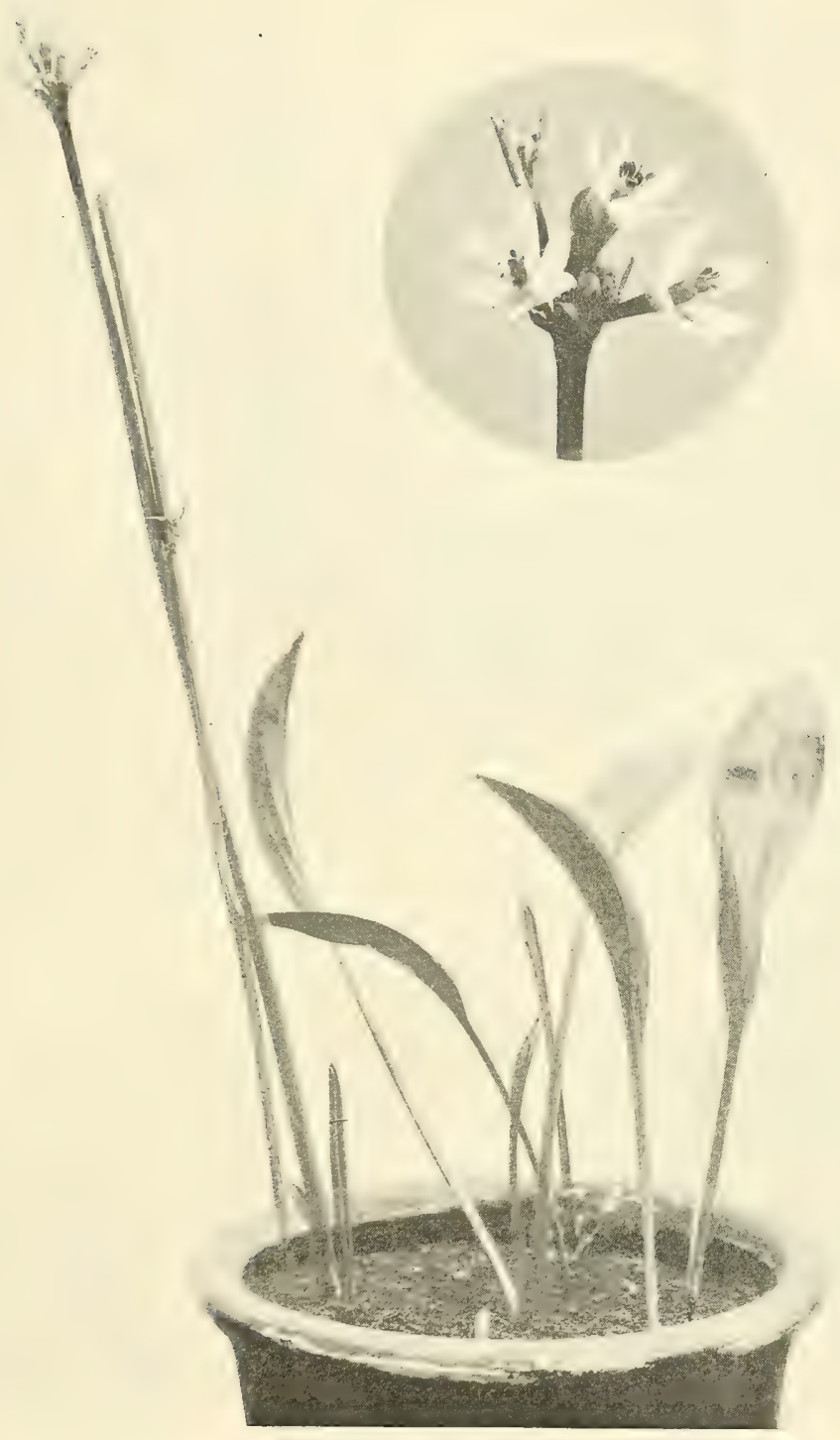

535. Calostemma album, $R . B r$. 
Tribe III.-Agaveæ.

Doryanthes, Correa.

excelsa, Corr.-The late Mr. Walter Hill reported having seen a white flowering variety on Mount Lindsay, S.Q. var. Palmeri, Bail.

var. Guilfoylei, Bail.

\section{Order CXXXII.-TACCACEÆ.}

Tacca, Forst.

pinnatifida, Forst._- " Be-ung-gal" of Bloomfield River, "Peang-gul" of Butcher's Hill, "Ung-ke" of Morehead River, "Niu” of Batavia River, and "An-tith-a” of Red Island natives. Probably some of these names belong to one or other variety, or perhaps only to a certain part of the plant. The rootstock furnishes an important article of food to the South Sea Islanders as well as to the Queensland aborigines. (Fig. 533.)

var. Brownii (Seem.), Bail.-Much smaller in all its parts than the normal form. (Fig. 534.)

var. aconitifolia, F.v.M.

\section{Order CXXXIII.-DIOSCORIDEÆ.}

Dioscorea, Limn.

sp., Bail ? nr. D. Kumaoensis, Kunth. (Fig. 536.)

transversa, R. Br.-Yam. "Am-pu" of Red Island, "Kowar" of Rockhampton, and "Guiaka" or "Gyaki" of Barron River natives.

sativa, Limn._- Kun-janga" of Butcher's Hill, "Wi-ka” of Morehead River, "Karro" of Mitchell River, and "Tarm" of Brisbane River natives.

var. elongata, Bail._."Wokai” of Cooktown natives. var. rotunda, Bail._- "Kalkur" of Cooktown natives.

*bulbifera, Limn.-An ornamental species common in Queensland gardens and now often met with as a stray from cultivation. Tropical Asia.

Petermannia, F.v. M.

cirrosa, F.v. M.

SERIES III.-CORONARIEA.

\section{Order CXXXIV.-ROXBURGHIACE E.}

Stemona, Lour. = Roxburghia, Jones.

javanica, Kunth., var. ? australiana, Benth. (Fig. 537.) 


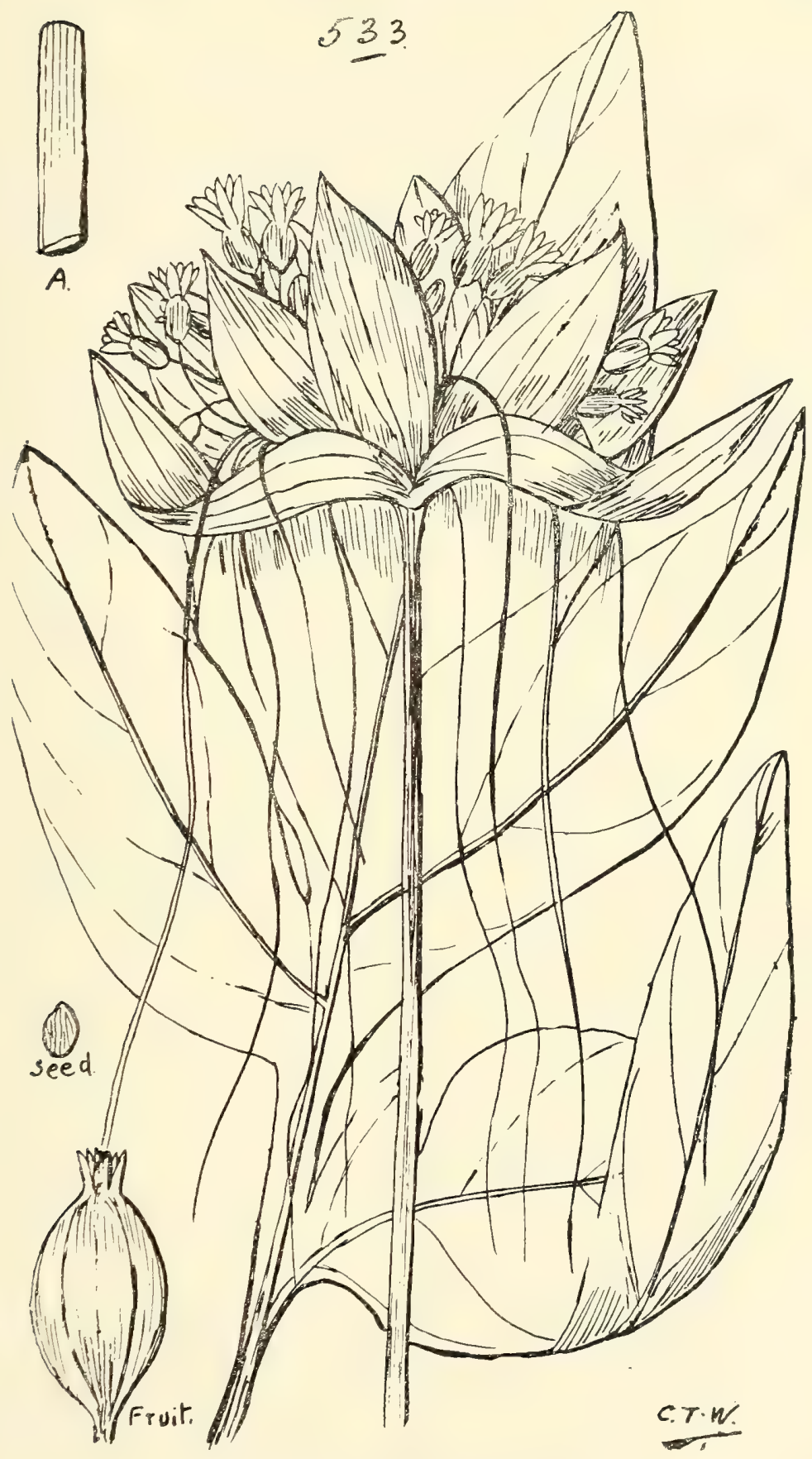

533. TaCCA pinnatifida, Forst.

(A) Portion of petiole. 


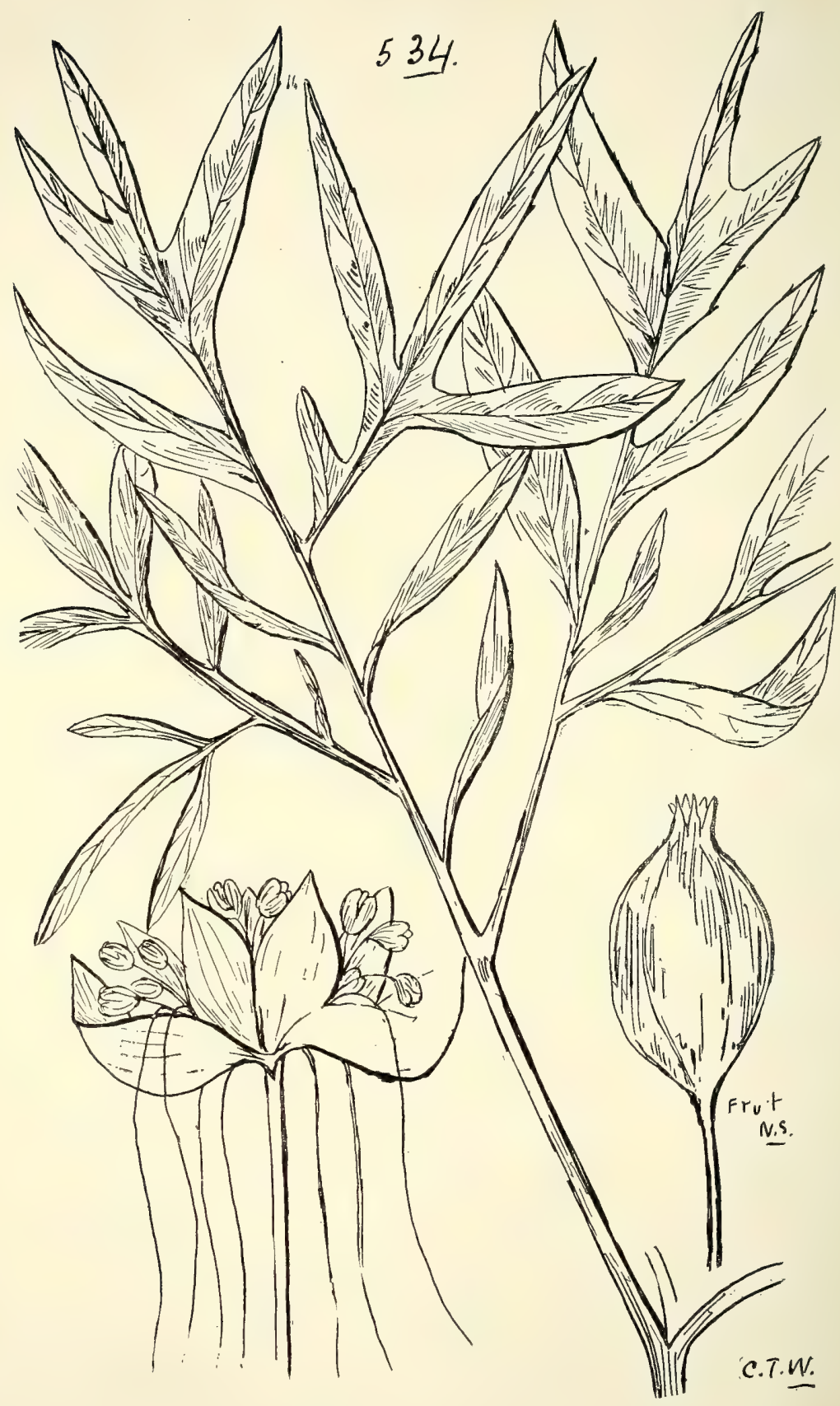

534. Tacca Pinnatifida, Forst., var. Brownit (Seem.), Bail. 


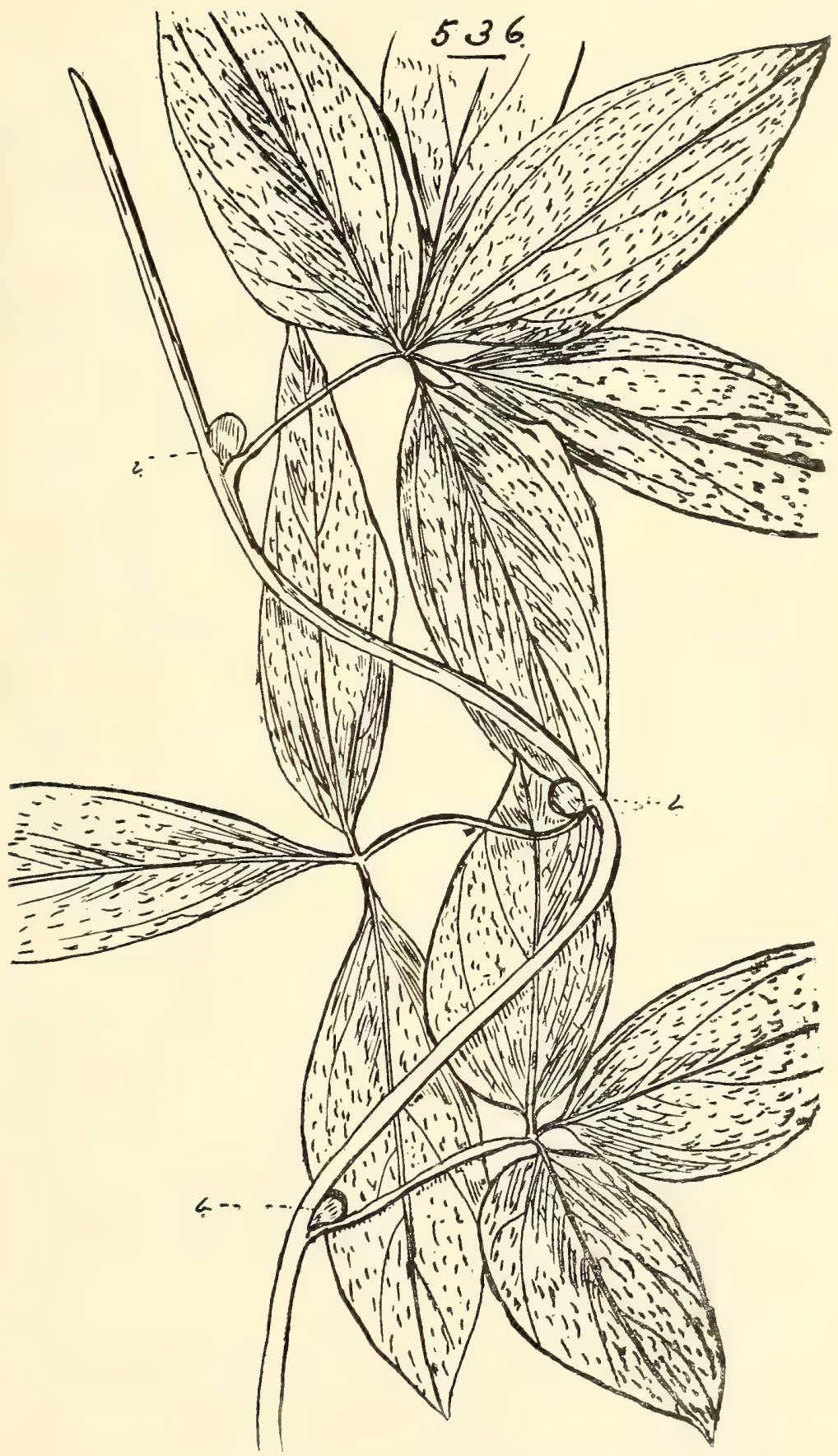

536. Dioscorea sp., nr. D. Kúmaoensis, Kunth. 


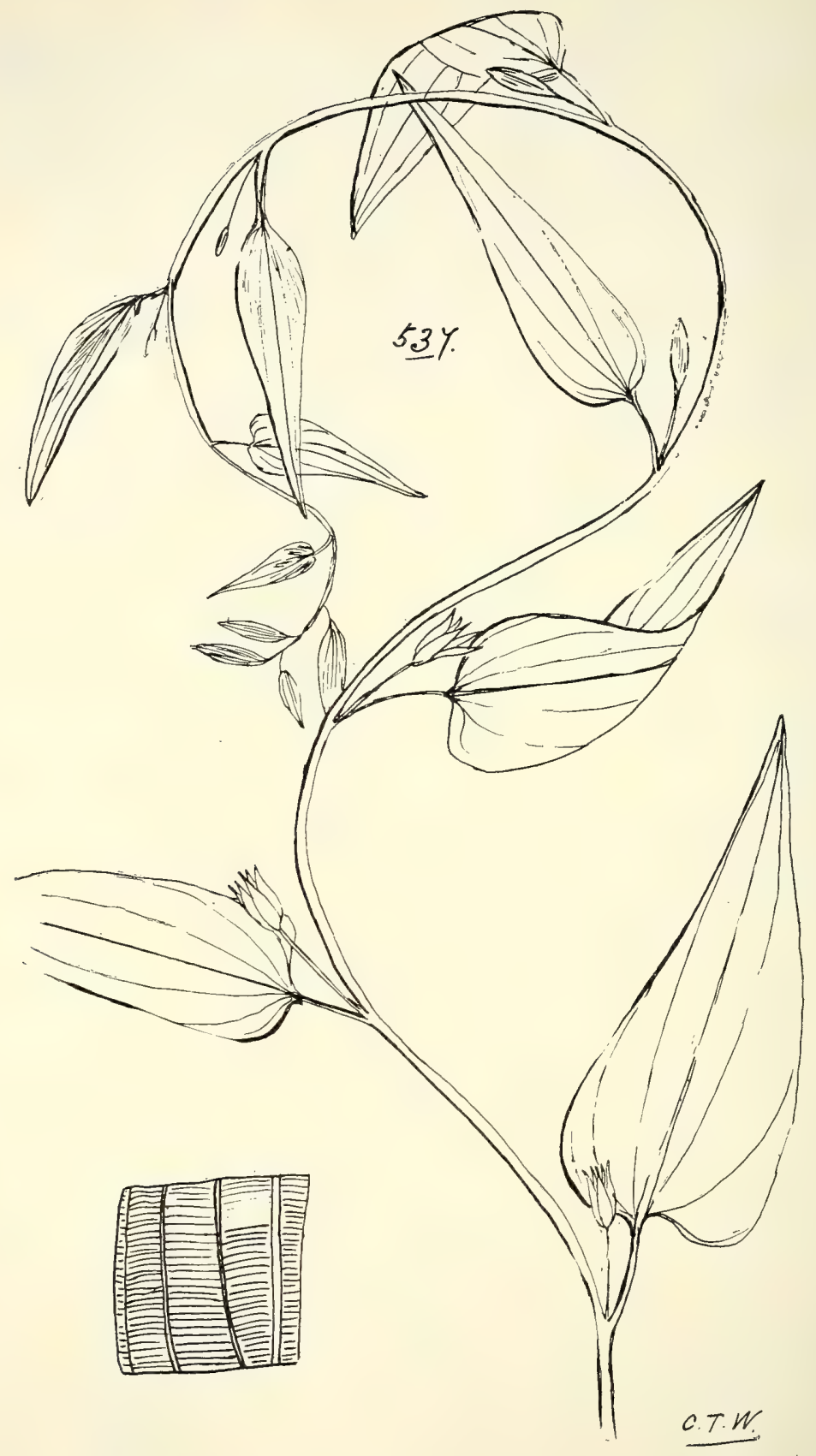

537. Stemona Javanica, Kunth, var. ? australiana, Benth. 


\section{Order CXXXV.-LILIACEÆ.}

Trĩbe I.-SMILAceÆ.

Smilax, Limn.

glycyphylla, Sm:-Supposed useful in scurvy.

australis, R. Br.-There are doubtless several forms of this species, but it requires, to work them out, that the specimens should be collected with the greatest care.

Rhipogonum, Forst.

album, R. Br.-Supplejack of some localities.

var. leptostachya, Benth.

discolor, F. v. M.

Danesii, Domin. in Fedde. Repert. x. (I9I I) 60.

Fawcettianum, F.v. M.

Elseyanum, F.v. M.

Tribe II.-Asparage⿸.

Asparagus, Linn.

racemosus, Willd.-A very troublesome weed.

TRIBE III,-LuZurJage $\approx$.

Eustrephus, R. Br.

latifolius, R. B.r.

var. angustifolia, Benth.

Geitonoplesium, A. Cunn.

cymosum, A. Cunn.-This is sometimes seen with variegated foliage.

Tribe IV.-Polygonateæ.

Drymophila, $R . B r$.

Moorei, Baker.

Tribe V.-Hemerocallem.

Blandfordia, Sm.

grandiflora, R. Br.

flammea, Hook.

var. aurea, Benth.

TRIBE VI.-DRACANEÆ.

Dracæna, Limn

angustifolia, Roxb.

var. Honoriæ, Bail.-Leaves green bordered with white.

worthy of cultivation. (Fig. 537 bis.)

Cordyline, Comm.

terminalis, Kunth.- "Churoga" of Tully River natives.

var. cannæfolia, Benth. 


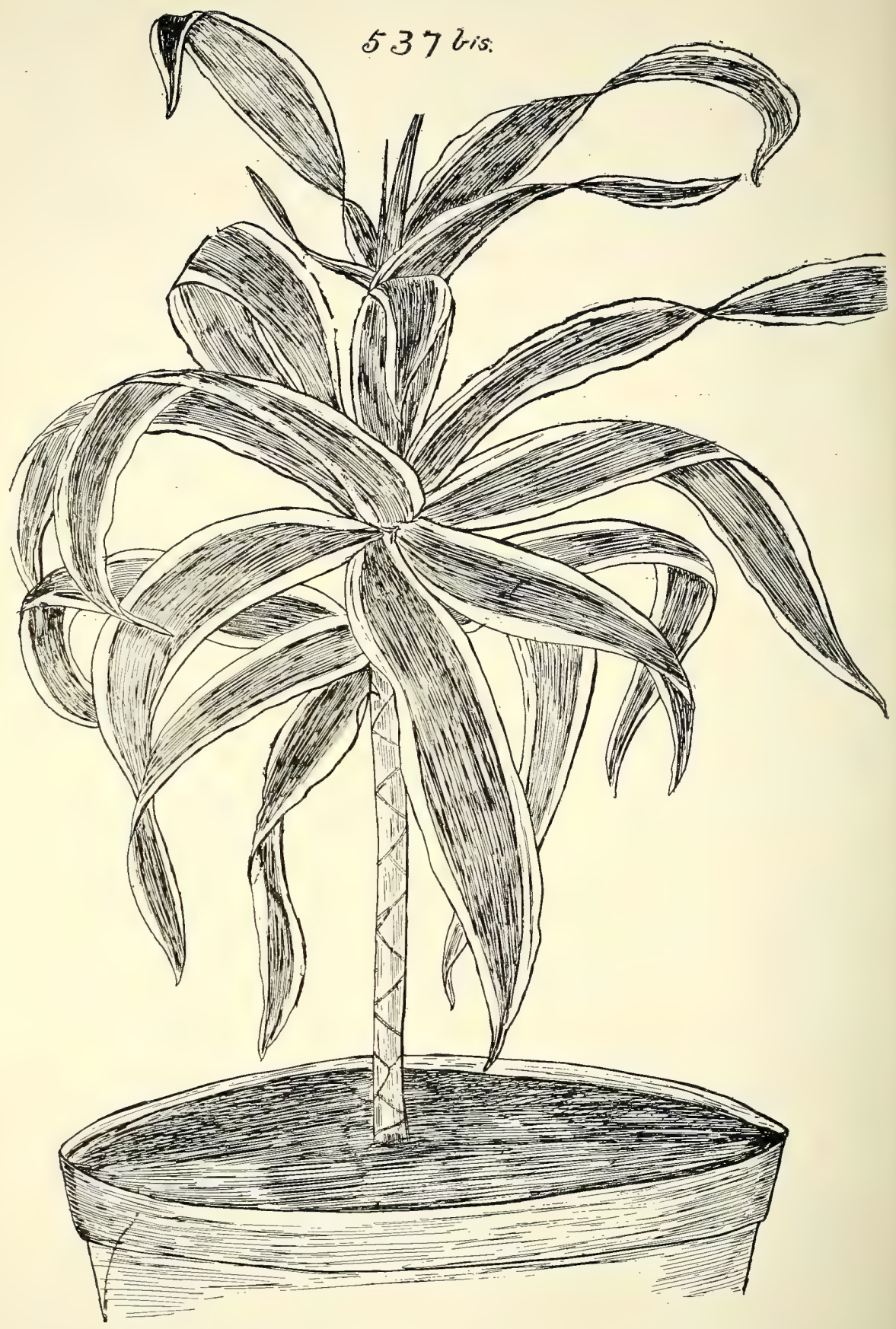

537 bis. Dracena nngustifolia, Roxb., var. Honorize, Bail. Plant reduced. 


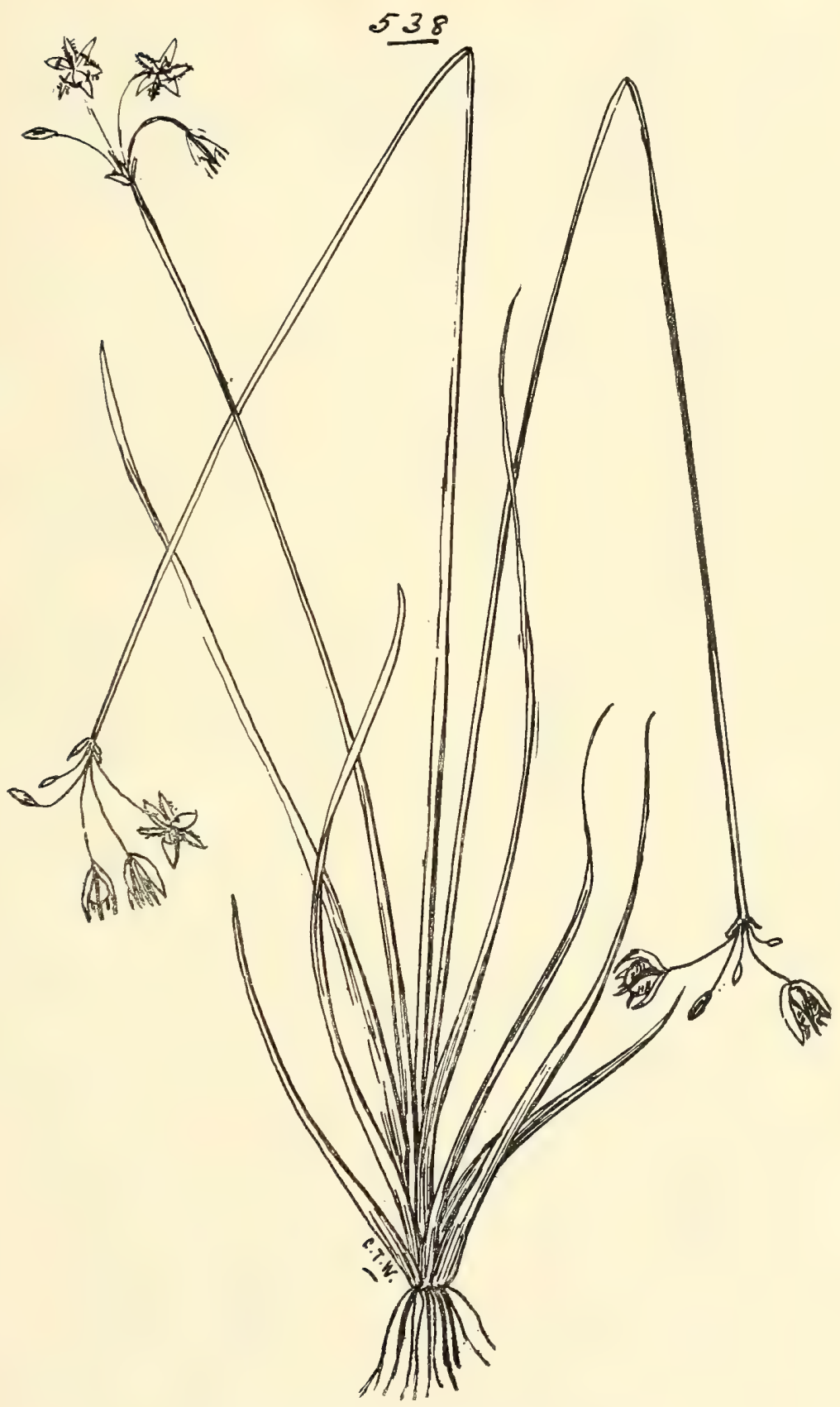

538. Thysanotus chrysantherus, $F, v, M$. 


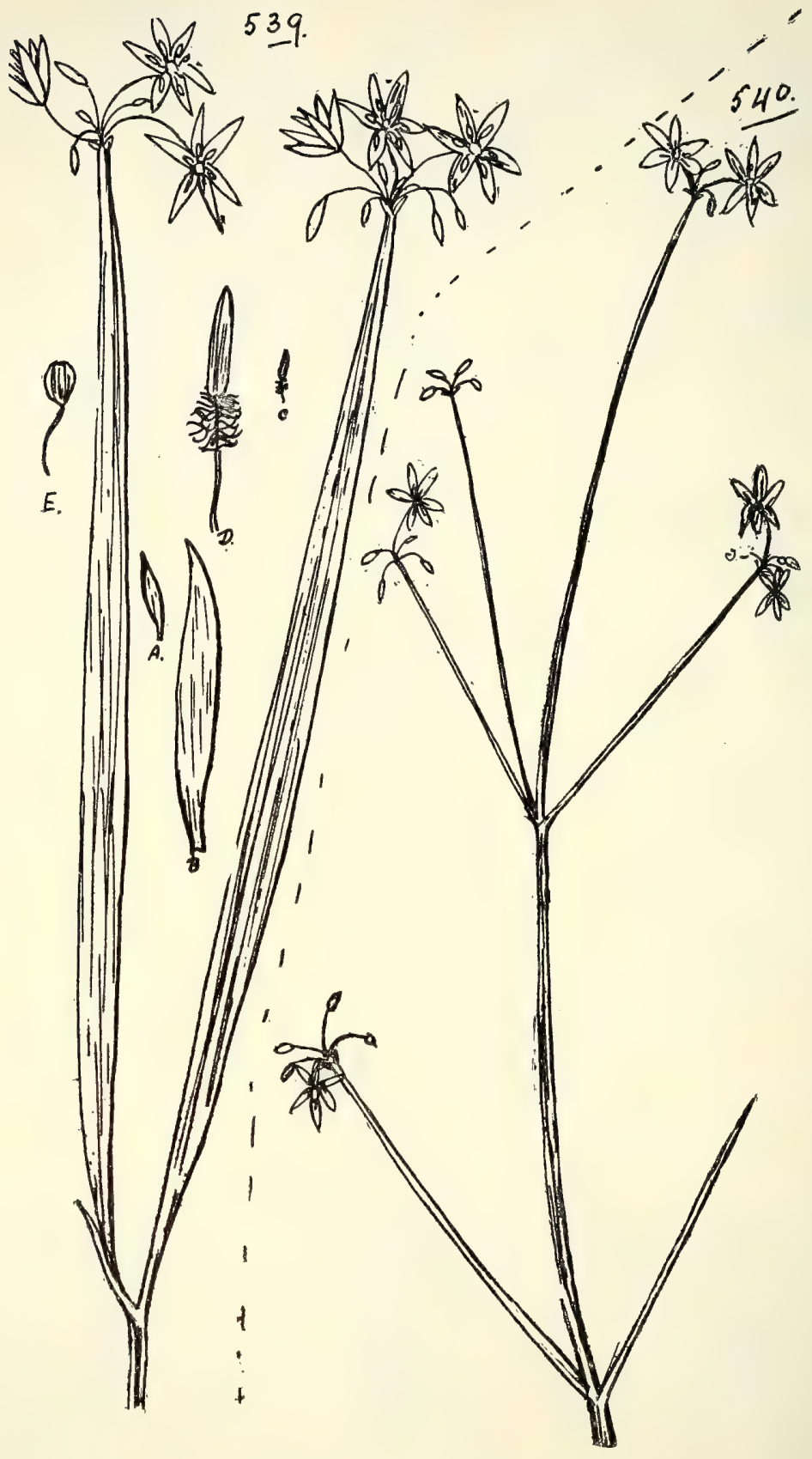

539. Tricoryne platyptera, Reichb.

540. T. ANCEPS, R. $\mathrm{Br}$. 
Cordyline-contd.

terminalis-contd.

var. Manners-Suttoniæ, Benth.

var. hedychioides, Benth.

var. Baileyi, Bail.-Foliage variegated.

Murchisoniæ, F.v.M.

stricta, Endl.

Tribe ViI.-Asphodeleæ.

*Asphodelus, Linn.

fistulosus, Linn.-Europe. A troublesome weed in some parts.

Bulbine, Linn.

bulbosa, Haw.-Poisonous to stock.

semibarbata, Haw.-Native Onion or Leek; poisonous to stock.

Thysanotus, R. Br.

Section Hexandre.

chrysantherus, F.v.M. (Fig. 538.)

tuberosus, $R$. Br.-Fringe Violet.

Baueri, R. Br.

Arthropodium, R. Br.

paniculatum, $R \cdot B r$.

minus, $R . B r$.

dianellaceum, F.v. M.

Dichopogon, Kunth.

strictus, Baker.

Sieberianus, Kunth.

Cæsia, R. Br.

vittata, $R . B r$.

var. chlorantha, Benth. $=$ C. chlorantha, F.v. M.

parviflora, $R \cdot B r$.

Corynotheca, F.v.M.

lateriflora, $F, \tau . M$.

Dianella, Lam.-Blueberry plants.

lævis, $R$. Br.-Some plants produce double flowers.

var. aspera, Benth.

revoluta, R. Br.

cærulea, Sims.

var. congesta, Benth--Some plants produce double flowers. ensifolia, Red Lil.

Stypandra, R. Br.

glauca, $R . B r$.

cæspitosa, $R . B r$. 


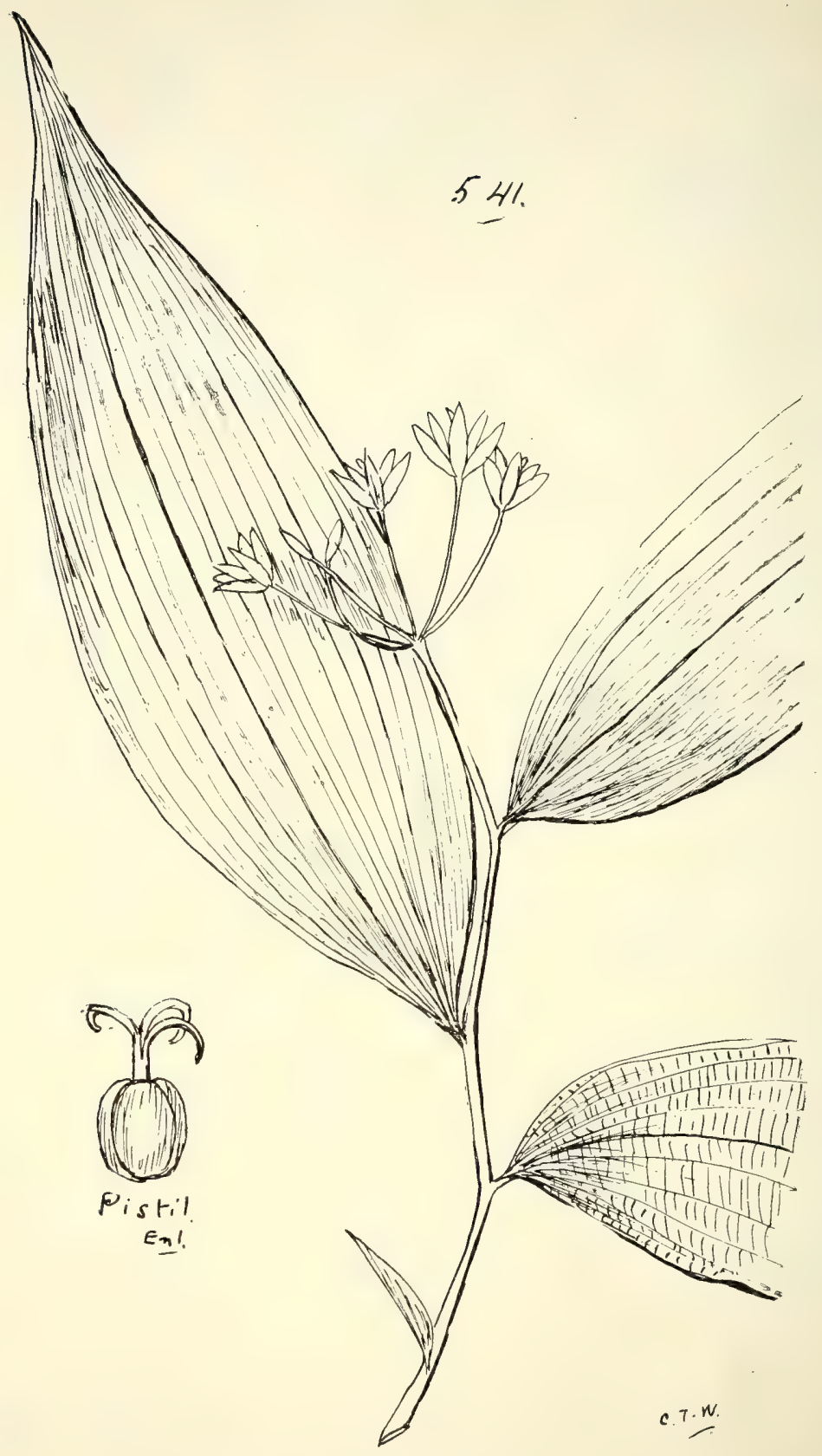

541. Schelhammera pedunculata, F. $v . M$. 


\section{Tribe VIII.-Johnsoniex.}

Tricoryne, R. Br.

platyptera, Reichb. (Fig. 539.)

anceps, R. Br. (Fig. 540.)

muricata, Baker.

elatior, R. Br.

simplex, R. Br.

Laxmannia, R. Br.

gracilis, R. Br. = Bartlingia gracilis, F. v. M.

Borya, Labill.

septentrionalis, $F \cdot v \cdot M$.-The tufts of young leaf-growth are of a pretty pink colour.

Sowerbæa, $S m$.

juncea, $S m$.

Tribe IX.-Allied.

*Nothoscordum, Kunth.

fragrans, Kunth.-North America.

Tribe X.-AnguillarieÆ.

Burchardia, R. Br.

umbellata, R. Br.

Anguillaria, R. Br.

dioica, R.Br. $=$ Wurmbsea dioica, F. v. M.

Iphigenia, Kunth.

indica, Kunth.

Tribe XI.-Uvularie底.

Schelhammera, R. Br.

multiflora, $R . B r$.

pedunculata, F.v.M. (Fig. 54I.)

Kreysigia, Reichb.

multiflora, Reichb.

\section{Order CXXXVI.-PONTEDERIACEA.}

*Pontederia, Willd.

cordata, Pursh-Pickerel weed. An American plant met with as a stray from cultivation.

*Eichhornia, Kunth.

speciosa, Kunth. Water Hyacinth; a noxious and now widespread water-weed. South America.

Monochoria, Presl.

cyanea, F.v.M. (Fig. 542.) 


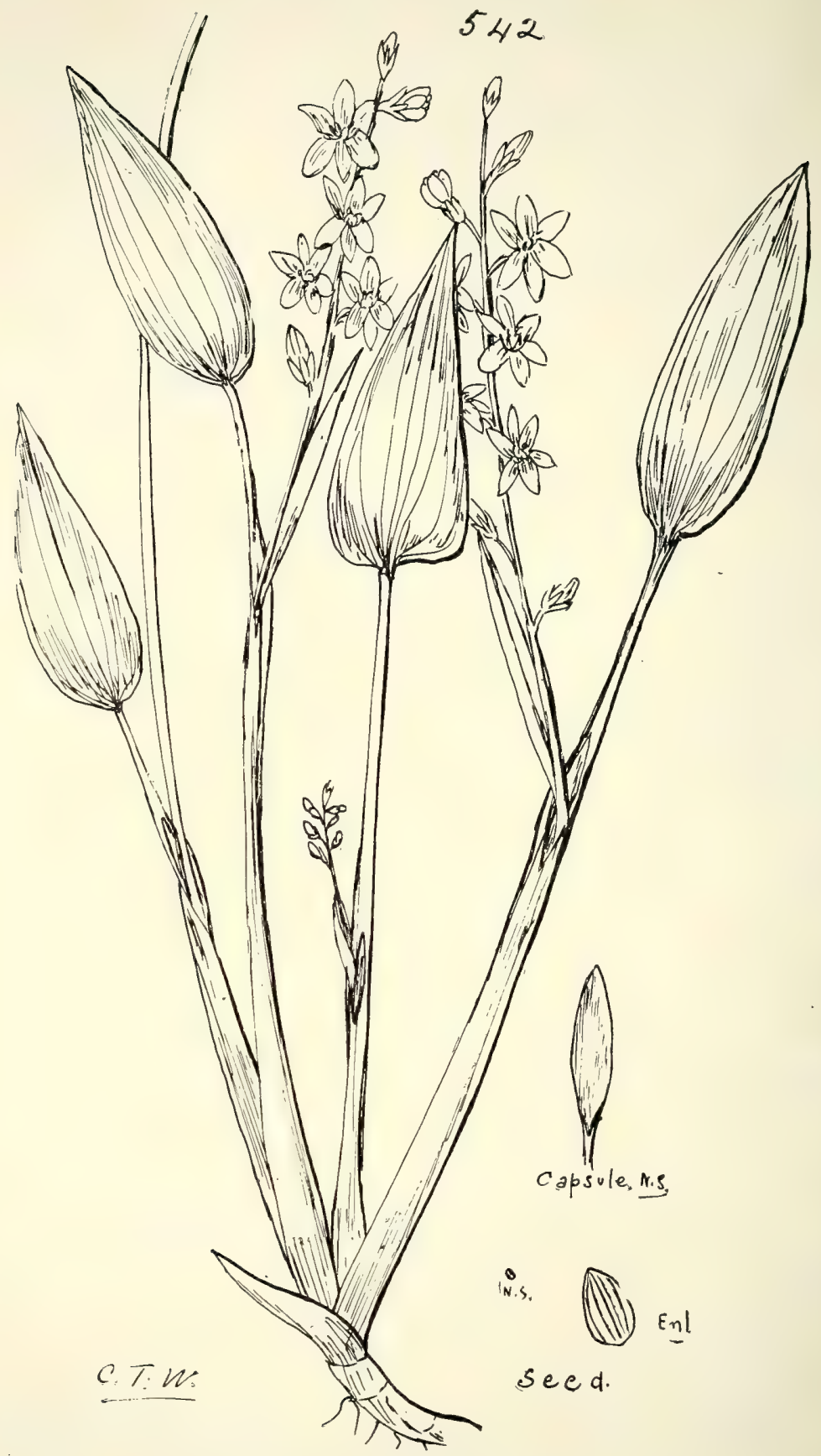

542. Monochoria Cyanea, $F . v . M$. 


\section{Order CXXXVII.-PHILYDRACEÆ.}

Philydrum, Banks.

lanuginosum, Banks.

Helmholtzia, F..$M$.

glaberrima, T. Caruel.

acorifolia, F.v.M.- "Kuranda" of Barron River natives.

(Fig. 543.)

\section{Order CXXXVIII.-XYRIDEF.}

Xyris, Limn.

complanata, R. Br.-Hatpin Grass. (Fig. 544.)

var. bracteata, Benth.

var. ? leptocaulis, Benth.

panciflora, Willd. $=$ X.paludosa, R. Br., which some botanists

retain as a species.

var. albifios, Bail. (Fig. 545.)

gracilis, $R . B r .=X$. juncea, R. Br., retained by some as a species.

operculata, Labill.

\section{Order CXXXIX.-COMMELINACEA.}

Tribe I.-Pollieæ.

Pollia, Thunb.

macrophylla, Benth.

crispata, Benth.-The foliage is sometimes variegated.

Tribe II.-COMMeline.e.

Commelina, Limn.

undulata, R. Br.

ensifolia, R. Br.-Called "Scurvy-grass" in some parts of Queensland.

cyanea, $R$. Br. $=$ C. communis, F. v. M., non Linn. Plants are sometimes seen with variegated foliage.

var. alba, Bail.

lanceolata, $R . B r$.

Aneilema, R. Br.

acuminatum, R. Br.

biflorum, R.Br. (Fig. 546.)

sclerocarpum, F.v.M.

siliculosum, R. Br.- "A-un-du" of Batavia River natives.

calandrinoides, $F . v \cdot M$.

gramineum, R. Br.

var. album, Bail.

giganteum, R. $\mathrm{Br}$.

var. gracilis, Benth.

$2 \mathrm{M}$ 


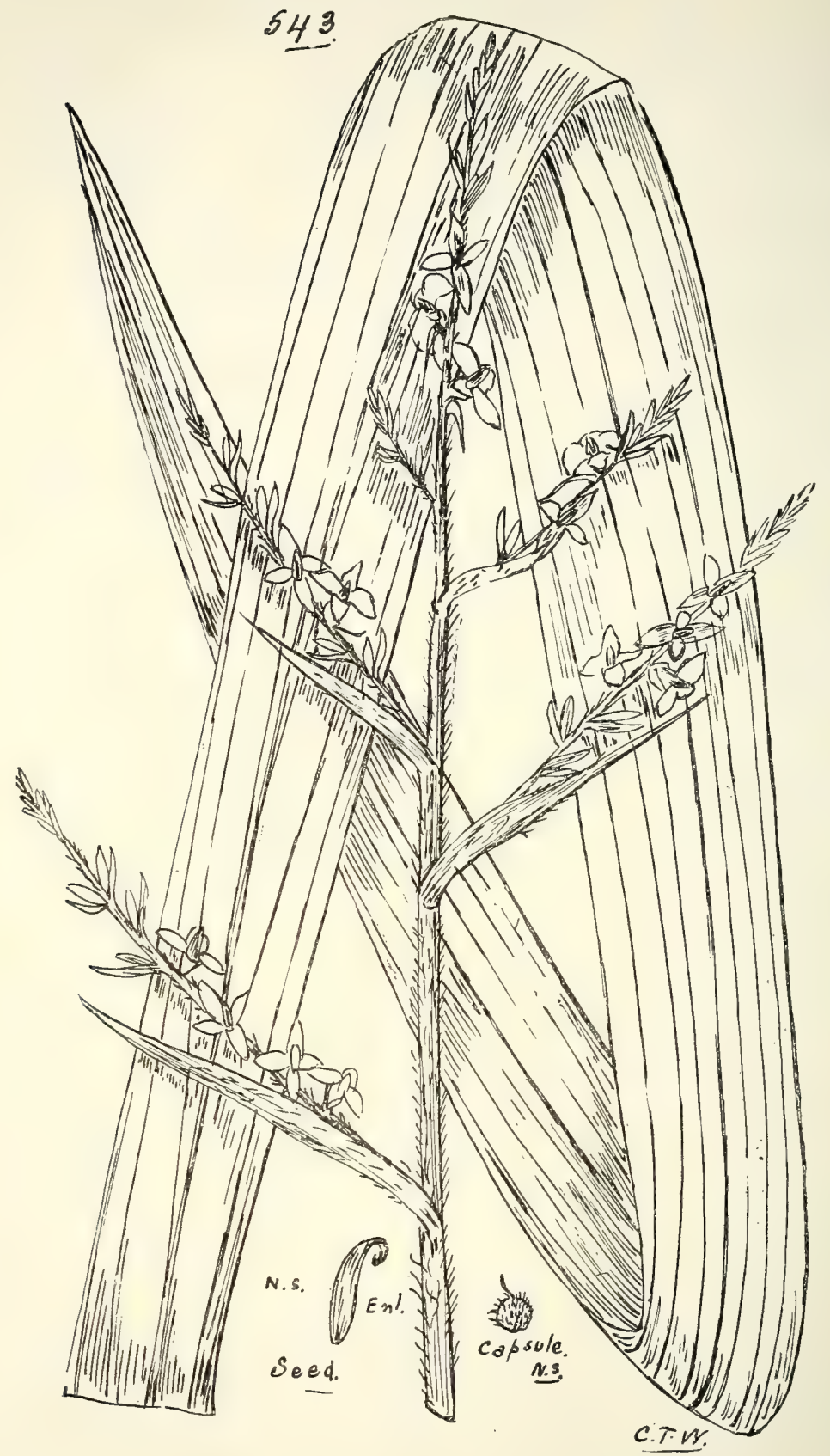

543. Helmholtzia acorifolia, F. v. $M$. 


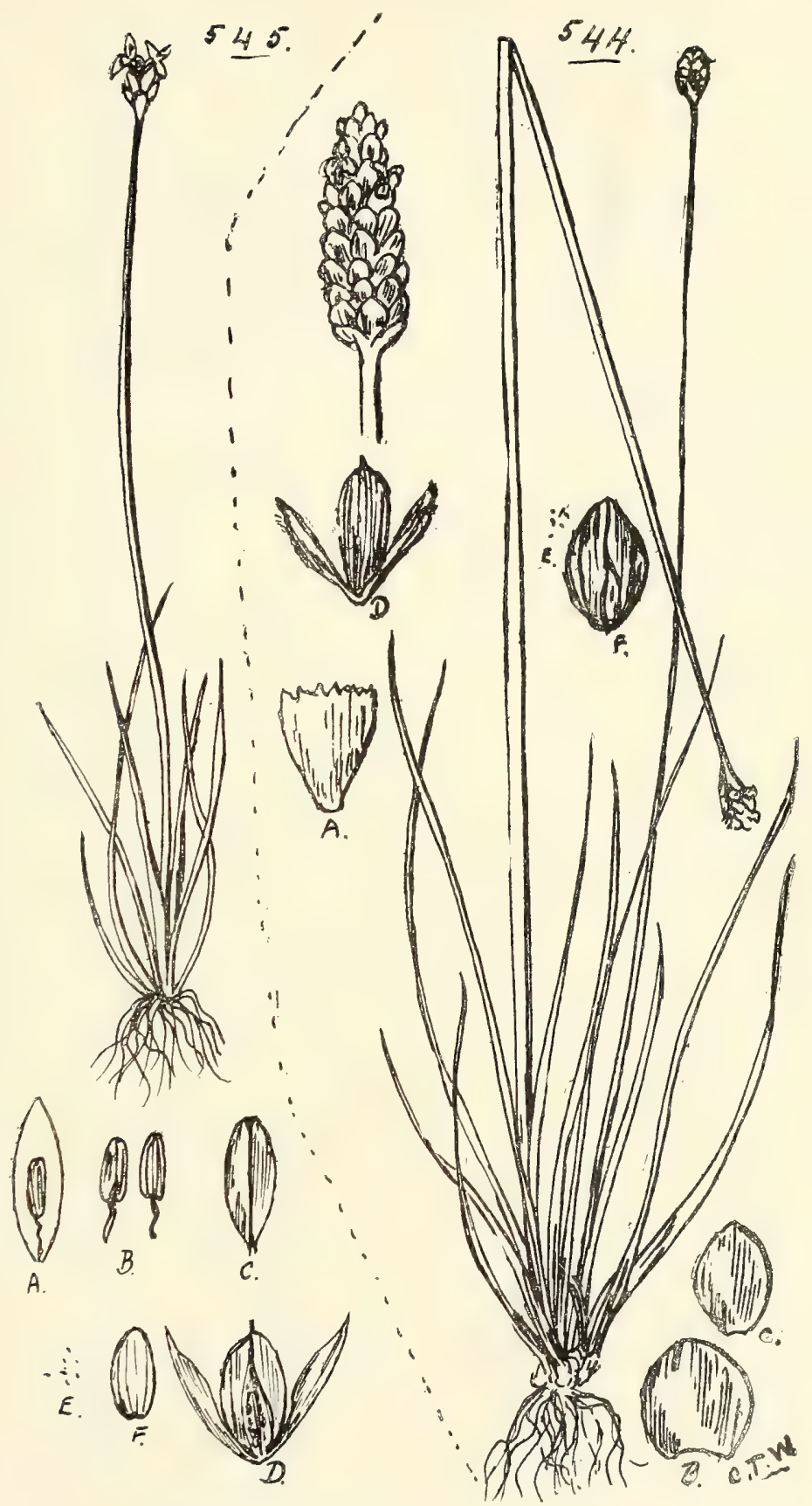

544. Xyris Complanata. R. Br.

(A) Perianth segment, (B) an outer scale, (C) an inner scale, (D) capsule, (E) seeds, (F) a single seed. (A) - (D) and (F) enl.; (E) nat. size.

545. X. PAuciflora, Willd, var. Albiflos, Bail.

(A) Perianth segment and stamen, (B) stamens, (C) bracteole, (D) capsule, (E) and (F) seed. (A)-(D) and (F) enl.; (E) nat. size. 


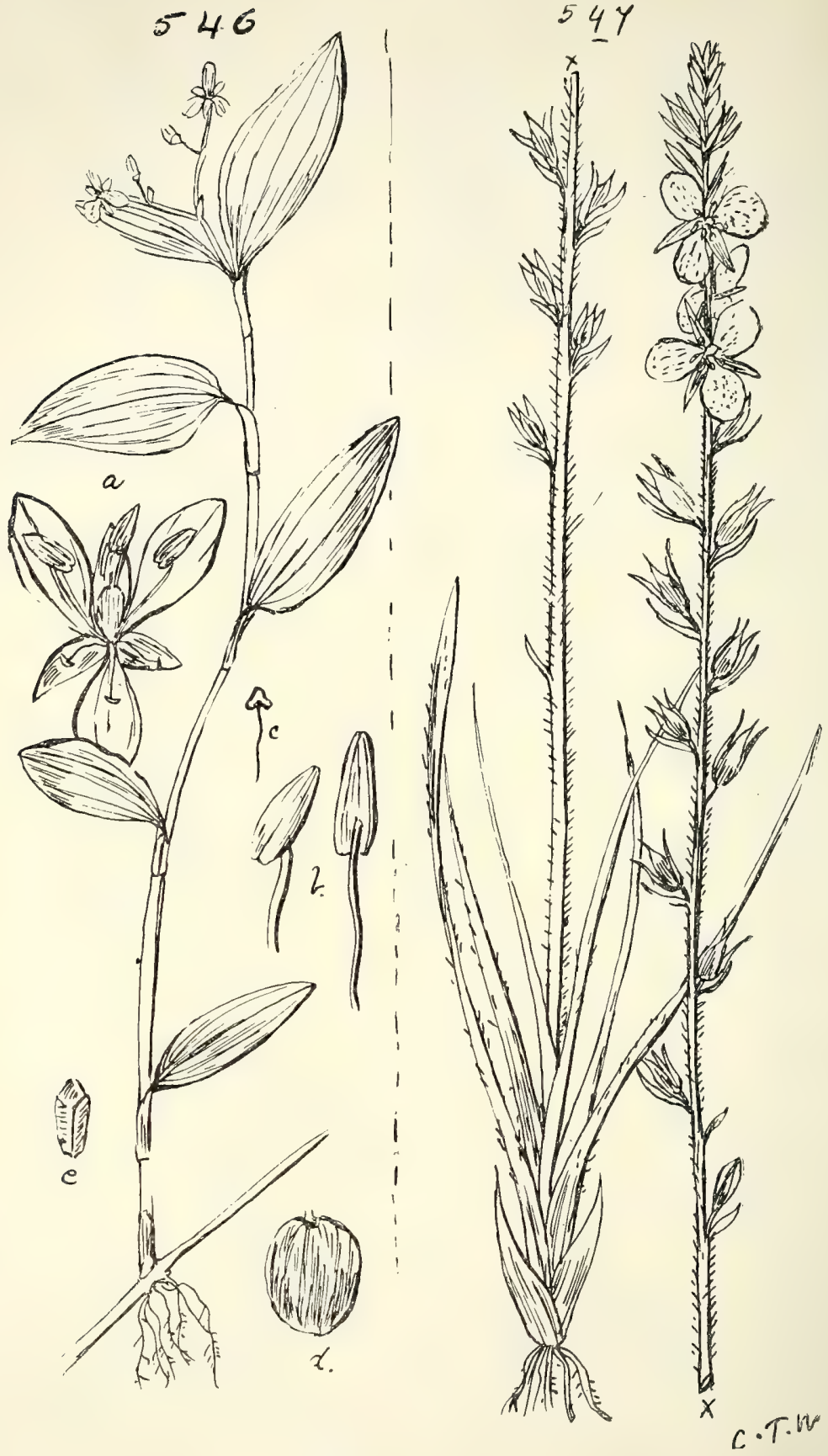

546. Aneilema biflorum, $R . B r$.

(a) Flowes laid open, (b) perfect anthers, (c) filament with abortive anther. 547. Cartonema parviflorum, Hassk. 
Tribe III.-Tradescantie.e.

Cyanotis, Don.

axillaris, Roem. et Schult.

Floscopa, Lour.

paniculata, Hassk. = Tradescantia paniculata, Roxb.

*Tradescantia, Linn.-Spiderworts.

fluminensis, Vell., var. tenella, C. B. Ciarke--Tropical America.

Cartonema, R. Br.

Baileyi, Bail.

spicatum, R. Br.

parviflorum, Hassk. (Fig. 547.)

brachyantherum, Benth.

\section{SERIES IV.-CALYCIN正.}

\section{Order CXL.-FLAGELLARIEAE.}

Flagellaria, Limn.

indica, Linn.- Supplejack. "Pain-ki" of Tully River and "Yurol" or "Yeroll" of Stradbroke Island natives. The stems were used by the natives in tree-climbing. They peeled off the rind, after which the two ends of the vine could be knotted together to form a kind of hoop, which they used to climb the trunks of tall trees and to sit in when chopping out the nests of bees (H. Schneider). var. gracilicaulis, Bail. (Fig. 548.)

\section{Order CXLI.-JUNCACEE.}

Tribe I.-Xerote压.

Xerotes, Banks.

Section I.-Euxerotes.

Series I.-Glumeratæ.

Banksii, R. Br.

longifolia, R. Br. = Lomandra longifolia, Labill._- Dilli" of

Brisbane River and "Chindirigan" of Tully River natives. var. montana, Benth.

confertifolia, Bail., Q1. Agric. J1. xxv. (I9IO) II. (Fig. 549.)

Series II.-Fasciculatæ.

multiflora, R. Br.-" Mat1-u" of Bloomfield River natives. sororia, F. v.M.

Series III.-Sparsifloræ.

effusa, Lindl.

filiformis, $R \cdot B r$. 
Xerotes-contd.

Section II.-Cephalogyne.

elongata, Benth.

Section III.-Typhopsis.

leucocephala, R. $B r$.

Xanthorrhæa, $S m$.

macronema, F.v.M.-Long-stamened Grass-tree.

minor, R.Br.- "Bulnan" of Cairns natives.

pumilio, R. Br. (Fig. 550.)

hastilis, R. Br. _ "Tchuckah" of Bundaberg and "Goonangulla" of Mackay natives.

arborea, R. Br.-."Kwanja" of Palmer River, "Ang-ga-tan" and "Rarnka" of Princess Charlotte Bay, "Pungga" of Cape Bedford, and "Ngang-ir" of Bloomfield River natives.

bracteata, R. Br.

quadrangulata, F.v.M. - "Tchagun" of Mount Perry natives.

T'ribe II.-Eujunce压.

Juncus, Limn.

planifolius, $R . B r$.

bufonius, Linn.

homalocaulis, F.v.M.

communis, E. Mey.-Common Rush. "Wirree" of Bundaberg natives.

vaginatus, $R \cdot B r$.

pauciflorus, R. Br.

pallidus, R. $B r$.

maritimus, Lam.

prismatocarpus, $R . B r$.

capillaceus, Hook.f.

Luzula, DC.

campestris, DC.-Glowworm Grass.

\section{Order CXLII.-PALM E.}

Areca, Linn.

Tribe I.-Arece.e.

Alicæ, F.v.M._-"Goolanjurie" of Cairns natives. (Fig. 55I.)

Orania, Zippel.

Beccarii, Bail. = Areca appendiculata, Bail.

Hydriastele, H.Wendl. et Drude.

Wendlandiana, F.v.M.

Douglasiana, Bail. (Fig. 552.) 


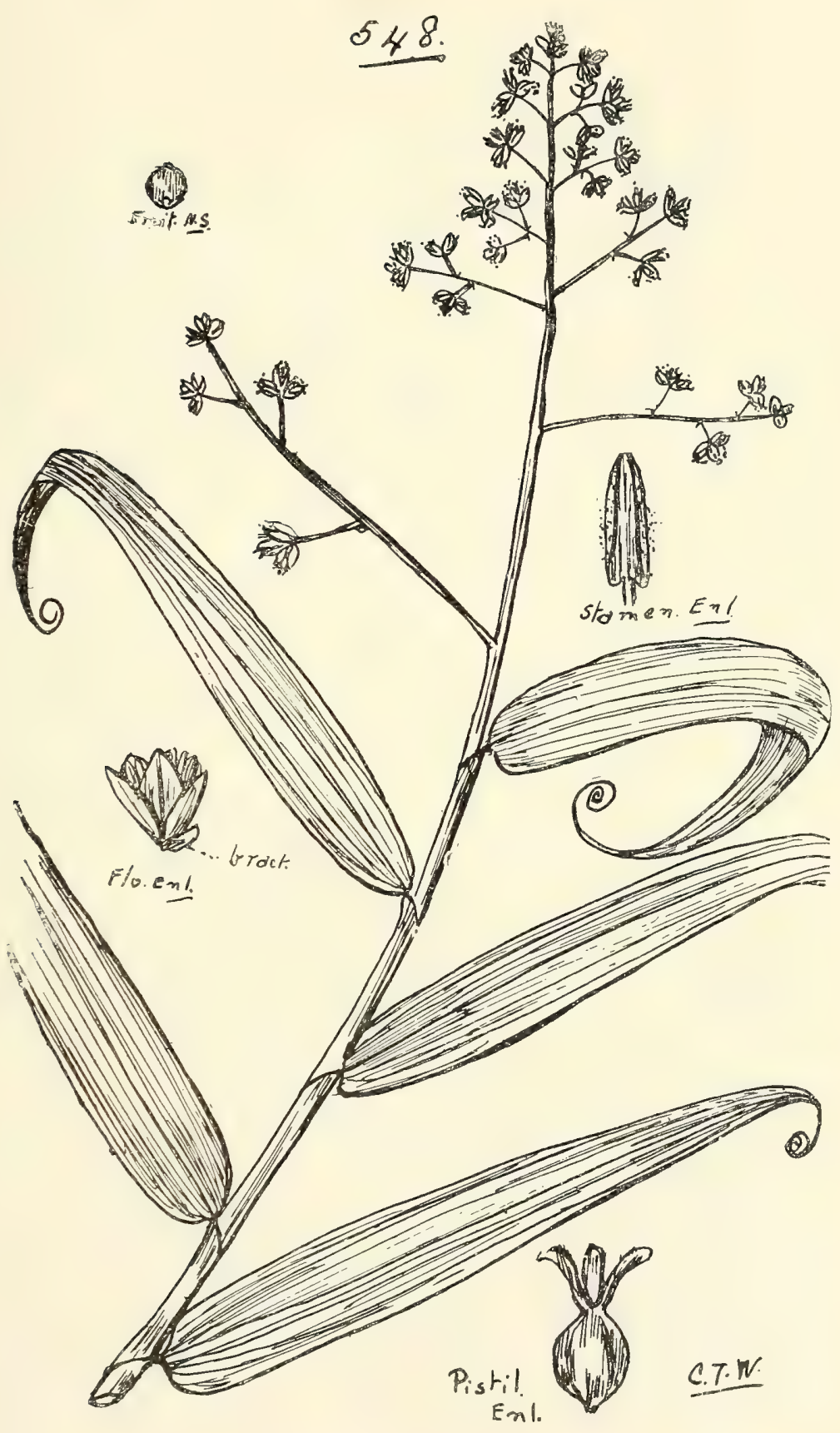

548. Flagellaria indica, Linn., var. Gracilicaulis, Bail. 


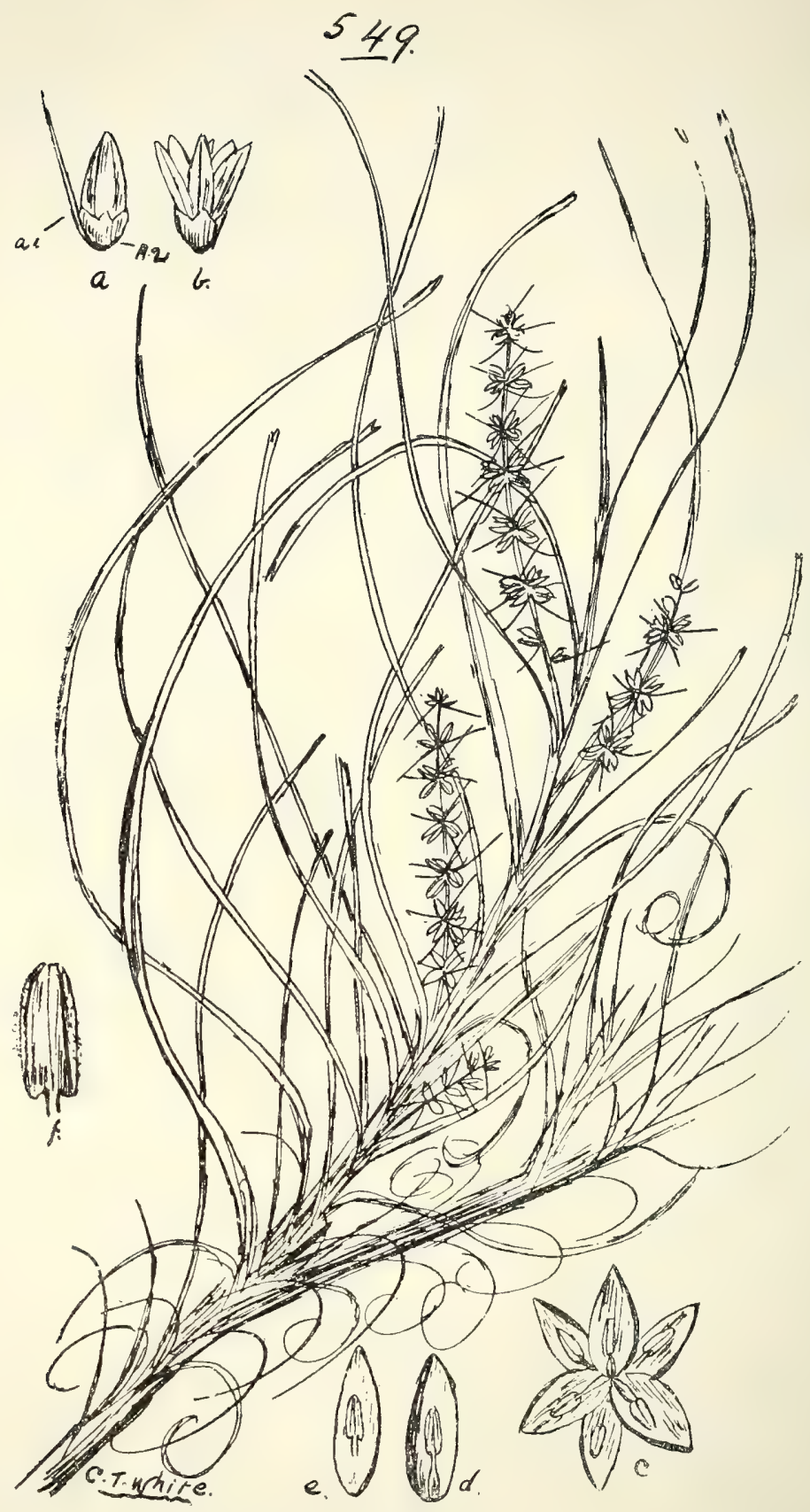

549. Xerotes confertifolia, Bail.

(a) Male flower-bud, (ar) bract, (az) bracteoles, (b) male flower, (c) male flower laid open, (d) inner perianth segment, (e) outer perianth segment, (f) anther. 


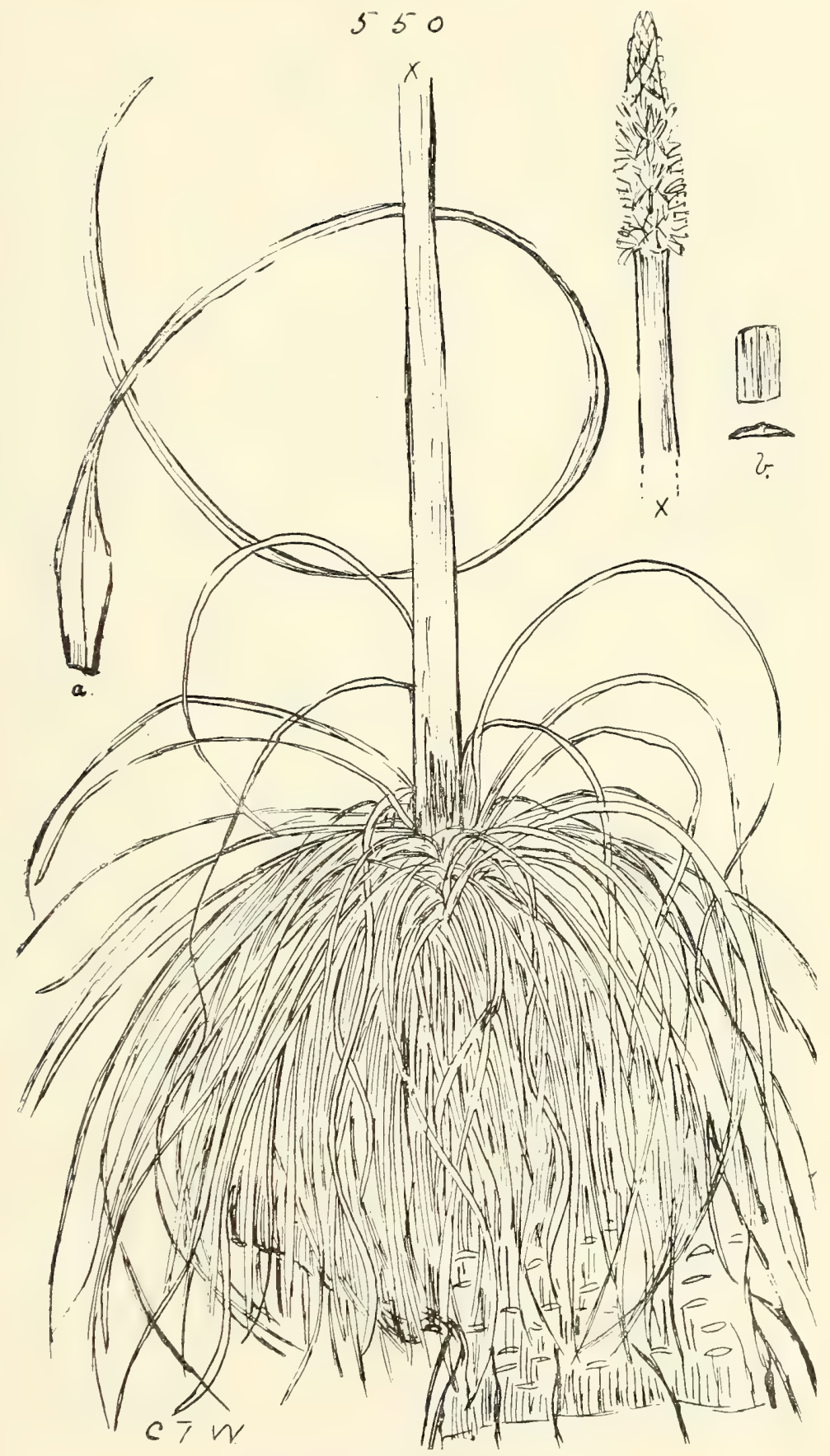

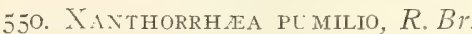

(a) Single leaf, nat. size, (b) portion of leaf and section, slightly enlarged. 


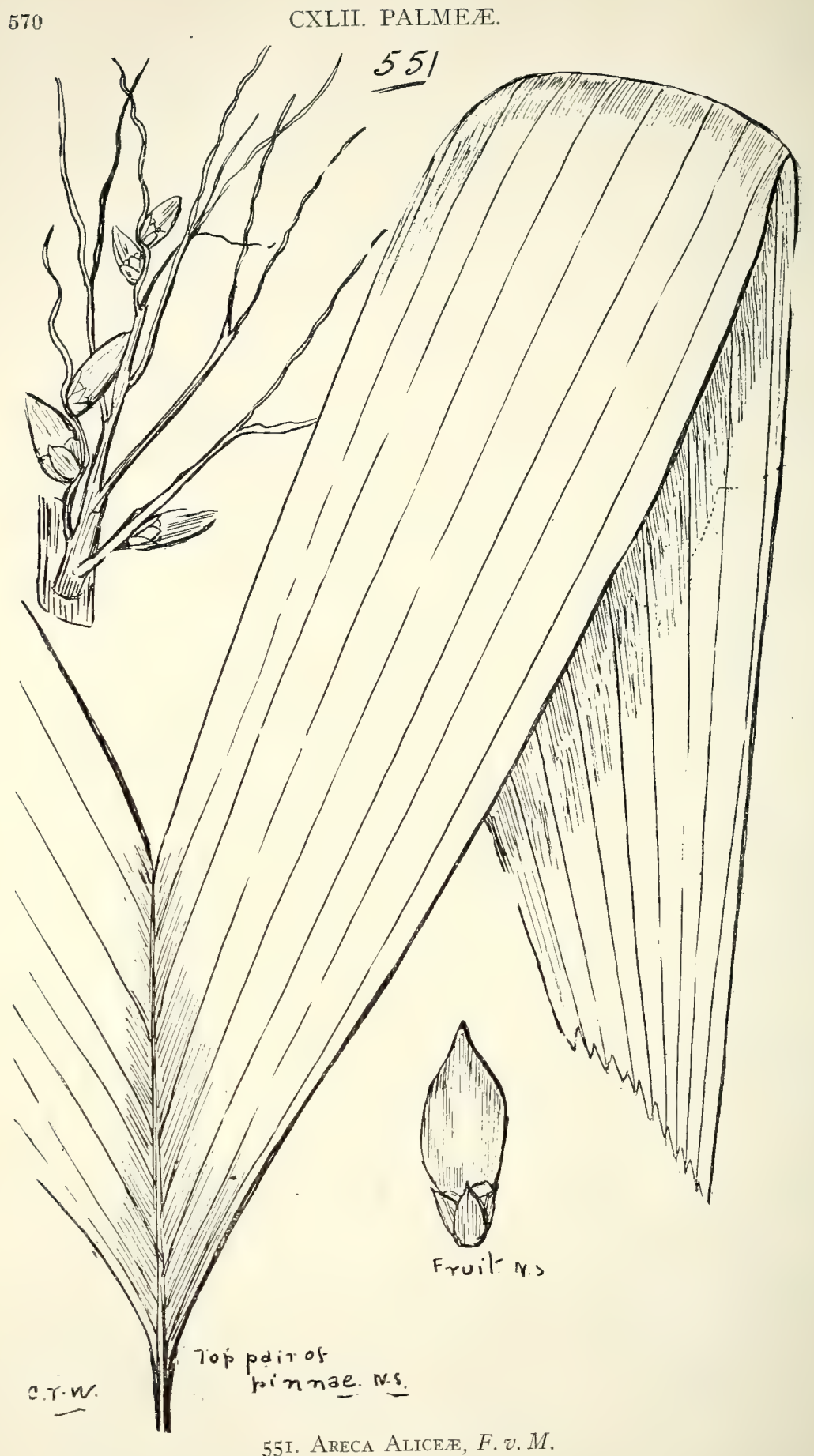




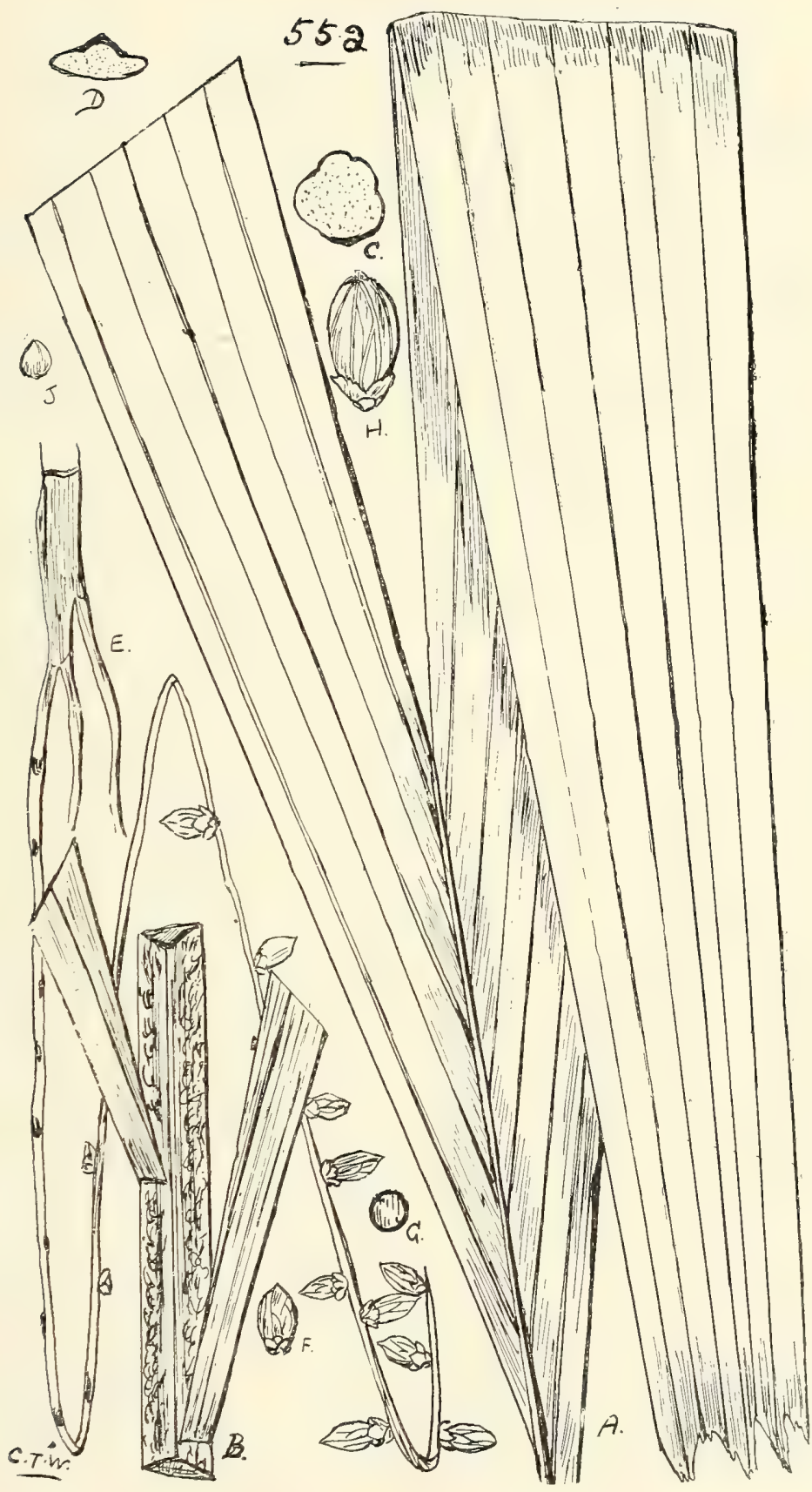

552. Hydriastele Douglasiana, Bail. 


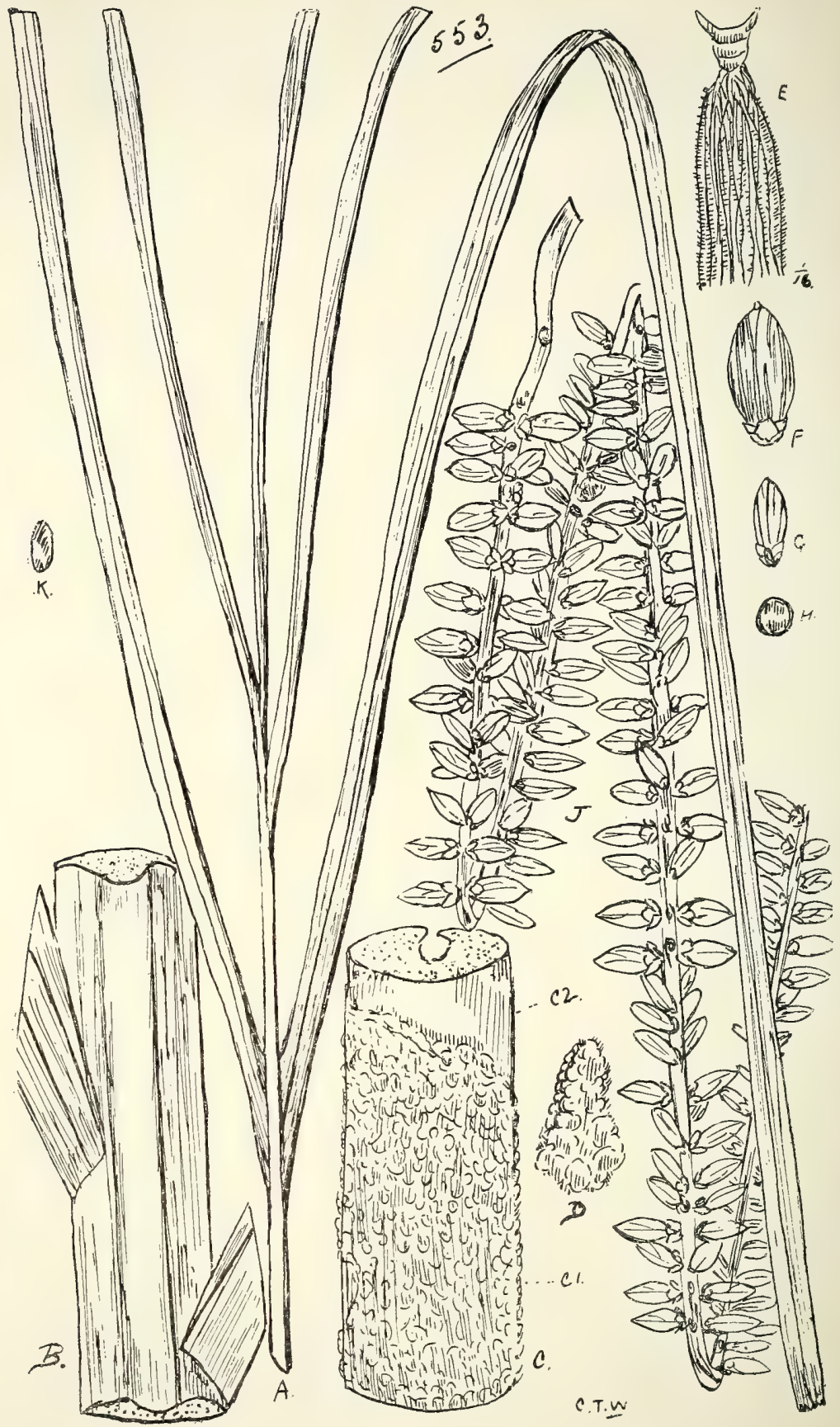

553. Gulubia costata, Becc.

(A) Apical leaf-segments, (B) thachis and bases of two leaf-segments, (C) portion f rof petioles and sheaths, (E) inforescence, (F) and (G) portion, (D) wool from bases of petioles and. (A), (B), (C), (D), (G), (H), fruit, (H) sect. fruit, (J) nat. size; (E) much reduced (I/I6 nat. size); (F) enl. 
Gulubia, Becc. in Ann. Jard. Buitenz. ii. I3I.

costata, Becc., 1.c. I34 = Hydriastele costata, Bail. (Fig.

\section{3.)}

Archontophonix, Wendl. et Drude.

Alexandræ, F.v.M._- "Koobin-karra" of Dunk Island,

"Borum-bru" of Cairns, and "Ko-pangara" of Tuliy

River natives.

Beatricæ, F.v.M.-Step Palm.

Cunninghamii, $W$ endl.-Sometimes seen with beautiful variegated foliage. "Piccabeen" or "Pikki" of Moreton' Bay and "Wal-garri" of Cairns natives. Often sold by nurserymen as Seaforthia Cunninghamii.

Ptychosperma, Labill.

elegans, Blume $=$ Seaforthia elegans, R. Br.

Jardinei, Bail. = Archontophonix Jardinei, Bail., Q1. Agric.

Journ. ii. I29.

Drymophlous, Zippel.

Normanbyi, Benth. et Hook. f.-Black Palm. "Dowar" of

Cape Bedford natives. The dark-coloured stems split up and used for making walking-sticks.

Calyptrocalyx, Blume.

australasicus, Scheff.=Ptychosperma laccospadix, Benth.

Bacularia, F.v.M.

monostachya, F.v.M. = Kentia monostachya, F.v. M.-

Walking-stick Palm. "Midgen" of Brisbane River natives. Sometimes plants are met with beautiful variegated foliage.

minor, F.v.M.- "Jak-ar-ungle" of Cairns natives.

Palmeriana, Bail. (Fig. 554.)

Caryota, Linn.

Rumphiana, Mart., var. Alberti, Bail.

Nipa, Wurmb.

fruticans, Wurmb., var. Neameana, Bail. - "Ki-bano" of Cardwell natives (R.B.H.), "Tacannapoon" of Pascoe River natives (Meston).

TRIBE II.-PhENICE.E.

*Phœenix, Limn.

dactylifera, Linn.-Date Palm. North Africa; Arabia.

Corypha, Linn.

TRIBE III.-CORYPHE⿸.

elata, Roxb.-According to Professor A. J. Ewart, Proc. Roy. Soc. Vic. n.s. xxiv (I9I2) 256, specimens of this Palm, determined by Dr. O. Beccari, have been collected on the Lower Gilbert River. 
Licuala, Rumph.

Muelleri, Wendl. et Drude.--" Moor-goo-doo" of Cairns and "Moi-yur" of Bloomfield River natives. In its native habitat, the most beattiful of our Fan-palms.

Livistona, R. $B r$.

Muelleri, Bail._- "Bel-em-buna” of Cairns natives.

Benthami, Bail._- Dre-amberi” of Batavia River natives.

Mariæ, F.v.M.

australis, Mart.-Cabbage-tree Palm. "Konda" of Rockhampton and "Binkar" of Brisbane natives.

TRIBE IV.--LePIDOCARYET.

Calamus, Linn.

Muelleri, Wendl. and Drude.-Small Lawyer Cane. "Taigam" of Brisbane River natives. Lengths of 66 feet of the stems are often used in measuring land, instead of the usual chain.

caryotoides (A. Cunn.), Beccari.-Ground Palm.

australis (Mart.), Beccari $=$ C. Jamboolum, Bail.; C. obstruens, F. v. M.- "Jamboolum" of Barron River natives. Large Lawyer Cane.

Moti, Bail. - "Moti" of Barron River, "Mollukan" of Atherton, and "Bai-kal " of Tully River natives. Large Lawyer Cane.

radicalis, Wendl. and Drude.

Tribe V.-BORASSEæ.

Borassus, Limn

flabellifer, Linn.-Palmyra Palm.

For the Queensland habitat of this Palm I am entirely dependent on Mr. F. L. Jardine, who has a male tree growing near his house at Somerset, which he informs me he brought when it was quite young from a locality in Cape York Peninsula about 40 miles from the coast; he further stated that there was quite a clump of the Palms from where he obtained his plant.

\section{TRIBE VI.-COCOINEE.}

*Cocos, Linn.

nucifera, Limn.-Cocoanut Palm. "Cooreemboola" of Mackay natives. 


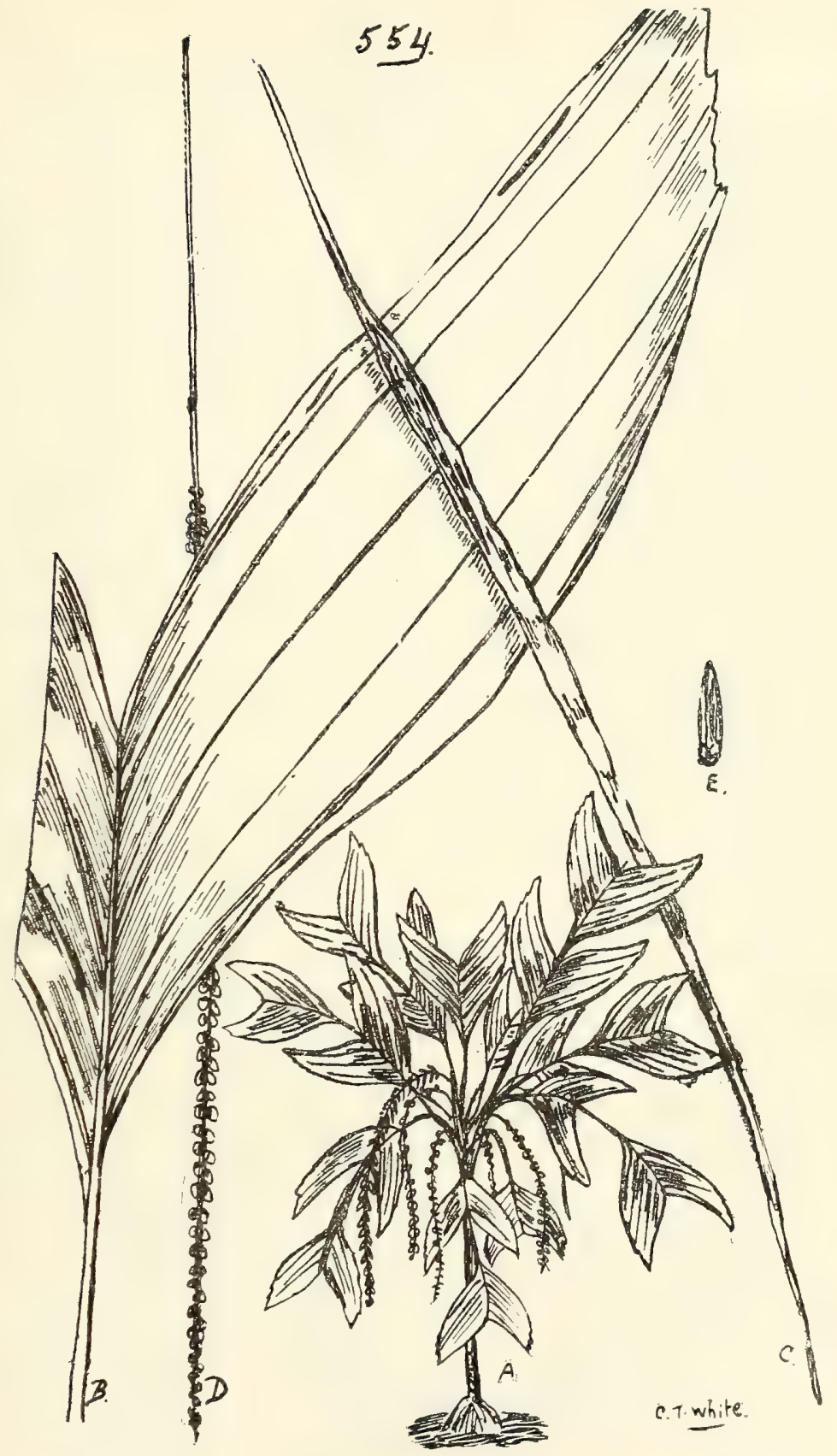

554. Bacularia Palmeriana, Bail.

(A) Plant, (B) pair of leaf-segments, (C) spathe, (D) spike, (E) young fruit. (A) much reduced; (B)-(E) nat, size. 


\section{Order CXLIII.-PANDANACE}

Tribe I.-Pandanee.

Pandanus, Limn.--Screw Pine.

\section{Section I.-Keura.}

odoratissimus, Linn. f.-_- "Pim-nar" of Dunk Island natives. aquaticus, F.v.M.- "Can-kee" of Dunk Island, "Ju-kee" of Cairns, and "A-koo" of Mitchell River natives.

pedunculatus, R.Br.-Often called Breadfruit in Queensland. "Wynnum" of Moreton Bay and "Camboor" of Cairns natives.

Solms-Laubachii, F.v.M.

\section{Section II.-Acrostigma.}

monticola, F.r.M._." Yagul” of Cairns natives. (Fig. 555.) Freycinetia, Gaudich.

insignis, Blume.-Recorded for Queensland by F. v. Mueller in 2nd Syst. Census Austr. Plants (I889).

Gaudichaudii, R. $B r$.

excelsa, F. v.M.

\section{Order CXLIV.-TYPHACEA.}

Typha, Limn.

angustifolia, Linn.-Bulrush. "Yimbun" of Brisbane River natives. The spikes off this plant were gathered by the early colonists of South Australia and the down used for stuffing pillows, under the name of "Murray Down."

Sparganium, Linn.

angustifolium, R. Br., not of Michx.-Australian Bur Reed.

\section{Order CXLV.-AROIDEZ.}

Tribe I.-ARINEE.

Typhonium, Schott.

alismifolium, F.v. M.

Brownii, Schott._- Gin-bur" of Morehead River, "Rur-oonba" of Musgrave, "Kam-mi" of Cooktown, and "Merrin” of Rockhampton natives.

angustilobum, F.v.M.- "Wu-ri” of Pennefather River and

"Wanjallo" of Mitchell River natives. (Fig. 556.) Millari, Bail. 

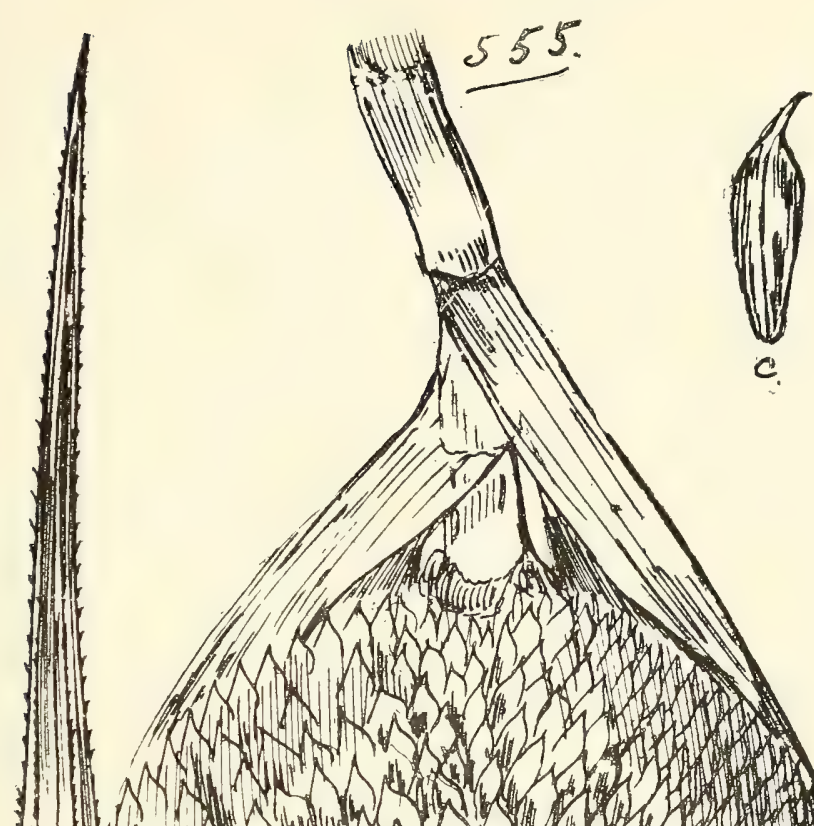

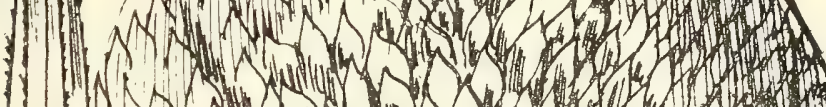

1 N N

14 Now

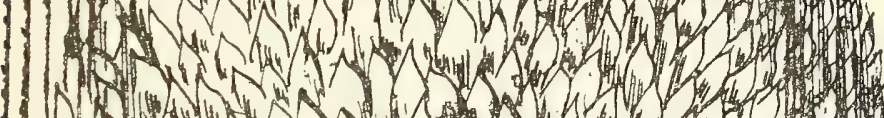

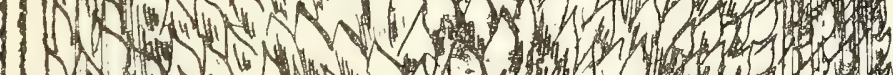

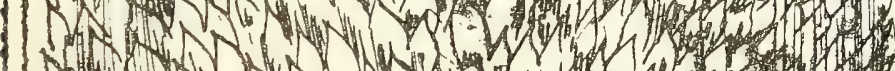

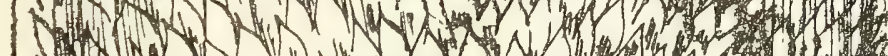

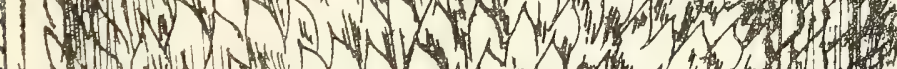

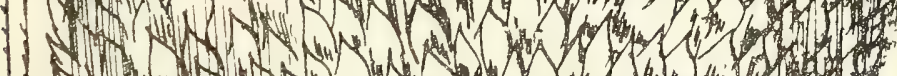

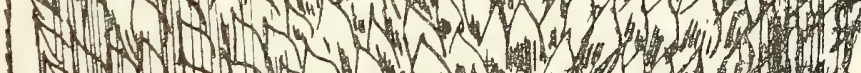

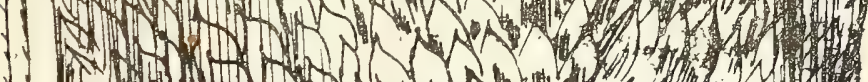

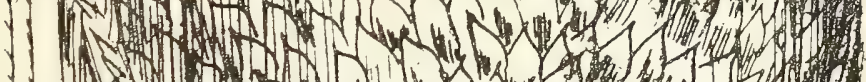
3 - 1 . - 1 H. -1 - 1 .

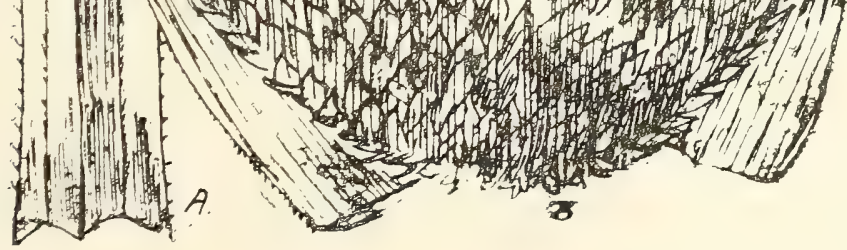

555. Pandanus monticola, $F, v, M$.

(A) Portion of leaf, (B) fruit, (C) drupe; all nat. size. 
578

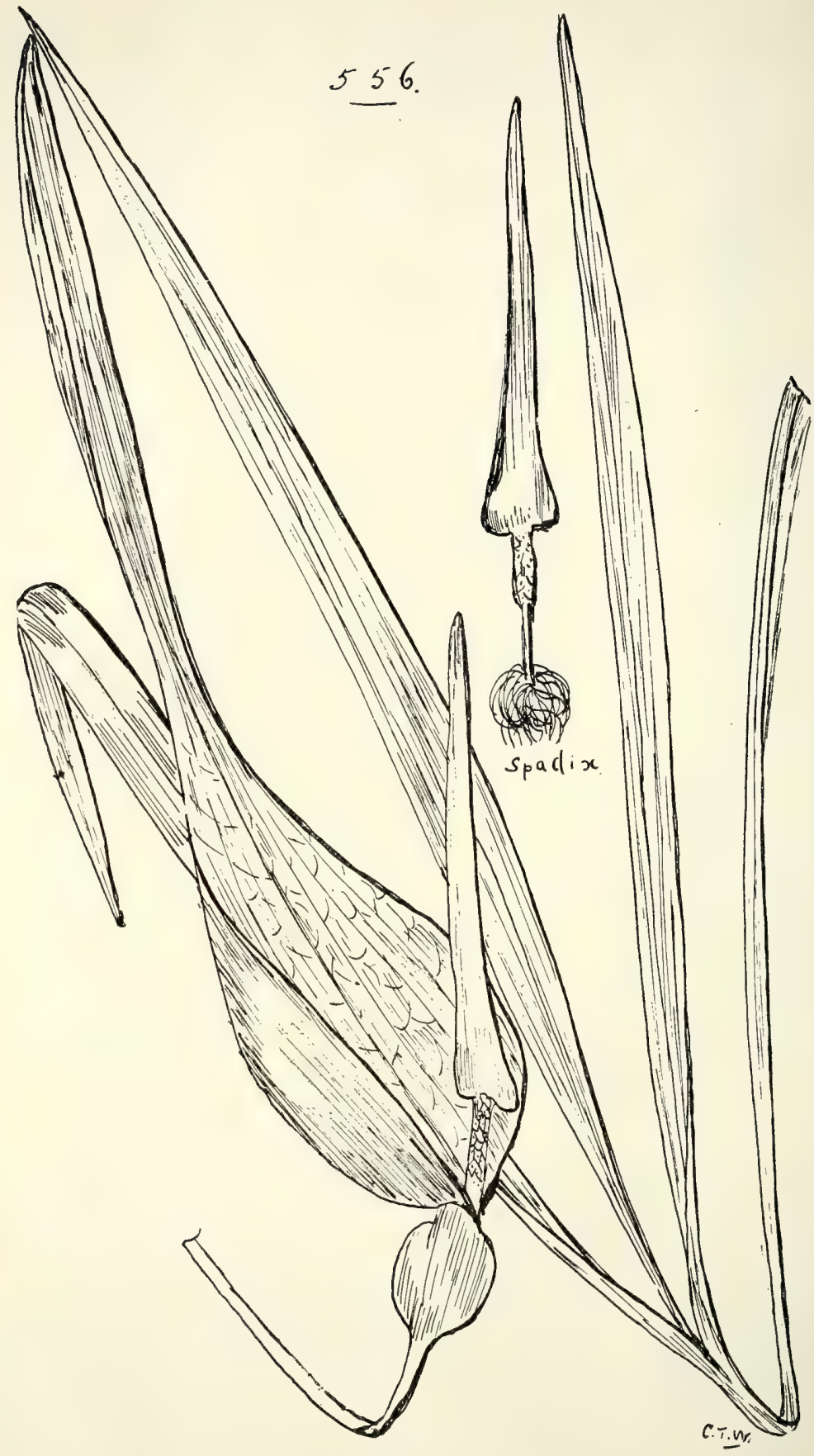

556. Typhonium angustilobum, F.v.M. 
Tribe II.-Pythoniez.

Amorphophallus, $B l$.

galbra, Bail._. Bandilin" of Cooktown natives. "Galbra," native name of plant at Cairns, "Jambolla" of the inflorescence.

angustilobum, Bail._-"Pun-ti-li” of Annan River natives.

Colocasia, Schott.

Tribe III.-Colocasieæ.

antiquorum, Schott.-Taro or Tara. "Pen" of Middle Morehead River and "Panar" of Cooktown natives. The acrid juice of the petioles of several forms of this species is a common domestic remedy, on account of its styptic and astringent properties. The petiole is slightly roasted and the juice expressed. I have seen purulent discharges from the ears in children stopped by a single application (Dymock). In Queensland, old running sores have been cured by the simple application of the fresh leaf.

Alocasia, Schott.

macrorrhiza, Schott. - Cunjevoi. "Dhoo-ee" of Burnett River, "Mur-gan" of Mount Cook, and "Koom-bi" of Tully and Atherton natives.

var. brisbanensis, Bail.

\section{Tribe IV.-Callee.}

Rhaphidophora, Hassk.

australasica, Bail.

Lovellæ, Bail._-"Naja" of Tully River natives.

$$
\text { Tribe V.-OrontieÆ. }
$$

Pothos, Linn.

longipes, Schott, Aroid. 23, t. 47.- "Koi-yo" of Tully River and "Ku-yu" of Atherton natives.

Brownii, Domin, Fedde. Repert. x. (I9II) 59.

The above two species are included under P. Loureiri in Bentham's Flora Australiensis and many other works.

Gymnostachys, R. Br.

anceps, R. Br.- "Boorgay" of Russell River natives. The leaves of this plant produce a very strong fibre. (Fig. 557.)

\section{Order CXLVI.-LEMNACE $\nexists$.}

Wolffia, Horkel. arrhiza, Wimm., var.—Rootless Duckweed. (Fig. 558.) 
Lemna, Linn.-Duckweed.

trisulca, Linn. (Fig. 559.)

minor, Linn. (Fig. 560.)

oligorrhiza, Kurz.-The surface of still water is often covered with this plant. (Fig. 56r.)

Series VI.-APOCARP王.

\section{Order CXLVII.-TRIURIDEÆ.}

Sciaphila, Blume.

australasica, Hemsl.

\section{Order CXLVIII.-ALISMACEÆ.}

TRIBE I.-ALISMEA.

Alisma, Linn.-Water Plantain.

acanthocarpum, F.v.M.

oligococcum, F.v. M.

reniforme, Don.

Damasonium, Juss.

australe, Salisb.

Tribe II.-Butomex.

Butomopsis, Kunth

lanceolata, Kunth. = Butomus lanceolatus. Roxb.

\section{Order CXLIX. - NAIADACE巴.}

Tribe I.-JunCagineE.

Triglochin, Linn.-Arrow-grass.

$$
\text { Section I.-Eutriglochin. }
$$

striata, Ruiz et Pav.

centrocarpa, Hook.

var. calcitrapa, Benth.

Section II.-Cycnogeton.

procera, R.Br.- "Wanna" of Cape Bedford, "Beega" of

Cardwell, and "Anamum" of Musgrave River natives (R.B.H.).

var. dubia, Benth.

Maundii, F.v.M. = Maundia triglochinoides, F.v. M., Fragm. i. 23. (Fig. 562.) 


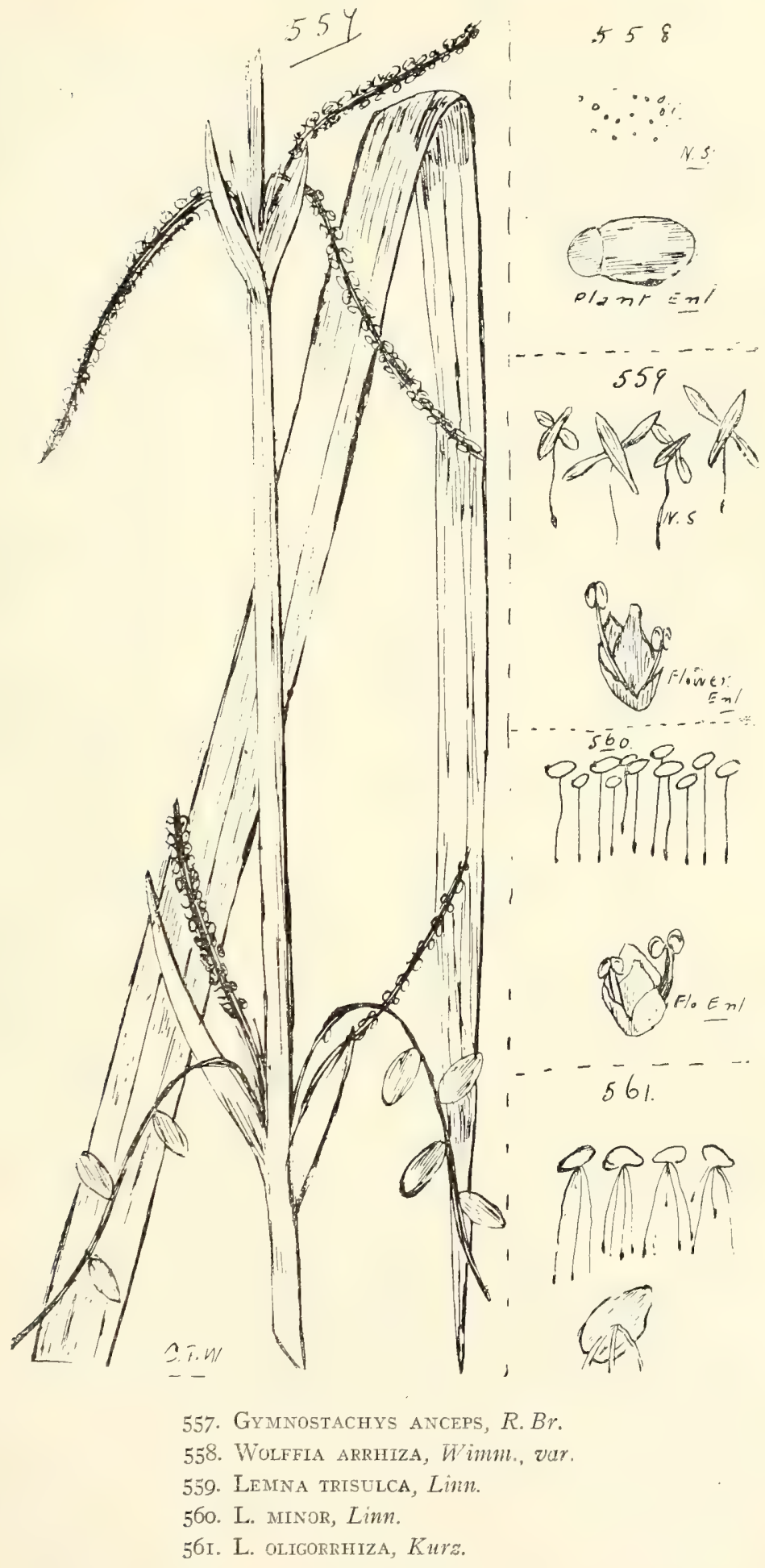




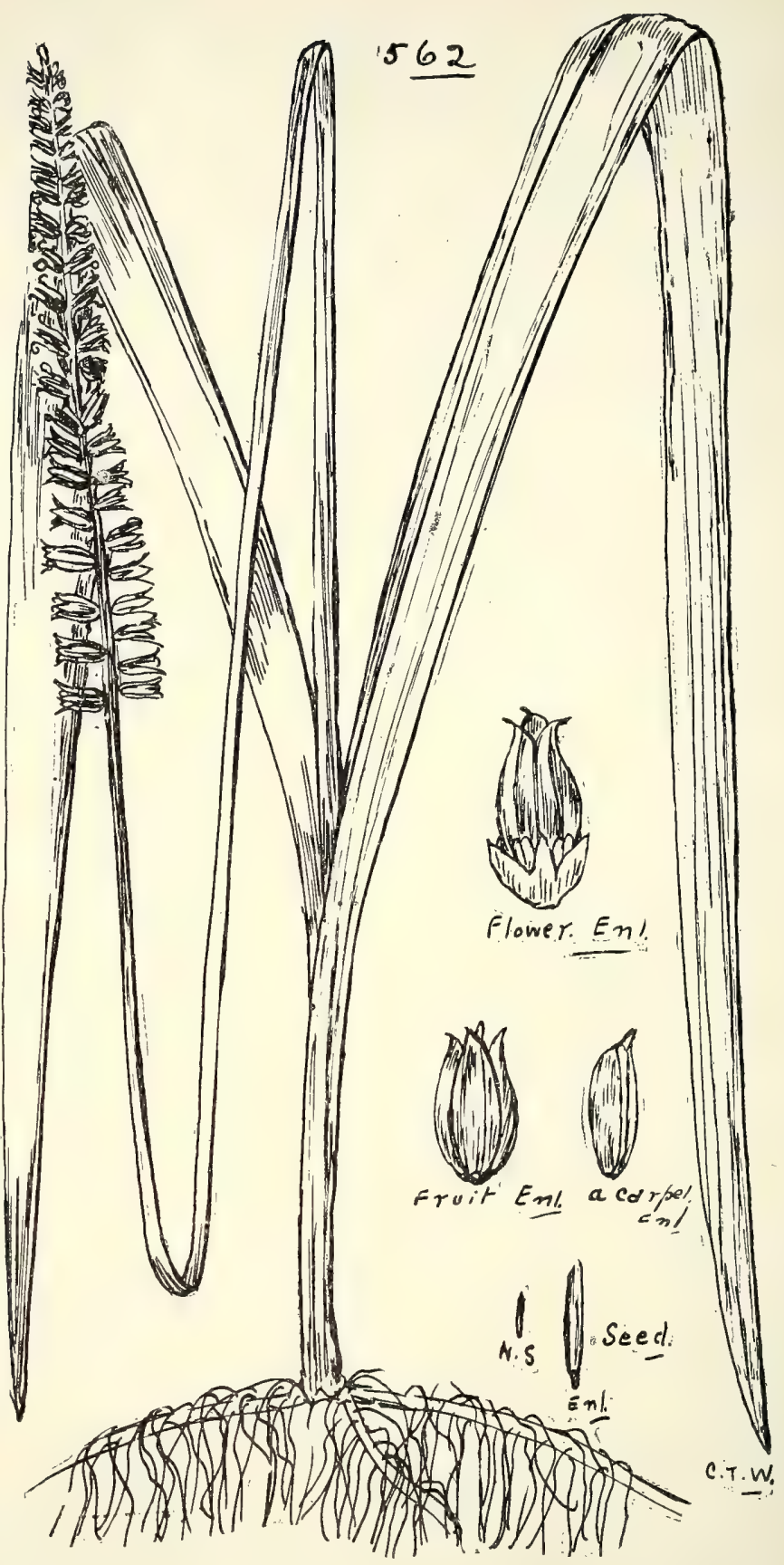

562. Triglochin Maundit, F.v. $M$. 
Tribe II.-AponogetoneT.

Aponogeton, Thunb.

monostachyus, Linn._- "Kàpäbina" of Bloomfield River natives.

elongatus, F.v.M.

\section{TRIBE III.-POTAMEA.}

Potamogeton, Linn.--Pond-weed.

\section{Section $I$.}

natans, Linn.

tricarinatus, F.v.M. (Fig. 563.)

Tepperi, A. Bennett.

javanicus, Hassk. $=$ P. tenuicanlis, F. v. M.

Section II.

perfoliatus, Limn.

var. minor, Bail., n. var.-Stems flat, narrow. Leaves almost orbicular, the largest seen about 8 lines diam., very dense near the ends of the branches. Peduncles about I inch long. Spikes dense, 3 to 5 lines long.

Hab.: Tambourine Mountain (J.H. Simmonds).

crispus, Linn.

obtusifolius, Mert. et Koch.

Section III.

pectinatus, Linn.--Fennel Pondweed.

Ruppia, Linn.

maritima, Linn.

Tribe IV.-Zostere⿸.

Zostera, Limn.-Grass-wrack.

nana, Roth.-Dugong Grass.

Tribe V.-NAIAdeE.

Naias, Linn.

major, Allioni.

var. intermedia, A. Br.

tenuifolia, R. Br.

Browniana, A. B. Rendle, Trans. Linn. Soc. 2nd ser. Bot. iv. (I899) 420.

graminea, Delile.

Leichhardtii, Magnus.

Hab.: Australia (Leichhardt), (Magnus); Rendie (? Qucensland). 


\section{Tribe VI.-CymodoceA.}

Cymodocea, Konig. ciliata, Ehrenb. serrulata, Aschers.

isoetifolia, Aschers.

\section{Order CL.-ERIOCAULEÆ.}

Eriocaulon, Linn.-Pipewort.

\section{Series $I$.}

Flowers mostly 3-merous.

setaceum, Linn.-Sir J. D. Hooker, in F1. Brit. Ind., considers the Australian plant to be E. bifistulosum, Van Heurck. (Fig. 564.)

australe, $R . B r$.- Hat-pin plant.

Smithii, R. Br.

nanum, R. Br. (Fig. 565.)

cinereum, R. $B r$.

pusillum, R. Br.

pallidum, R. Br.

nigricans, R. Br. (Fig. 566.)

Series II.

Flowers 2-merous.

spectabile, F.v.M

scariosum, R. $B r$.

fistulosum, R. $B$ r.

depressum, R. Br.

deustum, R. Br.

\section{Order CLI. - CENTROLEPIDEÆE.}

Centrolepis, Labill.-Bristlewort:

polygyna, Hieron.

pusilla, Roem. et Schult.

aristata, Roem. et Schult.

Banksii, Roem. et Schult.

fascicularis, Labill.

exserta, Rom. et Schult.

var. rubra, Bail. (Fig. 567.)

\section{Order CLII.-RESTIACE $Æ$.}

Lepyrodia, R. $B r$.

scariosa, R. Br.

interrupta, F.v. $M$. 


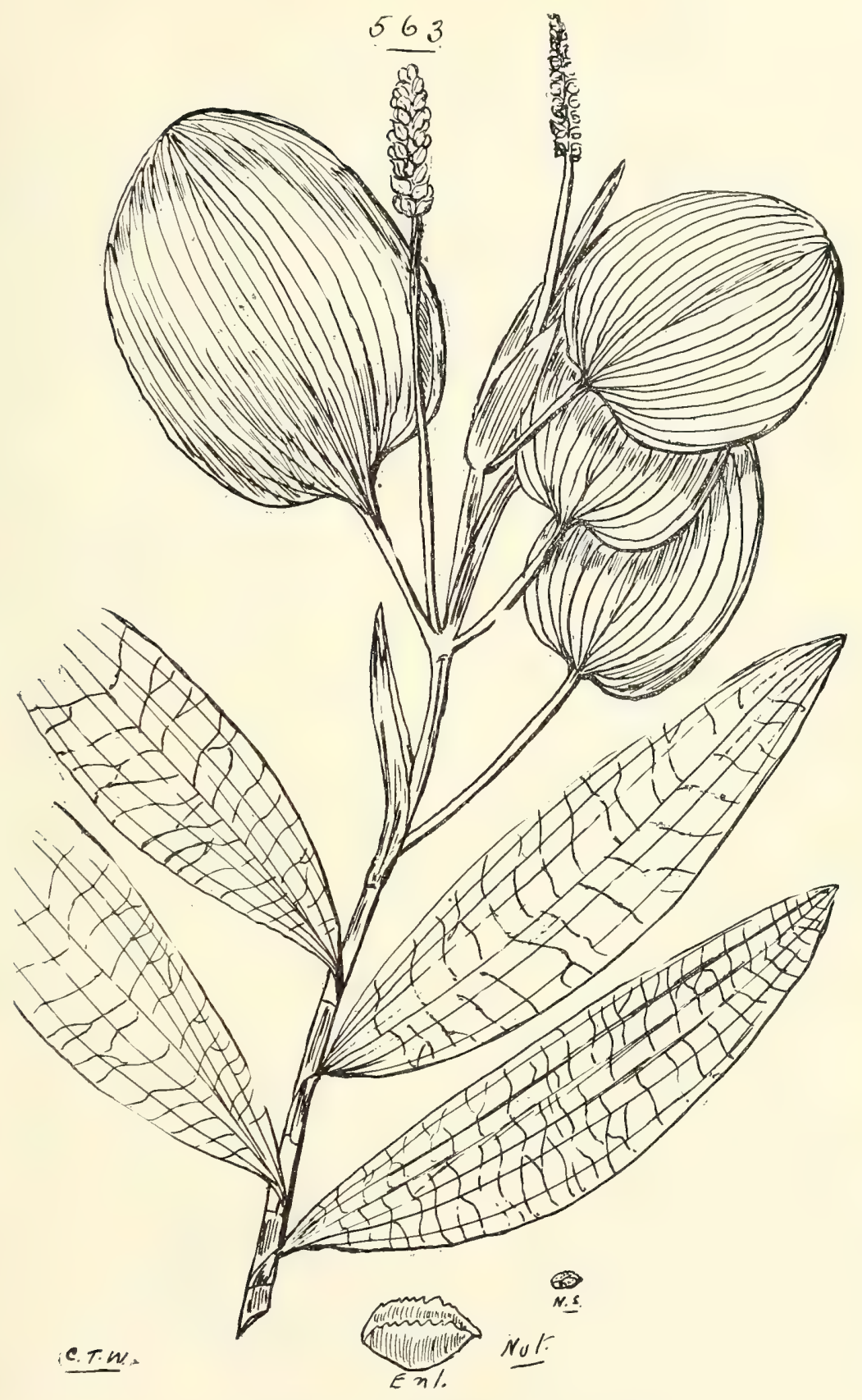

563. Potamogeton tricarinatus, $I . v . M$. 


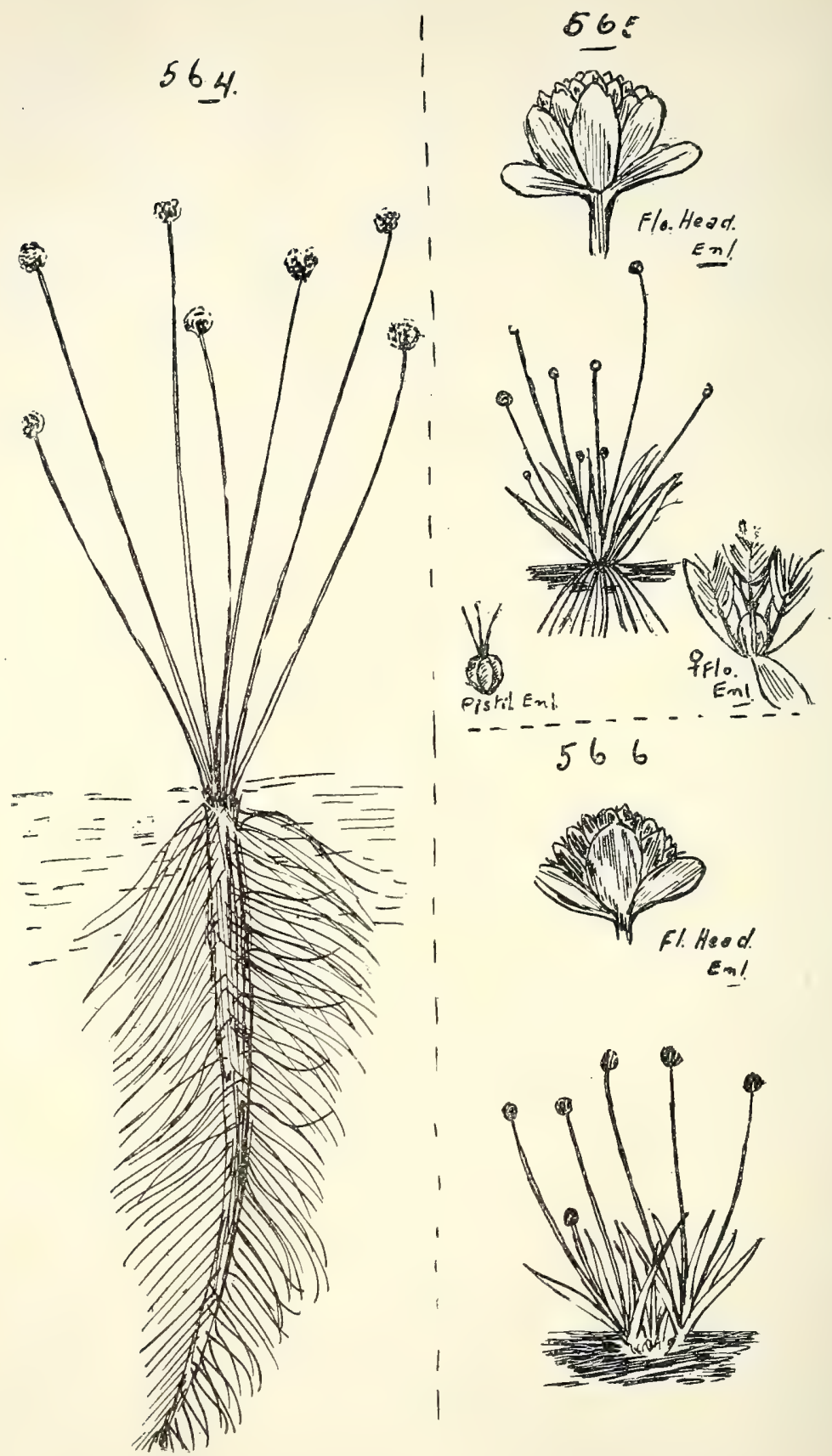

564. Ertocalion setaceum, Linn.

565. E. NANUM, $R$. $B r$.

5\%6. E. NIGRICANS, R. $\mathrm{Br}$. 


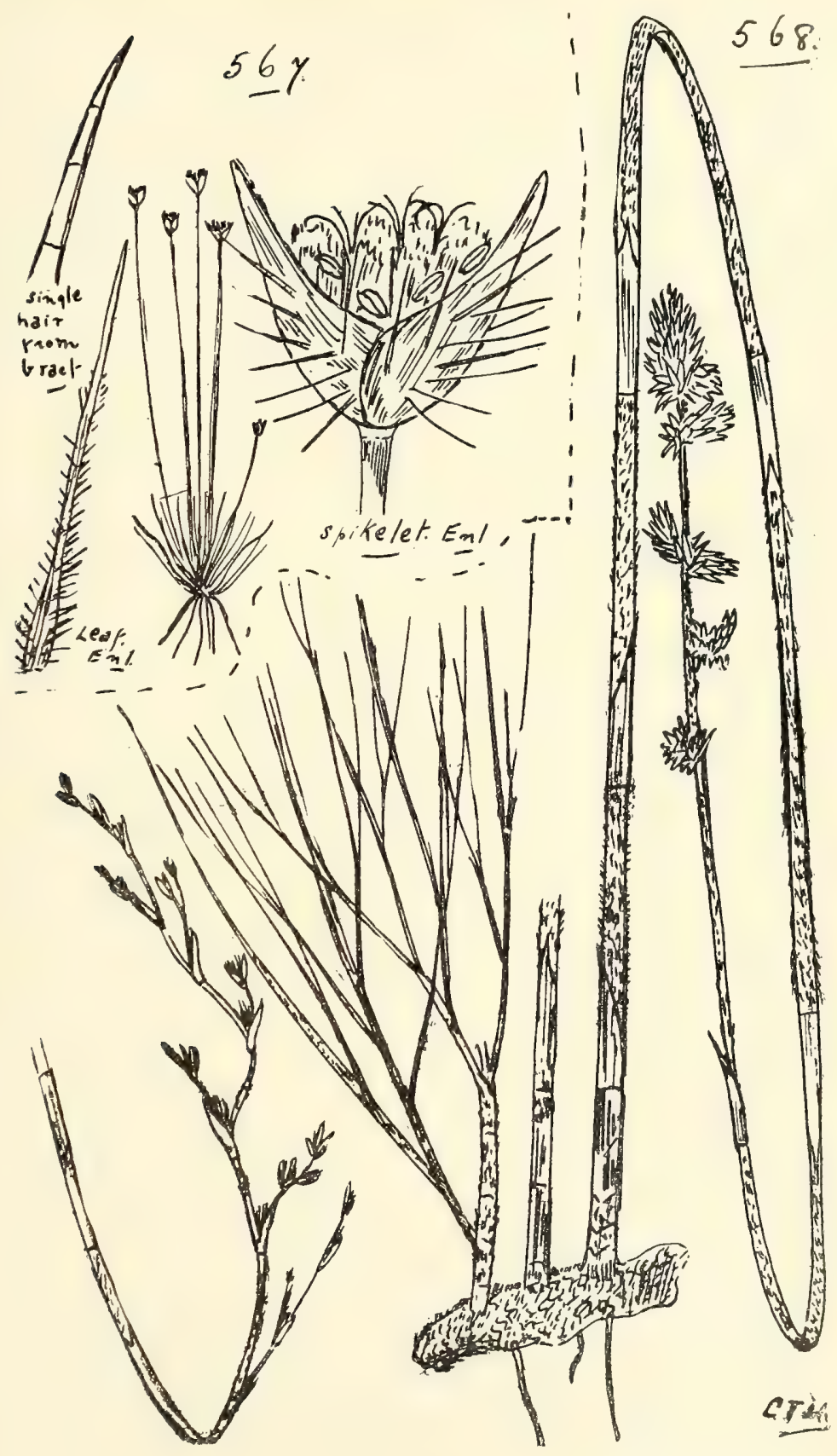

567. Centrolepts exserta, Røm. et Schult, var, Rubra, Bail. 568. Leptocarpus Schultzit, Benth. 
Restio, Linn.

$$
\text { Section I.-Stachygynia. }
$$

tropicus, R. Br.

dimorphus, R. Br.

gracilis, R. Br.

complanatus, R. Br.

tetraphyllus, Labill.-Feather-plant.

Leptocarpus, $R \cdot B r$.

Section I.-Diplanthesis.

tenax, R. Br.-Fraser's Island (Hon. Miss Lovell).

Section II.-Homoanthesis.

ramosus, $R \cdot B r$.

elatior, R. Br.

spathaceus, R. Br.

Schu1tzii, Benth. (Fig. 568.)

Hypolæna, R. $B r$.

Section I.-Calorophus.

lateriflora, Benth.

Section II.-Euhypolona.

fastigiata, R. Br.

\section{Order CLIII.-CYPERACEA.}

Cyperus, Linn.

Tribe I.-Scirpe玉.

Section I.-Pycreus.

pumilus, Linn. (Fig. 569.)

eragrostis, $V a h l$.

flavescens, Linn.

globosus, All.

unioloides, R. Br.

polystachyus, Rottb.

var. laxiflora, Benth.

flavicomus, Mich.

Section II.-Juncellus.

pygmæus, Rottb.

cephalotes, Vahl. (Fig. 570.)

platystylis, R. Br.

alopecuroides, Rottb.

Section III.-Eucyperus.

pulchellus, R. $B r$.

gracilis, R. Br.

var. ? rigidella, Benth. 
Cyperus-contd.

enervis, R. $B r$.

var. laxus, Benth.

debilis, R. Br.

lævis, $R . B r$.

castaneus, Willd.

cuspidatus, H.B.ct K. (Fig. 57I.)

squarrosus, Limn.

var. congestus, Benth.

var. cylindraceus, Benth.

difformis, Limn.

tetraphyllus, $R$. $B r$.

trinervis, R. Br.

Haspan, Limn.

flavidus, Retz.

concinnus, R. Br.

filipes, Benth.

pedunculosus, F.v.M.

vaginatus, $R . B r$.

textilis, Thun.

holoschænus, R. Br.

dactylotes, Benth.

Gilesii, Benth.

fulvtis, $R$. $B r$.

carinatus, R. $B r$.

alterniflorus, R. Br.

pilosus, $V a h l$.

ornatus, $R \cdot B r$.

Iria, Linn.

eleusinoides, $K u n t h$.

distans, Limn. $f$.

corymbosus, Rottb.

Section IV.-Papyrus.

rotundus, Lim.-Nut Grass; a noxious weed. I am glarl to be enabled to say something in favour of this pest of cultivation: The native companion bird is fond of the nuts; and both the Greeks and Romans use them in their medicines.

var. carinalis, Benth.

var. pallidus, Benth.

Tambo $($ Wuth $)=$ C. bifax, C. B. Clarke.

tenuiflorus, Rottb.

lucidulus, Klein.

congestus, Vahl.

subulatus, $R, B r$. 


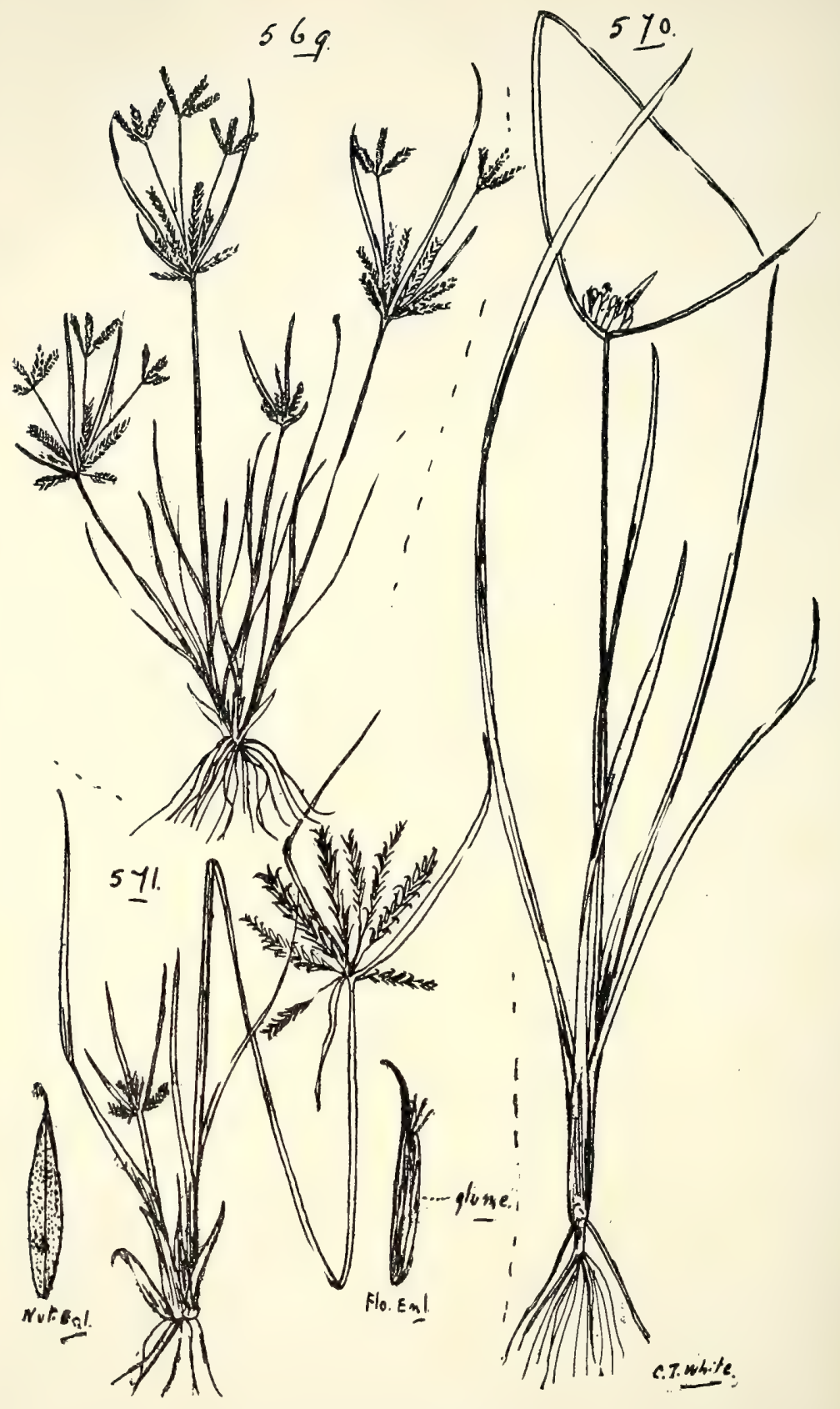

569. Cyperus pumilus, Linn.

570. C. cephalotes, Vahl.

57I. C. cuspidatus, H. B. et $K$. 
Cyperus-contd.

sporobolus, R. Br.

angustatus, $R . B r$.

novæ-hollandiæ, Backel.

Gunnii, Hook.

lucidus, R. Br.

pennatus, Lam.

exaltatus, Retz.

digitatus, Roxb. $=$ C. auricomus, Benth.

Section V.-Diclidium.

ferax, Rich.

esculentus, Limn._ "Mangaru" of Boulia, "Makora" of Cloncurry, "Ari-ira” of Musgrave, "Panje-a" of Batavia River, "Bulkuru" of Tully River, and "Kaya" of Rockhampton natives. A somewhat common weed on downs country, resembling the Nut-grass, but not difficult to eradicate.

Bowmanni, F.v. M.

trichostachys, Benth.

leiocaulon, Benth.

scaber, Benth.

Section VI.-Mariscus.

decompositus, F.v.M.

Armstrongii, Benth.

umbellatus, Benth.

var. laxiflora, Benth.

conicus, Backel. (Fig. 572.)

var. ramosus, Benth.

Kyllinga, Rottb.-Kyllingia of some authors.

intermedia, R. Br.

monocephala, Rottb.

var. latifolia, Benth.

cylindrica, Nees. (Fig. 573.)

triceps, Rottb.

Heleocharis (Eleocharis), R. Br.-Spike Rush.

Section I.-Limnocharis.

sphacelata, R.Br._- Ma-bil" of Cooktown and "Kaya" of

Rockhampton natives. Tubers used for food.

spiralis, R. Br.

variegata, Kunth.

fistulosa, Schult. (Fig. 574.) 


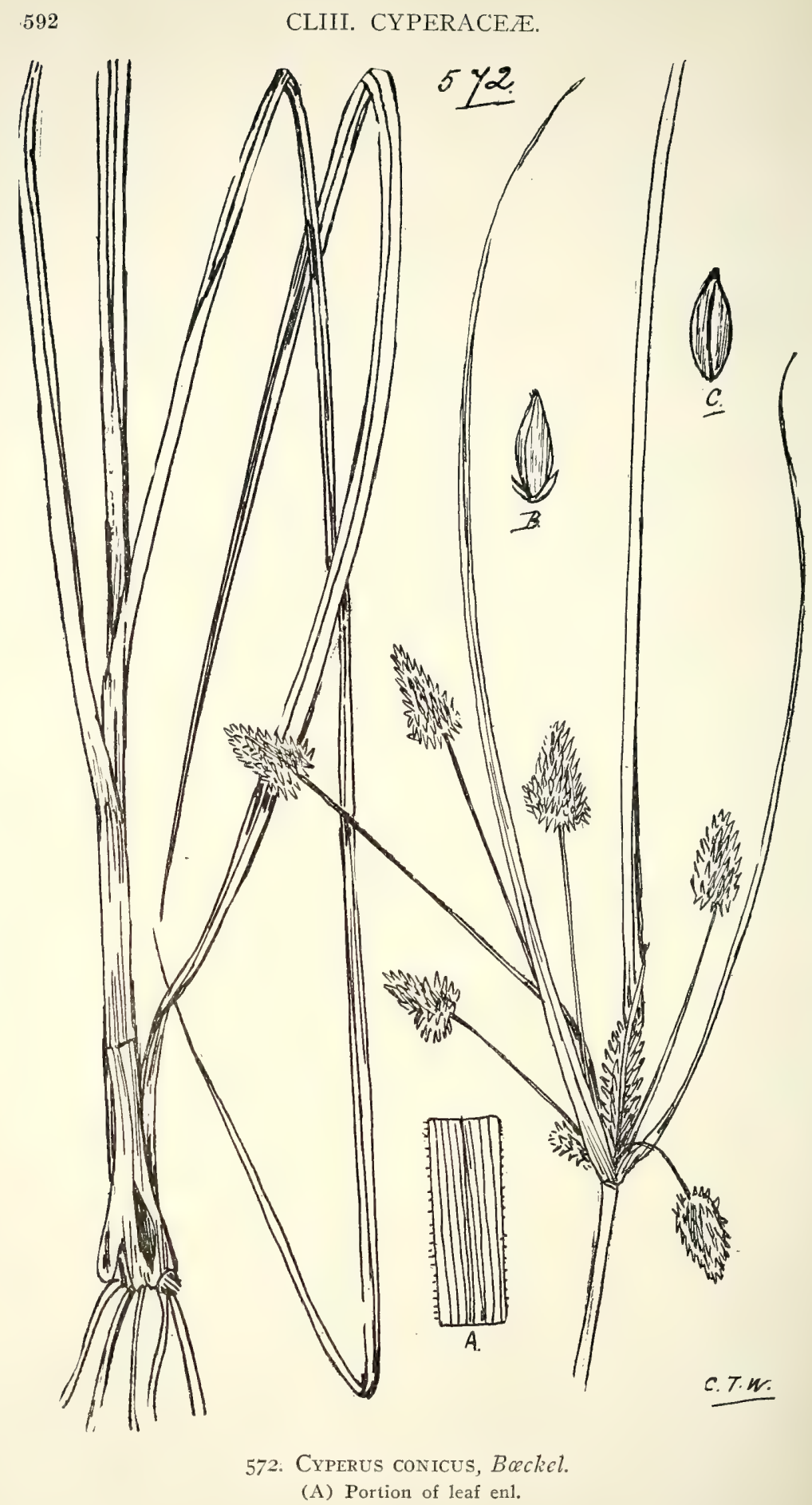


CLIII. CYPERACE $\mathrm{E}$.

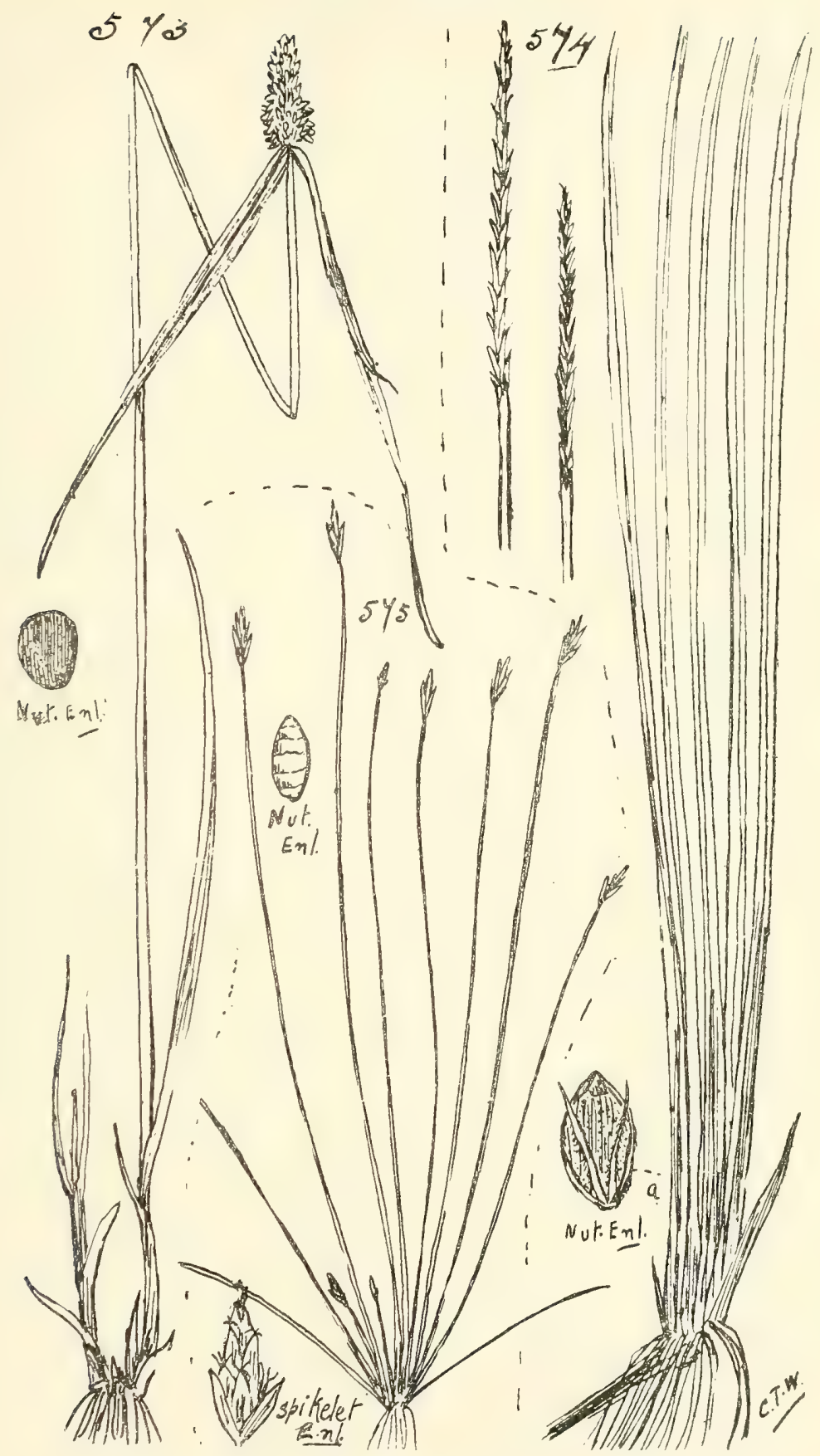

573. Kyllinga cylindrica, Nees.

574. Heleocharis fistulosa, Schult.

575. Fimbristylis acuminata, Vahl, var, setacea, Bentlo. 
Heleocharis-contd.

\section{Section II.-Scirpidium}

cylindrostachys, Backel.

tetraquetra, Nees.

acuta, R. Br.

var. pallens, Benth.

atricha, R. Br.

\section{Section III.-Heleogenus}

capitata, $R$. $B r$.

atropurpurea, Kunth.

var. setiformis, Benth.

Fimbristylis, Vahl.

Section I.-Heleocharoides.

acicularis, R. Br.

acuminata, Vahl., var. setacea, Benth. (Fig. 575.)

punctata, R. Br.

rhyticarya, F.v.M.

nutans, $V$ ahl.

pauciflora, R. Br.

Macgillivrayi, C. B. Clarke, Kew Bull. Add. series viii. (I908) 24.

polytrichoides, R. $B r$.

subbulbosa, Benth.

tetragona, R. Br.

pterygosperma, R. Br.

sphærocephala, Benth.

xyridis, R. Br.

var. rigidula, Benth.

Section II.-Abildgaardia.

squarrulosa, F.v. M.

monostachya, Hassk.

Dallachyi, F.v. M.

recta, Bail.

Section III.-Dichelostylis.

velata, $R . B r$.

æstivalis, $V a h l$.

var. macrostachya, Benth.

dichotoma, Vahl.

diphylla, $V a h l$.

var. gracilis, Benth.

ferruginea, $V$ ahl.

var. foliata, Benth.

denudata, R. $B r$.

cæspitosa, R. Br. 
Fimbristylis-contd.

Section IV.-Trichelostylis.

Series I.—Oligostachyæ.

leptoclada, Benth.

Series II.-Polystachyæ.

obtusangula, F. v.M.

miliacea, Vahl.

microcarya, F.v.M.

cyperoides, $R$. Br.

Series III.-Glomeratæ.

furva, R. $B r$.

cymosa, R. Br.

sericea, R. Br.

Neilsoni, F.v.M.

Series IV.-Capitatæ.

capitata, R. Br.

Section V.--Oncostylis.

barbata, Benth.

capillaris, A. Gray.

Scirpus, Linn.

Section I.-Isolepis.

fluitans, Linn.

setaceus, Linn.

squarrosus, Linn.

inundatus, Spreng.

prolifer, Rottb.

supinus, Linn.

articulatus, Linn.

nodosus, Rottb.

Section II.-Euscirpus.

debilis, Pursh.

mucronatus, Linn.

lacustris, Linn.

littoralis, Schrad.

maritimus, Linn.

var. fluviatilis, Benth.

polystachyus, $F$. v. $M$.

grossus, Linn.

Fuirena, Linn.

umbellata, Rottb.

glomerata, Lam. 


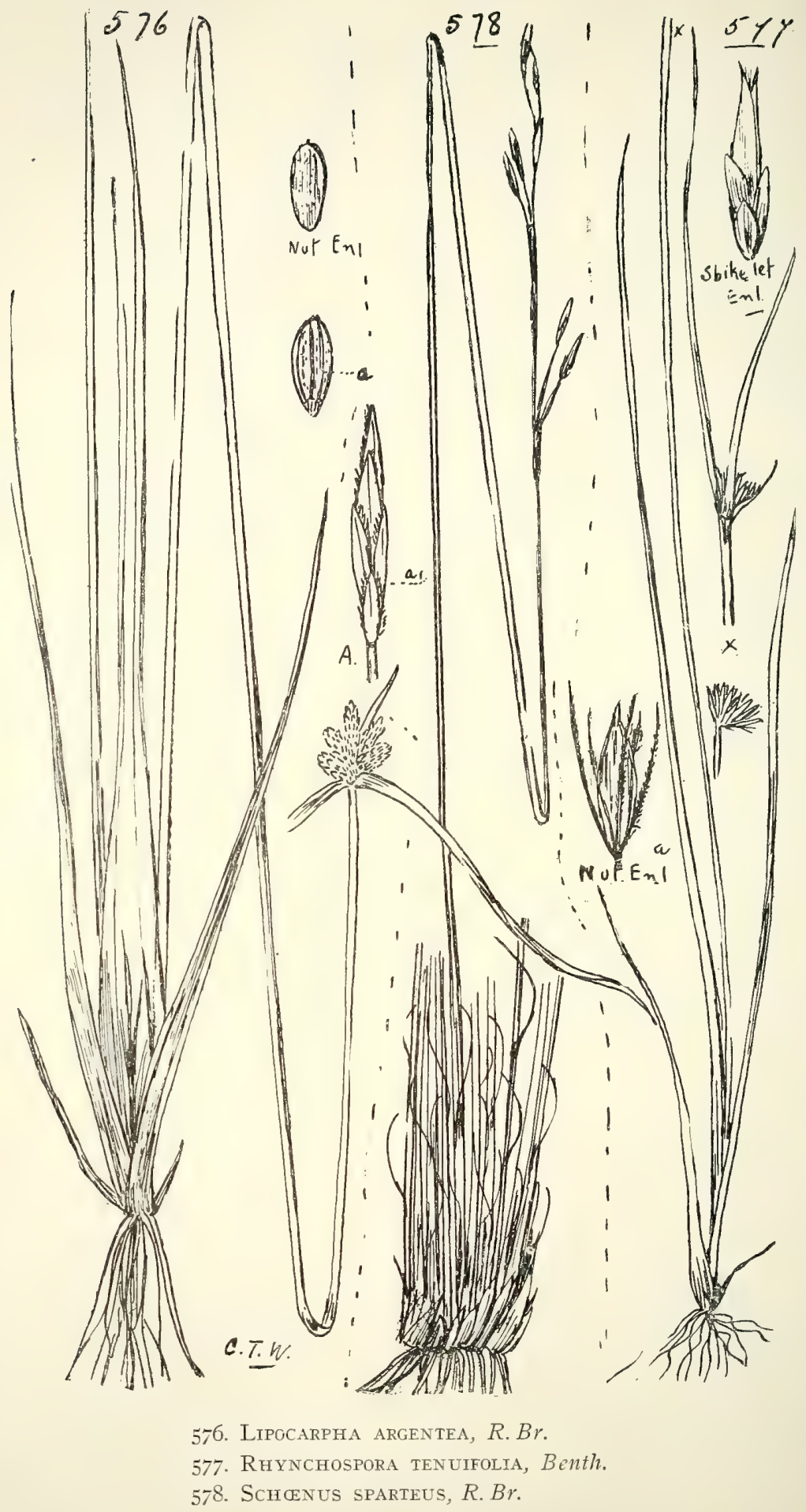


Lipocarpha, R. Br.

argentea, $R . B r .=$ Hypclyptum argenteum, Vahl. (Fig. 576.)

microcephala, Kunth $=H$. microcephalum, R. Br.

TRIBE II.-HYPOLYTREE.

Hypolytrum, Rich. = Hypalyptum, Vah1.; Hypelytrum, Poir.; Hyppalytrum, H. B. and K.

latifolium, Rich.

Mapania, Aubl.

hypolytroides, F.v.M.

Scirpodendron, Kurz.

costatum, Kurz.

Exocarya, Benth.

scleroides, Benth. = Cladium scleroides, F.v. M.; Scleria ustulata, Bail., 3rd Supp1. Syn. Q1. F1.

Lepironia, Rich.

mucronata, Rich. = Chondrachne articulata, R. Br.-"Chakata" of Cape Grafton natives. The stems are used in China for making mats, \&c.

Chorisandra, R. Br.

Section I.-Euchorisandra.

sphrerocephala, R. Br.

Section II.-Cymbaria.

cymbaria, R. Br.

Tribe III.-RhynCHOSPOREA.

Remirea, Aubl.

maritima, Aubl., var. pedunculata, Benth.-A binder of the sand on the sea-shore.

Arthrostylis, R. Br.

aphylla, R. Br.

Kennyi, Bail. (Fig. 576 bis.)

Rhynchospora, Vahl.

aurea, $V a h l$.

glauca, $V$ ahl.

Wallichiana, Kunth.

longisetis, R. Br,

tenuifolia, Benth. (Fig. 577.)

Leæ, C. B. Clarke, Kew Bull. Add. Series viii. (Igo8) 34.

Mesomelæna, Nees.

deusta, Benth. = Chotospora deusta, F.v. M.

sphærocephala, Benth. 
Schœenus, Limn.

Series I.-Isoschonea.

ericetorum, R.Br.

Series II.-Laterales.

nitens, Hook.

Series III.-Calostachyo.

calostachyus, Benth.

scabripes, Benth.

Series IV.-Paniculate.

brevifolius, R. $B r$.

melanostachys, R. Br.

sparteus, R. Br. (Fig. 578.)

vaginatus, $F . v . M$.

falcatus, $R$. $B r$.

punctatus, R. Br.

Series $V$.Laxa.

indutus, F.v. M.

Series VI.-Microcarpa.

Brownii, Hook.

Elynanthus, Nees.

capillaceus, Benth.

Tricostularia, Nees.

paludosa, Benth. = Chetospora paludosa, R. Br.

Lepidosperma, Labill.

Series I.-Longitudinales.

exaltatum, R. $B r$.

Series II.-Densiflora.

concavum, R. Br.

var. pyramidatum, Benth.

Scries III.-Stenostachyce.

laterale, $R . B r$.

var. angustum, Benth.

Cladium, R. Br.

Section I.-Eucladium.

mariscus, R. Br.

Section II.-Baumea.

articulatum, R. $B r$.

glomeratum, $R$. Br.

teretifolium, R. Br. 
CLIII. CYPERACEA.

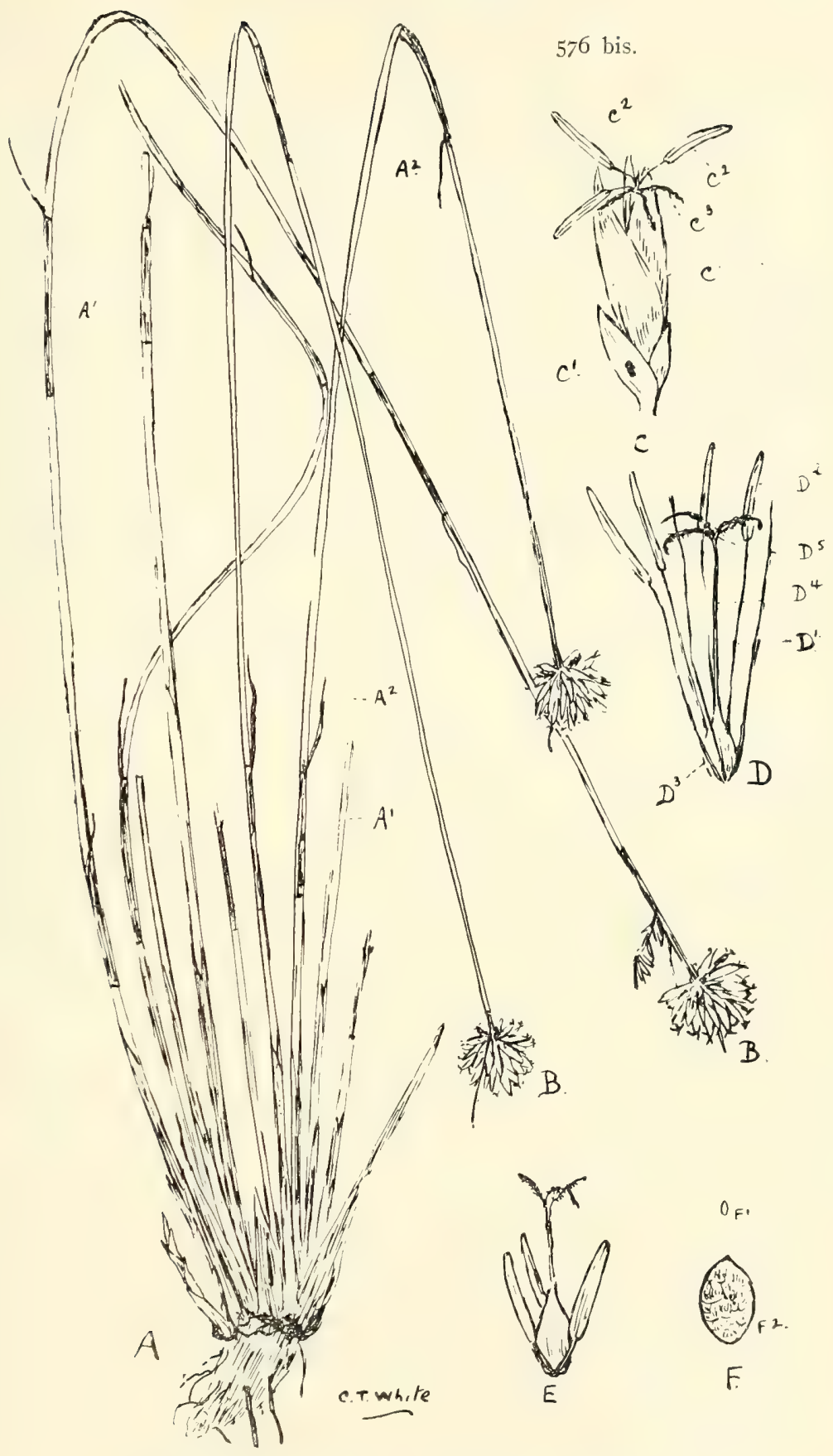

576 bis. Arthrostylis Kennyi, Bail., sp. nov.

(A) Plant nat. size, (Ar) leaf-sheaths, (Az) laminæ of leaf-sheaths, (B) terminal head of spikelets, (C) spikelet, ( $\left.C_{1}\right)$ glumes, (C2) stamens, ( $\left.C_{3}\right)$ stigmatic lobes, (D) stamens and pistil, (DI) filaments, (D2) anthers, (D3) ovary, (D4) style, (D5) stigmatic lobes, (E) pistil and three short stamens. (C) - (E) enl.; (FI) seed, nat. size; ( $\left.\mathrm{F}_{2}\right)$ seed, en1. 
Cladium-contd.

tetraquetrum, Hook. $f$. var. planifolium, Benth.

schœnoides, R. Br. var. elongatum, Benth.

Gunnii, Hook.f. junceum, R. Br.

Gahnia, Forst.

Section I.-Lampocarya.

aspera, Spreng. = Lampocarya aspera, R. Br.-The seeds might be used in ornamental work.

melanocarpa, R. Br.

Section II.-Eugahnia.

psittacorum, Labili.- "Yerer" of Cape Bedford natives. The seeds should be useful for ornamental work.

Caustis, R. Br.

pentandra, R.Br.

flexuosa, R.Br.

Tribe IV.-SCLERIEÆ.

Scleria, Berg.-The white seed might be used in fancy ornamental work.

Series I.-Axillares.

caricina, Benth. (Fig. 579.)

pygmæa, R. Br.

Series II.-Laxk.

rugosa, $R . B r$.

laxa, R.Br. (Fig. 58o.)

Series III.-Subspicate.

Brownii, Kunth.

lithosperma, Willd.

var. linearis, Benth.

Series IV.-Polystachyce.

tesselata, Willd., var. debilis, Benth.

Benthamii, C. B. Clarke, Kew Bull. Add. Series viii. (Igo8) $5^{8}=$ S. tesselata, Benth., F1. Austr. vii. p. 430 ; non Willd.

margaritifera, Willd.

Græffeana, Backel.

hebecarpa, Nees.

suffulta, C. B. Clarke, Kew Bull. Add. Series viii. (I908) 58. chinensis, Kunth. 


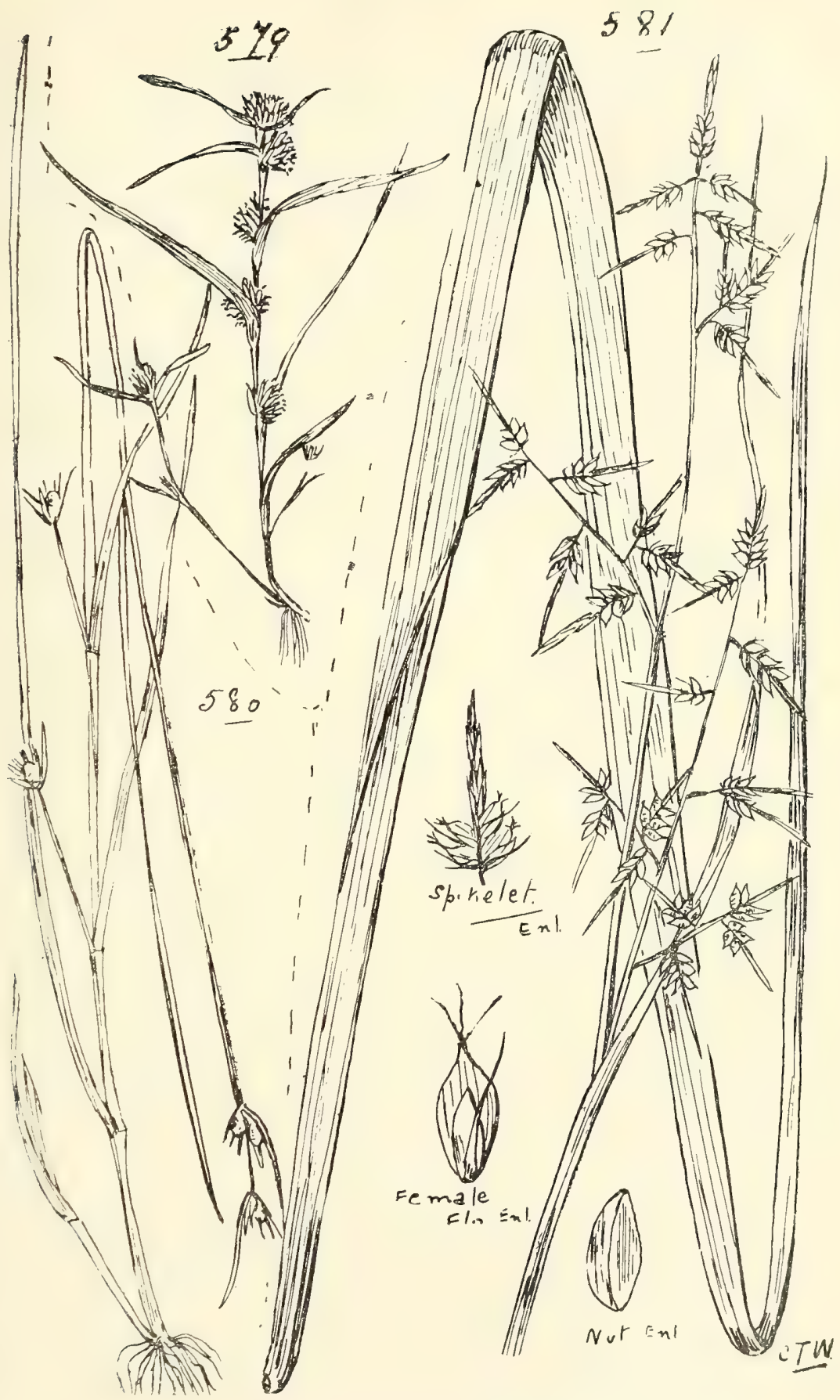

579. Scleria Caricina, Benth.

580. S. LAXA, R. Br.

58r. Carex fissilis, Boott. 
Scleria-contd.

Series $V .-D i o i c a$.

sphacelata, F. v. M.

Carex, Linn.

Tribe V.-CARICEA.

inversa, R. Br.

var. major, Benth. $=$ C. lophocarpus, C. B. Clarke, Kew Bull. Add. Series viii. (Igo8) 69.

chlorantha, R. Br.

paniculata, Linn.

var. subdiaphana, Benth.

declinata, Boott.

fissilis, Boott. (Fig. 58I.)

gracilis, R. $B r$.

contracta, F. v. M.

vulgaris, Fries.

acuta, Linn.

lobolepis, F.v.M.

pumila, Thunb.

breviculmis, R. Br.

maculata, Boott.

Brownii, Tuckerm.

longifolia, R. Br.

pseudocyperus, Linn.

\section{Order CLIV.-GRAMINE王.}

\section{Series A.-Panicaceæ.}

Tribe I.-PANicE瓜.

Paspalum, Linn.

Galmarra, Bail.-Russell River Grass; very productive of good feed. (Fig. 582.)

*ailatatum, Poir.-Caterpillar Grass. Brazil.

Polo, Bail--Being a more slender grass than the foregoing, and would likely form a useful pasture species. (Fig. 583.)

scrobiculatum, Limn.-Ditch Millet.

distichum, Linn.-Water Couch Grass.

*var. normale, Bail.-Found near fresh water; probably not indigenous.

var. Turleyi, Bail., Q1. Agric. J1. xx. (Igo8) p. I8I, tab. xvi.--A variegated form.

var. littorale, Bail.-Found on brackish, swampy land.

brevifolium, Fliigge.

var. propinquum, Bail. 
Paspalum-contd.

minutiflorum, Steud.

conjugatum, Berg.

platycaule, Poir.-Carpet Grass; considered an excellent pasture-grass for the tropics.

Eriochloa, Humb. et Kunth.

punctata, Hamilt.-Early Spring Grass; excellent for pasture. or hay.

annulata, Kunth.-Useful like the last.

Isachne.

decumbens, Bail. (Fig. 584.)

australis, R. Br.

myosotis, Nees. (Fig. 585.)

Panicum, Linn.--Some species contain prussic acid. Most species good for stock, and would form excellent hay, but for that material should be cultivated, and, like other grasses, be cut when in flower.

\section{Section I.-Digitaria.}

cœnicolum. F. v. M.

nematostachyum, Bail., Q1. Bot. Bull. xvi. (I903) 2.

divaricatissimum, R. Br.

var. glaberrimum, Benth.

var. normale, Benth.

var. radiatum, Benth.

macractinium, Benth.-Branches of panicle very long, often exceeding a foot.

papposum, R. Br.

var. leiostachyum, Benth.

sanguinale, Limn--Summer Grass; excellent for hay, but a. weed in cultivation. A young growth of this grass (cultivated) Mr. J. C. Brünnich, Queensland Agricultural Chemist, found to contain a large amount of prussic acid. didactylum, Kunth. = Digitaria didactyla, Willd.-Blue Couch. First met with near Nudgee early in I906, and from the specimens sent to me at the time considered only a form of $P$. glabrum, Gaud., differing only in the pubescence of the lower portions of the plant. The grass differs very much when seen at different stages of growth, for instance, when seen on a lawn, or closely fed, or when allowed to grow up for hay or seed. I do not consider it a Queensland grass, as, after knowing the Australian pastures for over 70 years, I had never previous to 1906 seen the species; and after reading Dr. Staph's paper in Kew Bulletin No. 6 of IgI I, p. 256 , follow him as above. 
Panicum-contd.

tenuissimum, Benth.

parviflorum, $R . B r$.-Spikelets glabrous. There are several forms of this grass, all good pasture kinds. var. pilosa, Benth.

Baileyi, Benth.-Spikelets hairy. (Fig. 586.)

Section II.-Trichachne.

gibbosum, R. Br.

leucophœum, H.B. et $K$.

Section III.-Paspaloidece.

rarum, R. Br. (Fig. 587.)

argenteum, R. Br.-Silver-spiked Grass; a decorative as well as a useful pasture-grass. (Fig. 588 .)

holosericeum, R. Br.

flavidum, Retz.-Warrego Summer Grass.

var. tenuior, Benth.

globoideum, Domin in Fedde. Repert. x. (I9II) IIg. gracile, R. Br.

jubiflorum, Trin. $=P$. distans, Trin. Sp. Gram. t. 172.-Shot Grass.

prostratum, Lam.

*muticum, Forsk.-Bancroft Grass; contains prussic acid.

A native of North Africa, Arabia, and neighbouring places; now met with in most warm countries. Gives a large quantity of fodder, but is of a very straggling habit ( see Q1. Agric. J1. xxi. (1908) 8. P1. 3, 4).

helopus, Trin.

Gilesii, Benth.

piligerum, F.v.M.-An excellent hay-grass, and this and the two following should be grown for that purpose.

polyphyllum, R. Br.

distachyum, Linn.

reversum, $F . v \cdot M$.

Section IV.-Echinochloa.

colonum, Linn.-A good fodder-grass (see Q1. Agric. J1. iv. (1899) 364, P1. 104).

crus-galli, Linn.-Cockshin Grass.

var. sabulicolum (Trin.), Doell.-Excellent for fodder; very succulent; an annual.

* frumentaceum, Roxb.-The Deccan Grass. Excellent for fodder (see Q1. Agric. J1. xvi. (Ig06) p. 450, P1. 24). 


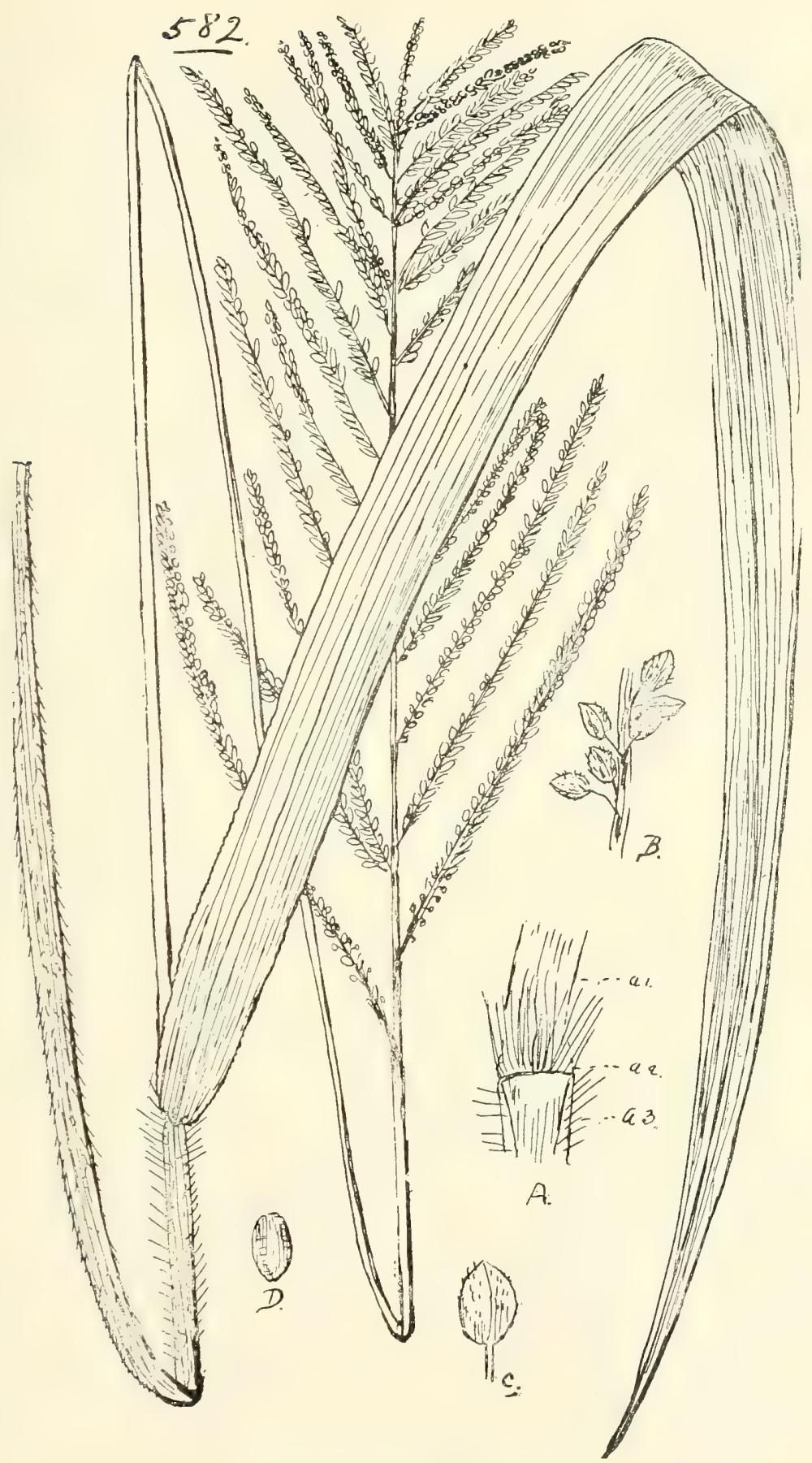

582. Paspalum Galmarra; Bail.

(A) I3ase of leaf and portion of leaf-sheath, (ai) base of leaf, (az) ligula, (a3) top of leaf-sheatin, (B) portion of a spike of the panicle, (C) a single spikelet, (D) grain. (A) - (D) enl. 


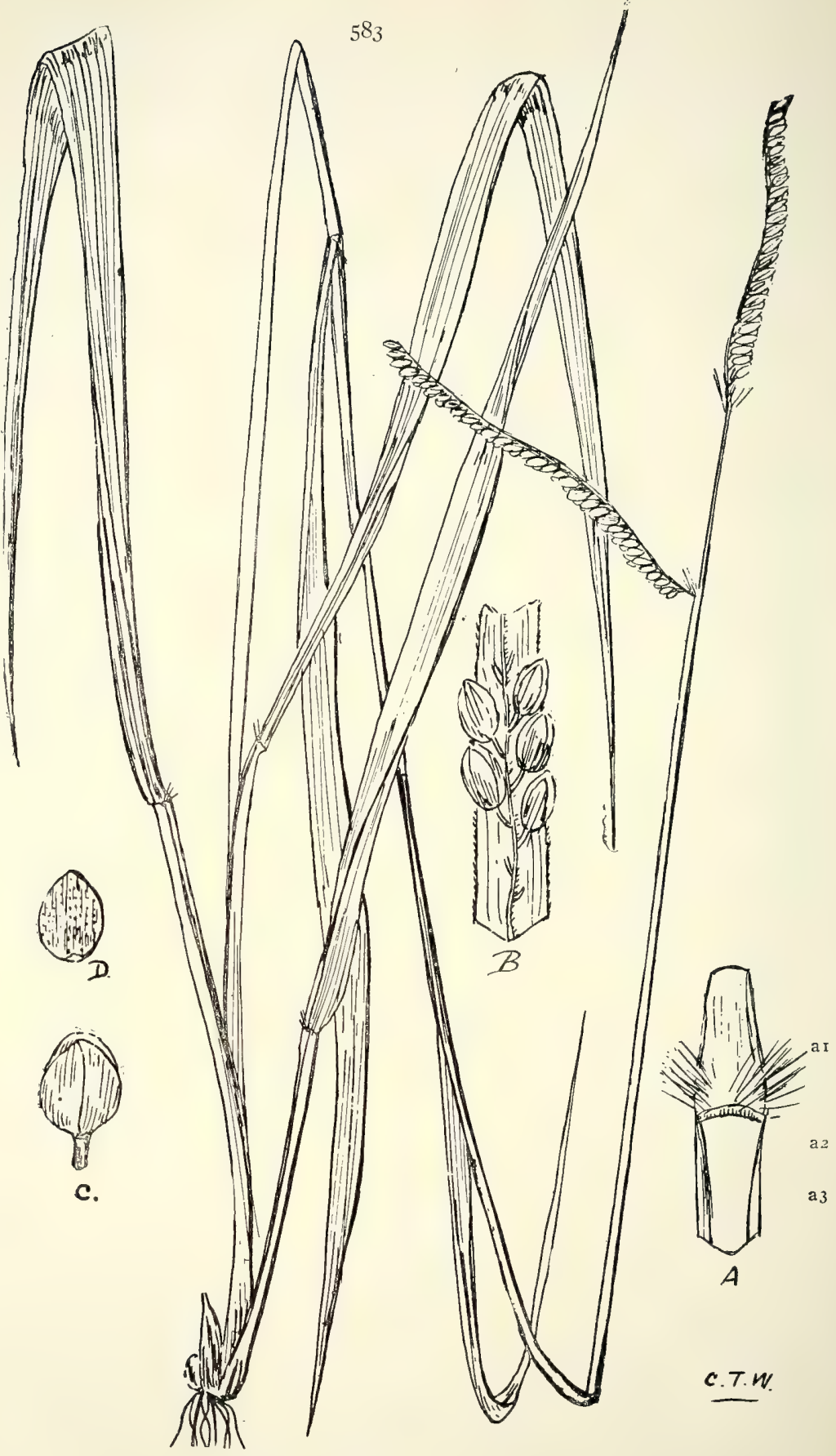

583. Paspalum Polo, Bail.

(A) Base of leaf and portion of leaf-sheath, (ar) base of leaf, (a2) ligula, (a3) top portion of leaf-sheath, (B) portion of spike, (C) a spikelet, (D) a grain. (A)(D) enl. 


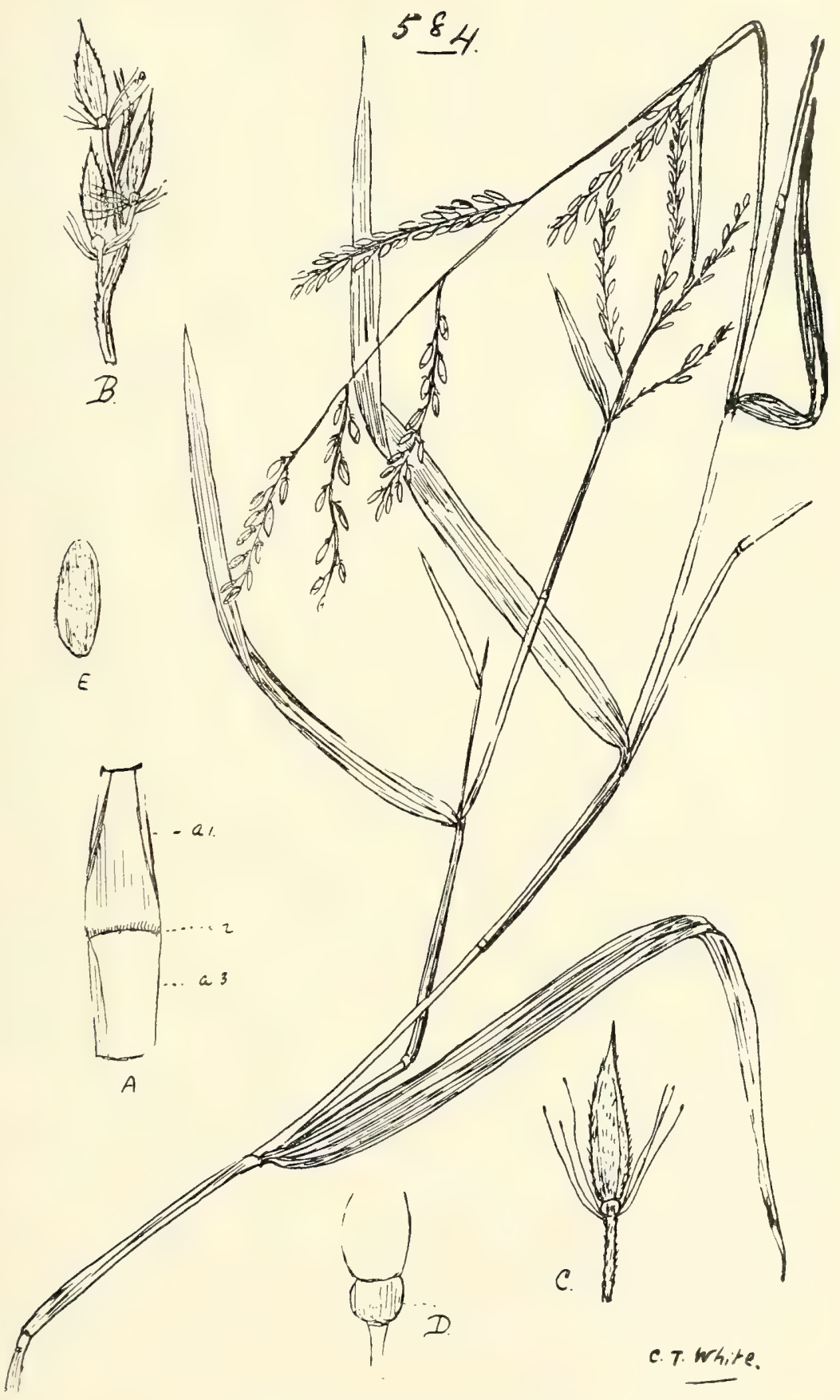

584. Eriochloa decumbens, Bail.

(A) Base of leaf and portion of leaf-sheath, (ar) base of leaf, (az) ligula, (a3) top portion of 1caf-sheath, (B) portion of a panicle-branch, (C) spikelet, (D) base of spikelet, (E) grain. (A)-(E) enl. 


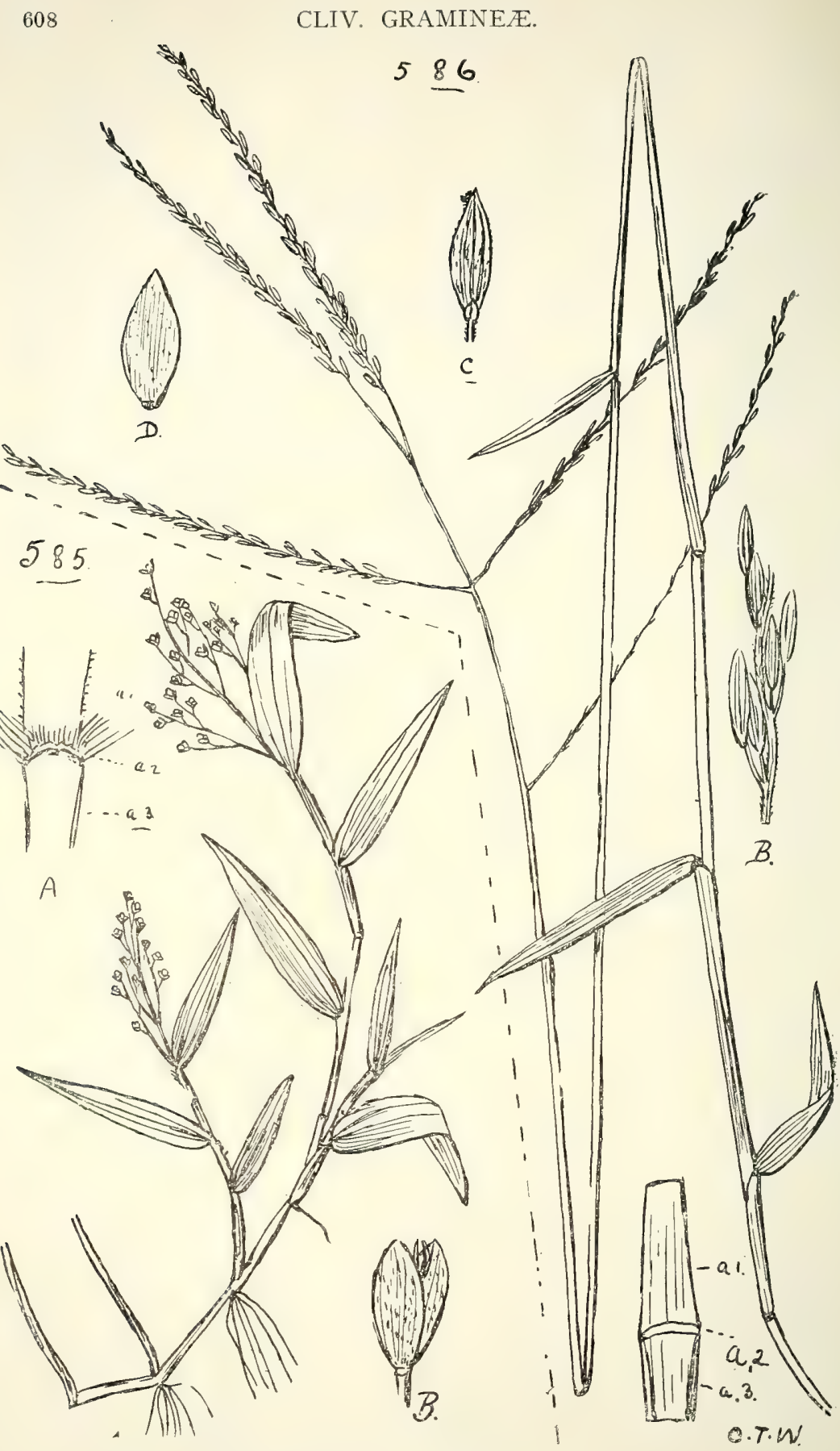

585. Isachne myosotis, Nees.

(A) Base of leaf and portion of leaf-sheath, (aI) base of leaf, (a2) ligula, (a3) topportion of leaf-sheath, (B) a spikelet. (A) and (B) enl.

586. Panicum Batleyi, Benth.

(a1) Base of leaf, (a2) ligula, (a3) top of leaf-sheath, (B) portion of panicle, (C) a spikelet, (D) grain. (A)-(D) enl. 
CLIV. GRAMINEA.

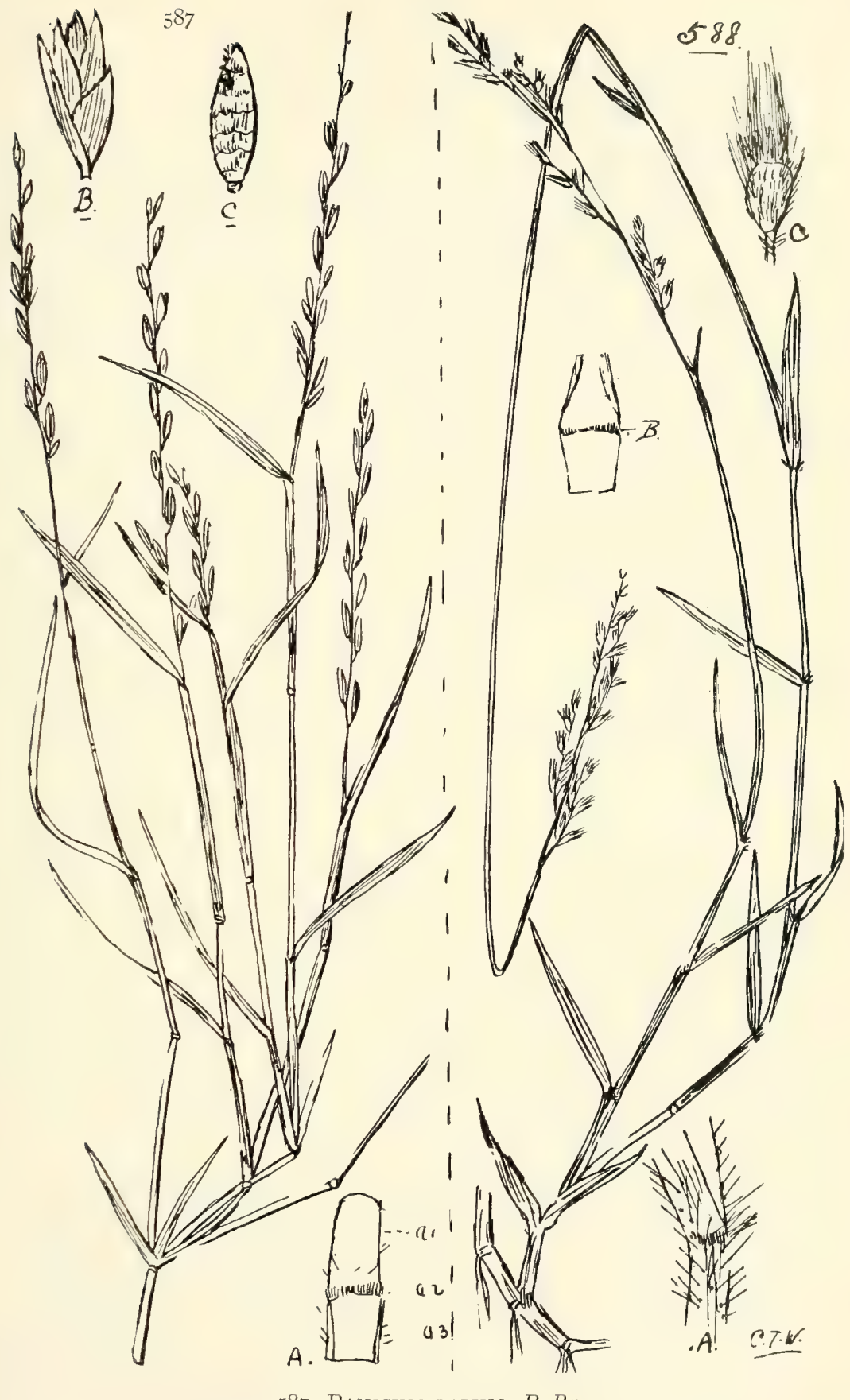

587. Panicum Rarum, $R B r$.

(A) Base of leaf and portion of leaf-sheath, (ar) base of leaf, (az) ligula, (a3) top portion of leaf-sheath, (B) spikelet, (C) fruiting glume. (A), (B), and (C) en].

588. P. ARGENTEUM, R. $B r$.

(A) Base of leaf and portion of leaf-sheath, (B) ligula, (C) spikelet. (A), (B), and (C) enl. 
Panicum-conta.

Section V.-Hymenachne.

myosuroides, $R . B r$.

indicum, Linn.

myurus, Lam. = Hymenachne myurus, Beauv.-A thickstemmed water-grass, greedily eaten by stock.

Section VI.-Eupanicum.

foliosum, R. Br.-A good fodder-grass; bears good foliage and abundance of grain.

adspersum, Trin.

inæquale, $F \cdot v \cdot M$.

uncinulatum, R.Br.-A scrub-grass; the spikelets purplish, bearing hooked hairs.

pauciflorum, R. Br.

repens, Linn.

*maximum, Linn.-Guinea Grass; contains prussic acid. Tropical Africa.

pygmæum, R. Br.-A dwarf coast-grass.

trichoides, Swartz.

hermaphroditum, Steud. $=$ P. pilipes, Nees. and Arn.; P. carinatum, Pres1.

marginatum, R. Br.

var. strictum, Benth.

var. majus, Benth.

lachnophyllum, Benth.

obseptum, Trin.

Buncei, F.v.M.

bicolor, R. Br.-A good pasture-grass.

melananthum, F.v.M.

*miliaceum, Linn.-India and Africa.

Prenticeanum, Bail.-A very tall scrub-grass of the Queensland tropics, and an excellent fodder. (Fig. 589.)

effusum, R. Br.

Mitchelli, Benth.

decompositum, R.Br.-Barley Grass; an excellent pasturegrass. "Tindil" of Cloncurry natives.

proliferum, Lam.

trachyrhachis, Benth.-Coolibar Grass. “Oo-kin” of Mitchell River natives.

var. tenuior, Benth.

Benthami, Domin in Fedde. Repert. x. (IgI I) 59.

prolutum, F.v.M.

queenslandicum, Domin in Fedde. Repert. x. (I9II) 58 . 
*Tricholæna, Schrad.

Teneriffæ, Parlat.-Red Natal Grass. North Africa. A grass of many warm countries.

Ichnanthus, Sw.

pallens, Munro = Panicum vicinum, Bail. (Fig. 59o.)

Axonopus, Beauv.

semialatus, Hook. $f$. = Panicum semialatum, R. Br.-Cockatoo Grass ; excellent pasture-grass. "Lo-thi " of Batavia River natives.

Oplismenus, Beauv.-All the species thrive under the shade of trees.

compositus, Beauv. -The foliage often variegated.

setarius, Rom. et Schult.

var. æmulus, Benth.

var. imbecillis, Benth.

Arundinella, Raddi.

nepalensis, . Trin.

Setaria, Beawv.

*italica, Beauv. - Panicum of Queensland farmers. China and Japan. Forms of this species are stated by various writers to have been cultivated for food as far back as 2700 B.C., and are supposed to have formed the main bread supply of the prehistoric peoples. The grain has been said to act as a diuretic and astringent, and to alleviate rheumatic pains.

glauca, Beaur.-Pigeon Grass of America; a good fodder.

var. minor, Bail., n. var.-This is the common form in

Tropical Queensland (see Q1. Flora, p. 1839).

macrostachya, H.B. et K.-A good fodder.

viridis, Beauv.-Comparatively rare in Queensland.

var. australis, Bail., $n$. var.-This may be given as a variety, on account of its strong, hard, flattened stems, which at times bear thick bunches of leaves at the nodes; the length also varies from long to quite short; should prove a useful fodder.

Hab.: Prairie Tableland (J. R. Chisholm).

Cenchrus, Lim.

australis, R. Br.-Hillside Burr Grass.

elymoides, F.v.M.-A coarse tropical fodder-grass.

Pennisetum, Rich.

compressum, R. Br.-Swamp Foxtail.

arnhemicum, F. v. $M$.

Plagiosetum, Benth.

refractum, Benth. = Pennisetum refractum, F.v. M. 
Chamæraphis, R. Br.

spinescens, Poir.-Water Grass. This is a useful grass, for, although its floating stems may pass often out of the reach of sheep, this habit of growth has a great value, for it is found near waterholes liable to become dry, and as the water dries up the floating runners rest upon the land, and root from the nodes, and soon cover with nutritious grass spots which would otherwise be bare.

var. parvispicula, Benth.

paradoxa, Poir.

Spinifex, Linn.

hirsutus, Labill.-A common coast-grass. The female inflorescence used by the native children as a toy for running along the coast sands.

longifolius, R. Br.

paradoxus, Benth.

Xerochloa, R. $B r$.

imberbis, R. Br.

barbata, R.Br. (Fig. 59I.)

*Stenotaphrum, Trin.

americanum, Schrank.-Buffalo Grass.

Thuarea, Pers.

sarmentosa, Pers.-Beach Grass.

Tribe II.-ORYZEA.

Oryza, Linn.

sativa, Linn. - Wild Rice. " Anbó-a" and "Kwang-an" of Princess Charlotte Bay and " Mokomurdo" of Cloncurry natives. Two forms are met with in our tropical swamps, the one having a dark and the other a light coloured inflorescence. Both forms produce an excellent fodder.

Leersia, Swartz.

hexandra, Sw.-Rice Grass; often found in shallow, scant water.

Leptaspis, R. Br.

Banksii, R.Br.-A beautiful and very ornamental tropical species. (Fig. 592.)

Tragus, Hall.

TRIBE III.-ZOYSIEE.

racemosus, Desf. = Lappago racemosa, Willd.-Small Burr Grass; a winter grass often met with on stony land. In this State there two or more forms. The most distinct one is found near the Hamilton River; it has a more dense inflorescence and broader leaves than the others, and approaches the variety brevispicula, Doell., of Brazil. 


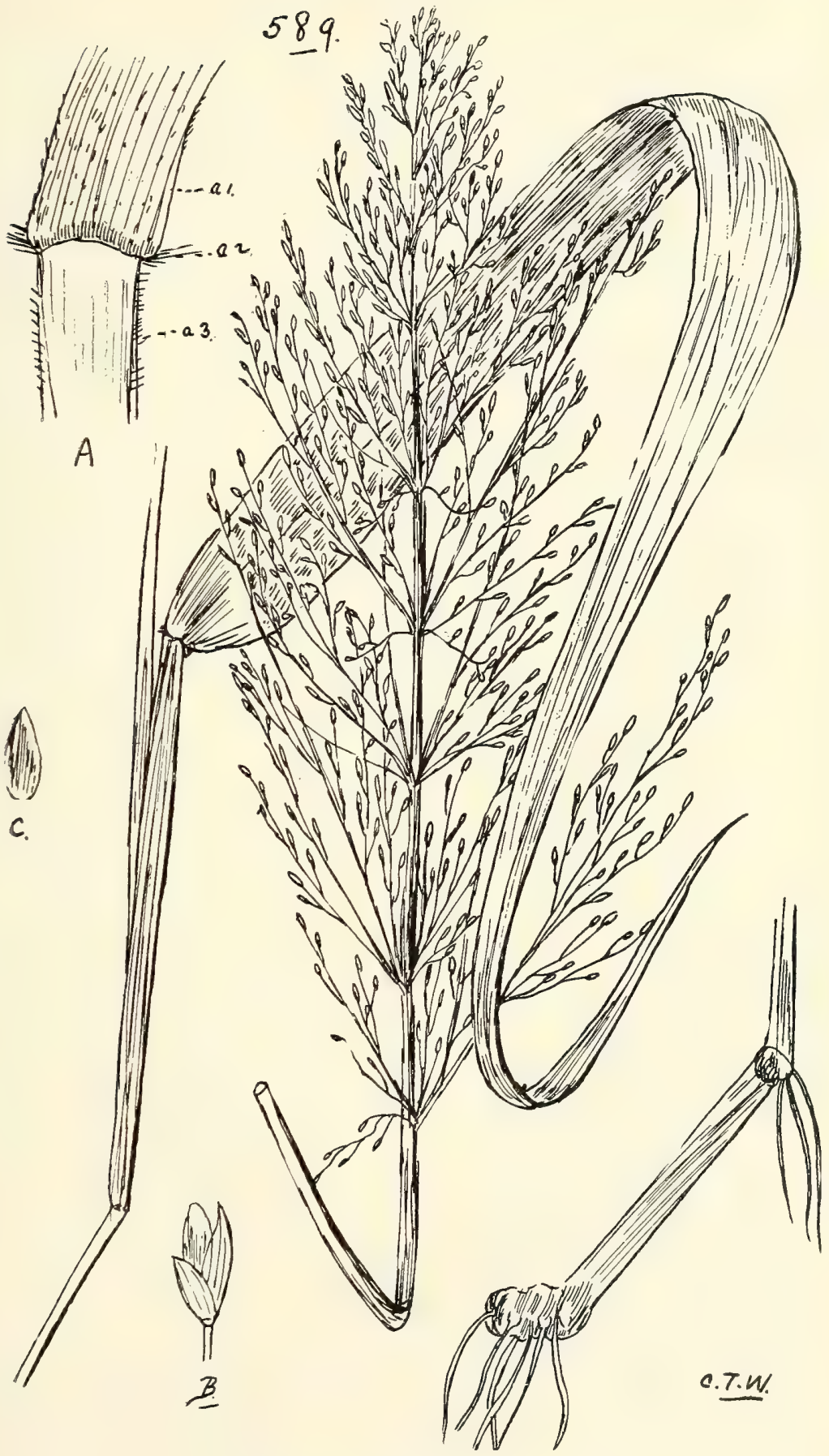

589. Panicum Prenticeanum, Bail.

(A) Base of leaf and portion of leaf-sheath, (ar) base of leaf, (az) ligula, (a3) top of leaf-sheath, (B) a spikelet, (C) fruiting glume. (A)-(C) enl. 


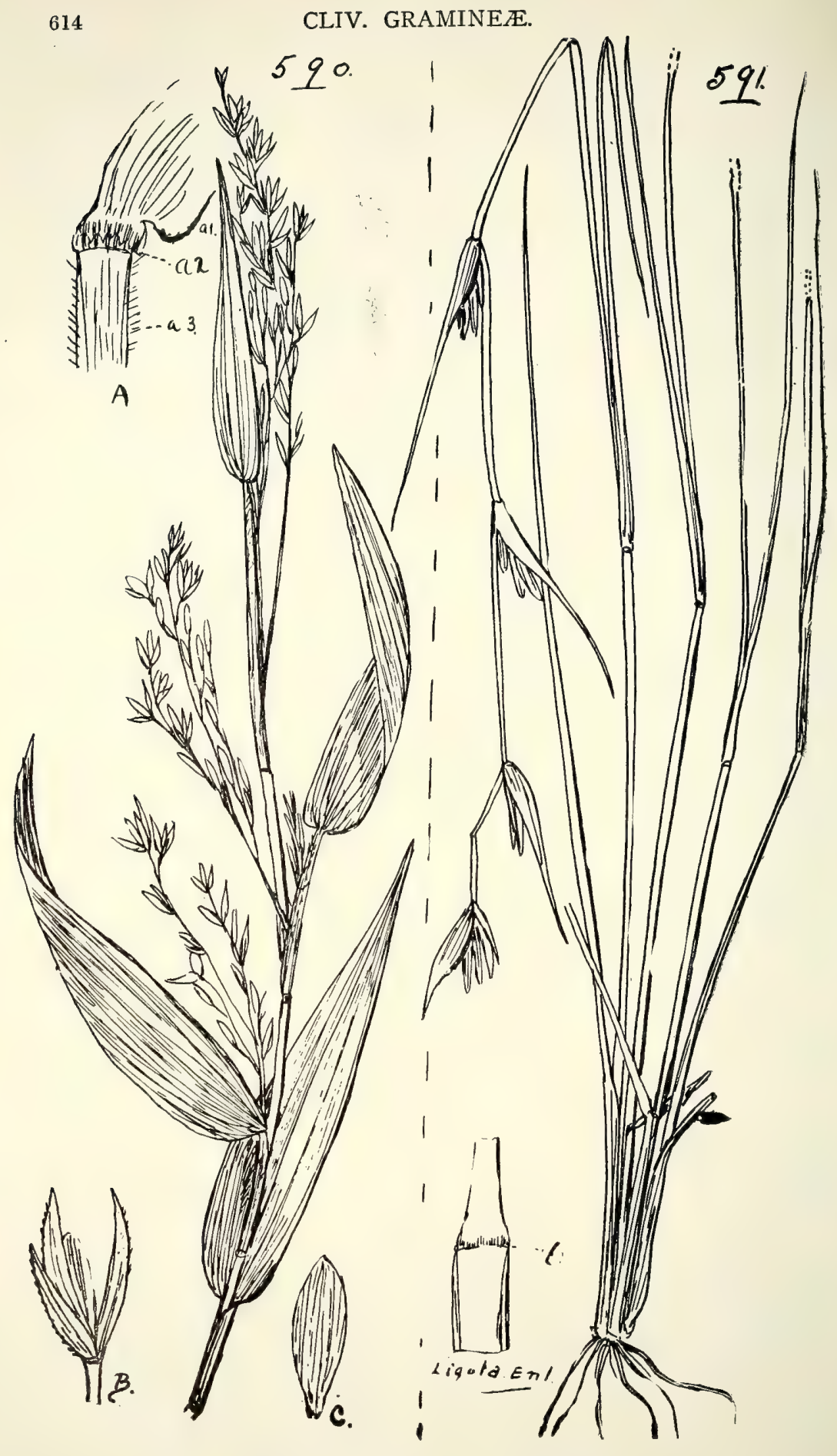

590. Ichnanthus pallens, Munro.

(A) Base of leaf and portion of leaf-sheath, (ar) base of leaf, (a2) ligula, (a3) top of leaf-sheath. 


$$
v^{2}
$$


Neurachne, R. Br.

Mitchelliana, Nees.-Mulga Grass; considered a good winter grass.

Perotis, Ait.

rara, R. Br.-Comet River Grass. Before seeding, forms a good leafy pasture.

Zoysia, Willd.

pungens, Willd-Coast Couch.

Tribe IV.-ANDROPOGONEÆ.

Subtribe I.-Maydece.

*Coix, Linn.

Lachryma Jobi, Linn.-Job's-tears. Tropics generally; yields a good fodder (see Q1. Agric. J1. iv. (I899) p. I96, Pl. 9I.)

Polytocha, R. Br. = Chionachne, R. Br.

barbata, Stapf. $=$ C. barbata, R. Br.

cyathopoda, Bail. = C. cyathopoda, F. v. M. - The common food of large birds. When taken out of their crops and sent to Brisbane I have found such seed to germinate freely.

Sclerachne, Bail. $=$ C. Sclerachne, Bail.-A troublesome Spear Grass at Somerset.

*Euchlæna, Schrad.

luxurians, Dwv. et Aschers.-Téosinté, or Guatemala Grass. America.

*Zea, Willd.-Fruit a Caryopsis.

Mays, Willd--Maize; contains prussic acid. Peru. In Greece the silky styles and stigmata are used as a decoction in diseases of the bladder; also, in America it is used under the name of Corn Silk.

Subtribe II.-Dimeriea.

Dimeria, R. Br.

acinaciformis, R. Br.

ornithopoda, Trin. $=$ D. tenera, Trin.,; D. psilobasis, F.v. M. glabriuscula, Bail. (Fig. 593.)

Subtribe III.-Saccharece.

Imperata, $C y r$.

arundinacea, Cyr.-Blady Grass. "Dirnbur" of Cape Bedford natives. Known in commerce as "Lalang Grass," and considered valuable for paper-making. 
Pollinia, Trin.

articulata, Trin.

var. minor, Benth.

irritans, Benth.

fulva, Benth.-Browntop. Equals the Mitchell Grass as a drought-resister, and on account of its sweetness has often been called Sugar-grass.

argentea, Trin.-Silvertop.

Subtribe IV.-Ischemea.

Eremochloa, Buse.

muricata, Hack.-This and the following species, Bentham

(F1. Austr. vii. 52I) included under Ischcemum pectina-

tum; both will often be seen to thrive on dry rocky land. binaculata, Hack.

Ischæmum, Linn.

triticeum, R. Br.

australe, $R . B r$.

var. villosum, Benth.

muticum, Linn.

ciliare, Retz., var. podostachyum, Benth.

fragile, R. Br.

laxum, R. Br.

Pogonatherum, Beauv.

saccharoideum, Beauv.

Arthraxon, Beauv.

ciliare, Beauv.- The proper name for Andropogon achinatus, Heyne.

Subtribe $V$.-Apludea.

Apluda, Linn.

mutica, Linn.

Subtribe VI.-Rottboelliece.

Rottboellia, Linn. f.-The Queensland species form good fodder in the tropical parts.

formosa, R.Br.

exaltata, Linn.

muricata, Retz.

ophiuroides, Benth.

rariflora, Bail., Q1. Bot. Bull. viii. (I893) 86. (Fig. 594.)

Ophiurus, R. Br.

corymbosus, Gartn.

Manisurus, Sw.

granularis, Sw-A good pasture-grass. 


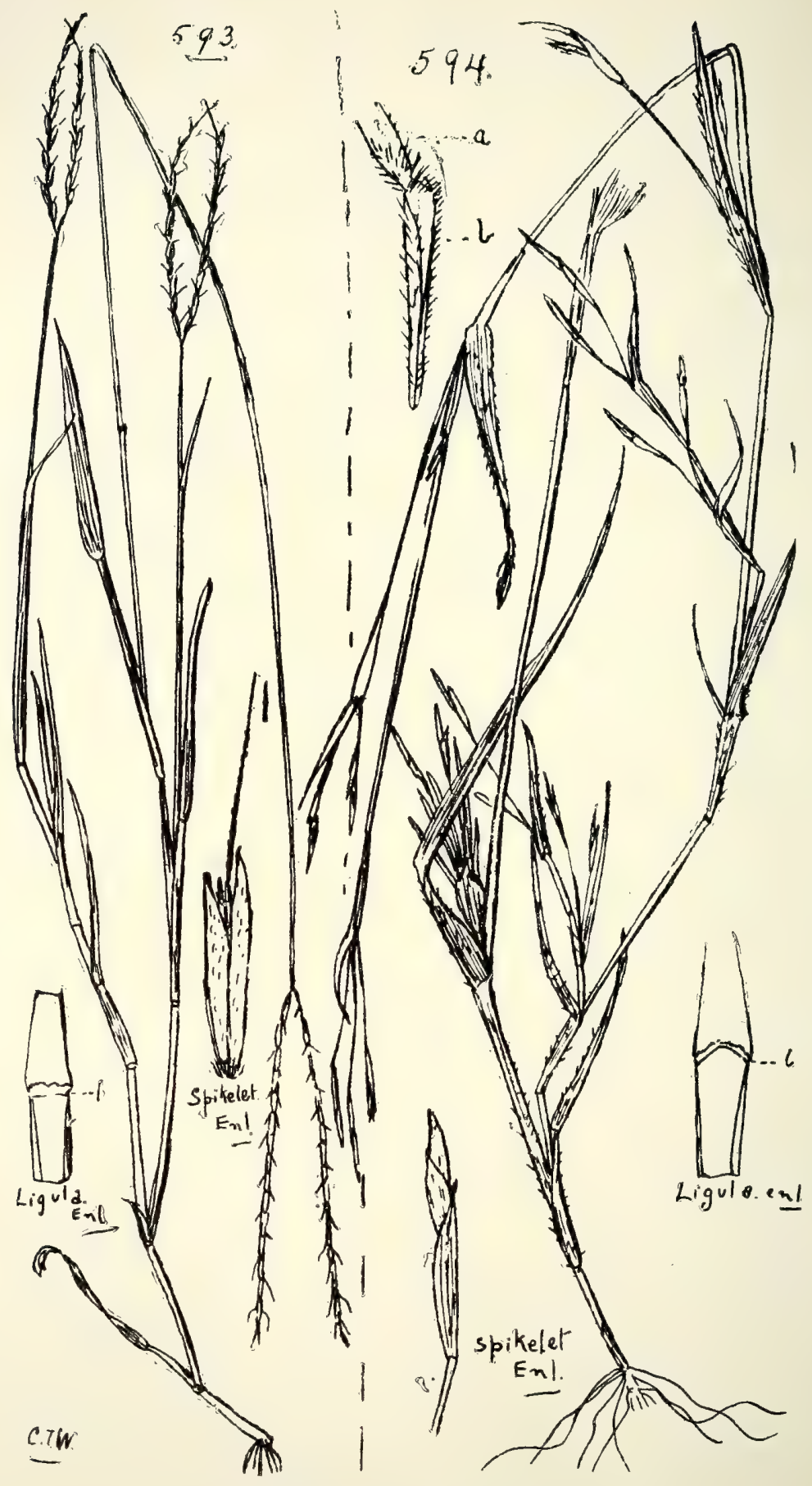

593. Dimeria glabriuscula, Bail.

594. Rottboellia rariflora, Bail.

(a) Base of leaf, enl., (b) top portion of leaf-sheath, enl. 
Hemarthria, R. Br.

compressa, R. Br.--There are two forms of this grass, the one much smaller than the other.

Subtribe VII.-Euandropogonea.

Elionurus, $W$ illd.

citreus, Munro.-Lemon Grass.

Heteropogon, Pers.

contortus, Roem. et Schult.-Bunch Spear Grass. This is by no means a desirable grass either for farm or run. Cattle, however, do eat it when it is young; but it has been stated that in Ceylon they do not touch it. Called "Spear Grass" in India.

insignis, Thw.-Tall Spear Grass.

Andropogon, Linn.

\section{Section I.-Gymnandropogon.}

erianthoides, F.v.M.-Satintop; one of our best pasturegrasses; forms a close, short, leafy bottom.

sericeus, R. Br.-Blue Grass; excellent for pasture.

var. polystachyus, Benth.-Tassel Blue Grass.

var. geniculatus, Bail., Q1. Agric. J1. xxvi. (I9I I) I28.-

A good pasture-grass.

affinis, R. $B r$.

pertusus, Willd.-A good pasture-grass, common on hilly country.

annulatus, Forsk.-A good pasture-grass.

var. monostachya, F.v. M.

intermedius, $R . B r$ - - A good grass producing a large quantity of excellent fodder.

Section II.-Cymbopogon.

procerus, $R \cdot B r$.

exaltatus, R. $B$ r.

lanatus, $R . B r$.

bombycinus, $R$. Br.

Nardus, Linn., var. grandis, Hack. = A. schœnanthus, var Martini, Benth.

refractus, $R$. Br.-Barbed-wire Grass; a very common, coarse pasture-grass.

filipendulus, Hochst. $=$ A. lachnatherus, Benth.

Section III.-Schizachyrium.

fragilis, $R . B r .=A$. brevifolius, $\mathrm{Sw}$.

Chrysopogon, Trin.

Gryllus, Trin.-A good summer grass.

var. pallidus, Benth. 
Chrysopogon—contd.

parviflorus, Benth.-Scented Golden-beard; produces a large quantity of coarse fodder.

var. spicigera, Benth.

var. flavescens, Bail.-A very ornamental grass and a useful fodder.

aciculatus, Trin.-On the Mulgrave known as "Mackie's Pest."

elongatus, Benth.

var. filipes, Benth.

Sorghum, Pers.-As many of this genus are grown for fodder, the following note by a chemist may be given:- " When young, say from three to seven weeks, the amount of prussic acid is really dangerous, especially if of rank growth."

*halepense, Pers.-Johnson Grass; contains prussic acid. Mediterranean and adjoining localities; now in most warm countries. Not a desirable species in cultivation paddocks.

plumosum, Beauv.-Excellent for fodder.

fulvum, Beauv.-Excellent for fodder.

laxiflorum, Bail.-Forms a good pasture. (Fig. 595.)

Anthistiria, Linn.

ciliata, Lim.-Kangaroo Grass. Sir J. D. Hooker (F1. Brit. Ind. vii. p. 2II) places the Australian grass under $A$. imberbis, Retz., and points out that $A$. ciliata is of an annual and $A$. imberbis of a perennial growth. We have three forms of this grass. The common tropical one is of an entangling, scrambling habit; of the two Southern forms the one is of a more or less deep-green. and the other is quite glaucous.

frondosa, $R, B r .=A$. arguens, Willd.

avenacea, F. v. M. - The tall Oat Grass of our Downs country. Iseilema, Hack.

Mitchellii, Anders. = Anthistiria membranacea, Lindl. Landsborough Grass, Red Gulf Grass, Flinders Grass, and Barcoo Grass; an excellent fodder grass, annual.

Series B.-Poaceж.

Microlæna, R. Br.

Tribe V.-Phalaride 2 .

stipoides, $R$. Br.-Meadow Rice Grass; will thrive under the shade of trees.

**Phalaris, Linn.

arundinacea, $\operatorname{Linn}$.-Is met with sometimes as a stray from cultivation. Northern Temperate Regions. 


$$
595
$$

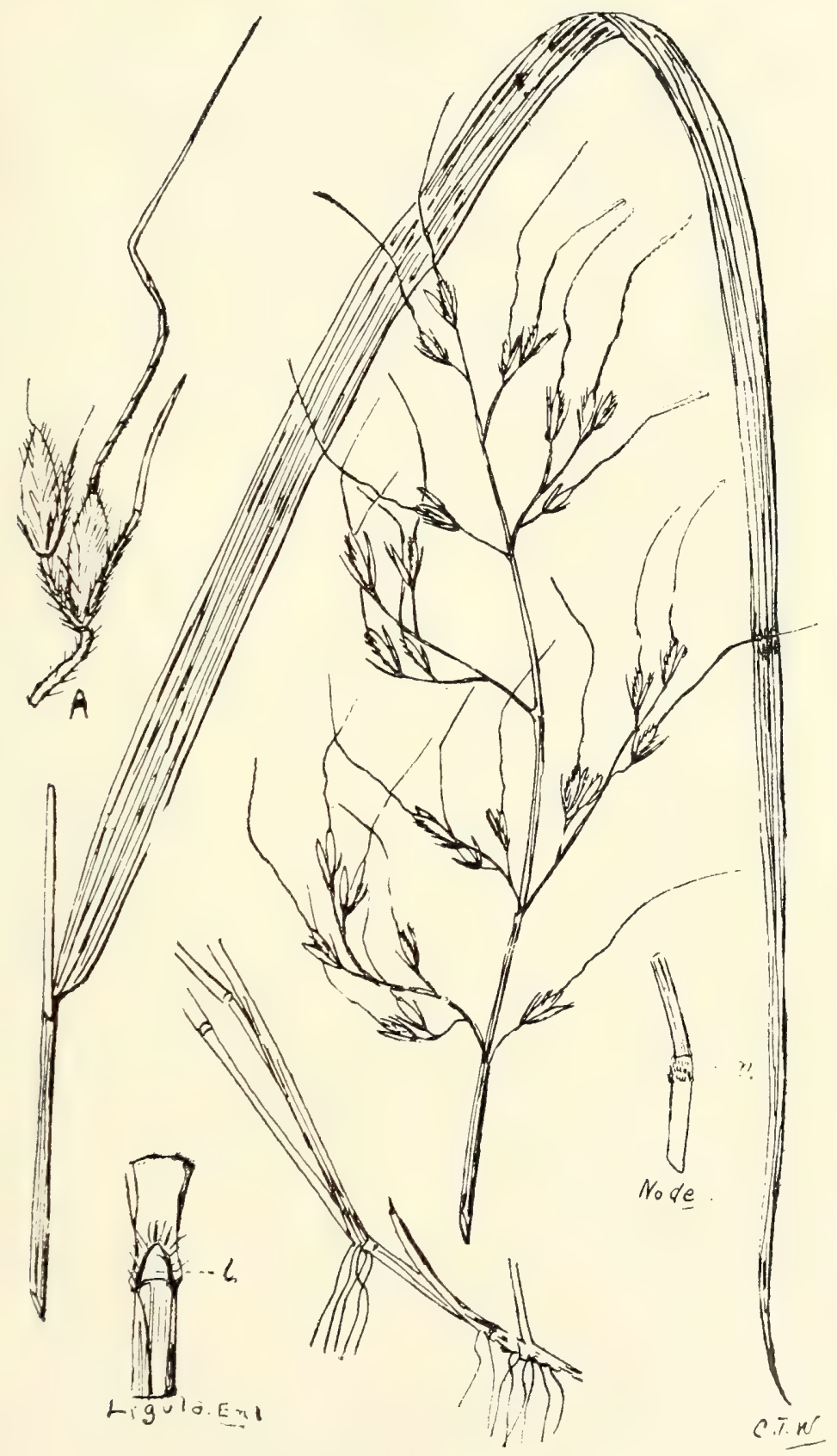

595. Sorghum LAXIFlorum, Bail.

(A) Spikelets, enl. 
Phalaris-contd.

intermedia, Nees., var. angusta, Chapm.-Californian Timothy Grass. North America.

nodosa (Linn.), R. and S., Trin. Sp. Gram. vol. i. t. 8I $=P$. bulbosa, Tenore; P. tuberosa, Linn.-Known in Queensland as P. commutata. Mediterranean Region.

canariensis, Limn.-Canary-seed Grass. South Europe and North Africa.

\section{Tribe VI.-Agrostidex.}

Subtribe I.-Stipea.

Aristida, Linn.-Three-awned Spear Grasses. On the whole these are not desirable grasses.

Section I.-Arthratherum.

hygrometrica, R. Br.

stipoides, $R$. Br.

arenaria, Gaudich.

\section{Section II.-Chætaria.}

Behriana, F.v. M.

leptopoda, Benth.-The only one of the genus we have of much use for pasture.

vagans, Cav.-A grass of forest land.

var. gracillima, Benth.

var. compacta, Benth.

Leichhardtiana, Domin in Fedde. Repert. ix: (I9II) p. 55I $=$ A. ramosa, var. (?) leptathera, Benth.

ramosa, $R . B r$.

var. compacta, Benth.

calycina, R. Br.

Adscensionis, Linn.$=$ A. depressa, Retz.

utilis, Bail., Q1. Agric. J1. xviii. (I907) 340.-Hat Grass; lately used in hat-making at Cooktown.

Stipa, Linn. - Some species of this genus have been proved to contain prussic acid.

elegantissima, Labill.

micrantha (Cav. ?) R. Br. = S. verticillata, Nees.-Bamboo Grass.

semibarbata, R. Br.

pubescens, R. Br.

setacea, R.Br.-Southern Spear Grass. Some idea of the penetrating power of the seeds of grasses of this genus may be obtained when it is pointed out that at one time it was no uncommon thing to see the seeds thick on a 
roast of mutton when on the table. Such a joint before being cooked would have the appearance of being sprinkled with carraway-seeds. Thus it will be seen that the seeds must have travelled through the wool and skin of the animal and lodged in the thin inner skin. Such was common about 70 years ago in South Australia. All the genus have somewhat similar seed. The South Australian one referred to is $S$. setacea, a grass not uncommon from Warwick to Stanthorpe, and, except for the above feature, an excellent sheep-grass.

aristiglumis, F.v.M.-A good pasture-grass, the awns not being as troublesome as most of the other kinds.

scabra, Lindl.

Streptachne, R. Br.

stipoides, $R$. $B r$.

Subtribe II.--Phleoidea.

*Phleum, Linn.

tenue, Schrad.-South Europe.

Alopecurus, Linn.

geniculatus, Linn.-The Water Foxtail; a good winter grass.

Subtribe III.-Euagrostece.

Echinopogon, Beauv.

ovatus, Beauv. = Cinna ovata, Kunth.

Amphipogon, R.Br.

strictus, R. Br.

Polypogon, Desf.

monspeliensis, Desf.

var. Rosei, Bail., Q1. Agric. J1. xxv. (1910) I65.

Sporobolus, R. Br.

Benthami, Bail.-A viviparous grass, excellent for pasture, found near water inland.

virginicus, Kunth.-Found on brackish land along the coast. var. minor, Bail.

indicus, R. Br.-Rat's-tail Grass. "Yak-kapari" of Boulia natives. This grass and the variety are when old very tough, thus often rejected by stock.

var. elongatus, Bail. =S. elongatum, R. Br.

diander, Beauv.

*pilifera, var. major, Kunth.-A Brazilian grass spreading in the State.

pulchellus, R. Br.-A good sheep-grass.

australasicus, Domin in Fedde. Repert. ix. (I9I I) 553. 
Sporobolus-contd.

Lindleyi, Benth.- "Unkeely-inkeela" of Georgina, "Yak-kaberry" of Cloncurry, and "Yak-kapari" of Boulia natives. A good sheep-grass.

actinocladus, F.v.M.- "Ka-too-ra" of Boulia and "Coocheramunda" of Georgina natives. A good sheep-grass.

Agrostis, Linn.

scabra, Willd.

*alba, Linn.-India.

Deyeuxia, Clarion.

Fosteri, Kunth.-A good winter grass.

quadriseta, Benth.

scabra, Benth.

Dichelachne, Endl.

crinita, Hook.-A good pasture-grass for winter and early spring.

sciurea, Hook.

Tribe VII.-AveneE.

Subtribe I.-Ariece.

Micraira, F.v.M.

subulifolia, F.v.M.-Mountain Couch. (Fig. 596.)

Cœlachne, R. Br.

pulchella, $R$. Br.-An ornamental grass.

Eriachne, R. Br.-Most species good for pasture.

stipacea, F.v.M.

Armitii, F.v.M.

squarrosa, R. Br.

glauca, R. $B r$.

rara, $R . B r$.

ciliata, $R . B r$.

avenacea, R. Br.

aristidea, F.v.M.

Muelleri, Domin in Fedde. Repert. x. (I9I I) 1 I8.

pallescens, R. Br.

melicacea, F. v. M.-The Queensland form is a fragrant grass, and on account of its scent should be a useful addition when cutting grass for hay.

pallida, $F \cdot v \cdot M$.

mucronata, $R \cdot B r$

obtusa, R. Br.

Aira, Limn.

caryophyllea, Linn.-North Africa and Temperate Asia. An ornamental grass, scarcely indigenous in Queensland. 


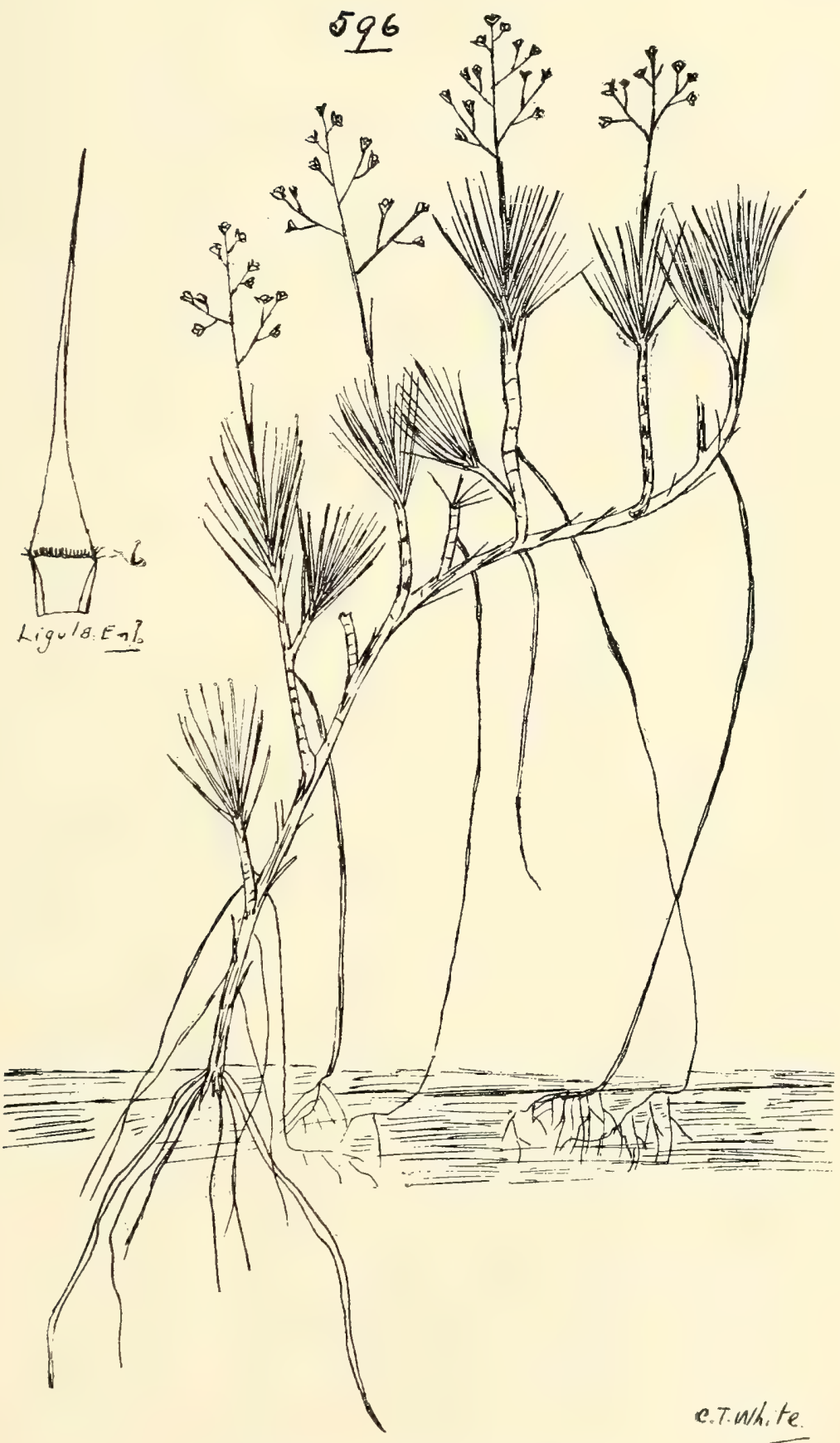

596. Micraria subulifolia, F. v. $M$. 


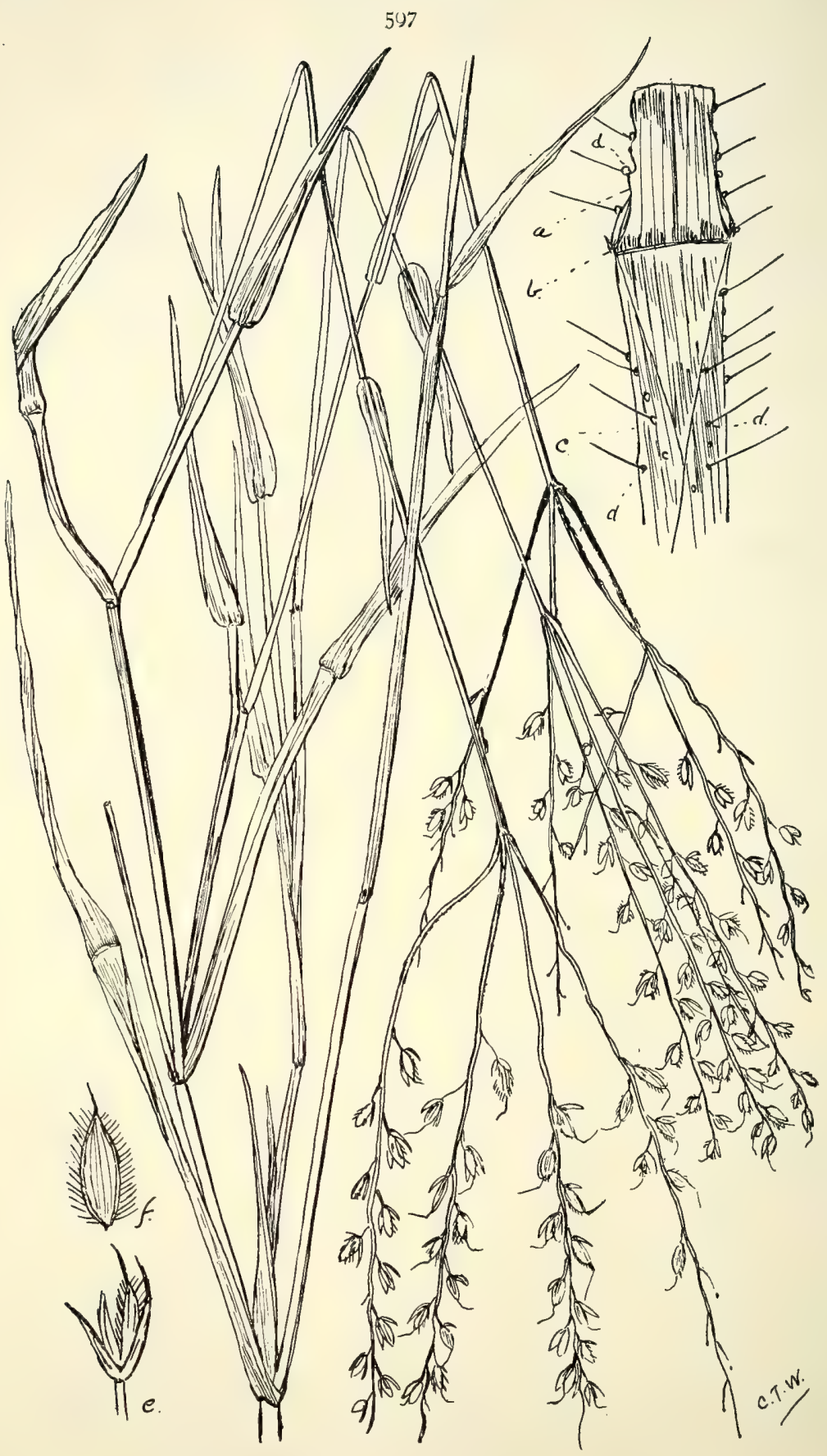

597. Eriachne melicacea, F. v. M., var.

(a) Base of leaf, (b) ligula, (c) top of leaf-sheath, (d) tubercles at base of hairs, (e) a spikelet, (f) a flowering glume. All enlarged. 
*Holcus, Linn.

Subtribe II.--Euavenece.

lanatus, Linn.-Yorkshire-fog. Some say, a weed of little value as a fodder; others, again, say the word is from "Fogan," old name for Moss.

*Avena, Limn.

fatua, Limn.-Wild Oats of the Mediterranean regions.

Anisopogon, R. Br.

avenaceus, $R$. $B r$.

Danthonia, $D C$.-All the kinds are good pasture-grasses.

Section Monachathera.

bipartita, F.v.M.-A good pasture-grass.

Section Eudanthonia.

(A11 Queensland species of this section are included by Mueller in his species $D$. penicillata.)

pallida, R.Br.-White-topped Grass; an excellent pasture species.

longifolia, R. Br.

racemosa, R. Br.-Excellent for downs pasture.

var. obtusata, F.v. M.

var. biaristata, Benth.

var. multiflora, Benth.

pilosa, R. Br.

semiannularis, R. Br.

\section{Tribe VIII.-ChLORIDEÆ.}

Cynodon, Pers.

dactylon, Pers.-Common Couch; a rather troublesome weed in cultivation, but for feeding stock it would be hard to find its equal.

tenellus, $R . B r .=$ Microchloa tenella, Domin.

convergens, F.v.M. $=$ M. convergens, Domin.

Chloris, Linn.-The species are usually found useful pasturegrasses.

unispicea, F.v.M.-Some Tarampa specimens bore three spikes on the same root with the single-spiked stalks.

pumilio, R. Br. (Fig. 598.)

pectinata, Benth.-An excellent pasture-grass.

divaricata, R.Br.-Star Grass.

acicularis, Lindl.

truncata, $R . B r$.

ventricosa, $R . B r$.-Blue Star Grass.

var. tenuis, Benth. 
Chloris-contd.

virgata, $S w .=C$. barbata, var. decora, Benth.-Rhodes Grass.

barbata, Sw.-Not so common in Queensland as C. virgata.

*Gayana, Kunth.-Rhodes Grass. Tropical Africa.

*distichophylla, Lagasca.-Tassel Grass. A South American species, both ornamental and useful.

scariosa, F. v. M.--Very ornamental. (Fig. 599.)

Astrebla, F.v.M.

pectinata, F.v.M.-Common Mitchell Grass; the common Comb-ear variety.

var. triticoides, Bail., Q1. Bot. Bull. xiii. (1896) I5.Wheat-ear Mitchell Grass; the tall, hard, coarse variety.

var. curvifolia, Bail., l.c.-Curly Mitchell Grass. This and the last are excellent pasture-grasses.

var. elymoides, Bail., l.c.-Weeping Mitchell Grass.

Eleusine, Gartn.

aristata, Ehrenb. =E. glancophylla, Munro; E. scindica,

Duthie.-An excellent lawn grass, and useful for growing under the shade of trees; cannot say if really indigenous, but have received specimens from Port Darwin and places in this State where it could hardly have been introduced.

ægyptiaca, Pers.-Button Grass. "Ya-ra-ka” of natives.

indica, Gartn.-Crowsfoot Grass; a common weed in cultivation; is usually a very tough, coarse fodder and will not stand the cold weather.

var. monostachya, Bail.

verticillata, Roxb.

Leptochloa, Beauv: (Included in Eleusine by Mueller.)

subdigitata, Trin.-Cane Grass. A shrubby species found growing around inland water.

chinensis, Nees.-A good pasture-grass.

polystachya, Benth.

\section{Tribe IX.-Festuceæ.}

Subtribe I.-Pappophorea.

Pappophorum, Schreb.

nigricans, $R . B r$. (including the two pretty forms $P$. gracile and $P$. purpurascens of R. Brown).- "Twill-ka" of Georgina River natives. avenaceum, Lindl. 
Subtribe II.-Arundinea.

Phragmites, Trin.

communis, Trin.-Common Reed. The Northern form is of a more delicate texture in all its parts, and is relished by stock.

Subtribe III.-Sesleriece.

Elytrophorus, Beauv.

articulatus, Beauv. (Fig. 6oo.)

Lamarckiana, Monch.

aurea, Mcnch.-An ornamental grass often met with as a stray from garden culture; contains prussic acid. Mediterranean Region.

Kœleria, Pers.

Subtribe IV.-Eragrostea.

phleoides, Pers.

Triodia, R.Br.-The first growth of all our kinds, after being burnt off, is relished by stock.

Mitchelli, Benth.-Warrego Spinifex.

pungens, R. Br.

Cunninghamii, Benth.

irritans, R. Br.-Porcupine Grass.

Diplachne, Beauv.

loliiformis, F.v.M.

Muelleri, Benth.

fusca, Beanv.-A good pasture-grass furnishing good succulent feed; often met with near brackish water.

parviflora, Benth.

Triraphis, R. Br.

mollis, $R$. Br.-A good pasture-grass.

plingens, $R . B r$.

Eragrostis, Beauv.-Love Grasses; good pasture-grasses in most situations.

Section I.-Chaunostachya.

interrupta, Beauv., var. tenuissima, Staph. = E. tenella, Beauv.; Poa tenella, Linn.

trachycarpa, Domin in Fedde. Repert. ix. (I9II) $55^{2}=$ nigrä, Nees., var. trachycarpa, Benth.

imbecilla, Benth.

leptocarpa, Benth.

megalosperma, F.v.M.

pilosa, Beauv--Weeping Love Grass; an annual, and a common garden weed, but a good pasture-grass.

leptostachya, Steud. 


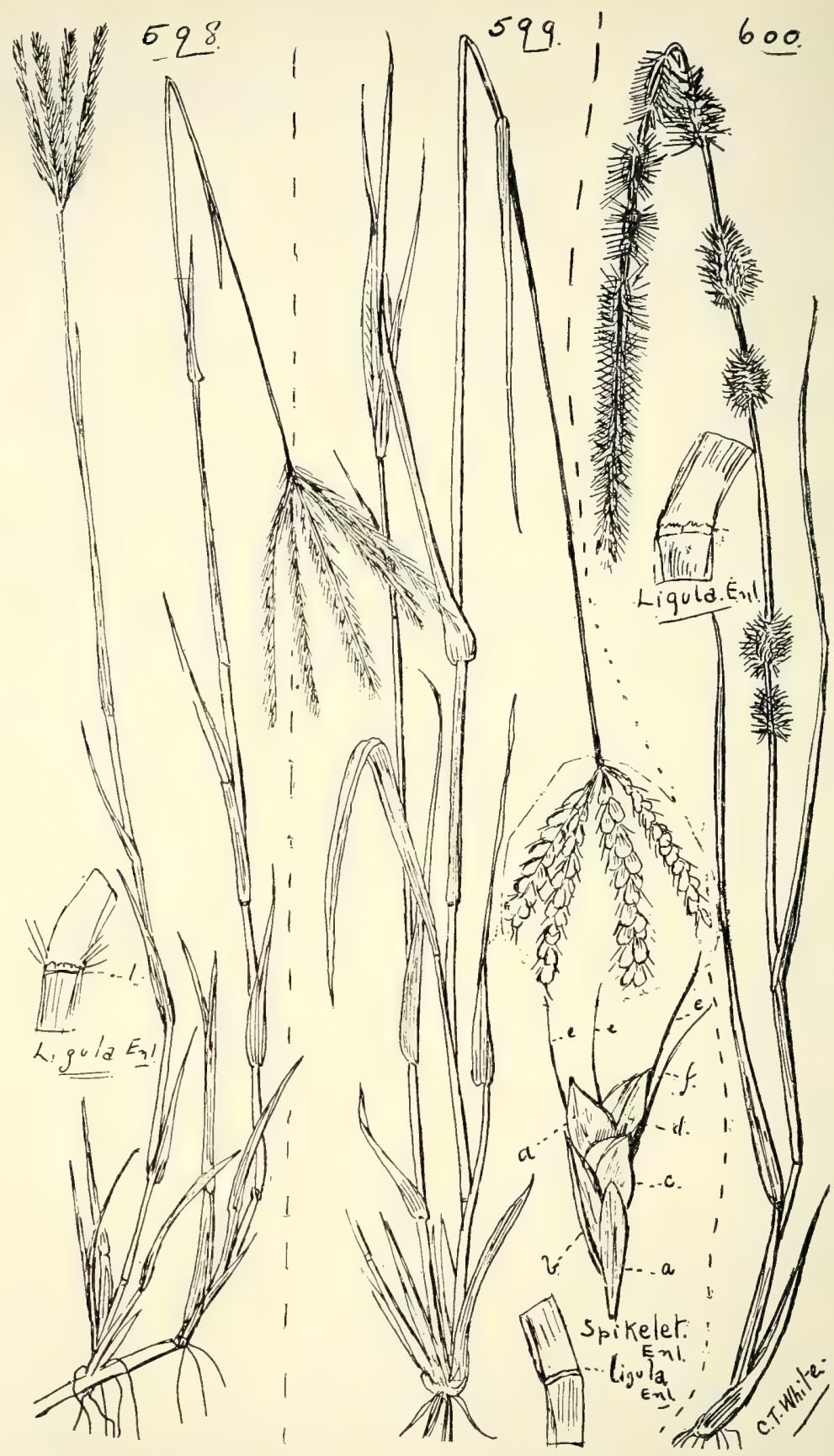

598. Chloris pumilio, $R . B r$.

599. C. scariosa, F.v. $M$.

(a) Ist (lowest) glume, (b) and glume, (c) 3rd (flowering) glume, (d), (e), and (f) terminal empty glumes and awns. (a) -(f) enl. 


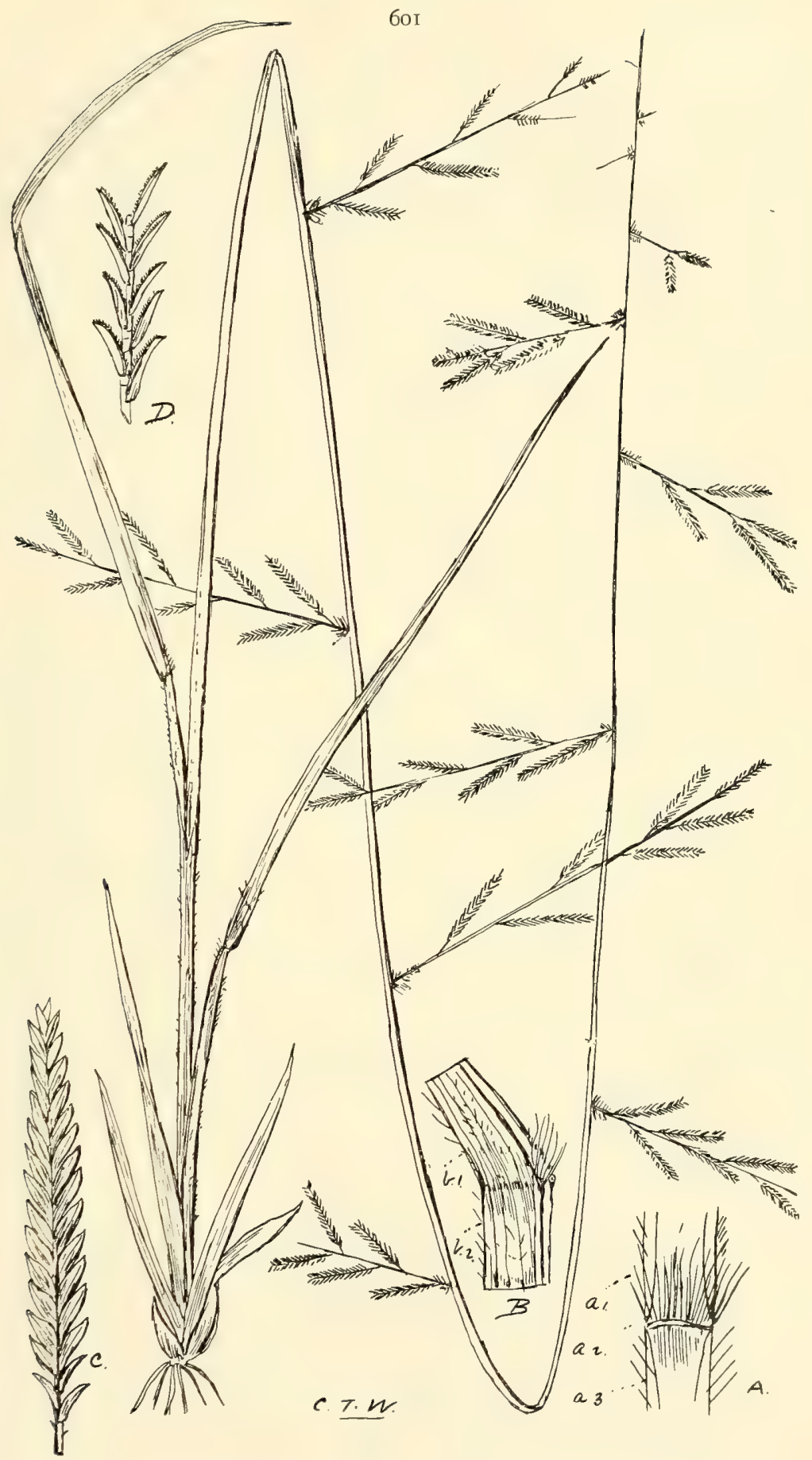

6oi. Eragrostis Brownit, Nees, var. pUbescens. Bail.

(aI) Base of leaf, (az) ligula, (a3) top of leaf-sheath, (bi) base of leaf, (b2) top of leaf-sheath, (C) spikelet, (D) rhachis of spikelet and outer glumes. 
Eragrostis-contd.

Section II.-Megastachya.

diandra, Steud.

Brownii, Nees. $=$ Poa polymorpha, R. Br.-Common Love Grass; excellent for pasture.

var. interrupta, Benth.-There are two distinct forms of this variety; the one most frequently met with on the coast attains the length of several feet; the one common in our pastures is more upright in growth, with slender stems, and the cluster of spikelets much smaller.

var. patens, Benth.

var. schistacea, Bail., Q1. Bot. Bull. xvi. (I903) 2.

var. pubescens, Bail.-A very delicate grass. (Fig. 6or.) concinna, Steud.

speciosa, Steud.-By some this is placed as a form of $E$. Brownii. It is a tropical grass, and one of the best fodder species for those parts. I do not, however, find any of my specimens with obtuse glumes.

laniflora, Benth.

eriopoda, Benth.

chætophylla, Steud.

Rankingi, Bail.-A delicate but good pasture-grass.

stricta, Bail.--Only known from a fragmentary specimen from the Walsh River.

Section III.-Cylindrostachya.

lacunaria, $F \cdot v \cdot M$.

falcata, Gaudich.

stenostachya, Steud.

bifaria, var. australiana, Bail.-Only known from the Walsh River.

Ectrosia, R. Br.-All are good pasture-grasses.

leporina, $R$. Br.-Hare's-tail Grass.

var. micrantha, Benth.

Gulliveri, F.v.M.

Heterachne, Benth.

Brownii, Benth.-A good pasture-grass.

Gulliveri, Benth.-A good pasture-grass.

\section{Subtribe V.-Centothecee.}

Centotheca, Desv.

lappacea, Desv.-The common Scrub Burr Grass of the tropical scrubs.

var. biflora, Benth. 
*Dactylis, Limn.

Subtribe VI.-Eufestucea.

glomerata, Limn.-Cock's-foot Grass. Europe.

*Briza, Linn.-The species are very ornamental; some contain prussic acid.

minor, Limn.-Small Quaking Grass. Europe; now widely spread.

maxima, Linn.-Large Quaking Grass. Africa; now widely spread.

Poa, Limn.

cæspitosa, Forst.-A good pasture-grass.

var. latifolia, Benth.-An excellent mountain fodder.

var. australis, Benth.

nodosa, Nees.

*annua, Linn.-Europe. Of little value for fodder; early introduced into Australia.

*arachnifera, Torr.-Texas Blue Grass. This excellent grass has become naturalised, and is spreading on some parts of the Darling Downs.

Glyceria, R. Br.

Fordiana, F.v.M.

fluitans, R. $\mathrm{Br}$.

ramigera, $F$. v. $M$.

latispicea, $F . v . M$.

Festuca, Linn.

bromoides, Limn.

littoralis, Labill.

Bromus, Linn.

arenarius, Labill.-Barley Grass; by some considered a gooa pasture-grass.

*sterilis, Limn--Europe.

*unioloides, H.B. et K.-Prairie Grass; excellent for winter pastures. America.

*mollis, Linn.-Europe.

*Lolium, Linn.

TRIBE X.-HORDEE.

perenne, Limn.-Perennial Rye Grass ; a well-known pasturegrass. Europe and Temp. Asia.

temulentum, Linn.-Drunken Darnel. The "Tares" of Scripture; a bad weed in the wheatfield. Europe and Temp. Asia.

*Triticum, Limn.

vulgare, Vill.-Common Wheat; often met with as a stray from cultivation. 
Agropyrum, J. Gaertn.

scabrum, Beauv.-A good pasture-grass.

*Hordeum, Linn.

murinum, Linn.-Mouse Barley and Wall Barley. It is stated that at the time the seed is ripe this annual grass contains more nutritive matter than at the time of flowering-a contrary result to all other annual grasses. However, it is one of the worst of grasses for fodder.

vulgare, Linn.

var. hexastichon. - Six-rowed Barley. Stray from field culture.

var. distichon. - Two-rowed Barley. Stray from field culture.

Lepturus, R. Br.

repens, $R . B r$.- A tropical coast grass.

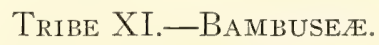

Arundinaria, Mich.

Coboni, Bail.-A Queensland erect Bamboo.

Bambusa, Schreber.

Moreheadiana, Bail.-A climbing Bamboo. Of these two native Bamboos, the flowers are unknown.

\section{Class IV.-ACOTYLEDONS or CRYPTOGAMS.}

\section{Order CLV. - LYCOPODIACEÆ (Club-Mosses).}

Isoetes, $\operatorname{Linn}$.

Muelleri, A. Braun.

Lycopodium, Linn.

varium, R. Br.

phlegmarioides, Gaudich. $=$ L. tetrapterygium, Bail.-Tasselled Club-Moss.

phlegmaria, Linn.-Tasselled Club-Moss.

Claræ, Bail.-_"Goorigen" of Barron River natives. (Fig. 602.)

Drummondii, Spring $=$ L. carolinianum, Linn.

cernuum, Linn.

densum, Labill.

laterale, $R . B r$.

Selaginella, Spring.

Preissiana, Spring.

uliginosa, Spring.

australiensis, Baker.

Bakeriana, Bail. = S. leptostachya, Bail. (Fig. 6o3.) 
Selaginella-contd.

brisbanensis, Bail.

flabellata, Spring.

proniflora, Baker $=$ Lycopodium Belangeri, Bory.

pumilio, Spring.

Azolla, Linn.

pinnata, R. Br.

rubra, R.Br.-Red Water-Moss.

Tmesipteris, Bernh.

tannensis, Bernh. = Psilotum truncatum, R. Br.

Psilotum, Szo.

triquetrum, Sw.-A tropical and subtropical species of the

New and Old World.

var. falacinum, Domin in Fedde. Repert. ix. (I9I I) p. 55I. complanatum, Sw.-A tropical species. (Fig. 604.)

\section{Order CLVI. - MARSILEACE $\approx$ (Pepperworts).}

Marsilea, Linn.

Brownii, A.Br.

angustifolia, R. $B r$.

hirsuta, R. Br.

Drummondii, A. Braun $=$ M. Macropus, Hook.-Nardoo. (Fig. 605.)

var. Muelleri, $A \cdot B r$.

var. elata, $A \cdot B r$.

\section{Order CLVII.-FILICES (Ferns).}

(For figures of the Queensland Ferns see "Lithograms of the Ferns of Queensland.”)

Tribe I.-Ophioglossez.

Fronds not circinate, the barren ones leaflike, the fertile spikelike, simple or branched, the stipes (stalk) of both frequently combined at the base. Spore-cases globular, 2-valved, without any ring, sessile in 2 rows or in small clusters on the spike or its branches.

Ophioglossum, Linn.

vulgatum, Linn.-Adder's-tongue.

var. gramineum, Bail.

var. minutum, Bail.

pendulum, Linn.-Ribbon Fern; spikes sometimes forked.

Botrychium, Sw.

ternatum, Sw.-Grape Fern.

Helminthostachys, Kaulf.

zeylanica, Hook. 

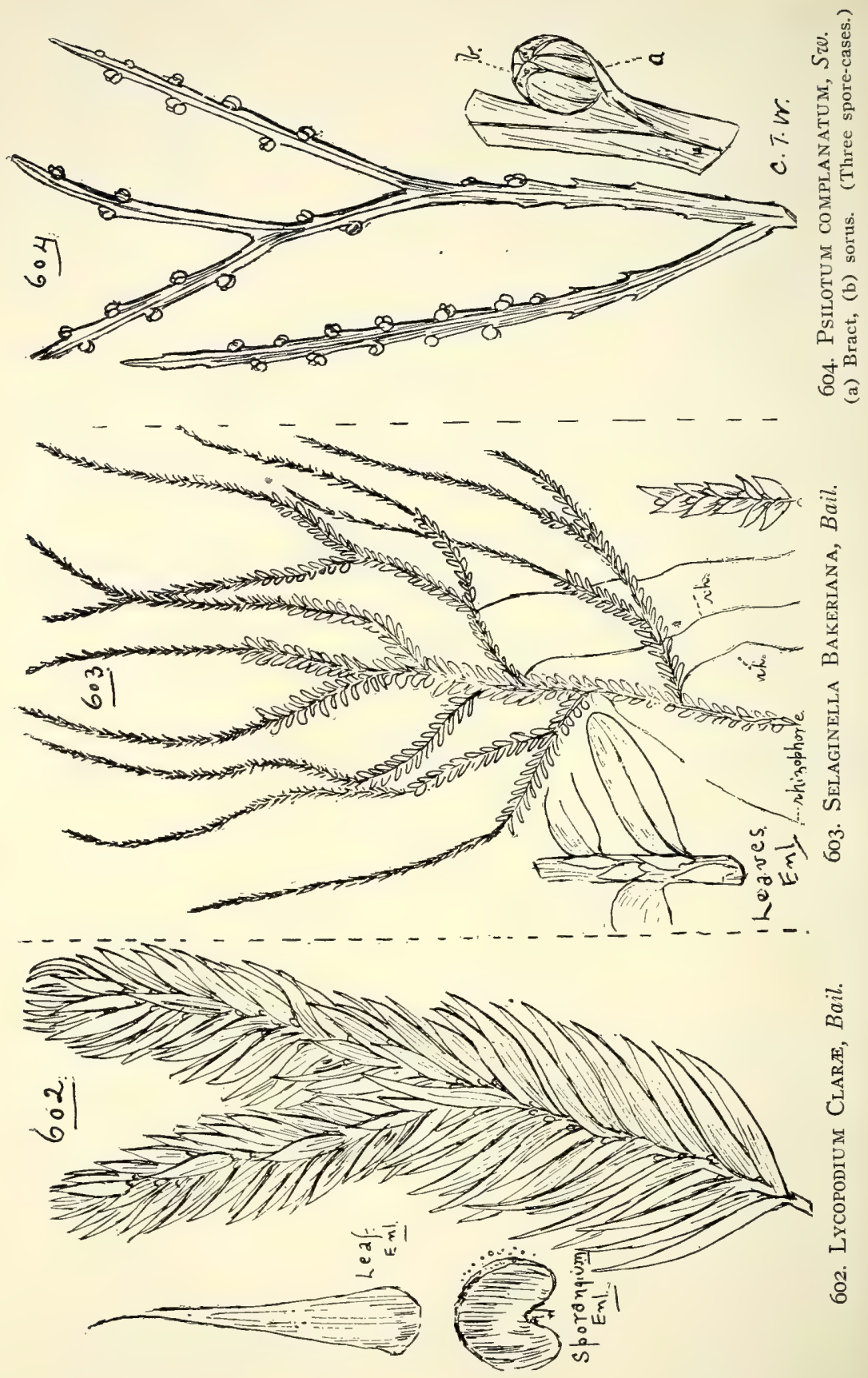


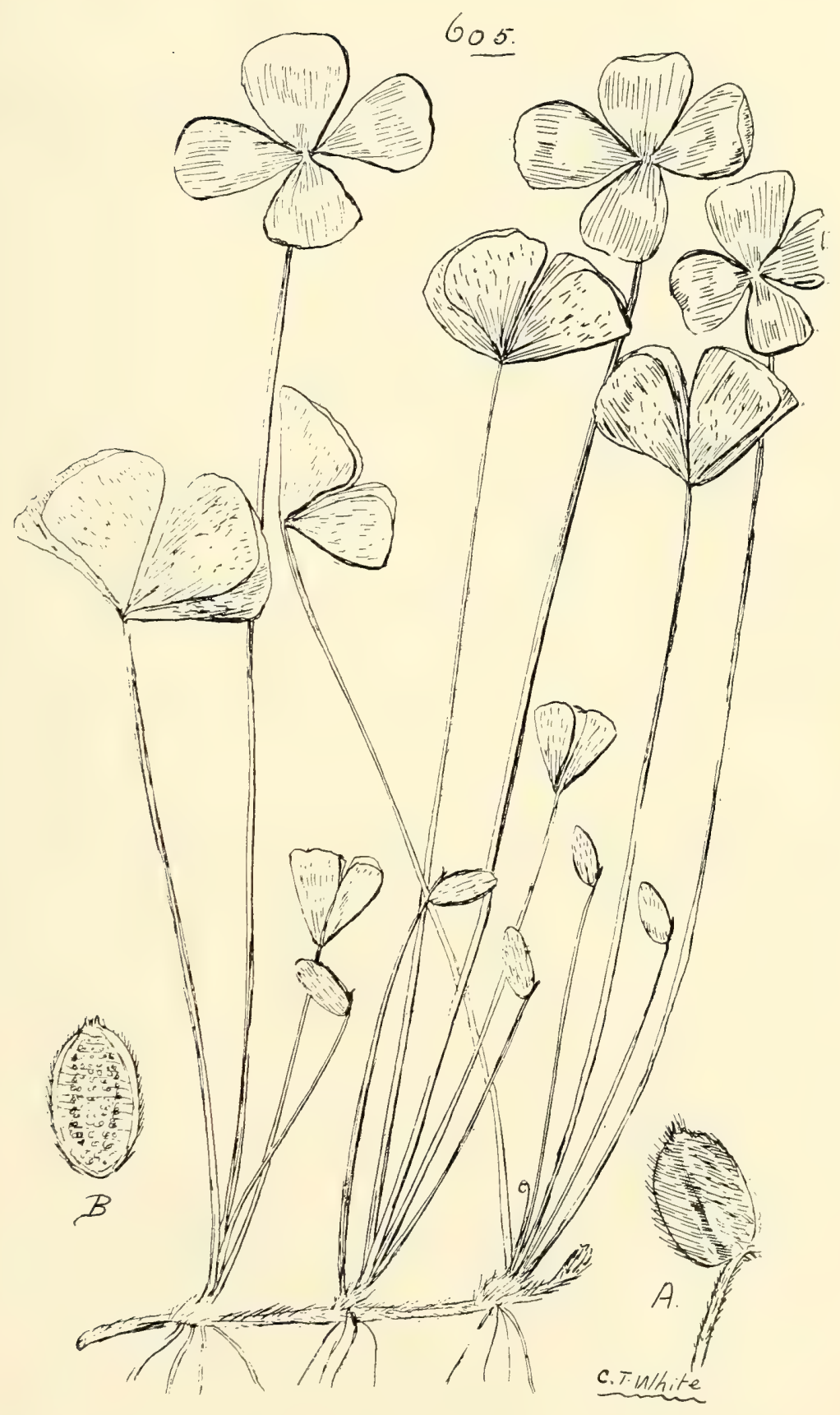

605. Marsilea Drummondi, A. Braun.

(A) Involucre, (B) section: (A) and (B) enl. 


\section{Tribe II.-Marattie $\approx$.}

Spore-cases without any perfect ring, opening in 2 valves or in a longitudinal slit, sessile or united, in 2 rows, in sori forming marginal lobes to the segments or placed on their under surface.

Lygodium, Sw.-Snake's-tongue Fern. All climbers, often called "Climbing Maidenhair."

dichotomum, Sw.

scandens, $S w$.

reticulatum, Schkuhr.

japonicum, $S w$.

Schizæa, Sm.

bifida, Sw.

dichotoma, Swe.

Forsteri, Spreng.-Often found growing among the upper roots at the base of Palm-trees.

Angiopteris, Hoffm.

avecta, Hoffm.-This tropical plant is met with in the scrubs of the North Coast line.

Marattia, $S m$.

fraxinea, Sm.-Potato Fern.

Tribe III.-OSMunde...

Spore-cases globular or nearly so without any or with an imperfect or transverse ring, opening in 2 valves or irregularly, few, sometimes solitary, rarely numerous and clustered in sori (a heap of sporangia), on the under surface of the segnents or pinnules.

Ceratopteris, Brongn.

thalictroides, Brongn.-Water Fern.

Platyzoma, R. $B r$.

microphyllum, R. Br.-Braid Fern.

Gleichenia, $S m$.

circinata, Sw.-Parasol Fern.

dicarpa, $R . B r$.

flabellata, R. Br.-Umbrella Fern; contains saponin.

flagellaris, Spreng.

dichotoma, Hook.

Todea, Willd.

barbara, T: Moore.-Swamp Fern-tree.

(Leptopteris) Fraseri, Hook. et Grev.-Crape Fern. Near the summit of Bellenden-Ker is the only Queensland habitat I know of this plant. 
Tribe $I^{\top}$.-Hymenophyllez.

Spore-cases depressed, with a transverse ring, sessile or nearly so on a columnar receptacle within a cup-shaped or 2-lobed indusium, embedded in or protruding from the margin of the frond. Fronds of a thin membranous half-pellucid texture.

Trichomanes, Linn.-Bristle Ferns.

Motleyi, Bosch. = T. Sayeri, F. v. M. and Baker in Ann. Bot. v. (1891) 195, Hook. Ic. P1. 2229.

vitiense, Baker. yandinense, Bail.

peltatum, Baker.-On stems of trees in the tropical scrubs. muscoides, Sw: parvulum, Poir. digitatum, Swartz. cuspidatum, Willd.

Kurzii, Bedd. minutum, $B l$. proliferum, $B l$. Wildii, Bail., Bot. Bull. iv. pyxidiferum, Linn. Filicula, Bory. Barnardianum, Bail. venosum, R. Br. pallidum, $B l$. caudatum, Brackenr. apiifolium, Presl. parviflorum, Poir. javanicum, $B l$. rigidum, Szes.

var. laxum, Bail. johnstonense, Bail. maximum, Blume.

Hymenophyllum, Sm.-Film Ferns.

flabellatum, Labill. javanicum, Spreng. tunbridgense, $S m$. var. exsertum, Bail. trichomanoides, Bail.

Tribe V.-CyatheEe.

Fern-trees. Fronds large compound. Spore-cases small, with a more or less bblique ring, sessile on a slightly raised receptacle, in globular sori on the under surface of the fronds. 
Cyathea, Sm.

Lindsayana, Hook.-Fern-tree.

arachnoidea, Hook.-Fern-tree.

Alsophila, R. $\mathrm{Br}$.

Rebeccæ, F.v.M.-Fern-tree.

var. commutata, Bail., 3rd Suppl. Syn. Q1. F1. p. 9I.-Wig

Fern tree.

Loddigessii, Kunze.-Fern-tree.

excelsa, R. Br.-Fern-tree.

australis, R. Br.-Common Fern-tree. "Kalldi” of Atherton natives.

var. glauca, Bail., Q1. Agric. J1. xxviii. (I9โ2) p. 203.

Leichhardtiana, F.v.M.-Hard-stemmed Fern-tree.

Woollsiana, F. v. M. viii. (I874) I79; Benth., F1. Austr. vii. 712. Of this I have never seen a specimen, and there seems some doubt as to its differing from $A$. Leichhardtiana.

Robertsiana, F.v.M.-Fern-tree; stem smooth.

Tribe VI.-POLYPODIE $æ$.

Habit various. Spore-cases small, with a longitudinal or scarcely oblique ring, numerous and stipitate in sori or patches on the underside or rarely the margins of the frond.

Series $A$.

Dicksonia, L'Her.-Sori covered, at least when young, with an indusium.

antarctica, Labill.-Woolly Fern-tree.

Youngiæ, C. Moore.-Fern-tree.

davallioides, $R . B r$.

Davallia, Sm.

solida, $S w$.

elegans, Sw.

pyxidata, Cav.-Hare's-foot Fern.

pedata, $S m$. = Humata pedata, J. Sm.

dubia, R.Br.-Mountain Bracken.

var. hirsuta, Bail., Q1. Agric. J1. xxviii. (I912) 204.

speluncæ, Baker.

tripinnata, F.v.M.-One of the most beautiful of our Ferns; abundant on Bellenden-Ker.

Vittaria, $S m$.

elongata, Sw.-Tape Fern.

(Tæniopsis) falcata, Kunze. 
Vittaria-contd.

(Tæniopsis) wooroonooran, Bail., 3rd Suppl. Syn. Q1. Fl. I874.-Dr. K. Domin (Fedde. Repert. x. (IgII) p. 57) places this as a variety of $\mathrm{V}$. pusilla, Bl. (= falcata, Fee), and further states that the typical $V$. falcata has not been found in Queensland.

Lindsaea, Dry.

linearis, Sre'.

dimorpha, Bail., Q1. Ferns 1874.

cultrata, Src.

flabellulata, Dry.

lobata, Poir.

microphylla, Sw.

incisa, Prentice in Trim. Journ. Bot. xi. (I873) 295.

Fraseri, Hook.

ensifolia, Szo.

var. heterophylla, Benth.

lanuginosa, Wall.-Epiphyte. Tropical.

Adiantum, Limn.-Maidenhair.

lunulatunı, Burm.

var. dolabriforme, Bail.-Thursday Island (T. Tate). Approaches very closely $A$. dolabriforme, Hook.

æthiopicum, Linn.

forma queenslandiæ, Bail., $n$. form.-Growth erect, fronds scattered, stipites tall, robust, as well as the rhachises very dark-coloured; pinnt1les from small to very large.

forma assimile ( $\mathrm{Sw}$.), Bail. = A. assimile, Sw.-Growth dense, delicate, sometimes weeping, stipites slender, as well as the rhachises of a reddish-brown colour.

forma variegatum, Bail., $n$. form.--Pinnules beautifully pencilled with yellow.

Hab.: Bundaberg (Miss Burkitt).

formosum, R. Br.

var. leptophyllum, Domin in Fedde. Repert. ix. (I9II)

p. 55 I.

affine, Willd.

var. intermedium, Benth.

diaphanum, Bl.

hispidulum, Sw--Five-finger Maidenhair.

var. Fitzalani, Bail., n, var.-This differs from the normal form in being almost glabrous.

Hab.: Daintree River (E. Fitzalan).

Hypolepis, Bernh.

tenuifolia, Bernh.

Cheilanthes, Sw.

tentuifolia, Sw-Curly Fern.

$2 \mathrm{R}$ 
Cheilanthes-contd.

tenuifolia-contd.

var. Sieberi, Bail.

var. nudiuscula, Bail.

caudata, R. Br. (Fig. 606 bis.)

tenuissima, Bail., Q1. Agric. J1. xvii. (I906) 28. (Fig. 606 ter.)

Pteris, Linn. (Including Platyloma, Litobrochia, and Pellæa.)

geraniifolia, Raddi.

paradoxa, Baker.

falcata, R. Br.

var. nana $=$ Pellca nana, Hook.

rotundifolia, Forst.

longifolia, Limn.

ensiformis, Burm.

umbrosa, R. Br.

quadriaurita, Retz.

tremula, R. $B r$.

aquilina, Limn., var. esculenta, Hook.-Bracken; contains prussic acid; suspected of being poisonous to stock. var. lanuginosa, Hook.

incisa, Thunb. $=P$. vespertilionis, Labill.-Bat's-wing Fern. marginata, Borg.

comans, Forst.

Lomaria, Willd. (Including Stegania and Onoclea.)

Patersoni, Spreng.

vulcanica, $B l$.

discolor, Willd.

lanceolata, Spreng.

capensis, Willd. = L. procera, Spreng.-Red-cabbage Fern of some of the Southern States.

euphiebia, Kunze.

Blechnum, Linn.

cartilagineum, $S w$.

var. tropica, Bail., Fern World of Australia (I88I) p. $50=$ B. nitidum, Bail. (non Presl.), Q1. Ferns (1874).

serrulatum, Rich.-At one time largely used as a native food.

"Bungwall" of Moreton Bay, "A1-utha" of Red Island, and "Dugal" of Tully River natives.

Whelani, Bail., 3rd Supp1. Syn. Q1. F1. (I890) p. 92. orientale, Linn.- "Baral " of Cape Bedford natives.

Monogramme, Schkuhr.

Junghuhnii, Hook. = Diciidopteris angustissima, Brackenr. 


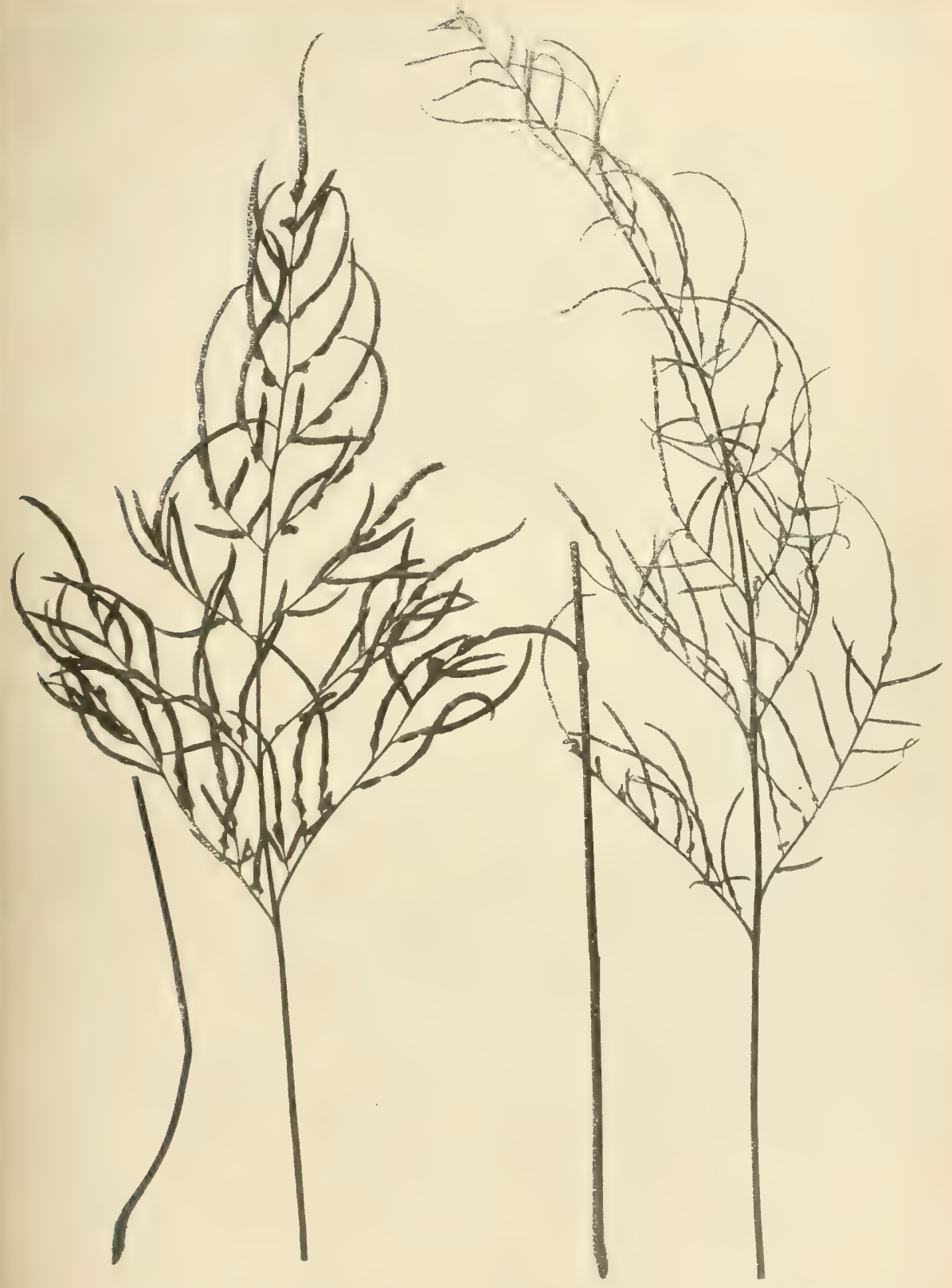


Doodia, R. Br. (Included in Woodwardia by Mueller.) aspera, R. $E r$. var. heterophylla, Bail., Fern World of Austr. (I88I) p. 5I, Syn. Q1. F1. ( I883) 704. caudata, R. Br. var. media, Benth.

Asplenium, Linn.-Spleenwort.

Section I.-Euasplenium.

nidus, Limn.-Bird's-nest Fern.

var. multilobum, Bail., Q1. Agric. J1. i. (I897) 370, with Plate. (Fig. 607.)

simplicifrons, F.v.M. var. laciniatum, Bail., Bot. Bull. (I896) xiii. p. I6, P1. I. attenuatum, R. Br.

var. multilobum, F.v.M.

var. Schneideri, Bail., Syn. Q1. F1. (I883) 706.

var. integrum, Bail., l.c.

flabellifolium, Cav.

paleaceum, R. Br.

falcatum, Lam.

var. Whittlei, Bail.

Wildii, Bail., Bot. Bu1l. iv.

marinum, Linn., var. difforme, Hook.; Bail., Ist Suppl. Syr. Q1. F1. p. 63.

obtusatum, Forst.

resectum, $S m$, var. australiense, Bail.

furcatum, Thunb.

affine, Sic.

laserpitiifolium, Lam.

Hookerianum, Colens, var., Bail.

Section II.-Darea.

bulbiferum, Forst.

flaccidum, Forst.

Section III.-Athyrium.

umbrosum, J.Sm.-Caraway-seed Fern.

var. tenera, Bail, Syn. Q1. F1. (1883) 708.

Section IV.-Diplasium.

Prenticei, Bail., Proc. Linn. Soc. N. S. Wales, iv. (I880) 37. japonicum, Thunb.

sylvaticum, Presl.

maximum, Don.

polypodioides, Metten.

Section V.-Anisogonum.

decussatum, Siv. 
Aspidium, Sw.

Section I.-Nephrolepis.

cordifolium, $S w .=$ A. tuberosum, Bory. exaltatum, Szo.

var. longipinna, Benth.

ramosum, Beauv.-Climbers.

var. lineare, Bail., 3rd Supp1. Syn. Q1. F1. (г890) 93.

var. etmundi, Bail. = A. cumundi, Bail.

Section II.-Nephrodium.

unitum, Sw._ "Tchungooin" of Bundaberg natives.

var. propinquum, Bail.- "Ngar-pul" of Cooktown natives.

pteroides, Sw.

var. terminans, Bail.

molle, Sw._ "Binung" of Bundaberg natives.

var. didymosorus, Benth.

var. decurtatum, Bail., Bot. Bull. v.-Only known from Stradbroke Island.

truncatum, Gaudich.

var. Keffordii, Bail. = A. molle, var. Keffordii, Bail., Q1.

Agric. Il. (Igo8) p. 242. (Fig. 608.)

Section III.-Sagenia.

confluens, Metten.

Section IV.-Polystichum.

aculeatum, Sw-Lady Fern.

aristatum, Siv.

Section V.-Lastrea.

decompositum, Spreng.

acuminatum, Lorve, var. villocum, Bail.

tenerum, Spreng.

Series B.-No indusium.

Polypodium, Linn

Series I.-Dianeura.

australe, Metten.

Hookeri, Brackenr.

albosetosum, Bail., 3rd Suppl. Syn. Q1. F1. (I890) 94.

blechnoides, Hook.

fuscopilosum, F.v.M. et Baker.

tenellum, Forst.-Climber.

punctatum, Thunb.-Climber.

pallidum, Brackenr.

aspidioides, Bail., Syn. Q1. F1. (I883) 7I4.

var. tropica, Bail., Litho. Ferns Q1. (I892) I46, Q1. F1.

p. Ig82. 
CLVII III.TCIS.

$60 \%$

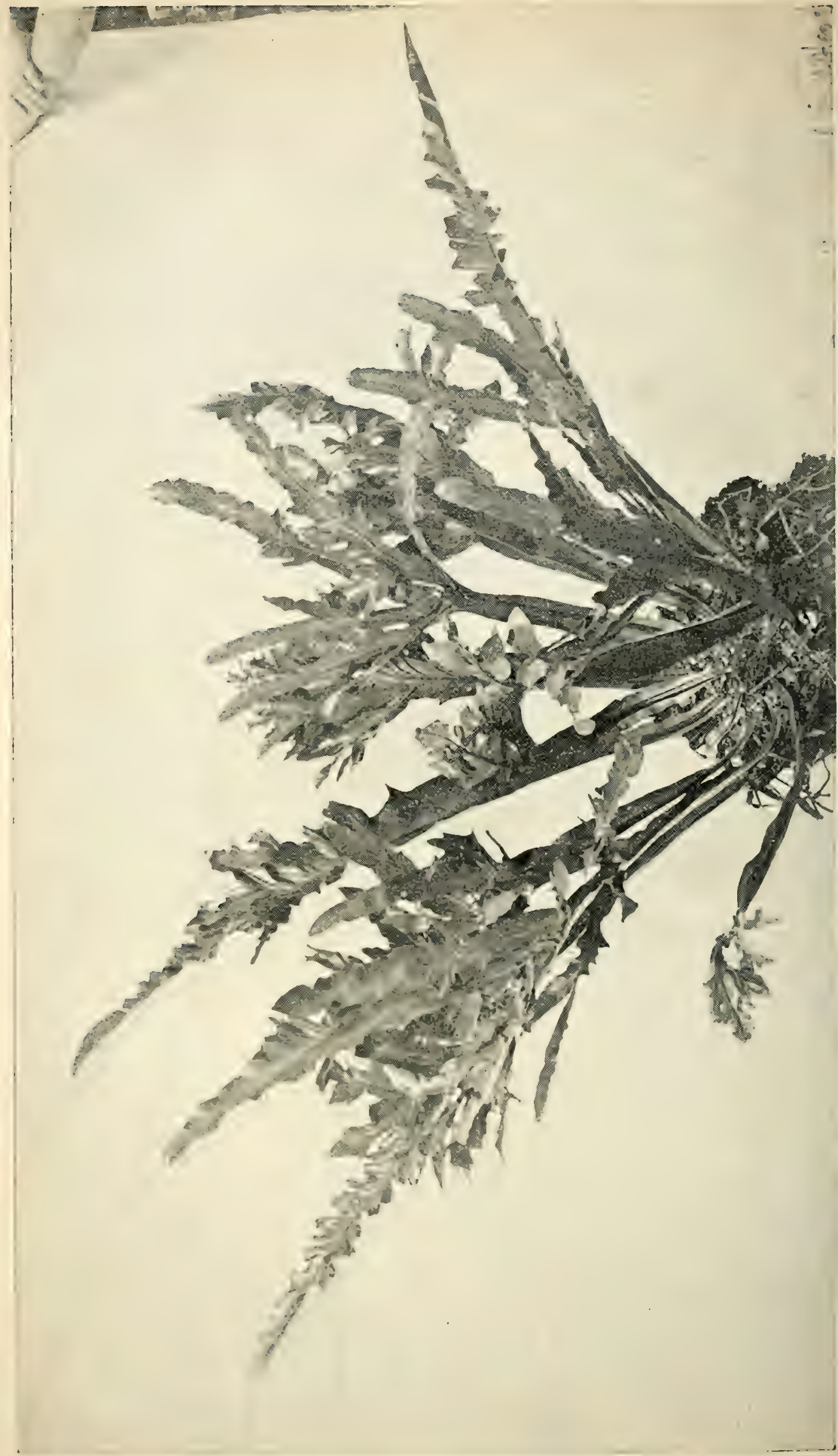

हु' 
CLVII. FILICES.

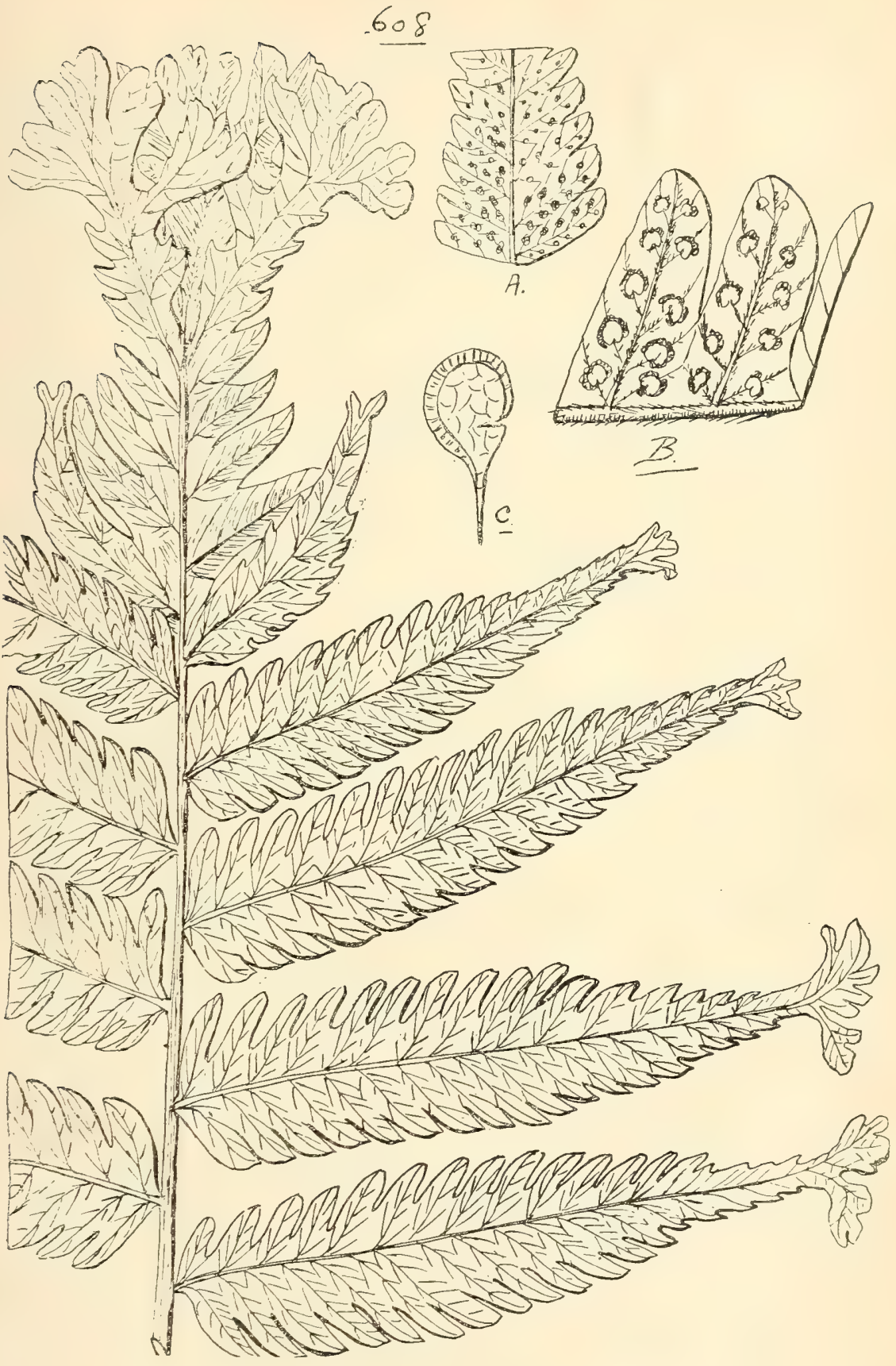

608. Aspidium truncatum, Gaudich., var. Keffordit, Bail.

(A) and (B) Portion under surface of frond, (C) sporangium. (A) nat. size; (B) and $(C)$ enl. 
Polypodium-contd.

Series II.-Synneura.

proliferum, Presi.

urophyllum, Wall.

Hillii, Baker.

pœecilophlebium, Hook.

Series III.-Dictyophlebia.

serpens, Forst.-Climber.

confluens, R. Br.-Climber.

var. lobatum, Bail., Litho. Ferns Q1. (I892) I52.

acrostichoides, Forst.

attenuatum, R. Br.

superficiale, Bl., var. australiense, Bail., Bot. Bull. iv. (I89r)

$2 \mathrm{I}$, with a figure.

simplicissimum, F..$M$.

nigrescens, $B l$.

phymatodes, Limn.

pustulatum, Forst.-Climber.

scandens, Forst.-Climber.

verrucosum, $W$ all.

subauriculatum, $B l$.

rigidulum, Swe.

var. Vidgeni, Bail., Syn. Q1. F1. (r883) 718, Litho. Ferus

Q1. ( 1892 ) I66. (Fig. 608 bis.)

var. Whitei, Bail., Q1. Agric. J1. xxvii. ( I9I I) 306, Pl 22.

(Fig. 609.)

zar. diversipinne, Bail., Q1. Agric. J1. xxviii. (I9I2) 74,

P1. I6. (Fig. 609 bis.)

var. cristatum, Bail., Ist Suppl. Syn. Q1. F1. (1886) 65.

quercifolium, Linn.

var. normale, Bail., Bot. Bull. v. (I892) 28.

var. Linnzi, Bail., l.c.

irioides, Poir.

var. lobatum, Bail., Syn. Q1. F1. (I883) I64, Bot. Bull. xiii.

P1. II. (Fig. 6Iо.)

forma ramosum, Hort. (Fig. 6II.)

forma cristatum, Bail., Q1. Agric. J1. xxvi. (I9II) I99,

P1. 2I, Bot. Bull: xiii. Plate III. (Fig. 6г 2.)

Knightex, Hort. Sander.

Notholæna, R. Br.

pumilio, R. Br.

vellea, R. $B r$.

distans, $R . B r$.

fragilis, Hook. 
Notholrena-contd.

Prenticei, Luerssn.

glabra, Brackenr.

Grammitis, Szo.

Reynoldsii, F.v.M.

Muelleri, Hook.

rutæfolia, R. Br.-Blanket Fern.

pinnata, F.v. M.

membranacea, $B l$.-Climber.

ampla, F.v.M.-Climber.

Meniscium, Schreb.

triphyllum, Sw,--Recorded for Queensland by F. v. Mueller.

Antrophyum, Kaulf. reticulatum, Kaulf.

Acrostichum, Linn.

conforme, Siv.-Deer's-tongue.

scandens, J. Sm.-Climber.

sorbifolium, Linn., var. leptocarpum, Benth-Climber. This

tropical plant is met with at Eumundi.

repandum, $B l$.

Taylori, Bail. in Rep. of Q1. Accli. Soc. April I884.

neglectum, Bail., Syn. Q1. F1. (I883) 722.

aureum, Linn.- “Dai-ingal” of Cape Grafton natives.

spicatum, Linn.-Frond of normal form, entire. (Fig. 612 bis.)

var. Schneideri, Bail., n. var.-Fronds 12 to i 8 in. long, divided into from 2 to 5 narrow lobes; bearing fertile spikes as in the normal form. Found in the hilly parts of Queensland; first met with about twenty years ago, but neglected to be published until preparing the present work. (Fig. 913.)

Hab.: Macpherson Range (Tryon and Schneider); Taylor's Range (F.M.B.).

pteroides, $R . B r$.

Platycerium, Desv.

alcicorne, Desv.-Elk's-horn.

var. Hillii, Bail. = P. Hillii, T. Moore, Gard. Chron.

grande, J. Sm.-Stag's-horn. The liquid composing what may be termed "tears" of the young fronds of this and other species has a sweetish taste, and on testing with Fehling's solution yielded the brick-red precipitate characteristic of grape sugar. It affords an interesting instance of nectar-excretory tissues among the Ferns R. Diimer. Annals of Eotany xxv. (I9II) I205. 

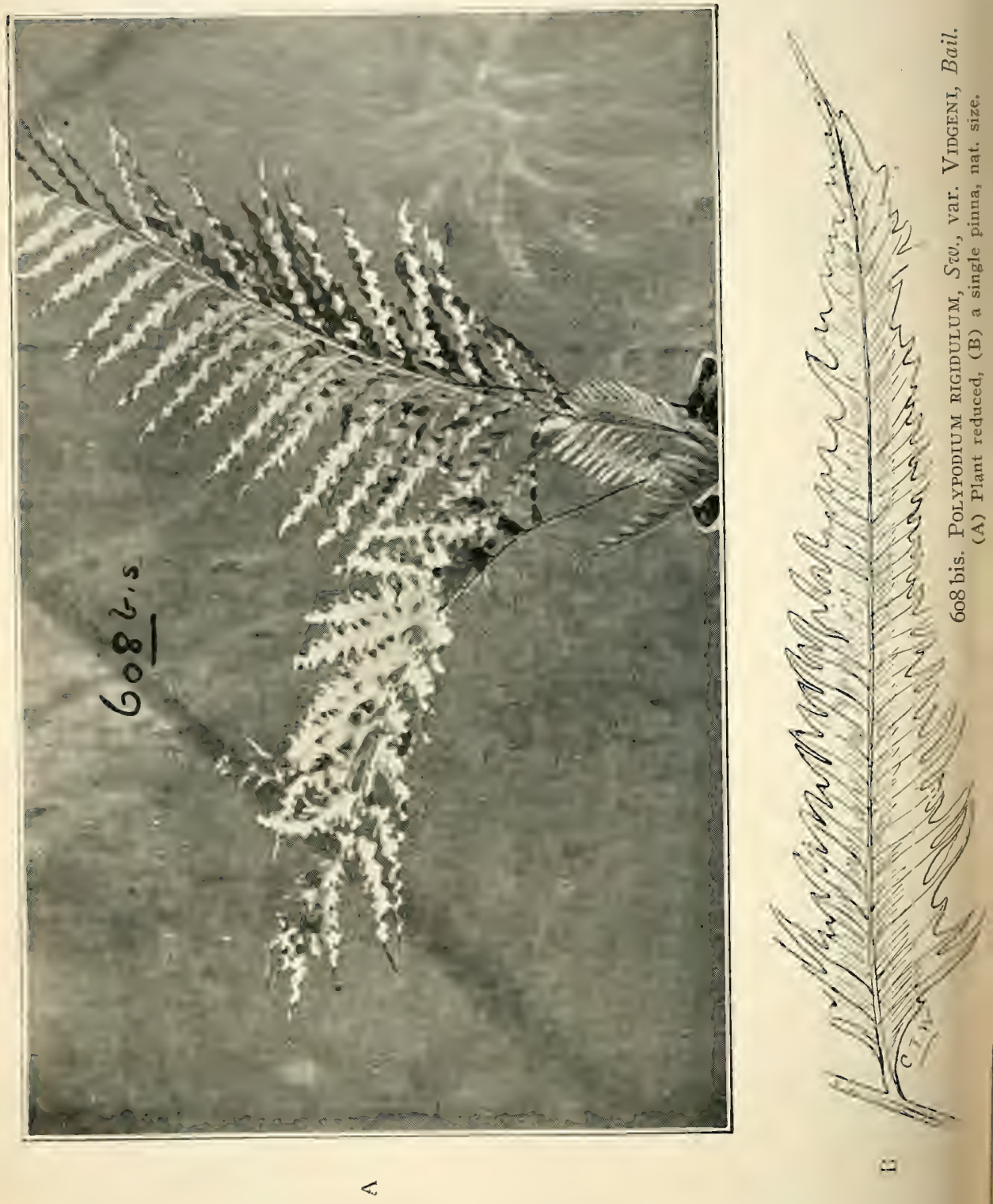
609 bis

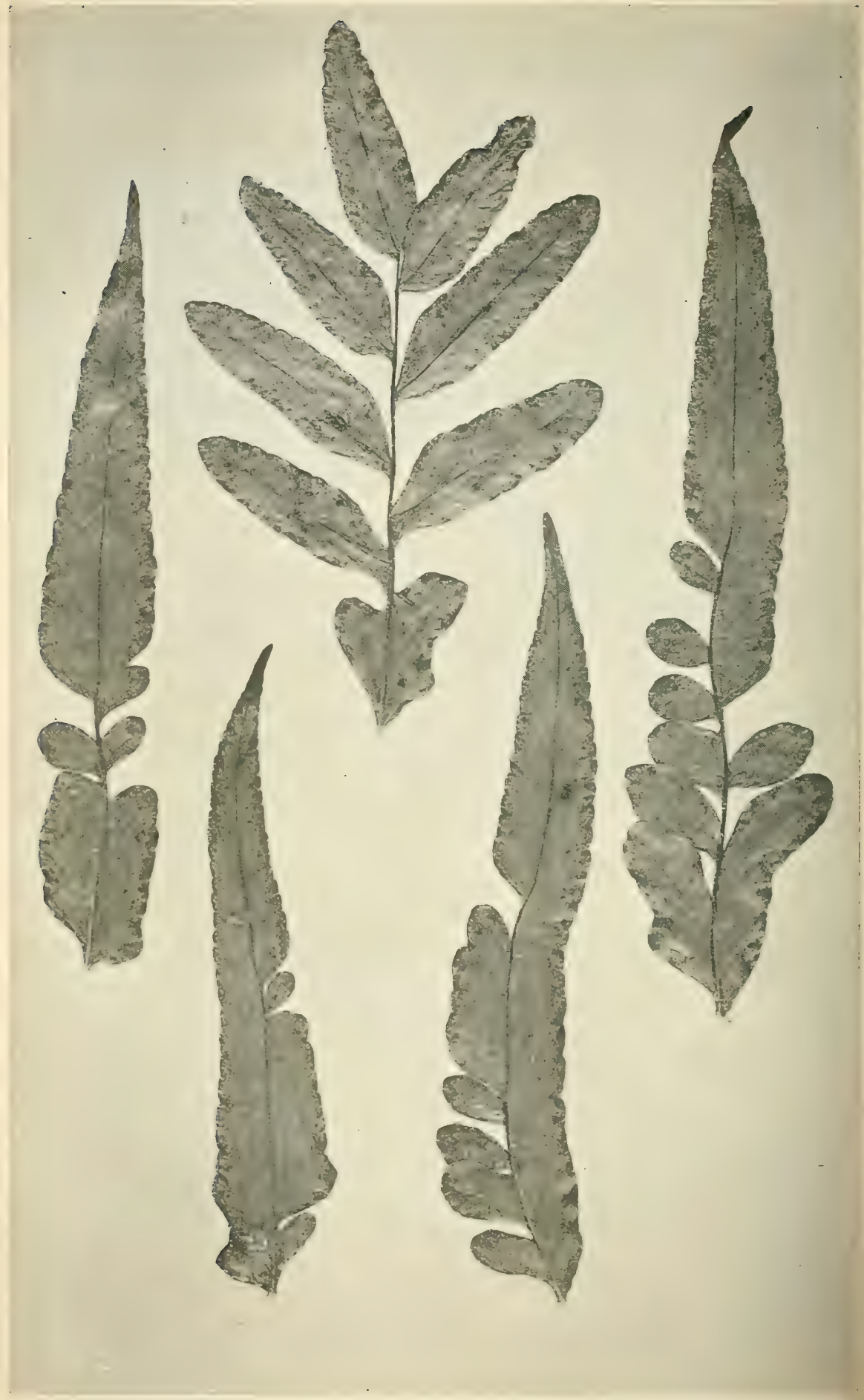

609 bis. Polypodium rigidulum, Sw., var. diversipinne, Bail.

(Five pinnæ from one frond.) 
610
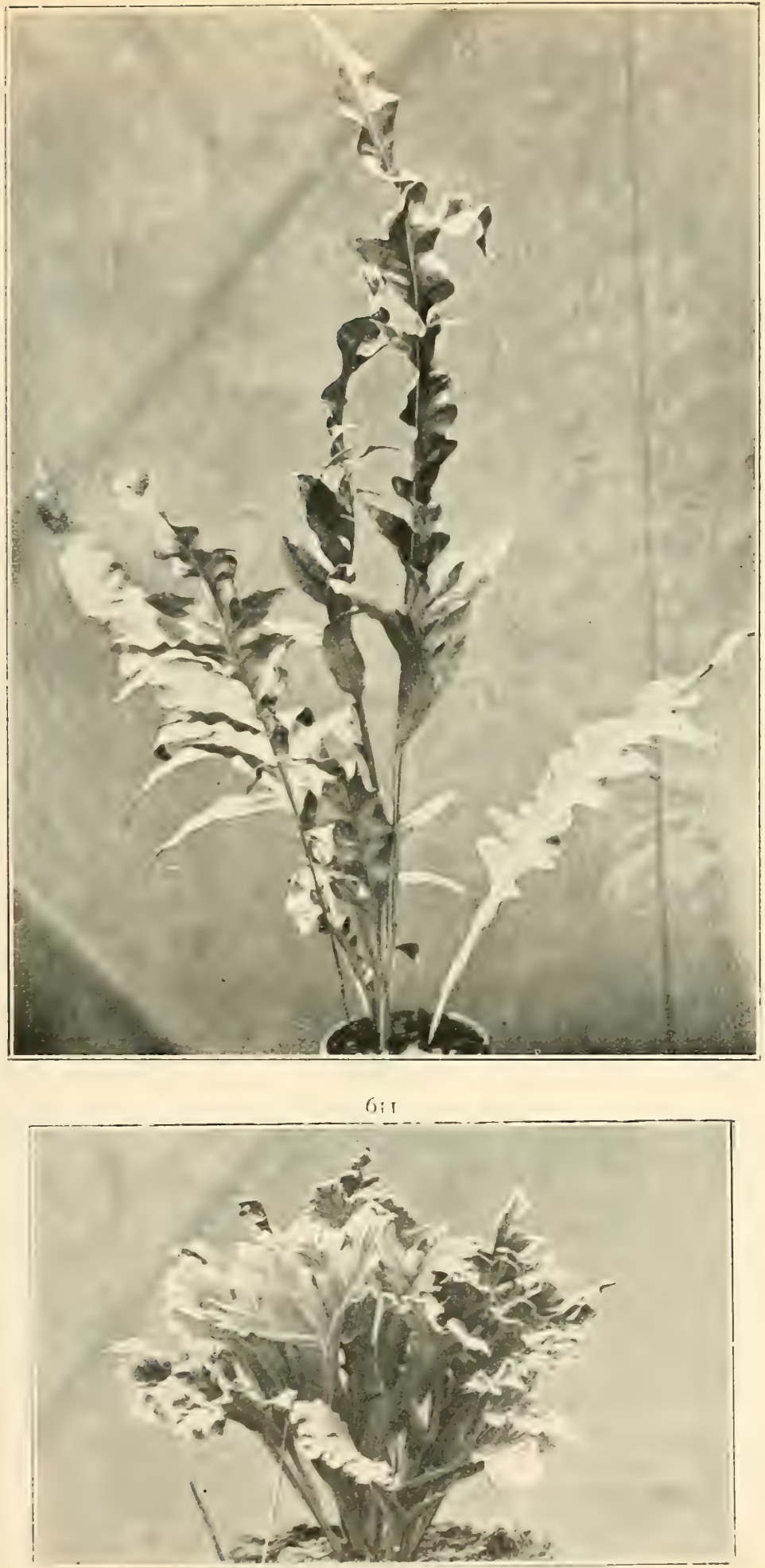

6io. Polypodium irioldes, var. lobatum, Bail.

6ri. P. irioides, var, lobatum, forma ramosum, Hort. 


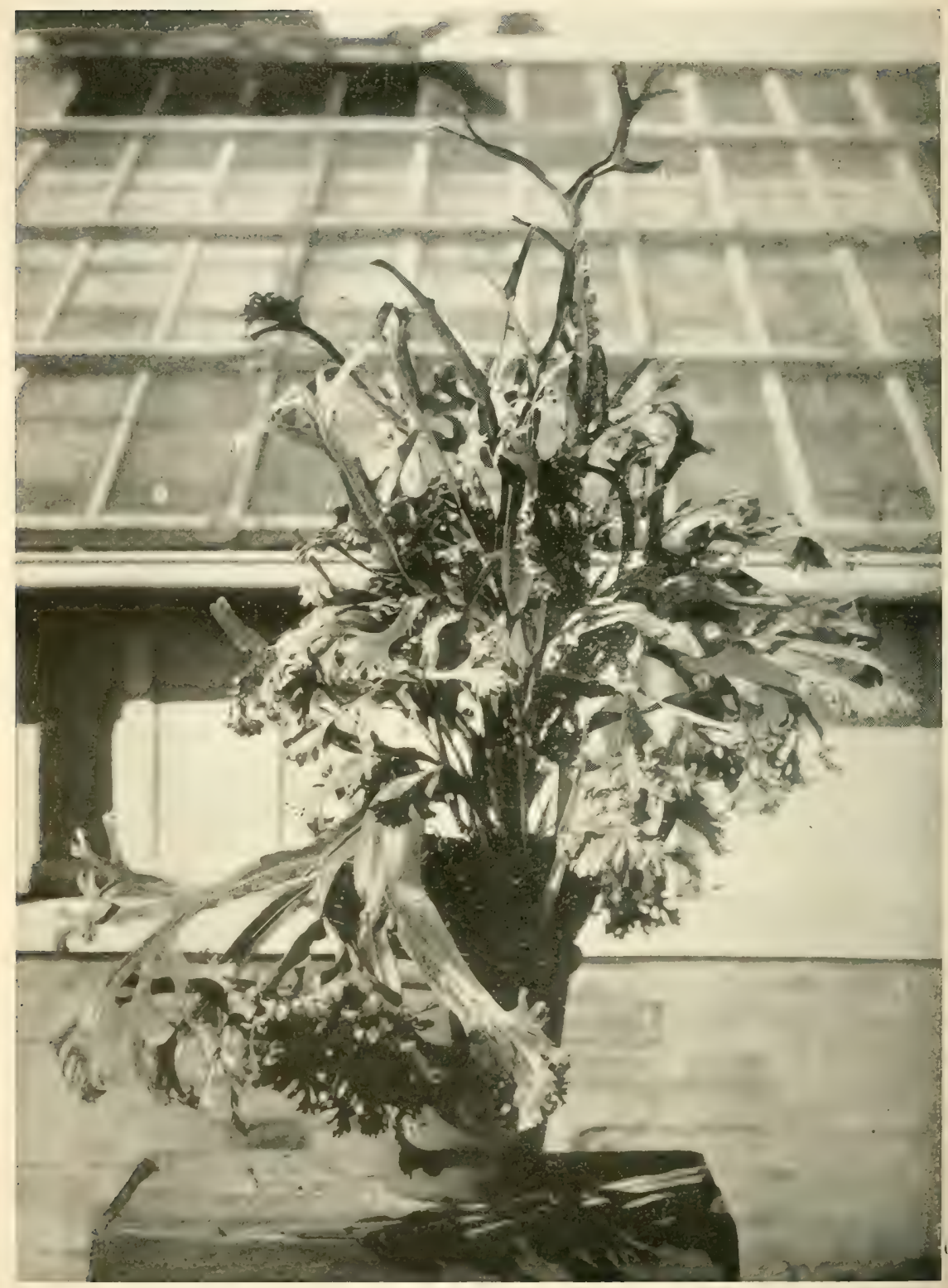

6i2. Polypodum irioides, Poir., var. lobatum, Bail., forma cristatum, Bail. 
CLVIT. FILICES.

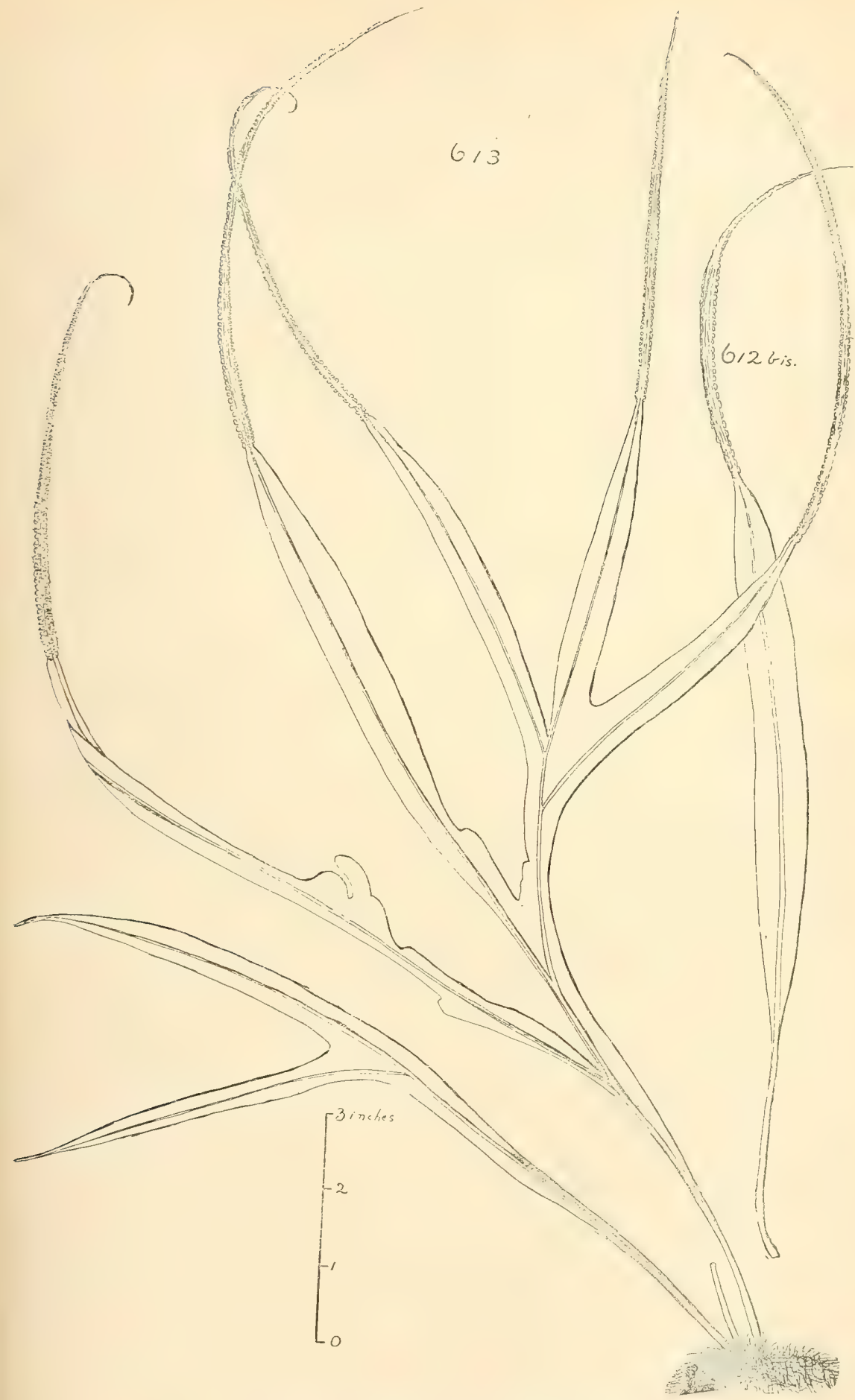

612 bis. Acrostichum spicatum, Limn.

6i3. A. spicatum, vat. Schneideri, Bail.

(Both reduced.) 


\section{Order CLVIII.-MUSCI (Mosses).}

I.-Dicrane.e.

Anisothecium, Mitten.

pycnoglossum, Broth .

Archidium, Bridel.

brisbanicum, Broth.

Bruchia, Schw.

Whiteleggei, C.M.

Ditrichium, Timm.

Muelleri, Hampe.

Baileyi, C.M. (Fig. 6I4.)

affine, C.M.

Hab.: Queensland (Watts and Whitelegge).

viride, C.M.

Dicranella, C. $M$.

Dietrichiæ, C.M.

tenax, C. $M$.

var. longipes, C.M.

tricruris, C.M.

Baileyana, C. $M$.

Trematodon, Rich.

longescens, C.M.

suberectus, Mitten.

brachyphyllus, C. M.

Baileyi, Broth. (Fig. 6r5.)

Ceratodon, Bridel.

purpureus, Bridel.

stenocarpus (Mont.), Br. $=$ C. purpureus, var. palustris, Mont.

Holomitritu, Bridel.

Dietrichiæ, C. $M$.

Muelleri, Hampe et C. M.

perichætiale, Brid.

corticola, Broth.

Dicranum, Hedrvig.

dicarpum, Hornsch.

austrinum, Mitten.

suberectum, Hampe. (Fig. 6i6.)

calymperidium, C.M.

Baileyanum, C.M.

Pœcilophyllum, Mitten.

Leichhardti, Hampe et C.M. 
Leucoloma, Brid.

clavinervis, C. $M$.

Sieberianum, Hsch.

forma rigida, Broth.

austro-scoparium, C. $M$.

subintegrum, Broth.

serratum, Broth. (Fig. 6I7.)

clavinerve. C. $M$.

Baueræ, C.M.

Sclerodontium, Schwoegr.

pallidum, Hook.

Leucophanes, Brid.

at1strale, Broth.

apiculatum, Hampe.

Campylopus, Bridel.

introflexus, Hedru.

pudicus, Hornsch.

Woollsii, C.M. (Fig. 6r8.)

torquatus, Mitten.

subtorquatus, C.M., var. flavifolius, C.M.

Dietrichiæ, C.M.

Whiteleggei, C.M.

appressifolius, Mitt.

viridicatus, C.M.

Grimmia, Ehrhart.

II.-GRIMMIE.⿸.

Eugrimmia.

trichophylla, Grev., Scott. Crypt. F1. ii. (I824) Ioo.

Guembelia.

leucophrea, Grev.

\section{Rhacomitrium.}

Sundaica, C. $M$.

pulvinata, Hook. et Tayl.

var. obtusa, Brid. et Sch.=G. cygnicolla, Tayl., Hook.

Lond. J1. Bot. v. ( I 846 ) 45, ct. 449.

leiocarpa, Tayl.

Glyphomitrium, Bridel.

Muelleri, Mitten. (Fig. 6r9.)

commutatum, C.M.

III.-LEUCOBRYET.

Octoblepharum, Hedre.

albidum, Linn.

Leucobryum, Hampe.

brachyphyllum, Hampe.

Hab.: Rockingham Bay (Dallachy), (Watts and Whitelegge). 
Leucobryum-contd.

candidum, Schregr.

conocladulum, C.M.

Hab.: Rockingham Bay (Dallachy), (Watts and Whitelegge).

Baileyanum, C.M. (Fig. 620.)

vesiculosum, C. $M$.

Hab.: Rockingham Bay (Dallachy), (Watts and Whitelegge). strictifolium, Broth.

Hab.: Rockingham Bay (Dallachy), (Watts and Whitelegge). Wattsii, Broth.

\section{IV.-SYRRHOPODONTEA.}

Syrrhopodon, Schwogr.

fimbriatus, C. M. =S. fimbriatulus, C. M.

mamillosus, C. $M$.

novæ-valesiæ, $C . M ., f$. microcarpa.

(Thyridium) undulatus, Broth., Aus. Mosses No. I47.

(Thyridium) subfasciculatus, Hampe.

Calymperes, Bridel.

Kennedyanum, Hampe.

Motleyi, Mitt.

repens, Harvey.

latifolium, Hampe.

(Hyophilina) panduræfolium, Broth., Aus. Mosses No. Ioo. (Hyophilina) nigrescens, Broth., Aus. Mosses No. I48.

$$
\text { V.-Tortuleж. }
$$

Acaulon, C.M.

brisbanicum, C.M.

Phascum, Linn.

perpusillum, C.M.

cylindricum, Tayl.

Gymnostomum, Hedw.

calcareum, Nees. et Hornsch.

Hymenostomum, R. Br.

pertostile, C.M.

Weisia, Hcdw.

flavipes, Hook. f. and Wils.

Hab.: Queensland (Watts and Whitelegge).

nuda, Mitten.

viridula, Brid.

pimpamæ, C.M. (Fig. 621.)

graciliseta, Broth.

perlinearis, C. M. 
Weisia-contd.

(Hyophila) pugionifolia, C.M. (Fig. 622.)

(Hyophila) angustifolia, C.M.

(Hyophila) squarrosa, Broth.

(Hyophila) tenera, Broth.

Leptodontium, Hanpe.

australe. C.M.

Tortula. Hedw.-Screw Moss.

austro-muralis, C. $M$.

calycina, Schro.

subcalycina, C.M.

(Barbula) Wildii, Broth.

(Barbula) calycina, Schwegr.

(Desmatodon) nervosa, Bridel $=T$. atrovirens $(\mathrm{Sm}$.$) Lindb.$

Tortella, C. $M$.

perhumilis, Broth.

\section{VI.-ORTHOTRICHET.}

Macromitrium, Bridel.

Macrocoma.

encalyptorum, C.M. (Fig. 623.)

Dæmellii, C.M.

Goniostoma.

pusillum, Mitt.

microstomum, Hook. et Grev.

Scottiæ, C.M.

pallido-virens, C.M.

Archeri, Mitt.

caloblastum, C.M.

caloblastoides, C.M.

dimorphım, C.M.

mucronulatum, C.M. $=$ M. Watsii, Broth., Aus. Mosses No. Io6. (According to Whitelegge and Watts, Cens. Musc. Aus.) (Fig. 624.)

Hartmanni, C.M.

hemitrichoides, Schwogr.

intermedium, Mitten.

diaphanum, C.M.

Baileyi, Mitten. (Fig. 625.)

aurescens, Hampe.

lingulare, Mitten.

Whiteleggei, Broth. 


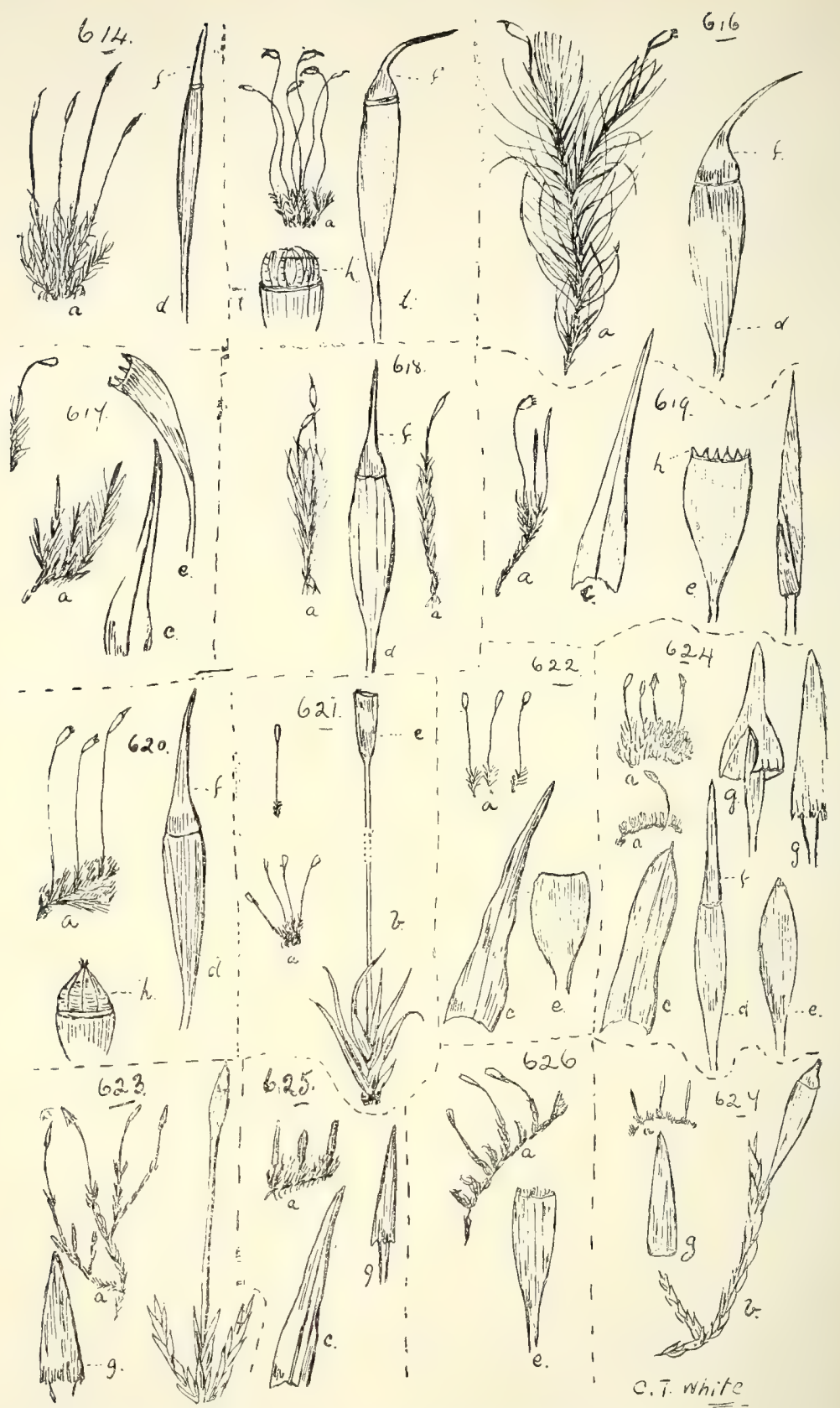

6if. Ditrichum Baileyi, C.M.

615. Trematodon Baileyi, Broth.

6i6. Dicranum suberectum, Hampe.

617. LeUCOLOMA SERRATUM, Broth.

6is. Campylopus Woolisit, C.M.

6ro. Glyphomitrium Muelleri, Mitt.

620. Leucobryum Baileyanum, C.M.
621. WEISTA PIMPAM

622. W. PUGIONIFOLIA, C.M.

623. Macromitrium eucalyptorum, C.M.

624. M. mucronulatum, C.M.

625. M. BaIleyi, Mitt.

626. Schlotheimia Bailèi, Broth.

627. Aulacopilum Hodgkinsoniz, Hampe et C.M.

(a) Plant, (b) portion of plant, (c) a leaf, (d) capsule, (f) operculum, (g) calyptra, (h) peristome. (a) nat. size; (b) - (h) enl. 
Macromitrium-contd.

\section{Leiostoma.}

involutifolium, Hook

carinatum, Mitten.

viridissimum, Mitt.

sordio-virens, C. M.

cylindromitrium, C.M.

platyphyllaceum. C. $M$

incurvulum, C. $M$.

indistinctum, $C . M$.

Sayeri, Mitten m.s.

weisioides, C. $M$.

Hab.: Russell River (Sayer), (Whitelegge and Watts).

Schlotheimia, Brid.

Baileyi, Broth. (Fig. 626.)

Powellia, Mitten.

involutifolia, Mitten.

\section{VII.-Splachn.e.}

Tayloria, Hook

octoblepharis, Hook.

Aulacopilum, Wilson.

Hodgkinsonix, Hampe et C. M. (Fig. 627.)

Wildia, Muell. Arg.

Solmsiellacea, C. M. et Broth. (Fig. 628.)

$$
\text { VIII.-FunarieÆ. }
$$

Ephemerum, Hampe.

fimbriatum, C.M.

Leptangitim, Mont.

repens, Hook. = Gigaspermum repens, Lindb.

Physcomitrium, Bridel.

brisbanicum, C.M.

minutulum, C.M.

nodulifolium, Mitten.

Tryoni, Broth. (Fig. 629.)

subserratum, Hampe.

Goniomitrium, Hook, et Wil.

acuminatum, $H$. et $W$.

Entosthodon, Schwcegr.

cuspidatus, F. Kiar. (Fig. 63o.)

ampullaceus, C.M.

apophysatus, Tayl. 
Funaria, Schreber.

elata, Mitt.

Smithhurstii, Broth.

glabra, Taylor.

crispula, $J . H$. et Wils.

\section{Eufunaria.}

hygrometrica, Hedw.

calvescens, Scha.

papillata, Hampe.

Plagiodus.

sphærocarpa, C.M.

IX.-BartramieT.

Philonotis, Bridel.

Slateri, Hampe.

pallida, Hampe.

pseudo-mollis, C.M. (Fig. 63I.)

X.-Bryete.

Wilsoniella, C. $M$.

Karsteniana, C. $M$.

Bryum, Linn.

semperlaxum, C. $M$.

Preissianum, Hampe.

coronato-affine, C. $M$.

dichotomum, Hedw.

angliophyllum, C.M.

synoicum, C. $M$.

subcrispatulum, C. $M$.

subpachypoma, Hp. et C. M.

subatropurpureum, C. $M$.

var. minor, Broth.

subtomentosum, $H p . e_{t}^{t} C . M$.

olivaceum, Hampe.

subfasciculatum, Hampe.

Commersonii, Bridel.

subleptothecium, C. $M$.

viridulum, $C . M$.

Baileyi, Broth. (Fig. 632.)

leptothecium, Taylor.

breviramulosum, Hampe.

chrysoneuron, C. $M$.

leptothrix, C.M.

minutissimum, C. $M$.

argente1m, Linn., var. 
Bryum-contd.

pimpamæ, C.M.

subviolaceum, C.M.

austro-argenteum, Brolh.

viridissimum, Broth.

Tryoni, Broth. (Fig. 633.)

(Rhodobryum) pusillum, Broth.

immarginatum, Broth.

humipetens, C. $M$.

Plagiobryum, Lindl.

Wildii, Broth. (Fig. 634.)

Leptotheca.

Gaudichaudii, Schwegr.

Rhizogonim, Bridel.

brevifolium, Broth.

Pyrrhobryum.

spiniforme, Bruch.

spininervi, Brid.

Hookeri, C. $M$.

parramattense, C.M.

var. nanum.

\section{Eurhizogonium.}

undulatum, Lindberg.

taxiforme, Hampe.

XI.-Hypopterygie T.

Hypopterygitum, Bridel.

Muelleri, Hampe.

Euhypopterygium.

discolor, Mitten.

viridulum, Mitten.

rotulatum, Hedwo. (Fig. 635.)

lopidium pinnatum, Hampe.

$$
\text { Xit.-Rhicopile.e. }
$$

Rhacopilum, Bridel.

convolutaceum, C.M.

æruginosum, $C M$.

tomentosum, Brid. (Fig. 636.)

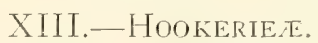

Distichophyllum, D. et $M$.

Baileyanum, C.M. (Fig. 637.) 
Hookeria, Sm.

lepida, Mitten.

(Callicostella) Baileyi, Broth. (ling. 638.)

(Euhookeria) Karsteniana, Broth.

XIV.-NECKEREA.

Hedwigia, Ehrhart.

Jaratzkæ, C.M.

novæ-valesiæ, $C . M$.

Splachnobryum.

Baileyi, Broth. (Fig. 639.)

Lasia, Bridel.

subproducta, C.M.

australis, C.M.

Cryphæa, Bridel.

tenella, Hornsch.

Eucryphoea.

viridissima, C. $M$.

ovalifolia, C. $M$.

Philudora.

Muelleri, Hampe.

flexinervis, Broth.

brevidens, C. $M$.

Lepidopilum, Bridel.

australe, Broth.

Ptychomnion, J.H. et Wils.

aciculare, Labill.

Bescherellia, Duby.

brevifolia, Hampe.

Garovaglia, Endl.

\section{Euptychium.}

cuspidata, Mitt.

Muelleri, Hampe.

neocaledonica, Schyr.

Eudotrichella.

lepida, C.M. (Fig. 640.)

Dietrichiæe, C. $M$.

brisbanica, C. $M$.

Cladomnion.

setosa, Hook. et Wils.

longicuspes, Broth., Aus. Mosses No. 161. 

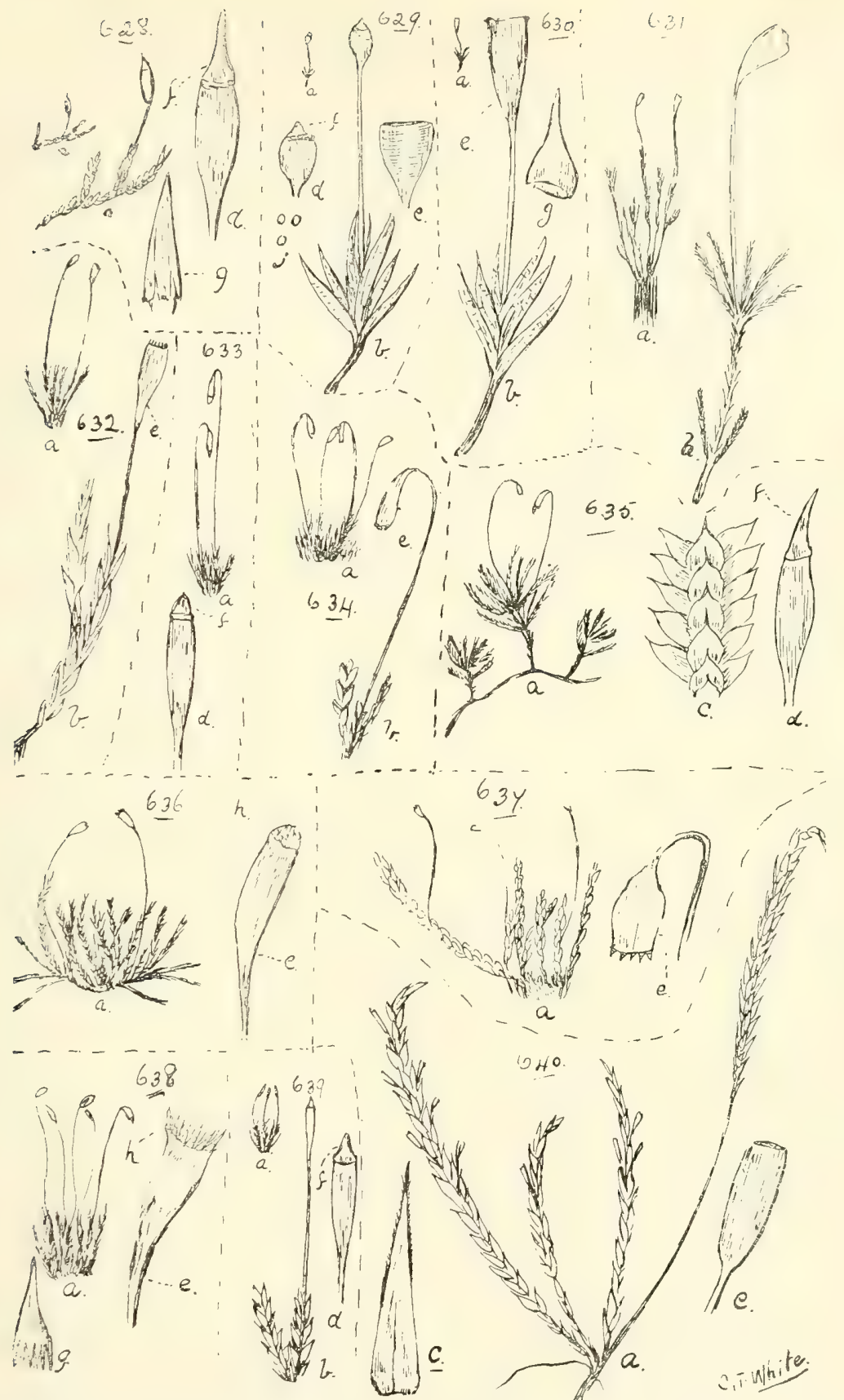

628. Wildia Solirstellacea, C.M. et Broth. 629. Physcomitrium Tryoni, Broth. 630. ENtosthodon cuspidatus, F. Kiar. 63i. Pitilonitis pseudomolis, C.M. 632. Bryum Baileyi, Broth. 633. B. TrYoni, Broth. 634. Plagiobryum Wilisi, Broth.
63.5. HypopterygiUm rotulatum, Hedw. 636. Rhacopilum tomentosum, Brid. 637. Distichophyllum Baileyanum, C.II. 638. Hookeria Baileyi, Broth. 639. Splachnobrium Baileyi, Brotil. 640. Garovaglia lepida, C.M.

(a) Plant, (b) portion of plant, (c) leaves, (d) capsule with operculum, (e) capsule without operculum, (f) operculum, (g) calyptra, (j) spores. (a) nat. size; (b) - (j) enl. 
Pterobryum, Hornsch.

\section{Calyptothecium.}

australianum, Mitten.

acutum, Mitten.

\section{Trachyloma.}

planifolium, Hook.

recurvulum, $C . M$.

Braithwaitea.

sulcatum, Hook.

longicuspes, Broth.

Meteorium, Bridel.

kermadecensis, C. $M$.

Eavesianum, Hampe.

filipendulum, J. Hook.

amblyacis, C.M.

Reginæ, Hampe.

trichophoroides, Hampe.

cinclidotus, C. $M$.

dimorphum, C.M.

compressum, Mitten.

Baileyi, Broth. (Fig. 64I.)

filum, $C . M$.

sigmatophyllum, C.M.

flexicaule, Tayl.

(Papiliaria) intricata, Mitten.

Barbella, C.M.

trichophoroides (Hampe), Broth.

Phyllogonium, Bridel.

Hampeanum, C.M.

cymbifolioides, C.M.

Neckera, Hedru.

Hodgkinsonæ, C.M.

Baileyana, C. $M$.

pennata, Haller.

hymenodonta, C.M.

(Rhystophyllum) Lepineana, C.M. (Fig. 6.42.)

Filotrichella.

perpinata, Broth., Aus. Mosses No. I 66.

Leptohymenium, Schwe.

papuanum, Broth., Aus. Mosses No. I27.

Homalia, Brid.

Baileyana, C.M. 
Porotrichum, Bridel.

vagum, Hornsch.

Schlosseri, C.M.

ramulosum, Mitten.

brisbanicum, C.M. (as Thamniella). (Fig. 643.)

deflexum, Mitten.

(Thamniella) molle, Broth.

XV.-Sematophyllex.

Rhaphidorrhynchum, Schimper.

contiguum, $J$. Hook.

Acanthocladium, Mitt.

extenuatum, Bridel.

XVI.-Stereodontel.

Entodon, C. $M$.

pallidus, Mitten.

Hartmanni, C.M.

mackayensis, C.M. (Fig. 644.)

Dæmelii, C.M.

flaccidisetus, C.M.

toowoombæ, C.M.

latifolius, Broth.

Acroceratium, Mitten.

cymbifolioides, C. $M$.

politum, J. Hook.

Isopterygitum, Mitt.

candidum, C. Muell.

austro-pusillum, C. M.

robustum, Broth.

Baileyanum, C.M. (Fig. 645.)

byssicaule, $C . M$.

Teysmanni, Broth.

Amblystegium, Schimper.

leptopterygiodes, C.M.

subrelaxum, Broth., Aus. Mosses No. 184.

Ectropothecium, Mitt.

Slateri, Hampe.

Hillianum, Hampe.

reticulatum, $D$. et $M$.

umbilicatum, C.M. (Fig. 646.)

XVII.-HyPnET:

Fäbronia, Raddi

Baileyana, C.M. (Fig. 647.) 


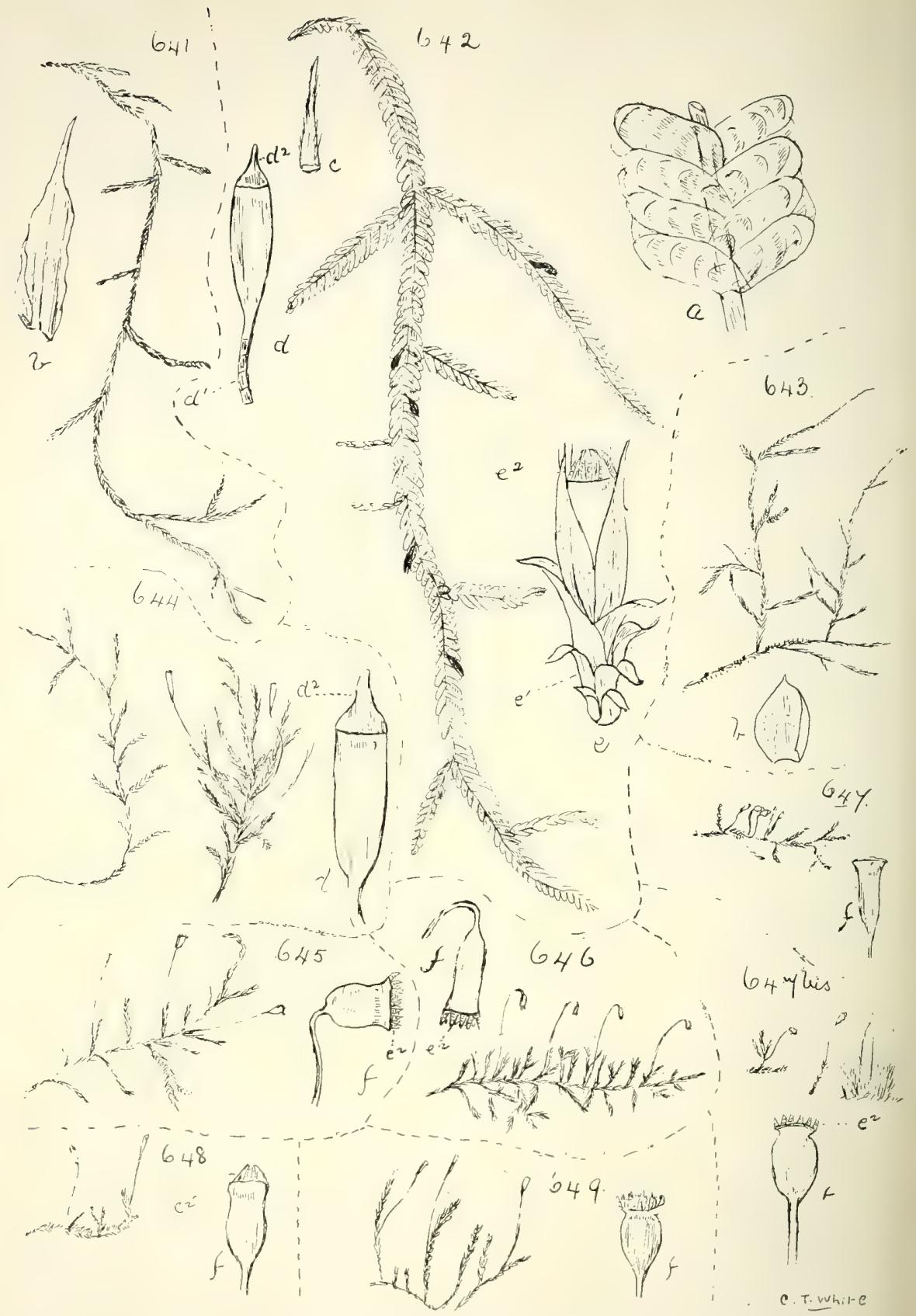

641. Meteorium Baileyi, Broth.

642. Neckera Lepineana, $C . M$.

(After Dozy et Molkenboer in part.)

643. Porotrichum brisbanicum, C. $M$.

644. Entodon MACKAYENSis, $C . M$.

645. Isoterygium Baileyanum, C. $M$.

646. Ectropothecie m umpilicatum, C. $M$.
647. Fabronia Baileyana, C. $M$.

647 bis. F. BRACH YDONTIA, C. $M$.

648. RhynCostegium NANO-PENNATUm, Broth. 649. Rhaphidostegium ovale, Broth.

(a) Leaves, (b) single leaf, (c) calyptra, (d) capsule with operculum, (dI) pedicel, (d2) operculum, (e) capsule with perianth, (cI) periantil, (ez) peristome, (f) capsule without operculum. (a) - (f) enlarged. 
Fabronia-contd.

obtuso-acuminata, C.M.

sphærocarpa, C. $M$.

Scottix, C.M.

var. denticulata, C. $M$.

brachydontia, C.M. (Fig. 647 bis.)

brachyphylla, C.M.

Leskea, Hedw.

calochlora, C.M.

Anacamptodon, Brid.

Fortunei, Mitt.

Anomodon, Hook. et Tayl.

brevissimus, Broth.

Rhynchostegium, Schimper.

erythropodium, Hampe.

glauco-viride, Hampe.

nano-pennatum, Broth. (Fig. 648.)

Stereodon, Brid.

stubchrysogaster, Broth., Aus. Mosses No. I7\&.

Rhaphidostegium.

ovale, Broth. (Fig. 649.)

aciculare, C.M.

acicula, C.M.

austro-circinale, Broth.

exalare, C.M.

Hypnodendron, C.M.

arcuatum, Hedw.

curvato-comosum, C.M.

flagellare, Angstr. (Fig. 650.)

pterobryoides, C.M.

eflagellare, Angstr.

Eurhynchium, Sch.

remotifolium, Grev.

Thuidium, Schimper.

sparsum, J. Hook. et Wils

ramentosum, Mitt.

nano-delicatulum, Hampe.

læviusculum, Mitt.

plumuliforme, Hampe.

protensulum, C.M.

suberectum, Hampe.

suberectum, var. byssoides, C. $M$.

pennula, C. $M$.

pennula, var., Broth. (Fig. 651.) 
Thuidium-contd.

obtusi-rameum, Broth. erectum, Hpe. et C. $M$. pallido-limbatum, Broth. (Fig. 652.)

liliputanum, Broth.

Chætomitrium, $D z$ e et $M . B$. nematosum, Broth.

Trichosteleum.

Kerianum, Broth. (Fig. 653.)

Hypnum (Dill.), Hedw.

(Aptychus) brachytheciella, C. $M$.

(Aptychus) exalare, C. $M$.

(Cupressina) umbilicatum, C.M.

(Drepanocladus) strictiusculum, C. M.

(Heterophyllum, Schgr.) Keysii, Kior. (Fig. 654.)

(Thacomium) microflagellare, C.M.

(Rhyncostegium), convolutifolium, Broth.

(Rhyncostegium) tenuifolium, Hedw:

\section{XVIII.-Skitophyllez.}

Fissidens, Bridel.

maceratus, Mitt.

hyophilus, Mitt.

Dietrichize, C.M.

undato-decurrens, C. $M$.

oblongifolius, Hook. et Wils.

incurvo-bryoides, C. $M$.

Whiteleggei, C.M.

liliputano-incurvatus, C. $M$.

liliputano-bryoides, $C . M$.

tenellus, Hook. et Wils.

obtuso-acuminatus, C. $M$.

Kerianus, C.M. (Fig. 655.)

arboreus, Broth.

grandisetis, Broth.

Wildii, Broth. (Fig. 656.)

(Eufissidens) calodictyon, Broth.

densifolius, Broth.

(Conomitrium) splachnoides, Broth.

(Conomitrium) Muelleri, Hampe.

Hab. : In still pools, Coomera River (Tryon and White). Det. at Royal Bot. Gard. Kew, Eng.

leuconeurus, C. $M$.

microlecythis, $C . M$. 
CLVIII. MUSCI.

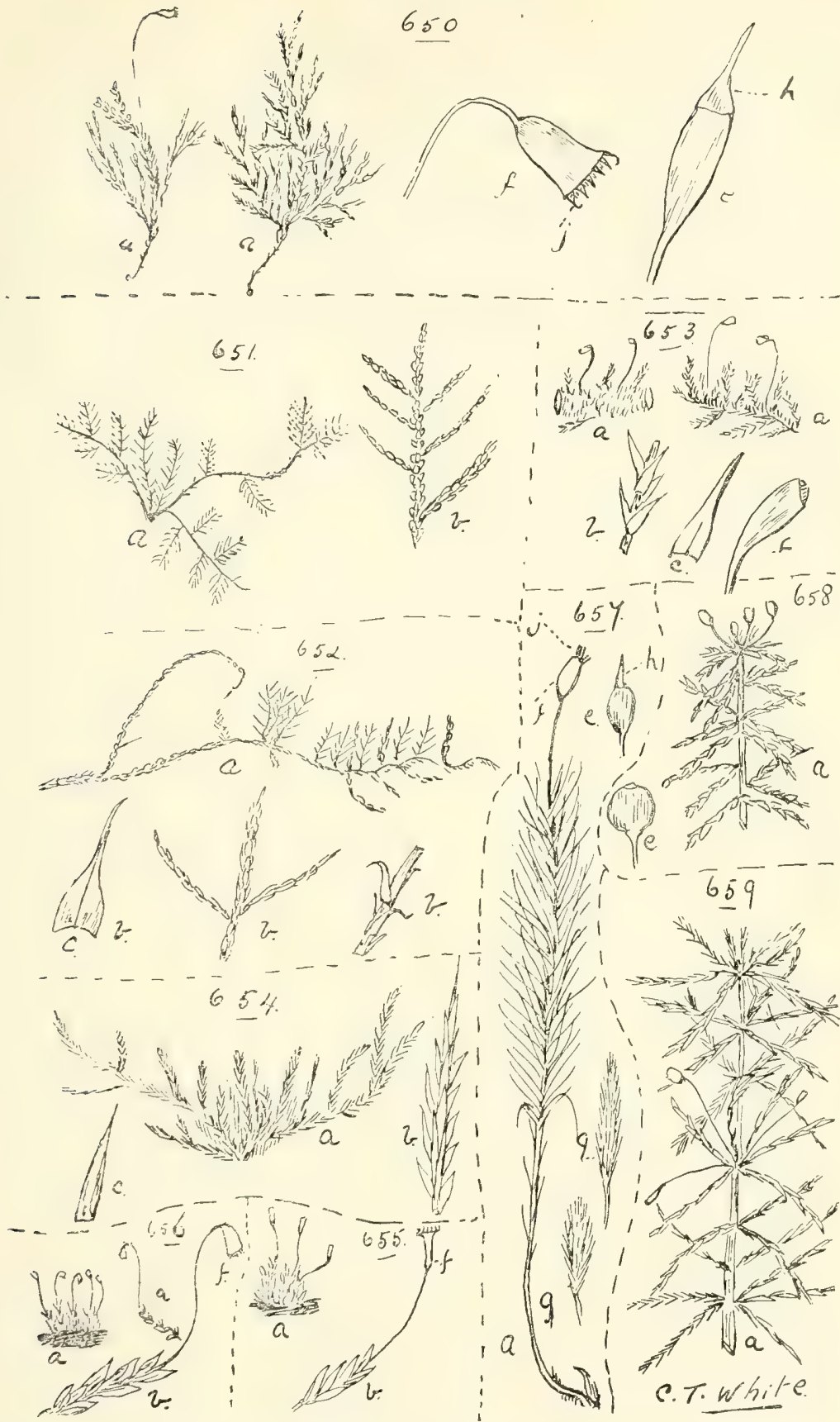

650. Hypiodexdron flagellare, Angstr.

65 I. THUJDIUM PENNULA, var., Broth.

652. T. PALLIDO-LIMEATUM, Broth.

653. Trichostelium Kerianum, Broth.

654. Hypnum Keysin, Kiar.
655. Fissidens Kerianus, C.M

656. F. WILDII, Broth.

657. Dawsonia POLytRichoides, R. Br. 658. Sphagnum CYMBifolium, Dillen. 659. S. CUSPIDATUM, Eh\%.

(a) Plant, (b) portion of plant, (c) and (d) leaves, (e) capsule with operculum, (f) capsule without operculum, (g) capsule with calyptra, (h) operculum, (j.) peristome. (a) and (g) nat. size; (b), (c), (d), (e), (f), (h), and (j) enl. 
XIX.-POLytricheÆ.

Polytrichadelphus, C. $M$. australasiæ, Hampe.

Polytrichum, Dill.

Camaræ, C.M.

Dawsonia, R. Br.

polytrichoides, R.Br. (Fig. 657.)

longiseta, Hampe.

superba, Grev.

XX.-SPHAGNE压.

Sphagnum, Dillen.-Bog Moss.

cymbifolium, Dillen. (Fig. 658.)

cymbophyllum, F..$M$.

cuspidatum, Ehrh. (Fig. 659.)

Naumanni, C. $M$.

Scortechinii, C.M., ex Warnst. in Hedw. I897.

\section{Order CLIX.-HEPATIC开 (Liverworts).}

A.-Folios A:.

Lembophyllum.

vagum, Hornsch.

Plagiochila, Nees. et Mont.

Mittenii, Steph.

Baileyana, Steph. (Fig. 660.)

pendula, $H p e$.

arbuscula, $L$. et $L$.

flexuosa, Steph.

Teysmanni, Lac.

Brotheri, Steph.

fasciculata, Steph.

Lyallii, Mitt.

Dicksoni, Hook. et Tayl.

Lophocolea, Nees.

heterophylloides, Nees.

Jungermannia, Linn.

hirtella, Web.

Porella, Dill.

Cranfordi, Steph. (Fig. 66I.)

Stangeri, $L d b g$.

Nardia, Gray.

fragilis, Steph. (Fig. 662.)

montana, Steph. 
Chiloscyphus, Corda.

argutus, Nees.

Balantiopsis.

diplophylla, Mitt. (Fig. 663.)

Physiotium, Nees.

articulatum, Lindenb.

Muelleri, Gottsch.

Bazzania, Gray.

filiformis, Steph.

acereta, $L$. et $L$.

Mitteni, Steph. = Mastigobryum Mittenii, Steph. (Fig. 664.)

Lepidozia, Nees.

capillaris, Lindenb.

patentissima, Hook, et Tayl.

capilligera, Lindl.

procera, Mitt.

Mastigobryum, Nees.

phoea, Gottsch.

novæ-hollandiæ, Nees.

Cephalozia, Dunn.

dentata, Raddi.

Cephaloziella, Spruce.

Brotheri, Steph.

Phragmicoma, Du Mortier.

Thozetiana, Gottsch. et Muell.

phaca, Gottsch.

Eavesiana, Gottsch. et Muell.

plicatiscypha, Hook. et Tayl.

Keppelina, Gottsch.

Radula, Nees.

javanica, Gottsch.

reflexa, $N$. et $M$.

Mittenii, Steph.

acutiloba, Steph. (Fig. 665.)

Gottscheana, F.v. M.

buccinifera, Tayl.

Lejeunia, Maria Libert.

fimbriata, Gottsch.

adusta, Gottsch.

occulta, Gottsch.

tumida, Mitt.

Eulejeunea, Steph.

Armitii, Steph. (Fig. 666.)

flava, Sw. 

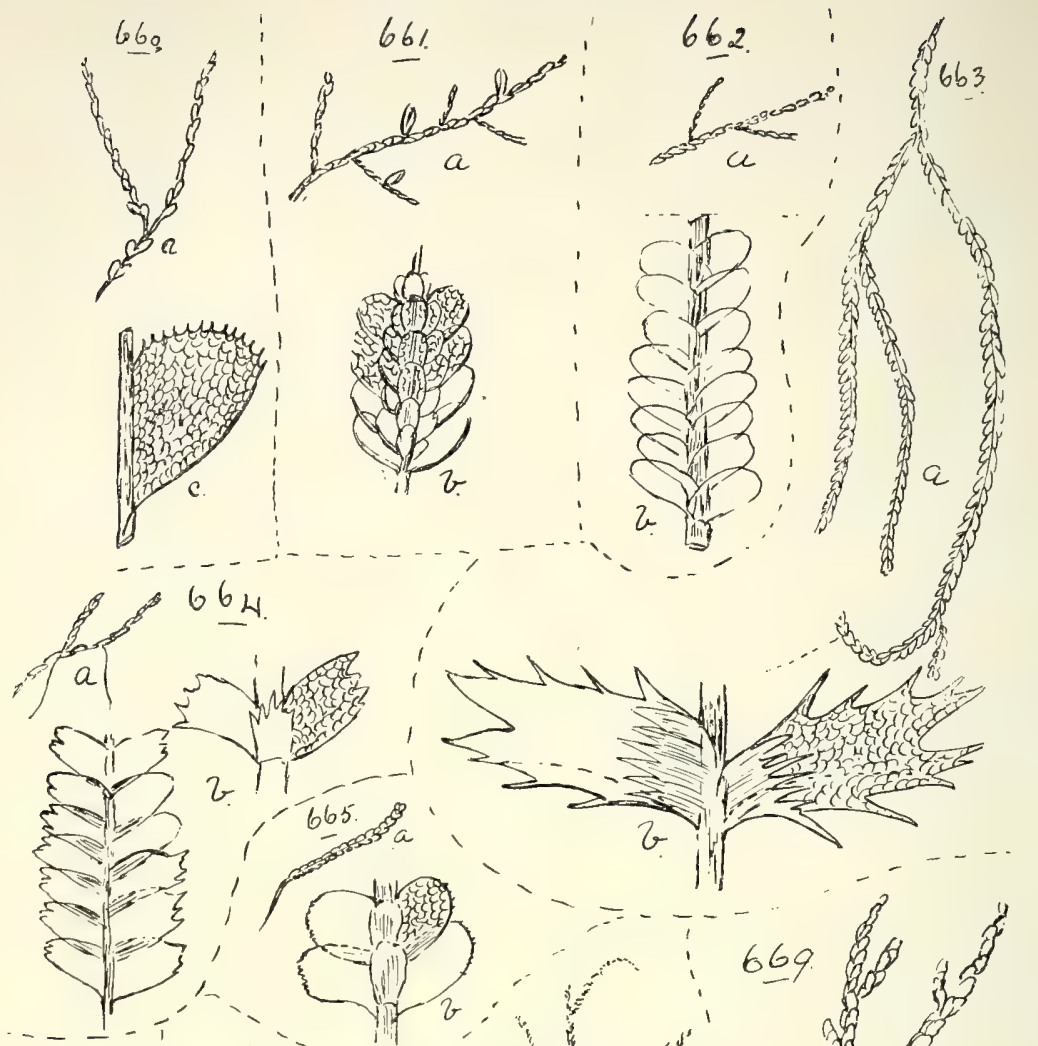
Cololejeunea.

trichomanes, Gottsch.

Schistocheila.

cristata, Steph. (Fig. 667.)

Brotheri, Steph.

Trichocolea, Nees.

pluma, Nees.

Hydrolejeunea.

Sayeri, Steph.

sacculifera, Steph.

Euosmolejeunea.

Baileyana, Steph. (Fig. 668.)

Acrolejeunea.

Wildii, Steph.

novæ-guineæ, Steph.

Leptolejeunea.

denticulata, Steph.

Mastigolejeunea.

phoea, Gottsch.

Ptycholejeunea.

Stephensoniana? Mitt.

Pycnolejeunea.

longidens, Steph.

Madotheca, Du Mort.

Stangeri, Gottsch. (Fig. 669.)

Godofrediana, Gottsch.

striata, Mitt.

Frullania, Raddi.

nodulosa, Nees.

serrata, Gottsch.

rubella, Gottsch.

spinifera, Tayl.

monocera, Tayl.

reptans, Mitt.

pentapleura, Tayl.

fugax, Tayl.

squarrosa, Nees.

seriata, Gottsch.

Wildii, Steph. (Fig. 670.)

exigua, Steph.

Hampeana, Nees.

squarrosula, Tayl.

Ptychanthus, Nees.

squarrosus, Mont. (Fig. 67I.) 
B.-Frondos 2 .

Fossombronia, Raddi.

papillata, Steph.

Aneura, Dumort.

multifida, Dumort.-On water-tubs at Bowen Park. (Fig. 672.)

Noteroclada, Tayl.

confluens, Tayl. (Fig. 673.)

Steetzia, Lehm.

Lyellii, Nees.

Symphyogyna, Mont. et Nees.

podophylla, Nees.

irregularis, Steph. (Fig. 674.)

flabellata, Mont.

Metzgeria, Raddi.

hamata, Lindenb. (Fig. 675.)

furcata, Nees.

australis, Steph.

Sarcomitrium, Mitt.

multifidum, Mitt.

C.-CARNOSE.

Riccia, Micheli.

natans, Linn. (Fig. 676.)

fluitans, Limn. (Fig. 677.)

multifida, Steph.

muscicola, Steph.

cartilaginosa, Steph.

vesiculosa, $C$. et. $P$.

Plagiochasma, Lehm. et Lindl.

australe, Nees.

Marchantia, Linn.

polymorpha, Linn. (Fig. 698.)

tubularis, Nees.

pallida, Steph.

Asterella, Pallis.

hemispharica, Beauv.

Reboulia, Nees.

hemisphærica, Raddi. (Fig. 679.)

Fimbriaria, Nees.

australis, Hook. et Tayl.

longibarbata, Steph.

Drummondii, Tayl. (Fig. 680.)

Whiteleggeana, Steph. 


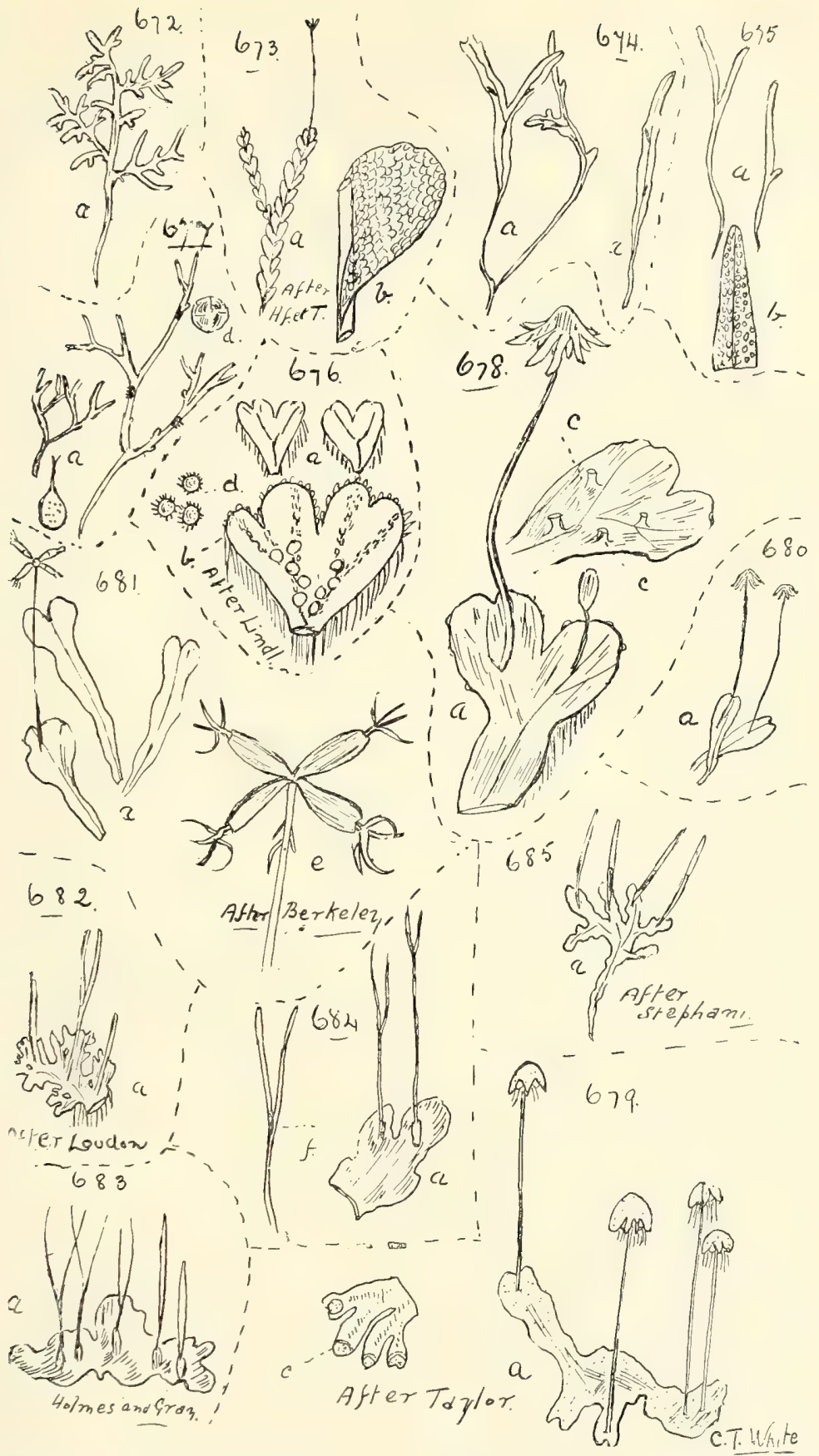

672. Aneura multifida, Dumort.

673. Noteroclada confluens, Tayl.

674. SYMPHYOGYNA IRREGULARIS, Steph.

675. Metzgeria hamata, Lindenb.

676. Riccia Natans, Linn.

677. R. FLUITANS, Limn.

678. Marchantia polymorpha, Linn.
679. Reboulia hemisph mica, Raddi. 680. Fimbriaria Drummondi, Raddi. 681. LUNULARIA VULGARIS.

682. Anthoceros pUnCtATUS, Limn. 683. A. LEVIS, Linn

684. A. Brotheri, Steph.

685. Dendroceros Muelleri, Steph.

(a) Plant, (b) leaf or portion of leaf, (c) gemmæ, (d) spores, (e) involucres and sporangia, (f) capsule. (a), (c), and (f) nat. size; (b), (d), and (e) enl. 
Lunularia, Mich.-Species of this genus in Europe are sometimes troublesome to cultivators of plants in pots.

vulgaris, Mich. (Fig. 68I.)

\section{D.-Anthocerotæ.}

Anthoceros, Micheli.

punctatus, Linn. (Fig. 682.)

lævis, Linn. (Fig. 683.)

Brotheri, Steph. (Fig. 684.)

Dendroceros, Nees.

Muelleri, Steph. (Fig. 685.)

crispatus, Hook. ?

\section{Order CLX.-CHARACE五 (Stoneworts).}

Nitella, Agardh.

myriotricha, $A . B r$.

oligospira, $A . B r$.

var. australiensis, Nordst.

microcarpa, A. Br., subspecics Glaziovi (Zeller), Nordst.

polyglochin, $A . B r . f$.

conglobata, var. Lhotzkyi, $A . B r$.

cristata, A.Br.

diffusa, $A . B r$.

? leptosoma, Nordst.

Sonderi, A.Br.

hyalina (DC.), Kutz.= Chara hyalina, DC. (Fig. 68б.) var. brachyactis, Nordst.

gelatinosa, $A . B r$.

var. podystachya, $A . B r$.

pseudoflabellata, $A . B r$.

var. muscosa, Nordst.

tasmanica, F.v.M.

partita, Nordst. (Fig. 687.)

Hookeri, $A . B r$.

Lychnothamnus (Rupr.), Leonh.

macropogon (A. Br.), Nordst., Hedwigia, I888.-Drawn from specimens received from Messrs. H. \& J. Groves. (Fig. 688.)

Chara, Linn.

australis, R. Br.

var. nobilis, $A$. $B r$.

var. lucida, $A . B r$.

dichopitys, R. $B r$.

var. Preissii, A.Br. 
CLX. CHARACE王.

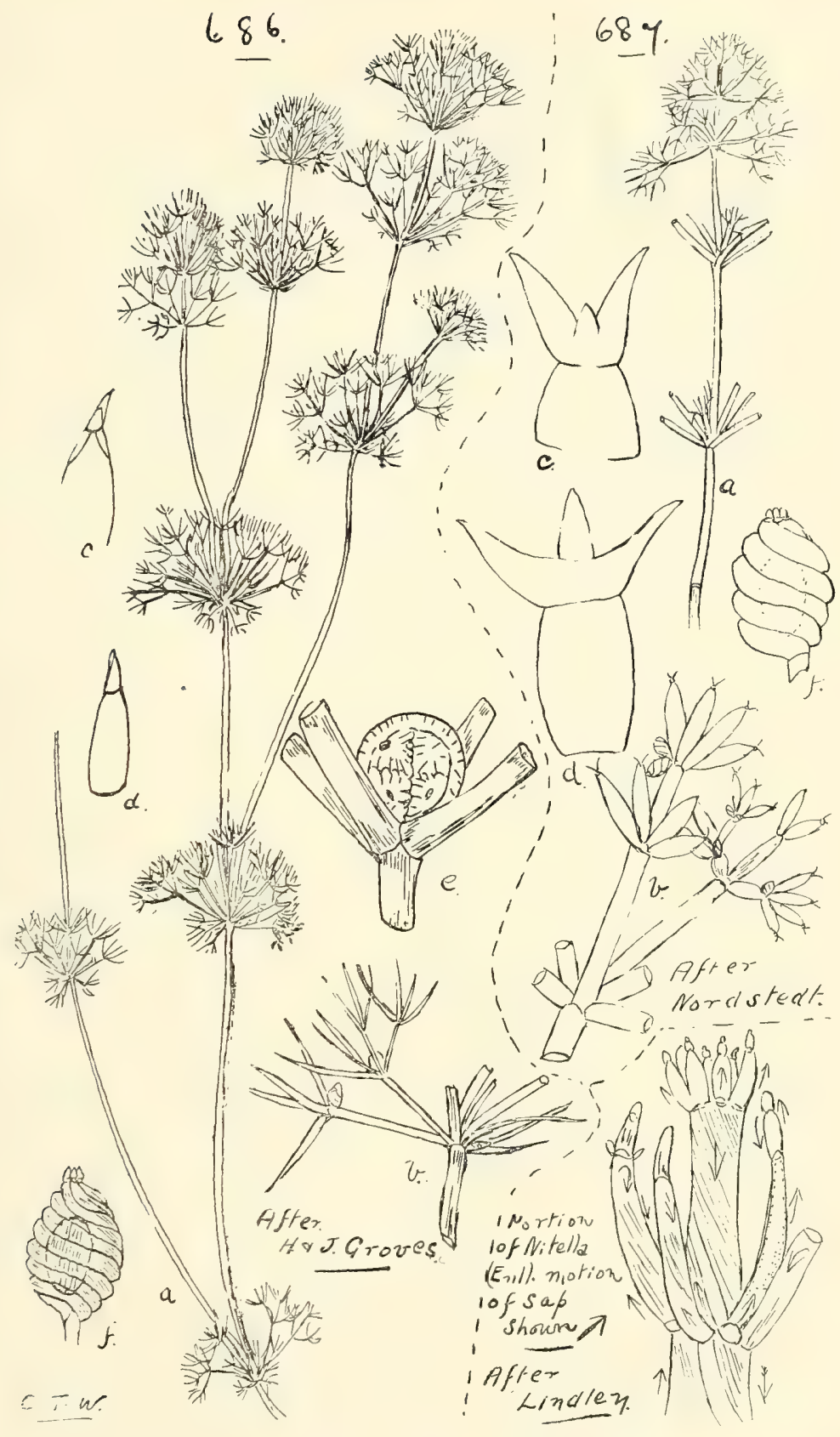

686. Nitella hyalina $(D C$.$) , Kutz.$ 687. N. Partita, Nordst.

(a) Portion of plant, (b) portion of a whorl, (c) apices of terminal rays, (d) young do., (e) antheridium, (f) fruit. 


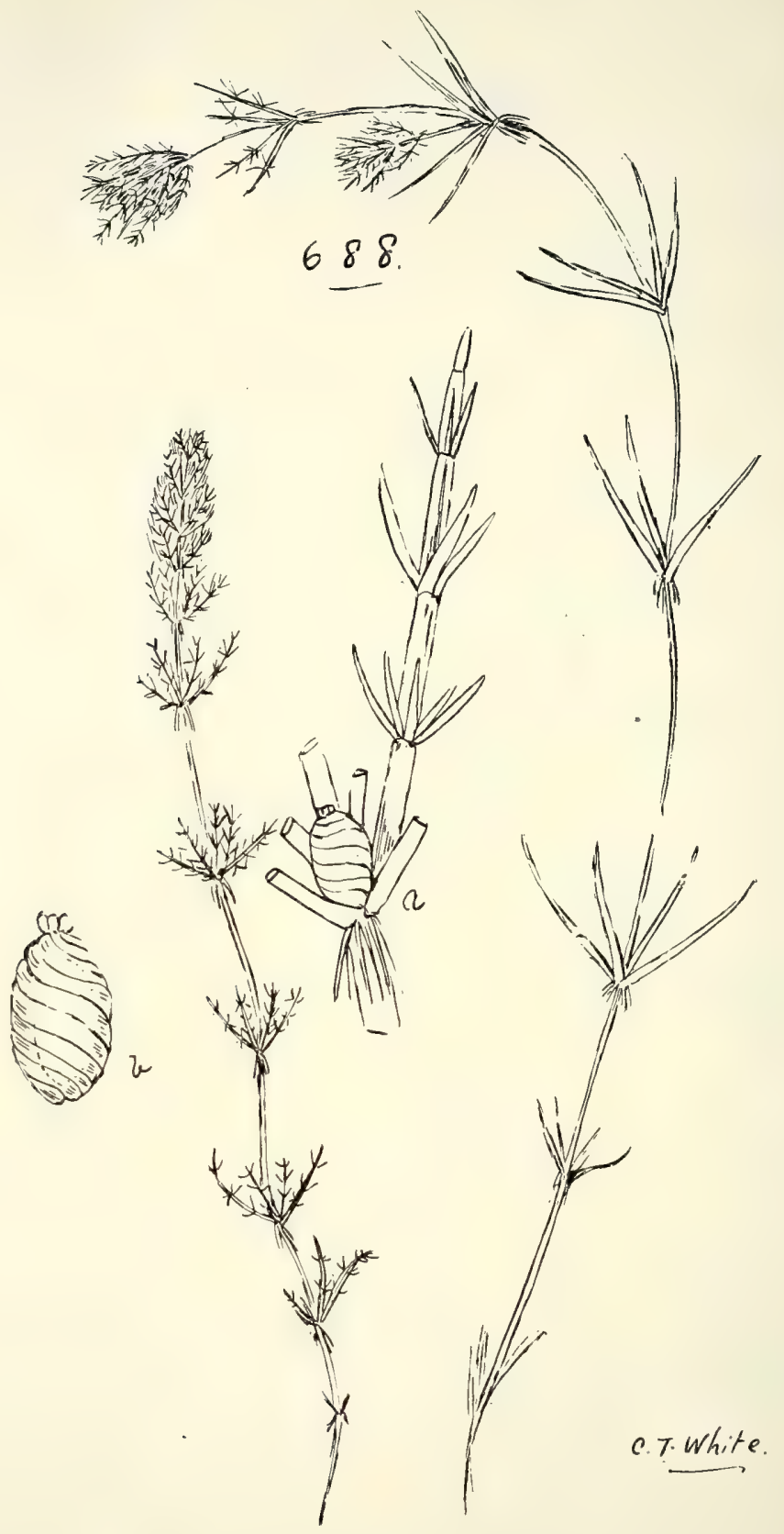

688. Lychnothamnus macropogon (A. Br.), Nordst.

(a) Portion of a whorl, enl., (b) fruit, enl. 


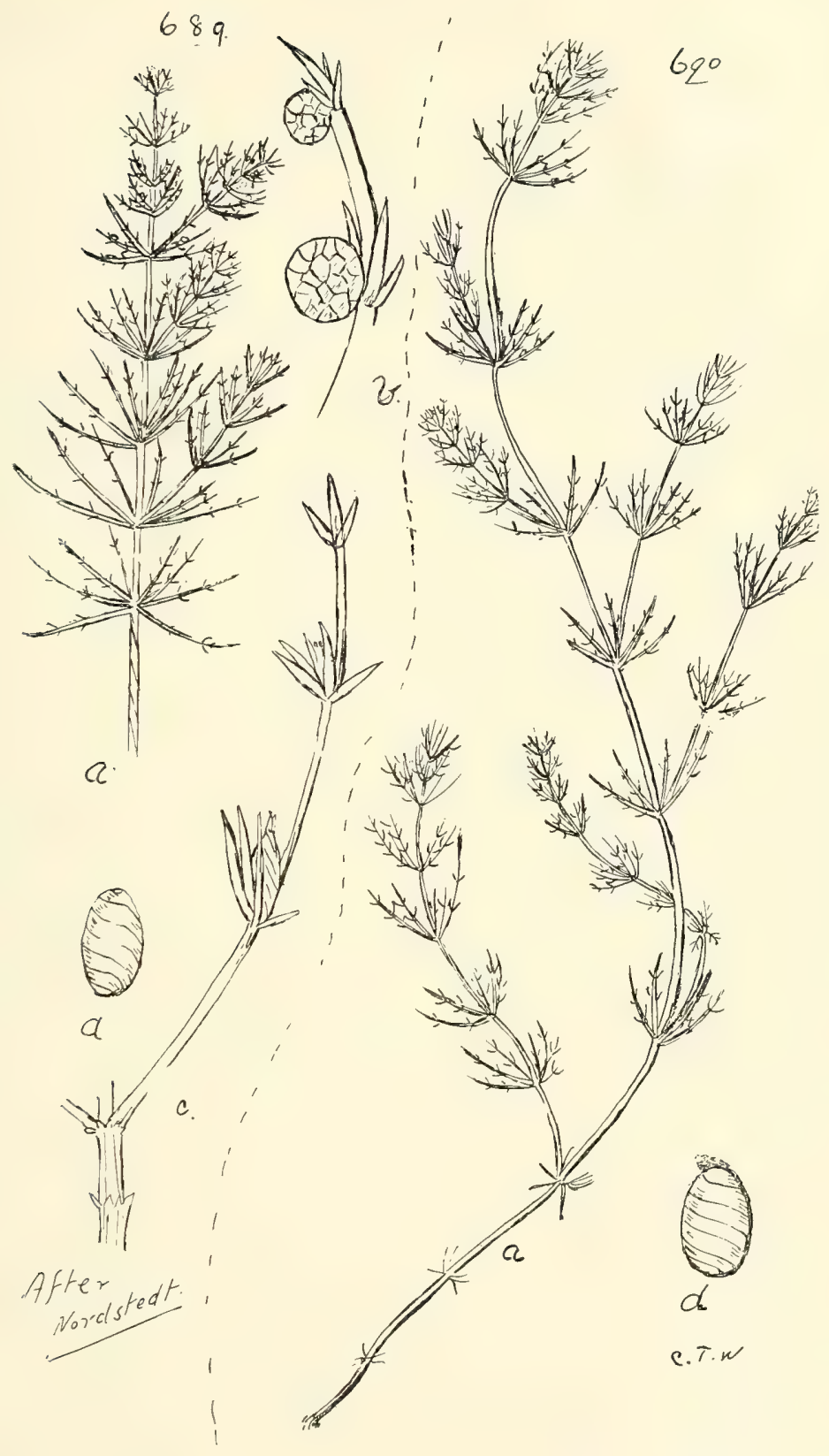

689. Chara submollusca, Nordst.

690. C. grmnopitys, $A . B r$.

(a) Portion of plant, nat. size, (b) antheridia, enl., (c) portion of a whorl, enl., (d) fruit, enl. 
Chara-contd.

leptosperma, A.Br., var.?

gymnopus, $A . B r$.

var. ceylonica (Klein), A. Br.

submoilusca, Nordst. (Fig. 689.)

gymnopitys, A.Br. (Fig. 69o.)

var. æquistriata, Nordst.

var. æquistriata-micrantha, Nordst.

var. æquistriata-polyphylla, Nordst.

var. æquistriata-tylacantha, Nordst.

Benthami, A. Br.-Messrs. H. \& J. Groves, who determined the species for me, say, "The plant is not always unistipulate as Braun described it, having often two stipulodes to each branchlet."

Hab.: Corinda (C. T. White).

fragilis, Desv., forma microptila.

\section{Order CLXI.-LICHENES (Lichens).}

\section{Family I.-COLLEMACEI.}

Sirosiphon, Kütz.

TRIBE I.-Lichinei.

Stirtoni, J. F. Shirl. = S. pluvinatum, Stirton.

Obryzum (Wallr.), Tul.

scabrosum, Stirton.

Synechoblastus (Trev.), Wainio.

microcarpus, Muell. Arg.

glaucothalmus, Muell. Arg.

Tribe II.-Coliemei.

Collema, Ach.

byrsinun, Ach. (Fig. 6gr.)

furvum, $A c h$.

leucocarpum, Tayl.

nigrescens, $H u d$.

hypolasium, Strn.

Gwytheri, Strn.

Leptogium, Ach.

phyllocarpum, $N y l$.

var. isidiosum, $N y l$.

var. dædalum, $N y l$.

tremelloides, Fries.

var. pichneum, Ach.

var. azureum (Ach.), Mnt

var. isidiosa, Muell, Arg.

diaphanum, Mont. 
Leptogium-contd.

corrugatulum, $N y l$.

marginellum, Sw.

hypotrachyum, Muell. Arg.

byssinum, Hoffm. = Physma byssinum, Massalongo.

bullatulum, Muell. Arg.

denticulatum, Wilson.

sphinctrinum, $N y l$.

carneolum, Wilson.

atroviride, Wilson.

chlorômelum, $N y l$.

Physma, Mass.

bursinum, var. amphiurum, Muell. Arg.

Myriangium, Mont. and Berk.

Duriæi, Mont. and Berk. (Fig. 692.)

\section{Family II.-LICHENACEI.}

\section{Series A.-Epiconioidei.}

Tribe I.-Caliciei.

Calicium, Ach.

hyherellum, var. validius, C. Knight. (Fig. 693.)

curtum, Borr.

chlorosporum, Wilson.

fulvo-fuscum, Wilson.

trachilinum, Ach. $=$ C.hymenosporum, Wilson; C. glebosum

var. concinnum, Wils.

var. queenslandiæ, Wilson.

atronitescens, Wilson.

victoriæ, C. Knight.

var. albo-carneum, Witson

var. jejunum, Wilson.

prætenue, Wilson.

glebosum, Muell. Arg.

var. concinnum, Wilson.

var. glaucescens, Wilson.

stubtile, Ach.

pachypus, J.M.

quercinum, var. lenticulare, 4 ch

Acolium, Fée.

buelliaceum, Muell. Arg.

Trachyllia, Fries.

tricincta, Wilson. 
Pyrgillus, $N y l$.

javanicus, $N y l .=$ Calicium javanicum, Mnt.; C. stenosporum, Wilson; Pyrgillus australiensis, Wilson.

caliciisporus, Wilson.

fallax, Wilson.

Tribe II.-TyLOPHOREI.

Tylophoron, $N y l$.

triloculare, Muell. Arg., Q1. Bot. Bull. viii. ( 1893) 92.

Tribe III.-SphærophoreI.

Sphærophoron, Pers.

compressum, Ach. (Fig. 694.)

australe, Laur. $=$ S. complanatum, Hook. et Tayl.; S. insigne,

Laur.; S. australe, var. insigne, Muell. Arg.

Series B.-Cladodiei.

Tribe IV.-Benomycei.

Bæomyces, Pers.

roseus, Pers.

\section{Tribe V.-Cladoniei.}

Thysanothecium, Berk. et Mont.

hyalinum, $N y l$.

Hookeri, Berk. et Mont.

Cladonia, Hoffm.

aggregata, Eschw.

var. straminea, Muell. Arg.

retipora, Flarke. (Fig. 695.)

cervicornis, Ach.

squamosa, $H$ off $m$.

furcata, Hoffm.

var. foliolosa, Muell. Arg. = C. pityrea, var. foliolosa,

Muell. Arg.

macilenta, Hoffm.

var. flabellulata, Muell. Arg., Q1. Bot. Bull. viii. (I893) 93.

Flœrkeana, Fries.-Red-cup Moss. (Fig. 696.)

gracilis, Hoffm. (Fig. 697.)

fimbriata, Schorer.

var. tenella, Muell. Arg.

var. radiata, Fries.

var. antilopea, Muell. Arg.

var. chordalis, Floerke $=$ var. subcornuta, Nyl., and var.

fibula, Hoffm.

war. pulverulenta (Del.), Muell. Arg. = C. borbonica, Nyl. 
Cladonia-contd.

lepidula, Kremp.

pityrea, Floerke.

elegantula, Muell. Arg.

squamulosa, Muell. Arg.

delicata, Florke.

cariosa, Fiarke.

rangiformis, var. sorediophora, Wainio.

ochrochlora, var. ceratodes, Floerke.

degenerans, var. pleolepis, Flocrke.

Heterodea, $N y$.

Muelleri, $N y l .=$ Trichocladia Baileyi, Stirton. (Fig. 6y8.)

Tribe VI.-Stereocaulei.

Stereocaulon, Schreb.

ramulosum, Ach. (Fig. 699.)

var. microcarpoides, Muell. Arg.

arbascula, $N y l$.

nanum, Ach.

proximum, var. naudatum, Muell. Arg.

\section{Series C.-Ramalodei.}

Tribe ViI.-Siphulei.

Eumitria, Stirton.

Baileyi, Stirton $=$ Usnea barbata, var. asperrima, Muelk Arg. ; U. Baileyi, Zahl. (Fig. 7oo.)

elegans, Stirton.

Thamnolia, Ach.

vermicularis, Szo.

Tribe ViII.-Usneei.

Usnea, Dillen.-Old Man's Beard.

barbata, Ach.

var. scabrida (Tay1.), Muell. Arg.

var. hirta, $F r$.

var. strigosa, Krph.

trichodea, Ach.

intercalaris, Krempelh.

longissima, Ach.

elegans, Stirton. (Fig. 7or.)

articulata, Ach. (Fig. 702.)

angulata, H. et Tayl.

dasypogoides, $N y l$.

dasypogon, Ach.

florida, Limn. 

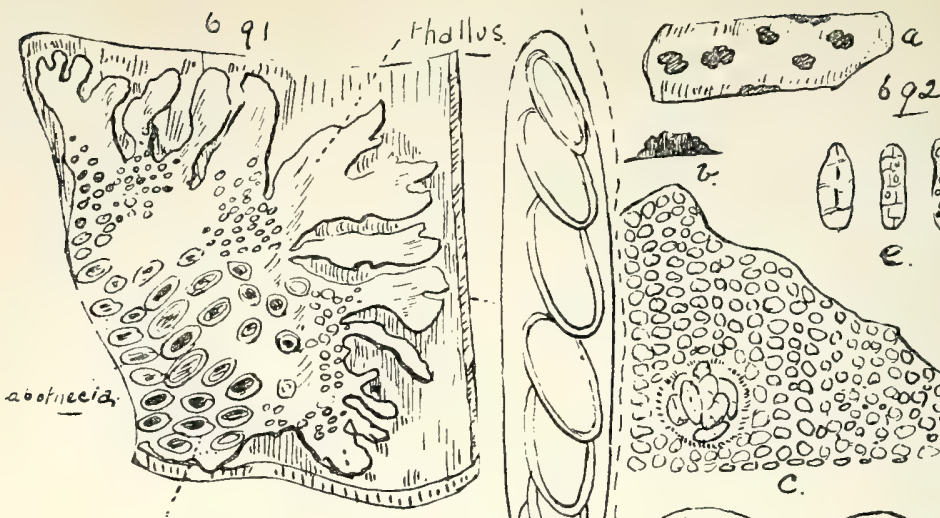
- iporthecia

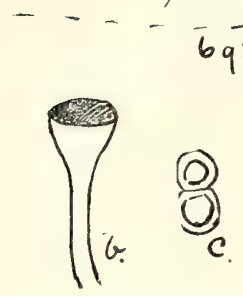

Z.c. After knight: a

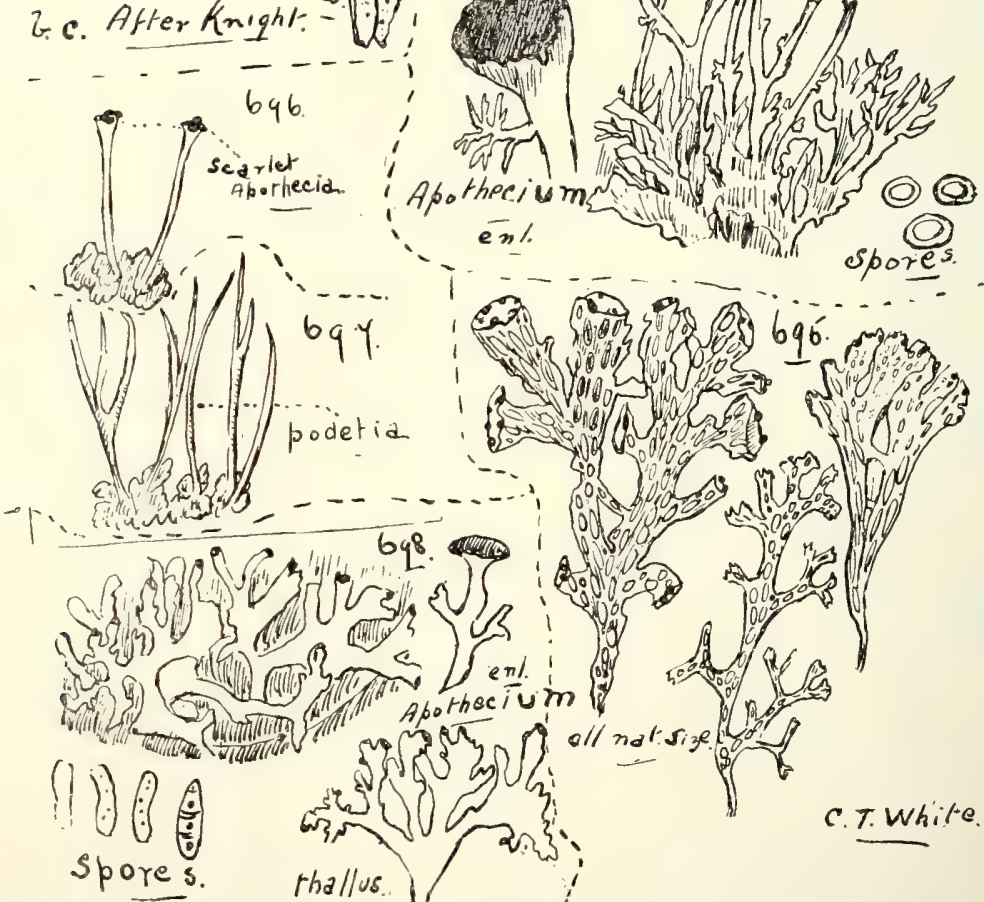

69i. Collema byrsinum, $A c h$.

692. Myriangium Duriei, Mont.

(a) Plants, (b) sect. of plant, (c) thin sect. thallus in fruit, (d) theca, (e) spores. (a) and (b) nat. size; (c)-(e) enl.

693. Calicium hyherfllum, var. validius, C. Knight.

(a) Plants, (b) apothecium, (c) spore. (a) nat. size; (b) and (c) enl.

694. SPH EROPHORON COMPRESSUm, Ach.

695. Cladonia retipora, Florke.

696. C. Flemkeana, Fries.

697. C. GRACILIS, Hoffm.

698. Heterodea Muelleri, $N y l$. 
Usnea-contd.

cornuta, Flot.

hirta, Fr.

ceratina, Ach.

sublurida, Stirton.

Tribe IX.-Ramalinei.

Ramalina, Ach.

farinacea, $N y l$.

var. nervulosa, Muell. Arg. (Fig. 7o3.)

var. squarrosa, Muell. Ary.

minuscula, var. alba, C. Knight.

fraxinea, Ach.

var. tæniæformis, Ach.

geniculata, Tayl.

gracilis, $N y l$.

pusilla, Le Prev.

intermedia, $D e l$.

inflata, Hook. et Tayl.

var. gracilis, $N y l$.

var. olivacea, Muell. Arg.

yemensis, Ach .

scopulorum, Ach.

calicaris, Fries.

exiguella, Stirton.

perpusilla, Stirton.

complanata, Ach.

rutilans, Stirton.

Eckloni, var. tenuissima, Mey. et Flot.

var. membranacea (Mnt.), Laur.

var. ovalis, Tayl.

dendriscoides, $N y l$.

var. minor, Muell. Arg.

leiodea, $N \mathrm{y} l$. (Fig. 704.)

\section{Tribe X.-Cetrariei.}

Platysma, Hoff $m$.

glaucum, Fries.

eriophylium, C. Knight $=$ Erioderma Knightii, Shirley. (Fig. 705.)

Series D.-Phyllodei.

Tribe XI.-Peltigerei.

Nephromium, Ach.

lævigatum, Ach.

tropicum, Muell. Arg. 
Peltigera, Ach.

polydactyla, Hoffm.

var. dissecta, Muell. Arg.

Tribe XiI.-PARMeliei.

Stictina, Nyl.

gilva, Thun.

quercizans, Ach. $=S$. tomentella, Leighton (non Hunıb.),

Shirley Lichens, F1. Q1. p. 54.

lutescens, Tayl. $=S$. subsinuosa, var. lutescens, Krph.

marginifera, Tayl.

punctillaris, Muell. Arg.

fragillima, $B a b$.

crocata, Ach.

var. esorediata, Muell. Arg. $=S$. intricata, Stirton (non

Del.), Shirley Lich., F1. Q1. p. 50. (Fig. 706.)

rutilans, Stirton.

brevipes, Muell. Arg. $=S$. marginifera, Tayl.

cinnamonea, Rich.

esorediata, Muell. Arg.

limbata, $S m$.

macrophylla, $N y l$.

Montagneana, $N y l$.

cyphellulata, Muell. Arg.

subtomentella, C. Knight.

impressula, Muell. Arg.

var. sublævis, Muell. Arg.

filicina, Ach.

Dozyana, Mont.

tomentella, C. Knight.

suborbicularis, Muell. Arg. = S. subtomentella, C. K.;

S. macrophylla, Shirley. (Fig. 707.)

argyracea, $\mathrm{Nyl}$, forma isidiosa, Muell. Arg.

punctilaris, Muell. Arg.

fuliginosa, Muell. Arg.

Freycinetii, var. isidulosa, Muell. Arg.

Sticta, Ach.

endochrysea, var. Urvillei, Muell. Arg. $=$ S. Urvillei, Del.

var. flavicans, Muell. Arg. = S. flavicans, Hk. et T.

Camaræ, Muell. Arg. (Fig. 708.)

variabilis, Ach.

var. papyracea, Muell. Arg.

retigera, Ach.

pulmonacea, Ach.

var. hypomela, Del. 
Sticta-contd.

filix, Hoffm.

var. myrioloba, Muell. Arg.

Urvillei, Delise.

aurata, $A c h$.

physcosporoides, F. v. M. et Muell. Arg.

fragillissima, Muell. Arg.

hypoleuca, Muell. Arg.

Karstenii, Muell. Arg.

var. linearis, Muell. Arg.

Sayeri, Muell. Arg.

sulphurea, Scherer.

flavissima, Muell. Arg.

glaucescens, Kremfelt.

dissimulata, $N y l$.

dissimilis, $N y l$.

aurata, var. pallida-glaucescens, C. Knight.

var. microphylla, Muell. Arg.

Billardieri, Delise.

dichotomoides, $N y l$.

impressa, Hook. et Tayl. = S. physciospora, Nyl.; S. Bennetii, Muell. Arg.

demutabilis, Krph.

carpolomoides, $N y l$.

glaucescens, $K r p h$.

Seemani, $B a b$.

Parmosticta, Stirton.

rubrina, Stirton.

Ricasolia, De Not.

crentlata, $N y l$.

Hartmanni, Muell. Arg.

hypoleuca, Muell. Arg.

plurimiseptata, C. Knight.

sublævis, $N y l$.

rhaphisphora, C. Knight.

patinifera, Muell. Arg.

erosa, Eschw. = R. stenospora, Nyl.

Ravenelii, Tuck.

Schæreri, $N y l .=$ Sticta Schareri, Mont. (Fig. 709.)

Parmelia, Ach.

latissima, Fée.

var. ciliata, $N y$.

mundata, $N y l$.

brisbanensis, Stirton.

adpressa, Kremp. $=$ P. amplexula, Stirton.

$2 \mathrm{~V}$ 
Parmelia-contd.

laceratula, $N \%$.

var. minor, Shirley.

reperata, Stirton.

perlata, Ach.

var. olivaria, $A c h .=P$. olivetorum, Ach.

var. ciliata, $D C$.

caperata, Ach.

revoluta, Floerke.

molliuscula, Ach. (Fig. 7Io.)

perforata, Ach. (Fig. 7II.)

var. ciliata, $N y l$.

pretervisa, Muell. Arg. = P. tinctorum, Despr.; P. perlata, var. pratervisa, Muell. Arg.

limbata, Laurer.

var. minor, C. Knight.

var. endococcinea, Muell. Arg.

conspersa, Ach.

var. exasperata, Muell. Arg.

var. hypocleistoides, Muell. Arg.

olivetorum, Ach.

austro-africana, Stirton.

sinuosa, S. $M$.

euplecta, Stirton.

erubescens, Stirton.

corallina, Ach.

eciliata, $N y l$.

hospitans, Muell. Arg.

Mongestii, Scharer.

ochroleuca, Muell. Arg.

pruniata, Muell. Arg.

rutidota, Tayl.

forma sorediosa, Muell. Arg. = P. ochroleuca, f. sorediosa,

Muell. Arg.

virens, Muell. Arg.

sphærospora, C. Knight.

tenuirima, var. corallina, Muell. Arg.

hypoxantha, var. major, Muell. Arg.

gracilis, Muell. Arg.

olivacea, Linn.

nitescens, Stirton.

cyathina, Stirton.

platycarpa, Stirton.

permutata, Stirton.

tiliacea, Ach. 
Parmelia-contd.

tiliacea-contd.

var. rugulata, Muell. Arg. $=$ P. caperatula, Stirton (non Nyl.), Shirl. Lich. F1. Q1. p. 42.

var. affixa, Strn.

var. stenophylla, Muell. Arg.

var. sulphurosa, Muell. Arg. $=$ P. tiliacea, forma asperator, Muell. Arg.

meizospora, $N y l .=P$. tiliacea, var. meizospora, $\mathrm{Nyl}$.

gracilis, Muell. Arg.

cetrata, Ach., var. sorediifera, Wainio $=P$. cristulata, Fée. Hookeri, Tayl. $=$ P. sublevigata, $\mathrm{Nyl}$.

tenuirima, Tayl.

ablata, Strn.

cetrarioides, Del.

physodes, Ach.

var. pulverata, Muell. Arg. = P. subphysodes, Kemp.

placorhodioides, $N y l$.

eciliata, $N y l$.

encausata, Ach.

corrugis, var. sorediata, Muell. Arg. =P. hypotropa, var. sorediata, Muell. Arg.

Anaptychia, Trev.

leucomolæna, Trev. = Physcia lencomela, Mich.

comosa, Trev. = Physcia comosa, var. alata, Wils.

speciosa, var. sorediosa, Muell. Firg.

var. hypoleuca, forma sorediifera, Muell. Arg.

Physcia, Nyl.

chrysopthalma, $D C$. (Fig. 7I2.)

var. leucoblephara, Muell. Arg.

var. Sieberianus, Laur.

var. alatus, Shirley.

var. denudata, Hoffm.

crispa, $N y l$.

var. Ravenelii, Tuck.

speciosa, Fries $=$ Lichen speciosus, Wulf.

var. soredioidea, Wainio.

var. hypoleuca, $A c h .=P$. hypoleuca, Nyl.

angustifolia, $N y l .=P$. leucomela, var. angustifolia, $M$. et $F$. flavicans, $D C$.

var. croceus (Ach.), $N y l$.

picta, $N y l$. (Fig. 7Iз.)

var. isidiophora, $\mathrm{Nyl}$.

var. sorediata, Muell. Arg.

stublurida, Stirton. 
Physcia-contd.

confluens, Mont.

stellaris, Fr.

var. acrita, $N y l$.

var. radiata, Ach.

aipola, Ach.

var. acrita, Ach.

barbifera, $N y l$.

adglutinata, Florke.

glanco-virescens, $N y$.

obesa, var. cæsio-crocata, Tuck.

sparsa, Tayl.

excelsior, Strn.

Tribe XiII.-Gyrophorei.

Pyxine, Fries.

Meissnerii, Tuck. (Fig. 7I4.)

var. endoleuca, Muell. Arg.

forma sorediosa, Muell. Arg.

cocoës, $N y l$.

var. sorediata, $N y l$.

var. endoxantha, Muell. Arg.

obscurior, Stirton.

retirugella, $N y l$.

picta, Tuck.

confluens, Fr.

endochrysina, $N y l$.

subvelata, Stirton.

rugulosa, Stirton.

subcinerea, Stirton.

Heppia, Nog.

brisbanensis, Wilson.

Series E.-Placodiet.

Tribe XIV.-Lecanorer.

Psoroma, Fries.

dispersum, Stirton.

Karstenii, Muell. Arg.

sphinctrinum, $N y l$.

cæsium, Muell. Arg.

Pannaria, Delise.

flexuosa, C. Knight. (Fig. 7т5.)

isidoides, Muell. Arg.

integrata, $\mathrm{Nyl}$.

Mariana, Muell. Arg. 

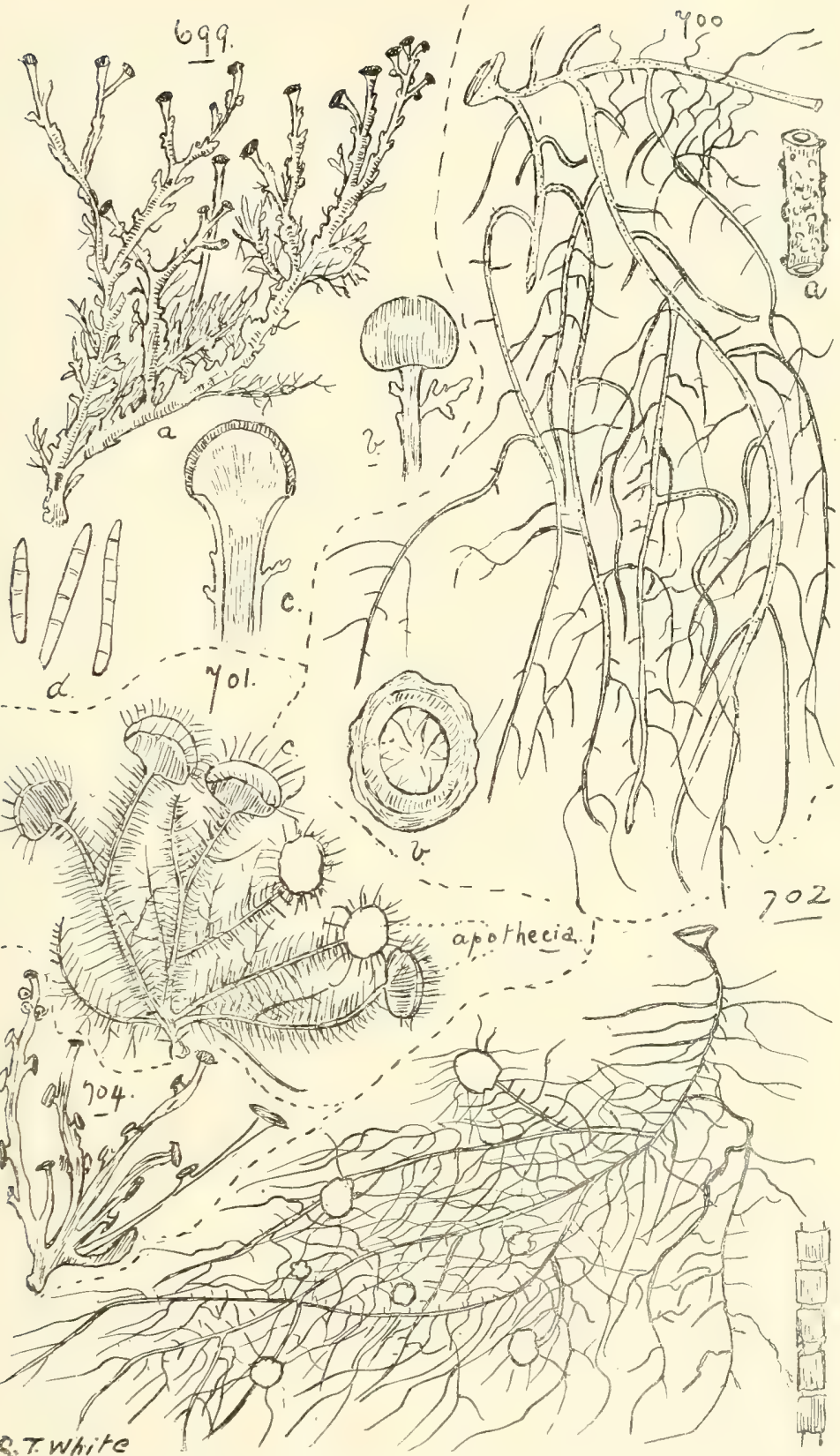

699. Stereocaulon ramulosum, Ach.

(a) Plant, (b) aptherium, (c) sect. do., (d) spores. (a) nat. size; (b)-(d) enl. 700. Usnea barbata, Ach., var. AsperRima, Muell. Arg.. (a) Port, stem, (b) sect, do. (a) and (b) enl.

7OI. U. ELEGANS, Stirt.

702. U. articulata, Ach

704. Ramalina leiodea, $N y l$. 
Pannaria-contd.

Mariana-contd. forma isidiosa, Muell. Arg. $=$ P. pannosa, f. isidiodea, Muell. Arg.

fulvescens, Mont.

brisbanensis, C. Knight.

rubiginosa, Del., var.

rordida, C. Knight.

cervina, Kremph.

pholidota, $N y l$.

pannosa, Delise. (Fig. 716.)

var. accolens, Strn.

molybdæa, Ach.

triplophylla, $N y l$.

ciliolata, Mont., sterilis. (See Q1. Agric. J1. v. (I899) 40.)

elatior, Strn.

terrestris, Strn.

Dichonema, Nees.

irpicinum, $N y l$.

sericeum ( $\mathrm{Sw}_{\mathrm{w}}$ ), Mnt.

Lecanora, $N y l$.

vitellina, Ach.

Tribe I.-Acraspora.

Tribe II.-Eulecanora.

subfusca, $\operatorname{Linn}$.

var. chlarona, Ach.

var. coilocarpa, $N y l .=L$. coilocarpa, Ach.

var. umbrina, Ehreb.

var. cinereo-carnea, Tuck.

var. compacta, Muell. Arg.

var. allophana, Ach. = var. horisa, Fée.

var. conjugens, Muell. Arg.

var. gangalea, Ach. $=$ L. gangalea, Ach.

var. chlarotea, Nyl.

var. distans, $N y l$.

var. testaceo-pallida, Muell. Arg.

var. subgranulata, $N y l .=L$. subgranulata, $\mathrm{Nyl}$.

plumosa, Muell. Arg.

albella, Pers.

rutilescens, Stirton.

pulverata, Stirton.

conyzæa, $N y l$.

atra, Huds.

var. virens, Muell. Arg.

var. immarginata, C. Knight. 
Lecanora-contd.

atra-contd.

var. serialis, Muell. Arg.

pinguis, Tuck.

queenslandiæ, C. Knight.

sorediifera, Fée.

melanommata, C. Knight.

sordida, Fries.

rhodopthalma, Muell. Arg.

Tribe III.-Rinodina.

continua, C. Knight.

thiomela, Nyl.

diffractella, Muell. Arg.

exigua, Ach.

minutula, Muell. Arg.

xanthomelana, Muell. Arg.

flavido-fusca, Muell. Arg.

Tribe IV.-Hamatomma.

punicea, Ach. (Fig. 7I7.)

var. collata, Stirton.

zar. infusea, Stirton.

Babingtonii, Mass.

Tribe $V$.-Lecanorastrum.

parella, Ach. (Fig. 7I8.)

var. phæoleuca, Nyl. (Fig. 7I9.)

pallescens, Linn.

subundulata, C. Knight.

tartarea, $\operatorname{Linn}$.

aurantiaca, Lightf.

rutilescens, Strn.

parella, Linn.

alligata, Strn.

phæantha, $N y l$.

interjecta, Muell. Arg.

granifera, Ach.

lacteola, Muell. Arg.

subimmersa, Muell. Arg.

connivens, Muell. Arg.

cæsio-rubella, Ach.

rhypoderma, Muell. Arg.

subpurpurea, Strn.

phæoplaca, Strn.

Knightiana, Muell. Arg.

melacarpella, Muell. Arg.

albellaria, Muell. Arg. 


\section{Coccocarpia.}

aurantiaca, Mont. et V. D. Bosch. $=$ Leptogium coruleum, Wilson.

pellita, Muell. Arg.

var. isidiophylla, Muell. Arg.

var. incisa, Muell. Arg.

var. smaragdina, Muell. Arg.

plumbea, Leight.

molybdæa, Pers.

Amphiloma, Fries.

gossypinum, $N y l$.

murorum, Karber.

glaucescens, Rev.F.R.M.Wils.

Placodium, $D C$.

cirrhochroum, Ach., forma thallus leprossis, C. Knight.

plurilocellare, Muell. Arg.

galactinum, var. dispersum, Pers.

glauco-lividum, Muell. Arg.

clavigerum, Stirton.

Callopisma, Notaris.

cinnabarinum, Ach.

var. opacum, Muell. Arg.

sanguinolentum, Krempel.

aurantiacum, $N y l$.

rubens, Muell. Arg.

conjungens, Muell. Arg.

Pertusaria, $D C$.

pustulata, Duby.

leutescens, Kremp.

melaleuca, Duby'.

trypetheliiformis, $N y l$.

var. Hartmanni, Muell. Arg.

xanthoplaca, Muell. Arg.

leucostigma, Muell. Arg.

dermatodes, $N y l$.

lactea, $N y l$. $=P$. sorediata, C.K.

gibberosa, Muell. Arg.

pertusella, Muell. Arg.

subflavens, Muell. Arg.

leioplacoides, Muell. Arg.

pilulifera, Pers.

porinella, $N y l$. (Fig. 720.)

leioplacella, $N y$.

minuta, C. Knight. 
Pertusaria-contd.

petrophyes, C. Knight. (Fig. 72I.)

thiospoda, C. Knight.

velata, Turn.

leiotera, Muell. Arg.

leioplaca, Ach.

var. gibbosa, Muell. Arg.

var. octospora, $N y l$.

var. minor, Schar.

persulphurata, Muell. Arg.

meridionalis, var. xanthostoma, Muell. Arg.

subvaginata, $N y l .=$ Variolaria globulifera, Fée; $V$. communis, Fée.

Wulfenii. $D C$.

macra, Muell. Arg.

albinea, Muell. Arg.

straminea, Muell. Arg.

irregularis, Muell. Arg.

amblyogona, Muell. Arg.

leucoxantha, Muell. Arg.

confluens, Muell. Arg.

subrigida, Muell. Arg.

leiocarpella, Muell. Arg.

elliptica, Muell. Arg.

leucostoma, Muell. Arg.

rhodotropa, Muell. Arg.

sulphurata, Muell. Arg.

globulifera, $N y l$.

commutata, Muell. Arg. = Variolaria commutata, Fée

depressa, var. octomera, Muell. Arg.

undulata, Muell. Arg.

plicatula, Muell. Arg.

multipuncta, Turn.

Phæotrema, Muell. Arg.

consimile, Muell. Arg.

cricotum, Muell. Arg. = Ocellularia cricota, Wilson

Thelotrema, Ach.

expansum, C. Knight. (Fig. 722.)

australiense, Muell. Arg.

laceratulum, Muell. Arg.

megalophthalmum, Muell. Arg.

trypethelioides, C. Knight. (Fig. 723.)

compunctum, $S m$.

heterosporum, C. Knight.

Wightii, $N y l$. 


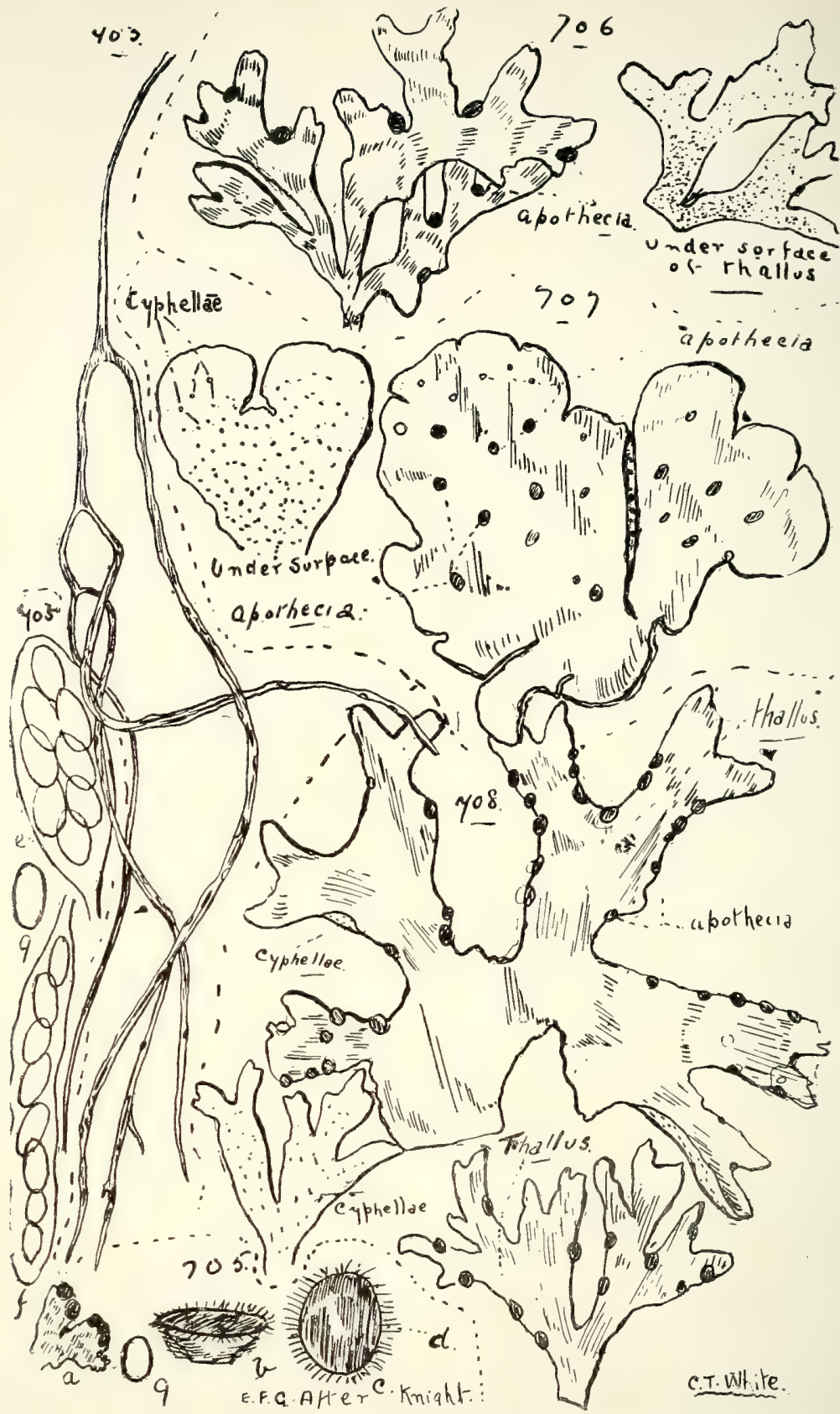

703. Ramalina farinacea, Nyl., var. nervulosa, Muell. Arg. 705. Platysma eriophyllum, Knight.

(a) Port. of thallus with apothecia, (b) apothecium, side view, (d) do. viewed from above, showing ray of filaments, (e) and (f) two forms of asci, (g) spores. (a) nat. size; (b)-(g) enl.

706. Stictina crocata, var. esorediata, Muell. Arg.

707. S. suborbicularis, Muell. Arg.

708. Sticta Camare, Muell. Arg. 
Thelotrema-contd.

microporellum, $N y l$.

olivaceum, Mont.

phæosporum, $N y l$ :

tærebratulum, $N y l$.

inturgescens, Muell. Arg.

argenteum, Muell. Arg.

megalosporum, Muell. Arg.

bicuspidatum, Muell. Arg.

endoxanthum, Muell. Arg.

rimulosum, Muell. Arg.

cupulare, Muell. Arg.

cyphelloides, Muell. Arg.

microphtalnum, Muell. Arg. = Ocellularia terebratulum, J. Shirley.

profundum, Shirley $=$ Ascidium profundum, Stirton.

Ascidium, Fée.

depressum, Mont.

octoloculare, C. Knight.

monosporum, C. Knight.

Diploschistes, Norm.

actinostomus, $Z$ ahl. $=$ Urceolaria actinostoma, Schr.; U. nove-zealandice, C. Knight; Lecidea clausa, C. Knight.

Leptotrema, Muell. Arg.

fallax, Muell. Arg.

albo-coronata, C. Knight.

integrum, Muell. Arg.

polycarpum, Muell. Arg.

patulum, Muell. Arg.

diffractum, Muell. Arg.

compunctum, Nyl. (See Q1. Bot. Bu1l. viii. (1893) I03.)

nitidulum, Muell. Arg.

æmulum, Muell. Arg.

Tribe XV.-Lecidiei.

Coenogonitim, Ehrenb.

interplexum, $N y l$.

botryosum, C. Knight.

Linkii, Ehrenb. (Fig. 724.)

rigidulum, Muell. Arg.

confervoides, $N y l .=C$. interpositum, Nyl.

moniliforme, Tuck. = Biatorinopsis torulosa, Muell. Arg.

implexum, $N y l$. 
Gyalectidium, Muell. Arg.

phyllocharis, Muell. Arg. = Lecidea phyllocharis, Nyl. (Fig. 726$.

filicinum, Muell. Arg.

Lecidea, $A c h$.

Tribe Psora.

foliata, Stirton.

var. subcorallina, Muell. Arg.

var. atrovirens, C. Knight.

parvifolia, Pers.

var. fibrillifera, $N y l$.

var. subgranulosa, Muell. Arg.

subhyalina, Stirton.

breviuscula, $N y l$.

rhypoderma, C. Knight. (Fig. 725.)

Tribe Eulecidea.

speirea, Ach.

contigua, Fries.

albo-cærulescens, $W$ ulf. $=L$. submubila, Stirton.

confluens, Fries.

meiospora, $N y l$.

impressa, Krph.

var. angulosa, Muell. Arg.

ferax, Muell. Arg., var. geographica, Muell. Arg.

Tribe Lecidella.

nesophila, Muell. Arg.

Tribe Biatora.

aberrata, Stirton.

aspidula, Krempel.

var. dispersa, Muell. Arg.

russula, $A c h$.

aurigera, Stivton.

bacidioides, Muell. Arg.

ludibunda, Muell. Arg.

subsimilis, $N y l$.

phæocarpa, C. Knight.

scabrida, C. Knight.

leioplaca, Muell. Arg.

mutabilis, Fée.

piperis, Spreng.

var. melanocarpa, Muell. Arg. = L. subuletorum, var. brasiliensis, Eschw. 
Lecidea-contd.

angloensis, Muell. Arg.

leptoloma, Mucll. Arg.

exigua, Chaub.

insulana, Muell. Arg.

Tribe Biatorella.

conspersa, Fries.

hæmatina, Muell. Arg. = L. plumbeella, Muell. Arg.; Lecanora (Aspicilia) lavissima, C. Knight.

Tribe Patellaria.

planella, $N y l$.

reniformis, J.F. Shirl.

leucoblephara, $N y$.

australiensis, Muell. Arg.

ventricosa, Muell. Arg.

inflexa, C. Knight.

brisbanensis, C. Knight.

domingensis, Ach.

var. coralloidea, Muell. Arg.

var. ventricosa, Muell. Arg.

var. inexplicata (Nyl.), Muell. Arg.

incompta, var. spissa, J. F. Shirl.

contraria, Muell. Arg.

superula, $N y l$.

effusa, $S m$.

furfurella, Muell. Arg.

subproposita, Muell. Arg.

alutacea, Krph.

Raffii, Stirton.

flavicans, Muell. Arg.

rhodocardia, Muell. Arg.

triseptata, Hepp.

tuberculosa, Muell. Arg. $=$ L. chloritis, Tuck.

melanodermia, Muell. Arg.

millegrana, Tayl $=$ Patellaria heterochroa, Muell. Arg.

var. fusco-nigrescens, Muell. Arg.

albo-flavicans, Muell. Arg.

intermixta, Muell. Arg.

entodiaphana, C. Knight.

melaclina (Nyl.), Muell. Arg.

sphæroides, Dicks.

multiseptata, $N y l$.

Tatiensis, Mont. (Fig. 727.)

var. epiglauca, $N y l$. 


\section{Lecidea-contd.}

ferruginea, Huds.

Tribe Blastenia.

ochroleuca, Muell. Arg.

Tribe Buellia.

exilis, Krempel.

stellulata, Tayl.

substellulata, C. Knight.

atro-alba, Ach.

parasema, Fries.

var. saprophila, Korb.

var. rugulosa, Korb.

var. vulgata, Th. Fries.

lactea, Korb. = L. saxatilis, Sch. = Buellia spuria, Arn.

demutans, Stirton.

myriocarpa, $D C$. (Fig. 728.)

disciformis, Fr.

var. cinereo-ferruginea, C. Knight.

glomerella, Strn.

placomorpha, Strn.

subdisciformis, Leight.

lauri-cassiæ, Fée.

modesta (Krph.), Muell. Arg.

innata, Muell. Arg.

subareolata, Muell. Arg.

tetrapla, var. nigro-cincta, Muell. Arg.

subarenaria, Muell. Arg.

rimulosa, Muell. Arg.

amblygona, Muell. Arg.

macrosporoides, Muell. Arg.

subrepleta, Strn.

subconnexa, Strn.

restituta, Strn.

Tribe Rhizocarpon.

geographica, var. cyclopia, $N y l$.

Tribe Heterothecium.

vulpina, Tuck., var. glaucescens, $N y l .=L$. domingensis, var. gyrosa, Stirton.

forma corallinum, Muell. Arg.

leucoxantha, Mass.

parabola, Nyl., var. subvulpina, Muell. Arg.

Sayeri, Muell. Arg. 
Lecidea-contd.

pulchrum, Muell. Arg.

biferum (Nyl.), Muell. Arg.

fusco-luteum, Dicks. = Heterotheceum fusco-luteum, Muell.

Arg.

\section{Tribe Nesolechia.}

coccocarpiæ, Muell. Arg.

subcærulea, Stirton, Q1. Agric. J1. v. ( I899) 40.

luteola, var. conspondens, $N y l$.

vinicolor, Strn.

sanguinolenta, Strn.

nodulosa, Strn.

Dermatocarpon, Muell. Arg.

miniatum, Th. M. Fries. = Lecidia miniata, Linn.

Thalloidima, Muell. Arg.

cæruleo-nigricans, Muell. Arg. = Lecidea caruleo-nigricans,

Leight.

Biatorinopsis, Muell. Arg.

lutea, Muell. Arg.

zonata, Muell. Arg.

Ocellularia, Muell. Arg.

Bonplandi, Spreng.

leucotyla, $N y l$.

zeorina, Muell. Arg.

pulchra, Muell. Arg.

diffractella, Muell. Arg.

Baileyi, Muell. Arg.

goniostoma, Muell. Arg.

xantholeuca, Muell. Arg.

leucotylia, Muell. Arg.

phlyctioides, Muell. Arg.

endomelæna, Muell. Arg.

jugalis, Muell. Arg.

platychlamys, Muell. Arg.

annulosa, Muell. Arg.

\section{Tribe XVI.-Graphidel.}

Opegrapha, Ach.

Bonplandi, Fée.

var. abbreviata, Muell. Arg.

inalbescens, Muell. Arg.

intrusa, Stirton.

lucina, Muell. Arg.

plurilocularis, $N y l$. 
Opegrapha-contd.

plurilocularis-contd.

var. pruinosa, Muell. Arg.

var. obfuscata, Muell. Arg.

grossulina, Muell. Arg.

interveniens, Muell. Arg.

varia, var. diaphora, $N y l$.

vulgata, var. subsiderella, $N y$ ?

platygraphoides, Muell. Arg.

lactella, Muell. Arg.

prosodea, Ach.

minutula, Muell. Arg.

microcarpella, Muell. Ars.

Platygranha, $N \mu l$.

Shirleyana, Mueī̄. Arg.

Melaspilea, $N y l$.

opegraphoides, iv"yi.

asteriscus, Muell. Arg.

congregans, Muell. Arg.

congregantula, Muell. Arg.

stellaris, Muell. Arg.

Phæographis, Muell. Arg.

leiogrammodes, Muell. Arg.

pseudomelana, Muell. Arg.

Graphis, Ach.

mucronata, Stirton. (Fig. 729.)

eludens, Stirton.

circumfusa. Stirton.

malacodes, $N y l$.

Afzelii, Ach. (Fig. 730.)

persulcata, Stirton.

argopholis, C. Knight.

Parmeliorum, C. Knight. (Fig. 731.)

assimilis, $N y l$.

crassilabra, Muell. Arg.

duplicata, Ach.

propinqua, Muell. Arg.

rimulosa, Mont.

var. pulverulenta, $N y l$.

var. brachycarpa, Muell. Arg.

Sayeri, Muell. Arg.

scripta, $N y l$.

var. recta, $N y l$.

striatula, $N v l$.

subtenella, Muell. Arg. 

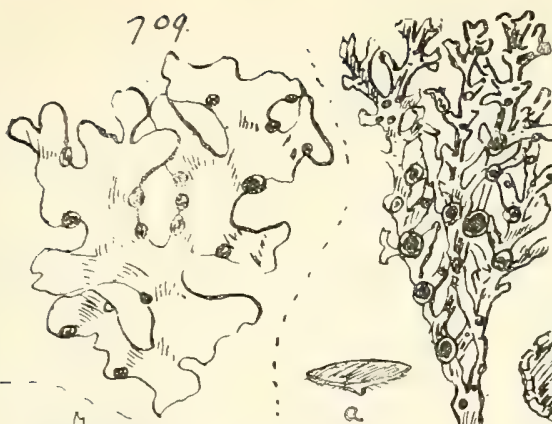

(4)

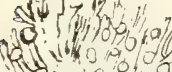

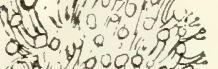
19 230 ith 200 ing $0=0$ yi2.

is

(9)

(-6)

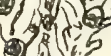

$3=$

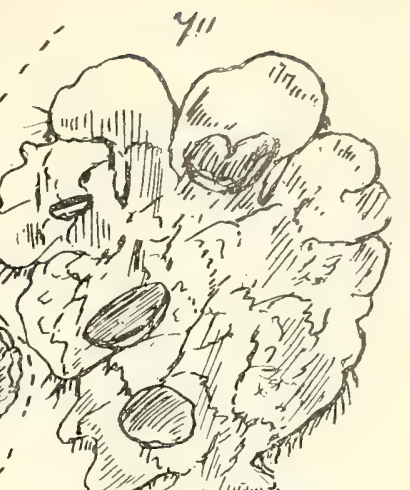

a.
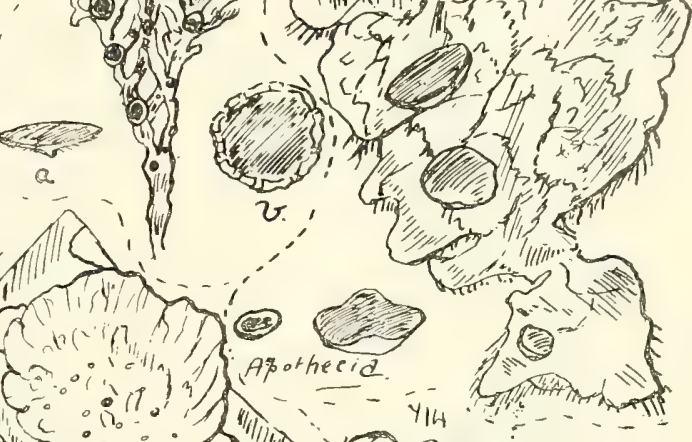

ele

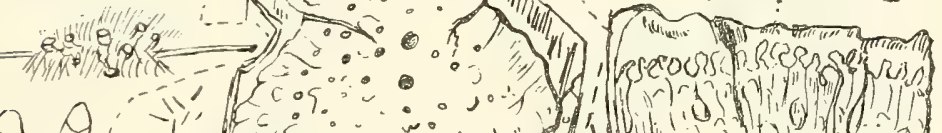

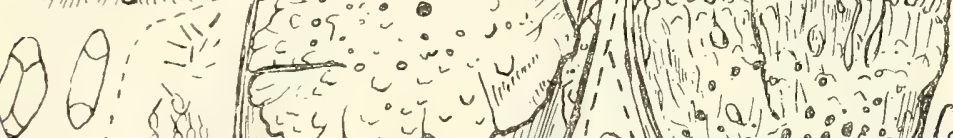

spores.

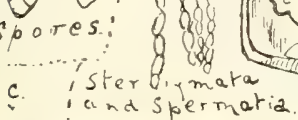

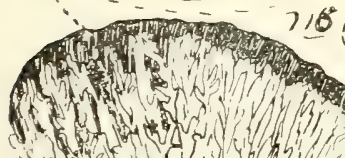

$716:$

$-4,5$

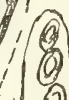

(0)

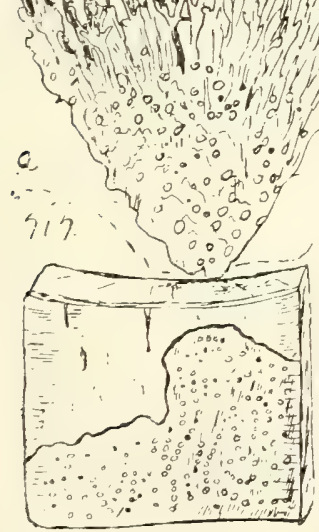

709. RiCASOLIA SCH FRERT, Nyl.

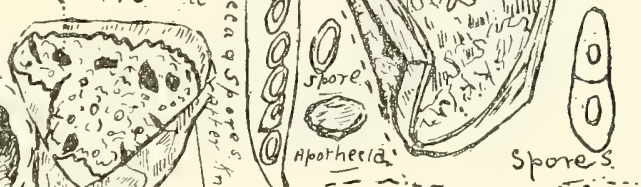
spores.
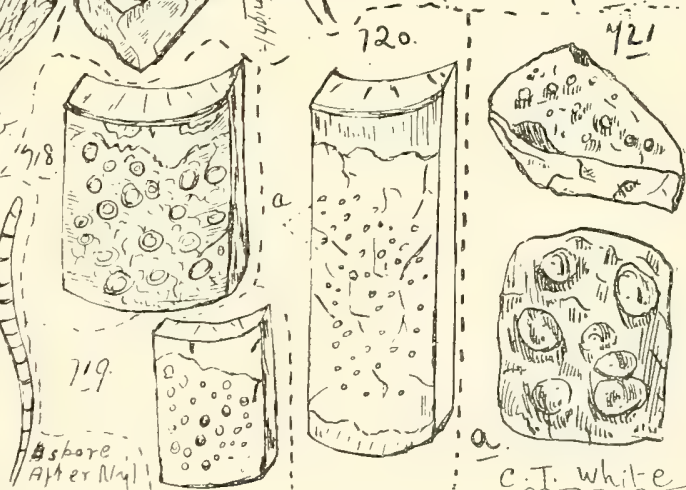

7IO. PARMelia MOLlituscula, Ach
(a) Apothecium, side view, (b) do. viewed from above. (a) and (b) enl.

iIt. P. PERFORATA, Ach.

iI3. P. PICTA, Nyl.

7i5. Pannaria flexuosa, C. Knight.

w, (b) do. viewed from above. (a) and (b) enl.
7i2. Physcia chrysopthalma, DC.

7i4. Pyxine Meissneri, Tuck.

7i6. P. pannosa, Delile.

(a) Thallus, (b) apothecia, (c) black hypothalline border.

717. Lecanora punicea, Ach.

7I8. L. parella, Ach.

7i9. L. parella, var, phæoleuca, $N y l$. 720. Pertusaria porinella, $N y l$.

(a) Apothecia.

721. P. PETROphyes, C. Knight.

(a) Port. plant enl. 
Graphis-contd.

tenella, Ach.

vermifera, Muell. Arg.

innata, C. Knight.

glaucoderma, $\mathrm{Nyl}$.

Lineola, Ach.

emersa, Muell. Arg.

albo-nitens, Muell. Arg.

lævigata, Muell. Arg.

robustior, Muell. Arg.

duplicata, Ach.

var. sublævis, Muell. Arg. $=$ G. striatula, var. sublavis, Nyl.

isidiosa, Muell. Arg.

Baileyana, Muell. Arg., Q1. Bot. Bu1l. viii. (I893) Iо3.

descissa, Muell. Arg.

vinosa, Muell. Arg.

albissima, Muell. Arg.

immersella, Muell. Arg.

epimelæna, Muell. Arg.

xanthospora, Muell. Arg.

subvelata, Strn.

semiaperta, Muell. Arg.

Graphina, Muell. Arg.

trachyspora, Muell. Arg.

Hartmanni, Muell. Arg.

polyclades, Kremp.

saxicola, Muell. Arg.

subtartarea, Muell. Arg.

palmicola, Muell. Arg.

sophistica, $N y l$.

var. recta, Muell. Arg.

fisso-furcata, Leigh. (Fig. 732.)

repleta, Stirton.

pertenella, Stirton.

simulans, Leigh.

tenurima, Shirley.

egenella, Muell. Arg.

Phæographina, Muell. Arg.

chrysentera, Muell. Arg.

contexta, Muell. Arg.

cæsio-pruinosa, Muell. Arg. = Leiogramma lateritium, Eschw.

var. monospora, Muell. Arg.

quasiæcola, Muell. Arg. = Graphis exserta, Nyl.; Graphina pyelodes, Wilson. 
Helminthocarpum, Muell. Arg.

Lojkanum, Muell. Arg.

Baileyanum, Muell. Arg.

Tremotylium, Muell. Arg.

a1xstralianum, Mucl. Arg.

nitidultim, Muell. Ary.

Arthonia, Ach.

Ricasoliæ, Muell. Arg.

cinnabarina, $W$ all. (Fig. 733.)

delicatula, Muell. Arg.

gracilenta, Muell. Arg.

gracillima, Muell. Arg.

Thozetiana, Muell. Arg.

vulgaris, var. astroidea, C. Knight.

cinereo-argentea, C. Knight.

gregaria, var. purpurea, Eschw. = Conioloma coccineum, Eschw., var. purpureum, Eschw.

gregaria, var. adspersa (Mnt.), Nyl. = Leiogramma tenella, Eschw.; Conioloma coccineum, Eschw.

subgyrosa, $N y l$.

leptospora, Muell. Arg.

gracilior, Muell. Arg.

amœna, Muell. Arg.

rubella (Fée), $N y l$.

subcondita, Strn.

conspersula, Strn.

varia, $A$ ch.

albofarinosa, Strn.

Arthothelium, Muell. Arg.

puniceum, Muell. Arg.

microsporum, Muell. Arg.

polycarpum, Muell. Arg.

macrotheca, Muell. Arg.

Mycoporellum, Muell. Arg.

perexiguum, Muell. Arg.

Glyphis, Ach.

favulosa, Ach.

var. intermedia, Muell. Arg.

var. depauperata, Muell. Arg.

labyrinthica, Ach.

medusulina, $N y l$.

confluens, Mont. (Fig. 734.)

colliculosa, C. Knight. 
Glyphis-contd.

verrucosa, C. Knight.

cyclospora, Muell. Arg.

cribosa, Fée.

Chiodecton, Ach.

ochraceo-fuscescens, C. Knight.

hypoleucum, C. Knight. (Fig. 735.)

sphærale, Ach. $=$ C. stromaticum, C. Knight.

farinaceum, Fée.

effusum, Fée.

rubro-cinctum, $N y l$.

sublævigatum, Kremp.

endoleucum, Muell. Arg.

hamatum, $N y l$.

virens, Muell. Arg.

Sarcographa, Muell. Arg.

subtricosa, J.M. = S. actinota, Wils.

forma pulverulenta, Wils.

oculata, Muell. Arg.

Asterotrema, Muell. Arg.

punctuliforme, Muell. Arg.

Tribe XVII.-Xylographide.

Diplogramma, Muell. Arg.

australiensis, Muell. Arg.

Series F.-Pyrenodei.

Tribe XVIII.-Pyrenocarpei.

Strigula, Fries.

nemathora, Mont.

complanata, Fée.

elatior, Stirton. (Fig. 736.)

elegans, var. Nematora, Muell. Arg.

elegans, var. pertenuis, Muell. Arg.

elegans, var. eumorpha, Muell. Arg.

Glaziovii, Muell. Arg.

Ccphaleuros virescens, mentioned in my and Adden. 3rd Suppl. Syn. Q1. Flora, is only one of a genus considered now only an abnormal development of the filamentous element of Strigula, and is commonly met with on fernfronds growing in our dense tropical scrubs; it is said to be a serious pest in the tea plantations of India.

Parmentaria, Fries.

papillata, C. Knight.

umbilicata, C. Knight.

subumbilicata, C. Knight.

plana, C. Knight. 
Parmentaria-contd.

subplana, C. Knight.

gregalis, C. Knight.

pallida, C. Knight. (Fig. 737.)

microspora, Muell. Arg.

Baileyana, Muell. Arg.

astroidea, $F_{\text {ée }}=$ Verrucaria astroidea, Fée; Heupleria pentagastrica, Muell. Arg.

subastroidea, Muell. Arg.

toowoombensis, Muell. Arg.

interlatens, Muell. Arg.

grossa, Muell. Arg.

Campylothelium, Muell. Arg.

nitidum, Muell. Arg.

defossum, Muell. Arg.

Pleurothelium, Muell. Arg.

australiense, Muell. Arg.

Parathelium, Nivl.

decumbens, Muell. Arg., Q1. Bot. Bull. viii. (I893) I05.

Trypethelium, Ach.

mastoideum, Ach.

tropicum, Muell. Arg.

var. nigratum, Muell. Arg.

catervarium, Tuck.

scoria, Fée.

Sprengelii, Ach.

papillosum, Ach.

Eleuteriæ, var. citrinum, Muell. Arg.

oligocarpum, Muell. Arg.

anomalum, Ach.

virgineum, Muell. Arg.

infuscatum, Muell. Arg.

exiguellum, Strn.

Plagiothelium, Stirton.

australiense, Stirton.

Bathelium, Muell. Arg.

chrysocarpum, Muell. Arg.

Melanotheca, Fée.

cruenta, Muell. Arg. = M. rubra, C. Knight.

cinnabarina, C. Knight.

rubescens, C. Knight $=$ Microthelia Shirleyana, Muell. Arg.

Achariana, Fée.

subsimplex, Muell. Arg.

oxyspora, Muell. Arg. 
Bottaria, Mass.

umbilicata, Muell. Arg. = Trypethelium umbilicatum, C. Knight.

Tomasellia, Mass.

dispora, Muell. Arg.

aciculifera, Muell. Arg.

queenslandica, Muell. Arg.

Endocarpon, Hedw.

Baileyi, Stirton.

Pleurotrema, Muell. Arg.

pyrenuloides, Muell. Arg.

Verrucaria, Pers.

coarctata, Strn.

fibrata, Strn.

Porina, Ach.

Subtribe Verrucaricce.

bacillifera, Muell. Arg.

exasperata, C. Knight.

africana, Muell. Arg. = P. limitata, C. Knight.

rudis, Muell. Arg. = P. mastoidea, var. rudis, Muell Arg. mastoidea, Ach.

persimilis, Muell. Arg.

præstantior, Muell. Arg.

phæophthalma, J.F. Shirl. = P. brisbanensis, Muell. Arg. subargillacea, var. nigrata, Muell. Arg.

tetraceræ, Ach.

Araucarieæ, Muell. Arg.

variegata, Fée.

glauca, Muell. Arg.

bellendica, Muell. Arg.

rhapidiospora, Muell. Arg. $=$ Verrucaria lepidospora, C. Knight.

internigricans, Muell. Arg.

fulvula, Muell. Arg.

pallida, Muell. Arg.

Phylloporina, Muell. Arg.

epiphylia (Nyi.) Muell. Arg.

Clathroporina, Muell. Arg.

enteroxantha (C. Knight), Shirley = Porina enteroxantha,

C. Knight.

endochrysa, Mont. 
Clathroporina-contd.

pustulosa, Krph. (Fig. 738.)

meiospora (C. Knight), Shirley = Porina meiospora, C. Knight.

tomentella, Muell. Arg. = Porina farinosa, C. Knight.

olivacea, Muell. Arg. = Porina enteroxantha, C. Knight. robusta, Muell. Arg.

desquamans, Muell. Arg.

forma sorediosa, Muell. Arg., Q1. Bot. Bull. viii. (I893)

I05.

flavescens, Muell. Arg.

Arthopyrenia, Muell. Arg.

cinereo-pruinosa, Krph.

picea, J. F. Shirl.

gravastella, Krph.

zostra, C. Knight.

rhaphispora, C. Knight.

suboculata, Muell. Arg.

Cinchonæ,. Muell. Arg.

atomaria, Muell. Arg.

oculata, Muell. Arg.

fallacior, Muell. Arg.

limitans, Mucll. Arg. = Verrucaria limitans, Nyl.

consobrina, Muell. Arg. = Verrucaria consobrina, Nyl.

extans, Muell. Arg.

Pseudopyrenula, Muell. Arg.

sulphurascens, Muell. Arg.

nitidiuscula, $N y l$.

ceratina, Fée.

Pyrenula, Fée.

convexa, Muell. Arg.

defossa, Muell. Arg.

finitima, Muell. Arg.

segregata, Mueil. Arg.

flaventior, Stirton.

immersa, Muell. Arg.

Baileyi, C. Knight. (Fig. 739.)

circumrubens, $N y$ l., var. rubrotecta, Stirton.

velata, Muell. Arg.

micromma, Mont.

porinoides, Ach.

Kunthii, Fée.

marginata, Trev.

bicuspidata, Muell. Arg.

nigro-cincta, Muell. Arg. 


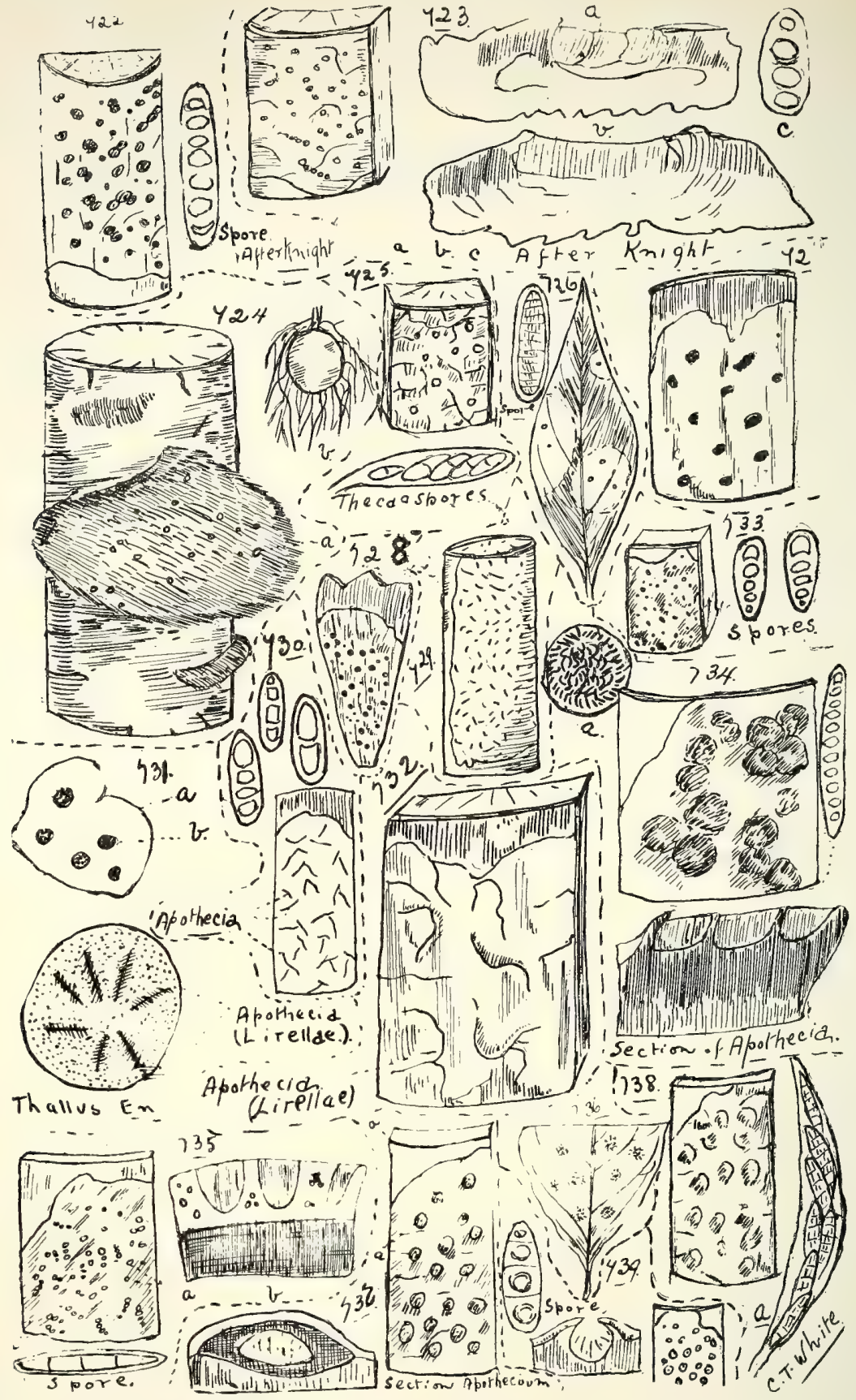

722. Thelotrema expansum, C. Knight. 723. T. trypethelioides, $C$. Knight.

(a) Sect. verruca with more than one apothecium, (b) do. do,, with only one apothecium, (c) spore. (a)-(c) ent.

724. Cenogonium Linkir, Ehrenb. 725 . Lecidea RHypoderima, $C$. Knight.

(a) Apothecium, nat. size, (b) apothecium and thallus filaments, eni.

726. Gyalectidium phyllocharis, Nyl. 727. Lecidea tatiensis, Mont.

728. L. MYrIocarpa, $D C$. 729. Graphis muCronata, Stirton.

730. G. AfzeliI, Ach.

729. Graphis muCronata, Stirton.
73I. G. PARMELIARU M, C. Knight.

(a) Thallus of Parmelia, (b) the parasite.

732. Graphina Fisso-furcata, Leight. 733. Arthonia cinnabarina, Wall.

734. Glyphis confluens, Mont. 735. Chiodecton hypoledcun, $C$. Knight. (a) Stroma and lirellæx, enl.

736. Strigula elatior, Stirton.

(a) Verruce, (b) sect. several verruca.
(a). Parmentaria Palima, C. Knight.

738. Clathroporina pustulosa, Krph. 739. Pyrenula Baileyi, C. Knight. 
Pyrenula-contd.

melaleuca, Muell. Arg.

nitidens, Muell. Arg.

mamillana (Ach.), Trev.

Warmingii, Muell. Arg.

mastophora (Nyl.), Muell. Arg.

adacta, Fée.

var. cinerascens, Muell. Arg.

Bonplandiæ, Fée $=P$. dispersa, Muell. Arg.; P. aspidistea,

Muell. Arg.

subcongruens, Muell. Arg.

velatior, Muell. Arg.

pinguis, Fée.

var. emergens, Muell. Arg.

nitida, Ach.

oxyspora, Muell. Arg.

indusiata, Muell. Arg.

sexlocularis, Muell. Arg.

microcarpoides, Muell. Arg.

mastophorizans, Muell. Arg.

Polyblastia, Muell. Arg.

velata, Muell. Arg.

tichospora, C. Knight.

nudata, Muell. Arg.

gregantula, Muell. Arg.

geminella, Muell. Arg.

var. cinerescens, Muell. Arg.

Anthracothecium, Muell. Arg.

sinapispermum, $N l$.

libricolum, Muell. Arg.

variolosum, Pers.

subvariolosum, C. Knight.

desquamans, Muell. Arg.

oligosporum, Muell. Arg.

Doleschalli, Muell. Arg.

macrosporum, Hepp.

strigosporum, Muell. Arg.

confine, Muell. Arg.

œenudatum, Muell. Arg. var. ochrotropum, Muell. Arg.

Thwaitesii, Muell. Arg. = Verrucaria Thwaitesii, Leight. amphitropum, Muell. Arg.

pyrenuloides (Mnt.), Muell. Arg.

oculatum, Muell. Arg.

oligósporum, Muell. Arg.

aurantium, Muell. Arg. = Verrucaria aurantium, Eschw. 
Microthelia, G. Körber.

miculiformis, Muell. Arg.

obovata, Muell. Arg.

brisbanensis, Muell. Arg.

alba, Muell. Arg.

queenslandiæ, Muell. Arg.

subregans, Muell. Arg.

\section{ABNORMAL LICHENS.}

Lepraria, Ach.

candelaria $=$ Lichen candelarius, Westr.

flava, Ach. = Lichen flava, Schreb.-The common yellowishgreen growth on fences, \&c.

\section{Order CLXII.-FUNGI.}

Many marked "Edible" are only so in a young state, and have been used in either Europe or America. Tastes differ greatly in this matter. One may consider only the common mushroom safe to use, while others consider only the Boleti and truffles are safe. It may, however, be seen from this list that we have in Queensland a very large number which it is quite safe to use as food, only be it understood that all fungi must be eaten only when fresh, for, as Dr. M. C. Cooke says, "high game" is not applicable to fungi.

\section{HYMENOMYCETES.}

\section{Family I.-Agaricini.}

Agaricus, Linn.

\section{Series I.-Leucospori.}

(Spores white.)

Subgenus Amanita.

ossideus, Fries.

vernus, Bull.-Poisonous. (Fig. 740.)

murinus, Cke. et Mass.

ananiceps, Berk.

Subgenus Amanitopsis.

vaginatus, Bull.-Edible, but not easily distinguished from poisonous kinds.

farinaceus, Cke. et Mass. (Fig. 74I.)

\section{Subgenus Lepicta.}

procerus, Scop.-Edible. The Parasol Mushroom of Europe. gracilentus, Kromb.-Edible. (Fig. 742.)

mastoideus, Fries.-Edible.

naucinus, Fries.-Edible.

var. sphærosporus, Cke. et Mass. 
Agaricus-contd.

bubalinus, Berk.

excoriatus, Schoeff.-Edible fawn-coloured Parasol Mushroom.

leontoderes, $B$. et $B r$.

cheimonoceps, B. et Curt.

granulosus, Batsch.

aspratus, Berk.

clypeolarius, Bull.

cepæstipes, Sow.-Edible.

var. cretaceus, Bull.-Edible.

dolichaulos, $B$. et $B r$.-Parasol Mushroom of Southern Queensland; the cap sometimes 9 in. across, on stalks. of 9 in. (Fig. 743.)

megalotheles, Kalchb.

rhytipelta, Kalchb. et F. v. M.

stenophyllus, Cke. et Mass. (Fig. 744.)

Beckleri, Berk.

bubalinus, Berk.

cretaceus, Bull.-Edible.

fimetarius, Cke. et Mass.-On dung.

ochrophyllus, Cke. et Mass.

membranaceus, Cke. et Mass.

aureus, Mass., Kew Bull. I912, p. I89. (Fig. 745.)

Subgenus Schulzeria.

revocans, Cke. et Mass.

Subgenus Armillaria.

melleus, $V$ ahl.-Edible. In America and Europe, known to be destructive to grape vines and other plants, causing. root-rot. Under the name "Stringy Rot," McAlpine (Fungus Dis. Pot. in Aus. p. 95) records this fungus as a serious potato pest in Tasmania, New South Wales, and Victoria. (Fig. 746.)

fulgens, Cke. et Mass.

\section{Subgenus Tricholoma.}

sulphureus, Bull.

sordidus, Fries.

civilis, Fries.

resplendens, Fries.

rheicolor, Berk.

Subgenus Clitocybe.

cerussatus, Fries.-Edible.

subsplendens, Cke. et Mass.

canaliculata, Cke. et Mass. (Fig. 747.)

laccatinus, Berk.

pruinosus, Lasch.

laccatus, Scop.-Edible. 
CLXII. FUNGI.

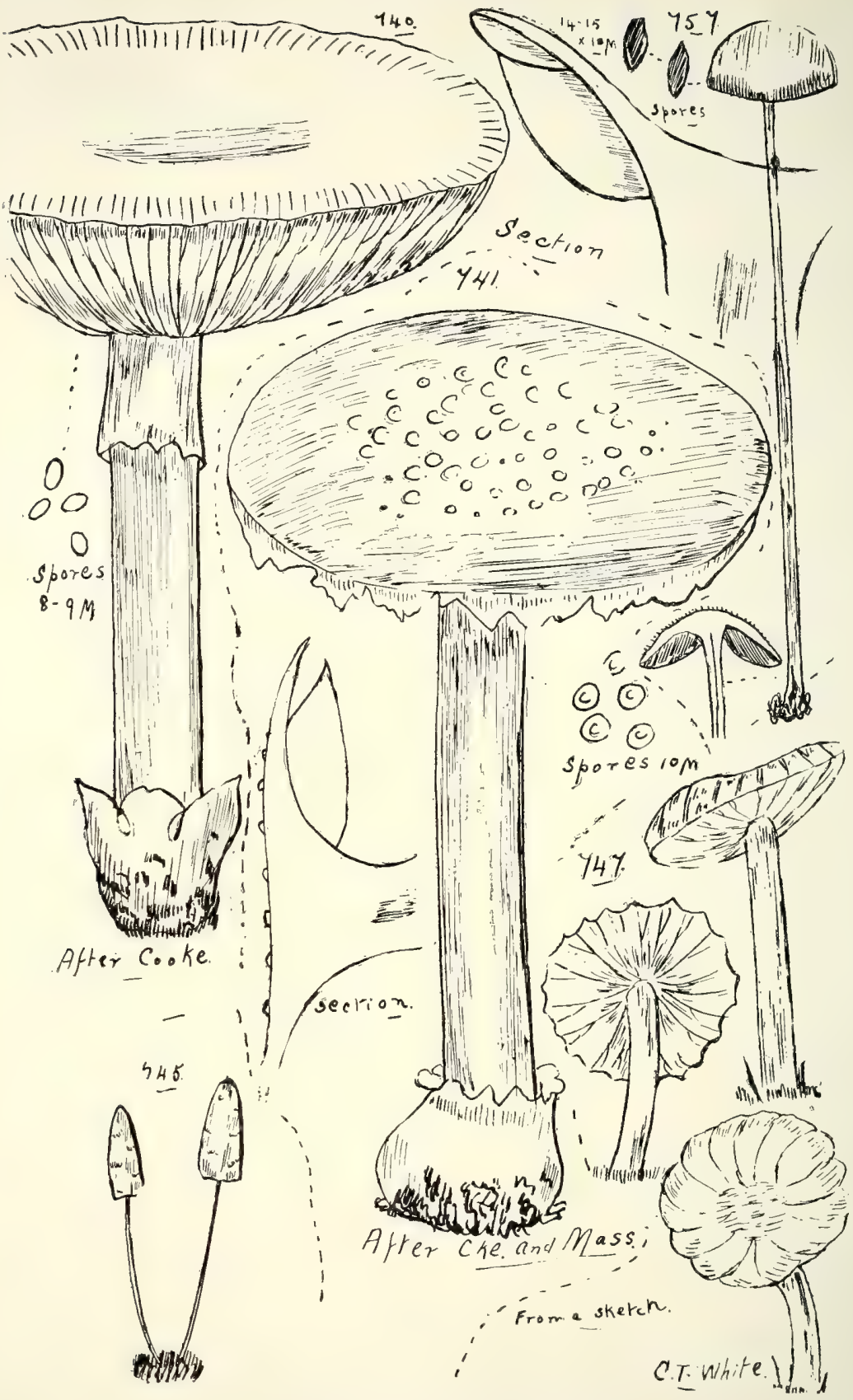

740. Agaricus vernus, Bull.

74I. A. farinaceus, Mass.

745. A. aureus, Mass.

747. A. canaliculatus, Mass.

757. A. veluticeps. Che et Mass. 
Agaricus-contd.

Subgenus Collybia.

coagulatus, B. et Br.

radicatus, Relh.-Edible.

musciputa, Cke. et Mass.

fusipes, Bull.-Edible.

tuberosus, Bull.-On dead fungi.

nummularius, Bull.

dryophilus, Bull.

Subgenus Mycena.

silenus, $B$. et $B r$.

stylobates, Pers.

epipterygius, Fries.

tenerrimus, Berk.-On stems of Fern-trees. (Fig. 748.)

corticola, Schum.

rhipterygius, Fries.

\section{Subgenus Omphalia.}

scyphiformis, Fries.

oniscus, Fries.

umbelliferus, Linn. (Fig. 749.)

fibula, Bull.

gomphomorphus, Berk.

Subgenus Pleurotus.

cyphellaformis, Berk.

sordulentus, Berk. et Br.

illuminans, $F . v . M$.

corticatus, Fries.

applicatus, Batsch.

Guilfoylei, Berk.

lenticula, Kalchb.

semisupinus, $B$. et $B r$.

flabellatus, Berk. et Br.

semiliber, $B$. et $B r$.

Thozetii, Berk. et F. v. M.

salignus, Fries.-Edible.

mitis, Pers.-Edible.

limpidus, Fries.

ostreatus, Fries.-Edible Oyster Mushroom.

diversipes, Berk.

sulciceps, Cke. et Mass.-Found at the base of a paling fence, Brisbane. (Fig. 750.)

striatulus, Fries.

euphyllus, Berk. 


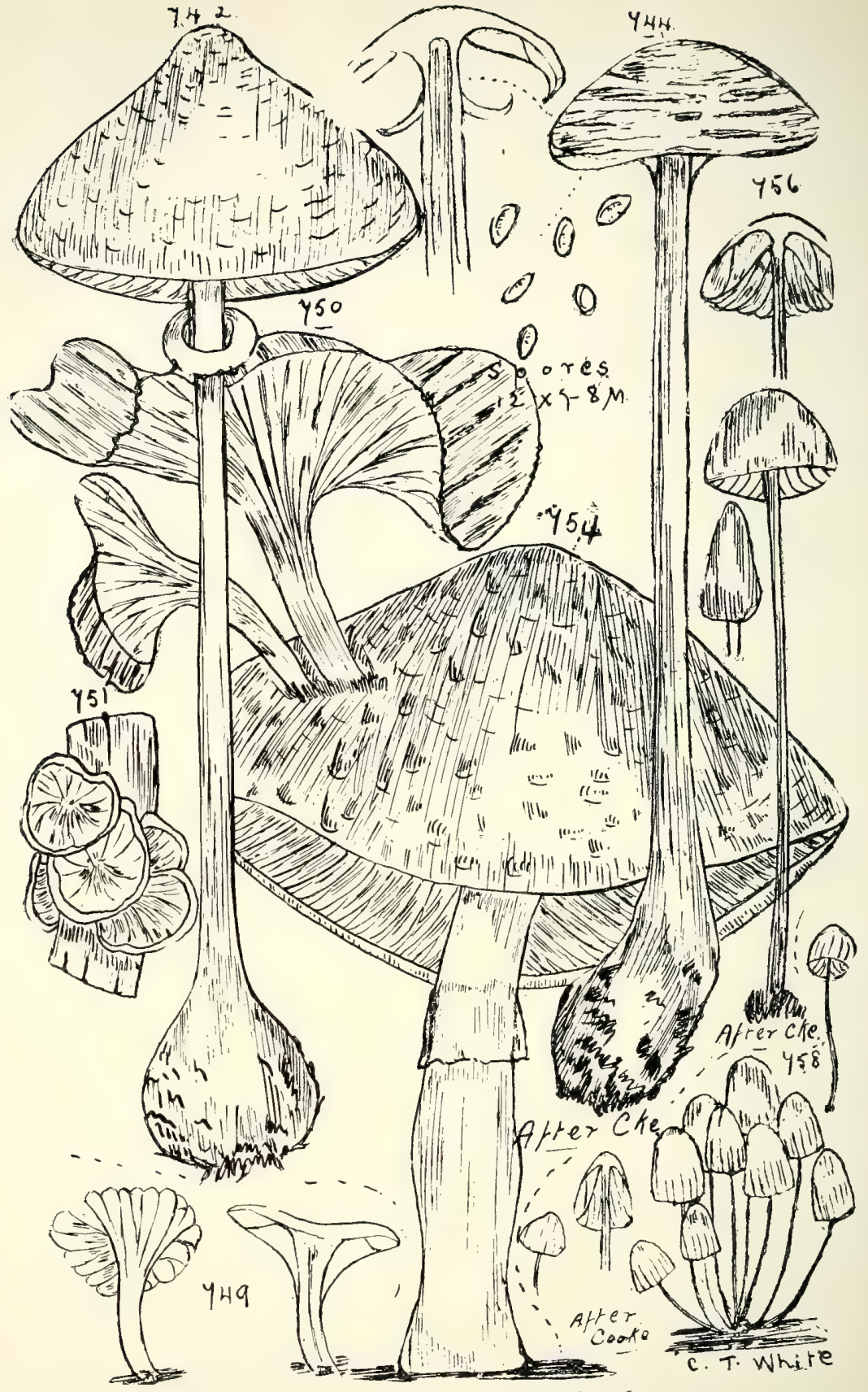

742. Agaricus gracilentus, Kromb.

744. A. stenophyllus, Cke. et Mass.

749. A. uMBelliferus, Linn.

750. A. sulciceps, Cke ei Mass.

751. A. variabilis, Pers.

754. A. sylvaticus, Schef.

756. A. Fimicola, Fries.

758. A. Disseminatus, Pers. 
Agaricus-contd.

SERIES II.-HyporHODII.

(Spores pink or salmon-coloured.)

parvulus, $W$ einm.

Subgenus Volvaria.

Subgenus Clitopilus.

cancrinus, Fries.

Subgenus Claudopus.

variabilis, Pers. (Fig. 75I.)

Series III.-Dermini.

(Spores various shades of reddish brown, brown, red, or yellowish brown.)

Subgenus Pholiota.

pliylicigena, Berk. (Fig. 752.)

flammans, Fries.

adiposus, Fries.

nudipes, Fries.

Subgenus Hebeloma.

Subgenus Flammula.

sapineus, Fries.

var. terrestris, Cke. et Mass.

picreus, Fries.

Baileyi, $B$. et $B r$.

spumosus, Fries.

gymnopodius, Bull.

avellanus, Cke. et Mass.

purpureo-nitens, Cke. et Mass.

Subgenus Naucoria.

semiorbicularis, Buill.

Bowmani, Berk.

anguineus, Fries.

escharoides, Fries.

melinoides, Bull.-On roadside among grass, Brisbane River.

Subgenus Galera.

peroxydatus, Berk.

Subgenus Tubaria.

furfuraceus, Pers. (Fig. 753.)

var. trigonophyllus, Lasch. 
Agaricus-contd.

mollis, Schaeff.

Subgenus Crepidotus.

interceptus, Berk.

stromaticus, Cke. et Mass.

Series IV.-Pratelli.

(Spores blackish purple or purplish brown, rarely fuscous.)

Subgenus Psalliota.

arvensis, Schoeff.-Edible Horse Mushroom, the best for ketchup.

sylvaticus, Schoeff. (Fig. 754.)

versipes, $B$. et $B r$. - At the base of Bamboo-stems in Brisbane Botanic Gardens.

campestris, Linn.-The Common Mushroom; edible.

var. sylvicola, Vitt.

Subgenus Stropharia.

semiglobatus, Batsch.

Subgenus Hypholoma.

adustus, Cke. et Mass. (Fig. 755.)

Subgenus Deconisa.

nucisedus, Fries.

fatu1us, Fries.

Subgenus Psathyra.

gossypinus, Fries.

Series V.-Coprinarit.

(Spores black.)

Subgenus Pancolus.

papilionacet1s, Bull.

fimiputris, Bull.

fimicola, Fries.-On wallaby-dung. (Fig. 756.)

eburneus, Cke. et Mass.-On horse-dung.

veluticeps, Cke. et Mass. (Fig. 757.)

Subgenus Psathyrella.

hiascens, Fries.

disseminatus, Pers.-On decomposing garden rubbish. (Fig. 758.)

acerinus, Fries. 


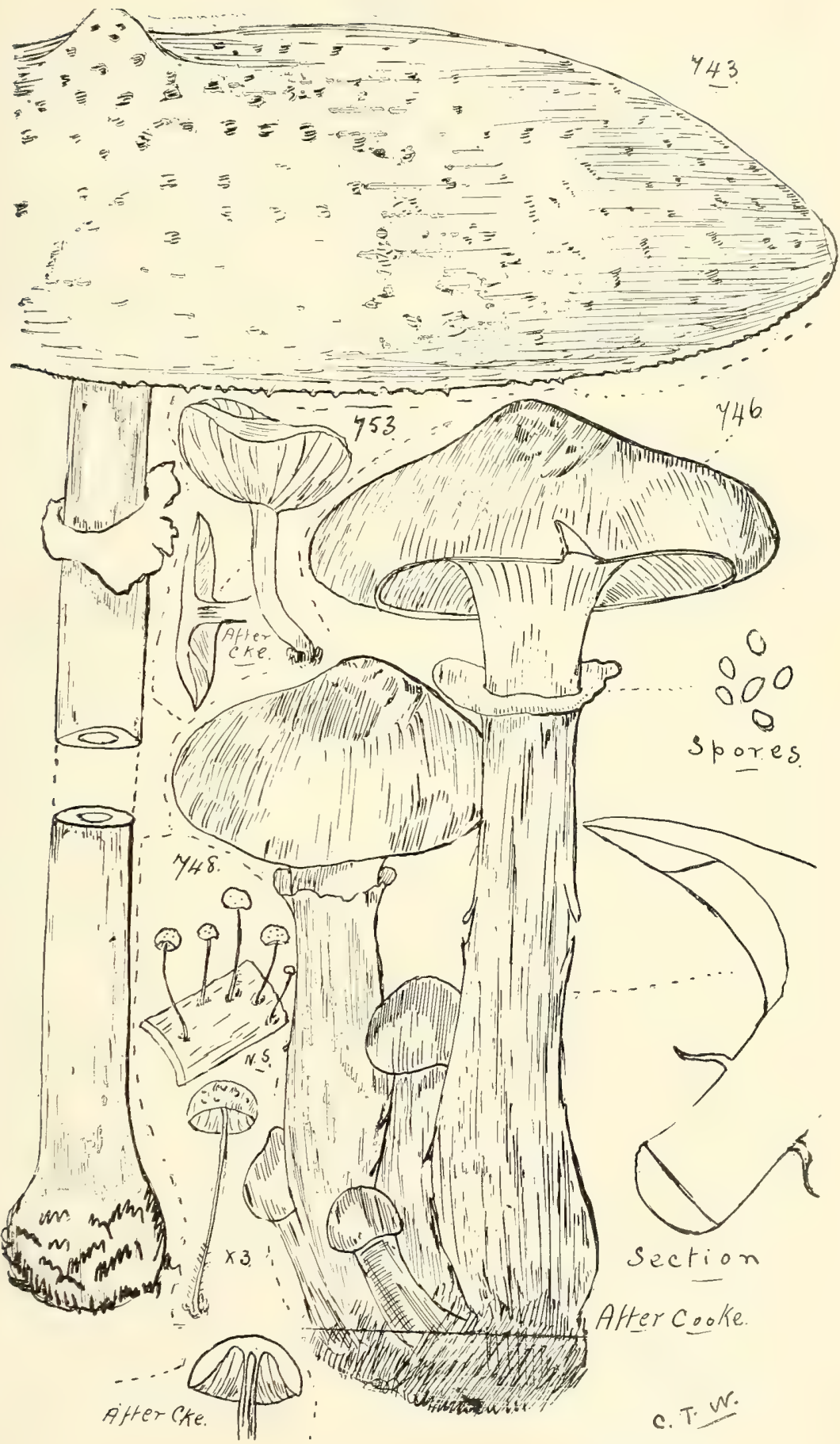

743. Agaricus dolichaulos, Berk. et $B r$.

746. A. MElleus, $V a h l$.

748. A. TENerRimus, Berk.

753. A. Furfuraceus, Pers. 
Coprinus, Fries.

gigasporus, Mass., Ann. Bot. x. (1896) $157=C$. picaceus, Cooke (non Fries), Hanbk. Aus. Fungi, p. 68.-On cowdung.

fimetarius, Fries.

micaceus, Fries.-On dead Mulberry-stump.

truncorum, Schaff.

australiensis, Mass., Ann. Bot. x. ( 1896$)$ I7 I $=C$. deliquescens, Cooke (non Bull.), Hanbk. Aus. Fungi, p. 69.-On dung.

tomentosus, Fries.

stercorarius, Fries.

ephemerus, Fries.

plicatilis, Curt. (Fig. 759.)

Bolbitius, Fries.

fragilis, Fries.

Hiatula, Fries.

Wynniæ, Berk. and Br.-A small luminous Fungus. (Fig. 760.)

Cortinarius, Fries.

cinnabarinus, Fries.

Paxillus, Fries.

hirtulus, Kalchb.

crassus, Fries.

Muelleri, Berk.

(Tapinia) paradoxus, Kalchb.

Hygrophorus, Fries.

porphyrinus, B. et $\mathrm{Br}$. (Fig. 761.)

virgineus, $W$ ulf.

miniatus, Fries.-Edible.

sciophanus, Fries.

conicus, Fries.

scarlatinus, Kalchb.

Lactarius, Fries.

quietus, Fries.-Edible.

piperatus, Fries.-Eaten on the continent of Europe but not in England.

Rassula, Fries.

sanguinea, Fries.

australiensis, Cke. et Mass.

rubra, Fries.

Linnæi, Fries.

fragilis, Fries. 
Rassula-contd.

emetica, Fries.

var. Clusii, Fries.-Poisonous.

subalbida, Brot.

Cantharellus, Adams.

cibarius, Fries.-The Chanterelle; edible, but not generally liked.

aureolus, Cke. et Mass. (Fig. 762.)

aurantiacus, Fries.

foliolum, Kalchb.

Arrhenia, Fries.

(Apus) cupularis, Fries = Cantharellus cupularis, Fries.

Marasmius, Fries.

urens, Fries.

pilopus, Kalchb.

erythropus, Fries.

cauticinalis, Fries.

putredinis, $B$. and $C$.

hæmatocephalus, Mont. $=M$. erythrocephalus, Mont.

rotula, Fries.

confertus, $B$. et $B r$.

subsupinus, Berk.

Muelleri, Berk.

rhyticeps, Kalchb.

rufo-pallidus, Kalchb.

epileucus, Berk.

calobates, Kalchb.

eucalypti, Berk.

opacus, $B$. et $C$.

stylobates, $B$. et $C$.

fœetidus, Sow.

ramealis, Bull.

aciculiæformis, $B$. et $B r$.- On the roots of an Orchid in cultivation.

pellucidus, $B$. et $B r$.

Muelleri, Berk.

equicrinus, B. et F. v. M.-Horsehair Fungus. (Fig. 763.)

bicolor, Sacc., Syl1. v. $2218=M$. dichrous, B. et C.

exocarpi, Berk.

affixus, Berk.

badius, Berk.

rhyssophyllus, Mont.

calopus, Fries.

lanaripes, Cke. et Mass. (Fig. 764.)

floriceps, B. ct $C$. 


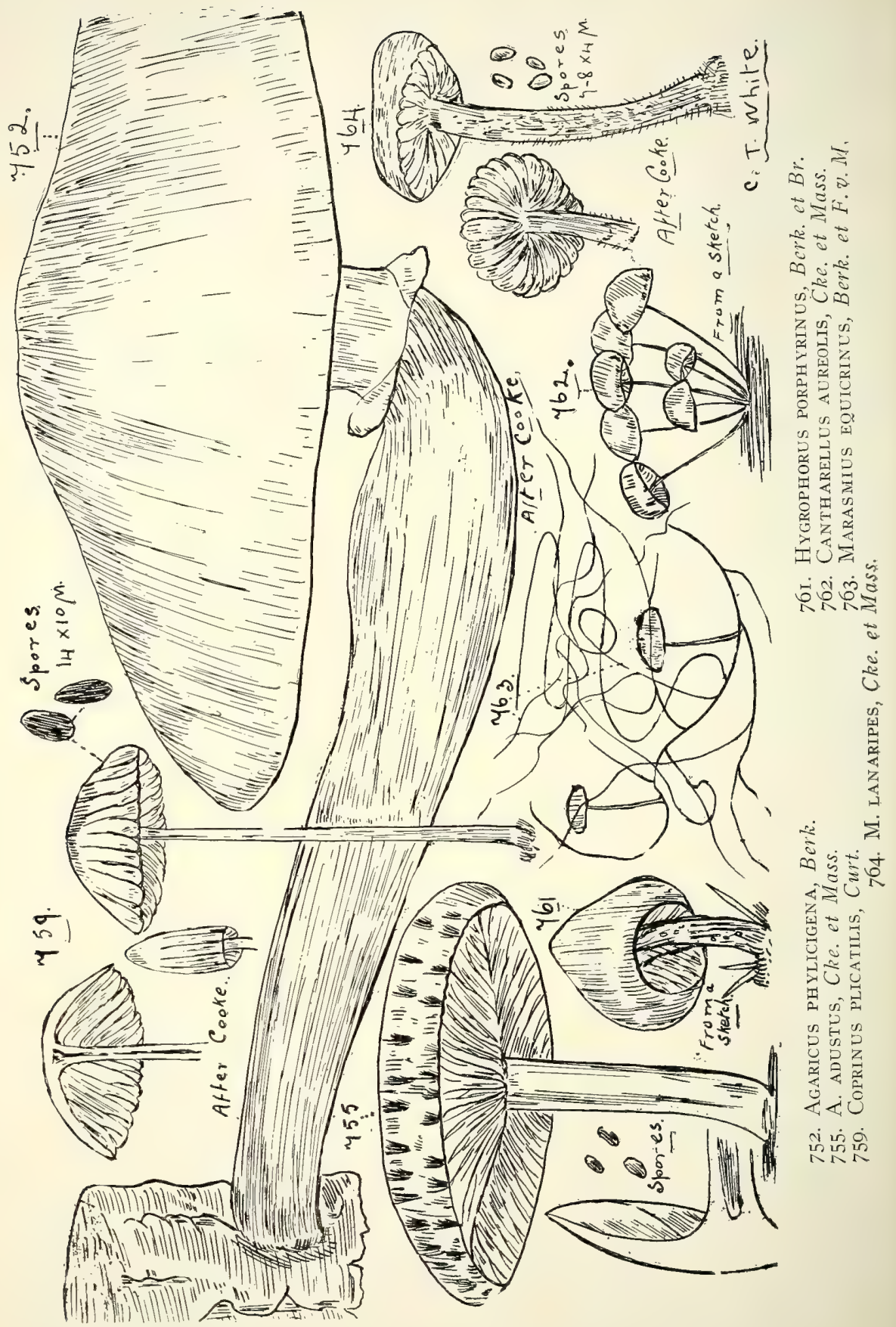


Lentinus, Fries.

Lecomtei, Fries.

strigosus, Fries.

pergamenus, Fries.

fulvus, Berk.

fusco-purpurens, Kalchb.

tener, Klotsch.

villosus, Klotsch.

fasciatus, Berk.

subdulcis, Berk.

subnudus, Berk.

exilis, Klotsch.

vulpinus, Fries.

Dunalii, Fries.

eugrammus, Mont.

cretaceus, Berk.

Shannii, Berk.

blepharodes, B. et Curt.

siparius, Berk. et Br.

calvescens, Berk.

tigrinus, Fries.-Edible.

catervarius, $B$. et $B r$. (Fig. 765.)

exasperatus, B. et Br. (Fig. 766.)

Kurzianus, Currey.

manipularis, Berk. et $B r$.

punctaticeps, B. et $B r$.

fusipes, Cke. et Mass.

suffrutescens, Brotere.

cochleatus, Fries.

cyathus, $B$. et $B r$.

lepideus, Fries.

Schomburgkii, Berk.

holopogonius, Berk.

radicatus, Cke. et Mass.

hyracinus, Kalchb.

lateritius, Berk.

gracilentus, Cke. et Mass.

Panus, Fries.

incandescens, $B$. et $\mathrm{Br}$. = Agaricus Gardneri, Cooke (non Berk.), Hanbk. Aus. Fungi, p. 3I, No. I49.-The large luminous Fungus.

angustatus, Berk.

viscidulus, $B$. et $B r$.

cinnabarinus, Fries.

eugrammus, Mont. 

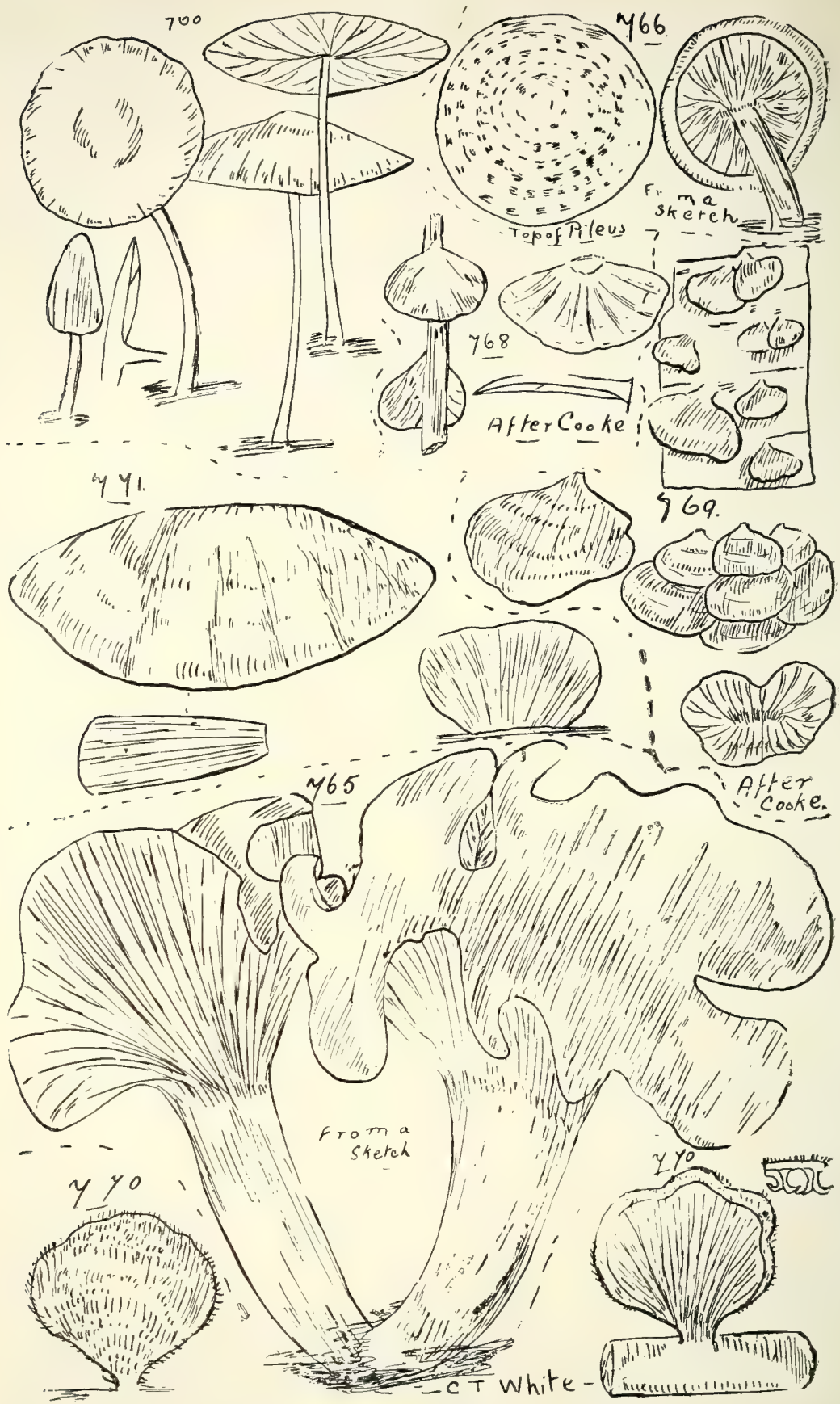

760. Hyatula Wynnie, Berk. et $B r$.

765. Lentinus catervarius, Berk. et Br.

766. L. exasperatus, Berk. et Br.

768. Xerotus tener, Berk. et Br.

769. Trogia crispa, Fries.

77o. Schizophyllum commune, Fries.

77 r. Lenzites striata, Fries. 


\section{Panus-contd.}

patellaris, Fries.

conchatus, Fries.-Luminous.

tortulosus, Fries.--Edible, but only when very young.

suborbicularis, $B$. et $B r$.-First found on some large whalebones in grounds of Queensland Museum. (Fig. 767 .) rivulosus, Berk.

Xerotus, Fries.

albidus, $B$. et $B r$.

lateritius, $B$. et $C$.

Rawakensis, Fries.

proximus, $B$. et $B r$.

fulvus, $B$. et $B r$.

Drummondi, Berk.

tener, B. et Br. (Fig. 768.)

Bertierii, Mont.

Archeri, Berk.

Trogia, Fries.

crispa, Fries. (Fig. 769.)

Schizophyllum, Fries.

cummune, Fries.-Fork-gill Fungus; very common on wood.

(Fig. 770.)

var. multifidum, Fries.

Lenzites, Fries.

betulina, Fries.

var. velutina, Berk.

deplanata, Fries.

acuta, Berk.

faventinus, Cald.

Beckleri, Berk.

Berkeleyi, Lév.

striata, Fries. (Fig. 771.)

Palisoti, Fries.

applanata, Fries.

aspera, Klotsch. = Doedalea aspera, Klotsch., Sacc. Syll. v. 644.

Guilfoylei, Berk.

nivea, Cke.

repanda, Fries.

abietina, Fries.

FAMILY II.-PoLy POREI.

Boletus, Dill.-Many of this genus show various colours when broken, but such may generally be considered unfit for food.

luteus, Linn.-Edible. 


\section{Boletus-contd.}

pachypus, Fries.

edulis, Bull.-Edible; the favourite for cooking on the continent of Europe.

Thozetii, Berk.

hædinus, B. et Br. = B. pachypus, B. and Br.

elegans, Schum.--Edible.

granulatus, Linn.-Edible.

badius, Fries.-Edible.

sanguineus, With.

subsimilis, Fries.

chrysenteron, Fries.

subtomentosus, Linn.

æstivalis, Fries.-Edible.

luridus, Fries.

felleus, Bull.

æreus, Bull. $=$ B. aneus, Fries.-Edible.

lacunosus, Cke. et Mass. (Fig. 772.)

Strobilomyces, Berk.

nigricans, Berk.

floccopus, Rosth.

palescens, Cke. et Mass.

velutipes, Cke, et Mass. (Fig. 773.)

rufescens, Cke. et Mass.

ananæceps, Berk. = Boletus ancenaceps, Berk.

Polyporus, Fries.

Hartmanni, Cke.

$$
\text { A.-Ovina. }
$$

tumulosus, Cke. - Eaten by the Queensland natives ( $D r$. Joseph Bancroft).

Mylittæ, Cke. and Mass.-Stone Fungus. Only the sclerotium so far found in Queensland.

myclodes, Kalchb.

brumalis, Fries.

$$
\text { B.-Lenti. }
$$

orbicularis, Sauter $=$ P. rhipidium, Berk.

arcularius, Fries $=P$. agaricus, Berk.

tricholoma, Mont.

similis, Berk.

alveolarius, Bosc.

stipitarius, B. et Curt. $=$ P. Armitii, F. v. M. and Kalch.

$$
\text { C.-Spongiosa. }
$$

Schweinitzii, Fries.

tabulæformis, Berk. 


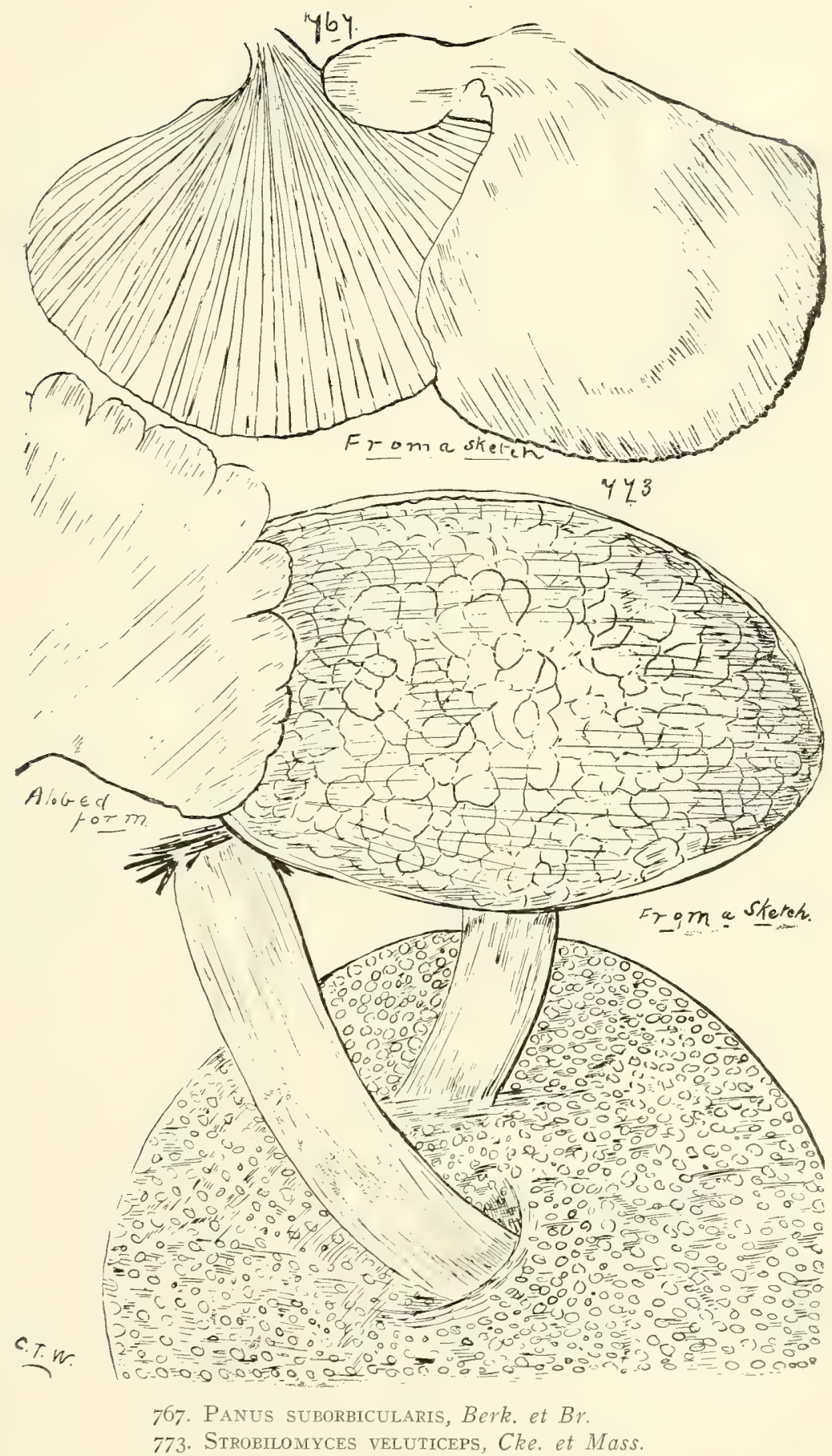


Polyporus-contd.

biennis, Bull.

rufescens, Fries.

proteiporus, Cke.

D.-Melanopodes.

squarrosus, Fries.

melanopus, Fries.

picipes, Fries.-Edible. (Fig. 774.)

infernalis, Berk.

elegans, Buli.

varius, Fries.

Guilfoylei, $B$. et $B r$.

phlebophorus, Berk. and $B r$.

dictyopus, Mont.

$$
\text { E.-Petaloides. }
$$

grammocephalus, Berk. (Fig. 775.)

var. Emerici, Berk.

var. minor, Cke.

platotis, $B$. et $B r$.

dorcadideus, $B$. et $B r$.

fusco-lineatus, $B$. et $\mathrm{Br}$.

$$
\text { F.-Frondosi. }
$$

intybaceus, Fries.-Edible.

confluens, Fries. $=P$. artemidorus, Lenz., and $P$. aurantius, Trog.

$$
\text { G.-Lobati. }
$$

anthracophilus, Cke.

$$
\text { H.-Imbricati. }
$$

sulphureus, Fries.-Edible.

retiporus, Cke.

epileucus, Fries.

$$
\text { I.-Mollis. }
$$

corrivalis, Berk.

chioneus, Fries.

argentatıs, Cke.

$$
\text { J.-Dichroi. }
$$

nidulans, Fries.

fædatus, Berk.

rubidus, Berk.

gilvus, Schw.

scruposus, Fries.

var. isidiodes (Berk.), Cooke.

adustus, Fries. 


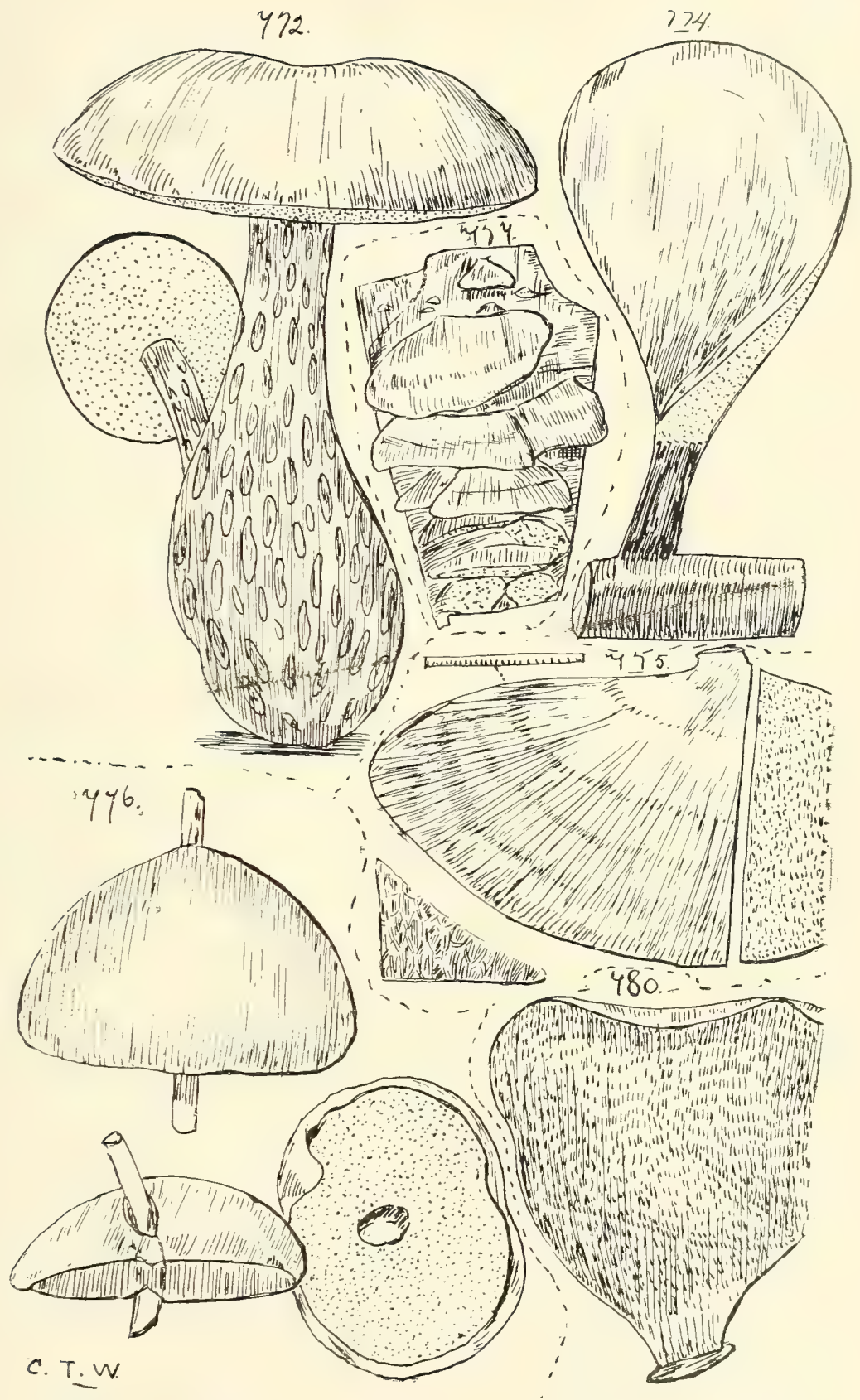

772. Boletus lacunosus, Che. et Mass.

774. Polyporus picipes, Frics.

775. P. GRammocephalus, Berk.

776. P. FRUTICUM, Berk. et Curt.

777. P. Anebus, Berk.

780. Polystictus elongatus, Berk 
Polyporus-contd.

$$
\text { K.-Hispidi. }
$$

hispidus, Fries.

fruticum, Berk. et Curt. (Fig. 776.)

pelliculosus, Berk.

substuppeus, Berk. et Cke.

\section{L.-Suberosi.}

betulinus, Fries.

portentosus, Berk.-Punk. In the early days of South Australia this fungus was used by the natives and also by the colonists for carrying fire.

$$
\text { M.-Lignescentes. }
$$

hypopolius, Kalchb.

cartilagineus, $B$. et $B r$.

zonalis, Berk.

subzonalis, Cke.

cubensis, Mont.

testudo, B. et Br.

anebus, Berk. (Fig. 777.)

plebius, Berk.

Fomes, Fries.

$$
\text { A.-Mesopodes. }
$$

rudis, Berk.-Brisbane River. (Fig. 778.)

rugosus, Nees.

pullatus, Berk.

$$
\text { B.-Pleuropodes. }
$$

amboinensis, Fries.

var. gibbosus, Fries $=$ Polyporus gibbosus, Nees.

lucidus, Fries $=P$. laccatus, Pers., P. nitens, Batsch., and P. obliquatus, Bull.

lucidus, var. exquisitus. Kalchb.

$$
\text { C.-Merismoidei. }
$$

scabriusculus, Berk.

senex, Nees. $=P$. rhabarbarinus, Berk.

\section{D.-Fomentarii.}

scansilis, Berk.

marginatus, Fries.

dochmius, Berk.

concavus, Cke.

conchatus, Fries. 
Fomes-contd.

australis, Fries $=$ P.dubius, Jungh.

var. arculatum, Bres.

chilensis, Fries.

applanatus, Fries $=P$. dryadens, Rostk.

nigrolaccatus, Cke.

igniarius, Fries $=P$. pomaceus, Pers.-Hoof Fungus.

fulvus, Fries $=P$. novo-anglice, B. et Cke.

salicinus, Fries.

E.-Impoliti.

hemileucus, Berk. et Cke.

exotephrus, Berk.

pullus, Berk. et Mont.

contrarius, Berk. et Curt.

lineato-scaber, $B$. et $B r$.

spadiceus, Berk.

inflexibilis, Berk.

limitens, Berk.

pectinatus, Klot. $=P$. conchatus, Fries.

Curreyi, Berk.

strigatus, Berk.

ponderosus, Kalchb.

annosus, Fries.

F.-Lavigati.

compressus, Berk.

connatus, Fries.

ferreus, Berk.

scopulosus, Berk.

fasciatus, Fries.

carneus, Nees.

cinereo-fuscus, Curr. (Fig. 779.)

placodes, Kalchb.

Palliseri, Berk. = Trametes arcticus, Berk.

homalopilus, Mont. = Polyporus carneofulvus, Berk. cryptarum, Bull.

incrassatus, Berk. $=$ P. reniformis, Morg.

$$
\text { G.-Resupinati. }
$$

cryptarum, Fries $=$ Boletus cryptarum, Bull.

obliquus, Fries.

luridus, Kalchb.

bistratosus, B. et Cke.

Pentzei, Kalchb.

versicolor, P. Henn., Hedw. xliii. 75 . 
Polystictus, Fries.

$$
\text { A.-Perennes. }
$$

tomentosus, Fries.

luteo-nitidus, Berk.

perennes, Fries $=P$. confluens, Schum., and P. coriaceus, Bull.

oblectans, Berk. $=$ P. splendens, Peck.

bulbipes, Fries $=P$. cladonia, Berk.

$$
\text { B.-Sacri. }
$$

quadrans, B. et $B r$.

xanthopus, Fries $=P$. cupreo-nitens, Kalchb., and $P$. crassipes, Curr.

$$
\text { C.-Discipedes. }
$$

flabelliformis, Klot.

Adami, $C k e .=P$. dilatatus, Berk.

porphyrites, Berk.

dilatatus, Lév.

mutabilis, B. et Cke.

luteus, Nees.

stereinus, $B$. et Curt. $=$ P. cognatus, Kalchb.

carneo-nigra, Berk.

sideroides, Lév.

nephridius, Berk.

affinis, Nees. $=P$. Teysmanni, Berk.

brunneolus, Berk.

libum, Berk.

sanguineus, Fries.

rasipes, Berk.

$$
\text { D.-Prolificantes. }
$$

laceratus, Berk.

elongatus, Berk. (Fig. 780.)

Hodgkinsoniæ, Kalchb.

var. stipitatus, Broome.

gallopavonis, B. et $B r$.

$$
\text { E.-Funales. }
$$

Leoninus, Klotsch.

funalis, Fries.

$$
\text { F.-Stuposi. }
$$

cristatus, $\mathrm{Cke} .=$ Trametes cristata, Cke.

acutus, $C k e .=$ Trametes acuta, Cke.

extensus, Berk.

versatilis, Berk. $=$ Trametes versatilis, Berk. 


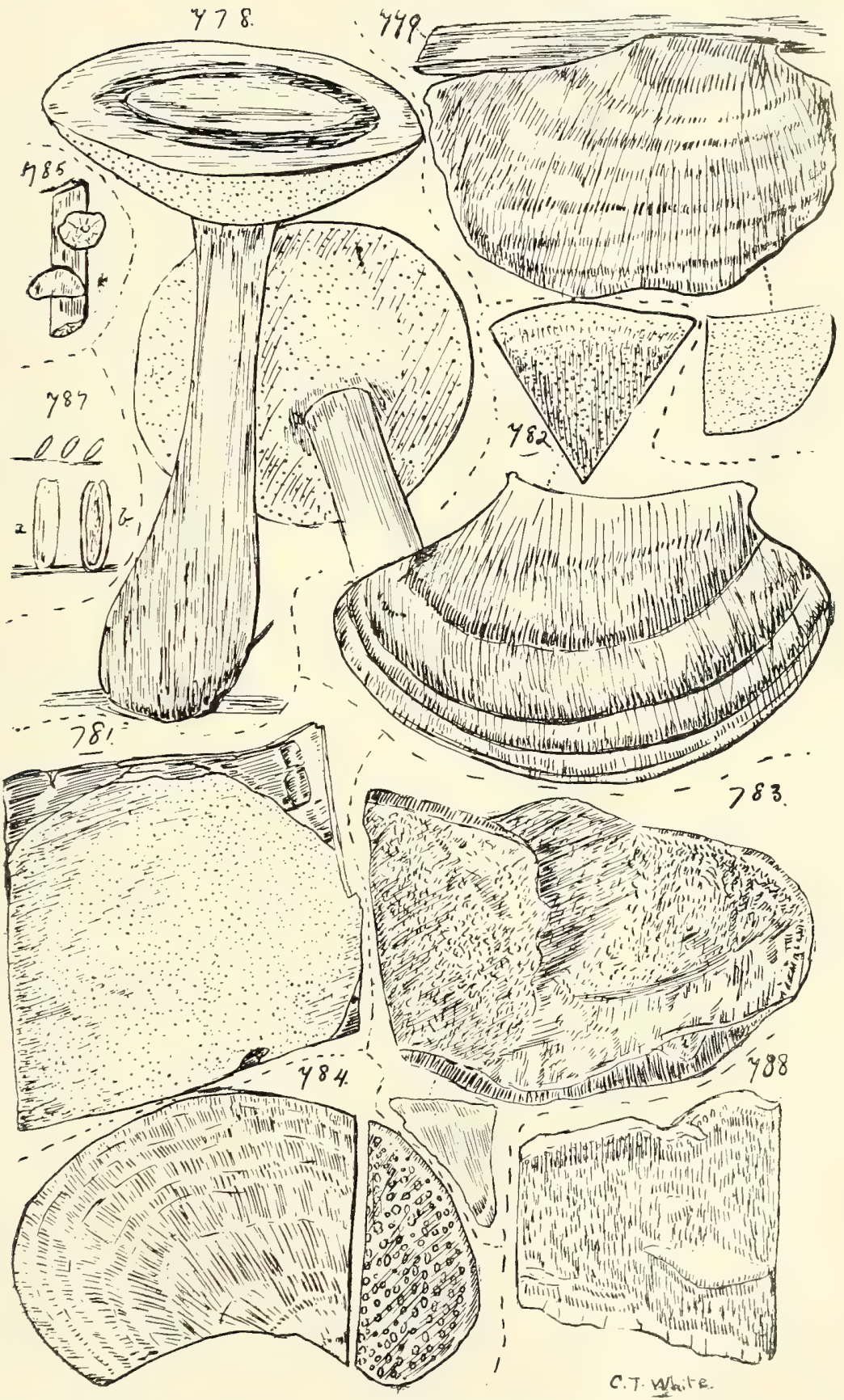

778. Fomes RUdis, Berk.

779. F. CINEREO-FusCus, Cur 781. Poria calcea, Berk. et Br. 782. Trametes PICTA, Berk. et $\mathrm{Br}$. 783. DAEDALEA INCOMPTA.
784. Hexagonia polygramma, Mont. 785. Laschia pustulata, Berk. 787. Solenia CANdida, Hoffm. (a) Cup enl., (b) sect. do.

788. IrPex flaves, Klot. 
Polystictus-contd.

pergamenus, Fries.

Feei, Fries.

lilacino-gilvus, Berk.

eucalypti, Kaichb.

cinnabarinus, Fries.

radiatus, Fries $=P$. fusco-gilous, Schw.

venustus, Berk.

floridanus, Berk.

\section{G.-Coriacei.}

versicolor, Fries $=P$. argyraceus, Pers.

obstinatus, $C k e .=$ Trametes obstinatus, Cke.

hirsutus, Fries.

velutinus, Fries $=P$. nigricans, Lasch.

glirinus, Kalchb. $=P$. murinus, Kalchb.

vellereus, Berk.

pinsitus, Fries.

trizonatus, Cke.

sterioides, Fries.

peradeniæ, $B$. et $B r .=P$. chrysoleucus, Kalchb.

vernicifluus, Berk.

vinosus, Berk.

occidentalis, Fries.

rigens, Sacc., Syll. = Trametes rigida, Berk. et M.

Persoonii, Fries $=$ Dadalea sanguinea, Klot.

aratus, Berk. = Trametes acupunctatus, Berk.

luteo-olivaceus, B. et Br.

var. tenuis.

tabacinus, Mont.

Hasskarlii, Lév. = P.ferrugineus, Jungh.

byrsinus, Mont.

cichoraceus, Berk. $=$ P. intybaceus, Berk.

xerampelinus, Kalchb.

breviporus, $C k e$.

bireflexus, $B$. et $B r$.

eriophora, B. et $B r$.

Broomei, Rabh.

Poria, Pers.

$$
\text { A.-Mollusci. }
$$

vulgaris, Fries $=P$. cellulosus, Wilbg.

calcea, B. et Br. (Fig. 78I.)

medulla-panis, Pers. $=$ P. bibulus, Pers.

callosa, Fries.

hyposclera, Berk. 
Polystictus-conti.

$$
\text { B.-Vaporarii. }
$$

vaporaria, Fries $=P$. cellaris, Lib.

victorix, Berk.

corticola, Fries.

$$
\text { C.-Rigidi. }
$$

sinuosa, Fries.

ferruginosa, Fries $=P$. corium, Kunze.

contigua, Fries $=$ P. croceus, Karst.

dictyopora, Cke.

$$
\text { D.-Reticulati. }
$$

Trametes, Fries.

Sprucei, Berk.

devexa, Berk.

picta, B. et Br. (Fig. 782.)

lactinea, Berk.

lavis, Berk.

pyrrhocreas, Berk.

versiformis, Berk.

Curreyi, Cke. $=$ T.umbrina, Curr.

cristata, Cke.

serpens, Fries.

ochroflava, Cke.

Muelleri, Berk.

ochrolenca, Bcrk. = Polyporus ochroleucus, Sacc. Syll. 5236. sinuosa, Fries.

gausapata, Berk. and Rav. = Irper gausapata, Berk. and Curt.

Sclerodepsis, Cke.

colliculosa, Cke. = Trametes colliculosa, Berk.

Drdalea, Fries.

sanguinea, Klot.

tenuis, Berk.

Bowmani, Berk.

unicolor, Fries.

latissima, Fries.

Sprucei, Berk.

incompta, Berk. (Fig. 783.)

scalaris, $B$. et $B r$.

subcongener, Berk.

sinulosa, Klot.

au1lacophyllus, Berk.

Hexagonia, Fries.

polygramma, Mont. (Fig. 784.) 
Hexagonia-contd.

Muelleri, Berk.

sericea, Fries.

umbrinella, Fries.

tenuis, Berk.

var. subtenuis, Berk.

crinigera, Fries.

Wightii, Klot.

rigida, Berk.

similis, Berk.

decipiens, Berk.

Favolus, Fries.

Boucheanus, Klotsch.

scaber, Berk. and Broome.

rhipidium, Berk.

princeps, Berk.

Glæoporus, Mont.

leptopilus, Lév.

Laschia, Fries.

Thwaitesii, B. et Br.

pustulata, Berk. ( Fig. 785.)

cæspitosa, Berk.

tremellosa, Fries.

Merulius, Haller.

corium, Fries.

aureus, Fries.

Baileyi, $B$. et $B r .-$ On the beams of a culvert-bridge near Brisbane. (Fig. 786.)

lacrymans, Schum.-Dry Rot; on rafters of a veranda in Brisbane.

serpens, Fries.

tenuissimus, B. et Br.-Parasitic on Hymenochæte.

binominatus, Mass., in litt.-In the heart of a Cypress Pine tree, Brisbane Botanic Gardens (F.M.B.).

Solenia, Pers.

candida, Hoffm.-At base of a paling fence, Brisbane. (Fig. 787.)

FAMily III.-HydneI.

Hydnum, Fries.

lævigatum, Fries.-Edible.

graveolens, Del.-Scent resembling the Melilot, and said to retain this odour for years.

mucidum, Fries.

merulioides, $B$. et $\mathrm{Br}$. 
Hydnum-iontd.

flavum, Klot.

Muelleri, Berk.

delicatulum, Klotsch.

gilvum, Berk.

ochraceum, Pers.

udum, Fries.

membranaceum, Bull.

tomentosum, Fries $=H$. cyathiforme, Schæff.

coralloides, Fries.-Edible.

'Tremellodon, Pers.

gelatinosum, Siop.

Sistotrema, Pers.

irpicinum, B. et $\mathrm{Br}$.

Irpex, Fries.

cervinus, Berk.

flavus, Klot. (Fig. 788.)

zonatus, Berk.

tabacinus, Berk. et Curt.

maximus, Mont.

sinuosus, Fries., var. cervicolor, Berk. and Br.-Wholly fawncoloured.

Radulum, Fries.

molare, Fries.-On stem of Peach-tree. (Fig. 789.)

Phlebia, Fries.

merismoides, Fries.

radiata, Fries.--On beams of a bridge near Brisbane.

reflexa, Berk.

coriacea, Berk.

Grandinia, Fries.

granulosa, Fries.

glauca, Cke.

cinereo-violacea, P. Henn., Hedw. xlii. 74.

FAMili IV.-Thelephorei.

Craterellus, Fries.

cornucopioides, Fries.-Edible; a great favourite with fungus-eaters, but rare in Queensland. (Fig. 790.) confluens, B. et Curt.

Cladoderris, Pers. dentritica, Pers.

Pritzelii, P. Hennings.

Lachnocladium, Lér. furcellatum, Lév. = Clavaria simulans, B. and Br. brasiliense, Lév. 


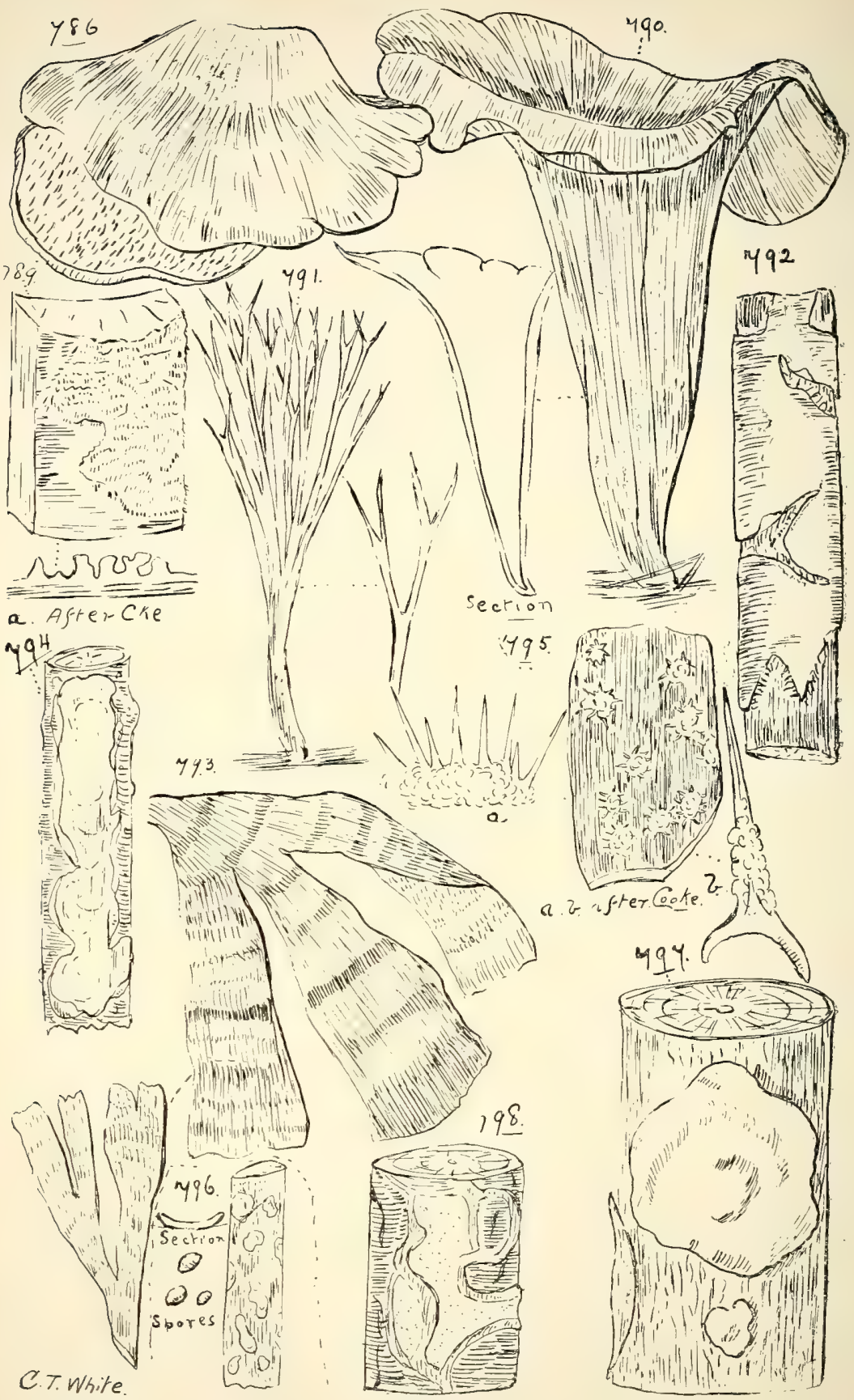

786. Merulius Baileyi, Berk. et Br.

789. Radulum molare, Fries.

790. Craterellus cornucopioides, Fries.

79I. Lach Nocladium flagelliforme, Berk.

792. Thelephora pedicellata, Schw.

793. Stereum radiato-fissum, Berk. et Br.
794. Hymenochete tenuissima, Berk.

795. Peniophora sparsa, Berk.

(a) Port. plant, enl., (b) a single metuloid, enl. 796. Aleurodiscus alpidus, Mass.

797. Corticium nudum, Fries.

798. Hyтochnus RUbro-cinctus, Ehrenb. 
Lachnocladitim-contd.

simulans, $B$. et $B r$.

rameale, $B$ erk.--On living branches of shrubs.

flagelliforme, Berk. (Fig. 79I.)

semivestitum, Berk.

Thelephora, Ehrenb.

Archeri, Berk.

multipartita, Schrwz.

pedicellata, Schw. = Septobasidium pedicellatum, Patouillard.-Said to be very destructive when it attacks fruittrees. (Fig. 792.)

congesta, Berk.

cristata, Fries.-Running over moss and grass.

caryophyllea, Fries.

palmata, Fries.

(Coniophora) atrovirens, B. et Br.-On branchlets of shrubs.

Stereum, Pers.-Shell Fungus.

caperatum, Berk. and Mont.

var. lamellatum (Berk. and Curt.), Cke.= Thelephora lamellata, Berk. et Curt.

var. spongipes (Berk.), Cke. = Thelephora spongipes, Berk., Fungi Brisb. ii. p. $6_{3}$, tab. xiv. fig. I, 2.

elegans, Meyer.

nitidulum, Berk.-Among grass.

Sowerbeii, Berk.

Thozetii, Berk.

prolificans, Berk. = S. Baileyanum, Berk. and Br.

radiato-fissum, Berk. and Br. (Fig. 793.)

spathulatum, Berk.

Leichhardtianum, Lév.

lobatum, Fries $=$ S. perlatum, Berk., S. Boryanum, Fries, S. luteo-badium, Fries, and $S$. ostrea, Nees.

involutum, Klotsch.

semilugens, Klch.

fasciatum, Fries.

gausapatum, Fries.

complicatum, Fries.

hirsutum, Fries.

illudens, Berk. $=S$. decipiens, Berk.

ochroleucum, Fries.

purpureum, Pers.-In Europe a destructive pest to friuttrees, known as the "Silver-leaf disease."

simulans, Berk. and Br.

spadiceum, Fries.

worticosum, Fries. 
Stereum-contd.

molle, Lérr.

sulfureum, Fries $=$ S. citrinum, Berk. and Ray.

amænum, Lév.

Hymenochæte, Lév.

rubiginosa, Lév.

phæa, Berk.

cacao, Berk.

tenuissima, Berk. (Fig. 794.)

innata, Cke. et Mass.

rhabarbarina, Berk.

Schomburgkii, Berk.

olivacea, Cke.

leonina, Berk. and Curt.

Peniophora, Cke.

papyrina (Mont.), Cke.

puberula, Klot. $=$ Stereum puberulum, Berk.

incarnata, Fries $=$ Corticium incarnatum, Sacc.

deglubens, Berk.

bambusicola, $B$. et $B r$.

sparsa, Berk. (Fig. 795.)

Aleurodiscus, Rabh.-White Fly Fungus.

amorphus, Pers. $=$ Corticium amorphum.

albidus, Mass. (Fig. 796.)

Corticium, Fries.

Archeri, Berk.

calceum, Fries.

miniatum, Cke.

atrovirens, Fries.

nudum, Fries.-On bark of Lemon-trees. (Fig. 797.)

læve, Fries.

scutellare, $B$. and $C$.

cæruleum, Fries.

arachnoideum, Berk.

viscosum, Pers.

anthochroum, Fries.

ochroleucum, Berk.

reticulatum, $B$. et $B r$., Ceylon Fungi $=C$. Marescalchianum,

Manc. and Sacc.

Coniophora, $D C$.

olivaceum, Fries $=$ Corticium olivaccum, Fries.

Hypochnus, Fries.

rubro-cinctus, Ehrenb. (Fig. 798.) 
Cyphella, Fries.

longipes, Cke. et Mass.-Tobacco-pipe Fungus; on the bark of trees. (Fig. 799.)

Schneideri, Broome.

capula, Fries.

villosa, Pers.-On dead stumps of Castor-oil plants.

Cora, Fries.

æruginosa, Nees. = Dichonema (Dictyonema) arnginosum, Nees. (Fig. 80o.)

$$
\text { Family V.-Clavariet. }
$$

Clavaria, Linn.

flava, Schoeff.-Edible Yellow Fairy Club.

botrytis, Pers.-Edible.

cristata, Pers.-Edible.

aurea, Schff.-Edible.

fastigiata, $D C$.-Edible.

formosa, Pers.-Edible.

rugosa, Bull.-Edible.

Kunzei, Fries.

stricta, Pers.

lætissima, Pers.

Colensoi, Berk.

portentosa, $B$. et $B r$.

rufa, Fl. Dan.

miltina, Berk.

argillacea, Pers.

fusiformis, Sow.

Archerii, Berk.

mucida, Fries.

Baileyi, Mass. (Fig. 8or.)

Muelleri, Berk.

Calocera, Fries.

cornea, Fries.

guepinoides, Berk.

\section{Family VI.-Tremeldinede.}

Sub-Family Auricularice.

Auricularia, Bull.

albicans, Berk. (Fig. 802.)

lobata, Fries.

mesenterica, Fries.

pusio, Berk.

delicata, Fries.

Exidia, Fries.

albida, Huds. = Tremella albida, Huds. 


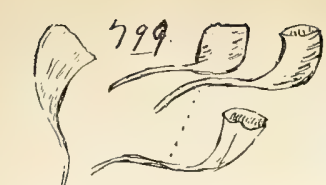

349. Froma sketch.

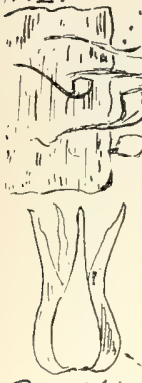

Basidia: 804

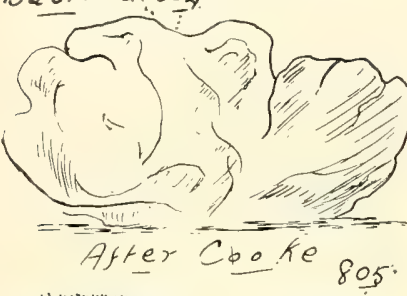

(1) (2)

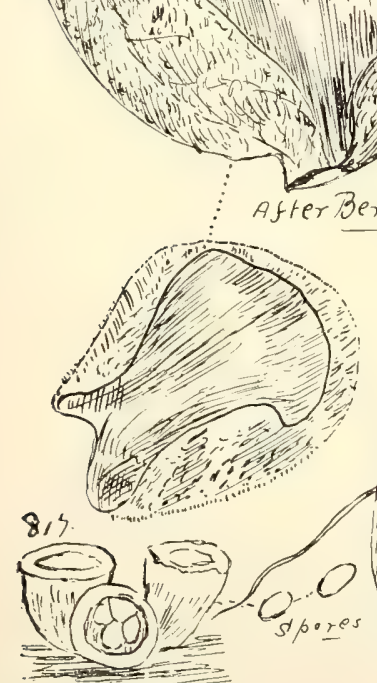

799. Cyphella longtpes, Cke. et Mass. Soo. Cora eruginosa, Nees.

8or. Clavaria Baileyi, Mass.

802. Auricularia albicans, Berk.

\&o3. Ulocola foliacea, Pers.

804. Tremella mesenterica, Retz.

So5. Hirneola rufa, Fries.

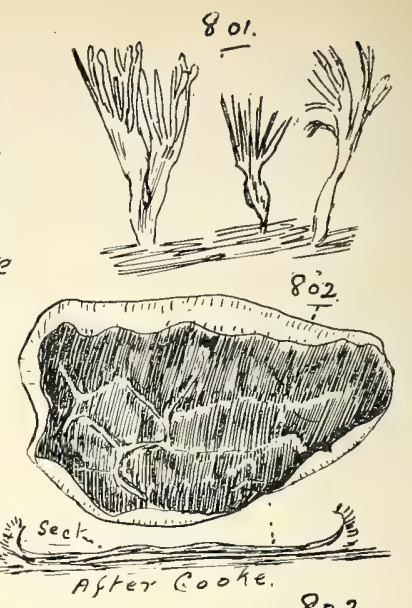

803
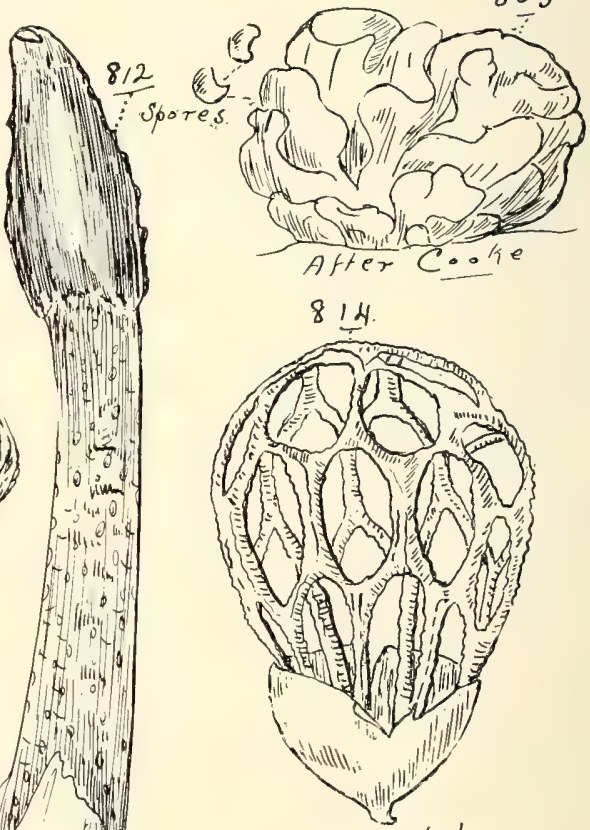

After Berteley $8 \overline{16}$

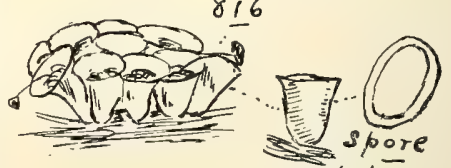

$$
\text { After Berkeley. }
$$

c. T. whire.

806. DaCRyMyCes. DeLIOUESCENS, Retz.

807. Guepinia PEZIZ eformis, Berk.

8iz. Ithyphallus atrominiatus, Bail.

8i4. Clathrus pusillus, Berk.

8i6. Cyathus fimetarius, $D C$.

8i7. Crucibulum vulgare, Tul. 
Ulocolla, Bref.

foliacea, Pers. = Tremella foliacea, Fries. (Fig. 803.)

Tremella, Fries.

fuciformis, Berk.

lutescens, Fries.-Edible. Brain Fungus.

mesenterica, Rets.-Edible. (Fig. 804.)

microscopica, $B$. et $B r$.

Hirneola, Fries.-Species of this genus are usually found on decaying stems of Urticaceous trees, as Fig-trees, \&c.

auricula-judaæ, Fries.--Ear Fungus. Edible by Chinese.

polytricha, Fries.-Ear Fungus. Edible by Chinese, and at one time largely exported from New Zealand to China. (See Q1. Agric. J1. II. (1898) to).

rufa, Fries. (Fig. 805.)

fusco-succinea, Mont.

hispidula, Berk.

auriformis, Fries $=H$. Lusueurii, Lév.

Dacrymyces, Nees.

sacchari, $B$. et $B r .-$ On burnt stumps of sugar-cane.

deliquescens, Duby. (Fig. 806.)

Guepinia, Fries.

spathularia, Fries.-Very common on damp fences, \&c.

pezizæformis, Berk. (Fig. 807.)

\section{GASTEROMYCETFS.}

Family I.-Phalloidet.

Lysurus, Fries.

australiensis, Cke. et Mass. (Fig. 808.)

tenuis, Bail. (Fig. 8og.)

Dictyophora, Desu.-Netted Stinkhorn Fungus.

phalloidea, Desv. = Phallus indusiatus, Vent.

tahitensis, Schlecht. $=$ P. Damonum, Rumph., Hooker in Hook. et Arn. Bot. Beechy's Voy. p. 78, tab. xx. (Fig. 809 bis.)

speciosa, Klot.

merulina, Berk.

multicolor, B. et $B r$. (Fig. 809 ter.)

Mutinus, Fries $=$ Cynophallus, Fries.-Stinkhorn Fungus.

Watsoni, Berk.

sulcatus, Cke. et Mass.

calyptratus, $B$. et $B r$.

discolor, Kalch.

annulatus, Bail. 
Hardyi, Bail. = M. pentagonus, Bail., Q1. Bot. Bull. x. (I895) p. 35, P1. I; M. pentagonus, var. Hardyi, Bail., Q1. Agric. J1. xvi. (I906) p. 494, P1. 29.-The first found of this species was truly pentagonal; the next had 6 angles; and since then Mr. J. H. Simmonds, of Brisbane, sent me a number of these plants, the prominent angles of whose stems varied from 4 to 6 ; hence it would be well to drop the name $M$. pentagonus, and to keep M. Hardyi, Bail. Angles of stems from 4 to 6. (Fig. 8Io.) papuasius, Kalchb.

Ithyphallus, Fries.-Dead-men's-fingers.

novæ-hollandiæ (Corda), Ed. Fischer in Sacc. Syll. Fung. vii. Io $=$ Phallus nove-hollandie, Corda.

aurantiaca, Mont.

quadricolor, B. et.Br. (Fig. 8ro bis.)

calyptratus, Berk.

operculatus, Bail. (Fig. 8I I.)

impudicus, Fries.

atrominiatus, Bail. (Fig. 8I2.)

Clathrus, Mich.-Net Fungus.

triscapus, Turp. (Fig. 8I3.)

crispus, Turp.

cibarius, Fisch. = Ileodictyon cibarius, Tul.

gracilis, Schl. = Ileodictyon gracile, Berk.

pusillus, Berk. (Fig. 8I4.)

Aseröe, Labill.

Limbs of the receptacle horizontal.

rubra, Labill.-Star Fungus.

var. pentactina, Endl.

Limbs of the receptacle erect.

poculiforma, Bait.-Goblet Fungus. Limbs of receptacle varying from 7 to $I$ I. (Fig. 8I5.)

FAMily III.-Nidulariace 7 :

Cyathus, Hall.-Bird's-nest Fungus.

campanulatus, Corda.

intermedius, $T u l$.

ambiguus, $T u l$.

Lusueurii, Tul.-On an old gunny-bag.

vernicosus, $D C$.

fimicola, Berk.

pezizoides, Berk.

pusio, Berk.

Montagnei, Tul.

fimetarius, $D C$. (Fig. 8I6.)

Baileyi, Massee.-On dung. 


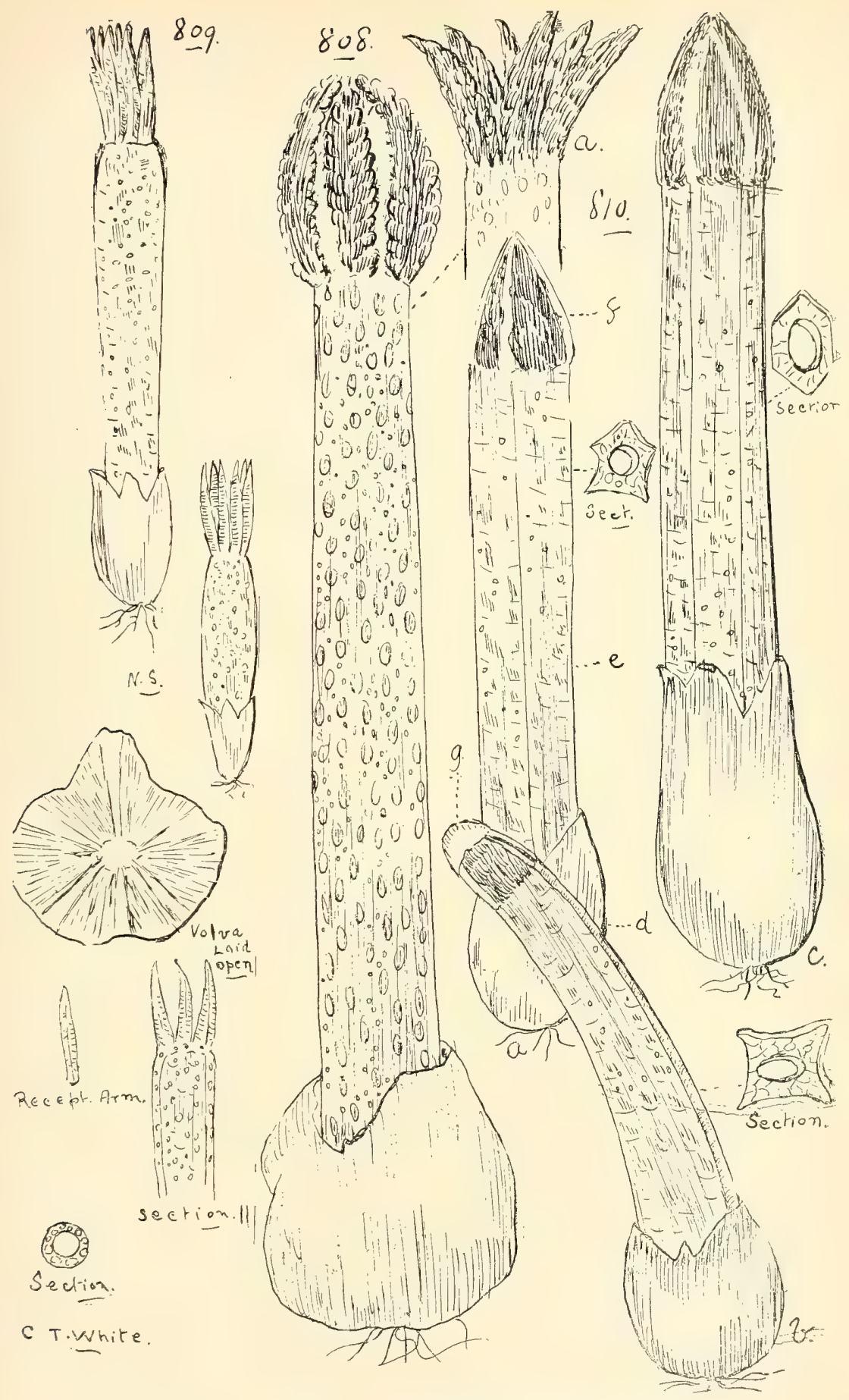

808. Lysurus australiensis, Cre. et Mass.

(a) Lobes of receptacle opened out.

809. L. TeNuis, Bail.

8io. Mutinus Hardyi, Bail.

(a) Plant with 5-angled stem, (b) do. with 4-angled stem, (c) do, with 6-angled stem. 
CLXII. IUNGI.

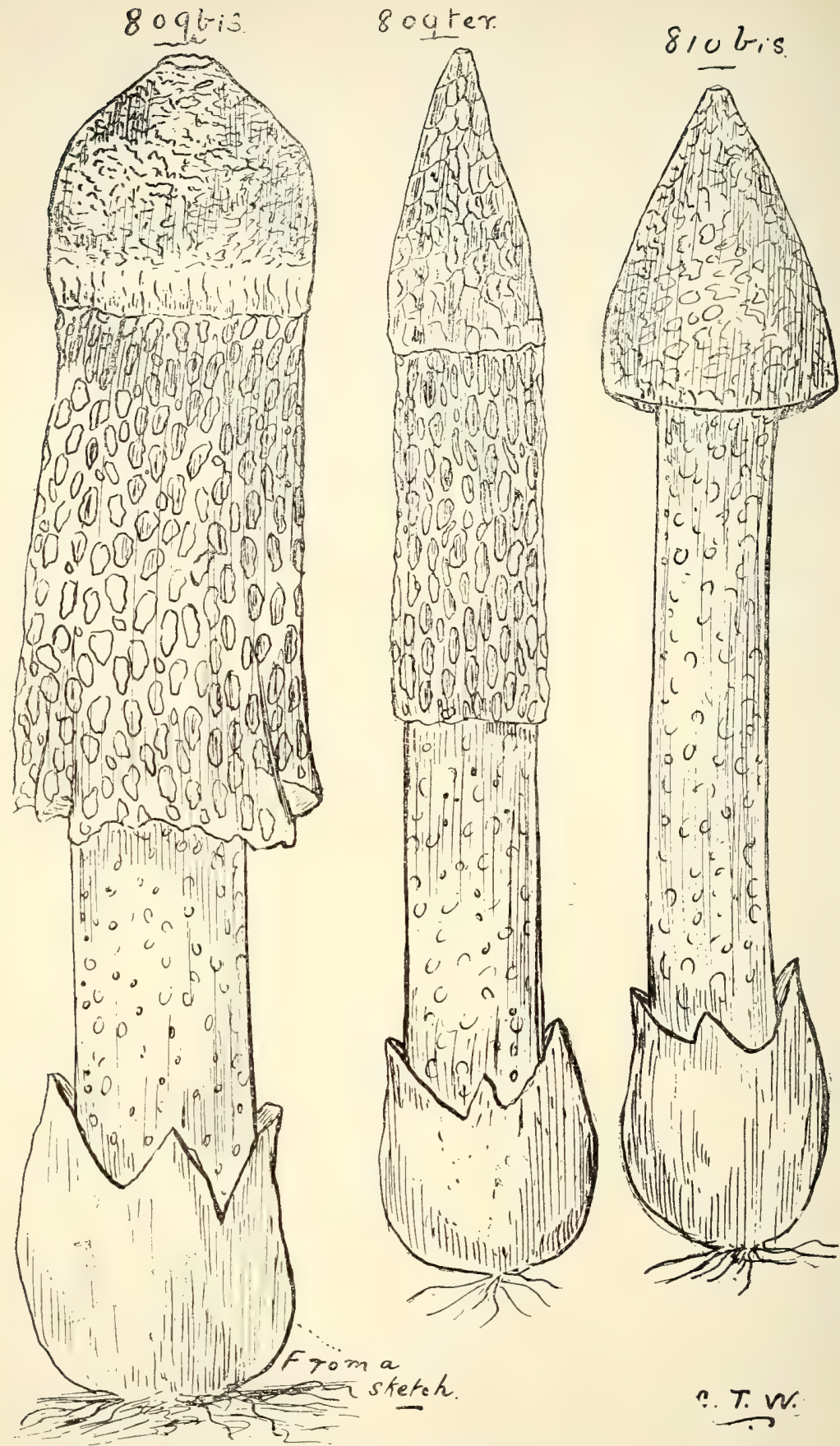

Eog bis. Dictyophora phalloidea, Desv.

809 ter. D. Multicolor, Berk. et Br.

Sio bis. TthyPhallus Quadricolor, Berk. et Br. 


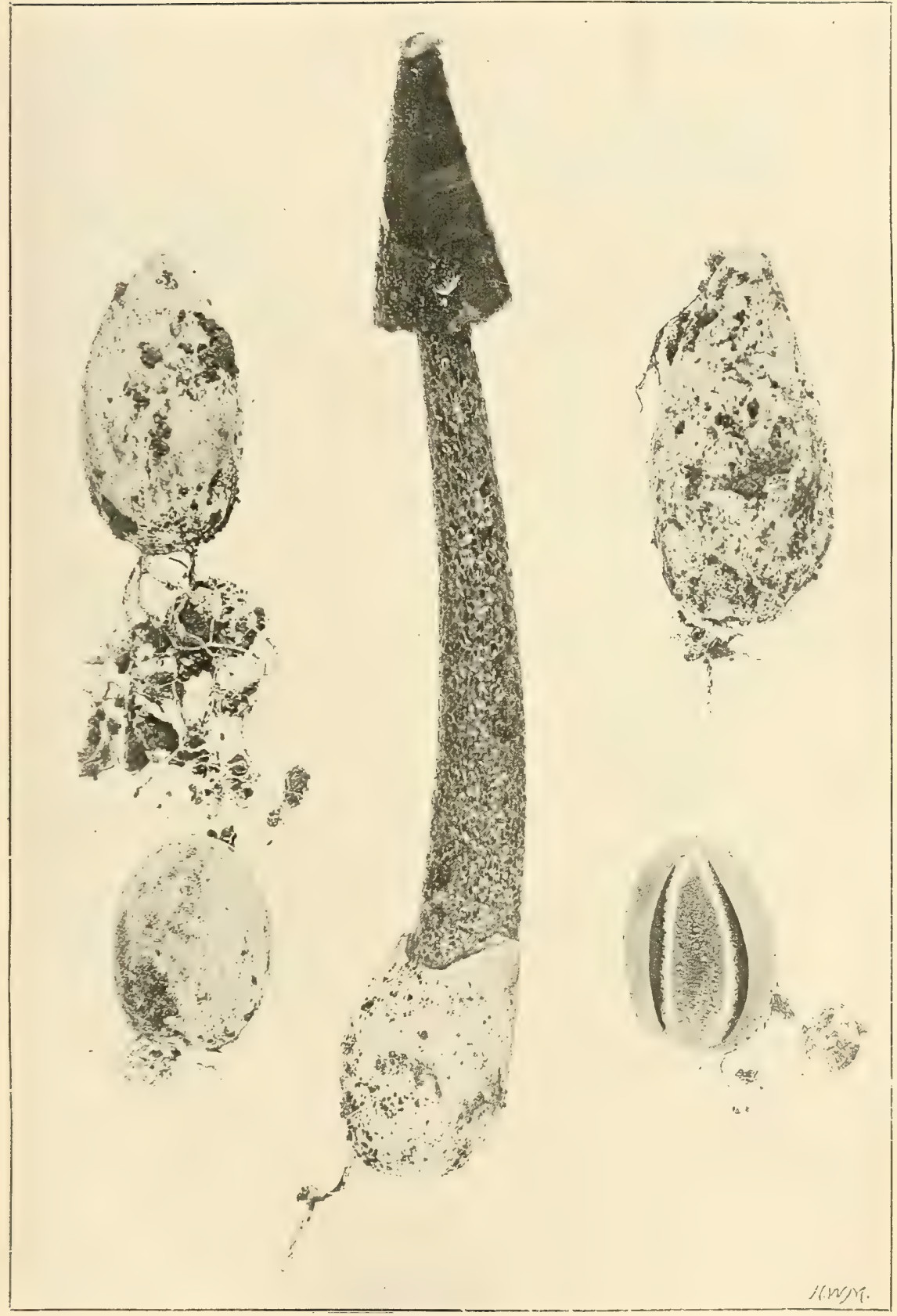

81T. Ithyphar.lus operculatus, Bail. 
CLXII. FUNGI.

$8 \mathrm{I} 5$
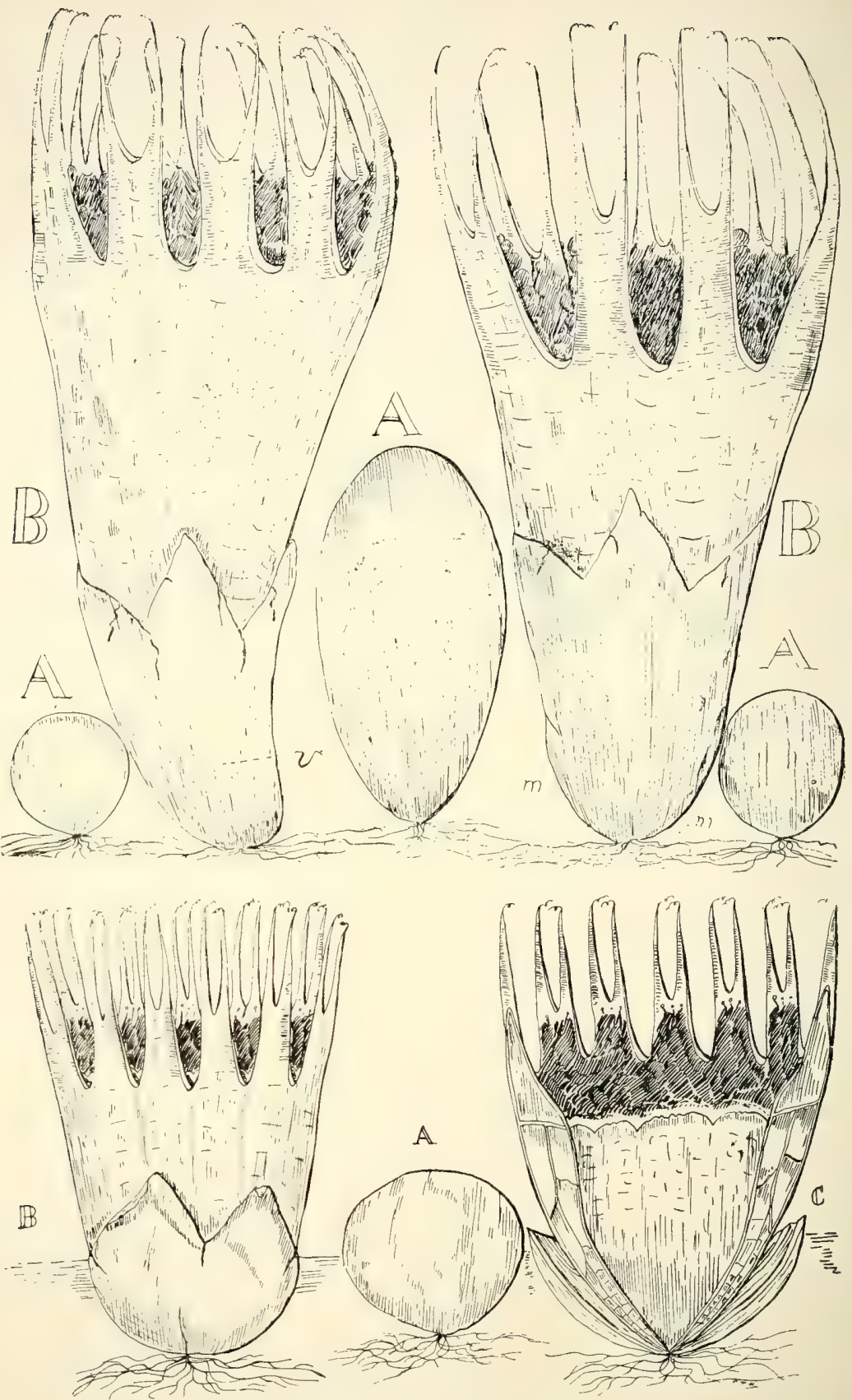

8i5. Aseröe poculiforma, Bail.

(A) Young plant, (B) full-grown do., (C) section do. Top figures nat. size; lower reduced about one-half. 
Crucibulum, Tul.

vulgare, Tul.-On wood. (Fig. 8r7.)

Protoglossum, Mass.

luteum, Mass. (Fig. 8I8.)

Tylostoma, Pers.-Stalked Puffball.

leprosum, Kalchb.

mammosum, Fries. (Fig. 8I9.)

Wightii, Berk.

Battarea, Pers.

phalloides, Dicks.

Mesophellia, Berk.

arenaria, Berk. (Fig. 820.)

ingratissima, Berk. et F.v.M.

F.MILY II.-LYCOPERDACEA.

Sub-Family Podarinea.

Podaxis, Desv. = Podaxon, Fries.

indica, Spreng. = Podaxon pistillaris, Linn. (Iig. 82 I.) carcinomalis, Linn. = Ant-hill Fungus.

Geaster, Mich.-Earth-stars.

minimus, Schw.

Guilfoylei, F.v.M. et Berk.

saccatus, Fries.

hygrometricus, Pers.

Archeri, Berk.

lignicola, Berk.--On wood.

striatus, $D C$.

lageniformis, Vitt.

floriformis, $V$ itt.

australis, Berk. (Fig. 822.)

striatulus, Kalchb.

fimbriatus, Fries.

subiculosum, Berk.-On rotten wood.

Speggazinianus, De Toni.

limbatus, Fries.

rufescens, Pers.

dubius, Berk.

Diploderma, Link.

suberosum, Cke. et Mass.

Bovista, Pers.

Muelleri, Berk.

cervina, Berk.

olivacea, $C$. and $M$. 
Lycoperdon, Tournef.

lilacinum, Berk. = Bovista lilacina, Berk.

bovista, Linn. = L. giganteum, Batsch.-Edible.

coprophilum, Cke. et Mass.

Gunnii, Berk.

substellatum, Berk.

gemmatum, Batsch.-Edible.

pusillum, Batsch. (Fig. 823.)

tephrum, B. et $B r$.

braziliensis, Fries.

cælatum, Fries.

australe, Berk.

pyriforme, Schff.

Scleroderma, Fries.

Bovista, Fries.

Geaster, Fries.

pandanaceum, F.v.M.

verrucosum, Pers.

vulgare, Fries.

australe, Mass.

umbrina, Cke. et Mass. (Fig. 824.)

(Areolaria) strobilina, Kalch.

Mycenastrum, Desv.

corium, Berk.

olivaceum, Cke. et Mass.

phæotrichum, Berk.

Xylopodium, Mont.

ochroleucum, Cke. et Mass. (Fig. 825.)

Polysaccum, Fries.

pisocarpum, Fries (including P. olivaceum, Fries).

var. acaule, Cke.

microcarpum, Cke. et Mass.

australe, Lév. (Fig. 826.)

tuberosum, Mich.

album, Cke.et Mass.

Octaviania, Vitt.

Family Hymenogastraceæ.

australiensis, Berk. $=H y$ dnanginm australiense, Berk. et Br.

(Fig. 827.)

Hymenogaster, Vitt.

lycoperdineus, $V$ itt.

Hydnangium, Wallr.

brisbanensis, Berk. (Fig. 828.) 


\section{ASCOMYCETEE}

A.TUBEROIDEE.

Elaphomyces, Nees.

Leveiilei, Tul. (Fig. 829.)

$$
\text { B.-DISCOMYCETEAE. }
$$

Family Henvellex.

Morchella, Dill.

deliciosa, Fries.-Edible; found amongst rotten bark. (Fig. 830.)

Leotia, Fries.

lubrica, Pers.--Edible. (Fig. 831.)

Geoglossum, Pers.-Earth-tongues.

glabrum, Pers. (Fig. 832.)

\section{Family Pezizeæ.}

Geopyxis, Pers.

aluticolor, Berk. (Fig. 833.)

Peziza, Dill.

cochleata, Bull.-Earth-cups; edible. (Fig. 834.)

Otidea, Pers,

apophysata, $C$. and $P$.

Discina, Fries.

repanda, $W$ allr. = Peziza (Discina) repanda, Wallr.

Humaria, Fries.

Hartmanni, Phillips = Periza Hartmanni, Phillips.

scatigena, Berk. and Cke. = Peziza scatigena, B. and C.

granulata, Bull. = Peziza granulata, Bull.

Thozetii, Berk. = Peziza (Geoscypha) Thozetii, Berk. (Fig. 835.)

Phillipsia, Berk.

subpurpurea, Berk. and Br. (Fig. 836.)

polyporoides, Berk.

Sarcoscypha, Fries.

(Plectania) melastoma, Fckl. = Peziza mclastoma, Fckl. (Fig. 837.)

Trichoscypha, Cke.

Hindsii, Berk.

tricholoma, Mont. (Fig. 838.)

Lachnea, Fries.

(Sepultaria) vinoso-brunnea, Berk. and Br.

(Sepultaria) confusa, Cke. = Sphorospora confusa, Cke.

(Scutellinia) scutellata, Lim. = Scutellinia scutellata, Linn. 


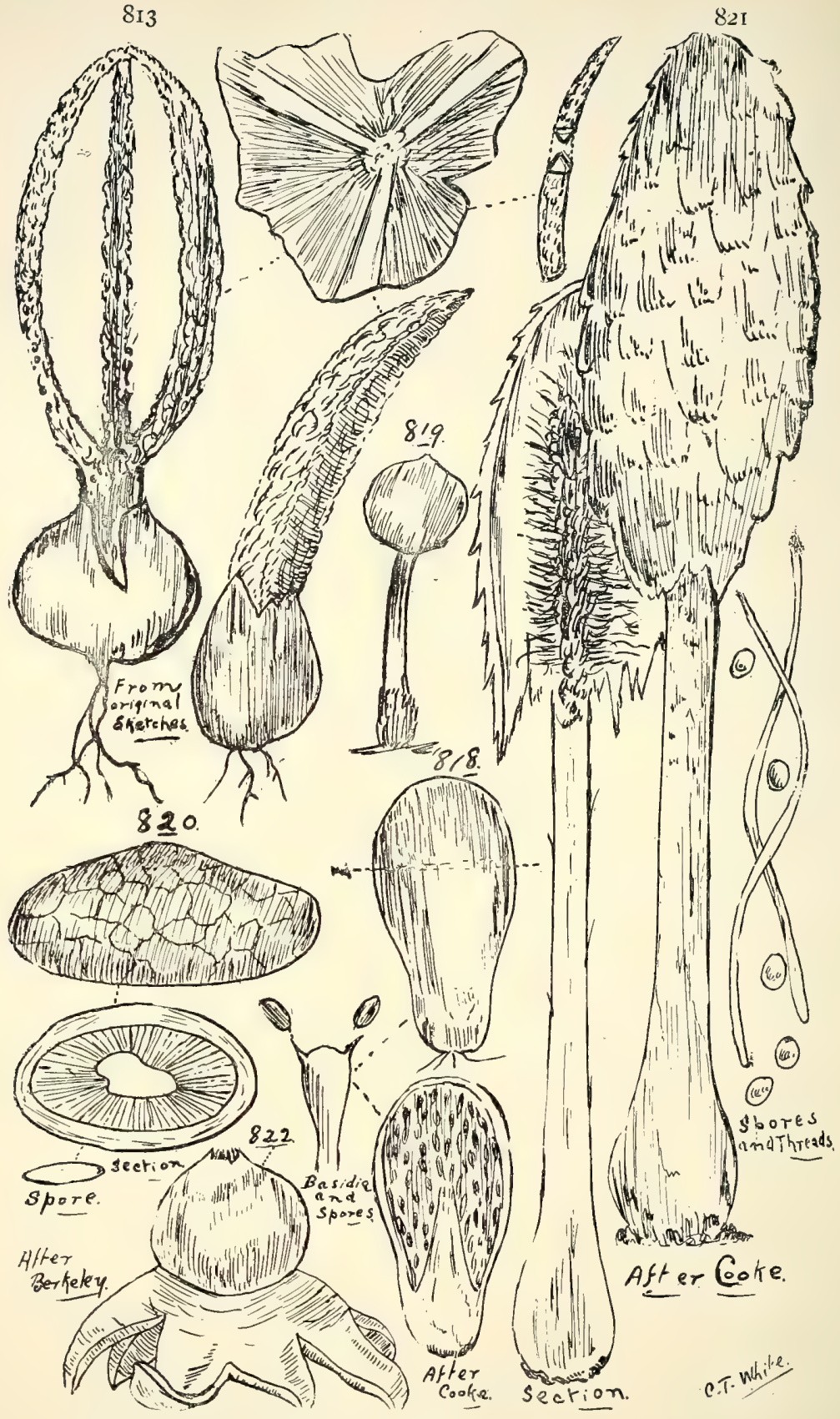

8i3. Clathrus triscapus, Turp.

Two plants in different stages of development; volva laid open, and section of one of the arms of the receptacle.

8i8. Protoglosium luteum, Mass.

8ig. Tylostoma mammosum, Fries.

82o. Mesophellia arenaria, Bcrk, (After Cooke.)

821. Podaxis INDiCA, Spreng.

822. Geaster australis, Berk. 
Lachnea-contd.

(Scutellinia) coprogena, Berk. = Peziza (Scutellinia) coprogena, B. and Br.

(Scutellinia) erinacea, Schw. = Peziza erinacea, Schw. (Fig. 839.)

Helotium, Fries.

citrinum, Hedw. (Fig. 840.)

epiphyllum, Pers.

kurandæ, $P$. Hennings.

Chlorospenium, Fries.

æruginosum, Tul. (Fig. 84I.)

Dasyscypha, Fries.

lachnoderma, Berk. = Lachnella lachnoderma, Berk.

terrestris, Berk, and Br. = Helotium terrestre, B. and Br.

Belonidium, Mont.

parasiticum, Cke. and Mass.--On the subiculum* of Asterina, growing upon leaves of Tarrietia trifoliata.

Erinella, Sacc.

Pritzeliana, P. Hennings.

\section{Family Ascobolex.}

Ascobolus, Pers.

australis, Berk.-Found growing deeply sunk in cowdung.

Philipsii, Berk.-On cowdung. (Fig. 842.)

Baileyi, Berk. and Broome.-On dung.

\section{Family Dermate.}

Urnula, Fries.

campylospora, Berk. = Peziza campylospora, Berk., and Geopyxis cinereo-nigra, B. and Br.-On rotten wood.

Cenangium, Fries.

lichenoideum, Bcrk. and Br. (Fig. 843.)

Family Bulgariex.

Ombrophila, Fries.

bulgarioides, Sacc.

Orbilia, Fries.

decipiens, Phill. = Calloria decipiens, Phill. - On rotten twine.

fusco-pallida, P. Hennings.

Bulgaria, Fries.

cyathiformis, P. Hennings.

Coryne, Tul.

Moffatæ, Mass (ined.). Ex Bail., Q1. Agric. J1. xvi. (I905) I93.

* Subiculum: A felted or byssoid stratum of hyphæ, bearing perithecia. 


\section{Family Stictex.}

Stictis, Pers.

radiata, Linn.

Pseudopeziza, Fckl.

\section{Family Phacidiei.}

medicaginis, Lib.-On Lucerne, Indooroopilly and Beaudesert (Tryon). (Fig. 844.)

Exoascus, Fckl.

\section{Family Gymnoascaceæ.}

deformans, Berk. = Ascomyces deformans, Berk.-Peachleaf Curl. As a cure for this disease, growers are advised to spray with Messrs. Bickford and Sons' Bordeaux paste, as used in South Australia with such good effect. (Fig. 845.)

Ailographum, Lib.

$$
\text { C.-HYSTERIACEZE. }
$$

melioloides, Cke. and Mass.-On leaves of native shrubs.

Glonium, Muhl.

cypericola, P. Hennings.-On Sedges.

Lembosia, Lév.

graphioides, Sacc, and Berl.-On leaves of Olea paniculata.

Tribliadlella, Sacc.

rufula, Spreng.-On bark. (Fig. 846.)

Rhytidhysterium, Spreng.

Scortechinii, Sacc. and Berl.

Hysterographium, Corda.

hiascens, Rehm. = H. macrum, Sacc. and Berl. (Fig. 847.)

\section{PYRENOMYCETEE.}

Family Hypocreace $\circledast$.

Epichlöe, Fries.

Sub-Family Hypocreoidea.

cinerea, Berk. and Br.-On grass-stems. (Fig. 848.)

Hypocrea, Fries.

Subgenus Hypocrella.

(Hypocrella) discoidea, Berk. and Br.-On leaves of plants cultivated in glass bowls. (Fig. 849.)

(Hypocrella) axillaris, Cke.-On Eragrostis stricta.

Sphærostilbe, Tul.

Sub-Family Nectria.

cinnabarina, Tul.--On bark.

hypocreoides, $K$. and $C$.-On bark.

dubia, Berk.-On bark of Egiceras (River Mangrove). 


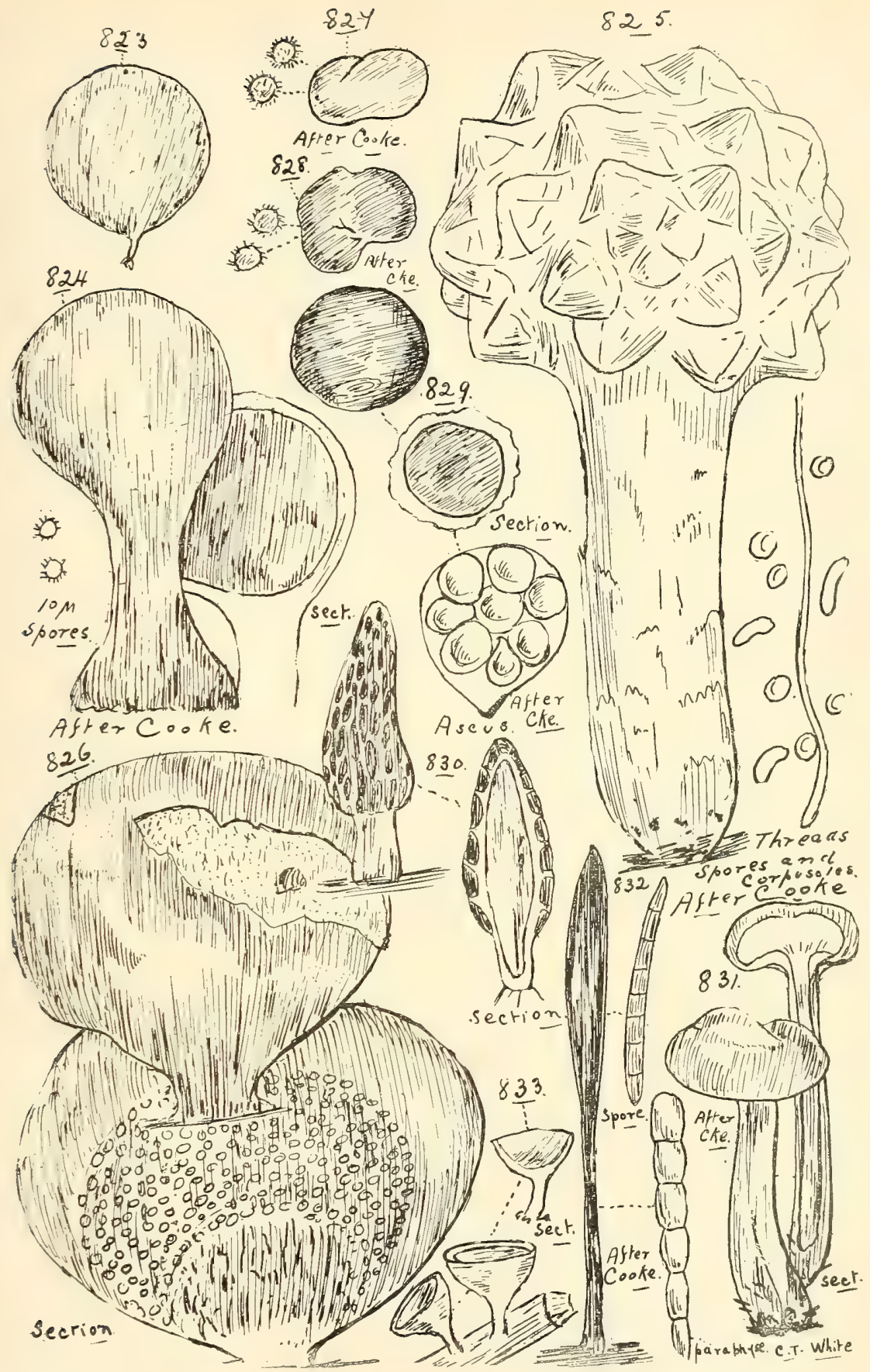

823. Lycoperdon pusillum, Batsch.

824. Scleroderma umbrina, Cke. et Mass. 825. Xylopodium ochroleucum, Cke. et Mass. 826. Polysaccum australe, Lév.

827. Octaviania australiensis, Berk. 828. Hydnangium brisbanensis, Berk.
829. Elaphomyces Leveillei, $T u l$. 83o. Morchella deliciosa, Fries. 831. Leotia lubrica, Pers. 832. Geoglossum glabrum, Pers. 833. Geopyxis aluticolor, Berk. 
Nectria, Fries.

coccinea, Fries.-On bark. (Fig. 850.)

episphæria, Tode.

Calonectria, De Not.

otagensis (Linds.), Sacc.-On twigs of Capparis Mitchelli.

Hypomyces, Fries.

chrysospermus, Tul.-On caps of Polyporus.

aurantius, Tul.-On caps óf Polyportss. (Fig. 851.)

membranaceus, Berk. and Br.-On caps of Polyporus.

stercicola, P.Hennings.-On Stereum lobatum.

Paranectria, Sacc.

Pritzeliana, P. Hennings.

Gibberella, Sacc.

Saubinetii, Mont., var. calami, P. Hennings.-On Lawyercane.

(Lisiella) passifloræ, Cke. and Mass. (Fig. 852.)

Megalonectria, Speg.

pseudotrichia (Schw.), Speg., var. australiensis, P. Hewnings.

Hypoderma, DC.

virgultorum (DC.), Sacc. $=$ Hysterium rubi, Pers.

Dielseilla, P. Hennings.

Pritzelii, P.Henn.-On leaves of Agathis Palmerstoni.

FAmily Xyiarieæ.

Xylaria, Hill.

australis, Cke.

involuta, Klotsch. $=X$. tabacina, Kicke.

grammica, Mont. $=$ X. ectogramma, Berk.

rhopaloides, Kunze.

rhytidophlæa, Mont.

zealandica, Cke.

scopiformis (Kunze), Mont.-On decaying capsules of Flindersia australis.

ovispora, Cke. and Mass.

gracilis, Klot.

polymorpha, Grev.

var. hypoxlea, Nitschke.

cerebriformis, Cke.

cynoglossa, Cke.

lobata, Cke.

castorea, Berk.

allantoidea, Berk.

escharoidea, Berk. 
Xylaria-conta.

fistulosa, Lév.

dealbata, $B$. and $C$.

anisopleura, Mont.

ianthino-velutina, Mont.-On capsules of a Flindersia from Northern Queensland.

cretacea, Berk, and Br.

elastica, Cke.

cinnabarina, Cke. and Mass.

fulvella, $B$. and $C$.

tuberiformis, Berk.

aphrodisiaca, Well. and Curr.

rhizophila, Cke. and Mass.-On roots of herbaceous plants. (Fig. 853.)

hypoxylon, Grev.

obtusissima, Berk.

carpophila, Pers.

Poronia, Willd.

adipus, Mont.-On dung.

pileiformis, Berk. (Fig. 854.)

Rhopalopsis, Cke.

(Kretzschmaria) australiensis, P.Hennings.

(Kretzschmaria) confusum, B. and $C$.

(Kretzschmaria) cetrarioides, Well. and Curr.

(Kretzschmaria) angolense, Well. and Curr. (Fig. 855.)

Ustulina, Tul.

vulgaris, Tul. (Fig. 856.)

Nummularia, Tul.

Baileyi, Berk. and Broome. (Fig. 857.)

lutea (A. and S.), Fries.

microplaca, $B$. and $C$. = Anthostoma microplacum (B. and C.), Sacc., i. 298.

Bulliardi, Tul.

Daldinia, De Not.

concentrica, Bolt. = Hypoxylon concentricum, Bolt. (Iiig. 858.)

var. Escholzii, Ehrenb.

var. minor, Berk. and $B r$.

var. varians, Berk. and $B r$.

Hypoxylon, Bull.

placentæforme, Berk. and $C$.

rutilum, $T u l$.

fuscum, Pers.

multiforme, Fries.

annulatum, Schw. 


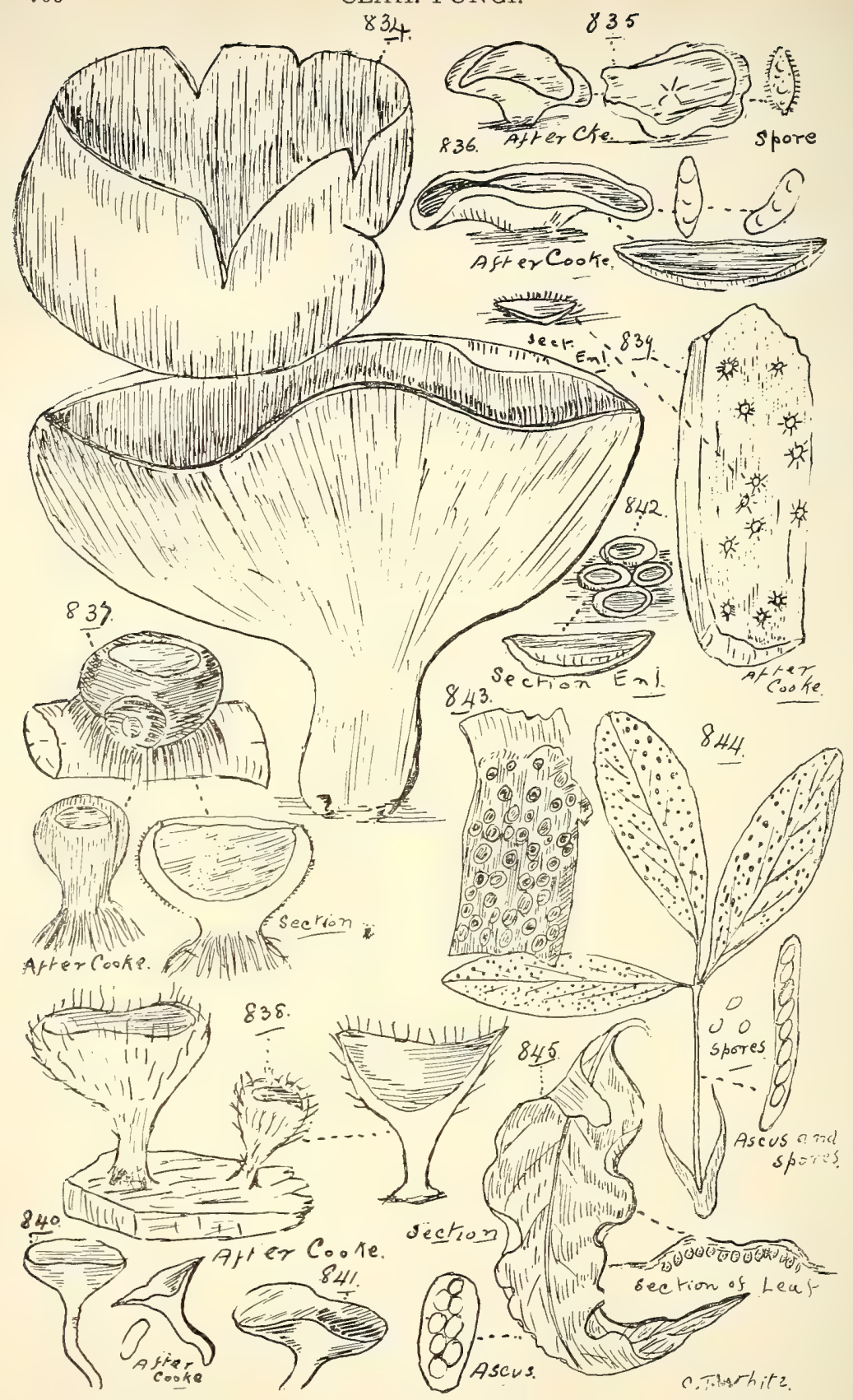

834. Peziza cochleata, Buli.

840. Helorium citrinum, $H \varepsilon d z$.

84i. Chlorospenium aeruginosum, Tul.

842. Ascobolus Phillifsit, Berk.

843. Cenangium lichenoideum, Berk. et Br.

844. Pseudopeziza medicaginis, Lib.

845. Exoascus deformans, Berk. 
Hypoxylon-contd.

rubiginostm (Fries), Pers.

hrematostroma, Mont.

marginatum, Schros.

atrosphæricum, Cke. and Mass.

hæmatites, Lév.

capnodes, Berk.

flavo-fuscum, Berk. and Br. (Fig. 859.)

oodes, Berk. and Br.

punctulatum, Berk. and $B r$.

serpens, Fries.

ellipticum, Cke. and Mass.

(Macroxylon) stratosum, Sacc.

FAMILY DOTHIDEACEA.

Sub-Family Dothideoidei.

Phyllachora, Fckl.

graminis, Pers.-On leaves of Kangaroo Grass (Anthistiria ciliata).

rhytismoides, Corda.-On leaves of Ficus opposita and Hibiscus tiliaceus.

alphinix, Cke. and Mass.--On leaves of Alpinia carulea. (Fig. 860.)

fimbristylis, Berk. and $B r$. = Dothidea fimbristylis, B. and Br.-On Fimbristylis.

(Dothidella) apiculata, Sacc. and Berl.--On leaves of Litsea dealbata.

symploci, Patouill.-On leaves of Symplocus Thrvaitesii.

(Montagnella) rugulosa, Cke.-On Eucalyptus leaves.

subcircinans, Speg.?-On living leaves of Tea-tree (Melalenca leucadendron).

Hab.: Cabbage-tree Creek and Coolangatta (C. T. White). (Det. by G. Massee.) Also on leaves of Myrtaceous plants in South America.

Dothidea, Fries.

(Bagnisiella) catervaria, Berk.--On the leaves of a Fig-tree. aspidea, Berk.

nitidula, Berk. and $\mathrm{Br}$.

Rhytisma, Fries.

Sub-Family Rhytismoidea.

hypoxanthum, Berk. and Br.-On leaves. (Fig. 86г.)

filicinum, Berk. and Br.-On Fern-fronds. (Fig. 862.)

Sub-Family Stigmatoidece.

Trabutia, Sacc. and Roum.

Pithecolobi, Cke. and Mass., Bot. Bull. xiii. 32.-On Pithecolobium grandiflorum. (Fig. 863.) 
Trabutia-contd.

eucalypti, Cke. et Mass.-On leaves of Eucalyptus siderophloia, var. rostrata.

Family Melogrammex.

Sarcoxylon, Cke.

compunctum, Jungh.

Gibellia, Sacc.

dothideoides, Sacc. and Berl.-On bark.

Melogramma, Tul.

rubricosa, Fries.-On bark.

Diatrype, Fries.

\section{Family Diatrypere.}

stigma, Hoffm.

chlorosarca, Berk. and Br.

Valsa, Fries.

\section{Family Valsex.}

echidna, Cke.-On bark.

Family Eulyper.

Cryptosphærella, Sacc.

macrozamix, Berk. and Br. = Spharia macrozamia, Berk. and Broome.-On fruit of Macrozamia Hopei. (Fig. 864.)

Eutypa, Tul.-Species of this genus have in the West Indies been destructive to Cacao (Theobroma) and other trees. polyscia, Berk. and Br.-On fruit of Bottle-gourd. 865.)

flavovirens, Hoffm.

Tarrietix, P. Hennings.

\section{Family Cucurbitariæ.}

Cucurbitaria, Gray.

Pritzeliana, P. Hennings.

Family Superficiales.

Byssophæria, Cke.

(Scortechinia) acanthostroma, Mont.

Coniochæta, Sacc.

queenslandiæ, P. Hennings.

Chætomium, Kunze.

cymatotrichum, Cke.-On leaves of Solanum Dallachyi. (Fig. 866.)

Rosellinia, De Not. Sub-Family Rosellinia.

mammoidea, Sacc. 
Rosellinia-contd.

tremellicola, Cke. and Mass.-On Tremella fuciformis. (Fig. 867.)

sericata, Cke. and Mass.

calami, P. Hennings.-On Lawyer Palm (Calamus).

Family Lophiostomaceæ.

Lophiostoma, Fries.

Schomburgki, Berk.

Family Obtecte.

Massaria, Fries.

eucalypti, F. Tassi.-On branchlets and petioles of Eucalyptus Watsoniana.

Leptosphæria, Ces. et de Not.

juncina (Auersw.), Sacc.-On dead stems of Juncus pallidus.

Didymosphæria, Sacc.

conoidella, Sacc, and Berl.-On branches of Capparis sarmentosa.

Banksix, Cke. et Mass.-On leaves of Banksia integrifolia, var. paludosa.

\section{Family Caulicole.}

Phomatospora, Sacc.

(Physalospora) gregaria, Sacc.-On stems of Ricinis communis (Castor Oil plant).

stigmatea, Fries.

seminata (B. and Rav.), Sacc. - On pods of a prostrate Desmodium. (Fig. 868.)

Didymella, Sacc.

bryoniæ, Fckl.-On Cucurbitaceous plants. (Fig. 869.)

Metasphæria, Sacc.

$s p$. Wakefield.-On breathing roots of White Mangrove (Avicennia officinalis), Moreton Bay (C.T. White).

Pleospora, Rabh.

vulgaris, Niessl.-On dead stems of Noogoora Burr (Xanthinm strumarium).

sp., Massee.-On bark of Citrus-trees, Brisbane River (C. T. White).

Hypospila, Fries.

eucalypti, Wakefield, Kew Bull. I9I2, p. I90.-On Eucalyptus leaves.

Family Follicole.

Læstadia, Auers.

xerotifolia, Cke. and Mass.-On leaves of Xerotes longifolia. destructiva, Berk. and Br. = Sphorella destructiva, B. and Br.-On leaves of Lucerne (Medicago sativa). (Fig. 7OI.) 


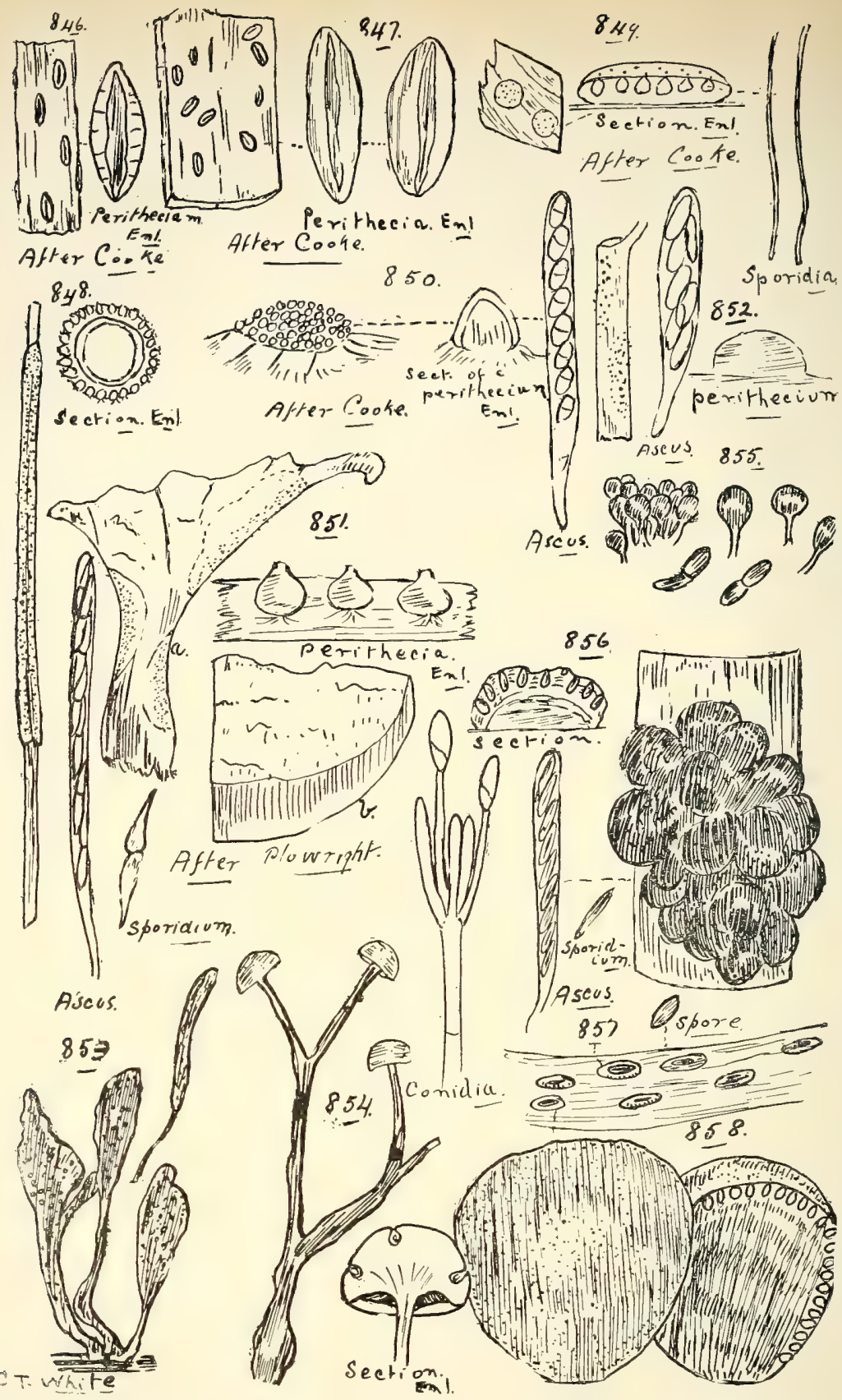

846. Tribliadella rufula, Spreng. 848. Eptchlöe cinerea, Berk, et $B r$.

847. Hysterographium hiascens, Rehm. 849. Hypocrea discoldea; Berk. et Br.

850. NeCTRIA COCCINEA, Fries.

Cluster of perithecia nat. size, sect. of perithecium enl., and ascus enl.

85i. Hypomyces aURANTIUS, Tul.

(a) Hypomyces on Panus, (b) conidia on an old Polyporus, both nat. size.

Perithecia, ascus, sporidium, and conidia enl.

852. GibBerella passiflore, Cke. et Mass.

Perithecium oil twig nat. size, and ascus and perithecium enl.

853. Xylaria rhizophila, Cke. et Mass.

854. Poronia PILEIIFORMIS, Berk.

855. RHOPALOPSIS ANGOLENSE, Well. et Curr.

856. Ustulina vulgaris, Tul.

857. Nummularia Baileyt, Berk. et Br.

858. Daldinia concentrica, Bolt. 
Lrestadia-contd.

dammaræ, Berk. and Br. = Spharia dammarce, B. and Br.On leaves of Agathis robusta.

litseæ, Berk. and Br. = Sphoria litsea, B. and Br.-On leaves of Litsea dealbata.

(Physalospora) sacchari, Berk. and Br. = Sphoria sacchari, B. and Br.-On Sugar-cane.

Trichosphæria, Tuck.

Sacchari, Massee.-Rind Fungus of Sugar-cane. On Sugarcane (Saccharum officinarum); at one time so prevalent in the West Indies as almost to paralyse the sugar industry $(K$. Bancroft $)$. As a preventive of this pest spreading, it has been recommended that the cuttings to be planted be soaked in dilute Bordeaux mixture. (Fig. 870 bis.)

Sphærella, De Not.

nubilosa, Cke.-On living leaves of Eucalyptus spp.

rubiginosa, Cke.-On leaves of Pittosporum rubiginosum. smilacicola, Schre:- On leaves of Smilax and Dioscorea. banksiæ, Cke. and Mass.-On leaves of Banksia. (Fig. 871.) Fragariæ (Tul.), Sacc.-Strawberry-leaf Blight. Generally prevalent on Strawberry plants throughout the southern portion of Queensland (Tryon).

\section{FAmily Microthyriace}

Myiocopron, Speg.

orchidearum (Mont.), Sacc.-On dead leaves and racemes of Cymbidium albuciflorum.

Microthyrium, De Not.

melaleucæ, P. Hennings. - On leaves of Melalenca leucadendron.

Micropeltis, Mont.

applanata, Mont. $=$ M. depauperata, Sacc. and Berl.

Seynesia, Sacc.

banksix, P. Hennings.-On leaves of Banksia latifolia. (Fig. 872.)

petiolicola, P. Hennings.-On leaves of a Dysoxylon.

Pemphidium, Mont.

dilatatum, Bcrk. et Br. = Myiocopron dilatatum, Sacc.-On dead petioles of Cabbage-tree Palm (Livistona australis).

\section{Family Perisporiace..}

Podosphæra, Kunzc.

tridactyla (Wallr.), De Bary--Apple Mildew.

Uncinula, Lév.

australiana, D. McAlpine.-Powdery Mildew. Very dense on leaves of Lagerstrœmia. (Fig. 873.)

Erysiphe, Hedw.

vitigera, Cke. and Mass.-Oidium condition. Powdery Mildew. On Grape-vines (Tryon). 
Erysiphe-contd.

communis, Wallr. = E. polygoni, DC.-The conidial form on plants of Lathyrus odoratus (Sweet Peas).

graminis, DC.-Powdery Mildew (conidial stage). On Wheat (Triticum sativum) (Tryon).

Sphærotheca, Lév.

Castagnei, Lév.-On leaves of Cucumber and Water Melon plants.

pannosa, Lér.-Rose Mildew. On Rose-leaves. (Fig. 875.) Eurotium, Link.

herbariorum, Link.-On damp herbarium specimens.

lateritium, Mont.-On Peperomia plants.

Asterina, Lév.

Baileyi, Berk. and Br.-On leaves of Hakea lora. (Fig. 876.) pelliculosa, Berk.-On leaves of Trema aspera.

platystoma, Cke. and Mass.--On leaves of Bean-tree (Castanospermum australe).

microthyroides, Wint.-On leaves of Eucalyptus siderophloia, var. rostrata.

hoveaefolia, Cke. et Mass.-On leaves of Hovea longifolia. (Fig. 877.)

(Asterella) alsophilæ, Cke. and Mass.-On fronds of the Wig Fern tree (Alsophila Rebecce).

(Asterella) eupomatiæ, P. Hennings.--On leaves of Eupomatia laurina.

reptans, Berk. and Curt.-On leaves of a Eugenia.

cryptocaryæ, Cke.-On leaves of Cryptocarya glancescens.

Dimerosporium, Fckl.

parvulum, Cke.-On leaves of the Peach-leaf Poison-bush

(Trema aspera). (Fig. 878.)

tarrietix, Cke, and Mass. - On the foliage of Tarrietia trifoliolata.

secedens, Sacc,-On living leaves.

Meliola, Fries.

corallina, Mont.-On various leaves in dense scrubs.

amphitricha, Fries.-Very common on the phyllodia of Acacia

Cunninghamiii and on the leaves of many other plants.

:musæ, Mont.-On various leaves in dense scrubs.

orbicularis, $B$. and $C$.-On various leaves and bark of shrubs. densa, Cke.-On Eucalyptus foliage.

loganiensis, Sacc, and Bert.-On leaves of a Smilax. polytricha, Kalch. and Cke.

microthecia, Thuem.-On leaves of Medicosma Cunninghamii. (Fig. 879.) 


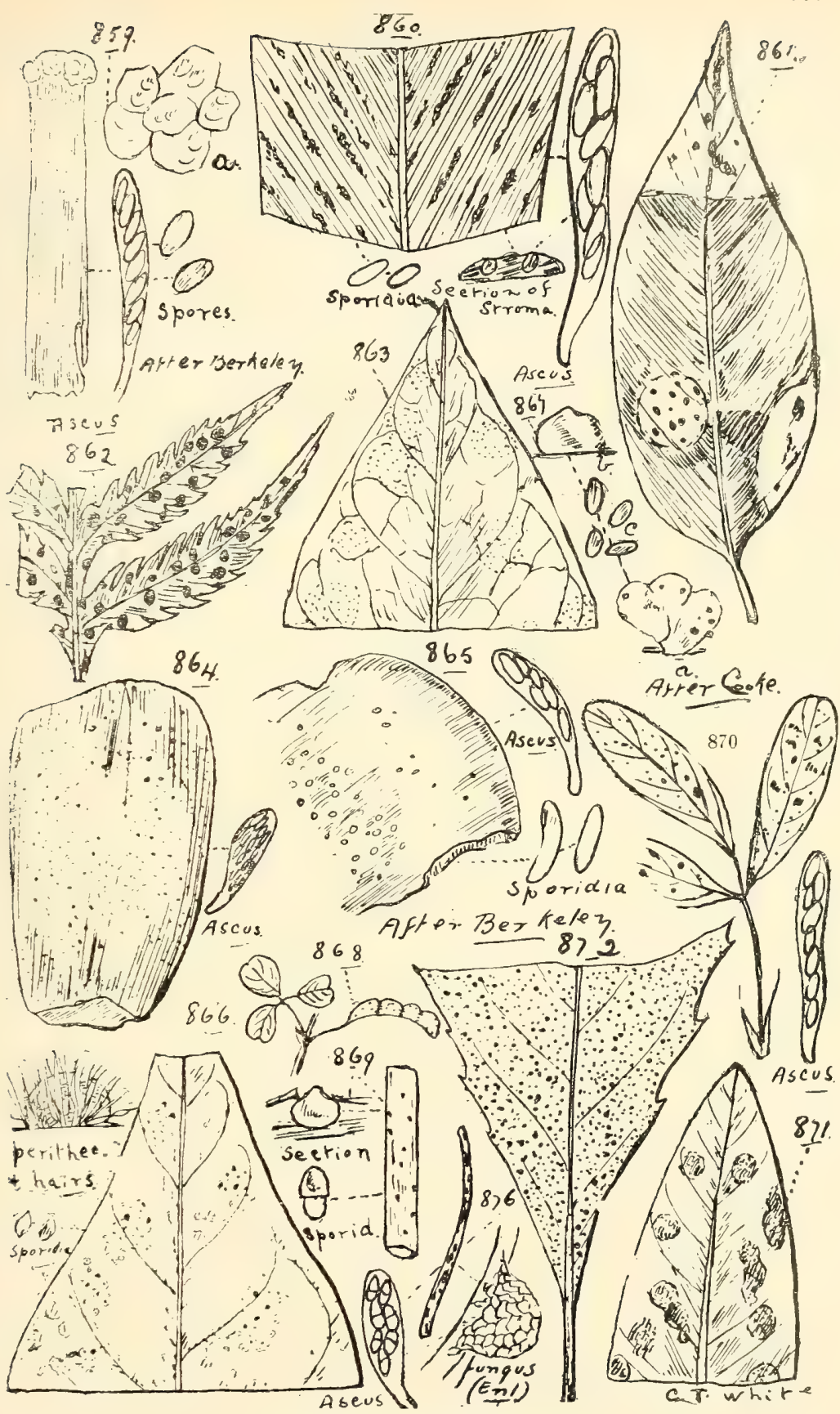

859. Hypoxylon flavo-Fuscum, Berk. et $B r$.

860. Phyllachora alpinie, Cke, et Mass. 864. Cryptosphærella Macrozamiæ, 86r. Rhytisma hypoxanthum, Berk. et $B r$. 862. R. FILICINUM, Berk. et $B r$. 863. TRABUtia PITHECOLOBI, Cke. et Mass.

865. Eutypa POLYSCia, Bcrk, et Br. 867. Roselinina tremellicola, Cke. et Mass.

(a) Perithecia on Tremella, nat. size, (b) a perithecium enI, (c) spores enl.

868. Phomatospora seminata, Sacc.

869. DidYMELla BRYONiE, Fickl.

870. L.estadia destructiva, Berk. et Br. 87i. SPH erella banksie, Cle. et Mass. 872. Seynesia banksie, P. Henn. 876. Asterina Baileyi, Berk. et $B r$. 
Meliola-contd.

eucalypti, Cke.-On Eucalyptus leaves.

octospora, Cke.-On leaves of Tristania conferta.

mollis, Berk. and Br.-On leaves of the Brisbane Box (Tristania conferta). (Fig. 880.)

tetraceræ, Thum.-On leaves of Tetracera Wuthiana. Dr.

M. C. Cooke considers this latter a Dimerosporium.

Capnodium, Mont.

citricolum, McAlp.-On Citrus spp. (Fig. 88r.)

citri, $B$. and Desm.-On Citrus leaves.

elongatum, B. and Desm.-On Grape foliage and twigs.

australe, Mont.-On the foliage of a Cycas.

salicinum, Mont.-On the foliage of a Zanthoxylum.

Corynelia (Achar.), Fries.

clavata (Linn.), Sacc. $=$ C. uberata, Fries. - On leaves of Podocarpus pedunculatus.

Antennaria, Link.

Robinsoni, B. and M.-On the foliage of Baccharis halimifolia and other plants.

semiovata, Berk. and Br.--On foliage of Ceratonia siliqua (the Carob or Algaroba Bean) and Ferns.

\section{PHYCOMYYETEE.}

Family Mucoracez.

Pilobolus, Tode.

crystallinus, Tode.-Glass-bead Fungus. On horsedung. (Fig. 882.)

Phycomyces, Kunze.

nitens, Kunze $=$ Mucor phycomyces, Berk. - Shining or Seaweed Fungus. On old boards and fatty substances.

Circinella, Tieg. and Mon.

umbellata, Tieg. and Mon., forma morelix = Helicostylum morelice, Berk. and Br.-On the dung of a carpet snake kept in a box.

\section{Family Peronosporacex.}

Cystopus, Lér.

candidus, Lév.-On Sencbiera didyma. (Fig. 883.)

Peronospora, Corda.

cubensis, Berk. et Curtis.-On Cucumber plants (Tryon).

Schleideni, Ung.-On Onion plants (Tryon).

parasitica (Pers.), De Bary.-On Brassica sp. (Tryon).

(Fig. 884.)

hyoscyami, De Bary.-On Tobacco leaves. 
Fhytophthora, De Bary.

infestans (Mont.), De Bary = Peronospora infestans (Mont.), M. C. Cooke; Botrytis infestans, B. et Br.The Potato Disease; until lately unknown in Queensland. Found by the State vegetable pathologist, Mr. H. Tryon, who also met with it on Tomato plants.

\section{Family Chytridiacem.}

Synchytrium, De Bary.

sp., Massee (immature).-On leaves of Medicago denticulata, Jimbour (C.J. Groyther).

Family Protomycetaceæ.

Protomyces, Unger.

macrosporus, Unger.-On Hydrocotyle.

\section{HYPODERMEAE.}

Ustilago, Pers.

\section{Section Ustilaginez.}

australis, Cke. = Sorosporium eriachnes, Thuem.; D.

McAlpine, Smuts of Aus. p. I78.-On Eriachne sp.

axicola, Berk. = Cintractia axicola (Berk.), Cornu.;

McAlpine, Smuts of Aus. p. I65.-On Fimbristylis

diphylla. (Fig. 885.)

bursa, Berk. = Tolyposporium bursum, McAlpine, Smuts of

Aus. p. Ig6.-On the inflorescence of Anthistiria frondosa.

leucoderma, Berk. = Cintractia leucoderma, P. Henn.; McAlpine, Smuts of Aus. p. I72.-On a Sedge, a form of the common Nut-grass.

segetum, Bull. = U. carbo, var destruens, Tul.-The Corn

Smut. On Wheat-ears; pustules covered by the epidermis.

bromivora (Tul.), Walldh-On Anthistiria ciliata.

sorghi (Link.), (Pass.) = Cintractia sorghi-rulgaris (Tul.), Wint.; McAlpine, Smuts of Aus. p. I73.-On Sorghum inflorescence.

Sacchari, Rabenh.-On Sugar-cane.

emodensis, Berk. - This, Dr. McAlpine, in The Smuts of Australia, p. I63, says, is an error. It should be Melanopsichium austro-americanum (Speg.) Beck.-On stems of Polygonum. (Fig. 886.)

Maydis, Corda.-On Maize (Zea Mays).

cesatii, $W$ alldh.-The same author in same work says that this

should be Sorosporium paspali, McAlpine.-On Paspalum scrobiculatum.

pertusa, Tracy and Earle.-On Setaria macrostachya. 


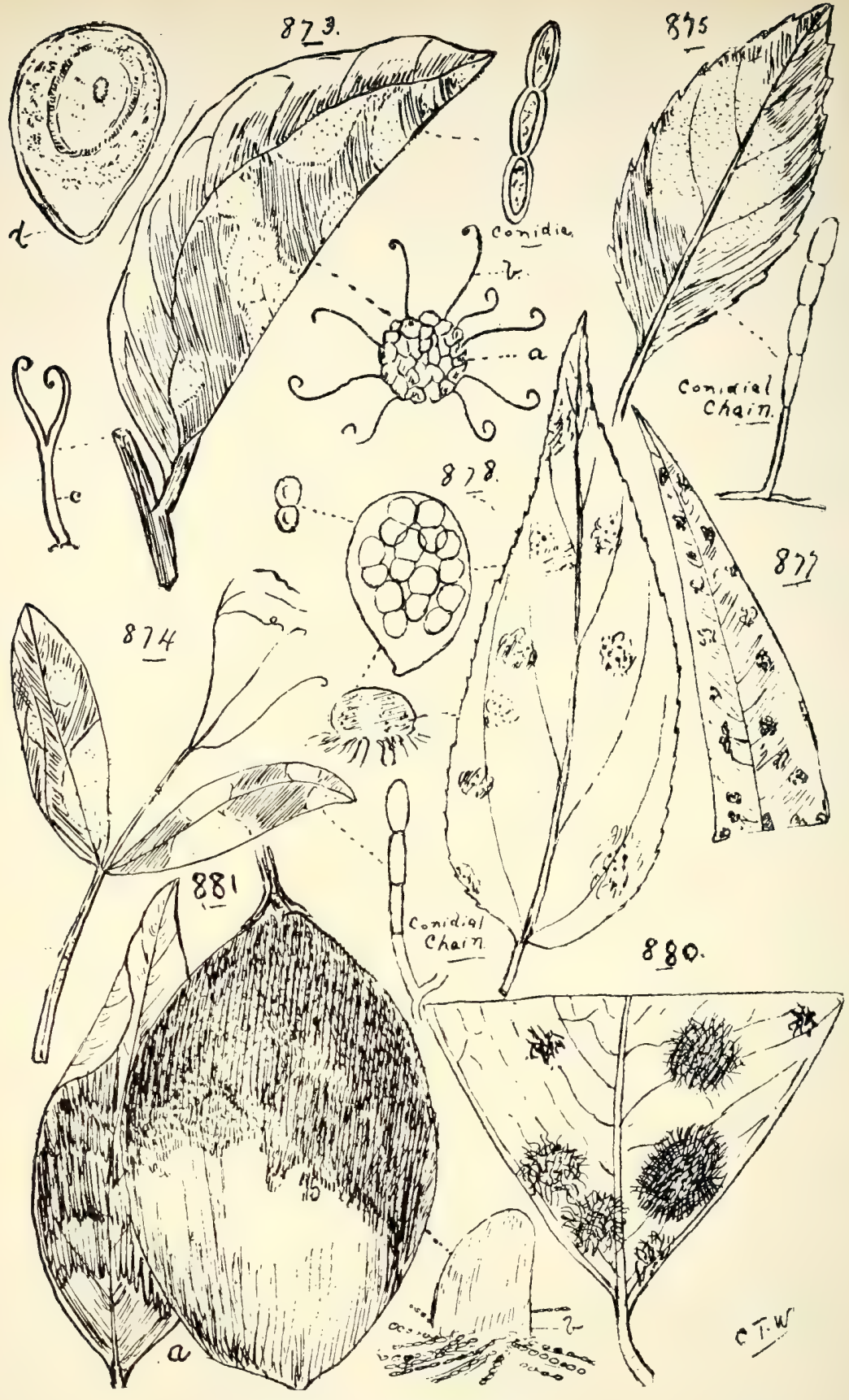

873. Uncin ula australiana, $M c A l p$.

(a) Perithecium with appendages, (b) an appendage, (c) a forked appendage, (d) immature ascus with sporidium; (d) after McAlpine.

874. ERYSiPHe COMniUnis, Wallr.

875. SPHzinotheCA PANNOSA, Lér.

877. Asterina hOveæfolia, Cke, et Mass.
878. Dimerosporium parvulum, $C k e$. 88o. Meliola mollis, Berk. et Br. 
Cintractia, Cornu.

patagonica, Cke. et Mass.-A bad pest on the Prairie Grass (Bromus unioloides). (Fig. 887.)

sp., Massee.-On Panicum effusum. Percy Islands ( $H$. Tryon).

Tilletia, Tul.

caries, $T u l_{0}=T$. tritica, Winter.-Bunt. In ovaries of Wheat. (Fig. 888.)

epiphylla, Berk. and Br. = Puccinia maydis, Bereng., according to McAlpine, "Smuts of Australia."-On leaves of Maize.

Entyloma, De Bary.

eugeniarum, Cke. and Mass.-On leaves of a native Eugenia. (Fig. 889.)

Sphacelotheca, De Bary.

hydropiperis, Schum. = Ustilago candollei, Tul.-On ovaries of Polygonum.

Thecaphora, Fing.

inguinans, Berk. and Br. $=T$. globuligera, B. and Br.-On Leersia hexandra.

Sorosporium, Rud.

setariæ, McAlpine.-On Setaria glanca.

consanguineum, Ell. and Everh. = Ustilago aristide, Peck.D. McAlpine (Smuts of Aus., p. I75) considers this the fungus attacking Aristida sp. in Australia, included by Cooke under Ustitago segetum.

tumifaciens, McAlpine, Smuts of Aus. p. I84.-On Stipa sp. and Stipa pubescens.

Urocystis, Rabenh.

tritici, Koern.-On Wheat.

Graphiola, Poit.

phonicis, Poit.-On leaves of Date-palm (Phonix dactylifera). (Fig. 890.)

Cerebella, Ces.

paspali, Cke. and Mass.-On Paspalum scrobiculatum. (Fig. 89i.)

andropogonis, Cke.-On Heteropogon contortus.

Uromyces, Link.

\section{SeCtion UREdineÆ.}

betæ, Pers.-On leaves of Red Beet (Beta vulgaris).

Fabæ, Pcrs.-On Broad Bean plants (Vicia Faba). (Fig. 892.)

amygdali, Cke.-On Peach-leaves. D. McAlpine and some other writers consider this to be only the uredo stage of Puccinia pruni, Pers. 
Uromyces-contd.

fusiporus, Cke and Mass.-On Acacia salicina phyllodia.

phyllodix, Cke. and Mass. $=U$. phyllodiorum, McAlp. (in part).-On Acacia phyllodia. (Fig. 892 bis.)

junci, Tul. (uredo stage).-On stems of Juncus pallidus. (Fig. 893.)

diploglottidis, Cke. and Mass. - On leaves of Diploglottis Cunninghamii.

puccinioides, Berk. and F.v.M.-On Goodenia.

striatus, Schret.-On Lucerne (Tryon).

bicinctus, McAlpine.-On phyllodes and pods of Acacia fasciculifera.

caryophyllinus, Schrolt. $=U$. dianthi, Niess1.—On stems and leaves of Dianthus and Carnation.

Uromycladium, McAlpine.

alpinum, McAlpine.-On phyllodia and pods of Acacia linifolia.

Tepperianum, McAlpine.-On the large galls formed by insects on Acacia Cunninghamii, very common round about Brisbane (C.T. White); also on A. flavescens, A. ixiophylla, A. pendula, and A. torulosa (McAlpine, Annales Mycologici, iv. (I906) p. 322).

Hemileia, Berk. et Br.

Woodii, Kalch.-On living leaves of Gardenia edulis.

Melampsora, Cast.

nesodaphnes, Berk. and Br.-On fruits of Cinnamomum Oliveri (not Beilschmiedia obtusifolia) (McAlpine, Rusts of Aus. p. 208).

phyllodiorum, Berk. and $B r .=$ Uromyces phyllodiorum (Berk.), McAlpine, under which name he includes Uromyces phyllodic, Cke. et Mass.--On phyllodia of Acacia.

Cronartium, Fries.

asclepiadeum, Fries.-On Jacksonia scoparia.. McAlpine (Rusts of Aus. p. I90) records this fungus as $C$. Jacksonia, P. Henn.

\section{DIDYMOSPOR E.}

Puccinia, Pers.

helianthi, Schw.-On Sunflower leaves.

caulinicola, Corda.-On Hypocharis glabra. According to D. McAlpine (Rusts of Aus. pp. I59 and 208), the fungus on this host is $P$. hypocharidis, Oudem.

graminis, Pers.-On Rye, Wheat, Leersia hexandra, and other grasses. (Fig. 894.) 
Puccinia-contd.

coronata, Corda.-On Oats (Avena sativa) (Tryon).

straminis, De Bary $=$ P. rubigo-vera, DC.-Red Rust. On

Hemarthria compressa and cereals. My form of this fungus on the grass Hemarthria, Mr. McAlpine (Rusts of Aus. p. II 7 ) records as P. cacao, McAlpine (Uredo rottbollic, Dietel).

triticina, Eriks.-On Wheat. (Fig. 895.)

acetosæ, Schum.$=$ P. rumicis, Lasch.-On Rumex Brozmii

(Queensland Dock). McAlpine (Rusts of Aus. p. I74)

refers this to $P$. Ludwigii, Tepper. (Fig. 896.)

Maydis, Bereng. = sorghi, Schwz. (in part).- On leaves of Maize (Zea Mays).

wurmber, Cke. and Mass.-On Anguillaria dioica.

caricis, Rab.-According to McAlpine (Rusts of Aus. p. I34), this specimen is P. cyperi, Arthur.-On common Nutgrass (Cyperus rotundus); also the æcidium stage (Ecidium urtica) on Nettle (Urtica) leaves.

malvacearum, Mont.-On Hollyhock and Malva. (Fig. 897.) heterospora, Berk. and $C$. = Uromyces Thwaitesii, B. and

Br.; U. pulcherrimus, B. and C.-On Hibiscus.

carissæ, Cke. and Mass.-On leaves of Carissa ovata. (Fig. 898.)

distincta, McAlpine.-On leaves and inflorescence of the English Daisy. (Fig. 899.)

sclerolænæ, Mass., Kew Bull. I9Io, p. 3.- On leaves of Sclerolena biflora. (Fig. 900.)

purpurea, Cke. = Uredo sorghi, Fckl.--On leaves of Johnson Grass and Sugar-cane.

pruni, Pers.--On Prunus spp. (Tryon).

Hamaspora, Korn.

longissima, Korn. = Phragmidium longissimum, Thuem.-On leaves of Rubus moluccanus.

\section{UREDINE\& INFERIORES.}

Acidium, Pers.

plectroniæ, Cke.-On Canthium coprosmoides.

compositarum, Mart.-On Compositæ.

apocyni, Schwein. = E. apocynatum, Schw. D. McAlpine (Rusts of Aus. p. I83) refers this to the genus Cæoma, and is named by him C. apocyni, McAlpine.-On Tabernæmontana leaves.

nymphoides, DC. = E. nymphoidearum, Berk.-On Limnanthemum indicum. The teleuto stage of this has been shown to be Puccinia scirpi, DC., not yet, however, found in Queensland. (Fig. 901.) 


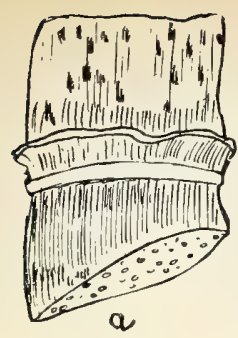

$870 \mathrm{~b}, \mathrm{~s}$
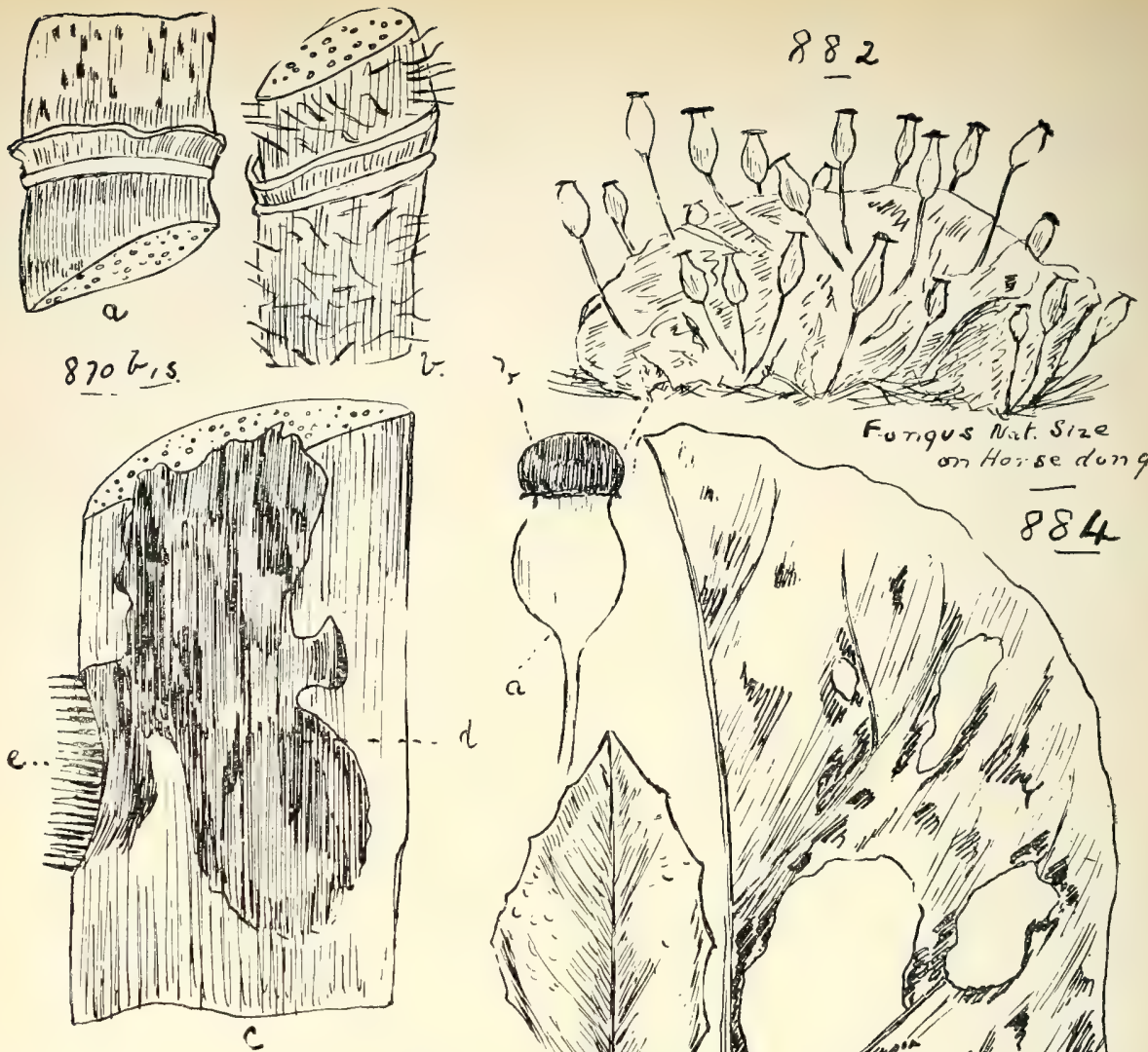

1
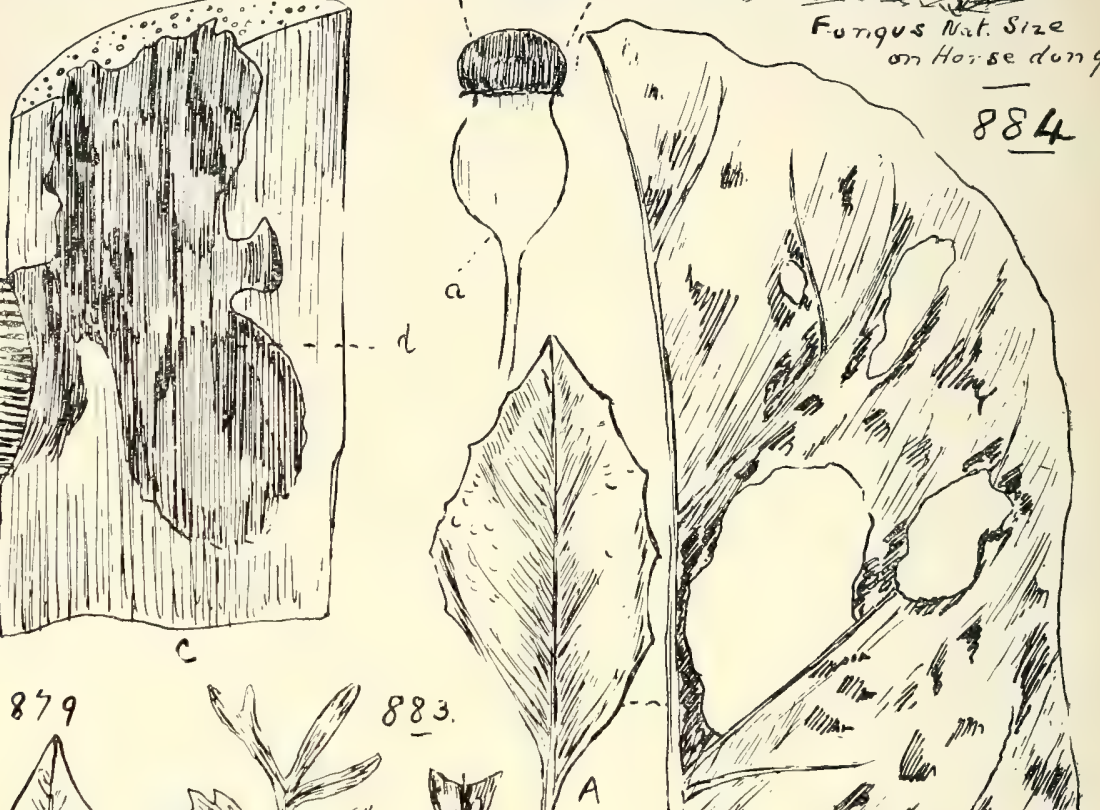

A
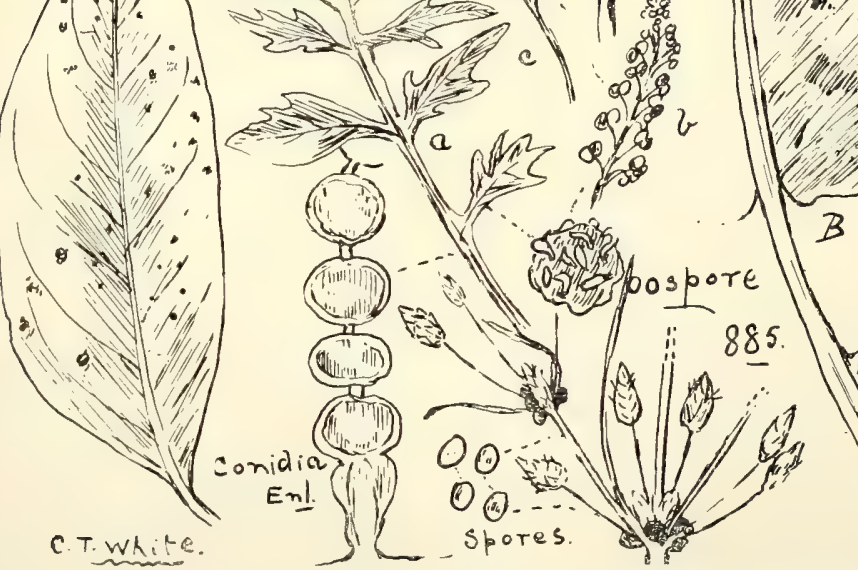

870 bis. Trichospheria SaCchari, Massec.

(a) Portion of a cane showing melanconium stage (Melanconium Sacchari) in a roung conchtion (b) do the centre showing the formation of (d) internal macroconidia.

879. Meliola microthecia, Thuem.

882. Pilobolus chystallines, Tode

(a) Inflated portion of the stem bearing the spore-mass (b). 883. Cystopus candidus, Lév.

(a) On leaf of Senebiera didyma, (b) on silicules of same, (c) on silicule of Capsella bursa-pastoris.

884. Peronospora parasitica (Pers.), De Bary.

(A) On leaf of young plant, (B) portion of a badly diseased leaf, (C) Conidiophore 
Ecidium-contd.

goodeniacearum, Berk.-On Scævola and Goodenia plants. (Fig. goI bis.)

deeringiæ, Cke. and Mass. - On leaves of Deeringia celosioides.

torquens, McAlp.-On Acacia Farnesiana.

Rrestelia, Rebent.

polita, Berk.-On Jacksonia scoparia.

Uredo, Pers.

leguminum, Desm.-On pods of Acacia,

cichoracearum, DC.-On Bidens pilosa.

clematidis, Berk. = Cooma clematidis, Thuem.-On Clematis. pallidula, Cke. and Mass.-On stems and pods of Cassia. (Fig. 902.)

rumicis, Schum.-On Muhlenbeckia gracillima. (Fig. 903.) crepidis-japonicæ, Lindroth.-On leaves of Crepis japonica. Kuehnii, Krueger.-On Sugar-cane.

Fici, Cast.-On Ficus carica (Tryon).

\section{FUNGI IMPERFECT $\not$.} SPH FROPSIDEE.

Family Sphæroideæ.

Section Hyalospora.

Phyllosticta, Pers.

opuntiæ, Sacc. and Speg.-On Prickly Pear. Very common round about Brisbane (C. T. White). Det. by E. M. Wakefield, Kew, Eng. "A common leaf-affection" (Tryon, Ann Rept. Q1. Dept. Agric. \& Stock (Igog-Io), Brisbane, p. 84).

leucadendri, P. Hennings.-On Melalenca leucadendron. circumscissa, Cke.-Shot-hole Fungus. On leaves of Peachtree (Prumus persica). (Fig. 904.)

acaciæe, Cke.-On Acacia phyllodia.

neurospilea, Sacc. and Berl.-On leaves of Vitis antarctica. uvariæ, Berk.--On leaves of Uvaria membranacea. fragricola, Desm.-On Strawberry leaves (Fragaria). flavidula, Sacc.-On leaves of Callistemon lanceolatus. cordylines, Sacc. and Berl.-On leaves of Cordyline terminalis. evodiæ, Cke.-On leaves of Evodia accedens. (Fig. 905.) palmicola, Cke.-On the leaves of young Palms.

scabiosa, McAlpine.-On Lemon leaves.

sterculiæ, Wint.-On leaves of Flame-tree (Stcrculia accrifolia).

sp., Wakefield.-Common on leaves of Monstera deliciosa in Queensland gardens (C. T. White). 
Phoma, Fries.

disoxyli, P. Hennings.-On a Dysoxylon.

rosarum, Dur. and Mont.-On Rose prickles and twigs.

notha, Berk.-On twigs of native shrubs.

purpurea, Cke. and Mass. - On Tristania and Eucalyptus leaves.

diploglottidis, Cke. and Mass.-On leaves of Diploglottis Cunninghamii.

bambusina, Speg.-On seedling Sugar-cane.

plagia, Cke. and Mass.-On young plants of a native Palnn.

cordylines, Thuem.-On Crinum pedunculatum. (Fig. 906.)

graminis, West.-On grass-stems.

folliculorum (Lév.), Sacc.-On follicle of Marsdenia.

ampelina, B. and Cke. = Sphaceloma ampelinum, De Bary.The Black Spot or Bird's-eye Rot of Grape-vines.

sycophila, Mass.-On native Fig (Ficus). (Fig. 907.)

citricarpa, McAlpine.-On Citrus fruit.

sp., Wakefield.-On Prickly Pear (Opuntia vulgaris), Cabbage-tree Creek (C.T. White).

Macrophoma (Sacc.), Berk. et Vogl.

$s p$. Massee.-On leaves of a native Eugenia, Coolangatta (C. T. White).

Aposphæria, Berk.

melaleucæ, P. Hennings.-On leaves of Melaleuca leucadendron.

Asteromella, Pass, and Thum.

epitrema, Cke.-On Trema aspera.

homalanthi, Cke. and Mass.-On Homalanthus populifolius.

Chatophoma, Cke.

eutricha, Sacc. and Berl. - On leaves of Castanospermum Cunninghamii (the Bean-tree).

Vermicularia, Fries.

herbarum, West.

Dothiorella, Sacc.

pericarpica, Sacc.-On fruit of Macrozamia.

Cytispora, Fries.

verrucula, Sacc. and Berl.-On branches.

Sphæropsis, Lév.

Section Phaospora.

tricorynes, Berk. and Br.-On Tricoryne anceps and Doryanthes leaves.

rosarum, Cke. and Ellis.-On Rose-branches.

malorum, Peck.-This Apple malady has recently been met with at Stanthorpe (H. Tryon). 


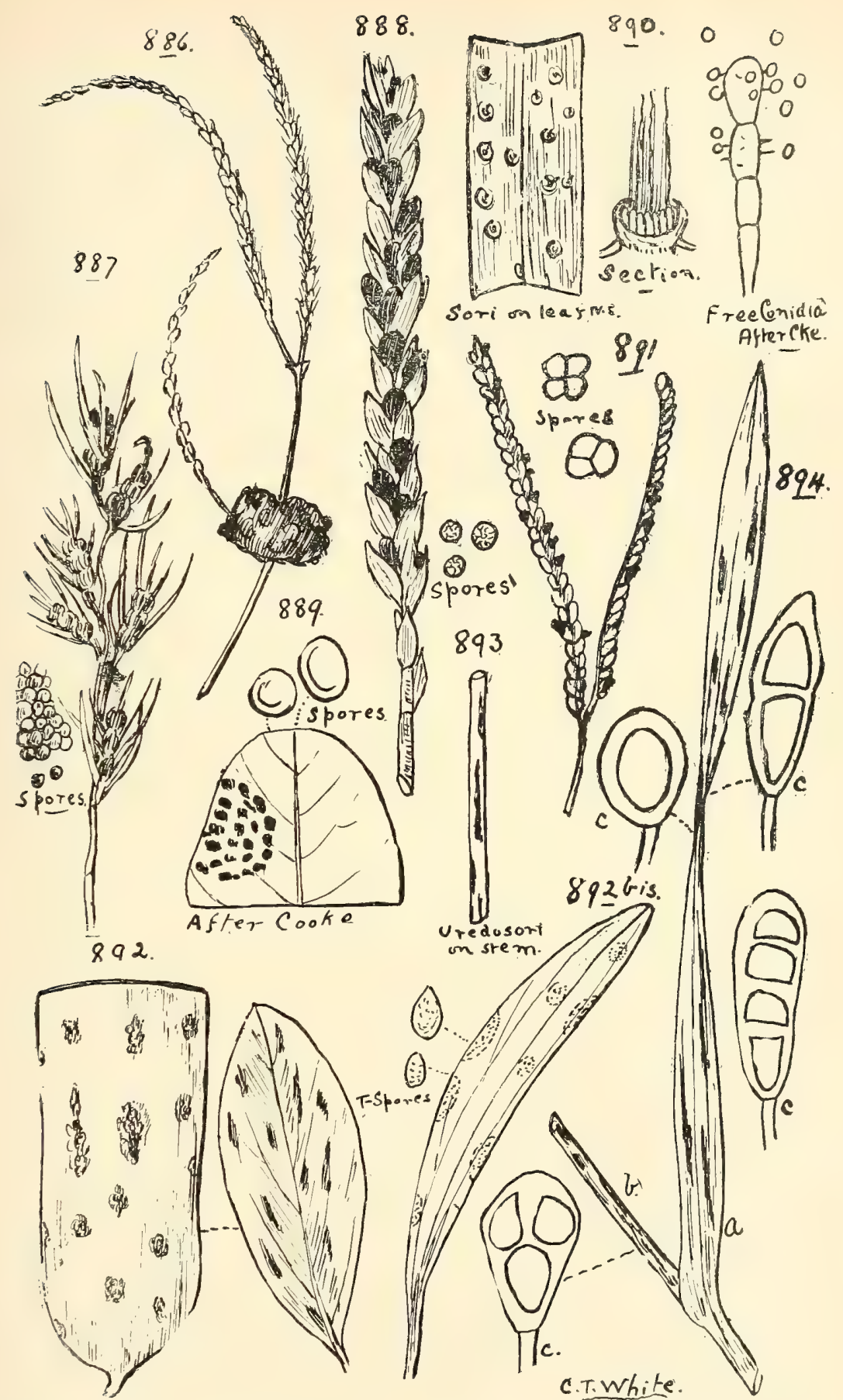

886. Ustuligo emodensis, Berk.

80i. Cerebella paspali, Cke et Mass.

887. Cintractia patagonica, Cke. et Mass. 892. Uromyces Fabe, Pers.

888. Tilletia caries, $T u l$.

892 bis. U. PHyllodik, Cke, et Mass.

889. Entyloma eugeniarum, Cke. et Mass.893. U. Junci, Tul.

890. Graphiola phenicis, Poit.

894. Puccinia graminis, Pers.

(a) Uredosori on leaf, (b) teleutosori on stem, (c) I-4 celled teleutospores, 
Coniothyrium, Corda.

xanthoræ⿸, P. Hennings.-On Xanthorrhæa (Grass-tree).

$s p$., Wakefield.-On Prickly Pear (Opuntia vulgaris), Cabbage-tree Creek (C.T.White).

Diplodia, Fries.

Section Phaodidyma.

calamicola, P. Hennings. - On Calamus Muelleri (Small Lawyer-cane).

lichenopsis, Cke. and Mass.-On Acacia complanata. (Fig. 908.)

phyllodiorum, Penz.-On Acacia phyllodia.

marsdeniæ, Cke. and Mass.-On follicle of Marsdenia sp.

pinea (Desm.), Kick.r.-Since the notices in Q1. Agric. J1. xxvi. (I9I2) Pp. I28 and I99, this fungus has been found attacking a large tree of Pinus longifolia in the Brisbane Botanic Gardens.

\section{Section Hyalodidyma.}

Ascochyta, Lib.

brunnea, Cke. and Mass. - On leaves of the Brisbane Box and other native trees.

apiospora, Cke. and Mass.-On leaves of Myrtaceous plants. (Fig. 909.)

Robillarda, Sacc.

sessilis, Sacc.-On Grape-vine leaves.

Actinonema, Fries.

rosæ, Lib.—On Rose-leaves. (Fig. 910.)

Diplodina, West.

dendrobii, Cke. and Mass.-On leaves of Dendrobium speciosum.

Watsoniana, Tassi.-On Eucalyptus Watsoniana.

Darluca, Cast.

filum, Cast.--Parasite on Uredo. (Fig. 9II.)

Section Dicty'ospore.

Camarosporium, Schutz.

punctiforme, Cke. and Mass.-On the fruit of a Saltbush.

Dichomera, Cke.

persooniæ, P. Hennings.-On Persoonia salicina.

Septoria, Fries.

\section{Section Scolecosporce.}

calami, $P$. Hennings.-On leaves of Calamus caryotoides. oleandrina, Sacc.-On leaves of Oleander. rosæ, Desmz.-On Rose-leaves. 
Septoria-contd.

lycopersici, Speg. - Leaf-freckle. On Tomato plants: (Tryon).

Dianthi, Desm.-On Carnation plants (Tryon).

Phlyctrena, Mont. and Desm.

passifloræ, Cke. and Mass.-On twigs of Passiflora edulis.

Gamospora, Sacc.

eriosporoides, Sacc. and Berl.-On leaves.

FAmily Nectrioider.

Aschersonia, Mont.

tahitensis, Mont.-On leaves of native shrubs in scrubs.

oxyspora, Berk.-On leaves of Cimamomum Oliveri.

australiensis, P. Hennings.-On leaves of Callistemon lanceolatus.

\section{Family Leptostromace e.}

Melasmia, Lér.

eucalypti, Cke. and Mass.-On leaves of a Eucalyptus.

tecomatis, Cke. and Mass.-On leaves of Tecoma jasminoides.

(Fig. 912.)

Actinothecium, Ces.

Scortechinii, Sacc, and Berl.-On leaves of Smilax.

Melophia, Sacc.

Woodsiana, Sacc. and Berl.-On the phyllodia of Acacia harpophylla.

Hainesia, Ell. et Sacc.

$$
\text { Family Melanconizei. }
$$

aurantiaca, Mass.-On fruit of Endiandra insignis. (Fig9I3.)

Gloosporium, Mont.

intermedium, Sacc.-On leaves of Hoya australis.

citricolum, Cke. and Mass.-On Orange leaves. (Fig. 9I4.)

periculosum, Cke. and Mass.-On fruit of Rhodomyrtus macrocarpus. Very poisonous. It is this fungus, probably, which causes blindness and often death to persons eating fruit containing it in quantities. (Fig. 915.)

cinnamomi, Cke. and Mass. - On leaves of Cimnamomun Oliveri.

glaucum, Cke. and Mass.-On the leaves of scrub plants, forming large bluish patches.

tristaniæ, Mass., Kew. Bull. I912, p. I90.-On leaves of Tristania suaveolens.

Denisonii, Sacc. and Berl.-On leaves of Macrozamia Denisonii.

Lindemuthianum, Sacc. and Magn.- On pods of French Beans. (Fig. 916.) 


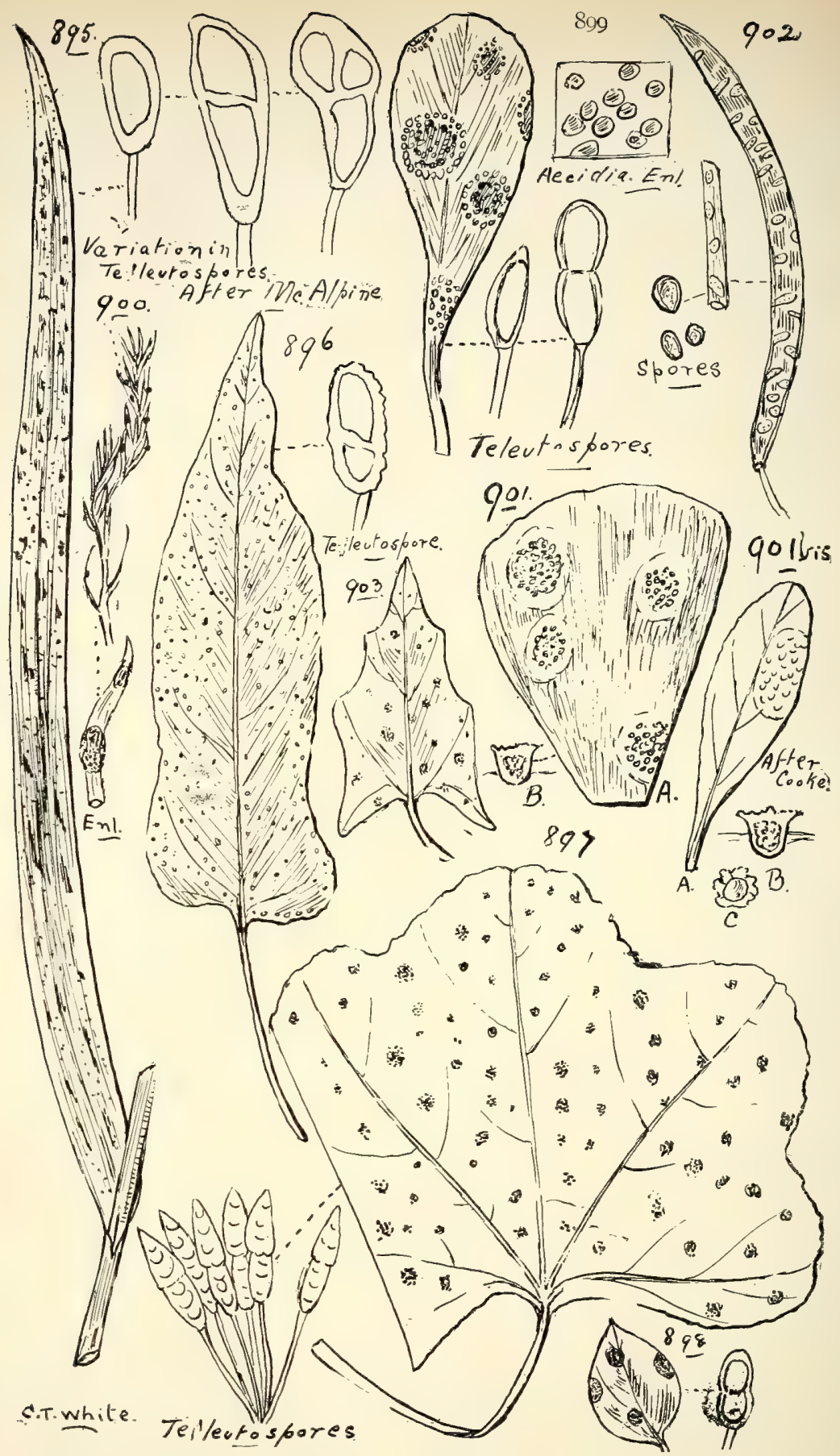

895. Puccinia triticina, Eriks.

896. P. ACETOS瓜, Schum.

897. P. malvacearum, Mont.

898. P. CARISS a, Cke. ct Mass.

899. P. DISTINCTA, McAlp.

900. P. SCLEROLENE, Mass.

90I. FCidium Nymphordes, $D C$.

(a) Portion of leaf of Limnanthemum with æcidia, nat. size, (b) sect. of an xcidium, enl.

goi bis. A. GOODEnIACEARUM, Berk.

(A) Leaf of Goodenia with æcidia, nat. size, (B) sect. of an æcidium, enl., (C) xcidiospore. 
Gloeosporium-contd.

alphitonix, Cke, and Mass.-On leaves of Alphitonia excelsa. palmarum, Cke. and Mass.-On leaves of Archontophonix Cunninghamii. (Fig. 917.)

fructigenum, Berk.-On Pear-fruit (Pyrus) and others. musarum, Cke. and Mass.-On Banana fruit (Musa). The diseased fruit has the appearance at first of containing a quantity of treacle, but afterwards becomes hard and dry. pestiferum, Cke. and Mass.-On Grape-vine twigs.

carpophilum, Mass.-On fruit of Xanthophyllum Macintyrii.

(Fig. 9I8.)

ampelophagum (Pass.), Sacc.-On Grapes. (Fig. 9I9.)

lagenarium, Pass.-On Mango fruit (Mangifera indica).

cucurbitarum, Berk. and Br.-On Water Melon and Banana fruit.

Raciborskii, P. Henn.-On Mango.

versicolor, Berk. and Cooke.-The Bitter Rot of Apples.

Kurzeanum, Niessl.-On base of plants of the Everlasting

Pea (Laihyrus latifolius) plants. For this disease the

following has been recommended:- When first observed wash the diseased parts with water in which sulphate of iron has been dissolved, say about 2 lb. of the sulphate in 5 gallons of water.

Colletotrichum, Corda.

falcatum, Went.-Red Rot of Sugar-cane (Tryon). (Fig.

glœosporioides, Penz.-On Lemon leaves.

$s p .$, Wakefield.-On leaves of young Flindersia sp., Buderim Mountain (C. T. White).

Asterostomella, Speg.

paraguensis, Speg.; forma, P. Hennings.-On leaves of Santalum lanceolatum.

homalanthi, Cke. and Mass.-On Homalanthus populifolius.

Coryneum, Nees.

papilliferum, P. Hennings.-On dead wood.

Pestalozzia, De Not.

uvicola, Speg.-On fruit and leaves of Grape-vines.

versicolor, Speg.-On leaves of Cupania anacardioides.

funerea, Desm.-On leaves of Elæodendron, Myrtus, and many other plants, and on fruit of Eugenia Smithii.

Guepini, Desm.-On leaves of Alphitonia excelsa. In India said to be one of the most destructive fungus blights of the Tea-plant and in Ceylon a common leaf-disease of Para Rubber.

vermiformis, Mass.-On leaves of a native Eugenia. 
Septogloeum, Sacc.

arachidis, Racib.-On leaves of Pea Nut (Arachis hypogaa), Rockhampton (Tryon).

erysiphoides, Fries.-On Pumpkin leaves.

\section{HYPHOMYCETE 压.}

\section{Family Mucedinee.}

Ramularia, Unoger.

\section{Section Phragmospore.}

citri, Penz.

Section Amerosporce.

Oospora, Wallr.

Subsection Micronemeæ.

aphides, Cke. and Mass.-On aphides upon Pumpkin leaves. scabies, Thaxter.-Potato Scab.

Oidium, Link.

leucoconium, Desm.-On Rose-leaves, \&c.

Tuckeri, Berk.-Powdery Mildew, on leaves of Grape-vine (Vitis). (Fig. 92I.)

Trichoderma, Pers.

Subsection Macronemeæ.

viride, Pers. - On bark.

Aspergillus, Mich.

glaucus, Link.-On putrescent organic substances.

Cookei, Sacc. $=$ A. mucoroides, Cke.-On dead plants and leaves.

entomophilus, Cke.-On dead insects of various kinds.

Sterigmatocystis, Cram. Viert.

nigra, $V$. Tiegh.-On fruit of Citrus Garrazayi.

Penicillium, Link.

glaucum, Link.-Met with on the top of brine used for meatpickle, fruit, \&c. The myceloid condition of this plant constitutes what is known as the Vinegar plant.

Sporotrichum, Link.

densum, Link.-On dead insects, \&c., on plants.

Botrytis, Mids.

cinerea, Pers.-On Strawberry plants, causing Root and Stem Rot, Brisbane district (Tryon).

Sepedonium, Link.

chrysospermum, Link.-On Boleti.

Nematogonium.

aureum, Berk.-On bark of Maba humilis.

\section{Family Dematieæ.}

Section Amerosporce.

Coniosporium, Link.

Subsection Micronemeæ.

atroeffusum, P. Hennings. 
Torula, Fries.

herbarum, Link.--On Acacia phyllodia.

Hormiscium, Kunse.

stilbosporum, Corda.-On plant-twigs.

Stachybotrys, Corda.

lobulata, Berk.-On wall-paper which had become damp in a Brisbane hotse.

Periconia, Bon.

nigrella, Berk.-On Andropogon.

Section Didymospora.

Bispora, Corda.

Subsection Micronemeæ.

monilioides, Corda.

Subsection Macronemeæ.

Fusicladium, Bon.

dendriticum, Wallr. = Cladosporium dendriticum, Wallr.On Pear-leaves (Pyrus communis). Apple Scab. (Fig. 922.)

Scolecotrichum, Kunze.

atriellum, Cke. and Mass.-On twigs of Passiflora edulis.

Cladosporium, Link.

herbarum, Link.-On Maize-leaves,

var. epixylinum, Corda.-On wood.

oligocarpum, Corda.-On Polyporus portentosus.

stenosporum, Berk. and C.-On Albizzia Lebbeck. asteroma, Fckl.

var. minor, Cke.-Brown Spot; on Grape-vine leaves.

hypophyllum, Fckl. - On leaves of a Serjania in Brisbane Botanic Garden.

epiphyllum, Pers.-On dead leaves of Camphor Laurel (Cimnamomum camphora) and Passion-vine (Passiflora edulis).

papyricolum, Berk. and Br. = Streptothrix atra, B. et C.-On damp wall-paper.

Section Phragmosporce.

Helminthosporium, Link.

Ravenelii, Curt.-On the inflorescence of Sporobolus and other grasses.

macrocarpum, Grev.-On the branchlets of Passiflora edulis. puccinioides, Sacc, and Berl.-On leaves of Tristania laurina. inconspicuum, Cke. and Ell.-On leaves of Maize (Zea).

Brachysporium, Sacc.

oligocarpum, Corda.-On stems of Passiflora edulis. 


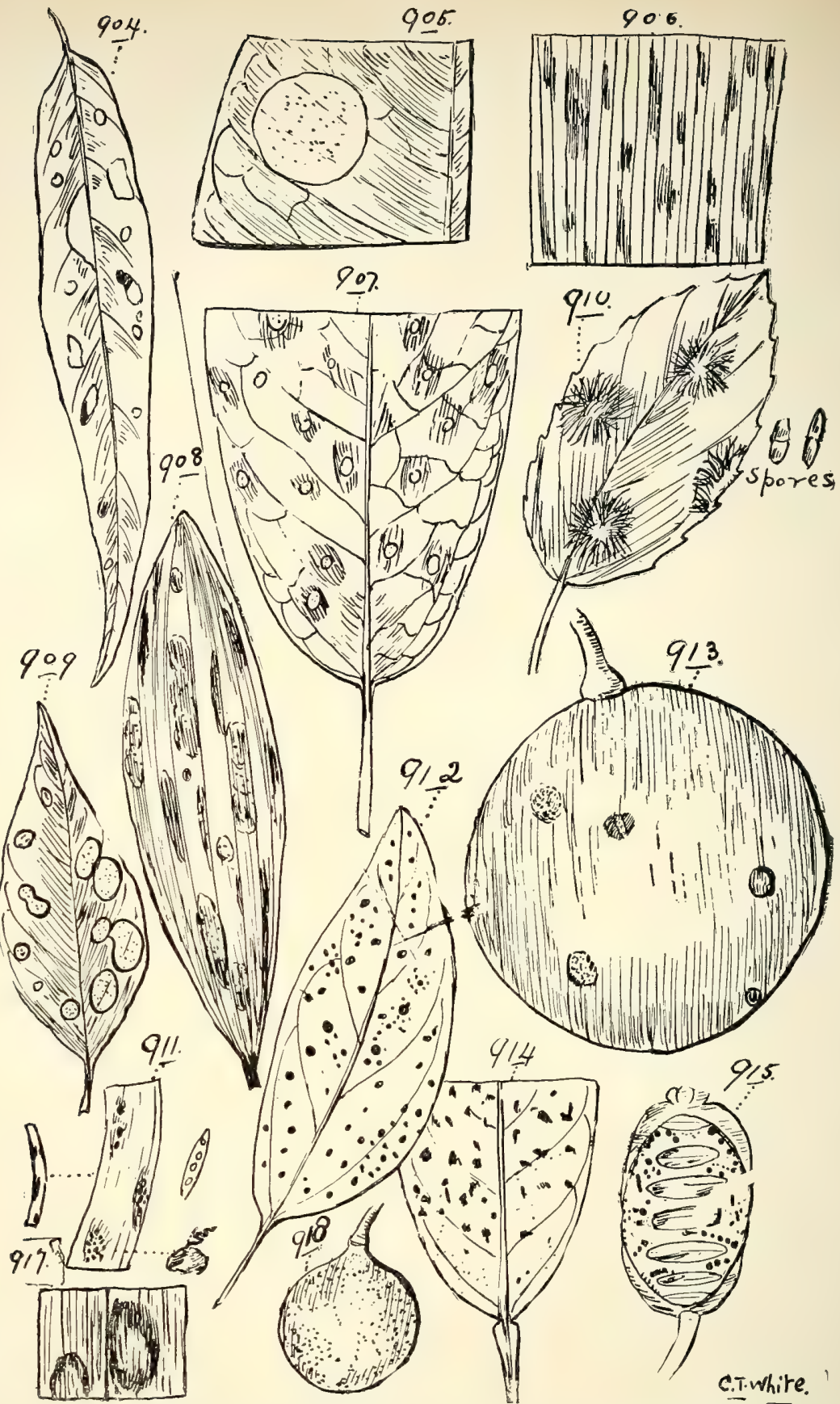

904. Phyllosticta circumscissa, Cke.

905. P. EVODIE, Cke.

906. Phoma cordylines, Thuem.

907. P. SYCOPHILA, Mass.

908. Diplodia Lichenopsis, Cke. et Mass. 909. Ascochyta aptosyora, Cke. et Mass. 9io. Actinonema ros $E$, Lib.

9i i. Darluca filum, Cast.

On uredosori of a uredine, nat. size and enl.; a perithecium enl. and spore with 4 sporidioles enl.

9i2. Melasmia tecomatis, Cke. et Mass. 9i4. Glcosporium citricolum, Cke. et Mass. 913. Hainesia aurantiaca, Mass. 
Cercospora, Fries.

Bryonix, Wakefield.-On leaves of Bryonia laciniosa.

Bloxami, Berk. and Br.-On Turnip leaves (Brassica).

viticola, Cess.-On Grape-vine leaves. (Fig. 923.)

medicaginis, Ell. et Ev.-On leaves of Lucerne (Medicago sativa), causing Leaf Blotch, Beaudesert (H. Tryon).

Raciborskii, Sacc. and Syd.--On leaves of Tobacco (Nicotiana Tabacum), Bowen district (H. Tryon). Also a pest of the Tobacco plant in Java.

solanacea, Sacc. and Berl.-On leaves of Solanum verbasci folium.

calanicola, P. Hennings.-On leaves of Calamus caryotoiaes. circumscissa, Sacc.-Shot-hole Fungus. On leaves of a Plum-tree. (Fig. 924.)

armoraciæ, Sacc.-On Horse-radish leaves (Cochlearia Armoracia). (Fig. 925.)

nicotiana, Ell. and Ev.-On Tobacco plants, causing Leaf Spot or White Spot (Tryon).

gossypina, Cke.-On Cotton.

coffeicola, Berk. and Cke.-On Coffee.

sp., Massee.-On leaves of Teucrium argutum, Stannary Hills (Dr.T.L. Bancroft).

\section{Section Dictyospora.}

Subsection Micronemex.

Sporodesmium, Link.

triseptatum, McAlpine.-On leaf of Lemon.

$s p .$, E. M. Wakefield.-On dead panicles of Yucca aloifolia

(see Appendix).

Macrosporium, Fries.

commune, Rabh.-On stems and leaves.

nigricantium, Atks.-On Cotton plants.

tomato, Cke. and Rav. = M. solani, Cke.-On Tomato fruit (Lycopersicum). The present fungus, which, as stated, infests the fruit at about its maturity, has nothing to do with the plants suddenly dying off when in healthy, vigorous growth. These deaths are not, in my opinion, due to any fungus; it is a mere matter of cause and effect. It will be noticed that at such times there has been an unusual fall of rain, and thus the soil is full of water; the plants become overcharged with liquid, often far beyond their power of utilisation; decomposition takes place, and the whole cellular matter of the root and stem forms a rotten mass, generally at the base of stem and upper part of root. From the same cause water-melons die off here and there in a field of these plants, often after an hour's rain. Here the parts 
Macrosporium-contd.

tomato-contd.

of the plants affected are the tips of the roots. The water has laid at the roots for perhaps a very short time, but these roots are most tender, and when overcharged with water rot at once, as one can easily prove by digging up one of these plants the next day after the rain. This may be called, for want of a better term, " vegetable dropsy." Fungi are not the cause, but a fungus may be found afterwards making use of the decomposing vegetable matter. Pelargoniums and other soft-wooded plants often meet the same fate during the summer months. H. Tryon, Annual Rept. Q1. Dept. Agric. and Stock ( I908-9) i I2, records $M$. solani, Cke., as very prevalent on Potato plants in many parts of Southern Queensland. (Fig. 926.)

peponicolum, Rabh.-On Papaw fruit (Carica).

Alternaria, Nees.

Brassicæ (Berk.), Sacc.-On Cabbage and Cauliflower leaves (H. Tryon).

Fumago, Pers.

vagans, $P$ ers. - On vine-leaves.

varians, Cke.-On leaves of Rhodomyrtus macrocarpa.

Family Stilbeæ.

Series Hyalostilbex.

Stilbum, Tode.

cinnabarinum, Mont.-On bark.

Pritzeliella, P. Hennings.

cærulea, $P$. Hennings.-On the chrysalis of a butterfly.

Pilacre, Fries.

Petersii, $B$. and $C$.

Isaria, Pers.

graminiperda, B. and Miill., var. fuciformis, Berk.-On grass. Considered injurious to the Perennial Rye-grass (Lolium perenne). Thus fungus is the Hypochnus fuciformis (Berk.), McAlpine. This author does not consider it injurious to stock, and has met with it on Medicago and utner plants.

Ceratium, Alb. and Schw.

hydnoides, $A$. and $S$.- On Eucalyptus.

arbuscula, Berk. and Br.-On rotten wood.

Series Phacostilbece.

Harpographium, Sacc.

quaternarium, Cke. and Mass.-On Passiflora edulis. 
Podosporium, Schwein.

australiense, $P$. Hennings.

Antromycopsis, Pat. and Trab.

? squamosus, $P$. Hennings.- On wood.

Isariopsis, Fries.

clavispora, B. and C.-On Grape-vine leaves (Vitis).

Family Tuberculariez.

Tubercularia, Tode.

Series Tuberculariece Mucedinea.

leguminum, Cke. and Mass.-On pods of a Cassia.

acinorum, Cavara.-On Grape-fruit (Vitis), causing Fruit Rot.

Illosporium, Mart.

flavellum, Berk. and Br.-On Lichens.

flaveolum, Sacc.-On wood.

Fusicolla, Bon.

incarnata, Cke. and Mass.-First found on dead leaves; and recently on fallen leaves of a Menispermaceous plant, Enoggera Scrub, and along with Phyllosticta sterculice on leaves of the Flame-tree (Sterculia acerifolia), Sandgate (C. T. White). Det. by E. M. Wakefield.

Thozetia, Berk. and Mrïll.

nivea, Berk.-On wood.

Bactridium, Kunze.

flavum, Kunze.-On wood.

Fusarium, Link.

cucurbitarieæ (Pat.), Sacc.-On Cucumbers, Bowen district (Tryon).

rubicolor, Berk. and Br.-On leaf-galls of Eucalyptus.

heterosporum, Link.-On Wheat-ear. (Fig. 927.)

decipiens, Cke. and Mass.-On leaves of Ficus aspera.

elongatum, Cke.-On branches of Custard Apple (Anona).

limonis, Briosi.-On Citrus. Collar Rot.

(Fusisporium) longisporum, Cke. and Mass.-On Argyreia twigs.

(Fusisporium) solani (Mart.), Sacc.-On Potato (Solanum tuberosum), also on Tomato (Lycopersicum esculentum). In about the early sixties this was very abundant in the potatoes exposed for sale in the Brisbane shops, but since it has not been anything like so abundant in this State. At the time mentioned shopkeepers used to make a practice of cutting one or two potatoes in halves to show that they were free from the disease. After boiling, the thready mycelium which formed a dark circle in the 


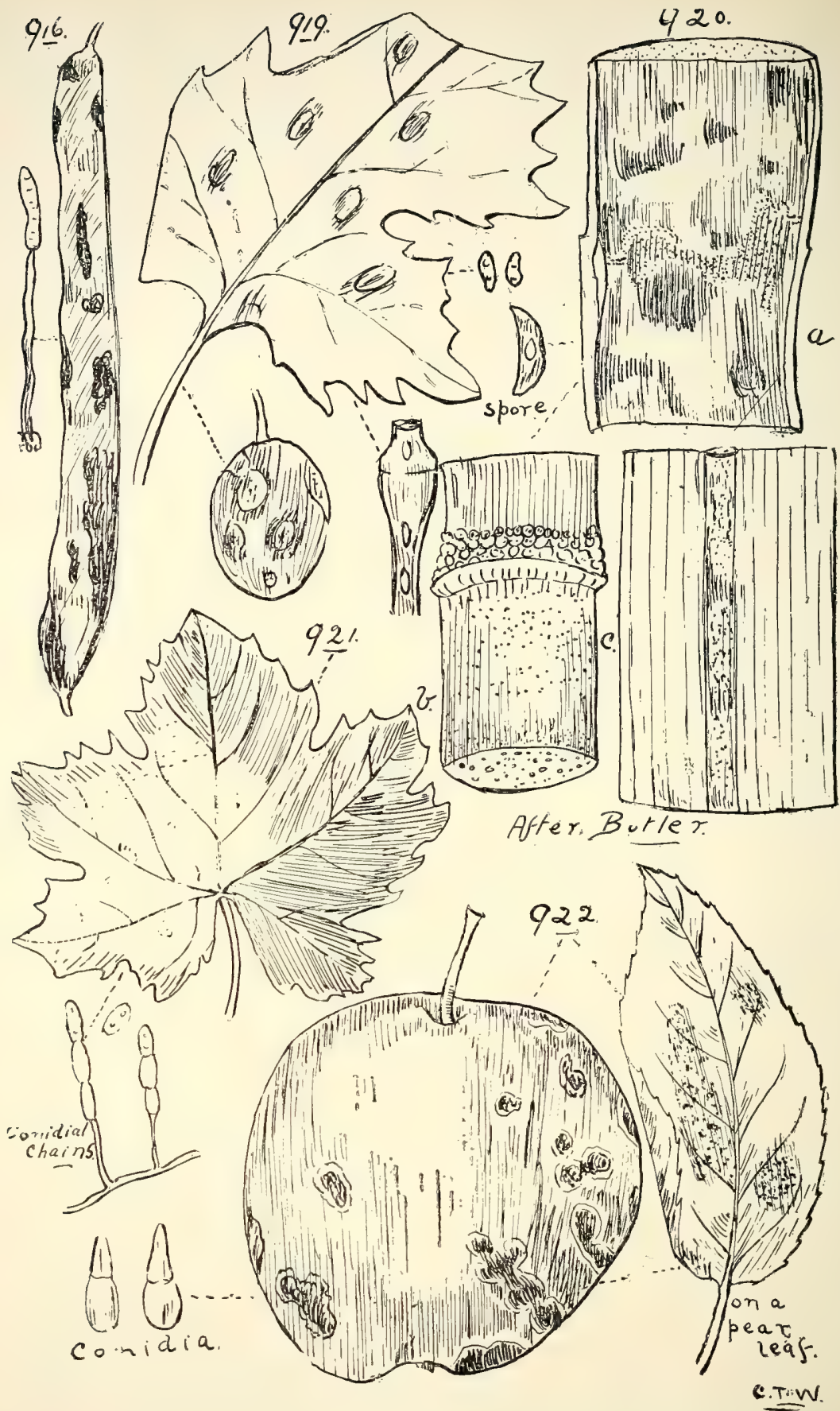

916. Gleosforium Lindemuthiandm, Sacc. and Magn.

919. G. A MPELOPHAGUM, Sacc.

920. Colletotrichum falcatum, Went.

(a) Section of cane showing discolouration due to "Red Rot," (b) a node of same showing the stromata at the eyes above the node and the waxy layer below, (c) midrib of sugar-cane leaf attacked by Colletotrichum.

921. Oidium Tuckeri, Berk.

922. Fusicladium dentriticum, Wallr. 
Fusarium-contd.

(Fusisporium) solani-contd.

tuber was easily separated with a fork from the floury portions of the potato.

(Selenospora) sarcochroum, Sacc.-On the bark of Orangetrees.

(Selenospora) hypocreoideum, Cke. and Mass.-On leaves of Ficus aspera.

Microcera, Desm.

coccophila, Desm.-On the cocci of Orange and Lemon trees (Citrus).

rectispora, Cke. and Mass.-On the cocci of Orange-trees.

Series Tuberculariea Dematiea.

Epicoccum, Link.

micropus, Corda.-On the foliage of young Quercus.

scabrum, Corda.-On Potato plants (Solanum).

Strumella, Sacc.

hysterioides, Cke. and Mass.-On branchlets.

sacchari, Cke.-On Sugar-cane.

vitis, McAlpine.-On Grape-fruit, causing Fruit Rot (Tryon).

Actinomma, Sacc.

gastonis, Sacc.-On phyllodia of Acacia.

Myrothecium, Tode.

roridum, Tode.-On old twine used in tying up plants.

inundatum, Tode.-On putrid Agarics.

Schizomycetes.

Bacillus, Cohn.

solanacearum, E. F. Smith-Bacteriosis of Potato (Solanum tuberosum). Also found on Tomato and other Solanaceous plants (H. Tryon).

Pseudomonas, E. F. Smith.

campestris, E. F. Smith.-Lately found attacking Cabbage plants at Redbank Plains (H. Tryon).

Micrococcus (Hall.), Cohn.

crepusculum (Ehrenb.), Cohn.-Found in putrescent liquids.

\section{MYXOMYCETEE.}

Section Peritrichez.

Tubulina, Pers.

Subsection Tubulinece.

cylindrica, Bull.-On rotten wood.

var. nitidissima, Berk. $=$ T. nitidissima, Berk.-On Eucalyptus microtheca.

Clathroptychium, Rost.

Subsection Cribraria.

rugulosum, Wall.-On dead twigs. 


\section{Section Columellifer $x$.}

Stemonitis, Gled.

\section{Subsection Stemonitea.}

fusca, Roth.-On rotten wood. (Fig. 928.)

Friesiana, $D e$ Bary $=$ Comatricha Friesiana, Rostfi. - On rotten wood.

ferruginea, Ehr.-On rotten wood.

Perichæna, Fries.

$$
\text { Subsection Arcyrio. }
$$

applanata, Cke. and Mass. $=$ Hemiarcyria applanata, C. and Mass.-On rotten Cycas, with Lachnea erinacea.

Lycogala, Mich.

epidendrum, Bux.-On a rotten pine-log.

Arcyria, Hill.

\section{Family Trichiacea.}

punicea, Pers.-On dead Passiflora edulis.

ferruginea, Sauter.-On dead Eucalyptus.

incarnata, Pers. $=$ A. adnata, Sacc.-On rotten wood.

nutans, Bull.-On rotten wood.

cinerea, Bull. = A. pomiformis, Rost.-On rotten wood.

Trichia, Haller.

Subsection Trichice.

varia, Pers.-On rotten wood.

contorta, Ditm.-On rotten wood.

affinis, De Bary.-On rotten wood.

\section{Section Lithodermex.}

\section{Subsection Didymex.}

Chondrioderma, Rostft.

difforme, Pers. = Physarum album, Fries.-On bark, leaves, and grass.

Didymium, Schrad.

farinaceum, Schrad-On dead leaves, pineapple skins, \&c.

spumarioides, Fries = Chondrioderma spumarioides, Fries.On moss and grass.

pezizoideum, Mont. = Chondrioderma pezizoideum, Jungh.On wood of Erythrina.

australis, Grev.--On old Auricularia.

Spumaria, Pers.

alba, Bull.-On grass. (Fig. 929.)

Diachæa, Fries.

leticopoda, Bull. = D. elegans, Fries.-On an Ophiopogon in bush-house at Indooroopilly. 


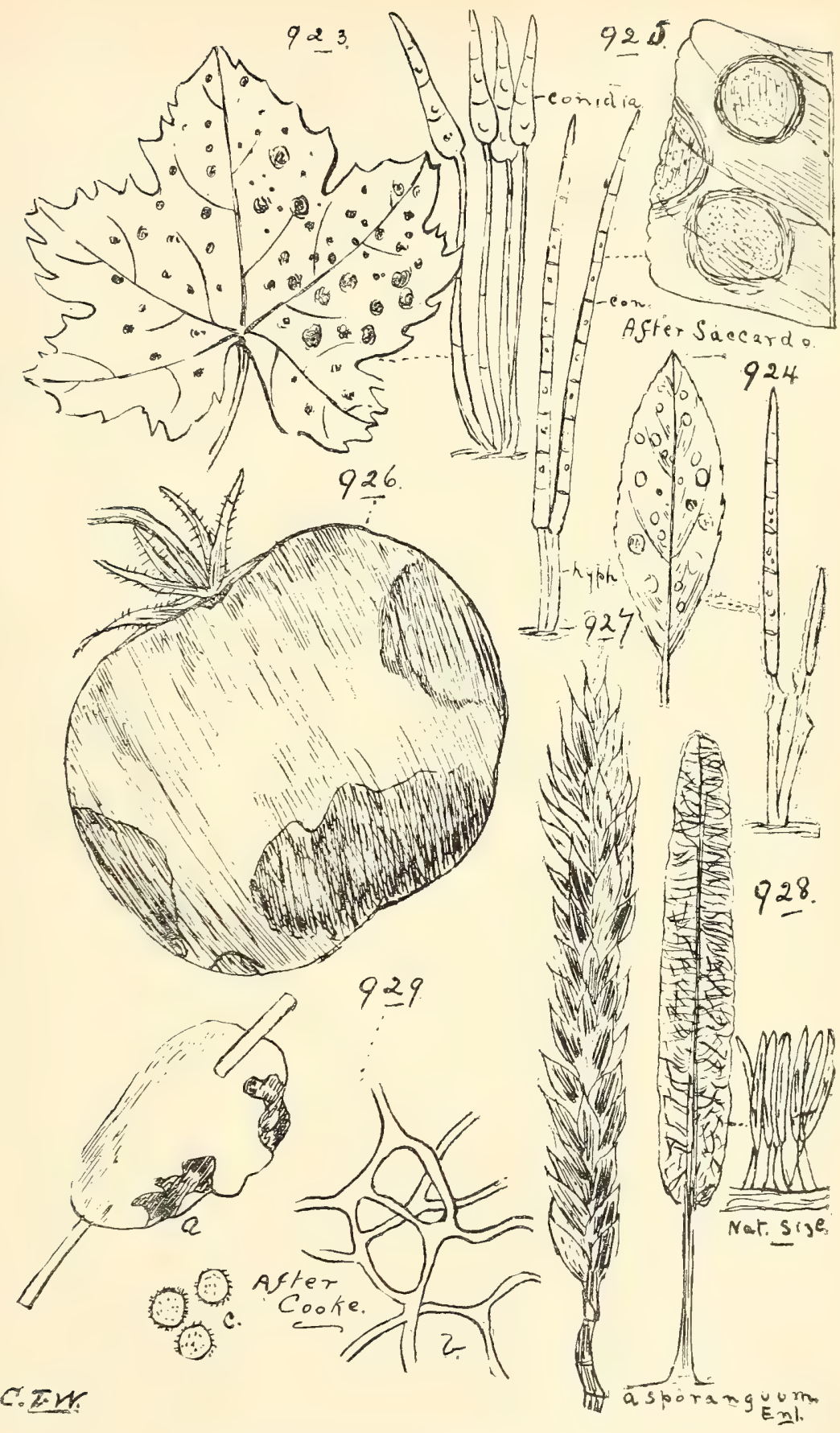

923. Cercospora viticola, Ces.

924. C. CirCumscissa, Sacc.

925. C. ARMORACIE, Sacc.

926. Macrosporium tomato, Cke. et Rar:

927. Fusarium heterosporum, Limk.

928. Stemonitis Fusca, Roth.

929. Spumaria alea, Bull.

(a) Ethalium, nat. size, (b) portion of capillitium, enI., (c) spores, enI. 
Physarum, Pers.

\section{Subsection Physaria.}

rufibasis, Berk. and $\mathrm{Br}$. = Tilmadoche rufipes, Berk. - On bark and moss.

didermoides, Rostft.-On scales of onions and maize or corn bracts (Zea).

Tilmadoche, Fries.

nutans, Pers.-On rotten wood.

mutabilis, Rostft. $=T$. viridis, Sacc.-On rotten wood. Fuligo, Hall.

varians, Sommf. $=$ F. septica, Sacc., and Ethalium septicum, Fries.-Soot Fungus.

Xylostroma, Tode.

\section{Mycelia.}

giganteum, Tode.-Found often in the layers of Bloodwood timber like white chamois leather.

Rhizomorpha, Roth.

Harrimannii, Kunze.-Resembling the coarse, hairy skin of some animal; often seen on logs in damp scrubs.

corynephora, Kunze.-Silver-cord Fungus. In damp scrubs, clothing thin exposed roots and twigs, hence called Silver-cord.

There are also several kinds of Sclerotia met with which have at times caused considerable damage to both ornamental and economic plants.

\section{Order CLXIII.-ALGÆ.}

[K. placed after a species signifies that it has been deternined for me by the Kew (England) authorities.

\section{CHLOROPHYCE正。}

ORDER CONFERVOIDEA.

Family Coleochetaceж.

Coleochæte, Bréb., De-Toni, Syll. Alg. i. 7.

Baileyi, Mabius, Q1. Bot. Bull. vi. 6.

conchata, Mobius, Q1. Bot. Bull. vi. 8 .

divergens, Pringsh., var. minor, Hansg., De-Toni, Syll. Alg. i. 8 .

orbicularis, Pringsh., De-Toni, Syll. Alg. i. 8.

scutata, De-Toni, Syll. Alg. i. 9.

var. minor (Hansg.), Moebius, Q1. Bot. Bull. xi.

irregularis, Pringsh., De-Toni, Syll. Alg. i. 9.

nitellarum, Jost.-Stannary Hills (Dr. T. L. Bancroft).

Borge.)

Chætopeltis, Berth., De-Toni, Syll. Alg. i. I I.

minor, Mob., Q1. Bot. Bull. vi. Io. 
Family Mrcoideaced.

Mycoidea, Cunningh., De-Toni, Syll. Alg. i. I3.

parasitica, Cunningh., De-Toni, Syll. Alg. i. I3.-On Citrus leaves, Gympie (H. Tryon). Not uncommon on various plants in warm countries.

\section{Family Edogoniaced.}

Bulbochæete, $A g$., De-Toni, Syll. Alg. i. I6.

setigera (Roth. ), Ag., De-Toni, Syll. Alg. i. 20.

gigantea, Pringsh., De-Toni, Syll. Alg. i. 22.

elatior, Pringsh., De-Toni, Syll. Alg. i. 23.

pygmæa (Pringsh.), Wittr., De-Toni, Syll. Alg. i. 25.

Edogonium, Link, De-Toni, Syll. Alg. i. 3I. fragile, Wittr., De-Toni, Syll. Alg. i. 36.

crispum (Hass.) Wittr., De-Toni, Syll. Alg. i. 37--Barron

River (Dr. T. L. Bancroft). (O. Borge.) (Fig. 930.)

nodulosum, Wittr., De-Toni, Syll. Alg. i. 40.

excisum, Wittr. and Lund., De-Toni, Syll. Alg. i. 45.

œlandicum, Wittr., De-Toni, Syll. Alg. i. 46.-Barron River

(Dr. T. L. Bancroft). (O. Borge.). (Fig. 93I.)

longicolle, Nordst., var. senegalense, Nordst., De-Toni, Syll.

Alg. i. 48.

undulatum (Bréb.), A. Br., De-Toni, Syll. Alg. i. 49.

var. Mœbiusii, W. Schmidle, Q1. Bot. Bull. xv. 5.

forma a, Hirn.-Barron River (Dr. T. L. Bancroft).

Borge.)

cyathigerum, Wittr., De-Toni, Syll. Alg. i. 5o.

flavescens (Hass.), Wittr., De-Toni, Syll. Alg. i. 52.

crassiusculum, Wittr., De-Toni, Syll. Alg. i. 54.

? birmanicum, Wittr., De-Toni, Syll. Alg. i. 55 .

echinospermum, A. Br., De-Toni, Syll. Alg. i. 63 .

Franklinianum, Wittr., De-Toni, Syll, Alg. i. 69.

cardiacum (Hass.), Kuetz., De-Toni, Syll. Alg. i. 70.

Pringsheimii, Cram., De-Toni, Syll. Alg. i. 7I.

punctato-striatum, De Bary', De-Toni, Syll. Alg. i. 72.

Boscii (Le Clere), Bréb., De-Toni, Syll. Alg. i. 72.

grande, Kuetz., De-Toni, Syll. Alg. i. 74 .

tapeinosporum, Wittr., De-Toni, Syll. Alg. i. 76.-Barron

River (Dr. T. L. Bancroft). (O. Borge.)

pachydermatosporum, Nordst., De-Toni, Syll. Alg. i. 82.

hians, Nordst. et Hirn.-Stannary Hills (Dr. T. L. Bancroft). (O. Borge.)

sp., Borge. (Description, Q1. Bot. Bu11. xv. p1. I3, fig. Ir.)

\section{Family Cylindrocapsacex.}

Cylindrocapsa, Reinsch., De-Toni, Syll. Alg. i. 92. geminella, Wolle., De-Toni, Syll. Alg. i. $93=$ Glootceinm Loitlesbergereanum, Hansg., Q1. Bot. Bull. xv. Io. 
Family Ulvaceæ.

Monostroma, Thur., De-Toni, Syll. Alg. i. 98.

sp., A. D. Cotton. K. - On rocks, Brisbane River (C. T. White).

Ulva, Linn., De-Toni, Syll. Alg. i. I Io.

Lactuca (Linn.), De-Toni, Syll. Alg. i. III.-Oyster Green or Sea Lettuce.

reticulata, Forsk., De-Toni. Syll. Alg. i. I I3.

Enteromorpha, Link, De-Toni, Syll. Alg. i. I 8 .

Gunniana, J. Ag., De-Toni, Syll. Alg. i. I I9. K.

intestinalis (Linn.), Link, De-Toni, Syll. Alg. i. I23.

compressa (Linn.), Grev., De-Toni, Syll. Alg. i. I26.

clathrata (Roth.), J. Ag., De-Toni, Syll. Alg. i. I33.

Schizomeris, Kuetz., De-Toni, Syll. Alg. i. I39.

Leibleinii, Kuetz., De-Toni, Syll. Alg. i. I40.-Mosman River (F. Muir). (West.)

FAmily Ulotrichiace e.

Schizogonium, Kuetz., De-Toni, Syll. Alg. i. I53.

thermale (Menegh.), Kuetz., De-Toni, Syll. Alg. i. I54 = Hormospora transversalis, Bréb., Q1. Bot. Bull. xi. I7.

Hormiscia, Fries, De-Toni, Syll. Alg. i. I 59.

subtilis (Kuetz.), De-Toni, Syll. Alg. i. ${ }_{59}=$ Ulothrix subtilis, Kuetz.

zonata (Web. and Mohr.), Aresch., De-Toni, Syll. Alg. i. I63 $=U$. zonata, Kuetz.

Uronema, Lagerh., De-Toni, Syll. Alg. i. г 76.

confervicolum, Lagerh., De-Toni, Syll. Alg. i. I76.

Aphanochæte, Berth., De-Toni, Syll. Alg. i. I79.

repens, Berth., De-Toni, Syll. Alg. i. I79.

Chætosphæridium (Klebahn), Mobius. (See Q1. Bot. Bull. vi. I8.)

Pringsheimii, Klebahn.

? Huberi, Mobius, Q1. Bot. Bull. xi. 22.

globosum (Nordst.), Kleb.-Barron River (Dr. T. L. Bancroft). (O. Borge.)

Herposteiron, Nag., De-Toni, Syll. Alg. i. I8o.

confervicolum, Naeg., De-Toni, Syll. Alg. i. I8I.

forma typica, Mabius, Q1. Bot. Bull. vi. I9.

forma bicellularis, Macbius, Q1. Bot. Bull. vi. 20.

Chætophora, Schrank., De-Toni, Syll. Alg. i. I82.

tuberculosa (Roth.), Hook., De-Toni, Syll. Alg. i. I84.

Stigeoclonium, Kuetz., De-Toni, Syll. Alg. i. 195.

tenue (Ag.), Rabenh., De-Toni, Syll. Alg. i. 197.

protensum (Dillw.), Kuetz., De-Toni, Syll. Alg. i. I99.

amœnum, Kuctz., De-Toni, Syll. Alg. i. 202.

var. novizelandicum, Nordst., De-Toni, Syll. Alg. i. 202. 
Stigeoclonium-contd.

australense, Mobius, Q1. Bot. Bull. vi. 2 I.

Askenasyi, $W$. Schmidle, Q1. Bot. Bull. xv. 6.

Conferva, Limn., De-Toni, Syll. Alg. i. 2 I5.

bombycina (Ag.), Lagerh., De-Toni, Syll. Alg. i. 216.

var. pallida, Kuetz., De-Toni, Syll. Alg. i. 2 I6.

Microspora, Thur., De-Toni, Syll. Alg. i. 225.

pachyderma (Wille.), Lagerh., De-Toni, Syll. Alg. i. 227.

abbreviata (Rabenh.), Lagerh., De-Toni, Syll. Alg. i. 228.

\section{Family Chroolepidacez.}

Trichophilus, Weber van-Bosse., De-Toni, Syll. Alg. i. 249.

sp., Moebius, Q1. Bot. Bull. xi. 24.

Family Cladophorace飞.

Chætomorpha, Kuetz., De-Toni, Syll. Alg. i. 265. tortuosa (Dillw.), Kuetz., De-Toni, Syll. Alg i. 266.

Rhizoclonium, Kuetz., De-Toni, Syll. Alg. i. 278.

hieroglyphicum (Ag.), Kuetz., De-Toni, Syll. Alg. i. 28I.

riparium (Roth.), Harv., De-Toni, Syll. Alg. i. 278. K.-

And a form slightly different from the European plant.

Brisbane River (C.T. White). (A. D. Cotton.)

Cladophora, Kuetz., De-Toni, Syll. Alg. i. 287.

Kilneri (Sond.), F.v. M., Fragm. xi. Io6.

parvula, Mobius, Q1. Bot. Bull. xi. 25.

fracta (Dillw.), De-Toni, Syll. Alg. i. 288.

subsimplex, Kuetz., De-Toni, Syll. Alg. i. 28 .

glomerata (L.), Kuetz., De-Toni, Syll. Alg. i. 295. K. (Fig. 932.)

crinalis, Harv., De-Toni, Syll. Alg. i. 309. K.

fusca, Martens, De-Toni, Syll. Alg. i. 3i I. K.

albida (Huds.), Kuets, De-Toni, Syll. Alg. i. 321. K.

fascicularis (Mert.), Kuetz., De-Toni, Syll. Alg. i. 316=

C. cristata (Zan.), F. v. M., Fragm. xi. Suppl. 40.

fertilis, E. Aske., Q1. Bot. Bu1l. xi. 5I.

Blodgettia, Harv., De-Toni, Syll. Alg. i. 357.

australis (J. Ag.), Cotton. K.

Spongocladia, Aresch., De-Toni, Syll. Alg. i. 360.

vaucheriæformis, Aresch., De-Toni, Syll. Alg. i. 360. K.

Boodlea, Murray et De-Toni. De-Toni, Syll. Alg. i. 363.

sp., A. D. Cotton. K.

Struvea, Sond., De-Toni, Syl1. Alg. i. 364.

delicatula, Kuetz., De-Toni, Syll. Alg. i. $366=$ Cladophora anastomosans, Hass., Syn. Q1. F1. 802.

Anadyomene, Lamour., De-Toni, Syll. Alg. i. 367.

Brownii (Gray), J. Ag., De-Toni, Syll. Alg. i. $370=A$. Muelleri, Sond, in Syn. Q1. F1. 802. 
Dictyosphæria, Decaisne, De-Toni, Syll. Alg. i. 37 I. favulosa (Mert. ?), Decaisne, De-Toni, Syll. Alg. i. 37 I.

Valonia, Gin., De-Toni, Syll. Alg. i. 373.

Egagropila, var. australis, J. Ag., De-Toni, Syll. Alg. i. 377. $\mathrm{K}$.

confervoides, Harv., De-Toni, Syll. Alg. i. 378.

fastigiata, Harv., De-Toni, Syll. Alg. i. 379.

Order SIPHONE王.

Family Vaucheriaceæ.

Vaucheria, DC., De-Toni, Syll. Alg. i. 393.

sessilis (Vauch.), DC., De-Toni, Syll. Alg. i. 398.

geminata (Vauch.), DC., De-Toni, Syll. Alg. i. 399.

sp., Schmidle, Q1. Bot. Bull. xv. 8.

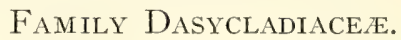

Dasycladus, $A g$., De-Toni, Syll. Alg. i. 4ro.

australasicus (Sond.), Cram., De-Toni, Syll. Alg. i. 4I I =

Chlorocladus australasicus, Sond. Bail Syn. Q1. F1. 8or.

Bornetella, Mun. Chalm., De-Toni, Syll. Alg. i. 4 I4. nitida, Harv., De-Toni, Syll. Alg. i. 4I 5.

Acetabularia (Tourn.), Lamour., De-Toni, Syll. Alg. i. 4I7.

calyculus, Quay and Gaimard., De-Toni, Syll. Alg. i. 4I8.

Kilneri, J. Ag., De-Toni, Syll. Alg. i. 4I8.

crenulata, Lamour., De-Toni, Syll. Alg. i. 419.

Polyphysa (Lamarck), Lamour., De-Toni, Syll. Alg. i. 42 I.

$s p$.

? Pleiophysa, Sond., De-Toni, Syll. Alg. i. 423.

spicata (Kuetz.), Sond., De-Toni, Syll. Alg. i. 423.

Halicoryne, Harv., De-Toni, Syll. Alg. i. 422.

Wrightii, Harv., De-Toni, Syll. Alg. i. 423. K. (Fig. 933.)

Family Bryopsidaceæ.

Bryopsis, Lamour., De-Toni, Syll. Alg. i. 427.

myura, J. Ag., De-Toni, Syll. Alg. i. 434.

muscosa, Lamour., De-Toni, Syll. Alg. i. 435.

Family Caulerpace.e.

Caulerpa, Lamour., De-Toni, Syll. Alg. i. 44I.

Webbiana, Mont., De-Toni, Syll. Alg. i. 444. parvifolia, Harv., De-Toni, Syll. Alg. i. 448. biserrulata, Sond., De-Toni, Syll. Alg. i. 448. taxifolia (Vah.), De-Toni, Syll. Alg. i. 452. plumaris, Forsk., De-Toni, Syll. Alg. i. 453. falcifolia, Harv. and Bail., De-Toni, Syll. Alg. i. 454.

Freycinetii, Ag., De-Toni, Syll. Alg. i. 458.

thujoides, J. Ag., De-Toni, Syll. Alg. i. 462. 


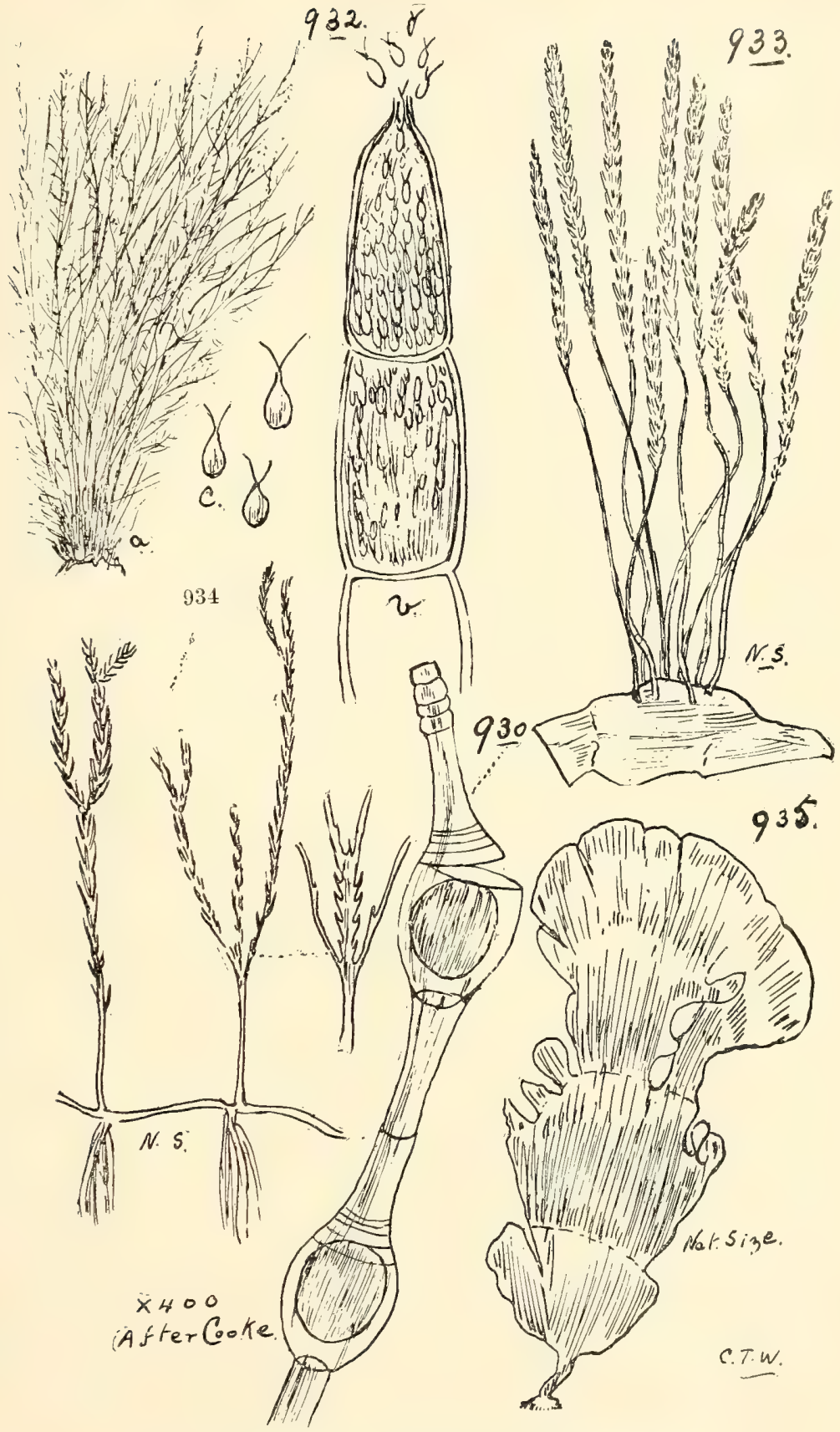

930. EDOGONIUM CRISPUM, Wittr. (With oogonia.)

932. Cladophora glomerata, Kuetz.

(a) Plant nat. size, (b) tip of a branch with zocgonidia $x 200$, (c) zoogonidia $\times 320$.

933. Halicoryne Wrightil, Harv.

934. Caulerpa cupressotnes, $A g$.

935. Udotea argentea, Zanard. 
Caulerpa-contd.

cupressoides (Vahl.), Ag., De-Toni, Syll. Alg. i. 46r ; var. lycopodium (Weber). K. (Fig. 934.)

tristicha, J. Ag., De-Toni, Syll. Alg. i. $463=$ C. serrata, Kuetz. in Syn. Q1. F1. 8oo.

fontinaloides, J. Ag., De-Toni, Syll. Alg. i. 465.

hypnoides (R. Br.), Ag., De-Toni, Syll. Alg. i. 470.

complanata, J. Ag., var. oligophylla, J. Ag., De-Toni, Syll.

Alg. i. 473 .

læte-virens, Mont., De-Toni, Syll. Alg. i. 474.

racemosa (Forsk.), J. Ag., De-Toni, Syll. Alg. i. 475.

var. laxa, J. Ag., De-Toni, Syll. Alg. i. 475.

Chemnitzia (Esp.), Lamour., De-Toni, Syll. Alg. i. 477.

peltata, Lamour., De-Toni, Syll. Alg. i. 478.

Kilneri, J. Ag., De-Toni, Syll. Alg. i. 484.

cactoides (Turn.), Ag., De-Toni, Syll. Alg. i. 485.

Family Spongodiacem.

Codium, Stackh., De-Toni, Syll. Alg. i. 488.

spongiosum, Harv., De-Toni, Syll. Alg. i. 489.

lineare, Ag., species, De-Toni, Syll. Alg. i. 495.

elongatum, $A g$., De-Toni, Syll. Alg. i. 496.

(And a thin form from Dunk Island. K.)

var. multiramea, F. v. M., Fragm. xi. Suppl. 38.

\section{Family Udoteace.}

Penicillus, Lamarck., De-Toni, Syll. Alg. i. 500.

Arbuscula, Mont., De-Toni, Syll. Alg. i. 502.

Udotea, Lamour., De-Toni, Syll. Alg. i. 505.

Palmetta, Decaisne, De-Toni, Syll. Alg. i. 506.

argentea, Zanard, De-Toni, Syll. Alg. i. 5 Io. K. (Fig. 935.)

flabellata, Lamour., De-Toni, Syll. Alg. i. 5 Io.

orientalis, Gepp. K.

Halimeda (Kuetz.), E. M. Holmes.

Avrainvillea, Decaisne, De-Toni, Syll. Alg. i. 5I3.

comosa, Bail. and Harv., De-Toni, Syll. Alg. i. 5I5 = Chlorodesmis comosa, B. and H., Bail. Syn. Q1. F1. 8oI.

Halimeda, Lamour., De-Toni, Syll. Alg. i. 5 I8.

Tuna (Ell. and Soland.), Lamour., De-Toni, Syll. Alg. i. 5 I8.

papyracea, Zanard, De-Toni, Syll. Alg. i. 5I9.

macroloba, Decaisne, De-Toni, Syll. Alg. i. 520.

Opuntia (Linn.), Lamour., De-Toni, Syll. Alg. i. 522.

forma triloba, Decaisne, De-Toni, Syll. Alg. i. 522.

incrassata (E11.), Lamour., De-Toni, Syll. Alg. i. 522.

tridens (Soland.), Lamour., De-Toni, Syll. Alg. i. 524.

cuneata, Hering, De-Toni, Syll. Alg. i. 526. K. (Fig. 936.)

discoidea (Esper. ?), Decaisne, De-Toni, Syll. Alg. i. 527. 


\section{Order PROTOCOCCOIDE死.}

\section{Family Volvocace.e.}

Volvox (Linn.), Ehrenb., De-Toni, Syll. Alg. i. 535.

globator (Linn.), Ehrenb., De-Toni, Syll. Alg. i. 535.

aurens, Ehrenb., De-Toni, Syl1. Alg. i. 536.

Pandorina, Bory., De-Toni, Syll. Alg. i. 538.

Morum (Muell. ?), Bory., De-Toni, Syll. Alg. i. 539.

\section{Family Palmellace}

Scenedesmus, Meyen., De-Toni, Syll. Alg. i. 563.

bijugatus (Turp.), Kucti., De-Toni, Syll. Alg. i. $563=S$. obtusus, Meyen.

quadricauda (Turp.), Bréb., De-Toni, Syll. Alg. i. 565.

denticulata, Lagerh., var. linearis, Hansg., De-Toni, Syll. Alg.

i. 565.-Nundah (C. T. White). Det. by G. S. West.

obliquus (Turp.), Kuetz. $=S$. acutus, Meyen., De-Toni,

Syll. Alg. i. 566.-Sandgate Lagoon (C. T. White).

Det. by G. S. West.

brasiliensis, Bohlin.-Barron River (Dr. T. L. Bancroft).

(O. Borge.)

var. horridus, Kirchn., De-Toni. Syll. Alg. i. 566.-Enoggera Reservoir (Dr. T. L. Bancroft). (O. Borge.)

(Fig. 937.)

Sorastrum, Kuetz., De-Toni, Syll. Alg. i. 568.

crassispinosum, (Hansg.), Bohlin-Barron River (Dr. T. L.

Bancroft). (O. Borge.)

Colastrum, Noeg., De-Toni, Syll. Alg. i. 570.

sphæricum, Nreg., De-Toni, Syll. Alg. i. 570.

var. compacta, Mobius, Q1. Bot. Bull. vi. 27.

var. subpulchrum (Lag.), Schmidle, Q1. Bot. Bu1l. xv. 9.

pulchrum, Schmidle, Beitr. Alg. Schwarzwald, Q1. Bot. Bull.

xv. 9. (By Dr. G. S. West considered only to be

C. cambricum, Archer.)

var. intermedium, Bohlin,-Barron River (Dr. T. L. Bancroft). (O. Borge.)

Pediastrum, Meyen., De-Toni, Syll. Alg. i. 572.

Boryanum (Turp.), Menegh, var rugulosum, G. S. West.Sandgate Lagoon (C. T. Whitc). Det. by G. S. West, who remarks (in litt.) "one very large cœnobium was noted with a diameter of $240 . "$

duplex, Meyen., De-Toni, Syll. Alg. i. 578 .

Tetras (Ehrenb.), Relfs., De-Toni, Syll. Alg. i. 58 I. 
Sciadium, $A$. Br., De-Toni, Syll. Alg. i. 585 .

Arbuscula, A. Br., De-Toni, Syll. Alg. i. 585 .

Ophiocytium, Nagg., De-Toni, Syll. Alg. i. 590.

majus, Nacg., De-Toni, Syll. Alg. i. 590.

parvulum (Perty.), A.Br., De-Toni, Syll. Alg. i. 591.-Brisbane Botanic Gardens (C. T. White). Det. by G. S. West.

capitatum, Wolle., De-Toni, Syll. Alg. i. 592.

var. longispinum (Mob.), Lemmerm.-Enoggera Reservoir

(Dr. T. L. Bancroft). (O. Borge.)

Rhaphidium, Kuetz., De-Toni, Syll. Alg. i. 592.

polymorphum, Fresen., De-Toni, Syll. Alg. i. 592.

var. aciculare (A. Br.), Rabenh. $=R$. aciculare, A. Br.;

Ancistrodesmus falcatus, Corda, var. aciculum, De-Toni, Syll. Alg. i. 593.-Brisbane Botanic Gardens (C. T.

White). Det. by G. S. West.

var. falcatum, Corda, De-Toni, Syll. Alg. i. 593.

convolutum (Corda), Rabenh., De-Toni; var. lunare, De-

Toni, Syll. Alg. i. 594 (Kirchneriella lunaris, Schmidle in Q1. Bot. Bull. vi. 29).

Tetræedron, Kuetz., De-Toni, Syll. Alg. i. 597.

minimum (A. Br.), Hansg. = Polyedrium minimum, A. Br., De-Toni, Syll. Alg. i. 6or.-Brisbane Botanic Gardens (C. T. White). Det. by G. S. West.

regulare, Kuetz., De-Toni, Syll. Alg. i. 6o4; forma majus, Reinsch. $=$ T. vulgare, Ktzg., Q1. Bot. Bu1l. xv. 9.

gigas (Wittr.), Hansg., forma tetrædricum, Nordst., De-Toni, Syll. Alg. i. 606.

enorme (Ralfs.), Hansg., De-Toni, Syll. Alg. i. 6o8.-Nundah (C. T. White). Det. by G. S. West.

Reinschiella, De-Toni, Syll. Alg. i. 6ı 2.

longispina, Moebius, Q1. Bot. Bull. xi. 32.

Schizochlamys, A. Br., De-Toni, Syll. Alg. i. 643. gelatinosa, A. Br., De-Toni, Syll. Alg. i. 644.

Palmodactylon, Nog., De-Toni, Syll. Alg. i. 644. subramosum, Noeg., De-Toni, Syll. Alg. i. 644.

Tetraspora, Link, De-Toni, Syll. Alg. i. 646.

explanata, $A g$., De-Toni, Syll. Alg. i. 647.

Dictyosphærium, Naeg., De-Toni, Syll. Alg. i. 659. pulchellum, Wood, De-Toni, Syll. Alg. i. 660.

Nephrocytium, Nag., De-Toni, Syll. Alg. i. 662.

Agardhianum, Nog., De-Toni, Syll. Alg. i. 663. 
Glœocystis, Norg., De-Toni, Syll. Alg. i. 668.

vesiculosa, Noeg., De-Toni, Syll. Alg. i. 668.

gigas (Kuetz.), Lagerh., De-Toni, Syll. Alg. i. 670.-Beaudesert (Dr. T.L. Bancroft). (G. S. West.) (Fig. 938.)

Botryococcus, Kuetz., De-Toni, Syll. Alg. i. 674.

Braunii, Kuetz., De-Toni, Syll. Alg. i. 674.

Protococcus, Ag., De-Toni, Syll. Alg. i. 699.

infusionum (Schrank.), Kirchn., De-Toni, Syll. Alg. i. 702.

\section{Order CONJUGAT E.}

\section{Family Zygnemace.}

Mougeotia, Ag., De-Toni, Syll. Alg. i. 7 I I.

lætevirens (A. Br.), Wittr., De-Toni, Syll. Alg. i. 718.

Zygnema, Ag., De-Toni, Syll. Alg. i. 727.

Rhynchonema, Hansg., De-Toni, Syll. Alg. i. 729.

leiospermum, De Bary, De-Toni, Syll. Alg. i. 730.

insigne (Hassall), Kuetz., De-Toni, Syll. Alg, i. 730.

cruciatum (V auch.), Ag., De-Toni, Syll. Alg. i. 732.

tenuissimum, Grun., De-Toni, Syll. Alg. i. 735.

pectinatum (Vauch.), Ag., De-Toni, Syll. Alg. i. 736.

ericetorum (Kuetz.), Hansg., De-Toni, Syll. Alg. i. 737.

Spirogyra, Link, De-Toni, Syll. Alg. i. 74I.

longata (Vauch.), Kuetz., De-Toni, Syll. Alg. i. 743.

decimina (Muell.), Kuetz., De-Toni, Syll. Alg. i. 749.-

Corinda (C.T. White). Det. by G. S. West.

nitida (Dillw.), Link, De-Toni, Syll. Alg. i. $75^{\circ}$.

australiensis, Moebius, Q1. Bot. Bull. xi. 34 .

rivularis, Rabenh., De-Toni, Syll. Alg. i. $75^{2}$.

inflata (Vauch.), Rabenh., De-Toni, Syll. Alg. i. 766.-Upper

Walsh River (Dr. T. L. Bancroft). (O. Borge.) (Fig.

939.)

maxima, (Hassall), Wittr., De-Toni, Syll. Alg. i. 756.

var. minor, Mabius, Q1. Bot. Bull. xi. 35 .

crassa, Kuetz., De-Toni, Syll. Alg. i. 757.

bellis (Hassall), Crouan, De-Toni, Syll. Alg. i. 762.

punctata, Cleve, De-Toni, Syll. Alg. i. 765 ; var. tenuior,

Moebius, Q1. Bot. Bull. vi. 36 .

calospora, Clev. Srensk., De-Toni, Syll. Alg. i. 773.

stictica (Engl. Bot.), De-Toni, Syll. Alg. i. $774=$ Sirogo-

nium strictum, Kizy., Q1. Bot. Bull. xv. Io.

Baileyi, W. Schmidle, Q1. Bot. Bull. xv. Io. 


\section{Family Desmidiacere.}

Desmidium, Ag., De-Toni, Syll. Alg. i. 779.

Swartzii, Ag., De-Toni, Syll. Alg. i. 780.

quadrangulare, Kuetz., De-Toni, Syll. Alg. i. 780.

aptogonium, Bréb., De-Toni, Syll. Alg. i. 78I.

var acutius, Nordst., De-Toni, Syll. Alg. i. 782.

Baileyi (Ralfs.), De Bary, De-Toni, Syll. Alg. i. 782.

quadratum, Nordst., De-Toni, Syll: Alg. i. 784 .

coarctatum, Nordst., De-Toni, Syll. Alg. i. 785 .

bengalicum, Turn., Algæ of East India, p. I47, tab. xix., fig. I-3.

Hyalotheca, Ehrenb., De-Toni, Syll. Alg. i. 785.

dissiliens (Smith), Bréb., De-Toni, Syll. Alg. i. 785.

dubia, Kuetz., var. subconstricta, Hansg., De-Toni, Syll. Alg.

i. 787 .

mucosa (Mert.), De-Toni, Syll. Alg. i. 787.

hians, Nordst., De-Toni, Syll. Alg. i. 788.

Sphærozosma, Corda, De-Toni, Syll. Alg. i. 788. excavatum, Ralfs., De-Toni, Syll. Alg. i. 790.

Onychonema, Wallich., De-Toni, Syll. Alg. i. 795. leve, Nordst., De-Toni, Syll. Alg. i. 796.

filiforme, Ehrenb., De-Toni, Syll. Alg. i. $796=$ Sphorozosma filiforme, Ehrenb., Q1. Bot. Bull. vi. 38 .

Gymnozyga, Ehrenb., De-Toni, Syll. Alg. i. 797.

moniliformis, Ehrenb., De-Toni, Syll. Alg. i. 797.

var. gracilescens, Nordst., De-Toni, Syll. Alg. i. 798.

var. minima, Borge.-Upper Walsh River (Dr. T. L. Bancroft). Det. by O. Borge. (Fig. 940.)

Gonatozygon, De Bary, De-Toni, Syll. Alg. i. 8or.

Ralfsii, De Bary, De-Toni, Syll. Alg. i. 8or.

Kinahani (Archer), Rabenh., De-Toni, Syll. Alg. i. 802.-Mosman River (F. Muir). (West.)

aculeatum, Hast.-Barron River (Dr. T. L. Bancroft). (O.

Borge.)

Cylindrocystis, Menegh., De-Toni, Syll. Alg. i. 8I5.

Brebissonii, Mencgh., De-Toni, Syll. Alg. i. 8I5; var. turgida,

Schmidle, Q1. Bot. Bull. xv. 34.

pyramidata, W. and G. S. West.-Mosman River (F. Muir). (West.)

Closterium, Nitzsch., De-Toni, Syll. Alg. i. 8I7. gracile, Bréb., De-Toni, Syll. Alg. i. 818. juncidum, Ralfs., De-Toni, Syll. Alg. i. 820 . acerosum (Schrank.), Ehrenb., De-Toni, Syll. Alg. i. 824. striolatum, Ehrenb., De-Toni, Syll. Alg. i. 826. 


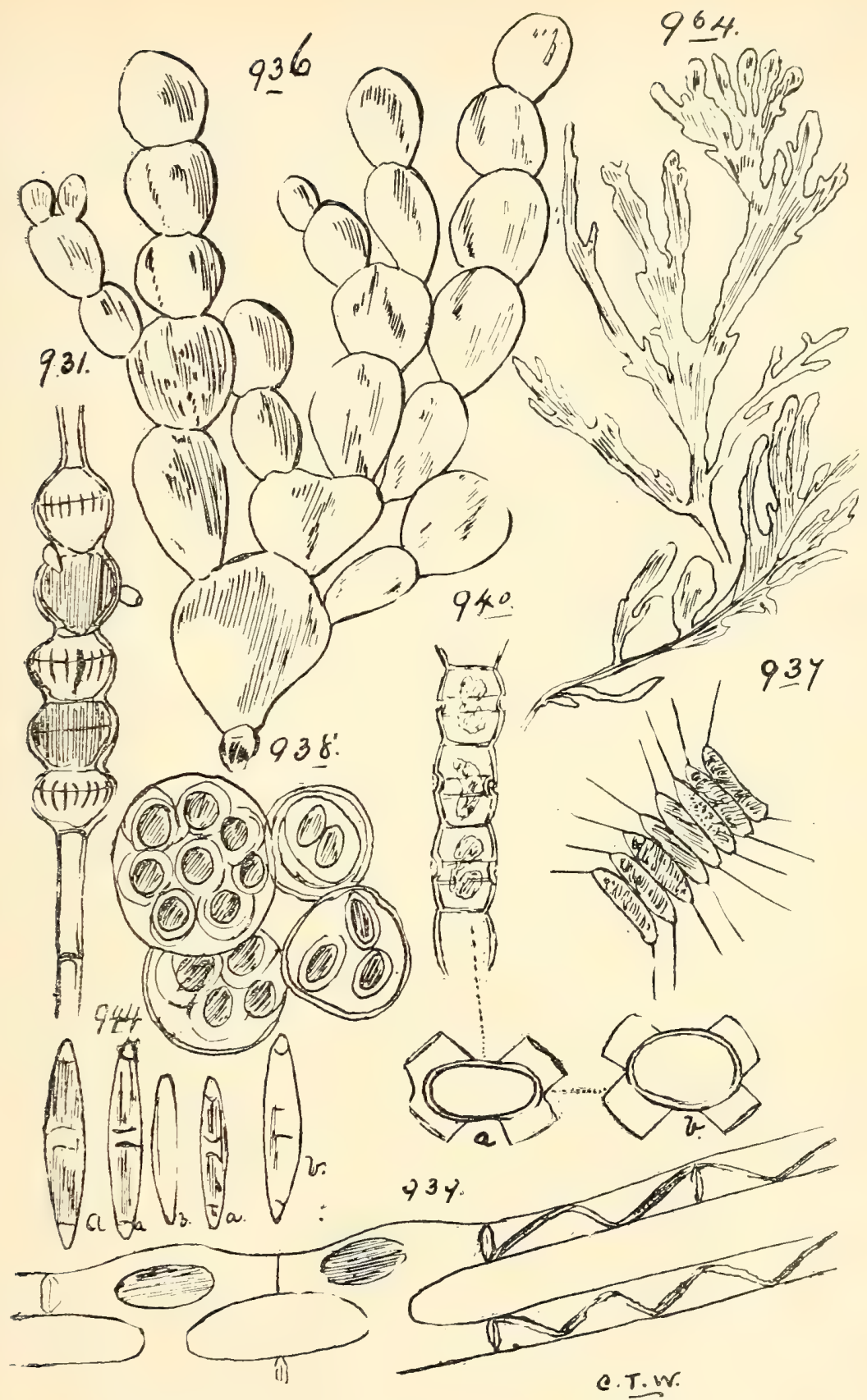

931. EDOgONIUM ELANDicum, Wittr.

x 390 (after Borge).

936. Halimeda cuneata, Hering.

937. Scenedesmus quadricauda, Bréb., var. HorRidus, Kirchn. x 500 (after Wolle).

938. GLGoCystis GIGAS, Lagerh.

Cells $\times 400$ (after Cooke).

939. Spirogyra inflata, Rabenh.

Filaments in fruit $\times 250$, showing lateral copulation (after Wolle).

940. Gymnozyga moniliformis, Ehrenb., var. minima, Borge.

944. Pinium Navicula, Bréb.

(a) Living fronds, (b) empty fronds.

964. Zonarta crenata, $J$. Ag. 
Closterium-contd.

prælongum, Bréb., De-Toni, Syll. Alg. i. 830.-Nundah (C. T. White). Det. by G. S. West.

Lunula (Muell.), Nitssch., De-Toni, Syll. Alg. i. 831. var. maximum, Borge.-Enoggera (Dr. T. L. Bancroft).

(O. Borge.)

intermedium, Ralfs., De-Toni, Syll. Alg. i. 834 .

Cornu, Ehrenb., De-Toni, Syll. Alg. i. 835.

var. brasiliense (Börgesen), Schmidle, Q1. Bot. Bull. xv. 33 . acutum (Lyngb.), Bréb., De-Toni, Syll. Alg. i. 836.

lineatum, Ehrenb., De-Toni, Syll. Alg. i. 838.

Dianæ, Ehrenb., De-Toni, Syll. Alg. i. 838.

Venus, Kuetz., De-Toni, Syll. Alg. i. 84I.-Mosman River (F. Muir). (West.)

parvulum, Nag., De-Toni, Syll. Alg. i. 84I.

calosporum, Wittr., De-Toni, Syll. Alg. i. 842.-Mosman River (F. Muir). (West.)

incurvum, Bréb., De-Toni, Syll. Alg. i. 843; var. majus, Wittr., De-Toni, Syll. Alg. i. 843.

Ehrenbergii, Menegh., De-Toni, Syll. Alg. i. 844.

moniliferum (Bory) Ehrenb., De-Toni, Syll. Alg. i. 845.Mosman River (F. Muir). (West.)

Leibleinii, Kuetz., De-Toni, Syll. Alg. i. 846.-Maroochie

(Dr. T. L. Bancroft); det. by O. Borge. Nundah

(C. T. White) ; det. by G. S. West. (Fig. 94I.)

Ralfsii, Bréb., De-Toni, Syll. Alg. i. 847.

var. typicum, Klebs., Q1. Bot. Bull. xv. 33 .

Kuetzingii, Bréb., De-Toni, Syll. Alg. i. 850.

setaceum, Ehrenb., De-Toni, Syll. Alg. i. 850 .

Penium, Bréb., De-Toni, Syll. Alg. i. 855.

margaritaceum (Ehrenb.), Bréb., De-Toni, Syll. Alg. i. 855 .

-Stannary Hills (Dr. T. L. Bancroft). (O. Borge.)

(Fig. 942.)

cucurbitinum, Biss., var. subpolymorphum, Nordst., De-Toni,

Syll. Alg. i. 856.-Stannary Hills (Dr. T. L. Bancroft).

(O. Borge.) (Fig. 943.)

closterioides, Ralfs., De-Toni, Syll. Alg. i. 860.

Navicula, Bréb., De-Toni, Syll. Alg. i. 861.-Barron River

(Dr. T. L. Bancroft). (O. Borge.) (Fig. 944.)

lamellosum, Bréb., De-Toni, Syll. Alg. i. 864.

sp., Moebius, Q1. Bot. Bull. xi. $3^{8}$.

Tetmemorus, Ralfs., De-Toni, Syll. Alg. i. 866.

Brebissonii (Menegh.), Ralfs., De-Toni, Syll. Alg. i. 866. var. attenuatus, Nordst., De-Toni, Syll. Alg. i. 867. 
Tetmemorus-contd.

Brebissonii-contd.

var. tenuissima, Moebius, Q1. Bot. Bull. vi. 42.

granulatus, Bréb., De-Toni, Syll. Alg. i. 867.

Triploceras, Bailey, De-Toni, Syll. Alg. i. 869.

verticillatum, Bail., De-Toni, Syll. Alg. i. 869; var. ornatum

= Docidium verticillatum, var. ornatum, Borge, Q1. P.ot.

Bu11. xv. 3I.

gracile, Bail., De-Toni, Syll. Alg. i. 870.

Docidium, Bréb., De-Toni, Syll. Alg. i. 871.

Baculum, Bréb., De-Toni, Syll. Alg. i. 872.

coronulatum, Grun., De-Toni, Syll. Alg. i. 872.

burmense, Josh., De-Toni, Syll. Alg. i. 874.

tessellatum, Josh., De-Toni, Syll. Alg. i. 876.

? cylindricum, Turn., Bail. Q1. Bot. Bull. xv. 30.

horridum, Borge., Q1. Bot. Bull. xv. 30.

elegans, Borge., Q1. Bot. Bull. xv. 3I.

australianum, Borge., Q1. Bot. Bull. xv. 31.

subundulatum, Borge., Q1. Bot. Bull. xv. 29.

Disphinctium, Naeg., De-Toni, Sv1l. Alg. i. 877.

Cucurbita (Bréb.), De-Toni, Syll. Alg. i. 88I.

? excavatum (Nordst.), De-Toni, Syll. Alg. i. 884. (Under

Cosmarium in Q1. Bot. Bull. xv. 20.)

cylindrus (Ehrenb.?), Noeg., De-Toni, Syll. Alg. i. 888.

(Under Cosmarim in Q1. Bot. Bull. xv. 20.)

Ralfsii (Kuetz.), Hansg., De-Toni, Syll. Alg. i. 888.

Pleurotænium, Narg., De-Toni, Syll. Alg. i. 895.

rectum, Delp., De-Toni, Syll. Alg. i. 896; forma australis,

Schmidle, Q1. Bot. Bull. xv. 32.

tenue, Schmidle, Q1. Bot. Bull. xv. 32.

Ehrenbergii (Ralfs.), De-Toni, Syll. Alg. i. 896. (Under

Docidium in Q1. Bot. Bull. xv. 3o.)

var. elongatum, West.-Barron River (Dr. T. L. Bancroft).

(O. Borge.)

ovatum, Nordst., De-Toni, Syll. Alg. i. 896.

var. inermis, Mobius, Q1. Bot. Bull. xi. 39.

maximum (Reinsch.), Lund., De-Toni, Syll. Alg. i. 899.-

Brisbane Botanic Gardens (C. T. White). Det. by

G. S. West.

nodosum (Bail.), Lund., DeToni, Syll.-Alg. i. 9or.= Docidium

nodosum, Bail., Q1. Bot. Bull. xv. 30.

var. mammillatum, Borge., Q1. Bot. Bull. xv. 30.

basiundulatum, $W$. and $G$. West.

cristatum (Turn.), Borge, forma, Borge, Trop. Sussw.

Chloroph. p. I5, t. I, f. I I.-Barron River (Dr. T. L.

Bancrofi ). (O. Borge.) (Fig. 945.) 
Pleurotænium-contd.

Stuhlmannii (Hieron.), Schmidle, forma, Borge, Desm. erst. Regnellsch. Exp. p. 83, t. 2, fig. 5.-Barron River ( $D r$. T. L. Bancroft). (O. Borge.) (Fig. 946.)

Pleurotæniopsis, Lund., De-Toni, Syll. Alg. i. 906.

De-Baryi (Archer), Lund., De-Toni, Syll. Alg. i. 906.

(Under Cosmarium in Q1. Bot. Bull. xv. 22.)

turgida (Bréb.), Lund., De-Toni, Syll. Alg. i. 907. (Under Cosmarium in Q1. Bott. Bull. xv. 22.)

tessellata (Delp.), De-Toni, Syll. Alg. i. 908; var. Nordstii, Mobius, Q1. Bot. Bull. vi. 45. (As a Cosmarium.)

? javanica (Nordst.), De-Toni, Syll. Alg. i. 91 $2=$ Cosmarium javanicum, Nordst.

ampla, Nordst., De-Toni, Syll. Alg. i. 91 $2=$ Cosmarium amplum, Nordst., Q1. Bot. Bull. xv. 29.

Xanthidium, Ehrenb., De-Toni, Syll. Alg. i. 9I6. fasciculatum, Ehrenb., De-Toni, Syll. Alg. i. 9I8. superbum, Elfu., De-Toni, Syll. Alg. i. 926. octonarium, Nordst., De-Toni, Syll. Alg. i. 927. multicorne, Borge., Q1. Bot. Bull. xv. I9. bifurcatum, Borge., Q1. Bot. Bull. xv. I9. Smithii, Archer, De-Toni, Syll. Alg. i. 924. var. variabile, Nordst., De-Toni, Syll. Alg. i. 924.

Cosmarium, Corda, De-Toni, Syll, Alg. i. 93 I. granatum, Bréb., De-Toni, Syll. Alg. i. 93I.

var. gibbosum, W. Schmidle, Q1. Bot. Bull. xv. 23.

moniliforme (Turp.), Ralfs., De-Toni, Syll. Alg. i. 932; forma, Schmidle, Q1. Bot. Bull. xv. 25. nitidulum, De-Not., De-Toni, Syll. Alg. i. 935. Meneghinii, Bréb., De-Toni, Syll. Alg. i. 937. (Including

C.crenulatum, Næg., Q1. Bot. Bull. xv. 24.)

var. concinnum, Rabenh., De-Toni, Syll. Alg. i. 938.

forma, Mobius, Q1. Bot. Bull. vi. 46.

Regnellii, Wille, De-Toni, Syll. Alg. i. 939.-Beaudesert

(Dr. T. L. Bancroft). (G. S. West.) impressulum, Elfv., forma, Schmidle, Q1. Bot. Bull. xv. 23. crenatum, Ralfs., De-Toni, Syll. Alg. i. 94I.

venustum (Bréb.), Arch., De-Toni, Syll. Alg. i. 945.

forma, Borge., Q1. Bot. Bull. xv. 25.

forma minor, Wille., Schmidle, Q1. Bot. Bull. xv. 25. var. hypohexagonum, West.

abruptum, Lund., De-Toni, Syll. Alg. i. 947.-Burnett River (Dr. T. L. Bancroft); Nundah (C. T. White). Det. by G. S. West. 
Cosmarium--contd.

minutum, Delp., De-Toni, Syll. Alg. i. 948; forma, Schmidle, Q1. Bot. Bu1l. xv. 25.

sulcatum, Nordst, var. glabrum, Borge.--Upper Walsh River

(Dr. T. L. Bancroft). (O. Borge.) (Fig. 947.)

subtumidum, Nordst., De-Toni, Syll. Alg. i. 950.

forma, Schmidle, Q1. Bot. Bull. xv. 24.

pseudoprotuberans, Kirchn, De-Toni, Syll. Alg. i. 95 I.

zar. angustius, Nordst., De-Toni, Syll. Alg. i. 951.-Stan-

nary Hills (Dr. T. L. Bancroft). (O. Borge.) (Fig. 948.)

var. tumidum, Borge,-Barron River (Dr. T. L. Bancroft). (O. Borge.) (Fig. 949.)

ellipsoidetum, Elfv., De-Toni, Syll. Alg. i. 953.

Elfvingii, Racib., De-Toni, Syll. Alg. i. $953=$ C. hexagonum, Elfv.

sexangulare, Lund., De-Toni, Syll. Alg. i. 954.

Lundellii, Delp., De-Toni, Syll. Alg. i. 956; var. corruptum (Turn.), W. and G.S. West.-Barron River (Dr. T. L. Bancroft), det. by O. Borge.; Mosman River ( $F$. Muir); Sandgate Lagoon (C. T. White), det. by G. S. West.

Seelyanum, Wolle., De-Toni, Syll. Alg. i. $95^{8}$.

Regnesii, Reinsch., De-Toni, Syll. Alg. i. 96г.

var. montanum, W. Schmidle, Q1. Bot. Bull. xv. 27.

punctulatum, Bréb., De-Toni, Syll. Alg. i. 96r.

var. subpunctulatum (Nordst.), Börges.-Upper Walsh

River (Dr. T. L. Bancroft). (O. Borge.)

forma typica, W. Schmidle, Q1. Bot. Bull. xv. 27.

forma, W. Schmidle, Q1. Bot. Bu11. xv. 27.

orthostichum, Lund., De-Toni, Syll. Alg. i. 963.

pyramidatum, Bréb., De-Toni, Syll. Alg. i. 969; forma,

Borge, Desm. erst. Regnellsch. Exp. p. 94, t. 3, fig. 8, sed minor.-Barron River (Dr. T. L. Bancroft). (O. Borge.) (Fig. 950.)

pachydermum, Lund., De-Toni, Syll. Alg. i. 970.

trachypleurum, Lund., De-Toni, Syll. Alg. i. 973; var. minus, Racib., De-Toni, Syll. Alg. i. 974.

obsoletum (Hantzsch.), Reinsch., De-Toni, Syll. Alg. i. 974. rar. punctatum, Mask., De-Toni, Syll. Alg. i. 975.

forma major, Lund., Q1. Bot. Bull. xv. 26.

perforatum, Lund., De-Toni, Syll. Alg. i. 977.--Stannary Hills (Dr. T. L. Bancroft). (O. Borge.) (Fig. 95 I.) 
Cosmarium-contd.

Botrytis (Bory.), Menegh., De-Toni, Syl1. Alg. i. 979.

var. tumidum, Wittr., Schmidle, Q1. Bot. Bull. xv. 28.

tetraophthalmum (Kuetz.), Bréb., De-Toni, Syll. Alg. i. 98I.

reniforme (Ralfs.), Archer, De-Toni, Syll. Alg. i. 982.

var. compressum, Nordst., De-Toni, Syll. Alg. i. 983.

Brebissonii, Menegh., De-Toni, Syll. Alg. i. 983.

Quadrum, Lund., De-Toni, Syll. Alg. i. 985; var. minus,

Nordst., forma, Schmidle, Q1. Bot. Bull. xv. 29.

Portianum, Archer, De-Toni, Syll. Alg. i. 986.

subspeciosum, Nordst., var. validium, Nordst., De-Toni, Syil.

Alg. i. 986.-Stannary Hills (Dr. T. L. Bancroft), O.

Borge. (Fig. 952.)

var. simplex, West.-Mosman River (F. Muir). (West.)

binum, Nordst., De-Toni, Syll. Alg. i. 993 ; var. australiensis,

Borge., Q1. Bot. Bull. xv. 23.

latum. Bréb., De-Toni, Syll. Alg. i. 999.-Stannary Hills (Dr. T. L. Bancroft). (O. Borge.) (Fig. 953.)

subcrenulatum, Hantzsch., De-Toni, Syll. Alg. i. Iooo.-

Nundah (C. T. White). Det. by G. S. West.

Phaseolus, Bréb., De-Toni, Syll. Alg. i. IOoI ; var. elevatum, Nordst., De-Toni, Syll. Alg. i. Ioor.

Blyttii, Wille., De-Toni, Syll. Alg. i. IoI3.

var. australicum, W. Schmidle, Q1. Bot. Bull. vi. 28.

angulatum (Perty), Rabenh., De-Toni, Syll. Alg. i. Ior7.

forma major, Grunow, Turner, Alg. E. Ind. p. 56.

neapolitanum, Bals., De-Toni, Syll. Alg. i. I020; var. austra-

licum, W. Schmidle, Q1. Bot. Bull. xv. 28.

subcostatum, Nordst., forma minor, West.-Mosman River (F. Muin). (West.)

angustatum (Wittr.), Nordst., De-Toni, Syll. Alg. i. I044.

Reinschii, Archer, De-Toni, Syll. Alg. i. I045.

subpunctulatum, Nordst., De-Toni, Syl1. Alg. i. I049.

? pseudobroomei, Wolle., De-Toni, Syll. Alg. i. I055.

denticulatum, Borge., Q1. Bot. Bull. xv. 2 I.

securiforme, Borge., Q1. Bot. Bull. xv. 2I.

Askenasyi, W. Schmidle, Alg. Sumatr., p. 304, P1. iv. fig. 7; Borge., Q1. Bot. Bu1l. xv. 22.

dubium, Borge., Q1. Bot. Bull. xv. 23.

palustre, Turn.; $a$ circulare, $b$ ovale; Fresh-water Alg. of

East India, p. 60.

glyptodermum, $W$. and G. S. West, Trans. Linn. Soc. (2nd ser. Bot.) v. 69.- Sandgate Lagoon (C. T. White). Det. by G. S. West, who remarks in litt., "Originally described from Madagascar, this species has since been 
Cosmarium-contd.

glyptodermum-contd.

found in different parts of Africa, and in Ceylon. It is one of the most beautifully marked of all the species of this genus. The original description is incorrect in one point. The cell-wall is described as having depressions of two sizes. This is not the case, the larger marks being hollow outstanding granules, and only the smaller intervening triangular marks are depressions (or scrobiculations) on the exterior of the wall."

retusiforme, (Wille.), Gutzo-Mosman River (F. Muir). (West.)

subtriordinatum, $W$. and G. S. West, var. rotundatum, Schmidle-Mosman River (F. Muir). (West.)

sp., Moebius, Q1. Bot. Bull. xi. 40.

Arthrodesmus, Ehrenb., De-Toni, Syll. Alg. i. 1056.

convergens, Ehrenb., De-Toni, Syll. Alg. i. I058.

var. mucronatus, Borge., Q1. Bot. Bull. xv. 20.

subulatus, Kuetz., De-Toni, Syll. Alg. i. I059.-Brisbane Botanic Gardens (C. T. White). Det. by G. S. West. apiculatus, Joshua, De-Toni, Syll. Alg. i. Io6o.

Euastrum, Ehrenb., De-Toni, Syll. Alg. i. I065.

verrucosum, Ehrenb., De-Toni, Syll. Alg. i. I066; var. Mœbii,

Borge., Q1. Bot. Bull. xv. I5 = Micrasterias Möbii,

W. and G. S. West, J1. Linn. Soc. xxxiii. I897, p. I62.-

Dr. West (Jl. Bot. I9I2, p. 86) records $M$. Möbii, var. jaranica, Gutwinski, from the Mosman River Coll. (F. Muir.)

divergens, Josh., De-Toni, Syll. Alg. i. Iо76; var. australianum, Borge., Q1. Bot. Bull. xv. I5.

spinulosum, Delp., De-Toni, Syll. Alg. i. I080.

var. inermius, Nordst., De-Toni, Syll. Alg. i. I080.

var. ornatum, Borge., Q1. Bot. Bull. xv. I5.

binale (Turp.), Ralfs., De-Toni, Syll. Alg. i. Io84; var. b, Ralfs.

Didelta (Turp.), Ralfs., De-Toni, Syll. Alg. i. I093.

cuneatum, Jenn., De-Toni, Syll. Alg. i. ıоgб.

ansatum, Ralfs., De-Toni, Syll. Alg. i. Iog6.

var. maxima, Borge., Q1. Bot. Bull. xv. I5.

var. attenuatum, W. Schmidle, Q1. Bot. Bull. xv. I4.

forma biscrobiculata, W. Schmidle, Q1. Bot. Bull. xv. I4. circulare, Hass., De-Toni, Syll. Alg. i. I097. sinuosum, Lenorm., De-Toni, Syll. Alg. i. Iog8.

rostratum, Ralfs., De-Toni, Syll. Alg. i. I099; var. præmorsum, Nordst., De-Toni, Syll. Alg. i. I099. 


\section{Euastrum-contd.}

elegans (Bréb.), Kuetz., De-Toni, Syll. Alg. i. IIO2.

var. pseudelegans (Turn.), West-Barron River (Dr.

T. L. Bancroft). (O. Borge.)

compactum, Wolle., De-Toni, Syll. Alg. i. I I05.

denticulatum (Kirchn.), Gay, De-Toni, Syll. Alg. i. I Io6;

var. stictum, Börges.-Barron River (Dr. T. L. Bancroft). (O. Borge.)

Turneri, West, Fresh-water Alg. W. Irel. p. I4I.

umbonatum, West.

asperum, Borge., Q1. Bot. Bull. xv. I4.

sp., Borge., Q1. Bot. Bull. xv. I5.

sp., Borge., Q1. Bot. Bull. xv. I6.

Micrasterias, Ag., De-Toni, Syll. Alg. i. I Iog.

incisa (Bréb.), Kuetz., De-Toni, Syll. Alg. i. IIIo; var. typica, Turner, Alg. East India.

var. Wallichiana, Turner, Alg. East India.

oscitans, Ralfs., De-Toni, Syll. Alg. i. II Io; var. pinnatifida.

(Kuetz.), Rabenh., De-Toni, Syll. Alg. i. I I Iо.

decemdentata, Neeg., De-Toni, Syll. Alg. i. I I 16.

forma, W. Schmidle, Borge., Q1. Bot. Bull. xv. I3.

Mahabuleshwarensis, Hobson, De-Toni, Syll, Alg. i. I I22.

var. Wallichii (Grun.), West.-Barron River (Dr. T. L.

Bancroft). (O. Borge.)

forma novizelandica, Nordst.

foliacea, Bail., De-Toni, Syll. Alg. i. I I29.

denticulata (Bréb.), Ralfs., De-Toni, Syll. Alg. i. Irzo.

var. notata, Nordst., De-Toni, Syll. Alg. i. I I3I.-Upper

Walsh River (Dr. T. L. Bancroft). (O. Borge.) (Fig. 954.)

alata, Wall., De-Toni, Syll. Alg. i. I I 36.

sp., Borge, Q1. Bot. Bull. xv. I3.

Staurastrum, Meyen., De-Toni, Syll. Alg. i. I I36.

dejectum, Brób., De-Toni, Syll. Alg. i. I I37.

muticum, Bréb., De-Toni, Syll. Alg. i. I I77.

Submanfeldtii, $W$. and G. S. West, Trans. Linn. Soc. (2nd ser. Bot.), vi. I88.-Burnett River (Dr. T. L. Bancroft). Det. by G. S. West.

pseudosebaldi, Wille., De-Toni, Syll. Alg. i. II78; var. bicornis, Boldt., De-Toni, Syll. Alg. i. II79.

subsp. tonsum, Nordst., De-Toni, Syll. Alg. i. II79.-Enoggera Reservoir (Dr. T. L. Bancroft). (O. Borge.) (Fig. 955.)

orbiculare (Ehrenb.), Ralfs., De-Toni, Syll. Alg. i. II8o.

var. denticulatum, Wille., De-Toni, Syll. Alg. i. I I8I. 
Staurastrum-contd.

orbiculare-contd.

var. depressum, Roy. et Bisset, De-Toni, Syll. Alg. i. I I8I. var. Ralfsii, W. and G. S. West.-Nundah (C. T. White).

Det. by G. S. West.

pygmæum, Bréb., De-Toni, Syll. Alg. i. I I8I.

var. obtusum, Wille., De-Toni, Syll. Alg. i. II82.

dilatatum, Ehrenb., De-Toni, Syll. Alg. i. I 193.

var. obtusilobum, De Not.-Upper Walsh River (Dr. T.I.

Bancroft). (O. Borge.) (Fig. 956.)

forma australica, W. Schmidle, Q1. Bot. Bull. xv. I8.

Bieneanum, Rabenh., De-Toni, Syll. Alg. i. II97.

A form with the cell-wall almost minutely granulate

has been collected at Nundah by C. T. White; det. by

G. S. West.

var. ellipticum, Wille., De-Toni, Syll. Alg. I 197.

gracile, Ralfs., De-Toni, Syll. Alg. i. I209.

var. uniseriatum, West., Alg. Madagascar; Trans. Linn.

Soc. (2nd ser. Bot.) v. 77, P1. ix. fig. 3 .

bicorne, Hauptfl., De-Toni, Syll. Alg. i. I2Io; var. longe-

brachiatum, Borge., Q1. Bot. Bull. xv. I7.

paradoxum, Meyen., De-Toni, Syll. Alg. i. I2I I.

sagittarium, Nordst., De-Toni, Syll. Alg. i. I2I4.

sexangulare (Bulnh.), Lund., De-Toni, Syll. Alg. i. I224.

var. australica, $W$. Schmidle, Q1. Bot. Bull. xv. I9.

var. incurvum, Borge., Q1. Bot. Bull. xv. I9.

var. gemmescens, Plavf.-Barron River (Dr. T. L. Bancroft). (O. Borge.) (Fig. 957.)

margaritaceum, Ehrenb., var. hirtum, Nordst.-Mosman

River (F. Muir). (West.)

subpinnatum, W. Schmidle, Q1. Bot. Bull. xv. I8.

rectangulare, Borge., Q1. Bot. Bull. xv. I7.

elegans, Borge., Q1. Bot. Bull. xv. I7.

retusum, Turner, Alg. East India, p. I04, plate xiii. fig. I3.

var. granulatum, Borge., Q1. Bot. Bull. xv. I6.

approximatum, West, forma, Borge.

leptocladum, Nordst., De-Toni, Syl1. Alg. i. I233.

var. cornutum, Wille., De-Toni, Syll. Alg. i. I234.

Heimerlianum, Liitkem, forma, West. - Mosman. River

(F. Muir). (West.)

\section{BACILLARIE}

ORDER RHAPHIDE无.

FAMILY NAVICULACE.

Navicula, Bory., De-Toni, Syll. Alg. ii. 6.

nobilis, Kuetz., var. Dactylus (Ehrb.), I.H., De-Toni, Syll. Alg. ii. 9.-Mosman River (F. Mur). (West.) 


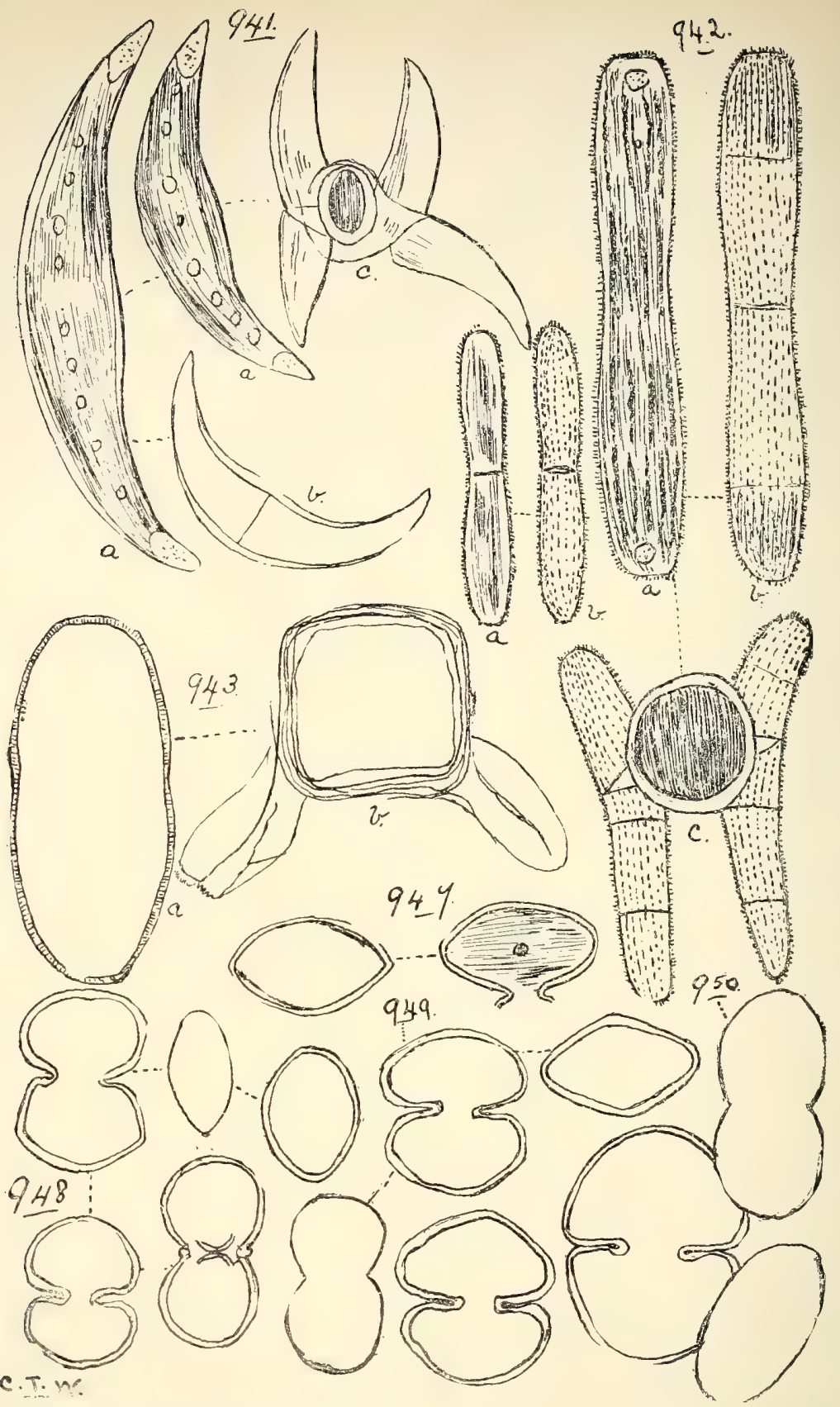

94t. Closterium Leiblenit, Kuetz.

(a) Living cells, (b) an empty cell, (c) zygospore. (After Cooke.)

942. Penium margaritaceum, Bréb.

(a) Living fronds, (b) empty fronds, (c) zygospore.

943. P. Cucurbitinum Biss., var. subpoly morpiedm, Nordst. (x 570). (After Nordstedt.)

947. Cosmarium sulcatum, Nordst., var Glabrum, Borge (x 740). (After Borge.)

948. C. PSeudoprotuberans, Kirchn., var. angustus, Nordst. (x 570$)$. (After Nordstedt.)

949. C. pSeudoprotuberans, Kirchn., var. tumidum, Borge (x 740). (After Borge.) 
Navicula—contd.

major, Kuetz, De-Toni, Syll. Alg. ii. Io.-Mosman River (F. Muir). (West.)

viridis (Nitzsch.), Kuetz, De-Toni, Syll. Alg. ii. II.-Mosman River (F. Muir); Sandgate Lagoon (C. T. White). Det. by G. S. West.

stauroptera, Grun., De-Toni, Syll. Alg. ii. 25.-Mosman River (F. Muir). (West.)

appendiculata (Ag.), Kuetz., De-Toni, Syll. Alg. ii. 28.Mosman River (F. Muir). (West.)

gracilis, Kuetz., De-Toni, Syll. Alg. ii. 40.-Mosman River (F. Muir). (West.)

viridula, Kuets., De-Toni, Syll. Alg. ii. 43.-Mosman River (F. Muir). (West.)

rhyncocephala, Kuetz., De-Toni, Syll. Alg. ii. 44.-Nundah

(C. T. White). Det. by G. S. West.

? diversa, Grev., De-Toni, Syll. Alg. ii. 79.

elliptica, Kuetz., De-Toni, Syll. Alg. ii. 89.-Corinda (C. T.

White). Det. by G. S. West.

Johnsoniana, Grev., De-Toni, Syll. Alg. ii. I24.

cuspidata, Kuetz., De-Toni, Syll. Alg. ii. I36.-Sandgate

Lagoon (C. T. White). Det. by G. S. West.

? Cistella, Grev., De-Toni, Syll. Alg. ii. I85.

Pleurosigma, W.Sm., De-Toni, Syll. Alg. ii. 23I.

attenuatum (Kuetz.), W.Sm., De-Toni, Syll. Alg. ii. $248=$ Gyrosigma attenuatum, Rabenh.-Brisbane Botanic Gardens and Corinda (C. T. White). Det. by G. S. West.

acuminatum (Kuetz.), Grun. = Gyrosigma lacustre, DeToni, Syll. Alg. ii. 252; P. lacustre, W. Sm.-Sandgate Lagoon (C. T. White). Det. by G. S. West.

Spencerii (Quek.), W. Sm., De-Toni, Syll. Alg. ii. $253=$ Gyrosigma Spencerii.-Brisbane Botanic Gardens, Nundah, and Corinda (C. T. White); Burnett River (Dr.T. L. Bancroft). Det. by G. S. West.

Stauroneis, Ehrenb., De-Toni, Syll. Alg. ii. 204.

Phœnicentron, Ehrenb., De-Toni, Syll. Alg. ii. 204.-Mosman River (F. Muir). (West.)

? biformis, Grun., De-Toni, Syll. Alg. ii. 216; forma australis,

De-Toni, Syll. Alg. = S, australis, Grev.

obesa, Grev., De-Toni, Syll. Alg. ii. 2 I7. 
Iirustulia, Ag., De-Toni, Syll. Alg. ii. 276.

rhomboides (Ehrenb.), De-Toni, var. saxonica (Rab.), DeToni, Syll. Alg. ii. $277=$ Vanhuerckia rhomboides, var. saronica, G. S. West.-Mosman River. (F. Muir); Sandgate Lagoon (C. T. White). Det. by G. S. West. vulgaris (Thwait.), De-Toni, Syll. Alg. ii. $280=\operatorname{Van}$. huerckia vulgaris, V.H.-Sandgate Lagoon (C. T. White). Det. by G. S. West.

\section{Family Amphitropidace⿸.}

Amphiprora, Ehr., De-Toni, Syll. Alg. ii. 328. oblonga, Grev., De-Toni, Syll. Alg. ii. 336. eximia, Grev., De-Toni, Syll. Alg. ii. 336. lata, Grev., De-Toni, Syll. Alg. ii. 337. ? Clepsydra, Grev., De-Toni, Syll. Alg. ii. 337. ? paradoxa, Grev., De-Toni, Syll. Alg. ii. 337. nitida, Grev., De-Toni, Syll. Alg. ii. 337. Jolisiana, Grev., De-Toni, Syll. Alg. ii. 340.

Rabenhorstiana, Grev., De-Toni, Syll. Alg. ii. 341. Brebissoniana, Grev., De-Toni, Syll. Alg. ii. 34I. Meneghiniana, Grev., De-Toni, Syll. Alg. ii. 34I. Kuetzingiana, Grev., De-Toni, Syll. Alg. ii. 34I. Thwaitesiana, Grev., De-Toni, Syll. Alg. ii. 342.

\section{FAmily Cymbellacez.}

Cymbella, Ag., De--Toni, Syll. Alg. ii. 349.

(Cocconema) lævis, Naeg. (Ehr.), Kirchn., De-Toni, Syll. Alg. ii. 352.-Mosman River (F. Muir). (West.)

(Cocconema) leptoceras (Ehr.), Rabenh., De-Toni, Syll. Alg. ii. 353.-Mosman River (F. Muir). (West.)

(Cocconema) lanceolata (Ehrenb.), Kirchn., De-Toni, Syll. Alg. ii. 362.-Beaudesert (Dr. L. T. Bancroft). (West.) (Fig. 958.)

(Cocconema) cymbiformis (Kuetz.), Bréb., De-Toni, Syll. Alg. ii. 363.-Mosman River (F. Muir). (West.) var. parva (W. Sm.), V.H., De-Toni, Syll. Alg. ii. $364=$ Cocconema parvum, W. Sm.-Beaudesert (Dr. T. L. Bancroft). (West.)

Encyonema, Kuetz., De-Toni, Syll. Alg. ii. 37 I.

turgidum (Greg.), Grun., De-Toni, Syll. Alg. ii. $372=$ Cocconema turgida, G. S. West.-Mosman River (F. Muir). (West.)

ventricosum (Ag.), Grun., De-Toni, Syll. Alg. ii. $373=$ Cocconema ventricosa, G. S. West.-Mosman River (F. Muir). (West.) 


\section{Family Cymbellacex.}

Amphora, Ehrenb., De-Toni, Syll. Alg. ii. 375.

flexuosa, Grev., De-Toni, Syll. Alg. ii. 378.

cofferformis (Ag.), Kuetz., De-Toni, Syll. Alg. ii. 389.-

Corinda (C. T. White). Det. by G. S. West.

undulata, Grev., De-Toni, Syll. Alg. ii. 394.

sinuata, Grev., De-Toni, Syll. Alg. ii. 394.

magnifica, Grev., De-Toni, Syll. Alg. ii. 395.

pulchra, Grev., De-Toni, Syll. Alg. ii. 395.

\section{Family Gomphonemace.e.}

Gomphonema, Ag., De-Toni, Syll. Alg. ii. 424.

Augur, Ehr., var. Gautieri, V.H., De-Toni, Syll. Alg. ii. 425.

-Beaudesert (Dr. L. T. Bancroft). (West.)

gracile, Ehrenb., De-Toni, Syll. Alg. ii. 426.-Mosman River (F. Muir). (West.)

parvulum, Kuetz., De-Toni, Syll. Alg. ii. 426.-Mosman River (F. Muir) ; Brisbane Botanic Gardens and Sandgate Lagoon (C.T.White). Det. by G. S. West.

tenellum, Kuetz.-Mosman River (F. Muir). (West.)

acuminatum, Ehrenb., De-Toni, Syll. Alg. ii. 423.-Burnett

River (Dr. T. L. Bancroft); Nundah (C. T. White). Det. by G. S. West.

\section{Family Cocconeidacem.}

Cocconeis, Ehrenb., De-Toni, Syll. Alg. ii. 442.

Placentula, Ehrenb., De-Toni, Syll. Alg. ii. 443.-Mosman River (F. Muir). (West.)

\section{Family Achnanthacee.}

Achnanthes, Bory., De-Toni, Syll. Alg. ii. 469.

crenulata, Grun., De-Toni, Syll. Alg. ii. 475.-Mosman River (F. Muir). (West.)

hungarica, Grun., De-Toni, Syll. Alg. ii. 478.--Mosman River (F.Muir). (West.)

\section{Family Nitzschiacex.}

Nitzschia, Hassal., De-Toni, Sy11. Alg. ii. 495. scalaris (Ehr.), W. Sm., De-Toni, Syll. Alg. ii. 519.-Mosman River (F. Muir). (West.)

Sigma, W. Sm., De-Toni, Syll. Alg. ii. 530, var.-Mosman River (F. Muir). (West.)

spectabilis (Ehr.), Ralfs., De-Toni, Syll. Alg. ii. 535.Corinda (C.T. White). Det. by G. S. West. 
Nitzschia-contd.

Palea (Kuetz.), W. Sm., var. debilis (Kuetz.), Grun., DeToni, Syll. Alg. ii. 54I.-Brisbane Botanic Gardens (C. T. White). Det. by G. S. West.

Lorenziana, Grun., var. incerta, Grun., De-Toni, Syll. Alg. ii. $55^{0}=N$. reversa, W. Sm.-Brisbane Botanic Gardens (C. T. White). Det. by G. S. West.

\section{ORDER PSEUDORHAPIDEÆ.}

\section{Family Surirellaceæ.}

Suriraya, Turp., De-Toni, Syll. Alg. ii. 567.

biseriata (Ehr.), Bréb., De-Toni, Syll. Alg. ii. 567.-Mosman River (F. Muir). (West.)

linearis, $W$. Sm., De-Toni, Syll. Alg. ii. 568.-Mosman River (F. Muir). (West.)

ovalis, Bréb., var. angusta (Kuetz.), V.H., De-Toni, Syll. Alg. ii. 580.-Mosman River (F. Muir). (West.)

Campylodiscus, Ehr., De-Toni, Syll. Alg. ii. 603.

Robertsianus, Grev., De-Toni, Syll. Alg. ii. бıб.

\section{FAMily Fragilariaceæ.}

Synedra, Ehr., De-Toni, Syll. Alg. ii. 65r.

Acus, Kuetz., De-Toni, Syll. Alg. ii. 656.-Beaudesert (Dr. T. L. Bancroft); Corinda (C. T. White). Det. by G. S. West. (Fig. 959.)

U1na (Nitzsch.), Ehrenb., De-Toni, Syll. Alg. ii. 653--Mosman River (F. Muir); Burnett River (Dr. T. L. Bancroft); Sandgate Lagoon (C.T. White). Det. by G. S. West.

z'ar. splendens (Kuetz.), Brun.-Beaudesert and Burnett River (Dr. T. L. Bancroft). Det. by G. S. West.

Desmogonium, Ehrenb., De-Toni, Syll. Alg. ii. 68 o.

Rabenhorstianum, Grun., De-Toni, Syll. Alg. ii. 680.-Mosman River (F. Muir). (West.)

\section{Family Eunotiacee.}

Cystopleura, Bréb., De-Toni, Syll. Alg. ii. 777.

turgida (Ehr.), Kunze = Epithemia turgida (Ehr.), Kuetz., De-Toni, Syll. Alg. ii. 777.-Beaudesert and Burnett River (Dr. T. L. Bancroft); Brisbane Botanic Gardens (C. T. White). Det by G. S. West. (Fig. 960.) 
Cystopleura-contd.

gibba (Ehr.), Kunze = Rhophalodia gibba (Kuetz.), O. Müller, De-Toni, Syll. Alg. ii. 780.-Beaudesert and Burnett River (Dr. T. L. Bancroft); Brisbane Botanic Gardens (C. T. White). Det. by G. S. West. (Fig. 96г.)

gibberula (Ehr.), Kunze = Rhophalodia gibberula (Kuetz.), O. Müller, De-Toni, Syll. Alg. ii. 786.-Beaudesert (Dr. T. L. Bancroft); Sandgate Lagoon (C. T. While); Mosman River (F. Muir). Det. by G. S. West. (Fig. 962.)

Eunotia, Ehrenb., De-Toni, Syll. Alg. ii. 790.

pectinalis (Kuetz.), Rabenh., De-Toni, Syll. Alg. ii. 793.Mosman River (F. Muir). (West.)

Formica, Ehrenb., De-Toni, Syll. Alg. ii. 795.-Mosman River (F. Muir). (West.)

diodon, Ehrenb., De-Toni, Syll. Alg. ii. 799.-Mosman River (F. Muir). (West.)

Pseudoeunotia, Grun., De-Toni, Syll. Alg. ii. 8o7.

lunaris (Ehr.), Grun., De-Toni, Syll. Alg. ii. 8o8.-Mosman River (F. Muir); Sandgate Lagoon (C. T. White). Det. by G. S. West.

flexuosa (Bréb.), Grun., De-Toni, Syll. Alg. ii. 8og.-Mosman River (F. Muir). (West.)

\section{Family Biddulphiace..}

Triceratium, Ehr., De-Toni, Syll. Alg. ii. 917.

Robertsianum, Grev., De-Toni, Syll. Alg. ii. 919.

\section{Family Eupodiscacee.}

Auliscus, Ehr., De-Toni, Syll. Alg. ii. 1025. intercedens, Janisch., De-Toni, Syll. Alg. ii. I046. lucidus, Rattray, De-Toni, Syll. Alg. ii. I056.

\section{Family Melosiracee.}

Lysigonium, Link., De-Toni, Syll. Alg. ii. I328.

varians (Ag.), De-Toni, De-Toni, Syll. Alg. ii. ${ }^{2} 329=$ Melosira varians, Ag.-Beaudesert (Dr. T. L. Bancroft); Brisbane Botanic Gardens (C.T. White). Det. by G. S. West. (Fig. 963.)

Cyclotella, Kuetz., De-Toni, Syll. Alg. ii. I35I.

Meneghiniana, Kuetz., De-Toni, Syll. Alg. ii. I354.-Corinda (C. T. White). Det. by G. S. West. 


\section{FUCOIDEE.}

\section{Order CYCLOSPORINA.}

\section{Family Sargassace⿸.}

Sargassum, Ag., De-Toni, Syll. Alg. iii. 6.

Peronii (Mert.), Ag., De-Toni, Syll. Alg. iii. I2.

decurrens (R. Br.), Ag., De-Toni, Syll. Alg. iii. I3.

Boryi, Ag., De-Toni, Syll. Alg. iii. I3.

scabripes, J. Ag., De-Toni, Syll. Alg. iii. I4.

fallax, Sond., De-Toni, Syll. Alg. iii. 29.

Amaliæ, Grun., De-Toni, Syll. Alg. iii. 30.

angustifolium (Turn.), Ag., De-Toni, Syll. Alg. iii. $3^{8}$.

carpophyllum, J. Ag., De-Toni, Syll. Alg. iii. 39.

var. leptophyllum, Asken., De-Toni, Syll. Alg. iii. 40.

flavicans (Mert.), Ag.,- De-Toni, Syll. Alg. iii. $40=$

S. amulum, Sond., Syn. Q1. F1. 783 .

var. moretonense, Grun., De-Toni, Syll. Alg. iii. 40.

fissifolia (Mert.), J. Ag., De-Toni, Syll. Alg. iii. 4I.

Swartzii (Turn.), Ag., De-Toni, Syll. Alg. iii. 44.

Binderi, Sond., De-Toni, Syll. Alg. iii. 47.

cristæfolium, Ag., De-Toni, Syll. Alg. iii. 5I.

var. condensatum, Sond.

spinifex, Ag., De-Toni, Syll. Alg. iii. 55.

lophocarpum, J. Ag., De-Toni, Syll. Alg. iii. 55.

ilicifolium (Turn.), Ag., De-Toni, Syll. Alg. iii. 56.

microcystum, J. Ag., De-Toni, Syll. Alg. iii. 57.

cinctum, J. Ag., De-Toni, Syll. Alg. iii. $59=S$. odontocarpum, Sond., Bail. Syn. Q1. F1. 784.

myriocystum, J. Ag., De-Toni, Syll. Alg. iii. 70.

parvifolium (Turn.), Ag., De-Toni, Syll. Alg. iii. $7 \mathrm{I}=$

S. ambigumm, Sond., Bail. Syn. Q1. F1. 784.

filifolium, $A g$. , De-Toni, Syll. Alg. iii. 72.

aquifolium (Turn.), Ag., De-Toni, Syll. Alg. iii. $75=$

S. obovatum, Grev., Bail. Syn. Q1. F1. 784.

stenophyllum, J. Ag., De-Toni, Syll. Alg. iii. 8o.

lanceolatum, J. Ag., De-Toni, Syll. Alg. iii. 8I.

Desvauxii (Mert.), Ag., De-Toni, Syll. Alg. iii. $82=S$. simu-

lans, Sond., Bail. Syn. Q1. F1. 784.

Dacciferum (Turn.), Ag., De-Toni, Syll. Alg. iii. 82.

Acinaria (Turn.), J. Ag., De-Toni, Syll. Alg. iii. 9r.

leptopodum, J. Ag., De-Toni, Syll. Alg. iii. 96.

spinuligerum, Sond., De-Toni, Syll. Alg. iii. 98.

Godeffroyi, Grun., De-Toni, Syll. Alg. iii. 98.

cystocarpum, Ag., De-Toni, Syll. Alg. iii. Ioo. 
Sargassum-contd.

granuliferum, Ag., De-Toni, Syll. Alg. iii. roI.

aciculare, Grun., De-Toni, Syll. Alg. iii. IOI.

gracile, J.Ag., De-Toni, Syll. Alg. iii. IO2.

polycystum, $A g$., De-Toni, Syll. Alg. iii. IO3.

Baccularia (Mert.), Ag., De-Toni, Syll. Alg. iii. IO4.

siliquosum, J. Ag., De-Toni, Syll. Alg. iii. I07.

Turbinaria, Lamour., De-Toni, Syll. Alg. iii. I24.

conoides, Kuetz., De-Toni, Syll. Alg. iii. I26.

ornata; J. Ag., De-Toni, Syll. Alg. iii. I 28.

Cystophyllum, J. Ag., De-Toni, Syll. Alg. iii. I52.

triode (Forsk.), J. Ag., De-Toni, Syll. Alg. iii. I53.

muricatum (Turn.), J. Ag., De-Toni, Syll. Alg. iii. I54.

\section{FAmily Fucacex.}

Hormosira, Endl., De-Toni, Syll. Alg. iii. I86.

Banksii (Turn.), Decne., De-Toni, Syll. Alg. iii. I87.

articulata (Forsk.), Zanard, De-Toni, Syll. Alg. iii. I88=

Cy'stoseira articulata, J. Ag., Bail. Syn. Q1. F1. 785.

Fucus (Tourn.), Linn., De-Toni, Syll. Alg. iii. I94.

vesiculosus, Limn., De-Toni, Syll. Alg. iii. 206.

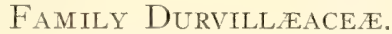

Sarcophycus, Kuetz., De-Toni, Syll. Alg. iii. 222.

potatorum (Labill.), Kuetz., De-Toni, Syll. Alg. iii. 222.

\section{ORDER TETRASPORIN 玉.}

\section{Family Dictyotace e.}

Gymnosorus, J. Ag., De-Toni, Syll. Alg. iii. 227.

variegatus (Lamour.), J. Ag., De-Toni, Syll. Alg. iii. $227=$

Zonaria variegata, Mart., Bail. Syn. Q1. F1. 786.

nigrescens (Sond.), J. Ag., De-Toni, Syll. Alg. iii. $228=$

Zonaria nigrescens, Sond., Bail. Syn. Q1. F1. 786.

Zonaria (Draparn.), Ag., De-Toni, Syll. Alg. iii. 229.

crenata, J. Ag., De-Toni, Syll. Alg. iii. 236. K. (Fig. 964.)

Stypopodium, Kuetz., De-Toni, Syll. Alg. iii. 239.

lobatum, Kuetz., De-Toni, Syll. Alg. iii. 239.

Taonia, J. Ag., De-Toni, Syll. Alg. iii. 240.

australasica, J. Ag., De-Toni, Syll. Alg. iii. 242.

Padina, Adans., De-Toni, Syll. Alg. iii. 242.

Pavonia (Linn.), Lamour., De-Toni, Syll. Alg. iii. 243.

Commersonii, Bory., De-Toni, Syll. Alg. iii. 244.

australis, Hauck., De-Toni, Syll. Alg. iii. 245. 
Spathoglossum, Kuetz., De-Toni, Syll. Alg. iii. 246.

Schrœderi (Mert.), J. Ag., De-Toni, Syll. Alg. iii. 249.

Haliseris, Targ-Jozz., De-Toni, Syll. Alg. iii. 253.

polypodioides (Desf.), Ag., De-Toni, Syll. Alg. iii. 254 ; var. denticulata, F.v. M., Fragm. xi. Suppl. p. 7.

Woodwardia (R. Br.), J. Ag., De-Toni, Syll. Alg. iii. 254.

Muelleri, Sond., De-Toni, Syll. Alg. iii. 255.

australis, Sond., De-Toni, Syll. Alg. iii. 255.

Dictyota, Lamour., De-Toni, Syll. Alg. iii. 260. nigricans, J. Ag., De-Toni, Syll. Alg. iii. 26r.

Bartayresiana, Lamour., De-Toni, Syll. Alg. iii. 262.

dichotoma (Huds.), Lamour., De-Toni, Syll. Alg. iii. 263.

sandvicensis, Sond., De-Toni, Syll. Alg. iii. 269.

radicans, Harv., De-Toni, Syll. Alg. iii. 273.

ciliata, J. Ag., De-Toni, Syll. Alg. iii. 274.

bifurca, J. Ag., De-Toni, Syll. Alg. iii. 279.

furcellata, $A g$., De-Toni, Syll. Alg. iii. 280.

prolificans, A. and E. S. Gepp., Journ. of Bot. Aug. 1906.

Dilophus, J. Ag., De-Toni, Syll. Alg. iii. 284.

fastigiatus (Sond.), J.Ag., De-Toni, Syll. Alg. iii. $288=$ Dictyota fastigiata, Sond., Bail. Syn. Q1. Fl. 787.

\section{ORdER PHAOZOOSPORIN无.}

\section{Family Laminariacee.}

Ecklonia, Hornem., De-Toni, Syll. Alg. iii. 353.

radiata (Turn.), J. Ag., De-Toni, Syll. Alg. iii. 354.-Moreton Bay.

forma exasperata (Harv.),F.v.M., Fragm. xi. 7.-Cooktown.

FAmily Sporochnacee.

Perithalia, J. Ag., De-Toni, Syll. Alg. iii. 377. inermis, R.Br., De-Toni, Syll. Alg. iii. 378. K.

\section{Family Stilophoraceæ.}

Stilophora, J. Ag., De-Toni, Syll. Alg. iii. 390.

rhizodes (Ehrh.), J. Ag., De-Toni, Syll. Alg. iii. 390. K. (Fig. 965.)

\section{Family Arthrocladiace.}

Chnoospora, J. Ag., De-Toni, Syll. Alg. iii. 464.

obtusangula (Harv.), Sond., De-Toni, Syll. Alg. iii. 465. ? implexa (Hering.), J. Ag., De-Toni, Syll. Alg. iii. 466. 
Family Encoliaceæ.

Colpomenia, Derb. and Sol., De-Toni, Syll. Alg. iii. 489.

sinuosa (Roth.), Derb. and Sol., De-Toni, Syll. Alg. iii. 489.

(Under Asperococcus, Lamour., in Syn. Q1. F1. 787.)

This is considered a troublesome oyster-pest.

Hydroclathrus, Bory., De-Toni, Syll. Alg. iii. 490.

cancellatus, Bory., De-Toni, Syll. Alg. iii. 490.

Family Sphacelariaceæ.

Sphacelaria, Lyngb., De-Toni, Syll. Alg. iii. 499.

furcigera, Kuetz., De-Toni, Syll. Alg. iii. 506.

Stypocaulon, Kuetz., De-Toni, Syll. Alg. iii. 5r6.

paniculatum (Suhr.), Kuetz., De-Toni, Syll. Alg. iii. 5I6 = Sphacelaria paniculata, Lyngb., Syn. Q1. F1. 787.

Family Ectocarpacee.

Ectocarpus, Lyngb., De-Toni, Syll. Alg. iii. 537.

simplicinoculus, Ag., De-Toni, Syll. Alg. iii. 538; var.

viliensis, Asken., Q1. Bot. Bull. xi. 57.

indicus, Sond., De-Toni, Syll. Alg. iii. 546.

siliculosus (Dillw.), Lyngb., De-Toni, Syll. Alg. iii. 549; var., Asken., Q1. Bot. Bull. xi. 56.

\section{FLORIDERE.}

Subclass BANGIOIDEA.

Family Compsopogonace.

Compsopogon, Mont., De-Toni, Syll. Alg. iv. 28.

cæruleus (Balb.), Mont., De-Toni, Syll. Alg. iv. 29.

\section{Subclass EU-FLORIDE王.}

\section{Order NEMALIONIN玉.}

\section{Family Helminthocladiace..}

Sub-Family Batwachospermea.

Batrachospermum, Roth., De-Toni, Syll. Alg. iv. 49. moniliforme, Roth., De-Toni, Syll. Alg. iv. 50. Dillenii, Bory., De-Toni, Syll. Alg. iv. 57. vagum, Ag., De-Toni, Syll. Alg. iv. 58 .

var. flagelliforme, Sirod., De-Toni, Syll. Alg. iv. 59. sp., Mobius, Q1. Bot. Bull. vi. 4. 


\section{Sub-Family Chantransiex.}

Chantransia (DC.), Schmitz., De-Toni, Syll. Alg. iv. 67. violacea, Kuetz., De-Toni, Syll. Alg. iv. 74. pygmæa, Kuetz., De-Toni, Syll. Alg. iv. 74. subtilis, Mobius, Q1. Bot. Bull. xi. Io, and De-Toni, Syll. Alg. iv. App. I865.

\section{Sub-Family Nemaliea.}

Liagora, Lamour., De-Toni, Syll. Alg. iv. 86. leprosa, J. Ag., De-Toni, Syll. Alg. iv. 87.

\section{Family Chatangiace旡.}

\section{Sub-Family Chotangiex.}

Brachycladia, Sond., De-Toni, Syll. Alg. iv. Io8. marginata, Soland., f. dilatata (Kuetz.), J. Ag., De-Toni, Syll. Alg. iv. I Io = Galaxauria dilata, Kuetz. K.

Galaxaura, Lamour., De-Toni, Syll. Alg. iv. I Io.

obtustata (Soland.), Lamour., De-Toni, iv. IIo. K. (Fig. g66.)

umbellata (Esper.), Lamour., De-Toni, Syll. Alg. iv. I I . fragilis (Lamk.), Kuetz., De-Toni, Syll. Alg. iv. Iı2. K. rugosa (Soland.), Lamour., De-Toni, Syll. Alg. iv. I I3. elongata, J. Ag., De-Toni, Syll. Alg. iv. II3. lapidescens (Soland.), Lamour., De-Toni, Syll. Alg. iv. II4.

Actinotrichia, Decne, De-Toni, Syll. Alg. iv. I 16. rigida (Lamour.), Decne., De-Toni, Syll. Alg. iv. II7.

Family Geididiacer.

Sub-Family Gelidiea.

Gelidium, Lamour., De-Toni, Syll. Alg. iv. I44. pusillum (Stackh.), Le Jol., De-Toni, Syll. Alg. iv. I47. K. rigidum (Vahl.), Grev., De-Toni, Syll. Alg. iv. I49. latifolium, Born., De-Toni, Syll. Alg. iv. I50 $=$ G. corneum, Grev., Bail. Syn. Q1. F1. 794.

\section{Order GIGARTININ在. \\ Family Gigartinaceæ. \\ Sub-Family Gigartinea.}

Gigartina, Stackh., De-Toni, Syll. Alg. iv. I96. brachiata, Harv., De-Toni, Syll. Alg. iv. 200. K.

Sub-Family Tylocarpea.

Gymnogongrus, Mart., De-Toni, Syll. Alg. iv. 240. assimilis (Sond.), F.v. M., Fragm. xi. Suppl. I05. 
Sub-Family Dicranemex.

Dicranema, Sond., De-Toni, Syll. Alg. iv. 268.

setaceum, Sond., De-Toni, Syll. Alg. iv. 272.

\section{Famili Rhodophyllidace.e. \\ Sub-Family Ci'stocloniea.}

Catenella, Grev., De-Toni, Syll. Alg. iv. 3I7.

Opuntia (Good. and Woodw.), Grev., De-Toni, Syll. Alg. iv. 3I8.

\section{Sub-Family Solieriece.}

Erythroclonium, Sond., De-Toni, Syll. Alg. iv. 35.3.

Sonderi, Harv., De-Toni, Syll. Alg. iv. 354. K.

Muelleri, Sond., De-Toni, Syll. Alg. iv. 355.

Solieria, J. Ag., De-Toni, Syll. Alg. iv. 365.

chordalis (Ag.), De-Toni, Syll. Alg. iv. 366.

Eucheuma, J. Ag., De-Toni, Syll. Alg. iv. 368.

spinosum (Linn.), J. Ag., De-Toni, Syll. Alg. iv. 369.

Thysanocladia, Endl., De-Toni, Syll. Alg. iv. 379.

densa, Sond., De-Toni, Syll. Alg. iv. 380.

laxa, Sond., De-Toni, Syll. Alg. iv. 383 .

\section{Order RHODYMENIN无.}

FAMily SpHarococcaceæ.

Sub-Family Ceratodictyea.

Ceratodictyon, Zanard, De-Toni, Syll. Alg. iv. 409. spongiosum, Zanard = Spongia cartilaginea. $\mathrm{K}$.

Gelidiopsis ? Schmitz., De-Toni, Syll. Alg. iv. 4 IO.

acrocarpa (Harv.), Schmitz., De-Toni, Syll. Alg. iv. 4I I =

Gelidium acrocarpum, Harv., Syn. Q1. F1. 794.

Sarcodia, J. Ag., De-Toni, Syll. Alg. iv. 4I3.

palmata, Sond., De-Toni, Sy11. Alg. iv. 4I4.

\section{Sub-Family Gracilariea.}

Gracilaria, Grev., De-Toni, Syll. Alg. iv. 428.-Agar-agar; edible. lichenoides (Linn.), Harv., De-Toni, Syll. Alg. iv. 430.-The substance Thao jelly is made from this plant. In India it is known as Ceylon Moss, and is considered as a nutritive, emollient, demulcent, and alterative (Dr. Djmock).

confervoides (Linn.), Grev., De-Toni, Syll. Alg. iv. 43I.

var. capillaris (Kuetz.), F.v.M., Fragm. xi. Supp1. 22.

var. secundata, F.v. M., Fragm. xi. Suppl. 22.

tænioides, J. Ag., De-Toni, Syll. Alg. iv. 432.

compressa (Ag.), Grev., De-Toni, Syll. Alg. iv. 438. 
Gracilaria-contd.

Textorii, Suring., De-Toni, Syll. Alg. iv. 449, A. and E. S.

Gepp. in Journ. Bot. 44, 256.

canaliculata (Kuetz.), Sond., De-Toni, Syll. Alg. iv. 455.

polyclada, Sond., De-Toni, Syll. Alg. iv. 455.

Lucasii, Gepp., J1. Bot. 44 (I906) 256. K.

Corallopsis, Grev., De-Toni, Syll. Alg. iv. 457.

minor (Sond.), J. Ag., De-Toni, Syll. Alg. iv. 459.

Urvillei (Mont.), J. Ag., De-Toni, Syll. Alg. iv. 459.

Cereus (J. Ag.), F.v. M., Fragm. xi. Suppl. I05.

Sub-Family Hypneex.

Hypnea, Lamour., De-Toni, Syll. Alg. iv. 469.

musciformis (Wulf.), Lamour., De-Toni, Syll. Alg. iv. 472.

Has been used as a vermifuge. K. (Fig. 967.)

seticulosa, J. Ag., De-Toni, Syll. Alg. iv. 476.

divaricata, Grev. De-Toni, Syll. Alg. iv. 478.

cornuta (Lamour.), J. Ag., De-Toni, Syll. Alg. iv. 478.

valentiæ (Grom.), Mont., De-Toni, Syll. Alg. iv. 479.

nidifica, J. Ag., De-Toni, Syll. Alg. iv. 479.

cervicornis, J. Ag., De-Toni, Syll. Alg. iv. 480.

pannosa, J. Ag., De-Toni, Syll. Alg. iv. 482.

rugulosa, Mont., De-Toni, Syll. Alg. iv. 484.

Family Rhodymeniacer.

Sub-Family Rhodymeniece.

Epymenia, Kuetz., De-Toni, Syll. Alg. iv. 524.

? cuneata (Harv.), J. Ag., De-Toni, Syll. Alg. iv. 526.

Sebdenia, Berth., De-Toni, Syll. Alg. iv. 53o.

ceylanica (Harv.), Heydr., De-Toni, Syll. Alg. iv. 530.

maculata (J. Ag.), De-Toni, Syll. Alg. iv. 53I = Halymenia maculata, J. Ag. K.

Chrysymenia, J. Ag., De-Toni, Syll. Alg. iv. 537.

Uvaria (Linn.), J. Ag., De-Toni, Syll. Alg. iv. 543.

var. leptopoda, J. Ag., De-Toni, Syll. Alg. iv. 543.

Champia, Desv., De-Toni, Syll. Alg. iv. 556 .

parvula (Ag.), J. Ag., De-Toni, Syll. Alg. iv. $55^{8}$.

\section{Sub-Family Plocamiece.}

Plocamium, Lamour., De-Toni, Syll. Alg. iv. 586.

hamatum, J. Ag., De-Toni, Syll. Alg. iv. 589.

coccineum (Huds.), De-Toni, Syll. Alg. iv. 59o. K.

Preissianum, Sond., De-Toni, Syll. Alg. iv. 591. K. 


\section{FAmily Delesseriacez.}

Sub-Family Delesseriece.

Hypoglossum, De-Toni, Syll. Alg. iv. 68 I.

sp., A. D. Cotton. K.

\section{Sub-Family Sarcomeniea.}

Caloglossa, Harv., De-Toni, Syll. Alg. iv. 728.

mnioides, Harv., De-Toni, Syll. Alg. iv. 729.-A form with smaller cells than in the typical plant. $\mathrm{K}$.

\section{Family Bonnemaisoniaceæ.}

Delisea, Lamour., De-Toni, Syll. Alg. iv. 760.

pulchra (Grev.), Mont., De-Toni, Syll. Alg. iv. 763.

Asparagopsis, Mont., De-Toni, Syll. Alg. iv. 769 .

armata, Harv., De-Toni, Syll. Alg. iv. 772.

\section{Sub-Family Laurencica.}

Laurencia, Lamour., De-Toni, Syll. Alg. iv. 777.

Forsteri (Mert.), Grev., De-Toni, Syll. Alg. iv. 779. K.

gracilis, Hook. and Harv, De-Toni, Syll. Alg. iv. 780.

nidifica, J. Ag., De-Toni, Syll. Alg. iv. 785 ; var. tenuioris, F. i. M., Fragm. xi. Suppl. 29.

divaricata, J. Ag., De-Toni, Syll. Alg. iv. 786.

dendroidea, J.Ag., De-Toni, Syll. Alg. iv. $787=$ L. Vieillardi,

Kuetz., Bail. Syn. Q1. F1. 790.

rigida, J. Ag., De-Toni, Syll. Alg. iv. 789.

papillosa (Forsk.), Grev., De-Toni, Syll. Alg. iv. 789.

var. thyrsoides, F.v.M., Fragm. xi. Suppl. 3 o.

obtusa (Huds.), Lamour., De-Toni, Syll. Alg. iv. 79I. K.

pannosa, Zanard, De-Toni, Syll. Alg. iv. 794.

thujoides (Kuetz.), F.v.M., Fragm. xi. Suppl. 30.

pinnatifida (Gmel.), Lamour., De-Toni, Syll. Alg. iv. 798.

concinna, Mont., De-Toni, Syll. Alg. iv. $806=$ L. coccinea,

Mont., typographical error in Syn. Q1. F1. 790.

\section{Sub-Family Chondriece.}

Acanthophora, Lamour., De-Toni, Syll. Alg. iv. 8I6. muscoides (Linn.), Bory., De-Toni, Syll. Alg. iv. 8I8=

A. ramulosa, Lind1., Bail. Syn. Q1. F1. 789.

dendroides, Harv., De-Toni, Syll. Alg. iv. 820. K.

Thierii, Lamour., De-Toni, Syll. Alg. iv. 820. K. orientalis, J. Ag., De-Toni, Syll. Alg. iv. 822.

Chondria, Ag., De-Toni, Syll. Alg. iv. 828.

dasyphylla (Woodw.), Ag., De-Toni, Syll. Alg. iv. 842. 


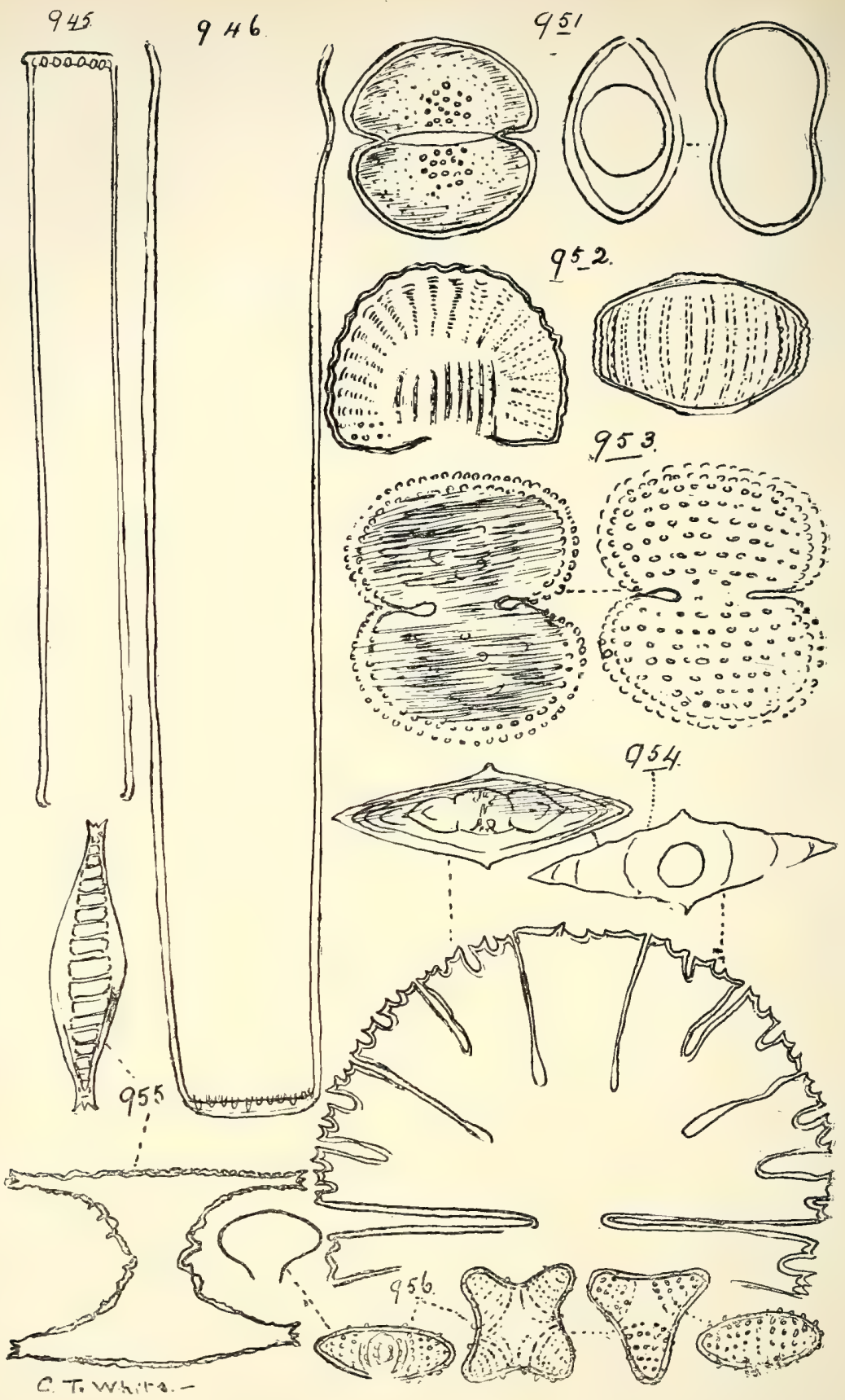

945. Pleurotenium cristatum, Borge (x 740). (After Borge.)

946. P. Stuhlmannir, Schmidle (x 405). (After Borge.)

951. Cosmarium Perforatum, Lund. (After Lundell.)

952. C. Subspeciosum, Nordst., var. Validius, Nordst. (x 570). (After Nordstedt.)

953. C. LAtum, Bréb. (After Cooke.)

954. Micrasterias denticulata, Ralfs, var. notata, Nordst. (After Nordstedt.)

955. Staurastrum PSeudosebaldi, Wille, subsp. tonsum, Nordst. (After Nordstedt.)

956. S. dilatatum, Ehrenb., var. obtusilobum, De Not. (After Nordst.) 
Sub-Family Polysiphoniea.

Polysiphonia, Grev., De-Toni, Syll. Alg. iv. 866.

mollis, Hook. and Harv., De-Toni, Syll. Alg. iv. 877.

purpureo-cærulea (Sond.), F.v.M., Fragm. xi. I05.

ferulacea, Suhr., De-Toni, Syll. Alg. iv. 892.

Digenea, Ag., De-Toni, Syll. Alg. iv. 96r.

simplex (Wulf.), Ag., De-Toni, Syll. Alg. iv. 963.

Tolypiocladia, Schmitz., De-Toni, Syll. Alg. iv. 963.

glomerulata (Ag.), Schmitz., De-Toni, Syll. Alg. iv. $964=$ Polysiphonia glomerulata, End1., Syn. Q1. F1. 789.

\section{Sub-Family Pterosiphoniece.}

Polexfenia, Harv., De-Toni, Syll. Alg. iv. 978.

lobata (Lamour.), Falkenberg, De-Toni, Syll. Alg. iv. 979 = Jeannerettia lobata, Hook. and Harv. K.

\section{Family Pterosiphoniex.}

Symphyocladia, Falkenb., De-Toni, Syll. Alg. iv. 989.

marchantioides (Harv.), De-Toni, Syll. Alg. iv. 989.

\section{Sub-Family Polyzoniea.}

Leveillea, Decaisne, De-Toni, Syll. Alg. iv. I033.

jungermannioides (Mont. and Hering.), Harv., De-Toni, Syll.

Alg. iv. I033. (L. Schimperi, Dcne, and L. gracilis,

Dcne., of Syn. Q1. F1. 788, both included.)

\section{Sub-Family Rytiphloce.}

Amansia, Lamour., De-Toni, Syll. Alg. iv. I082.

glomerata, Ag., De-Toni, Syll. Alg. iv. I086.

Dietrichiana, Grun., De-Toni, Syll. Alg. iv. Io87.

Dæmelii (Sond.), J.Ag., De-Toni, Syll. Alg. iv. Io88= Vidalia Damelii, Sond., Syn. Q1. F1. 788.

pumila (Soncl.), J.Ag., De-Toni, Syll. Alg. iv. Io88= Vidalia pumila, Sond., Syn. Q1. F1. 788.

Vidalia, Lamour., De-Toni, Syll. Alg. iv. I Ioo.

fimbriata (R. Br.), J. Ag., De-Toni, Syll. Alg. iv. IIo3.

spiralis, Iam., De-Toni, Syll. Alg. iv. I ro6. K.

Neurymenia, J. Ag., De-Toni, Syll. Alg. iv. I I Io.

fraxinifolia (Mert.), J. Ag., De-Toni, Syll. Alg. iv. Ir I2.

Lenormandia, Sond., De-Toni, Syll. Alg. iv. I I 2.

spectabilis, Sond. =L. latifolia, Harv., De-Toni, Syll. Alg. iv. $\operatorname{III7.~K.~}$ 
Sub-Family Dasyea.

Dasya, Ag., De-Toni, Syll. Alg. iv. I 183.

elongata, Sond., De-Toni, Syll. Alg. iv. I Ig6.

cuspidifera, Sond., De-Toni, Syll. Alg. iv. I2I3.

Heterosiphonia, Mont., De-Toni, Syll. Alg. iv. I2r6.

multiceps (Harv.), De-Toni, Syll. Alg. iv. I233 = Dasya multiceps, Harv., Syn. Q1. Fl. 789.

\section{Family Ceramiaceæ.}

Sub-Family Callithamniea.

Callithamnion, Lyngb., De-Toni, Syll. Alg. iv. I3II.

corymbosum (Sm.), Lyngb., De-Toni, Syll. Alg. iv. I329.

var. australis, Asken., De-Toni, Syll. Alg. iv. I330, Bail. Q1. Bot. Bull. xi. 58.

forma, Asken., Q1. Bot. Bull. xi. 59.

Sub-Family Spongocloniea.

Haloplegma, Mont., De-Toni, Sy11. Alg. iv. I364.

Duperreyi, Mont., De-Toni, Syll. Alg. iv. I365.

\section{Sub-Family Spyridiea.}

Spyridia, Harv., De-Toni, Syll. Alg. iv. I424.

filamentosa (Wulf.), Harv., De-Toni, Syll. Alg. iv. I427.

breviarticulata, J. Ag., De-Toni, Syll. Alg. iv. I427.

\section{Sub-Family Ceramiece.}

Ceramium, Wiggers., De-Toni, Syll. Alg. iv. I443.

tenuissimum (Lyngb.), J. Ag., De-Toni, Syll. Alg. iv. I450.

var. pygmæum (Kuetz. ?), De-Toni, Syll. Alg. iv. $1452=$

C. pygmaum? Kuetz., Bail. Q1. Bot. Bull. xi. 59.

clavulatum, Ag., De-Toni, Syll. Alg. iv. I49I = Centroceras clavillatum, Ag., Bail. Syn. Q1. Fl. 799.

\section{Family Grateloupiace.e.}

Halymenia, C. Ag., De-Toni, Syll. Alg. iv. I537.

lacerata, Sond., De-Toni, Syll. Alg. iv. I540.

Floresia (Clem.), Ag., De-Toni, Syll. Alg. iv. I545.

Prionitis, J. Ag., De-Toni, Syll. Alg. iv. I 586.

obtusa, Sond., De-Toni, Syll. Alg. iv. I 593.

Carpopeltis, Schmitz., De-Toni, Syll. Alg. iv. I604.

capitellata (Sond.), Schmitz., De-Toni, Syll. Alg. iv. I6o6.

(Under Cryptonemia in Syn. Q1. Fl. 797.) 
Cryptonemia, J. Ag., De-Toni, Syll. Alg. iv. I607.

luxurians (Mert.), J. Ag., De-Toni, Syll. Alg. iv. I6og.

undulata, Sond., De-Toni, Syll. Alg. iv. 16 I0.

Baileyi, E. M. Holmes in Litt. (I885).

Thamnoclonium, Kuetz., De-Toni, Syll. Alg. iv. I6I4.

Tissotii, Weber. K.

\section{Family Rhizophyldace}

Chondrococcus, Kuet:., De-Toni, Syll. Alg. iv. I673=Desmia, Lyngb., Bail. Syn. Q1. F1. 706.

Hornemanni (Mert.), Schmitz., De-Toni, Syll. Alg. iv. I674 = Desmia pulvinata, Ag., Bail. Syn. Q1. F1. 706.

Kilneri (J. Ag.), Dc-Toni, Syl1. Alg. iv. I676.

Harveyi (J. Ag.), De-Toni, Syll. Alg. iv. I676.

\section{Family Squamariacex. \\ Sub-Family Squamariea.}

Peyssonnelia, Decne., De-Toni, Syll. Alg. iv. I692. australis, Sond., De-Toni, Syll. Alg. iv. I699.

\section{Family Corallinacel.}

Lithothamnion, Phil., De-Toni, Syll. Alg. iv. I729. glaciale, Kjellm., De-Toni, Syll. Alg. iv. I730. membranaceum (Esp.), Fosl., De-Toni, Syll. Alg. iv. I758.

Melobesia, Lamour., De-Toni, Syll. Alg. iv. I764. farinosa, Lamour., De-Toni, Syll. Alg. iv. I764.

Dermatolithon, Fosl., De-Toni, Syll. Alg. iv. I77 1. pustulatum (Lamour.), Fosl., De-Toni, Syll. Alg. iv. I771.

Mastophora, Decaisne, De-Toni, Syll. Alg. iv. I774. plana (Sond.), Harv., De-Toni, Syll. Alg. iv. I775.

Amphiroa, Lamour., De-Toni, Syll. Alg. iv. I805. gracilis, Harv., De-Toni, Syll. Alg. iv. I8og. anceps (Lamarck), Decne., De-Toni, Syll. Alg. iv. I8I 5. granifera, Harv., De-Toni, Syll. Alg. iv. I8I9. K. (Fig. 968.)

Cheilosporum, Aresch., De-Toni, Syll. Alg. iv. 1822. spectabile, Harv., De-Toni, Syll. Alg. iv. I826.

Wardii (Harv.), De-Toni, Syll. Alg. iv. ${ }^{8} 828=$ Arthrocardia W araii, Aresch., Bail. Syn. Q1. F1. 79I. sagittatum (Lamour.), Aresch., De-Toni, Syll. Alg. iv. I832.

Corallina (Tournef.), Lamour., De-Toni, Syll. Alg. iv. I834. rubens, Linn., De-Toni, Syll. Alg. iv. I836. granifera, Ell. and Soland., De-Toni, Syll. Alg. iv. I845. Cuvieri, Lamour., De-Toni, Syll. Alg. iv. I848. 
Corallina-contd.

(Jania) fastigiata, Harv., De-Toni, Syll. Alg. iv. I854.

(Jania) micrarthrodia, Lamour., De-Toni, Syll. Alg. iv. I855; var. tenuissima (Sol.), F.v. M., Fragm. xi. Suppl. 21.

(Jania) natalensis, Harv., De-Toni, Syll. Alg. iv. 1856.

\section{MYYXOPHYCEA.}

\section{ORDER COCCOGONEA.}

\section{Family Chroococcaceds.}

Chroococcus Nag., De-Toni, Syll. Alg. v. 7 .

cohærans (Bréb.), Nag., De-Toni, Syll. Alg. v. 2I.-Nundah (C. T. White). Det. by G. S. West.

minor (Kuetz.), Nag., De-Toni, Syll. Alg. v. 23--Nundah

(C. T. White). Det. by G. S. West.

Synechococcus, Nceg., De-Toni, Syll. Alg. v. 26. æruginosus, Narg., De-Toni, Syll. Alg. v. 27.

Glœothece, Nag., De-Toni, Syll. Alg. v. 60. Baileyana, Schmidle, De-Toni, Syll. Alg. v. бі.

Clathrocystis, Henfrey, De-Toni, Syll. Alg. v. 94. æruginosa (Kuetz.), Henfrey, De-Toni, Syll. Alg. v. 94.

Merismopedium, Meyen., De-Toni, Syll. Alg. v. I04. glaucum (Ehrh.), Nag., De-Toni, Syll. Alg. v. 105. convolutum, Bréb., De-Toni, Syll. Alg. v. Io8. revolutivum, Asken., De-Toni, Sy11. Alg. v. rog.

\section{ORder HORMOGONEÆ.}

\section{Family Oscillatoriace瓜.}

Sub-Family Oscillatoriee.

E Oscillatoria, Vauch., De-Toni, Syll. Alg. v. I47. princeps, Vauch., De-Toni, Syll. Alg. v. I50. sancta (Kuetz.), Gom., De-Toni, Syll. Alg. v. I53.

var. caldariorum, Lagerh., De-Toni, Syll. Alg. v. I54. limosa (Roth.), Ag., De-Toni, Syll. Alg. v. I54,-Brisbane

Botanic Gardens (C. T. White). Det. by G. S. West. nigro-viridis, Thwaites, De-Toni, Syll. Alg. v. I6r. tenuis, Ag., De-Toni, Syll. Alg. v. I66. geminata (Menegh.), Gomont., De-Toni, Syll. Alg. v. I72.In a ditch, Brisbane (C. T. White). Det. by G. S. West. splendida, Grev., De-Toni, Syll. Alg. v. I73 = O. leptotricha, Kuetz., Bail. Q1. Bot. Bull. vi. 55.

chalybea (Mertens), Gom., De-Toni, Syll. Alg. v. I85.-In a ditch, Brisbane (C. T. White). Det. by G. S. West. 
Trichodesmium, Ehrenb., De-Toni, Syl1. Alg. v. 202.

erythræum, Ehrenb., De-Toni, Syll. Alg. v. 202. - The Red

Sea is said to derive its name from the scum formed by this plant. It emits a strong disagreeable odour, which affects the mucous membrane, sometimes causing inflammation of the eyes and troublesome sneezing.

Sub-Family Spirulinea.

Arthrospira, Stizenb., De-Toni, Syll. Alg. v. 206.

Jenneri (Kuetz.), Stiz., De-Toni, Syll. Alg. v. 206.-Nundah (C. T. White). Det. by G. S. West.

Spirulina, Turpin, De-Toni, Syll. Alg. v. 208.

princeps, $W$. and G. S. West, De-Toni, Syll. Alg. v. 2I I.Mosman River (F. Muir). (G. S. West.)

Sub-Family Lyngbyece.

Phormidium, Kuetz., De-Toni, Syll. Alg. v. 217.

molle (Kuetz.), Gom., De-Toni, Syll. Alg. v. 219.-Mosman River (F. Muir). (West.)

tenue (Menegh.), Gom., De-Toni, Syll. Alg. v. 227.-Nundah

(C. T. White). Det. by G. S. West.

solitare (Kuetz.), Rabenh., De-Toni, Syll. Alg. v. $236=$ Lyngbya solitaris, Kuetz., Bail. Q1. Bot. Bull. vi. 55.

Retzii (Ag.), Gom., De-Toni, Syll. Alg. v. 24I.-Mosman River (F. Muir). (G. S. West.)

Lyngbya, C. Ag., De-Toni, Syll. Alg. v. 258.

æstuarii (Mert.), Liebm., De-Toni, Syll. Alg. v. 262.

majuscula (Dillw. ?), Harv., De-Toni, Syll. Alg. v. 268.

ærugineo-cærulea (Kuetz.), Gum., De-Toni, Syll. Alg. v. 28I.

Sub-Family Schizotrichea.

Hydrocoleus, Kuetz., De-Toni, Syll. Alg. v. 3 I 5 .

lyngbyaceus, Kuetz., De-Toni, Syll. Alg. v. 317.

Microcoleus, Desm., De-Toni, Syll. Alg. v. 370.

tenerrimus, Gomont, De-Toni, Syll. Alg. v. 373.

paludosus (Kuetz.), Gomont, De-Toni, Syll. Alg. v. 376.

\section{Family Nostochace.e.}

\section{Sub-Family Heterocystidece.}

Nostoc, Vauch., De-Toni, Syll. Alg. v. 386.

paludosum, Kuetz., De-Toni, Syll. Alg. v. 390.

Linckia (Roth.), Bornet., De-Toni, Syll. Alg. v. 39I.

Passerinianum (De Not.), Bornet et Thuret, De-Toni, Syll.

Alg. v. 399. Hab.: In grass land, after rain. Coll., ?

Det. by A. D. Cotton.

cæruleum, Lyngbye, De-Toni, Syll. Alg. v. 4I6. 
Nodularia, Mertcns, De-Toni, Syll. Alg. v. 432.

spumigena, Mertens, De-Toni, Syll. Alg. v. 433.

var. genuina, Born. and Flah., De-Toni, Syll. Alg. v. 433.

Anabæna, Bory., De-Toni, Syll. Alg. v. 437.

Flos-aquæ (Lyngb.), Bréb., De-Toni, Syll. Alg. v. 44I.

Cylindrospermum, Kuetz., De-Toni, Syli. Alg. v. 47r.

licheniforme (Bory.), Kuetz., De-Toni, Syll. Alg. v. 476.

Microchæe, Thur., De-Toni, Syll. Alg. v. 482.

tenera, Thur., De-Toni, Syll. Alg. v. 482.

var, major, Mobius, De-Toni, Syll. Alg. v. 483.

\section{Family Scytonemace旡.}

Plectonema, Thur., De-Toni, Syll. Alg. v. 489.

Wollei, Farl., De-Toni, Syll. Alg. v. 489.

Tomasinianum (Kuetz.), Born., De-Toni, Syll. Alg. v. 490. - Upper Walsh River (Dr. T. L. Bancroft). Borge.) (Fig. 967.)

Scytonema, Ag., De-Toni, Syll. Alg. v. 498.

crispum (Ag.), Born., De-Toni, Syll. Alg. v. $498=$ S. cincinnatum, Thur., Bail. Q1. Bot. Bull. xi. 44.

subtile, Mabius, Q1. Bot. Bull. vi. 53 .

sp., Mœbius, Q1. Bot. Bull. xi. 45.

Tolypothrix, Kuetz., De-Toni, Syll. Alg. v. 540.

tenuis, Kuetz., De-Toni, Syll. Alg. v. 545 .

\section{Family Stigonemace..}

Hapalosiphon, Noeg., De-Toni, Syll. Alg. v. 564.

fontinalis (Ag.), Born., De-Toni, Syll. Alg. v. $568=H$. pumilus, Kuetz., Q1. Bot. Bull. vi. 5I.

Stigonema, Ag., De-Toni, Syll. Alg. v. 577.

hormoides (Kuetz.), Born., De-Toni, Syll. Alg. v. 577.

Capsosira, Kuetz., De-Toni, Syll. Alg. v. 59I.

Brebissonii, Kuetz., De-Toni, Syll. Alg. v. 592.

Family Rivulariaceæ.

Calothrix, Ag., De-Toni, Syll. Alg. v. 603.

scopulorum (Web. and M.), Agardh., De-Toni, Syll. Alg. v. 608.

æruginea( Kuetz.), Thuret., De-Toni, Syll. Alg. v. 6rз.

crustacea, Thuret, De-Toni, Syll. Alg. v. 6I4=Leibleinia australis, Kuetz., Bail. Syn. Q1. F1. 803.

fusca (Kuetz.), Born. et Flah., De-Toni, Syll. Alg. v. 6r7.Barron River (Dr. T. L. Bancroft). (O. Borge.) (Fig. 970.)

parietina (Næg.), Thuret, De-Toni, Syll. Alg. v. 62 I. 


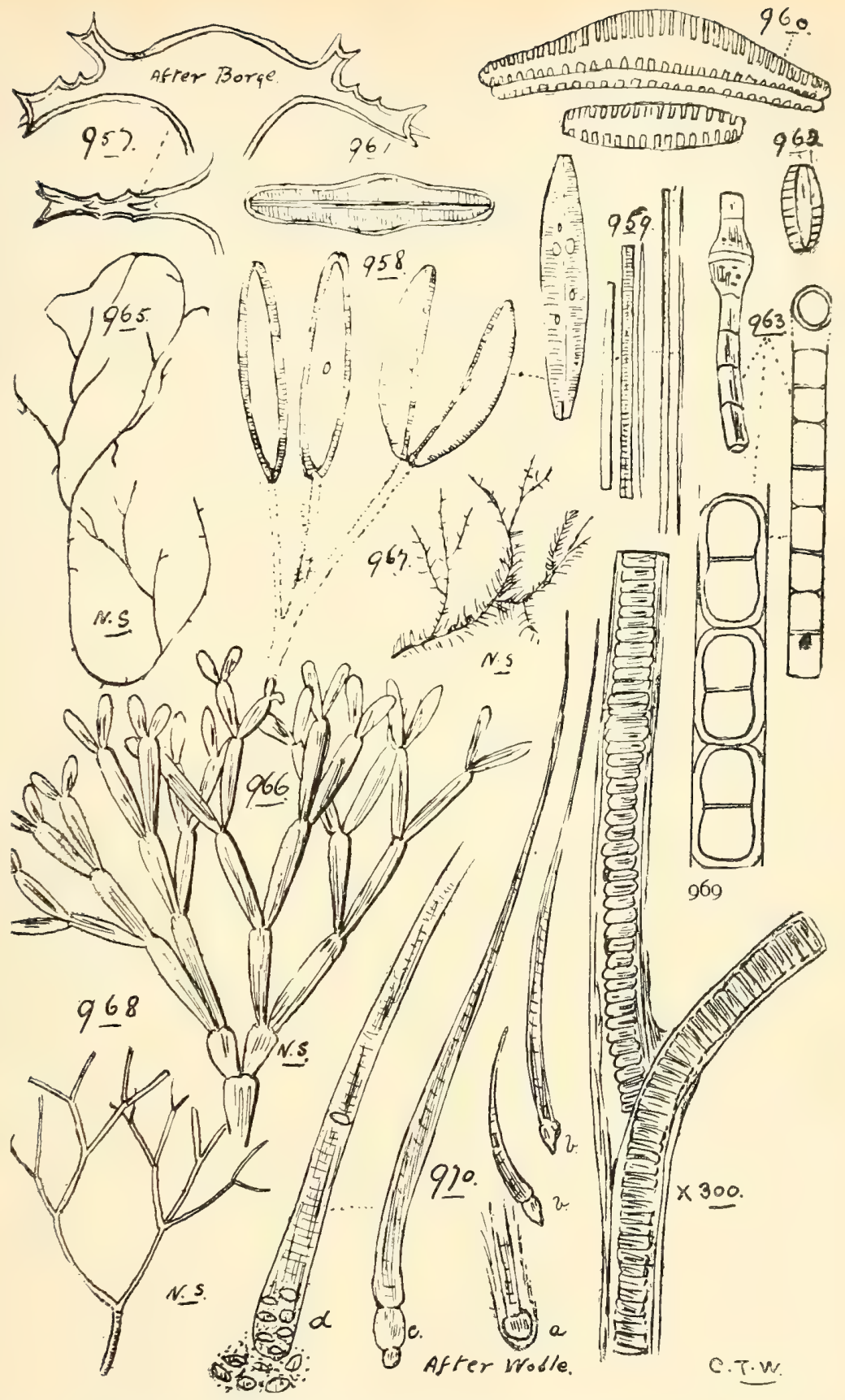

957. Staurastrum sexangulare, Lund, var. gemmescens, Play'f. (x 740). 958. Cymbella lanceolata, Kirchn. (After Rabenhorst.)

959. SyNedRA Act's, Kuetz. (After Rabenhorst.)

960. Cystopleura turgida, Kunze. (After Rabenhorst.)

96r. C. GIBBA, Kunze. (After Rabenhorst.)

962. C. Gibberula, Kunze. (After Rabenhorst.)

gó3. Lysigonium VARIANs, De-Toni.

965. STILOPHORA rHizOdES, $J . A g$.

966. Galaxa ura obtusata, Lamour.

367. HYPNEA MUSCIFORMIS, Lamour.

968. Amphíron granifera, Harv.

969. Plectonema Tomasinianum, Born. (After Cooke.)

070. Calothrix fusca, Born. et Flah.

(a) Form with spherical single heterocyst, (b) do. with single pyriform heterocyst, (c) do. with twin heterocysts, (d) the basal cells developing macrogonidia. (After Wolle.) 
Dichothrix, Zanard, De-Toni, Syll. Alg. v. 638.

Baneriana (Grunow), Born et Flah., De-Toni, Syll. Alg. v. 640.-Barron River (Dr. T. L. Bancroft). (O. Borge.)

Rivularia (Roth.), Ag., De-Toni, Syll. Alg. v. 648.

natans (Hedw.), Welwu., De-Toni, Syll. Alg. v. $648=$ Gloco trichia natans, Rabenh., Q1. Bot. Bull. xi. 42.

\section{Family Glaucophyceæ.}

Glaucocystis, Itzigs., De-Toni, Syl1. Alg. v. 693. Nostochinearum, Itzigs., De-Toni, Syll. Alg. v. 593.

Porphyridium, Noeg., De-Toni, Syll. Alg. v. 696.

cruentum (Ag.), Nag., De-Toni, Syll. Alg. v. 697. 


\section{ADDENDA AND CORRIGENDA.}

Considering the length of time this work has been in printing (three years), it might naturally be expected that this concluding part would be of considerable length; such, however, has been avoided by having all additions inserted, wherever possible, in their systematic places as the printing progressed.

December Igth, I9I2.

$$
\text { F.M.B. }
$$

\section{Order DILLENIACE $Æ$.}

P. I8.-Wormia alata: After "wood," add (Plate II.).

\section{Order CRUCIFERÆ.}

P. 33--Under Sisymbrium, for Iris, Linn, read Irio, Linn.

\section{Order TILIACEAE.}

P. 67.-Add to Aristotelia the following species:-

A. trilocularis, Bail., sp. now. The specimens received were but fragmentary, so but a short diagnosis can be here given:-A small tree, branchlets slender, ribbed, of a dark colour, and more or less clothed with short grey hairs. Leaves opposite, linearlanceolate, with a rather long tapering point, I I/2 to 3 in. long, 6 to 9 lines broad, pale on the underside. Peduncle about 6 lines, more under the fruit; pedicels very slender, 6 lines long under the flowers, twice that under the fruit. Sepals about one half the length of the petals, brown, incurved at the point, linear, seem about 5 or 6 ; petals white, same number as sepals. Stamens I2, 4 lines long; filaments flexuous, $2 \frac{1}{2}$ lines long; anthers emarginate, $\mathrm{I}^{\mathrm{T}} / 2$ line long. Style thick, about the length of the stamens. Fruit red, ovate, forming a point by the thickened base of the style, $1 / 2$ to $3 / 4$ in. long, 3-celled. This species differs principally from. $A$. megalosperma, F. v. M., in the number of fruit-cells.

Hab.: Babinda, Mrs. Rowan, Igr2.

\section{Order BURSERACEÆ.}

P. 85.-After Bursera australasica, Bail., add:-

Protium australasicum, Sprague, Kew Bull. I912, p. 370. This plant is placed by Mr. T. A. Sprague, 1.c., under the genus Protium, following Engler in DC. Monogr. Phan. vol. iv. As, however, I follow in all my publications as near as possible the Genera Plantarum of Bentham and Hooker, I leave it as heretofore.

\section{Order CELASTRINEÆ.}

P. 96.-After Celastrus Cunninghamii, F.v.M., add:zar. parvifolius, Bail., Q1. Agric. J1: xxix. (I9I2) p. I78, I1. 22. (Fig. 97I.) 


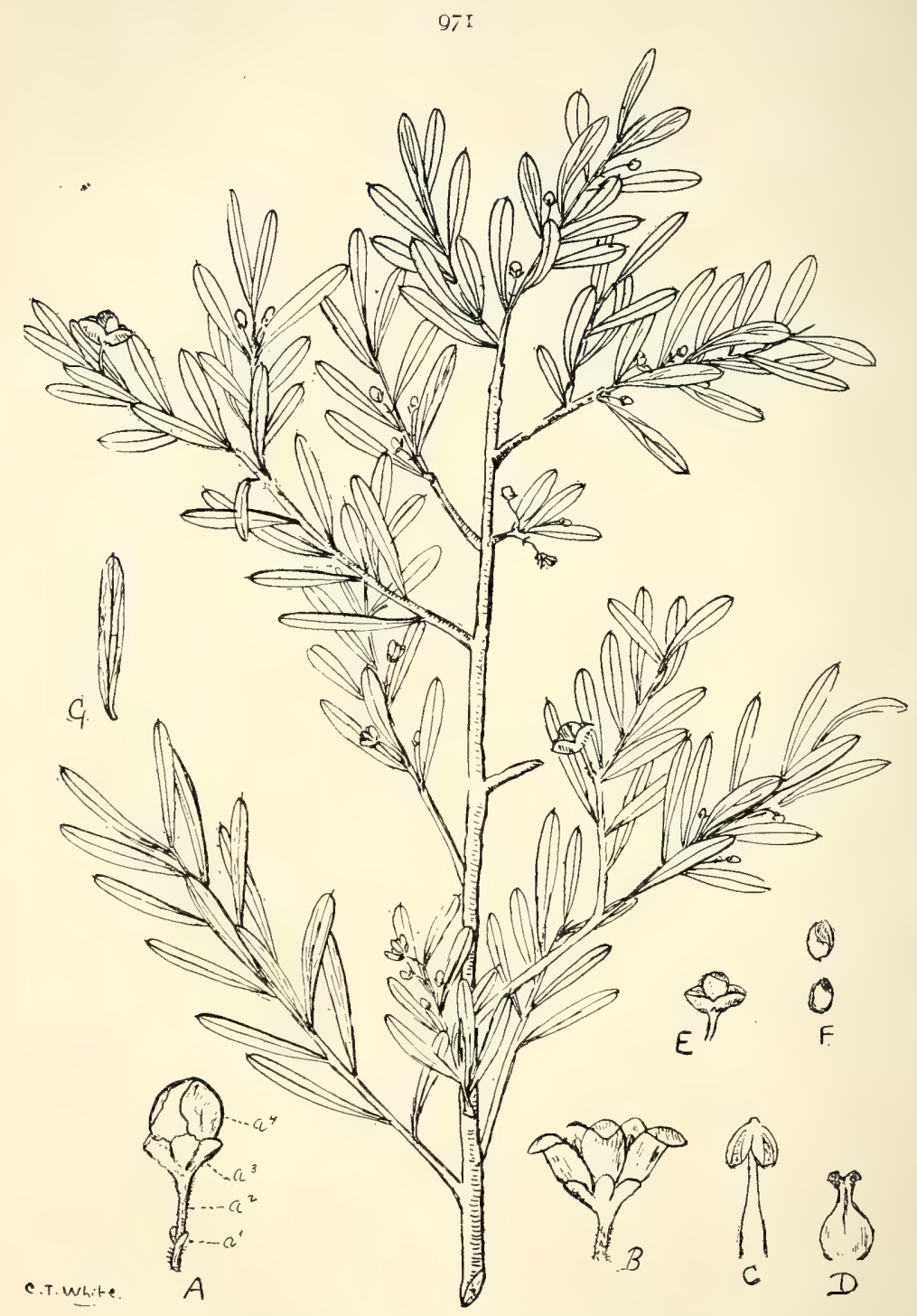

971. Celastrus Cunninghamit, F. v. M., var. parvifolius, Bail.

(A) Flower-bud, (a1) bracts, (a2) pedicel, (a3) calyx lobes, (a4) petals, (13) flower, (C) stamen, (D) pistil, (E) capsule, (F) seed with its arillus, (G) a single leaf to show veining. (A) -(D) enl.; (E) - (G) nat. size. 


\section{Order RHAMNACEÆ.}

P. Ioo.-Alphitonia: After excelsa, Reissek., add:var. franguloides.-A tree attaining $2 \frac{\mathrm{T}}{2} \mathrm{ft}$. in diameter; differs from all other Northern forms in its large oval-elliptical or oblong leaves, from 4 in. long and $1 / 2$ in. broad to $7 \mathrm{in.}$ long and 3 in. broad.

Hab.: Boar Pocket, near Barron River, J. F. Bailey, June, r899; Jordan Creek, Innisfail district, H. W. Mocatta, Nov., I9I2. Native name, "Maraticoola," H.W.M. This is the A. franguloides, Gray, Bot. Amer. Expl. Exped. i. 280, tab. 22.

\section{Order CONNARACE $Æ$.}

P. I24.-Tricholobus connaroides, F. v. M.: Judging from the fragmentary specimens received from time to time of the fruit and foliage of this plant it would appear that there are several distinct species or varieties in our Northern scrubs; in some the seeds are black with a yellow arillus, in other kinds the seeds are reddish or purplish and mottled, and show no arillus in the dried specimens.

In some black-seeded specimens recently received from Mrs. F. C. Rowan, collected at Babinda, the leaflets are very irregular and exceptionally large; the largest one measuring I4 in. in length and nearly 5 in. in breadth.

\section{Order LEGUMINOS ÆE.}

P. I25.-After Gompholobium virgatum, Sieb., add:-

var. emarginatum, Bail., n. var. (Fig. 972.) The present plant chiefly differs from the normal form in its larger and broader leaflets, which are deeply emarginate at the apex.

Hab.: Noosa, H. A. Longman, Oct. I9r2.

P. 139.-After Swainsona luteola, F.v.M., add:-

A short time ago I received specimens of this plant from Messrs. Statham Bros., of "Tregoning," Morven, with the following remarks:- "We had to destroy a mare and three foals, and there are now three others in as bad a condition and I have not much hope of them recovering; the cause of this is from eating a weed which causes them to slip their foals, and causes blindness, weakness in the loins, and, in fact, general uselessness of limbs."

P. I54.-Under Tribe Eumimosæ, before Mimosa, add:* Desmanthus, Willd.

virgatus, Willd.-A native of Tropical America now naturalised in many warm countries.

P. I58.-After Acacia linifolia, Willd., add-Brisbane Golden Wattle. 


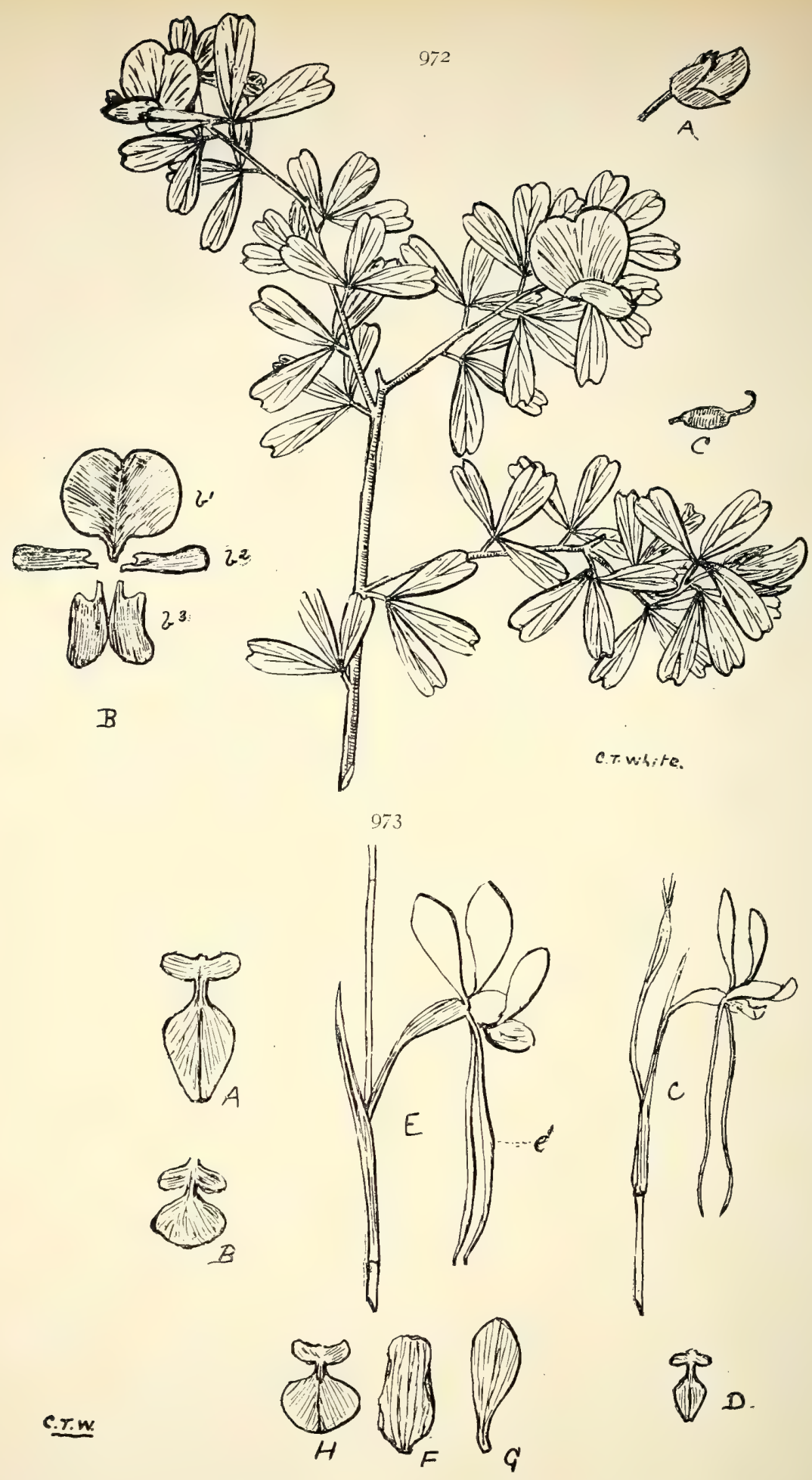

972. Gompholobium virgatum, Sieb., var. fmarginatum, Bail.

(A) Flower-bud, (B) petals, (bI) standard, (b2) wings, (b3) keel, (C) ovary. All nat. size.

973. Diuris punctata, Sm., and forms.

(A) Labellum of normal form, (B) a flabellate labellum, (C) flower of var. minor, Benth., (D) labellum of ditto, (E) flower of var. Blakneyæ, Bail., (er) dorsal sepals, (F) dorsal sepal of ditto, $(G)$ a petal of ditto, $(H)$ labellum of ditto. All nat. size. 
P. I63.-Acacia holosericea, A. Cunn.: I recently received specimens of this plant, collected in North Queensland by Mr. i. G. Bell, Chief Engineer for Railways, with the remark that the green pods rubbed in the hands produced a lather. The specimens were handed over to Mr. J. C. Brünnich, Agricultural Chemist, who reports - " The pods and seeds contain saponin, but no decicled test for saponin could be obtained in the leaves."

P. I64.-Acacia farnesiana, Willd.: For "Cussie pomade" read "Cassie pomade."

\section{Order UMBELLIFERÆ.}

P. 229.-Siebera Billardieri, Benth.: After var. crassifolia, Benth., add:-

z'ar. lanceolata, Benth.-Leaves lanceolate, acute, narrowed at the base, $\mathrm{I} / 2$ in. to above $\mathrm{I}$ in. long. Azorella lanceolata, Labill., P1. Nov. Holl. i. 74, t. 99; Trachymene lanceolata, Spreng., Bot. Mag. t. 3334.

Hab.: Helidon, F.M.B.; Macpherson Range, Tryon and Schneider; Cooroy, H. A. Longman.

\section{Order COMPOSIT EE.}

P. 272.-After Galinsoga add:*Tridax, Linn.

procumbens, Linn.-A native of Tropical America, for some years past naturalised in India and the East Indies; has recently established itself in Northern Queensland (see Q1. Agric. J1. xxix. ( I9I2) p. 484, Pl. 49).

P. 276.-Under Tribe Cichoriaceæ, before Microseris, add:-*Cichorium, Linn.

Intybus, Linn.-Chicory. Europe; Asia. Naturalised on the Darling Downs; Hermitage (J. Liverseed), Hendon (C. T. White).

P. 277.--Lactuca: After Scariola, Linn., add:saligna, Linn.-Europe; Western Asia. Naturalised in several localities on the Darling Downs, C. T. White.

\section{Order OLEACEÆ.}

P. 3II.-Notelæa longifolia, Vent., var. velutina, Bail.: After Killarney add-Coll. : J. Wedd.

\section{Order APOCYNACE}

P. 3I8.-Alstonia, after constricta, F.v. M., var. montmariensis, Bail., add:-

Follicles $3^{T / 2}$ to $4^{T / 2}$ in. long and 2 lines broad, submembranous. Seeds about 3 lines long, the hairs at each end slightly longer than the seed. When publishing this variety in Q1. Agric. 
I1. xxvi. (I9II) I98, the follicles had not been met with. Indeel, I have only this month (Nov. I9I2) received the material for the above note from my good friend Dr. Thos. L. Bancroft, Eidsvold.

P. 322.-Alyxia ilicifolia: For Bail. read F. v. M.

\section{Order BORAGINEAE.}

P. 347.-Lithospermum arvense, Linn.: For "Groundsel" read "Gromwell."

\section{Order CONVOLVULACEA.}

P. 347.-Ipomæa Calobra, Hill et F.v.M.: After "Weir" add-or "Gueeah" (of Balonne River natives).

\section{Order LABIAT E.}

P. 388.-After Leucas linifolia, Spreng., add:-India. In "Queensland Flora," p. I I99, I referred to having received specimens of what appeared to be this plant, some years ago, from Mr. E. Cowley, Kamerunga, with the remark that it was an introduced weed spreading in the Cairns district; but had heard nothing of it since. However, I have lately received specimens of this plant from Mr. H. A. Longman, with the remark that it was naturalised round about Palmwoods, North Coast line.

\section{Order PIPERACEÆ.}

P. 4I8.-After (Fig. 402) add (Plate XII.). After (Fig. 403) omit (Plate XII.).

\section{Order URTICACEE.}

Ficus, Linn.

P. 485.-The following Queensland Figs were described by Dr. O. Warburg in Fedde Repertorium, vol. i., pp. 7I-77, a work to which I had not access, and have to thank the Director, Royal Botanic Gardens, Kew, England, for type-written copies of Dr. Warburg's descriptions. At the same time I take the opportunity of including descriptions of two others, which brings my work on the Queensland Figs up to date.

P. 485.-After Ficus Parkinsoni add:-

A medium-sized tree, glabrous, branches slightly angular, branchlets thickish, a little fleshy, longitudinally corrugate, smooth, ochraceous, leafy towards the apex. Leaves alternate, scattered, sub-oblique, oval-oblong, apex very shortly subacuminate, obtuse or slightly acute, base rounded or narrow-obtuse, inconspicuously 3-5 nerved, coriaceous, entire, spreading, above a pleasant shining green, veins and veinlets very slightly raised, somewhat reticulate, beneath pale green, smooth, veins and veinlets immersed, reticulations minute, flat, about $3 \frac{3}{4}-6$ in. long, $2-3 \frac{1}{4}$ in. broad, penniveined, lateral nerves about 8 on either side of the midrib, spreading, 
slender; petioles canaliculate above, I3/4-2 $1 / 2$ in. long; stipules ovate, acute, caducous, appressed, puberulous on the back to 8 lines long. Peduncles axillary, in pairs, very slightly compressed, green, 3-4 lines long. Receptacles globose, glabrous, white-spotted, 8-I3 lines diam., when young pale green, afterwards whitish green, tinged with red, when mature dark purple, very slightly umbilicate, umbilical bracts at the apex 3, sub-rotund, small, obtuse, base bracteate, basal bracts small. Flowers very shortly pedicellate, pedicel bracteolate at the base, bracteole flaccid $1 / 2-3 / 4$ line long, male flowers $3 / 4$ line long, perianth unequally 3 -partite, $1 / 2$ line long, segments obtuse, imbricate, stamen I, filament short, stout; anther thick, bilocular; female flowers nearly I line long, perianth unequal $3-4$ fid, $1 / 2$ line long, style included, slender, elongate $3 / 4$ line long, stigma subulate, attaining I line in length and nearly $1 / 2$ line broad.

Hab.: Booby Island, Banks and Solander.

P. 485.-After F. virginea $a d d$ :-

A medium or large-sized tree, branchlets very slightly compressed or angular, smooth, towards the apex very slender and pubescent. Leaves alternate, scattered, elliptic or somewhat oval, apex a little acute or obtuse or sometimes shortly acuminate, base cuneate or obtuse, suboblique, coriaceous, entire, broad, smooth on both surfaces, above a dark green, shining, beneath a paler green, somewhat I-nerved, penniveined, veinlets anastomosing, reticulations minute, petiolate, $2-5$ in. long, $1 / 2-\mathrm{I}$ in. broad, petioles 4-Io lines long, canaliculate. Stipules ovate-lanceolate, caducous, 4 lines long. Peduncles in pairs, terete, short, thick, very thinly pubescent. Receptacle sub-globose, pisiform, glabrous, smooth, spotted, spots small, scattered, yellow or at length reddish, 3-4 lines diam. Basal bracts very short. Bracteoles of the flowers small, flaccid. Flowers small, subsessile, obovoid, perianth glabrous, 3-4 partite, segments ovate or obovate, stamen I, filament very short, anther short, broad, stigma obtuse or conical, attaining $\mathrm{T} / 2$ line in length.

Hab.: Booby Island, Torres Strait, Banks and Solander. Hiern.

The specimen seems nearest to F. engenioides, F. v. M.

P. 486.-After F. infectoria add:-

F. Pritzelii, Warb., Fedde Rep. I, p. 74.-A high-climbing liana, branchlets glabrous, stipules small not $\mathrm{T} / 2$ line long, glabrous, involute-lanceolate, caducous, petioles about I/2 in. long, canaliculate, glabrous, pale-coloured when dry, at the apex glandular, spotted on the back. Leaves somewhat coriaceous, glabrous, elliptic even to oblong, base rotundate, a little cordate or subcordate, apex rotundate then shortly apiculate acute; main nerves, about 12 on either side of the midrib, patent, slender, scarcely 
prominent on either surface, near the margin very much bent and joined; basal nerves not at all divergent nor thicker, reticulations very slender, but rather prominent. Receptacles axillary in pairs or single by abortion, subglobose but the apex depressed, glabrous, when dry fuscescent, base sometimes shortly stipitate, 4 to 5 lines in diameter, ostiole scales convex, prominent, pubescent, peduncles $\mathrm{I} / 2$ to 2 lines long, $\mathrm{I} / 2$ line broad, very slightly puberulous. Male flowers not at all standing out. Female flowers: sepals oblong, red, apex obtuse or rotundate, style filiform, stigma elongate, apex not at all thickened, bracts lanceolate.

Hab.: On stiff clay soils, about 1,66o feet above sea-level, Upper Barron River, L. Diels.

The species stands nearest to $F$. infectoria, Roxb., and its Australian representatives $F$. nesophila and $F$. Cunninghamii, but differs from them in that the leaves are much narrower and thicker, the undivided base-nerves, the finer and straighter lateral nerves, the very fine reticulations, and furthermore by the flattopped receptacle.-Warb. l.c.

P. 486.-After F. Thynneana $a d d:-$

F. Cairnsii, $W a r b$., Fedde. Rep. I, 74.-A small tree, branchlets glabrous, stipules lanceolate, glabrous, acute, 4 to 5 lines long, leaves coriaceous, broadly obovate, $I \frac{3}{4}$ to $3 \frac{3}{4} \mathrm{in}$. long, $\mathrm{I} \mathrm{I} / 2$ to 2 in. broad, base obtuse or rotund, rarely rotundate-cuneate, apex commonly rotundate, rarely obtuse or retuse; veins slender, somewhat prominent on both sides of the leaf, main nerves about 8 to Io, a little thicker, basal pair rather more prominent, ascending and marginal. Receptacles sessile axillary, in pairs in the upper portions of the branchlets, nearly $\mathrm{x} / 2 \mathrm{in}$. long, broadly pyriform, apex flattened, basal bracts 3 , connate at the base, I to I I 2 line long, obtuse, glabrous, ostiole flat. Male flowers monandrous, sepals 3, apex rotund, red with white margins, filament short, anthers broad, obtuse, base cordate. Female flowers: sepals 3, obtuse, red with white margins, style filiform, stigma oblong, scarcely thicker, papillose.

Hab.: In sandy alluvial soil not far from the seaside, Cairns, L. Diels.

F. Thynneana, Baii,, has, according to the description, much longer and narrower leaves, while the description of the receptacle and the habitat agree with our species. Dr. Diels thinks that F. Thynneana has been described after two species.-Warb. l.c.

I may here state that my original description of $\mathrm{F}$. Thynneana was drawn up from specimens collected by myself off a single tree, so could hardly be referred to two species.

P. 486.-Ficus retusa, Linn., var. nitida, King: Of the two trees referred to, the larger one, which was planted about I880, has a spread of $80 \mathrm{ft}$. and a height of about $40 \mathrm{ft}$., and the smaller one a spread of $66 \mathrm{ft}$. and a height of about $25 \mathrm{ft}$. 
P. 487.-After Ficus macrophylla add:-

F. cylindrica, Warb., Fedde. Rep. I, 72.-A small tree having the appearance of $F$. elastica. Branchlets thick, young branches densely brown-ferrugineous-tomentose, petioles $2-3 \frac{1}{2}$ in. long, ferrugineous-pubescent at length glabrous, leaves coriaceous oblong, apex gradually acuminate then almost obtuse, base obtuse, glabrous above, minutely ferrugineous-tomentose beneath soon glabrous, $5^{\mathrm{T} / 2-7^{\mathrm{T}} / 2}$ in. long, $2-2^{\mathrm{T} / 2}$ in. broad, main nerves thick, about 25 on either side of the midrib somewhat erect, basal nerves a little thicker and more ascending. Receptacles axillary (probably in pairs or single by abortion), very shortly stipitate; stipites about 3 lines long and broad, covered with a greyish tomentum, receptacles somewhat cylindrical, $\mathrm{I} 3 / 4-2$ in. long and 7 lines broad, apex truncate, glabrous and covered with large spots, ostiole small, somewhat sunk. Male flowers scattered over the interior and not at all confined to the apex of the receptacle, sepals 4 or 5 , red, cucullate, rotundate, all involute; anther I, very thick; gall-flowers gibbous, sepals obtuse; red, circumdate, style filiform, apex truncate, glabrous.

Hab.: Found singly on the edge of the forest, Upper Barron River, L. Dicls.

Related to F. macrophylla, but easily distinguished by its hairiness and characteristic receptacles which remind one of long acorns. - Warb. l.c.

\section{P. 504.-After Ficus glomerata add:-}

F. trichostyla, Warb., Fedde Rep. I, 77.-Branchlets in dried specimens angular, at first hispid but soon glabrous, stipules caducous, lanceolate, covered with an appressed grey. pubescence, acuminate, 5 lines long, petioles $4^{\mathrm{T} / 2-7}$ lines long, pilose at length glabrous. Leaves partly alternate, partly in inequal pairs but not at all opposite, pergamaceous, ovate, apex shortly acuminate, acute base somewhat oblique, rotundate margins slightly sinuate, $4^{-6}$ in. long, $2-3 \frac{\mathrm{T}}{4}$ in. broad, a little asperous on the upper surface with scattered appressed hairs, on the under surface white pilose on the main nerves, on lesser nerves appressed pilose; veins about 7 on either side of the midrib, basal pair smaller than the rest. Receptacles axillary in pairs or solitary, peduncles I $3 / 4-3$ lines long, slightly hairy, peduncles $\mathrm{I} 3 / 4-3$ lines long with 2 or 3 squamiform bracts at the apex, receptacle depressed-globose, 7 lines broad, 5 lines long, when young distantly albo-punctate, at the apex rather distinctly longitudinally 6-striate, smooth, ostiole large but not at all prominent. Female flowers stipitate, perianth small, byaline, style slightly hairy, stigma not at all thickened.

Hab.: Cairns, O. Warburg.

F. setistyla, Warb. in Fedde Rep. I, 77.-A small tree, branchlets glabrous smooth. Stipules narrow-lanceolate, glabrous, about 
I in. long, acute, deciduous. Petioles $3 / 4-I$ in. long, glabrous. Leaves membranaceous, oblong-elliptic, base often somewhat oblique, obtuse or subcuneate-acute, apex acuminate and then cuspidate, 4-8 in. long, 2-3 $\frac{\mathrm{T} / 2}{2}$ in. broad, glabrous, slightly rough, veins about Io on either side of the midrib, patent, nearly strict, prominent on both sides, reticulations very slender hardly discernible. Receptacles on special branches from the trunk I6-I8 in. long, upper peduncles 3-4 lines long covered with a pale appressed ferrugineous pubescence, apical bracts 3 , squamiform, obtuse receptacle depressed globose, nearly $\mathrm{I}$ in. broad, $3 / 4 \mathrm{in}$. long, densely covered with an appressed pale ferrugineous pubescence. Male flowers at the apex of the receptacle, stamen I, sepals cucullate, all involute; all flowers densely crowded, perianth infundibuliform, hyaline, style hairy, apex very shortly dilated and somewhat 2-lobed.

Hiab.: Damp spots in the scrub, banks of streams, \&c., Lower Russell River, L. Diels.

A tree belonging to the section Covellia, having bright reddishyellow hairy receptacles and a bristly style. The receptacle branches on the stem are pendant and often nearly 20 in. long.Tiarb. i.c.

\section{P. 504.-Add:-}

\section{Section IV.-Sycidium.}

F. Dielsii, Warb., Fedde Rep. I, 75.-A small tree, young branchlets grey-pubescent, soon glabrous. Stipules caducous, $1 / 2-2$ in. long, involute, lanceolate, acute, in the centre greytomentose. Petioles $3 / 4-\mathrm{I}$ in. long, grey pubescent at length glabrous. Leaves membranaceous, elliptic to oblong, base narrow, sub-cuneate, apex acuminate or shortly cuspidate, glabrous above, pubescent on the nerves on the upper surface, $33 / 4-6$ in. long, $\mathrm{I} 3 / 4-2^{\mathrm{I} / 4}$ in. broad, veins about Io on either side of the midrib, slender, oblique, ascending, somewhat curved at and scarcely joined near the margin, basal nerves 2 or 3 on either side of the midrib, the central ones more ascending, reticulations slender, very distinct on the under surface but not prominent. Receptacles numerous, borne on short-branching branches from the trunk, peduncles $1 / 2-3 / 4$ in. long, at the apex subincrassate, bracts 3 , squamiform, obtuse, $3 / 4$ line long and broad. Receptacles pyriform, covered with an appressed pubescence, about $1 / 2$ in. long, $3^{\mathrm{T} / 2-4}$ lines broad, apex rugose, ostiole impressed; inside among the flowers are numerous bracts or white hairs. Male flowers monandrous, perianth segments cucullate, filament thick, anther rotund-ovate, base nearly cordate. Female flowers (gall-flowers?) sessile, perianth segments 3 or 4 , red with a pale margin, obtuse or rotundate, often subcucullate; style short, lateral, white, narrow-infundibuliform.

Hab.: In stony bed of a creek, shady localities, Central Barron, I. Diels. 
Belonging probably to the section Sycidium. A well-characterised species, as shown by the scarcely rough leaves, hairy on the nerves on the under-surface, and by the short-branched fruitbearing branches from the trunk.-Warb. l.c.

F. subinflata, Warb., Fedde Rep. I, 76.-A small tree, with a light grey bark. Branches thick, internodes swollen, the hollow part rather narrow, $2^{\mathrm{T} / 2-3}$ lines broad, outside minutely prickly, stipules $3-3 \frac{\mathrm{T}}{2}$ lines long, lanceolate, acute glabrous. Leaves $3-5$ crowded together; petioles $1 / 4-1 / 2$ in. long, often rough, leaves subpergamentaceous, oblong apex nearly cuspidate, base narrow, rotund or subcordate, margins sub-undulate, veins asperous on the under surface and slightly so on the upper, main nerves patent, about to on either side of the midrib, much raised on the under surface, not at all confluent at the margins, basal pair short ascending. Receptacles axillary, in pairs or at length single, peduncles $3^{\mathrm{T} / 2-5}$ lines long, commonly slightly rough with minute aculei. Receptacles bearing female flowers about I in. diam., nearly globose, when mature depressed globose, about II/4 in. broad and $\mathrm{I}$ in. long, apex impressed, ostiole convex. Female flowers: perianth segments 4 or 5, white, oblong; style filiform, stigma papillose, broadly clavate. Seeds smooth.

Hab.: Edge of forest on clay soil, Upper Barron, L. Diels.

Stem light grey, foliage rough, ripe fruit streaked with red, sweet. The somewhat swollen internodular twigs, together with the crowded leaves, make it probable that this tree, which nay be referred to the section Sycidium, is an "Ant-plant."-Warb. l.c.

\section{Order ORCHIDEÆ.}

P. 535.-Cymbidium canaliculatum, R. Br., var. Sparkesii $=$ C. Sparkesii, Rendle in J1. Bot, xxxvi. (I898) 22I.-I hare recently seen specimens of this orchid grown by Mr. J. Silcock, near Brisbane, and the only difference I can find is in the deep, dull red colour of the flower, which "in reflected light appears almost black." In the "Queensland Flora," p. I547, under C. canaliculatum, I drew attention to the great variability in the colouring and markings of the flowers, and, as I had not seen a specimen of the present plant, left it unplaced; but since seeing Mr. Silcock's specimen I think it might be placed as above. The other species, C. Leai, described by Mr. Rendle, 1.c., from his description, I cannot separate from C. canaliculatum, R. Br.

P. 535.-Sarcochilus, after Hillii, F. v. M., add:-

minutiflos, Bail., sp. nov. (Fig. 974). On branchlets of shrubs and trees. Roots very long and slender, mostly white and more or less curled. Stem very short. Leaves several, slender, 2-4 in. long and about 2 lines broad, sometimes dotted. Racenes numerous, very slender, from 2-6 in. long, sometimes forked, bearing 


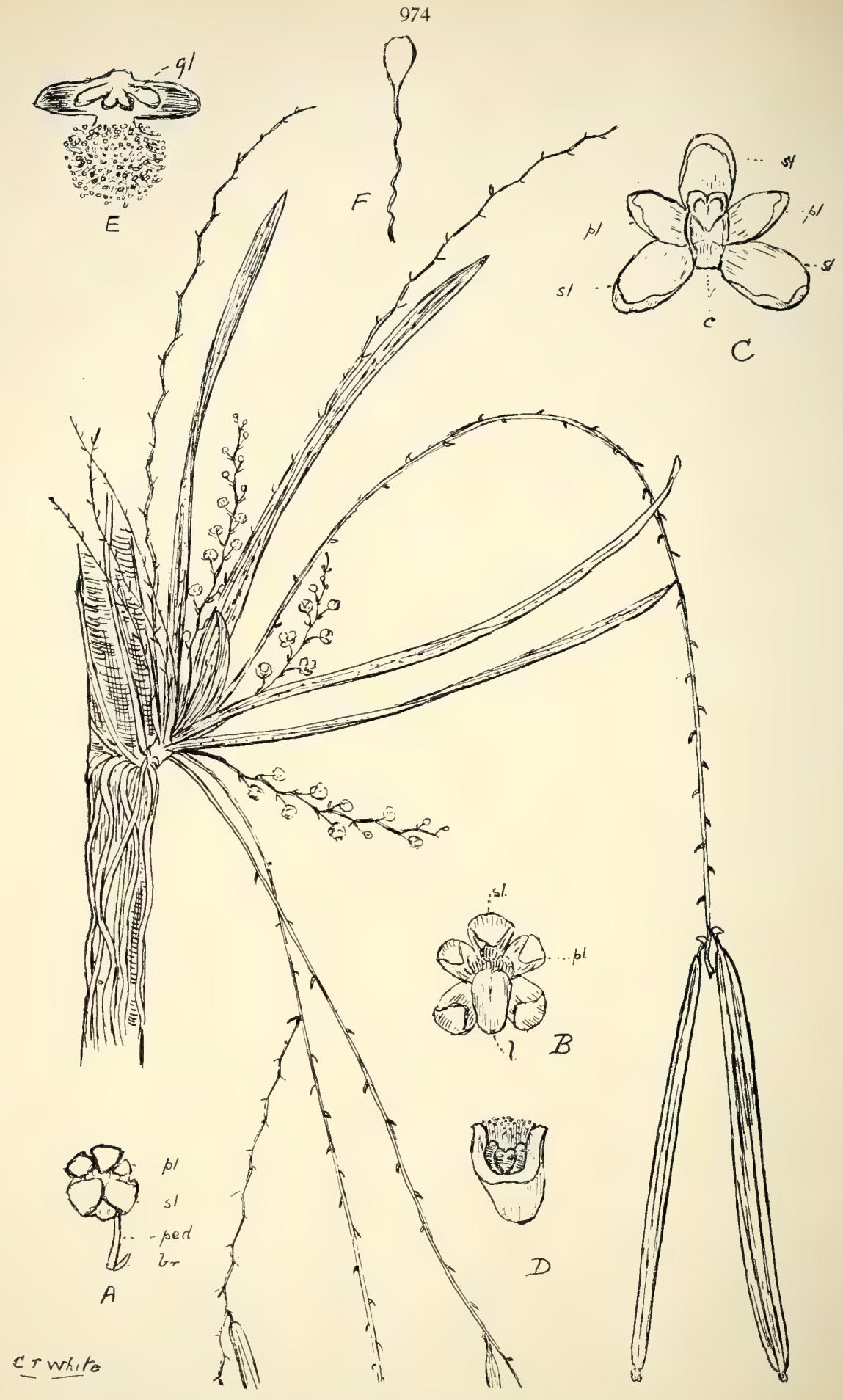

974. Sarcochilus minutiflos, Bail.

(A) Flower, (B) flower more fully opened, (C) flower, labellum removed, (D) labellum from the front, (E) lobes of the labellum; (F) a single hair from the middle lobe of the labellum. (A)-(F) enl. (br.) bract, (ped.) pedicel, (pl.) petal, (sl.) sepal, (1.) labellum, (c.) column, (g1.) gland or disc calli. 
throughout their whole length or nearly so very numerots minute flowers. Flowers on slender pedicels of about 2 lines, nearly globular from the incurving of the sepals and petals, of a greenish white sometimes tinged with pink, and less than 2 lines in diameter. Bracts minute. Sepals somewhat larger than the petals. Labellum small, the lateral lobes purplish, blunt, ovate-oblong, middle lobe stalked, for the most part composed of a globular mass of glandular white hairs. Disc calli orange yellow. Column short; anther-lid stained with purple. Capsule narrow, straight, $2-2^{1} / 2$ in. long.

Hab.: Eidsvold, Dr. T. L. Bancroft.

P. 537.-After Diuris punctata, Sm., add:-

var. minor, Benth. ? (F1. Austr. vi. 327).-Specimens of what I think is this variety I have recently received from Miss Morris, Ormiston, but the middle lobe of the labellum in our plant is not especially rhomboidal. (Fig. 973 C \& D.)

forma Blakneyæ, Bail., n. form.-This form principally differs from the normal plant in the seeming absence of dots in the flower and in the almost rotundate middle lobe of the labellum.

Hab.: Ravenshoe, Miss F. Blakney. (Fig. 973E) I collected a specimen in 1874 at Maroochie in which the labellum closely resembles that of this new form. This latter specimen, however, is in very bad condition. (Fig. 973в.)

\section{P. 537.-After Diuris add:-}

Orthoceras, R. Br.-Dorsal sepal erect, incurved, hoodshaped; lateral sepals narrow-linear, long and erect; petals short, erect, narrow. Labellum 3-lobed, the middle lobe larger and contracted at the base, a thick callus on the disk between the lateral lobes. Column very short, with lateral erect lobes not connected behind the anther. Anther erect or slightly incurved, tapering to the end, the 2-valved cells occupying the whole inner face. Pollenmasses granular or mealy. Rostellum very short. Terrestrial glabrous herb, with an ovoid tuber, few narrow leaves, and several sessile flowers. The genus is limited to the single Australian species, found also in New Zealand.

I. O. strictum, R. Br., Prod. 3I7 ; Benth., F1. Austr. vi. 332.Stem rigid, erect, I to $\mathrm{I} / 2 \mathrm{ft}$. high. Leaves several near the base, linear, 3 to 6 in. long, or one or two outer ones short and lanceolate, and 2 or 3 long sheaths with short erect laminæ above the leaves. Flowers distant, erect, in an interrupted spike, the subtending bracts sheathing, acute, sometimes scarcely exceeding the ovary, sometimes much longer than the dorsal sepal. Dorsal sepal broad and very concave, much incurved, acute or obtuse, about $1 / 2$ in. long, greenish or white outside, brown purple or yellowish inside; lateral sepals antenna-like, slightly clavate, 


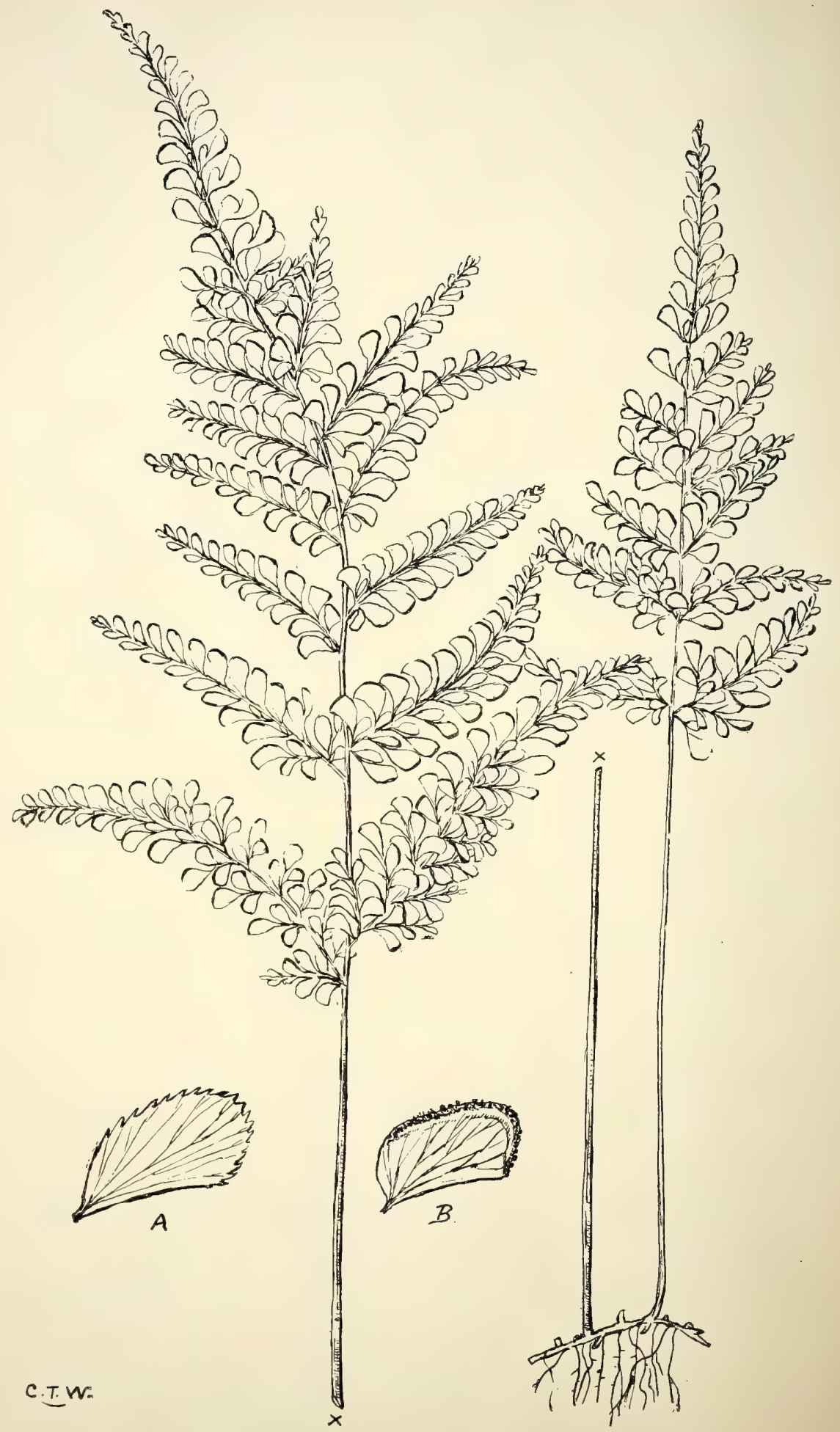

C75. Linds đea flabellulata, Dryand, var. Multipinnulata, Bail. (A) Sterile pinnule, (B) fertile pinnule. 
$3 / 4$ to nearly I in. long; petals thin, not 2 lines long, trincate notched or toothed at the end. Labellum 3 to 4 lines long, the lateral lobes broad and oblique, the middle lobe twice as long and ovate, the callus between the lateral lobes broad and prominent, but variable in shape. Lateral lobes of the column often nearly as long as the petals.-Lindl. Gen. and Sp. Orci. 5r2; Reichb. f. Beitr. I4; Fitzg. Aus. Orchids cum Ic. pict. O. Solandri, Lindl. 1.c. ; Diuris Nove-Zelandia, A. Rich. F1. Novæ Zel. I63, t. 25.

Hab.: Noosa, H. A. Longman, Oct. rgr2.

The Queensland plant represents a very robust growth in all its parts.

\section{Order GRAMINEA.}

P. 6o3.-Paspalum: After platycaule, Poir., add:*virgatum, Limn.-South America. This grass, for some few years past cultivated in Queensland, is now becoming naturalised in several localities.

P. 632.--Eragrostis: After stricta, Bail., add:*major, Host.-Stink Grass. South Europe; Asia. Naturalised and becoming very common in some localities on the Darling Downs, C. T. White. This grass possesses a very fœtid odour.

\section{Order FILICES.}

P. 64I.-After Lindsæa flabellulata, Dryand., add:-var. multipinnulata, Bail., Q1. Agric. I1. xxix. (I9I2) 349, Plate 36. (Fig. 975.)

P. 648.-After Polypodium confluens, R. Br., add:forma liberatum, Bail., n. form.-The present plant only differs from the normal form in the greater part of the sori being free not confluent, the confluent part occupying less than an inch.

Hab.: Kin Kin, Blackall Range, F. Allbon.

P. 649.-Acrostichum spicatum, Linn., var. Schneideri, Bail.Capt. C. R. W. K. van Alflerwerelt van Rosenburgh, in the "Bulletin du Jardin Botanique de Buitenzorg" (and ser.) viii. (I9I2) p. I9, describes without name a form of Hymenolepis (Acrostichum) spicatum from Mount Gedeh, Java, which is evidently identical with my var. Schneideræ.

\section{Order HEPATIC $\not$ E.}

P. 677.-Fig. 680, Fimbriaria Drummondii: For Raddi read Tayl. 


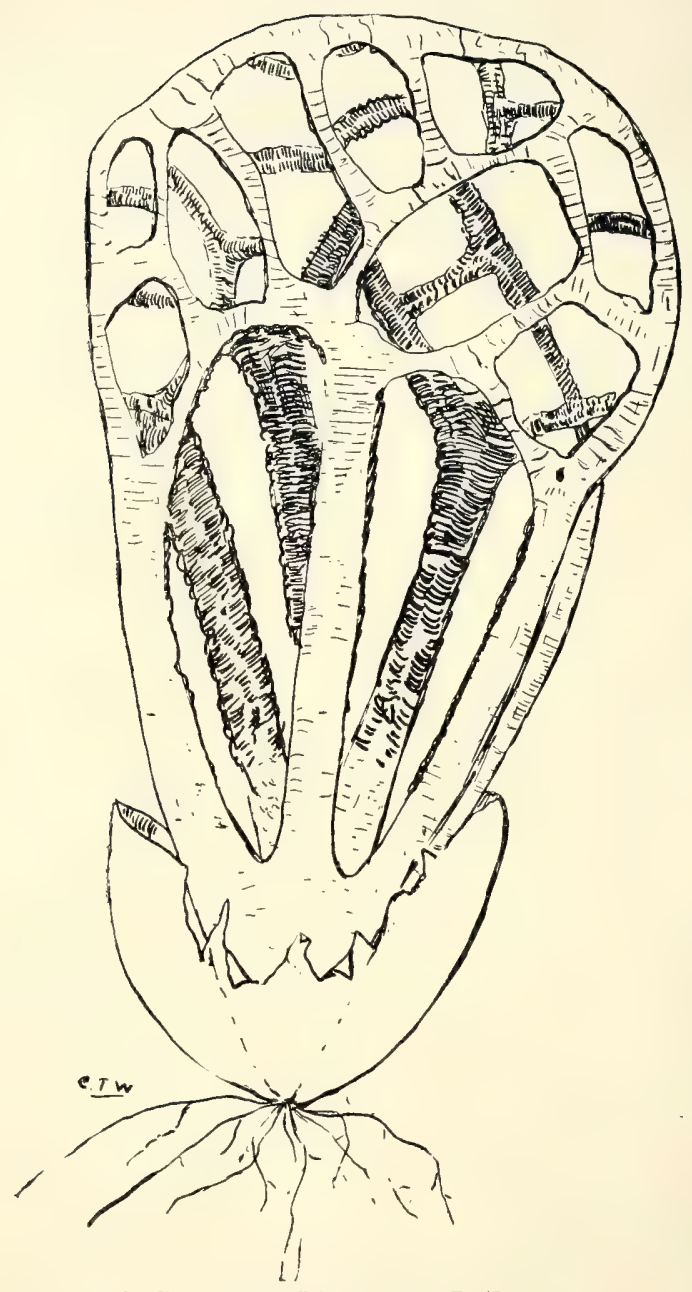

976. Clathrus Higginsit, Bail. 


\section{Order CHARACE王.}

P. 678.-After Nitella conglobata, var. Lhotzkyi, A. Br., add:I recently received specimens of this Nitella from Mr. A. Cameron, Murweh, with the account that it was becoming a troublesome weed in the bore-drains there. The species was determined as N. Lhotekyi, A. Br., by Messrs. H. and J. Groves, to whom I sent the specimen for determination.

\section{Order FUNGI.}

P. 742.-- Hymenochæte, after innata, Cke. et Mass., add:purpurea, Cke. et Morg.-On wood, Brisbane (Benj. Crow). Det. by Miss E. M. Wakefield, Royal Botanic Gardens, Kew, England.

P. 746.-Clathrus, after crispus, Turp., add:Higginsii, Bail., Q1. Agric. J1. xxix. (I912) p. 487. (Fig. 976.)

P. 751.-Battarea, Pers.: To the species add:Stevenii (Libosch.), Fries. Westbrook (J. Liverseed). Det. by Miss E. M. Wakefield, Kew.

P. 755--Ascobolus, Pers.: To the species add:furfuraceus, Pers.--On dung, Brisbane River (J. H. Simmonds). Det. by Miss E. M. Wakefield, Kew.

immersus, Pers.-On dung, Brisbane River (J.H. Simmonds): Det. by Miss E. M. Wakefield, Kew.

P. 778.-After Coniothyrium, Corda, add:-

Capnodiastrum, Speg.

orbiculatum, Cke. et Mass._-On living leaves.

P. 783.-Cladosporium, after epiphyllum, Pers., add:graminum, Corda.-On leaves of Black Bamboo (Phyllostachys nigra), Brisbane (C. T. White). Det. by Miss E. M. Wakefield.

P. 785.-Cercospora, Fries.: To the species add:daviesia, Cke. et Mass.-On leaves of Desmodium sp., Enoggera (C. T. White). Det. by Miss E. M. Wakefield, Kew.

P. 787.-Fusarium, Link.: To the species add:Yuccæ, Cke.-On dead panicles of Yucca aloifotia. A species of Sporodesmium also occurred in association with the above. Brisbane (C. T. White). Det. by Miss E. M. Wakefield, Kew. 
P. 790.-For Trichiacea read Trichiaceæ. After Trichia add:-

Family Plasmodiophorex.

Plasmodiophora, Woronin.

Brassicæ, Woronin.-On roots of Candytuft (Iberis umbellata). Det. by Miss E. M. Wakefield.

This disease is the well-known "Finger-and-toe" or "Clubroot" of Cruciferous plants (Cabbage, Turnip, \&c.), and has recently been found attacking very badly plants of Candytuft in Brisbane gardens; probably introduced with seed of European origin. In a paper by Mr. G. Massee in the "Proceedings of the Royal Society," and reprinted in the "Kew Bulletin," I895, pp. I $30-132$, he makes the following remarks:- "The germs of the disease are present in the soil that has produced a diseased crop, and retain their vitality for at least two years. For the purpose of sterilising infected soil, experiments prove that either a dressing of lime or a manure containing potash-salts is effective, the last being most valuable, as it not only destroys the germs in the soil but also arrests the disease in seedling plants, and at the same time supplies one of the ingredients necessary for the healthy growth of turnips."

P. 792.-After Rhizomorpha add:Ozonium, Link.

auricomum, Link,-On dead logs of Poinciana regia, Brisbane (C. T. White). Det. by Miss E. M. Wakefield.

\section{Order ALGA.}

P. 795-Chætomorpha: After tortuosa, Kuetz., add:aerea (Dillw.), Kuetz., De-Toni, Syll. Alg. i. 273.

Hab. Coolangatta, C. T. White. Det. by A. D. Cotton, Royal Botanic Gardens, Kew, England.

NUMBER OF KNOWN QUEENSLAND PLANTS.

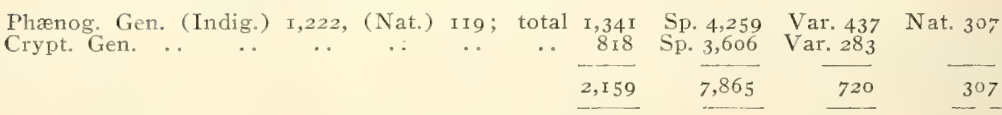




\section{LIST OF PLATES.}

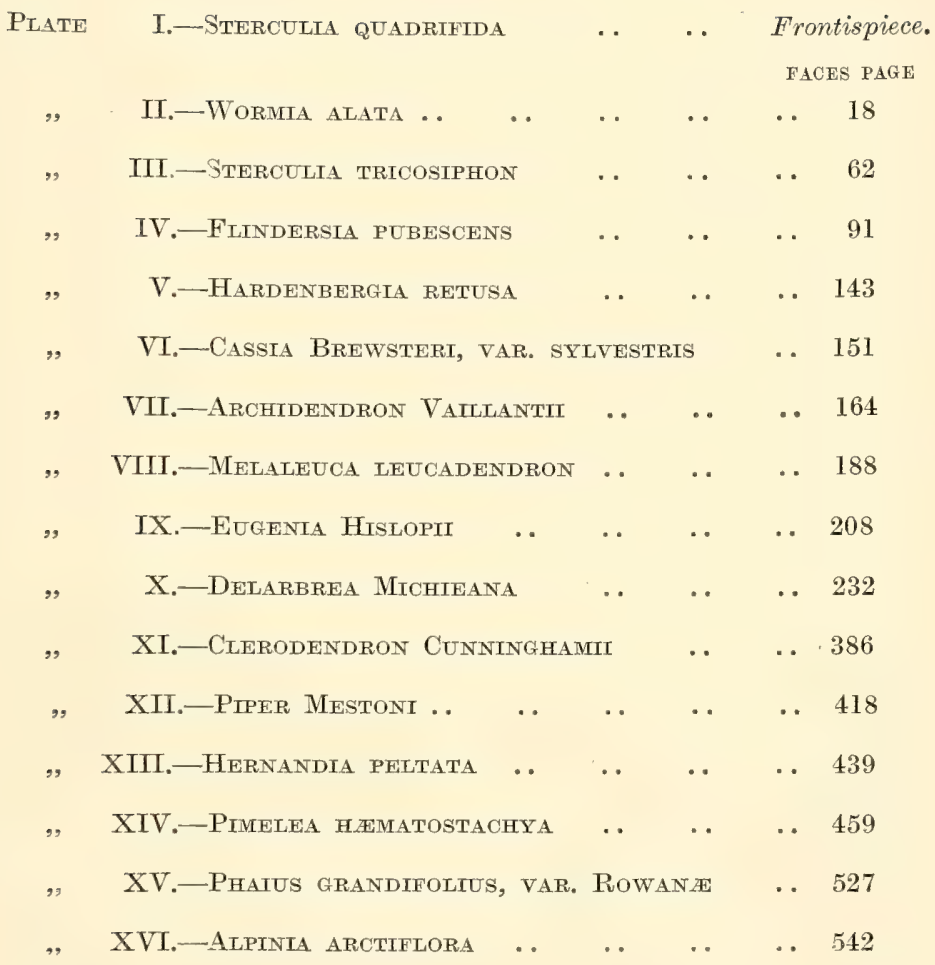





\section{Index of Systematic Names.}

The Orders are printed in Sualt Capitals, and the Genera incidentally mentioned in Italics.

Page.

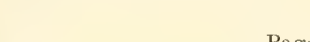

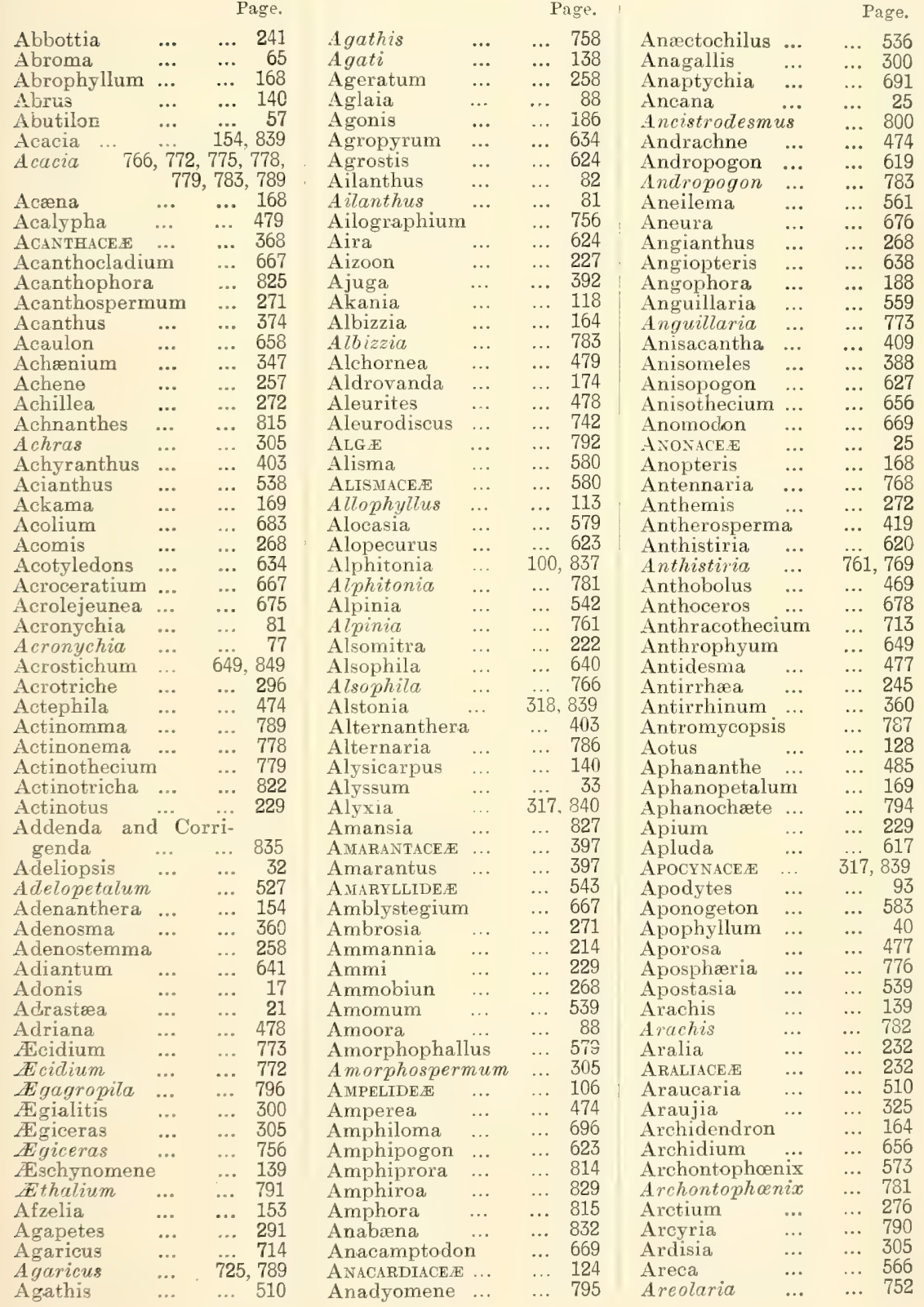


Page.

Argemone $\quad . .6 \quad \ldots \quad 33$

Argophyllum ... $\quad \ldots \quad 168$

$\begin{array}{llll}\text { Argyreia } \quad \ldots & \ldots & 347\end{array}$

$\begin{array}{llll}\text { Argyreia } & \ldots & \ldots & 787\end{array}$

$\begin{array}{llll}\text { Aristida } & \ldots & \ldots & 622\end{array}$

Aristida $\quad \ldots \quad \ldots 771$

Aristolochia $\quad \ldots \quad \ldots \quad 418$

ARISTOLOCHIACE \&

Aristotelia

Armillaria

AROIDEE

Arrhenia

Artanema

Arthonia

Arthopyrenia

Arthothelium ... ... 707

Arthraxon ... 617

Arthrocardia ... ... 829

Arthrochilus $\quad \ldots . \quad \ldots .538$

Arthrocnemum _... 409

Arthrodesmus ... ... 809

Arthropodium _.. 557

Arthrospira $\quad . .831$

Arthrostyles $\quad \ldots . \quad \ldots .597$

Arundinaria ... . .. 634

Arundinella $\quad \ldots \quad$... 611

$\begin{array}{llll}\text { Aschersonia } & \text {.. } & \text {... } & 779\end{array}$

Ascidium $\quad 699$

Asclepiadeez $\quad \ldots \quad \ldots \quad \ldots 325$

Asclepias

Ascobolus

Ascochyta

A SCOMYCETEX

Ascomyces

Aseroe

Asparagopsis

Asparagus $\quad \ldots . \quad \ldots .553$

Aspergillus $\quad . . . .782$

Asperococcus $\quad . . . \quad$... 821

Asperula

Asphodelus

$\begin{array}{llll}\text { Aspidium } & \ldots & \ldots & \ldots \\ & \ldots & 645\end{array}$

Asplenium

Asterella $\quad \ldots . . .766$

Asterella $\quad \ldots .6676$

Asterina $\quad . . .766$

Asterolasia $\quad \ldots \quad \ldots .76$

Asteromella ... ... 776

Asterostomella $\quad . .781$

Asterotrema ... ... 708

Astrebla $\quad . . .628$

Astrotriche $\quad \ldots . \quad \ldots .232$

Asystasia $\quad . .6374$

Atalantia $\quad . .6 \quad \ldots .82$

Atalaya $\quad \ldots . \quad \ldots .116$

Atherosperma $\quad$... 419

Atriplex $\quad \ldots \quad \ldots 408$

Atylosia $\quad \ldots . \quad \ldots .147$

Aulacopilum ... ... 661

$\begin{array}{llll}\text { Auliscus } & \ldots & \ldots & 817\end{array}$

Auricularia ... ... 743

Auricularia $\quad . . . \quad \ldots .790$

Australina $\quad \ldots .6505$

$\begin{array}{llll}\text { Avena } & \ldots & \ldots & 627\end{array}$

Avena $\quad \ldots \quad \ldots .773$

Avicennia $\quad \ldots \quad \ldots 387$

Avrainvillea ... ... 798

Axonopus $\quad$... $\quad \ldots 611$

Azolla $\quad \ldots . \quad \ldots 635$

Azorella $\quad \ldots \quad \ldots 839$

Babbagia $\quad$.. $\quad$... 409

Bacca $\quad \ldots . . . . .354$

Baccharis
Page.

$\begin{array}{llll}\text { Baccharis } & \ldots & \ldots & 768\end{array}$

BaCILLARIE

Bacillus $\quad \ldots \quad \ldots 789$

$\begin{array}{llll}\text { Backhousia } & \ldots & \ldots & 202\end{array}$

Bactridium $\quad \ldots \quad \ldots \quad 787$

Bacularia $\quad \ldots \quad \ldots \quad 573$

Brea

$\begin{array}{llll}\text { Bæckea } & \ldots & \ldots & 181\end{array}$

Bromyces $\quad \ldots \quad$... 684

$\begin{array}{llll}\text { Bagnisiella } & \ldots & \ldots & 761\end{array}$

Balanophora ... ... 470

BALANOPHOREE $\quad \ldots \quad 470$

Balanops $\quad \ldots \quad \ldots 480$

Balanopse王 $\quad \ldots \quad \ldots \quad 480$

Balantiopsis $\quad \ldots \quad \ldots 673$

Balls-Headleya $\quad$... 163

$\begin{array}{llll}\text { Baloghia } & \ldots & \ldots & 478\end{array}$

Balusta $\quad \ldots . \quad \ldots \quad 215$

$\begin{array}{llll}\text { Bambusa } \quad \ldots & \ldots & 634 \\ & & \end{array}$

Banksia $\quad \ldots . \quad \ldots \quad 455$

Banksia $\quad \ldots \quad 763,765$

Barbella $\quad \ldots \quad \ldots 666$

$\begin{array}{llll}\text { Barklya } & \ldots & \ldots & 148\end{array}$

Barringtonia ... $\quad \ldots .209$

Bartlingia $\quad$... $\quad$... 559

$\begin{array}{llll}\text { Bassia } & \ldots & \ldots & 404\end{array}$

Bathelium $\quad \ldots \quad \ldots .709$

Batrachospermum ... 821

$\begin{array}{lll}\text { Battarea } \quad \ldots & 751,851\end{array}$

$\begin{array}{llll}\text { Bauera } & \text {... } & \text {... } & 169\end{array}$

Bauhinia $\quad \ldots . \quad \ldots \quad 153$

Bazzania $\quad . .6 \quad \ldots 673$

Beilschmiedia $\quad$... 430

Beilschmiedia $\quad$... 772

Belonidium ... ... 755

Benincasa $\quad \ldots \quad \ldots 221$

BerCheMia $\quad \ldots . \quad \ldots \quad 100$

$\begin{array}{llll}\text { Bergia } & \ldots & \ldots & 49\end{array}$

Bertya

Bescherellia

Beta $\quad . .6-\cdots 771$

$\begin{array}{llll}\text { Beyeria } & \ldots & \ldots & 473 \\ \end{array}$

Biatora $\quad \ldots \quad$... 700

Biatorella , ... ... 701

Biatorinopsis ... ... 703

Biatorinopsis ... $\quad$.. 699

Bidens $\quad \ldots \quad \ldots 272$

$\begin{array}{llll}\text { Bidens } & \ldots & \ldots & 775 \\ \text { Bignoniace E } & \ldots & \ldots & 364\end{array}$

Billardiera $\quad \ldots \quad \ldots . \quad 43$

Biophytum ,.. $\quad$... 73

$\begin{array}{llll}\text { Bischoffia } & \ldots & \ldots & 477\end{array}$

$\begin{array}{llll}\text { Bispora } & \ldots & \ldots & 783\end{array}$

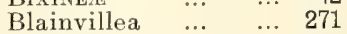

Blandfordia ... $\quad \ldots .553$

$\begin{array}{llll}\text { Blechnum } & \ldots & \ldots & 642 \\ & \ldots & & \end{array}$

Blennodia $\quad \ldots . . .34$

Blepharocarya $\quad \ldots .118$

$\begin{array}{llll}\text { Bletia } & \text {... } & \text {... } & 527\end{array}$

Blodgettia $\quad \ldots \quad \ldots 795$

Blumea $\quad \ldots . \quad \ldots 263$

Blyxa $\quad \ldots . \quad \ldots 518$

Boehmeria ... ... 505

Boerhaavia ... ... 396

$\begin{array}{llll}\text { Bolbitius } & \ldots & \ldots & 722\end{array}$

$\begin{array}{llll}\text { Boletus } & \ldots & \ldots & 727\end{array}$

$\begin{array}{llll}\text { Boletus } & \text {... } & \text {.. } 782\end{array}$

Bombax $\quad \ldots \quad-\ldots 61$

$\begin{array}{llll}\text { Bonnaya } & \ldots & \ldots & \\ \text { Bon } & \ldots & \ldots & 365\end{array}$

$\begin{array}{llll}\text { Boodlea } & \ldots & \ldots & 795\end{array}$

Boraginea $\quad \ldots \quad 338,840$

$\begin{array}{llll}\text { Borassus } & \ldots & \ldots & 574\end{array}$
Page.

$\begin{array}{llll}\text { Bornetella } & \ldots & \ldots & 796\end{array}$

$\begin{array}{llll}\text { Boronia } & \ldots & \ldots & 73\end{array}$

$\begin{array}{llll}\text { Borya } & \ldots & \ldots & 559\end{array}$

$\begin{array}{llll}\text { Bosistoa } & \ldots & \ldots & 77\end{array}$

$\begin{array}{llll}\text { Bossiæa } & \ldots & \ldots & 132\end{array}$

Botrychium _.. $\quad \ldots \quad 635$

$\begin{array}{llll}\text { Bottaria } & \ldots & \ldots & 710\end{array}$

Boussingaultia $\quad \ldots 441$

$\begin{array}{llll}\text { Bovista } & \ldots & \ldots & \\ & & & \end{array}$

$\begin{array}{llll}\text { Bowenia } & \ldots & \ldots & 518\end{array}$

Brachycome $\ldots . \quad \ldots .258$

Brachyloma ... ... 295

Brachysema ... ... 124

Brachysporium _.. 783

Brackenridgea $\quad \ldots \quad 85$

$\begin{array}{llll}\text { Brasenia } & \ldots & \ldots & 32\end{array}$

Brassaia $\quad \ldots \quad \ldots 236$

$\begin{array}{llll}\text { Breweria } & \ldots & \ldots & 353\end{array}$

Breynia $\quad \ldots \quad \ldots 477$

Bridelia $\quad . .6 \quad \ldots \quad 474$

Botryococcus ... $\quad \ldots \quad 881$

Botrytis $\quad \ldots . \quad \ldots 782$

Botrytis $\quad$... $\quad \ldots 769$

Bouchardatia $\quad$... 77

Bovista $\quad$... ... 752

$\begin{array}{llll}\text { Brachychiton } & . . & \ldots & \ldots\end{array}$

\begin{tabular}{llll} 
Brachycladia & $\ldots$ & $\ldots$ & 822 \\
\hline
\end{tabular}

$\begin{array}{lll}\text { Brassica } & \ldots & \ldots \\ \text { Brassica } & \ldots & 768,785 \\ & & \end{array}$

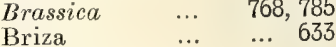

$\begin{array}{llll}\text { Brombya } & \ldots & \ldots & 77\end{array}$

Bromus $\quad \ldots \quad \ldots 633$

$\begin{array}{llll}\text { Bromus } & \ldots & \ldots & 771\end{array}$

$\begin{array}{llll}\text { Brucea } & \ldots & \ldots & 85\end{array}$

Bruchia $\quad \ldots \quad \ldots 656$

$\begin{array}{llll}\text { Bruguiera } & \text {... } & \text {... } & 175\end{array}$

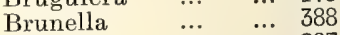

Brunonia $\quad \ldots \quad \ldots 287$

Bryonia $\quad \ldots . \quad \ldots 221$

Bryonia $\quad$.. $\quad \ldots \quad 785$

Bryophyllum ... ... 174

$\begin{array}{llll}\text { Bryopsis } & \ldots & \ldots & 796\end{array}$

Bryum $\quad \ldots . \quad \ldots .662$

Buchanania $\quad . . \quad \ldots \quad 119$

$\begin{array}{llll}\text { Buchnera } & \ldots & \ldots & 363\end{array}$

Buckinghamia $\quad$... 454

$\begin{array}{llll}\text { Buellia } & \ldots & \ldots & 702\end{array}$

Bulbine $\quad \ldots \quad \ldots 557$

Bulbochæte $\quad . . \quad$... 793

Bulbophyllum ... $\quad \ldots .526$

$\begin{array}{llll}\text { Bulgaria } & \ldots & \ldots & 755\end{array}$

Bupleurum $\quad \ldots . \quad \ldots 229$

Burchardia $\quad$... $\quad$... 559

Burmannia ... ... 519

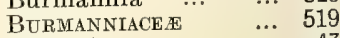

$\begin{array}{llll}\text { Bursaria } & \ldots & \ldots & 43\end{array}$

$\begin{array}{llll}\text { Bursera } & \ldots & \ldots & \\ \text { Bu }\end{array}$

Burseracee $\quad \ldots \quad$... 85

$\begin{array}{llll}\text { Burtonia } & \ldots & \ldots & 125\end{array}$

Butomopsis $\quad \ldots \quad \ldots \quad 580$

$\begin{array}{llll}\text { Butomus } & \ldots & \ldots & 580\end{array}$

$\begin{array}{llll}\text { Byblis } \quad \ldots . & \ldots & 174\end{array}$

Byssosphæria ${ }^{\cdots} \quad \ldots .762$

$\begin{array}{lllr}\text { Cabomba } & \ldots & \ldots & 32 \\ \text { CACTEA } & \ldots & \ldots & 222 \\ \text { Cadellia } & \ldots & \ldots & 85 \\ \text { Calandria } & \ldots & \ldots & 526 \\ \text { Caoma } & \ldots & 773 & 775 \\ \text { CESALPINEE } & \ldots & \ldots & 148 \\ \text { Cæasalpinia } & \ldots & \ldots & 148 \\ \text { Cæsia } & \ldots & \ldots & 557\end{array}$


Page.

Caladenia $\quad \ldots . \quad \ldots 538$

Calamus

Calamus

Calandrinia

574

Calanthe

Calceolaria

Caleana

Calicium

Callicarpa

Callicoma

Callistemon

Callistemon

Callithamnion

Callitriche

Callitris

Callopisma

Calloria

Calocephalus ...

Calocera

Calochilus

Caloglossa

Calogyne

Calonectria $\quad \ldots . \quad \ldots 758$

Calonyction speciosum 349

Calophyllum ... $\quad$... 54

$\begin{array}{llll}\text { Calostemma } & \ldots & \ldots & 543\end{array}$

$\begin{array}{llll}\text { Calothrix } & \ldots & \ldots & 832\end{array}$

$\begin{array}{llll}\text { Calotis } & \ldots & \ldots & 259\end{array}$

Calymperes ... ... 658

Calyptrocalyx ... 573

$\begin{array}{llll}\text { Calystegia } & \ldots & \ldots & 349\end{array}$

$\begin{array}{llll}\text { Calythrix } & \ldots & \ldots & 181\end{array}$

Camarosporium ... 778

Campandlacée $\quad$... 291

Campylopus ... ... 657

Campylothelium $\quad \ldots .7709$

$\begin{array}{llll}\text { Cananga } & \ldots & \ldots & 25\end{array}$

Canarium $\quad$... $\quad . . .866$

$\begin{array}{llll}\text { Canavalia } & \ldots & \ldots & 144\end{array}$

Candolleace

$\begin{array}{llll}\text { Canscora } & \text {... } & \text {.. } & 337\end{array}$

$\begin{array}{llll}\text { Cansjera } & \ldots & \ldots & 93\end{array}$

$\begin{array}{llll}\text { Cantharellus } & \ldots & \ldots & 723\end{array}$

$\begin{array}{llll}\text { Canthium } & \ldots & \ldots & 245 \\ & & & \end{array}$

Canthium ... ... 773

Capnodiastrum $\quad$... 851

$\begin{array}{llll}\text { Capnodium } & \ldots & \ldots & 768\end{array}$

Capparide

$\begin{array}{llll}\text { Capparis } & \ldots & \ldots & 36\end{array}$

Capparis $\quad \ldots \quad 758,763$

CAPRIFOLIACE E

$\begin{array}{llll}\text { Capsella } & \text {... } & \ldots & 341\end{array}$

Capsicum _... ... 357

Capsicum $\quad \ldots . \quad \ldots .354$

$\begin{array}{lll}\text { Capsosira } & \cdots & 295,418,358\end{array}$

$\begin{array}{lrl}\text { Capsule } & 295,418,358 \\ \text { Carallia } & . . & \ldots . .175\end{array}$

$\begin{array}{llll}\text { Carapa } & \ldots & \ldots & 88\end{array}$

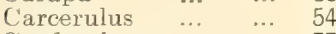

$\begin{array}{llll}\text { Cardamine } & \ldots & \ldots & 33\end{array}$

Cardiopteris ... $\quad . .993$

Cardiospermum $\quad \ldots \quad 112$

Carduus $\quad \ldots . \quad \ldots 276$

Cardwellia $\quad \ldots . \quad \ldots .454$

Carex $\quad$.... $\quad \cdots 602$

Careya $\quad . . . \quad . .203$

Cargillia $\quad \ldots .3 . . . .306$

$\begin{array}{llll}\text { Carica } & \text {... } & \text {... } 220\end{array}$

Carica $\quad . . . \quad \ldots 786$

Carissa $\quad \ldots . \quad \ldots .311$

Carissa $\quad \ldots . \quad \ldots 773$

Carnarvonia ... ... 454

Carpesium ...... 271

Carpopeltis $\quad . . \quad \ldots 828$
Page.

Carthamus

Cartonema

CaRYOPHYLLE 死

Caryopsis $\quad \ldots \quad \ldots 616$

Caryospermum _.. 96

$\begin{array}{llll}\text { Caryota } & \ldots & \ldots & 573 \\ \text { Casearia } & \ldots & \ldots & 215\end{array}$

Cassia

Cassia

Cassinia

Cassytha

Castanospermum

Castonospermum

Castanospora

Casuarina

CASTARINE.E

Catakidozamia

Catenella

Catosperma

Caulerpa

Caustis

Cedrela

Celastrinem

Celastrus

Celosia

Celsia

Celtis

$\begin{array}{llll}\text { Cenangium } & \ldots & \ldots & \\ & \ldots & \ldots & 755\end{array}$

$\begin{array}{llll}\text { Cenchrus } & \ldots & \ldots & \ldots 11\end{array}$

Centipeda

... 272

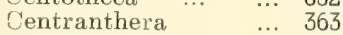

Centratherum $\quad . . .257$

'entroceras … $\quad . .828$

Centrolepide.

Centrolepis $\quad \ldots . \quad \ldots \quad 584$

Tephaleuros ... ... 708

$\begin{array}{llll}\text { Cephalozia } & \ldots & \ldots & 673\end{array}$

Cephaloziella $\ldots . \quad \ldots \quad 673$

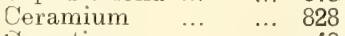

$\begin{array}{llll}\text { Cerastium } & \text {... } & \ldots & 48\end{array}$

Ceratium $\quad \ldots \quad \ldots 786$

Ceratodictyon $\quad \ldots .823$

Ceratodon $\quad$... ... 656

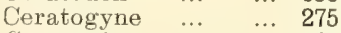

$\begin{array}{llll}\text { C'eratoniz } & \ldots & \ldots & 768\end{array}$

Ceratopetalum $\quad$... 169

Ceratophylleæ $\quad$... 508

Ceratophyllum $\quad 508$

$\begin{array}{llll}\text { Ceratopteris } & \ldots & \ldots & 638\end{array}$

Cerbera

Cercospora

Cerebella

Cereus

Ceriops

$\begin{array}{llll}\text { Ceropegia } & \ldots & \ldots & 175 \\ & \ldots & \ldots & 332\end{array}$

Cestrum $\quad . . . \quad \ldots 358$

Chætomitrium $\ldots . . .670$

Chretomium ... ... 762

Chætomorpha ... 795,852

Chætopeltis _... 792

$\begin{array}{llll}\text { Chretophoma } & \ldots & \ldots & 776\end{array}$

Chrotophora $\quad \ldots \quad$... 795

$\begin{array}{llll}\text { Chætospora } & \ldots & \ldots & 598\end{array}$

Chamæraphis $\quad$... 612

$\begin{array}{llll}\text { Champia } & \ldots & \ldots & 824\end{array}$

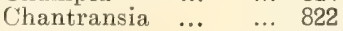

Chara

678

('HARACE.E … 678, 851

Chatosphæridium ... 794

$\begin{array}{llll}\text { Cheilanthes } & \text {... } & \ldots & 641 \\ \text { Cheilosporum } & & & 829\end{array}$

Cheiranthera $\quad . .443$
Page.

Chenolea _....... 408

Chenopodiace 2 .... 404

Chenopodium _... 404

Chilocarpus ... ... 317

Chiloglottis ... ... 539

Chiloscyphus ... ... 673

Chiodecton $\quad$.. $\quad . .7708$

Chionachne ... ... 616

Chionanthus ... ... 311

$\begin{array}{llll}\text { Chloanthes } & \ldots & \ldots & 381\end{array}$

Chloris $\quad \ldots \quad \ldots \quad 627$

Chlorodesmis ... ... 798

Chlorophyce

Chlorosplenium ... 755

Chnoospora ... $\quad . .8820$

Chondria $\quad$... $\quad$... 825

Chondrioderma ... 790

Chondrioderma … 790

Chondrococcus _... 829

Chondrodendron ... 29

Choretrum ... ... 469

Chorisandra ... ... 597

$\begin{array}{llll}\text { Chorizema } & \ldots & \ldots & 125\end{array}$

Chroococeus ... ... 830

Chrysophyllum _.. 305

Chrysopogon _... 619

Chrysymenia … 824

Chthonocephalus ... 271

Cichorium $\quad$... $\quad . .8839$

Cinnamomum $\quad . .431$

Cinnamomum $\quad 772,779$

Cintractia $\quad . . \quad \ldots \quad 771$

Cintractia $\quad \ldots . \quad \ldots 769$

Circinella $\quad \ldots \quad \ldots 768$

Cirrhopetalum _... 527

$\begin{array}{llll}\text { Cissampelos } & \ldots & \ldots & 29\end{array}$

Cissus $\quad \ldots . \quad \ldots \quad 106$

Citriobatus $\quad . .6 \quad \ldots \quad 43$

Citrullus $\quad \ldots \quad \ldots 221$

Citrus $\quad \because 768,776,782$

$\begin{array}{ll}\text { Citrus } & 768,776,782, \\ 787, & 793\end{array}$

Cladium _... ... 598

Cladoderris $\quad \ldots .7 . \quad \ldots 7739$

Cladodes $\quad$... $\quad . .4479$

Cladonia $\quad$... $\quad \ldots .684$

Cladophora $\quad$... $\quad . .795$

Cladosporium ... $\quad 783,851$

Cladosporium ... 783, 849

Clanophora ... ... 795

Claoxylon $\quad \ldots . \quad \ldots \quad 478$

Clathrocystis ... $\quad . .8830$

Clathroporina ... ... 710

Clathroptychium $\quad$... 789

$\begin{array}{lll}\text { Clathrus } & \ldots & 746,851\end{array}$

Claudopus $\quad$... $\quad$... 719

$\begin{array}{llll}\text { Clausena } & \ldots & \ldots & 82\end{array}$

$\begin{array}{llll}\text { Clavaria } & . . & \ldots & 743\end{array}$

Clavaria $\quad$... $\quad$... 739

$\begin{array}{llll}\text { Cleisostoma } & \ldots & \ldots & 536\end{array}$

Cleistanthus ... ... 474

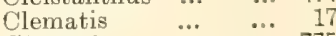

Clematis $\quad \ldots . \quad \ldots \quad 775$

Cleome $\quad \ldots . \quad \ldots .36$

Cleome $\quad \ldots .6 . . . .36$

Clerodendron ... $\quad \ldots \quad 386$

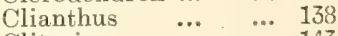

$\begin{array}{llll}\text { Clitoria } & \ldots & \ldots & 143\end{array}$

Closterium $\quad$... $\quad . . .802$

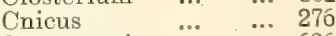

Coccocarpia ... $\quad . . .690$

Cocconeis $\quad \ldots \quad$... 415

Cocconema $\quad \ldots . \quad \ldots .817$

Cocculus $\quad \ldots . \quad \ldots .29$ 


\begin{tabular}{|c|c|c|c|c|c|c|c|c|c|c|}
\hline & & Page. & & & & age. & & & & age. \\
\hline Cochlearia . & & 785 & Cosmos & ... & $\ldots$ & 272 & Cyrtostylis & ... & $\ldots$ & 538 \\
\hline Cochlospermun & & 42 & Costus & $\ldots$ & $\ldots$ & 542 & Cytispora & ... & $\ldots$ & 776 \\
\hline Cocos & $\ldots$ & 574 & Cotula & ... & $\ldots$ & 272 & Cystophyllum & & $\ldots$ & 819 \\
\hline Codiæum & $\ldots$ & 478 & Crantzia & $\ldots$ & $\ldots$ & 229 & Cystopleura & ... & ... & 816 \\
\hline Codium & $\ldots$ & 798 & Craspedia & $\ldots$ & ... & 268 & Cystopus & ... & $\ldots$ & 768 \\
\hline Codonocarpus & & 411 & Crassulace e & $\ldots$ & $\ldots$ & 169 & & & & \\
\hline Coelachne & $\ldots$ & 624 & Cratægus & ... & $\ldots$ & 167 & Dacrymyces & $\ldots$ & $\ldots$ & 745 \\
\hline Coelastrum & $\ldots$ & 733 & Craterellus. & $\ldots$ & $\ldots$ & 739 & Dactylis & ... & $\ldots$ & 633 \\
\hline Coelebogyne & $\ldots$ & 479 & Cremocarpium & & $\ldots$ & 228 & Dædalea & $\ldots$ & & 737 \\
\hline Colospermum & & 249 & Crepidotus & $\ldots$ & $\ldots$ & 720 & Dadalea & $\begin{array}{l}\cdots \\
\cdots\end{array}$ & 727 & 736 \\
\hline Coenogonium & $\ldots$ & 699 & Crepis & $\ldots$ & $\ldots$ & 276 & Damia & $\ldots$ & $\ldots$ & 326 \\
\hline Coffea & $\ldots$ & 245 & Crepis & $\ldots$ & $\ldots$ & 775 & Dalbergia & $\ldots$ & $\ldots$ & 147 \\
\hline Coix & $\ldots$ & 616 & Cressa & $\ldots$ & $\ldots$ & 353 & Daldi & $\ldots$ & $\ldots$ & 759 \\
\hline Coldenia & $\cdots$ & 538 & Crinum & ... & $\ldots$ & 343 & Dallachya & .... & $\cdots$ & 100 \\
\hline Coleochare & $\ldots$ & 792 & Crinum & $\ldots$ & $\ldots$ & 776 & Damasonium & $\ldots$ & $\ldots$ & 580 \\
\hline Coleocoma & $\ldots$ & 263 & Cronartium & $\ldots$ & $\ldots$ & 772 & ara & $\ldots$ & $\ldots$ & 510 \\
\hline Coleus & $\cdots$ & 387 & Crotalaria & $\ldots$ & $\ldots$ & 132 & Dampiera & $\cdots$ & $\cdots$ & 287 \\
\hline Collema & $\ldots$ & 682 & Croton & $\ldots$ & $\ldots$ & 478 & honia & $\ldots$ & $\ldots$ & 627 \\
\hline Colletia & $\ldots$ & 106 & Crowea & $\ldots$ & $\ldots$ & 76 & ndra & $\ldots$ & $\ldots$ & 430 \\
\hline Colletotrichum & & 781 & Crucibulum & $\ldots$ & & 751 & ngia & $\begin{array}{l}\cdots \\
\ldots\end{array}$ & $\cdots$ & 454 \\
\hline Collybia & $\ldots$ & 717 & CRUCIFERE & $\ldots$ & 33 & 835 & $\mathrm{Da}$ & $\ldots$ & $\ldots$ & 778 \\
\hline Colocasia & $\ldots$ & 579 & Cryphæa & $\cdots$ & $\ldots$ & 664 & inia & ... & ... & 181 \\
\hline Cololejeunea & $\begin{array}{l}\cdots \\
\cdots\end{array}$ & 675 & Cryptandra & $\cdots$ & $\ldots$ & 106 & $\mathrm{Da}$ & $\cdots$ & $\cdots$ & 828 \\
\hline menia & $\ldots$ & 821 & Cryptocarya & $\ldots$ & $\ldots$ & 436 & & $\ldots$ & $\ldots$ & 828 \\
\hline brina & $\cdots$ & $\cdots$ & Cryptocarya & $\ldots$ & $\ldots$ & 766 & ladus & $\cdots$ & $\ldots$ & 796 \\
\hline Colutea & $\cdots$ & 138 & Cryptogams & $\ldots$ & .. & 634 & ypha & $\ldots$ & $\ldots$ & 755 \\
\hline Comatricha & $\ldots$ & 790 & Cryptonemia & ... & $\ldots$ & 829 & Dat & $\ldots$ & $\ldots$ & 358 \\
\hline RETACEE & $\ldots$ & 180 & ptonema & & $\ldots$ & 828 & & & $\ldots$ & 232 \\
\hline Con & ... & ... & osphærel & 110 & $\begin{array}{l}\cdots \\
\ldots\end{array}$ & 762 & lia & ... & $\cdots$ & 640 \\
\hline lyna. & ... & 561 & tostegia & $\ldots$ & ... & 325 & sonia & $\ldots$ & $\ldots$ & 169 \\
\hline ELYNACEE & & 561 & temma & & $\ldots$ & 276 & & $\ldots$ & $\ldots$ & 128 \\
\hline sonia & & 65 & tostylis & $\ldots$ & $\ldots$ & 537 & ia & $\ldots$ & $\ldots$ & 672 \\
\hline Comi & $\ldots$ & 257.839 & mis & $\begin{array}{l}\cdots \\
\cdots\end{array}$ & $\cdots$ & 221 & permum & $\cdots$ & $\ldots$ & 207 \\
\hline pogon & $\ldots$ & .. 821 & bita & $\ldots$ & $\ldots$ & 221 & ia & $\ldots$ & $\ldots$ & 397 \\
\hline taculum & & .. 325 & RBITACEE & $\cdots$ & $\cdots$ & 221 & $D e$ & $\cdots$ & $\cdots$ & 775 \\
\hline Conf & $\ldots$ & 795 & rbitaria & $\ldots$ & $\ldots$ & 762 & brea & $\ldots$ & $\ldots$ & 232 \\
\hline $\mathrm{CON}$ & $\ldots$ & $\ldots$ & Cudrania & $\ldots$ & $\ldots$ & 504 & Deli & $\ldots$ & $\ldots$ & 825 \\
\hline$o m a$ & $\ldots$ & $\ldots$ & $\mathrm{Cur}$ & ... & & 113 & obium & $\ldots$ & $\ldots$ & 519 \\
\hline phora & $\ldots$ & 742 & $C u_{2}$ & $\ldots$ & 113 , & 781 & robium & $\ldots$ & $\ldots$ & 778 \\
\hline Coniosporium & & 782 & CUPULIFERE & $\ldots$ & $\ldots$ & 508 & Iroceros & $\ldots$ & $\ldots$ & 678 \\
\hline Coniothyrium & & 778 & uligo & ... & ... & $5 \neq 5$ & mia & $\ldots$ & $\ldots$ & 96 \\
\hline CONNA] & & 124,835 & $\mathrm{Cu}$ & $\ldots$ & $\ldots$ & 539 & Den & $\ldots$ & $\ldots$ & 237 \\
\hline Conochæta & $\ldots$ & ... 762 & Cuscuta & ... & .. & 553 & atocarpor & & $\ldots$ & 703 \\
\hline mitrium & & 670 & Cut & $\ldots$ & $\ldots$ & 168 & natolithon & & $\ldots$ & 829 \\
\hline Conospermum & & $\ldots$ & Cyanocarpus & $\begin{array}{l}\cdots \\
\ldots\end{array}$ & $\ldots$ & 452 & Derr & $\ldots$ & $\ldots$ & 147 \\
\hline Conringia & $\ldots$ & & Cyanotis & $\ldots$ & $\ldots$ & 565 & anthus & $\ldots$ & $\ldots$ & 837 \\
\hline VULACE. & & 347,839 & nea & $\cdots$ & $\begin{array}{l}\cdots \\
\cdots\end{array}$ & 640 & & $\ldots$ & $\ldots$ & 829 \\
\hline rolvulus & $\ldots$ & .. 353 & thula & $\cdots$ & $\cdots$ & 403 & dium & $\begin{array}{l}\cdots \\
\cdots\end{array}$ & $\cdots$ & 802 \\
\hline Con & $\ldots$ & $\cdots$ & Cyathus & $\cdots$ & $\ldots$ & 746 & dium & $\cdots$ & & 139 \\
\hline Cor & $\ldots$ & $\ldots$ & DACE E & $\ldots$ & $\ldots$ & 513 & odium & & 763 & 851 \\
\hline Cor & ... & $\ldots$ & $\mathrm{Cyc}$ & $\cdots$ & & 513 & gonium & & $\ldots$ & 816 \\
\hline llina & $\ldots$ & -.. & C'ycas & ... & 768 & 790 & xia & $\ldots$ & $\cdots$ & 624 \\
\hline lopsis & $\cdots$ & -... & Cyclogyne & $\cdots$ & $\ldots$ & 138 & Dia & ... & ... & 790 \\
\hline $\mathrm{Co}$ & ... & $\ldots$ & Cyclotella & $\ldots$ & $\ldots$ & 81 & Dia & $\ldots$ & $\ldots$ & 557 \\
\hline Cor & ... & 338 & Cylindrocapsa & & $\cdots$ & 79 & & $\ldots$ & ... & 772 \\
\hline line & $\ldots$ & $\ldots$ & Cylindrocystis & & $\ldots$ & 80 & & $\ldots$ & $\ldots$ & 44 \\
\hline Cordyline & $\begin{array}{l}\cdots \\
\ldots\end{array}$ & $\begin{array}{l}\cdots \\
\cdots\end{array}$ & drosperm & aum & & 83 & Di & $\ldots$ & $\ldots$ & 762 \\
\hline Core & $\cdots$ & $\ldots$ & Cymbella & $\ldots$ & & 814 & achne & $\ldots$ & $\ldots$ & 624 \\
\hline rum & $\ldots$ & $\ldots$ & Cymbidium & $\ldots$ & 535, & 845 & nera & $\ldots$ & $\ldots$ & 778 \\
\hline & $\begin{array}{l}\cdots \\
\cdots\end{array}$ & $\begin{array}{l}\cdots \\
\cdots\end{array}$ & bidium & & $\ldots$ & 76 & dra & $\begin{array}{l}\cdots \\
\cdots\end{array}$ & $\cdots$ & 353 \\
\hline Cor & $\ldots$ & $\ldots$ & onotus & $\ldots$ & $\ldots$ & 27 & $m a$ & $\ldots$ & 534 & 743 \\
\hline Cor & $\ldots$ & $\ldots$ & ocea & $\begin{array}{l}\cdots \\
\ldots\end{array}$ & $\cdots$ & 58 & ggon & $\ldots$ & $\ldots$ & 557 \\
\hline ius & ... & $\begin{array}{l}\cdots \\
\cdots\end{array}$ & hum & $\begin{array}{l}\cdots \\
\cdots\end{array}$ & $\begin{array}{l}\cdots \\
\cdots\end{array}$ & 23 & Dichot & $\begin{array}{l}\cdots \\
\cdots\end{array}$ & $\cdots$ & 834 \\
\hline Cor & $\ldots$ & ... & don & $\begin{array}{l}\cdots \\
\ldots\end{array}$ & $\begin{array}{l}\cdots \\
\ldots\end{array}$ & 62 & cephala & & $\ldots$ & 258 \\
\hline nborchis & & $\ldots$ & lossum & & $\ldots$ & 34 & hia & & $\ldots$ & 640 \\
\hline Coryne & $\cdots$ & $\cdots$ & netra & & ... & 15 & & $\begin{array}{l}\cdots \\
\cdots\end{array}$ & $\begin{array}{l}\cdots \\
\ldots\end{array}$ & 396 \\
\hline Cor & $\cdots$ & $\begin{array}{l}\cdots \\
\cdots\end{array}$ & CEE & $\begin{array}{l}\cdots \\
\cdots\end{array}$ & $\cdots$ & 58 & Dicliptera & $\begin{array}{l}\cdots \\
\cdots\end{array}$ & $\cdots$ & 374 \\
\hline $\mathrm{Cor}$ & $\ldots$ & & $\mathrm{Cy}_{\mathrm{y}}$ & & $\ldots$ & 53 & Dicotyledons & & $\ldots$ & 18 \\
\hline leca & $\ldots$ & & & & & 77 & nella & & $\ldots$ & 656 \\
\hline Cor & $\ldots$ & $\ldots$ & Cyphella & $\begin{array}{l}\cdots \\
\ldots\end{array}$ & $\cdots$ & 74 & ema & $\begin{array}{l}\cdots \\
\ldots\end{array}$ & $\begin{array}{l}\cdots \\
\ldots\end{array}$ & 823 \\
\hline Corysanthes & $\ldots$ & $\ldots$ & Cyphomandra & & $\ldots$ & 35 & $\mathrm{um}$ & .. & $\ldots$ & 656 \\
\hline Cosmarium & ... & $\ldots 80$ & & & $\ldots$ & 257 & Dicrastyles & $\ldots$ & $\ldots$ & 381 \\
\hline Cosmarium & $\ldots$ & 805,80 & Cyrtandra & $\ldots$ & $\ldots$ & 364 & Dictidopteris & $\ldots$ & $\ldots$ & 642 \\
\hline
\end{tabular}


Page.

\begin{tabular}{|c|c|c|c|c|c|c|c|}
\hline Dictyonema & .. & & 743 & Dunbaria & & & 147 \\
\hline Dictyonema & ... & $\ldots$ & 743 & Durandex & $\cdots$ & $\ldots$ & 69 \\
\hline Dictyophora & $\ldots$ & $\ldots$ & 745 & Duranta & $\ldots$ & $\ldots$ & 382 \\
\hline Diotyosphæria & & $\ldots$ & 796 & Dysophylla & ... & $\ldots$ & 387 \\
\hline Dictyosphæriu & um & ... & 800 & Dysoxylon & $\ldots$ & & 86 \\
\hline Dictyota & $\ldots$ & $\ldots$ & 820 & Dysoxylon & $\ldots$ & 765 , & 776 \\
\hline Didiscus & $\ldots$ & $\ldots$ & 228 & Dysphania & ... & $\ldots$ & 396 \\
\hline Didymella & ... & $\cdots$ & 763 & & & & \\
\hline Didymium & $\ldots$ & ... & 790 & & & & \\
\hline Didymocarpus & & $\cdots$ & 364 & EBENACE & $\ldots$ & $\ldots$ & 306 \\
\hline Didymosphæri & & $\ldots$ & 763 & Ebermaiera & $\cdots$ & ... & 368 \\
\hline Dielseilla & $\cdots$ & ... & 758 & Ecballium & & $\ldots$ & 221 \\
\hline Digena. & ... & $\ldots$ & 827 & Echinocarpus & $\ldots$ & $\ldots$ & 67 \\
\hline Digitaria & $\ldots$ & .. & 603 & Echinopogon & & $\ldots$ & 623 \\
\hline Dillenia & $\ldots$ & $\ldots$ & 54 & Echinospermu & & $\ldots$ & 346 \\
\hline DILLENIACE & ... & $\cdots$ & 18 & Ecklonia & $\ldots$ & $\ldots$ & 820 \\
\hline Dillwynia & $\cdots$ & $\ldots$ & 129 & Eclipta & $\ldots$ & $\ldots$ & 271 \\
\hline Dilophus & ... & ... & 820 & Ectocarpus & & $\ldots$ & 821 \\
\hline Dimeria & $\ldots$ & ... & 616 & Ectropotheciu & & $\ldots$ & 667 \\
\hline Dimerosporiur & & $\ldots$ & 766 & Ectrosia & $\ldots$ & $\ldots$ & 632 \\
\hline Dimerosporiun & & $\ldots$ & 768 & Ehretia & $\ldots$ & $\ldots$ & 338 \\
\hline Dioscorea & $\ldots$ & $\cdots$ & 548 & Eichhornia & $\ldots$ & $\ldots$ & 559 \\
\hline Dioscorea & $\cdots$ & $\cdots$ & 765 & ELAEAGNACEE & $\cdots$ & $\ldots$ & 459 \\
\hline DIOSCORIDEE & $\cdots$ & $\cdots$ & 548 & Elæagnus & $\cdots$ & $\cdots$ & 459 \\
\hline Diospyros & $\cdots$ & $\cdots$ & 306 & Elæocarpus & $\cdots$ & $\cdots$ & 67 \\
\hline Diplachne & $\cdots$ & $\cdots$ & 629 & Elæodendron & & $\ldots$ & 96 \\
\hline Diplanthera & $\cdots$ & $\cdots$ & 368 & Elaodendron & $\cdots$ & $\ldots$ & 781 \\
\hline Diploderma & ... & $\cdots$ & 751 & Elaphomyces & $\cdots$ & $\cdots$ & 753 \\
\hline Diplodia & $\cdots$ & $\cdots$ & 778 & Elatine & $\ldots$ & $\ldots$ & 49 \\
\hline Diplodina & & i? & 778 & ELATINEE & ... & $\ldots$ & 49 \\
\hline Diploglottis & & 772 , & 775 & Elatostemma & $\ldots$ & $\ldots$ & 505 \\
\hline Diplogramma & & $\cdots$ & 708 & Elephantopus & $\ldots$ & $\ldots$ & 257 \\
\hline Diploschistes & $\cdots$ & $\cdots$ & 699 & Elettaria & $\ldots$ & $\cdots$ & 542 \\
\hline Diplospora & $\cdots$ & ... & 241 & Eleusine & $\cdots$ & $\ldots$ & 628 \\
\hline Diplotegia & $\cdots$ & $\cdots$ & 291 & Elionurus & $\ldots$ & $\ldots$ & 619 \\
\hline Dipodium & $\cdots$ & $\cdots$ & & Elynanthus & $\ldots$ & $\ldots$ & 598 \\
\hline Dipodium & ... & $\cdots$ & 535 & Elythophorus & $\ldots$ & $\ldots$ & 629 \\
\hline Discaria & $\cdots$ & $\cdots$ & $10 t$ & Embelia & $\ldots$ & $\ldots$ & 300 \\
\hline Dischidia & $\cdots$ & $\cdots$ & 332 & Embothrium & $\ldots$ & $\ldots$ & 455 \\
\hline Discina & $\cdots$ & ... & 753 & Emex & $\ldots$ & $\cdots$ & 417 \\
\hline Discina & ... & $\cdots$ & 753 & Emilia & $\ldots$ & $\ldots$ & 275 \\
\hline Disphinctium & $\ldots$ & $\cdots$ & 805 & Emmenosperm & num & $\ldots$ & 106 \\
\hline Dissiliaria & $\cdots$ & $\ldots$ & 477 & Encephalartos & & $\ldots$ & 513 \\
\hline Distichophyllu & $\mathrm{Am}$ & $\cdots$ & 663 & Enchylæna & $\ldots$ & $\ldots$ & 409 \\
\hline Distichostemor & & $\cdots$ & & Encyonema & $\ldots$ & $\ldots$ & 814 \\
\hline Ditrichium & ... & & 656 & Endiandra & $\cdots$ & $\cdots$ & 431 \\
\hline Diuris & $\cdots$ & 537, & 847 & Endiandra & $\cdots$ & $\cdots$ & \\
\hline Docidium & $\cdots$ & $\cdots$ & 805 & Endocarpon & $\cdots$ & $\ldots$ & 710 \\
\hline Docidium & $\therefore$ & $\ldots$ & 805 & Enhalus & ... & $\ldots$ & 519 \\
\hline Dodonæa & $\ldots$ & $\ldots$ & 11 & Enhydra & $\ldots$ & $\ldots$ & 271 \\
\hline Dolichandrone & & $\ldots$ & 36 & Entada & $\ldots$ & $\cdots$ & 153 \\
\hline Dolichos & $\ldots$ & $\ldots$ & & Enteromorpha & & $\ldots$ & 794 \\
\hline Doodia & $\cdots$ & $\ldots$ & 644 & Entodon & $\ldots$ & $\therefore$ & 667 \\
\hline Dopatrium & $\cdots$ & $\cdots$ & 361 & Entosthodon & ... & $\ldots$ & 661 \\
\hline Dorstenia & $\cdots$ & $\ldots$ & 485 & Entyloma & ... & $\cdots$ & 771 \\
\hline Doryanthes & ... & & 548 & EPACRIDE王 & $\ldots$ & $\ldots$ & \\
\hline Doryanthes & ... & 535, & 776 & Epacris & ... & $\ldots$ & \\
\hline Doryphora & $\cdots$ & $\ldots$ & 419 & Epaltes & $\ldots$ & $\cdots$ & 263 \\
\hline Dothidea & ... & $\ldots$ & 76 & Ephemerum & $\cdots$ & ... & 661 \\
\hline Dothidella & ... & $\ldots$ & 761 & Epichloe & $\ldots$ & $\ldots$ & \\
\hline Dothiorella & $\cdots$ & $\ldots$ & 77 & Epicoccum & $\cdots$ & $\cdots$ & 789 \\
\hline Dracæena & $\cdots$ & ... & 553 & Epilobium & $\cdots$ & $\cdots$ & \\
\hline Dracophyllum & & $\cdots$ & & Epipactis & $\ldots$ & $\ldots$ & \\
\hline Drakæa & & $\therefore$ & 53 & Epipogum & ... & $\ldots$ & 539 \\
\hline Drepanocladus & & $\ldots$ & 670 & Epithemia & $\therefore$ & & \\
\hline Drimys & $\ldots$ & $\therefore$ & 21 & Epymenia & $\ldots$ & & 824 \\
\hline Drosera & ... & $\therefore$ & 174 & Eragrostis & $\ldots$ & 629 , & 849 \\
\hline DROSERACEE & ... & ... & 174 & Eragrostis & ... & $\ldots$ & 756 \\
\hline Drupe & ... & $\therefore$ & 167 & Eranthemum & $\ldots$ & $\ldots$ & 374 \\
\hline Drymaria & $\cdots$ & $\ldots$ & & Erechthites & $\ldots$ & $\ldots$ & 275 \\
\hline Drymophila & & & 553 & Eremochloa & $\ldots$ & $\ldots$ & 617 \\
\hline Drymophlæus & & $\therefore$ & 57 & Eremophila & $\ldots$ & ... & 37 \\
\hline Duboisia & $\ldots$ & $\therefore$ & & Eria & $\ldots$ & $\ldots$ & \\
\hline
\end{tabular}

Page.

\begin{tabular}{|c|c|c|}
\hline Eriachne & & \\
\hline Eriachne & $\ldots$ & $\ldots$ \\
\hline ERICAOE E & $\ldots$ & $\ldots$ \\
\hline Erigeron & $\ldots$ & ... \\
\hline Erinella & $\ldots$ & $\ldots$ \\
\hline ERIOCAULEE & $\ldots$ & $\ldots$ \\
\hline Eriocaulon & ... & ... \\
\hline Eriochilus & ... & $\ldots$ \\
\hline Eriochlamys & $\ldots$ & $\ldots$ \\
\hline Eriochloa & $\ldots$ & $\ldots$ \\
\hline Eriodendion & ... & ... \\
\hline Erioderma & $\ldots$ & $\ldots$ \\
\hline Eriosema & $\ldots$ & $\ldots$ \\
\hline Eriostemon & $\ldots$ & $\ldots$ \\
\hline ichium & ... & ... \\
\hline Erod & $\ldots$ & $\ldots$ \\
\hline Ery & $\ldots$ & ... \\
\hline Eryngium & $\ldots$ & $\ldots$ \\
\hline Ery & $\ldots$ & $\ldots$ \\
\hline Erythræa & $\ldots$ & $\ldots$ \\
\hline Erythrina & $\ldots$ & $\ldots$ \\
\hline hrina & $\ldots$ & \\
\hline hroclonium & & \\
\hline Erythroploum & & $\ldots$ \\
\hline Erythroxylon . & & \\
\hline & ... 18 & \\
\hline Ethulia & $\cdots$ & $\cdots$ \\
\hline Euastrum & ... & ... \\
\hline Eucalyptus & & \\
\hline
\end{tabular}

Eucheuma .... ... 823

Euchlæna $\quad$... $\quad . .6616$

Fiu-Filoridare $\quad \ldots . \quad \ldots \quad 821$

Eugenia $\quad \ldots ., \ldots 208$

Eugenia $766,771,776,781$

Eulejeunea ... ... 673

$\begin{array}{llll}\text { Eulophia } & \ldots & \ldots & 535\end{array}$

$\begin{array}{llll}\text { Eumitria } & \ldots & \ldots & \ldots 85\end{array}$

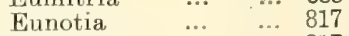

Eunotiace正 $\quad \ldots . \quad \ldots \quad 817$

Euonymus $\quad \ldots \quad \ldots 93$

Euosmolejeunea ... 675

Eupatorium ... ... 258

$\begin{array}{llll}\text { Euphorbia } & \ldots & \ldots & 470\end{array}$

EUPHORBIACE $E$ E $\quad \ldots \quad 470$

$\begin{array}{llll}\text { Euphoria } \quad \ldots & \ldots & 116\end{array}$

Euphrasia $\quad \ldots \quad \ldots 363$

$\begin{array}{llll}\text { Eupomatia } & \ldots & \ldots & 29\end{array}$

Eupomatia $\quad \ldots .7 .7 .766$

Eurhynchium $\quad \ldots 669$

Euroschinus ... ... 119

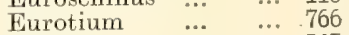

Eurycles ... ... 543

Eustrephus $\quad \ldots \quad \ldots . \quad \ldots 53$

$\begin{array}{llll}\text { Eutypa } & \text {.. } & \ldots & 762\end{array}$

Euxolus $\quad \ldots .3 .397$

$\begin{array}{llll}\text { Evodia } & \ldots & \ldots & 7 \\ & & & \end{array}$

Evodia $\quad \ldots . \quad \ldots 775$

Evolvulus $\quad \ldots \quad \ldots \quad 353$

Excæcaria $\quad \ldots . \quad \ldots .480$

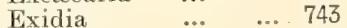

Exoascus $\quad \ldots .7 \% 756$

Exocarpus $\quad \ldots \quad \ldots 469$

Exocarya $\quad \ldots . \quad \ldots 597$

$\begin{array}{llll}\text { Eyrea } & \ldots & \ldots & \\ & & & \end{array}$

Fabronia $\quad \ldots 6 \quad \ldots 667$

$\begin{array}{llll}\text { Fagopyrum } & \ldots & \ldots & 612 \\ & & \ldots & \end{array}$

$\begin{array}{llll}\text { Fagræa } & \ldots & \ldots & 337\end{array}$

$\begin{array}{llll}\text { Fagus } & \ldots & \ldots & 508\end{array}$

$\begin{array}{llll}\text { Faradaya } & \ldots & \ldots & \\ & & & \end{array}$ 


\begin{tabular}{|c|c|c|c|}
\hline & & & age. \\
\hline Tatsia & & $\ldots$ & 236 \\
\hline Favolus & ... & ... & 738 \\
\hline Fenzlia & ... & ... & 207 \\
\hline Festuca & $\ldots$ & $\ldots$ & 633 \\
\hline FICOIDE $E$ & $\ldots$ & & 227 \\
\hline Ficus & & 485 , & 840 \\
\hline Ficus & 61,775 & 776 , & 789 \\
\hline Filices & & 635 & \\
\hline Fimbriaria & $\ldots$ & 676 , & 849 \\
\hline Fimbristylis & $\ldots$ & & 534 \\
\hline 'imbristylis & & 761 , & 709 \\
\hline idens & $\ldots$ & $\ldots$ & 570 \\
\hline Fit & $\ldots$ & $\ldots$ & 25 \\
\hline lagellaria & $\ldots$ & $\ldots$ & 565 \\
\hline IE $E$ & $\mathrm{E} \ldots$ & & \\
\hline Fla & . $\quad .$. & ... & 272 \\
\hline ngia & $\ldots$ & & \\
\hline Fle & & & \\
\hline ia & & & 90 \\
\hline lindersia & 758 & 759 , & 781 \\
\hline FLC & $\ldots$ & $\ldots$ & 821 \\
\hline Flo: & $\ldots$ & $\therefore$ & 565 \\
\hline Flu & $\ldots$ & $\ldots$ & 416 \\
\hline alum & $\ldots$ & $\ldots$ & 22 \\
\hline or & $\ldots$ & $\ldots$ & 732 \\
\hline nea & $\ldots$ & $\ldots$ & 478 \\
\hline onia & a ... & $\ldots$ & 676 \\
\hline ariacex & & $\ldots$ & 816 \\
\hline Fragaria & $\ldots$ & $\ldots$ & 168 \\
\hline ra & $\ldots$ & $\ldots$ & 775 \\
\hline Fra & & & 4 \\
\hline KENIACEA & & $\ldots$ & \\
\hline$a$ & $\ldots$ & $\ldots$ & 510 \\
\hline etia & .. & $\ldots$ & \\
\hline ania & $\ldots$ & $\ldots$ & 675 \\
\hline tulia & & $\ldots$ & 814 \\
\hline $\mathrm{CE} E$ & & $\ldots$ & \\
\hline Fuc & .. & ... & 819 \\
\hline osia & & ... & \\
\hline ena & . $\quad \ldots$ & $\ldots$ & \\
\hline & $\ldots$ & & \\
\hline Fumago & & .. & 786 \\
\hline & & & \\
\hline & $\ldots$ & & 662 \\
\hline & & 71 & 851 \\
\hline & $\ldots$ & & \\
\hline & $\ldots$ & 787 & \\
\hline Fusicladium & & $\ldots$ & \\
\hline usicolla & . & & \\
\hline usisporum & ... & & \\
\hline
\end{tabular}

Gahnia

$\begin{array}{lll}\ldots & \ldots & 144\end{array}$

Galbulus = Strobitus ‥ 822

Galbulimima ... ... 25

$\begin{array}{llll}\text { Galega } & \ldots & \ldots & \\ \text { Gralegl } & & \ldots & \end{array}$

Galeola $\quad 536$

Galinsoga $\cdots \quad \cdots \quad 272,837$

Galium $\ldots 257$

Gamopetale

Gamospora $\quad \ldots . \quad \ldots .779$

Ganophyllum _... 86

Garcinia $\quad \ldots \quad \ldots .54$

Gardenia $\quad \ldots .242$

Gardenia $\quad \ldots \quad 241,772$

Garovaglia .... ... 664

Garuga ...

Gastrodia … ... ... 539

Gastrolobium ... $\quad \ldots . .128$

Geaster $\quad \ldots . \quad \ldots 751$
Page.

Geijera $\quad \ldots \quad \ldots \quad 81$

Geissois $\quad \ldots . \quad \ldots .169$

Geitonoplesium ... 553

Gelidiopsis $\quad \ldots \quad$... 823

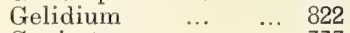

Geniostoma $\quad \ldots \quad \ldots 337$

GentianeE $\quad \ldots . \quad \ldots .337$

Geococcus $\quad \ldots \quad$... 34

Geodorum $\quad \ldots \quad \ldots \quad 535$

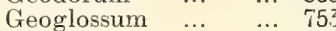

Geophila $\quad \ldots . \quad \ldots \quad 249$

Geopyxis $\quad \ldots \quad \ldots 753$

Geraniacem $\quad \ldots \quad \ldots \quad 72$

$\begin{array}{lllr}\text { Geranium } & \ldots & \ldots & 72 \\ \text { GeSNeRACEE } & \ldots & \ldots & 364\end{array}$

Gibellia $\quad \ldots . \quad \ldots \quad 762$

Gigartina $\quad \ldots \quad \ldots 822$

Gigaspermum ... $\quad . . .66$

Gilberella $\quad \ldots . \quad \ldots .758$

$\begin{array}{llll}\text { Gillbeea } & \ldots & \ldots & 169\end{array}$

Glaucocystis ... ... 834

Gleichenia $\quad \ldots \quad \ldots 638$

Glochidion $\quad \ldots . \quad \ldots .476$

Gloeocystis $\quad \ldots \quad \ldots 881$

$\begin{array}{llll}\text { Gloeoporus } & \ldots & \ldots & 738 \\ \text { Glo } & \cdots & & \end{array}$

$\begin{array}{llll}\text { Gloeosporium } & \ldots & \ldots & 779 \\ \text { Gloeothece } & \cdots & & 830\end{array}$

$\begin{array}{llll}\text { Gloothece } & \ldots & \ldots & 830 \\ \text { Glotoium } & \ldots & \ldots & 793\end{array}$

Glonium $\ldots . \ldots 756$

Glossocarya $\quad \ldots \quad \ldots \quad 386$

$\begin{array}{llll}\text { Glossodia } & \ldots & \ldots & 538\end{array}$

Glossogyne $\quad \ldots \quad \ldots .272$

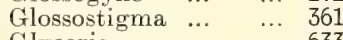

Glyceria $\quad \ldots \quad \ldots 633$

$\begin{array}{llll}\text { Glycine } & \ldots & \ldots & 143\end{array}$

$\begin{array}{lllr}\text { Glycosmis } & \ldots & & \\ \text { Glyphis } & & \cdots & 707\end{array}$

Glyphomitrium $\quad \ldots .657$

Gmelina $\quad \ldots \quad \ldots \quad 386$

Grnaphalium $\ldots \quad \ldots \quad 266$

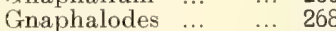

Gnephosis $\quad$... 260

Gomphandra ... ... 93

Gomphocarpus _.. 325

Gompholobium 125,837

Gomphonema ... ... 815

Gomphrena $\quad \ldots \quad$... 404

Gonatozygon ... $\quad$.. 802

Gongronema $\quad \ldots \quad \ldots \quad 326$

Croniomitrium ... 661

Goodenia

Goodenia

Goodenovien

$\begin{array}{llll}\text { Goodia } & \ldots & \ldots & 132 \\ \text { Goodyera } & & \ldots & \end{array}$

Goodyera $\quad \ldots . \quad \ldots \quad 536$

$\begin{array}{lllr}\text { Gossypium } & \ldots & \ldots & 61\end{array}$

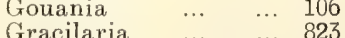

$\begin{array}{lll}\text { Gracilaria } & \ldots & \ldots 02823 \\ \text { Gramine } & \ldots & 602,849\end{array}$

Grammitis $\quad \ldots \quad \ldots \quad 649$

$\begin{array}{llll}\text { Grandinia } & \ldots & \ldots & 739\end{array}$

Graphina $\quad . . \quad \ldots 706$

Graphiola $\quad \ldots \quad$... 771

$\begin{array}{llll}\text { Craphis } & \ldots & \ldots & 704\end{array}$

Crraptophyllum _.. 375

$\begin{array}{llll}\text { Fratiola } & \ldots & \ldots & 361\end{array}$

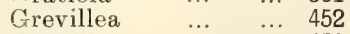

$\begin{array}{llll}\text { Grevillea } & \ldots & \ldots & 452\end{array}$

Grewia $\quad \ldots \quad \ldots 66$

Grimmia $\quad \ldots \quad \ldots \quad 657$

$\begin{array}{llll}\text { Grimanania } & \ldots & \ldots & 167\end{array}$

$\begin{array}{llll}\text { Truepinia } & \ldots & \ldots & 767 \\ & & \ldots & \end{array}$

Guettarda $\quad \ldots \quad \ldots 241$
Guettirda 245

$\begin{array}{lllr}\text { Guilfoylix } & \ldots & \ldots & 245 \\ & \ldots & \ldots & 85\end{array}$

Gulubia $\quad \ldots . \quad \ldots \quad 573$

Gunnia $\quad \ldots \quad \ldots .227$

Guttiferea $\quad \ldots \quad \ldots \quad 54$

Gyalectedium $\quad$... 700

Gymnanthera ... ... 325

Gymnena $\quad \ldots \quad \ldots \quad 326$

Gymnogongrus _.. 822

Gymnosorus $\quad \ldots \quad \ldots \quad \ldots 19$

Gymnosporia $\quad$... 96

Gymnostachys $\quad \ldots \quad 579$

Gymnostomum _.. 658

$\begin{array}{llll}\text { Gymnozyga } & \ldots & \ldots & 802\end{array}$

Gynandropsis ... $\quad \ldots .36$

Gymnospermeæ ... 510

$\begin{array}{llll}\text { Gynura } & \text {... } & \text {... } & 275\end{array}$

Gypsophila $\quad \ldots \quad \ldots \quad$... 441

Gyrocarpus $\quad \ldots \quad \ldots \quad 180$

$\begin{array}{llll}\text { Gyrosigma } & \ldots & \ldots & 813\end{array}$

Habenaria ... ... 539

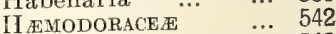

Hæmodorum ... $\quad \ldots .542$

$\begin{array}{llll}\text { Hainesia } & \ldots & \ldots & 779\end{array}$

Hakea $\quad$... $\quad$... 453

Hakea $\quad \ldots . \quad \ldots 766$

Halfordia $\quad \ldots \quad \ldots .81$

Halgania $\quad \ldots . \quad \ldots 338$

Halicorne $\quad \ldots \quad \ldots 796$

Halimeda $\quad \ldots \quad \ldots 798$

Haliseris $\quad$... $\quad \ldots .820$

Halophila $\quad$.. $\quad \ldots .519$

$\begin{array}{llll}\text { Haloplegma } & \ldots & \ldots & \ldots 28\end{array}$

HALORAGE

Haloragis $\quad \ldots \quad \ldots . \quad 174$

Halymenia $\quad \ldots . \quad \ldots .828$

Halymenia $\quad . . \quad \ldots \quad 824$

$\begin{array}{llll}\text { Hamaspora } & \ldots & \ldots & 773\end{array}$

$\begin{array}{lllr}\text { Hannafordia } & \text {... } & \ldots & 66\end{array}$

Hapalosiphon $\quad$... 832

Haplostichanthus ... 25

Hardenbergia $\quad$... 143

Harpographium ... 786

$\begin{array}{llll}\text { Harpullia } & \ldots & \ldots & 110\end{array}$

Harpullia $\quad \ldots . \quad \ldots .113$

Harrisonia $\ldots . \quad \ldots \quad 85$

Haussmannia ... $\quad \ldots .368$

$\begin{array}{llll}\text { Hearnia } & \ldots & \ldots & \\ & & \end{array}$

$\begin{array}{llll}\text { Hedera } & \ldots & \ldots & 236 \\ & \ldots & \ldots & 236\end{array}$

Hedraianthera $\quad$... 96

$\begin{array}{llll}\text { Hedwigia } & \text {... } & \text {.. } & 664\end{array}$

Hedacarya $\quad \ldots . \quad \ldots \quad 419$

$\begin{array}{llll}\text { Hedyotis } & \ldots & \ldots & 237\end{array}$

Heleocharis $\ldots . \quad \ldots .591$

$\begin{array}{llll}\text { Helichrysum } & \ldots & \ldots & 267\end{array}$

$\begin{array}{llll}\text { Helicia } & \ldots & \ldots & 452\end{array}$

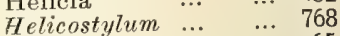

$\begin{array}{llll}\text { Helicteres } & \ldots & \ldots & 65 \\ & & \ldots & \end{array}$

Heliotropium ... $\quad$... 346

$\begin{array}{llll}\text { Helipterum } & \ldots & \text {.. } & 2 \xi 6\end{array}$

$\begin{array}{llll}\text { Hellenia } & \ldots & \ldots & \ldots \\ \end{array}$

Helmholtzia $\ldots . \quad \ldots .561$

THelminthocarpon $\quad \ldots \quad 707$

Helminthosporium ... 783

Telminthostachys $\quad \ldots .635$

Helotium $\quad \ldots \quad \ldots \quad 755$

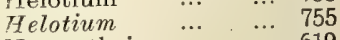

Hemarthria $\quad \ldots \quad \ldots 619$

Hemarthria $\quad \ldots \quad$... 773

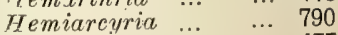

Hemicyclia $\ldots . \quad \ldots \quad 477$ 
Page.

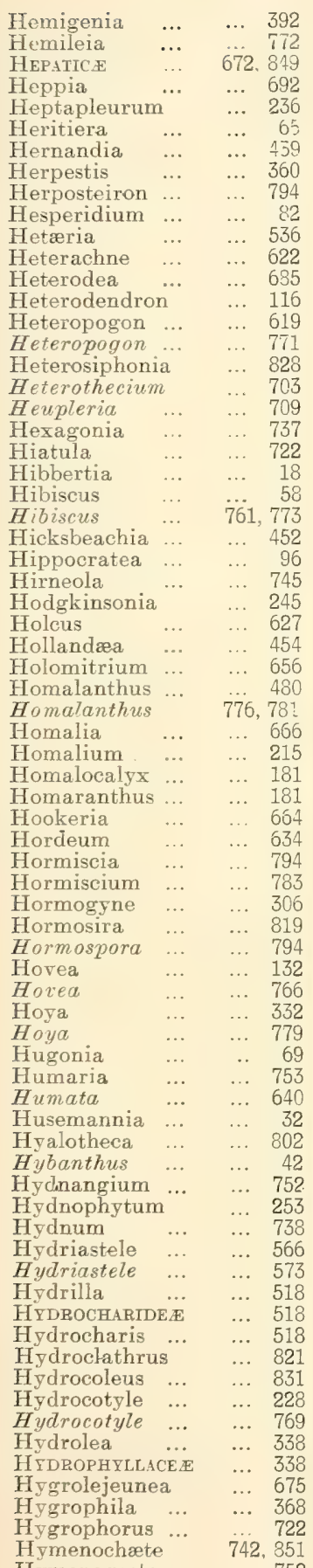

Page.

Hymenolepis ... _... 849

HYMENOMYCEIES _.. 714

Hymenophyllum _.. 639

Hymenosporum _.. 42

Hymenostomum $\quad \ldots .658$

HYPERICINE E ...

Hypericum ...

Hypnea ...

Hypnum ...

Hypocarpium ...

Hypochæris ...

Hypochoris

$\begin{array}{llll}\text { Hypochnus } & \ldots & \ldots & 772 \\ & \ldots & \ldots & 742\end{array}$

ïypochnus $\quad$... ... 786

Hypocrea $\quad \ldots \quad \ldots 756$

Hypocrella $\quad \ldots \quad \ldots 756$

Hypoderma ... ... 758

$\begin{array}{llll}\text { Hypoglossum } & \ldots & \ldots & 825\end{array}$

$\begin{array}{llll}\text { Hypcelyptum } & \text {... } & \text {.. } & 597\end{array}$

$\begin{array}{llll}\text { Hypoestes } & \text {... } & \text {.. } & 374\end{array}$

Hypolæna $\quad$.. $\quad \ldots \quad 588$

Hypolepis $\quad$... $\quad \ldots .641$

Hypolytrum $\quad \ldots \quad \ldots \quad 597$

Hypomyces ... ... 758

Hypopterygium ... 663

$\begin{array}{llll}\text { Hypoxis } & \ldots & \ldots & 543\end{array}$

Hypoxylon $\quad \ldots . \quad 759$

$\begin{array}{llll}\text { Hypserpa } & \ldots & \ldots & 27\end{array}$

$\begin{array}{llll}\text { Hyptiandra } & \ldots & \ldots & 85\end{array}$

$\begin{array}{llll}\text { Hysterium } & \ldots & \ldots & 758\end{array}$

Hysterographium $\quad \ldots \quad 756$

Hyposphila $\quad . . \quad \ldots 96$

Hypospila $\quad \ldots \quad$... 763

Iberis

Ichnanthus

Ichnocarpus

Ileodictyon

Ilex

(1)

$\begin{array}{llll}\text { ILLECEBRACE E } & \ldots & \ldots & 396\end{array}$

$\begin{array}{llll}\text { Illosporium } & \ldots & \ldots & 787\end{array}$

Imperata $\ldots . . .616$

$\begin{array}{llll}\text { Indigofera } & \ldots & \ldots & 136\end{array}$

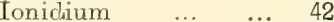

Iphigenia $\quad . . \quad 559$

Ipomæa $\quad \ldots \quad 347,840$

IRIDACE $E$ E

$\begin{array}{llll}\text { Irpex } & \ldots & \ldots & 739\end{array}$

$\begin{array}{llll}\text { Isachne } & \ldots & \ldots & 603\end{array}$

$\begin{array}{llll}\text { Tsaria } & \ldots & \ldots & 786\end{array}$

Isariopsis $\quad \ldots . \quad \ldots 787$

Ischremum $\quad$...

$\begin{array}{llll}\text { Iseilema } & \ldots & \ldots & 620\end{array}$

$\begin{array}{llll}\text { Isoetes } & \ldots & \ldots & 634\end{array}$

$\begin{array}{llll}\text { Isoetopsis } & \ldots & \ldots & 275\end{array}$

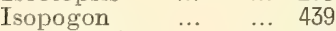

Isopterygium $\quad \ldots \quad \ldots 669$

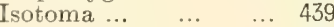

$\begin{array}{llll}\text { Tsotropis } & \ldots & \ldots & 125\end{array}$

Ithyphallus $\quad \ldots \quad$... 746

Ixiolæna $\quad \ldots . \quad \ldots \quad 267$

$\begin{array}{llll}\text { Ixora } & \ldots & \ldots & 245\end{array}$

Jacksonia

Jucksonia

Jambosa

Jania

Jasminum

Jatropha

Jeannerettia ...
Josephinia

Page.

Juncus $\quad \ldots . \quad \ldots 566$

Juncus $\quad \ldots \quad 763,772$

Jungermannia $\quad$.. 672

$\begin{array}{llll}\text { Jussiæa } & \ldots & \ldots & 215 \\ & \text { Justicia } & \ldots & \end{array}$

Justicia $\quad$... $\quad \ldots \quad 374$

Kayea

54

Kennedya $\quad \ldots \quad 65,143$

Kentia $\quad \ldots \quad \ldots 573$

Kentrophyllum _.. 276

Keraudrenia ... ... 66

Kermadecia $\quad \ldots \quad \dddot{452}, 453$

Kibara $\quad . .4 \quad . . .419$

Kirchneriella $\ldots . . . .8800$

Kleinhovia $\quad \ldots \quad \ldots 65$

$\begin{array}{llll}\text { Knoxia } & \ldots & \ldots & 245\end{array}$

$\begin{array}{llll}\text { Kochia } & \ldots & \ldots & 240 \\ & \ldots & \ldots & 408\end{array}$

Koeleria $\quad \ldots 6 \quad \ldots 629$

Kreysigia $\quad \ldots \quad$... 559

Kunzea $\quad \ldots . \quad \ldots$... 1871

Kyllinga $\quad \ldots \quad \ldots 591$

LABIAT正 $\quad \ldots \quad 387,840$

$\begin{array}{llll}\text { Labichea } & \ldots & \ldots & 152\end{array}$

Lachnea $\quad \ldots \quad \ldots 753$

Lachnea $\quad$... $\quad$... 790

Lachnella $\quad \ldots .7 .75$

Lachnocladium .... 739

Lactarius _.. ... 722

$\begin{array}{lll}\text { Lactuca } & \ldots & 277,839\end{array}$

Lagenaria $\quad \ldots \quad \ldots 221$

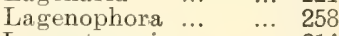

Lagerstrœmia ... $\quad$... 214

Lagerstromia $\quad$.. 765

$\begin{array}{llll}\text { Lagunaria } & \ldots & \ldots & 61\end{array}$

$\begin{array}{llll}\text { Lamarckia } & \text {.. } & \text {... } & 629\end{array}$

$\begin{array}{llll}\text { Lamium } & \ldots & \ldots & 388\end{array}$

$\begin{array}{llll}\text { Lam pocarya } & \text {... } & \text {... } & 600\end{array}$

Lamprolobium _.. 137

$\begin{array}{llll}\text { Lantana } & \text {... } & \ldots & 382\end{array}$

Laportea $\quad$... $\quad . .6504$

Lappago $\quad \ldots .6 \% 612$

$\begin{array}{llll}\text { Lapula } & \ldots & \ldots & 346\end{array}$

$\begin{array}{llll}\text { Laschia } & \ldots & \ldots & 738\end{array}$

$\begin{array}{llll}\text { Lasia } & \ldots & \ldots & 664\end{array}$

Lasianthus f.. ... 249

Lastadia $\quad \ldots \quad \ldots 763$

Lathyrus $\quad \ldots \quad 766,781$

Laurencia $\quad \ldots . \quad \ldots \quad 825$

LAURINEA $\quad \ldots . \quad \ldots .430$

$\begin{array}{llll}\text { Lavatera } & \ldots & \ldots & 54\end{array}$

Lawrencella $\quad . .5 \quad \ldots .57$

Laxmannia $\quad \ldots . \quad \ldots .559$

Lecanora $\quad$... 694.701

Lecidea $\quad \ldots \quad \ldots 700$

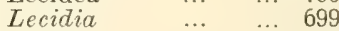

Ledgeria $\quad \ldots \quad \ldots 536$

Leea $\quad \ldots . \quad \ldots .112$

Leersia $\quad . . .6 \quad \ldots 612$

Leersia $\quad \ldots \quad 771,772$

Legnephora $\quad \cdots \quad$... 29

$\begin{array}{llll}\text { Legume } & \ldots & \ldots & 144\end{array}$

Leguninos.

$\begin{array}{llll}\text { Leibleinia } & \ldots & \ldots & 832\end{array}$

$\begin{array}{lllr}\text { Leichhardatia } & \ldots & 29 \\ \text { Leiogramma } & \ldots & 705,707\end{array}$

Lejeunia $\quad . .6 \quad \ldots 673$

Lémbophyllum $\quad$... 672 


\begin{tabular}{|c|c|c|c|c|c|c|c|c|c|c|c|}
\hline & \multicolumn{3}{|c|}{ Page. } & \multicolumn{4}{|c|}{ Page. } & \multicolumn{4}{|c|}{ Page. } \\
\hline Lembosia & $\cdots$ & $\cdots$ & 756 & Litobrochia & $\cdots$ & & 692 & Mangifera & $\cdots$ & & \\
\hline Lemna & $\cdots$ & ... & 580 & Litsea & $\ldots$ & & 431 & Manisuris & $\cdots$ & & \\
\hline LEMNACEæ & $\cdots$ & $\ldots$ & 579 & Litsea & ... & 761 , & 765 & Mapania. & & & \\
\hline Lenormandia & $\cdots$ & $\cdots$ & 827 & Livistona & $\ldots$ & $\ldots$ & 574 & Marasmius & $\ldots$ & & \\
\hline LENTIBULARIE $A$ & & $\ldots$ & 363 & Livistona & $\cdots$ & ... & 765 & Marattia & & & \\
\hline Lentinus & $\ldots$ & ... & 725 & Lobelia & $\ldots$ & ... & 291 & Marchantia & $\cdots$ & $\cdots$ & 676 \\
\hline Lenzites & $\ldots$ & $\ldots$ & 727 & Logania & $\cdots$ & ... & 337 & Marianthus & $\ldots$ & & \\
\hline Leonotis & $\ldots$ & $\cdots$ & 388 & LOGANIACEA & $\ldots$ & $\ldots$ & 332 & Marlea & & & \\
\hline Leotia & $\cdots$ & $\ldots$ & 753 & Lolium & $\ldots$ & $\ldots$ & 633 & Marrubium & & & \\
\hline Lepidium & $\ldots$ & $\ldots$ & 34 & Lomandr $x$ & $\cdots$ & $\cdots$ & 565 & Marsdenia & $\cdots$ & & \\
\hline Lepidopilum & $\ldots$ & $\cdots$ & 664 & Lomaria & ... & $\cdots$ & 642 & Marsdenia & $\ldots$ & 776 & 778 \\
\hline Lepidosperma & & $\ldots$ & 598 & Lomatia & $\ldots$ & ... & 454 & Marsilea & $\cdots$ & ... & \\
\hline Lepidozia & $\ldots$ & $\ldots$ & 673 & Lomentum & $\ldots$ & $\cdots$ & 139 & MarsileaCEA & $\ldots$ & $\therefore$ & \\
\hline Lepironia & $\ldots$ & $\ldots$ & 597 & Lonchocarpus & & $\ldots$ & 147 & Martynia & & & 000 \\
\hline Lepistemon & $\ldots$ & $\ldots$ & 349 & Lonicera & $\ldots$ & $\ldots$ & 236 & Massaria & $\ldots$ & $\ldots$ & 10. \\
\hline Lepraria & $\ldots$ & $\ldots$ & 714 & Lophiostoma & ... & $\cdots$ & 763 & \multicolumn{2}{|c|}{ Mastigobryum } & & \\
\hline Leptangium & $\ldots$ & $\ldots$ & 661 & Lophocolea & $\cdots$ & $\ldots$ & 672 & \multicolumn{2}{|c|}{ Mastigobryum } & & \\
\hline Leptaspis & $\ldots$ & $\ldots$ & 612 & LORANTHACEE & $\cdots$ & $\ldots$ & 460 & \multicolumn{2}{|c|}{ Mastigolejeunea } & ... & 675 \\
\hline Leptocarpus & $\ldots$ & $\ldots$ & 588 & Loranthus & $\ldots$ & ... & 460 & Mastophora & ... & & תח \\
\hline Leptoceras & $\ldots$ & $\ldots$ & 538 & Lotus & ... & $\cdots$ & 136 & Maundia & & & \\
\hline Leptochloa & $\ldots$ & $\cdots$ & 628 & ma & $\ldots$ & $\ldots$ & 305 & Mayepea & $\cdots$ & & 01 \\
\hline Leptodontrum & & $\ldots$ & 659 & wigia & $\ldots$ & $\cdots$ & 215 & $\mathrm{Ma}$ & $\cdots$ & $\ldots$ & 360 \\
\hline Leptogium & $\ldots$ & $\ldots$ & 682 & Luffa & ... & $\ldots$ & 221 & Medicago & & & 133 \\
\hline Leptogium & $\ldots$ & $\ldots$ & 696 & ia & $\cdots$ & $\ldots$ & 535 & Medicago & 763,7 & 769 & 785 \\
\hline Leptohymeni & $\mathrm{am}$ & $\ldots$ & 666 & nitzera & $\cdots$ & $\cdots$ & $78 \mathrm{C}$ & & & & \\
\hline Leptolejeunea & & $\ldots$ & 675 & alaria & $\cdots$ & $\cdots$ & 678 & icosma & $\ldots$ & & \\
\hline Leptomeria & ... & $\ldots$ & 469 & aus & $\ldots$ & $\ldots$ & 13 & Medicosma & & & 766 \\
\hline Leptopteris & $\ldots$ & $\ldots$ & 638 & Luzula & $\ldots$ & & 566 & Medinilla & & & ris \\
\hline Leptorhynehu & & $\ldots$ & 266 & Lychnis & $\cdots$ & $\ldots$ & 44 & Megalonectria & & $\cdots$ & 758 \\
\hline Leptosema & $\ldots$ & $\ldots$ & 12 & Lyehnothamn & us & & 678 & Melaleuca & & & 187 \\
\hline Leptospermu & & $\ldots$ & 186 & $\mathrm{~mm}$ & $\cdots$ & & 357 & Melaleuca & 761,7 & 765 & 775 , \\
\hline Leptospharia & & $\cdots$ & 763 & gala & $\cdots$ & ... & 790 & & & & \\
\hline Leptotheca & $\cdots$ & $\ldots$ & 662 & Lycoperdon & $\ldots$ & & 752 & Melanopsichiv & $\operatorname{um}$ & $\ldots$ & 769 \\
\hline Leptotrema & $\ldots$ & $\ldots$ & 699 & Lycopersicum & $\ldots$ & & 353 & Melampsora & $\cdots$ & $\ldots$ & 77 \\
\hline Lepturus & $\ldots$ & $\ldots$ & 634 & Lycopersicum & $\ldots$ & 785 & 787 & Melanotheca & & $\ldots$ & 709 \\
\hline Lepyrodia & $\ldots$ & $\ldots$ & 58 & LYCOPODIACEE & & $\ldots$ & 63 & nia & & & 779 \\
\hline Leschenaultia & & $\cdots$ & 282 & Lycopodium & $\ldots$ & $\cdots$ & 634 & spilea & $\because$ & $\cdots$ & 704 \\
\hline Lespea & $\ldots$ & $\ldots$ & 669 & pus & $\ldots$ & $\cdots$ & 388 & Melastoma & $\ldots$ & $\ldots$ & \\
\hline edeza & $\ldots$ & $\ldots$ & 140 & Lygodium & $\ldots$ & $\cdots$ & 63 & ASTOMACE & & ... & 209 \\
\hline Lettsomia & $\ldots$ & $\ldots$ & 347 & Lyngb ya & $\ldots$ & $\ldots$ & 831 & Melhania & ... & $\ldots$ & \\
\hline Leucas & $\ldots$ & 388 & 840 & igbya & $\ldots$ & $\cdots$ & 831 & Melia & & ... & \\
\hline bryum & $\ldots$ & ... & 657 & Lyonsia & $\ldots$ & $\cdots$ & 318 & IACEF & $\cdots$ & $\cdots$ & \\
\hline oloma & $\ldots$ & & 657 & arpus & ... & $\ldots$ & 202 & Meliacea & $\ldots$ & $\ldots$ & \\
\hline ophanes & $\cdots$ & $\cdots$ & 657 & nium & $\cdots$ & $\cdots$ & 817 & Melichrus & $\ldots$ & ... & 295 \\
\hline opogon & ... & $\cdots$ & 29 & Lysinema & $\ldots$ & $\cdots$ & 29 & pe & $\ldots$ & $\ldots$ & 77 \\
\hline osmia & $\cdots$ & $\cdots$ & 459 & Lysurus & $\ldots$ & $\ldots$ & 745 & lotus & $\ldots$ & $\ldots$ & 133 \\
\hline ea & $\cdots$ & $\cdots$ & 276 & LYTHRARIEE & $\ldots$ & $\ldots$ & 214 & ola & & $\therefore$ & 766 \\
\hline illea & $\ldots$ & $\cdots$ & 827 & Lythrum & $\cdots$ & $\cdots$ & 214 & besia & & & 829 \\
\hline Liagora & & & 822 & & & & & Melochia & & & \\
\hline Libertia & $\ldots$ & $\ldots$ & 542 & & & & & Melodinus & & $\cdots$ & 317 \\
\hline Lichen & $\ldots$ & $\ldots$ & 69 & Maba & & & 306 & Melodorum & & & \\
\hline HENES & .. & & 682 & Maba & $\cdots$ & $\cdots$ & 782 & ramma & & & 762 \\
\hline ala & $\ldots$ & $\ldots$ & 57 & Macadamia & $\ldots$ & & 440 & Melophia & & $\ldots$ & \\
\hline trum & $\ldots$ & $\ldots$ & 31 & Macadamia & $\ldots$ & $\ldots$ & 452 & thria & & $\ldots$ & \\
\hline CEA & ... & & 55 & ranga & $\ldots$ & $\ldots$ & 479 & ecylon & & & \\
\hline Limacia & $\ldots$ & $\ldots$ & 29 & rthuria & $\ldots$ & $\ldots$ & 227 & Meniscium & $\ldots$ & $\ldots$ & \\
\hline anthemu & & $\ldots$ & 338 & regoria & $\ldots$ & & 100 & MENISPERMACE & & & \\
\hline Limnanthemv & $m$ & & 773 & Mackinlaya & $\ldots$ & $\cdots$ & 23 & tha & & & 388 \\
\hline phila & $\ldots$ & $\ldots$ & 360 & romitrium & & $\cdots$ & 659 & smopediu & & & 830 \\
\hline sella & $\ldots$ & & 36 & ophoma & & & 776 & Merulius & & & 738 \\
\hline ia & $\ldots$ & & 360 & pteranth & hes & & 18 & mbryant & hemum & & 227 \\
\hline Lindsæa & $\ldots$ & 641 & 849 & osporium & & & 78 & melæena & $\ldots$ & $\ldots$ & 597 \\
\hline & $\ldots$ & $\ldots$ & 6 & ozamia & & & 513 & phellia & & $\ldots$ & 751 \\
\hline ciera & ... & & 311 & ozamia & 762 & 776 & 779 & phæria & & .. & $76 \overline{3}$ \\
\hline & & & 65 & Madotheca & ... & & 67 & ium & & & \\
\hline & ... & & & Mrsa & $\ldots$ & & 300 & ideros & & $\ldots$ & \\
\hline Lipocarpha & ... & & 59 & JOLIACE & $\ldots$ & & 21 & eria & $\ldots$ & $\ldots$ & 676 \\
\hline & & & 382 & Malaisia & $\ldots$ & & 485 & eurum & & & 15 \\
\hline Lisiella & ... & & & Mall & $\cdots$ & & 475 & ira & & & 62 \\
\hline Lissanthe & & & 295 & IGHIACE无 & $\ldots$ & & 69 & ntheum & $\ldots$ & $\ldots$ & 473 \\
\hline Listera & $\ldots$ & & 536 & Malva & & & 54 & sterias & & $\ldots$ & 81 \\
\hline Lithospermu & & 347 & & MaLvace $\approx$ & & & 54 & Microcarpeæ & & & 361 \\
\hline Lithothamnio & & ... & 829 & Malvastrum & & & 57 & Microcera & $\ldots$ & & \\
\hline
\end{tabular}


Page.

$\begin{array}{ll}\text { Microchrete } & \ldots \\ \text { Microchloa } & \ldots \\ \text { Microclisia } & \ldots \\ \text { Micrococcus } & \ldots \\ \text { Microcoleus } & \ldots \\ \text { Micropeltis } & \ldots \\ \text { Microlrena } & \ldots \\ \text { Micromelum } & \ldots \\ \text { Micromyrtus } & \ldots \\ \text { Microseris } & \ldots \\ \text { Microspora } & \ldots \\ \text { Microstemma } & \ldots \\ \text { Microstylis } & \ldots \\ \text { Microstylis } & \ldots \\ \text { Microthelia } & \ldots \\ \text { Microthelia } & \ldots \\ \text { Microthyrium } & \ldots \\ \text { Microtis } & \ldots \\ \text { Millettia } & \ldots \\ \text { Millotia } & \ldots \\ \text { Mimosa } & \ldots \\ \text { Mimosere } & \ldots \\ \text { Mimulus } & \ldots \\ \text { Mimusops } & \ldots \\ \text { Minuria } & \ldots \\ \text { Mirabilis } & \ldots \\ \text { Mirbelia } & \ldots \\ \text { Mitrasacme } & \ldots \\ \text { Mitrephora } & \ldots \\ \text { Modecca } & \ldots \\ \text { Modiola } & \ldots \\ \text { Mollinedia } & \ldots \\ \text { Mollugo } & \ldots \\ \text { Moluccella } & \ldots \\ \text { Momordica } & \ldots \\ \text { Monarda } & \ldots \\ \text { Monenteles } & \ldots \\ \text { Mongestia } & \ldots \\ \text { Monminaces } & \ldots \\ \text { Monochoria } & \ldots \\ \text { Monococcus } & \ldots \\ \text { Monocotyle } & \ldots \\ \text { Mo }\end{array}$

Monocotyledons

Monogramme ...

Monostroma

Monotaxis ..

Monotoca
Monstera

Montagnella

Morchella

Morgania

Morinda

... 249

$\begin{array}{llll}\text { Motherwellia } & \ldots . & \ldots & 38 \\ & & & \end{array}$

$\begin{array}{llll}\text { Mucor } & \text {... } & \text {... } & 768\end{array}$

Mucuna $\quad \ldots . \quad \ldots .144$

Muhlenbeckia ... $\quad \ldots \quad 417$

Muhlenbeckia ... ... 381

$\begin{array}{llll}\text { Mukia } & \ldots & \ldots & 222\end{array}$

$\begin{array}{llll}\text { Murraya } & \ldots & \ldots & \\ & & \end{array}$

$\begin{array}{llll}\text { Musa } & \ldots & \ldots & 52\end{array}$

Musa $\quad \ldots .787$

Muscr (Mosses) $\ldots . . .656$

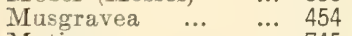

Mutinus $\quad \ldots .674$

Mycenastrum .... $\quad \ldots \quad 752$

$\begin{array}{llll}\text { Mycoidea } & \ldots & \ldots & 793\end{array}$

Mycoporellum $\quad \ldots 707$

Myiocopron ... ... 765

MYoporine $\quad \ldots \quad$... 375

Myoporum $\quad \ldots . \quad \ldots \quad 375$

$\begin{array}{llll}\text { Myosotus } & \ldots & \ldots & \\ & & \ldots & \end{array}$

$\begin{array}{llll}\text { Myosurus } & \ldots & \ldots & 17\end{array}$

$\begin{array}{llll}\text { Myriangium } & \ldots & \ldots & \ldots 83\end{array}$

\section{Page.}

Myriocephalus

Myriophyllum

... ... 419

Myristice E $\quad \ldots \quad$... 419

Myrmecodia $\quad . . \quad$... 253

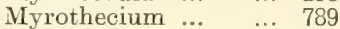

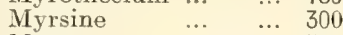

Myrsine e $\quad \ldots \quad \ldots \quad 300$

MYrtace E $\quad \ldots \quad \ldots 181$

$\begin{array}{llll}\text { Myrtus } & \ldots & \ldots & 207\end{array}$

$\begin{array}{llll}\text { Myrtus } & \ldots & \ldots & 781\end{array}$

Nageia

NaIt $\quad \ldots \quad$... 510

NATDACEE

Nalas $\quad \ldots \quad \ldots \quad 583$

Nardia

$\begin{array}{llll}\text { Nasturtium } & \ldots & \ldots & 672 \\ & \ldots & \ldots & 33\end{array}$

$\begin{array}{llll}\text { Nauclea } & \ldots & \ldots & \\ \text { Navilea } & \ldots & \ldots & 237\end{array}$

$\begin{array}{llll}\text { Navicula } & \ldots & \ldots & 811\end{array}$

$\begin{array}{llll}\text { Neckera } & \ldots & \ldots & 666\end{array}$

Nectria $\quad \ldots \quad \ldots 758$

$\begin{array}{llll}\text { Velitris } & \ldots & \ldots & 758\end{array}$

Nelsonia $\quad \ldots . \quad \ldots 368$

$\begin{array}{llll}\text { Nelumbium } & \ldots & \ldots & 32\end{array}$

Nematogonum .. 782

Neoræpera $\quad \ldots \quad$... 476

NepeNthace.

Nepenthes $\quad \ldots .4 . \quad \ldots .417$

Nephelium ... ... 116

Nephrocytium ... … 800

Nephromium ... $\quad \ldots .687$

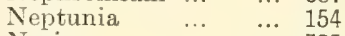

Nerium $\quad$... $\quad$... 325

Nesæa $\quad$... $\quad \ldots .214$

Nesogenes $\quad$... $\quad$... 382

Neurachne ... ... 616

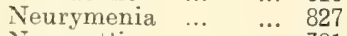

Newcastlia $\quad \ldots \quad \ldots 381$

$\begin{array}{llll}\text { Nicotiana } & \ldots & \ldots & 358\end{array}$

Nicrandra $\quad \ldots \quad \ldots . \quad 357$

Nipa $\quad \ldots \quad \ldots 573$

Nitella $\quad \cdots \quad 676,851$

Nitraria

Nitzschia

Nodularia

Nostoc

Notelæa

Noterocladia ...

Notholæna

Nothoscordum

Notothixos

Nuculanium

Nummularia

NyCtaGine瓜

Nymphæa

NymiphateE $\cdots$

$\begin{array}{llll}\text { Nyssanthes } & \ldots & \ldots & \text {... } \\ \text { No3 }\end{array}$

Oberonia

Obryzum

Ocellularia

Ocellularia

OCHNACEE

Ochrosia

Ocimum

Octaviania

$\begin{array}{llll}\text { Octoblepharum } & \ldots & \ldots & 657\end{array}$

Edogonium ... ... 793

Enanthe $\quad \ldots \quad \ldots \quad 229$

Enothera $\quad \ldots . \quad \ldots .215$

Oidium $\quad \ldots \quad 765,782$
Page.

Olactinez

$\begin{array}{llll}\text { Olax } & \ldots & \ldots & 93\end{array}$

$\begin{array}{llll}\text { Oldenlandia } & \ldots & \ldots & \ldots 37\end{array}$

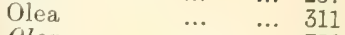

$\begin{array}{llll}\text { Olea } & \ldots & \ldots & 756\end{array}$

Oleace. $\quad \cdots \quad 311,339$

Oleander $\quad$... $\quad$... 778

$\begin{array}{llll}\text { Olearia } & \ldots & \ldots & 259 \\ \text { Ombrophila } & \ldots & \ldots & 755\end{array}$

Omphalea $\quad \ldots \quad$... 480

Onagrarie. $\quad \ldots \quad \ldots .215$

$\begin{array}{llll}\text { Onoclea } & \ldots & \ldots & 642\end{array}$

Onopordon ... ... 270

Onychonema ... ... 802

Oospora $\quad \ldots . \quad \ldots 782$

Opegrapha $\quad \ldots \quad \ldots 703$

$\begin{array}{llll}\text { Opercularia } & \ldots & \ldots & 253\end{array}$

Ophiocytium $\quad . . \quad \quad \ldots \quad 800$

Ophioglossum ... $\quad$... 635

Ophiopogon $\quad . . \quad \ldots \quad 542$

Ophiopogon $\quad$.. $\quad$.. $\quad 790$

$\begin{array}{llll}\text { Ophiorrhiza } & \ldots & \ldots & 237\end{array}$

Ophiurus $\quad \ldots \quad \ldots 617$

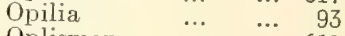

Oplismenus $\quad \ldots \quad \ldots 611$

Opuntia $\quad \ldots \quad \ldots 222$

Opuntia $\quad$... 776,778

Orania $\quad \ldots \quad \ldots 566$

$\begin{array}{llll}\text { Orbilia } & \ldots & \ldots & 755\end{array}$

ORCHIDEe $\quad \ldots \quad 519.845$

Orites $\quad . . \quad \ldots \quad 452$

$\begin{array}{llll}\text { Ormocarpum } & \ldots & \ldots & 139 \\ \text { Ornithochilus } & . . & & 536\end{array}$

Ornitrophe ... ... 536

$\begin{array}{llll}\text { Orobanche } & \ldots & \ldots & 113 \\ & & \ldots & 364\end{array}$

Orobanche巴

$\begin{array}{llll}\text { Orthoceras } & \ldots & \ldots & \ldots 47\end{array}$

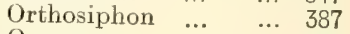

$\begin{array}{llll}\text { Oryza } & \ldots & \ldots & 612\end{array}$

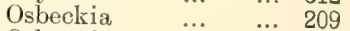

Osbornia $\quad \ldots \quad \ldots 202$

$\begin{array}{llll}\text { Oscillatoria } & \ldots & \ldots & 830\end{array}$

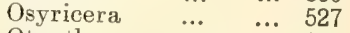

Otanthera $\quad \ldots . \quad \ldots .209$

$\begin{array}{llll}\text { Otidea } & \ldots & \ldots & 753\end{array}$

Ottelia $\quad \ldots . \quad \ldots 518$

$\begin{array}{llll}\text { Owenia } & \ldots & \ldots & 88\end{array}$

Owenia $\quad \ldots . \quad \ldots .124$

Oxalis $\quad \ldots . \quad \ldots \quad 73$

Oxylobium $\quad \ldots .6124$

$\begin{array}{llll}\text { Ozonium } & \ldots & \ldots & \ldots 52\end{array}$

$\begin{array}{llll}\text { Pachygone } & \ldots & \ldots & 32\end{array}$

$\begin{array}{llll}\text { Padina } & \ldots & \ldots & 819\end{array}$

$\begin{array}{llll}\text { Pagetia } & \ldots & \ldots & 77\end{array}$

Palma $\quad \ldots . \quad \ldots 566$

Palmeria $\quad \ldots \quad$... 419

Palmodactylon $\quad$... 800

Panax $\quad$... $\quad$... 232

Pandanaces $\quad \ldots \quad \ldots \quad 576$

$\begin{array}{llll}\text { Pandanus } & \ldots & \ldots & 576\end{array}$

$\begin{array}{llll}\text { Pandorina } & \text {... } & \text {... } & 799\end{array}$

Panicum $\quad$... ... 603

Panicum $\quad \ldots .6 \%$

Pannaria $\quad$... $\quad$... 692

$\begin{array}{llll}\text { Panus } & \ldots & \ldots & 725 \\ & \ldots & \ldots & 725\end{array}$

$\begin{array}{llll}\text { Papaver } & \ldots & \ldots & 32\end{array}$

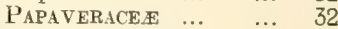

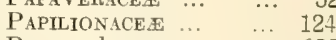

Pappophorum ... 628

$\begin{array}{llll}\text { Paranectria } & \ldots & \ldots & 758\end{array}$

$\begin{array}{llll}\text { Parathelium } & \ldots & \ldots & 758 \\ & & . . . & 709\end{array}$

Parkinsonia ... ... 151 
Page.

Parietaria $\quad \ldots \quad \ldots \quad 505$

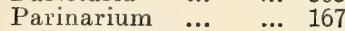

$\begin{array}{llll}\text { Parmelia } & \ldots & \ldots & 689\end{array}$

$\begin{array}{llll}\text { Parmentaria } & \ldots & \ldots & 708\end{array}$

$\begin{array}{llll}\text { Parmosticta } & \ldots & \ldots & 689\end{array}$

\begin{tabular}{llll} 
Parsonsia $\quad \ldots$ & $\ldots$ & 318 \\
\hline & &
\end{tabular}

Paspalum $\quad$... 602,849

Pxspalum $\quad \ldots \quad 769,771$

Passiflora $\quad \cdots \quad \ldots 220$

$\begin{array}{llll}\text { Passifiora } & 779,783,786 \\ \text { Passiflone. } & \ldots & \ldots & 220\end{array}$

$\begin{array}{llll}\text { Patellaria } & \ldots & \ldots & 701 \\ & & \end{array}$

Patersonia $\quad$... $\quad$... 543

$\begin{array}{llll}\text { Pavetta } \quad \ldots & \ldots & 245 \\ & & \end{array}$

$\begin{array}{llll}\text { Pavonia } & \ldots & \ldots & 58\end{array}$

Paxillus $\quad \ldots .678$

Pedalinee $\quad \ldots . \quad \ldots .368$

$\begin{array}{llll}\text { Pediastrum } & \ldots & \ldots & 799\end{array}$

Pelargonium ... ... 72

Pellaca

Peltigera

Peltophorum ... $\quad \ldots . .615$

Pemphidium ... $\quad . . .765$

$\begin{array}{llll}\text { Pemphis } & \ldots & \ldots & 214\end{array}$

Penicillus $\quad$... $\quad$... 798

Penicillium $\quad . .6 \quad \ldots .782$

Peniophora $\quad \ldots \quad$ …

Penium $\quad \ldots . \quad \ldots 804$

Pennantia

Pennisetum

Pentaceras

Pentapanax

Pentatropis

Peperomia

Peperomia

Peplidium

Peplis

Рepo

Pericampylus

Perichæna

Periconia

Perithalia

Pereskia

Peronospora

Peronospora

Perotis

Persea

Persoonia

Persoonia

Pertusaria

Pestalozzia

Petalostigma .

Petalostyles

Petermannia

Petrophila

Petunia

Peyssonnelia

Peziza

Phacelothrix

$\begin{array}{llll}\text { Phæographina } & \cdots & 527 \\ & & 706\end{array}$

Phreographis ... ... 704

$\begin{array}{llll}\text { Phæotrema } & \ldots & \ldots & \ldots 97\end{array}$

Phalænopsis ... ... 535

Phalaris $\quad$... $\quad \ldots \quad 620$

Phaleria $\quad \ldots .64 \%$

Phallus $\quad$... ... 745

Phascum $\quad$... $\quad \ldots .658$

Phaseolus $\quad \ldots . \quad \ldots .144$

Phebalium $\quad \ldots \quad$... 76

Phillipsia $\quad \ldots .675$

Philonotis $\quad$... ... 662

$\begin{array}{lllr}\text { Philotheca } & \ldots & \ldots & \ldots \\ \end{array}$
Page.

$\begin{array}{llll}\text { Philydracele } & \ldots & \ldots & 561\end{array}$

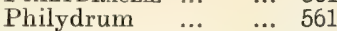

$\begin{array}{llll}\text { Phlebia } & \text {... } & \text {... } 739\end{array}$

Phlebocalymna $\quad$... 93

Phleum $\quad$... $\quad$... 623

Phlyctæna $\quad \ldots \quad$... 779

Phoenix $\quad$... $\quad$... 573

Phonix $\quad$... ... 771

$\begin{array}{llll}\text { Pholidia } & \ldots & \ldots & 375\end{array}$

$\begin{array}{llll}\text { Pholidota } & \ldots & \ldots & 527\end{array}$

$\begin{array}{llll}\text { Phoma } & \ldots & \ldots & 776 \\ \text { Phomatospora } & \ldots & 763\end{array}$

Phormidium ... ... 831

$\begin{array}{llll}\text { Photinia } & \ldots & \ldots & 167\end{array}$

Phragmicoma $\quad \ldots 673$

Phragmidium $\quad$... 773

$\begin{array}{llll}\text { Phragmites } & \ldots & \text {... } & 629\end{array}$

$\begin{array}{llll}\text { Phreatia } & \text {... } & \text {... } & 527\end{array}$

$\begin{array}{llll}\text { Phreatia } & \ldots & \ldots & 519\end{array}$

$\begin{array}{llll}\text { Phycomyces } & \ldots & \ldots & 768\end{array}$

Phyllachora $\quad . .6 \quad \ldots .761$

Phyllanthus ... ... 476

Phyllogonium ... 666

Phylloporina ... ... 710

Phyllostachys _.. 851

$\begin{array}{llll}\text { Phyllosticta } & \ldots & \ldots & 775\end{array}$

$\begin{array}{llll}\text { Phyllosticta } & \text {... } & \ldots & 787\end{array}$

$\begin{array}{llll}\text { Phyllota } & \ldots & \ldots & 128\end{array}$

Physalis

Physalospora

Physarum

Physarum

Physcia

Physcomitrium

Physianthus

Physiotium a...

$\begin{array}{llll}\text { Physma } & \ldots & \ldots & \ldots \\ \end{array}$

Phytolacea $\quad \ldots \quad \ldots \quad 411$

$\begin{array}{lll}\text { Phytolaccace e } & \ldots & 411 \\ \text { Phytophthora } & & 769\end{array}$

$\begin{array}{lll}\text { Phytophthora } & \ldots & 769 \\ \text { Picris } & \ldots & 505\end{array}$

$\begin{array}{llll}\text { Picris } & \ldots & \ldots & 505 \\ \text { Pilacre } & \ldots & \ldots & 786\end{array}$

Pilea

Pilobolus

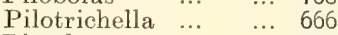

Pimelea $\quad . . .4 \quad \ldots 455$

Piper

PiPerace E

Piptocalyx

Pipturus

Pisonia

Pithecolobium

Pittospore.

... 43

$\begin{array}{llll}\text { Pityrodia } & \ldots & \ldots & 381\end{array}$

$\begin{array}{llll}\text { Pinus } & \ldots & \ldots & 778\end{array}$

Pircunia $\quad \ldots . \quad \ldots 411$

Pithecolobium $\quad \ldots \quad 761$

Pittosporum $\quad$... 765

$\begin{array}{lllr}\text { Placodium } & \ldots & \ldots & 696 \\ \text { Plagianthus } & \ldots & & 57\end{array}$

Plagiobryum $\quad \cdots \quad \ldots .663$

Plagiochasma $\quad$... 676

Plagiosetum ... ... 611

Plagiothelium _.. 709

$\begin{array}{llll}\text { Plagischila } & \ldots & \ldots & 672\end{array}$

Plantagineæ $\quad \ldots . \quad \ldots \quad 396$

Plantago $\quad \ldots . \quad \ldots \quad 396$

Plasmodiophora $\quad \ldots \quad 852$

$\begin{array}{llll}\text { Platycerium } & \text {... } & \ldots & 649\end{array}$

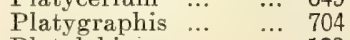

$\begin{array}{llll}\text { Platylobium } & \text {... } & \text {... } & 129\end{array}$

$\begin{array}{llll}\text { Platyloma } & \ldots & \ldots & 642\end{array}$
Page.

$\begin{array}{llll}\text { Platysma } & \text {... } & \text {... } 687\end{array}$

$\begin{array}{llll}\text { Platyzoma } & \ldots & \ldots & \ldots 38\end{array}$

Plectania $\quad$... $\quad \ldots \quad 753$

Plectonema $\quad \ldots \quad$... 832

Plectranthus ... ... 387

$\begin{array}{llll}\text { Plectronia } & \ldots & \ldots & 245\end{array}$

Pleiococca ... $\quad . . .81$

$\begin{array}{llll}\text { Pleiogynium } & \ldots & \ldots & 125\end{array}$

Pleiophysa $\quad \ldots \quad \ldots \quad 795$

$\begin{array}{llll}\text { Pleogyne } & \ldots & \ldots & 29\end{array}$

Pleospora $\quad \ldots . \quad \ldots 763$

Pleurocarpæa $\quad$... 257

Pleurosigma ... ... 813

Pleurotæniopsis $\quad \ldots .806$

Pleurotænium _.. 805

Pleurothelium ... 709

$\begin{array}{llll}\text { Pleurotrema } & \ldots & \ldots & 710\end{array}$

Plocamium ... $\quad$... 824

$\begin{array}{llll}\text { Pluchea } & \ldots & \ldots & 263 \\ & & \ldots & 263\end{array}$

Plumbagineæ $\quad$... 300

Plumbago $\quad \ldots \quad \ldots \quad 300$

Poa $\quad \ldots \quad \ldots 633$

$\begin{array}{llll}\text { Podaxis } & \text {... } & \ldots & 751\end{array}$

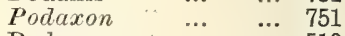

Podocarpus ... ... 510

Podocarpus $\quad$... $\quad$... 768

$\begin{array}{llll}\text { Podochilus } & \text {... } & \text {... } & 527\end{array}$

Podocoma $\quad$... $\quad \ldots \quad 262$

Podolepis $\quad \ldots \quad \ldots \quad 268$

$\begin{array}{llll}\text { Podolobium } & \ldots & \ldots & 125\end{array}$

$\begin{array}{llll}\text { Podopetalum ... } & \text {... } & 148\end{array}$

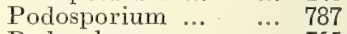

Podosphæra ... $\quad$... 765

Podostemonace

Poecilophyllum ... 556

Pogonatherum $\quad \ldots .617$

$\begin{array}{llll}\text { Pogonia } & \text {... } & \text {... } & 539\end{array}$

$\begin{array}{llll}\text { Poinciana } & \text {... } & \text {.. } 852\end{array}$

$\begin{array}{llll}\text { Polanisia } & \ldots & \ldots & 36\end{array}$

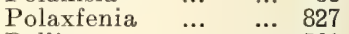

Pollia $\quad \ldots \quad \ldots \quad 561$

Pollinia $\quad \ldots .6 .617$

$\begin{array}{llll}\text { Polyalthia } & \ldots & \ldots & 25\end{array}$

Polyblastia $\ldots . . \ldots 713$

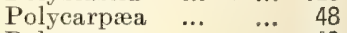

Polycarpon $\quad \ldots \quad$... 48

Polyedrium $\quad \ldots . \quad \ldots 800$

$\begin{array}{llll}\text { Polygala } & \ldots & \ldots & 43\end{array}$

Polvgale

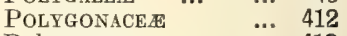

Polygonum ... $\quad \ldots \quad 412$

Polygonum $\quad$...769, 771

Polymeria ... ... 353

$\begin{array}{llll}\text { Polyosma } & \ldots & \ldots & 168\end{array}$

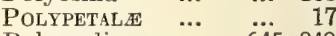

Polypodium $\quad \ldots \quad 6 \% \quad 645,849$

Polypogon ... ... 623

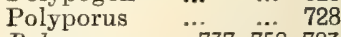

Polyporus $\quad 737,758,783$

Polysaccum ... ... 752

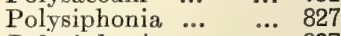

$\begin{array}{llll}\text { Polysiphonia } & \text {... } & \text {... } & 827\end{array}$

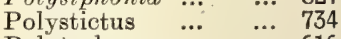

Polytocha ... ... 616

Polytrichadelphus ... 672

Polytrichum $\quad . . \quad$... 672

Pomaderris $\quad \ldots . \quad \ldots .106$

$\begin{array}{llll}\text { Pomax } & \ldots & \ldots & 253\end{array}$

Pome $\quad \ldots \quad \ldots . \quad \ldots .6167$

Pongamia $\quad \ldots . \quad \ldots .148$

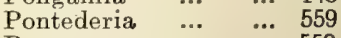

Pontederiace e $\quad$... 559

$\begin{array}{llll}\text { Poranthera } & \ldots & \ldots & 473\end{array}$ 
Page.

Porella

Poria

Porina

Poronia

Porospermum

Porotrichum ...

Portulaca

Portulacee

Potamogeton

Pothos

Pouzolzia

Powellia $\quad \ldots .6661$

Prasophyllum ... ... 537

Pratia ... ... ... 291

Premna ... $\quad$.. $\quad \ldots \quad 386$

Prinlelice.

Prionites $\quad \ldots \quad \ldots \quad 828$

Pritzeliella $\quad \ldots .7 . \quad \ldots \quad 786$

Prostanthera $\quad . . . \quad$... 392

Proteace.

Protium $\quad$... ... 835

Protococcus ... $\quad$... 801

Protoglossum … $\quad \ldots .751$

Protomyees $\quad \ldots . \quad \ldots \quad 769$

\begin{tabular}{llll} 
Prunus & $\ldots$ & $\ldots$ & 167 \\
\hline Prumus & & &
\end{tabular}

Pseudalagium ... 236

Pseudanthus ... $\quad \ldots .473$

Pseudo-Eunotia $\quad$... 817

Pseudomonas ... $\quad$... 789

Pseudomorus ... ... 485

Pseudopeziza _.. 756

Pseudopyenula $\quad$... 77

$\begin{array}{llll} & \text { Psidium } & \ldots & \ldots\end{array}$

Psilotum $\quad$... $\quad \ldots \quad 635$

Psoralea. $\quad \ldots . \quad \ldots \quad 136$

Psoroma $\quad$... ... 692

$\begin{array}{llll}\text { Psychotria } & \ldots & \ldots & 249\end{array}$

Pterigeron ...

Pteris ... ... $\quad \ldots, 642$

$\begin{array}{llll}\text { Pterobryum } & \ldots & \ldots & 665\end{array}$

Pterocaulon ... ... 266

Pterolobium ... $\quad \ldots \quad 151$

Pterospermum _.. 65

Pterostylis $\quad \ldots . \quad \ldots .537$

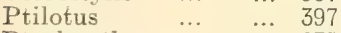

Ptychanthus ... ... 675

Ptycholejeunea ... 675

Ptychomnion ... ... 664

Ptychosperma ... ... 573

Puccinia

Puccinia

Pultencea

Pultenæa

Pycnolejeunea $\cdots \quad$... 375

Pycnospora ... ... 140

$\begin{array}{llll}\text { Pygeum } & \ldots & \ldots & 167\end{array}$

Pvrenula $\quad \ldots . \quad \ldots 711$

$\begin{array}{llll}\text { Pyrgillus } & \ldots & \ldots & 684\end{array}$

$\begin{array}{llll}\text { Pyrus } & \ldots & \ldots & 167\end{array}$

Pyrus $\quad 783$

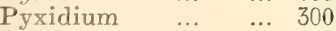

$\begin{array}{llll}\text { Pyxine } & \text {... } & \text {... } 692\end{array}$

Quercus $\quad \ldots \quad \ldots 789$

Quintinia $\quad . . . \quad \ldots .168$

Radula ... ... ... 673

Radulum $\quad \ldots . \quad \ldots 739$

Rrestelia
Page.

Ramalina $\quad \ldots \quad \ldots 687$

Ramularia

Randia $\quad \ldots . \quad \ldots 24$

$\begin{array}{llll}\text { RanunCulace } & & \ldots & 17 \\ \text { Ranunculus } & . . & \ldots & 18\end{array}$

$\begin{array}{llll}\text { Ranunculus } & \ldots & \ldots & 18 \\ \text { Raphanus } & \ldots & \ldots & 36\end{array}$

Raphistrum ... ... 30

$\begin{array}{llll}\text { Rassula } & \ldots & \ldots & 722\end{array}$

Ratonia $\quad \ldots . \quad \ldots .113$

Retonia $\quad . . . . . .110$

Reboulia $\quad \ldots .67676$

Regma _.. ... 470

Reinschiella $\ldots . \quad \ldots 800$

Reinwardtia ... $\quad \ldots \quad$ by

Remirea. $\quad . .6 \quad \ldots \quad 597$

Restiace

Restio $\quad \ldots \quad \ldots \quad 588$

Rhacopilum ... ... 665

Rhagodia $\quad \ldots . \quad \ldots .404$

RHavNE.E $\quad \ldots \quad 100,837$

$\begin{array}{llll}\text { Rhamnus } & \ldots & \ldots & 100 \\ & & & \end{array}$

Rhamphicarpa … 363

$\begin{array}{llll}\text { Rhaphidium ... } & \ldots & 800 \\ \text { Rhaphidophora } & \ldots & 579\end{array}$

Rhaphidorrhynchum 667

Rhaphidostegium ... 669

Rhaphiolepis ... … 167

Rhipogonum ... ... 553

Rhizoclonium ... ... 793

Rhizogonium ... ... 663

Rhizomorpha ... ... 792

Rhizophora ... $\quad . . .175$

RHIZOPHORE E ... ... 175

Rhodamnia ... ... 207

Rhododendron $\quad \ldots \quad 290$

Rhodomyrtus ... 207

Rhodomyrtus $\quad 779,786$

Rhudosphæra ... ... 119

Rhophalodia ... $\quad$.. 817

Rhopalopsis $\quad \ldots \quad$... 759

Rhus 119

Rhynchosia $\quad \ldots \quad \ldots .6147$

Rhynchospora $\quad \ldots \quad 597$

Rhynchostegium ... 669

Rhytidhysterium ... 756

Rhytisma $\quad \ldots . \quad \ldots 761$

Ricasolia $\quad$... $\quad$... 689

Riccia $\quad \ldots \quad \ldots 66$

Richardsonia ... ... 253

Ricinocarpus _... 473

Ricinus $\quad . . . \quad \ldots \quad 480$

Ricinus $\quad$... $\quad \ldots 763$

Rivina $\quad \ldots \quad \ldots 441$

Rivularia ... 834

Rivulariaceæ ... ... 834

Robillarda ... $\quad . . .778$

Rochelia $\quad . .3 \quad \ldots 347$

Roperia $\quad . .3 \quad \ldots .36$

Rettlera $\quad . .3 \quad \ldots 364$

Rosa $\quad$... $\quad \ldots .168$

ROSACE E

Rosellinia ... ... 762

Rottboellia ... ... 617

Rourea

Roxburghia ... ... 548

ROXBURGHIACE E _.. 548

RUBIACE

$\begin{array}{llll}\text { Rubus } & \ldots & \ldots & \\ \text { Rub } & \ldots & \ldots & 167\end{array}$

Rubus $\quad \ldots . \quad \ldots 773$

Ruellia $\quad \ldots \quad \ldots \quad 374$

Rulingia $\quad \ldots .6065$

Rumex

$\begin{array}{llll}\text { Rumex } & \ldots & \ldots & \\ \text { Rum } & \ldots & \ldots & 773\end{array}$
Page.

Tiumplidia $\quad \ldots \quad \ldots \quad 536$

$\begin{array}{llll}\text { Rungia } & \text {... } & \text {... } & 374\end{array}$

$\begin{array}{llll}\text { Ruppia } & \ldots & \ldots & 583\end{array}$

RUTACE. $\quad$...

$\begin{array}{llll}\text { Rutidosis } & \ldots & \ldots & 268\end{array}$

Iivtidosis $\quad$... $\quad$... 268

$\begin{array}{llll}\text { Ryssopterys } & \text {... } & \text {... } & 69\end{array}$

Saccharum $\quad \ldots \quad \ldots 765$

Saccolabium ... $\quad$... 535

Saccopetalum ... $\quad$... 29

$\begin{array}{llll}\text { Sagina } & \ldots & \ldots & 48\end{array}$

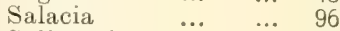

Salicornia $\quad$... $\quad$... 409

$\begin{array}{llll}\text { Salomonia } & \ldots & \ldots & 43\end{array}$

Salsola $\quad \ldots . \quad \ldots \quad 411$

Salvia $\quad \ldots . \quad \ldots \quad 388$

$\begin{array}{llll}\text { Samadera } & \ldots & \ldots & 85\end{array}$

$\begin{array}{llll}\text { Samara } & \ldots & \ldots & 81\end{array}$

$\begin{array}{llll}\text { Samara } & \ldots & \ldots & 300\end{array}$

Sambucus $\quad \ldots . \quad \ldots 236$

Samolus $\quad \ldots . \quad \ldots \quad 300$

SAMYDACE

SANTALACE

Santalum $\quad$.. $\quad$... 469

Santalum $\quad \ldots . \quad \ldots .781$

SAPINDACEE $\quad \ldots \quad \ldots \quad \ldots 112$

Sapindus $\quad \ldots \quad \ldots .116$

Saponaria $\quad \ldots \quad \ldots . \quad \ldots 44$

SAPOTACE $\mathbf{E}$... $\quad$... 305

Saprophyte ... $\quad \ldots .536$

Sarcocephalus _.. 237

Sarcochilus ... 5335,845

Sarcodia $\quad \ldots \quad \ldots 823$

Sarcographa ... ... 708

Sarcomitrium $\quad \ldots .676$

Sarcopetalum ... $\quad . . .29$

Sarcophycus ... ... 819

Sarcoscypha $\ldots . \quad \ldots 753$

Sarcostemma ... ... 326

Sarcoxylon $\quad \ldots .67 \%$

$\begin{array}{llll}\text { Sargassum } & \ldots & \ldots & \end{array}$

Saurauja $\quad \ldots \quad \ldots .541$

Saussurea $\quad \ldots \quad \ldots 276$

SAXIFRAGE

$\begin{array}{llll}\text { Scævola } & \ldots & \ldots & 287\end{array}$

Scervolx $\quad$... $\quad . .7773$

Scandix $\quad \ldots \quad \ldots 229$

Scenedesmus ... $\quad \ldots \quad 799$

Schelhammera $\quad . .559$

$\begin{array}{llll}\text { Schistocheila } & \ldots & \ldots & 675\end{array}$

Schistocarpæa $\quad$... 100

$\begin{array}{llll}\text { Schizæa } & \ldots & \ldots & 638\end{array}$

Schizochlamys $\quad \ldots .680$

Schizogonium $\quad$... 794

Schizomeria $\quad$... $\quad$... 169

Schizophyllum $\quad \ldots \quad 727$

$\begin{array}{llll}\text { Schleichera } & \text {... } & \text {... } & 86\end{array}$

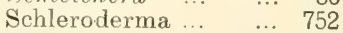

Schleroderma ... ... 773

Schlothemia $\quad \ldots \quad \ldots 661$

Schmidelia $\quad \ldots \quad \ldots \quad 113$

$\begin{array}{llll}\text { Schonus } & \ldots & \ldots & 598\end{array}$

Seiadium $\quad \ldots \quad \ldots 800$

Sciaphila $\quad \ldots \quad \ldots \quad \ldots 50$

Scirpodendron $\quad \ldots \quad 597$

$\begin{array}{llll}\text { Scirpus } & \ldots & \ldots & 595\end{array}$

SCITAMINE

Scleranthus $\quad \ldots \quad \ldots \quad 396$

Scleria $\quad \ldots \quad \ldots 600$

$\begin{array}{llll}\text { Sclerodepsis } & \ldots & \ldots & 737 \\ \text { Sclerodontium } & \ldots & 657\end{array}$ 


\begin{tabular}{|c|c|c|c|c|c|c|c|c|c|}
\hline & & Page. & & & Pag & & & & age. \\
\hline Sclerolæna & $\cdots$ & 409 & Sorosis & & 485 & Sticta & $\cdots$ & .. & 689 \\
\hline Selerolana & $\cdots$ & 775 & Sorosporium & ... & 771 & Stictina & ... & ... & 688 \\
\hline Sclerotia & $\ldots$ & 792 & Sorosporium & $\cdots$ & 769 & Stictis . & ... & $\ldots$ & 756 \\
\hline Scolecotrichum & & 783 & Sowerbæa & .. & 559 & Stigeoclonium & & $\cdots$ & 794 \\
\hline Scolopia & $\ldots$ & 42 & Sparganium .. & & 576 & Stilbum & ... & $\cdots$ & 786 \\
\hline Scoparia & $\ldots$ & 361 & Sparthothamnu & & 381 & Stilophora & $\ldots$ & ... & 821 \\
\hline Scorpiurus & ... & 139 & Spathodea... & .. & 364 & Stipa & ... & $\cdots$ & 622 \\
\hline Scortechinia & ... & 762 & Spathoglottis .. & & 527 & Stipa & $\ldots$ & $\cdots$ & 771 \\
\hline Scozonera & $\ldots$ & 277 & Spathoglossum & & 820 & Strangea & ... & $\ldots$ & 452 \\
\hline SCROPHULARINE & $\mathrm{E} E \mathrm{E}$ & 358 & Spergula ... & .. & 48 & Streptachne & ... & ... & 623 \\
\hline Scutellaria & $\ldots$ & 388 & Spergularia & .. & 48 & Streptothrix & $\ldots$ & $\ldots$ & 783 \\
\hline Scutellinia & $\ldots$ & 753 & Spermacoce & .. & 253 & Striga & $\ldots$ & $\ldots$ & 363 \\
\hline Scyphiphora & $\ldots$ & 241 & Sphacelaria & .. & 821 & Stigonema & ‥ & ... & 832 \\
\hline Scytonema & $\cdots$ & 832 & Sphacelaria & .. & 801 & Strigula & $\cdots$ & $\cdots$ & 708 \\
\hline Seaforthia & $\ldots$ & 573 & Sphaceloma .. & .. & 776 & Strobilomyces & & $\ldots$ & 728 \\
\hline Sebæa & $\ldots$ & 337 & Sphacelotheca & & 771 & Strobilus . & $\ldots$ & ... & 510 \\
\hline Sebastiana & $\ldots$ & 480 & Sphæranthus .. &.. & 263 & Strongylodon. & $\cdots$ & $\cdots$ & 143 \\
\hline & $\cdots$ & 824 & Sphærella & .. & 765 & Strumella . & ... & $\ldots$ & 789 \\
\hline Secamone & $\ldots$ & 324 & Spharella & .. & … 763 & Struvea & ... & ... & 795 \\
\hline Securinega & $\ldots$ & 476 & Sphoria $\quad$.. & .. & 762,765 & St & ... & $\ldots$ & 337 \\
\hline elaginella & $\cdots$ & 634 & Sphærococcacex & & .. 823 & artina & ... & ... & 266 \\
\hline Selenospora & $\ldots$ & 789 & Sphærolobium & & ... 128 & Sturmia & $\cdots$ & $\ldots$ & 519 \\
\hline Semecarpus & $\ldots$ & 119 & phoron & & 684 & IDIE ÆE & ... & $\cdots$ & 277 \\
\hline Senebiera & $\cdots$ & 34 & Sph & .. & 776 & ium & ... & $\cdots$ & 277 \\
\hline iera & $\ldots$ & 768 & $S^{\prime} p h$ & $\cdots$ & 753 & andra & ... & $\ldots$ & 557 \\
\hline io. & ... & 275 & $\mathrm{Sp}$ & .. & 756 & phelia & $\cdots$ & $\cdots$ & 295 \\
\hline nium & $\ldots$ & 782 & erotheea .. & .. & 766 & ulon . & ... & $\cdots$ & 821 \\
\hline asidium & & 741 & sma .. & ... & 802 & popodium . & $\ldots$ & $\ldots$ & 819 \\
\hline Septoria & $\ldots$ & 778 & osma .. & $\cdots$ & 802 & RACACE E . & $\cdots$ & ... & 306 \\
\hline gloeum & ... & 782 & n $\quad$. & $\cdots$ & 672 & la & $\ldots$ & $\ldots$ & 409 \\
\hline Sepultaria & $\cdots$ & 753 & Spi & $\cdots$ & 272 & ana & $\cdots$ & $\ldots$ & 85 \\
\hline & $\ldots$ & 66 & ex $\quad .$. & $\cdots$ & 612 & raya & ... & & 816 \\
\hline Serj & ... & 783 & nthemum & & 169 & sona & & 130, & 837 \\
\hline Ses: & $\ldots$ & 363 & inthes .. & ... & ... 536 & Symphyocladia & & $\ldots$ & 827 \\
\hline Sesk & ... & 138 & aium .. & .. & ... 801 & Symphyogyne & & $\ldots$ & 676 \\
\hline Sesu & ... & $22 i$ & a & ... & 801 & ocos & ... & $\ldots$ & 306 \\
\hline Set: & $\ldots$ & ‥ 611 & Spirulina & $\cdots$ & 831 & & ... & ... & 761 \\
\hline Set: & $\cdots$ & 769,171 & Splachnobryum & & 664 & tantha & ... & $\ldots$ & 257 \\
\hline hesia & ... & ... 765 & lias ... & $\cdots$ & 124 & $\mathrm{rp}$ & ... & $\cdots$ & 202 \\
\hline Sic & ... & 222 & ocladia.. & $\ldots$ & 795 & ia & $\cdots$ & $\ldots$ & 202 \\
\hline Sida & $\ldots$ & 57 & bolus .. & .. & 623 & ytrium & $\ldots$ & $\ldots$ & 769 \\
\hline roxylon & $\ldots$ & ‥ 305 & Sporobolus. & $\cdots$ & ... 783 & Synconus & $\ldots$ & $\cdots$ & 48 \\
\hline era & ... & 228,837 & Sporodesmium & & 785,851 & hoblastus & & $\cdots$ & 682 \\
\hline Siegesbeckia & $\ldots$ & .. 271 & trichum. & $\ldots$ & .. 782 & Synechococcus & & ... & 830 \\
\hline Sil & $\ldots$ & $\cdots$ & gelia .. & $\cdots$ & 296 & Synedra & ... & ... & 816 \\
\hline Sili & $\cdots$ & $\ldots$ & Spi & $\cdots$ & 790 & Sy & $\ldots$ & $\ldots$ & 83 \\
\hline Sili & $\ldots$ & $\ldots$ & botrys .. & $\ldots$ & 783 & ppodon. & $\ldots$ & ... & 658 \\
\hline um & ... & 276 & Stac & $\cdots$ & 388 & Syzygium & ... & $\ldots$ & 208 \\
\hline JBE $\mathrm{E}$ & $\ldots$ & $\therefore$ & tarpheta & & 382 & & & & \\
\hline Siphonodon & $\ldots$ & 96 & sia. & & 100 & & & & \\
\hline nium & $\cdots$ & 801 & HOUSIEE & & $\cdots$ & & & & \\
\hline phon & $\ldots$ & 682 & Stat & $\ldots$ & $\ldots$ & Tabe & and & $\cdots$ & \\
\hline ema & $\ldots$ & .. 739 & astrum & $\ldots$ & $\ldots$ & Tabernomonta & ana & $\cdots$ & Th \\
\hline brium & $\ldots$ & 33,835 & roneis & $\ldots$ & $\ldots$ & Tacca & $\cdots$ & $\cdots$ & \\
\hline Sisyrinchium & $\ldots$ & .. 542 & Steetzia & $\ldots$ & 676 & $\mathrm{ACE}$ 层 & & $\ldots$ & \\
\hline Sium & $\ldots$ & 229 & & $\cdots$ & 642 & Tæniophyllum & & $\cdots$ & 536 \\
\hline Sloanea & $\ldots$ & ... & Stellaria & $\cdots$ & $\ldots$ & Tagetes & $\ldots$ & ... & 272 \\
\hline Smilax & ... & $\ldots \quad 553$ & Stemodia & $\cdots$ & $\cdots$ & Talinum & $\cdots$ & $\cdots$ & \\
\hline Smilax & & 766,779 & Ste & $\ldots$ & $\ldots$ & a,$\ldots$ & & & 819 \\
\hline ithia & & ... 139 & nitis . & $\cdots$ & $\cdots$ & Tapeinocheilos & & $\ldots$ & 54 \\
\hline NACEE & ... & $\ldots$ & themum & & $\cdots$ & Taraxacum & ... & $\cdots$ & 27 \\
\hline Solanum & & $\ldots 354$ & us. & & 45 & ietia & $\ldots$ & $\ldots$ & \\
\hline & 762 & 785,787 & etalum. & & $\cdots$ & rietia. & $\ldots$ & ... & 76 \\
\hline & $\ldots$ & .. 738 & aphrum & & 612 & annia & ... & $\cdots$ & \\
\hline Solieria & $\cdots$ & $\ldots$ & Step & $\ldots$ & $\cdots$ & oria & $\ldots$ & ... & 66 \\
\hline & & ... & Sterculia & $\cdots$ & 6 & & $\ldots$ & $\ldots$ & 364 \\
\hline & ... & ... & Sterculia $\quad$. & ... & 775,787 & & ... & ... & 779 \\
\hline ratia & ... & ... & STERCULIACE . & ... & ... 62 & rnia & & $\cdots$ & \\
\hline ratia & $\ldots$ & & & $\cdots$ & $\ldots$ & letonia & ... & $\cdots$ & 132 \\
\hline & $\ldots$ & ... & Stereocaulon & ... & $\ldots$ & Tephrosia & ... & ... & \\
\hline jia & $\ldots$ & $\ldots$ & Stereum & ... & $\ldots$ & Terminalia & $\cdots$ & $\cdots$ & \\
\hline rum & $\ldots$ & ... & & $\ldots$ & $\ldots$ & TERNSTREMIACI & EE & $\cdots$ & \\
\hline & $\ldots$ & 620 & Sterigmatocyst & tis & $\ldots$ & Tetmemorus & & $\cdots$ & \\
\hline Sorghum & $\ldots$ & $\therefore 769$ & Sticta & ... & $\ldots$ & Tetracera & $\cdots$ & $\cdots$ & \\
\hline
\end{tabular}




\begin{tabular}{|c|c|c|c|c|c|c|c|c|c|}
\hline & & & age. & & & Page. & & & Page. \\
\hline Tetracera & ... & $\cdots$ & 768 & Trichinium & & 397 & Usnea & & 685 \\
\hline Tetraedron & ... & ... & 800 & Trichocladia & $\cdots$ & 346 & Usnea & $\ldots$ & 685 \\
\hline Tetragonia & ... & ... & 227 & Trichocolea & ... & 675 & Ustilago & $\ldots$ & 769 \\
\hline Tetraspora & ... & $\ldots$ & 800 & Trichoderma & ... & 346 & Ustilago & $\ldots$ & 771 \\
\hline Tetratheca & ... & $\ldots$ & 43 & Trichodesma & ... & 782 & Ustulina & $\ldots$ & 759 \\
\hline Teucrium & ... & $\ldots$ & 392 & Trichodesmiun & & 831 & Utricularia & ... & 363 \\
\hline Teucrium & ... & ... & 785 & Tricholæna & & $\ldots 611$ & Utriculus & ... & 397 \\
\hline Thalloidima & $\ldots$ & ... & 703 & Tricholobus & $\ldots$ & 124,837 & Uvaria & ... & -.. \\
\hline Tham niella & $\ldots$ & ... & 667 & Tricholoma & $\ldots$ & .. 715 & Uvaria & $\ldots$ & 775 \\
\hline Thamnocloniu & & ... & 829 & Trichomanes & ... & 639 & & & \\
\hline Thamnolia & $\ldots$ & ... & 685 & Trichophilus & $\ldots$ & 795 & & & \\
\hline Thecaphora & $\ldots$ & ... & 701 & Trichosanthes & & 221 & & & \\
\hline Helephora & $\cdots$ & ... & 741 & Trichoscypha & ... & 753 & VACCINIACE E & & \\
\hline Thelephora & $\cdots$ & $\cdots$ & 741 & Trichosphæsia & & $\ldots$ & Vallisneria & $\begin{array}{l}\cdots \\
\cdots\end{array}$ & 518 \\
\hline Thelotrema & $\cdots$ & $\cdots$ & 697 & Tricoryne & $\cdots$ & $\ldots$ & Valonia & $\ldots$ & 796 \\
\hline ymitra & $\cdots$ & $\cdots$ & 537 & Tricoryne & ... & $\cdots$ & Valsa & ... & ... \\
\hline Theobroma & ... & $\cdots$ & 762 & Trichosteleum & & 670 & Vandellia & $\ldots$ & ... \\
\hline sium & .. & ... & 469 & Tricostularia & ... & 598 & Vanhuerclia & ... & $\ldots$ \\
\hline pesia & $\cdots$ & $\cdots$ & 61 & Tridax & $\cdots$ & $\cdots$ & Variolaria & $\ldots$ & 697 \\
\hline pidium & $\cdots$ & $\cdots$ & 263 & Trifolium & $\cdots$ & $\cdots$ & Vaucheria & ... & ... \\
\hline Ihlasp1 & $\cdots$ & $\cdots$ & $\begin{array}{r}50 \\
332\end{array}$ & Triglochin & ... & $\cdots$ & Velleia & $\ldots$ & ... \\
\hline Thozetia & … & $\cdots$ & 332 & Trigonella & $\cdots$ & 130 & Ventilago & $\ldots$ & ... \\
\hline Thozetia (fun & igus) & $\cdots$ & 787 & Trio & ... & 629 & Verbascum & ... & ... \\
\hline lkeldia & $\cdots$ & $\cdots$ & 409 & Triploceras & ... & ... & na & $\cdots$ & ... \\
\hline xspermum & & $\cdots$ & 535 & telus & $\cdots$ & $\cdots$ & BENACE. & $\ldots$ & $\ldots$ \\
\hline Thryptomene & $\cdots$ & $\cdots$ & 181 & Trir & ... & $\cdots$ & Verbesina & ... & 271 \\
\hline ea & $\cdots$ & $\cdots$ & 612 & Tristania & & $\ldots \quad 201$ & icularia & $\ldots$ & ... \\
\hline lium & $\cdots$ & $\cdots$ & 669 & Tristania. & 768 & , 776, 779 & Ionia & $\ldots$ & 257 \\
\hline Thunbergia & $\cdots$ & $\cdots$ & 368 & Tristellateia & $\cdots$ & ... 69 & nica & $\ldots$ & ... \\
\hline Threanotus & $\cdots$ & $\cdots$ & 557 & Tristichocalyx & & $\cdots$ & aria & $\ldots$ & 710 \\
\hline THYUEL ÆACE & & $\ldots$ & 455 & Triti & $\cdots$ & $\cdots$ & ecaria & $\ldots$ & 709,711 \\
\hline Thysanocladia & & $\cdots$ & 823 & Triticum & $\cdots$ & $\cdots$ & Verticordia & ... & ... 181 \\
\hline anotheciu & $\mathrm{am}$ & & 684 & Triumfetta & $\cdots$ & ... & a & ... & ... \\
\hline TiLI & $\cdots$ & & 835 & TRIURIDEA & ... & $\ldots$ & Vidalia & ... & ... \\
\hline Tillæa & $\cdots$ & $\cdots$ & 169 & Trochocarpa & $\cdots$ & $\cdots$ & Vidalia & ... & $\ldots$ \\
\hline Tilletia & $\cdots$ & $\cdots$ & 771 & Trog & $\ldots$ & ... & & ... & $\ldots$ \\
\hline loche & $\cdots$ & $\cdots$ & 791 & Trypethelium & ... & ... & aresia & $\ldots$ & $\ldots$ \\
\hline & ... & $\cdots$ & 245 & thelium & $\cdots$ & $\cdots$ & Villarsia & ... & $\ldots$ \\
\hline Tinospora & $\cdots$ & $\cdots$ & 29 & Tubercularia & $\cdots$ & ... & inaria & $\cdots$ & $\ldots$ \\
\hline Tinesipteris & $\cdots$ & ... & 635 & Tubulin & $\cdots$ & ... & & $\cdots$ & $\ldots$ \\
\hline Todea & $\cdots$ & $\cdots$ & 638 & naria & $\cdots$ & $\cdots$ & Vincetoxicum & $\cdots$ & ... \\
\hline Tolypiocladia & $\cdots$ & ... & 827 & Tur & ... & $\ldots$ & Viola & ... & $\cdots$ \\
\hline Tolyposporium & & $\cdots$ & 769 & Tylophora & ... & $\ldots$ & ARIEA & $\cdots$ & ... \\
\hline hrix & $\cdots$ & ... & 832 & loron & $\cdots$ & ... & $\mathrm{m}$ & $\ldots$ & ... \\
\hline Tomasellia & $\cdots$ & ... & 710 & Tylostoma & $\cdots$ & $\ldots$ & & ... & $\cdots$ \\
\hline Torenia & $\cdots$ & $\cdots$ & 361 & Typha & ... & $\cdots$ & Vit & $\ldots$ & 106 \\
\hline Tor & ... & ... & 659 & TYPHACE & $\cdots$ & $\cdots$ & Vitis & $\ldots$ & 775,787 \\
\hline Tor & ... & $\cdots$ & 659 & Typhonium & $\cdots$ & $\cdots$ & Vittadina & ... & ... 26 \\
\hline Torula & $\cdots$ & $\cdots$ & 783 & & & & Vittaria & $\ldots$ & ... \\
\hline efortia & $\cdots$ & $\cdots$ & 346 & & & & Volvox & $\ldots$ & $\ldots$ \\
\hline Trabutia & $\cdots$ & $\cdots$ & 761 & & & & & & \\
\hline Trachyllia & $\cdots$ & $\cdots$ & 683 & Udote & $\cdots$ & $\cdots$ & & & \\
\hline Trachymene & $\cdots$ & $\cdots$ & 22 & Ulex & $\cdots$ & $\cdots$ & & & \\
\hline ntia & $\begin{array}{l}\cdots \\
\ldots\end{array}$ & $\ldots$ & & Ulocolla & ... & $\cdots$ & & & \\
\hline Tra & $\cdots$ & $\cdots$ & 480 & Tlothrix & $\cdots$ & $\ldots$ & Wa & $\cdots$ & \\
\hline Tragopogon & ... & $\cdots$ & 277 & Ulva & $\cdots$ & ‥ 794 & Wa & $\cdots$ & $\cdots$ \\
\hline & $\cdots$ & $\cdots$ & 612 & ELLIFER丑 & $\cdots$ & 228,83 & leria & & ... \\
\hline etes & $\cdots$ & & 737 & Uncar & $\ldots$ & ... 237 & & .. & $\cdots$ \\
\hline metes 733 , & 734 & 736 , & 737 & Unc & $\ldots$ & $\ldots$ & Wedelia & ... & 271 \\
\hline Trema & $\ldots$ & & 485 & Un & $\ldots$ & & nmannia & $\ldots$ & $\ldots$ \\
\hline Trema & $\cdots$ & 766 , & 776 & Ura & ... & $\cdots$ & & .. & $\cdots$ \\
\hline ANDREE & $\ldots$ & $\ldots$ & 43 & Urceolaria & ... & $\ldots$ & Wendlandia & ... & $\cdots$ \\
\hline atodon & $\ldots$ & ... & 656 & Uredo & ... & ... 775 & Westringia & ... & ... \\
\hline Tre & ... & & 745 & Uredo & ... & 773,778 & strøemia & ... & $\ldots$ \\
\hline Tre & $\cdots$ & 743 , & 763 & & $\cdots$ & ... 5 & & & $\cdots$ \\
\hline mellodon & ... & $\ldots$ & 739 & Urnula & ... & ... 755 & Wilsoniella & & 662 \\
\hline motylium & $\ldots$ & $\ldots$ & 707 & Urocystis & ... & ‥ 771 & aria & & ... \\
\hline & $\cdots$ & $\ldots$ & 22 & Uromyces & $\cdots$ & 772,773 & & & $\cdots$ \\
\hline Tribliadella & $\cdots$ & ... & 756 & Uromyces & $\ldots$ & ... 771 & Woodwardia & & 644 \\
\hline Tribulus & ... & $\cdots$ & 72 & Uromycladiun & & $\ldots$ & Woollsia & & 296 \\
\hline eratium & $\cdots$ & $\cdots$ & 817 & Uronema & $\cdots$ & $\cdots$ & & & 18,83 \\
\hline & 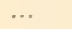 & & 720 & & & .. 504 & htia & & .. 325 \\
\hline Trichilia & ... & & 119 & URTICACEE & & 480,839 & II urmbsea & & 559 \\
\hline
\end{tabular}




\section{INDEX OF SYSTEMATIC NAMES.}

\begin{tabular}{|c|c|c|c|c|c|c|c|c|c|c|c|}
\hline & & & age. & & & & age. & & & & age. \\
\hline Xanthidium & $\therefore$ & $\ldots$ & 806 & Ximenesia & ... & ... & 271 & Zea & & & 616 \\
\hline Xanthium & $\ldots$ & $\cdots$ & 271 & Xylaria & $\ldots$ & $\ldots$ & 758 & Zea & 76 & 783, & 791 \\
\hline Xanthium & $\ldots$ & $\cdots$ & 763 & Xylomelum & ... & $\ldots$ & 440 & Zehneria & ... & $\ldots$ & 222 \\
\hline Xanthophyllun & & $\ldots$ & 44 & Xylopodium & $\ldots$ & $\ldots$ & 752 & Zieria & ... & $\cdots$ & 73 \\
\hline$X$ anthophyllun & & $\ldots$ & 781 & $\mathrm{X} y$ losma & $\cdots$ & $\ldots$ & 12 & Zingiber & $\ldots$ & $\cdots$ & 539 \\
\hline Xanthorrhæa & $\ldots$ & $\ldots$ & 566 & Xylostroma & $\ldots$ & ... & 792 & Zinnia & $\ldots$ & $\ldots$ & 271 \\
\hline X anthorrhoa & $\ldots$ & $\ldots$ & 778 & XYRIDEA & $\ldots$ & $\cdots$ & 511 & Zizyphus & $\cdots$ & $\ldots$ & 100 \\
\hline Xanthosia & $\ldots$ & $\ldots$ & 229 & Xyris & $\ldots$ & $\ldots$ & 561 & Zonaria & $\ldots$ & $\ldots$ & 819 \\
\hline Xanthostemon & & $\ldots$ & 202 & & & & & Zornia & $\ldots$ & $\ldots$ & 139 \\
\hline Xerochloa & $\ldots$ & $\ldots$ & 612 & Yucca & $\ldots$ & $\ldots$ & 851 & Zosteria & ... & $\ldots$ & 583 \\
\hline Xerotes & $\ldots$ & $\ldots$ & 565 & & & & & Zoysia & ... & ... & 616 \\
\hline Xerotes & ... & $\ldots$ & $76 \overline{3}$ & Zaleya & $\ldots$ & $\ldots$ & 227 & Zygnema & $\ldots$ & $\ldots$ & 801 \\
\hline Xerotus & $\ldots$ & $\ldots$ & 727 & Zanthoxylum & $\ldots$ & $\ldots$ & 81 & ZYGOPHYLLE & $\ldots$ & $\ldots$ & 72 \\
\hline Ximenia & ... & ... & 91 & Zanthoxylum & $\ldots$ & ... & 768 & Zygophyllum & $\ldots$ & ... & 72 \\
\hline
\end{tabular}




\section{Index of Vernacular Names, Notes, etc.}

\begin{tabular}{|c|c|c|c|c|c|c|c|c|}
\hline & & & & & age & & & age. \\
\hline gubber & $\cdots$ & 452 & Ara-ra & ... & 144 & Baral & & 642 \\
\hline & $\cdots$ & 167 & ... & $\ldots$ & 591 & Barbadda $\quad \ldots$ & & \\
\hline cia Cedar & .... & 164 & ... & $\ldots$ & 305 & Barbadoes Gooseber & & \\
\hline alla & ... & & al & ... & 77 & Barbed-wire Grass & & \\
\hline & $\cdots \quad \cdots$ & 249 & iga & ... & 153 & Barley Grass ... & & \\
\hline Adder's-tongu & ae Fern & 635 & imby & $\cdots$ & 476 & Barley, Malting & or & \\
\hline $\begin{array}{l}\text { Aeroplanes, } \\
\text { suitable for }\end{array}$ & Wood & & $\begin{array}{ll}\text { Arrongg } & \ldots \\
\text { Ar-roo-in } & \ldots\end{array}$ & $\begin{array}{l}\ldots \\
\ldots\end{array}$ & 455 & 'Two-rowed ... & ... & \\
\hline $\begin{array}{l}\text { suitable for } \\
\text { Agar-agar }\end{array}$ & $\ldots$ & $\begin{array}{l}143 \\
823\end{array}$ & $\begin{array}{ll}\text { Ar-roo-in } & \ldots \\
\text { Arrow-grass } & \ldots\end{array}$ & & $\begin{array}{l}453 \\
580\end{array}$ & $\begin{array}{l}\text { Barley, Six-rowed } \\
\text { Barley, Wall or Mou }\end{array}$ & & \\
\hline & $\cdots$ & 96 & Arsenic Plant & $\cdots$ & 21 & a & ... & \\
\hline & $\ldots$ & 396 & Artificial Flowers & $\ldots$ & 287 & inya & $\ldots$ & \\
\hline & ... & 153 & Arumba $\quad \ldots$ & & 518 & & $\ldots$ & 09 \\
\hline ata & ... & 332 & Asthma, Cure for 2 & 221,3 & 358, & $\ldots$ & $\ldots$ & 525 \\
\hline coo & $\cdots$ & 576 & 1)othmo & 470 & 473 & Batham $\quad \ldots$ & ... & \\
\hline an 1 & & & Asthma-plant ... & & 473 & Bathurst Burr & ... & if \\
\hline a. Bea & an & 768 & Astringent properti & ies & & -wing Fern & $\cdots$ & \\
\hline Indi & $\cdots$ & 200 & commonly & net & & Baven $\quad$... & $\cdots$ & \\
\hline limond, Indi & Tohnstone & 180 & $\begin{array}{l}\text { with in Acacias (15 } \\
\text { and Eucalypts }\end{array}$ & $\begin{array}{l}\text { 54) } \\
93)\end{array}$ & & $\begin{array}{l}\text { Beach Grass } \ldots \\
\text { Bean Capers }\end{array}$ & & 612 \\
\hline River & $\ldots \quad \ldots$ & 69 & Atchoourgo ... & $\ldots$ & 188 & Bean-tree & 148 & \\
\hline$a$ & $\ldots$ & 349 & $\ldots$ & $\cdots$ & 306 & Bean-tree of $\mathrm{Obu}$ & um & \\
\hline & $\ldots$ & 136 & du $\quad \ldots$ & $\ldots$ & 561 & Obum & ... & 1 \\
\hline & $\ldots$ & 271 & ralian Bugle & $\ldots$ & 392 & Bear's breech ... & & 74 \\
\hline & $\cdots$ & 642 & Australian Centaury & & 337 & traw, Australi & ian & 257 \\
\hline m-angka & a-ina & 266 & Australian Heaths & & 296 & ew-rie $\quad .$. & $\ldots$ & \\
\hline & $\cdots \quad \cdots$ & 200 & ralian Mint & & 388 & & $\cdots$ & \\
\hline $\begin{array}{l}\text {-mega } \\
\text { merican }\end{array}$ & Ẅalnut, & 136 & $\begin{array}{l}\text { Australian Mudwort } \\
\text { Australian Peppe }\end{array}$ & & 361 & $\begin{array}{l}\text { ch, Negro-head } \\
\text { ch, Queensland }\end{array}$ & & \\
\hline & & 124 & vine $\quad \cdots$ & & 112 & $\begin{array}{l}\text { Beech, Queensland } \\
\text { Beech, Wood rese }\end{array}$ & em: & \\
\hline - & $\cdots$ & 396 & Australian Speedwel & ells & 361 & bles English & ... & 440 \\
\hline & $\cdots$ & 548 & Australian Wat & ter & & Beefwood $\quad \ldots$ & $\cdots$ & \\
\hline & $\ldots$ & $\begin{array}{l}375 \\
580\end{array}$ & Horehound & .. & 388 & $\cdots$ & $\cdots$ & \\
\hline & $\cdots$ & $\begin{array}{l}580 \\
332\end{array}$ & & & 404 & aid & & \\
\hline & ... & 612 & $\begin{array}{l}\text { r's Buttons } \\
\text { sis of Pot: }\end{array}$ & & 789 & fora & & 411 \\
\hline & ... & 539 & $\ldots$ & & 513 & old & & 411 \\
\hline -ga & ... & & $\ldots$ & & 574 & & & (1) \\
\hline & $\cdots$ & 10 & & & 158 & & & 486 \\
\hline$-\tan$ & $\cdots$ & 566 & al-balgal & & 73 & Bent work, & ood & \\
\hline & $\cdots$ & 62 & $a \quad \ldots$ & ... & 14 & eful for $\ldots$ & .. & 305 \\
\hline 11 Fung & gus & 751 & mo ... & ... & 48 & & & \\
\hline house & $\cdots, \cdots$ & 253 & con-vine $\quad .$. & ... & 112 & Betel-leaf, & ute & \\
\hline $\begin{array}{l}\text { idote for } \\
\text { sh }\end{array}$ & Poison- & & Gum $\quad \ldots$ & $\cdots$ & 43. & $\ldots$ & .. & \\
\hline & $\begin{array}{l}\cdots \\
\cdots\end{array}$ & & $\operatorname{li}^{2} \mathrm{~A}$ & $\cdots$ & $\begin{array}{l}375 \\
221\end{array}$ & $\cdots$ & & \\
\hline tic & $\begin{array}{l}\cdots \\
\cdots\end{array}$ & 548 & $\begin{array}{l}n \text { App } \\
n \text { of C }\end{array}$ & $\begin{array}{l}\cdots \\
\ldots\end{array}$ & & $\cdots$ & & \\
\hline & $\ldots$ & 542 & $n$ of $c$ & & 17 & $\begin{array}{l}\ldots \\
\ldots\end{array}$ & & 542 \\
\hline lant, an. & .. & & oo, $\mathrm{Bla}$ & & & $\begin{array}{l}\cdots \\
\ldots\end{array}$ & & 175 \\
\hline Berry. & & 4 & $\mathrm{oo}, \mathrm{Cli}$ & ... & & ... & & 50 \\
\hline e, Black & & 305 & oo, Ere & ... & 634 & n-billan & & 311 \\
\hline $\begin{array}{l}\text { le, Fraze } \\
\text { ad }\end{array}$ & er's Is- & $81-2>-1$ & 00 Gra & & 622 & Bill & & \\
\hline ple, Fungu & us Dis- & & a Fru & & $\begin{array}{r}96 \\
781\end{array}$ & $\begin{array}{l}\text { d-cues, } \\
\text { ble for }\end{array}$ & & \\
\hline & & & , Nat & & 542 & & & \\
\hline Ma & $\operatorname{cov} \theta$ & & a (Wilds) & of & & -plant & ... & \\
\hline Sca & $\cdots$ & 783 & bane childre & & 25 & & & 175 \\
\hline , Scrub & & 783 & oft Grass & & 604 & i (introduced) & & 271 \\
\hline of Tam & nbour & & oft's $\quad$ t & & & & & \\
\hline & & 209 & oft's & & & reeds & 349 & \\
\hline Thorn & or Mads & 358 & $\ldots$ & ... & & & $\ldots$ & 318 \\
\hline le-tree & & & $\operatorname{lin}$ & $\ldots$ & & ... & ... & \\
\hline Sle, Wood & rese & & & $\cdots$ & & $\cdots$ & $\cdots$ & \\
\hline mill & .. & $\begin{array}{l}477 \\
200\end{array}$ & $\tan$ of Carins & & $\begin{array}{l}485 \\
486\end{array}$ & able f & for & 396 \\
\hline & & 143 & Bara & & 430 & Bird's-nest F & & \\
\hline
\end{tabular}




\begin{tabular}{|c|c|c|c|c|c|c|c|}
\hline & & age. & & & & & ge. \\
\hline Bird's-nest Fungus . & ... & 746 & Boomban & 431 & Bulkuru & & 591 \\
\hline e $\quad \ldots$ & ... & & & 504 & $\cdots$ & ... & 88 \\
\hline ... & ... & 318 & ... & 200 & rush & $\ldots$ & 576 \\
\hline Birthwort & .. & 418 & ... & 88 & an & ... & \\
\hline Birula & $\ldots$ & 485 & Booral & 439 & Bumbar $\quad \ldots$. . & & 455 \\
\hline Bishop's-weed & ... & 229 & Boorbal & 116 & Bunch Spear Grass . & ... & 619 \\
\hline Bitamon & $\ldots$ & & Boorgay $\quad .$. & 579 & gadinnia ... . & $\cdots$ & 09 \\
\hline Bitter-bark & $\ldots$ & 318 & Boorgoolbean ... & 245 & kiam & & 535 \\
\hline itter-cress & ... & 33 & Boorgun $\quad \ldots$ & $\cdots$ & wall & ... & \\
\hline Bitter-rot & & 781 & $\ldots$ & 249 & ... & $\cdots$ & 124 \\
\hline Bitter-weed, & & & oda & 508 & Bunji-bunji & ... & 91 \\
\hline can & $\ldots$ & 271 & ogun & 542 & Bun-jin-oo & $\ldots$ & 513 \\
\hline Black Apple ... & ... & & Bootharoo .... & 387 & Bunkerman $\quad \ldots$ & $\cdots$ & \\
\hline Black Bamboo & $\ldots$ & 851 & Bordeaux Mixture & 765 & Bunt, Winter ... & & \\
\hline Black Bean ... & $\ldots$ & 144 & Bordeaux Mixture, t & to & Bunya Bunya & ... & \\
\hline lack Box ... & $\ldots$ & 19 & make ... : & :. & gari $\ldots$ & $\cdots$ & 510 \\
\hline Black Mustard & $\ldots$ & 34 & Bore Drains, a Pest i & 851 & Burdekin Plum & & 124 \\
\hline Black Myrtle ... & $\cdots$ & 306 & Boree $\quad \ldots$ & 159 & Burdock, Common : & & 276 \\
\hline Black Palm & $\ldots$ & 57 & im-bru & 573 & Burmese unit weigh & & 140 \\
\hline lack Pine & $\ldots$ & 510 & Bottle-brush $\quad$... & 187 & Bur Reed ... & & 576 \\
\hline Black Walnut & & 430 & Bottle Gourd ... & 221, 762 & Burr Grass, Hillsio & & 11 \\
\hline Blackberry of & & & Bottle-tree (broa & & Burr Grass, Small. & ... & \\
\hline & & & & 6 & , Serub ... & & \\
\hline Blackberry, British & & & Bottle-tree & & owa & & \\
\hline $\begin{array}{c}\text { Blackberry, } \\
\text { land }\end{array}$ & & 167 & oison-plant & 360 & $\begin{array}{l}\text { Burthargona } \\
\text { Buttercup }\end{array}$ & $\begin{array}{l}\cdots \\
\cdots\end{array}$ & \\
\hline Helid & Ion & 167 & Box, Bastard Gu & & Buttercup-tree. & $\cdots$ & 201 \\
\hline utt & $\ldots$ & 19 & leaved $\quad \ldots$ & 194 & Button Grass ... & ... & 28 \\
\hline d Susan & $\ldots$ & 368 & Box, Brisbane ... & 201,778 & Button Orchid & ... & 32 \\
\hline pot of Grape & $\ldots$ & 776 & Box, Gur & $\ldots \quad 194$ & Buyan $\quad \ldots$ & $\ldots$ & 112 \\
\hline ood $\quad \ldots$ & $\ldots$ & 159 & Box, Poplar ... & 194,195 & Byamurra & $\ldots$ & \\
\hline ladder Ketmia & $\cdots$ & 5 & Box, Stanthorpe & 195 & & & \\
\hline derwort $\ldots$ & $\cdots$ & & Box, Wood resemblin & $n g$ & Cabbage, & & \\
\hline $\begin{array}{l}\text { Grass } \\
\text { ret Fern }\end{array}$ & $\cdots$ & 610 & glish & 91 & Disease of $\ldots$ & & \\
\hline $\begin{array}{l}\text { ret Fern ... } \\
\text { ing-tree ... }\end{array}$ & $\cdots$ & 649 & thorn , ... & ... & Cabbage, Fungus & on & \\
\hline $\begin{array}{l}\text { g-tree } \\
\text { ss, Causing. }\end{array}$ & $\cdots$ & 460 & 'Thozet's ... & $\cdots$ & bage Gum & & \\
\hline $\begin{array}{l}\text { ss, Causing } \\
\text { Europe }\end{array}$ & $\cdots$ & & $\begin{array}{l}\text { Bpa-Burry } \\
\text { Bramble Indian }\end{array}$ & $\cdots$ & $\begin{array}{l}\text { Cabbage-tree } \mathrm{Pa} \\
\text { Cabbage of } \mathrm{Tr}\end{array}$ & & \\
\hline ope & $\begin{array}{l}\cdots \\
\ldots\end{array}$ & & $\begin{array}{l}\text { Bramble, Indian } \\
\text { Bramble, Queenslan }\end{array}$ & nod & $\begin{array}{c}\text { Cabbage of } \mathrm{T} \\
\text { coast }\end{array}$ & & 287 \\
\hline & $\begin{array}{l}\cdots \\
\cdots\end{array}$ & 200 & $\begin{array}{l}\text { Bramble, Queenslan } \\
\text { Bracken }\end{array}$ & -.. & $\begin{array}{cc}\text { coast } & \cdots \\
\text { Cacao } & \ldots\end{array}$ & $\ldots$ & 762 \\
\hline od, Scrub & $\ldots$ & 47 & gain. & $\begin{array}{l}\cdots \\
\cdots\end{array}$ & ns Hickory & $\ldots$ & 91 \\
\hline dwood, White & ... & 20 & d Fern & ... & Cairns Lancewood & ... & 163 \\
\hline Bean & $\ldots$ & 147 & in Fruit ... & $\cdots$ & Calf's-snout $\quad . .$. & $\ldots$ & 60 \\
\hline Bean (flat) & $\cdots$ & & Fungus & 745 & Callarie & ... & \\
\hline luebell, Native & $\cdots$ & 29 & lian Cherry & ... & ra & ... & 347 \\
\hline y plant & $\cdots$ & 55 & Bread-fruit $\quad .$. & 576 & Caltrops $\quad \ldots$ & $\because ;$ & \\
\hline & ... & 40 & Breathing processes & & Calves with "Scou & & \\
\hline lue Couch Grass & $\ldots$ & 60 & & 38 & re for $\quad \ldots$ & .. & \\
\hline lue Grass $\ldots$ & & & low $\quad .$. & $\cdots$ & ooor $\quad \ldots$ & $\cdots$ & \\
\hline lue Grass, Tassell & & 61 & Brisbane Box ... & 201 & el Fodder... & $\therefore$ & \\
\hline lue Grass, Texas & $\ldots$ & & gane Gol & & hor Laurel & & \\
\hline lue Gum .... & .. & 19 & attle & 837 & dian Flea-bane & & \\
\hline Waterlily & $\ldots$ & 3 & Bristle Fern ... & $\ldots$ & y-seed Grass & ... & \\
\hline bo $\quad \ldots$ & $\ldots$ & & e-wort $\quad \ldots$ & ... & $\operatorname{ood} \ldots$ & $\cdots$ & 237 \\
\hline ... & $\ldots$ & & Bean & $\cdots$ & it $\quad \ldots$ & $\ldots$ & 478 \\
\hline & $\ldots$ & & -weed $\quad .$. & ... & ood & $\ldots$ & \\
\hline$\cdots$ & $\ldots$ & & Rapes & $\ldots$ & uft & $\ldots$ & \\
\hline$\varepsilon s \quad \ldots$ & ... & 67 & gorre $\ldots$ & $\ldots$ & $\ldots$ & ... & \\
\hline -bogum & $\ldots$ & & nspot of Grape & bes & Grass & $\ldots$ & \\
\hline$\quad \ldots$ & $\ldots$ & & n-top Grass & & kee $\quad \ldots$ & $\cdots$ & \\
\hline$\ldots$ & $\ldots$ & & es, to prever & & onball-tree & $\cdots$ & \\
\hline ... & $\ldots$ & & olouration fros & om & $\ldots$ & ... & \\
\hline ... & $\ldots$ & 249 & $n \quad \ldots$ & $\ldots$ & seberry & $\ldots$ & \\
\hline & $\ldots$ & 7 & & 41 & e Weed ... . & ... & \\
\hline$\cdots$ & ... & 69 & wheat, Climbin & ng & caway-seed Fern & & \\
\hline .. & $\ldots$ & 186 & o Burr ... & $\ldots$ & & & \\
\hline$\ldots$ & $\ldots$ & & o Grass ... & $\ldots$ & vell Maple & & 91 \\
\hline$\ldots$ & .. & & groo $\quad \ldots$ & $\ldots$ & ation $\quad \ldots$ & 772 & \\
\hline & & 51 & e, Australian & $\ldots$ & Carob Bean. & & \\
\hline & & & $\ldots$ & ... & Carpet Grass ... & ... & \\
\hline & & & . & $\ldots$ & Carpet Snake, Fung & rus & \\
\hline & $\ldots$ & 77 & on & ... & dung of $\ldots$ & $\cdots$ & \\
\hline olarchoo & $\cdots$ & 20 & 3ul-boora & $\ldots$ & Carrabbe & $\cdots$ & \\
\hline
\end{tabular}


Page.

Carrabean $\quad . .$.
Carr-lee $\quad .$.
Carrot
Carrot, Native
Carrot-wood ...
Cartalogoor $\ldots$.
Cascarilla Bark
Cassie Pomade
France

Castor Oil plant

Catamarans, Wood used for $\quad \ldots \quad \ldots 180$

Catchfly $\quad$... $\quad \ldots .44$

Caterpillar Grass ... 602

Caterpillar Pod ... 139

Cathartic $\quad \ldots \quad 347,349$

Cat-o'-nine-tails ... 453

$\begin{array}{llll}\text { Cat's-ear } \quad \ldots & \ldots & 276\end{array}$

$\begin{array}{lllr}\text { Cat's-head } & \ldots & \ldots & 72 \\ \text { Cat's-moustach } & & & 387\end{array}$

$\begin{array}{llll}\text { Cat's-moustache } & \ldots & 387 \\ \text { Cattle-bush } & \text {... } & \ldots & 116\end{array}$

Cauliflower, on leaves of

$\begin{array}{llll}\text { Caustic-creeper } & \text {... } & 470 \\ \text { Caustic-plant } & \ldots & 473\end{array}$

Caustic-plant, Steven's 473

Caustic-vine $\quad$... $\quad . .3326$

Cayenne Pepper ... 357

Cedar, Acacia ... 164

Cecar, Red ... ... 88

Cedar, White ... ... 86

Chain Measure $\quad . . .5574$

$\begin{array}{llll}\text { Chainfruit } & \text {... } & \text {... } & 317\end{array}$

$\begin{array}{llll}\text { Chakata } & \ldots & \ldots & 597\end{array}$

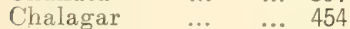

Chambin $\quad . . . \quad \ldots .113$

Chamois-leather Fun-

$\begin{array}{cccc}\text { gus } & \ldots & \ldots & 792 \\ \text { Chantarelle } & \ldots & \ldots & 723\end{array}$

Charcoal-tree, a $\quad \ldots .485$

$\begin{array}{llll}\text { Chargir } & \text {... } & \text {... } & 118\end{array}$

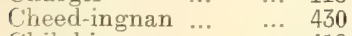

$\begin{array}{llll}\text { Chib-bi } & \text {... } & \text {... } & 418\end{array}$

$\begin{array}{llll}\text { Chichm } & \ldots & \ldots & 152\end{array}$

$\begin{array}{llll}\text { Chicory } & \ldots & \ldots & \ldots 39\end{array}$

Chickweed, European 48

Chickweed, Mouse-ear 48

Chillagoe Disease, sup-

posed cause of 133, 139

\section{Chille}

Chinana $\quad \cdots \quad \cdots \quad 455$

Chindirigan $\quad \ldots \quad \ldots .565$

Chinese Burr ... ... 66

Chingum $\quad \ldots \quad \ldots 538$

$\begin{array}{llll}\text { Chiroo } & \ldots & \ldots & 325\end{array}$

Chisel-handles, Wood useful for ... ... 169

Choolo-choolo $\cdots \quad \ldots .202$

Choopool ... 510

Chrysalis, Fungus on 786

$\begin{array}{llll}\text { Chukin } & \text {... } & \text {.. } 386\end{array}$

$\begin{array}{llll}\text { Chunka } & \text {... } & \text {... } 477\end{array}$

$\begin{array}{llll}\text { Chupolla } & \ldots & \ldots & 510\end{array}$

Churnwood ...

Churoga $\quad \ldots . \quad \ldots 55 z$

Cigar Boxes, "Wood $\begin{array}{lllr}\text { for } & \ldots & \ldots & 44 \\ \text { Cismatan } & \ldots & \ldots & 152\end{array}$

Citron-scented، Gum ... 201

Citrus trees, Fungus on

Citrus, Alga on leaves of
Page.

$\begin{array}{llll}\text { Cleavers } & \ldots & \ldots & 257\end{array}$

Climbing Buckwheat 412

Climbing, Stems used for $\quad$... ... 565

Clover, Cluster _... 136

Clover, Hop ... $\quad \ldots .136$

Clover, White ". ... 136

Club Mosses ... ... 634

Club Root ... ... 849

Cluster Fig ... ... 504

Cobbler's-pegs 262, 272

Cocci, Fungus on ... 789

Cochineal-plant ... 222

Cockatoo Grass $\quad$... 611

$\begin{array}{llll}\text { Cock's-comb } & \text {... } & \text {... } & 397\end{array}$

Cock's-foot Grass ... 633

Cockshin-Grass _.. 604

Cockspur Thorn ... 504

Cocoanut Palm ... 574

Coffee-blight $\quad . . \quad \ldots \quad 785$

$\begin{array}{llll}\text { Collar-rot } & \ldots & \ldots & 787\end{array}$

Comet Grass ... .... 616

Condy's Fluid ... ... 128

$\begin{array}{llll}\text { Convavola } & \ldots & \ldots & 62\end{array}$

$\begin{array}{llll}\text { Coobiaby } & \ldots & \ldots & 237\end{array}$

Coobine or Coobyn ... 518

Coocheramunda $\quad \ldots .624$

Cood-mo-ro $\quad$... ... 504

Cooktown Loquat .... 207

Coolibar $\quad$... $\quad . . .195$

Coolibar Grass ... 610

$\begin{array}{llll}\text { Coolooli } & \text {... } & \ldots & 510\end{array}$

$\begin{array}{llll}\text { Coonam } & \ldots & \ldots & 510\end{array}$

Coondoo $\quad$... $3 . . .305$

Coongera $\quad \ldots . \quad \ldots \quad 480$

$\begin{array}{llll}\text { Cooraloo } & \ldots & \ldots & 480 \\ & \ldots & \ldots & 113\end{array}$

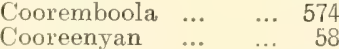

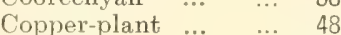

Coral-tree $\quad . . \quad 143,163$

Corduroy $\quad$... $\quad \ldots .116$

Coriander $\quad$... $\quad$... 229

Cork-tree, Bat's-wing 143

$\begin{array}{llll}\text { Cork-vine } & \text {... } & \text {... } & 151\end{array}$

Corkwood ... $\quad \ldots 358$

Corn Cockle ... $\quad$... 44

Cornflower ... ... 276

Corn Gromwell ... 347

Corn or Maize ... 616

Corn Silk $\quad . .6 \quad \ldots .616$

Corn-smut $\quad \ldots \quad \ldots 769$

Corn Sowthistle ... 277

Cotton, Australian ... 61

Cotton Blight …785

Cotton-bush ... 408, 478

Cotton-leaved Physic-

$$
\text { nut }
$$

$\begin{array}{lllr}\text { Cotton-tree } & \ldots & \ldots & 61\end{array}$

Couch Grass ... ... 627

Couch Grass, Coast ... 616

Couch Grass, Moun-

tain $\quad$... ... 624

Couch Grass, Water 602

Country Almond ... 180

Cowdung Fungus ... 755

$\begin{array}{llll}\text { Cow-herb } & \ldots & \ldots & 44\end{array}$

Cow-pea $\quad \ldots \quad \ldots .144$

Cows, reputed poison$\begin{array}{llll}\text { ous to } & \ldots & \ldots & 287\end{array}$

$763 \quad$ Crab's-eyes ... ... 140

Crape Fern ... $\quad \ldots \quad 638$

793 | Crane's-bill ...
Page.

Crow Poison ... ... 220

Crow's Apple ... ... 88

Crow's Ash ... ... 91

Crow's-foot EIm of

Atherton $\quad \ldots \quad \ldots \quad 62$

Crow's-foot Grass … 628

Cucumber $\quad 766,768,787$

Cudgerie $\quad$... $\quad$... 439

$\begin{array}{llll}\text { Cudweed } & \ldots & \ldots & 266\end{array}$

$\begin{array}{llll}\text { Cullonen } & \ldots & \ldots & 386\end{array}$

$\begin{array}{llll}\text { Cunjevoi } & \ldots & \ldots & 579\end{array}$

Cupid's Flower ... 349

$\begin{array}{llll}\text { Curarine } & \text {... } & \text {... } & 439\end{array}$

$\begin{array}{llll}\text { Curgura } & \ldots & \ldots & 193\end{array}$

Curiy Fern ... ... 641

Curly Pine Palm ... 513

Currant, Frazer Is-

land ... ... 469

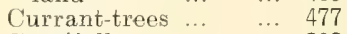

$\begin{array}{llll}\text { Currijello } & \ldots & \text {... } & 202\end{array}$

Currumgul $\quad \ldots \quad \ldots \quad 477$

Custard Apple $\quad \ldots 787$

Cypress Cherry ... 470

Cypress Pine ... ... 510

Daalgaal $\quad \ldots \quad \ldots 510$

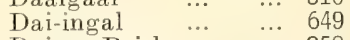

Daisy, Brisbane ... 258

Daisy Burx $\quad \ldots \quad$... 259

Daisy, Downs ... $\quad$... 258

Daisy, English _.. 773

Daisy, Yellow Burr ... 259

Dalby Myall ... ... 159

Dampy-ampy ... $\quad . . .535$

Dandelion, European 277

Dardatra $\quad . .7 \quad \ldots .72$

Darling Pea ... ... 138

$\begin{array}{llll}\text { Darreen } & \text {... } & \text {... } & 159\end{array}$

Darrinjar $\quad \ldots \quad \ldots 215$

Date Palm … 573, 771

Davidsonian Plum $\quad 164,200$

$\begin{array}{llll}\text { Dead-finish } & \ldots & 164,200 \\ \text { Dead-hen } & \ldots & \ldots & 364\end{array}$

Deadmen's-fingers ... 746

Deafness, Cure for ... 237

Deccan Grass ... ... 604

Deep-yellow wood ... 119

Deer's-tongue Fern ... 649

De-hoor $\quad \ldots \quad \ldots 487$

$\begin{array}{llll}\text { Deng-ul-ka } & \ldots & \ldots & 485 \\ & & \ldots & \end{array}$

Denna $\quad . . . \quad \ldots .125$

Desert Poison-bush,

$\begin{array}{lll}\text { Antidote for } \quad \ldots & 128\end{array}$

Devil's Apple ... ... 387

Devil's Thorn ... ... 417

$\begin{array}{lllr}\text { Dewtie } \quad \cdots & \cdots & 62\end{array}$

Dhoo-ee $\quad . . . \quad 579$

Diabetes, supposed

Cure for ... ... 208

Diaphoretic $\quad \ldots \quad$... 419

Diarrhoea, Cure for

Di-eni

Digitalis, Sübstitute

for $\quad \ldots \quad \ldots 220$

$\begin{array}{llll}\text { Dilka } & \ldots & \ldots & 220 \\ & \ldots & \ldots & 163\end{array}$

Dilli $\quad \ldots . \quad \ldots \quad 565$

Dilly-boolen $\quad \ldots \quad$... 88

$\begin{array}{llll}\text { Dim } & \ldots & \ldots & 65\end{array}$

Din-din $\quad \ldots . \quad \ldots 207$

Dirnbur $\quad \ldots . \quad \ldots 616$

D iscolouring of

Bruises, to prevent $17 t$ 
Distaff Thistle, Yel-

low ...

Ditch Millet $\ldots . .6 \% 60$

Diuretic, considered 33

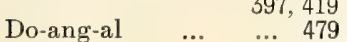

Do-anjin-jin $\quad \ldots \quad \ldots 140$

Docks $\quad \ldots . \quad \ldots \quad 412$

Dodder, to prevent

the growth of $\ldots 350$

Dodders $\quad$... $\quad \ldots .353$

Dodders, Laurel ... 413

Dog Mustard ... ... 36

Dog-weed of America 271

Dogwood ... ... 125

Doolbi $\quad \ldots . \quad \ldots .6124$

Doolin ..... 124

Doomba Oil tree ... 54

Dor-churn ... ... 539

Dorndorli $\quad . . . \quad \ldots .486$

Dortalana ... $\quad . .4 \quad 486$

Doubah ... ... 332

Dowar ... ... 573

Downs Lime ... ... 82

Draiputo _... ... 180

Dre-amberi ... $\quad$... 574

Dropsy, Vegetable ... 786

Dropwort, Water ... 229

Drunken Darnel ... 633

Dry-rot $\quad . .6 \quad \ldots .738$

Duck Orchid ... ... 537

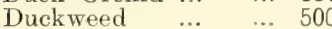

Dugong Grass ... 583

Dugal $\quad \ldots . \quad \ldots .642$

Dugulla ... ... 306

Dundarum ... 510

Dundathu Pine .... 510

Dundul ... ... 159

$\begin{array}{llll}\text { Dun-jo } & \ldots & \ldots & 245\end{array}$

Durandool ... ... 460

Durin 180

Dysentery, Cure for 66. $85,193,508,535$

Eandi

Early Spring G̈rass $\cdots \quad 603$

Ears of Vishnu ... 143

$\begin{array}{llll}\text { Earth-cups } & . . & \ldots & 753\end{array}$

Earth-stars ... ... 751

Earth-tongues … 753

Ebony _... ... 306

Ebony-heart ... $\quad$.. 69

Eczema, Cure for 151, 397

Eeger . ... ... 119

Egaie $\quad . . . \quad \ldots .387$

Eiderberry, Yellow ... 236

Eldin ... ... 88

Elemi-tree, Queensland 86

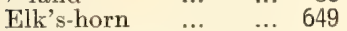

Ellangowan Poisonbush 375

Emetic $\quad \ldots \quad \ldots .148$

Emu, a favourite

food of $\quad$... $\quad \ldots .275$

Emu Apple ... $\quad \ldots .68$

Emu Grass ... ... 136

Elm, Wood resemb. ling English

Endabari 349

English Apple," Wood resembling

English Beech

Page.

English Box

English Elder, Wood

like

96, 235

English Lime ... ... 77

English Sycamore 67,245

Epumer-an ... $\quad$... 387

$\begin{array}{llll}\text { Eramba } & . . & \ldots & 476 \\ & & \end{array}$

$\begin{array}{lllr}\text { Esiangkara } & \ldots & \ldots & 29\end{array}$

Esie $\quad \ldots . \quad \ldots .208$

Esya-ro $\quad$... $\quad$ 208

Eucalyptus, Fungus on Leaves of

Evelyn Teak ....

Evening Primrose $\ldots 2.215$

Everlasting Pea, Fungus on

$\begin{array}{llll}\text { Expectorant, an } & \ldots & 77\end{array}$

Eyes, Cure for Inflam mation of

Eyes, causes Inflam. mation of ...

$\begin{array}{llll}\text { Eye-bright } & \ldots & \ldots & 363\end{array}$

Eye-plant ... ... 358

Eyeyaba

147

Fairy Clubs ... $\quad \ldots 743$

Fat-hen 404

Feather-plant ... $\quad \ldots .588$

Federal weed ... ... 275

Fennel 229

Tennel Pond-weed ... 533

Fenugreek, Sweet ... 136

Fern Fronds ... ... 761

Ferns $\quad . . .6 . . .6635$

Fern-tree, Common ... 640

Eern-tree, Hard-

stemmed ... ... 640

Fida $\quad \ldots . \quad \ldots .542$

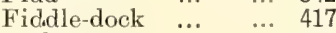

Field Poppy ... ... 32

Fig, Cluster ... ... 487

Fig, Commercial ... 775

Fig, Hill's Weeping 486

Fig, Moreton Bay ... 487

Fig, Rough or Purple 487

Fig-leaves, Fungus on 761

Film Fern ... ... 639

Finger and Toe 849

Finger Lime ... $\quad$... 82

Fire-wheel $\quad . . . \quad \ldots . \quad 454$

Fish-hooks, Native ...

Fitzalan's Ironbark... 194

Five-finger Fern ... 641

Flame-tree 62, 775, 787

Flannel Flower ... 229

Flax, Common ... 69

Flinders Grass $\quad$... 620

Flos Adonis ... ... 17

Fly Orchid ... $\quad \ldots .537$

Forest Mahogany ... 200

Forget-me-not, Australian

Fork-gill Fungus

Formic Acid ... $\quad$... 504

Four-o'clock $\ldots . .396$

Fowls, Fruit harmful

$\begin{array}{crr}\text { to } & & \\ \text { Fox-tail Grass, Swamp } & 611\end{array}$

Frazer Island Apple... 81

Frazer Island Currant 469

Page.

French Beans... 144, 779

French Flax ... ... 69

$\begin{array}{llll}\text { Frogbit } \quad . . & \text {.. } & 518\end{array}$

Fringe Violet ... ... 557

Fringe Water-lily ... 338

Fuchsia, Queensland 381

Fumitory $\quad$... ... 33

Furze-bush $\quad \ldots . \quad \ldots .133$

Gaja $\quad \ldots \quad \ldots 200$

Galactopoietic …4 480

Galbra $\quad \ldots . \quad \ldots .579$

Galls, Fungus on 772,787

Galmarra.

Gambier, Native $\quad \ldots 237$

Gang-ga-gur ... .... 112

Gaygar $\quad \ldots . \quad \ldots \quad 194$

Geebung $\quad \ldots \quad \ldots 4440$

Gellmerra $\quad \ldots . \quad \ldots \quad 430$

Genus, species, variety, and form, re-

garding $\quad \ldots \quad \ldots \quad g$

Georgina Gidgee $\quad \ldots .159$

Germander $\quad \ldots \quad \ldots .392$

Gherkin Orchid $\ldots 326$

$\begin{array}{llll}\text { Ghittoe } & \ldots & \ldots & 81\end{array}$

$\begin{array}{llll}\text { Gidgee } & \ldots & \ldots & 159\end{array}$

Gin-bur $\quad \ldots . \quad \ldots .576$

Gingelly Oil ... $\quad \ldots \quad 368$

Ginger $\quad$... $\quad \ldots \quad 539$

Gir-irjal $\quad \ldots . \quad \ldots .430$

Glass-bead Fungus ... 768

Glasswort f... ... 409

Glow-worm Grass ... 566

$\begin{array}{llll}\text { Gnanjeen } \quad \ldots & \ldots & 21\end{array}$

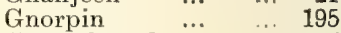

Goat's-beard ... ... 277

Goat's Rue ... ... 137

Goblet Fungus : :... 746

Gogo-vine $\quad \ldots . \quad \ldots .153$

$\begin{array}{lllll}\text { Goitcho } & \ldots & \ldots & 396\end{array}$

Gold and Silver

Flower $\quad \ldots \quad \ldots 236$

Golden-beard Grass ... 620

Golden Candlestick ... 455

Golden Mistletoe ... 469

Golden Wattle, Bris-

bane ... ... ... 837

Gold-spangled Wood 454

Goolanjurie ... $\quad \ldots \quad 566$

Goolaway . ... $\quad \ldots \quad 431$

Goombargne ... $\quad$... 245

Goomurrie ... .... 143

Goona-ngulla $\ldots . . \quad \ldots .56$

Go-onje $\quad \ldots . \quad \ldots \quad 209$

Goorigen _.. $\quad \ldots \quad 634$

Gooroombah $\quad \ldots \quad \ldots . \quad \ldots \quad 419$

Gooseberry Cucumber 221

Goose Grass $\quad \ldots \quad$... 257

$\begin{array}{llll}\text { Gorarba } & \ldots & \ldots & 62\end{array}$

Gotoobah $\quad \ldots \quad \ldots .91$

Gou-unya $\quad \ldots . \quad \ldots 201$

Grain or bread food of prehistoric man 611

Gram, Madras or Horse

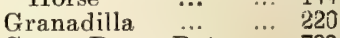

Grape Berry Rot ... 739

Grape Fern ... ... $\$ 3.5$

Grape Vine, Fungusblight of $778,781,783$, 


\begin{tabular}{|c|c|c|}
\hline & & age. \\
\hline Grapes, Native & $\ldots$ & 106 \\
\hline Grass, Fungus & on & \\
\hline & $\ldots$ & 376 \\
\hline Grass-tree $\quad \ldots$ & $\ldots$ & 566 \\
\hline Grass Wrack ... & & 583 \\
\hline $\begin{array}{c}\text { Grease Nut or } \\
\text { gerie }\end{array}$ & $\begin{array}{l}\text { ud- } \\
\ldots\end{array}$ & 439 \\
\hline Green Wattle ... & $\ldots$ & 163 \\
\hline Greyanger $\quad \ldots$ & $\ldots$ & 230 \\
\hline Grey Gum & $\cdots$ & 200 \\
\hline Gromwell $\quad \ldots$ & $\ldots$ & 840 \\
\hline Ground Cherry & $\ldots$ & 249 \\
\hline Ground Palm ... & $\ldots$ & 574 \\
\hline Groundsel, Europ & an & 276 \\
\hline Groundsel-tree & $\ldots$ & 262 \\
\hline Guatemala Grass & $\ldots$ & bio \\
\hline Guava $\quad$... & ... & \\
\hline Gueeah & $\cdots$ & 840 \\
\hline Guiaka $\quad$... & ... & 548 \\
\hline Guinea Grass ... & $\ldots$ & 010 \\
\hline Gulf Grass, Red & $\cdots$ & 20 \\
\hline Gulgong .... & $\ldots$ & $1 \mathrm{~s} b$ \\
\hline Gurr Arabic Su & sti- & \\
\hline tute for $\ldots$ & ... & $4 b s$ \\
\hline Gum-trees i & $\cdots$ & 105 \\
\hline Gum-topped Box & $\ldots$ & 194 \\
\hline Gumin Gumin & ... & \\
\hline Gundey Bluey & $\ldots$ & 158 \\
\hline Gundur-Gundur & ... & 473 \\
\hline Gunpowder-plant & $\ldots$ & 505 \\
\hline Gunthamarrah & $\ldots$ & 209 \\
\hline Guttapercha-tree & ... & \\
\hline Gunyoo $\quad \cdots$ & $\cdots$ & 518 \\
\hline Gyaki & $\cdots$ & \\
\hline Gymple & ... & \\
\hline
\end{tabular}

Hairy Vetch ... ... 140

Hair-trigger or Spring-

$\begin{array}{lll}\text { back plant } \ldots & \ldots & 277 \\ \text { Hand of Mary } & \ldots & 386\end{array}$

$\begin{array}{lr}\text { Hand of Mary } \quad \ldots . & 386 \\ \text { Hare's-ear } & 33,229\end{array}$

Hare's-ear Treacle-

mustard ... ... 33

Hare's-foot Fern ... 640

Hare's-tail Grass $\ldots .632$

Harpee $\quad$... $\quad . . .543$

Hat Grass $\quad \ldots .6 .622$

Hatpin Grass $\quad \ldots 561$

Hatpin-plant ... ... 584

Hatpins, Seeds used

$\begin{array}{cccc}\text { in making ... } & \ldots & 128 \\ \text { Hawk's-beard } & & \ldots & 276\end{array}$

$\begin{array}{lll}\text { Hawk's-beard } & \ldots & 276 \\ \text { Hawk Weed ... } & \ldots & 276\end{array}$

Hawthorns ... ... 167

Hawthorn, Indian ... 167

Hay Weed ... ... 271

Heart Pea ... ... 112

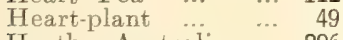

Heaths, Australian ... 296

Hedge Acacia _.. 157

Hedge Mustard ... 33

Hedge Nettle _.. 388

Hemp Agrimony ... 258

Herbert River Cherry 477

Herpes, Cure for ... 148

Henbit Nettle... $\quad$... 388

Hexham Scent ... 133

Hickory, Cairns ... 91

Hickory Wattle ... 163

Hogweed ... 396, 412

Hollyhock ... ... 773

Holly, Wood resemb-

ling English
Page.

Honey, supposed to

Poison ... ... 175

Honeysuckle $\ldots . \quad \ldots 236$

Honeysuckle, Austra-

$\begin{array}{cccc}\text { lian } & \ldots & \ldots & 455 \\ \text { Hood Bindweed } & \ldots & 349\end{array}$

Hood Orchid ... ... 537

Hoof Fungus ... ... 733

Hoop Pine ..... .510

Hop-bushes ... ... 118

Horehound, Austra-

lian … ... 388

Horehound, White ... 388

Hornwort ... ... 508

Horsehair Fungus ... 723

Horse Mushroom ... 720

Horse Radish, Blight on $\quad \ldots \quad \ldots 785$

Horses, causes B Blindness in Vermifuge

Horses,
for

Hound's-tongue ... 346

Hyacinth of Tasmania

537

Indian Almond $\quad \ldots \quad 180$

$\begin{array}{llr}\text { Indian Hawthorn } & \ldots & 167 \\ \text { Indian Tulip-tree } & & 61\end{array}$

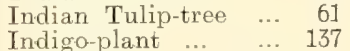

Indigo-plant, ' West Indian

Inflammation of the Eyes, Cure for $\quad$... 276

Ink-plant, Red ... 411

Tnkstains, to remove 100

Insect Stings, Cure for 346

Insects, Fungus on ... 782

Investigator's Tree... 400

Tnecacuanha. White 253

Ironbark, Black ... 194

Ironbark, Grey .... 194

Ironbark, Lemon-

scented $\quad$... $\quad$... 194

Ironbark, Narrow-

leaved $\quad$... ... 194

Ironbark, "Silver-

leaved ... $\quad$... 194

Iron-gum tree ... $\quad \ldots . .6195$

Ironwood ... ... 153

Ironwood or Pine

$\begin{array}{llrr}\text { Acacia } & \text {... } & \text {... } & 157\end{array}$

Tronwood, Scrub ... 207

Tronwood Wattle $\quad . . .159$

$\begin{array}{llll}\text { Ithnee } & \ldots & \ldots & 58\end{array}$

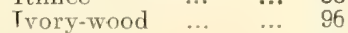

I-wa-wal $\quad \ldots .6163$

Taboo'um

574

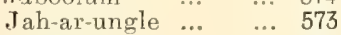

Jalcan Jalcan ... 505

Jaln-ba $\quad$... $\quad$... 119

Jambolla $\quad \ldots . \quad \ldots 55$

$\begin{array}{llll}\text { Je-jo } & \ldots & \ldots & 477\end{array}$

Jequerity $\quad \ldots .6140$

Jerusalem Thorn ... 151

Jerusalem Oak ... 404

$\begin{array}{llll}\text { Jiddo } & \text {... } & \text {.... } & 542\end{array}$

Jimmie-iimmie $\quad . . .88$

Jimmy Low ... $\quad \ldots .200$
Page.

Jinbul $\quad \ldots . \quad \ldots \quad 195$

Jingull $\quad$... $\quad \ldots .106$

Jinjalga $\quad \ldots \quad \ldots \quad 180$

$\begin{array}{llll}\text { Jinjil } & \ldots & \ldots & 200\end{array}$

Job's-tears $\quad$... $\quad \ldots .616$

Johnson Grass $\quad$ 620, 773

Johnstone River Al-

$\begin{array}{llll}\text { mond } & \text {... } & 6 & 69\end{array}$

Johnstone $\quad \cdots$ River

Hardwood ... ... 202

Johnstone River 153

Teak _... ... 153

Jointed Charlock ... 36

Jool-lun $\quad$... $\quad$... 543

$\begin{array}{llll}\text { Joonda } & \ldots & \ldots & 167\end{array}$

Jo-ora $\quad \ldots . \quad \ldots \quad 193$

Jow-war $\quad \ldots .4646$

Jujube $\quad$... $\quad$... 100

$\begin{array}{llll}\text { Ju-kee } & \ldots & \ldots & 576\end{array}$

Julkin $\quad$... $\quad \ldots .518$

Jun-jun $\quad$... $\quad$... 539

Kabir $\quad$... $\quad$... 455

$\begin{array}{llll}\text { Kai-go } & \ldots & \ldots & 208 \\ & & \ldots & \end{array}$

Kai-inuma $\quad \ldots . \quad \ldots .485$

Kalaara $\quad \ldots . \quad \ldots 207$

Kalan $\quad \ldots \quad \ldots 662$

Kal-boo-roon-ga $\quad$... 349

Kalburadji ... ... 207

Kaldi $\quad$... $\quad \ldots .640$

Kalkur $\quad \ldots . \quad \ldots .548$

Kal-lar $\quad \ldots . \quad \ldots .6147$

Kamdoothal ... $\quad \ldots \quad 40$

$\begin{array}{llll}\text { Kame } & \ldots & \ldots & \ldots \\ & \ldots & 66\end{array}$

Kamela-tree $\quad . . \quad$... 479

Kammama $\quad \ldots \quad \ldots 513$

Kam-mi : $\quad \ldots \quad \ldots 576$

Kana-wara $\quad \ldots .3 . \quad \ldots 306$

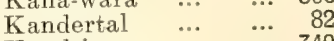

$\begin{array}{llll}\text { Kand-ja } & \ldots & \ldots & 349\end{array}$

Kangabberoo .... ... 91

Kangaroo Grass ... 620

$\begin{array}{llll}\text { Kangga } & \text {... } & \text {... } & 112\end{array}$

\begin{tabular}{lllr} 
Kaooroo & $\ldots$ & $\cdots$ & 32 \\
\hline
\end{tabular}

Kapabina $\quad \cdots \quad \cdots 42,61$

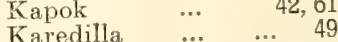

Karey $\quad \ldots . \quad \ldots .317$

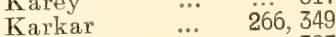

Kar-kar $\quad$... $\quad \ldots \quad 387$

Kar-kor $\quad \ldots .3 . \quad \ldots 349$

Karoo $\quad \ldots .6209$

$\begin{array}{llll}\text { Kar-pe } & \text {... } & \text {... } & 504\end{array}$

Karro $\quad \ldots \quad \ldots 548$

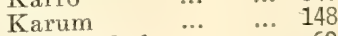

Katakarkal ... $\quad \ldots .69$

Ka-too-ra $\quad$... $\quad$... 628

Kauri Pine ... ... 510

Kavor-kavor ... ... 245

$\begin{array}{lllr}\text { Kaya } & \ldots & \ldots & 591 \\ & & \end{array}$

\begin{tabular}{llll} 
Kedgy-kedgy & $\ldots$ & $\ldots$ & $\ldots$ \\
\hline
\end{tabular}

$\begin{array}{llll}\text { Keena } & \ldots & \ldots & 54 \\ \text { Kel-lan } & \ldots & \ldots & 62\end{array}$

Kennedy's Heath ... 186

Kerdolo $\quad$... $\quad$... 144

Ke-ril $\quad \ldots \quad \ldots 487$

Kerosene-tree _.. 81

$\begin{array}{llll}\text { Kery } & \ldots & \ldots & 43\end{array}$

$\begin{array}{llll}\text { Ketey } & \ldots & \ldots & 62\end{array}$

Ki-bano $\quad \ldots . \quad \ldots \quad 573$

Kidney-wallum $\quad$... 510

Ki-gera $\quad \ldots \quad \ldots \quad 513$ 


\begin{tabular}{|c|c|c|c|}
\hline & & & $\mathrm{g}$ \\
\hline i-gunno & & $\ldots$ & 510 \\
\hline & & $\ldots$ & \\
\hline imalo & & $\ldots$ & 513 \\
\hline inginga-kil & amul & & \\
\hline not Grass & $\ldots$ & $\ldots$ & \\
\hline ie-yan & $\ldots$ & $\ldots$ & 386 \\
\hline & $\ldots$ & $\ldots$ & 430 \\
\hline & $\ldots$ & $\ldots$ & \\
\hline & $\ldots$ & $\ldots$ & \\
\hline o-ya & $\ldots$ & $\ldots$ & \\
\hline & $\ldots$ & $\ldots$ & \\
\hline & $\ldots$ & $\ldots$ & \\
\hline$-\mathrm{mol}$ & $\cdots$ & $\ldots$ & 543 \\
\hline a-ru & $\ldots$ & $\ldots$ & \\
\hline & ... & $\ldots$ & \\
\hline -an & $\ldots$ & $\ldots$ & \\
\hline gangn & $\ldots$ & $\ldots$ & \\
\hline yara & & $\ldots$ & \\
\hline oobin-karra & & $\ldots$ & 573 \\
\hline o & & $\ldots$ & \\
\hline mon $W$ & ood & $\ldots$ & 237 \\
\hline o-loomo & & $\ldots$ & \\
\hline barra & & $\ldots$ & 4. \\
\hline Loom-be & & $\ldots$ & 579 \\
\hline Koomeroo K & Coom & 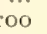 & \\
\hline ndeeba & $\ldots$ & $\ldots$ & \\
\hline & $\ldots$ & $\ldots$ & \\
\hline -je-rung & & $\cdots$ & \\
\hline on & & $\ldots$ & \\
\hline & $\ldots$ & $\ldots$ & \\
\hline arrie & $\cdots$ & $\cdots$ & \\
\hline & $\ldots$ & $\ldots$ & \\
\hline ka-bidga & & $\ldots$ & 2 \\
\hline & $\ldots$ & $\ldots$ & 31 \\
\hline o-rat & $\ldots$ & $\ldots$ & 431 \\
\hline th & $\ldots$ & $\ldots$ & \\
\hline gara & $\ldots$ & & 573 \\
\hline & $\ldots$ 。 & 40 & 148 \\
\hline & $\ldots$ & $\ldots$ & 287 \\
\hline & ... & $\ldots$ & \\
\hline & $\ldots$ & $\ldots$ & 147 \\
\hline $\operatorname{lng}$ & $\ldots$ & $\ldots$ & \\
\hline & $\ldots$ & $\ldots$ & \\
\hline kull & $\ldots$ & ... & $16 ?$ \\
\hline & $\ldots$ & $\ldots$ & \\
\hline & $\ldots$ & $\ldots$ & \\
\hline & $\cdots$ & $\ldots$ & \\
\hline & $\ldots$ & $\ldots$ & \\
\hline gal & $\ldots$ & $\ldots$ & 1 \\
\hline & ... & $\ldots$ & \\
\hline & $\ldots$ & $\ldots$ & \\
\hline rtcho & $\ldots$ & $\ldots$ & \\
\hline iji & $\ldots$ & $\ldots$ & 5 \\
\hline noo-roo & & & 387 \\
\hline uat, A & ustre & $\operatorname{ian}$ & 8 \\
\hline anga & $\ldots$ & $\ldots$ & 548 \\
\hline erberry & $\ldots$ & $\ldots$ & \\
\hline & $\ldots$ & $\ldots$ & \\
\hline & ... & … & \\
\hline & $\ldots$ & $\ldots$ & \\
\hline & $\begin{array}{l}\cdots \\
\cdots\end{array}$ & & \\
\hline & $\cdots$ & ... & \\
\hline ijong & $\ldots$ & $\ldots$ & \\
\hline jong, & Brown & & \\
\hline oombah & ... & $\cdots$ & 410 \\
\hline & $\ldots$ & $\ldots$ & \\
\hline & & & \\
\hline & $\cdots$ & & 50 \\
\hline oree & ... & $\ldots$ & \\
\hline
\end{tabular}

Laburnum, Sea-coast 148 Lady Fern ... ... 645
Page.

Lady's Slipper plant 360 Lalang Grass ... ... 616 Lamb's-tails $\quad . . \quad \ldots 411$ Lancewood … 162, 163 Landsborough Grass 620 Langcion's Hardwood 202 Lantern Flower ... 57 $\begin{array}{llll}\text { Lar } & \text {... } & \text {... } & 159\end{array}$ $\begin{array}{llll}\text { Larchanama } & \ldots & \ldots & 175\end{array}$ Laurel Dodders $\quad \ldots .431$ Lawyer Cane ... ... 574 Lawyer Cane, Fungus on $758,763,778$ Laxative $\quad \ldots \quad \ldots 33$

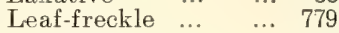
Leek, Native ... ... 557 Leichhardt's Box ... 194 Leichhardt-tree _.. 237

Lemon Grass ... . ... 619 Lemon-scented Ironbark

Lemon-trees, Fungi on 775 , $781,785,789$

Lenn $781,785,789$

Leopard-tree 91

Lettuce, Prickly . ... 277

Lichens, Fungus on 682, 787

Lignum $\quad \ldots \quad 381,417$

Lignum-vitæ $\quad \ldots \quad \ldots \quad 386$

Lilac of South Australia

Lillypilly

Lime, Finger

Lime, Mount White 82

Lime, Wood resembling English

Little Hood

Logan Apple ... $\quad \ldots . \quad 81$

London Rocket .. 33

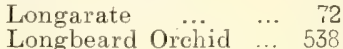

$\begin{array}{lll}\text { Longbeard Orchid } & \ldots & 538 \\ & & \end{array}$

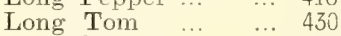

Longullah

Looking-glass tree ... 65

$\begin{array}{llll}\text { Loosestrife } & \text {... } & \text {... } & 214\end{array}$

$\begin{array}{llll}\text { Loquat } & \ldots & \ldots & 167\end{array}$

$\begin{array}{llll}\text { Lorwara } & \ldots & \ldots & 112\end{array}$

$\begin{array}{llll}\text { Lo-thi } & \ldots & \ldots & 611\end{array}$

Love Apple ... ... 353

Love Grasses …

$\begin{array}{lll}\text { Love-lies-bleeding } \quad \ldots . & 397 \\ \text { Lucerne } & & \end{array}$

Lucerne Lucerne Blight 756, 763, 772

Lucerne Pest, a … 353

$\begin{array}{llll}\text { Lu-luka } & \ldots & \ldots & 249\end{array}$

Luminous Fungus, a 727

Luminous Fungus,

Large

Luminous Fungus Small

Luya's Hardwood ... 202

Maapa

539

Mabil

591

Macassar Kernels ... 85

Mackie's Pest... ... 620

Mad Apple ... ... 358

Madras Gram ... 144
Madwort

Mahogany, Tom Rus-

sell's $\quad$... ... 202

Mai $\quad \ldots . \quad \dddot{148}, 479$

Maidenhair, Climbing 638

Maidenhair Fern ... 641

Maidenhair, Five-

fingered ... ... 641

Maiden's-blush wood 67 ,

615, 7119

Maize, Fungus on 615, 773 ,

Magura $\quad \ldots \quad \ldots 487$

Mahogany, Swamp... 201

$\begin{array}{llll}\text { Makila } & \text {... } & \text {.. } & 167\end{array}$

Makora $\quad$... $\quad$... 591

Malacca Bean ... 387

Malaga $\quad$... $\quad \ldots .144$

Malkan $\quad \ldots \quad \ldots . \quad 144$

Mallban $\quad . . \quad \ldots 485$

Mallee, Red or White 193

Mallets, Wood useful

$\begin{array}{llll}\text { for } & \ldots & \text {... } & 169\end{array}$

Mallow $\quad \ldots . \quad \ldots \quad 54$

Mamboo $\quad$... $\quad \ldots .125$

$\begin{array}{llll}\text { Man-djar } & \ldots & \ldots & 61\end{array}$

Mangaru . ... ... 591

Mango $\quad$... 119, 781

Mangosteen $\ldots, \quad \ldots \quad 54$

Mangrove, Apple ... 175

Mangrove, Black ... 175

Mangrove, Grey ... 175

Mangrove, Milky ... 480

Mangrove, Red or 175

Orange … ‥ 175

Mangrove, River ... 305

Mangrove, White ... 387

$\begin{array}{llll}\text { Man-guru } & \ldots & \ldots & 25 \\ \text { Manin } & \ldots & \ldots & 88\end{array}$

$\begin{array}{llll}\text { Manin } & \ldots & \ldots & 88 \\ \text { Manni } & \ldots & \ldots & 40\end{array}$

Maple, Cardwe.l $\ldots .91$

Mara, cardwe.. $\quad \ldots .6147$

$\begin{array}{llll}\text { Mara } & \ldots & \ldots & 147 \\ \text { Ma-ra } & \ldots & \ldots & 513\end{array}$

Marada $\quad \ldots . \quad \ldots 300$

Maraguigi $\quad \ldots . \quad \ldots .6113$

$\begin{array}{llll}\text { Marara } & \ldots & \ldots & 169\end{array}$

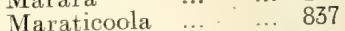

Mares, Injurious to 835

Marking-nut tres ... 119

Mar-ko ... … 245

Maroochie Nut ... 440

Marragiddie ... ... 431

Marsh Watercress ... 33

$\begin{array}{llll}\text { Marum } & \ldots & \ldots & 187\end{array}$

Marvel of Peru ... 396

Matchbox Bean ... 153

Маu-u $\ldots . . \ldots 565$

Mbau-nu $\quad \ldots . \quad \ldots \quad 112$

McKenzie Bean ... 144

Meadow Rice Grass... 620

Measuring Chain, a substitute for ... 574

Medick Burr ... ... 133

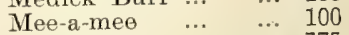

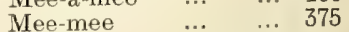

Me-la-ba $\quad \ldots . \quad \ldots .480$

Melilot $\quad \ldots . \quad \ldots .133$

Melilot Fungus, scented like ... 738

Mel-joor-ang ... $\quad \ldots .175$

Melon, Water ... 221

$\begin{array}{lllr}\text { Me-mama } & \ldots & \ldots & 49\end{array}$

$\begin{array}{llll}\text { Merangara } & \ldots & \ldots & 25\end{array}$ 
Page.

Merikin . ... ... 241

Merrany $\quad$... $\quad$... 169

Merrin $\quad \ldots \quad \ldots 576$

Messmate ... 194, 195

Meston's Mangosteen 54

Metrorrhagia, Rem-

$\begin{array}{llll}\text { edy for } & \text {... } & \text {... } & 258\end{array}$

Mi

Midgen

... 573

Miera $\quad \ldots .61 \%$

Mijah $\quad \ldots \quad \ldots .40$

Mildew, Apple ... 765

Mildew, Grape-vine... 782

Mildew, Powdery, on

Grape-vine

Mildew, Powdery, on

Lagerstromia

Mildew, Powdery, on

$$
\text { Wheat }
$$

Milkbush

Milk Taints ... 34,411

Milkwort … 43

Milky Mangrove ... 480

Millai-millai ... $\quad$... 459

$\begin{array}{llll}\text { Millgar } & \ldots & \ldots & 325\end{array}$

Mint

Mintie

Mistletoe

Mitchell Grass

Mock Orange

Mo-i

Moiary

Moi-kin

Moinjal

Moi-yur

Moko-murdur

Mokor-ja

Mollukan

Molucea Balm

Mondo

Mondoleir $\quad \ldots . \quad \ldots .440$

Mongo $\quad$... $\quad . .305$

Mon-jim $\quad \ldots \quad \ldots \quad 163$

Mooda $\quad \ldots \quad \ldots .387$

Mooi-goo-doo .... 574

Moo-jee $\quad \ldots \quad \ldots \quad 180$

$\begin{array}{llll}\text { Moolar } & \text {... } & 195\end{array}$

Mooleeah $\quad$... $\quad . . .486$

Moo-nah $\quad \ldots .6 .518$

Moonah $\quad$... ... 188

Moonflower ... ... 349

$\begin{array}{llll}\text { Moonya } & \ldots & \ldots & 317\end{array}$

Moorool ... ... 208

$\begin{array}{llll}\text { Moorum } & \ldots & \ldots & \ldots\end{array}$

Moranggal ... $\quad \ldots \quad 318$

Mor-bir $\quad . . . \quad \ldots .112$

Moregi $\quad \ldots . . . . .5542$

Moreton Bay Ash ... 200

Moreton Bay Chest-

nut or Bean-tree ... 148

Moreton Bay Fig ... 487

Moreton Bay Pine ... 510

Morgogaba $\quad . . \quad \ldots .542$

$\begin{array}{llll}\text { Morna } & \ldots & \ldots & \ldots\end{array}$

$\begin{array}{llll}\text { Mor-ngi } & \ldots & \ldots & \ldots \\ & \text {... } & 188\end{array}$

Morning-glory _.. 347

$\begin{array}{llll}\text { Mornimi-an } & \text {... } & \ldots & 144\end{array}$

Mosses $\quad . .6 \quad \ldots 656$

Moth Mullein _.. 358

Moti $\quad \ldots \quad \ldots .574$

Mountain Bracken ... 640

Mountain Couch ... 624
Page.

Mourning Flowers oî the Northern Aborigines

143

Mouse Barley .... 634

Mouse-ear Chickweed 48

Mouse-tail ... $\quad . . . \quad 17$

Moving Plant ... 140

Mowbulan White-

$\begin{array}{llll}\text { wood } & \ldots & \ldots & 236\end{array}$

$\begin{array}{llll}\text { Moweii } & \ldots & \ldots & 151\end{array}$

$\begin{array}{llll}\text { Mucki } & \ldots & \ldots & 337\end{array}$

Mudwort. Australian 361

$\begin{array}{llll}\text { Mugurpul } \quad \ldots & \text {.. } & 88\end{array}$

$\begin{array}{llll}\text { Mulga } & \ldots & \ldots & 162\end{array}$

Mulga Grass ... ... 616

$\begin{array}{llll}\text { Mullar } & \text {.. } & \ldots & 164\end{array}$

$\begin{array}{llll}\text { Mullet } & \text {... } & \text {... } & 194\end{array}$

Milpup 40

Mumble $\quad$... $\quad$... 43

Mumps, Remedy for 504

Mungal _.. ... 195

Muntenpin _.. .. 477

Mun-tyal

$\begin{array}{llll}\text { Murdarka } & \ldots & \ldots & 305\end{array}$

Mur-gan $\quad \ldots \quad 579$

Murgon $\quad \ldots \quad \ldots \quad 116$

$\begin{array}{llll}\text { Murite } & \ldots & \ldots & 338\end{array}$

Murl-kue-kee ... ... 203

$\begin{array}{llll}\text { Murpe } & \ldots & \ldots & 317\end{array}$

Murray Down ... 576

Mushroom, Common 720

Mushroom, Horse ... 720

Mushroom, Oystex ... 717

Muskwood ... ... 236

Mustard, Black ... 34

Mustard, Hedge 33

Mustard, Treacle … 33

$\begin{array}{llll}\text { Myopia } & \text {... ... } 207\end{array}$

Mysore Thorn _.. 151

Na-bun-yu-ban _.. 124

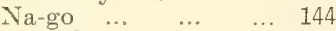

Na-gobar $\quad \ldots \quad \ldots \quad 153$

$\begin{array}{llll}\text { Nai-ya } & \ldots & \ldots & 112\end{array}$

Naja

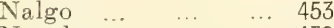

Nanchee $\quad$... ... 439

Nankeen Dye Wood... 81

$\begin{array}{llll}\text { Nannan } & \text {... } & \text {... } & 485 \\ & & \end{array}$

$\begin{array}{llll}\text { Nappala } & \text {... } & \text {... } & 478\end{array}$

Napum-napum _.. 148

$\begin{array}{llll}\text { Nardoo } & \ldots & \ldots & 635\end{array}$

$\begin{array}{llll}\text { Nar-gul } & \ldots & \ldots & 147\end{array}$

Narm-boon-bong ... 200

Narran $\quad$... ... 158

Narroo $\quad$... $\quad \ldots .91$

Native Fruits, Iegarding our ... 13

Native Fuchsia $\quad$... 381

Native Gambier ... 237

Native Grass used as a toy

Native Lime

Native Names

Native Orange

Native Parsnip _... 228

Native Peach of S.A. 469

Native Pear ... ... 209

Native Rosella ... 58

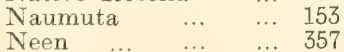

$\begin{array}{llll}\text { Neram } & \ldots & \ldots & 167\end{array}$
Page.

Net Fungus ... ... 746

Netted Stinkhorn

Fungus $\ldots . \quad \ldots 745$

Nettle, English ... 504

Nettle, Twining ... 480

Nettle-tree ... ... 505

New Zealand Spinach 227

Ngalki ... $\quad$... $\quad$... 386

$\begin{array}{llll}\text { Ngam-boi } & \ldots & \ldots & 386\end{array}$

Ngang-ir $\quad$... $\quad$... 566

$\begin{array}{lllr}\text { Ngar-golly } \quad \ldots & \ldots & 58\end{array}$

Ngar-pul $\quad$... ... 645

$\begin{array}{llll}\text { Ngarwowoya } & . . & \ldots & \ldots\end{array}$

$\begin{array}{llll}\text { Ngean-jerry } & \ldots & \ldots & 138\end{array}$

$\begin{array}{llll}\text { Ngo-go-ro } & \ldots & \ldots & 180\end{array}$

$\begin{array}{llll}\text { Ngobi } & \ldots & \ldots & 77\end{array}$

$\begin{array}{llll}\text { Ngorbai } & \text {... } & \text {... } & 305\end{array}$

$\begin{array}{llll}\text { Ngorkuru } & \ldots & \ldots & 180\end{array}$

$\begin{array}{llll}\text { Ngoru } & \ldots & \ldots & \ldots \\ & \ldots & \ldots & 306\end{array}$

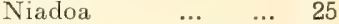

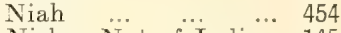

Nicker Nut of India 145

Nightshade $\quad \ldots . \quad \ldots \quad 353$

$\begin{array}{llll}\text { Nijar } \quad \ldots & \ldots & \ldots & \ldots 13\end{array}$

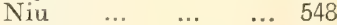

$\begin{array}{llll}\text { Nonda-tree } & \ldots & \ldots & 167\end{array}$

Noogoora Burr ... 271

Noogoora Burr, Fun-

$\begin{array}{llll}\text { gus on } & \ldots & \ldots & 763\end{array}$

Noomaic $\quad \ldots . \quad \ldots \quad 487$

$\begin{array}{llll}\text { Noongi } & \text {... } & \text {... } & 124\end{array}$

Nor-ro $\quad$... $\quad \ldots .220$

Noyau Seeds ... $\quad . . .347$

Nun-flower ... ... 537

Nurrum-nurrum _.. 116

Nut Grass _.. 589, 773

Nutmeg, Queensland 419

Oak, Stringy or

Thready Bark .... 508

Oak, Forest ... ... 508

Oak, Swamp ... ... 508

Oaks, Shea ... ... 505

Oars, Wood useful for 66

Oat Grass, Tall ... 620

Oat Fungus ... ... 773

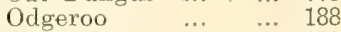

$\begin{array}{llll}\text { Ogarrah } & \ldots & \ldots & 159\end{array}$

Ointment for Sores ... 85

Old-maid Flower ... 318

Old-man Saltbush ... 408

Old-man's Beard ... 685

Oleander, Fungus on 778

Olive, Native... ... 311

Olm-bah $\quad \ldots \quad \ldots 201$

Onion, Native ... 557

Onion, Orchid ... 525

Onion-tree $\quad$... $\quad \ldots \quad 88$

Onogona $\quad \ldots . \quad \ldots 263$

On-tho $\quad \ldots . \quad \ldots \quad 542$

Oo-kin ... $\quad \ldots . \quad \ldots 610$

Oolgar $\quad \ldots . \quad \ldots \quad 477$

Oolpanje $\quad \ldots . \quad \ldots \quad 237$

Ool-peen $\quad \ldots . \quad \ldots .542$

Oombar $\quad \ldots . \quad \ldots .194$

Oon-doo $\quad \ldots . \quad \ldots .154$

Oon-doroo ... ... 354

Oon-gaary $\quad \ldots \quad \ldots .545$

Ooray ... ... ... 169

Opium, substitute for 277

Orange, Fungus on... 779 


\begin{tabular}{|c|c|c|c|}
\hline & & & age. \\
\hline Orange Man & grove & $\ldots$ & 175 \\
\hline Orange, Nat & ive & ... & 82 \\
\hline Ori $\quad \ldots$ & $\ldots$ & $\cdots$ & 200 \\
\hline Oringorin & $\ldots$ & $\cdots$ & 469 \\
\hline Ouraie & $\ldots$ & $\ldots$ & 66 \\
\hline Ox-tail & $\ldots$ & $\ldots$ & 271 \\
\hline Ox-tongue & $\ldots$ & $\ldots$ & 276 \\
\hline Oyster Green & $\ldots$ & $\ldots$ & 794 \\
\hline Oyster Mush & room & $\ldots$ & 717 \\
\hline Oyster Pest, & an & $\cdots$ & 821 \\
\hline & & - & \\
\hline Paiamara & & $\ldots$ & 317 \\
\hline Pain-ki & $\ldots$ & $\ldots$ & 565 \\
\hline Pal-la-ga & & $\ldots$ & 430 \\
\hline $\begin{array}{l}\text { Palm, } \\
\text { tree }\end{array}$ & Cabba & ge- & 765 \\
\hline Palm, Walki & ing-stic & & 573 \\
\hline Palms, Fur & agus & on & 776 \\
\hline Palmyra Pal & $\mathrm{m}$ & & 574 \\
\hline Pam-mo & $\ldots$ & $\ldots$ & 66 \\
\hline Panar & & $\ldots$ & 579 \\
\hline Pandara & $\ldots$ & $\ldots$ & 504 \\
\hline Pand-ja & $\ldots$ & $\ldots$ & 147 \\
\hline Panga-panga & $\ldots$ & ... & 167 \\
\hline Panicum & $\ldots$ & $\cdots$ & 611 \\
\hline Panje-a & $\therefore$ & $\ldots$ & 591 \\
\hline Papajarin & $\ldots$ & & 241 \\
\hline Papaw & & 220 & 786 \\
\hline Para Rubbe & er, I & eaf & \\
\hline
\end{tabular}

Disease of ... $\quad$... 781

Parasol Fern ... … 658

Parasol Mushroom 741, 715

Pareira Brava plant,

Spurious ... $\quad \ldots . \quad 29$

$\begin{array}{llll}\text { Parpa } & \ldots & \ldots & 504\end{array}$

\begin{tabular}{llll} 
Parpangata & $\ldots$ & $\ldots$ & 153 \\
\hline & & $\ldots$ & 387
\end{tabular}

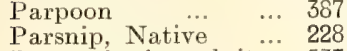

Parson-in-the-pulpit ${ }^{\cdots} \quad 537$

Passion-fruit ... ... 220

Peach-leaf Curl $\quad$... 756

Peach-leaf Poison-

$\begin{array}{ccc}\text { bush } & \text {... 485, } 766\end{array}$

Peach-leaves, Fungus

$\begin{array}{llll}\text { on } \quad . . & \text {... } & 756,771\end{array}$

Peach, Native $\quad . . .469$

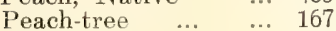

Pe-ang-gul ... $\quad \ldots .548$

$\begin{array}{llll}\text { Pea-bush } & \ldots & \ldots & 138\end{array}$

Pea-nut $\quad . . . \quad 139,782$

Pear-fruit, Fungus on 781

Pear, Native ... 209

Pear, Wooden ... 440

Pearlwort ... ... 48

Pee-been ... ... 202

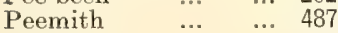

Pegunny $\quad \ldots . \quad \ldots .153$

Peirir $\quad \ldots .6262$

Pellitory $\quad \ldots . \quad \ldots .505$

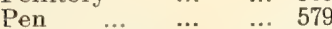

Pencil Cedar ... $\quad 86,305$

Pencil Orchid … ... 526

Penda $\quad$... $\quad$... 202

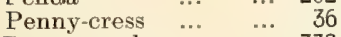

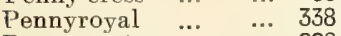

Pennywort ... $\quad . . .228$

Pennywort, Indian ... 228

Peppers $\quad . . . \quad$.... 418

Pepper-vine $\quad \ldots \quad \quad 112,418$

Pepperwort $\quad \ldots \quad$... 34
Page.

$\begin{array}{llll}\text { Physic Nut } & \text {... } & \text {... } & 478\end{array}$

Physic Nut, Cotton-

leaved

Piccabeen Palm ... 573

$\begin{array}{lll}\text { Pickerel-weed } & \ldots & 559\end{array}$

$\begin{array}{llll}\text { Pie-melon } & . . & \ldots & 221\end{array}$

Pigeon-berry A Ash ... 311

Pigeon Grass ... ... 611

Pig's-face $\quad \ldots . \quad \ldots .227$

Pigs, supposed poisonous to $\quad \ldots \quad$... 430

$\begin{array}{llll}\text { Pigweed } & \ldots & \ldots & 48\end{array}$

$\begin{array}{llll}\text { Pikki } & \ldots & \ldots & 573\end{array}$

Pim-nar $\quad \ldots \quad \ldots 576$

Pimpernel ... ... 300

Pine-apple, Native ... 513

Pine, Acacia ... ... 157

Pine, Bunya ... ... 513

Pine, Cypress ... 510

Pine, Dundathu ... 510

Pine, Moreton Bay or Hoop $\quad \ldots \quad$... 510

Pine, Mt. Spurgeon Black Kauri … 510

Pine, Queensland $\begin{array}{cccc}\text { Kauri } & \ldots & \ldots & 510 \\ & & \ldots & \end{array}$

$\begin{array}{llll}\text { Pine, She } & \ldots & \ldots & 510 \\ \text { Pine, Stringybark } & . . & 510\end{array}$

Pink ... ... $\quad$... 44

$\begin{array}{llll}\text { Pinkheart } & \ldots & \ldots & 77\end{array}$

Pink Water-lily ... 32

Pink Nose, Reputed Cause of

Pinkoen Oil

$\begin{array}{llll}\text { Pipewort } & \ldots & \ldots & 478 \\ & & & \end{array}$

Pitcher-bearing plant 332

Pitcher-plants $\quad$... 417

Planes, Wood useful for

Plantain

169

... 396

Poison-berry ... $\quad \ldots . .358$

Poison-bush ... ... 128

Poison-tree ... $\quad . . .153$

Poison-tree, River ... 480

Pomegranate ... ... 215

Pomegranate, Native 40

Pomera ... 455

Pond-weed $\quad \ldots .6135$

Pondir-pondir $\quad$... 140

Poodgee-podgee $\quad$... 479

Pool-boo-nong .... 207

Pooma ... $\quad . . . \quad \ldots .32$

Poona Oil $\quad . . . \quad \ldots .148$

Poona Spar tree ... 54

$\begin{array}{llll}\text { Pooragri } & \ldots & \ldots & 510\end{array}$

$\begin{array}{llll}\text { Poorga } & \ldots & \ldots & 18 \\ \text { Poplar Box } & \ldots & \ldots & 194\end{array}$

Poplar Box …

leaved $\quad$... $\quad \ldots .195$

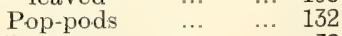

Poppy $\quad \ldots . \quad \ldots . \quad 32$

Poppy, Prickly $\quad$... 53

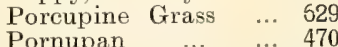

Potato, Diseases of... 769 .

782, 786, 789

Potato Fern ... ... 638

Potato, Sweet... $\quad \ldots .347$

$\begin{array}{lll}\text { Prairie Grass ... } & 633,771 \\ \text { Prickly Lettuce } & \text {... } & 277\end{array}$

$\begin{array}{llll}\text { Prickly Lettuce } & \text {... } & 277 \\ \text { Prickly } & 222\end{array}$
Page.

Prickly Pear, Fungus on 775, 776, 778

Prickly Poppy ... 33

Prickly Thistle ... 411

Prolapsis ani of Chil-

dren, remedy for 202, 258

Prussic Acid in

Queensland plants 3

Pudding Gum ... 200

Pudginjacker $\quad$... 208

Puffball, Stalked ... 750

Pulkun $\quad$... $\quad \ldots .112$

Pumplin $\quad \ldots \quad 221,781$

Punburra $\quad$... $\quad$... 119

$\begin{array}{llll}\text { Pungga } & \ldots & \ldots & 119 \\ & & \ldots & 566\end{array}$

$\begin{array}{lllll}\text { Punk } & \ldots & \ldots & \ldots & 732\end{array}$

Pun-ti-li $\quad$... $\quad \ldots 559$

Purgative $\quad$ 143, 151,417

Purging Nut ... ... 478

$\begin{array}{llll}\text { Purpletop } & \ldots & \ldots & 382\end{array}$

Purslane $\quad . . . \quad . . .48$

Putrescent Liquids,

Fungus in ... ... 789

Quaking Grass _.. 633

Quandong, Brisbane 67

Queensland Beech ... 386

Queensland Bramble 167

Queensland Ebony ... 153

Queensland Kauri

Pine ... ... $\quad . .5510$

Queensland Nut $\ldots . .4440$

Quince $\quad \ldots . \quad \ldots .6167$

Quinine $\quad \ldots . \quad \ldots c 318$

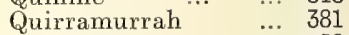

Quootham $\quad . . \quad \ldots . \quad 58$

Rabbits, said to be refused. by ... .... 476

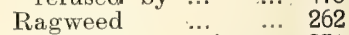

Ragweed, American 271

Rancootan ... ... 124

$\begin{array}{llll}\text { Ran-na } & \ldots & \ldots & 167\end{array}$

$\begin{array}{lllll}\text { Rape } & . . & \ldots & \ldots & 34\end{array}$

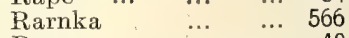

Rarum 40

Raspberry, Queens-

land ... $\ldots . \quad \ldots-167$

Rat's-tail Grass _... 623

Rattle-pods ... $\quad$... 132

Rdai-ite . ... $\quad$... 387

Red Apple ... ... 209

Red Ash ... ... 100

Red Beech, Herberton 91

Red Beech, John-

stone River $\quad$... 62

Red Beet ... ... 771

Red Bottle-brush ... 187

Red Cabbage Fern ... 642

Red Cedar ... ... 88

Red-cup Moss ${ } \quad$... 684

Red Finger Lime ... 80

Red Gulf Grass ... 620

Red Gum ... ... 195

Redhead $\quad . . . \quad \ldots .335$

Red-ink plant ${ }^{\prime .} \quad$... 411

Red Mangrove 65, 175

Red Natal Grass ... 611

Red. Oak $\quad$... $\quad$... 454

Red Rot $\quad . . . \quad \ldots .7781$

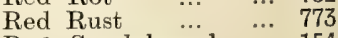

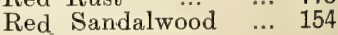


Page.

Red Sea-scum 831

Red Silky Oak … 455

Red Stringybark ... 200

Red Walnut ... ... 430

Red Water-moss ... 635

Redwood, Scrub ... 169

Reed, Common … 629

Rheumatism, Cure for 144

$\begin{array}{lll}\text { Rhodes Grass } \quad 194, & 504 \\ \text { Ribbon } & \cdots & 628 \\ & & 635\end{array}$

Ribbon Hern _... 635

Rib Grass .. ... 396

Rice Grass $\quad \ldots \quad \ldots 612$

Rice, Native ... ... 612

Rice-paper plant ... 236

Rickets plants 513

Ringworm, Cure for 151,

200,271

Ringy Rosewood

River Buttercup

River Mangrove

... 162

305, 756

$\begin{array}{llll}\text { River Poison-tree } & \ldots & 480 \\ \text { Roangga } & \ldots & \ldots & 200\end{array}$

Rokwara $\quad \ldots . \quad \ldots .154$

Roley-poley ... ... 409

Roman Wormwood ... 271

Rose of Jericho, Queensland … 364

Rosella, Native ... 58

Rosemary, Shrub ... 267

Rose-mildew ... 766, 782

Rose-plants, Fungi on 766 . $776,778,782$

Rose-prickles, Fungus

$\begin{array}{llll}\text { on } & \ldots & \ldots & 776 \\ \text { Rosewood } & \ldots & \ldots & 162\end{array}$

Rosewood … ‥ 162

$\begin{array}{llll}\text { Ru Rosewood, Ringy ... } & 142 \\ \text { Ru ... } & \ldots & \text {... } & 143\end{array}$

Rubber, Leaf " Disease of Para

Rubber-plants, Indigenous

Running Sores, Cure for $\quad . .6 \quad \ldots . \quad \ldots \quad 579$

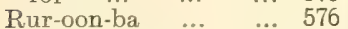

Rush Broom ... $\quad . . .128$

Russell River Grass... 602

Russian Thistle ... 411

Rusty Gum ... ... 188

Rye $\quad \ldots \quad \ldots \quad \ldots 772$

Rye Grass $\quad \ldots \quad$ 633, 786

Sacred Balm ... ... 387

Sacred Lotus ... ... 32

Saliva, Bark pro$\begin{array}{lll}\text { motes flow of } & \ldots & 77 \\ 277\end{array}$

Saltbush, Fungus on Fruit of ... ... 778

Saltbush, Old-man ... 408

Saltbushes $\quad \ldots \quad 404,408$

Salt, Plant encrusted with

Sandalwood, Bastard 375

Sandalwood, Red ... 154

Sandalwood, Scented 469

Sapistan ... ... 338

Saponin, contain 154,305,

Sarsaparilla, Bush-

$\begin{array}{ccr}\text { man's } & \ldots & 9,143 \\ \text { Sassafras } & \ldots & 419,431\end{array}$
Page.

Satinwood. Cairns ... 88

Satin-top Grass ... 619

Scabies, Cure for ... 148

Scented Golden-beard 620

Scented Sandalwood 469

Sciatica, supposed

Cure for ... ... 470

Scorpion-pod ... ... 139

Scotch Thistle

(Queensland) ... 276

Scours, Cure for .... 193

Scour-weed $\quad . . \quad \ldots 542$

Scribbly Gum _.. 193

Screw Pine ... ... 576

Scrub Crab-apple ... 305

Scrub Poison-tree ... 480

Scrub Redwood ... 169

Scurvy, supposed

useful in ... ... 553

Scurvy Grass .... ... 561

Sea Blite 409

Sea-coast Laburnum 148

Sea Heath ... ... 44

Sea Lavender .... 300

Sea Lettuce ... ... 794

Sea-weed Fungus ... 768

Seed, Germination of 11

$\begin{array}{llll}\text { Self-heal } & \text {... } & \text {... } & 388\end{array}$

Sensitive Orchid ... 538

Sensitive Plant, Com-

mon ... ... ... 154

$\begin{array}{llll}\text { Serpentary } & \ldots & \ldots & 413\end{array}$

Sharp Dock ... ... 417

Shea Oak ... $\quad \ldots .505$

Sheep-poison ... $\quad$... 332

Sheep's Sorrel ... 417

Shell Fungus ... .... 741

Shepherd's-needle $\quad \ldots .229$

Shepherd's-purse $\quad$... 34

Shot Grass ... ... 604

Shot-hole Fungus 775,785

Shuttlecock Flower ... 215

Sida Weed ...

Silk-cotton tree $\quad . .661$

Silkwood ... 91

Silky Oak, Brown 453, 454

Silky Oak, Northern 454

Silky Oak, Red ... 455

Silky Oak, Southern 453

Silky Oak, White ... 454

Silver-cord Fungus ... 792

Silver-leaf Disease

(of Europe)... ... 741

Silver-spiked Grass ... 604

Silver-top Grass ... 617

Silver Wattle ... 158

Six-o'clock $\ldots . \ldots 358$

Skin-diseases, Cure for $\quad \ldots \quad 148,228$

Skull-cap, Queensland 388

Smartweed ... ... 412

Smooth-stemmed Ferntree

Smut, Corn ... $\quad$.... 769

Snake-bite, Cure for 112 ,

Snake-root, Australian f... ... 237

Snake-root of Commerce

Snake's-tongue Fe... 418

Snakewood ... ... 337

Sneezing, Causes ... 831
Page.

Snuff-plant ... ... 272

Solid Wood ... ... 85

Solomon Islands Indigo $\quad \ldots . \quad \ldots .139$ Soot Fungus ... ... 790

Sorrel, Sheep's _.. 417

Sorrel, Wood ... ... 73

Sour Cherry ... ... 209

Sour Grass ... ... 73

Spade Flower... ... 42

Spear Grass, Bunch 619

Spear Grass of

Somerset ... ... 616

Spear Grass, Tall ... 619

Spear Grass, Threeawned

Spear-points, "Wood useful for ... 153, 159

Spearwood ... ... 162

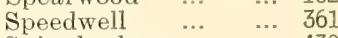

Spice-bark $\quad \ldots . \quad \ldots 430$

Spider Flower … 36

Spider Orchid $\quad \ldots 538$

$\begin{array}{llll}\text { Spiderwort } & \text {... } & \text {... } & 565\end{array}$

Spike Rush ... ... 591

Spinach, Substitute

for $\quad \ldots \quad$... 48

Spinifex Grass $\quad$... 629

Spinifex Shrub ... 262

$\begin{array}{llll}\text { Spleenwort } & \ldots & \ldots & 644\end{array}$

Sports $\quad \ldots . \quad \ldots \quad 5$

Spotted Gum ... . ... 201

Spotted Lily ... ... 526

Spotted Medick Burr 133

Spotted tree ... $\quad \ldots \quad 91$

Spurious Pareira

Brava plant $\quad \ldots .29$

$\begin{array}{llll}\text { Spurry } & \ldots & \ldots & 48\end{array}$

Squirting Cueumber, indigenous ... ... 222

Squirting Cucumber,

introduced $\quad . .2221$

Stagger-weed $\quad \ldots .388$

Stag's-horn Fern ... 649

Stanthorpe Box ... 195

Star of Bethlehem ... 349

Star Burr ... ... 271

Star of Jerusalem ... 277

Star Fungus ... ... 751

Star Grass ... ... 627

Star Grass, Blue ... 627

Stavewood ... ... 62

St. Barnaby's Thistle 276

Step Palm ... ... 573

Steven's Caustic-plant 473

Stinging-tree, Gym-

pie ... ... ... 505

Stinging-tree, ' Large 505

Stinging-tree, shin-

ing leaf ... ... 505

Stings of Insects, Cure

for $\quad \ldots \quad \ldots 346$

Stink Grass …..... 849

Stinkhorn Fungus ... 745

Stinking Rodger ... 272

St. John's Wort ... 49

Stone Fungus ... ... 728

$\begin{array}{llll}\text { Stoneworts } & \ldots & \ldots & 678\end{array}$

Stork's-bill $\quad \ldots \quad \ldots .72$

Strasburg Turpentine,

substitute for … 202

Strawberry Blight 765, 775 . 


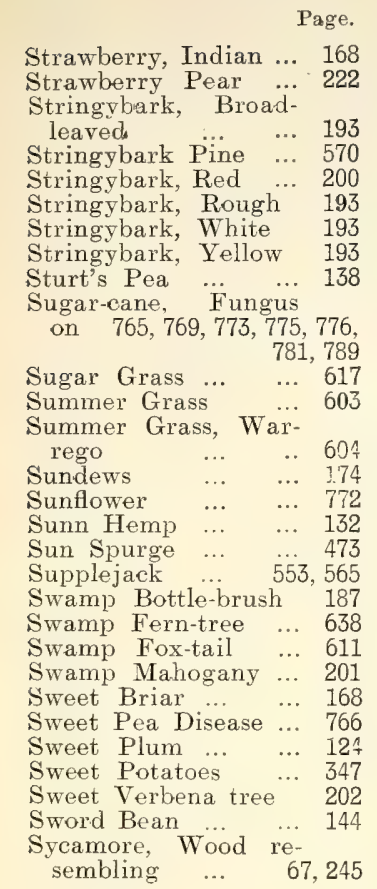

$\begin{array}{lccr}\text { Ta-anji } & \ldots & \ldots & 386 \\ \text { Tacamahac } & \ldots & \ldots & 54 \\ \text { Tacannapoon } & \ldots & \ldots & 573 \\ \text { Tagon Tagon } & \ldots & \ldots & 387 \\ \text { Tai-gam } & \ldots & \ldots & 574 \\ \text { Takking } & \ldots & \ldots & 112 \\ \text { Tallow-wood } & \ldots & \ldots & 183 \\ \text { Talwalpin } & \ldots & \ldots & 61 \\ \text { Tamarind, } & \text { Native } & \ldots & 112 \\ \text { Tandaji } & \ldots & \ldots & 144 \\ \text { Tandi } & \ldots & \ldots & 542 \\ \text { Tandoor } & \ldots & \ldots & 194 \\ \text { Tangber } & \ldots & \ldots & 469 \\ \text { Tang-gul } & \ldots & \ldots & 412 \\ \text { Tannanolen } & \ldots & \ldots & 180 \\ \text { Tannin, Rich } & \text { in } & 154 & 175 \\ \text { Tantoon } & \ldots & \ldots & 186 \\ \text { Tape Fern } & \ldots & \ldots & 640 \\ \text { Tape Grass } & \ldots & \ldots & 518 \\ \text { Tara } & \ldots & \ldots & 579 \\ \text { Ta-ra } & \ldots & \ldots & 163 \\ \text { Tarampa } & \ldots & \ldots & 112 \\ \text { Tarbugai } & \ldots & \ldots & 209 \\ \text { Tares of } & \text { Scripture } & \ldots & 633 \\ \text { Tar-kal } & \ldots & \ldots & 478 \\ \text { Tarm } & \ldots & \ldots & 548 \\ \text { Taro } & \ldots & \ldots & 579 \\ \text { Taromeo } & \ldots & \ldots & 62 \\ \text { Taroom } & \ldots & \ldots & 40 \\ \text { Tarpoon } & \ldots & \ldots 39 \\ \text { Tasselled } & \text { Blue } & \text { Grass } & 619 \\ \text { Tasselled Clubo-moss } \ldots & 634 \\ \text { Tchagum } & \ldots & \ldots & 566 \\ \text { Tchalli } & \ldots & \ldots & 518 \\ \text { Tchaln-ji } & \ldots & \ldots & 66 \\ \text { Tchannan } & \ldots & \ldots & 96 \\ \text { Tcheergum } & \ldots & \ldots & 193 \\ & & & \end{array}$

Page.

Tcherda

Tchilgar

$\begin{array}{llll}\text { Tchoomeroo } & \ldots & \ldots & 455 \\ \end{array}$

$\begin{array}{llll}\text { Tchuckah } & \ldots & \ldots & 566\end{array}$

$\begin{array}{llll}\text { Tchumba } & \ldots & \ldots & 200\end{array}$

$\begin{array}{llll}\text { Tchungooin } & \ldots & \ldots & 645\end{array}$

$\begin{array}{llll}\text { Tchuntchee } & \text {... } & \text {.. } & 387\end{array}$

Teak, Evelyn .... ... 54

Teak, Johnstone

River

$\begin{array}{cccc}\text { the } & \ldots & \ldots & 708 \\ \text { Tea-tree, Broad-leaf } & 186\end{array}$

Tea-tree of Captain

Cook's voyages ... 185

Tea-tree, Fungus on

Leaves of ... 761

Tea-tree, Paper-

barked $\quad \ldots \quad \ldots 188$

$\begin{array}{llll}\text { Tecan } & \ldots & \ldots & 188 \\ & \ldots & \ldots & 118\end{array}$

$\begin{array}{llll}\text { Tee } & \ldots & \ldots & 118 \\ & \ldots & \ldots & 193\end{array}$

$\begin{array}{llll}\text { Tee-doo } & \ldots & \ldots & 187\end{array}$

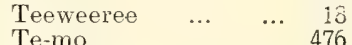

Téosinté $\quad$... ... 616

Teredo, Wood said to $\begin{array}{llll}\text { resist the } & \ldots & \ldots & 163\end{array}$

Terra Japonica _... 237

Texas Blue Grass ... 633

Thal-ango-thera $\quad \ldots \quad 77$

$\begin{array}{llll}\text { Thalmera } & \ldots & \ldots & 153\end{array}$

$\begin{array}{llll}\text { Thandorah } & \ldots & \ldots & 700\end{array}$

$\begin{array}{llll}\text { Thankoin } & \ldots & \ldots & 306\end{array}$

Thao Jelly $\quad \ldots . . \quad$.. 823

$\begin{array}{llll}\text { Thappin } & \ldots & \ldots & 460\end{array}$

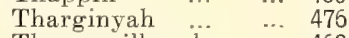

Tharra-gilberah _... 469

$\begin{array}{llll}\text { Thil-la-wo } & \ldots & \ldots & 505\end{array}$

Thistle, Plumed … 276

Thistle, Scotch (Queensland) $\quad \ldots \quad 276$

Thistle, Slender $\quad \ldots 276$

Thistle, Sow ... ... 277

Thistle, Spear ... 276

Thistle, Star ... ... 276

Thistle, St. Barnaby's 276

Thistle, Virgin Mary's 276

$\begin{array}{llll}\text { Thoogeer } & \ldots & \ldots & 40\end{array}$

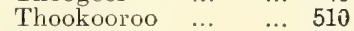

$\begin{array}{llll}\text { Thooromia } & \ldots & \ldots & 326\end{array}$

Thorn Apple .... ... 358

Thorny Acacia of Brisbane $\quad \ldots \quad \ldots \quad \ldots 151$

$\begin{array}{lllll}\text { Thorow-wax } & \ldots & \ldots & 229\end{array}$

$\begin{array}{llll}\text { Thow-i-ee } & \ldots & \ldots & 164 \\ \text { Thozet's Box } & \ldots & \ldots & 195\end{array}$

Three-awned Spear

Grasses $\quad \ldots \quad \ldots \quad 622$

Thukouro $\quad \ldots . \quad \ldots \quad 48$

$\begin{array}{llll}\text { Thurkoo } & \ldots & \ldots & 386\end{array}$

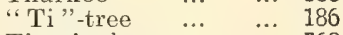

$\begin{array}{llll}\text { Tiger's-claw } & \ldots & \ldots & 368\end{array}$

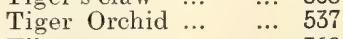

$\begin{array}{llll}\text { Til } & \ldots & \ldots & 368\end{array}$

Tilgul $\quad \ldots . \quad \ldots .486$

$\begin{array}{llll}\text { Till } & \ldots & \ldots & 486 \\ & \ldots & \ldots & 431\end{array}$

Timothy Grass, Californian $\quad$... $\quad . . \quad 622$

$\begin{array}{llll}\text { Tinda-burra } & \ldots & \ldots & 140 \\ & \ldots & & \end{array}$

$\begin{array}{llll}\text { Tindil } & \ldots & \ldots & 610\end{array}$

Toad Flax $\quad \ldots .3 . \quad \ldots .360$
Page.

Tobacco, Fungus on Leaves of ... 768, 785

Tobacco-pipe Fungus 743

Tobacco-pipe Gum-

tree $\quad \ldots . \quad \ldots \quad 195$

Tobacco-pipes, Fruit $\begin{array}{llll}\text { used for } & \text {... } & \text {... } & 195\end{array}$

Tobacco-plant _.. 358

Tobacco-tree ... $\quad \ldots \quad 358$

$\begin{array}{llll}\text { To-choon } & \ldots & \ldots & 539 \\ & & \ldots & \end{array}$

To-e $\quad \ldots . \quad \ldots .144$

Toeah $\quad \ldots .3 . \ldots .380$

Togoi $\quad \ldots . \ldots 5510$

Toi . ... ... 193

Toka $\quad \ldots . \quad \ldots \quad 237$

Tomato $\quad 353,769,779,785$

Tomato, Tree... ... 357

Tom-min $\quad \ldots \quad \ldots .180$

Tom Russell's Ma$\begin{array}{llll}\text { hogany } & \ldots & \ldots & 202\end{array}$

Tonic, a useful ... 418

$\begin{array}{llll}\text { Tonic-herb } & \ldots & \ldots & 337\end{array}$

$\begin{array}{llll}\text { Toolbar } & \ldots & \ldots & 124\end{array}$

$\begin{array}{llll}\text { Tooloo } & \ldots & \ldots & 159\end{array}$

Top-kie $\quad \ldots . \quad \ldots .477$

$\begin{array}{llll}\text { Torakal } & \ldots & \ldots & 177\end{array}$

Tor-chun _.. $\quad \ldots .539$

Towel Gourd ... $\quad \ldots .221$

$\begin{array}{llll}\text { Towra } & \ldots & \ldots & 77\end{array}$

Traveller's Joy _.. 17

Treacle Mustard ... 33

Tree, Fern ... ... 640

Tree of Heaven $\quad$... 82

Tree, Swamp Fern ... 638

Tree Tobacco ... ... 358

$\begin{array}{llll}\text { Tree Tomato .... } & \ldots & 357\end{array}$

$\begin{array}{llll}\text { Treine } & \ldots & \ldots & 96\end{array}$

Tronganongan ... 477

Troublesome in Boredrains $\quad \ldots \quad \ldots 847$

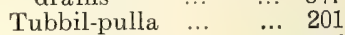

Tuckeroo $\quad \ldots . \quad \ldots .113$

Tuggan Tuggan … 453

$\begin{array}{llll}\text { Tulip-flower } & \ldots & \ldots & 454\end{array}$

Tulip-tree (Indian) ... 61

$\begin{array}{llll}\text { Tulipwood } & \text {... } & \ldots & 118\end{array}$

Tumkullum $\quad \ldots . \quad \ldots \quad 479$

$\begin{array}{llll}\text { Tunduli } & \ldots & \ldots & 486\end{array}$

$\begin{array}{llll}\text { Tun-jol } & \ldots & \ldots & 159\end{array}$

Turkey-bush $\quad \ldots \quad \ldots . \quad 152$

$\begin{array}{lll}\text { Turnip } & \cdots & 34,785\end{array}$

Turnipwood ... ... 118

Turpentine-tree $\quad \ldots .202$

$\begin{array}{llll}\text { Twill-ka } & \ldots & \ldots & 628\end{array}$

Twining Nettle ... 480

Uine $\quad \ldots . \quad \ldots \quad 535$

Ulcers, Cure for $\quad \dddot{8} 6,209$

$\begin{array}{llll}\text { Ulee-ree } & \text {... } & \ldots & 54\end{array}$

$\begin{array}{llll}\text { Ulorin } & \ldots & \ldots & 317\end{array}$

Umbrella Fern ... 638

Umbrella Handles,

Wood useful for ... 163

$\begin{array}{lll}\text { Umbrella-tree } & \ldots & 236\end{array}$

$\begin{array}{llll}\text { Undoour } & \ldots & \ldots & 306\end{array}$

$\begin{array}{llll}\text { Un-dor-a } & \ldots & \ldots & 543\end{array}$

$\begin{array}{llll}\text { Ung-ke } & \ldots & \ldots & 548 \\ \text { Und } & \ldots & \ldots & 548\end{array}$

$\begin{array}{llll}\text { Ungwe } & \ldots & \ldots & 477\end{array}$

Unicorn-plant $\quad \ldots 368$

$\begin{array}{lll}\text { Unit of Weight, a } & \ldots & 140\end{array}$

Unkeelyinkeela $\quad$.. 624

$\begin{array}{llll}\text { U-o-ba } & \ldots & \ldots & 543\end{array}$

$\begin{array}{llll}\text { Urar } & \ldots & \ldots & 543 \\ & & & \end{array}$ 
Page.

$\begin{array}{lcrr}\text { Uroka } & \ldots & \ldots & 88 \\ \text { Urooh } & \ldots & \ldots & 311 \\ \text { Urrgula } & \ldots & \ldots & 200 \\ \text { Urr-tee } & \ldots & \ldots & 162 \\ \text { Ura-ursi, } & \text { substitute } & \\ \text { for } & \ldots & \ldots & 186\end{array}$

Vegetable Dropsy ... 786

Venus's-comb 229

Vermifuge for Horses

Vervain 477, 510

Vinegar-plant .... 140

Violet, Native ... 40

Virgin's-bower $\quad$... 17

Waargoon-waargoon 208

Wackay ... ... 148

Wai-gen $\quad$... $\quad \ldots .139$

Wal-garri $\quad \ldots . \quad \ldots 573$

Walkaran $\quad \ldots .6208$

Walking-stick PaIm 573

Wall Barley ... ... 634

Wallflower Poison-

bush 128

Wall-paper, Fungus on 783

Wallum _... ... 455

Walno-jo $\quad \ldots .635$

Walnut, $\quad$ Black

Queensland ... ... 430

Walnut, Wood resem-

bling American ... 474

Wanakan ... ... 305

Wanchon $\quad \ldots .6208$

Wanga $\quad$.... 88,430

Wanjillo $\quad . . . \quad \ldots 576$

Wanna $\quad . .5 \quad \ldots 580$

Wannakai $\quad \ldots . \quad \ldots 207$

Wa-pan $\quad \ldots .65$

Wappo-wappo $\quad . . .112$

War-booga $\quad \ldots \quad \ldots \quad 332$

Warra-anji $\quad . .2 \quad \ldots .245$

Warrego Spinifex ... 629

Warrego Summer Grass $\quad$... ... 604

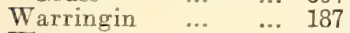

Warrew $\quad . . . \quad \ldots .164$

War-roon ... ... 163

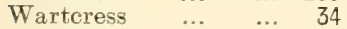

Washerman's plant of India

Water Couch .... ... 602

Watercress ... 33

Water Dropwozt ... 229

Water Fern ... ... 638

Water Foxtail Grass 623

Water Grass ... ... 612

Water Gum ... 208, 201

Water Horehound ... 388

Water Hyacinth ... 559

Water-lily, Blue … 32
Page.

Water-lily, Pink ... 32

Water-lily, Yellow ... 32

Water-lily, White ... 32

Water Melon 221, 766, 781

Water Milfoil ... 175

Water Moss, Red ... 635

Water Parsnip ... 229

Water Plantain ... 580

Water Purslane ... 214

Watershield ... ... 32

Water Starworts ... 175

Waterwort ... ... 49

$\begin{array}{llll}\text { Waxflower } & \text {... } & \text {... } & 332\end{array}$

Wedeely ... ... 318

Weeping Fig, Hill's 486

Weeping Love-grass 629

Weeping Myall ... 159

Weeping Tea-tree $\quad . . .188$

Weir 347

Whalebone-wood ... 241

Wheat ... 633, 773

Wheat, Blight on 766,771 ,

$\begin{array}{lr} & 772 \\ \text { Wheat-ear Fungus ... } & 787\end{array}$

Wheat Orchid $\quad . . .526$

White Apple ... ... 208

White Beech of Bunya

Mountains ... ... 67

White Bloodwood ... 201

White Cedar ... ... 86

White Fly Fungus ... 742

White Horehound ... 388

White Mangrove 387, 763

White Melilot ... 133

White Pine $\quad \ldots . \quad \ldots \quad 318$

White Rot $\ldots . .26 .228$

White Silky Oak ... 454

White-topped Grass ... 627

Whitewood ... 116, 368

Whitewood (Mowbu-

lan) $\quad \ldots \quad \ldots 236$

Wiepa $\quad \ldots . \quad \ldots .167$

Wig Fern tree $\quad \ldots .640$

$\begin{array}{llll}\text { Wi-ka } & \ldots & \ldots & 548\end{array}$

Wilda $\quad . .64 \quad \ldots 45$

Wild Oat $\quad . .6 \quad \ldots 627$

Wild Pea ... ... 140

Wild Radish ... ... 36

Wild Rice $\quad \ldots .612$

$\begin{array}{llll}\text { Wilga } & \ldots & \ldots & 81\end{array}$

Willgar $\quad \ldots .40477$

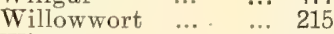

Wire-grass $\quad \ldots . \quad \ldots .412$

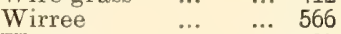

Wo-a $\quad$... $\quad$... 25

Wo-in-ya $\quad \ldots . \quad \ldots .6167$

$\begin{array}{llll}\text { Wokai } & \ldots & \ldots & \\ \text { Wot } & \text {... } & 548\end{array}$

Woller-Woller $\quad$... 187

$\begin{array}{llll}\text { Wonda } & \ldots & \ldots & 419\end{array}$

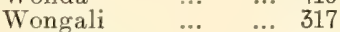

Wong-arrah ... $\quad$... 159

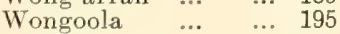

Wonkara $\quad \ldots .6200$
Page.

Wo-o $\quad$... $\quad \ldots \quad 504$

Wooden Pears .... 440

Woolah ... ... 67

Woolly Fern tree ... 640

Woollybutt ... ... 195

Woombun-Woombun 34

Woomerahs, Wood

used for ... 153, 159

Woonara ...

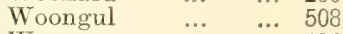

$\begin{array}{llll}\text { Woorgun } & \text {... } & \text {... } & 194\end{array}$

$\begin{array}{llll}\text { Wooroola } & \ldots & \ldots & 194\end{array}$

$\begin{array}{llll}\text { Woota } & \ldots & \ldots & \end{array}$

Worms in Horses, to

expel $\quad \ldots \quad 510,477$

Wormwood, Roman ... 271

Woundwort ... ... 388

Wunna Wunnarumpin 508

Wu-ri $\quad \ldots \quad \ldots \quad 570$

Wurri $\quad \ldots . \quad \ldots .54$

$\begin{array}{llll}\text { Wynnum } & \ldots & \ldots & 576\end{array}$

$\begin{array}{llll}\text { Yackaber } & \ldots & \ldots & 453\end{array}$

Yadthor $\quad$... $\quad$... 164

Yagul $\quad \ldots \quad \ldots 576$

Yak-ka-berry ... $\quad \ldots .624$

Yak-kapari $\quad . .6 \quad 623,624$

$\begin{array}{lll}\text { Yam } \quad \ldots & 332,548\end{array}$

Yamberin $\quad . . \quad \ldots 525$

Yam-vine of $\mathrm{Hugh}$ -

$\begin{array}{llll}\text { enden } & \text {... } & \text {... } & 29\end{array}$

$\begin{array}{llll}\text { Yapunyah } & \ldots & \ldots & 194\end{array}$

Ya-ra-ka $\quad$.. $\quad \ldots 628$

$\begin{array}{llll}\text { Yaroong } & \ldots & \ldots & \ldots \\ \text { Yar } & \ldots & 112\end{array}$

$\begin{array}{llll}\text { Yarra } & \ldots & \ldots & 195\end{array}$

Yar-ro-kal $\quad \ldots .6241$

Yedgengen $\quad \ldots \quad \ldots .249$

Yeenga $\quad \ldots \quad \ldots 535$

Yellow Distaff Thistle 276

Vellow Elderberry ... 236

Yellow-fruited Bram-

ble ... ... 167

Yellow-jacket 194, 195

Yellow Plum ... ... 91

Yellow Stringybark 193

Yellowtop $\quad . . . \quad \ldots . \quad 151$

Yellow Weed ... $\quad \ldots . .271$

Yellowwood 91, 202, 132

Yellowwood of Port

$\begin{array}{llll}\text { Curtis } & \text {... } & \text {... } & 132\end{array}$

$\begin{array}{llll}\text { Yerer } & \ldots & \ldots & 132 \\ \text { Yeroll } & \ldots & \ldots & 598\end{array}$

Yeroll $\quad \ldots . \quad \ldots .565$

$\begin{array}{llll}\text { Yikri } & \ldots & \ldots & 86\end{array}$

Yimbun $\quad \ldots . \quad \ldots \quad 576$

Yin-yin $\quad \ldots \quad \ldots 148$

Yoo-a-bal $\ldots . \quad \ldots .163$

Yorkshire Fog …6 627

$\begin{array}{llll}\text { Yuara } & \text {... } & \ldots & 543\end{array}$

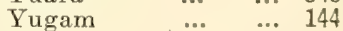

Yurol $\quad \ldots .6565$

Zula $\quad$... $\quad \ldots 519$ 




SMITHSONIAN INSTITUTION LIBRARIES 